UNITED STATES DEPARTMENT OF THE INTERIOR

GEOLOGICAL SURVEY

Phase Data Catalogue for IASPEI Events

by

Bruce W. Presgrave

U.S. Geological Survey

Denver, Colorado

Open-File Report 85-398

1985

This report is preliminary and has not been edited or reviewed for conformity with U.S. Geological Survey editorial standards and stratigraphic nomenclature. 


\title{
PHASE DATA CATALOGUE FOR IASPEI EVENTS
}

\author{
by Bruce W. Presgrave
}

\begin{abstract}
This report provides a catalogue of relocated hypocenters and a listing of reported phase arrivals for a series of 51 events chosen for discussion during a special session on the Analysis of Selected Earthquakes which will be convened by the Commission on Practice at the 1985 IASPEI General Assembly in Tokyo. The basic data set was obtained from the phase data tapes of the International Seismological Centre and from the Preliminary Determination of Epicenters Monthly Listing data base of the U.S. Geological Survey, National Earthquake Information Center (NEIC). To this data set, additional data were added from several sources, and the events were relocated using the standard programs used by NEIC to produce the Monthly Listings and Earthquake Data Report (EDR). The results are presented in standard PDE and EDR format, along with station distribution maps for each of the events.
\end{abstract}




\section{Introduction}

The purpose of this report is to provide a catalogue of relocated hypocenters and a listing of reported phase arrivals for a series of events chosen for discussion during a special session on the Analysis of Selected Earthquakes which will be convened by the Commission on Practice at the 1985 IASPEI General Assembly in Tokyo. This report is intended to be a companion report to the Waveform Catalogue for IASPEI Events by M. D. Zirbes and B. J. Moon (U.S. Geological Survey Open-File Report 85-218).

The goal of this special session is to provide a focus on modern scientific practice in the areas of earthquake quantification, data exchange, digital seismology, and algorithms. Participants have agreed to analyze a selection of fifty-one recent well-recorded world-wide earthquakes representing a range of sizes, locations, focal depths, and source characteristics.

\section{The Data Base}

The basic data set was obtained from the phase data tapes of the International Seismological Centre (ISC) for events in 1980 through 1982 and from the Preliminary Determination of Epicenters (PDE) Monthly Listing data base of the U.S. Geological Survey, National Earthquake Information Center (NEIC) for events in 1983 and 1984. Data were extracted from these data bases using the retrieval program FARSE, written by G. J. Dunphy of NEIC.

To this data set additional readings were added from several sources. Hypocenter information and much local and regional phase data were supplied by personnel of the U.S. Geological Survey, National Center for Earthquake Research, in Menlo Park for the earthquakes in California and Alaska. Data for the Columbia Bay, Alaska earthquakes of July 12 and September 7, 1983, were supplied by C.D. Stephens; data for the Eureka, California earthquake of November 8, 1980 and the Coalinga, California earthquake of May 2, 1983, were supplied by J.P. Eaton; and data for the Mammoth Lakes, California earthquake of May 25, 1980, were supplied by R.S. Cockerham. Local phase data from the Adak Network were supplied by E.R. Engdahl, of NEIC, for the Andreanof Islands, Alaska earthquake of June 9,1983 . In addition to the contributions listed above, additional phase data were entered from selected network bulletins for many of the events, especially those occurring in 1983 and 1984 for which the ISC data tapes were not yet available. Finally, additional phase data were read by the author from the NEIC U.S. Telemetered Network for 31 of the events, including all the events within or near the United States. Table 1 presents a summary of the number of stations reported for each event as well as the number used in each hypocenter computation by the NEIC PDE Monthly Listing, the ISC Bulletin and this report.

While checking network bulletins for additional data, it was discovered that the Japan Meteorological Agency (JMA) had determined that the 1ASPEI event of January 1, 1984 was indeed two events located in nearly the same place about 4 seconds apart. The PDE Monthly Listing had reported only one event at this time in the area. The conclusion of the JMA that there were really two events was confirmed by checking the data of the 
U.S. Telemetered Network for that time period. The main shock, which is really the event IASPEI intended to have on its list, has a magnitude of $6.6 m_{b}$ and is preceded by a foreshock of magnitude approximately $5.3 \mathrm{~m}_{b}$. In this study, both events were recomputed and published as an aid to interpreting the main event, even though the foreshock is not really part of the IASPEI event list.

\section{Recomputation}

All events in the list were recomputed using the standard programs which are included in the NEIC Seismic Data Analysis System (SEDAS) for routine publication of NEIC's Quick Epicenter Determinations (QED), PDE, PDE Monthly Listing and Earthquake Data Report (EDR). Hypocentral parameters for seven of the events were constrained to solutions furnished by agencies who have regional networks and/or special crustal models for the areas in which those events occurred. The seven events were the California and the Columbia Bay, Alaska earthquakes furnished by Stephens, Eaton and Cockerham, as listed previously; the Hawaii earthquake of November 16, 1983 supplied by the staff of the U.S. Geological Survey, Hawaiian Volcano Observatory; and the New Brunswick Canada earthquake of January 9, 1982 which was constrained to a special solution prepared by James Dewey of NEIC.

The remaining forty-five events were recomputed using generally standard procedures followed by NEIC geophysicists for publication of the routine bulletins. The revised event list of the IASPEI events is presented in standard PDE format in Table 3 and a listing of all phase data is given in standard EDR format in Table 4. In addition, a listing of the hypocenters and associated phase data is available on magnetic tape and may be obtained by interested organizations upon request to the National Earthquake Information Center. Detailed descriptions of procedures and symbols used in routine listings of the PDE and EDR may be found in the January and July issues of the PDE Monthly Listing, and each issue of the EDR. For convenience, summaries of these explanations are included at the end of Tables 3 and 4 .

In addition to the traditional PDE and EDR for these events, two special data presentations were prepared for this special study. Table 2 shows the size and orientation of the semi-major and semi-minor axes of the horizontal projection of the error ellipsoid and the marginal confidence interval for depth for each of the events. The values shown represent the 90-percent confidence levels for each parameter. As a visual aid to recognizing the station distribution pattern for each event, azimuthal equi-distant plots were made with vectors between the epicenter and each station associated to the event within 105 degrees of the epicenter. Stations used in the computation were plotted with a diamond symbol while stations associated but not used in the computation were designated by an " $\mathrm{x}$ ". To show distribution near the hypocenter, a second plot was made for each event for stations within 10 degrees of the event. In cases where no stations were within 10 degrees of the hypocenter, the regional plot uses a sufficiently large radius to show at least the closest station. For comparison, these special plots have a dashed circle plotted at the normal 10-degree radius. These plots are presented in Figures 1 through 26. 
TABLE 1

Number of Stations Used in Computation and Total Number Reported by PDE Monthly Listing. ISC Bulletin and This Publication

Event

1980

Jan 01

May 25

Jun 29

Jul 29

Oct 10

Oct 24

Nov 08

Nov 23

1981

Jan 18

Jan 23

Jul 06

Oct 28

Nov 22

Nov 27

1982

Jan 03

Jan 09

Aug 05

Sep 06

Dec 13

1983

Feb 13

Apr 03

Apr 04

Apr 11

Apr 18

May 02

May 26

Jun 01

Jun 02

Jun 09

Jun 21

Jun 24

Jul 12

Aug 06

Aug 17

Sep 07

Sep 12

Oct 04

Oct 09

Oct 17

Oct 22

Oct 30

Nov 16

Nov 24

Nov 30

Dec 22

Dec 30

1984

Jan 01

Jan 01

Feb 07

Mar 05

Mar 19

Mar 24
PDE Monthly
Used/Rep.

$16: 42: 40$

$16: 33: 44$

$07: 20: 07$

$14: 58: 41$

$12: 25: 23$

$14: 53: 34$

$10: 27: 33$

$18: 34: 53$

$18: 17: 26$

$21: 13: 48$

$03: 08: 34$

04:34:17

$15: 05: 22$

$17: 21: 45$

$14: 09: 50$

$12: 53: 51$

$20: 32: 48$

$01: 47: 03$

$09: 12: 48$

$01: 40: 11$

02:50:02

$02: 51: 34$

08:18:10

$10: 58: 48$

$23: 42: 37$

$02: 59: 59$

$01: 59: 54$

20:12:50

$18: 46: 02$

$06: 25: 26$

$09: 06: 45$

$15: 10: 04$

$15: 43: 52$

$10: 55: 54$

$19: 22: 05$

15: 42:08

$18: 52: 12$

$11: 25: 38$

$19: 36: 22$

$04: 21: 35$

$04: 12: 28$

16:13:00

$05: 30: 36$

$17: 46: 00$

$04: 11: 29$

23:52:39

$09: 03: 37$

$09: 03: 41$

$21: 33: 20$

$03: 33: 50$

$20: 28: 38$

$09: 43: 59$

$249 / 349$
$246 / 246 F$
$222 / 272$
$257 / 296$
$203 / 310$
$326 / 369$
$314 / 384$
$265 / 346$

$220 / 277$

$188 / 268$

$153 / 269$

$100 / 137$

$298 / 336$

$315 / 341$

$219 / 291$
$276 / 276 F$
$165 / 315$
$413 / 496$
$303 / 303 F$

$194 / 290$

$318 / 423$

$426 / 470$

$350 / 373$

$369 / 428$

$471 / 471 \mathrm{~F}$

$481 / 558$

$343 / 400$

$343 / 379$

$357 / 383$

$468 / 552$

$311 / 361$

$300 / 341$

$301 / 410$

$413 / 480$

$377 / 411$

$380 / 407$

$285 / 360$

$249 / 289$

$279 / 366$

$132 / 318$

$357 / 407$

$394 / 394 \mathrm{~F}$

$364 / 538$

$402 / 495$

$343 / 394$

$485 / 525$
ISC Bullet in
Reported *

432

355

507

451

514

510

510
506

513

379

510

272

469

606

412

391

456

676

419

Current List

Used/Rep.

$354 / 451$

$579 / 579 \quad F$

$436 / 506$

$383 / 449$

$430 / 514$

$457 / 540$

$828 / 828 \quad F$

$432 / 504$

$416 / 509$

$306 / 383$

$402 / 515$

$198 / 284$

$435 / 470$

$572 / 611$

$300 / 414$

$393 / 393 \mathrm{~F}$

$257 / 526$

$599 / 679$

$372 / 435$

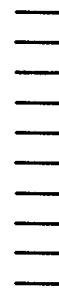

$283 / 389$

$370 / 512$

$494 / 543$

$414 / 449$

$420 / 487$

$569 / 569 \quad F$

$590 / 685$

$461 / 529$

$417 / 463$

$477 / 509$

$589 / 664$

$362 / 412$

$518 / 518 \quad F$

$385 / 492$

$530 / 591$

$534 / 534 F$

$455 / 491$

$348 / 442$

$331 / 390$

$372 / 447$

$145 / 385$

$406 / 463$

$474 / 474 F$

$414 / 588$

$439 / 525$

$412 / 465$

$536 / 580$

$346 / 367$

$432 / 456$

$376 / 548$

$600 / 693$

$486 / 575$

$518 / 658$

Notes:

* Since ISC uses a weighting scheme for its solutions, the number of stations used has no meaning. ISC data for 1983 and 1984 were not yet available when this report was in preparation.

F Furnished solution

nr This foreshock was not reported on the PDE Monthly. 


\section{Results}

The results of this data collection and recomputation effort are presented in Tables 2,3 and 4 and Figures 1 through 26 on the following pages. However, a few additional remarks appear to be in order. First, it is interesting to note the rather dramatic differences in the station distribution patterns for different events, even though each is large and recorded throughout the world. Although this is not unexpected because of the uneven distribution of land masses on the earth's surface, this clearly demonstrates that it is dangerous to assume that large events in different parts of the world will be equally well located. Of course, this effect is even more pronounced for events of smaller magnitude.

Second, it should be noted that for two events in the list, a procedure was followed which departs from standard PDE and EDR operations. Normally, the depth of the Santa Cruz Islands earthquake of August 5, 1982 would have been restrained by the geophysicist to some depth such as 10 or $33 \mathrm{~km}$, since the free depth was $5+/-23.5 \mathrm{~km}$. For this paper, however, it was decided that the free depth was more useful for interpreting the event though it was obviously poorly constrained.

Finally, the Banda Sea earthquake of Nov 24, 1983 has an $M_{s}$ magnitude listed despite the well-constrained depth of $199 \mathrm{~km}$. Normally, $M_{s}$ magnitudes are not listed for events whose depth (plus marginal confidence interval of depth, if applicable) is greater than $50 \mathrm{~km}$. This was done to draw attention to a difficulty observed by NEIC geophysicists on many occasions-namely, that the magnitudes which are traditionally reported for large intermediate and deep earthquakes grossly underestimate the true size of the event. This problem arises because the traditional short-period $m_{b}$ magnitude saturates at about magnitude 6.5 regardless of depth. It is also known that the uncorrected $M_{s}$ magnitude underestimates the true size of intermediate and deep earthquakes, but as shown by the Banda Sea event, this underestimate is considerably less than the underestimate caused by reporting only the $m_{b}$ magnitude. Therefore, until other magnitudes such as long-period $m_{b}$ or moment magnitude are reported routinely, perhaps the uncorrected $M_{s}$ magnitude is the best indicator available at the present time for the true size of large intermediate events, despite the limitations.

\section{Acknowledgements}

The author wishes to thank E.R. Engdahl, R.E. Needham and R.P. Buland for their many helpful suggestions with the analysis of the data and the preparation of the manuscript. The author also wishes to express thanks to the many scientists, technicians and communications personnel around the world who provided the basic seismogram interpretations without which this report, and indeed most studies of global seismology, would be impossible.

\section{References}

Zirbes, M.D. and B.J. Moon, Waveform Catalogue for IASPEI Events, U.S. Geological Survey, Open-File Report 85-218. 
TABLE 2

Size and Orientation of Semi-Major and Semi-Minor Axes of Horizontal Projection of Error Ellipsoid and Marginal Confidence Interval of Depth for IASPEI Events

\begin{tabular}{|c|c|c|c|c|c|c|}
\hline \multicolumn{2}{|c|}{ Event } & \multicolumn{2}{|c|}{$\begin{array}{cc}\text { Semi-Major Axis } \\
\text { Az } & \text { Size } \\
(\mathrm{deg}) & (\mathrm{km})\end{array}$} & $\begin{array}{c}\text { Sem } i-1 \\
\text { Az } \\
(\text { deg) }\end{array}$ & $\begin{array}{l}\text { Axis } \\
\text { Size } \\
(\mathrm{km})\end{array}$ & $\begin{array}{l}\text { Dep CI } \\
\text { Size } \\
(\mathrm{km})\end{array}$ \\
\hline $\begin{array}{l}1980 \\
\text { Jan } 01 \\
\text { May } 25 \\
\text { Jun } 29 \\
\text { Jul } 29 \\
\text { Oct } 10 \\
\text { Oct } 24 \\
\text { Nov } 08 \\
\text { Nov } 23\end{array}$ & $\begin{array}{l}16: 42: 40 \\
16: 33: 44 \\
07: 20: 07 \\
14: 58: 41 \\
12: 25: 23 \\
14: 53: 34 \\
10: 27: 33 \\
18: 34: 53\end{array}$ & $\begin{array}{c}167.7 \\
F \\
133.9 \\
26.8 \\
151.7 \\
210.7 \\
F \\
17.1\end{array}$ & $\begin{array}{l}4.7 \\
2.8 \\
4.0 \\
3.0 \\
3.6 \\
3.0\end{array}$ & $\begin{array}{r}77.7 \\
F \\
223.9 \\
116.8 \\
61.7 \\
120.7 \\
F \\
107.1\end{array}$ & $\begin{array}{l}2.4 \\
1.9 \\
2.2 \\
2.3 \\
3.0 \\
2.1\end{array}$ & $\begin{array}{l}G \\
F \\
1.9 \\
D \\
D \\
4.4 \\
F\end{array}$ \\
\hline $\begin{array}{l}1981 \\
\text { Jan } 18 \\
\text { Jan } 23 \\
\text { Jul } 06 \\
\text { Oct } 28 \\
\text { Nov } 22 \\
\text { Nov } 27\end{array}$ & $\begin{array}{l}18: 17: 26 \\
21: 13: 48 \\
03: 08: 34 \\
04: 34: 17 \\
15: 05: 22 \\
17: 21: 45\end{array}$ & $\begin{array}{r}110.1 \\
39.2 \\
203.2 \\
145.1 \\
72.4 \\
56.7\end{array}$ & $\begin{array}{l}4.5 \\
4.3 \\
4.0 \\
7.7 \\
3.6 \\
2.8\end{array}$ & $\begin{array}{r}200.1 \\
129.2 \\
113.2 \\
55.0 \\
162.4 \\
146.7\end{array}$ & $\begin{array}{l}2.8 \\
2.9 \\
3.6 \\
6.5 \\
2.5 \\
2.1\end{array}$ & $\begin{array}{l}4.2 \\
G \\
4.6 \\
G .8 \\
\vec{D}\end{array}$ \\
\hline $\begin{array}{l}1982 \\
\text { Jan } 03 \\
\text { Jan } 09 \\
\text { Aug } 05 \\
\text { Sep } 06 \\
\text { Dec } 13\end{array}$ & $\begin{array}{l}14: 09: 50 \\
12: 53: 51 \\
20: 32: 48 \\
01: 47: 03 \\
09: 12: 48\end{array}$ & $\begin{array}{c}148.7 \\
F \\
210.6 \\
117.3 \\
56.5\end{array}$ & $\begin{array}{l}5.1 \\
6.1 \\
3.4 \\
3.7\end{array}$ & $\begin{array}{l}58.7 \\
F \\
120.6 \\
207.3 \\
146.5\end{array}$ & $\begin{array}{l}3.2 \\
4.9 \\
2.8 \\
3.0\end{array}$ & $\begin{array}{r}\text { G } \\
F \\
23.5 \\
3.5 \\
3.8\end{array}$ \\
\hline $\begin{array}{ll}1983 \\
\text { Feb } & 13 \\
\text { Apr } & 03 \\
\text { Apr } & 04 \\
\text { Apr } & 11 \\
\text { Apr } & 18 \\
\text { May } & 02 \\
\text { May } & 26 \\
\text { Jun } & 01 \\
\text { Jun } & 02 \\
\text { Jun } & 09\end{array}$ & $\begin{array}{l}01: 40: 11 \\
02: 50: 02 \\
02: 51: 34 \\
08: 18: 10 \\
10: 58: 48 \\
23: 42: 37 \\
02: 59: 59 \\
01: 59: 54 \\
20: 12: 50 \\
18: 46: 02\end{array}$ & $\begin{array}{c}187.0 \\
223.5 \\
211.8 \\
160.5 \\
199.0 \\
F \\
121.4 \\
162.4 \\
68.0 \\
170.7\end{array}$ & $\begin{array}{l}4.6 \\
3.8 \\
3.6 \\
3.1 \\
3.8 \\
2.8 \\
3.9 \\
4.2 \\
4.0\end{array}$ & $\begin{array}{r}97.0 \\
133.5 \\
121.8 \\
70.5 \\
109.0 \\
F \\
211.4 \\
72.4 \\
158.0 \\
80.7\end{array}$ & $\begin{array}{l}3.1 \\
2.5 \\
2.8 \\
2.8 \\
2.3 \\
1.8 \\
2.9 \\
2.8 \\
2.1\end{array}$ & $\begin{array}{l}6.0 \\
4.0 \\
5.2 \\
4.3 \\
5.0 \\
F \\
3.1 \\
D \\
D \\
4.4\end{array}$ \\
\hline $\begin{array}{ll}\text { Jun } & 21 \\
\text { Jun } & 24 \\
\text { Jul } & 12 \\
\text { Aug } & 06 \\
\text { Aug } & 17 \\
\text { Sep } & 07 \\
\text { Sep } & 12 \\
\text { Oct } & 04 \\
\text { Oct } & 09 \\
\text { Oct } & 17\end{array}$ & $\begin{array}{l}06: 25: 26 \\
09: 06: 45 \\
15: 10: 04 \\
15: 43: 52 \\
10: 55: 54 \\
19: 22: 05 \\
15: 42: 08 \\
18: 52: 12 \\
11: 25: 38 \\
19: 36: 22\end{array}$ & $\begin{array}{r}105.9 \\
216.3 \\
F \\
21.8 \\
144.5 \\
F \\
19.1 \\
74.6 \\
77.6 \\
167.8\end{array}$ & $\begin{array}{l}2.4 \\
3.2 \\
3.2 \\
3.6 \\
3.1 \\
5.3 \\
5.0 \\
4.4\end{array}$ & $\begin{array}{r}15.9 \\
126.3 \\
F \\
111.8 \\
54.5 \\
F \\
109.1 \\
164.6 \\
167.6 \\
77.8\end{array}$ & $\begin{array}{l}1.7 \\
2.8 \\
2.0 \\
2.5 \\
2.0 \\
3.7 \\
3.3 \\
2.3\end{array}$ & $\begin{array}{l}G \\
3.5 \\
F \\
G \\
8.3 \\
F \\
D \\
3.6 \\
4.0 \\
D\end{array}$ \\
\hline $\begin{array}{ll}\text { Oct } & 22 \\
\text { Oct } & 30 \\
\text { Nov } & 16 \\
\text { Nov } & 24 \\
\text { Nov } & 30 \\
\text { Dec } & 22 \\
\text { Dec } & 30\end{array}$ & $\begin{array}{l}04: 21: 35 \\
04: 12: 28 \\
16: 13: 00 \\
05: 30: 36 \\
17: 46: 00 \\
04: 11: 29 \\
23: 52: 39\end{array}$ & $\begin{array}{r}29.2 \\
188.0 \\
F \\
66.1 \\
19.9 \\
142.1 \\
190.0\end{array}$ & $\begin{array}{l}8.5 \\
3.1 \\
4.5 \\
4.4 \\
3.8 \\
3.3\end{array}$ & $\begin{array}{r}119.2 \\
98.0 \\
F \\
156.1 \\
109.9 \\
52.1 \\
100.0\end{array}$ & $\begin{array}{l}5.9 \\
1.9 \\
3.5 \\
3.3 \\
2.6 \\
1.8\end{array}$ & $\begin{array}{c}D \\
3.6 \\
F \\
D \\
G \\
4.6 \\
2.6\end{array}$ \\
\hline $\begin{array}{l}1984 \\
\text { Jan } 01 \\
\text { Jan } 01 \\
\text { Feb } 07 \\
\text { Mar } 05 \\
\text { Mar } 19 \\
\text { Mar } 24\end{array}$ & $\begin{array}{l}09: 03: 37 \\
09: 03: 41 \\
21: 33: 20 \\
03: 33: 50 \\
20: 28: 38 \\
09: 43: 59\end{array}$ & $\begin{array}{r}141.4 \\
146.5 \\
37.3 \\
76.6 \\
10.1 \\
134.6\end{array}$ & $\begin{array}{l}3.9 \\
3.1 \\
4.8 \\
2.6 \\
3.2 \\
3.5\end{array}$ & $\begin{array}{r}51.4 \\
56.5 \\
127.3 \\
166.6 \\
100.1 \\
224.6\end{array}$ & $\begin{array}{l}2.4 \\
2.2 \\
3.8 \\
2.2 \\
1.6 \\
2.3\end{array}$ & $\begin{array}{l}1.5 \\
0.9 \\
D \\
0.9 \\
D \\
3.9\end{array}$ \\
\hline $\begin{array}{l}\text { tes: } \\
\text { F - } \\
D= \\
G=\end{array}$ & $\begin{array}{l}\text { restra } \\
\text { restra }\end{array}$ & r. & & & & \\
\hline
\end{tabular}


TABLE 3

PRELIMINARY DETERMINATION OF EPICENTERS

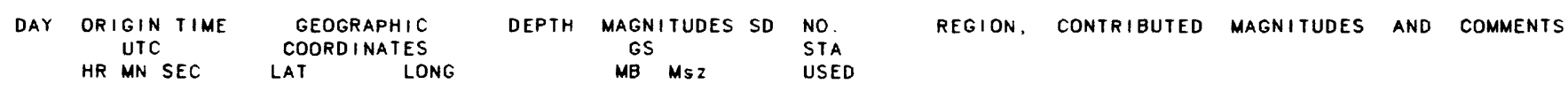

JAN 1980

$\begin{array}{llllllllllll}01 & 1642 & 40.0 & 38.790 \mathrm{~N} & 27.785 \mathrm{~W} & 10 & \mathrm{G} & 6.0 & 6.8 & 1.2 & 354\end{array}$

AZORES ISLANDS. At least 56 people killed, 400 or more injured ond extensive damage (VIII) on Terceira. At leost 4 people killed, some injured ond extensive domage on Son Jorge. Damage also reported on Graciosa.

MAY 1980

$\begin{array}{llllllllllll}25 & 16 & 33 & 44.1 \& & 37.598 & \mathrm{~N} & 118.829 \mathrm{~W} & 8 \mathrm{G} & 6.2 & 6.2\end{array}$

579 CALIFORNIA-NEVADA BORDER REGION. LGS>. SEVEn peOPle injured, damage ( $V \mid I)$ and rockslides in the Mammoth Lokes-Crowley Loke area, Collifornia. Rockslides olso occurred in Yosemite Notionol Park. Totol damoge estimated at 2 million dollars. Surface foulting and ground crocks observed in the epicentral oreo. Felt in an area of opproximately $272,000 \mathrm{sq}$. $\mathrm{km}$. Of Colifornia ond Nevodo.

JUN 1980

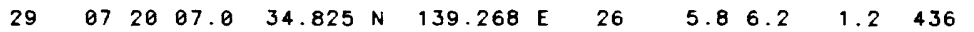

NEAR S. COAST OF HONSHU, JAPAN. Eight people injured and consideroble domoge ( $V$ JMA) and londslides on the izu Peninsula and on Oshima. Felt (IV JMA) at Kumagaya, Mishimo. Toteyoma, Tokyo ond Yokohoma; (111 JMA) at Chichibu, Choshi, I rozaki, Kowgouchi-ko, Kofu and Utsunomiyo; ( 11 JMA) at Gifu, Kakioka, Koruizawa, Maeboshi. Matsumota, Mito. Nagoya, Ononoma, Shizuoka ond on Hochijo-jimo. Locoi tsunomi generated in Sogami Boy. Maximum wave height (peok-to-trough) was $57 \mathrm{~cm}$. ot Okodo.

JUL 1980

$29 \quad 1458 \quad 41.0 \quad 29.656 \mathrm{~N} \quad 81.108 \mathrm{E} \quad 19 \mathrm{D} \quad 6.16 .6 \quad 1.0 \quad 383$

NEPAL One hundred fifty to 200 people killed, mony injured ond extensive damage in western Nepol. At leost $13 \mathrm{killed}, 40$ injured ond domage in the Pithorogarh orea, Indio. Felt strongly ot Kothmandu, Nepal and in the Delhi orea, India.

OCT 1980

$\begin{array}{llllllllllllll}10 & 12 & 25 & 23.8 & 36.213 \mathrm{~N} & 1.395 \mathrm{E} & 11 & \mathrm{D} & 6.3 & 7.2 & 1.2 & 430\end{array}$

ALGERIA. At least 5000 people killed, 9000 injured and extensive domage in the $E$ I Asnom oreo. Felt throughout northwestern Algerio and in southeostern Spain. Approximotely $42 \mathrm{~km}$. of surface rupture observed.

$\begin{array}{lllllllllllll}24 & 14 & 53 & 34.6 & 18.225 & \mathrm{~N} & 98.195 \mathrm{~W} & 67 & 6.5 & 6.9 & 1.1 & 457\end{array}$ CENTRAL MEXICO. At least 300 people reported killed. many injured, opproximotely 150,000 homeless and extensive domoge ( $I x$ ) in central Mexico, mainly in the Huajuapan de Leon areo Felt throughout central and southern Mexico ond in Guatemala

NOV 1980

$\begin{array}{llllllllllll}08 & 10 & 27 & 33.08 & 41.088 & \mathrm{~N} & 124.643 \mathrm{~W} & 13 \mathrm{G} & 6.2 & 7.3\end{array}$

828 NEAR COAST OF NORTHERN CALIF. LGS>. SIX peOPIE injured and damoge ( $V I I$ ) in the Fields Londing orea. One person injured ot Fortuna. Si ight domoge (VI) at many communities olong the coost from Rio Dell,

California to Brookings, Oregon. Totol damage estimated at $1.75 \mathrm{million}$ dollors. Smoll landslides and liquefaction occurred olong the Eel River ond the Big Lagoon sond spit. Felt in an oreo of approximately $97.000 \mathrm{sq}$. $\mathrm{km}$. from narthern Oregon to the Son Francisco Boy areo, as well as in ports of western Nevado.

$\begin{array}{llllllllllllll}23 & 18 & 34 & 53.2 & 46.884 & \mathrm{~N} & 15.325 \mathrm{E} & 8 & \mathrm{D} & 6.1 & 6.8 & 1.4 & 432\end{array}$ SOUTHERN ITALY. Over 3800 people killed, about 1900 missing, 7750 injured, 250,000 nomeless and extensive damage.

JAN 1981

$\begin{array}{llllllllllllll}18 & 18 & 17 & 26.0 & 38.649 \mathrm{~N} & 142.836 \mathrm{E} & 48 & 6.2 & 7.0 & 1.2 & 416\end{array}$

NEAR EAST COAST OF HONSHU, JAPAN. FEIT (IV JMA) Ot Miyoko, Sendai and Morioka; (III JMA) ot ofunato, Ishinomaki, Fukushima, Hachinohe, Onahama, Aki to. Shirakowo, Sakota and Utsunomiya; (1I JMA) at Urakawa, Maebashi, Tokyo. Tateyamo. Yokonomo and Kofu. Local tsunomi generated with moximum wave heights (peak to trough) $20 \mathrm{~cm}$. ot Urakawa and ofunato, $15 \mathrm{~cm}$. ot Aikawa, $14 \mathrm{~cm}$. at Hachinohe, $13 \mathrm{~cm}$. at $H i$ roo and $12 \mathrm{~cm}$. ot Miyako ond Orunoto.

$\begin{array}{llllllllllllll}23 & 21 & 13 & 48.1 & 30.967 & \mathrm{~N} & 101.134 & \mathrm{E} & 10 & \mathrm{G} & 5.8 & 6.8 & 1.2 & 306\end{array}$

JUL 1981

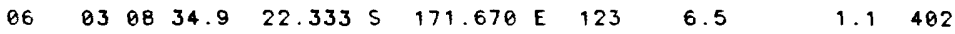

OCT 1981

$\begin{array}{lllllllllllllll}28 & 04 & 34 & 17.0 & 31.363 \mathrm{~S} & 110.694 \mathrm{~W} & 10 & \mathrm{G} & 6.1 & 6.3 & 1.3 & 198\end{array}$

$\begin{array}{lllllllllll}\text { NOV } & 1981 \\ 22 & 15 & 05 & 22.2 & 18.750 \mathrm{~N} & 120.829 \mathrm{E} & 36 & 6.3 & 6.5 & 1.1 & 435\end{array}$

$\begin{array}{lllllllllllll}27 & 17 & 21 & 45.3 & 42.877 & \mathrm{~N} & 131.247 & \mathrm{E} & 544 & \mathrm{D} & 5.8 & 1.2 & 572\end{array}$

JAN 1982

$\begin{array}{lllllllllllll}03 & 14 & 09 & 50.5 & 0.945 \mathrm{~S} & 21.877 \mathrm{~W} & 10 \mathrm{G} & 5.8 & 6.5 & 1.0 & 300\end{array}$

$09 \quad 125351.8 \& 46.984 \mathrm{~N} \quad 66.656 \mathrm{~W} \quad 10 \mathrm{G} \quad 5.85 .4$
SICHUAN PROVINCE, CHINA. ADOUt 150 peOple killed, 300

injured ond extensive damage in the Dawu orea.

LOYALTY ISLANDS REgION. FeIt (IV) at Noumeo and (11) ot Koumoc. New Coledonio. Proboble multiple event. Strong phose observed on mony stotions about 11 seconds after

\section{EASTER I SLAND REGION}

LUZON, PHILIPPINE ISLANDS. Domoge in the Bocarra-looog area. Felt throughout western Luzon.

E USSR-N.E. CHINA BORDER REG.
CENTRAL MID-ATLANTIC RIDGE

NEW BRUNSWICK. 〈SPEC>. SIight damage (VI) in ports of New Brunswick and ot Bridgewoter, Caribou, Eoston, 
AUG 1982

$\begin{array}{lllllllll}05 & 20 & 32 & 48.9 & 12.573 & 5 & 165.968 \text { E } & 5\end{array}$

SEP 1982

$\begin{array}{llllllllll}06 & 01 & 47 & 03.8 & 29.414 & \mathrm{~N} & 140.397 & \mathrm{E} & 185\end{array}$

6.07 .1

6.5

14257

DEC 1982

$\begin{array}{llllllllllll}13 & 09 & 12 & 48.5 & 14.707 \mathrm{~N} & 44.278 \mathrm{E} & 3 & 6.0 & 5.9 & 1.2 & 372\end{array}$

FEB 1983

APR 1983

$04 \quad 02 \quad 51 \quad 34.3 \quad 5.739 \quad \mathrm{~N}$

$\begin{array}{llllll}11 & 08 & 18 & 10.1 & 10.426 \mathrm{~N}\end{array}$

$62.775 w 40$

6.7

6.15 .9

1.1494

$1.1 \quad 414$

18

MAY 1983

$62 \quad 2342 \quad 37.7 \& \quad 36.219 \mathrm{~N} \quad 120.317 \mathrm{~W} \quad 10 \quad 6 \quad 6.36 .5$

26

$025959.4 \quad 40.465$ N 139.133 E 23

6.87 .8

1.2590

JUN 1983

\begin{tabular}{|c|c|c|c|c|c|c|c|c|c|c|c|c|c|}
\hline 1 & 01 & 59 & 54.6 & 17.035 & $s$ & 174.601 & $w$ & 180 & 0 & 6.3 & & 0.9 & 461 \\
\hline 02 & 20 & 12 & 50.2 & 9.501 & $\mathrm{~s}$ & 71.219 & $w$ & 593 & 0 & 5.9 & & 1.0 & 417 \\
\hline$\forall 9$ & 18 & 46 & 02.9 & 51.441 & N & 174.120 & $w$ & 34 & & 6.2 & 6.0 & 1.0 & 477 \\
\hline & 86 & 25 & 26.4 & 41.339 & $N$ & 139.085 & $E$ & 5 & G & 6.7 & 7.0 & 1.2 & 589 \\
\hline
\end{tabular}

$\begin{array}{lllllllll}21 & 06 & 25 & 26.4 & 41.339 & \mathrm{~N} & 139.085 & \mathrm{E}\end{array}$ $\begin{array}{lllllllllll}13 & 01 & 40 & 11.9 & 39.994 & \mathrm{~N} & 75.210 & \mathrm{E} & 20 & 5.7 & 6.3\end{array}$

Haynesvilte, Monticello. Presque isle. Dakfield and Stockholm. Moine. Felt in the Maritime Provinces, parts of Ouebec and throughout much of New England.

SANTA CRUZ ISLANOS

SOUTH OF HONSHU, JAPAN. Felt ( 111 JMA) ot Toteyama and Fukushimo. Felt (II JMA) on Chichi-jimo. Hochijo-jimo and Oshimo and ot Yokohama, Choshi, Tokyo, Kumagayo. Mi to, Utsunomiyo. Onahamo, Kakioka, Sendai, Morioko, Miyoko ond ofunoto.

WESTERN ARABIAN PENINSULA. Unconfirmed reports of 2800 people killed, 1500 injured, 700,000 homeless and obout 300 villages destroyed or bodly damaged in remen. Moximum intensity VIll in the Dawran-Risobah areo. Felt throughout Yemen and in the Najran areo, Soudi Arabia. Landslides occurred in the epicentral area, os well os extensionol ground crocks trending north-northwest in zones up to $15 \mathrm{~km}$. in length. This is the first instrumentally located hypocenter in the Ohamar region of remen.

SOUTHERN XINJIANG, CHINA. Several peaple injured and moderote damage in the Wuqia area. Felt (IV) in the Andizhon-Naryn area, USSR. Felt also (1II) ot Frunze and (11) of Tashkent, USSR.

COSTA RICA. Five people died from heort ottocks, one person killed by a collapsing house, and several people injured in southeastern Costa Rica. Also felt strongly in southwestern Panama.

NORTHERN SUMATERA. About 100 people injured ond domoge in the Bonda Aceh areo. Felt at Pinang. Moloysio.

NEAR COAST OF VENEZUELA. Felt (V) an Trinidod. Felt in the nor theastern Venezuela states of Sucre. Monogas, Anzoategui ond Bolivar. Also felt in the eostern suburbs of Corocas.

SOUTHERN IRAN. Felt strongly in southeostern Iron. Also felt of Korochi, Pakiston.

569 CENTRAL CALIFORNIA. <GS>. Forty-five people injured and estimoted $31 \mathrm{million}$ dollars damage in the Coalinga oreo. Maximum intensity Vill. Some damoge at Avenal and other surrounding communities. Felt from Los Angeles to Socramento and from Son Froncisco to Reno.

NEAR WEST COAST OF HONSHU, JAPAN. One hundred four people killed, 163 injured and extensive domage to dwellings, roods and vessels caused by earthquake and a tsunami along the Japan Seo coast from southern Hokkaido to the Ni igato areo. Honshu. Many of the cosuolties and much of the damage occurred on the ogo Peninsulo. Tsunomi damage occurred os for owoy os romoguchi Prefecture in southwestern Honshu. along the Jopan Seo coast of USSR, and along the eostern and southern coasts of South Korea, where 3 odditional people were killed. Felt (V JMA) ot Akito, Fukauro and Mutsu. Felt on Hokkaido and throughout northern and centrol Honshu. Estimated tsunami heights were $14 \mathrm{~m}$. ot Minehama, Honshu, 2-6 $\mathrm{m}$. along southern Hokkaido and northern Honshu, up $108 \mathrm{~m}$. olong the coost of USSR. and $4 \mathrm{~m}$. olong the coost of South korea.

TONGA ISLANDS. Felt (II) ot APIO, SOMOO Islands. PERU-BRAZIL BORDER REGION

ANOREANOF ISLANDS, ALEUTIAN IS. FEIt (III) on Adak.

HOKKAIDO, JAPAN REGION. SOme domoge in narthern Honshu. Felt (IV JMA) at Aomori. Mori. Fukaura and Esasni; ( 111 JMA) ot Hakodote, Akita, Muroran, Hachinohe, Morioka ond Mutsu; ( 11 JMA) at Suttsu. Kutchon, Otaru, Miyako, Urokowo, Sakato, Onahomo and Tomokomai. One meter tsunomi ot Akito, Noshiro and wokami. Fifty cm. tsunami reported in many oreos olong the west coast of northern Honshu.

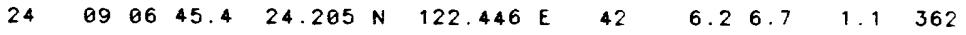

TAIWAN REGION. Felt on Toiwan. Felt ( $|1|$ JMA) on I riomote-jimo and ( 1 I JMA) on Ishigaki-shima and ronaguni-jimo, Ryukyu Islands.

JUL 1983

$\begin{array}{lllllllllllllll}12 & 15 & 10 & 04.38 & 61.028 \mathrm{~N} & 147.147 \mathrm{~W} & 18 & 6 & 6.36 & 4\end{array}$

Caloge (VI) at Valdez. Fel (V) at Chitino. Chugiak, Coaper Landing, Copper Center. Cordova, Fort Richardson, Gakono, Girdwood, Moose Pass. Skwentno, Sutton. Whititier and Willow. Felt (IV) at Anchorage, Polmer and Seward. Feit (iil) at Fairbanks ond rokutot. Also felt $(111)$ at Whitehorse, Yukon Territory.

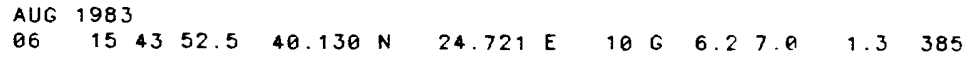

AEGEAN SEA. Slight damage ( $V I$ ) on Limnos and to four monasteries in the Mount Athos area. Also telt (VI) on Thasos and Lesvos. Felt (V) throughout much of nor thern Greece. Felt strongly in southern Bulgaria and nor thwestern Turkey.

NEAR EAST COAST OF KAMCHATKA. Felt $(I V-V)$ at Petropavlovsk-komchatskiy. 
SEP 1983

OCT 1983
28.137 E 199 D 6.57 .0
534
DEC 1983

$\begin{array}{lllllllllllll}22 & 04 & 11 & 29.0 & 11.862 \mathrm{~N} & 13.537 \mathrm{~W} & 10 & 6.4 & 6.3 & 1.0 & 412\end{array}$

\section{3}

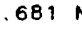

\section{6} Felt (V) ot Anchoroge, Chugiok. Copper Center, Cooper Londing. Homer, Moose Poss, Polmer, Soldotno, Sutton, Wasillo and Willow.

AFGHANISTAN-USSR BORDER REGION. SIight damage in the Koshmir Valley. Felt (V) at Khorog. Pyandzh, Shaartuz, Dust $i$ and Gissor; (IV-V) at Kulyab. Nurek, Dushanbe. Ordzhonikidzeabod, Tashkent, Garm, Ura-Tyube,

Pendzhikent and Leninobad; (IV) ot Ishkoshim, Rushan, Kalininabod, Kurgan-Tyube, Samorkand and Andizhon; (III-IV) ot Obigarm and Chimkent; (III) at Dzhirgotal and Noryn; $(1|-| \mid 1)$ at Dzhambul and Talgar; and (11) at Frunze, USSR. Also telt in the Peshowar-Lahare orea, Pakiston.

NEAR COAST OF NORTHERN CHILE. At leost 5 people killed, 24 injured and extensive damage (VIII) in the Copiopo-Chanorol areo. A 1.5 meter uplift near Chanorol wos reported and some roads were blocked by landslides. Minor tsunami recorded at valparaiso. Felt in Chile from lquique to Santiago. Felt in Argentino and at Lo Paz, Bolivio and Soo Paulo, Brazil. This oppears to be

NEAR COAST OF NORTHERN CHILE. Felt (V) ot Copiopo, (IV) ot Chonaral and $(111)$ in the Antofogasta orea. NORTH ATLANTIC OCEAN

SOUTH SANDWICH ISLANDS REGION

TURKEY. At leost 1342 people killed, 534 seriously injured, more thon 25,000 homeless and 50 villages completely destroyed in Kars and Erzurum Pravinces.

474 HAWAII. LHVO-P>. Six people injured and considerable domage ( $V I I I)$ in parts of Kopopolo, Hawai Volconoes National Park, Volcono, Kau and Puna districts and Hilo. Landslides and rockfolls occurred, telephone and electrical service disrupted and water mains broken in severol areas of the island. Total domoge estimated at 6 to 6.5 million dallors. Also felt on Moui, Oohu and KoUO i

BANDA SEA. Felt on Alor, Flares, Sumbo ond Timor. Also felt in Australia from Dorwin to Perth. Nate the large Ms mognitude for this event, despite the well-constroined depth of $199 \mathrm{~km}$

CHAGOS ARCHIPELAGO REGION. Some domage (VI) to buildings ond piers on Diego Gorcia. Five foot rise in wove height in the lagoon and significant wave domoge neor the southeostern tip of the island. Forty $\mathrm{cm}$. tsunami ot Victorio, Seychelles Large zone of discolored seo woter observed 35 to 40 nautical miles northwest of Diego Gorcio.

NORTHWEST AFRICA. At least 443 people killed, 200 reported missing. 150 seriously injured and extensive damage in the Gaoul-Koumbia oreo, Guineo. Felt in Guineo-Bissou. Senegal, The Gambio and Sierro Leone. HINDU KUSH REGION. Twelve people killed, 483 injured ond extensive domoge in the Kabul and Samangon oreas. Afghaniston. Faurteen people killed, hundreds injured and moderote domage in the Peshowor orea. Pokiston. Same damage (VII) in Tojikiston, USSR. Felt in much of northwestern Afghanistan, northern Pakistan, northern Indio and in Tajikistan, Uzbekiston ond Kirghizio, USSR.

NEAR S. COAST OF SOUTHERN HONSHU. Foreshock. This event was not included on the original IASPEl list, but is I isted here os on aid to interpreting the moin event which follows. The mognitude shown for this event may be high but the event can be seen cleorly on stations in the United States as for away os 90 degrees.

NEAR S. COAST OF SOUTHERN HONSHU. FeIt (IV JMA) at Toteyamo, Yokohamo, Tokyo, Utsunomi yo and Onahamo; ( I ) JMA) on Oshimo and of Chichibu, Kumagaya, Choshi, Mito, Shirokowo. Chiba, Kokioko, Nikko, Fukushima and Sokota. Felt from Hakkoido to northeostern Kyushu and on the Bonin Islands.

FEB 1984

MAR 1984

$\begin{array}{llllllllllll}05 & 03 & 33 & 50.8 & 8.148 & N & 123.775 & E & 646 & 6.6 & 1.0 & 600\end{array}$

$\begin{array}{lllllllllllllll}19 & 20 & 28 & 38.2 & 40.341 & \mathrm{~N} & 63.359 & \mathrm{E} & 15 & \mathrm{D} & 6.5 & 7.0 & 1.0 & 486\end{array}$
SOLOMON ISLANDS. Damage in southern Guadalcanal and some lands! ides reported. Felt throughout the Solomon Islands, (VI) at Haniaro and (III) ot Roboul, New Britoin

MINDANAO. PHILIPPINE ISLANDS. FeIt (II RF) on Mindanoo, Leyte and Ponoy.

UZBEK SSR. At leost 100 people injured and extensive domage $(I x)$ in the Gazli areo. Damage (VII-VIII) ot Dzhongeldy and ( $V I I$ ) in the Bukhoro oreo. Felt (VII) at Chardzhou; ( $V I)$ at Navai and Mary; (V-VI) ot Samorkand: (V) at Dzhizok ond Ashkhobod; (IV) ot Toshkent and uushanbe. ( $|1|-\mid V)$ ot Andizhan; (111) ot Leninabad. Uro-Tyube, Chimkent and Dzhambul: $(1|-| 1 \mid)$ at Kulyab. Khorog ond Frunze. Felt also at Moshhod. Iron. 
KUR:L ISLANDS. Fell (V) of Yuzhno-Kurilsk ond (IV) on Shikotan Feli ( 11 JMA) ot Nemuro, Obihiro and Urakawa, Hokkoido and at Hochinohe ond Miyako. Honshu. Fourteen $\mathrm{cm}$. Isunomi ot Nemuro. Hokkaido. Possible multiple event

EXPLANATION OF ABBREVIATIONS AND SYMBOLS APPEARING IN THIS TABLE

Abbreviotions in Heoding

MB - Body wove magnitudes.

Msz - Vertical surface wove magnitudes.

UTC - Coordinated Universal Time. HR MN SEC - Hour, minute, second.

SD - Standard Deviation from the arithmetic meon of residuols.

No. Sta. - Number of stations reporting $P$ or PKP phoses used in computation.

Symbols and Abbreviatians Used in Comments

AGS Alasko Seismic Project, U.S. Geological Survey, Menlo Park, Colitornio.

U.S. Geological Survey, Menlo Pork. California.

HVO Howaitan Volcano Observotary

JMA Japan Meteoralogical Agency (generally used to indicate 7-point Japanese Intensity Scole).

RF Rossi-forel Intensity Scole

SPEC An NEIS solution bosed on use of dense local networks, a lacal crustal model, or other methods not routinely applied in calculating the hypocenter parameters.

Roman Used to indicote intensity (when not followed by RF or JMA they refer to the Modified

Numerols Mercalli Scole or ony 12-point intensity scale closely related to it).

- " Geographic degrees, minutes, seconds.

-p Supplied hypocenter is a preliminory computotion.

Any additianol 3 to 5 letter codes enclosed in porentheses or ongle brackets refer to individual station codes. These cades may be found in Geologicol Survey Circular 791 , Seismegraph Statian cades and ChargeterisIiss (1978).

\section{Symbols Following Depth}

Symbols Following origin Time

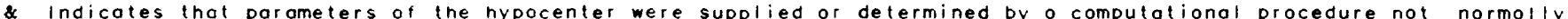
used by the Notional Eorthquake informotion Service (NEIS). The source or noture of the determinotion is indicated by a 2 to 5 letter cade enclosed by angle brackets and oppeoring in the first line of comments. A "-p" appended to the code indicates that the computation is preliminary. These codes are included with the list of abbreviations above

COMPARISON OF RATINGS OF INTENSITY SCALES APPEARING IN PRELIMINARY DETERMINATION OF EPICENTERS

U.S.A. Modified
Mercalli (M.M.), 1931

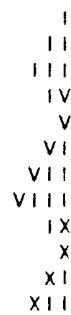

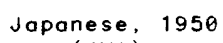

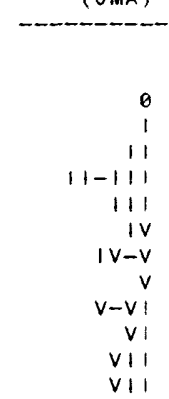

Rossi-Forel, 1873 (RF) 


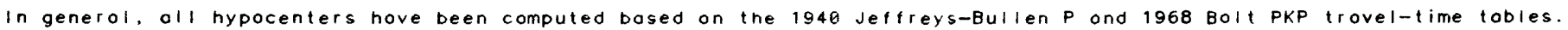

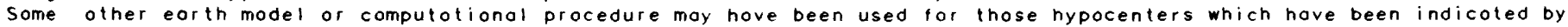
on ompersond ( $(2)$ following the origin time.

\section{NEIS MAGNITUDES}

All mognitudes ore NEIS mognitudes unless otherwise indicoted. Averoge mognitudes ore computed by o $25 \%$ trimmed meon os described by Rosenberger, J. L. ond Gasko. M.. 1983, "Comparing locotion estimotors: trimmed meons, medions, ond trimean" in Understonding Bobust ond Explorglery Rojo Anglysis, ed. Hooglin. D.C.,

Mosteller. F., ond Tukey. J.W., John Wiley, New Yark.

These surfoce wove mognitudes ore computed from the 1.A.S.P.E.I formulo:

$M S=\log (A / T)+1.66 \log D+3.3$

\section{where:}

A is the moximum ground omplitude in micrometers (microns) of the verticol component of the surfoce wove within the period ronge $18 \leq T \leq 22$.

$T$ is the period in seconds.

$D$ is the distonce in geocentric degrees (stotion to epicenter) ond $20^{\circ} \leq D^{\circ} \leq 160^{\circ}$

No depth corrections ore opplied, and Ms mognitudes ore not generolly computed tor depths greoter thon 50 $\mathrm{km}$. The Ms volue published is the overoge of the individual station mognitudes from reported $T$ ond A dota.

If the uncertointy of the computed depth is considered greot enough thot the depth could be less thon $50 \mathrm{~km}$. on MS volue moy still be published, computed by the 1.A.S.P.E.1. formula ond nes corrected for depth.

In generol. the Ms magnitude is more reliable than the MB mognitude os o meons of yielding the relative "size" of a shollow-tocus eorthquake.

These compressionol body wove (P-wave) magnitudes are camputed occording to the tormulo:

$M B=\log (A / T)+O(D, h)$

defined by Gutenberg ond Richter (1956) except that $T$, the period in seconds, is restricted to $0.1 \leq T \leq 3 . \theta$ ond $A$, the ground omplitude in micrometers, is not necessorily the maximum in the $P$ group. 0 is o tunction of distance (D) and depth (h) where $D \geq 5^{\circ}$.

\section{REFERENCES}

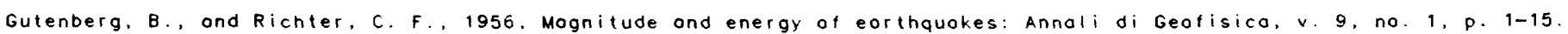

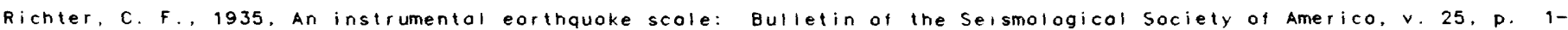
32 . 


\section{Figure 1}

JAN 01, 1980 16:42:40 AZORES ISLANDS 105 Degree Radius

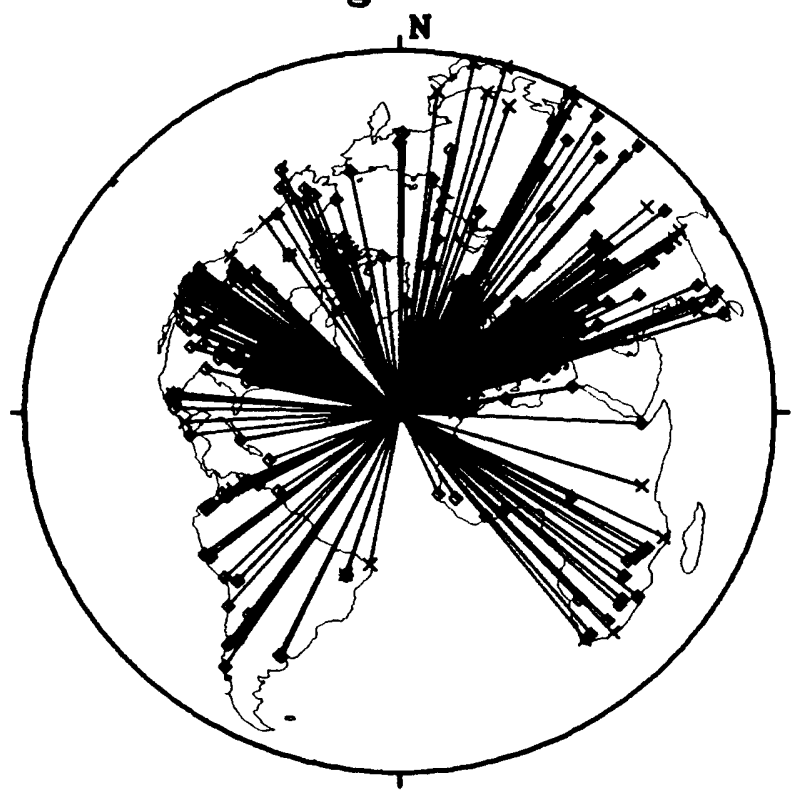

MAY 25, 1980 16:33:44

CALIFORNIA-NEVADA BORDER REGION 105 Degree Radius

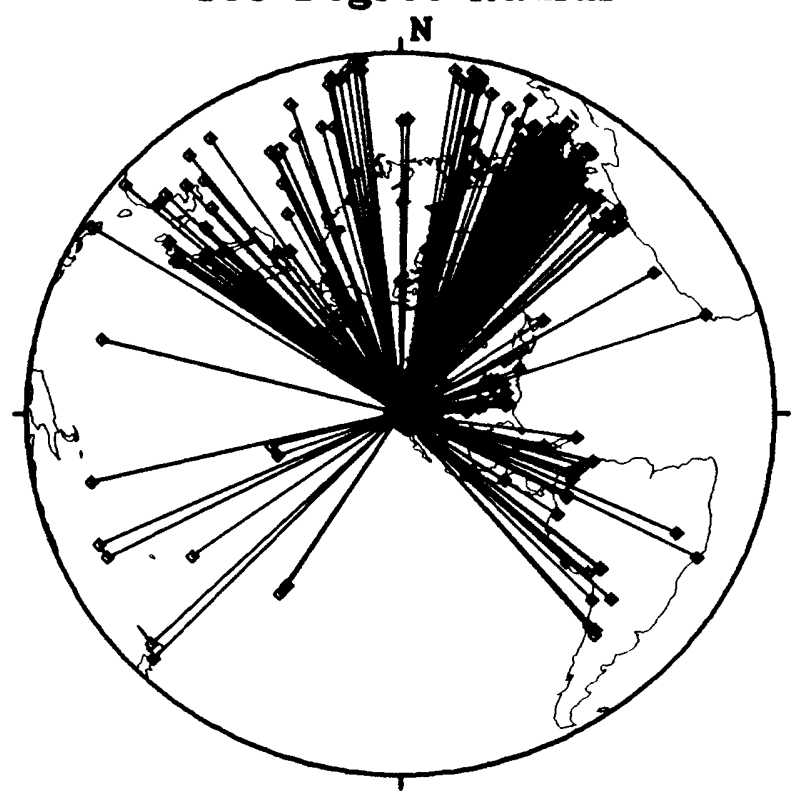

JAN 01, 1980 16:42:40 AZORES ISLANDS 10 Degree Radius

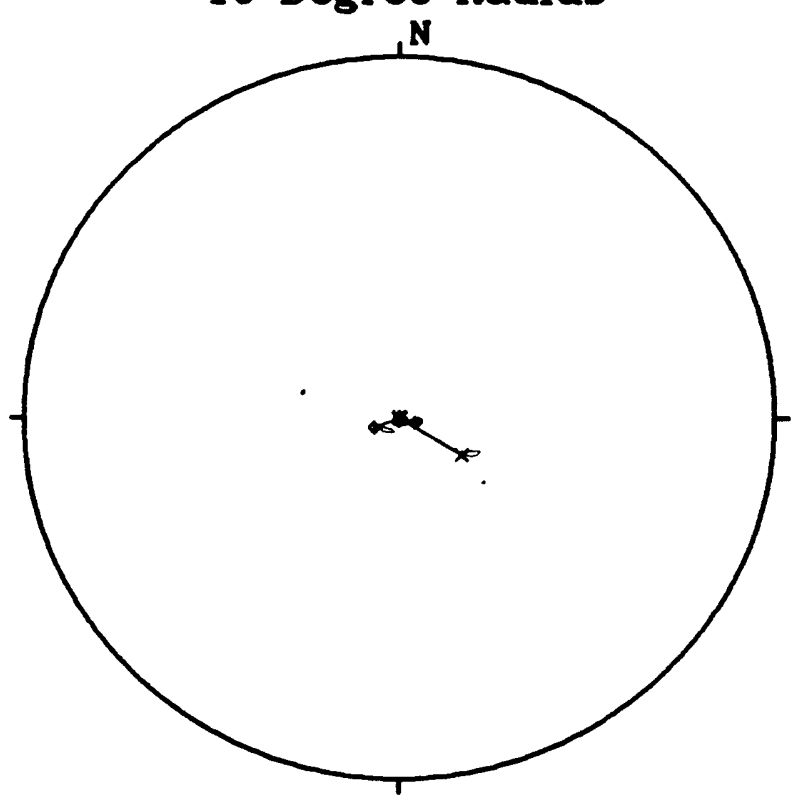

MAY 25, 1980 16:33:44

CALIFORNIA-NEVADA BORDER REGION 10 Degree Radius

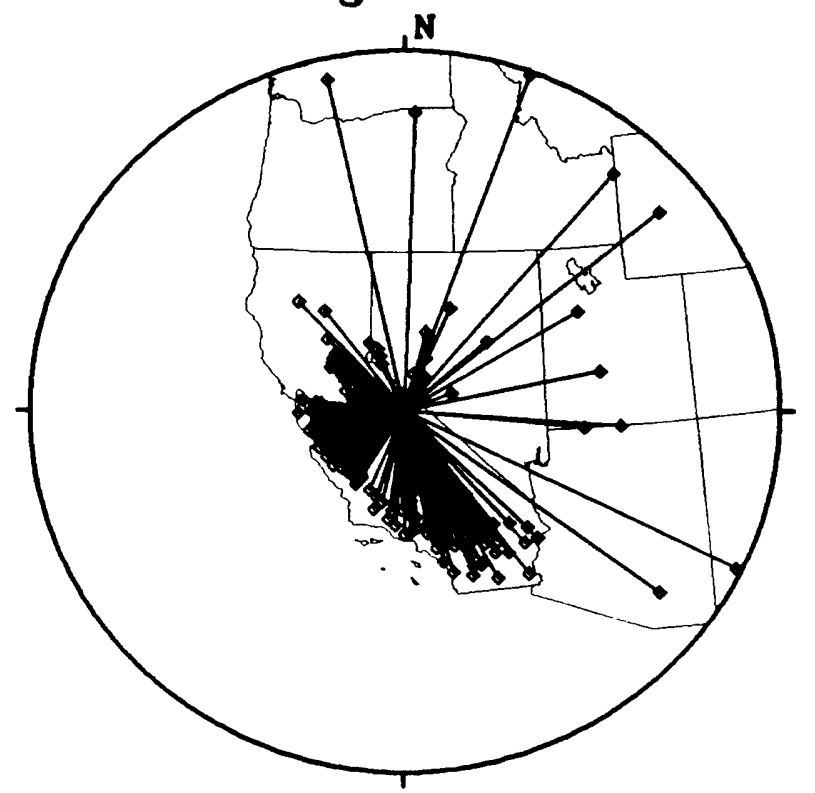


JUN 29, 1980 07:20:07

NEAR S. COAST OF HONSHU, JAPAN 105 Degree Radius

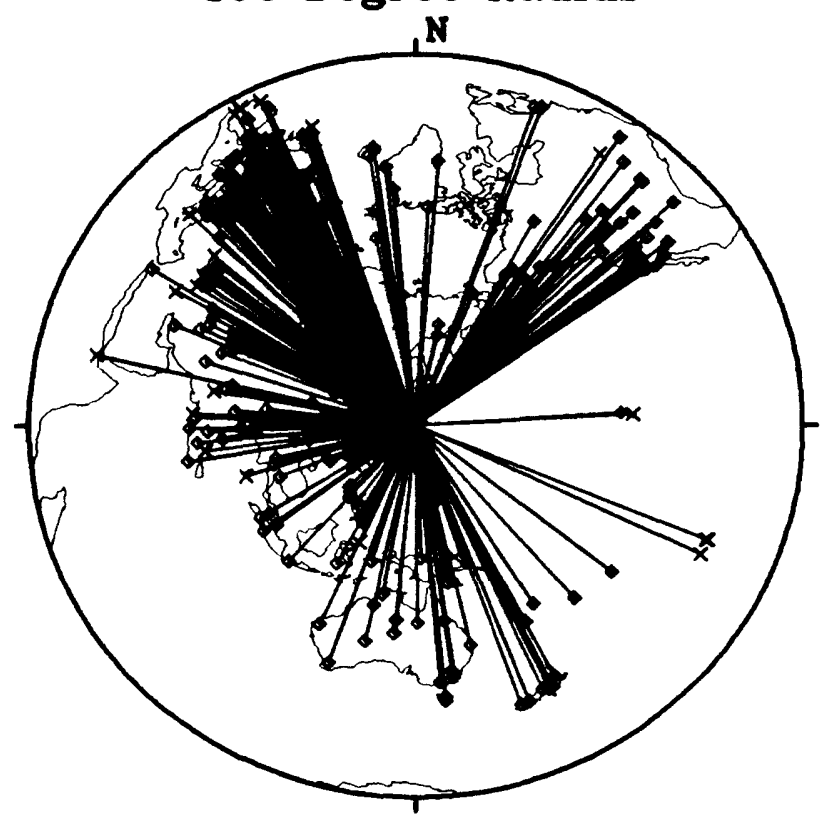

JUL 29, 1980 14:58:41 NEPAL

105 Degree Radius

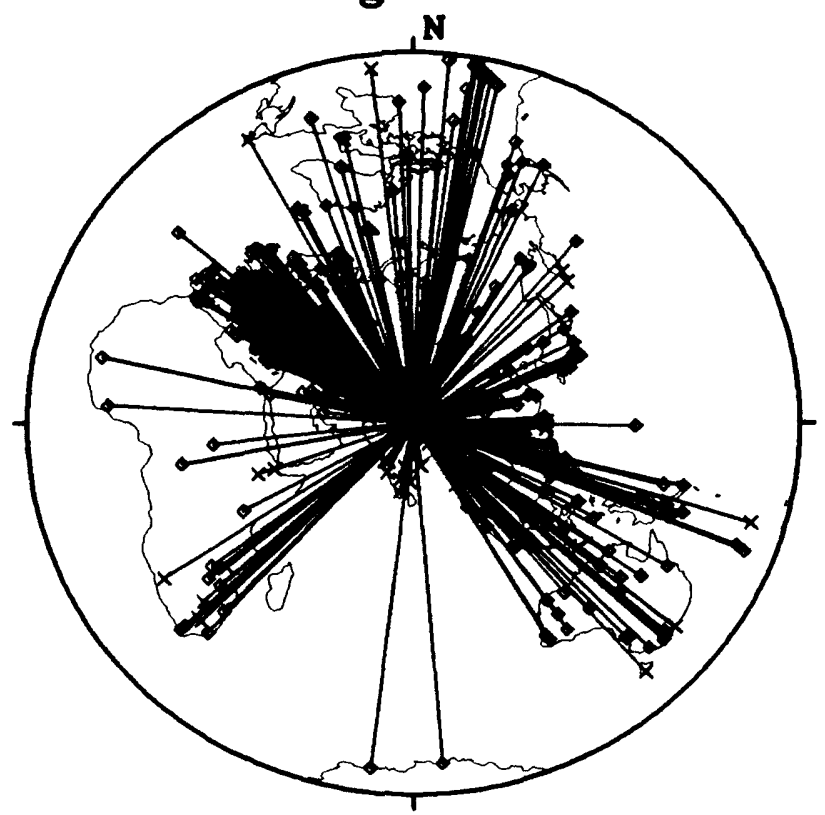

JUN 29, 1980 07:20:07

NEAR S. COAST OF HONSHU, JAPAN 10 Degree Radius

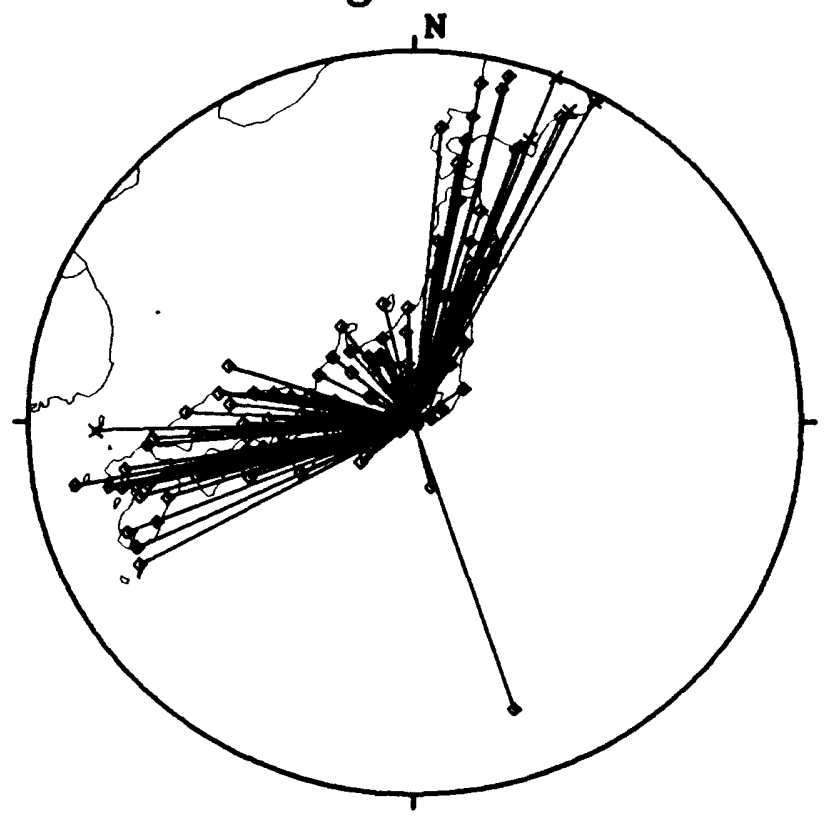

JUL 29, 1980 14:58:41 NEPAL

10 Degree Radius

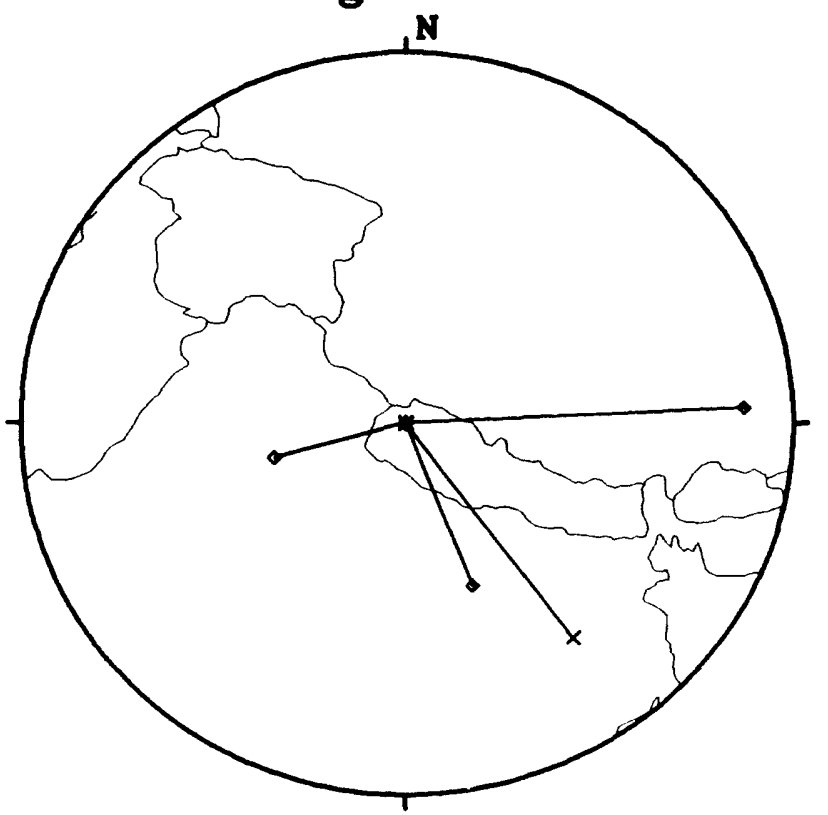


Figure 3

OCT 10, 1980 12:25:23

ALGERIA

105 Degree Radius

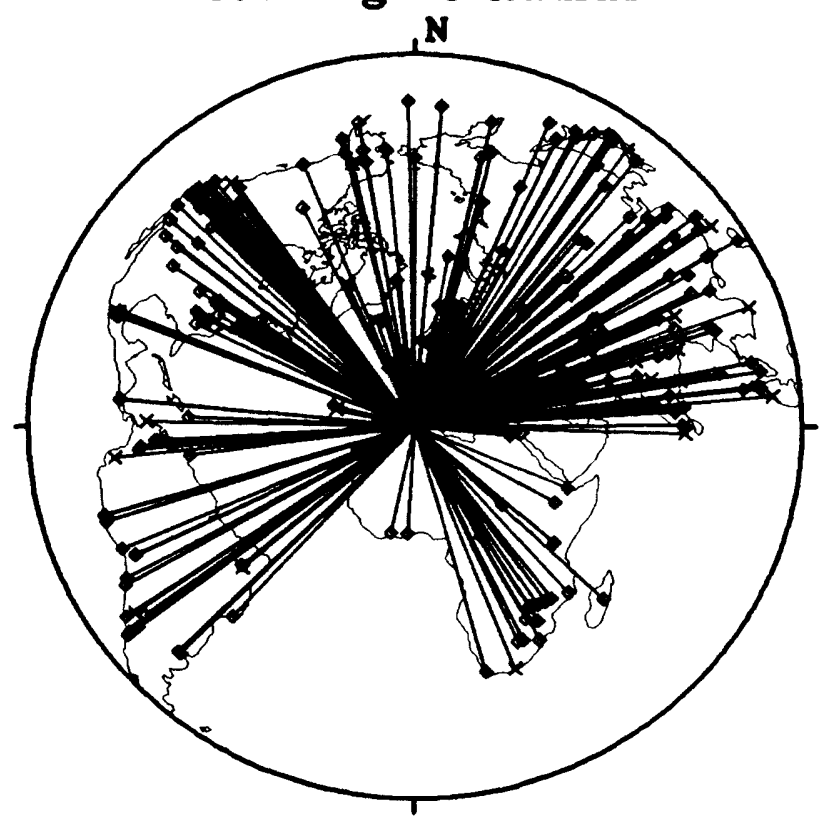

OCT 24, 1980 14:53:34

CENTRAL MEXICO

105 Degree Radius

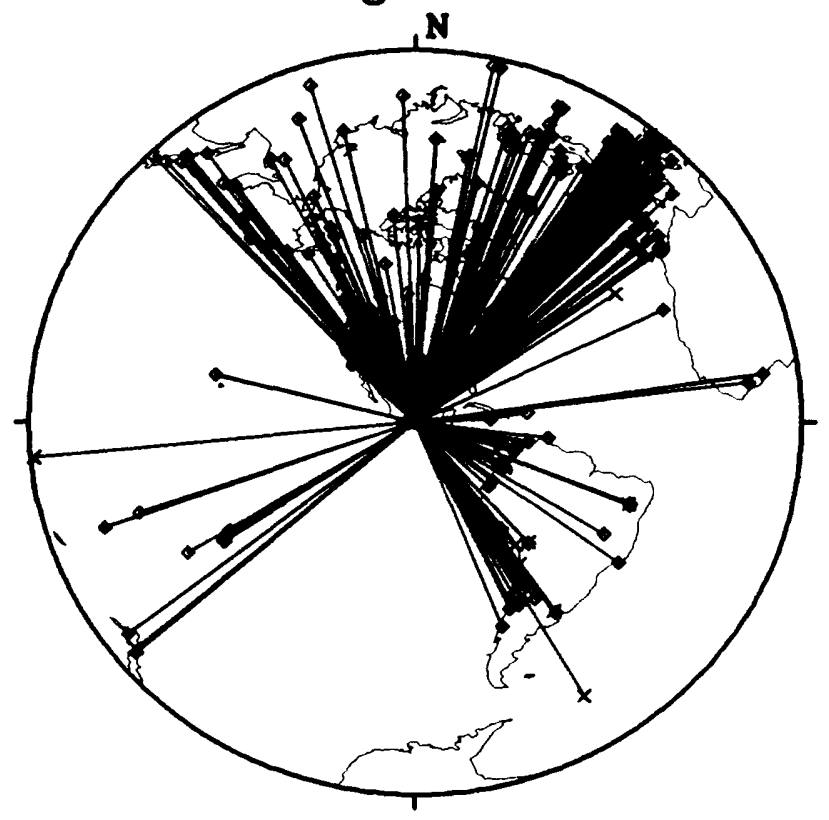

OCT 10, 1980 12:25:23

ALGERIA

10 Degree Radius

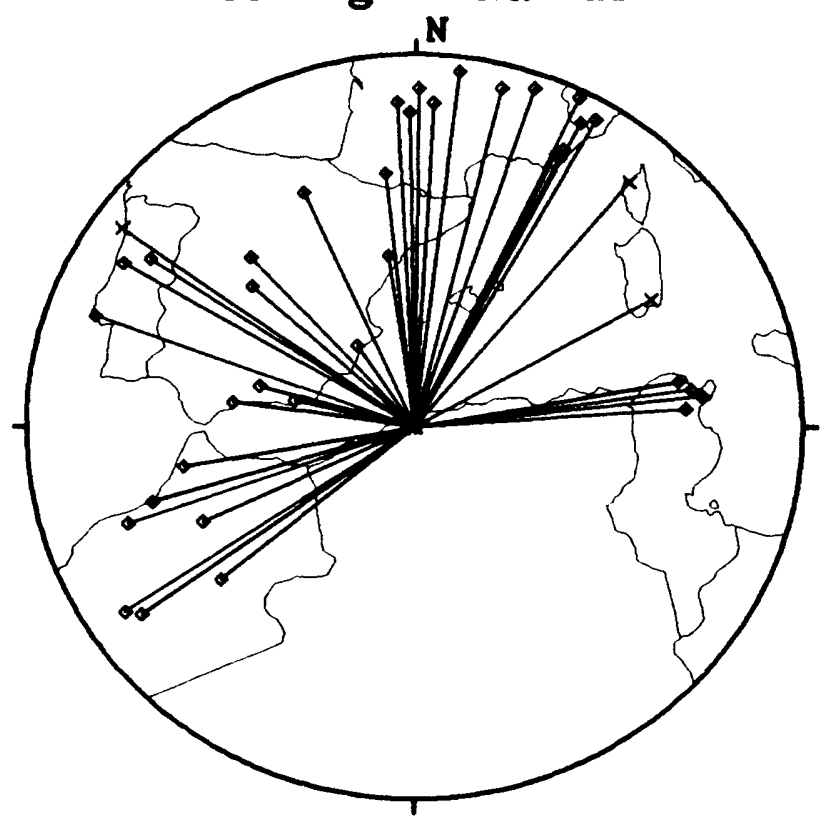

OCT 24, 1980 14:53:34

CENTRAL MEXICO

10 Degree Radius

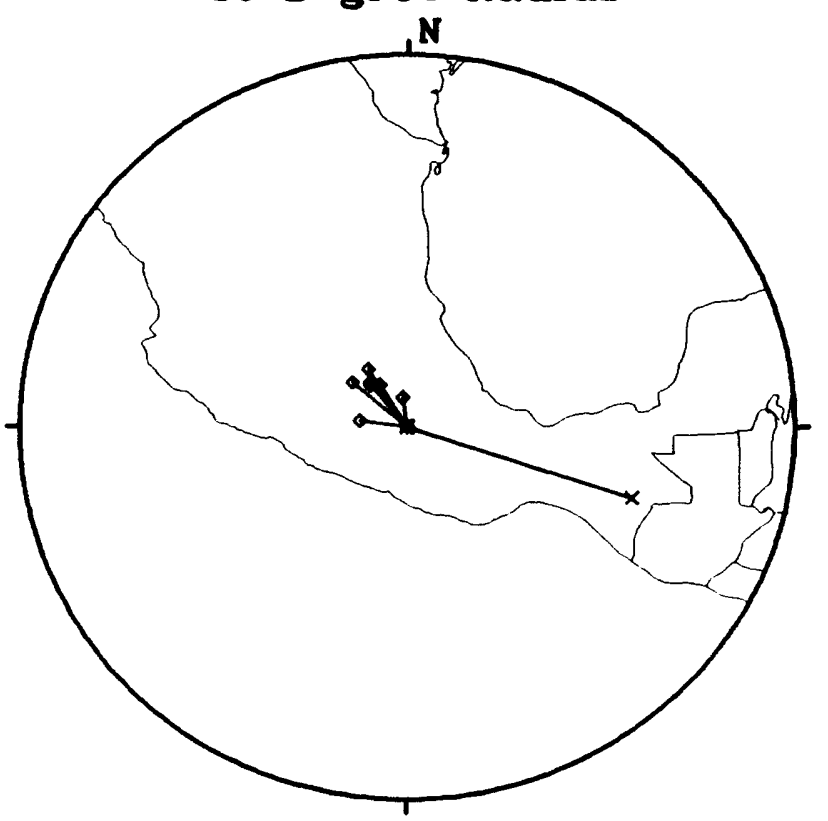


NOV 08, 1980 10:27:33 NEAR COAST OF NORTHERN CALIF. 105 Degree Radius

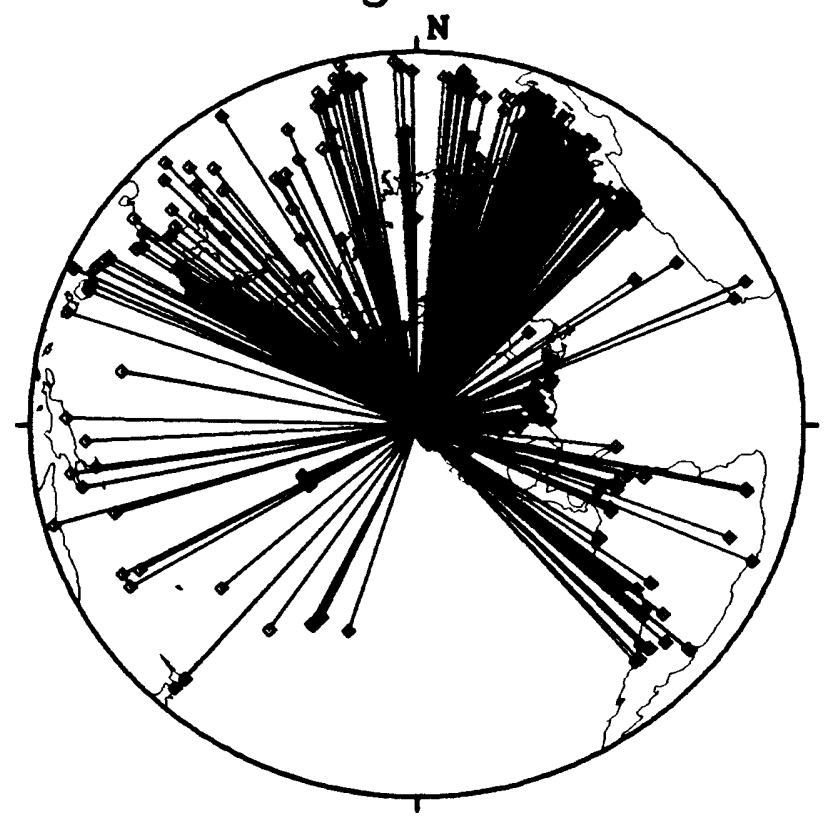

NOV 23, 1980 18:34:53 SOUTHERN ITALY 105 Degree Radius

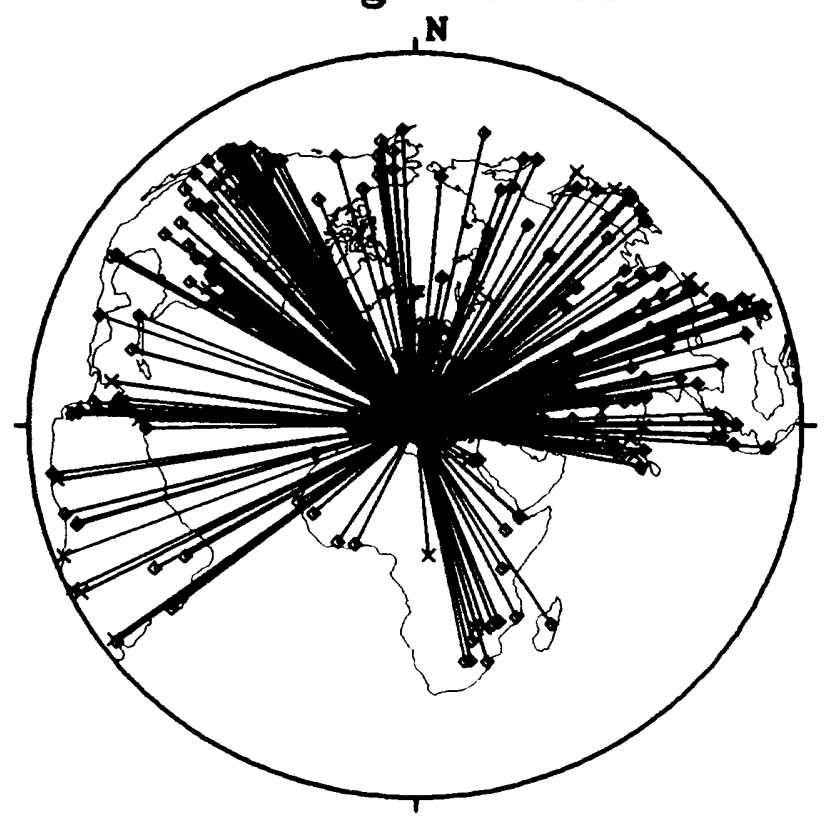

NOV 08, 1980 10:27:33 NEAR COAST OF NORTHERN CALIF. 10 Degree Radius

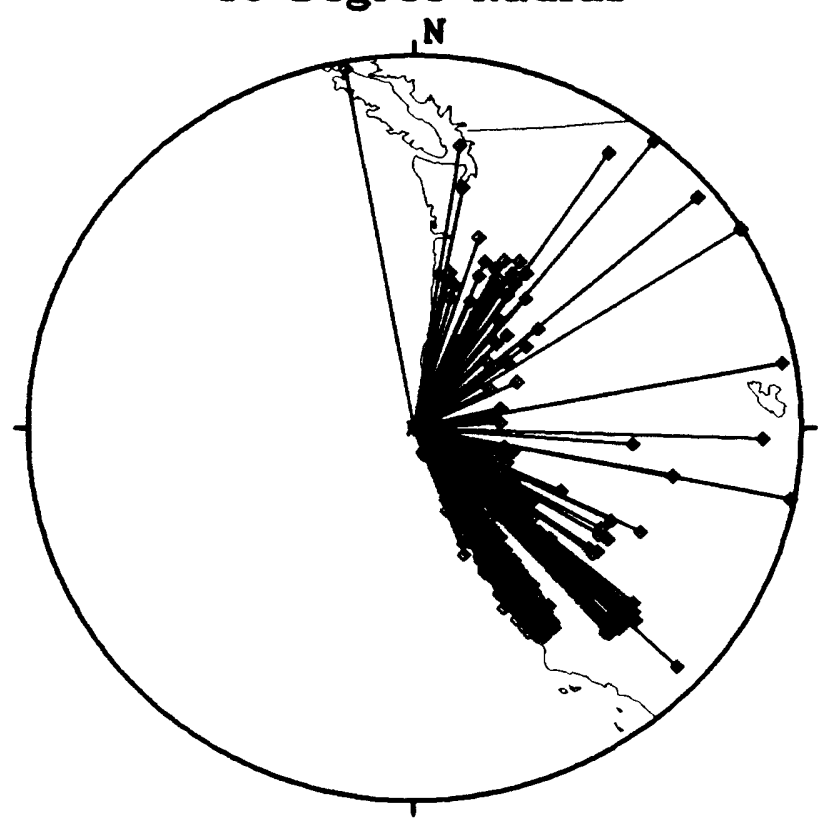

NOV 23, 1980 18:34:53 SOUTHERN ITALY 10 Degree Radius

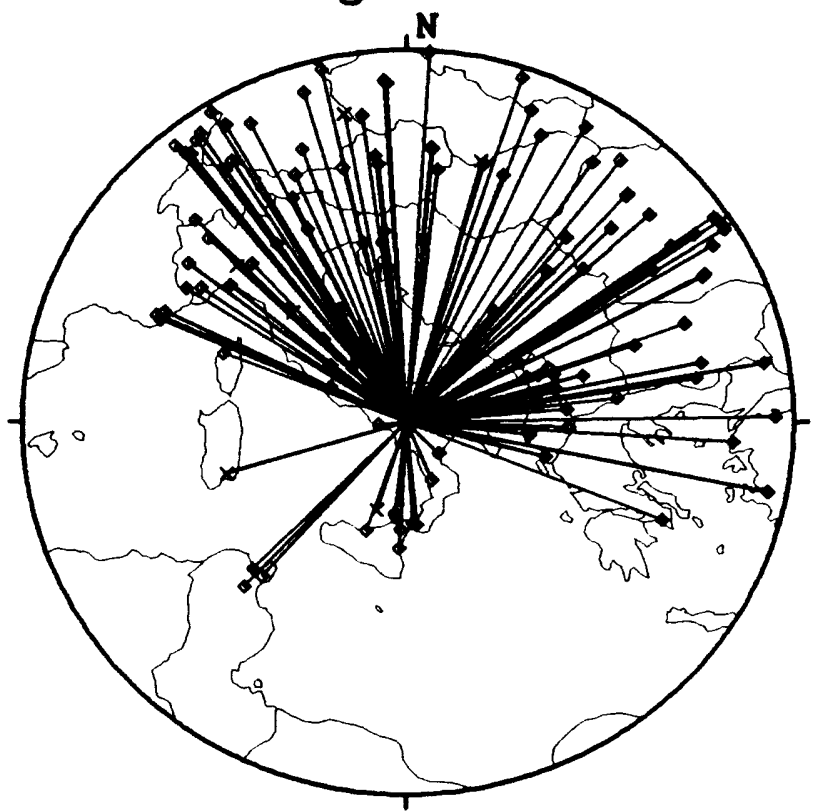


JAN 18,1981 18:17:26 NEAR EAST COAST OF HONSHU, JAPAN 105 Degree Radius

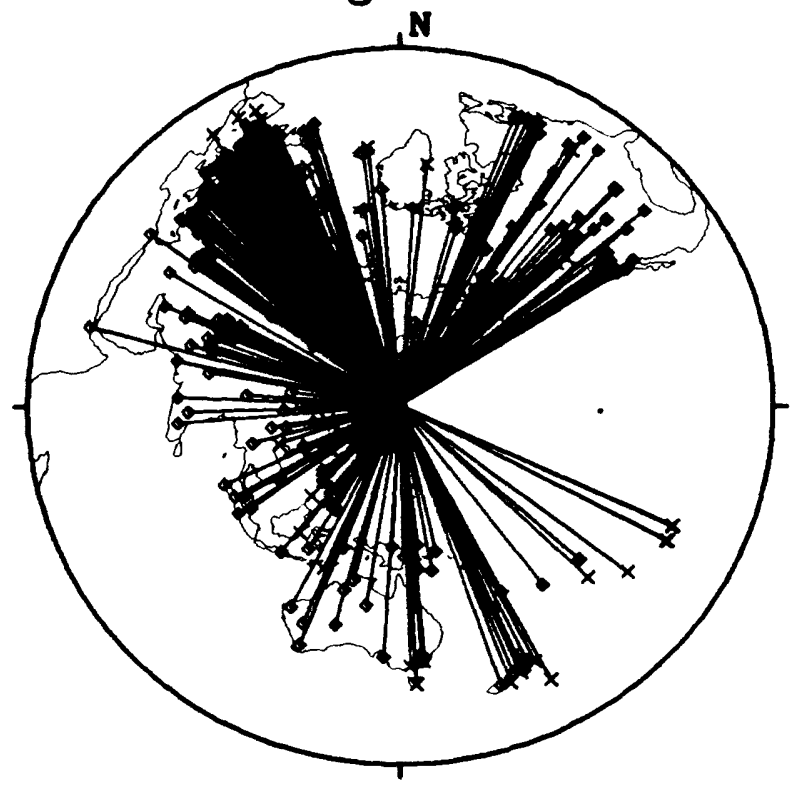

JAN 23, 1881 21:13:48 SICHUAN PROVINCE, CHINA 105 Degree Radius

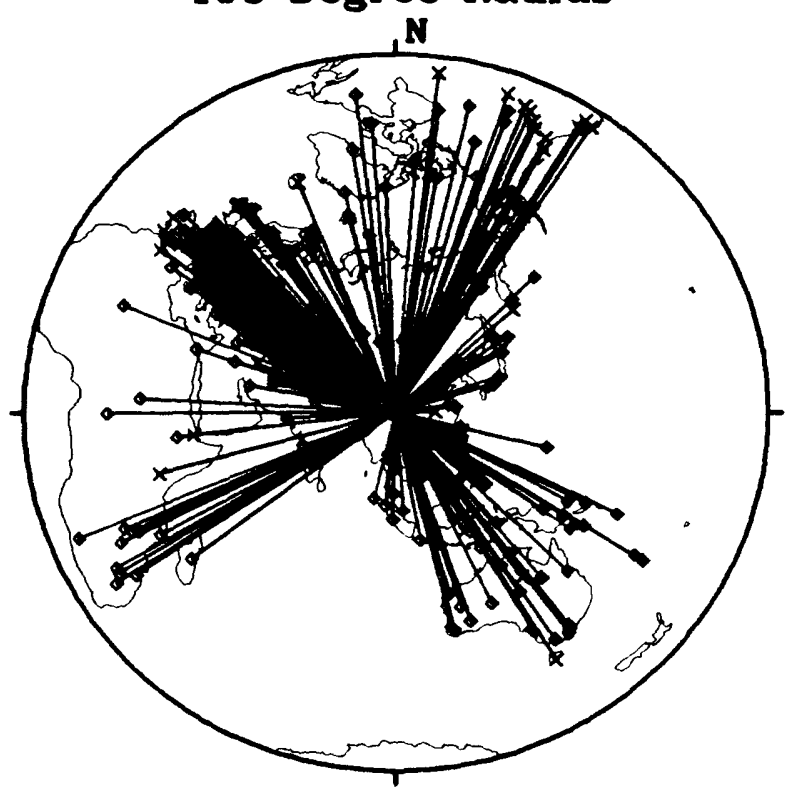

JAN 18,1981 18:17:26 NEAR EAST COAST OF HONSHU, JAPAN 10 Degree Radius

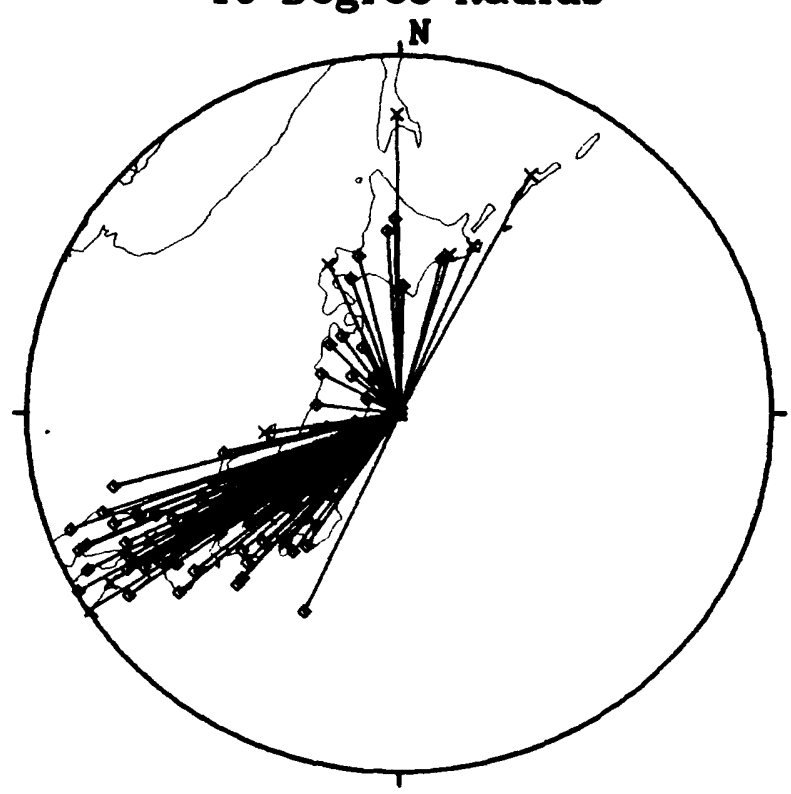

JAN 23, 1981 21:13:48 SICHUAN PROVINCE, CHINA 10 Degree Radius

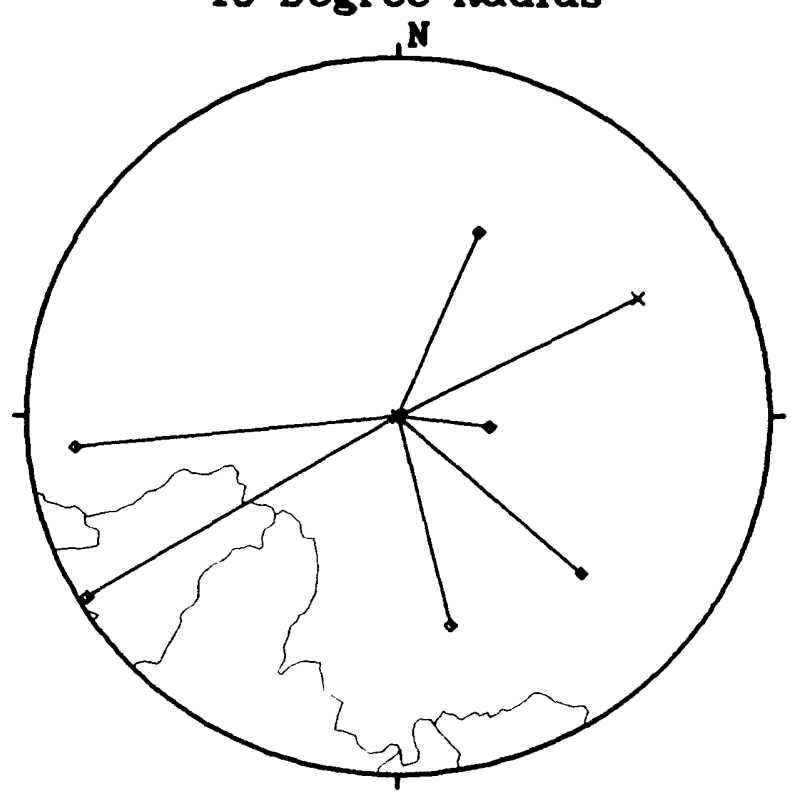


Figure 6

JUL 06, 1981 03:08:34

LOYALTY ISLANDS REGION 105 Degree Radius

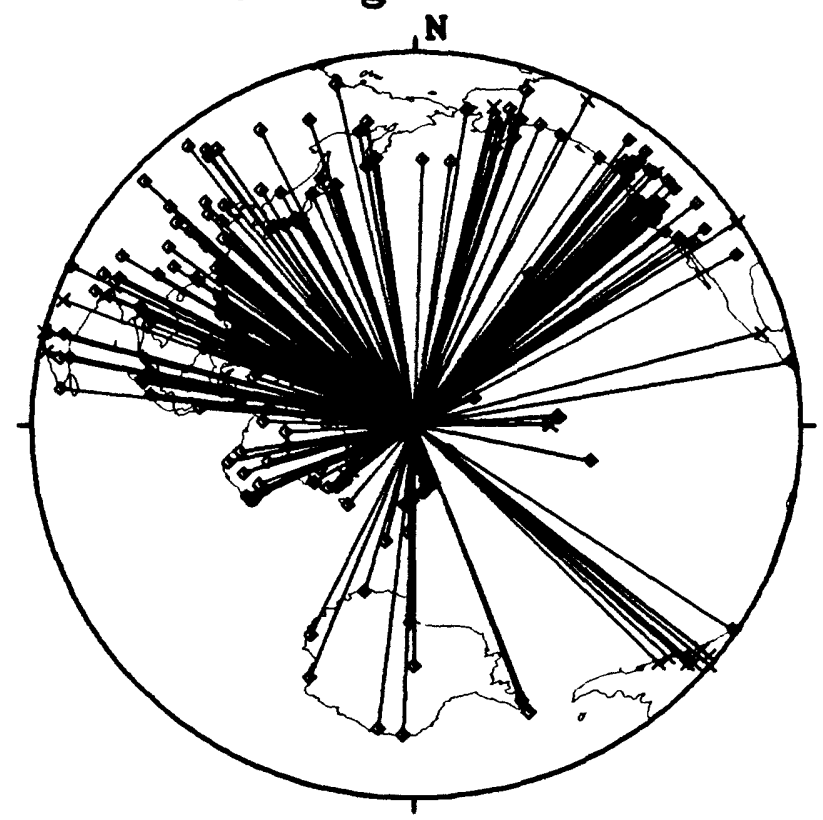

OCT 28, 1981 04:34:17 EASTER ISLAND REGION 105 Degree Radius

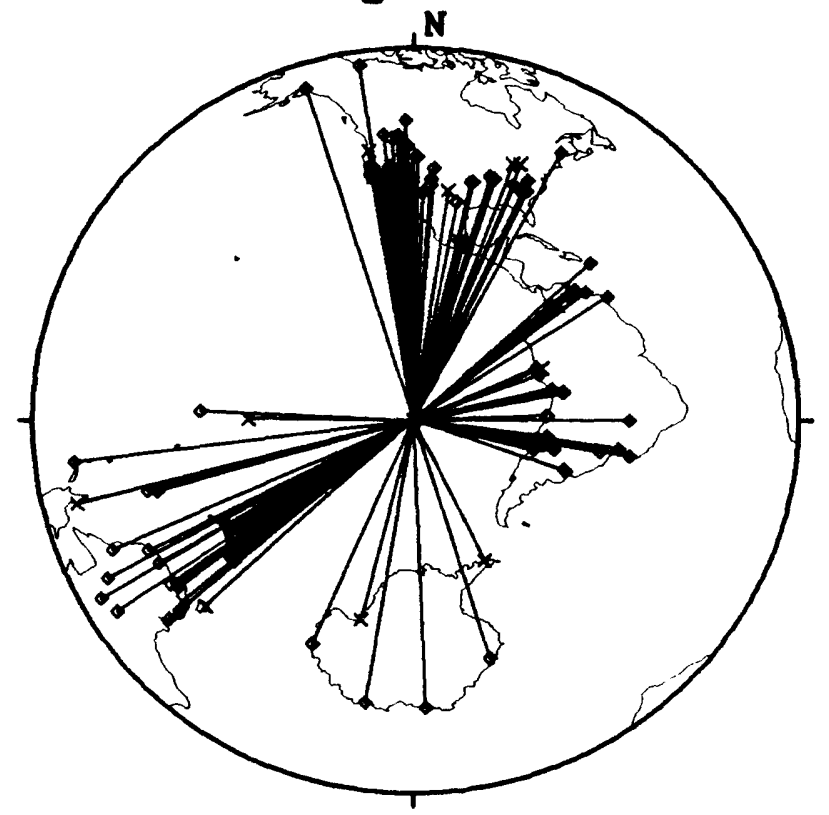

JUL 06, 1981 03:08:34 LOYALTY ISLANDS REGION 10 Degree Radius

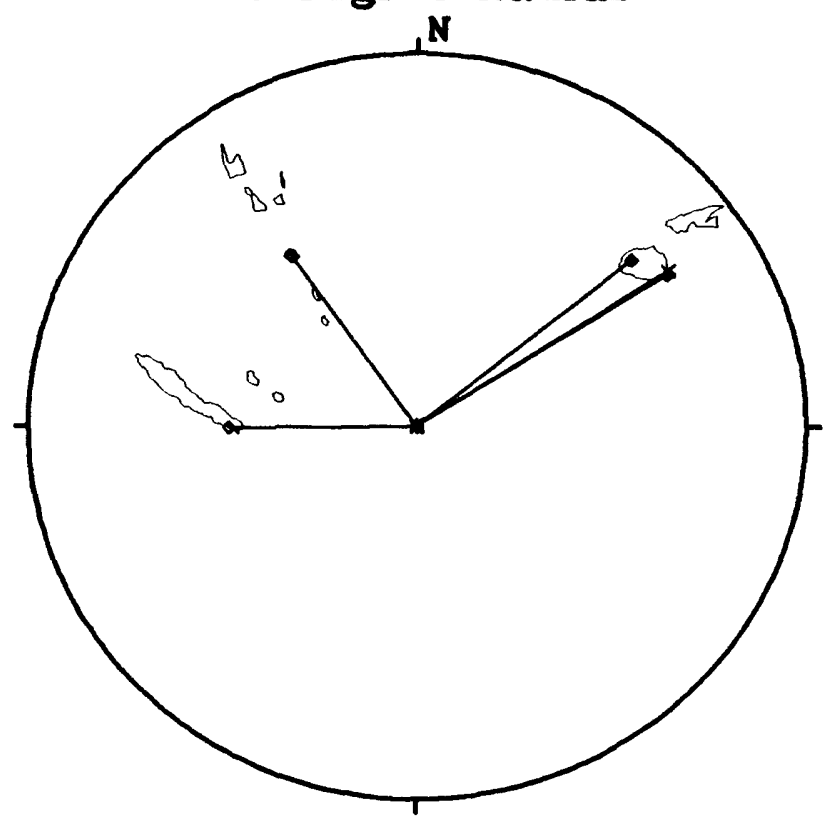

OCT 28, 1981 04:34:17 EASTER ISLAND REGION 35 Degree Radius

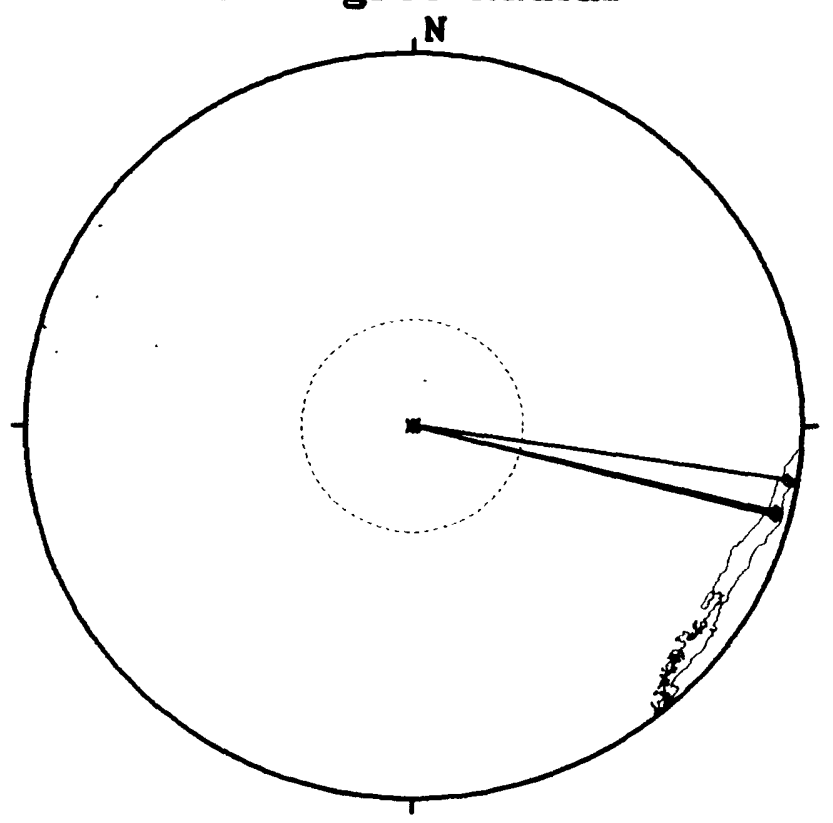


Figure 7

NOV 22, 1981 15:05:22

LUZON, PHILIPPINE ISLANDS 105 Degree Radius

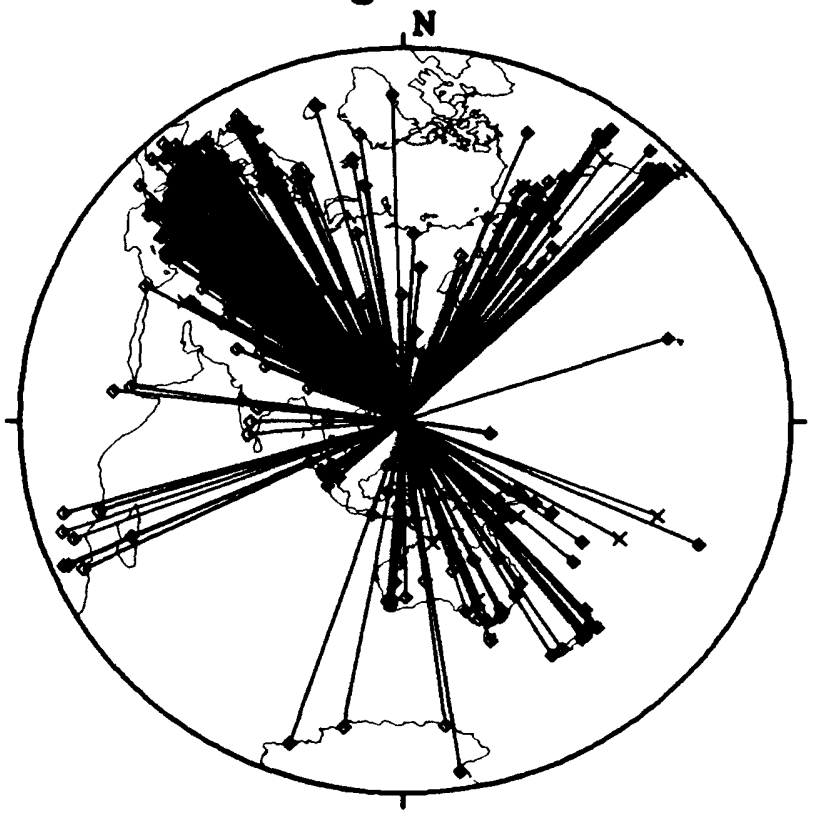

NOV 27, 1981 17:21:45

E. USSR-N.E. CHINA BORDER REG. 105 Degree Radius

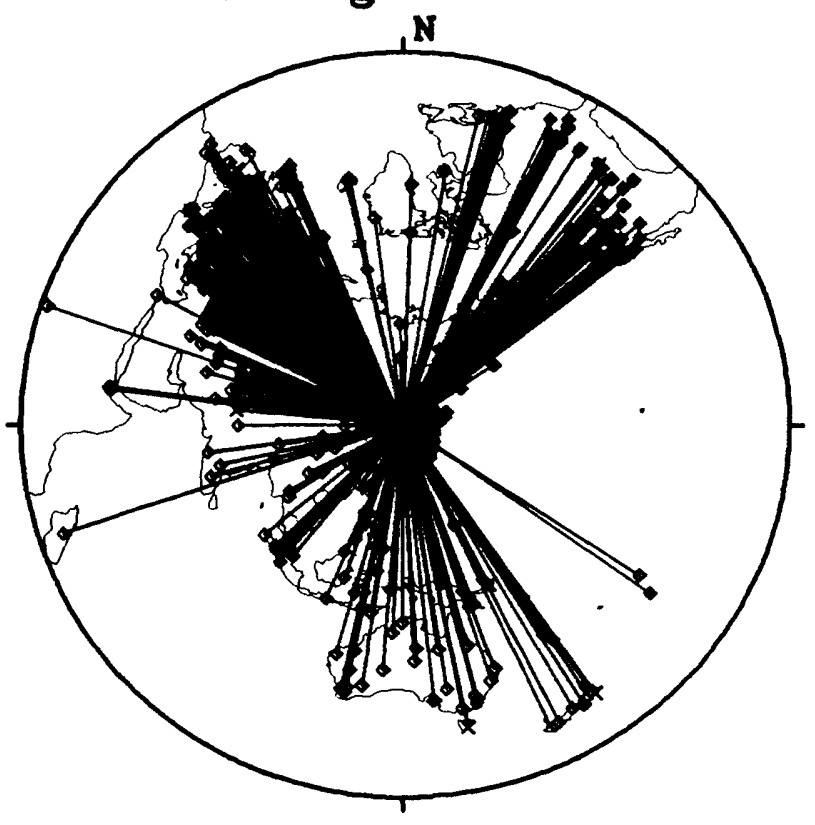

NOV 22, 1981 15:05:22 LUZON, PHILIPPINE ISLANDS 10 Degree Radius

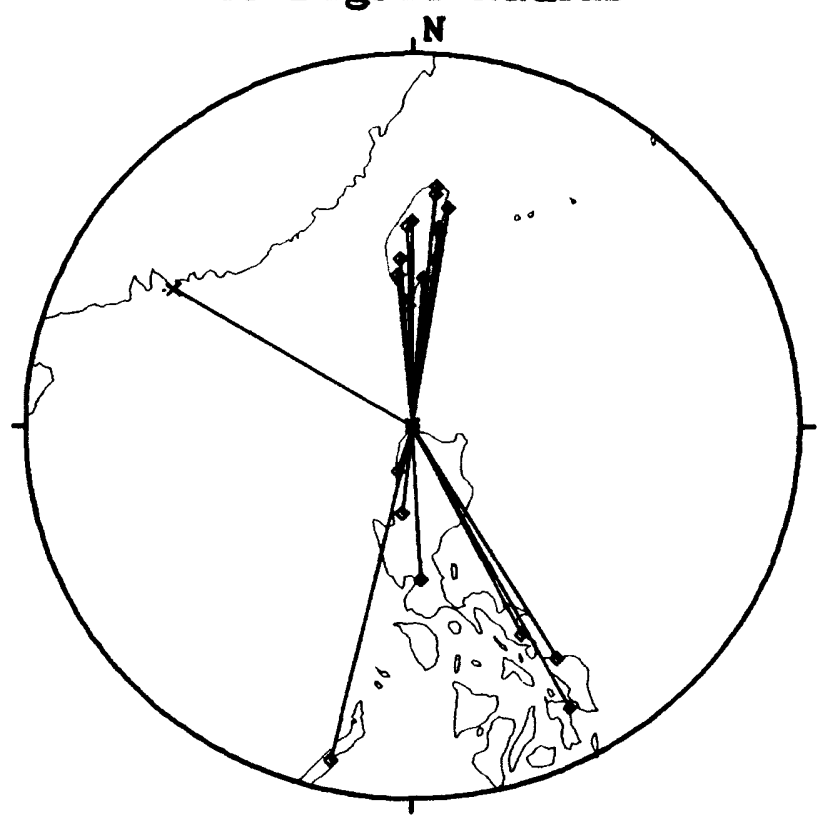

NOV 27, 1981 17:21:45

E. USSR-N.E. CHINA BORDER REG. 10 Degree Radius

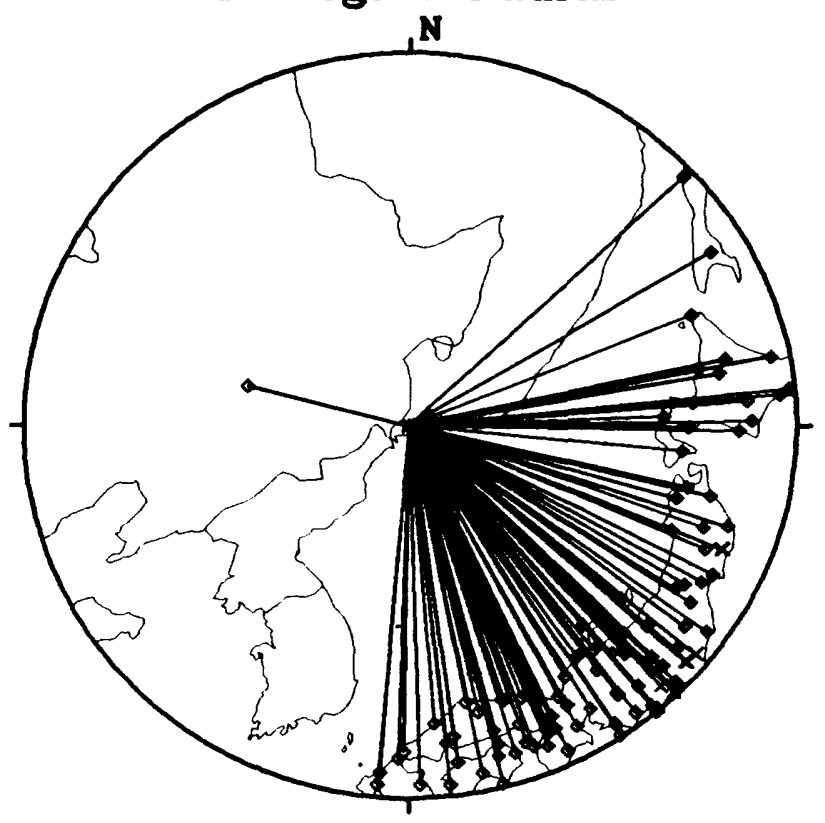


Figure 8

JAN 03, 1982 14:09:50

CENTRAL MID-ATLANTIC RIDGE 105 Degree Radius

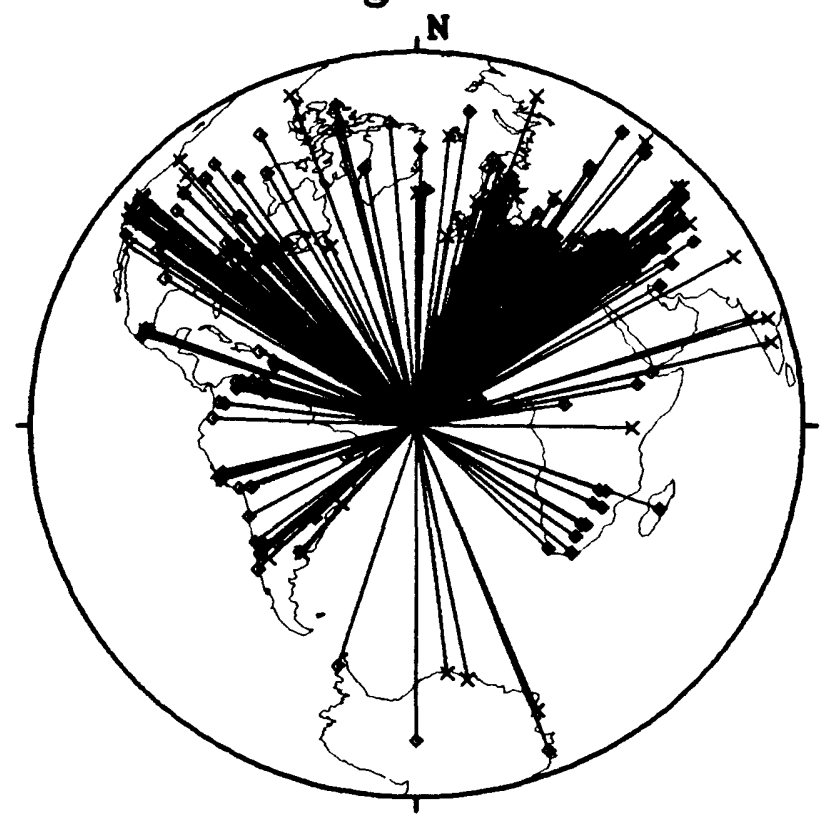

JAN 09, 1982 12:53:51

NEW BRUNSWICK

105 Degree Radius

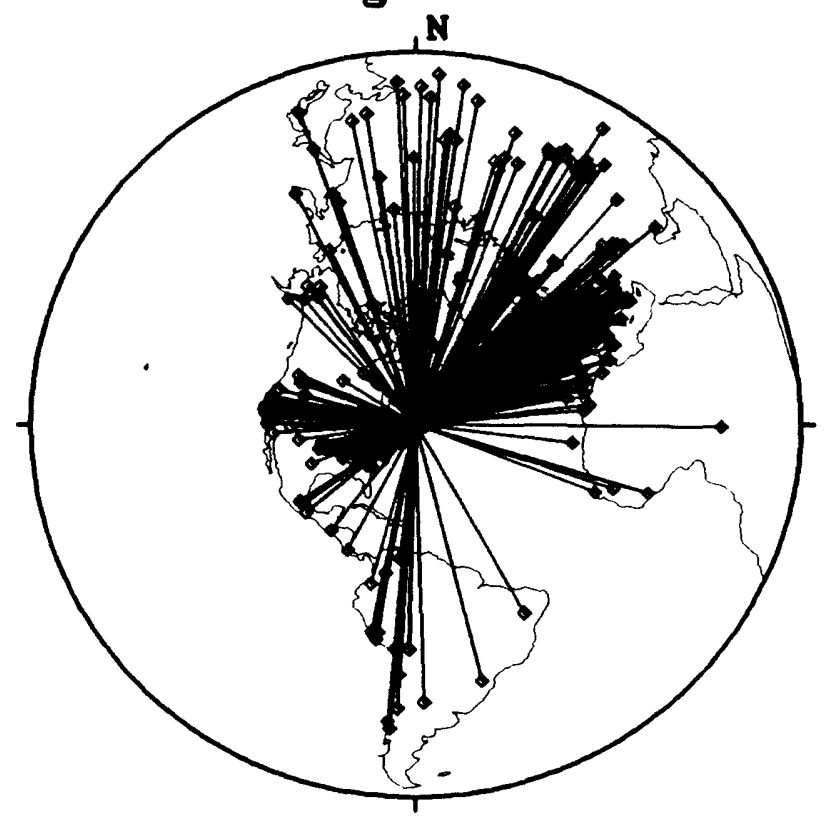

JAN 03, 1982 14:09:50 CENTRAL MID-ATLANTIC RIDGE 20 Degree Radius

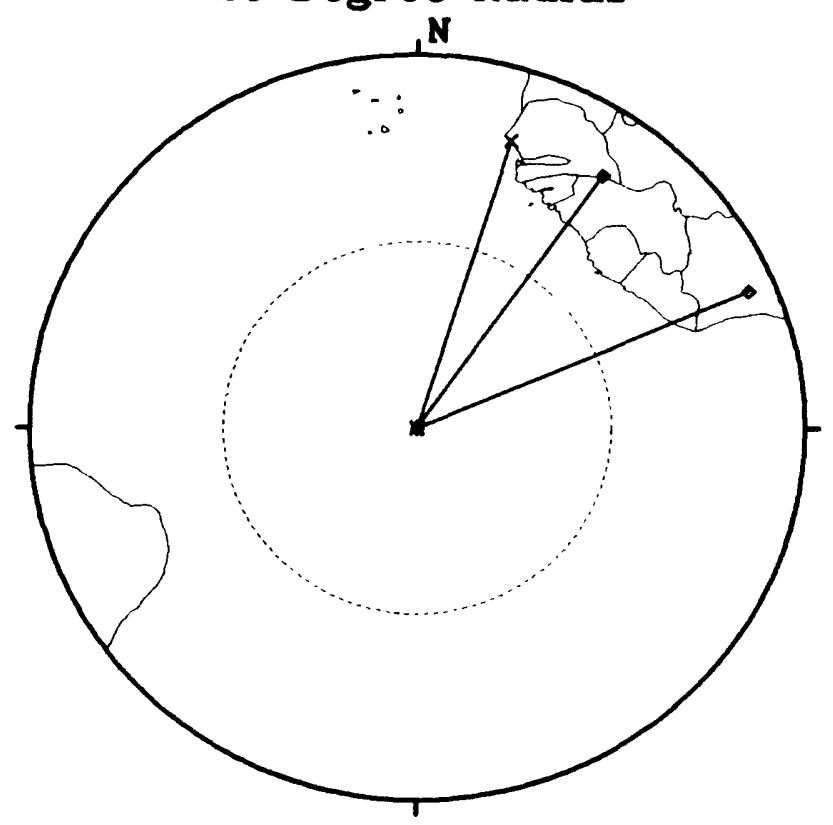

JAN 09, 1982 12:53:51 NEW BRUNSWICK 10 Degree Radius

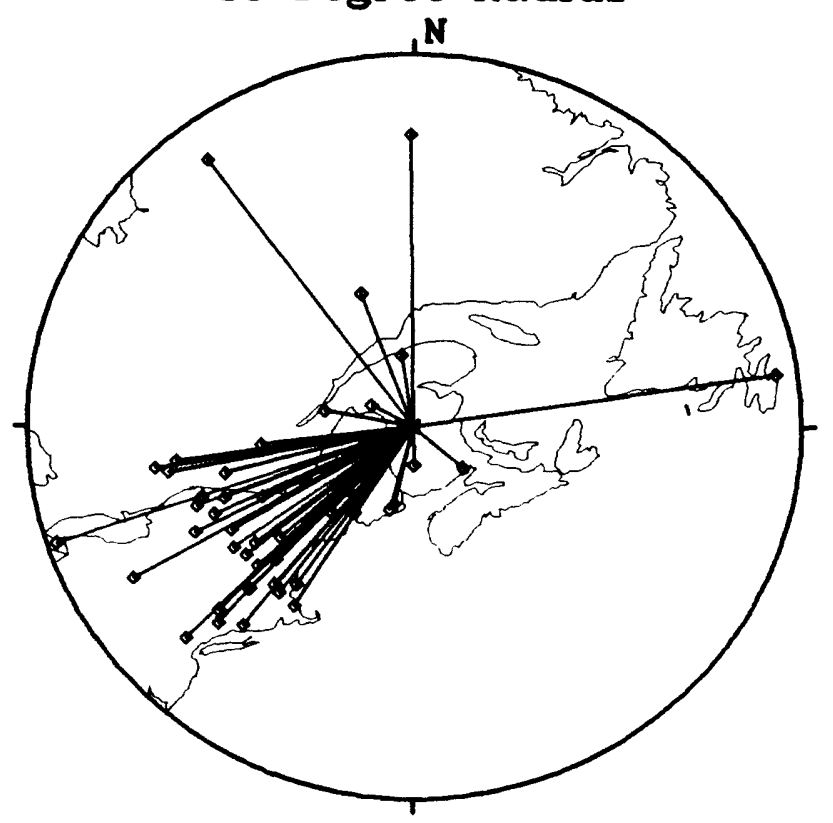


Figure 9

AUG 05, 1982 20:32:48

SANTA CRUZ ISLANDS 105 Degree Radius

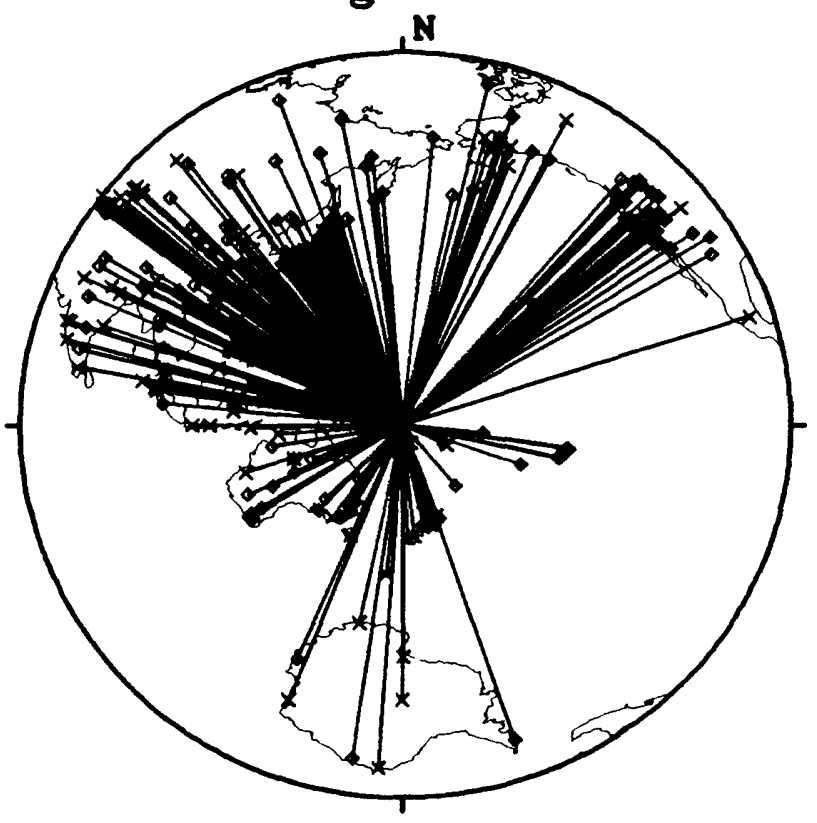

SEP 06, 1982 01:47:03 SOUTH OF HONSHU, JAPAN 105 Degree Radius

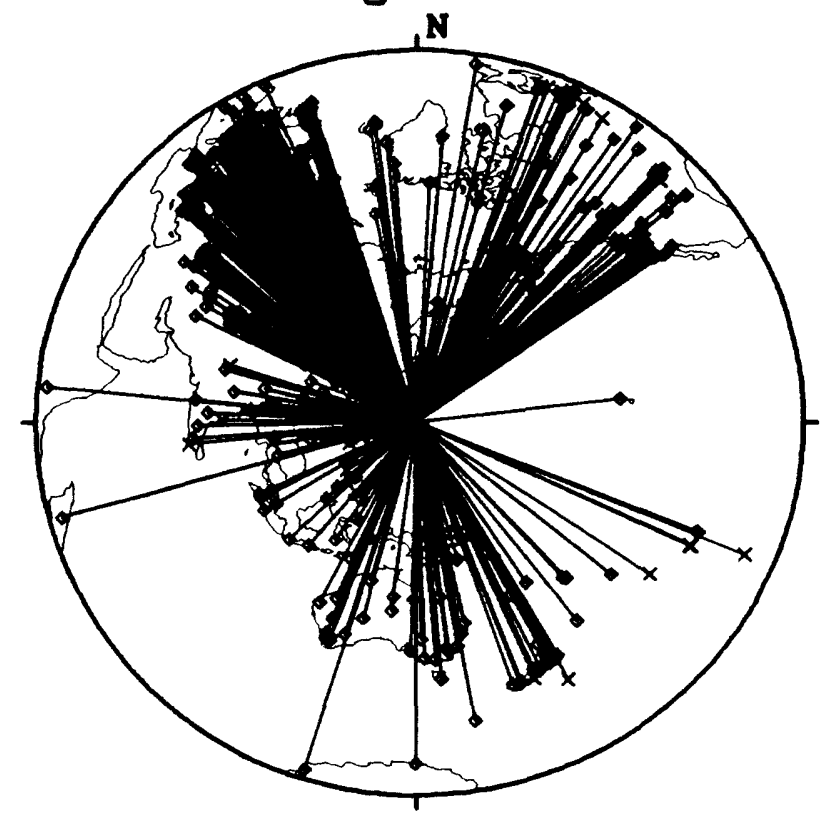

AUG 05, 1982 20:32:48 SANTA CRUZ ISLANDS 10 Degree Radius

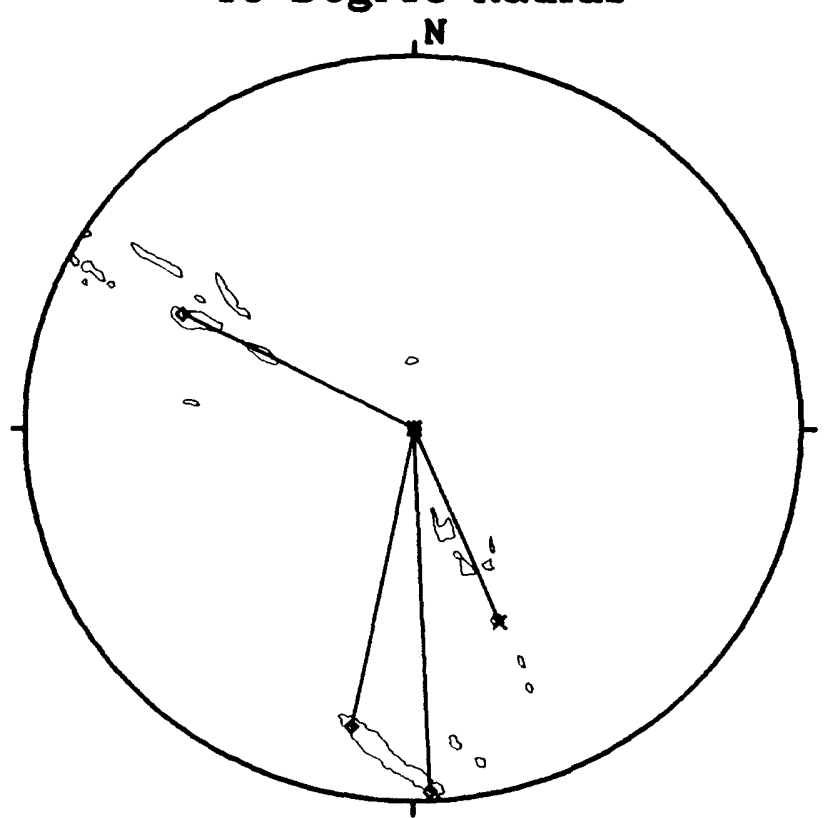

SEP 06, 1882 01:47:03 SOUTH OF HONSHU, JAPAN 10 Degree Radius

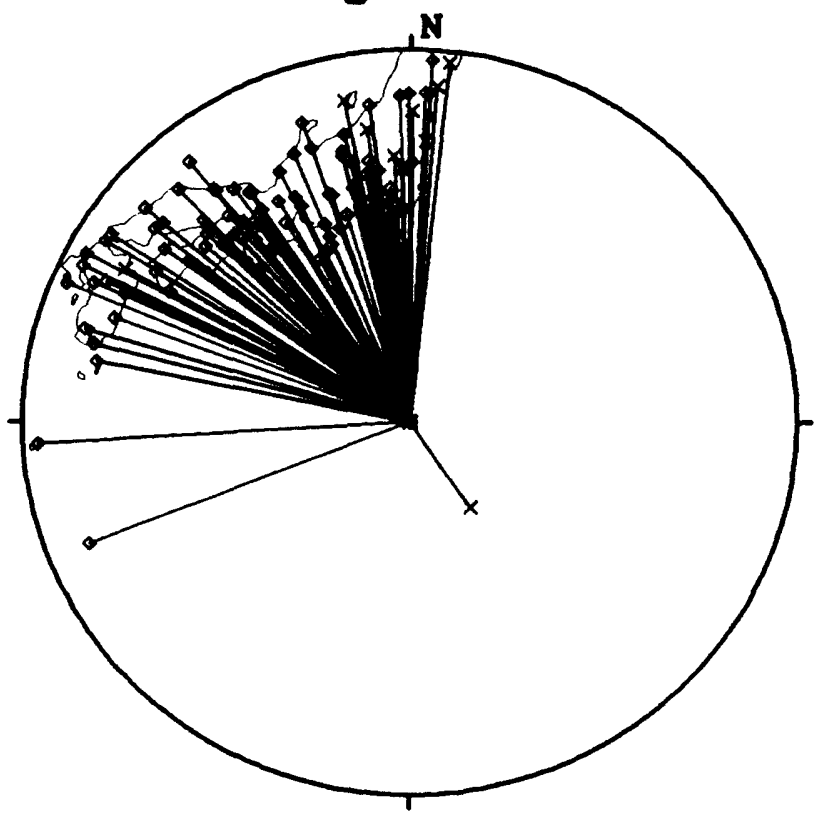


Figure 10

DEC 13, 1982 09:12:48 WESTERN ARABIAN PENINSULA 105 Degree Radius

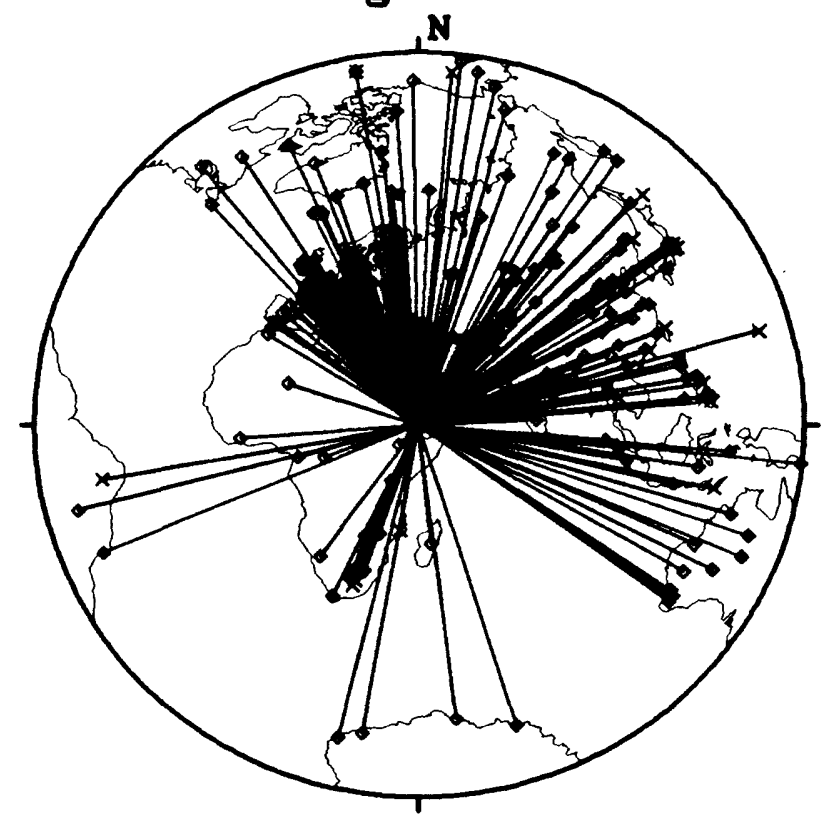

FEB 13, 1983 01:40:11 SOUTHERN XINJIANG, CHINA 105 Degree Radius

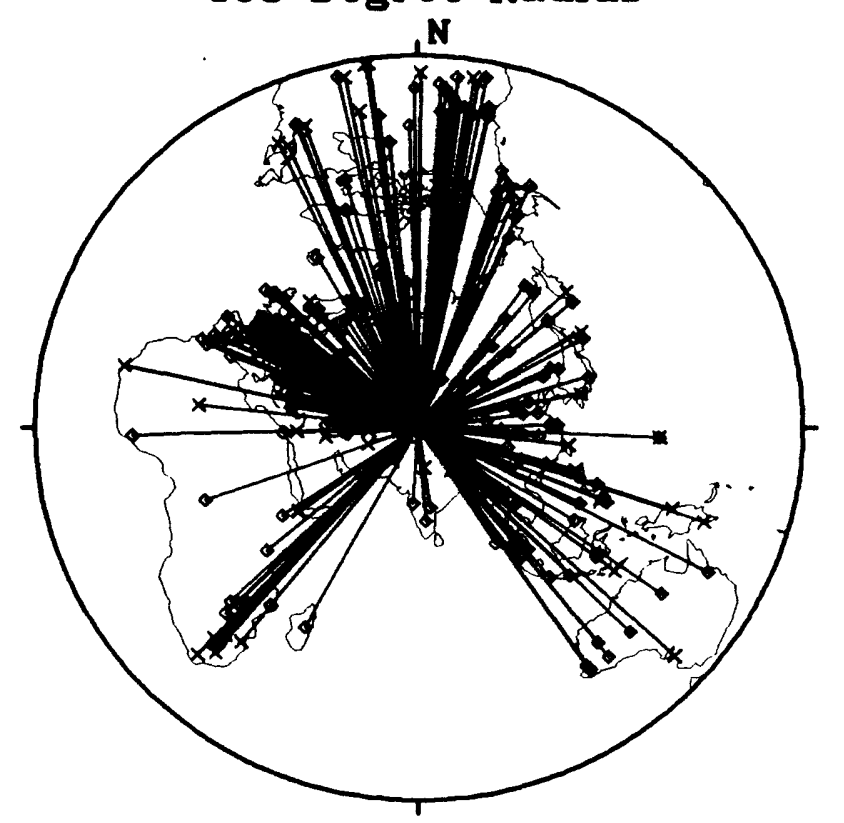

DEC 13, 1982 09:12:48 WESTERN ARABIAN PENINSULA 10 Degree Radius

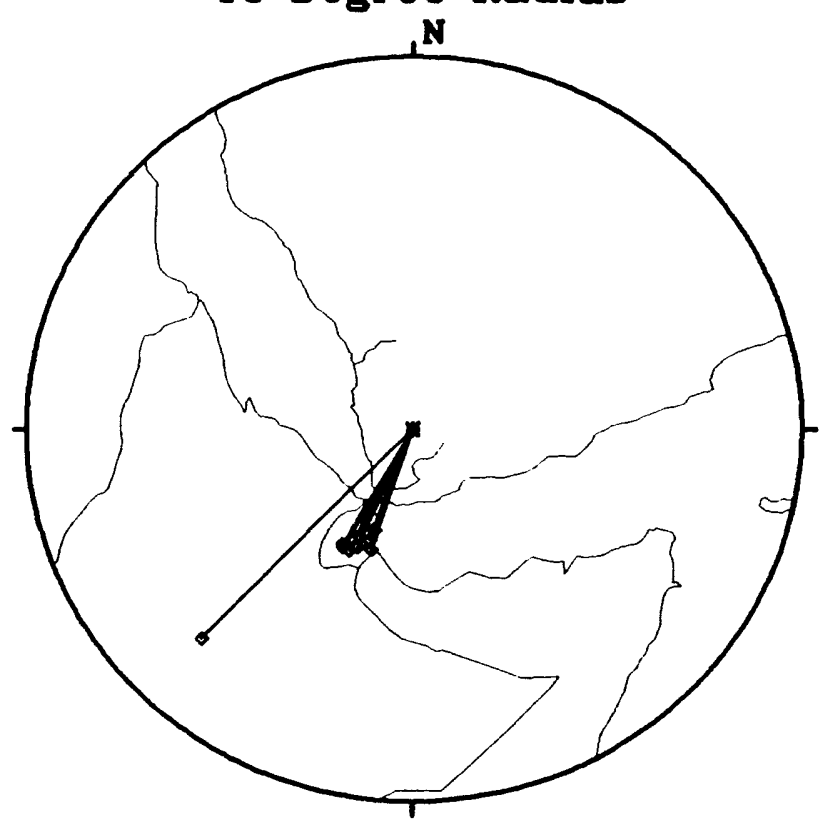

FEB 13, 1983 01:40:11 SOUTHERN XINJIANG, CHINA 10 Degree Radius

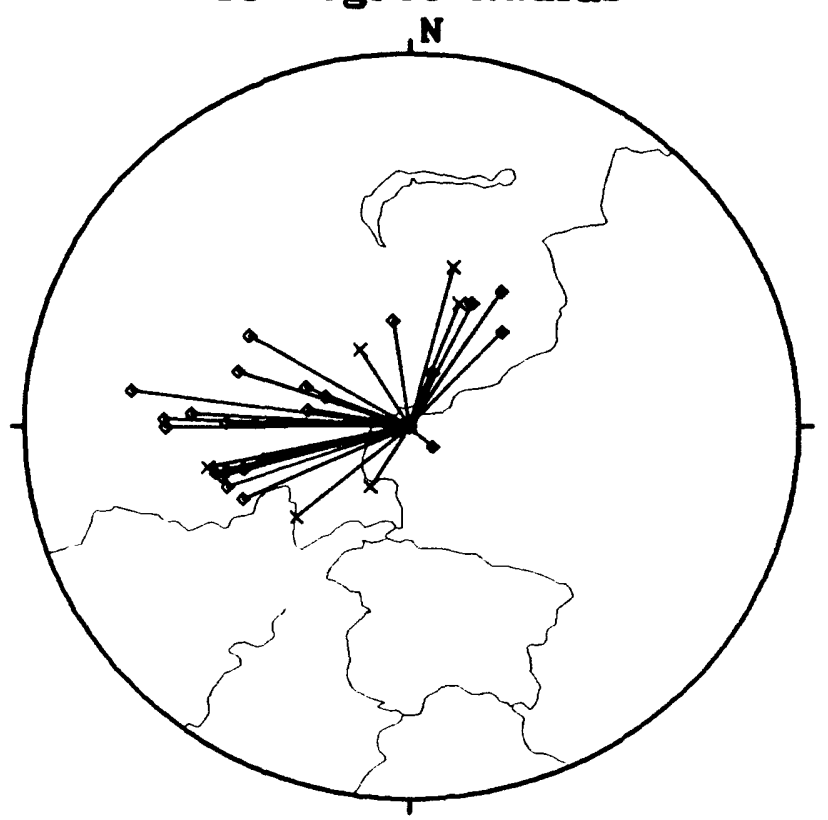


Figure 11

APR 03, 1983 02:50:02 COSTA RICA

105 Degree Radius

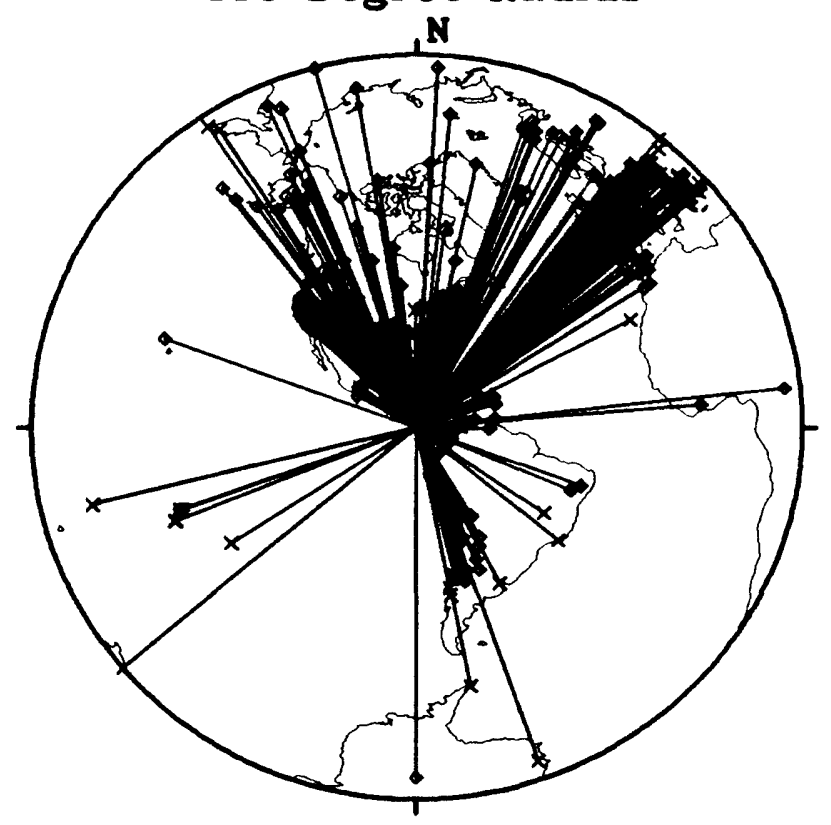

APR 04, 1983 02:51:34 NORTHERN SUMATERA 105 Degree Radius

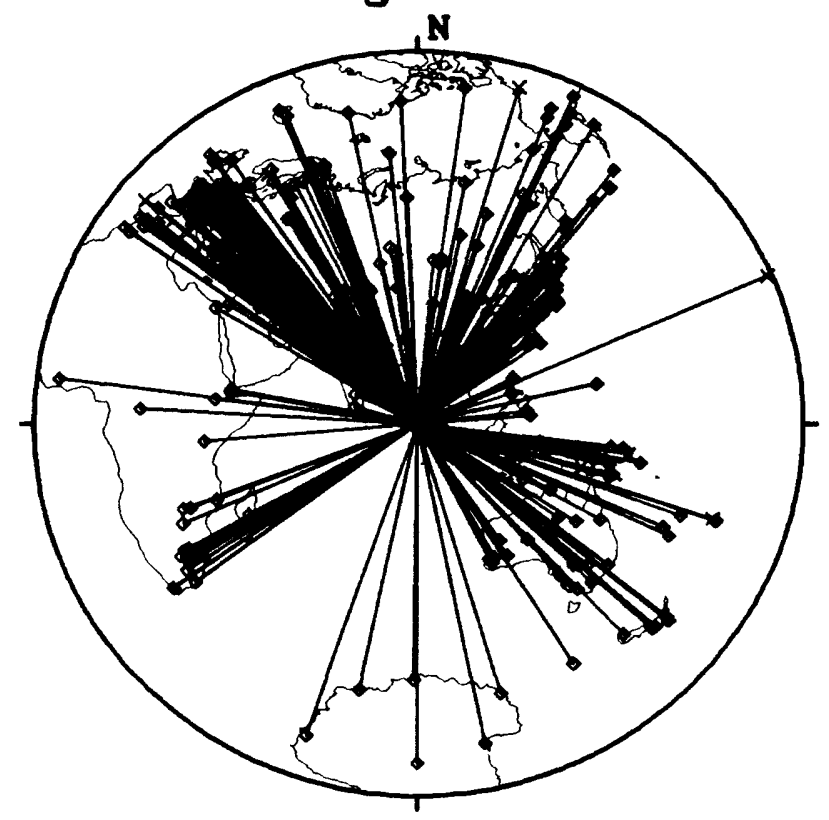

APR 03, 1983 02:50:02 COSTA RICA

10 Degree Radius

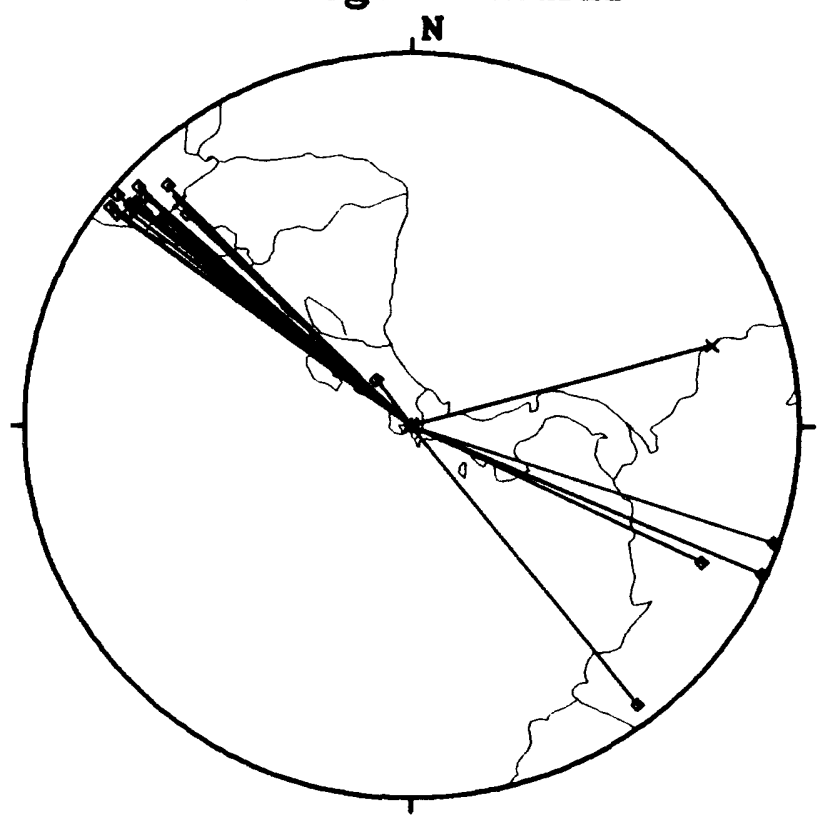

APR 04, 1983 02:51:34 NORTHERN SUMATERA 10 Degree Radius

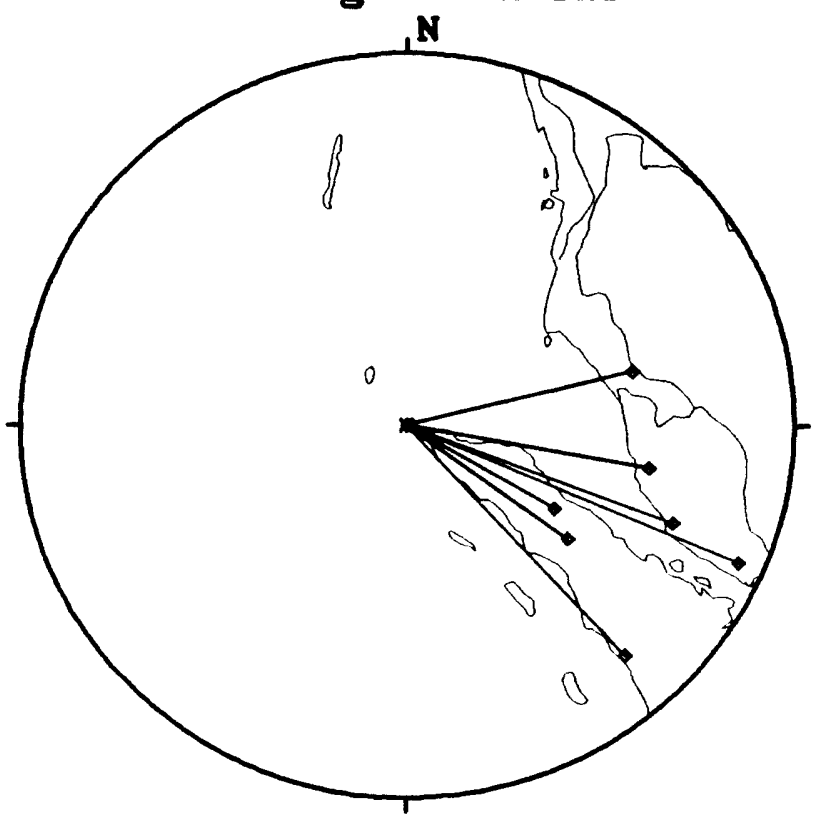


Figure 12

APR 11, 1983 08:18:10 NEAR COAST OF VENEZUELA 105 Degree Radius

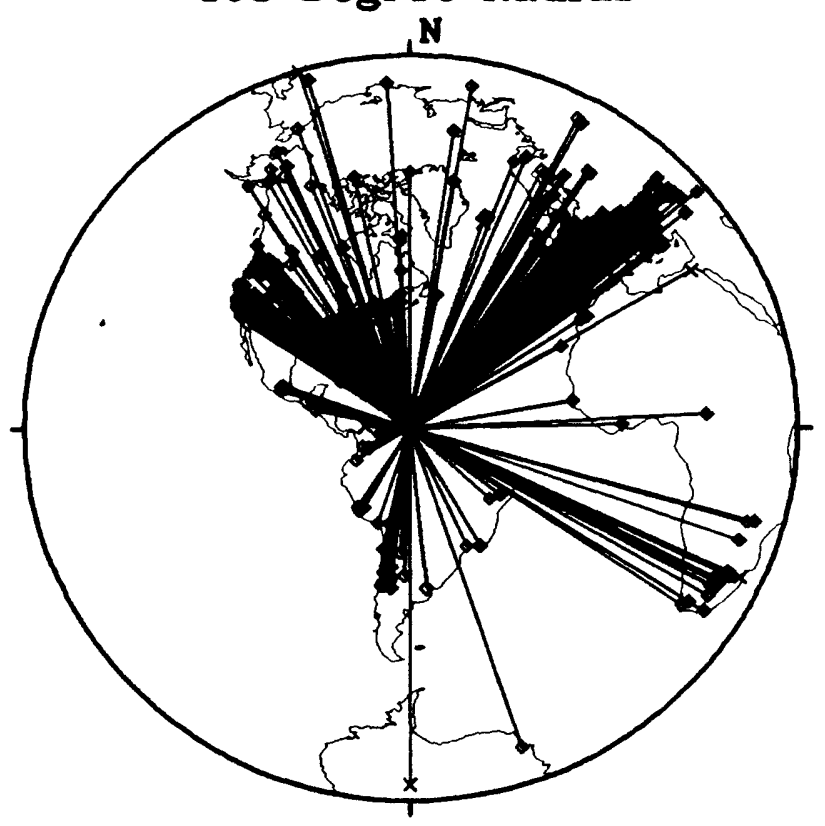

APR 18, 1983 10:58:48 SOUTHERN IRAN 105 Degree Radius

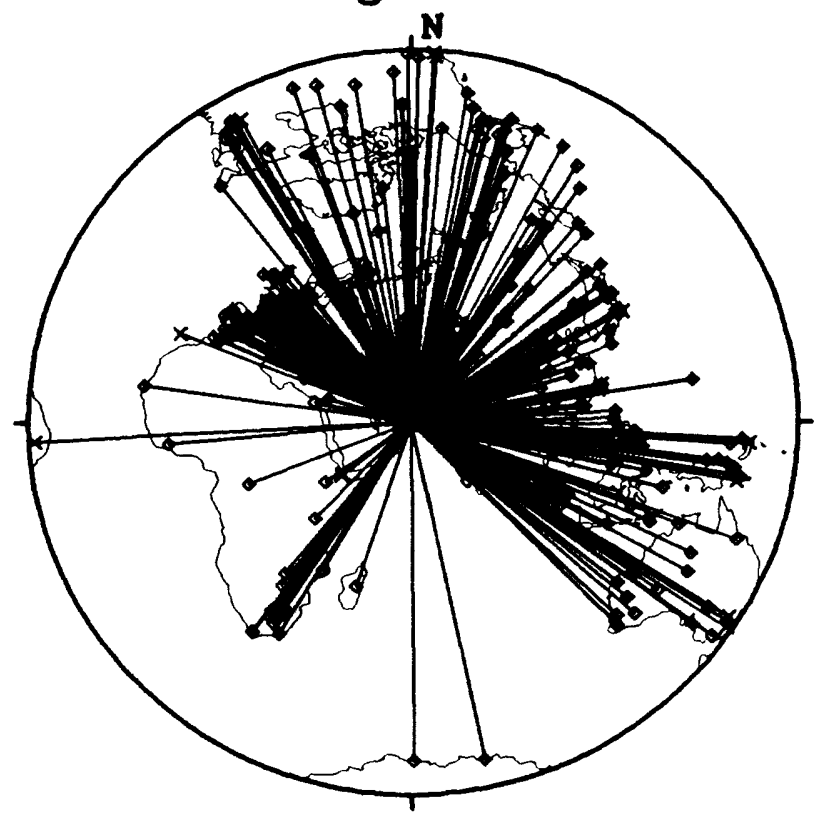

APR 11, 1983 08:18:10 NEAR COAST OF VENEZUELA 10 Degree Radius

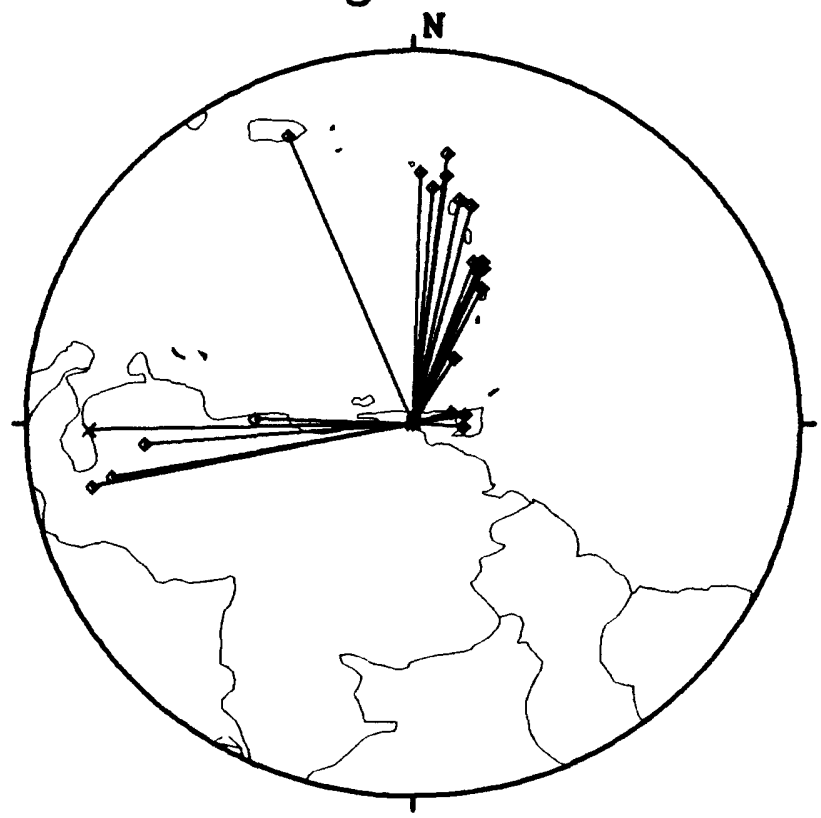

APR 18, 1983 10:58:48 SOUTHERN IRAN 10 Degree Radius

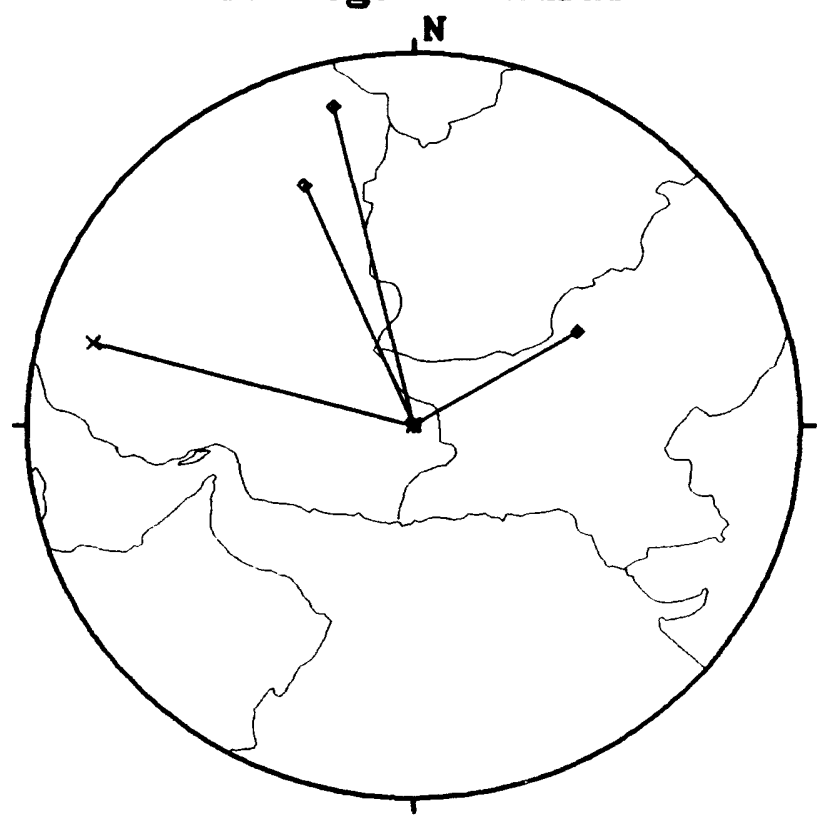



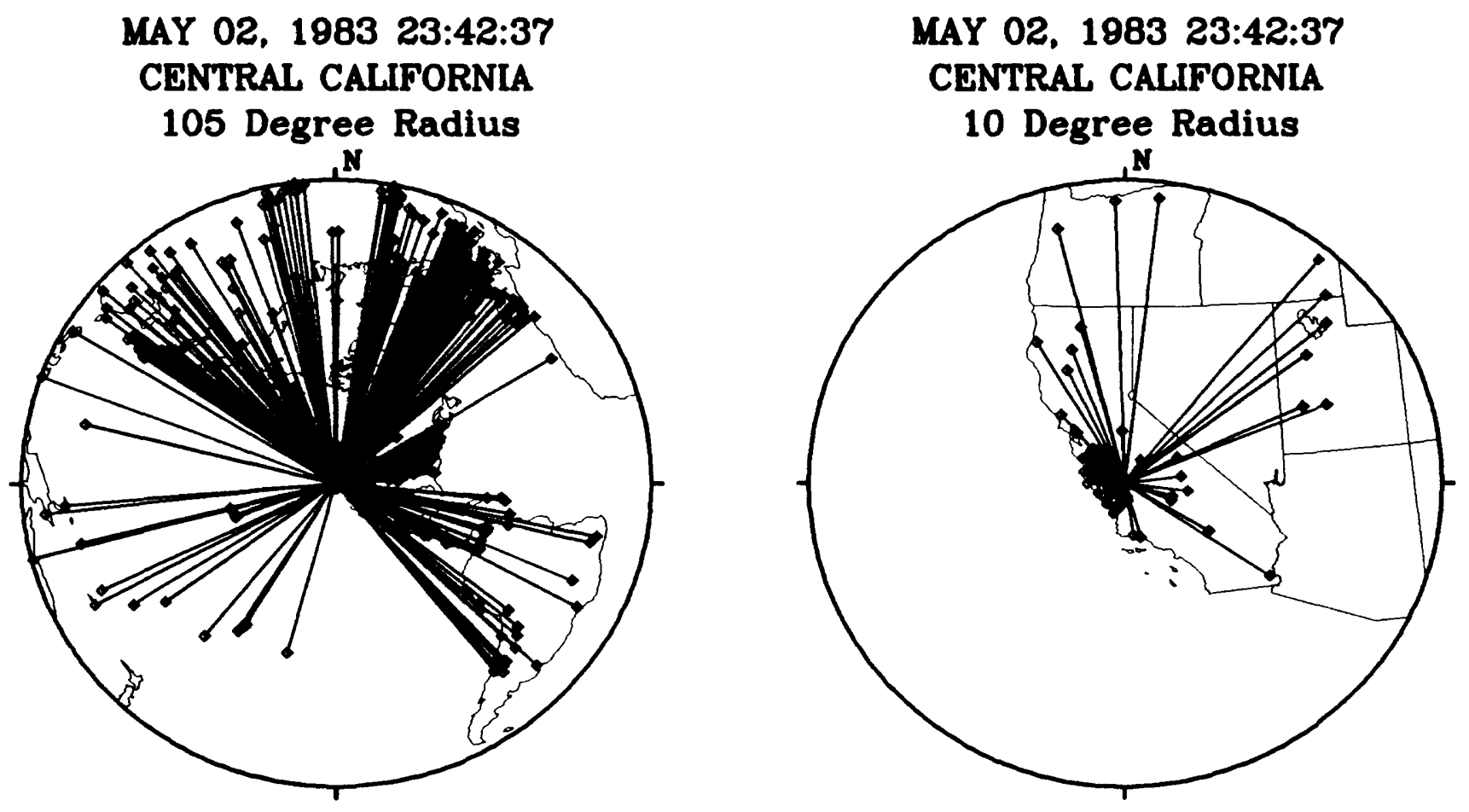

MAY 26, 1983 02:59:59

NEAR WEST COAST OF HONSHU, JAPAN 105 Degree Radius

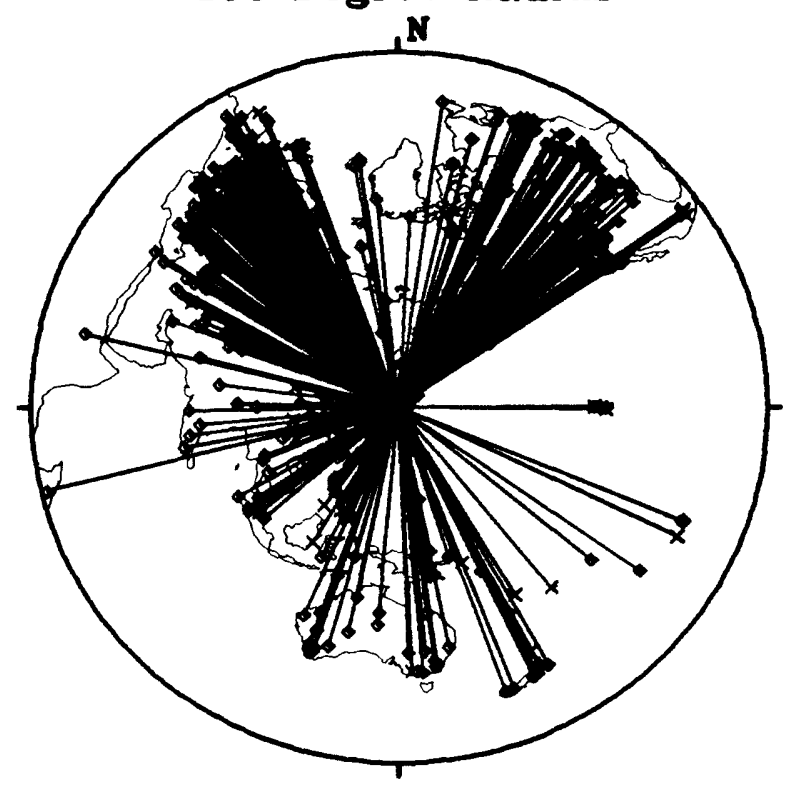

MAY 26, 1983 02:59:59

NEAR WEST COAST OF HONSHU, JAPAN 10 Degree Radius

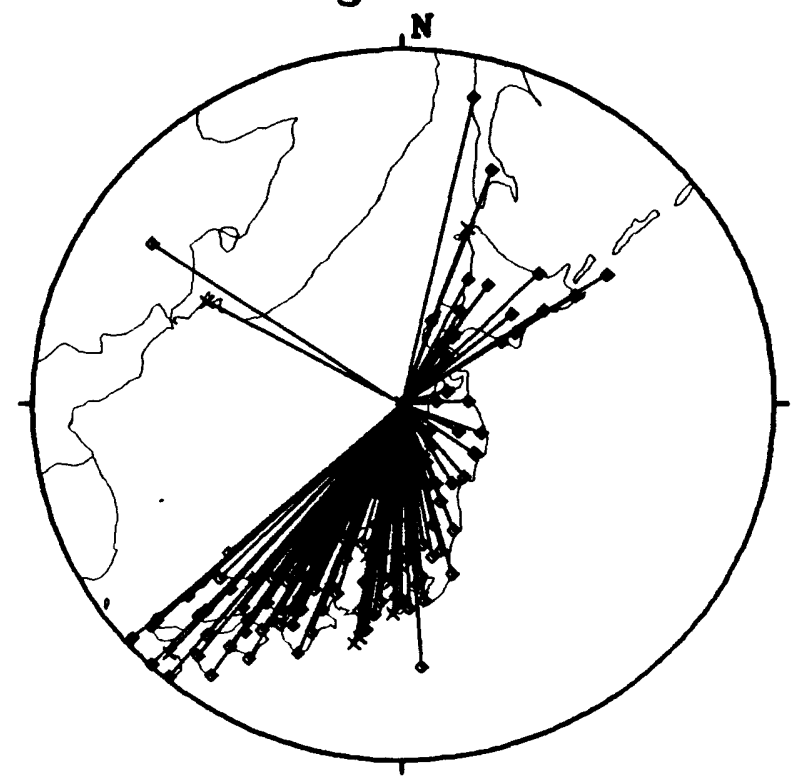


Figure 14

JUN 01, 1983 01:59:54 TONGA ISLANDS 105 Degree Radius

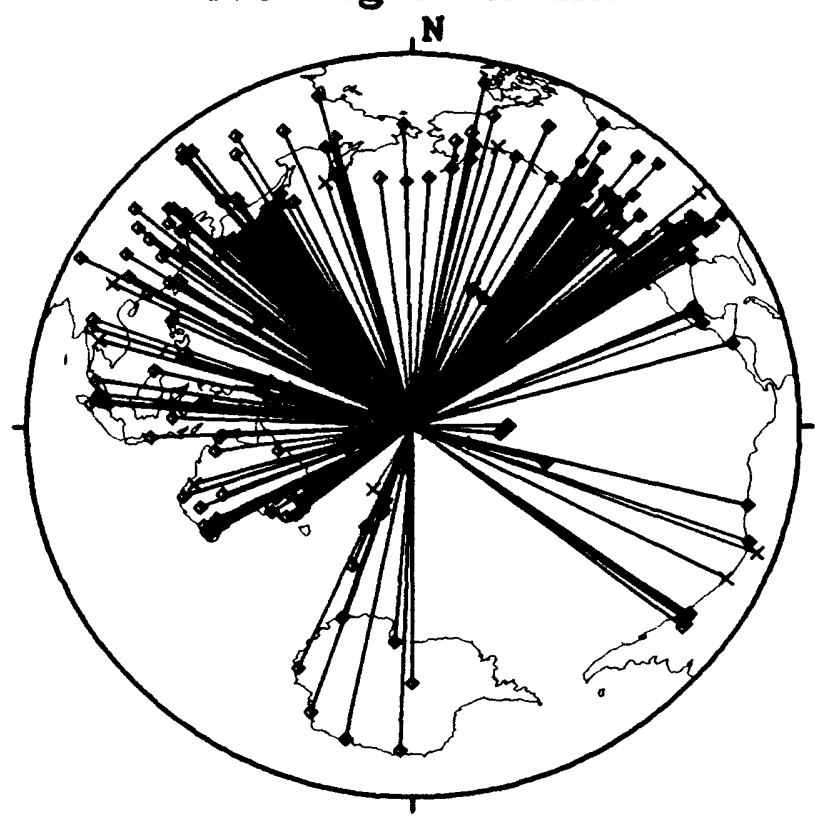

JUN 02, 1983 20:12:50 PERU-BRAZIL BORDER REGION 105 Degree Radius

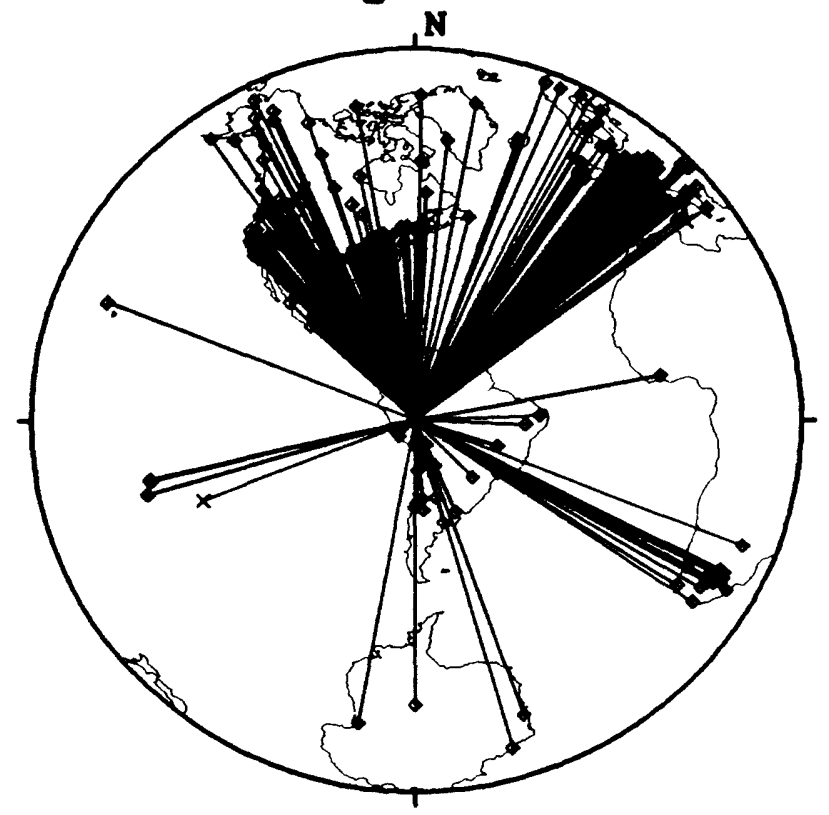

JUN 01, 1983 01:59:54 TONGA ISLANDS 10 Degree Radius

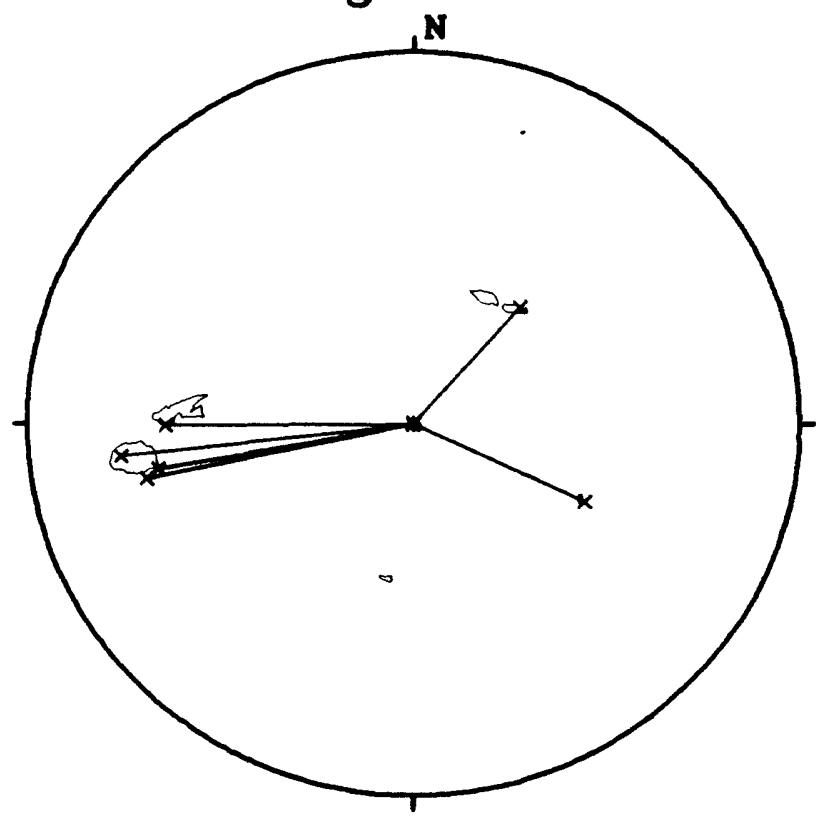

JUN 02, 1983 20:12:50 PERU-BRAZIL BORDER REGION 10 Degree Radius

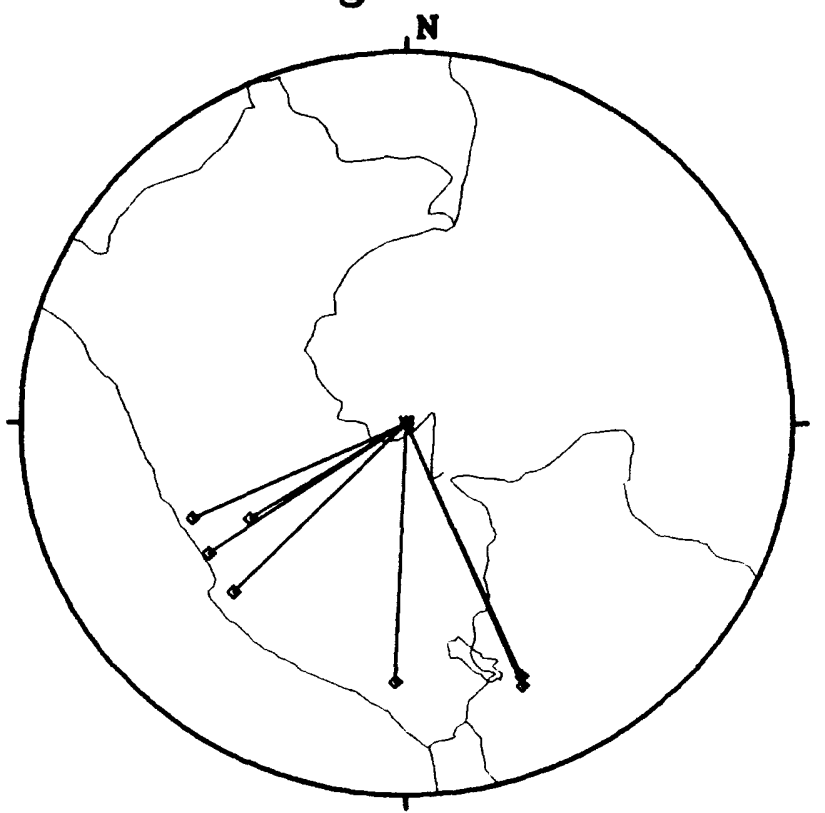


JUN 09, 1983 18:46:02 ANDREANOF ISLANDS, ALEUTIAN IS. 105 Degree Radius

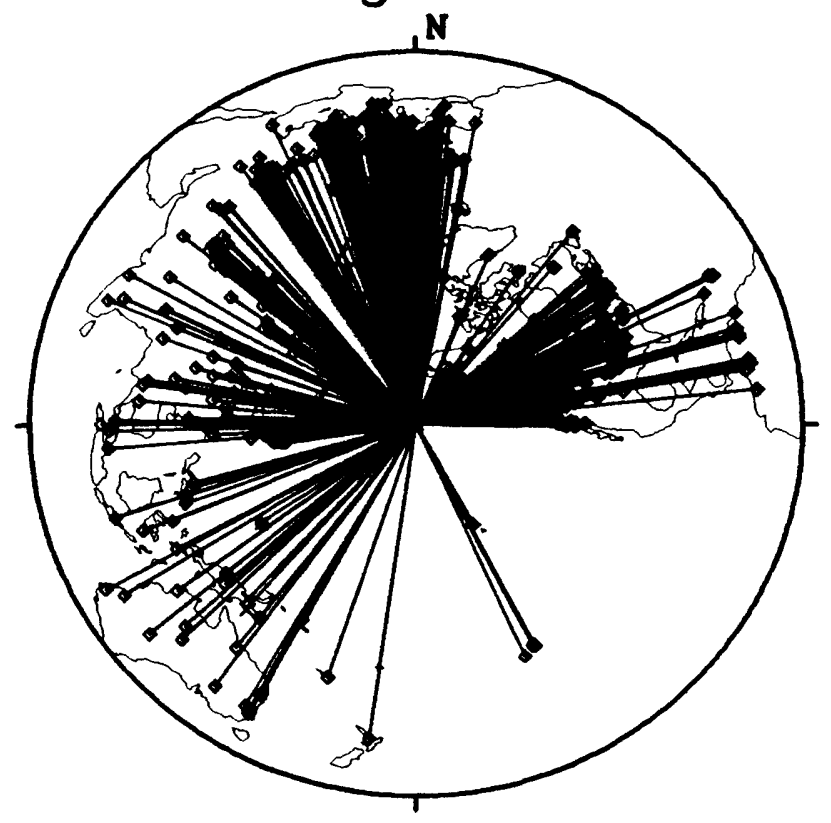

JUN 21, 1983 06:25:26 HOKKAIDO, JAPAN REGION 105 Degree Radius

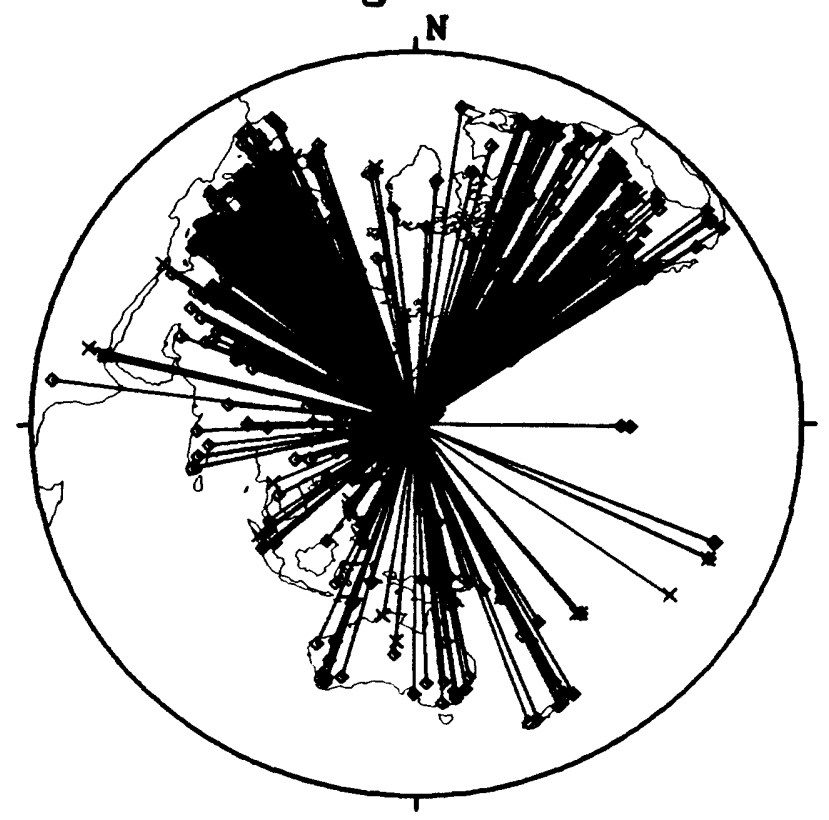

JUN 09, 1983 18:46:02 ANDREANOF ISLANDS, ALEUTLAN IS. 10 Degree Radius

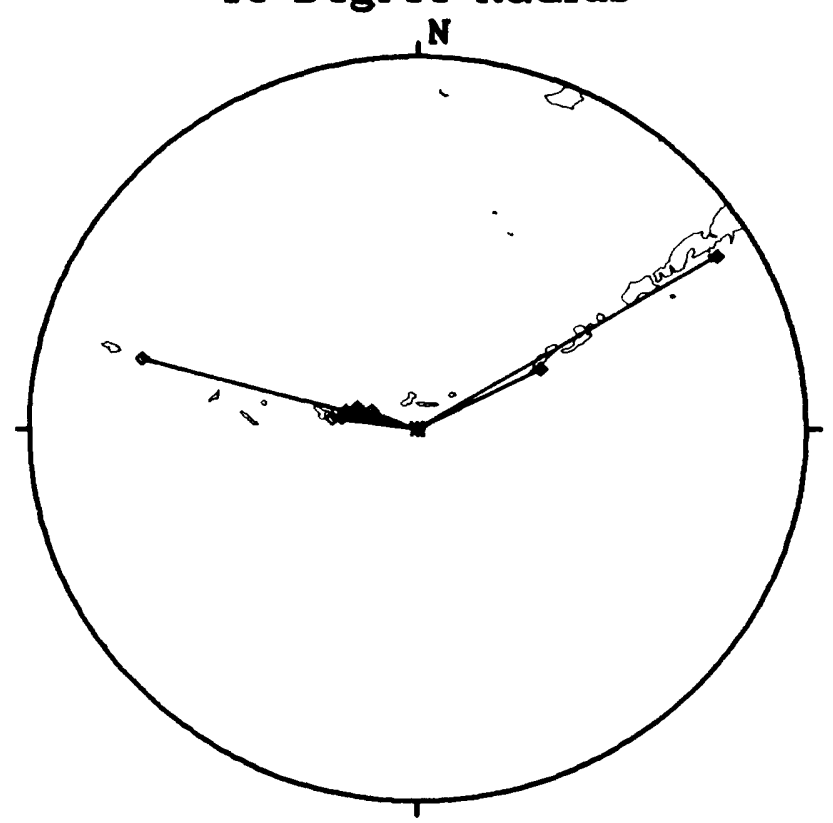

JUN 21, 1983 06:25:26 HOKKAIDO, JAPAN REGION 10 Degree Radius

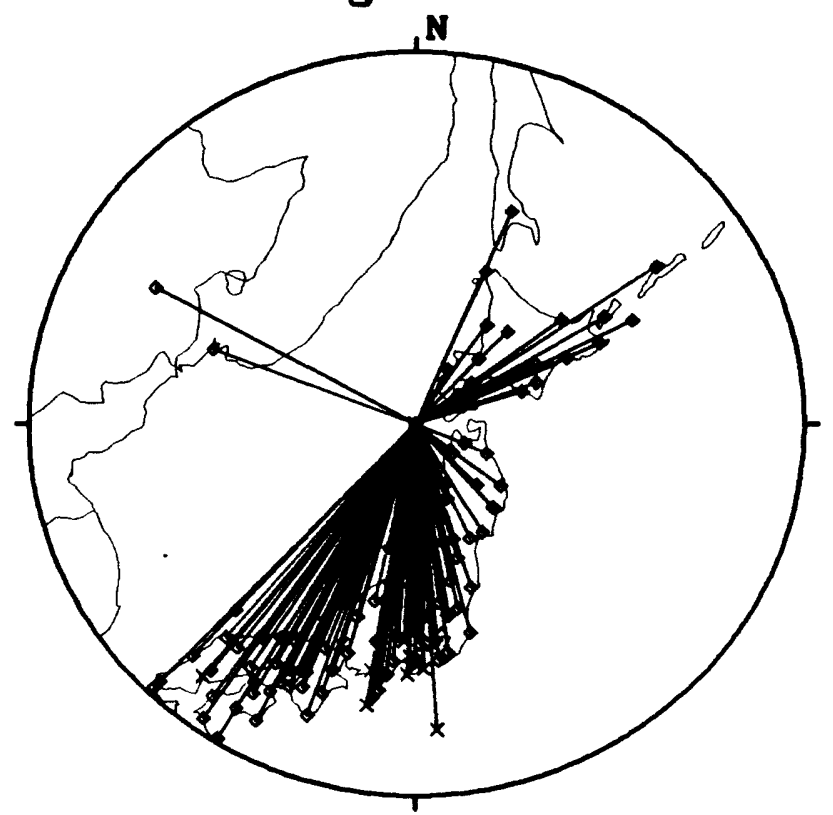


JUN 24, 1983 09:06:45 TAIWAN REGION 105 Degree Radius

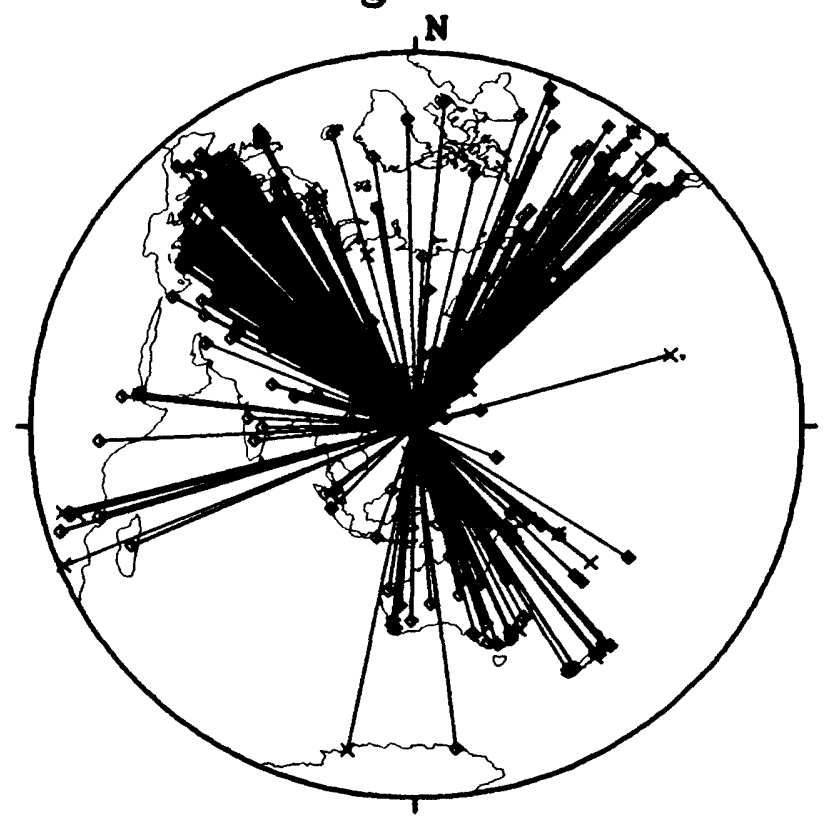

JUL 12, 1983 15:10:04 SOUTHERN ALASKA 105 Degree Radius

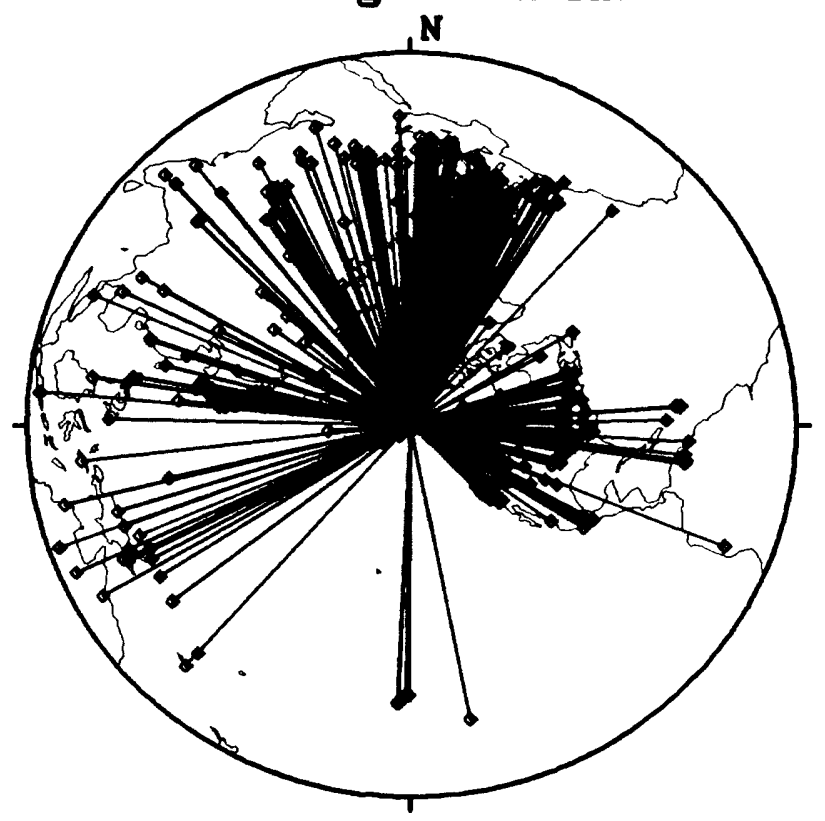

JUN 24, 1983 09:06:45 TAIWAN REGION

10 Degree Radius

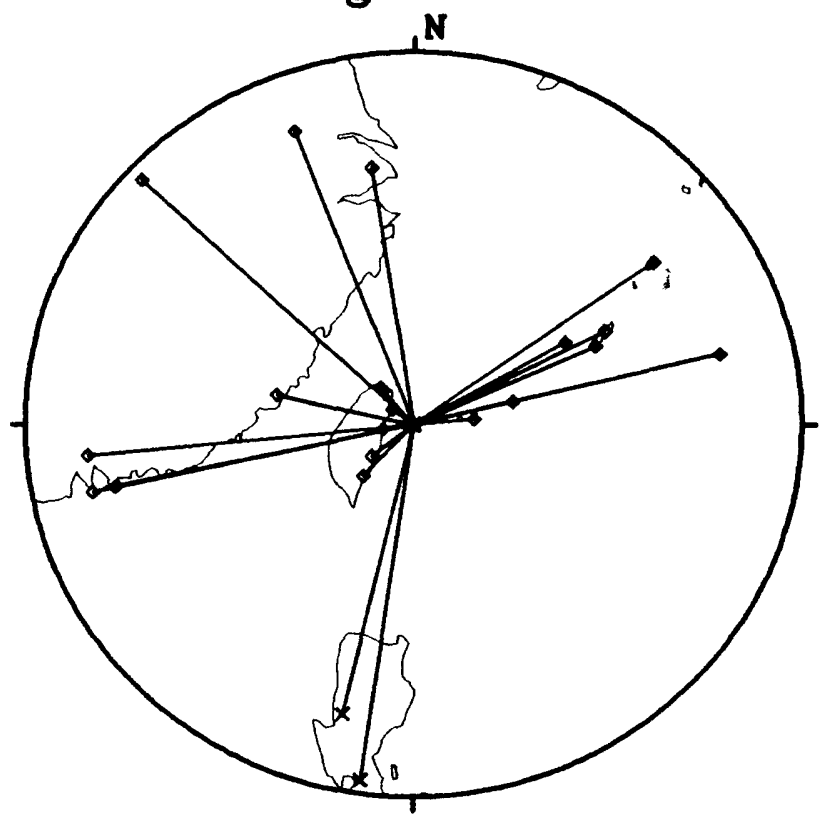

JUL 12, 1983 15:10:04 SOUTHERN ALASKA 10 Degree Radius

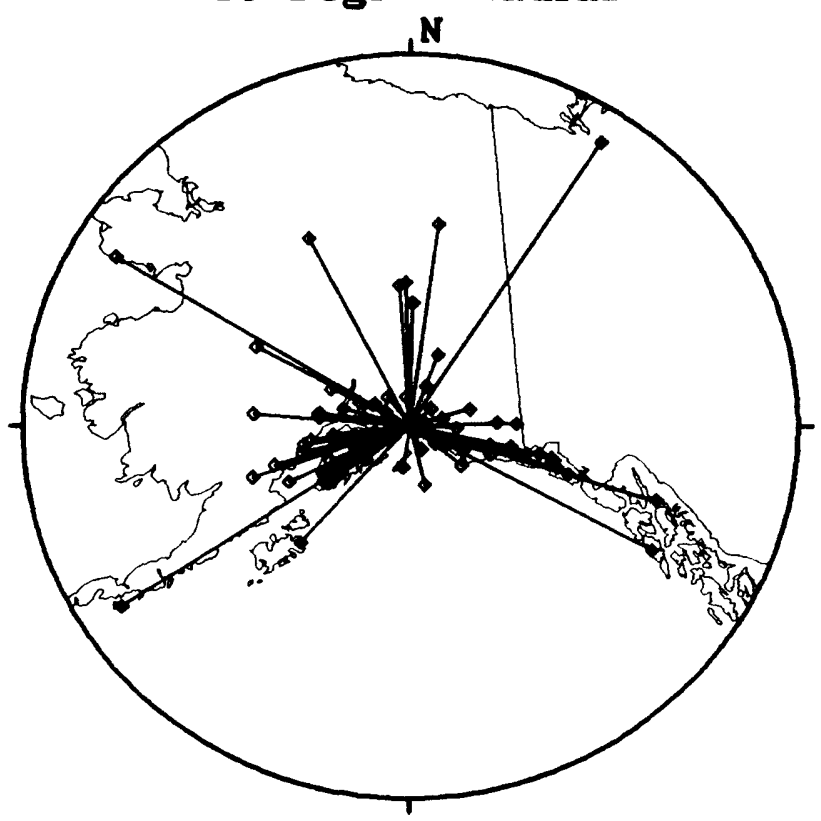


Figure 17

AUG 06, 1983 15:43:52

AEGEAN SEA

\section{Degree Radius}

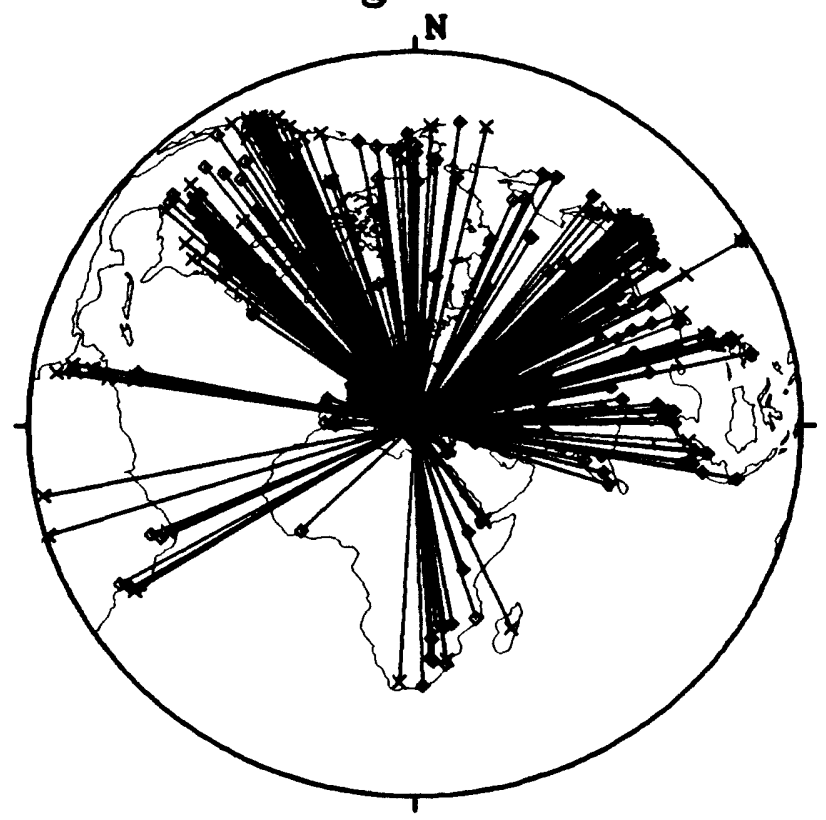

AUG 17, 1983 10:55:54 NEAR EAST COAST OF KAMCHATKA 105 Degree Radius

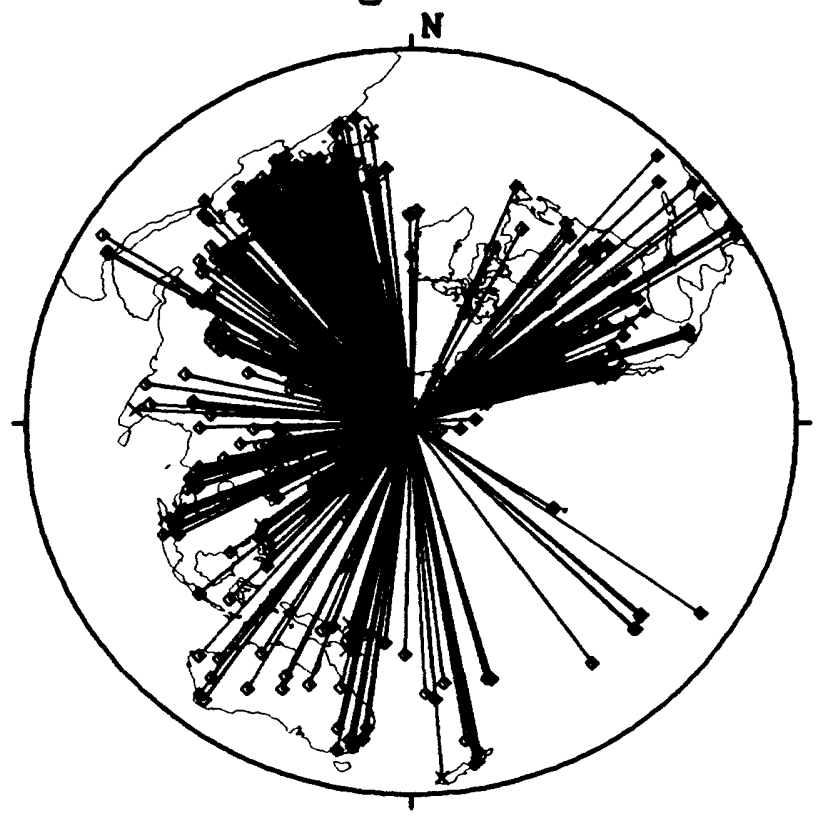

AUG 06, 1983 15:43:52 AEGEAN SEA

10 Degree Radius

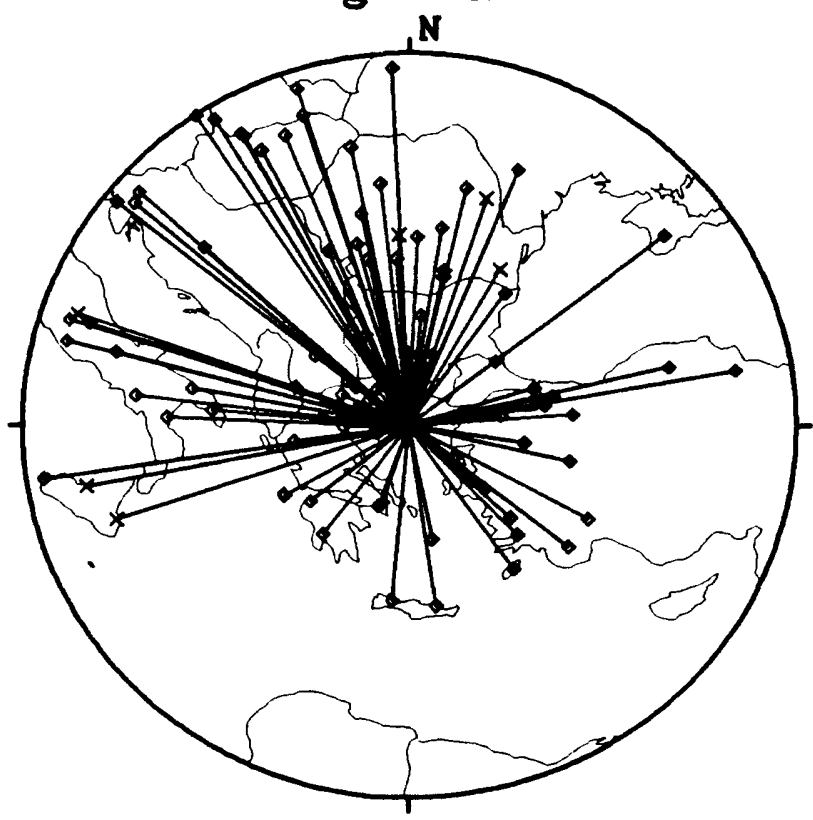

AUG 17, 1983 10:55:54 NEAR EAST COAST OF KAMCHATKA 10 Degree Radius

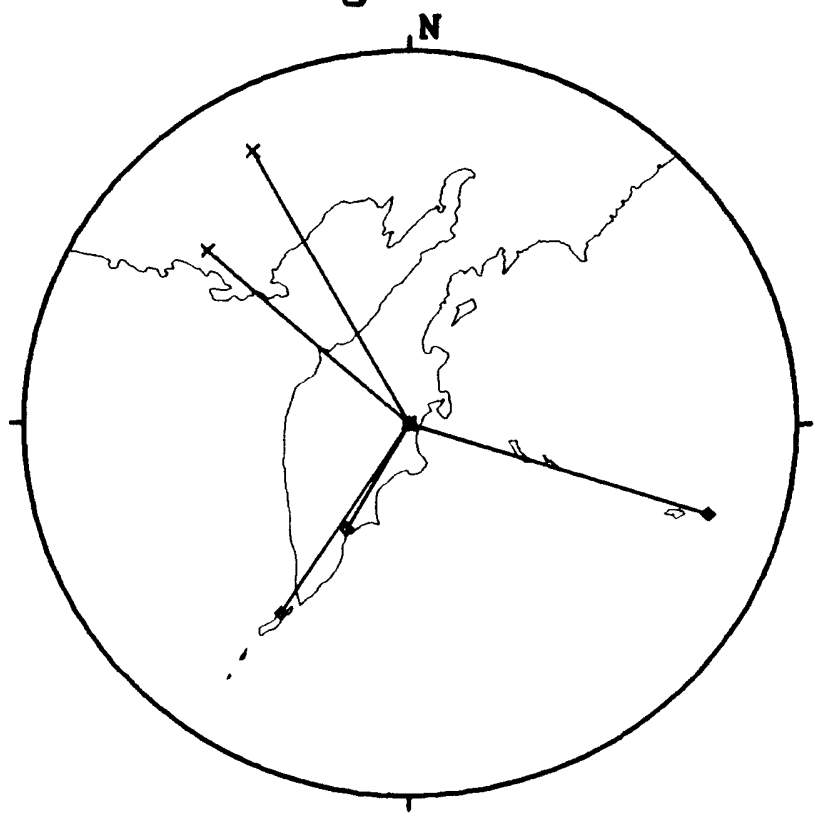


Figure 18

SEP 07, 1983 19:22:05 SOUTHERN ALASKA 105 Degree Radius

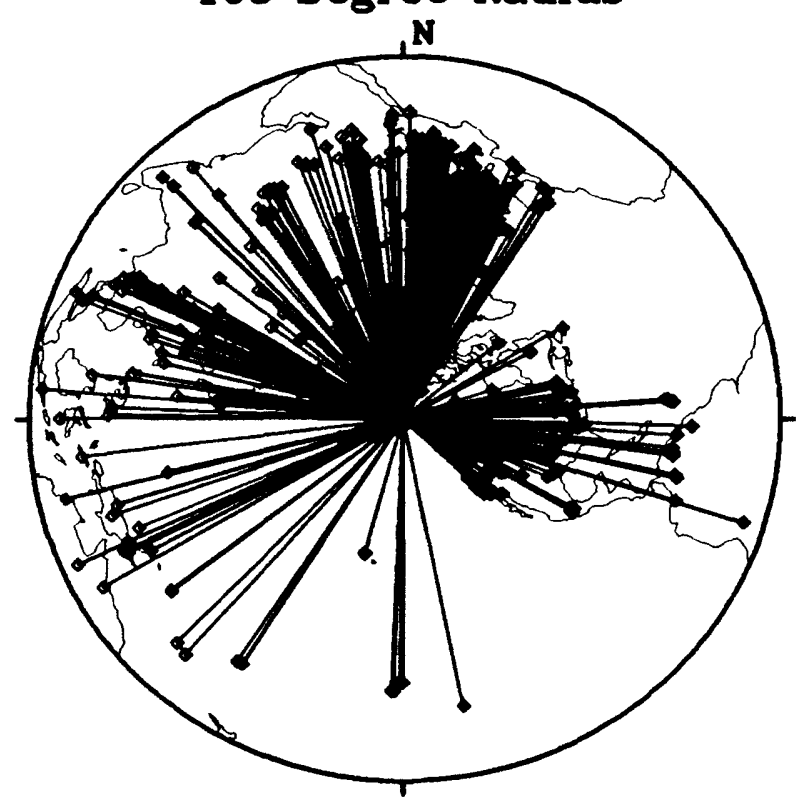

SEP 12, 1983 15:42:08 AFGHANISTAN-USSR BORDER REGION 105 Degree Radius

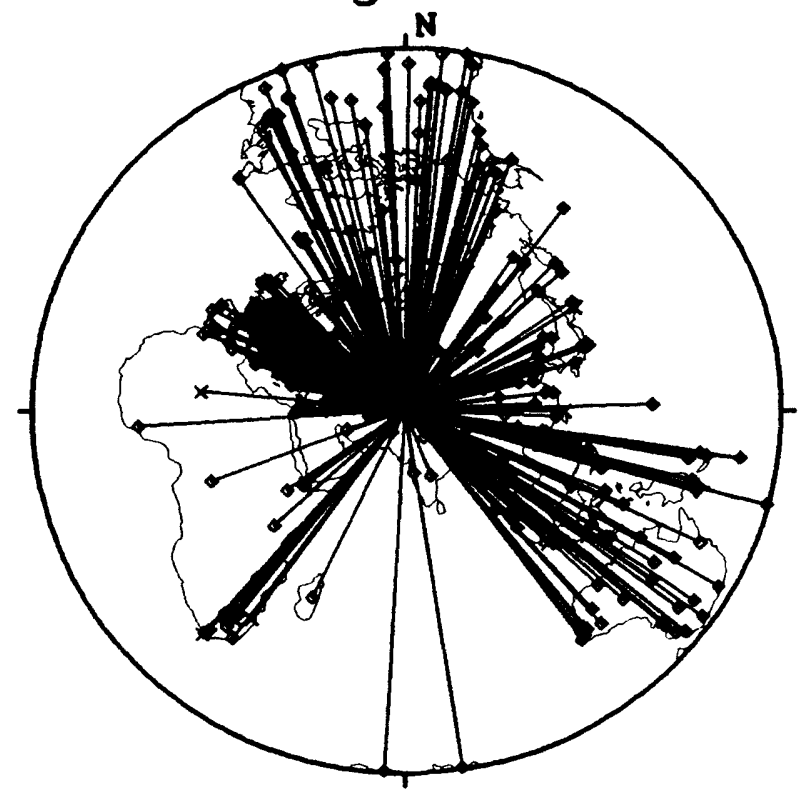

SEP 07, 1983 19:22:05 SOUTHERN ALASKA 10 Degree Radius

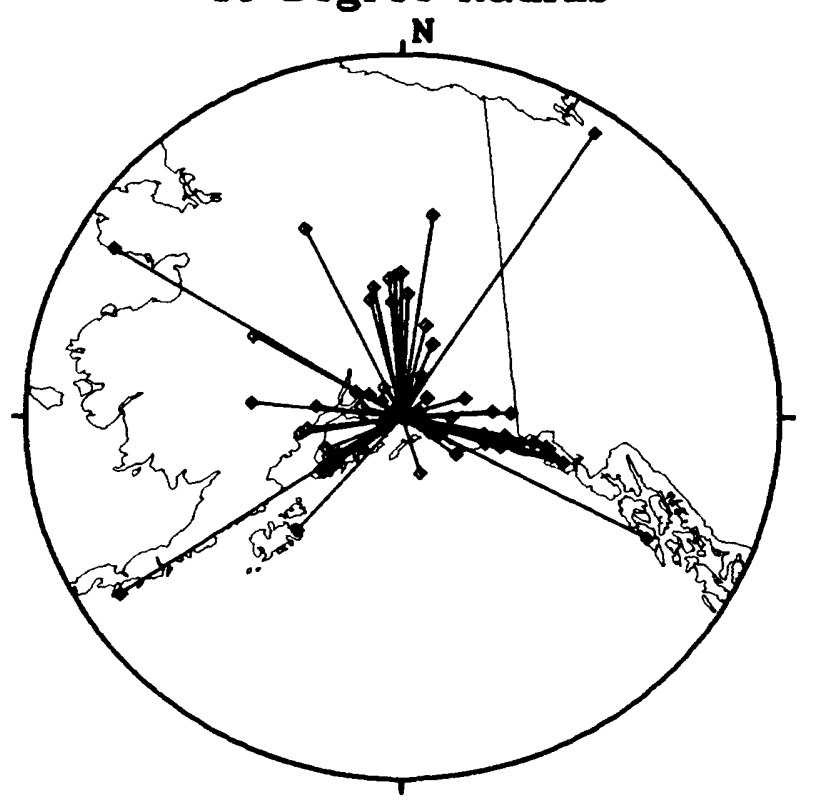

SEP 12, 1983 15:42:08 AFGHANISTAN-USSR BORDER REGION 10 Degree Radius

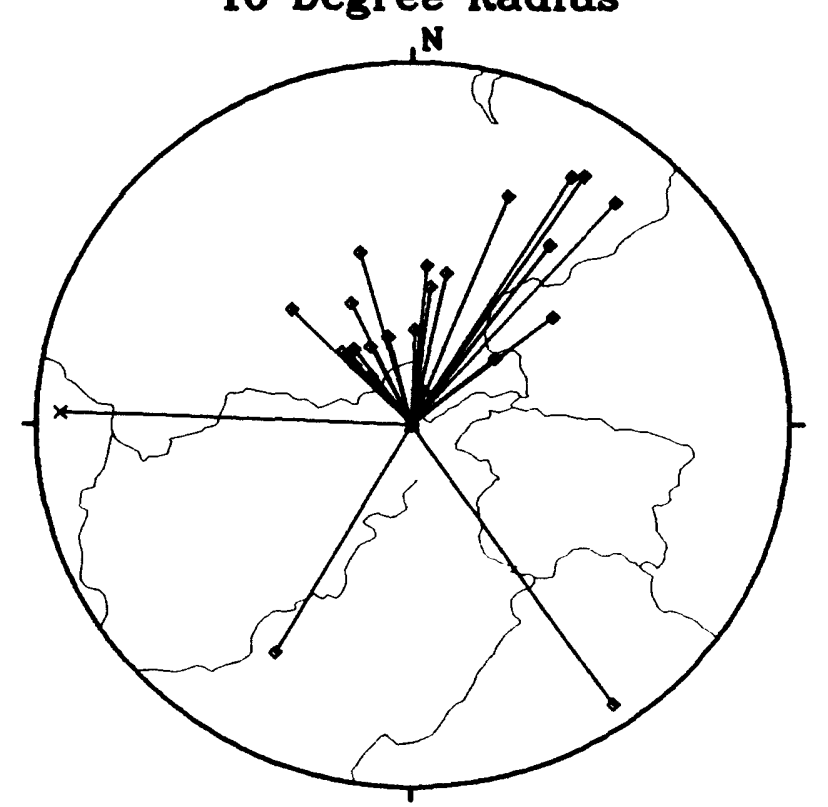


OCT 04, 1983 18:52:12 NEAR COAST OF NORTHERN CHILE 105 Degree Radius

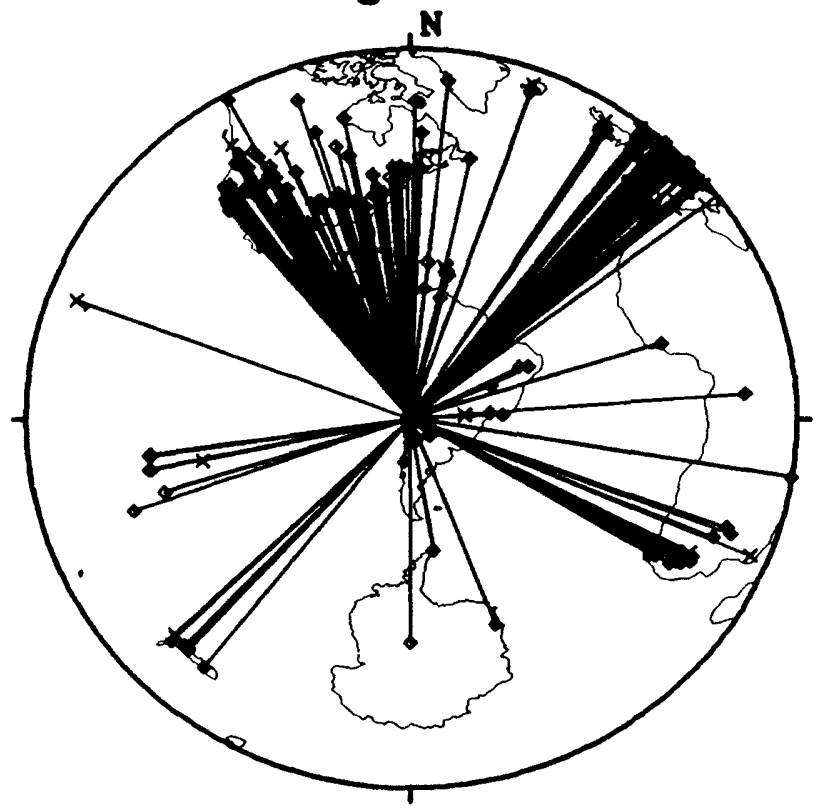

OCT 09, 1983 11:25:38

NEAR COAST OF NORTHERN CHILE 105 Degree Radius

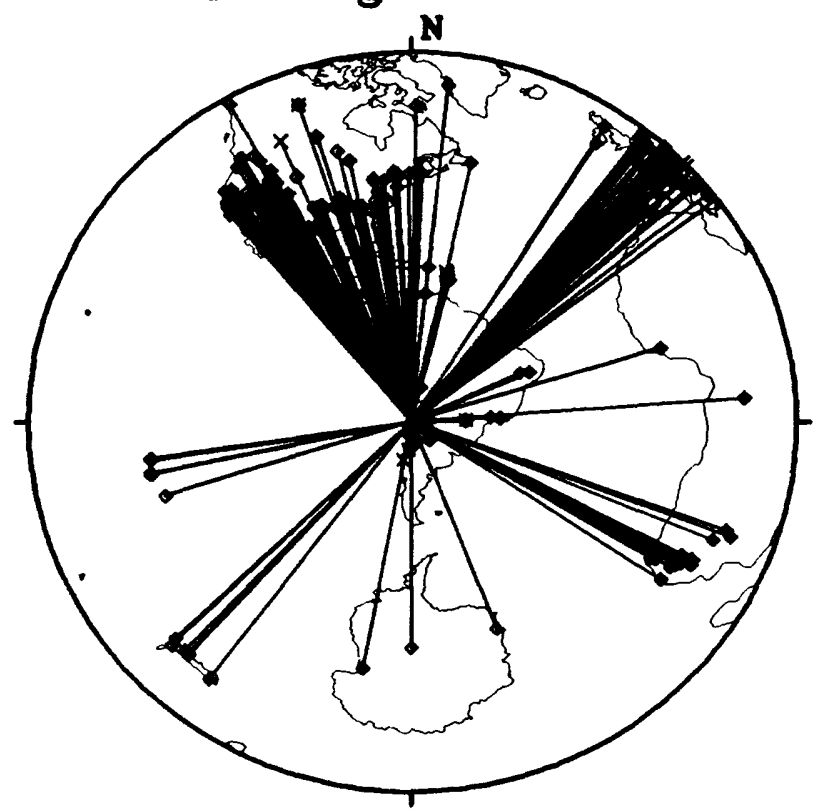

OCT 04, 1983 18:52:12 NEAR COAST OF NORTHERN CHILE 10 Degree Radius

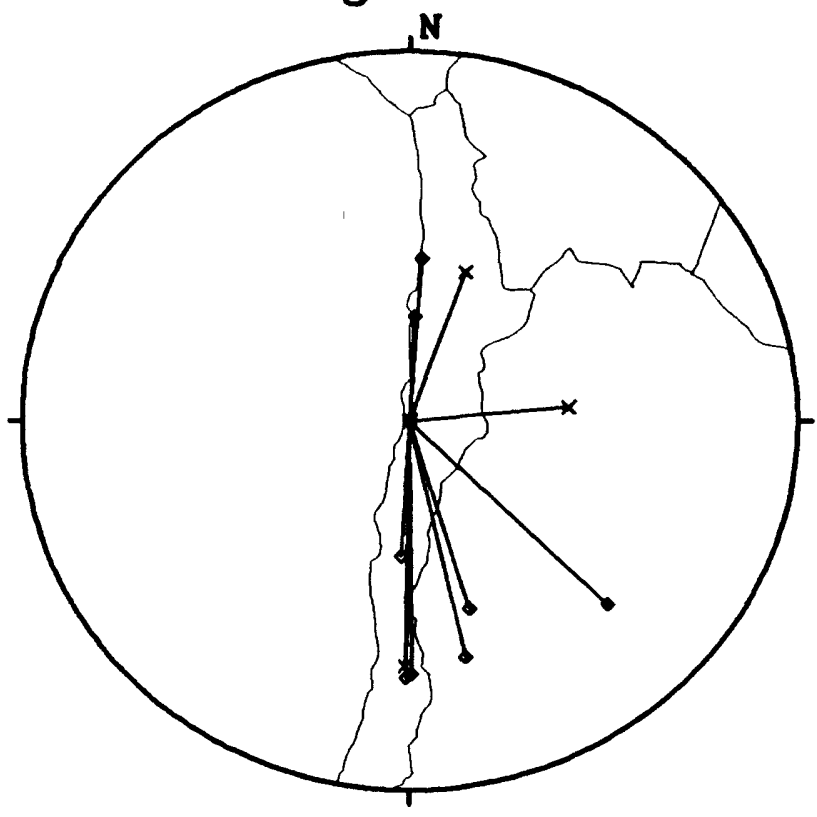

OCT 09, 1983 11:25:38 NEAR COAST OF NORTHERN CHILE 10 Degree Radius

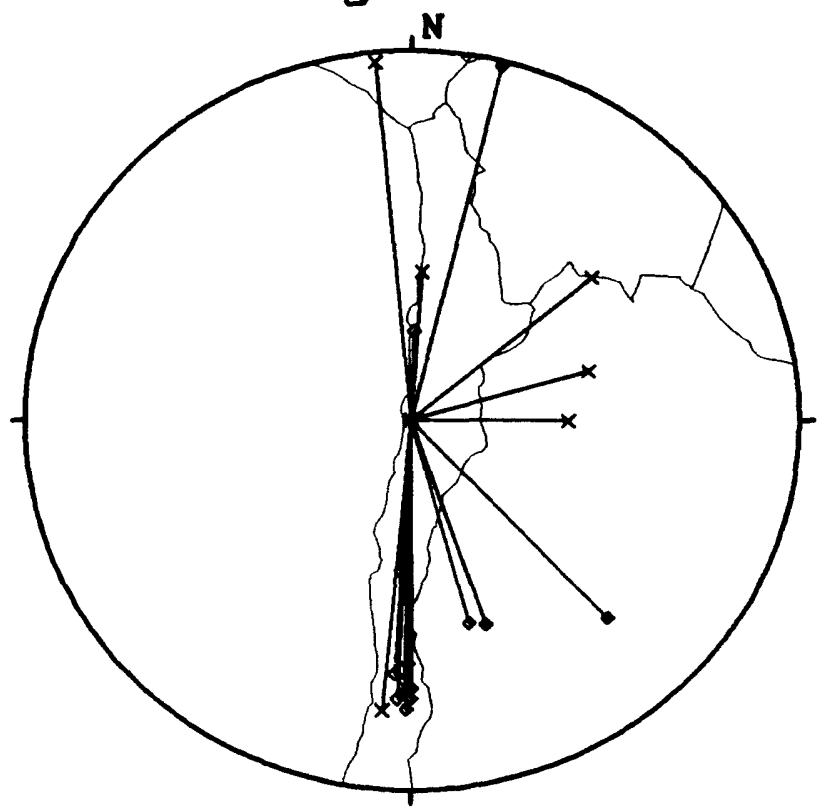


Figure 20

OCT 17, 1983 19:36:22 NORTH ATLANTIC OCEAN 105 Degree Radius

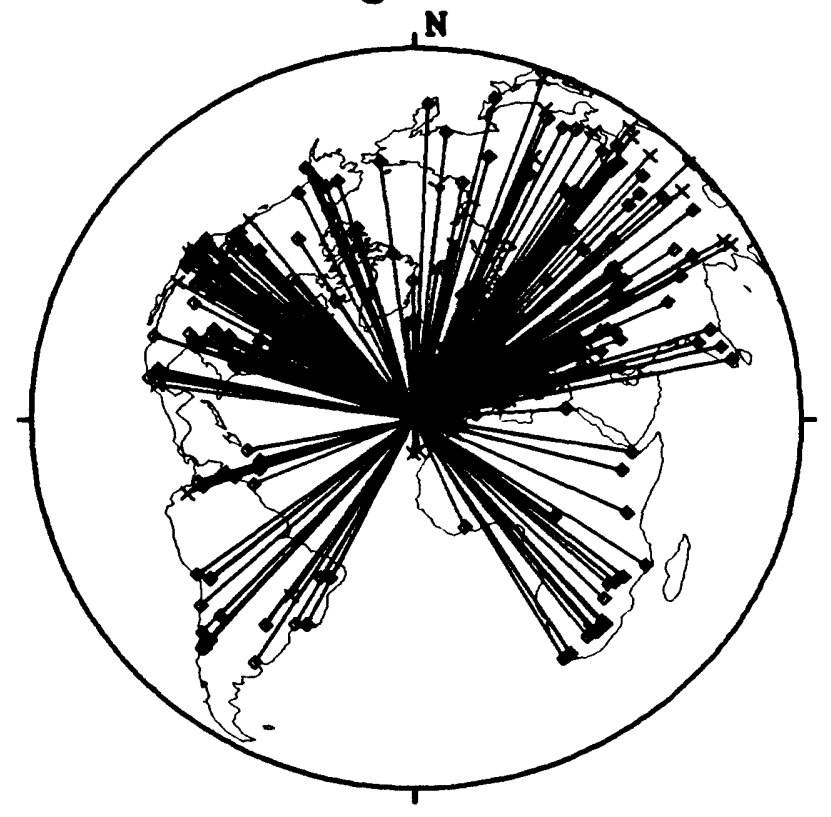

OCT 17, 1983 19:36:22 NORTH ATLANTIC OCEAN 10 Degree Radius

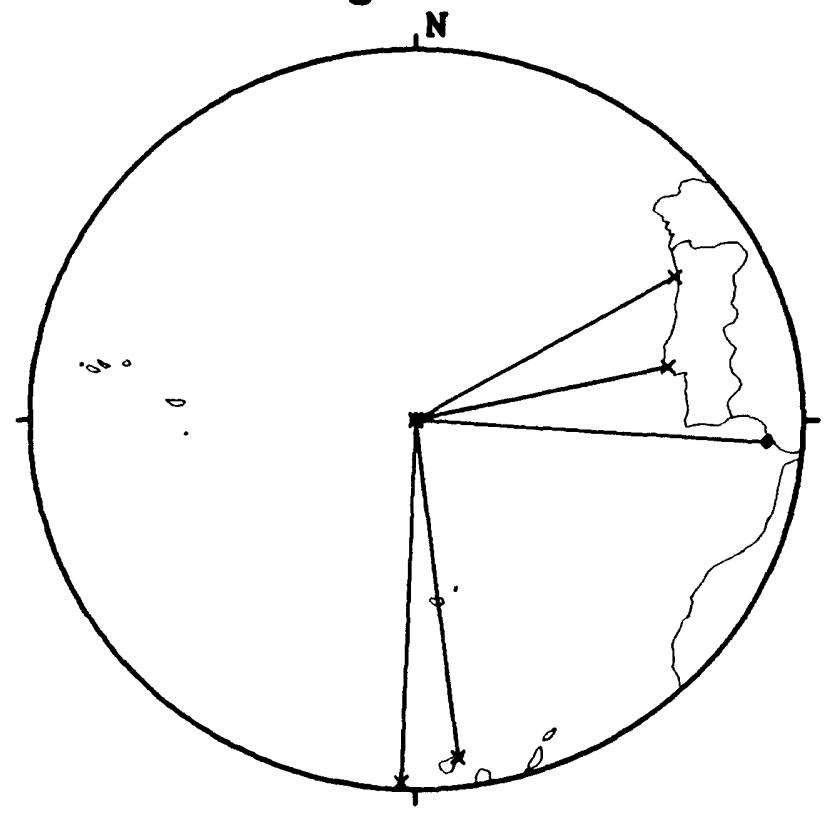

OCT 22, 1983 04:21:35 SOUTH SANDWICH ISLANDS REGION 105 Degree Radius

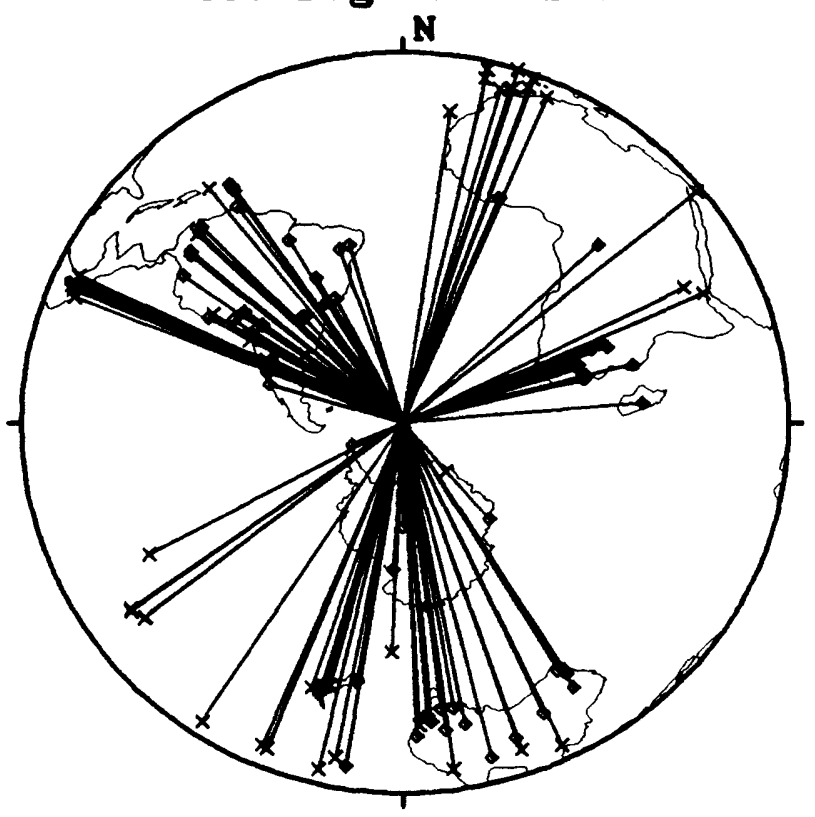

OCT 22, 1983 04:21:35 SOUTH SANDWICH ISLANDS REGION 20 Degree Radius

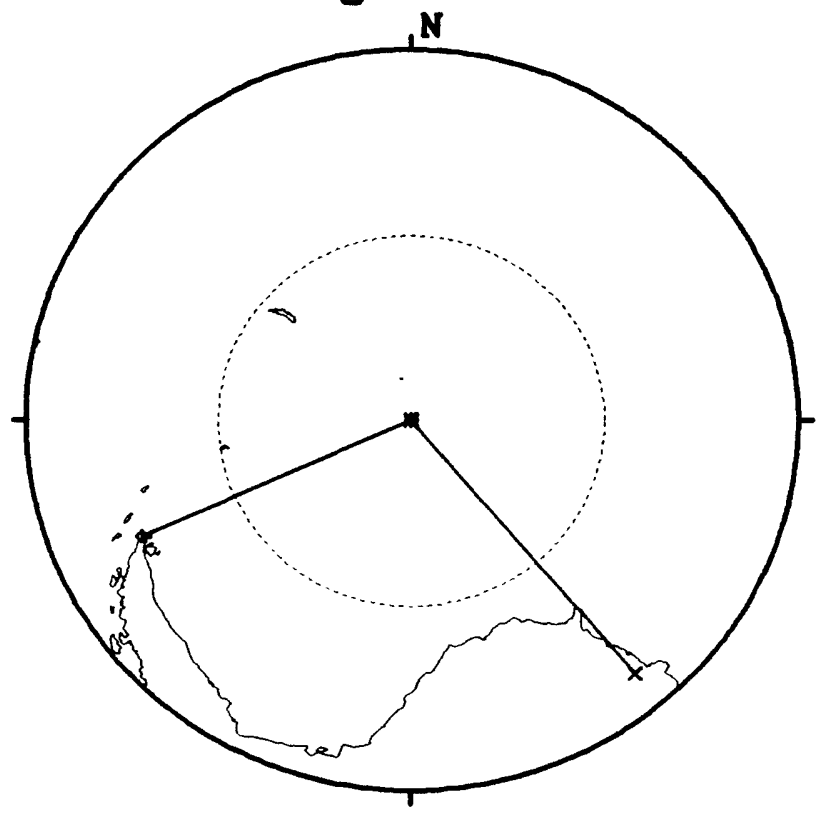


Figure 21

OCT 30, 1983 04:12:28 TURKEY

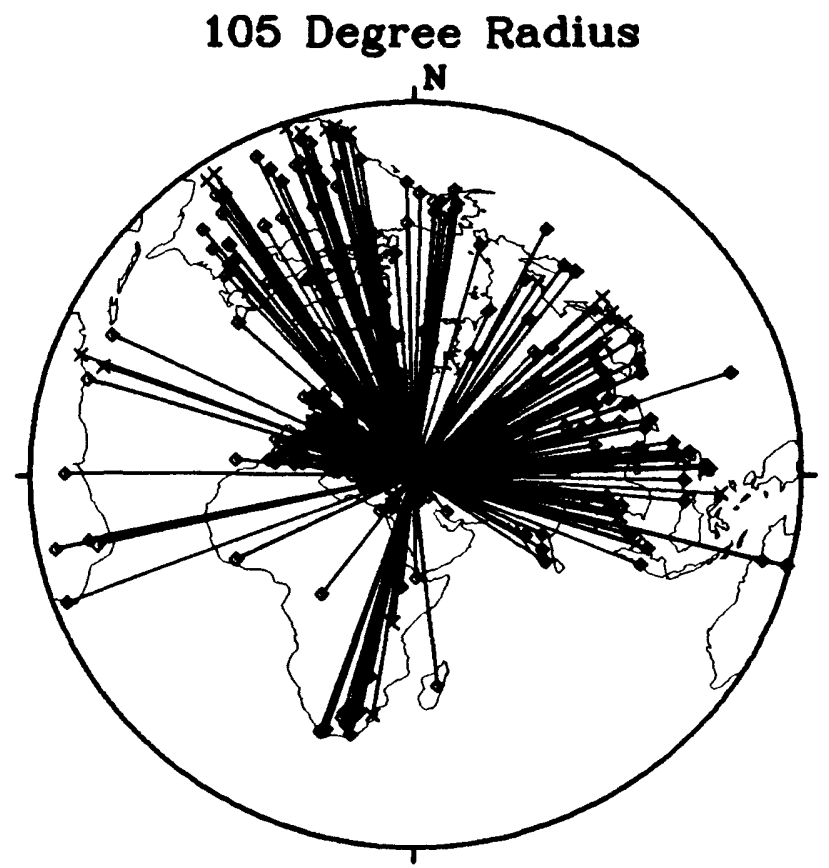

NOV 16, $198316: 13: 00$ HAWAII 105 Degree Radius

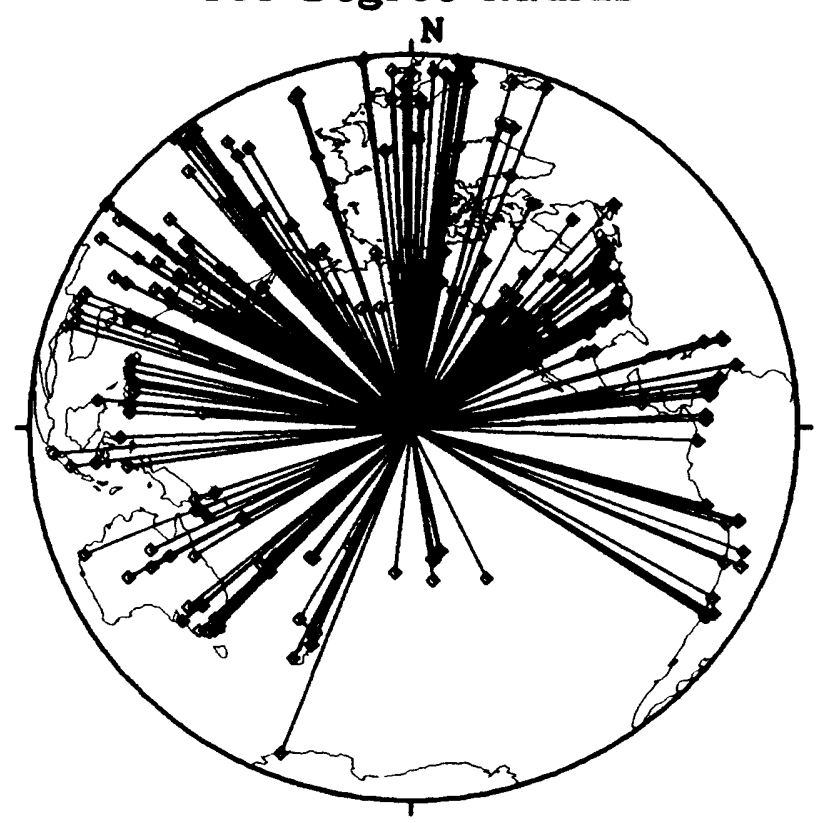

OCT 30, 1983 04:12:28 TURKEY 10 Degree Radius

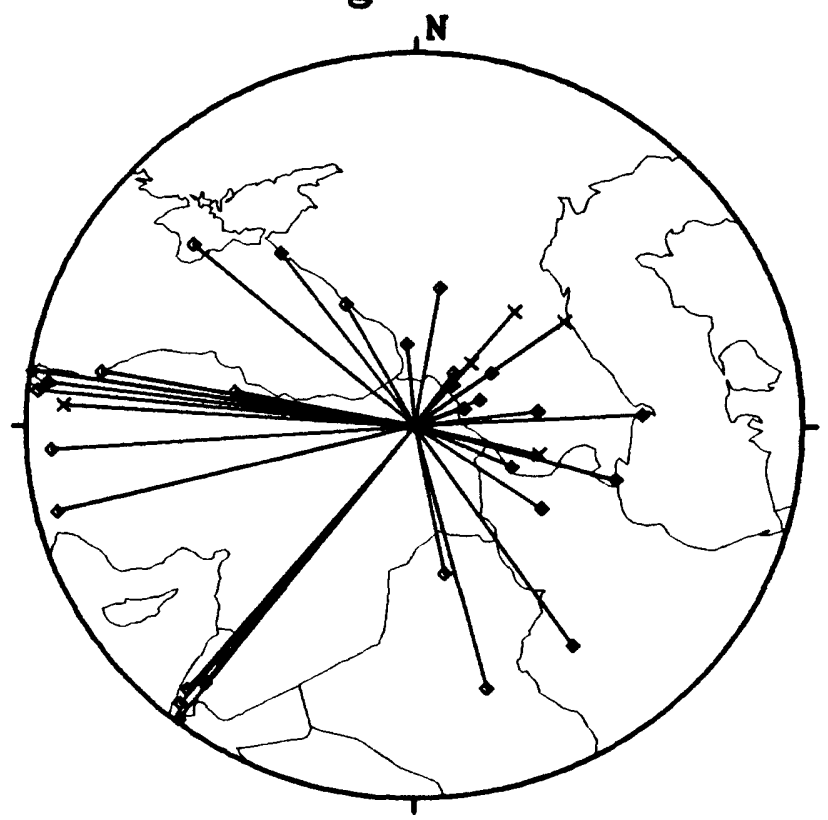

NOV $16,198316: 13: 00$ HAWAII

10 Degree Radius

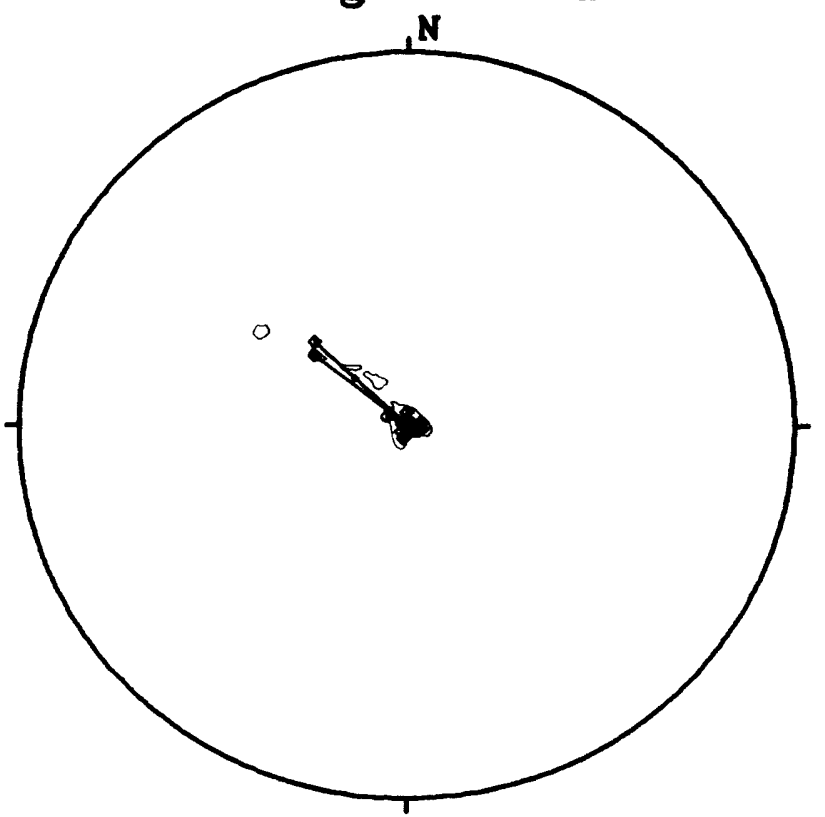


Figure 22

NOV 24, 1983 05:30:36

$$
\text { BANDA SEA }
$$

105 Degree Radius

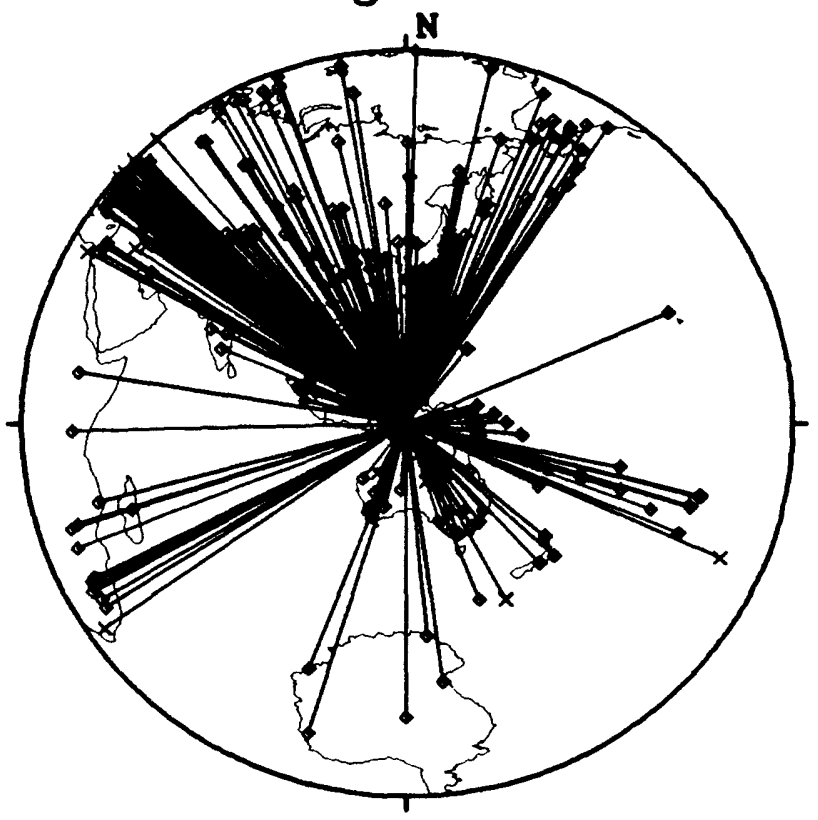

NOV 30, 1983 17:46:00 CHAGOS ARCHIPELAGO REGION 105 Degree Radius

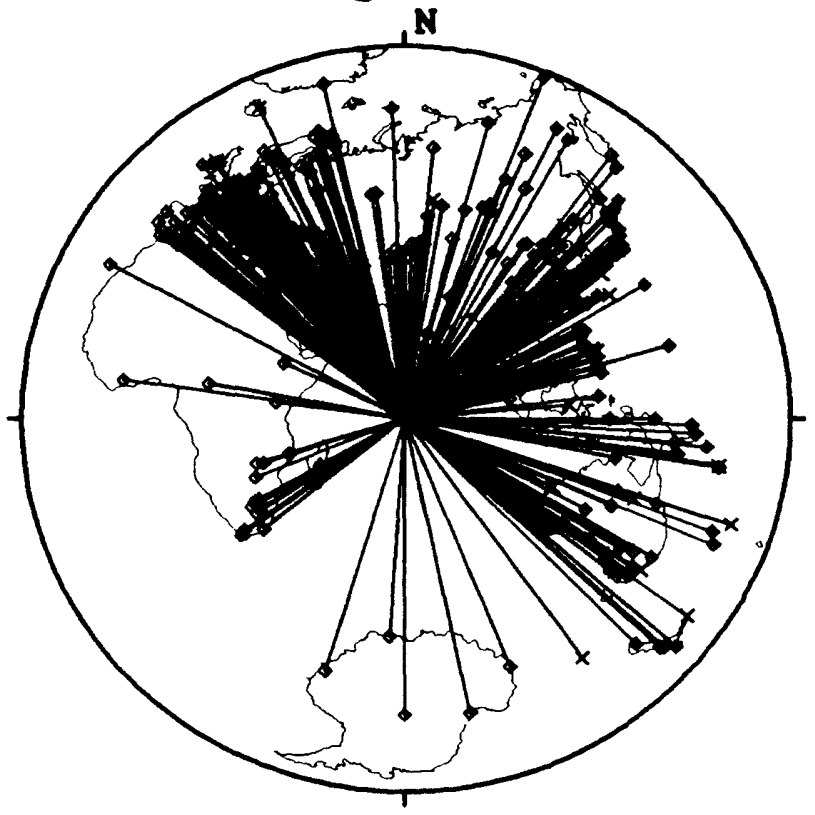

NOV 24, 1983 05:30:36 BANDA SEA

10 Degree Radius

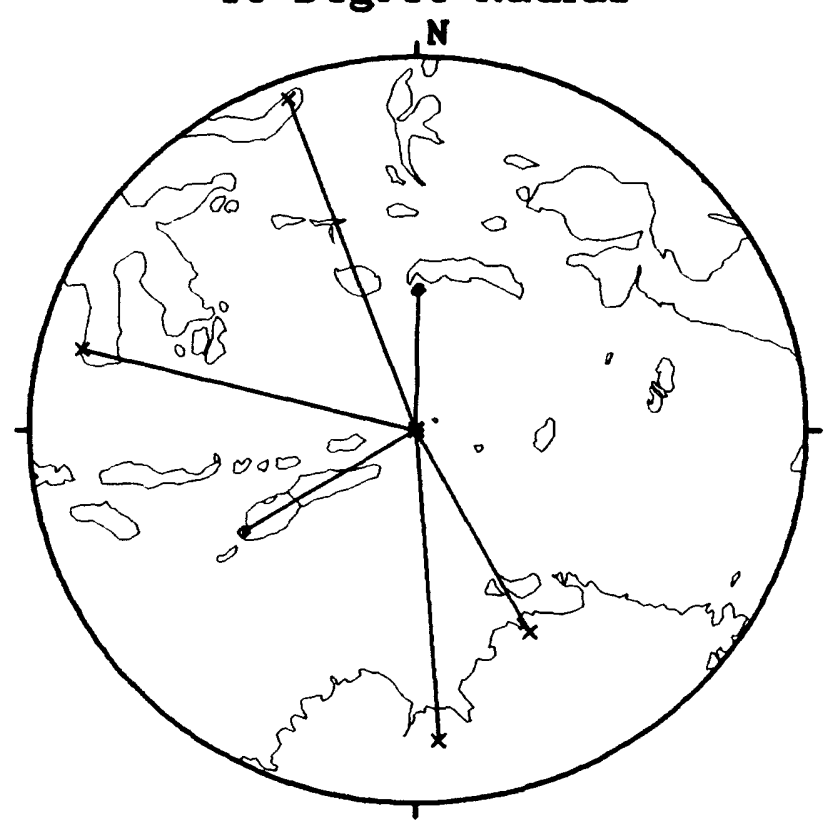

NOV 30, 1983 17:46:00 CHAGOS ARCHIPELAGO REGION 20 Degree Radius

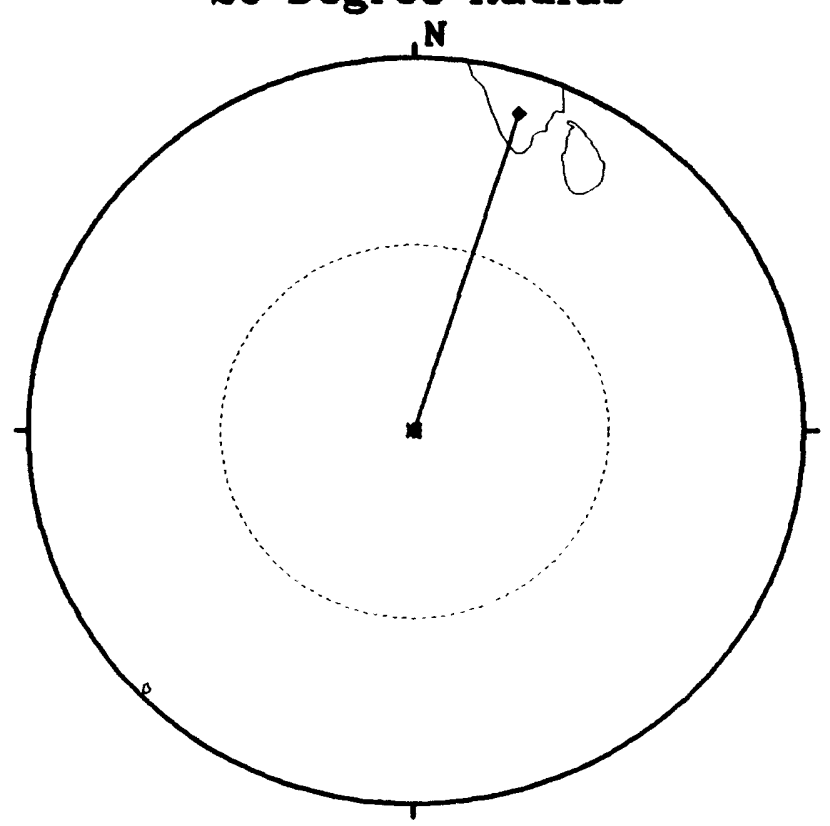


Figure 23

DEC 22, 1983 04:11:29 NORTHWEST AFRICA 105 Degree Radius

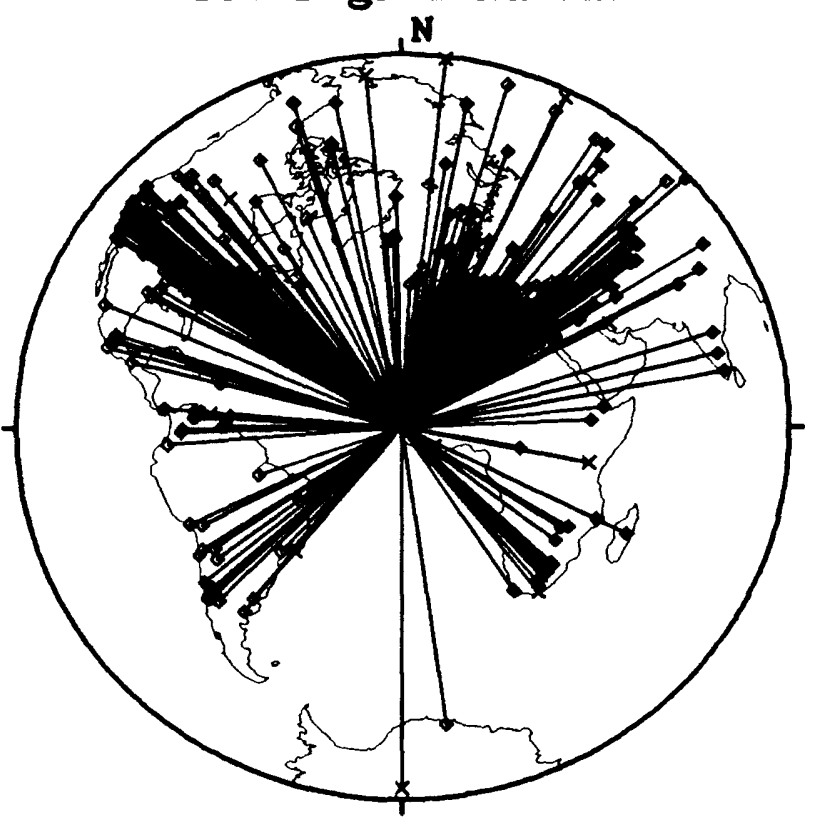

DEC 30, 1983 23:52:39 HINDU KUSH REGION 105 Degree Radius

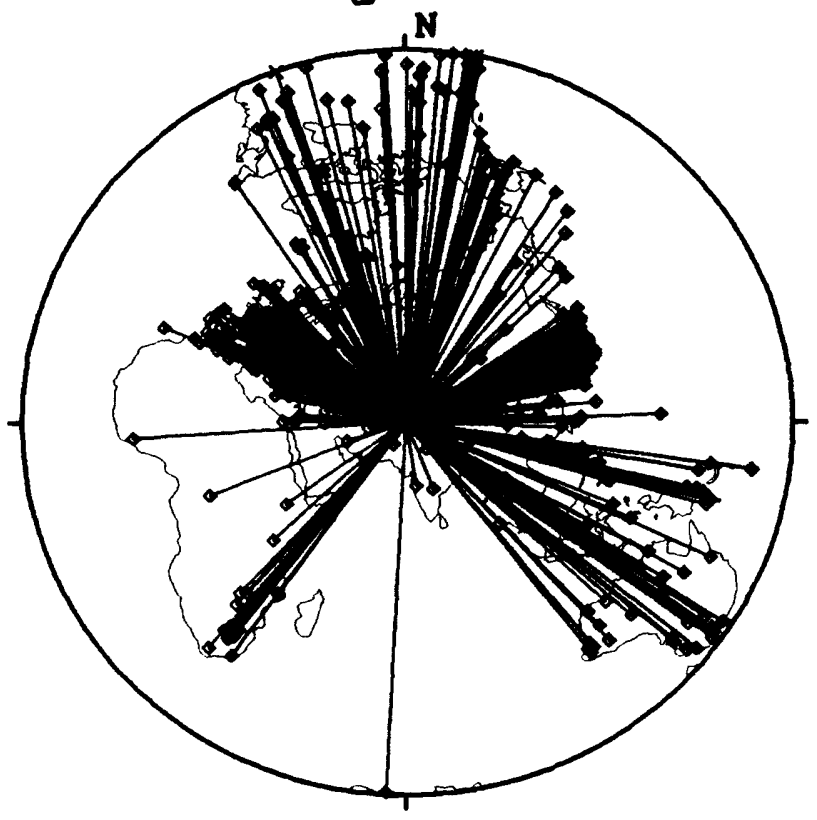

DEC 22, 1983 04:11:29 NORTHWEST AFRICA 10 Degree Radius

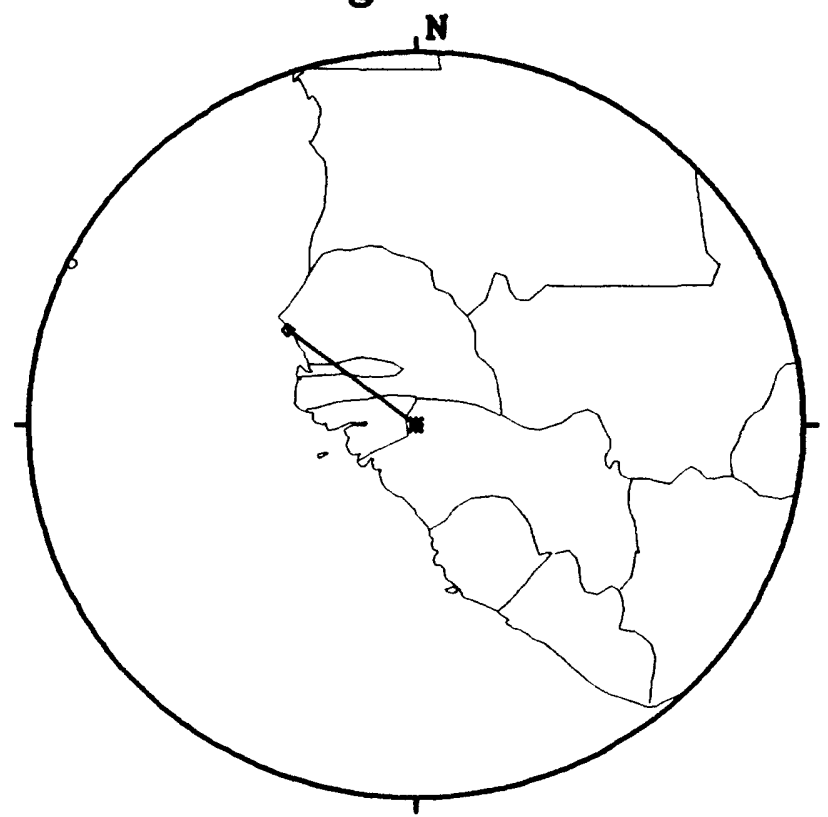

DEC 30, 1983 23:52:39 HINDU KUSH REGION 10 Degree Radius

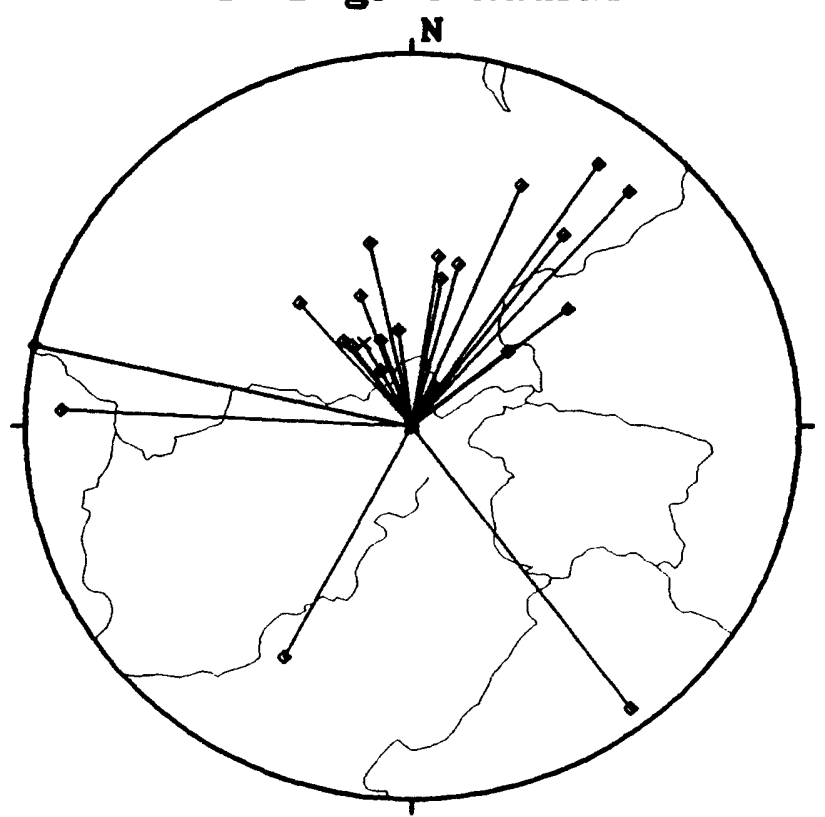


JAN 01, 1984 09:03:37

NEAR S. COAST OF SOUTHERN HONSHU

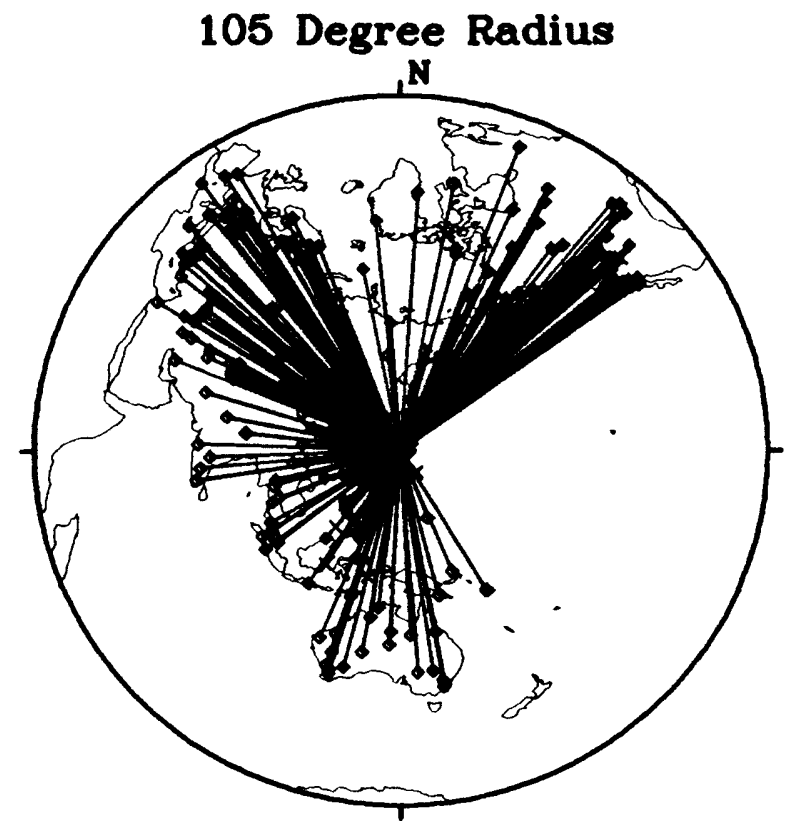

JAN 01, 1984 08:03:37

NEAR S. COAST OF SOUTHERN HONSHU 10 Degree Radius

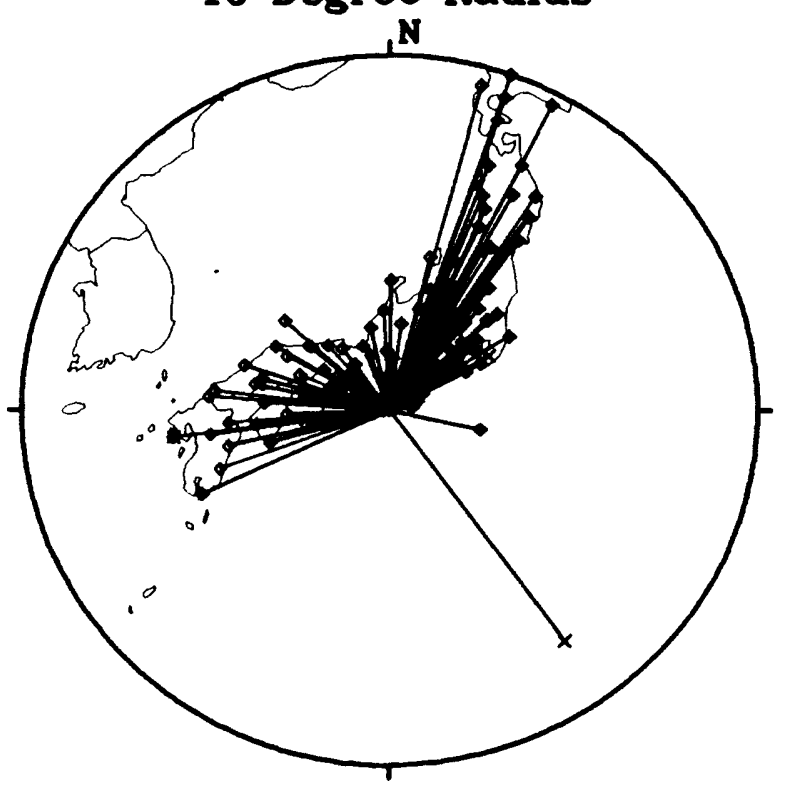

JAN 01, 1984 09:03:41

NEAR S. COAST OF SOUTHERN HONSHU

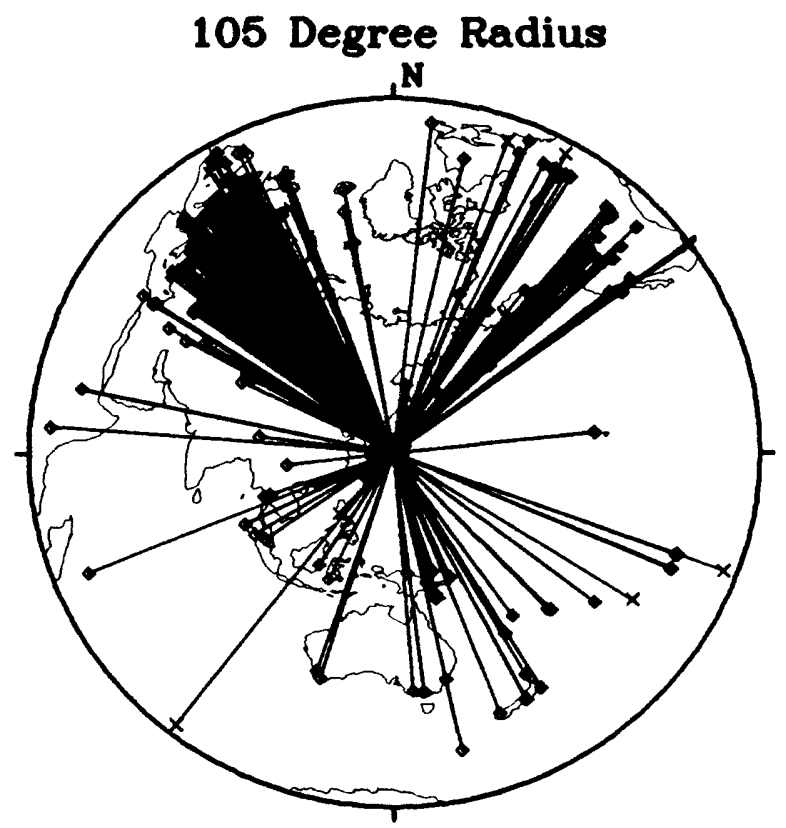

JAN 01, 1984 09:03:41

NEAR S. COAST OF SOUTHERN HONSHU 10 Degree Radius

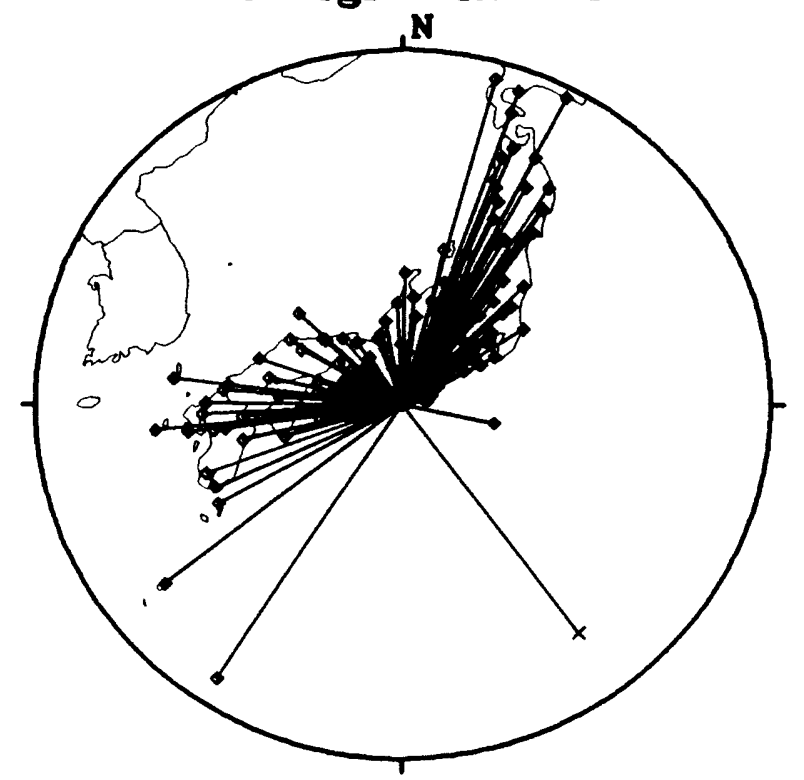


FEB 07, 1984 21:33:20 SOLOMON ISLANDS 105 Degree Radius

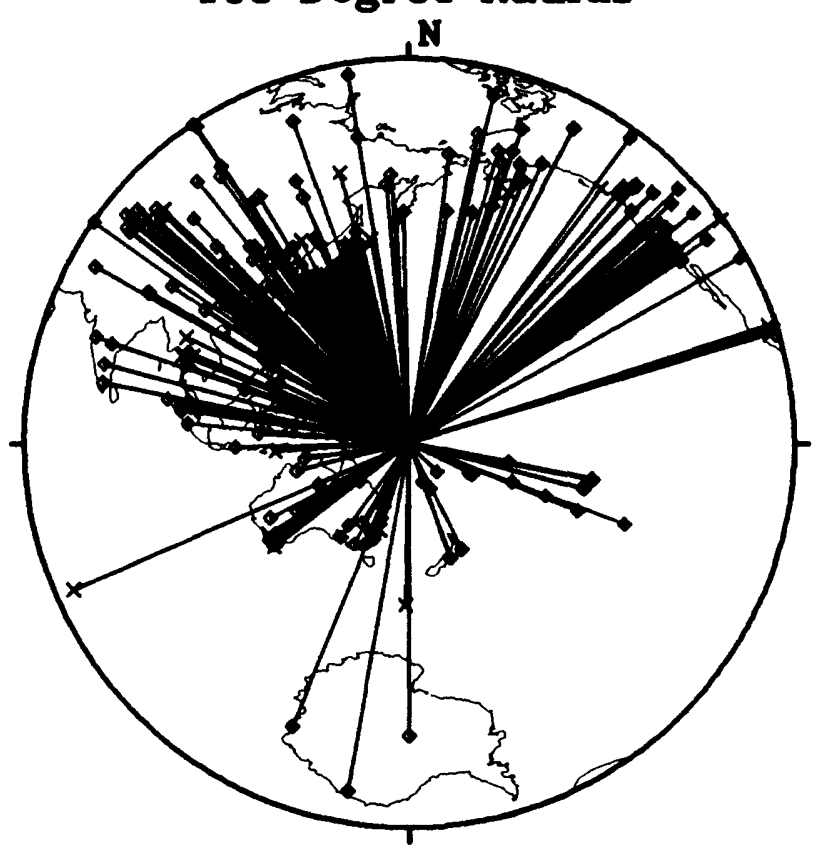

MAR 05, 1984 03:33:50 MINDANAO, PHILIPPINE ISLANDS 105 Degree Radius

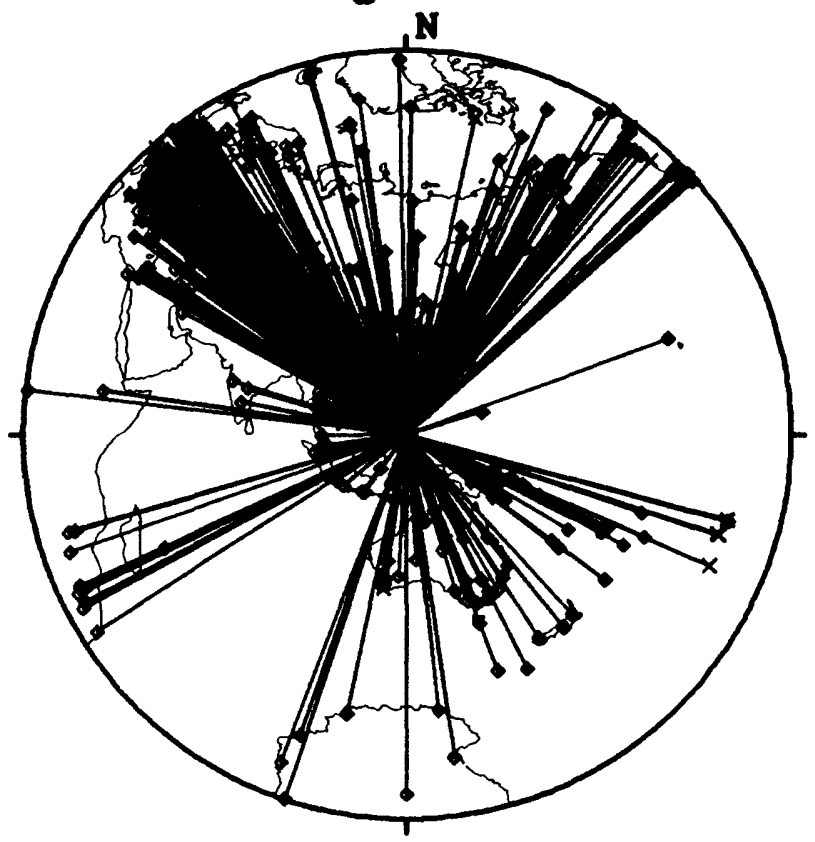

FEB 07, 1984 21:33:20 SOLOMON ISLANDS 10 Degree Radius

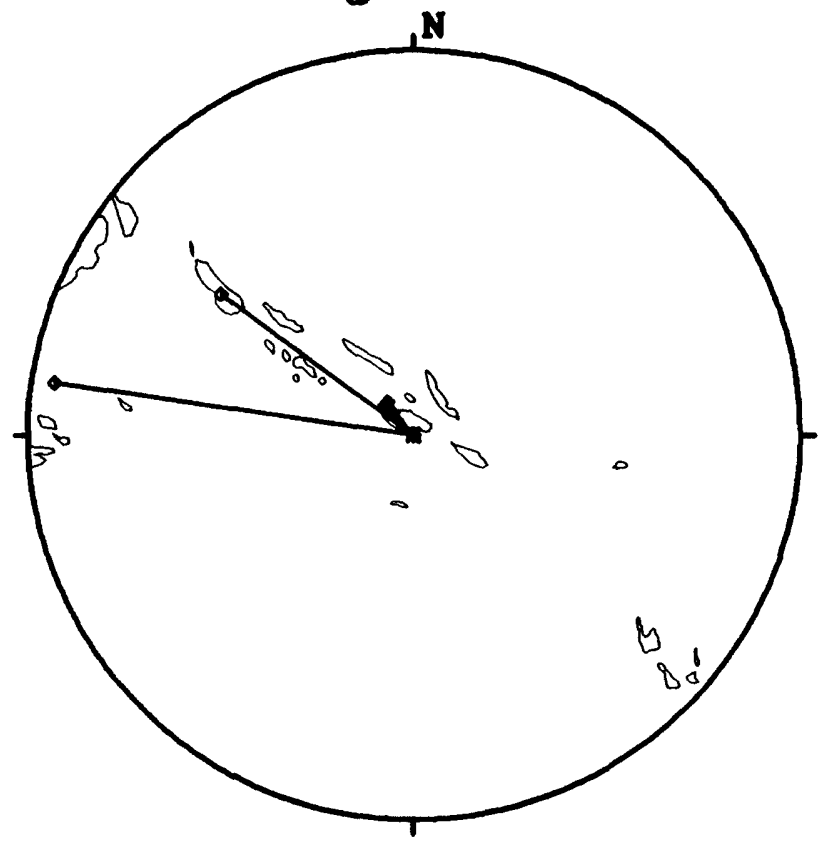

MAR 05, 1884 03:33:50 MINDANAO, PHILIPPINE ISLANDS 10 Degree Radius

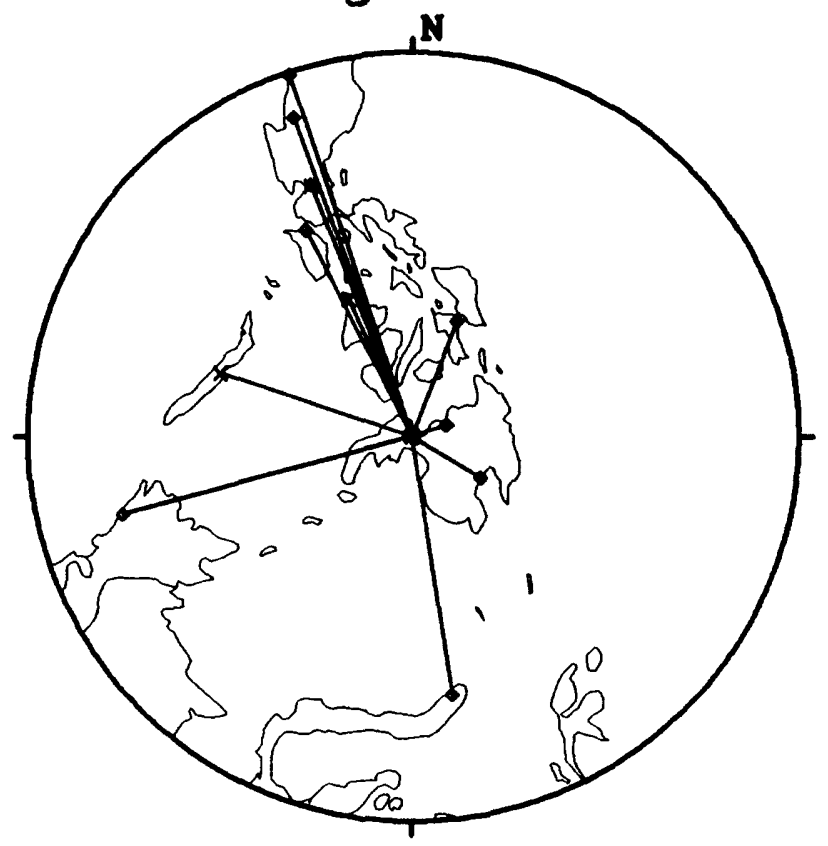


MAR 19, 1984 20:28:38 UZBEK SSR

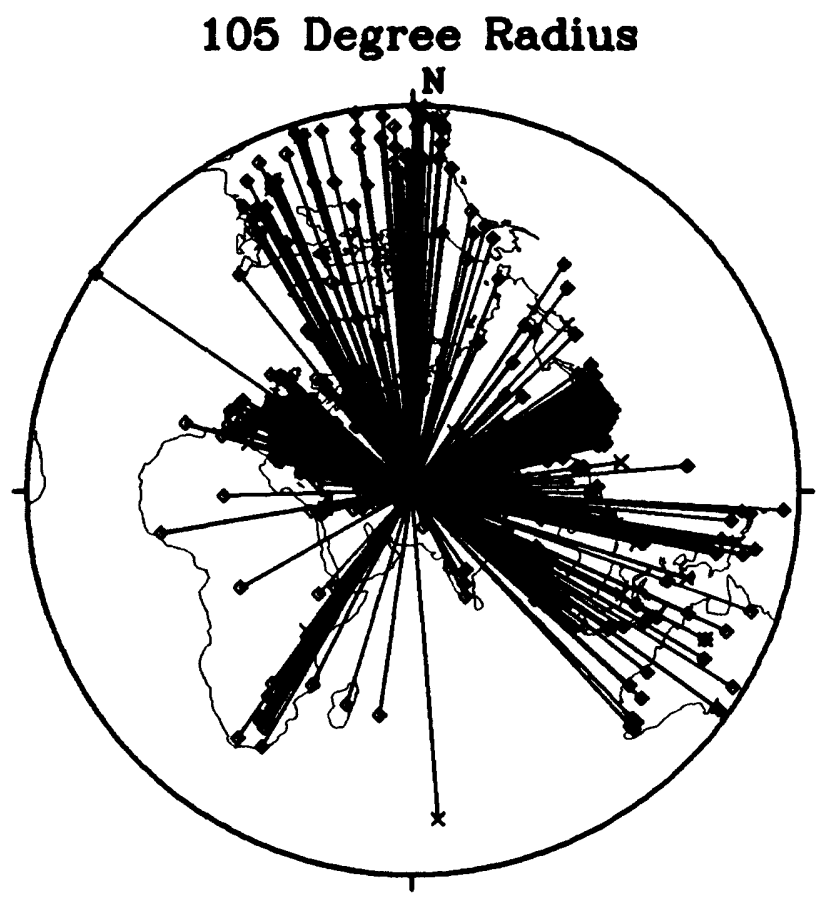

MAR 24, 1984 09:43:59 KURIL ISLANDS 105 Degree Radius

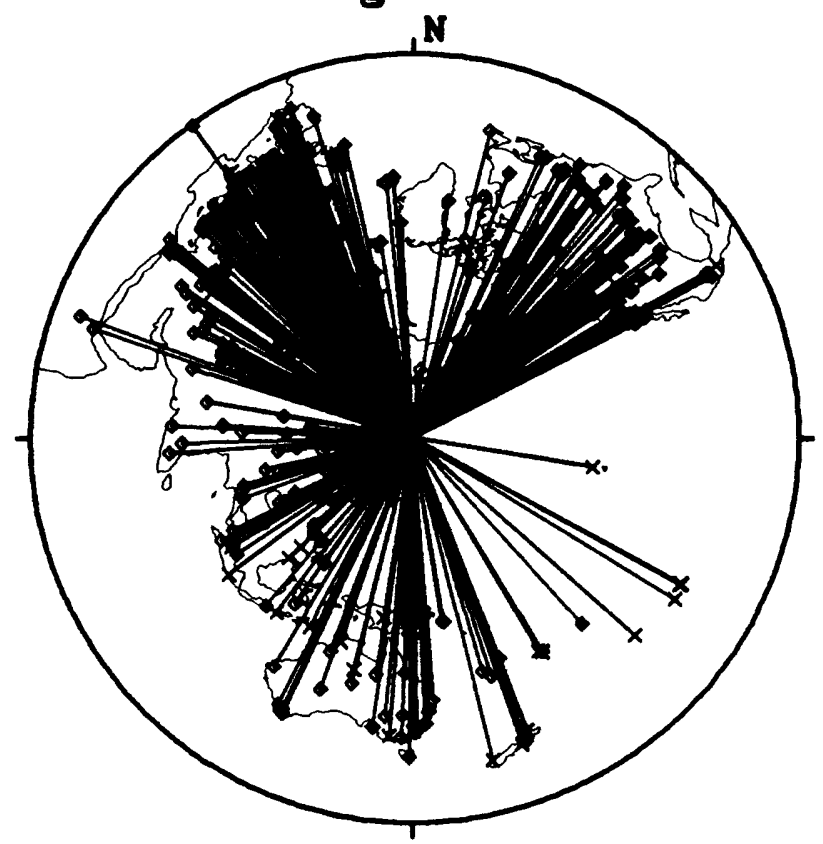

MAR 19, 1984 20:28:38 UZBEK SSR 10 Degree Radius

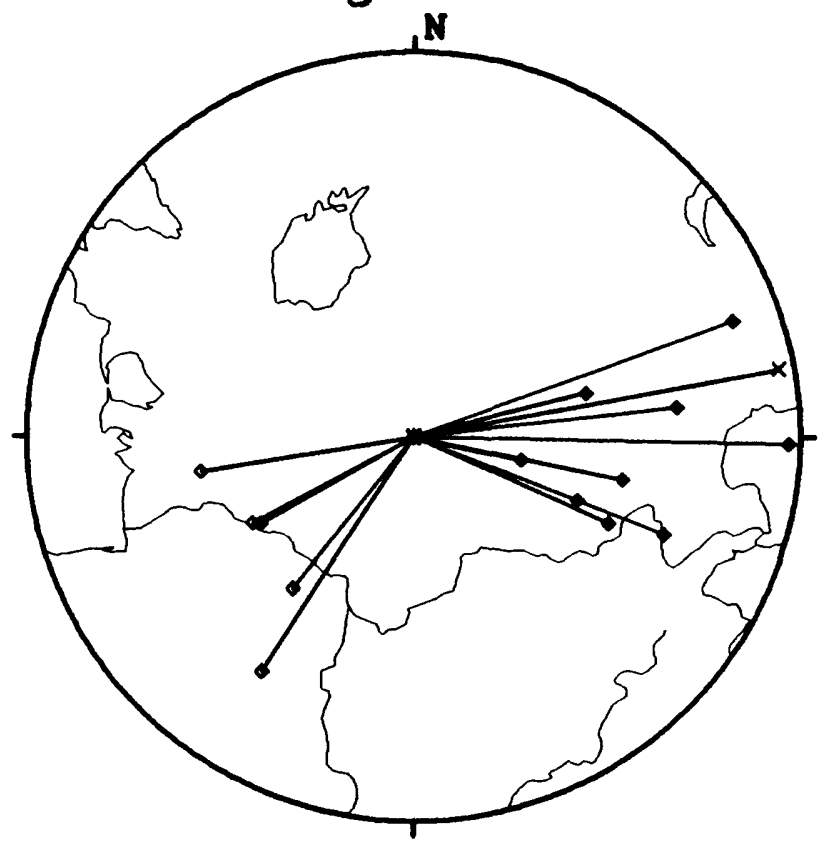

MAR 24, 1984 09:43:59 KURIL ISLANDS 10 Degree Radius

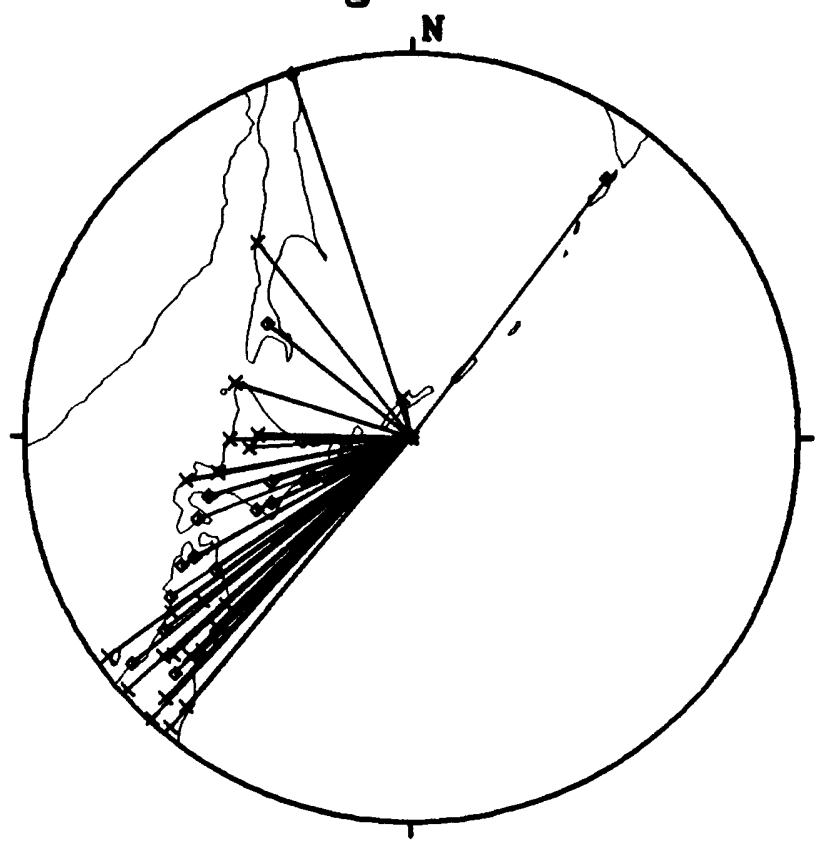


JAN 01,1980 16h $42 \mathrm{~m} 40.07 \pm 0.08 \mathrm{~s}$ । MZF $38.790 \mathrm{~N} \pm 2.2 \mathrm{~km} 27.785 \mathrm{~W} \pm 1.2 \mathrm{~km}$ | EAU DEPTH $=10.0 \mathrm{~km}$ (geophysicist)

$6.0 \mathrm{mb}$ ( 91 obs.) 6.8Msz ( 13 obs.) | EBL AZORES ISLANDS $(465)$ | At leost 56 people killed, 400 , ED or mare injured and extensive domoge (VIII) on Terceiro. At IEBH leost 4 people killed, some injured ond extensive domoge on ELO Son Jorge. Domoge olso reported on Grocioso.

\begin{tabular}{|c|c|c|c|c|c|c|c|}
\hline & & & & & & & I EGL \\
\hline$A D H$ & 0.45 & 107 & $\mathrm{Pg}$ & 42 & 48.90 & -0.3 & 1 \\
\hline HOR & 0.71 & 249 & $\mathrm{Pg}$ & 42 & 54.50 & 0.4 & A AVF \\
\hline PDA & 1.97 & 121 & $P n$ & 43 & 09.40 & $-4.4 x$ & i \\
\hline TEN & 14.07 & 134 & iP & 45 & 56.80 & $-4.8 x$ & EDU \\
\hline & & & $e(S)$ & 48 & 44.00 & & 1 \\
\hline LIS & 14.55 & 84 & $\mathrm{PC}$ & 46 & 02.50 & $-5.3 x$ & SSF \\
\hline & & & iPP & 46 & 15.00 & & 1 \\
\hline & & & is & 48 & 28.50 & & I SMF \\
\hline & & & LR & 49 & 16.00 & & | \\
\hline PTO & 14.90 & 75 & $P$ & 46 & 05.00 & $-7.4 x$ & LOR \\
\hline & & & $p P$ & 46 & 86.70 & & 1 \\
\hline & & & s & 48 & 33.80 & & SSB \\
\hline Col & 15.02 & 78 & $\mathbf{P}$ & 46 & 07.30 & $-6.7 x$ & DOU \\
\hline & & & s & 48 & 48.70 & & 1 \\
\hline MTE & 15.69 & 78 & $\mathbf{P}$ & 46 & 15.00 & $-7.8 x$ & 1 \\
\hline & & & s & 48 & 54.00 & & \\
\hline FAR & 15.74 & 90 & $\mathbf{P}$ & 46 & 18.48 & $-5.0 x$ & 1 \\
\hline & & & s & 49 & 07.40 & & I UCC \\
\hline SFS & 17.25 & 91 & iP & 46 & 43.60 & 0.5 & 1 \\
\hline & & & $\mathbf{i}$ & 46 & 49.50 & & \\
\hline & & & iPP & 47 & 03.00 & & REY \\
\hline & & & $i$ & 47 & 40.00 & & LRG \\
\hline & & & is & 49 & 24.00 & & 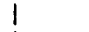 \\
\hline VAL & 17.96 & 37 & ePc & 46 & 50.00 & -1.2 & I MBO \\
\hline & & & $S$ & 49 & 56.00 & & 1 \\
\hline & & & LO & 50 & 31.00 & & \\
\hline & & & LR & 51 & 11.00 & & i \\
\hline GUD & 18.27 & 77 & iP & 46 & 52.00 & $-3.3 x$ & I \\
\hline TOL & 18.39 & 79 & $\mathrm{PC}$ & 46 & 52.50 & $-4.2 x$ & i \\
\hline & & & is & 50 & 12.00 & & | LMR \\
\hline MAL & 18.59 & 89 & IPd & 46 & 59.80 & 0.7 & 1 \\
\hline LGR & 19.51 & 71 & IPd & 47 & 09.40 & -1.0 & SPF \\
\hline & $1.3 \mathrm{~s}$ & & $4.50 \mathrm{~nm}$ & & & $.6 \mathrm{mb} \times$ & I \\
\hline & & & iPP & 47 & 25.40 & & HAU \\
\hline & & & is & 50 & 31.40 & & \\
\hline STJ & 20.13 & 304 & $e P$ & 47 & 17.00 & 0.2 & WLF \\
\hline & $1.4 \mathrm{~s}$ & 999 & 9.00 & & & $.0 \mathrm{mb}$ & 1 \\
\hline DKM & 20.68 & 39 & eP & 47 & 21.70 & -0.8 & DBN \\
\hline & $1.4 \mathrm{~s}$ & $270 e$ & 0.00 & & 6 & $.4 \mathrm{mb}$ & I \\
\hline DDK & 20.72 & 38 & eP & 47 & 21.90 & -1.0 & 1 \\
\hline DMU & 0.76 & 37 & eP & 47 & 23.00 & -0.4 & MEM \\
\hline & $1.7 \mathrm{~s}$ & 5070 & $0.00 \mathrm{~nm}$ & & 6 & $.6 \mathrm{mb}$ & I \\
\hline ALI & 21.32 & 83 & iPC & 47 & 29.00 & -0.2 & I \\
\hline SGR & 1.42 & 57 & $e^{P}$ & 47 & 31 & 0.9 & I \\
\hline LPF & 1.42 & 56 & $e P$ & 47 & 29.40 & -0.7 & BSF \\
\hline EPF & 1.63 & 70 & eP & 47 & 29.90 & -2.4 & 1 \\
\hline & $1.4 \mathrm{~s}$ & $184 E$ & 0.08 & & 6 & $3 \mathrm{mb}$ & HEE \\
\hline GRR & 21.64 & 55 & eP & 47 & 32.20 & -0.1 & i \\
\hline & $1.2 \mathrm{~s}$ & 998 & 0.00 & & 6 & $.1 \mathrm{mb}$ & BAF \\
\hline MFF & 21.68 & 60 & eP & 47 & 31.70 & -1.1 & $\mathrm{ECH}$ \\
\hline EBR & 21.78 & 76 & iP & 47 & 33.60 & -0.2 & 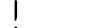 \\
\hline & & & is & 51 & 22.60 & & CDF \\
\hline & & & e & 51 & 29.00 & & I \\
\hline & & & e & 51 & 50 & & WLS \\
\hline FLN & 21.98 & 54 & eP & 47 & 36.90 & 1.1 & STR \\
\hline & $1.4 \mathrm{~s}$ & 735 & 5.00 & & & $.9 \mathrm{mb}$ & I \\
\hline LFF & 22.04 & 65 & eP & 47 & 35.20 & -1.2 & WTS \\
\hline & $1.3 \mathrm{~s}$ & $106 e$ & $0.00 \mathrm{~nm}$ & & 6 & $.1 \mathrm{mb}$ & 7 \\
\hline MLS & 22.17 & 70 & iPd & 47 & 38.00 & 0.3 & 1 \\
\hline SSC & 22.17 & 55 & eP & 47 & 38.00 & 0.3 & WIT \\
\hline & $1.0 \mathrm{~s}$ & 59 & $4.00 \mathrm{~nm}$ & & & $.0 \mathrm{mb}$ & 1 \\
\hline LPO & 22.34 & 65 & eP & 47 & 38.10 & -1.3 & $1 \mathrm{ZUL}$ \\
\hline & $1.4 \mathrm{~s}$ & 1018 & $0.00 \mathrm{~nm}$ & & 6 & $.1 \mathrm{mb}$ & BบB \\
\hline WOL & 22.42 & 47 & $P d$ & 47 & 42.80 & 2.8 & I CVF \\
\hline & $0.9 \mathrm{~s}$ & 244 & $4.00 \mathrm{~nm}$ & & & $.7 \mathrm{mb}$ & STU \\
\hline RJF & 22.63 & 64 & eP & 47 & 40.60 & -1.7 & 1 \\
\hline & $1.4 \mathrm{~s}$ & 521 & $1.00 \mathrm{~nm}$ & & & $.8 \mathrm{mt}$ & $S A L$ \\
\hline LSF & 22.77 & 61 & eP & 47 & 42 & -1.0 & $O G A$ \\
\hline CWF & 22.98 & 44 & $P d$ & 47 & 47.30 & 1.8 & GAP \\
\hline & $1.0 \mathrm{~s}$ & 221 & $1.00 \mathrm{~nm}$ & & & $.6 \mathrm{mb}$ & KDS \\
\hline CAF & 2.98 & 65 & eP & 47 & 44.10 & -1.6 & $F I R$ \\
\hline TCF & 3.24 & 61 & eP & 4 & 47.70 & -0.5 & i \\
\hline$E A B$ & 23.35 & 34 & iPc & 47 & 49.70 & 0.6 & FUR \\
\hline & $1.3 \mathrm{~s}$ & 1046 & $0.00 \mathrm{~nm}$ & & 6 & $.2 \mathrm{mb}$ & \\
\hline
\end{tabular}

23.4962 P $4750.00 \quad-0.6 \quad$

$\begin{array}{llllll}4750.00 & -0.6 & \text { I } & 5344.00\end{array}$ $1.3 \mathrm{~s} * * * * * * \mathrm{~nm} \quad 7.2 \mathrm{mb} \times \mid \quad 1.4 \mathrm{~s} \quad 140.00 \mathrm{~nm} \quad 5.6 \mathrm{mb}$ $23.68 \quad 36 \mathrm{eP} \quad 4752.40 \quad 0.0 \quad 2 \quad 2 \mathrm{~s} \quad 371.00 \mathrm{um} \quad 7.2 \mathrm{MszX}$ $1.3 \mathrm{~s} \quad 876.00 \mathrm{~nm} \quad 6.2 \mathrm{mb}$ $23.70 \quad 36 \mathrm{eP} \quad 4753.10 \quad 0.6$

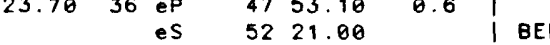
4853.68 $1.3 \mathrm{~s} 1240.00 \mathrm{~nm} \quad 6.3 \mathrm{mb} \quad$ I MOX $30.05 \quad 54 \mathrm{eP} \quad 4851.00 \quad-0.3$ $23.8034 \mathrm{iPc} 4754.20 \quad 0.7 \quad 3.4 \mathrm{~s} 4750.00 \mathrm{~nm}$ $1.3 \mathrm{~s} \quad 872.00 \mathrm{~nm} \quad 6.2 \mathrm{mb} \quad \mathrm{N}^{2} \quad 17 \mathrm{~s} \quad 374.00 \mathrm{um}$ $\begin{array}{llllllllllll}23.82 & 39 \mathrm{PC} & 4800.00 & 6.3 \times & \text { HOF } & 30.17 & 55 & \mathrm{iPd} & 48 & 51.70 & -0.7\end{array}$ $\begin{array}{llllll}23.87 & 36 \mathrm{iPC} & 4754.40 & 0.2 & 1.0 \mathrm{~s} & 550.00 \mathrm{~nm}\end{array}$

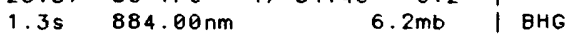
$\begin{array}{lllllll}24.09 & 61 \mathrm{eP} & 47 & 56.00 & -0.4 & \text { WET }\end{array}$ $1.0 \mathrm{~s} 203.00 \mathrm{~nm} \quad 5.7 \mathrm{mb}$ | RMP $24.1535 \mathrm{ePC} \quad 4756.70 \quad-0.2$ $1.3 \mathrm{~s} \quad 748.00 \mathrm{~nm} \quad 6.1 \mathrm{mb}$ $\begin{array}{ll}1.0 \mathrm{~s} & 550.00 \mathrm{~nm} \\ 30.63 & 60 \mathrm{eP}\end{array}$

30.7157 IPd

$30.83 \quad 71 \mathrm{ePd}$

iPP

$31.0153 \mathrm{ePc}$

i

PP

s

SS

SSS

SSS

$\begin{array}{lc}31.06 & 295 \mathrm{eP} \\ 1.0 \mathrm{~s} & 91.00 \mathrm{~nm}\end{array}$

$31.1657 \mathrm{P}$

$1.1 \mathrm{~s} \quad 46.00 \mathrm{~nm}$

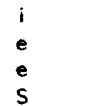

31.2564 iPO

$31.27 \quad 70 \mathrm{eP}$

$31.30 \quad 51$ IPd

$31.48 \quad 36$ iP

$31.48 \quad 59$ iPd

$31.55 \quad 54 \mathrm{iPd}$

$P P$

$\begin{array}{lll} & S \\ 31.55 & 44 & \mathrm{ePc}\end{array}$

$1.0 \mathrm{~s} \quad 360.09 \mathrm{~nm}$ iPP

is

$31.5760 \mathrm{iPd}$

$50 \quad 02.00$

5348.00

4902.50 4911.00 $49 \quad 15.00$

5405.00

0225.00

$\begin{array}{cccccc}31.77 \quad 63 \mathrm{eP} & 4904.00 & -2.5 \\ & \mathrm{i} & 4913.40 & \end{array}$

eS $\quad 5413.40$

$\begin{array}{llllll}31.84 & 4 & \text { IPC } & 49 & 07.20 & 0.4\end{array}$

$1.3 \mathrm{~s} \quad 577.00 \mathrm{~nm}$

IPP $50 \quad 13.00$

$i \quad 5405.00$

is $\quad 5421.00$

$\begin{array}{lllll}31.85 & 55 \mathrm{PC} & 4907.00 & -0.1\end{array}$

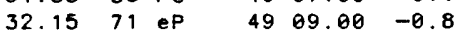

$\begin{array}{llllll}32.60 & 34 \mathrm{P} & 49 & 15.28 & 1.7\end{array}$

$\begin{array}{lllll}32.68 & 294 \mathrm{eP} & 49 & 13.30 & -1.1\end{array}$

$1.2 \mathrm{~s} \quad 71.50 \mathrm{~nm} \quad 5.5 \mathrm{mb}$

$32.95289 \mathrm{eP}^{\mathrm{P}} \quad 4913.40 \quad-3.3 \times$

32.9559 eP $4914.00 \quad-2.7$

PP $\quad 5028.00$

$\begin{array}{rrrrrr} & & i & 54 & 39.00 & \\ 32.98 & 59 & 02 & 00.00 & \\ & \text { eP } & 49 & 14.00 & -2.9 \\ & & \text { PP } & 50 & 30.00 & \end{array}$

$\begin{array}{ccc}P P & 5030.00 \\ e & 0200.00\end{array}$

$\begin{array}{lllll}32.99 & 290 \mathrm{eP} & 49 & 16.10 & -1.0\end{array}$

33.0354 iPd 4918.00

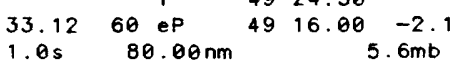

4905.00

$1.7 \mathrm{~s} \quad 38.50 \mathrm{~nm} \quad 5.1 \mathrm{mb}$

$0.8 \mathrm{~s} \quad 57.00 \mathrm{~nm} \quad 5.6 \mathrm{mb}$ 
$61 \mathrm{~d} 16 \mathrm{~h}$

\begin{tabular}{|c|c|c|c|c|c|c|c|c|c|c|c|}
\hline GDH & 33.51 & 344 & iPd & 49 & 22.00 & 0.7 & I SJG & 39.15 & 249 & iP & 50 \\
\hline & & 594 & $4.00 \mathrm{~nm}$ & & & $.4 \mathrm{mb}$ & i & $1.1 \mathrm{~s}$ & 633 & $3.00 \mathrm{~nm}$ & \\
\hline & & & is & 54 & 32.00 & & i & $20 s$ & & $9.22 \mathrm{um}$ & \\
\hline & & & iss & 56 & 43.00 & & i & & & is & 56 \\
\hline HFS & 33.57 & 37 & iP & 49 & 20.60 & -1.4 & 1 & & & i & 58 \\
\hline & 1. $1 \mathrm{~s}$ & 101 & $1.00 \mathrm{~nm}$ & & & $.7 \mathrm{mb}$ & I CMP & 39.17 & 63 & eP & 50 \\
\hline & $15 \mathrm{~s}$ & 223 & $3.00 \mathrm{um}$ & & 7 & $\operatorname{\theta g}=2 \mathrm{X}$ & 1 & & & iPP & 51 \\
\hline$B L Y$ & 33.72 & 65 & $\mathbf{P}$ & 49 & 23.30 & -0.2 & 1 & & & es & 56 \\
\hline QUA & 33.75 & 291 & eP & 49 & 23.20 & -0.5 & I KIR & 39.21 & 27 & $P$ & 50 \\
\hline & $1.3 \mathrm{~s}$ & 149 & $9.00 \mathrm{~nm}$ & & 5 & $.8 \mathrm{mb}$ & $i$ & & & $\mathbf{i}$ & 50 \\
\hline UCT & 33.76 & 290 & eP & 49 & 23.30 & -0.5 & I TRO & 39.47 & 24 & iP & 50 \\
\hline & $1.5 \mathrm{~s}$ & 315 & $5.00 \mathrm{~nm}$ & & 6 & $.0 \mathrm{mb}$ & I PVL & 39.67 & 66 & $\mathrm{PC}$ & 50 \\
\hline MNT & 34.26 & 296 & eP & 49 & 27.00 & -1.1 & I MLR & 39.76 & 62 & $\mathrm{Pc}$ & 50 \\
\hline & $0.9 \mathrm{~s}$ & & $5.00 \mathrm{~nm}$ & & 5 & $.5 \mathrm{mb}$ & I ATH & 39.94 & 74 & $e P c$ & 50 \\
\hline PNY & 34.28 & 295 & $e P$ & 49 & 27.00 & -1.3 & $i$ & & & ePP & 52 \\
\hline SRO & 34.30 & 60 & iP & 49 & 28.00 & -0.4 & i & & & es & 56 \\
\hline & $11 \mathrm{~s}$ & 208 & $3.00 \mathrm{um}$ & & 7 & $1 \mathrm{MszX}$ & I MRG & 39.96 & 288 & iPc & 50 \\
\hline & & & e (PP) & 50 & 48.00 & & I Buc & 40.07 & 64 & eP & 50 \\
\hline & & & e (S) & 54 & 51.50 & & 1 & & & es & 56 \\
\hline & & & i & 55 & 04.10 & & $1 \mathrm{KDZ}$ & 40.14 & 68 & iPd & 50 \\
\hline BCT & 34.66 & 289 & eP & 49 & 30.80 & -0.7 & | VRI & 40.24 & 62 & eP & 50 \\
\hline & $1.0 \mathrm{~s}$ & 97 & $7.50 \mathrm{~nm}$ & & 5 & $.6 \mathrm{mb}$ & I BAC & 40.24 & 61 & eP & 50 \\
\hline FRB & 34.71 & 329 & ePd & 49 & 36.00 & $4.3 x$ & i & & & e & 52 \\
\hline & $1.2 \mathrm{~s}$ & 682 & $2.00 \mathrm{~nm}$ & & 6 & $.4 \mathrm{mb}$ & i & & & e & 56 \\
\hline BUD & 34.79 & 60 & eP & 49 & 31.00 & -1.6 & I CLE & 40.63 & 291 & iPd & 50 \\
\hline KRA & 35.33 & 56 & ePc & 49 & 35.90 & -1.3 & $\mathrm{i}$ & & & $\mathrm{s}$ & 56 \\
\hline & $1.4 \mathrm{~s}$ & 814 & $4.00 \mathrm{~nm}$ & & 6 & $.4 \mathrm{mb}$ & I KUK & 40.84 & 136 & $\mathrm{PC}$ & 50 \\
\hline & $12 \mathrm{~s}$ & 286 & $5.00 \mathrm{um}$ & & 7 & $2 M \leqslant 2 X$ & I KJF & 41.00 & 33 & ip & 50 \\
\hline & $12 \mathrm{~s}$ & 193 & $3.00 \mathrm{um}$ & & & & i & $0.8 \mathrm{~s}$ & & $0.20 \mathrm{~nm}$ & \\
\hline & $12 \mathrm{~s}$ & 251 & $1.00 \mathrm{um}$ & & & & i & & & $\mathrm{i}$ & 50 \\
\hline & & & $i$ & 49 & 43.60 & & I & & & ePP & 52 \\
\hline & & & is & 55 & 10.00 & & 1 & & & eS & 56 \\
\hline UPP & 5.36 & 38 & iP & 49 & 35.70 & -1.6 & I BLA & 41.03 & 285 & ¿P & 50 \\
\hline & $1.5 \mathrm{~s}$ & 399 & $9.00 \mathrm{~nm}$ & & 6 & $.1 \mathrm{mb}$ & 1 & $1.2 \mathrm{~s}$ & 237 & $7.00 \mathrm{~nm}$ & \\
\hline & & & $i$ & 49 & 41.80 & & 1 & & & es & 56 \\
\hline & & & is & 54 & 56.00 & & I TRN & 41.04 & 236 & eP & 50 \\
\hline SPC & 35.54 & 57 & eP & 49 & 41.00 & 1.8 & i & $1.2 \mathrm{~s}$ & 195 & $5.00 \mathrm{~nm}$ & \\
\hline & $10 \mathrm{~s}$ & 101 & $1.00 \mathrm{um}$ & & 6 & $9 M \leq z X$ & I SOD & 41.30 & 28 & $P$ & 50 \\
\hline & & & $i$ & 49 & 47.20 & & i & & & $\mathrm{i}$ & 50 \\
\hline & & & $i$ & 51 & 09.00 & & 1 & & & es & 56 \\
\hline GPD & 5.55 & 289 & eP & 49 & 38.20 & -1.0 & I CFR & 41.35 & 62 & eP & 50 \\
\hline$N \mid E$ & 35.58 & 57 & $\mathrm{PC}$ & 49 & 40.00 & 0.6 & I KIS & 41.50 & 60 & $P d$ & 50 \\
\hline & & & $i$ & 49 & 47.00 & & 1 & & & ipp & 50 \\
\hline & & & $i$ & 49 & 55.00 & & 1 & & & is & 56 \\
\hline OT T & 35.74 & 296 & eP & 49 & 40.00 & -0.7 & I PUL & 41.66 & 40 & $\mathrm{Pc}$ & 50 \\
\hline & & & $P P$ & 49 & 47.00 & $24 \mathrm{kmx}$ & i & & & $i s P$ & 50 \\
\hline JOS & 35.76 & 58 & eP & 49 & 40.00 & -0.9 & I DMK & 41.84 & 67 & eP & 50 \\
\hline & $1.6 \mathrm{~s}$ & 250 & $9.00 \mathrm{~nm}$ & & 5 & $.8 \mathrm{mb}$ & I AAM & 41.96 & 293 & ePd & 50 \\
\hline BEO & 36.03 & 64 & $P$ & 49 & 43.00 & -0.2 & I KEV & 42.12 & 25 & iP & 50 \\
\hline & & & iPP & 51 & 07.50 & & i & $0.6 \mathrm{~s}$ & & $2.50 \mathrm{~nm}$ & \\
\hline & & & iPPP & 51 & 30.50 & & 1 & & & $\mathrm{i}$ & 50 \\
\hline & & & is & 55 & 30.20 & & 1 & & & ePP & 52 \\
\hline WAR & 36.05 & 52 & ePc & 49 & 46.00 & 2.7 & I & & & es & 56 \\
\hline & $16 \mathrm{~s}$ & 190 & $9.00 \mathrm{um}$ & & &.$\theta M s z X$ & I EDC & 42.32 & 70 & iP & 50 \\
\hline & & & e & 51 & 10.00 & & $1 \quad I Z M$ & 42.46 & 73 & eP & 50 \\
\hline & & & e & 51 & 50.00 & & I AN7 & 42.50 & 291 & eP & 50 \\
\hline & & & e & 53 & 02.00 & & I AN 4 & 42.70 & 290 & eP & 50 \\
\hline & & & e & 55 & 22.00 & & I AN12 & 42.71 & 291 & eP & 50 \\
\hline & & & e & 56 & 34.00 & & I AN1 & 42.79 & 291 & $e P$ & 50 \\
\hline$T I M$ & 36.45 & 63 & $P$ & 50 & 02.00 & $15.3 x$ & $15 T$ & 42.95 & 68 & ¡Pd & 50 \\
\hline UZH & 36.92 & 58 & $P d$ & 49 & 50.50 & -0.1 & 1 & & & i & 57 \\
\hline & & & is & 55 & 33.00 & & 1 & & & es & 00 \\
\hline OHR & 36.94 & 71 & iP & 49 & 51.40 & 0.5 & I ANB & 42.98 & 291 & eP & 50 \\
\hline & & & $i$ & 50 & 00.40 & & I ISK & 42.99 & 68 & eP & 50 \\
\hline & & & $i(s)$ & 55 & $46.4 \theta$ & & I AN9 & 42.99 & 291 & eP & 50 \\
\hline SKO & 37.22 & 69 & iPd & 49 & 52.90 & -0.4 & I AN10 & 3.04 & 291 & $e P$ & 50 \\
\hline & & & $\mathrm{i}$ & 49 & 58.50 & & I AN11 & 43.17 & 291 & eP & 50 \\
\hline & & & i & 50 & 17.00 & & $1 \mathrm{KBS}$ & 43.25 & 10 & iPC & 50 \\
\hline & & & $i$ & 50 & 43.00 & & 1 & & & i & 50 \\
\hline & & & iPP & 51 & 21.20 & & 1 & & & $\mathrm{~s}$ & 57 \\
\hline & & & $i$ & 53 & 45.00 & & I ACM & 43.44 & 294 & eP & 50 \\
\hline DEV & 37.60 & 62 & $\mathrm{PC}$ & 49 & 58.00 & 1.6 & i & & & epp & 50 \\
\hline UME & 37.72 & 33 & $P$ & 49 & 57.50 & 0.4 & I PRM & 43.62 & 281 & eP & 50 \\
\hline & & & $i$ & 50 & 04.70 & & I APA & 43.86 & 29 & $P C$ & 50 \\
\hline BMR & 37.80 & 59 & eP & 50 & 00.00 & 2.0 & 1 & & & es & 57 \\
\hline LVV & 37.98 & 56 & $P d$ & 50 & 00.00 & 0.5 & I TKL & 44.10 & 284 & iP & 50 \\
\hline & & & is & 55 & 53.00 & & $A L T$ & 44.37 & 71 & $i P_{C}$ & 50 \\
\hline VAY & 38.19 & 70 & iPd & 50 & 01.40 & 0.0 & | ORT & 44.42 & 285 & eP & 50 \\
\hline CANY & 38.36 & 293 & eP & 50 & 02.70 & -0.1 & I CAR & 44.84 & 242 & $i P c$ & 50 \\
\hline IC & 8.42 & 141 & iPc & 50 & 02.20 & -1.4 & i & $1.2 \mathrm{~s}$ & 100 & $0.00 \mathrm{~nm}$ & \\
\hline OF & 38.45 & 67 & iPd & 50 & 05.00 & 1.4 & I NAT & 44.97 & 191 & $i P C$ & 51 \\
\hline RA & 38.73 & 64 & eP & 50 & 08.00 & 2.1 & | ELL & 45.00 & 74 & iP & 50 \\
\hline NUR & 38.93 & 39 & iP & 50 & 07.80 & 0.5 & I OBN & 45.18 & 47 & $P C$ & 51 \\
\hline & $1.5 \mathrm{~s}$ & 280 & $.00 \mathrm{~nm}$ & & & $.7 \mathrm{mb}$ & 1 & & & is & 57 \\
\hline & & & $i$ & 50 & 14.90 & & I RCC & 45.19 & 260 & $e P$ & 50 \\
\hline & & & ePP & 51 & 36.00 & & I BCK & 45.26 & 73 & iPc & 51 \\
\hline & & & es & 56 & 04.00 & & ALE & 45.32 & 354 & $e^{P}$ & 50 \\
\hline
\end{tabular}




\begin{tabular}{|c|c|c|c|c|c|c|c|c|c|c|c|}
\hline & & & es & 00 & 58.00 & & I ORV & 69.11 & 304 & iP & 53 \\
\hline BDW & 59.71 & 302 & iPd & 52 & 45.00 & -2.3 & i WDC & 69.21 & 306 & iP & 53 \\
\hline & $1.0 \mathrm{~s}$ & & $2.40 \mathrm{~nm}$ & & 5 & $.5 \mathrm{mb}$ & PMR & 69.30 & 334 & eP & 53 \\
\hline & & & epP & 52 & 54.10 & $30 \mathrm{~km} x$ & i & $1.0 \mathrm{~s}$ & 125 & $5.00 \mathrm{~nm}$ & \\
\hline MSA & 59.97 & 295 & $e(P)$ & 52 & 49.50 & 0.1 & i & $20 \mathrm{~s}$ & 52 & $2.00 \mathrm{um}$ & \\
\hline & & & $i p P$ & 52 & 57.80 & $27 \mathrm{kmx}$ & 1 & $20 \mathrm{~s}$ & 22 & $2.50 \mathrm{um}$ & \\
\hline I NK & 59.98 & 336 & $e P$ & 52 & 47.00 & -1.5 & i & $20 \mathrm{~s}$ & 44 & 4.00 um & \\
\hline & $1.9 \mathrm{~s}$ & 2190 & $0.00 \mathrm{~nm}$ & & & $0 m b$ & I JAS & 69.30 & 302 & IPO & 53 \\
\hline COM & 60.10 & 267 & iP & 53 & 49.00 & $58.8 x$ & I PEC & 69.31 & 297 & eP & 53 \\
\hline DCI & 60.18 & 304 & eP & 52 & 59.70 & $9.2 x$ & | FRI & 69.34 & 301 & iP & 53 \\
\hline$O A O$ & 60.49 & 243 & $i P c$ & 52 & 53.50 & 0.4 & I NVS & 69.70 & 35 & $\mathbf{P}$ & 53 \\
\hline$R \times F$ & 60.50 & 311 & $\mathrm{iPC}$ & 52 & 53.20 & 0.7 & I PAS & 69.87 & 298 & iPO & 53 \\
\hline MSO & 60.69 & 308 & ePd & 52 & 53.20 & -0.6 & i & $2.5 \mathrm{~s}$ & 1500 & $0.00 \mathrm{~nm}$ & \\
\hline & $1.1 \mathrm{~s}$ & 246 & $6.00 \mathrm{~nm}$ & & & $2 \mathrm{mb}$ & $i$ & $20 \mathrm{~s}$ & 57 & $7.00 \mathrm{um}$ & \\
\hline $\mathrm{N}$ & $18 \mathrm{~s}$ & 175 & $5.00 \mathrm{um}$ & & & & i & $20 \mathrm{~s}$ & 69 & $9.00 \mathrm{um}$ & \\
\hline E & $21 \mathrm{~s}$ & 161 & 1. 00 um & & & & i & $20 \mathrm{~s}$ & & 3.00 um & \\
\hline LDM & 60.79 & 310 & $i P C$ & 52 & 54.40 & 0.0 & i & & & ePP & 56 \\
\hline NCE & 60.81 & 242 & eP & 52 & 55.60 & 0.1 & i & & & es & 03 \\
\hline YKM & 60.85 & 311 & iPC & 52 & 55.50 & 0.6 & I FHC & 70.00 & 307 & eP & 53 \\
\hline TEH & 61.17 & 66 & eP & 52 & 54.50 & -2.8 & TAS & 70.25 & 53 & Po & 53 \\
\hline$A L O$ & 61.20 & 293 & ePd & 52 & 58.00 & 0.5 & i & & & is & 03 \\
\hline MOT & 61.50 & 288 & iP & 52 & 58.20 & -1.5 & I ARN & 70.36 & 302 & $e P$ & 53 \\
\hline NEW & 61.95 & 311 & iP & 53 & 01.00 & -1.3 & I MHC & 70.43 & 302 & IPO & 53 \\
\hline$z$ & $z \quad 195$ & 265 & $5.00 \mathrm{um}$ & & & $.4 M s Z$ & | PR I & 70.45 & 301 & IPO & 53 \\
\hline$N$ & $19 s$ & 105 & $5.00 \mathrm{um}$ & & & & I TTA & 70.52 & 338 & eP & 53 \\
\hline$E$ & 195 & 180 & 0.00 um & & & & I BKS & 70.52 & 303 & eP & 53 \\
\hline NR I & 62.43 & 21 & $\mathrm{Pc}$ & 53 & 06.40 & 1.3 & i & $0.8 \mathrm{~s}$ & 34 & $4.00 \mathrm{~nm}$ & \\
\hline KAT & 62.89 & 60 & Po & 53 & 09.50 & 1.0 & i & $20 \mathrm{~s}$ & 67 & $7.00 \mathrm{um}$ & \\
\hline & & & is & 01 & 43.00 & & i & $20 \mathrm{~s}$ & 112 & 2.00 um & \\
\hline PNT & 62.91 & 313 & $e P$ & 53 & 07.50 & -1.1 & i & $20 \mathrm{~s}$ & 48 & $8.00 \mathrm{um}$ & \\
\hline & $1.8 \mathrm{~s}$ & 578 & $8.00 \mathrm{~nm}$ & & 6 & $.5 \mathrm{mb}$ & i & & & e & 54 \\
\hline VHO & 63.13 & 271 & iP & 53 & 08.00 & -2.6 & 1 & & & e & 54 \\
\hline$I+T$ & 63.34 & 274 & ip & 53 & 16.00 & $3.8 x$ & i & & & ePP & 56 \\
\hline SVM & 63.60 & 292 & eP & 53 & 13.00 & -0.6 & i & & & es & 03 \\
\hline & & & $i p P$ & 53 & 21.00 & $26 \mathrm{kmX}$ & i & & & e & 03 \\
\hline||$P$ & 63.64 & 274 & ip & 53 & 19.00 & $4.9 x$ & i & & & e & 05 \\
\hline 110 & 63.67 & 275 & eP & 53 & 13.20 & -1.1 & I NAI & 71.25 & 108 & iPc & 54 \\
\hline TAC & 63.82 & 274 & eP & 53 & 23.00 & $7.7 x$ & i & $2.6 \mathrm{~s}$ & 538 & $8.00 \mathrm{~nm}$ & \\
\hline GCA & 63.82 & 297 & eP & 53 & 14.20 & -0.7 & I ILT & 71.39 & 349 & Po & 54 \\
\hline $1 \mathrm{IM}$ & 63.85 & 274 & iP & 53 & 18.00 & 2.5 & 1 & & & isp & 54 \\
\hline BRV & 64.43 & 344 & $e(P)$ & 53 & 18.60 & 0.4 & i & & & es & 03 \\
\hline & & & e & 53 & 27.20 & & ELT & 72.10 & 35 & $e P$ & 54 \\
\hline 111 & 64.61 & 274 & ip & 53 & 20.00 & -0.4 & i & & & es & 03 \\
\hline $\mathrm{SHI}$ & 65.07 & 71 & iPd & 53 & 22.50 & -0.7 & I GAR & 72.23 & 54 & $P$ & 54 \\
\hline & & & $e(s)$ & 02 & 14.00 & & i & & & isP & 54 \\
\hline PGC & 65.43 & 313 & eP & 53 & 20.50 & $-4.4 x$ & I SLA & 72.38 & 216 & Po & 54 \\
\hline TUC & 65.64 & 293 & eP & 53 & 26.40 & -0.3 & I KUL & 72.51 & 55 & Po & 54 \\
\hline 2 & $18 \mathrm{~s}$ & 22 & $2.46 \mathrm{um}$ & & & $4 \mathrm{Msz}$ & i & & & is & 03 \\
\hline BMN & 65.86 & 303 & IPd & 53 & 27.20 & -0.9 & I FRU & 72.59 & 49 & eP & 54 \\
\hline & $1.0 \mathrm{~s}$ & 57 & $7.60 \mathrm{~nm}$ & & & $7 \mathrm{mb}$ & $K D C$ & 73.26 & 333 & $e P$ & 54 \\
\hline & & & $\mathrm{i}$ & 53 & 35.20 & & I ANT & 73.88 & 220 & $e^{P}$ & 54 \\
\hline PRN & 65.98 & 299 & $e^{P}$ & 53 & 28.50 & -0.4 & i & & & is & 03 \\
\hline ZOBO & 66.52 & 223 & $\mathbf{P}$ & 53 & 32.10 & -0.8 & I KHO & 73.90 & 55 & eP & 54 \\
\hline MAIO & 66.57 & 62 & $P d-$ & 53 & 33.00 & 0.4 & I WIN & 74.11 & 137 & iPc & 54 \\
\hline $\mathrm{COL}$ & 66.57 & 336 & eP & 53 & 32.00 & -0.1 & i & $1.0 \mathrm{~s}$ & 50 & $0.00 \mathrm{~nm}$ & \\
\hline & & & es & 02 & 26.00 & & I NRN & 74.26 & 49 & eP & 54 \\
\hline FBA & 66.57 & 336 & $e^{P}$ & 53 & 32.50 & 0.4 & I PRZ & 75.01 & 47 & eP & 54 \\
\hline & $1.0 \mathrm{~s}$ & 50 & $0.00 \mathrm{~nm}$ & & 5 & $.7 \mathrm{mb}$ & I OUE & 75.15 & 63 & eP & 54 \\
\hline LPB & 66.72 & 222 & $\mathrm{PC}$ & 53 & 33.00 & -1.1 & i & & & es & 04 \\
\hline & $1.5 \mathrm{~s}$ & 417 & $7.00 \mathrm{~nm}$ & & 6 & $4 \mathrm{mb}$ & $\mathrm{KSH}$ & 75.47 & 51 & $P$ & 54 \\
\hline & & & $i$ & 53 & 35.00 & & i & & & $\mathbf{P}$ & 54 \\
\hline & & & $s$ & 02 & 28.00 & & i & & & $\mathrm{s}$ & 04 \\
\hline SIT & 66.81 & 325 & eP & 53 & 38.50 & $4.9 x$ & $i$ & & & ScS & 04 \\
\hline PNL & 66.95 & 329 & $e^{P}$ & 53 & 35.90 & 1.3 & I KRI & 77.07 & 124 & $\mathrm{P}+$ & 54 \\
\hline TNP & 67.09 & 301 & ePd & 53 & 34.10 & -2.0 & I YAK & 77.90 & 11 & $\mathrm{Pc}$ & 54 \\
\hline HUA & 67.24 & 232 & iPd & 53 & 37.00 & -0.4 & $i$ & & & es & 04 \\
\hline IMA & 67.50 & 339 & eP & 53 & 38.00 & -0.1 & SDN & 77.92 & 335 & $e^{P}$ & 54 \\
\hline MNV & 67.53 & 302 & iP & 53 & 37.50 & -1.2 & MTD & 78.32 & 123 & eP & 54 \\
\hline ARO & 67.80 & 93 & iP & 53 & 40.00 & -0.6 & $B A A$ & 78.36 & 205 & $P$ & 54 \\
\hline & & & es & 02 & 57.26 & & $i$ & & & $\mathrm{~s}$ & 04 \\
\hline WCN & 68.11 & 303 & $e^{P}$ & 53 & 41.80 & -0.6 & 1 & & & LQ & 15 \\
\hline GLA & 68.13 & 295 & eP & 53 & 42.00 & -0.4 & $i$ & & & LR & 19 \\
\hline NNA & 68.14 & 233 & IPC & 53 & 41.80 & -0.8 & LPA & 78.46 & 205 & iPC & 54 \\
\hline & $1.2 \mathrm{~s}$ & 284 & $4.00 \mathrm{~nm}$ & & & $3 \mathrm{mb}$ & 1 & $20 s$ & & $6.30 \mathrm{um}$ & \\
\hline & & & $i$ & 53 & 51.50 & & $i$ & & & is & 04 \\
\hline & & & es & 02 & 42.00 & & SEY & 78.66 & 360 & $P C$ & 54 \\
\hline $\operatorname{LM2}$ & 68.35 & 233 & iP & 53 & 46.50 & 2.7 & 1 & & & isP & 54 \\
\hline ARE & 68.51 & 225 & $e^{P}$ & 53 & 45.00 & -0.2 & 1 & & & es & 04 \\
\hline & & & es & 02 & 47.00 & & BUL & 78.88 & 127 & eP & 54 \\
\hline & & & eSS & 07 & 01.00 & & TET & 79.19 & 121 & $P$ & 54 \\
\hline & & & eLO & 10 & 33.00 & & 1 & & & PP & 57 \\
\hline VPEM & 68.64 & 299 & $e(P)$ & 53 & 47.10 & 1.4 & WMO & 79.22 & 42 & $P$ & 54 \\
\hline & & & i & 53 & 53.70 & & 1 & & & $P$ & 54 \\
\hline$T I K$ & 68.64 & 8 & $e^{P}$ & 53 & 45.00 & 0.1 & MOY & 79.42 & 30 & $e P c$ & 54 \\
\hline$M I N$ & 68.74 & 305 & ip & 53 & 44.90 & -1.4 & 1 & & & es & 04 \\
\hline KPK & 68.97 & 304 & $e^{P}$ & 53 & 47.10 & -0.5 & I CFA & 79.51 & 214 & $P C$ & 54 \\
\hline & & & $i$ & 53 & 54.00 & & $i$ & & & $\mathrm{~s}$ & 04 \\
\hline
\end{tabular}


01d $16 \mathrm{~h}$

\begin{tabular}{|c|c|c|c|c|c|c|c|c|c|c|c|}
\hline & & & PP & 80 & 17.60 & & 1 & & $20 s$ & & $7.00 \mathrm{um}$ \\
\hline & & & eS & 07 & 39.60 & & 1 & & $20 \mathrm{~s}$ & & 0.00 um \\
\hline$T I A$ & 98.64 & 28 & $\mathbf{P}$ & 56 & 26.10 & $5.5 x$ & 1 & PMG & 150.40 & 10 & ePKP \\
\hline & & & PP & 80 & 25.90 & & 1 & & $19 \mathrm{~s}$ & & 1.00 um \\
\hline GYA & 102.36 & 41 & Pdiff & 56 & 38.90 & 1.3 & 1 & DRV & 151.23 & 170 & PKP \\
\hline & & & $P$ & 56 & 40.90 & & 1 & LUG & 153.36 & 326 & PKPd \\
\hline & & & PP & 00 & 51.40 & & 1 & PVC & 154.76 & 322 & PKP \\
\hline WHN & 102.52 & 33 & Pdiff & 56 & 39.60 & 1.6 & I & WRA & 155.68 & 45 & PKP \\
\hline & & & P & 56 & 42.60 & & 1 & & $1.6 \mathrm{~s}$ & 61 & $1.18 \mathrm{~nm}$ \\
\hline & & & PP & $\theta 0$ & 53.60 & & 1 & WB2 & 155.69 & 44 & ePKP \\
\hline NJ2 & 102.99 & 29 & Pdiff & 56 & 45.30 & $5.3 x$ & 1 & & & & $i$ \\
\hline & & & PP & 80 & 59.30 & & 1 & & & & ePP \\
\hline MAT & 103.93 & 12 & ePdiff & $f 56$ & 49.00 & $4.8 x$ & 1 & ASP & 158.33 & 51 & ePKP \\
\hline & $1.5 \mathrm{~s}$ & & $1.10 \mathrm{~nm}$ & & & $6.2 \mathrm{mb}$ & 1 & & & & e \\
\hline & $z 19 \mathrm{~s}$ & & 4.51 um & & & 6. $0 \mathrm{Msz}$ & 1 & ISO & 158.94 & 35 & ePKP \\
\hline SSE & 104.71 & 27 & Pdiff & 56 & 50.70 & $3.0 x$ & 1 & & & & e \\
\hline & & & eP & 56 & 53.70 & & 1 & KOU & 159.06 & 327 & PKPC \\
\hline SHK & 104.83 & 17 & ePdiff & 56 & 52.70 & $4.5 x$ & 1 & NOU & 159.55 & 319 & PKPd \\
\hline & & & e & 01 & 14.70 & & 1 & GNZ & 159.88 & 262 & ePKP \\
\hline $\mathrm{OZH}$ & 109.22 & 32 & Pdiff & 57 & 14.80 & $6.9 x$ & 1 & WTZ & 160.57 & 265 & ePKP \\
\hline & & & iPP & 01 & 43.80 & & 1 & & & & e \\
\hline ANP & 110.17 & 30 & Pdiff & 57 & 19.00 & $6.7 x$ & 1 & CTA & 160.66 & 17 & PKP \\
\hline & & & $\mathrm{S}$ & 11 & 12.00 & & I & & & & i \\
\hline SNA & 110.36 & 171 & $e(P K P)$ & 01 & 07.00 & $-5.8 x$ & I & & & & iPP \\
\hline TCU & 110.77 & 31 & PKP & 02 & 04.30 & $49.3 x$ & 1 & & & & ISKKP \\
\hline HEN & 12.72 & 32 & PKP & 01 & 29.00 & $10.3 x$ & 1 & & & & iss \\
\hline TS I & 15.12 & 63 & EPKPC & 00 & 52.00 & $-31.6 x$ & 1 & KRP & 161.69 & 265 & ePKP \\
\hline$P \mid P$ & 115.93 & 34 & ePKP & 01 & 33.40 & $8.4 x$ & 1 & & & & e \\
\hline & & & es & 02 & 32.60 & & 1 & & & & e \\
\hline SI & 15.94 & 63 & PKPd & 01 & 1.50 & $6.3 x$ & 1 & MNG & 162.01 & 257 & ePKP \\
\hline I PM & .83 & 60 & ePKPc & 01 & 2 & $-2.3 x$ & 1 & & & & e \\
\hline$B A G$ & 117.61 & 35 & PKP & 01 & 9.00 & 0.5 & I & WEL & 162.56 & 255 & ePKP \\
\hline & & & e & 02 & 38.00 & & 1 & $M J Z$ & 165.36 & 243 & ePKP \\
\hline BAG & 7.61 & 35 & $d i f f$ & 57 & 44.00 & -1.7 & 1 & BRS & 168.62 & 357 & PKP- \\
\hline MSP & 117.67 & 35 & ePKP & 01 & 39.56 & $10.8 x$ & I & & & & $i$ \\
\hline & & & es & 02 & 40.90 & & 1 & & & & $\mathrm{i}$ \\
\hline PGP & 120.34 & 36 & PKP & 01 & 41.50 & $8.0 x$ & 1 & & & & e( ) \\
\hline LGP & 121.87 & 33 & EPKPC & 01 & 41.50 & $5.1 x$ & I & & & & e \\
\hline & & & es & 02 & 50.20 & & I & & & & ePP \\
\hline RUV & 122.35 & 276 & ePKP & 01 & 47.00 & $9.7 x$ & 1 & & & & e \\
\hline & $1.2 \mathrm{~s}$ & 30 & $3.00 \mathrm{~nm}$ & & & & 1 & & & & eSKKKS \\
\hline PPR & 122.49 & 40 & ePKP & 01 & 4.50 & $6.9 x$ & 1 & TAU & 174.46 & 140 & ePKP \\
\hline & & & es & 03 & 08.00 & & 1 & & & & e \\
\hline AW & 25.43 & 152 & ePKP & 01 & 48.00 & $6.3 x$ & 1 & & & & e \\
\hline CGP & 126.48 & 35 & ePKP & 01 & 53.28 & $7.8 x$ & 1 & TOO & 174.56 & 79 & ePKP \\
\hline GUMO & 127.45 & 9 & elPdif & 58 & 32.00 & $2.7 x$ & 1 & & & & e \\
\hline MPP & 127.55 & 33 & ePKP & 01 & 55.50 & $7.9 x$ & 1 & SAV & 175.17 & 129 & ePKP \\
\hline & $1.5 \mathrm{~s}$ & 320 & $0.00 \mathrm{~nm}$ & & & & 1 & CAN & 175.69 & 38 & ePKP \\
\hline DAV & 128.08 & 34 & PKP & 01 & 51.00 & $2.5 x$ & 1 & & & & e \\
\hline & & & es & 04 & 00.00 & & 1 & & & & ePP \\
\hline SPA & 128.60 & 180 & ePKPd & 01 & 47.00 & -1.1 & I & & $S \cdot D .=1$ & 1.2 & on 354 \\
\hline & $1.0 \mathrm{~s}$ & 35 & $5.00 \mathrm{~nm}$ & & & & 1 & & & & \\
\hline & $20 \mathrm{~s}$ & 25 & $5.60 \mathrm{um}$ & & & $6.9 \mathrm{Msz}$ & 1 & MAY & 25, & 1980 & $16 \mathrm{~h} 3$ \\
\hline & & & $i$ & 04 & 08.20 & & 1 & & $.598 \mathrm{~N}$ & & 11 \\
\hline LEM & 128.77 & 63 & $e(P K P)$ & 01 & 53.00 & $3.0 x$ & 1 & & $\mathrm{PTH}=$ & $8.0 k$ & $(g e$ \\
\hline & & & eS & 83 & 56.00 & & 1 & & $2 \mathrm{mb}<54$ & 4 obs & s.) \\
\hline MNI & 132.78 & 39 & $e(P K P)$ & 02 & 07.80 & $9.7 x$ & 1 & CALIF & FORN IA-N & NEVAD & $D A \quad 8 O R D$ \\
\hline AF I & 139.72 & 298 & ePKP & 02 & 23.00 & $12.6 x$ & 1 & & $\langle G S\rangle, \quad S$ & Seven & n peopl \\
\hline & & & PP & 05 & 00.00 & & 1 & & damage & ( $v) !$ & 1) and \\
\hline & & & e & 06 & 00.00 & & I & & the Man & moth & h Lokes \\
\hline & & & e & 12 & 10.00 & & 1 & & orea, & Calif & fornio. \\
\hline & & & e & 17 & 36.00 & & 1 & & 015000 & ccurr & red in \\
\hline & & & SKKS & 18 & 33.00 & & 1 & & Nationo & $01 \mathrm{~Pa}$ & ark. To \\
\hline & & & SS & 23 & 44.00 & & 1 & & estimot & ted a & at $2 \mathrm{mi}$ \\
\hline & & & SSP & 24 & 08.00 & & 1 & & Surfoce & e fou & ulting \\
\hline & & & SSS & 28 & 45.00 & & 1 & & cracks & obse & erved $i$ \\
\hline & & & LO & 40 & 00.00 & & 1 & & epicent & trol & oreo. \\
\hline & & & LR & 48 & 00.00 & & 1 & & of appr & roxim & mately \\
\hline SBA & 140.35 & 185 & PKP & 02 & 06.00 & $-3.7 x$ & 1 & & of Coli & iforn & nio and \\
\hline & & & PP & 05 & 10.00 & & 1 & & & & \\
\hline & & & PKS & 85 & 37.00 & & 1 & CLKR & 0.01 & 152 & IPd \\
\hline & & & e & 24 & 00.00 & & I & ORC & 0.14 & 75 & iPc \\
\hline JAY & 142.40 & 19 & ePKP & 82 & 18.20 & $2.9 x$ & I & LMCR & 0.16 & 325 & iPc \\
\hline & 1. & & $5.90 \mathrm{~nm}$ & & & & l & MONR & 0.46 & 5 & iPc \\
\hline & & & es & 05 & 29. & & I & BONR & 0.55 & 49 & $i P c$ \\
\hline OM & 143.16 & 8 & ePKP & 02 & 20.00 & $3.5 x$ & 1 & $T$ IN & 0.72 & 138 & $\mathrm{Pg}$ \\
\hline VG & 143.93 & 2 & EPKP & 02 & 17.00 & -0.8 & 1 & FRI & 0.93 & 229 & $\mathrm{iPC}$ \\
\hline RAB & 145.56 & 0 & PKPC & 02 & 20.00 & -0.6 & 1 & MNA & 0.99 & 32 & iPd \\
\hline WAB & 145.97 & 15 & PKP & 02 & 21.50 & -0.1 & 1 & RYN & 1.06 & 13 & $i P c$ \\
\hline$M D G$ & 146.11 & 12 & ePKP & 02 & 16.00 & $-5.5 x$ & 1 & FERR & 1.12 & 27 & $I P C$ \\
\hline MTN & 148.10 & 42 & ePKP & 02 & 26.00 & 1.4 & I & MMWM & 1.17 & 294 & iPd \\
\hline KNA & 49.17 & 48 & ePKP & 02 & 31.00 & $4.7 x$ & 1 & MBFM & 1.22 & 274 & iPc \\
\hline SVA & 149.26 & 305 & ePKP & 02 & 33.50 & $7.2 x$ & 1 & MSTM & 1.29 & 284 & iPd \\
\hline SWV & 149.86 & 88 & ePKP & 02 & 32.00 & $5.1 x$ & 1 & CWC & 1.30 & 152 & $i P *$ \\
\hline HNR & 149.96 & 345 & EPKP & 02 & 34.00 & $6.5 x$ & 1 & JAS & 1.32 & 286 & iPc \\
\hline MUN & 150.01 & 88 & ePKP & 02 & 32.00 & 4. $9 x$ & 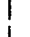 & TNP & 1.36 & 69 & IP* \\
\hline & 2 & 57 & o0um & & & $M s z$ & & $\mathrm{CRH}$ & 1.40 & 288 & $i P d$ \\
\hline
\end{tabular}


$25 d \quad 16 \mathrm{~h}$

\begin{tabular}{|c|c|c|c|c|c|c|c|c|c|c|c|}
\hline $\mathrm{CDC}$ & 2.19 & 256 & ePc & 34 & 22.79 & 1.4 & I ADL & 3.24 & 159 & ePd & 34 \\
\hline HSPM & 2.19 & 258 & iPc & 34 & 22.94 & 1.5 & I SDW & 3.30 & 154 & ePd & 34 \\
\hline BCGM & 2.20 & 247 & iPc & 34 & 22.73 & 1.3 & I BLU & 3.31 & 164 & $e^{P d}$ & 34 \\
\hline WJPM & 2.20 & 173 & iPd & 34 & 23.13 & 1.5 & I TWL & 3.32 & 177 & ePc & 34 \\
\hline LRC & 2.23 & 233 & iPc & 34 & 22.90 & 1.8 & 1512 & 3.39 & 180 & ePd & 34 \\
\hline MNR & 2.23 & 271 & ePc & 34 & 22.77 & 0.8 & I MWC & 3.42 & 169 & ePo & 34 \\
\hline BMCM & 2.23 & 246 & iPc & 34 & 22.85 & 0.8 & I RDM & 3.46 & 157 & ePd & 34 \\
\hline WKR & 2.24 & 218 & iPc & 34 & 22.86 & 0.8 & I ROD & 3.47 & 148 & iPd & 34 \\
\hline CT LM & 2.24 & 272 & ePc & 34 & 23.07 & 1.0 & I $M I N$ & 3.49 & 323 & ePnd & 34 \\
\hline CBSM & 2.24 & 276 & iPc & 34 & 23.47 & 1.3 & I PEM & 3.51 & 167 & ePd & 34 \\
\hline PMCM & 2.24 & 214 & iPc & 34 & 22.91 & 0.8 & $15 s 2$ & 3.55 & 162 & ePd & 34 \\
\hline SAO & 2.25 & 249 & ipnc & 34 & 23.20 & 1.0 & I SIL & 3.63 & 153 & ePd & 34 \\
\hline PIVM & 2.25 & 222 & iPc & 34 & 23.04 & 0.8 & PCF & 3.64 & 166 & ePc & 34 \\
\hline MHC & 2.25 & 264 & iPnc & 34 & 23.60 & 1.2 & I GAV & 3.72 & 163 & ePd & 34 \\
\hline BMHM & 2.26 & 247 & IPC & 34 & 23.10 & 0.7 & I HDG & 3.77 & 146 & IPd & 34 \\
\hline COVM & 2.27 & 270 & ePc & 34 & 23.05 & 0.6 & I RVR & 3.79 & 161 & ePd & 34 \\
\hline$A D R$ & 2.27 & 260 & ePc & 34 & 24.00 & 1.5 & | RMR & 3.84 & 151 & ePd & 34 \\
\hline HSFM & 2.27 & 251 & iPC & 34 & 23.79 & 1.2 & I RCH & 3.85 & 148 & ePd & 34 \\
\hline SHG & 2.27 & 239 & iPc & 34 & 23.57 & 1.0 & I VPD & 3.87 & 167 & ePd & 34 \\
\hline APRM & 2.27 & 305 & ePd & 34 & 23.74 & 1.2 & 1000 & 3.88 & 164 & ePd & 34 \\
\hline DIXR & 2.28 & 15 & ePd & 34 & 22.60 & -0.1 & I LED & 3.91 & 142 & epd & 34 \\
\hline PSAM & 2.28 & 227 & ePc & 34 & 23.58 & 0.9 & I MRVC & 3.92 & 150 & ePd & 34 \\
\hline ARWM & 2.28 & 307 & ePC & 34 & 23.47 & 0.8 & I PEC & 3.94 & 159 & ePn & 34 \\
\hline CSAM & 2.28 & 273 & ePc & 34 & 23.70 & 1.0 & I WWR & 4.81 & 153 & ePd & 34 \\
\hline CMNM & 2.29 & 272 & ePc & 34 & 21.40 & $-1 \cdot 3$ & I VG2 & 4.10 & 156 & ePd & 34 \\
\hline FRP & 2.29 & 249 & IPC & 34 & 23.63 & 0.8 & $1 W D C$ & 4.15 & 317 & iPnd & 34 \\
\hline CSR & 2.29 & 255 & iPc & 34 & 23.63 & 0.9 & I TPC & 4.15 & 146 & ePd & 34 \\
\hline COE & 2.29 & 262 & ePc & 34 & 24.30 & 1.5 & I SPM & 4. 18 & 137 & ePd & 34 \\
\hline HBTM & 2.30 & 252 & iPc & 34 & 24.20 & 1.3 & I INS & 4.24 & 149 & ePd & 34 \\
\hline JHC & 2.30 & 244 & iPc & 34 & 23.53 & 0.6 & I SNS & 4. 29 & 166 & ePd & 34 \\
\hline AFRM & 2.32 & 302 & ePc & 34 & 24.82 & 1.7 & I KEE & 4.33 & 155 & ePd & 34 \\
\hline ANZ & 2.32 & 253 & iPc & 34 & 24.08 & 0.9 & I PNMC & 4.37 & 145 & ePd & 34 \\
\hline HGWM & 2.33 & 256 & iPc & 34 & 24.09 & 0.8 & I CTW & 4.59 & 147 & ePd & 34 \\
\hline $\mathrm{CBO}$ & 2.33 & 259 & ePc & 34 & 24.34 & 1.0 & | TTM & 4.60 & 134 & ePd & 34 \\
\hline HJGM & 2.33 & 251 & ePc & 34 & 24.08 & 0.7 & I VSTC & 4.62 & 163 & ePd & 34 \\
\hline MHR & 2.34 & 265 & ePc & 34 & 24.81 & 1.3 & I HOT & 4.65 & 156 & ePd & 34 \\
\hline BSRM & 2.34 & 247 & iPc & 34 & 24.90 & 1.3 & i cor & 4.70 & 153 & ePd & 34 \\
\hline YEG & 2.34 & 203 & iPc & 34 & 24.57 & 1.0 & $1 \mathrm{BC2}$ & 4.79 & 144 & ePd & 34 \\
\hline ALNM & 2.35 & 305 & ePc & 34 & 24.80 & 1.2 & I KNB & 4.82 & 95 & eP & 34 \\
\hline CBC & 2.35 & 254 & iPc & 34 & 24.69 & 1.0 & | LTM & 4.86 & 138 & $e P c$ & 34 \\
\hline SVC & 2.36 & 263 & ePc & 34 & 25.20 & 1.4 & i JULC & 4.89 & 158 & ePd & 34 \\
\hline CVR & 2.37 & 267 & ePc & 34 & 24.92 & 1.0 & i RVS & 4.99 & 134 & ePd & 35 \\
\hline$M T C$ & 2.37 & 276 & ePc & 34 & 25.20 & 1.2 & SUP & 5.24 & 151 & ePc & 35 \\
\hline DIL & 2.37 & 252 & iPc & 34 & 24.47 & 0.5 & I MSU & 5.33 & 78 & eP & 35 \\
\hline JRRM & 2.37 & 258 & iPc & 34 & 24.78 & 0.8 & i & & & i & 35 \\
\hline PKC & 2.37 & 255 & ePc & 34 & 24.97 & 1.0 & I DUG & 5.36 & 59 & ip & 35 \\
\hline PSHM & 2.38 & 213 & ePc & 34 & 25.00 & 1.0 & I GLA & 5.59 & 143 & ePd & 35 \\
\hline CVLM & 2.39 & 272 & ePc & 34 & 25.67 & 1.5 & I GCA & 5.80 & 94 & eP & 35 \\
\hline DOO & 2.39 & 274 & ePc & 34 & 25.38 & 1.2 & 1 & & & $i$ & 35 \\
\hline AVRM & 2.39 & 307 & ePc & 34 & 24.35 & 0.2 & I MFW & 8.30 & 2 & ep & 35 \\
\hline JSTM & 2.40 & 262 & ePc & 34 & 25.13 & 0.9 & i TUC & 8.45 & 126 & eP & 35 \\
\hline PRS & 2.40 & 239 & iPnc & 34 & 25.30 & 1.0 & $\mid D C 1$ & 8.64 & 40 & eP & 35 \\
\hline PJLM & 2.40 & 232 & iPC & 34 & 25.45 & 1.1 & I BDW & 8.77 & 51 & $i P C$ & 35 \\
\hline HCOM & 2.40 & 254 & ePc & 34 & 25.05 & 0.7 & I LON & 9.41 & 347 & iPd & 36 \\
\hline CPNM & 2.41 & 272 & ePc & 34 & 25.68 & 1.2 & I & $2.0 \mathrm{~s}$ & 1510 & $0.00 \mathrm{~nm}$ & \\
\hline MS.J & 2.42 & 269 & ePc & 34 & 25.73 & 1.1 & I SVM & 9.87 & 116 & ep & 36 \\
\hline CACM & 2.42 & 256 & ePc & 34 & 25.58 & 1.0 & I MSO & 9.91 & 20 & iPc & 36 \\
\hline ABJM & 2.43 & 311 & eP & 34 & 25.48 & 0.7 & i & $1.3 \mathrm{~s}$ & 2130 & $.00 \mathrm{~nm}$ & \\
\hline JFS & 2.43 & 157 & ePd & 34 & 25.14 & 0.2 & MSA & 10.24 & 90 & eP & 36 \\
\hline EUC & 2.44 & 258 & ePc & 34 & 25.67 & 0.8 & I ALO & 10.33 & 101 & eP & 36 \\
\hline JHLM & 2.44 & 259 & ePc & 34 & 25.83 & 0.8 & I GMW & 10.36 & 345 & $e^{P}$ & 36 \\
\hline AMC & 2.44 & 261 & ePc & 34 & 25.83 & 0.9 & i & & & $\mathrm{i}$ & 36 \\
\hline CRGC & 2.46 & 197 & ePC & 34 & 26.36 & 1.1 & I NEW & 10.73 & 6 & ePd & 36 \\
\hline Вмтс & 2.46 & 176 & iPd & 34 & 27.29 & 1.9 & I GOL & 10.73 & 75 & ep & 36 \\
\hline HCZM & 2.47 & 255 & ePc & 34 & 25.90 & 0.6 & I LDM & 11.15 & 12 & iPd & 36 \\
\hline BPCM & 2.47 & 246 & ePc & 34 & 25.92 & 0.6 & I $Y K M$ & 11.48 & 10 & iPd & 36 \\
\hline TMB & 2.57 & 193 & ePd & 34 & 28.01 & 1.2 & I PGC & 11.55 & 345 & ep & 36 \\
\hline GCC & 2.59 & 258 & iPnc & 34 & 27.50 & 0.5 & I RXF & 11.58 & 12 & iPd & 36 \\
\hline BKS & 2.71 & 277 & iPn & 34 & 29.60 & 0.8 & I PNT & 11.73 & 357 & $e^{p}$ & 36 \\
\hline FTC & 2.72 & 181 & ePd & 34 & 30.74 & 1.7 & I LD3 & 12.80 & 42 & ePc & 36 \\
\hline$A B L$ & 2.76 & 187 & ePd & 34 & 30.65 & 0.9 & i & & & $\mathrm{i}$ & 36 \\
\hline GSC & 2.81 & 144 & ePd & 34 & 30.30 & 0.0 & SES & 13.96 & 21 & eP & 37 \\
\hline PKM & 2.81 & 197 & iPc & 34 & 31.09 & 0.7 & I MOT & 14.07 & 115 & ip & 37 \\
\hline$P C C$ & 2.83 & 269 & iPnc & 34 & 30.80 & 0.4 & I LUB & 4.37 & 101 & $e P$ & 37 \\
\hline ORV & 2.86 & 314 & iPnd & 34 & 31.00 & 0.0 & I PHC & 14.47 & 338 & ep & 37 \\
\hline SWM & 2.88 & 176 & ePd & 34 & 33.38 & 2.1 & I EBS & 15.14 & 55 & $P$ & 37 \\
\hline EUR & 2.93 & 49 & iPn & 34 & 32.20 & 0.1 & EDM & 16.08 & 12 & eP & 37 \\
\hline LHU & 2.94 & 173 & ePd & 34 & 33.62 & 1.5 & I JCT & 17.28 & 109 & ip & 37 \\
\hline RYS & 2.98 & 188 & ePd & 34 & 34.37 & 1.6 & i & & & is & 41 \\
\hline FLSC & 3.00 & 151 & ePd & 34 & 33.52 & 0.3 & I TUL & 18.53 & 88 & iPct & 38 \\
\hline SBB & 3.02 & 164 & ePC & 34 & 32.84 & -0.3 & 1 & $2.0 \mathrm{~s}$ & 5140 & $0.00 \mathrm{~nm}$ & \\
\hline BMN & 3.09 & 23 & $i P_{n}$ & 34 & 34.00 & -0.3 & 1 & $22 \mathrm{~s}$ & 70 & . 70 um & \\
\hline LJB & 3.10 & 165 & ePd & 34 & 35.06 & 0.7 & I & & & es & 41 \\
\hline LRRC & 3.13 & 168 & ePd & 34 & 35.82 & 1.1 & i & & & e & 01 \\
\hline ECF & 3.14 & 184 & ePd & 34 & 36.68 & 1.8 & RLO & 19.09 & 87 & iPc & 38 \\
\hline I RC & 3.22 & 174 & ePd & 34 & 37.66 & 1.6 & HKT & 20.55 & 105 & eP & 38 \\
\hline JNH & 2 & 67 & ePd & 34 & 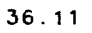 & 0 & $\mathrm{FC}$ & 20.63 & 29 & $P$ & 38 \\
\hline
\end{tabular}




\begin{tabular}{|c|c|c|c|c|c|c|c|c|c|c|c|}
\hline & & & $i$ & 42 & 47.00 & & I SAP & 71.7 & 310 & eP & 45 \\
\hline & & & es & 49 & 56.00 & & $I D C N$ & 71.87 & 36 & iPc & 45 \\
\hline LGN & 50.65 & 110 & eP & 42 & 55.00 & 9.0 & i & $1.0 \mathrm{~s}$ & & $4.08 \mathrm{~nm}$ & \\
\hline UAV & 1.75 & 111 & $e^{P}$ & 43 & 01.50 & 6.9 & $\angle P B$ & 71.92 & 129 & $P$ & 45 \\
\hline rov & 1.99 & 109 & $\mathrm{eP}^{\mathrm{P}}$ & 42 & 58.20 & 2.0 & i & & & is & 54 \\
\hline FUO & .99 & 117 & eP & 42 & 59.00 & 2.4 & EDI & 72.02 & 32 & eP & 45 \\
\hline$B O G$ & 35 & 118 & eP & 43 & 01.50 & 2.2 & 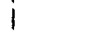 & $0.9 \mathrm{~s}$ & & $0.00 \mathrm{~nm}$ & \\
\hline BOCO & 2.39 & 118 & $e(P)$ & 43 & 00.80 & 1.1 & SOD & 72.12 & 13 & eP & 45 \\
\hline PSO & 2.61 & 123 & $e^{P}$ & 43 & 02.00 & 0.7 & i & & & $i$ & 45 \\
\hline CAR & .70 & 106 & eP & 43 & 07.50 & -1.5 & $E B L$ & 72.18 & 32 & eP & 5 \\
\hline cum & 5.84 & 104 & ep & 43 & 19.30 & -5.2 & i & $0.9 \mathrm{~s}$ & 32 & $2.00 \mathrm{~nm}$ & \\
\hline SEY & 56.78 & 327 & $\mathrm{Pc}_{\mathrm{c}}$ & 43 & 31.40 & 0.7 & BER & 72.19 & 26 & iP & 45 \\
\hline & & & isP & 43 & 37.00 & & DLE & 72.24 & 36 & eP & 45 \\
\hline & & & es & 51 & 30.00 & & $E G L$ & 72.25 & 32 & eP & 45 \\
\hline PET & 56.87 & 314 & $\mathrm{Pd}$ & 43 & 32.00 & 0.6 & i & $0.9 \mathrm{~s}$ & 29 & $9.08 \mathrm{~nm}$ & \\
\hline & & & esP & 43 & 36.00 & & DDK & 72.27 & 36 & eP & 45 \\
\hline & & & es & 51 & 25.00 & & D DKM & 72.39 & 36 & iPc & 45 \\
\hline KTG & 57.31 & 23 & $i P c$ & 43 & 34.70 & 0.3 & 1 & $1.8 \mathrm{~s}$ & 56 & $6.88 \mathrm{~nm}$ & \\
\hline & $2.0 \mathrm{~s}$ & 565 & $5.00 \mathrm{~nm}$ & & & $.3 \mathrm{mb}$ & EKA & 72.42 & 33 & $\mathrm{Pc}$ & 45 \\
\hline & & & $i$ & 44 & 00.00 & & i & $0.9 \mathrm{~s}$ & 15 & $5.80 \mathrm{~nm}$ & \\
\hline & & & is & 51 & 40.00 & & NAO & 73.67 & 23 & $P$ & 45 \\
\hline MGD & 58.45 & 324 & ePc & 43 & 43.00 & 0.5 & i & $1.9 \mathrm{~s}$ & 608 & $0.00 \mathrm{~nm}$ & \\
\hline & & & esP & 43 & 44.50 & & I UME & 73.85 & 18 & $P$ & 45 \\
\hline & & & es & 51 & 50.00 & & i & & & $i$ & 45 \\
\hline RUV & 58.97 & 213 & ep & 43 & 53.00 & 6.5 & I HFK & 4.01 & 33 & $P$ & 45 \\
\hline & $1.2 \mathrm{~s}$ & 198 & $0.00 \mathrm{~nm}$ & & & $.1 \mathrm{mb}$ & ¿ $B O D$ & 5.86 & 334 & eP & 45 \\
\hline VAH & 59.12 & 213 & eP & 43 & 54.00 & 6.5 & I HFS & 75.89 & 22 & $P$ & 45 \\
\hline & $1.2 \mathrm{~s}$ & 155 & $5.00 \mathrm{~nm}$ & & & $.8 \mathrm{mb}$ & i & $1.8 \mathrm{~s}$ & 46 & $6.00 \mathrm{~nm}$ & \\
\hline SKR & 59.20 & 312 & eP & 43 & 46.00 & -1.8 & i & $24 \mathrm{~s}$ & & $5.88 \mathrm{um}$ & \\
\hline REY & 59.74 & 30 & $e^{P}$ & 43 & 58.20 & 6.9 & KJF & 75.15 & 14 & iPd & 45 \\
\hline KES & 60.28 & 10 & iPd & 43 & 56.40 & 1.5 & $i$ & $1.1 \mathrm{~s}$ & 38 & $8.18 \mathrm{~nm}$ & \\
\hline & & & $i$ & 44 & 00.00 & & i & $z 20 \mathrm{~s}$ & 17 & $7.50 \mathrm{um}$ & \\
\hline AKU & 60.54 & 28 & ip & 43 & 58.60 & 1.8 & i & $\mathrm{N} 20 \mathrm{~s}$ & & $9.60 \mathrm{um}$ & \\
\hline & $1.9 \mathrm{~s}$ & 1000 & $0.00 \mathrm{~nm}$ & & & $.6 \mathrm{mb}$ & i & E $20 \mathrm{~s}$ & & $7.58 \mathrm{um}$ & \\
\hline$z$ & $16 \mathrm{~s}$ & & $9.50 \mathrm{um}$ & & &.$\theta M s z X$ & i & & & i & 45 \\
\hline N & $18 \mathrm{~s}$ & & $5.00 \mathrm{um}$ & & & & i & & & ePP & 48 \\
\hline E & $18 \mathrm{~s}$ & 15 & $5.00 \mathrm{um}$ & & & & I ANT & 76.89 & 135 & $\mathbf{P}$ & 45 \\
\hline & & & $i$ & 44 & 03.60 & & 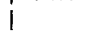 & & & $s$ & 55 \\
\hline TIK & 61.27 & 340 & $\mathrm{Pc}$ & 44 & 01.30 & -0.4 & i & & & $\mathrm{ScS}$ & 55 \\
\hline & & & is $P$ & 44 & 06.50 & & 1 & & & SS & 00 \\
\hline & & & es & 52 & 25.00 & & I TSK & 76.46 & 305 & eP & 45 \\
\hline KHE & 62.02 & 1 & $\mathrm{Pc}$ & 44 & 08.00 & 1.3 & UPP & 76.47 & 21 & $\mathbf{P}$ & 5 \\
\hline & & & es & 52 & 32.00 & & i & & & $i$ & 45 \\
\hline TVo & 62.08 & 213 & eP & 44 & 19.00 & 11.2 & i & & & is & 55 \\
\hline & $1.2 \mathrm{~s}$ & 178 & $0.00 \mathrm{~nm}$ & & & & DDR & 77.18 & 306 & eP & 5 \\
\hline NNA & 63.11 & 133 & $P$ & 44 & 16.00 & 1.4 & I VLA & 77.19 & 315 & eP & 5 \\
\hline YAK & 66.51 & 331 & $P_{c}$ & 44 & 36.70 & 0.7 & SRY & 77.37 & 305 & ep & 5 \\
\hline & & & es & 53 & 31.20 & & MAT & 77.49 & 306 & eP & 5 \\
\hline KUR & 66.58 & 310 & $e^{P}$ & 44 & 37.00 & 0.2 & i & $1.6 \mathrm{~s}$ & 307 & $7.00 \mathrm{~nm}$ & \\
\hline & & & es & 53 & 36.00 & & i & $20 \mathrm{~s}$ & & 3.19 um & \\
\hline YSS & 68.71 & 313 & ePd & 44 & 52.00 & 1.9 & i & & & es & 55 \\
\hline & & & esP & 44 & 56.10 & & I OYM & 77.50 & 305 & eP & 45 \\
\hline & & & es & 53 & 57.00 & & I NUR & 77.75 & 18 & ip & 45 \\
\hline ARE & 69.79 & 131 & eP & 44 & 57.08 & -0.5 & i & $1.0 \mathrm{~s}$ & 640 & $0.00 \mathrm{~nm}$ & \\
\hline & & & es & 54 & 20.00 & & i & $z 22 s$ & & 4.81 um & \\
\hline & & & e LO & 02 & 40.00 & & STS & 77.90 & 45 & ep & 45 \\
\hline KEV & 70.08 & 12 & eP & 44 & 59.00 & 0.9 & LN & 3.11 & 37 & $i P C$ & 45 \\
\hline & & & $i$ & 45 & 04.68 & & i & $.3 \mathrm{~s}$ & 1420 & $0.00 \mathrm{~nm}$ & \\
\hline & & & es & 54 & 12.00 & & I GRR & 78.20 & 37 & iPc & 45 \\
\hline & & & ePS & 54 & 44.08 & & i & $2.3 \mathrm{~s}$ & 1640 & $0.00 \mathrm{~nm}$ & \\
\hline$K I R$ & 70.50 & 15 & $\mathbf{P}$ & 45 & 01.08 & 0.2 & DBN & 78.22 & 31 & iPd & 45 \\
\hline & & & $i$ & 45 & 02.28 & & & Z $18 \mathrm{~s}$ & & $8.80 u m$ & \\
\hline & & & $i$ & 5 & 86.80 & & i & & & $\mathrm{i}$ & 45 \\
\hline & & & $i$ & 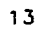 & 84.78 & & i & & & iPP & 48 \\
\hline KMU & .17 & 309 & $e^{P}$ & . & 06.30 & 1.8 & 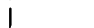 & & & is & 55 \\
\hline VAL & 1.31 & 38 & ePd & 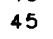 & 07.00 & 1.1 & COP & 78.23 & 26 & ePd & 45 \\
\hline & & & PP & 47 & 52.80 & & i & $z \quad 20 \mathrm{~s}$ & & $6.00 \mathrm{um}$ & \\
\hline & & & $s$ & 54 & 32.00 & & i & & & iPP & 48 \\
\hline & & & ss & & 12.8 & & & & & is & 55 \\
\hline & & & sss & 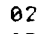 & 33.00 & & & & & iPS & 56 \\
\hline & & & LR & 87 & 26.00 & & wi & & 30 & $e^{P}$ & 5 \\
\hline EAB & .36 & 32 & eP & 5 & 85.90 & -0.2 & LPF & 36 & 37 & $i P c$ & 45 \\
\hline & $9 \mathrm{~s}$ & & 2.00 & & & $3 \mathrm{mb}$ & & $.9 \mathrm{~s}$ & 651 & $1.00 \mathrm{~nm}$ & \\
\hline AF I & 71.36 & 235 & eP & 45 & 12.00 & 5.2 & ssc & 78.42 & 36 & iPc & 45 \\
\hline & & & $s$ & & & & & $2.3 \mathrm{~s}$ & 1130 & $000 \mathrm{~nm}$ & \\
\hline & & & ss & 58 & 52.00 & & I UCC & 78.84 & 33 & $\mathrm{Pc}$ & 45 \\
\hline & & & sS & 02 & & & & & & PP & 8 \\
\hline & & & e & 06 & 00.00 & & & & & $\mathrm{~s}$ & 5 \\
\hline ELO & .44 & 32 & ep & 4 & 06.90 & 2 & WTS & 78.97 & 31 & iPd & 5 \\
\hline & & & 2.00 & & & & & & & & 45 \\
\hline NR I & .48 & 350 & $\mathrm{Pc}$ & 45 & 06.00 & -0.7 & & & & ePP & 48 \\
\hline & & & $i s P$ & 45 & 12.00 & & PTO & 30 & 46 & $e^{P}$ & 45 \\
\hline$E B H$ & 67 & 32 & eP & 45 & 08.20 & 0.2 & i & & & es & 55 \\
\hline & & & 2.08 & & 5 & $6 \mathrm{~m}$ & & & & es & 55 \\
\hline MU & 71.68 & 35 & iPc & 45 & 09.50 & 1.3 & i & & & ess & 00 \\
\hline & $1.0 \mathrm{~s}$ & 58 & $8.00 \mathrm{~nm}$ & & 5. & $6 \mathrm{~m}$ & DOU & 79.46 & 33 & $\mathrm{Pc}$ & 45 \\
\hline $0 \mathrm{~B}$ & 71.70 & 128 & $P$ & 45 & 08.80 & -0.6 & . & & & SKS & 56 \\
\hline
\end{tabular}




\begin{tabular}{|c|c|c|c|c|c|c|c|c|c|c|c|}
\hline & & & & $\mathbf{i}$ & 46 & 17.00 & & I & & & e \\
\hline & & & & e & 47 & 02.00 & & I NVS & 85.93 & 347 & $P$ \\
\hline & & & & es & 56 & 36.80 & & i & & & es \\
\hline I RK & & 82.93 & 335 & eP & 46 & 09.00 & -1.6 & I SVE & 85.95 & 0 & $P d$ \\
\hline & & & & es & 56 & 28.00 & & I & & & isP \\
\hline $8 \cup 8$ & & 82.96 & 33 & P & 46 & 12.00 & 1.1 & 1 & & & es \\
\hline SSB & & 83.01 & 36 & iPd & 46 & 12.80 & 1.6 & I SOP & 86.23 & 28 & $\mathbf{P}$ \\
\hline MLS & & 83.01 & 40 & iPd & 46 & 18.00 & 6.8 & 1 & & & ePCP \\
\hline ZUL & & 83.08 & 33 & P & 46 & 12.50 & 1.0 & 1 & & & e \\
\hline CEN & & 83.19 & 139 & $e(P)$ & 46 & 14.00 & 1.8 & 1 & & & e \\
\hline PRA & & 83.47 & 28 & iP & 46 & 15.00 & 1.6 & I SPC & 86.29 & 26 & eP \\
\hline & & & & e & 46 & 21.00 & & 1 & $z \quad 15 \mathrm{~s}$ & 14 & $4.80 u m$ \\
\hline & & & & PP & 49 & 36.00 & & 1 & & & $\mathrm{i}$ \\
\hline & & & & PPP & 51 & 28.00 & & I ARU & 86.34 & 1 & eP \\
\hline & & & & s & 56 & 50.00 & & 1 & & & isp \\
\hline & & & & PS & 57 & 42.00 & & 1 & & & es \\
\hline SFS & & 83.50 & 48 & iP & 46 & 16.00 & 2.2 & I UER & 86.65 & 340 & $P c$ \\
\hline & & & & iPP & 49 & 52.00 & & I GUA & 86.73 & 284 & $e(P)$ \\
\hline & & & & is & 56 & 44.00 & & I GUMO & 86.73 & 285 & $e(P)$ \\
\hline KSP & & 83.51 & 27 & eP & 46 & 15.00 & 1.4 & 1 & & & es \\
\hline PRU & & 83.57 & 28 & Pd & 46 & 16.20 & 2.3 & TRI & 86.75 & 31 & IPd \\
\hline & & $1.8 \mathrm{~s}$ & 87 & $.50 \mathrm{~nm}$ & & & $7 \mathrm{mb}$ & 1 & & & iPP \\
\hline & & & & $\mathrm{i}$ & 46 & 21.70 & & 1 & & & es \\
\hline & & & & es & 56 & 50.00 & & 1 & & & $\mathrm{i}$ \\
\hline & & & & $i$ & 57 & 38.00 & & I SRO & 86.77 & 27 & iPd \\
\hline FUR & & 83.76 & 31 & eP & 46 & 17.50 & 2.6 & 1 & $20 s$ & 15 & 5.90 um \\
\hline & & & & es & 56 & 50.00 & & 1 & $20 s$ & 20 & 0.20 um \\
\hline $\mathrm{KHC}$ & & 83.90 & 29 & $P d$ & 46 & 17.80 & 2.1 & 1 & & & e \\
\hline & & & & esp & 46 & 23.00 & & 1 & & & is \\
\hline WAR & & 83.93 & 23 & eP & 46 & 17.00 & 1.3 & I LJU & 86.79 & 30 & $e^{P}$ \\
\hline & $Z$ & $20 \mathrm{~s}$ & 12 & $.30 u m$ & & & $3 M s z$ & 1 & & & es \\
\hline & & & & e & 49 & 23.00 & & I ELT & 86.94 & 345 & eP \\
\hline & & & & e & 56 & 55.00 & & I JOS & 86.98 & 26 & ePd \\
\hline$M D Z$ & & 84.17 & 140 & $e(P)$ & 46 & 16.40 & -0.7 & 1 & $1.5 \mathrm{~s}$ & 90 & $0.00 \mathrm{~nm}$ \\
\hline EBR & & 84.24 & 42 & eP & 46 & 19.00 & 1.6 & I LVV & 86.98 & 23 & Pd \\
\hline & & & & $(P P)$ & 49 & 43.00 & & 1 & & & es \\
\hline & & & & $(\mathrm{S})$ & 56 & 49.00 & & | PRT & 87.08 & 34 & $\mathbf{P}$ \\
\hline ORO & & 84.33 & 34 & $\mathbf{P}$ & 46 & 20.50 & 2.5 & I & & & is \\
\hline Mor & & 84.52 & 336 & ePc & 46 & 21.00 & 2.4 & I CVF & 87.10 & 36 & eP \\
\hline CRT & & 84.53 & 47 & $i P C$ & 46 & 23.00 & 3.9 & I FIR & 87.23 & 34 & $\mathbf{P}$ \\
\hline OGA & & 84.68 & 32 & iPd & 46 & 22.50 & 2.7 & I & & & $\mathrm{s}$ \\
\hline RAC & & 84.82 & 26 & $\mathbf{P}$ & 46 & 22.00 & 1.8 & I PSZ & 87.25 & 26 & $\mathbf{P}$ \\
\hline ZAK & & 84.87 & 334 & Pd & 46 & 22.50 & 2.1 & I UZH & 87.46 & 25 & $\mathrm{Pd}$ \\
\hline & & & & es & 56 & 42.00 & & 1 & & & is \\
\hline MOS & & 84.88 & 13 & $P d$ & 46 & 23.00 & 2.6 & I ZAG & 87.55 & 30 & eP \\
\hline & & & & $i s p$ & 46 & 26.00 & & 1 & & & es \\
\hline & & & & es & 56 & 52.00 & & | BJI & 87.73 & 321 & eP \\
\hline$B A O$ & & 84.92 & 114 & eP & 46 & 22.20 & 0.9 & 1 & $17 \mathrm{~s}$ & & $3.40 \mathrm{um}$ \\
\hline KMR & & 84.99 & 30 & iPd & 46 & 23.80 & 2.7 & 1 & $19 \mathrm{~s}$ & & 5.10 um \\
\hline & & & & e & 23 & 20.00 & & I & & & eSKS \\
\hline STV & & 85.09 & 36 & $P$ & 46 & 25.50 & 3.7 & I HNR & 88.82 & 257 & eP \\
\hline MOA & & 85.21 & 30 & iPc & 46 & 23.50 & 1.3 & 1 & & & es \\
\hline & & & & e & 46 & 25.00 & & I MNS & 88.97 & 34 & $\mathbf{P}$ \\
\hline OBN & & 85.23 & 14 & eP & 46 & 23.00 & 0.9 & I AOU & 89.31 & 33 & $\mathbf{P}$ \\
\hline & & & & is & 56 & 58.00 & & | RMP & 89.44 & 34 & $\mathbf{P}$ \\
\hline LRG & & 85.24 & 37 & eP & 46 & 24.20 & 1.8 & 1 & & & ePP \\
\hline & & $2.3 \mathrm{~s}$ & 1070 & $.00 \mathrm{~nm}$ & & & $7 \mathrm{mb}$ & I & & & es \\
\hline ROB & & 85.30 & 35 & $\mathbf{P}$ & 46 & 24.50 & 1.7 & I TIM & 89.59 & 27 & $\mathbf{P}$ \\
\hline SPF & & 85.31 & 36 & eP & 46 & 24.30 & 1.5 & BEO & 90.10 & 28 & $\mathbf{P}$ \\
\hline & & $2.1 \mathrm{~s}$ & 718 & $3.00 \mathrm{~nm}$ & & & $5 \mathrm{mb}$ & I TIA & 90.13 & 318 & $P$ \\
\hline LMR & & 8540 & 37 & eP & 46 & 24.80 & 1.6 & DUI & 90.33 & 33 & $P$ \\
\hline & & 2.35 & 982 & $.00 \mathrm{~nm}$ & & & $6 \mathrm{mb}$ & 1 & & & e \\
\hline$A \perp M$ & & 85.42 & 46 & iPd & 46 & 31.20 & 7.8 & I MBO & 90.48 & 71 & $e P$ \\
\hline & & $2.4 \mathrm{~s}$ & & $.00 \mathrm{~nm}$ & & & $4 \mathrm{mb} x$ & i & & & e \\
\hline & $\mathrm{N}$ & $21 \mathrm{~s}$ & & .80 um & & & & 1 & & & e \\
\hline KRA & & 85.44 & 25 & ePd & 46 & 24.60 & 1.3 & I & & & e \\
\hline & & $2.2 \mathrm{~s}$ & 1000 & $.00 \mathrm{~nm}$ & & & $6 \mathrm{mb}$ & I & & & e \\
\hline & $Z$ & $20 \mathrm{~s}$ & & .90 um & & & $\theta M S Z$ & 1 & & & $e$ \\
\hline & $N$ & $24 \mathrm{~s}$ & & .00 um & & & & I KIS & 90.94 & 21 & $\mathrm{PC}$ \\
\hline & $E$ & $24 \mathrm{~s}$ & & .00 um & & & & I & & & is \\
\hline & & & & e & 46 & 25.60 & & CMP & 91.31 & 25 & eP \\
\hline & & & & e & 46 & 29.20 & & $M L R$ & 91.41 & 24 & eP \\
\hline & & & & e & 46 & 41.60 & & SSE & 91.62 & 312 & eP \\
\hline & & & & e & 49 & 42.00 & & 1 & $20 \mathrm{~s}$ & & 2.12 \\
\hline & & & & e & 51 & 42.00 & & I & $18 \mathrm{~s}$ & & $1.67 \mathrm{um}$ \\
\hline & & & & es & 57 & 00.00 & & I & & & es \\
\hline & & & & e & 02 & 07.40 & & I NOU & 92.03 & 243 & $P d$ \\
\hline & & & & e & 02 & 09.50 & & I NJ2 & 92.28 & 314 & $P$ \\
\hline & & & & e & 02 & 16.30 & & I BUC & 92.42 & 24 & $e^{P}$ \\
\hline PCN & & 85.52 & 34 & $\mathbf{P}$ & 46 & 33.00 & 9.2 & I KOU & 92.48 & 246 & $\mathbf{P d}$ \\
\hline VKA & & 85.66 & 28 & IPd & 46 & 26.50 & 2.1 & I TIR & 92.83 & 30 & $P$ \\
\hline & & & & PP & 46 & 32.00 & $17 \mathrm{kmx}$ & I SKO & 92.91 & 29 & eP \\
\hline & & & & PPP & 49 & 52.00 & & I & & & $\mathrm{i}$ \\
\hline & & & & S & 57 & 01.00 & & 1 & & & iPP \\
\hline VIE & & 85.69 & 28 & ePd & 46 & 27.00 & 2.5 & I & & & eSKS \\
\hline & & & & PP & 46 & 32.00 & $16 \mathrm{kmx}$ & I & & & is \\
\hline & & & & 5 & 57 & 00.00 & & I SOF & 92.99 & 27 & eP \\
\hline
\end{tabular}


KRI $\quad 145.39 \quad 62$ ePKP $53 \quad 26.00 \quad 1.2$

CER $\quad 145.84 \quad 96$ EPKP $5326.50 \quad 1.5$

SUR 146.7193 PKPC 5913.00

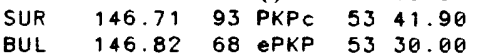

147.6156 PKP 5331.00

148.1854 PKP+ 5332.00

$148.94 \quad 87$ PKPd 5340.00

$149.70 \quad 77$ PKPC $53 \quad 38.50$

$149.71 \quad 84$ PKP $53 \quad 38.50$

$\begin{array}{lrlll}149.93 & 181 & \text { EPKP } & 53 & 36.00 \\ 150.34 & 47 & \text { PKP } & 53 & 40.00\end{array}$

$153.05 \quad 74$ PKP 5348.00

$\begin{array}{llll}157.99 & 36 & \text { ePKP } 53 & 49.70\end{array}$

579 obs. associoted

N 29,1980 07h $20 \mathrm{~m} \mathrm{07.02t0.15s}$

$34.825 \mathrm{~N} \pm 1.1 \mathrm{~km} 139.268 \mathrm{E} \pm 1.1 \mathrm{~km}$ | SHR

DEPTH $=26.1 \pm 1.0 \mathrm{~km}$

$5.8 \mathrm{mb}$ ( 71 obs.) $6.2 \mathrm{Msz}$ ( 14 obs.) TKD

NEAR S. COAST OF HONSHU, JAPAN (230)

Eight people injured and

considerable domage ( $V$ JMA) and TOY

landslides on the Izu Peninsulo

and on Oshima. Felt (IV JMA) ot HIK
Kumagayo, Mishimo, Toteyoma,

Tokyo and Yokohoma; ( 111 JMA) ot I OWA

Chichibu, Choshi, Irozaki,

Kowoguchi-ko, Kofu ond

Utsunomiya; ( 11 JMA) of Gifu,

Kokioka, Koruizowo, Moebashi.

Motsumoto, Mito, Nogoyo,

Onohomo, Shizuoko and on

Hachijo-jimo. Locol tsunomi

generoted in Sagomi Boy. Maximum KYO

wove height (peak-to-trough) was

$57 \mathrm{~cm}$. at okodo.

OSH $\quad 0.11125 \mathrm{P*}$

0. 19309 P:-

$0.26327 \quad P *-$

$0.40316 \mathrm{P*-}$

0. $41237 \mathrm{P}$.

$0.4778 \mathrm{P}$.

0.59358 i $P_{*}$

YOK

$0.6927 \mathrm{P}$.

$0.72282 \mathrm{P}$ *

$0.78 \quad 0 \quad \mathrm{iP} . \mathrm{C}$

$0.79329 \mathrm{P}$ *

$0.8163 \mathrm{iP*c}$

$0.90256 \mathrm{P}$

eS

$0.9525 \mathrm{P*t}$

$1.02326 \mathrm{P*}$ eS

$\begin{array}{llll} & & & \text { eS } \\ \text { TKO4 } & 1.23 & 249 & \text { Pnt } \\ \text { CHJJ } & 1.24 & 350 & \text { Pn- }\end{array}$

$\begin{array}{llll}\text { CHJJ } & 1.24 & 350 & \mathrm{Pn}- \\ \text { TKO3 } & 1.26 & 239 & \mathrm{Pnt}\end{array}$

HMM $\quad 1.28265 \mathrm{Pnt}$

IIDJ $\quad 1.29301 \mathrm{Pn}^{-}$

KMG

IID $\quad 1.36301 \mathrm{Pn}-$

$\begin{array}{llll}\text { TKO2 } & 1.53 & 235 \mathrm{Pn} \\ \text { TSK } & 1.54 & 26 & \mathrm{Pn}\end{array}$

KAKJ $1.5628 \mathrm{Pnt}$

$\mathrm{CHO}$

MAE $\quad 1.58354 \mathrm{Pnt}$

KAZ $\quad 1.62339 \stackrel{\mathrm{Pn}}{\mathrm{Pn}}$

IKO1 $\quad 1.75233 \mathrm{Pn}$

MTM

$\begin{array}{lll}1.75 & 233 \mathrm{Pn} \\ 1.77 & 324 \mathrm{Pn}\end{array}$

S $\mathrm{Pn-}$ \begin{tabular}{lll|l}
20 & 11.30 & -0.6 & FKS
\end{tabular}

$2010.10 \quad-2.6$

\begin{tabular}{lll|l}
20 & 11.30 & -2.3 & $\mathrm{NI}$
\end{tabular}

$20 \quad 13.80$

$20 \quad 13.70$

$\begin{array}{ll}20.60 \\ 20 & 16.70\end{array}$

2018.00

2028.00

$20 \quad 17.40$

2020.50

$20 \quad 31.90$

2020.00

2031.20

\begin{tabular}{lrr|l|l|}
20 & 22.50 & 0.1 & WKY
\end{tabular}

2023.00

2039.00

2024.30

2038.00

$\begin{array}{ll}20 & 24.00 \\ 20 & 36.00\end{array}$

2026.80

$20 \quad 52.60$

$20 \quad 29.60$

$20 \quad 27.70$

2030.40

$20 \quad 48.20$

$20 \quad 29.50$

2030.20

2048.60

2030.40

2648.60

$20 \quad 33.60$

$\begin{array}{ll}20 & 32.00 \\ 20 & 32.30\end{array}$

$\begin{array}{ll}20 & 32.30 \\ 20 & 33.70\end{array}$

2054.30

$20 \quad 34.20$

$20 \quad 57.40$

2035.00

2054.80

2036.00

$20 \quad 39.10$

2103.20
2039.00

\begin{tabular}{l|l}
-1.1 & OKA \\
-1.0 &
\end{tabular}

\begin{tabular}{l|l}
0.2 & MRT \\
0.6 & $M I Z$
\end{tabular}

$0.7 \quad$\begin{tabular}{l|l}
$\mathrm{HJH}$ \\
$\mathrm{OFU}$
\end{tabular}

\begin{tabular}{l|l}
0.3 & OFUJ \\
2.8 &
\end{tabular}

YONJ \begin{tabular}{lll|l}
20 & 20.50 & -1.4 & OIC \\
20 & 21.00 & -1.1 & YAM
\end{tabular} \begin{tabular}{r|l}
15.2 & MIT \\
2.9 & MAJO \\
2.8 & MAT
\end{tabular}

2.8

2.7 NAG

\begin{tabular}{l|l}
9.8 & \\
7.0 & NGN
\end{tabular}

7.01

5.7 I TKY

\begin{tabular}{r|l}
7.5 & \\
11.8 & MTMJ
\end{tabular}

$6.5 \mid \mathrm{GIF}$

TSU

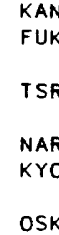

1.78 is 2103.50

$1.8332 \mathrm{Pnt} 2036.00$

2056.40

1.92334 iPnd 2039.10

$1.92334 \mathrm{Pn}-$

2039.00

$1.92 \quad 281 \mathrm{Pn}-$

03.00

$2.03335 \mathrm{Pn}-$

2106.40

$20 \quad 41.90$

2111.20

2. $11309 \mathrm{Pn}$

$2.12326 \mathrm{Pn}$ -

$2.13286 \mathrm{Pn}$

$\begin{array}{ll}20 & 43.00 \\ 21 & 12.60\end{array}$

$20 \quad 42.60$

$\begin{array}{lll}20 & 43.20\end{array}$

2112.00

$2.27268 \mathrm{Pnt}$ is

$2.42 \quad 18 \mathrm{Pn}$

2. $42355 \mathrm{Pnt}$

2. $42340 \mathrm{Pn}-$

2. $5032 \mathrm{Pnt}$

$2.52319 \mathrm{Pn}-$

$2.52281 \mathrm{Pn}-$

2113.10

$20 \quad 45.00$

$20 \quad 45.50$

$20 \quad 47.70$

$21 \quad 17.00$

$\begin{array}{ll}20 & 46.00 \\ 20 & 48.30\end{array}$

2122.00

$20 \quad 48.70$

2120.70

2. $65254 \mathrm{Pnt}$

$2.74310 \mathrm{Pn}-$

$20 \quad 48.70$

2118.40

$2.77297 \mathrm{Pn}-$

2052.20

2052.00

2127.00

$2.78286 \mathrm{Pn}$

2051.00

$2.83268 \mathrm{Pn}$

$2.91275 \mathrm{Pn}$

2126.90

2052.20

2053.30

298267 Pnt 2127.30

2054.00

$\begin{array}{llll} & \text { is } & 21 & 28.70 \\ 3.08 & 18 \mathrm{Pnt} & 2055.00\end{array}$

$\begin{array}{ll}20 & 55.00 \\ 21 & 33.00\end{array}$

$3.08 \quad 357 \mathrm{Pnt}$

2056.50

2135.80

$3.09268 \mathrm{Pnt}$

2055.80

2133.00

2055.00

2059.00

2139.00

$20 \quad 57.20$

2133.00

$3.21246 \mathrm{Pn}-$

2059.00

$3.24283 \mathrm{Pn}$

$3.29346 \mathrm{Pn} \quad 2058.50$

$3.34259 \mathrm{ePnc} 2058.00$

$3.4010 \mathrm{Pnt}$

$3.44261 \mathrm{Pnt}$

2101.00

2141.90

$3.5314 \mathrm{Pn} \quad 2102.00$

$\begin{array}{lll} & \mathrm{S} & 2152.80 \\ 3.6720 \mathrm{Pnt} & 2102.80\end{array}$

() 2148.00

$3.71282 \mathrm{Pn} \quad 2104.00$

$3.76271 \mathrm{Pn} \quad 2105.10$

2155.80

$\begin{array}{llll}3.95 & 24 \mathrm{Pn} & 2108.00\end{array}$

2202.40

$3.95260 \mathrm{Pnt}$

2107.60

2154.90

$4.06 \quad 8 \mathrm{Pn}$

2110.00

2112.00

2111.00

2203.00

2112.10

2200.90

2114.00

2114.00

$4.40260 \mathrm{Pnt}$

$4.41270 \mathrm{Pn}$

2205.20

4. $51251 \mathrm{Pn}$ -

2115.10

2212.50

4.5519 ipnc 2115.70

4. $569 \mathrm{Pnt}$

2116.40

4. $6624 \mathrm{Pn}$

2117.00

2212.00

$4.6624 \mathrm{Pnt}$

2116.30

4. 78276 es

2211.00

$\begin{array}{lll}4.78 & 276 & P n \\ 4.92 & 257 & P n+\end{array}$

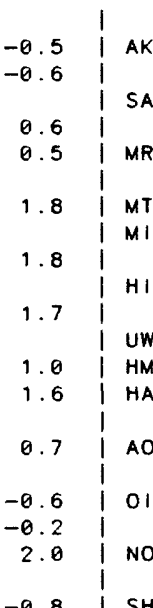

AK I

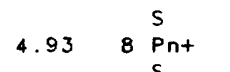

2219.80

2123.50

2220.80

2223.00

2124.00

2224.00

2125.00

$\begin{array}{lllll}5.27 & 23 \mathrm{Pn} & 21 & 25.00 \\ & \mathrm{~S} & 22 & 29.20 \\ 5.65 & 267 \mathrm{Pn} & 21 & 32.00\end{array}$

$\begin{array}{lllll}5.27 & 23 \mathrm{Pn} & 21 & 25.00 \\ & \mathrm{~S} & 22 & 29.20 \\ 5.65 & 267 \mathrm{Pn} & 21 & 32.00\end{array}$

$\begin{array}{rrr}5.65267 \mathrm{Pn} & 2132.00 \\ \mathrm{eS} & 2233.00\end{array}$

$\begin{array}{llll}5.79 & 256 \mathrm{Pn} & 21 & 34.00\end{array}$

$5.92273 \mathrm{Pn} \quad 2137.00$

$\begin{array}{ccll}5.97 & 17 \mathrm{Pnt} & 21 & 36.80 \\ & & 22 & 51.00\end{array}$

$\begin{array}{rrrr} & & \text { ES } & 2251.00 \\ 6.10 & 11 \mathrm{Pn} & 21 & 39.00\end{array}$

$\begin{array}{llll}6.54258 \mathrm{Pn} & 21 & \mathrm{eS} & 22.50 \\ & & \end{array}$

$\begin{array}{llll} & S & 2305.30 \\ 6.70 & 253 \mathrm{Pn} & 2147.00\end{array}$

eS 2308.00

$6.77266 \mathrm{Pn} \quad 2147.00$

$6.94265 \mathrm{Pn} \quad 2149.00$

$\begin{array}{llll} & S & 2305.20\end{array}$

$7.09256 \mathrm{Pnt} 2151.00$

$\begin{array}{cccc}7.09256 \mathrm{Pnt} & 21 & 51.00 \\ \mathrm{eS} & 23 & 15.00\end{array}$

$\begin{array}{ll}7.17248 \mathrm{Pn} & 2154.40\end{array}$

$\begin{array}{ll}\mathrm{S} & 2317.40\end{array}$

$7.40254 \mathrm{Pn} \quad 2157.00$

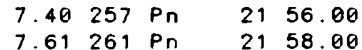

2328.60

$\begin{array}{ll}21 & 59.00 \\ 22 & 01.60\end{array}$

0.7 | MRRJ

$7.7210 \mathrm{Pn}$

$\begin{array}{rrrr}7.82 & 20 \mathrm{Pn} & 2202.00 \\ & \mathrm{eS} & 23 & 31.00 \\ 7.92 & 245 \mathrm{Pn} & 2203.00\end{array}$

$\begin{array}{llll}7.82 & 20 \mathrm{Pn} & 22 & 02.00 \\ & \mathrm{eS} & 23 & 31.00 \\ 7.92 & 245 \mathrm{Pn} & 22 & 03.00\end{array}$

0.4

KAGJ

$7.92245 \mathrm{Pn}$

$\begin{array}{ll}22 & 03.00 \\ 22 & 01.80\end{array}$

$\begin{array}{cccc}7.99 & \text { eS } & 36 & 34.80 \\ \mathrm{P} & 22 & 04.80\end{array}$

$7.995 \mathrm{Pn} 2205.00$

$\begin{array}{ccc}8.07242 \mathrm{P} & 2334.00 \\ & 2206.00\end{array}$

eS 2345.00

$8.10258 \mathrm{P} \quad 2206.20$

es 2337.40

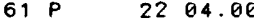

$\begin{array}{llll}8.18 & 21 \mathrm{P} & 2344.50 \\ 8.25 & 269 & \mathrm{P} & 2203.00\end{array}$ 


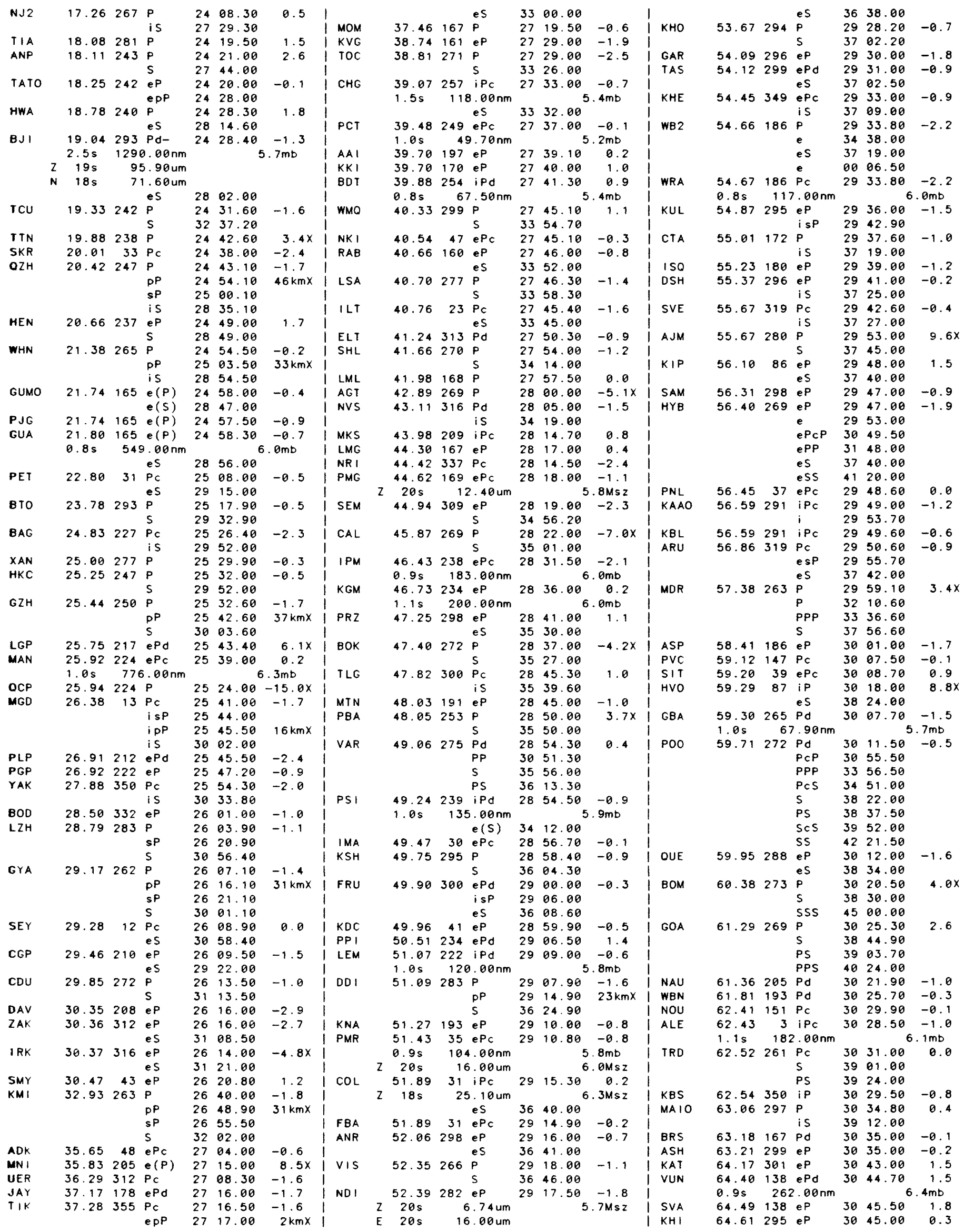


29d B7h

\begin{tabular}{|c|c|c|c|c|c|c|c|c|c|c|c|c|}
\hline KEV & & $\begin{array}{l}64.89 \\
0.8 \mathrm{~s}\end{array}$ & $\begin{array}{r}339 \\
49\end{array}$ & $\stackrel{i P}{9.60 \mathrm{~nm}}$ & 30 & 45.20 & $\begin{array}{l}-0.5 \\
.7 \mathrm{mb}\end{array}$ & $\begin{array}{l}\text { I MHC } \\
\text { I SAV }\end{array}$ & $\begin{array}{l}76.13 \\
76.52\end{array}$ & $\begin{array}{r}54 \\
174\end{array}$ & $\begin{array}{l}\text { iP } \\
\text { eP }\end{array}$ & $\begin{array}{l}31 \\
31\end{array}$ \\
\hline & & & & $\mathbf{i}$ & 30 & 58.30 & & I SAO & 76.58 & 55 & iP & 31 \\
\hline & & & & iPP & 33 & 11.20 & & I JAS & 76.59 & 53 & $i P c$ & 31 \\
\hline & & & & es & 39 & 24.00 & & 1 & & & $\mathbf{e}$ & 32 \\
\hline SOD & & 66.32 & 337 & $P$ & 30 & 53.90 & -1.1 & 1 & & & PP & 34 \\
\hline & & & & iPP & 33 & 19.80 & & I BUT & 76.77 & 43 & $e^{P}$ & 31 \\
\hline YKA & & 66.59 & 29 & $P$ & 30 & 57.40 & 0.7 & 1 & & & e & 32 \\
\hline AFI & & 67.12 & 127 & $P$ & 31 & 02.00 & 1.2 & I СМТ & 76.82 & 42 & ePc & 31 \\
\hline & & & & s & 40 & 03.00 & & 1 & & & e & 32 \\
\hline TRO & & 67.23 & 341 & iP & 30 & 59.80 & -0.9 & I KON & 77.11 & 336 & iP & 31 \\
\hline$K J F$ & & 67.74 & 334 & iP & 31 & 03.00 & -1.0 & I BMN & 77.26 & 50 & ePc & 32 \\
\hline & & $0.9 \mathrm{~s}$ & 111 & $1.00 \mathrm{~nm}$ & & & $.0 m b$ & 1 & $1.0 \mathrm{~s}$ & 25 & $5.00 \mathrm{~nm}$ & \\
\hline & & & & $\mathrm{i}$ & 31 & 10.00 & & I KIS & 77.39 & 319 & $\mathrm{Pc}$ & 32 \\
\hline & & & & iPP & 33 & 38.80 & & 1 & & & is & 41 \\
\hline & & & & es & 39 & 58.00 & & | PR I & 77.44 & 55 & iPC & 32 \\
\hline DAG & & 67.82 & 355 & $i P c$ & 31 & 02.90 & -1.4 & | FR I & 77.61 & 54 & iPc & 32 \\
\hline & & $0.9 \mathrm{~s}$ & & $4.00 \mathrm{~nm}$ & & & $.9 \mathrm{mb}$ & I TAU & 77.71 & 174 & eP & 32 \\
\hline & & & & is & 40 & 05.00 & & 1 & & & es & 41 \\
\hline$K I R$ & & 67.95 & 339 & $P_{C}$ & 31 & 04.00 & -1.3 & I MNV & 77.81 & 52 & iPc & 32 \\
\hline & & & & i & 31 & 10.10 & & I BER & 77.89 & 339 & ip & 32 \\
\hline & & & & is & 40 & 02.00 & & I WAR & 77.94 & 326 & eP & 32 \\
\hline & & & & $\mathbf{i}$ & 59 & 26.00 & & 1 & & & e & 35 \\
\hline MOS & & 67.98 & 323 & $P d$ & 31 & 02.00 & $-3.6 x$ & 1 & & & e & 42 \\
\hline & & & & es & 40 & 06.00 & & I LVV & 78.12 & 323 & Pd & 32 \\
\hline & & & & $\mathrm{S}$ & 40 & 12.00 & & 1 & & & is & 42 \\
\hline MAK & & 68.46 & 308 & eP & 31 & 12.08 & $3.2 x$ & I AKU & 78.28 & 351 & iP & 32 \\
\hline & & & & is & 40 & 16.08 & & 1 & $2.0 \mathrm{~s}$ & 900 & $0.00 \mathrm{~nm}$ & \\
\hline OBN & & 68.77 & 323 & $P_{c}$ & 31 & 10.80 & 0.3 & 1 & $20 s$ & & 4.50 um & \\
\hline & & & & is & 40 & 14.00 & & 1 & & & $\mathrm{i}$ & 32 \\
\hline SHE & & 68.79 & 305 & eP & 31 & 11.00 & 0.1 & I EUR & 78.59 & 50 & iP & 32 \\
\hline PUL & & 69.20 & 329 & $P d$ & 31 & 13.00 & -0.1 & 1 & $1.0 \mathrm{~s}$ & 36 & $6.50 \mathrm{~nm}$ & \\
\hline & & & & isP & 31 & 18.00 & & I CFR & 78.91 & 318 & eP & 32 \\
\hline & & & & is & 40 & 16.00 & & I COP & 79.10 & 333 & iPct & 32 \\
\hline YOU & & 69.27 & 172 & $P$ & 31 & 11.30 & -2.5 & 1 & $0.9 \mathrm{~s}$ & 161 & $1.00 \mathrm{~nm}$ & \\
\hline GRO & & 69.43 & 309 & eP & 31 & 14.00 & -0.8 & 1 & & & iPP & 35 \\
\hline & & & & is & 40 & 25.00 & & 1 & & & is & 42 \\
\hline CAN & & 70.37 & 172 & $\mathrm{Pc}$ & 31 & 21.30 & 0.8 & | VR I & 79.26 & 319 & $e^{P}$ & 32 \\
\hline NWAO & & 70.50 & 200 & eP & 31 & 21.00 & -0.3 & I LD3 & 79.74 & 40 & ePc & 32 \\
\hline UME & & 70.59 & 336 & $P C$ & 31 & 20.38 & -1.2 & 1 & $0.9 \mathrm{~s}$ & 26 & $6.10 \mathrm{~nm}$ & \\
\hline & & & & $\mathrm{i}$ & 31 & 28.10 & & UZH & 79.75 & 323 & Pc & 32 \\
\hline & & & & is & 40 & 43.30 & & 1 & & & es & 42 \\
\hline PYA & & 70.76 & 311 & eP & 31 & 24.00 & 1.1 & I BMR & 79.79 & 322 & eP & 32 \\
\hline & & & & is & 40 & 43.50 & & I KRP & 79.83 & 152 & $P$ & 32 \\
\hline GRS & & $70 \quad 88$ & 305 & $\mathrm{Pc}$ & 31 & 22.80 & -1.1 & I MLR & 79.93 & 319 & eP & 32 \\
\hline LON & & 71.03 & 46 & iPc & 31 & 24.30 & -0.3 & I KRA & 79.93 & 325 & ePc & 32 \\
\hline & & $0.9 \mathrm{~s}$ & & $8.60 \mathrm{~nm}$ & & & $.4 \mathrm{mb}$ & 1 & $0.9 \mathrm{~s}$ & 77 & $7.18 \mathrm{~nm}$ & \\
\hline NUR & & 71.07 & 332 & $\mathbf{P}$ & 31 & 23.50 & -0.9 & 1 & $15 \mathrm{~s}$ & 66 & 6. 18 um & \\
\hline WAM & & 71.22 & 172 & $P$ & 31 & 25.70 & 0.2 & 1 & $28 s$ & 59 & 9.28 um & \\
\hline SHI & & 71.31 & 293 & ePd & 31 & 26.00 & -0.7 & 1 & $20 \mathrm{~s}$ & 79 & $9.70 \mathrm{um}$ & \\
\hline & & & & $e(S)$ & 40 & 44.00 & & 1 & & & e & 32 \\
\hline TAB & & 71.59 & 364 & $e^{P}$ & 31 & 32.00 & $3.8 x$ & 1 & & & e & 32 \\
\hline BKR & & 71.62 & 308 & $P d$ & 31 & 29.00 & 0.6 & 1 & & & e & 32 \\
\hline ERE & & 71.76 & 307 & $P_{C}$ & 31 & 33.00 & $3.9 x$ & 1 & & & e & 32 \\
\hline LEN & & 71.89 & 307 & ep & 31 & 30.00 & 0.0 & 1 & & & e & 33 \\
\hline 100 & & 72.26 & 175 & $P d$ & 31 & 32.00 & 0.2 & 1 & & & e & 35 \\
\hline $\mathrm{FHC}$ & & 72.74 & 52 & i P & 31 & 36.30 & 1.4 & 1 & & & e & 35 \\
\hline NEW & & 72.93 & 43 & iPc & 31 & 36.00 & 0.2 & 1 & & & es & 42 \\
\hline & & $1.0 \mathrm{~s}$ & & $5.00 \mathrm{~nm}$ & & & $.5 \mathrm{mb}$ & 1 & & & e & 43 \\
\hline & $z$ & $18 \mathrm{~s}$ & & $3.00 \mathrm{um}$ & & & $3 M s z$ & I REY & 80.25 & 352 & eP & 32 \\
\hline & $\mathrm{N}$ & $18 \mathrm{~s}$ & & $6.00 \mathrm{um}$ & & & & I PAS & 80.26 & 55 & iPc & 32 \\
\hline & $E$ & $18 \mathrm{~s}$ & & $8.00 \mathrm{um}$ & & & & 1 & $20 \mathrm{~s}$ & 14 & 4. $00 \mathrm{um}$ & \\
\hline SOC & & 73.06 & 311 & $\mathrm{Pc}$ & 31 & 40.00 & $3.4 x$ & 1 & $20 \mathrm{~s}$ & & 5. 00 um & \\
\hline WDC & & 73.80 & 52 & IPC & 31 & 41.60 & 0.7 & 1 & $20 s$ & & 1.00 um & \\
\hline UPP & & 74.16 & 333 & iPc & 31 & 41.60 & -1.0 & 1 & & & ePCP & 32 \\
\hline & & $1.4 \mathrm{~s}$ & 508 & $0.00 \mathrm{~nm}$ & & & $5.3 \mathrm{mb}$ & 1 & & & is & 42 \\
\hline & & & & is & 41 & 08.00 & & 1 & & & escs & 43 \\
\hline KTG & & 74.19 & 354 & iPc & 31 & 42.70 & 0.1 & I CJR & 80.37 & 321 & eP & 32 \\
\hline & & $0.9 \mathrm{~s}$ & & $4.00 \mathrm{~nm}$ & & & $5.6 \mathrm{mb}$ & 1 & & & e & 32 \\
\hline & & & & is & 41 & 18.00 & & $1 S P C$ & 80.38 & 324 & eP & 32 \\
\hline MIN & & 74.52 & 52 & $i P_{C}$ & 31 & 45.20 & -0.2 & 1 & & & $i$ & 32 \\
\hline SES & & 74.97 & 39 & $e^{P}$ & 31 & 47.00 & -0.6 & 1 & & & e & 32 \\
\hline ORV & & 75.02 & 52 & iPc & 31 & 48.20 & 0.2 & 1 & & & iPP & 35 \\
\hline BHD & & 75.42 & 300 & ePc & 31 & 55.08 & $4.6 x$ & 1 & & & $e(S)$ & 42 \\
\hline & & & & e & 41 & 37.50 & & I BDW & 80.45 & 44 & ePc & 32 \\
\hline BKS & & 75.44 & 54 & ePc & 31 & 51.10 & 0.6 & 1 & $1.1 \mathrm{~s}$ & 25 & $5.60 \mathrm{~nm}$ & \\
\hline & 2 & $22 \mathrm{~s}$ & & $1.20 \mathrm{um}$ & & & $.2 M \leqslant z X$ & I CMP & 80.56 & 319 & eP & 32 \\
\hline & $N$ & $22 \mathrm{~s}$ & & $3.50 u m$ & & & & $1 \mathrm{JOS}$ & 80.71 & 324 & ePc & 32 \\
\hline & $E$ & $22 \mathrm{~s}$ & & 1.70 um & & & & I KSP & 81.04 & 327 & iPc & 32 \\
\hline & & & & es & 41 & 34.00 & & 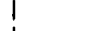 & & & ePP & 35 \\
\hline SIM & & 75.67 & 315 & ePd & 31 & 51.00 & -0.6 & 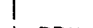 & & & e (S) & 42 \\
\hline & & & & es & 41 & 39.00 & & BRN & 81.21 & 330 & $\mathrm{ePc}$ & 32 \\
\hline GDH & & 75.80 & 5 & $i P c$ & 31 & 51.20 & -0.7 & 1 & & & i & 35 \\
\hline & & $1.0 \mathrm{~s}$ & & $6.00 \mathrm{~nm}$ & & 5 & $5 \mathrm{mb}$ & DEV & 81.28 & 321 & $e P$ & 32 \\
\hline & & & & is & 41 & 32.00 & & PSZ & 81.42 & 324 & $P$ & 32 \\
\hline NAO & & 75.82 & 337 & $P$ & 31 & 50.00 & -2.2 & COB & 81.57 & 155 & $P$ & 32 \\
\hline & & $0.9 \mathrm{~s}$ & & .8 & & & $.2 \mathrm{mb}$ & GZR & 81.67 & 320 & $e^{P}$ & 32 \\
\hline
\end{tabular}




\begin{tabular}{|c|c|c|c|c|c|c|c|}
\hline & & & $\mathbf{e}$ & 35 & 43.00 & & I DOK \\
\hline & & & es & 43 & 08.00 & & I BAF \\
\hline GRF & 84.09 & 329 & $i \mathrm{Pc}$ & 32 & 37.10 & 0.5 & I ALO \\
\hline & $1.1 \mathrm{~s}$ & 277 & $7.00 \mathrm{~nm}$ & & & $.4 \mathrm{mb}$ & I \\
\hline & & & e & 35 & 54.50 & & 1 \\
\hline & & & $i$ & 42 & 58.00 & & I DLE \\
\hline GRFO & 84.09 & 329 & $i P c$ & 32 & 37.30 & 0.7 & 1 \\
\hline & & & $\mathrm{i}$ & 32 & 44.50 & & I BSF \\
\hline MOA & 84.13 & 326 & $i P c$ & 32 & 37.40 & 0.5 & \\
\hline & & & e & 32 & 43.00 & & I HAU \\
\hline & & & PP & 35 & 56.00 & & 1 \\
\hline EIL & 84.17 & 302 & $\mathrm{Pc}$ & 32 & 36.50 & -0.9 & $I D C N$ \\
\hline VAY & 84.52 & 317 & ¡P & 32 & 39.20 & 0.3 & 1 \\
\hline DBN & 84.59 & 334 & $\mathrm{iPc}$ & 32 & 44.00 & $5.0 x$ & 1 ECP \\
\hline & $22 \mathrm{~s}$ & 13 & $3.70 \mathrm{um}$ & & & . 3MsZ & 1 \\
\hline & $120 \mathrm{~s}$ & 46 & $6.30 \mathrm{um}$ & & & & $101 x$ \\
\hline & $20 \mathrm{~s}$ & & $0.50 \mathrm{um}$ & & & & I FIR \\
\hline & & & e & 32 & 59.00 & & I ARO \\
\hline & & & e & 33 & 13.00 & & I MNS \\
\hline & & & e & 35 & 41.00 & & i \\
\hline & & & $i$ & 35 & 56.00 & & I \\
\hline & & & $i$ & 36 & 04.00 & & I LOR \\
\hline & & & is & 43 & 07.00 & & 1 \\
\hline & & & e & 43 & 36.00 & & I LBF \\
\hline EKA & 84.61 & 340 & $P d$ & 32 & 39.60 & 0.5 & 1 \\
\hline & $1.0 \mathrm{~s}$ & 20 & $0.90 \mathrm{~nm}$ & & & $.3 \mathrm{mb}$ & SSF \\
\hline SKO & 84.70 & 319 & ip & 32 & 45.00 & $5.2 x$ & i \\
\hline & & & is & 43 & 10.00 & & I RMP \\
\hline BNS & 84.74 & 332 & iPc & 32 & 40.00 & 0.2 & 1 \\
\hline$Z A G$ & 84.80 & 324 & ip & 32 & 45.80 & $5.6 x$ & I \\
\hline & & & ¿PCP & 33 & 08.10 & & I FLN \\
\hline & & & eSKS & 43 & 07.40 & & 1 \\
\hline GOL & 84.85 & 44 & ePc & 32 & 42.30 & 1.3 & $15 S C$ \\
\hline & $1.1 \mathrm{~s}$ & 22 & $2.50 \mathrm{~nm}$ & & & $3 \mathrm{mb}$ & 1 \\
\hline PMO & 84.93 & 112 & ep & 32 & 48.00 & $6.8 x$ & I SMF \\
\hline & $1.3 \mathrm{~s}$ & 160 & $0.00 \mathrm{~nm}$ & & & $.1 \mathrm{mb}$ & 1 \\
\hline BLY & 84.98 & 323 & iP & 32 & 41.20 & 0.0 & I VAL \\
\hline FUR & 85.22 & 328 & $i P_{c}$ & 32 & 42.90 & 0.5 & 1 \\
\hline & & & e & 36 & 07.00 & & 1 \\
\hline & & & es & 43 & 09.00 & & i \\
\hline HEE & 85.26 & 332 & $i P c$ & 32 & 42.60 & 0.2 & 1 \\
\hline & & & e & 32 & 49.50 & & I AVF \\
\hline & & & e & 00 & 44.00 & & i \\
\hline PPN & 85.33 & 115 & eP & 32 & 51.00 & $7.8 x$ & I GRR \\
\hline & $1.3 \mathrm{~s}$ & 128 & $0.00 \mathrm{~nm}$ & & & $.0 \mathrm{mb}$ & 1 \\
\hline LJU & 85.33 & 325 & $e P c$ & 32 & 43.00 & 0.1 & I LPF \\
\hline & & & ePP & 36 & 08.50 & & i \\
\hline & & & es & 43 & 08.50 & & I SSB \\
\hline RUV & 85.43 & 112 & eP & 32 & 51.00 & $7.3 x$ & I SGR \\
\hline & $1.3 \mathrm{~s}$ & 120 & $0.00 \mathrm{~nm}$ & & & $.0 \mathrm{mb}$ & I TCF \\
\hline MEM & 85.47 & 332 & $\mathrm{Pc}$ & 32 & 45.00 & 1.5 & $i$ \\
\hline & & & PP & 36 & 05.60 & & CVF \\
\hline & & & PS & 44 & 13.00 & & i \\
\hline CEY & 85.61 & 325 & ePc & 32 & 43.00 & -1.3 & I LSF \\
\hline & & & $\mathrm{i}$ & 32 & 48.00 & & 1 \\
\hline & & & ePP & 36 & 08.50 & & SPF \\
\hline & & & es & 43 & 08.50 & & i \\
\hline OHR & 85.64 & 318 & iP & 32 & 44.80 & 0.2 & LRG \\
\hline & & & i & 32 & 50.60 & & 1 \\
\hline STU & 85.65 & 329 & ePct & 32 & 44.50 & 0.1 & I LMR \\
\hline & $1.0 \mathrm{~s}$ & 160 & $0.00 \mathrm{~nm}$ & & & $.2 \mathrm{mb}$ & 1 \\
\hline TR I & 85.94 & 325 & $i(P) c$ & 32 & 44.30 & -1.6 & MFF \\
\hline & & & $i$ & 32 & 49.00 & & 1 \\
\hline & & & $i(P P)$ & 36 & 10.00 & & RJF \\
\hline & & & $e(S K S)$ & 43 & 04.00 & & $i$ \\
\hline & & & $i(s)$ & 43 & 14.00 & & I CAF \\
\hline UCC & 85.94 & 333 & Pd & 32 & 46.00 & 0.2 & LFF \\
\hline & & & $P C P$ & 32 & 50.10 & & 1 \\
\hline & & & PP & 36 & 08.20 & & I LPO \\
\hline ATH & 86.11 & 314 & ePc & 32 & 50.00 & $3.1 \mathrm{x}$ & 1 \\
\hline & & & ePP & 36 & 06.00 & & $\mathrm{ICHI}$ \\
\hline & & & es & 43 & 18.00 & & TUL \\
\hline BUH & 86.12 & 330 & ePc & 32 & 46.50 & -0.4 & i \\
\hline WLF & 86.17 & 332 & ePc & 32 & 53.00 & $6.0 x$ & 1 \\
\hline & & & $s$ & 43 & 18.00 & & RLO \\
\hline & & & ScS & 43 & 32.00 & & MLS \\
\hline TUC & 86.29 & 53 & ePc & 32 & 48.90 & 0.8 & EPF \\
\hline DOU & 86.43 & 333 & Pd & 32 & 48.30 & 0.0 & 1 \\
\hline & & & $P P$ & 36 & 14.00 & & I FVM \\
\hline CDF & 86.72 & 330 & IPd & 32 & 50.20 & 0.3 & JCT \\
\hline & $1.0 \mathrm{~s}$ & & $2.10 \mathrm{~nm}$ & & & $.6 \mathrm{~m}$ & \\
\hline DMU & $\begin{array}{l}86.92 \\
1.15\end{array}$ & $\begin{array}{r}341 \\
100\end{array}$ & $\begin{array}{l}\text { eP } \\
0.00 \mathrm{~nm}\end{array}$ & 32 & 50.60 & $\theta .0$ & 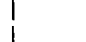 \\
\hline $\mathrm{ECH}$ & 86.92 & 330 & $P$ & 32 & $50.20^{\circ}$ & -0.6 & 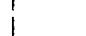 \\
\hline & & & is & 43 & 26.00 & & MIM \\
\hline & & & E & 06 & 00.00 & & TRM \\
\hline ZUL & 86.99 & 329 & $P$ & 32 & 50.00 & -1.1 & TOL \\
\hline
\end{tabular}

87.22340 eP $\quad 3251.80 \quad-0.2$ $87.29330 \mathrm{P} \quad 3252.60 \quad 0.0$

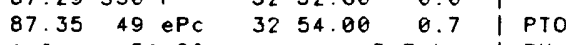

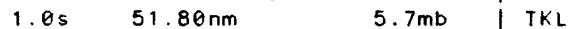
$z 18 \mathrm{~s} 9.11 \mathrm{um} \quad 6.2 \mathrm{Msz}$ MAL 87.36340 eP $3252.40-0.3$ । $1.2 \mathrm{~s} \quad 140.00 \mathrm{~nm} \quad 6.1 \mathrm{mb}$

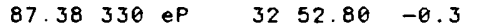
$\begin{array}{llll}1.1 \mathrm{~s} & 30.50 \mathrm{~nm} & 5.5 \mathrm{mb} & \text { LIS }\end{array}$ $87.42331 \mathrm{eP} \quad 3252.90 \quad-0.3$ $0.8 \mathrm{~s} \quad 18.10 \mathrm{~nm} \quad 5.4 \mathrm{mb}$ 87.51341 eP $32 \quad 52.90 \quad-0.6$ $1.3 \mathrm{~s} \quad 130.00 \mathrm{~nm} \quad 6.0 \mathrm{mb}$ SFS 88.30340 eP $32 \quad 56.80 \quad-0.4$ $1.2 \mathrm{~s} \quad 155.00 \mathrm{~nm}$ $8851329 \mathrm{P} \quad 3257.50 .2 \mathrm{mb}$

$88.57325 \mathrm{Pc} \quad 3304.00 \quad 5.3 \times$ BNG $88.70283 \mathrm{iPC} \quad 3305.50 \quad 5.6 \mathrm{X}$ 88.94323 iP $3300.00-0.5$ SBA \begin{tabular}{c|ccc|c|c} 
& $i$ & 33 & 05.50 & & MTD \\
89.01331 iPd & 363900.80 & & CIR \\
& 33 & 0.0 & MAW
\end{tabular} $1.1 \mathrm{~s} 37.30 \mathrm{~nm} \quad 5.6 \mathrm{mb}$ BUL $89.20331 \mathrm{iPd} \quad 3301.50-0.2$ $0.6 \mathrm{~s} 17.00 \mathrm{~nm} \quad 5.5 \mathrm{mb}$ PRE $89.32332 \mathrm{iPd} 3302.40 \quad 0.1$

$1.0 \mathrm{~s} \quad 26.00 \mathrm{~nm}$

89.36323 eP $\quad 3301.50-1.1$ ePP 3636.00

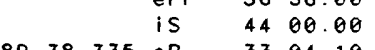
$89.38 \quad 335$ eP $3304.10 \quad 1.6$ \begin{tabular}{ccc|c}
$0.9 \mathrm{~s}$ & $29.40 \mathrm{~nm}$ & $5.6 \mathrm{mb}$ & \\
89.40 & $334 \mathrm{iPd}$ & $3302.60^{0.0}$ & KDS
\end{tabular} $0.8 \mathrm{~s} 29.40 \mathrm{~nm} \quad 5.6 \mathrm{mb} / \mathrm{KIC}$ 89.53331 eP $3303.10 \quad-0.2 \quad$ BCR $1.1 \mathrm{~s} 35.70 \mathrm{~nm} \quad 5.6 \mathrm{mb}$ WIN 89.57342 eP $3300.00-3.3 \times$ CAR (SKS) $43 \quad 32.00$ $\mathrm{SCS} 4354.00$ LO $56 \quad 44.00$ LR 0204.00 $\begin{array}{lllllll}89.60 & 331 & \text { IPd } & 33 & 03.70 & 0.1 & \text { TRN }\end{array}$

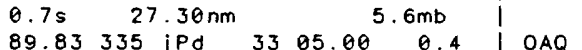

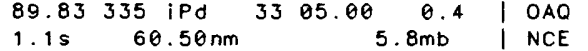
\begin{tabular}{l}
90.20335 iPd $3306.90 \quad 0.6$ \\
\hline SNA
\end{tabular} $1.2 \mathrm{~s} 68.00 \mathrm{~nm} \quad 5.8 \mathrm{mb}$ NNA $90.35330 \mathrm{eP} \quad 3306.50-0.7$ । 90.43334 (P) $3314.00 \quad 6.6 \times$ 90.46332 iPd $33 \quad 08.00 \quad 0.4 \quad$ HUA $1.4 \mathrm{~s} 55.30 \mathrm{~nm} \quad 5.6 \mathrm{mb}$ |A $1 \mathrm{~A}$ $90.62326 \mathrm{eP} \quad 3307.80-0.6 \quad$ ARE $0.9 \mathrm{~s} \quad 18.00 \mathrm{~nm} \quad 5.4 \mathrm{mb}$ \begin{tabular}{llll|l}
90.76 & 332 eP & $33 \quad 09.46$ & 0.4 & ZOBO
\end{tabular}

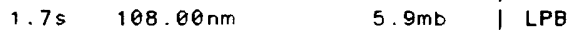
$90.81328 \mathrm{eP} \quad 3309.00 \quad-0.3$ $0.9534 .30 \mathrm{~nm} \quad 5.7 \mathrm{mb}$ | ANT 91.05328 iPd $33 \quad 10.20 \quad-0.1$ \begin{tabular}{llll|}
0.95 & $44.30 \mathrm{~nm}$ & $5.8 \mathrm{mb}$ \\
91.08 & $328 \mathrm{iPd}$ & 33 & $10.30^{5}-0.2$
\end{tabular} $1.1 \mathrm{~s} \quad 48.60 \mathrm{~nm} \quad 5.8 \mathrm{mb}$ 91.09333 iPd 3311.100 .6 ( CPP $1.1 \mathrm{~s} 50.00 \mathrm{~nm} \quad 5.8 \mathrm{mb}$ TLL 91.55332 iPd $3313.20 \quad 0.6$
1 PEL $1.3 \mathrm{~s} 50.20 \mathrm{~nm} \quad 5.7 \mathrm{mb}$ | SAN 91.65331 iPd $3314.30 \quad 1.2 \quad$ SLA 92.16332 iPd $3316.50 \quad 1.1$ | MDZ $0.9 \mathrm{~s} 42.50 \mathrm{~nm} 5.9 \mathrm{mb}$ CFA 92.20332 iPd $3316.60 \quad 1.0$ | CYA \begin{tabular}{lll|l}
$1.1 \mathrm{~s}$ & $25.70 \mathrm{~nm}$ & $5.6 \mathrm{mb}$ & BAO
\end{tabular} $\begin{array}{lllll}92.37 & 33 \mathrm{P} & 33 & 22.00 & 5.6 \times\end{array}$

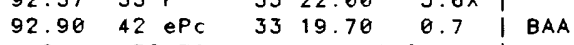
$1.2 \mathrm{~s} \quad 78.70 \mathrm{~nm} \quad 6.0 \mathrm{mb}$ $220 \mathrm{~s} 7.95 \mathrm{um} \quad 6.2 \mathrm{Msz}$ | LPA \begin{tabular}{rrrrr|l}
93.13 & $41 \mathrm{eP}$ & 33 & 20.50 & 0.5 & \\
93.68 & $331 \mathrm{ePC}$ & 33 & 22.60 & 0.1 & RDJ
\end{tabular} 93.91331 eP $3323.90 \quad 0.3$ $\begin{array}{lll}0.8 \mathrm{~s} & 21.80 \mathrm{~nm} & 5.6 \mathrm{mb}\end{array}$ $94.14 \quad 37 \mathrm{ePc} \quad 33 \quad 24.20 \quad-0.5$ $\begin{array}{llllll}94.48 & 48 & \text { iP } & 33 & 27.00 & 0.6\end{array}$ $1.0 \mathrm{~s} \quad 12.50 \mathrm{~nm} \quad 5.3 \mathrm{mb}$ $20 \mathrm{~s} \quad 10.60 \mathrm{um} \quad 6.3 \mathrm{Msz}$ $\begin{array}{rccccc} & & i & 37 & 12.50 & \\ 96.28 & 20 & 44 & 12.00 & \\ 96.84 & 21 & \text { eP } & 33 & 35.80 & 1.4 \\ 98.38 & 33.60 & 1.7\end{array}$ $\begin{array}{rrrrrr}96.84 & 21 & \mathrm{eP} & 33 & 38.60 & 1.7 \\ 98.32 & 332 & \mathrm{eP} & 33 & 43.00 & -0.7\end{array}$ is $45 \quad 42.00$ $\begin{array}{lllll}98.84336 P & \text { iSS } & 51 & 55.00 & \\ 93 & 43.00 & -2.9\end{array}$ $99.19 \quad 34$ eP $\quad 33 \quad 48.00 \quad 0.3$ 101.19331 ePdiff34 $01.00 \quad 4.5 x$ IPP $38 \quad 08.00$ iSKS $44 \quad 36.00$ is 4528.00

101.20335 Pdiff 3400.00 ePP $38 \quad 08.00$ S $\quad 4520.00$ LR $\quad 10 \quad 00.00$

$102.13 \quad 332$ iPdi $\{34 \quad 05.00$ $\begin{array}{lll}\text { iPP } \quad 38 & 14.00\end{array}$ eS 4542.00 e $\quad 57 \quad 30.00$

112.05292 ePKPd $38 \quad 44.20$ $1.0 \mathrm{~s} \quad 7.90 \mathrm{~nm}$

$\begin{array}{llllll}113.64 & 174 & \text { iPKP } & 38 & 45.20 & 1.7\end{array}$

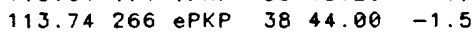

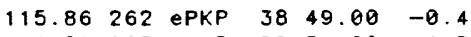

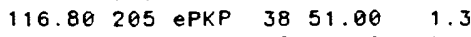
$\begin{array}{lllll}117.86 & 264 \text { iPKPc } 38 \quad 53.50 & 0.1\end{array}$

$0.8 \mathrm{~s} \quad 7.09 \mathrm{~nm}$

$120.83 \quad 259$ iPKPc $3900.50 \quad 1.6$ $1.0 \mathrm{~s} \quad 39.00 \mathrm{~nm}$

$z 20 \mathrm{~s} \quad 12.80 \mathrm{um} \quad 6.6 \mathrm{Msz}$ $\begin{array}{lllll}121.92 & 259 & \text { iPKPc } 39 & 00.30 & -0.7\end{array}$ $1.0 \mathrm{~s} \quad 16.00 \mathrm{~nm}$

$\begin{array}{lllll}124.64 & 180 & \text { iPKPC } 39 & 05.60 & 0.7\end{array}$ $0.9 \mathrm{~s} \quad 29.40 \mathrm{~nm}$

$20 \mathrm{~s} 6.60 \mathrm{um} \quad 6.3 \mathrm{Msz}$ $\begin{array}{llllll}125.65 & 325 & \text { iPKP } 39 & 07.60 & -0.7\end{array}$ $\begin{array}{llllll}126.80 & 313 & \text { iPKP } & 39 & 10.50 & -0.1\end{array}$ $\begin{array}{llllll}128.33 & 43 & \text { ePKP } & 39 & 13.00 & -0.5\end{array}$ 128.45268 PKP $3847.20-26.5 X$ $\begin{array}{llllll}128.54 & 34 & \text { ePKP } & 39 & 14.80 & 0.8\end{array}$

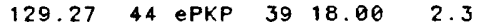
$\begin{array}{llllll}129.77 & 45 & \text { ePKP } & 39 & 20.50 & 3.9 \times\end{array}$ $\begin{array}{llllll}129.81 & 45 & \text { ePKP } & 39 & 19.00 & 2.2\end{array}$ $130.44 \quad 52$ ePKP $39 \quad 19.00 \quad 1.0$ $\begin{array}{llllll}130.72 & 27 & \text { ePKP } & 39 & 18.70 & 0.8\end{array}$

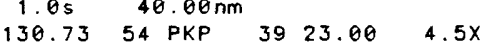
$131.06 \quad 54$ ePKP $3923.00 \quad 3.7 x$

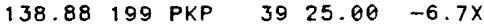
$\begin{array}{llllll}140.14 & 64 & \text { ePKP } & 39 & 30.50 & -5.2 x\end{array}$ $Z 20 \mathrm{~s} 9.75 \mathrm{um} \quad 6.6 \mathrm{Msz}$

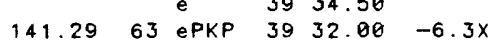
$\begin{array}{llll}146.43 & 162 & \text { e(PKP) } 3951.00 & 6.1 \mathrm{X}\end{array}$ $\begin{array}{lllll}146.98 & 64 & \text { ePKP } 3950.00 & 2.3\end{array}$ ESS $02 \quad 12.00$ $149.30 \quad 60$ PKP $3952.60 \quad 0.9$ 149.4960 PKPd 3953.80 $2.1 \mathrm{~s} \quad 1720.00 \mathrm{~nm}$

$\begin{array}{lll}151.93 & 75 & \text { PKP } 40 \quad 02.00\end{array}$

2. 0

$7.3 x$

PP 4359. SS 0322.00 SSS $09 \quad 14.00$

$\begin{array}{lllll}153.63 & 82 & \text { PKP } & 40 & 02.50\end{array}$ 154.2788 ePKP $40 \quad 05.00$ 155.1595 PKP $40 \quad 04.00$ $\begin{array}{lllll}155.23 & 95 \text { ePKP } & 40 & 07.00\end{array}$ 156.3272 PKPC $40 \quad 09.20$ 156.6093 e (PKP) $40 \quad 09.10$ $\begin{array}{lllll}156.79 & 90 & \text { PKP } 40 & 03.50\end{array}$ $157.76 \quad 80$ PKPC $40 \quad 07.80$ $159.8121 \mathrm{e}(\mathrm{PKP}) 40 \quad 07.00$ $\begin{array}{lllll} & & e & 41 & 46.00 \\ 165.39 & 94 & \text { PKP } & 40 & 17.10\end{array}$ $\begin{array}{llll}P P & 45 & 02.70\end{array}$ $\begin{array}{llllll}165.87 & 95 & \text { ePKP } & 40 & 38.00 & 27.7 X\end{array}$ $Z 20 \mathrm{~s} 7.80 \mathrm{um}$

$167.92 \quad 11$ PKP $40 \quad 18.00$ e 4124.80 $\begin{array}{llll}\text { ESKP } & 43 & 17.20\end{array}$ iPP $\quad 45 \quad 12.00$
PSKS eSKS $48 \quad 28.40$

S.D. $=1.2$ an 436 of 506 abs.

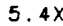

$6.8 x$ 5. $0 x$ 8. $2 x$ 8. $1 x$ 2.3 $5 \cdot 3 x$ 1.8

$7.2 x$ $5.8 x$

JUL $29,1980 \quad 14 \mathrm{~h} 58 \mathrm{~m} \mathrm{41.09 \pm 0.06 \textrm {s }}$ $29.656 \mathrm{~N} \pm 1.7 \mathrm{~km} \quad 81.108 \mathrm{E} \pm 1.2 \mathrm{~km}$ DEPTH $=18.8 \mathrm{~km}$ ( 13 depth phases) $6.1 \mathrm{mb}$ ( 65 obs.) $6.6 \mathrm{Msz}$ ( 22 obs.) NEPAL

(310) 
One hundred fifty to 200 people | UER killed, many injured and extensive damage in western Nepol. At least 13 killed, 40 injured and damage in the Pithoragorh orea, India. Felt strongly of Kathmondu. Nepol and in the Delhi area, India.

\begin{tabular}{|c|c|c|c|c|c|c|c|}
\hline NDI & 3.54 & 255 & $\begin{array}{l}\text { Pn- } \\
\text { isnn }\end{array}$ & $\begin{array}{l}59 \\
00\end{array}$ & $\begin{array}{l}36.50 \\
18.00\end{array}$ & 0.2 & $\begin{array}{l}\text { BTO } \\
\text { моY }\end{array}$ \\
\hline \multirow[t]{2}{*}{ VAR } & 4.66 & 158 & $P n$ & 59 & 54.60 & 2.4 & ZAK \\
\hline & & & Sn & $\theta 0$ & 55.60 & & BSI \\
\hline BOK & 7.16 & 143 & $P_{n}$ & 00 & 30.80 & $3.4 x$ & BAK \\
\hline LSA & 8.74 & 87 & $P_{c}$ & $\theta 0$ & 51.10 & 1.2 & \\
\hline \multirow{2}{*}{ SHL } & 10.39 & & $S$ & 02 & 29.50 & & IRK \\
\hline & 16.39 & 111 & $\begin{array}{l}\mathrm{Pc} \\
\mathrm{S}\end{array}$ & $\begin{array}{l}01 \\
03\end{array}$ & $\begin{array}{l}09.00 \\
02.00\end{array}$ & $-3.3 x$ & WHN \\
\hline KSH & 10.65 & 338 & $P_{c}$ & 01 & 13.80 & -2.0 & \\
\hline \multirow[t]{2}{*}{ KHO } & 11.16 & 317 & eP & 01 & 18.00 & $-4.9 x$ & \\
\hline & & & is & 03 & 29.60 & & KER \\
\hline KAAO & 11.33 & 299 & $i P_{c}$ & 01 & 22.00 & $-3.2 x$ & $\mathrm{GZH}$ \\
\hline KBL & 11.33 & 299 & $i P_{c}$ & 01 & 22.20 & $-3.0 x$ & \\
\hline vis & 12.05 & 170 & $P$ & 01 & 31.00 & $-3.8 x$ & TAB \\
\hline \multirow[t]{2}{*}{ OUE } & 12.30 & 276 & eP & $\begin{array}{l}03 \\
01\end{array}$ & $\begin{array}{l}36.00 \\
34.80\end{array}$ & $-3.5 x$ & \begin{tabular}{|l}
$\mid$ \\
1
\end{tabular} \\
\hline & & & es & 03 & 50.00 & & B J I \\
\hline \multirow[t]{3}{*}{ HYB } & 12.41 & 191 & ePd- & 01 & 34.00 & $-5.6 x$ & \\
\hline & & & $\begin{array}{l}\text { e } \\
\text { es }\end{array}$ & $\begin{array}{l}02 \\
03\end{array}$ & 00.00 & & \\
\hline & & & e & 04 & $\begin{array}{l}40.40 \\
56.00\end{array}$ & & HKC \\
\hline \multirow[t]{2}{*}{ NRN } & 12.46 & 342 & $P c$ & 01 & 38.50 & -2.1 & \\
\hline & & & es & 03 & 55.00 & & SVE \\
\hline \multirow[t]{2}{*}{ GAR } & 12.89 & 319 & eP & 01 & 40.00 & $-6.1 x$ & \\
\hline & & & es & 04 & 11.60 & & $T I A$ \\
\hline \multirow[t]{3}{*}{ POO } & 12.90 & 212 & Po & 01 & 49.20 & $3.0 x$ & \\
\hline & & & $P P$ & 01 & 56.00 & & \\
\hline & & & $\mathrm{s}$ & 04 & 14.00 & & ARU \\
\hline \multirow[t]{2}{*}{ PRZ } & 12.99 & & SS & 04 & 27.00 & & \\
\hline & 12.95 & 351 & $\begin{array}{l}P c \\
e S\end{array}$ & $\begin{array}{l}01 \\
04\end{array}$ & $\begin{array}{l}45.80 \\
09.40\end{array}$ & -1.6 & IPM \\
\hline BOM & 13.11 & 217 & Pd & 01 & 43.00 & $-5.9 x$ & GRO \\
\hline & & & s & 04 & 03.00 & & \\
\hline ANR & 13.17 & 330 & ePc & 01 & 44.00 & $-5.7 x$ & BHD \\
\hline DSH & 13.53 & 314 & $\begin{array}{l}\text { eS } \\
\text { Pc }\end{array}$ & 04 & $\begin{array}{r}09.00 \\
08.59\end{array}$ & & PS \\
\hline & & & is & 04 & $\begin{array}{l}48.50 \\
15.50\end{array}$ & $-6.0 x$ & I LEN \\
\hline TLG & 13.91 & 349 & $P$ & 01 & 58.00 & -1.5 & \\
\hline FRU & 14.15 & 340 & $\begin{array}{l}P c \\
\text { is }\end{array}$ & 02 & 00.50 & -2.1 & $\mathrm{~N} J 2$ \\
\hline WMO & 15.08 & 19 & Pc & $\begin{array}{l}04 \\
02\end{array}$ & 14.10 & -0.7 & \\
\hline TAS & 15.09 & 324 & ePc & 02 & 06.00 & $-8.8 x$ & I \\
\hline & & & is & 04 & 57.00 & & \\
\hline SAM & 15.30 & 315 & ePc & 02 & 12.00 & $-5.7 x$ & BKR \\
\hline & & & is & 04 & 59.80 & & \\
\hline GOA & 15.62 & 207 & $\mathrm{Pd}$ & 02 & 16.00 & $-5.9 x$ & PYA \\
\hline GBA & 16.33 & 193 & $P c$ & 02 & 21.20 & $-9.8 x$ & \\
\hline & $1.2 \mathrm{~s}$ & 389 & $9.00 \mathrm{~nm}$ & & 5 & $.4 \mathrm{mb}$ & $\mathrm{OZH}$ \\
\hline MDR & 16.60 & 183 & $P$ & 02 & 42.00 & $7.6 x$ & \\
\hline & & & s & 05 & 31.50 & & SSE \\
\hline TGI & 19.01 & 286 & $i P c$ & 03 & 04.10 & -0.4 & \\
\hline MAID & 19.29 & 296 & $\mathrm{Pct}$ & 03 & 06.20 & -1.5 & \\
\hline & & & es & 06 & $\begin{array}{l}35.60 \\
00.00\end{array}$ & & 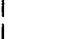 \\
\hline CHG & 19.49 & 120 & iPc & 03 & 08.10 & -2.0 & $K G M$ \\
\hline & & 343 & $\begin{array}{l}\text { 3. } 00 \mathrm{~nm} \\
\text { es }\end{array}$ & 06 & 40.00 & & \\
\hline CHTO & 19.49 & 120 & $i P_{c}$ & 03 & 07.50 & -2.6 & PPI \\
\hline KOD & 19.62 & 191 & eP & 03 & 08.00 & $-3.9 x$ & \\
\hline & & & $\begin{array}{l}\text { PPP } \\
\text { eS }\end{array}$ & 03 & 42.00 & & ICU \\
\hline KMI & 19.72 & 98 & PC & 03 & 12.20 & -0.7 & ANP \\
\hline & & & is & 06 & 52.00 & & \\
\hline CDU & 19.83 & 81 & Pc & 03 & 13.40 & -0.3 & $B O D$ \\
\hline $\mathrm{LZH}$ & 20.12 & 66 & $P_{c}$ & 03 & 16.60 & -0.3 & HEN \\
\hline & & & S & 97 & 00.60 & & TIN \\
\hline BDT & .51 & 123 & $i P_{c}$ & 03 & 20.30 & -0.5 & HWA \\
\hline ASH & 20.61 & 300 & $P c$ & 03 & 20.50 & -1.2 & 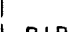 \\
\hline SEM & 20.74 & 358 & $P c$ & 03 & 22.00 & -1.0 & PIP \\
\hline PBA & 20.91 & 146 & $\mathrm{P}$ & 03 & 36.00 & $11.0 x$ & \\
\hline KAT & & & $\begin{array}{l}S \\
P_{C}\end{array}$ & $\begin{array}{l}07 \\
03\end{array}$ & 32.00 & & CN2 \\
\hline NAT & 22.53 & 302 & $\begin{array}{l}P c \\
\text { is }\end{array}$ & $\begin{array}{l}03 \\
07\end{array}$ & 47.00 & 0.4 & \\
\hline GYA & 22.76 & 92 & $\mathrm{Pc}$ & 03 & 44.20 & 0.5 & \\
\hline & & & $\begin{array}{l}\text { S } \\
\text { ep }\end{array}$ & & $\begin{array}{l}51.70 \\
44.00\end{array}$ & -1.1 & $s$ \\
\hline ELT & 23.88 & 8 & $P_{C}$ & 03 & 55.80 & 1.8 & KVT \\
\hline
\end{tabular}

$\begin{array}{llll}\text { ELT } & 23.88 \quad 8 \mathrm{PC}\end{array}$
$23.9320 \mathrm{PC}$ $23.93124 \mathrm{eP}$ $23.9972 \mathrm{PC}$

$24.82277 \begin{aligned} & S P \\ & \mathrm{ePC}\end{aligned}$

$25.283 \mathrm{PC}$

$25.68291 \mathrm{eP}$

$25.93 \quad 57 \mathrm{PC}$

$26.51 \quad 28 \mathrm{PC}$

$26.5933 \mathrm{PC}$

$27.52148 \mathrm{eP}$

$27.58301 \mathrm{PC}$

$28.36 \quad 31 \mathrm{PC}$

$28.7280 \mathrm{PC}$

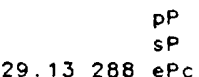

$29.5595 \mathrm{P}$

$29.94296 \mathrm{IPC}$

$30.11298 \mathrm{PC}$

$30.4161 \mathrm{iPct}$

$1.5 \mathrm{~s} \quad 642.00 \mathrm{~nm}$

$\begin{aligned} & \text { SP } \\ 30.5496 & \text { PS }\end{aligned}$

$30.72338 \mathrm{Pc}$

$30.80 \quad 68 \quad \mathrm{PC}$

$\begin{array}{rl}31.10 & 336 \\ S & 5 P\end{array}$

$31.10336 \mathrm{eP} \quad 0501.00$

$\begin{array}{lll}\text { es } & 10 \quad 06.50\end{array}$

$31.26 \quad 139 \mathrm{iPC}$

$1.1 \mathrm{~s} \quad 165.00 \mathrm{~nm}$

$31.34306 \mathrm{PC}$

31.43286 is

$31.70144 \mathrm{ePd}$

$0.8 \mathrm{~s} \quad 52.30 \mathrm{~nm}$

$32.18300 \mathrm{PC}$

$32.3976 \mathrm{PC}$

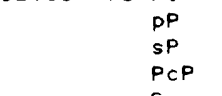

$S$

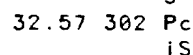

$33.35306 \mathrm{PC}$

$33.54 \quad 89 \mathrm{PC}$

$34.48 \quad 77 \mathrm{~S}$

$Z 20 \mathrm{~s} \quad 136.00 \mathrm{um}$

$\begin{array}{llr}\mathrm{N} & 20 \mathrm{~s} & 84.00 \mathrm{um} \\ \mathrm{E} & 20 \mathrm{~s} & 106.00 \mathrm{um}\end{array}$

34

$\begin{array}{ll}34.66 & 138 \mathrm{ePc} \\ 1.3 \mathrm{~s} & 465.00 \mathrm{~nm}\end{array}$

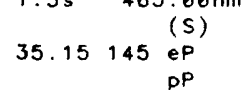

$35.5989 \stackrel{P P}{\text { is }}$

$36.82 \quad 87 \mathrm{P}$

$36.2930 \mathrm{PC}$

$36.35 \quad 93 \mathrm{eP}$

$36.4491 \mathrm{P}$

$36.46 \quad 89 \mathrm{P}$

$37.65 \quad 98 \quad$ iP

$1.1 \mathrm{~s} \quad 233.00 \mathrm{~nm}$

$37.7956 \mathrm{PC}$

37.79 SP
SP
PP

SP

$37.84 \quad 100 \mathrm{ePc}$

$1.0 \mathrm{~s} 41400 \mathrm{~nm}$ $38.09300 \mathrm{P}$
035600 0356.90 0355.50 $04 \quad 07.90$ 0405.00 $04 \quad 07.00$ 0829.00 0416.00 $04 \quad 15.10$ 0422.10 421.00 0421.50 0426.5 0914.00 0436.90 0929.50

0439.50 $04 \quad 46.50$ 0451.50 0446.60 0458.60 0452.00 0452.00

0950.80

0508.08 0953.00 0455.00
$10 \quad 00.00$ 0458.00

1000.00 0458.30 $05 \quad 10.60$ 0501.00 $\begin{array}{ll}01.30 & -0.7\end{array}$ 0503.00 1008.00 \begin{tabular}{l|l}
-1.9 & DST
\end{tabular} $0512.00^{5.5 m b} 1.9$ | YER $1024.00 \quad \mid$ EDC \begin{tabular}{lll|l}
05 & 12.30 & 0.5 & DMK
\end{tabular} $0519.2024 \mathrm{~km}$ | LEM 0524.20 BIR 0800.20 | AAE 1025.20 \begin{tabular}{lll|l}
05 & 15.70 & 2.2 & I MFT
\end{tabular} \begin{tabular}{ll|l|l}
10 & 32.70 & & $F O C$
\end{tabular} 1037.50 VRI

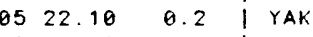
\begin{tabular}{lll|l}
10 & 42.60 & & YAK \\
05 & 30.20 & 0.3 & PUL
\end{tabular} $6.7 \mathrm{Msz}$ 1102.00 BuC EZN \begin{tabular}{ll|l}
11 & 32.00 & BUC 1
\end{tabular} $0806.00^{6.2 \mathrm{mb}}$ 0536.20 3820.00 $38 \quad 44.50$ \begin{tabular}{lll|l}
05 & 39.20 & -0.3 & BKB
\end{tabular} 0543.00 1126.00 DIM $\begin{array}{llll}05 & 45.00 & 0.0 & \text { PVL }\end{array}$ $0549.00 \quad 3.1 \times$ | CMP $0553.00 \quad 6.3 \times$ 05 $35.70-11.2 x$ DRA 1124.50 I LVV $\begin{array}{llll}05 & 57.70 & 0.8 & \text { । }\end{array}$ $0558.50^{5.9 \mathrm{mb}} 0.7$ | APA $0606.00 \quad 25 \mathrm{~km}$ CJR 0610.50 I DAV \begin{tabular}{lll|l}
07 & 30.50 & & \\
06 & 00.00 & 1.5 & $\mathrm{PPH}$
\end{tabular} $6.2 \mathrm{mb}$ SOF
$38.45 \quad 101$ iPCt $06 \quad 04.00 \quad 0.1$ $1.5 \mathrm{~s} \quad 500.00 \mathrm{~nm} \quad 6.1 \mathrm{mb}$

39.70103 is 1200.00 $\begin{array}{lllll}39.81306 \mathrm{PC} & 06 & 15.50 & 0.8\end{array}$ isP 0620.00 es $\quad 12 \quad 18.00$ $\begin{array}{llllll}39.86 & 251 & \text { isS } & 12 & 22.00 & \\ \text { i PC } & 06 & 12.30 & -3.2 x\end{array}$ $\begin{array}{llllll}39.92 & 282 & \mathrm{PC} & 06 & 17.00 & 1.2\end{array}$ $\begin{array}{llllll}39.99 & 4 & P C & 06 & 14.00 & -1.8\end{array}$ is 1222.00 $\begin{array}{ccccc}40.16 & 105 \mathrm{iPc} & 06 & 17.30 & -0.5 \\ 1.0 \mathrm{~s} & 76.00 \mathrm{~nm} & & 5.4 \mathrm{mb}\end{array}$

$\begin{array}{lllll}40.25323 \mathrm{PC} & 06 & 18.00 & -0.1\end{array}$ is 1228.00 $\begin{array}{llllll}40.31 & 112 & \text { iPd } & 06 & 18.80 & -0.2\end{array}$ $1.2 \mathrm{~s} \quad 421.00 \mathrm{~nm} \quad 6.0 \mathrm{mb}$

$\begin{array}{ccccc}40.55298 \text { iPC } & 06 & 21.00 & 0.1\end{array}$ 40.56118 iPC $06 \quad 23.30 \quad 2.0$ $1.0 \mathrm{~s} \quad 499.00 \mathrm{~nm} \quad 6.2 \mathrm{mb}$ $\begin{array}{lllll}40.59 & 322 \mathrm{PC} & 06 & 21.00 & 0.1\end{array}$ $\begin{array}{lllll}41.01299 \mathrm{P} & 06 & 26.30 & 1.6\end{array}$ $\begin{array}{llllll}42.39 & 294 & P & 06 & 35.30 & -0.8\end{array}$ $\begin{array}{llllll}42.42 & 57 \mathrm{PC} & 06 & 36.50 & 0.4\end{array}$ $\begin{array}{llllll}42.45299 & \text { is } & 12 & 56.50 & \\ 46 & 06 & 35.30 & -1.1\end{array}$ $\begin{array}{lllll}42.59299 P & 06 & 38.00 & 0.2\end{array}$ $\begin{array}{lllll}42.66297 \mathrm{P} & 06 \quad 38.00 & -0.3\end{array}$ 42.68103 ePd $0638.50 \quad 0.0$ $12 \mathrm{~s} 2.30 \mathrm{um} \quad 5.3 \mathrm{MszX}$ $\begin{array}{rllll}43.00293 \mathrm{P} & 06 & 42.10 & 1.0\end{array}$ $43.35298 P \quad 0644.30 \quad 0.3$ $43.37300 P \quad 0644.60 \quad 0.7$ $\begin{array}{llllll}43.58 & 70 & \mathrm{iPc} & 06 & 46.00 & 0.3\end{array}$ $1.4 \mathrm{~s} \quad 484.00 \mathrm{~nm} \quad 6.1 \mathrm{mb}$ $43.64308 \mathrm{PC} \quad 06 \quad 46.00 \quad 0.0$ $\begin{array}{llll}\text { isP } & 06 & 48.00 & \\ \text { ipP } & 06 & 50.00 & 13 \mathrm{~km} \\ \text { is } & 13 & 14.00 & \end{array}$ $43.77298 P \quad 0647.60$ $\begin{array}{lllllll}43.84300 P & 06 \quad 48.10 & 0.3\end{array}$ $44.25294 P \quad 0651.50 \quad 0.3$ $\begin{array}{lllll}44.31299 P & 06 & 51.40 & -0.2\end{array}$ $44.33301 \mathrm{P} \quad 0651.90 \quad 0.2$ 44.33141 ip $\quad 06 \quad 54.50 \quad 2.4$ $\begin{array}{llll}44.45307 \mathrm{P} & 06 & 55.00 & 2.4\end{array}$ 44.58251 eP $06 \quad 57.40 \quad 3.0 x$ 44.73105 iPc $0655.20 \quad 0.1$ $\begin{array}{lllll}44.73299 P & 06 & 54.80 & -0.2\end{array}$ $44.73 \quad 306$ eP $0756.00 \quad 61.1 x$ $\begin{array}{lllll}44.93296 \mathrm{P} & 06 & 56.20 & -0.4\end{array}$ $\begin{array}{lllll}45.06 & 307 \text { eP } & 06 & 58.00 & 0.5\end{array}$ $\begin{array}{lllll}45.13 & 30 \mathrm{PC} & 06 & 57.40 & -0.3\end{array}$ 45.33327 eS 1326.20 $\begin{array}{lll}45.33327 \mathrm{PC} & 0700.00\end{array}$ $45.49305 \mathrm{eS} \quad 1340.00$ $45.53298 P \quad 0701.50$ 45.54304 iP $\quad 07 \quad 04.00$ 45.60306 eP 0704.00 ePP $\quad 0850.00$ ePP 0851.00 $\begin{array}{rrrr} & \text { ES } & 1326.00 & \end{array}$

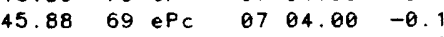
$\begin{array}{llllll}45.93 & 126 & \text { iPd } & 07 & 07.50 & 2.8\end{array}$ $1.0 \mathrm{~s} \quad 610.00 \mathrm{~nm}$ $45.94301 \mathrm{eP}$
$46.19303 \mathrm{PC}$ 46.24306 eP $0705.00 \quad 0.5$ $07 \quad 06.00 \quad-0.4$ $0708.00 \quad 1.1$

46.80305 P $47.04312 \mathrm{PC} \quad 0713.80 \quad 0.7$ is 1403.00 $\begin{array}{llll}22 & 337 \mathrm{Pd} & 07 \quad 14.50\end{array}$ $\begin{array}{llll}47.24308 \mathrm{eP} & 07 & 16.00\end{array}$ $47.40109 \mathrm{PC} \quad 07 \quad 17.00$ 47.49 is $\begin{array}{llll}47.56302 \mathrm{PC} & 07 & 18.00\end{array}$ $47.73 \quad 307$ ePd 0720.00
0.6

2. 1

2.7

2. 0

0.3

1.2
0.7

0. 3

0.6

1. 4 


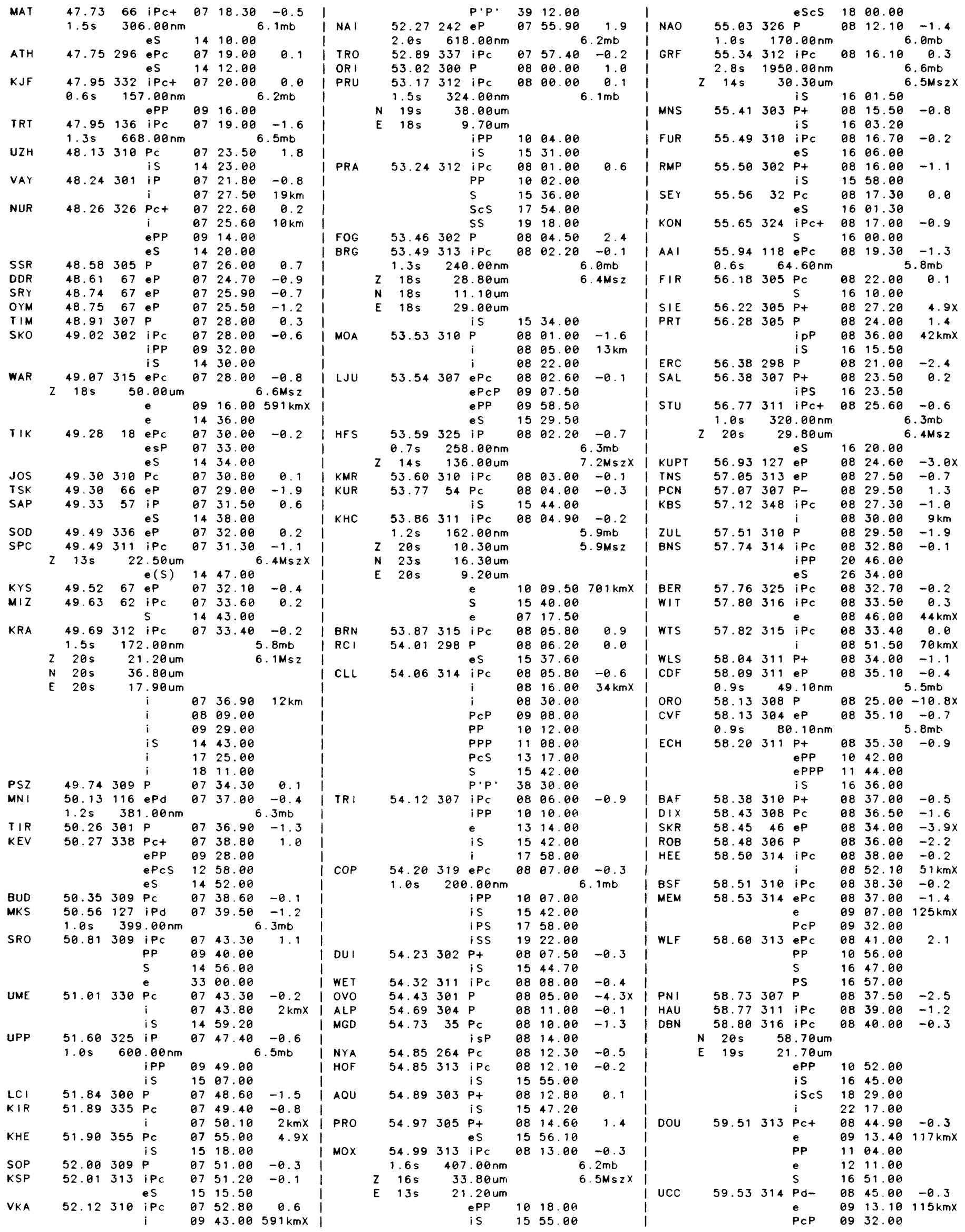




\begin{tabular}{|c|c|c|c|c|c|c|c|c|c|c|c|}
\hline & & & $\mathrm{s}$ & 16 & 56.00 & & I GUD & & 68.03 & 305 & iP \\
\hline & & & ScS & 18 & 36.00 & & I ALM & & 68.15 & 301 & iPc \\
\hline PET & 59.62 & 43 & eP & 08 & 42.00 & $-3.9 x$ & i & & $1.4 \mathrm{~s}$ & & $8.40 \mathrm{~nm}$ \\
\hline & & & esP & 88 & 44.00 & & 1 & $\mathbf{N}$ & $15 \mathrm{~s}$ & & $1.00 \mathrm{um}$ \\
\hline LMR & 59.65 & 305 & $e^{P}$ & 08 & 46.20 & 0.0 & 1 & & & & iPP \\
\hline & $1.2 \mathrm{~s}$ & 117 & $7.00 \mathrm{~nm}$ & & & $5.9 \mathrm{mb}$ & $1 \mathrm{TOL}$ & & 68.23 & 304 & - $\mathrm{PC}$ \\
\hline NPA & 60.07 & 228 & $P$ & 88 & 50.00 & 0.5 & i & & & & $\mathrm{i}$ \\
\hline CDR & 60.09 & 306 & $P$ & 88 & 48.80 & -0.5 & i & & & & iscs \\
\hline & & & pP & 08 & 59.60 & $36 \mathrm{kmx}$ & i & & & & iss \\
\hline LBF & 60.56 & 310 & $i P c$ & 08 & 51.70 & -0.8 & I SMY & & 68.71 & 41 & eP \\
\hline LOR & 60.57 & 310 & $i P c$ & 08 & 51.60 & -1.0 & I CRT & & 68.87 & 302 & $i P C$ \\
\hline GUMO & 60.71 & 91 & $e(P)$ & 08 & 52.50 & -1.5 & $i$ & & & & is \\
\hline & & & $e(S)$ & 17 & 05.00 & & I REY & & 69.12 & 333 & $e^{P}$ \\
\hline SMF & 60.73 & 310 & iPc & 08 & 53.20 & -0.4 & ICIR & & 69.37 & 229 & $\mathrm{Pt}$ \\
\hline SSF & 60.85 & 310 & iPc & 88 & 54.00 & -0.5 & I WAB & & 69.41 & 109 & $P$ \\
\hline AVF & 61.02 & 310 & $i P c$ & 88 & 55.10 & -0.5 & I SWV & & 69.63 & 149 & $P C$ \\
\hline PYM & 61.50 & 309 & $\mathrm{Pc}$ & 08 & 58.50 & -0.5 & 1 MAL & & 69.65 & 301 & iPc \\
\hline NAU & 61.55 & 144 & $\mathrm{Pc}$ & 08 & 58.20 & -1.2 & 1 & & & & ePP \\
\hline & & & $\mathrm{i}$ & 09 & 03.40 & $17 \mathrm{~km}$ & 1 & & & & is \\
\hline UZF & 61.67 & 309 & iPc & 09 & 00.00 & -0.1 & I MUN & & 69.77 & 149 & iPC \\
\hline $\mathrm{TCF}$ & 61.91 & 310 & iPc & 09 & 01.70 & 0.0 & i & & $1.0 \mathrm{~s}$ & $44 E$ & $0.00 \mathrm{~nm}$ \\
\hline CAF & 62.36 & 308 & iPc & 09 & 04.70 & 0.0 & i & $z$ & $20 \mathrm{~s}$ & & $9.00 \mathrm{um}$ \\
\hline LSF & 62.37 & 310 & iPc & 09 & 04.30 & -0.4 & i & $\mathrm{N}$ & $20 \mathrm{~s}$ & & 1.00 um \\
\hline RJF & 62.63 & 309 & iPc & 09 & 06.80 & 0.4 & 1 & E & $20 \mathrm{~s}$ & & $7.00 \mathrm{um}$ \\
\hline MEL & 62.79 & 139 & $\mathrm{PC}$ & 09 & 05.90 & -1.9 & I STS & & 70.27 & 309 & eP \\
\hline SSC & 62.85 & 312 & iPc & 09 & 07.10 & -0.7 & I WBN & & 70.55 & 137 & $\mathrm{Pc}$ \\
\hline EDI & 62.90 & 321 & $I P C$ & 09 & 07.50 & -0.5 & I MOM & & 70.59 & 104 & - $\mathrm{Pc}$ \\
\hline & $0.8 \mathrm{~s}$ & 148 & $3.00 \mathrm{~nm}$ & & & $6.2 \mathrm{mb}$ & I BUL & & 70.77 & 232 & P+ \\
\hline EBH & 63.00 & 321 & $i P_{c}$ & 09 & 08.10 & -0.6 & i & & & & is \\
\hline & $0.9 \mathrm{~s}$ & 122 & $2.00 \mathrm{~nm}$ & & & $6.1 \mathrm{mb}$ & I PTO & & 70.98 & 307 & $\mathrm{IPC}$ \\
\hline EKA & 63.02 & 320 & $\mathrm{Pd}$ & 09 & 08.80 & -0.1 & i & & & & is \\
\hline & $0.9 \mathrm{~s}$ & 182 & $2.00 \mathrm{~nm}$ & & & $6.2 \mathrm{mb}$ & I NWAO & & 71.04 & 148 & iPc \\
\hline LPO & 63.02 & 308 & $\mathrm{iPc}$ & 09 & 09.10 & 0.1 & i & & $0.9 \mathrm{~s}$ & $45 e$ & $0.00 \mathrm{~nm}$ \\
\hline & $0.7 \mathrm{~s}$ & 59 & $9.70 \mathrm{~nm}$ & & & $5.9 \mathrm{mb}$ & i & $Z$ & $20 s$ & & $0.00 \mathrm{um}$ \\
\hline$F L N$ & 63.04 & 313 & iPc & 09 & 08.20 & -0.9 & i & $N$ & $20 \mathrm{~s}$ & & 0.00 um \\
\hline & $0.9 \mathrm{~s}$ & 72 & 2. $20 \mathrm{~nm}$ & & & $5.8 \mathrm{mb}$ & i & $E$ & $20 \mathrm{~s}$ & & $1.00 \mathrm{um}$ \\
\hline ESK & 63.05 & 320 & iPc & 09 & 08.50 & -0.6 & I WRA & & 71.14 & 127 & $\mathrm{Pd}$ \\
\hline & $1.0 \mathrm{~s}$ & 200 & $0.00 \mathrm{~nm}$ & & & $6.2 \mathrm{mb}$ & $i$ & & $1.2 \mathrm{~s}$ & $63 e$ & $0.00 \mathrm{~nm}$ \\
\hline & & & e & 12 & 33.00 & & I WB2 & & 71.15 & 127 & $\mathrm{Pc}$ \\
\hline EAU & 63.06 & 321 & $i P c$ & 09 & 08.80 & -0.4 & i & & & & es \\
\hline & $1.1 \mathrm{~s}$ & 228 & $3.00 \mathrm{~nm}$ & & & $6.2 \mathrm{mb}$ & KLG & & 71.32 & 144 & - $\mathrm{Pc}$ \\
\hline LFF & 63.26 & 308 & iPc & 09 & 10.70 & 0.1 & I LIS & & 72.33 & 305 & $\mathrm{Pc}$ \\
\hline GRR & 63.38 & 312 & iPc & 09 & 10.40 & -0.9 & i & & & & iPP \\
\hline MFF & 63.40 & 310 & iPc & 09 & 10.80 & -0.7 & $i$ & & & & $\mathrm{~s}$ \\
\hline LPF & 63.59 & 312 & $i P c$ & 09 & 12.00 & -0.8 & I LML & & 72.80 & 108 & $P$ \\
\hline & $1.0 \mathrm{~s}$ & 66 & $5.30 \mathrm{~nm}$ & & & $5.7 \mathrm{mb}$ & ASP & & 73.38 & 130 & $P_{C}$ \\
\hline JAG & 63.60 & 345 & iPC & 09 & 10.80 & -1.6 & 1 & & & & e \\
\hline & $1.2 \mathrm{~s}$ & 169 & $9.00 \mathrm{~nm}$ & & & $6.1 \mathrm{mb}$ & і мвс & & 73.57 & 5 & iPc \\
\hline & & & is & 17 & 45.00 & & I JOZ & & 73.65 & 224 & $\mathrm{Pd}$ \\
\hline$S G R$ & 63.60 & 312 & $e P c$ & 09 & 11.90 & -0.9 & I PMG & & 74.41 & 111 & $\mathrm{Pct}$ \\
\hline BNG & 64.04 & 260 & $i P c$ & 09 & 14.50 & -1.8 & I LMG & & 74.94 & 110 & eP \\
\hline & 0.75 & 293 & $3.00 \mathrm{~nm}$ & & & $6.5 \mathrm{mb}$ & 1150 & & 75.33 & 124 & $P_{C}$ \\
\hline $\mathrm{CAO}$ & 64.05 & 260 & ePC & 09 & 14.00 & -2.3 & 1 & & & & e \\
\hline EPF & 64.08 & 306 & $i P_{c}$ & 09 & 15.20 & -0.9 & I RAB & & 75.76 & 103 & $\mathrm{PC}$ \\
\hline $1 \mathrm{TN}$ & 64.11 & 124 & $P d$ & 09 & 15.10 & -1.5 & 1 & & & & es \\
\hline CNA & 64.42 & 128 & $\mathrm{Pd}$ & 09 & 17.30 & -1.2 & I KSR & & 75.82 & 229 & iPC \\
\hline TET & 64.61 & 232 & $\mathrm{PC}$ & 08 & 24.00 & $-55.8 x$ & i & & $1.1 \mathrm{~s}$ & $16 e$ & $0.00 \mathrm{~nm}$ \\
\hline JAY & 65.25 & 108 & ePC & 09 & 22.00 & -2.1 & IMA & & 75.84 & 20 & $\mathrm{iPC}$ \\
\hline & 1.05 & 85 & $.90 \mathrm{~nm}$ & & & $5.9 \mathrm{mb}$ & I RES & & 75.89 & 359 & eP \\
\hline DDK & 65.33 & 319 & iPc & 09 & 23.60 & -0.3 & 1 & & $0.9 \mathrm{~s}$ & 124 & $4.00 \mathrm{~nm}$ \\
\hline & $1.5 \mathrm{~s}$ & 640 & $.00 \mathrm{~nm}$ & & & $6.6 \mathrm{mb}$ & I GDH & & 76.00 & 345 & $I P C$ \\
\hline DLE & 65.47 & 319 & iPc & 89 & 24.60 & -0.3 & 1 & & $1.8 \mathrm{~s}$ & $15 E$ & $6.00 \mathrm{~nm}$ \\
\hline & $1.5 \mathrm{~s}$ & 540 & $.00 \mathrm{~nm}$ & & & $6.5 \mathrm{mb}$ & i & $z$ & $20 \mathrm{~s}$ & 49 & $9.00 \mathrm{um}$ \\
\hline DMU & 65.50 & 319 & iPC & 09 & 25.00 & -0.1 & 1 & & & & iPP \\
\hline & $1.0 \mathrm{~s}$ & 610 & $0.00 \mathrm{~nm}$ & & & $6.7 \mathrm{mb}$ & 1 & & & & ¿PPP \\
\hline $\mathrm{ECP}$ & 65.70 & 318 & iPc & 09 & 26.00 & -0.3 & i & & & & is \\
\hline & 1 & 990 & $.00 \mathrm{~nm}$ & & & $6.7 \mathrm{mb}$ & I PRY & & 76.17 & 228 & $P$ \\
\hline $\mathrm{DCN}$ & 65.88 & 319 & iPC & 09 & 27.40 & -0.1 & I ESA & & 77.59 & 109 & $\mathrm{Pc}$ \\
\hline & & 820 & $.00 \mathrm{~nm}$ & & & $6.7 \mathrm{ml}$ & I $\mathrm{COL}$ & & 78.39 & 19 & iP \\
\hline$A L I$ & 66.16 & 302 & iPd & 09 & 30.00 & 0.5 & i & & $1.2 \mathrm{~s}$ & 253 & $3.00 \mathrm{~nm}$ \\
\hline & & & is & 18 & 18.00 & & $i$ & & & & e \\
\hline LGR & 66.25 & 307 & $i P C$ & 09 & 30.00 & -0.1 & i & & & & es \\
\hline & & & iPP & 11 & 57.40 & & I FBA & & 78.39 & 19 & $\mathrm{IPC}$ \\
\hline & & & is & 18 & 20.50 & & I INK & & 79.02 & 12 & IPC \\
\hline ATD & 66.42 & 233 & $\mathrm{P}+$ & 09 & 31.00 & -0.6 & $i$ & & $1.2 \mathrm{~s}$ & 377 & $7.00 \mathrm{~nm}$ \\
\hline MEK & 66.46 & 144 & $\mathrm{Pc}$ & 09 & 29.30 & -2.2 & I CTA & & 79.88 & 120 & $P$ \\
\hline ILT & 66.58 & 24 & $\mathrm{PC}$ & 09 & 31.50 & -0.3 & 1 & & & & is \\
\hline & & & es & 18 & 22.00 & & I HVD & & 79.95 & 226 & $\mathrm{Pc}$ \\
\hline KTG & 66.61 & 339 & iPc & 09 & 32.80 & 0.9 & | PKR & & 80.02 & 227 & $P$ \\
\hline & 1.15 & 96 & $.00 \mathrm{~nm}$ & & & $.9 \mathrm{mb}$ & I PMR & & 80.44 & 22 & ePc \\
\hline & & & iPP & 12 & 02.00 & & i & & $1.3 \mathrm{~s}$ & 151 & $1.00 \mathrm{~nm}$ \\
\hline & & & is & 18 & 28.00 & & I & $z$ & $19 \mathrm{~s}$ & & 1. $50 \mathrm{um}$ \\
\hline LE & 66.68 & 355 & eP & 09 & 32.00 & -0.3 & WIN & & 80.52 & 237 & iPC \\
\hline$K U$ & 67.02 & 334 & iPct & 09 & 35.50 & 0.9 & 1 & & $1.3 \mathrm{~s}$ & 108 & $8.00 \mathrm{~nm}$ \\
\hline & $0.9 \mathrm{~s}$ & 1650 & $.00 \mathrm{~nm}$ & & & $7.2 \mathrm{mb} \times$ & 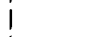 & 2 & $19 s$ & 29 & 9.50 um \\
\hline & & & e & 11 & 54.40 & $728 \mathrm{kmX}$ & GRM & & 81.15 & 224 & iPc \\
\hline RI & 67.83 & 234 & $\mathrm{P}+$ & 09 & 40.00 & -0.6 & 1 & & $1.2 \mathrm{~s}$ & 244 & $4.00 \mathrm{~nm}$ \\
\hline
\end{tabular}




\begin{tabular}{|c|c|c|c|c|c|c|c|c|c|c|c|}
\hline & & & SKS & 24 & 06.00 & & 1 & & ePP & 2224.00 & 1 \\
\hline & & & SKKS & 25 & 10.00 & & $i$ & & ePPP & $25 \quad 48.00$ & \\
\hline & & & PS & 27 & 47.00 & & $i$ & & ess & 4150.00 & \\
\hline & & & PKKP & 28 & 01.00 & & 1 & & eSSS & 4746.00 & \\
\hline & & & PPS & 29 & 08.00 & & CEN & $154.03 \quad 258$ & e (PKP) & 1830.00 & $-2.9 x$ \\
\hline & & & SS & 34 & 28.00 & & $\mathrm{TLL}$ & $155.67 \quad 262$ & PKP & 1837.00 & 1.6 \\
\hline & & & sss & 38 & 22.00 & & SAN & $155.68 \quad 254$ & ePKP & 1842.00 & $7.0 \times 1$ \\
\hline PAS & 113.98 & 17 & ePKP & 17 & 20.00 & -0.8 & LNV & $156.29 \quad 253$ & ePKP & 1837.00 & $1.3 \quad$ \\
\hline & $z \quad 20 \mathrm{~s}$ & 18 & $8.00 \mathrm{um}$ & & & $.7 \mathrm{Msz}$ & I TMU & 156.51240 & PKP & $18 \quad 37.00$ & 1.1 \\
\hline & $\mathrm{N} 20 \mathrm{~s}$ & & $0.00 \mathrm{um}$ & & & & 1 & S.D. $=1.0$ & on 383 & 3 of 449 of & bs. \\
\hline & E $20 \mathrm{~s}$ & 13 & $3.00 \mathrm{um}$ & & & & 1 & & & & \\
\hline & & & ePP & 18 & 18.00 & & $O C$ & T 10,1980 & $12 h_{2}$ & $25 \mathrm{~m} 23.81 \pm$ & $0.08 \mathrm{~s}$ \\
\hline & & & ePKKP & 28 & 01.00 & & 36 & $.213 \mathrm{~N} \pm 1.3$ & $3 \mathrm{~km}$ & $1.395 \mathrm{EI}$ & $9.2 \mathrm{~km}$ \\
\hline RLO & 114.43 & 357 & iPKP & 17 & 21.60 & 0.0 & $D E$ & PTH $=11.1 \mathrm{k}$ & $\mathrm{km}<1$ & 14 depth pl & hases) \\
\hline PRM & 114.71 & 345 & ePKPd & 18 & 22.20 & $60.0 x$ & 6 & $3 \mathrm{mb}(48$ obs & s.) & $2 \mathrm{Msz}(14$ & obs.) \\
\hline TUL & 114.72 & 357 & iPKP & 17 & 21.50 & -0.6 & ALGE & $R \mid A$ & & & $(396)$ \\
\hline & $1.2 \mathrm{~s}$ & & $3.00 \mathrm{~nm}$ & & & & i & At least 50 & 000 peo & ople kille & \\
\hline & $z 20 \mathrm{~s}$ & & 4. $16 \mathrm{um}$ & & & $.0 M s z$ & i & 9000 injure & ed ond & extensive & \\
\hline SNA & 115.43 & 202 & PKP & 17 & 22.70 & 0.3 & i & damage in $t$ & the EI & Asnom are & \\
\hline GLA & 195.83 & 15 & EPKPC & 17 & 25.80 & 1.4 & i & Felt throug & ghout n & northweste & \\
\hline TUC & 117.35 & 11 & ePKPd & 17 & 28.50 & 1.1 & i & Algeria and & d in so & outheasteri & \\
\hline$S B A$ & 117.85 & 166 & iPKP & 17 & 27.20 & 0.4 & i & Spoin. Appr & roximot & tely $42 \mathrm{~km}$ & of \\
\hline & & & e & 17 & 35.00 & & i & surface rup & pture o & observed. & \\
\hline & & & SKS & 24 & 28.00 & & i & & & & \\
\hline & & & PS & 28 & 38.00 & & $A L \mid$ & 2.61325 & $i P_{n}$ & 2606.00 & -0.6 \\
\hline & & & ss & 34 & 58.00 & & $A L M$ & $3.17 \quad 283$ & $i P_{n}$ & $26 \quad 13.00$ & -1.5 \\
\hline & & & LQ & 48 & 00.00 & & I CRT & 4.13285 & iPnd & $26 \quad 28.90$ & 0.7 \\
\hline & & & LR & 54 & 00.00 & & I EBR & $4.65 \quad 352$ & $e P_{n}$ & $26 \quad 35.00$ & -0.6 \\
\hline SPA & 119.49 & 180 & ePKPC & 17 & 29.70 & -0.7 & i & & e & 3756.00 & \\
\hline & $0.9 \mathrm{~s}$ & 25 & $5.20 \mathrm{~nm}$ & & & & I MAL & $4.71 \quad 278$ & $i P_{n}$ & 2635.60 & -0.8 \\
\hline & $220 \mathrm{~s}$ & 33 & $3.70 \mathrm{um}$ & & & $.0 \mathrm{Msz}$ & $i$ & & is & 2750.00 & \\
\hline MOT & 119.80 & 5 & iPKP & 17 & 26.20 & $-6.0 x$ & TOL & $5.64 \quad 312$ & Pnd & 2648.00 & -1.7 \\
\hline JCT & 120.19 & 1 & ePKP & 17 & 32.80 & -0.7 & I IFR & $6.00 \quad 245$ & $P_{n}$ & 2652.50 & -2.3 \\
\hline & $0.9 \mathrm{~s}$ & 77 & $7.70 \mathrm{~nm}$ & & & & BMK & 6.03260 & $P_{n}$ & 2656.00 & 0.9 \\
\hline & $\mathrm{Z} 20 \mathrm{~s}$ & 12 & $2.40 u m$ & & & $5 \mathrm{Msz}$ & GUD & $6.21 \quad 317$ & $i P_{n}$ & 2655.80 & -1.9 \\
\hline HKT & 120.65 & 357 & EPKP & 17 & 33.60 & 0.1 & HAD & $6.42 \quad 231$ & $P_{n}$ & 2659.00 & -1.7 \\
\hline SOB3 & 122.85 & 278 & PKP & 17 & 38.50 & 0.3 & EPF & $6.86 \quad 354$ & $P_{n}$ & 2706.40 & -0.4 \\
\hline & & & e & 17 & 41.60 & & I LGR & $6.93 \quad 335$ & iPnd & $27 \quad 10.00$ & 2.3 \\
\hline & & & e & 17 & 44.60 & & i & $0.9 \mathrm{~s}$ & $7.00 \mathrm{~nm}$ & & $.8 \mathrm{mb} \times$ \\
\hline & & & e & 17 & 51.60 & & i & & esn & 2830.00 & 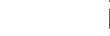 \\
\hline TRN & 126.05 & 312 & IPKPC & 17 & 45.70 & 1.3 & $\mathrm{KCHT}$ & 6.94 & $i P_{n}$ & 2705.60 & -2.3 \\
\hline & $0.9 \mathrm{~s}$ & 128 & $8.00 \mathrm{~nm}$ & & & & I CGL & $7.00 \quad 61$ & $P_{n-}$ & 2704.50 & $-4.4 x$ \\
\hline CAR & 129.57 & 318 & IPKPC & 17 & 50.00 & -1.3 & I $Z G N$ & $7.04 \quad 86$ & $i P_{n}$ & 2707.00 & -2.4 \\
\hline & $1.2 \mathrm{~s}$ & 144 & $4.00 \mathrm{~nm}$ & & & & I BMB & $7.06 \quad 254$ & $P_{n}$ & 2707.00 & -2.5 \\
\hline & $z 22 \mathrm{~s}$ & & $7.41 \mathrm{um}$ & & & . $3 \mathrm{MsZ}$ & I SBS & $7.24 \quad 82$ & $i P_{n}$ & $27 \quad 12.50$ & 0.4 \\
\hline ROJ & 130.02 & 264 & PKP & 17 & 49.00 & $-2.7 x$ & I $M B Z$ & $7.49 \quad 84$ & iPn & 2713.60 & -2.0 \\
\hline & & & iPP & 20 & 08.00 & & I AVE & $\begin{array}{lll}7.81 & 251\end{array}$ & $P_{n}$ & $27 \quad 19.00$ & -1.1 \\
\hline & & & iPKS & 21 & 19.20 & & i & & $\mathrm{Pg}$ & 2751.00 & \\
\hline & & & EPPP & 22 & 56.40 & & i LMR & $8.13 \quad 27$ & $p$ & $27 \quad 22.30$ & -2.1 \\
\hline & & & eSKS & 25 & 07.60 & & i & & sn & 2848.20 & \\
\hline & & & eSKKS & 27 & 02.40 & & I MTE & 8.17303 & $i p$ & 2724.00 & -1.2 \\
\hline & & & EPPS & 31 & 55.60 & & I LRG & $8.18 \quad 26$ & $\mathrm{p}$ & 2723.30 & -1.8 \\
\hline & & & eSS & 37 & 25.20 & & i & & sn & 2847.80 & \\
\hline 110 & 130.86 & 0 & ePKP & 17 & 54.40 & 0.5 & I FRF & 8.37 & $\mathrm{P}$ & 2725.60 & -2.3 \\
\hline BAO & 131.36 & 275 & ePKP & 17 & 53.50 & -1.1 & 1 & & sn & 2853.90 & \\
\hline IIT & 131.60 & 359 & ePKP & 17 & 55.60 & 0.4 & i LPO & $8.46 \quad 359$ & $P$ & 2728.00 & -1.1 \\
\hline 111 & 132.25 & 1 & ePKP & 17 & 57.40 & 1.0 & CVF & $8.58 \quad 40$ & $P$ & 2727.20 & $-3.7 x$ \\
\hline PPT & 132.36 & 87 & ePKP & 18 & 01.00 & $4.7 x$ & $i$ & & sn & 2856.80 & \\
\hline & $1.1 \mathrm{~s}$ & 90 & $0.00 \mathrm{~nm}$ & & & & BME & $8.69 \quad 234$ & $\mathrm{P}$ & 2729.00 & -2.4 \\
\hline VHO & 133.34 & 357 & iPKP & 18 & 00.00 & 1.6 & $1 \mathrm{col}$ & 8.69300 & $P$ & 2730.40 & -1.9 \\
\hline UAV & 133.52 & 321 & EPKP & 17 & 46.30 & $-12.6 x$ & CAF & 8.72 & $\mathrm{P}$ & 2731.30 & -1.4 \\
\hline & $0.8 \mathrm{~s}$ & & $7.00 \mathrm{~nm}$ & & & & LFF & $8.73 \quad 357$ & $P$ & 2731.80 & -1.0 \\
\hline LPS & 135.31 & 347 & ePKP & 17 & 46.50 & $-15.6 x$ & LIS & 8.75290 & $P_{C}$ & 2730.20 & -2.9 \\
\hline FUo & 137.54 & 321 & ePKP & 17 & 59.00 & $-7.7 x$ & i & & es & 2907.00 & \\
\hline LCR & 138.29 & 338 & PKPC & 18 & 07.70 & -0.2 & I 110 & $8.94 \quad 236$ & $\mathrm{P}$ & 2734.00 & -1.9 \\
\hline$A \mid A$ & 138.42 & 201 & $e(P K P)$ & 18 & 09.00 & $2.7 x$ & $R\lrcorner F$ & 9.08 & $p$ & 2736.60 & -1.1 \\
\hline $\mathrm{CHN}$ & 138.96 & 323 & ePKP & 18 & 09.00 & -0.1 & STV & $9.21 \quad 28$ & $P$ & 2739.00 & -0.6 \\
\hline OTP & 140.17 & 83 & EPKP & 18 & 16.00 & $5.1 x$ & PTO & $9.23 \quad 305$ & $P$ & 2736.30 & $-3.5 x$ \\
\hline & $1.2 \mathrm{~s}$ & 170 & $0.00 \mathrm{~nm}$ & & & & i & & $i$ & 2926.00 & \\
\hline PSO & 143.05 & 322 & iPKP & 18 & 13.50 & $-3.2 x$ & SSB & 9.36 & ep & 2741.70 & 0.1 \\
\hline LPA & 145.15 & 251 & PKPd+ & 18 & 20.20 & 1.1 & ROB & $9.46 \quad 30$ & $P$ & 2741.50 & -1.5 \\
\hline & $1.0 \mathrm{~s}$ & 1840 & $0.00 \mathrm{~nm}$ & & & & GRN & 9.60 & ip & $27 \quad 45.30$ & 0.3 \\
\hline & $z 20 \mathrm{~s}$ & 22 & $2.70 \mathrm{um}$ & & & $.9 \mathrm{Msz}$ & I PYM & 9.61 & $P d$ & 2744.50 & -0.5 \\
\hline ICE & 145.23 & 322 & PKP & 18 & 21.60 & 1.1 & I PNI & $9.79 \quad 26$ & $\mathrm{P}$ & $27 \quad 47.70$ & 0.2 \\
\hline BAA & 145.59 & 251 & PKP & 18 & 19.40 & -0.5 & $i$ & & es & 2937.00 & \\
\hline RKT & 147.12 & 87 & IPKP & 18 & 24.20 & 1.5 & I GEN & 10.01 & $\mathrm{P}$ & 2748.90 & -1.5 \\
\hline & 1.15 & 240 & $0.00 \mathrm{~nm}$ & & & & 1 & & es & 2939.00 & \\
\hline ZOBO & 148.80 & 288 & IPKP & 18 & 30 & 0.5 & LSF & 10.03 & $P$ & 2749.00 & -1.8 \\
\hline LPB & 148.92 & 288 & PKP & 18 & 27.50 & 1.2 & 1 & & sn & 2934.90 & \\
\hline SLA & 149.95 & 271 & PKPC & 18 & 28.40 & 1.9 & $M Z F$ & 10.03 & $P$ & 2749.50 & -1.3 \\
\hline CYA & 151.09 & 264 & PKPC & 18 & 30.20 & 1.4 & TCF & 10.08 & $P$ & 2749.80 & -1.7 \\
\hline ARE & 151.66 & 292 & PKPC & 18 & 31.50 & 1.3 & sis & $10.16 \quad 314$ & iPd & 2750.50 & -2.1 \\
\hline HUA & 151.95 & 304 & iPKPc & 18 & 33.80 & $2.9 x$ & $i$ & & sn & 3122.50 & \\
\hline NNA & 153.00 & 306 & PKP+ & 18 & 32.80 & 0.9 & GIB & 10.24 & P- & 2756.00 & 2.2 \\
\hline VCA & 153.23 & 264 & PKPC & 18 & 34.50 & 2.5 & 1 & & isn & $29 \quad 48.50$ & \\
\hline CFA & 153.59 & 258 & PKPC & 18 & 33.80 & 1.5 & i & & $\mathrm{i}$ & 3239.00 & \\
\hline ANT & 153.91 & 276 & PKP & 18 & 34.50 & 1.7 & I RMP & $10.42 \quad 54$ & $\mathrm{P}$ & 2756.00 & -0.2 \\
\hline
\end{tabular}


$10 d \quad 12 r$

STB $\quad 14.90 \quad 14 \quad \mathrm{IPC}$

TNS $\quad 14.91 \quad 18$ IPC

KOE $\quad 14.93 \quad 16 \mathrm{eP}$

MOA $\quad 15.03 \quad 35 \mathrm{iPC}$

HEE $\quad$ C $\quad 3747.03 \quad 11$ iPC $\quad 2908.00$

$\begin{array}{llllll}15.03 & 11 & \text { iPC } & 29 & 00.00 \\ & & i & 29 & 02.90\end{array}$

KMR $\quad 15.12 \quad 35 \mathrm{iPC} \quad 2900.30$

WOL $\quad 15.21354$ iPd 2900.20

$\begin{array}{llllll}\text { CLG } & 15.24 & 13 & \text { IPd } & 28 & 57.50\end{array}$

GRFO $\quad 15.25 \quad 25$ IP $\quad 2902.10$

GRF $\quad 15.25 \quad 25 \mathrm{ePC}$

$\begin{array}{ccc}\text { i } & 29 & 05.00 \\ \text { eS } & 32 & 03.50\end{array}$

$\begin{array}{llllll}\text { TIR } & 15.28 & 65 & \mathrm{P} & 28 & 59.40 \\ \text { BNS } & 15.32 & 14 & \text { iPd } & 29 & 06.40\end{array}$

$1.4 \mathrm{~s} \quad 6.20 \mathrm{~nm}$

$i^{20 \mathrm{~nm}} 32 \quad 47.00$

HOK $\quad 15.36 \quad 14$ iPd 2907.40

$\begin{array}{llllll}\text { WET } & 15.41 & 29 & \text { IPC } & 29 & 02.20\end{array}$

KHC $\quad 15.69 \quad 31$ i 2904.40

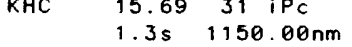

905.10 2906.40 2908.50 3455.50

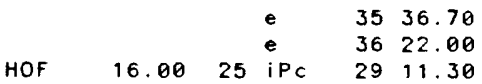

$\begin{array}{llll}16.00 & 25 \mathrm{i} C & 29 & 14.30\end{array}$

$\begin{array}{lllll}\text { SOP } \quad 16.05 \quad 40 \mathrm{PC} & 29 & 11.20\end{array}$ e $\quad 3004.00$

DBN $\quad 16.11 \quad 8$ iPd 2917.00

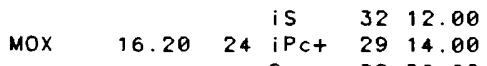

$\begin{array}{rrr}\text { MOX } & 3220.00 \\ & \mathrm{e} & 3730.00\end{array}$

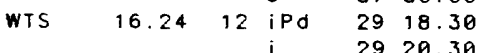

i $\quad 2922.60$
VKA

$\begin{array}{llllll}\text { VKA } & 16.31 & 38 & \text { IPC } & 29 & 15.78\end{array}$

$\begin{array}{lll}\text { i } & 29 & 18.00 \\ \text { PP } & 29 & 30.00\end{array}$

S $\quad 3218.00$

SS 3234.00

$\begin{array}{lllll} & & \mathrm{e} & 37 & 20.00 \\ \text { VIE } & 16.32 & 38 \mathrm{iPC} & 29 & 15.50\end{array}$

$\begin{array}{ll}\text { PRU } & 16.7531 \mathrm{PC} \\ & 2.9 \mathrm{~s} 5190 \mathrm{O} \mathrm{nm}\end{array}$

$\begin{array}{rll}S & 3246.00 \\ i & 2024.50\end{array}$

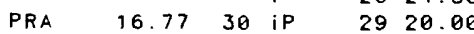

IEN $16.78248 \mathrm{~S} \quad 3222.00$

$\begin{array}{llllll} & 16.78 & 248 & \text { IPC } & 29 & 21.10 \\ & & & \text { iPCS } & 37 & 16.50\end{array}$

$\begin{array}{llllll}\text { BEO } & 16.82 & 53 \mathrm{P} & 29 & 21.40\end{array}$

$\begin{array}{lllllll} & & & & \text { iSg } & 32 & 41.00 \\ \text { ECP } & 16.89 & 343 & \mathrm{ePd} & 29 & 23.60\end{array}$

WIT $\begin{array}{llll}2.5 \mathrm{~s} & 9700.00 \mathrm{~nm} & \\ & & & \end{array}$

$\begin{array}{ccccc}17.01 & 11 \mathrm{eP} & 29 & 26.50 \\ & \mathrm{e} & 29 & 29.00\end{array}$

$2 \stackrel{i}{\mathrm{i}}$

HRE

2933.20

$17.03 \quad 42 \mathrm{eP}$

2926.00

SRO $\quad 17.06 \quad 42 \quad \mathrm{iP}$

$32 \quad 46.60$

2936.30

4100.00

BRG $17.21 \quad 28 \mathrm{iPC}$

$\begin{array}{llll}\text { CLL } & 17.23 \quad 25 & \mathrm{iPC}\end{array}$

2926.50

$\begin{array}{ll}29 & 27.00 \\ 29 & 29.00\end{array}$

2934.00

$29 \quad 45.00$

$32 \quad 35.00$

BUD $\quad 17.26 \quad 44 \mathrm{Pc} \quad 2927.20$

3118.00

$\begin{array}{rlll}\text { e } & 31 & 18.00 \\ \text { eS } & 3233.00\end{array}$

$\begin{array}{lllll} & & & & \end{array}$

TIM $\quad 17.70 \quad 51 \mathrm{eP} \quad 2931.00$

$\begin{array}{llllll}\text { VAL } & 17.77336 \text { iP } & 29 & 33.00 \\ & & \text { is } & 32 & 42.00\end{array}$

$\begin{array}{llllll}\text { DKM } & 17.87 & 345 & \text { iPd } & 29 & 35.90\end{array}$

$1.5 \mathrm{~s} 3800.00 \mathrm{~nm}$

ATH \begin{tabular}{l|l}
$2.9 x$ & \\
1.8 & OLE
\end{tabular}

\begin{tabular}{l|l}
1.8 & OLE \\
$3.3 \times$ & \\
1.3 & ODK
\end{tabular}

I SOF

$2.4: \begin{array}{l:l}\text { DCN } \\ \text { BRN }\end{array}$

\begin{tabular}{l|l}
1.5 & HAM \\
0.2 & HAM
\end{tabular}

$-2.8 \times$ GZR

\begin{tabular}{r|r}
1.6 & \\
-0.4 & DMU
\end{tabular}

JOS

$-1.6$

$8 \mathrm{mb} \times \mathrm{DEV}$

DEV
SPC

\begin{tabular}{c|c}
$5.4 \times$ & NIE \\
-0.5 & KRA
\end{tabular}

$-1.1$

$9 \mathrm{mb}$

I

\section{DRA}

$\left\{\begin{array}{l}\text { CEI } \\ \text { ESK }\end{array}\right.$

\begin{tabular}{l|l}
1.0 & EKA \\
0.4 & EKA
\end{tabular}

$K D Z$

PVL

$5.4 \times \quad \mathrm{DIM}$

UZH

1.2 EBL

EGL

$5.0 \times$

MDB

1.5 EAU

EZN

EDI

CMP

$0.3 \quad \mathrm{EBH}$

$6.2 \mathrm{mb}$ EAB

BUC 1

0.1 BUC

0.9

ELO

$0.7 \quad M L R$

2.2 MFT

Smb | COP

\begin{tabular}{l|l}
$3.5 \times$ & \\
& ISR \\
& EDC
\end{tabular}

\begin{tabular}{l|l|l}
2.8 & DMK \\
& WAR
\end{tabular}

$12.7 \times$ LVV

1.0 VRI

1.3 ODE

YER
CTT

PDA

DST

1.1 BIR

CFR

CFR
ISK

0.0 BKT

\begin{tabular}{l|l|l}
0.6 & IAS
\end{tabular}

$0.5) \mathrm{ADH}$

2.2 ALT

\begin{tabular}{l|l}
$3 \mathrm{mb}$ & GPA \\
-0.1 & $\mathrm{KIS}$
\end{tabular} is $\quad 3300.00$
17.96344 is

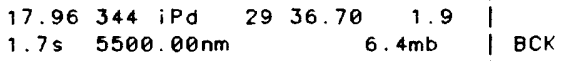
$\begin{array}{llllll}18.01 & 345 \text { iPd } 29 & 39.00 & 3.6 \times & \text { KON }\end{array}$ $\begin{array}{lllllll}18.11 & 62 & \mathrm{PC} & 29 & 32.00 & -4.9 \times & \text { BER }\end{array}$ $\begin{array}{lllllll}18.18 & 343 & \mathrm{iPd} & 29 & 39.40 & 1.9 & \mathrm{SYT}\end{array}$ $2.057900 .00 \mathrm{~nm} 6.5 \mathrm{mb}$ MGN \begin{tabular}{llllll|l}
18.23 & 24 & $\mathrm{iPC}$ & 29 & 41.00 & 2.8 & $\mathrm{HFS}$
\end{tabular} \begin{tabular}{llllll|l}
18.26 & $16 \mathrm{iPc}$ & 33 & 03.50 & & \\
18.60 & $4.1 \times$ & \\
& & & &
\end{tabular} $18.56 \quad 54 \mathrm{eP}$

18.61344 i 2942.00

18.61344 iPd

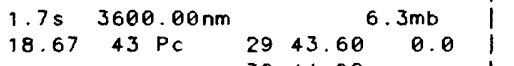
$29 \quad 43.90$

-0.4 NAO

\begin{tabular}{rrrrrr|l} 
& $e$ & 3044.00 & & UPP \\
& & 3 & 3148.00 & & \\
18.83 & 53 & $\mathrm{PC}$ & 29 & 31.00 & & \\
18.91 & 41 & $\mathrm{iP}$ & 29 & 49.00 & 0.4 & $\mathrm{SIM}$
\end{tabular}

\begin{tabular}{llllll|l}
18.91 & 41 & iP & 29 & 49.10 & 2.3 & \\
19.09 & 40 & iP & 29 & 50.50 & 1.7 & KVT
\end{tabular}

\begin{tabular}{rrrrrr|r}
19.09 & 40 & IP & 29 & 50.50 & 1.7 & KVT \\
19.28 & $38 \mathrm{eP}$ & 29 & 50.30 & -0.8 & NUR
\end{tabular}

$1.3 \mathrm{~s} \quad 2080.00 \mathrm{~nm}$

$2953.10^{6.2 \mathrm{mb}} 11 \mathrm{~km}$

$3001.60 \quad 11 \mathrm{~km}$ EIL

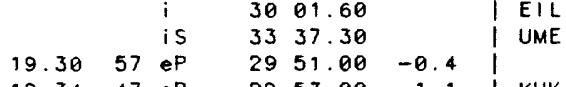

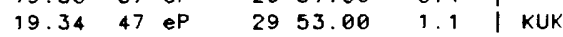

\begin{tabular}{ccc|c|c} 
& $\mathrm{eS}$ & 33 & 40.00 & PUL \\
$19.37352 \mathrm{iPd}$ & 29 & 53.50 & 1.4 & \\
$1.0 \mathrm{~s} \quad 2160.00 \mathrm{~nm}$ & & $6.4 \mathrm{mb}$ & SOC
\end{tabular}

$19.38352 \mathrm{Pd} 2955.40^{6.4 \mathrm{mb}} \mathrm{Kd}^{3.2 x} \mathrm{KIC}$

$0.8 \mathrm{~s} 234.00 \mathrm{~nm} \quad 5.5 \mathrm{mb}$ OBN

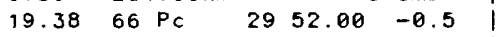

\begin{tabular}{llllll|l}
19.53 & 62 & $\mathrm{PC}$ & 29 & 52.00 & -2.1 & SUF
\end{tabular}

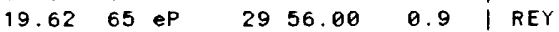

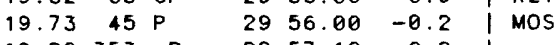

$19.80353 \mathrm{ePC} \quad 2957.10 \quad 0.2 \quad$

$0.6 \mathrm{~s} \quad 14.70 \mathrm{~nm} \quad 4.5 \mathrm{mb} \times \mathrm{AKU}$

19.85353 (PC

\begin{tabular}{llll|}
$0.6 \mathrm{~s}$ & $42.00 \mathrm{~nm}$ & $4.9 \mathrm{mb} \times \mid$ & \\
19.88 & $53 \mathrm{eP}$ & 29 & $56.00^{-1.8}$
\end{tabular}

$00-1.8$ | NYA

$19.91352 \quad \mathrm{iPc}$

$1.1 \mathrm{~s} \quad 2050.00 \mathrm{~nm}$

$19.9572 \mathrm{P}$

$\begin{array}{lll}19.96 & 352 \mathrm{ePC} \\ 0.6 \mathrm{~s} & 49.06 \mathrm{~nm}\end{array}$

$\begin{array}{ll}0.6 \mathrm{~s} & 49.06 \mathrm{~nm} \\ 20.01 & 56 \mathrm{Pd}\end{array}$

$20.31352 \mathrm{ePc} \quad 3002.30 \quad 0.0 \quad$ BKR

$0.6 \mathrm{~s} \quad 21.70 \mathrm{~nm} \quad 4.7 \mathrm{mb} \times \mid$ LEN

$20.36351 \mathrm{iPC} \quad 3005.50 \quad 2.8$ ।

$1.1 \mathrm{~s} 1780.00 \mathrm{~nm}$

$20.4259 \mathrm{eP} \quad 3004.00^{6.3 \mathrm{mb}} 0.5$

\begin{tabular}{llll|l}
20.48 & $59 \mathrm{eP}$ & 3004.00 & -0.1 & SOD
\end{tabular}

\begin{tabular}{cccc|c}
$20.56352 \mathrm{ePc}$ & $3006.80 \quad 2.0$ & \\
$1.1 \mathrm{~s}$ & $1090.00 \mathrm{~nm}$ & $6.1 \mathrm{mb}$ & GRO
\end{tabular}

$20.6776 \mathrm{P}$

$20.6956 \mathrm{eP}$

$3006.50^{6.1 \mathrm{mb}} 0.3$

\begin{tabular}{lllll|l} 
& is & 33 & 47.00 & -2.4 & TRO \\
20.74 & $69 \mathrm{P}$ & 3007.00 & 0.1 & BCAO
\end{tabular}

$\begin{array}{lllllll}20.88 & 18 & \text { iPd } & 30 & 09.40 & 1.3 & \text { GRS }\end{array}$

$1.1 \mathrm{~s} \quad 3040.00 \mathrm{~nm}$

$20.99 \quad 57 \mathrm{eP}$

$21.1571 \mathrm{P}$

$21.1967 \mathrm{P}$

$21.2135 \mathrm{eP}$

$21.3143 \mathrm{PC}$

$21.32 \quad 55 \mathrm{P}$

$21.52 \quad 56 \mathrm{eP}$

$21.56 \quad 80 \mathrm{P}-$

$21.6369 \mathrm{P}$

$21.65282 \mathrm{P}$

$21.7273 \mathrm{P}$

$22.0455 \mathrm{eP}$

$22.05 \quad 69 \mathrm{Pt}$

$22.10 \quad 58 \mathrm{eP}$

$22.1069 \mathrm{P}$

$22.12 \quad 71 \mathrm{P}$

$22.30 \quad 52 \mathrm{eP}$

$22.83285 \stackrel{\mathrm{PS}}{\mathrm{P}}$

$22.8874 \mathrm{P}-$

$22.90 \quad 80 \mathrm{P}+$

$23.0271 \mathrm{P}$

$23.0954 \mathrm{PC}$ is 3445.00 $\begin{array}{llllll}23.36 & 78 \mathrm{Pt} & 34 & 51.00 & \\ 24.05 & 18 & \text { iP } & 30 & 40.30 & 0.9\end{array}$ $\begin{array}{llllll}24.05 & 10 & \text { iP } & 30 & 40.30 & 0.8\end{array}$ $24.32 \quad 5$ iP $\quad 3044.30 \quad 2.2$ $24.4078 \mathrm{P}-\quad 3044.00 \quad 0.7$ $\begin{array}{lllll}24.46 & 70 \mathrm{P} & 30 & 42.90 & -0.8\end{array}$ $\begin{array}{llllll}25.22 & 14 & \text { eP } & 30 & 49.50 & -1.2\end{array}$

$\begin{array}{lll}1.0 \mathrm{~s} & 417.00 \mathrm{~nm} & 6.1 \mathrm{mb}\end{array}$

$z \quad 12 \mathrm{~s} \quad 806.00 \mathrm{um}$

$25.36 \quad 11 \mathrm{P} \quad 3051.70 \quad-0.4$

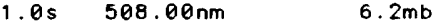

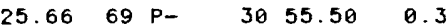

$\begin{array}{lllll}25.83 & 96 \mathrm{iPC} & 30 & 58.00 & 1.3\end{array}$

$\begin{array}{llllll}25.89 & 19 & \text { iS } & 34 & 22.00 & \\ & \text { P } & 30 & 55.00 & -2.0\end{array}$

$1.1 \mathrm{~s} 2400.00 \mathrm{~nm} \quad 6.8 \mathrm{mb}$

$\begin{array}{ccccc} & \text { is } & 35 & 28.50 & \\ 26.20 & 60 \mathrm{PC} & 30 & 59.50 & -0.5\end{array}$

$\begin{array}{llllll}27.38 & 69 & \text { iS } & 35 & 39.50 & \\ 28 & 31 & 10.80 & -0.2\end{array}$

$\begin{array}{llllll}28.50 & 24 & \mathrm{PC} & 31 & 20.20 & -0.6\end{array}$

$i \quad 3122.20 \quad 7 \mathrm{~km}$

\begin{tabular}{llllll}
28.85 & 94 & $\mathrm{PC}$ & 36 & 04.00 & \\
\hline & $\mathrm{PC}$ & 31 & 23.50 & -0.8
\end{tabular}

$\begin{array}{llllll}29.92 & 17 \mathrm{P} & 31 & 32.50 & -1.0\end{array}$

$\begin{array}{llll} & \text { ipP } \quad 31 \quad 35.60 \quad 11 \mathrm{~km}\end{array}$

$\begin{array}{lll}31 & 33.50 & -0.5\end{array}$

$\begin{array}{rrrrr}30.06 & 29 \mathrm{Pc} & 3134.00 & -0.8 \\ & \text { is } & 3639.00 & \end{array}$

$\begin{array}{llllll}30.10 & 64 & \text { PC } & 36 & 39.00 & 31 \\ 30.00 & -0.4\end{array}$

$\begin{array}{llllll}30.24 & 192 \mathrm{eP} & 31 & 34.00 & -2.8\end{array}$

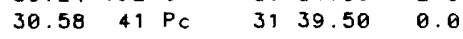

$\begin{array}{llllll}30.63 & 22 & \text { is } & 36 & 45.00 & \\ 31 & 39.00 & -0.9\end{array}$

$\begin{array}{lllll}31.31340 \mathrm{eP} & 3150.60 & 4.8 \mathrm{X}\end{array}$

$\begin{array}{lllll}31.35 & 40 \mathrm{PC} & 3147.00 & 0.8\end{array}$

$\begin{array}{rrrrr}31.67345 \text { i } & 3653.00 & \\ 1.15 & 9000.00 & & \end{array}$

$1.1 \mathrm{~s} 9000.00 \mathrm{~nm}$

$32.06133 \mathrm{Pt}$

$3154.20 \quad 15 \mathrm{~km}$

$\begin{array}{lll}31 & 52.60 & -0.3\end{array}$

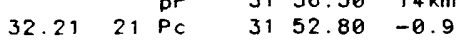

$i \quad 3155.00 \quad 8 \mathrm{~km}$

ePP $\quad 3300.00$

$\begin{array}{rrrrr} & \text { eS } & 3700.00 & \\ 32.52 & 63 \mathrm{PC} & 31 & 56.00 & -0.7\end{array}$

32 is 3719.00

$\begin{array}{llllll}32.96 & 67 & \mathrm{PC} & 32 & 00.60 & -0.1\end{array}$

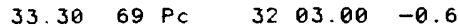

$\begin{array}{llllll} & \mathrm{eS} & 37 & 20.00 & \\ 33.46 & 13 \mathrm{P} & 3203.10 & -1.4\end{array}$

$\begin{array}{llll} & \text { ipP } \quad 32 & 06.10 & 10 \mathrm{~km}\end{array}$

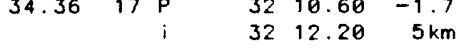

$\begin{array}{lllll}34.45 & 64 \mathrm{Pc} & 32 & 13.40 & 0.1\end{array}$

$\begin{array}{llllll}34.82 & 11 \text { IP } & 37 & 34.00 & \\ \text { iP } & 32 & 16.40 & 0.2\end{array}$

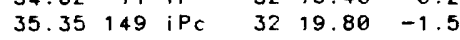

$1.5 \mathrm{~s} \quad 433.00 \mathrm{~nm}$

$35.36 \quad 71 \mathrm{Pc} \quad 3221.20 \quad-0.2$

$\begin{array}{llllll}35.60 & 73 & \mathrm{iPc} & 32 & 23.00 & -0.5\end{array}$

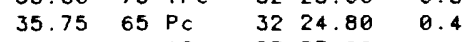

$\begin{array}{llllll}36.23 & 20 \mathrm{PC} & 37 & 57.00 & \\ 32.00 & -0.2\end{array}$

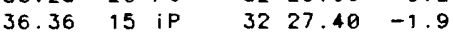

$1.0 \mathrm{~s} 1220.00 \mathrm{~nm} \quad 6.7 \mathrm{mb}$

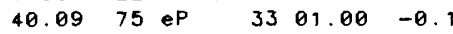

$\begin{array}{llllll}41.65 & 353 \mathrm{iPc} & 33 & 13.20 & 0.1\end{array}$

$0.9 \mathrm{~s} 244.00 \mathrm{~nm}$

3500.00

3902.00

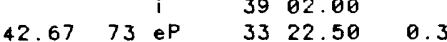

$\begin{array}{lllll}42.91 & 43 \mathrm{PC} & 33 & 21.80 & -1.9\end{array}$

$\begin{array}{llllll}42.96 & 69 & \mathrm{PC} & 39 & 51.00 & \\ 43 & 25.50 & 1.1\end{array}$

eS 3940.50

$\begin{array}{lllll}43.01 & 84 \mathrm{ePC} & 33 & 26.00 & 0.9\end{array}$

$43.40119 \mathrm{eP} \quad 3330.20 \quad 1.6$

40.08

is 4008.00

$\begin{array}{llllll}44.34 & 335 & \mathrm{iPC} & 33 & 50.00 & 14.8 x\end{array}$

$1.0 \mathrm{~s} \quad 100.00 \mathrm{~nm}$

$\begin{array}{lll}34 & 51.00296 \mathrm{kmx}\end{array}$

iPP 3511.00

iPCP 3520.00 


\begin{tabular}{|c|c|c|c|c|c|c|c|c|c|c|c|}
\hline & & & 1 & 39 & 04.00 & & 1 & & & e & 36 \\
\hline & & & is & 39 & 40.00 & & 1 & & & e (S) & 44 \\
\hline ARO & 44.69 & 113 & iPc & 33 & 39.70 & 1.0 & 1 & & & e & 44 \\
\hline & & & i & 33 & 43.80 & $14 \mathrm{~km}$ & 1 & & & e & 47 \\
\hline & & & e & 35 & 26.00 & & I CLE & 62.42 & 303 & $i P_{C}$ & 35 \\
\hline & & & escs & 43 & 46.00 & & i NDI & 62.94 & 73 & $\mathrm{iPC}$ & 35 \\
\hline & & & escs & 43 & 53.30 & & i & $0.9 \mathrm{~s}$ & 143 & $3.00 \mathrm{~nm}$ & \\
\hline MAIO & 46.22 & 72 & $\mathrm{PCt}$ & 33 & 49.00 & -1.7 & i & $20 s$ & & $0.80 \mathrm{um}$ & \\
\hline & & & es & 40 & 41.60 & & $i$ & & & is & 44 \\
\hline $\mathrm{KHI}$ & 46.25 & 75 & $i P_{C}$ & 33 & 50.20 & -0.9 & I BLA & 63.51 & 298 & eP & 35 \\
\hline TGI & 47.09 & 76 & eP & 33 & 56.80 & -1.0 & i & & & es & 44 \\
\hline KHE & 49.22 & 10 & $\mathrm{Pc}$ & 34 & 15.00 & 1.5 & I WMO & 63.66 & 54 & $\mathrm{PC}$ & 35 \\
\hline & & & es & 41 & 12.00 & & i & & & i PP & 38 \\
\hline NAI & 49.76 & 131 & iPc & 34 & 21.00 & 2.4 & I CIR & 63.68 & 148 & $\mathrm{P}+$ & 35 \\
\hline & $1.0 \mathrm{~s}$ & 130 & $0.00 \mathrm{~nm}$ & & & $5.9 \mathrm{mb}$ & 1 & & & iPn & 36 \\
\hline SAM & 50.81 & 65 & $\mathrm{Pc}$ & 34 & 26.00 & -0.2 & I UTO & 63.73 & 304 & ePc & 36 \\
\hline CBM & 51.52 & 305 & $e^{P}$ & 34 & 31.00 & -0.4 & I BOM & 64.32 & 85 & P- & 36 \\
\hline EMM & 51.67 & 302 & eP & 34 & 31.80 & -0.7 & 1 & & & $P \subset P$ & 36 \\
\hline & $1.0 \mathrm{~s}$ & 340 & $9.00 \mathrm{~nm}$ & & & $6.2 \mathrm{mb}$ & i & & & PP & 38 \\
\hline TAS & 51.97 & 62 & $\mathrm{Pc}$ & 34 & 35.00 & 0.0 & i & & & PPP & 40 \\
\hline & & & is & 42 & 00.00 & & i & & & $S$ & 44 \\
\hline DSH & 52.48 & 66 & $\mathrm{Pc}$ & 34 & 38.90 & 0.0 & I AN3 & 64.38 & 303 & eP & 36 \\
\hline & & & is & 42 & 10.40 & & I AN 12 & 64.47 & 303 & eP & 36 \\
\hline$M I M$ & 52.58 & 303 & eP & 34 & 39.00 & -0.5 & I ANG & 64.77 & 303 & eP & 36 \\
\hline GAR & 53.47 & 65 & eP & 34 & 44.00 & -2.3 & I AN10 & 64.86 & 303 & eP & 36 \\
\hline & & & esP & 34 & 46.40 & & I AN 11 & 64.96 & 303 & eP & 36 \\
\hline & & & es & 42 & 13.60 & & I POO & 65.35 & 84 & $\mathrm{P}+$ & 36 \\
\hline KBL & 54.14 & 70 & $e P$ & 34 & 36.00 & $-15.3 x$ & 1 & & & $P C P$ & 36 \\
\hline OUE & 54.26 & 76 & iPc & 34 & 50.40 & -1.9 & i & & & PP & 38 \\
\hline & & & es & 42 & 35.10 & & i & & & PPP & 40 \\
\hline ANR & 54.35 & 62 & $\mathrm{PC}$ & 34 & 52.80 & 0.2 & i & & & $\$$ & 44 \\
\hline & & & is & 42 & 38.00 & & i & & & ScS & 46 \\
\hline WES & 55.04 & 300 & eP & 34 & 55.70 & -1.8 & i & & & SS & 49 \\
\hline & $0.8 \mathrm{~s}$ & 1480 & $0.00 \mathrm{~nm}$ & & & 7. $1 \mathrm{mb}$ & I SEH & 65.54 & 78 & $\mathrm{P}+$ & 35 \\
\hline FRU & 55.24 & 59 & eP & 34 & 58.00 & -1.1 & I KSR & 66.27 & 155 & iPd & 36 \\
\hline & & & ipp & 34 & 59.40 & $5 \mathrm{~km}$ & i & $0.7 \mathrm{~s}$ & & $6.00 \mathrm{~nm}$ & \\
\hline & & & is & 42 & 49.40 & & I TIK & 66.30 & 16 & $\mathrm{Pc}$ & 36 \\
\hline HNH & 55.27 & 302 & eP & 35 & 00.00 & 0.8 & i & & & esP & 36 \\
\hline NRI & 55.49 & 25 & $\mathrm{Pc}$ & 35 & 00.00 & -0.5 & i & & & epP & 36 \\
\hline & & & is & 42 & 48.00 & & I PRM & 66.35 & 296 & ePd & 36 \\
\hline HDM & 56.19 & 300 & eP & 34 & 53.00 & $-12.8 x$ & I CAR & 66.40 & 266 & eP & 36 \\
\hline SEM & 56.49 & 49 & $P C$ & 35 & 07.50 & -0.4 & I PRE & 66.62 & 154 & iPc & 36 \\
\hline NVS & 56.91 & 43 & $\mathrm{Pc}$ & 35 & 09.00 & -1.9 & i & $0.8 \mathrm{~s}$ & 128 & $3.00 \mathrm{~nm}$ & \\
\hline & & & is & 43 & 07.00 & & 1 & & & $\mathrm{~s}$ & 45 \\
\hline TLG & 56.99 & 58 & $\mathbf{P}$ & 35 & 11.00 & -0.7 & I TKL & 66.62 & 298 & iPc & 36 \\
\hline & & & is & 43 & 00.00 & & $i$ & & & e & 04 \\
\hline KSH & 57.40 & 62 & $\mathrm{Pc}$ & 35 & 15.70 & 1.0 & I ORT & 66.88 & 298 & eP & 36 \\
\hline & & & PP & 37 & 18.70 & & $1 \mathrm{CPO}$ & 67.93 & 299 & $i P$ & 36 \\
\hline PRZ & 57.97 & 58 & eP & 35 & 19.00 & 0.3 & 1 & $2.9 \mathrm{~s}$ & 585 & $5.00 \mathrm{~nm}$ & \\
\hline & & & es & 43 & 21.40 & & $i$ & & & es & 44 \\
\hline ELT & 59.10 & 44 & $\mathrm{PC}$ & 35 & 23.70 & -2.5 & I CNG & 68.62 & 150 & eP & 36 \\
\hline KRI & 59.12 & 148 & $\mathrm{P}+$ & 35 & 25.00 & -1.9 & i & $1.2 \mathrm{~s}$ & 149 & $9.00 \mathrm{~nm}$ & \\
\hline MTD & 59.94 & 146 & $P+$ & 35 & 32.00 & -0.5 & $i$ & & & e & 36 \\
\hline & & & $i P_{n}$ & 36 & 21.00 & & 1 & & & $e(P \subset P)$ & 36 \\
\hline & & & iPP & 37 & 46.00 & & 1 & & & $e(P P)$ & 39 \\
\hline TET & 60.31 & 144 & eP & 35 & 33.00 & -1.9 & VAR & 69.11 & 73 & $P-$ & 36 \\
\hline & & & $e(P c$ & 36 & 23.00 & & 1 & & & $\mathrm{PCP}$ & 36 \\
\hline & & & $e(P P)$ & 37 & 46.00 & & 1 & & & PP & 38 \\
\hline & & & e & 40 & 26.00 & & $i$ & & & PPP & 40 \\
\hline & & & e & 42 & 35.00 & & 1 & & & PPS & 46 \\
\hline & & & $e(S)$ & 43 & 47.00 & & I TOV & 69.12 & 267 & eP & 36 \\
\hline CLK & 60.59 & 142 & $P+$ & 35 & 37.00 & 0.1 & I YKA & 69.12 & 333 & $\mathrm{P}$ & 36 \\
\hline & & & iPn & 36 & 26.00 & & I IRK & 69.33 & 40 & $\mathrm{Pc}$ & 36 \\
\hline SOB 4 & 60.66 & 230 & $\mathbf{P}$ & 35 & 37.90 & 0.5 & 1 & & & es & 45 \\
\hline & & & e & 35 & 40.80 & $10 \mathrm{~km}$ & I HYB & 69.72 & 83 & ePct & 36 \\
\hline & & & $e$ & 35 & 41.80 & & $i$ & & & $\mathrm{PCP}$ & 37 \\
\hline & & & e & 35 & 46.70 & & i & & & $\mathrm{pP}$ & 39 \\
\hline & & & e & 35 & 50.20 & & i & & & $\mathrm{S}$ & 45 \\
\hline & & & ePCP & 36 & 20.20 & & 1 & & & $P \cdot P$. & 05 \\
\hline SOB 3 & 60.85 & 230 & $e(P)$ & 35 & 43.30 & $4.6 x$ & I ZAK & 69.81 & 42 & $\mathrm{Pc}$ & 36 \\
\hline BHK & 61.07 & 71 & $\mathbf{P}$ & 35 & 43.00 & $3.0 x$ & I FVM & 70.11 & 303 & $e P$ & 36 \\
\hline & & & $p P$ & 35 & 51.20 & $27 \mathrm{kmx}$ & i & $20 \mathrm{~s}$ & 98 & $8.60 \mathrm{um}$ & \\
\hline & & & $\$$ & 44 & 03.00 & & I AVY & 70.18 & 133 & iPd & 36 \\
\hline SJG & 61.61 & 272 & iP & 35 & 44.00 & 0.1 & SDV & 70.32 & 267 & $e P$ & 36 \\
\hline & & & $i p P$ & 35 & 49.00 & $16 \mathrm{~km}$ & $i$ & $0.8 \mathrm{~s}$ & 60 & $0.00 \mathrm{~nm}$ & \\
\hline & & & is & 44 & 12.00 & & I UAV & 70.89 & 267 & $e P$ & 36 \\
\hline & & & iLO & 51 & 02.00 & & 1 & $0.4 \mathrm{~s}$ & 130 & $0.00 \mathrm{~nm}$ & \\
\hline BUL & 61.68 & 151 & $P_{+}$ & 35 & 42.00 & -2.4 & GBA. & 70.90 & 87 & $P d$ & 36 \\
\hline & & & $i P_{n}$ & 36 & 32.00 & & $i$ & $2.3 \mathrm{~s}$ & 1060 & $0.00 \mathrm{~nm}$ & \\
\hline TRN & 61.88 & 262 & eP & 35 & 47.00 & 1.3 & | CER & 71.21 & 164 & iPd & 36 \\
\hline & $1.0 \mathrm{~s}$ & 340 & $0.00 \mathrm{~nm}$ & & & $6.5 \mathrm{mb}$ & i & $1.2 \mathrm{~s}$ & 78 & 8. $10 \mathrm{~nm}$ & \\
\hline AJM & 61.88 & 77 & $P+$ & 35 & 47.00 & 1.4 & 1 & & & (S) & 46 \\
\hline & & & $\mathrm{PP}$ & 35 & 56.00 & $29 \mathrm{kmX}$ & I BOK & 72.03 & 73 & $P$ & 36 \\
\hline & & & $\mathrm{s}$ & 44 & 08.00 & & 1 & & & PP & 37 \\
\hline MRG & 62.12 & 300 & iPc & 35 & 47.40 & 0.4 & 1 & & & PCP & 37 \\
\hline & & & ePP & 38 & 01.00 & & i & & & PP & 39 \\
\hline$P A$ & & 138 & PC & 35 & 49.00 & 0 & RD & 7 & 223 & $P_{c}$ & 36 \\
\hline
\end{tabular}


$100 \quad 12 h$

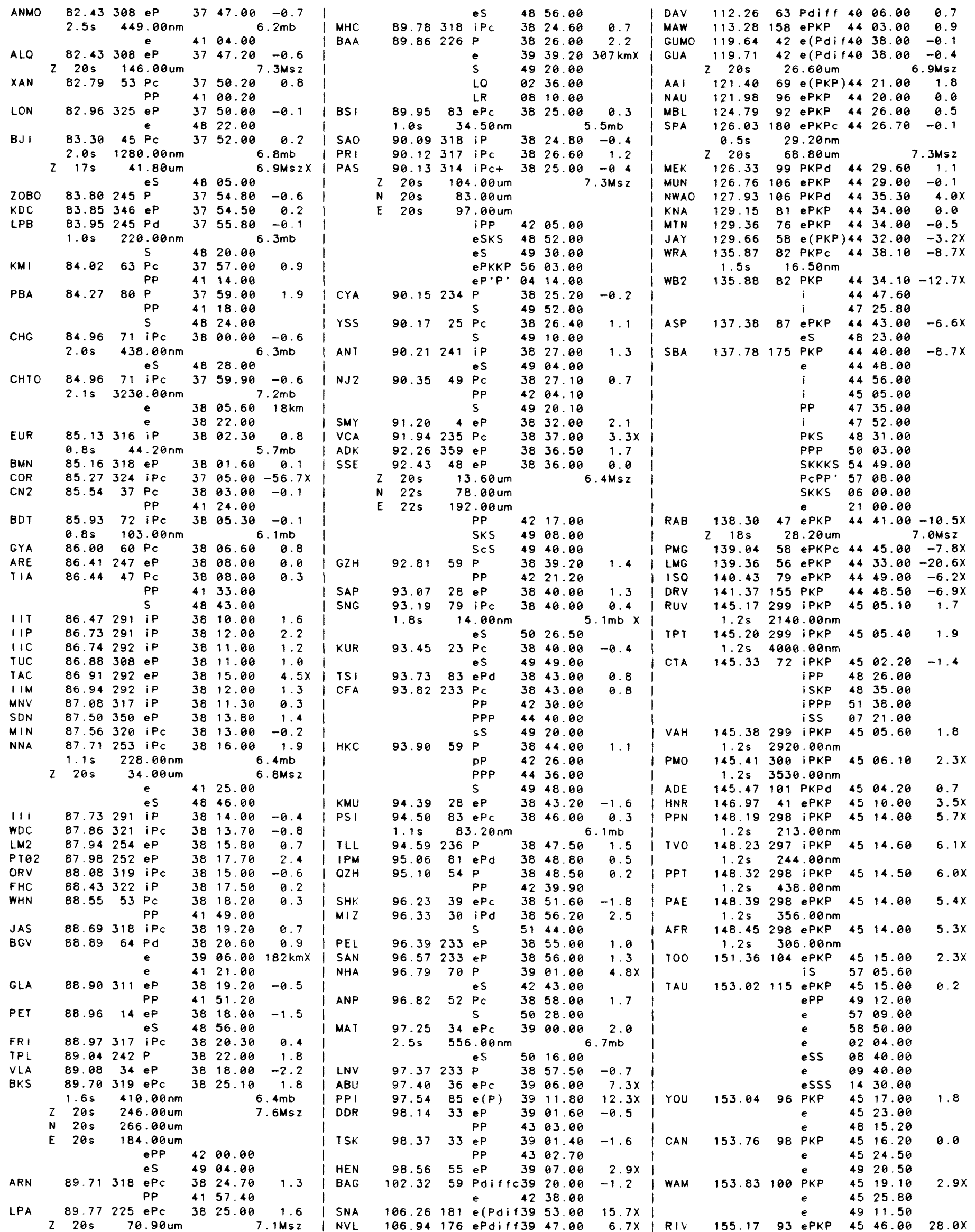




\begin{tabular}{|c|c|c|c|c|c|c|c|c|c|c|c|}
\hline AFI & & 156.96 & 343 & PKP & 45 & 23.00 & 2.0 & 1 & & & es \\
\hline & & & & PP & 49 & 24.00 & & I TUC & 18.04 & 323 & iPd \\
\hline & & & & PPP & 53 & 00.00 & & I RLO & 18.09 & 8 & iPd \\
\hline & & & & PKKP & 53 & 25.00 & & I WLA & 18.15 & 20 & eP \\
\hline & & & & e & 56 & 32.00 & & I ALO & 18.20 & 338 & iPd \\
\hline & & & & SKSP & 59 & 48.00 & & $\mathrm{MPH}$ & 18.36 & 22 & eP \\
\hline & & & & e & 02 & 59.00 & & MET & 18.37 & 22 & iPc \\
\hline & & & & SS & 09 & 16.00 & & $\mid$ GA & 18.40 & 49 & P \\
\hline & & & & SSP & 10 & 00.00 & & EBZ & 18.59 & 23 & eP \\
\hline & & & & SSS & 15 & 08.00 & & POW & 18.90 & 18 & ep \\
\hline & & & & LO & 29 & 00.00 & & I GRT & 19.57 & 22 & ¿P \\
\hline & & & & LR & 38 & 00.00 & & LST & 19.70 & 21 & ep \\
\hline RAR & & 57.79 & 307 & iPKP & 45 & 29.50 & $7.7 x$ & I MSA & 19.80 & 341 & iPd \\
\hline & & & & e & 46 & 05.00 & & I BBJ & 19.87 & 86 & eP \\
\hline & & & & PP & 49 & 39.00 & & I DON & 20.23 & 19 & ePd \\
\hline & & & & SSP & 10 & 28.00 & & STH & 20.32 & 87 & eP \\
\hline$P \vee C$ & & 58.26 & 36 & PKPC & 45 & 58.50 & $36.1 x$ & I HOJ & 20.38 & 87 & eP \\
\hline ou & & 58.38 & 48 & PKPC & 45 & 22.70 & 0.2 & I GWJ & 20.39 & 87 & eP \\
\hline NOU & & 61.01 & 47 & PKPC & 45 & 25.80 & B. 6 & I ELC & 20.56 & 21 & eP \\
\hline NUE & & 61.30 & 334 & ePKP & 46 & 17.00 & $51.4 \times$ & FVM & 20.83 & 17 & ip \\
\hline MSZ & & 66.72 & 134 & ePKP & 45 & 31.00 & 1.2 & I & $20 \mathrm{~s}$ & 135 & $.86 \mathrm{um}$ \\
\hline & & & & e & 45 & 35.00 & & I GLA & 21.00 & 318 & iP \\
\hline & & & & PP & 51 & 31.00 & & I PRM & 21.20 & 39 & iPd \\
\hline RHP & & 68.31 & 136 & ePKP & 45 & 32.00 & 0. 9 & I TYS & 2129 & 17 & eP \\
\hline & & & & e & 46 & 44.00 & & I ORT & 21.48 & 32 & eP \\
\hline & & & & PP & 50 & 33.00 & & I TKL & 21.56 & 33 & iPd \\
\hline WEL & & 72.76 & 136 & iPKP & 45 & 34.50 & 1.3 & 1 & & & ipp \\
\hline & & & & e & 46 & 54.00 & & 1 & & & es \\
\hline & & & & e & 47 & 03.00 & & I HBF & 21.69 & 44 & eP \\
\hline & & & & PKS & 49 & 38.00 & & I JSC & 22.60 & 40 & eP \\
\hline & & & & PP & 50 & 52.00 & & I GCA & 22.09 & 330 & iPd \\
\hline & & & & PPP & 55 & 19.00 & & I GOL & 22.28 & 345 & ip \\
\hline & & & & SKKS & 57 & 40.00 & & I & $20 s$ & 60 & $.80 \mathrm{um}$ \\
\hline & & & & SKKS & 00 & 10.00 & & I GLD & 22.30 & 346 & P \\
\hline & & & & SKSP & 01 & 15.00 & & 1 & $20 \mathrm{~s}$ & 110 & $.80 \mathrm{um}$ \\
\hline MNG & & 73.61 & 135 & iPKP & 45 & 35.40 & 1.7 & I LHS & 22.41 & 40 & iPC \\
\hline & & & & e & 47 & 03.00 & & I GA & 23.38 & 105 & ip \\
\hline & & & & PP & 51 & 03.00 & & I SD & 23.43 & 318 & iPd \\
\hline & & & & PCPP. & 54 & 08.00 & & I SCI & 23.45 & 313 & ip \\
\hline KRP & & 75.01 & 112 & ePKP & 45 & 40.00 & $5.9 x$ & I MSU & 3.61 & 332 & eP \\
\hline & & & & e & 47 & 11.00 & & I PAS & 23.86 & 316 & $P$ \\
\hline & & & & PP & 51 & 05.00 & & 1 & & & $P P$ \\
\hline & & & & e & 51 & 39.00 & & I & & & $P \subset P$ \\
\hline & & & & PCPP. & 54 & 23.00 & & 1 & & & $\mathrm{~s}$ \\
\hline GNZ & & 76.38 & 133 & PKP & 45 & 37.00 & $2.6 x$ & 1 & & & $S C P$ \\
\hline & & & & e & 47 & 12.00 & & I PRN & 24.16 & 326 & IPD \\
\hline & & & & PP & 51 & 11.00 & & I PUV & 24.17 & 36 & eP \\
\hline & S. & D & 2 & on 431 & 0 of & $514 \mathrm{ob}$ & os. & I PWV & 24.27 & 35 & iPc \\
\hline & & & & & & & & I NAV & 24.39 & 35 & iPd \\
\hline OC & & 24 , & 1980 & $14 \mathrm{~h}$ & $53 \mathrm{~m}$ & $34.65 \pm$ & $0.28 \mathrm{~s}$ & I BLA & 24.52 & 36 & ePd \\
\hline & .22 & $25 \mathrm{~N}$ & \pm 1.6 & $5 \mathrm{~km}$ & 98.1 & $195 w \pm$ & $1.5 \mathrm{~km}$ & 1 & $0.9 \mathrm{~s}$ & & 9. $64 \mathrm{~nm}$ \\
\hline & PTH & $H=\epsilon$ & 66.6 & \pm 2. & $4 \mathrm{~km}$ & & & BAV & 24.53 & 36 & eP \\
\hline & $5 \mathrm{mt}$ & b $(70$ & 8 obs & S. ) & & & & GLR & 24.54 & 324 & IPA \\
\hline CENT & RAL & L MEX & 100 & & & & $(523)$ & I HWV & 24.57 & 35 & iPd \\
\hline & & 1 leos & st $3 e$ & 90 peor & $p / e$ & reporte & & I AN8 & 25.01 & 26 & eP \\
\hline & & illed. & , mar & hy inj & ured & & & I AN11 & 25.12 & 25 & eP \\
\hline & & ipproxi & imate & $=1 \times 15$ & 0.00 & 90 homel & less & I AN10 & 5.13 & 25 & eP \\
\hline & & ind $\mathrm{ex}$ & tensi & ive don & noge & $=(1 x) i$ & in & I AN 4 & 25.15 & 26 & eP \\
\hline & & entral & I Mex & kico, $n$ & noin & ily in $t$ & the & I DUG & 25.27 & 333 & iPd \\
\hline & & luajuop & pon 0 & Je Leor & n or & ea. Fel & & I AN9 & 25.32 & 25 & eP \\
\hline & & hrough & hout & centro & al a & ind sout & hern & I AN3 & 25.46 & 26 & eP \\
\hline & & lexico & and & in Guc & a tem & nalo. & & I BV3 & 25.62 & 35 & eP \\
\hline & & & & & & & & $\mathrm{ICH}$ & 25.70 & 118 & ip \\
\hline $11 T$ & & 0.80 & 352 & iP & 53 & 52.70 & 1.7 & FRV & 25.71 & 39 & iP \\
\hline 111 & & 1.22 & 277 & iP & 53 & 54.80 & -1.5 & I TNP & 25.84 & 324 & iPd \\
\hline $11 P$ & & 1.31 & 329 & iP & 53 & 59.50 & 1.9 & I PBV & 6.06 & 40 & iPc \\
\hline $11 \mathrm{M}$ & & 1.44 & 320 & iP & 54 & 00.00 & 0.6 & $1 \mathrm{CVL}$ & 6.17 & 37 & eP \\
\hline MEX & & 1.44 & 320 & iP & 54 & 00.90 & 1.5 & I EUR & 6.19 & 328 & iP \\
\hline TAC & & 1.51 & 321 & iP & 54 & 02.00 & 1.7 & I GHV & 26.25 & 38 & eP \\
\hline CRX & & 1.83 & 310 & eP & 54 & 06.20 & 1.4 & I BDW & 26.31 & 341 & i Pd \\
\hline & & & & ePP & 54 & 23.70 & & I PHGM & 26.41 & 316 & eP \\
\hline $11 C$ & & 1.83 & 327 & iP & 54 & 06.10 & 1.3 & $|\mathrm{BE}|$ & 26.48 & 337 & eP \\
\hline COM & & 6.12 & 108 & iP & 55 & 00.00 & $-4 \cdot 8 x$ & I บTO & 26.49 & 25 & IPd \\
\hline$J C T$ & & 12.29 & 353 & iP & 56 & 29.50 & 0.7 & $1 \mathrm{ACM}$ & 26.51 & 21 & eP \\
\hline & 2 & $20 \mathrm{~s}$ & 60 & . 38 um & & & & I NA12 & 26.51 & 38 & eP \\
\hline MTX & & 14.91 & 351 & eP & 57 & 04.20 & 1.0 & I MRC & 26.54 & 33 & iP \\
\hline EPT & & 15.44 & 332 & $P$ & 57 & 10.00 & 0.0 & I PSO & 26.58 & 127 & eP \\
\hline SRA & & 15.59 & 119 & $P+$ & 57 & 12.70 & 0.7 & | FRI & 26.59 & 319 & iPd \\
\hline LUB & & 15.64 & 349 & eP & 57 & 13.00 & 0.4 & 1 & & & SCP \\
\hline SJS & & 15.99 & 119 & P- & 57 & 18.10 & 1.0 & 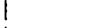 & & & e \\
\hline LCR & & 16.14 & 120 & $\mathrm{P}+$ & 57 & 18.40 & -0.6 & I MNV & 26.61 & 323 & ePd \\
\hline SVM & & 17.10 & 330 & iPc & 57 & 32.60 & 1.6 & I NAII & 26.63 & 38 & ep \\
\hline$\$ 10$ & & 17.53 & 5 & iPd & 57 & 36.80 & 0.7 & $10 A O$ & 26.66 & 131 & iPd- \\
\hline & & & & i & 57 & 52.10 & & I NA2 & 26.69 & 38 & ip \\
\hline TUL & & 17.74 & 6 & iPd- & 57 & 38.80 & 0.1 & PRI & 26.69 & 316 & iPd \\
\hline & Z & $20 \mathrm{~s}$ & 276 & .00 & & & & $\mid$ & & & PP \\
\hline & & & & $\mathbf{i}$ & 57 & 40.80 & & & & & $P \subset P$ \\
\hline
\end{tabular}




\begin{tabular}{|c|c|c|c|c|c|c|c|c|c|c|c|}
\hline & $1.6 \mathrm{~s}$ & 19 & $3.00 \mathrm{~nm}$ & & & $.7 \mathrm{mb}$ & 1 & & & $P P$ & 06 \\
\hline FCC & 40.59 & 3 & ePd & 01 & 07.70 & -1.4 & 1 & & & PCS & 09 \\
\hline ARE & 43.35 & 141 & eP & 01 & 28.00 & $-4 \cdot 5 x$ & 1 & & & $s$ & 12 \\
\hline & $1.5 \mathrm{~s}$ & 778 & $8.00 \mathrm{~nm}$ & & & $.3 \mathrm{mb}$ & i & & & scs & 13 \\
\hline $\mathrm{SCH}$ & 43.70 & 26 & ePd & 01 & 32.90 & -1.7 & 1 & & & SS & 16 \\
\hline 2080 & 45.29 & 137 & $P$ & 01 & 45.20 & $-3.3 x$ & 1 & & & LO & 20 \\
\hline LPB & 45.51 & 138 & $P d$ & 01 & 49.00 & -0.9 & 1 & & & LR & 23 \\
\hline & & & $\mathrm{s}$ & 08 & 20.00 & & I LPA & 65.23 & 144 & iPd & 04 \\
\hline$Y K C$ & 45.64 & 350 & iPd & 01 & 48.10 & -1.9 & i & $20 s$ & 109 & 9.00 um & \\
\hline & $0.6 \mathrm{~s}$ & 350 & $0.00 \mathrm{~nm}$ & & & $.4 \mathrm{mb}$ & 1 & & & $i p P$ & 04 \\
\hline YKA & 45.68 & 349 & $\mathbf{P}$ & 01 & 50.20 & -0.1 & 1 & & & is & 12 \\
\hline STJ & 47.14 & 41 & $e^{P}$ & 02 & 01.90 & -0.1 & I PDA & 65.44 & 56 & $P$ & 04 \\
\hline & $1.1 \mathrm{~s}$ & $15 \varepsilon$ & $8.00 \mathrm{~nm}$ & & & $.9 \mathrm{mb}$ & I ALE & 65.82 & 5 & iPd & 04 \\
\hline SIT & 47.59 & 333 & $e(P)$ & 02 & 07.00 & 1.6 & 1 & $1.0 \mathrm{~s}$ & 355 & $5.00 \mathrm{~nm}$ & \\
\hline TPL & 48.54 & 144 & $P$ & 82 & 21.00 & $7.9 x$ & I RDJ & 67.49 & 125 & $P d$ & 84 \\
\hline ANT & 49.72 & 146 & iP & 02 & 21.00 & -1.2 & I & & & iPP & 06 \\
\hline FRB & 49.77 & 17 & ePd & 02 & 21.00 & -1.1 & I & & & IPPP & 98 \\
\hline HON & 50.55 & 334 & iP & 02 & 28.90 & 0.8 & I & & & is & 13 \\
\hline I NK & 54.75 & 345 & eP & 02 & 59.00 & -0.3 & 1 & & & isss & 18 \\
\hline & $0.6 \mathrm{~s}$ & 385 & $5.80 \mathrm{~nm}$ & & 6 & $.6 \mathrm{mb}$ & I REY & 67.78 & 27 & eP & 84 \\
\hline$T L L$ & 54.87 & 151 & Pd & 03 & 03.20 & 2.3 & 1 & & & $\mathrm{i}$ & 04 \\
\hline VCA & 54.96 & 148 & $\mathbf{P}$ & 03 & 01.80 & 0.3 & I ADK & 68.81 & 319 & eP & 04 \\
\hline KDC & 55.96 & 328 & eP & 83 & 06.90 & -1.2 & I AKU & 69.47 & 26 & iPd- & 04 \\
\hline PMR & 55.98 & 333 & eP & 03 & 07.50 & -0.7 & 1 & $1.5 \mathrm{~s}$ & 7500 & $9.00 \mathrm{~nm}$ & \\
\hline & $24 \mathrm{~s}$ & 12 & $2.00 \mathrm{um}$ & & 5 & $.9 M s z X$ & I ILT & 69.69 & 337 & Pd & 04 \\
\hline CYA & 55.99 & 145 & $P d$ & 03 & 07.30 & -1.4 & 1 & & & esp & 05 \\
\hline$K \mid P$ & 56.08 & 284 & eP & 03 & 09.50 & 0.0 & 1 & & & is & 13 \\
\hline & & & es & 11 & 02.00 & & I DAG & 69.96 & 14 & iPd & 04 \\
\hline HON & 56.09 & 284 & $\mathrm{P}$ & 03 & 12.00 & 2.4 & 1 & $0.7 \mathrm{~s}$ & & $3.00 \mathrm{~nm}$ & \\
\hline RES & 56.51 & 1 & eP & 03 & 05.00 & $-6.8 x$ & 1 & & & $\mathrm{i}$ & 07 \\
\hline & $1.2 \mathrm{~s}$ & 528 & $0.00 \mathrm{~nm}$ & & & $.5 \mathrm{mb}$ & 1 & & & $\mathrm{i}$ & 09 \\
\hline $\mathrm{COL}$ & 57.05 & 337 & iPd & 03 & 14.50 & -1.4 & I RAR & 71.92 & 240 & eP & 04 \\
\hline FBA & 57.05 & 337 & eP & 03 & 13.90 & -2.0 & I & & & e & 05 \\
\hline CFA & 57.28 & 150 & $\mathrm{PC}$ & 03 & 16.00 & -1.9 & 1 & & & $P \subset P$ & 05 \\
\hline & & & $\mathrm{s}$ & 11 & 12.00 & & 1 & & & $\mathrm{~s}$ & 14 \\
\hline PEL & 57.45 & 153 & $\mathrm{Pd}$ & 03 & 17.10 & -1.9 & 1 & & & PS & 15 \\
\hline LNV & 57.83 & 154 & eP & 03 & 19.00 & -2.6 & 1 & & & $\mathrm{i}$ & 15 \\
\hline GDH & 57.90 & 17 & IPd- & 03 & 20.00 & -1.7 & I & & & e & 18 \\
\hline & $1.0 \mathrm{~s}$ & 280 & $0.00 \mathrm{~nm}$ & & 6 & $.3 \mathrm{mb}$ & I & & & LR & 26 \\
\hline & & & $\mathrm{i}$ & 05 & 56.00 & & I SMY & 74.34 & 321 & eP & 05 \\
\hline & & & is & 11 & 15.00 & & I VAL & 74.62 & 40 & iPd- & 05 \\
\hline MDZ & 58.05 & 151 & ip & 03 & 21.30 & -2.0 & 1 & & & PP & 08 \\
\hline & & & $i(S)$ & 03 & 39.40 & & 1 & & & PPP & 09 \\
\hline RUV & 58.75 & 239 & iP & 03 & 26.20 & -2.1 & 1 & & & $\mathrm{~S}$ & 14 \\
\hline & $1.0 \mathrm{~s}$ & 1088 & $0.00 \mathrm{~nm}$ & & 6 & $.9 \mathrm{mb}$ & 1 & & & $\operatorname{ses}$ & 15 \\
\hline TPT & 58.83 & 239 & ip & 03 & 27.20 & -1.7 & 1 & & & SS & 19 \\
\hline & $1.0 \mathrm{~s}$ & 1080 & $9.00 \mathrm{~nm}$ & & 6 & $9 \mathrm{mb}$ & I & & & LO & 23 \\
\hline TCA & 58.90 & 146 & Pd & 03 & 27.10 & -2.2 & 1 & & & LR & 27 \\
\hline VAH & 58.97 & 239 & iP & 03 & 28.00 & -1.9 & I TEN & 74.66 & 65 & $P$ & 05 \\
\hline & $1.0 \mathrm{~s}$ & 864 & $4.00 \mathrm{~nm}$ & & 6 & $.8 \mathrm{mb}$ & 1 & & & $S$ & 15 \\
\hline PMO & 59.07 & 239 & ip & 03 & 29.00 & -1.5 & $1 \mathrm{KBS}$ & 76.01 & 11 & ¿Pd & 05 \\
\hline & $1.0 \mathrm{~s}$ & 1080 & $.00 \mathrm{~nm}$ & & 6 & $.9 \mathrm{mb}$ & I DCN & 76.13 & 38 & IPd & 05 \\
\hline MBC & 59.07 & 354 & iPd & 03 & 27.90 & -1.9 & 1 & $1.7 \mathrm{~s}$ & 3350 & $.00 \mathrm{~nm}$ & \\
\hline & $1.0 \mathrm{~s}$ & 594 & $4.00 \mathrm{~nm}$ & & 6 & $.7 \mathrm{mb}$ & I DMU & 76.23 & 38 & IPd & 05 \\
\hline TTA & 59.47 & 333 & ePd & 03 & 31.00 & -1.9 & 1 & $1.3 \mathrm{~s}$ & 2948 & $9.00 \mathrm{~nm}$ & \\
\hline SDN & 59.50 & 324 & ep & 03 & 32.30 & -0.7 & I DLE & 76.58 & 38 & iPd & 05 \\
\hline I MA & 59.77 & 337 & eP & 03 & 32.50 & -2.4 & 1 & $1.7 \mathrm{~s}$ & 2340 & $3.00 \mathrm{~nm}$ & \\
\hline BAO & 59.81 & 121 & $e^{P}$ & 03 & 34.90 & -0.9 & I DDK & 76.67 & 38 & iPd & 05 \\
\hline TMU & 61.53 & 157 & $P$ & 03 & 45.00 & -2.0 & 1 & $1.7 \mathrm{~s}$ & 3128 & $.00 \mathrm{~nm}$ & \\
\hline TVO & 61.65 & 237 & ip & 03 & 46.30 & -1.9 & DKM & 76.75 & 38 & eP & 05 \\
\hline & $0.8 \mathrm{~s}$ & 189 & $.00 \mathrm{~nm}$ & & & $3 \mathrm{mb}$ & 1 & $1.7 \mathrm{~s}$ & 3120 & $.00 \mathrm{~nm}$ & \\
\hline PPN & 61.66 & 238 & iP & 83 & 47.66 & -0.6 & I ECP & 76.92 & 39 & iPd & 85 \\
\hline & $0.8 \mathrm{~s}$ & 194 & $+.00 \mathrm{~nm}$ & & 6 & $.3 \mathrm{mb}$ & 1 & $1.5 \mathrm{~s}$ & 3480 & $.00 \mathrm{~nm}$ & \\
\hline PPT & 61.79 & 238 & iP & 03 & 49.10 & -0.1 & I STS & 77.58 & 49 & iPd & 05 \\
\hline & $0.8 \mathrm{~s}$ & 135 & $5.00 \mathrm{~nm}$ & & 6 & $.1 \mathrm{mb}$ & I РTO & 77.95 & 51 & Pd & 05 \\
\hline PAE & 61.85 & 238 & iP & 03 & 49.80 & 0.3 & 1 & & & iPP & 08 \\
\hline & $0.8 \mathrm{~s}$ & 140 & $.00 \mathrm{~nm}$ & & & $.1 \mathrm{mb}$ & 1 & & & iPPP & 10 \\
\hline AFR & 61.94 & 238 & iP & 83 & 51.40 & 1.3 & 1 & & & is & 15 \\
\hline & $0.8 \mathrm{~s}$ & 200 & $.00 \mathrm{~nm}$ & & & $.3 \mathrm{mb}$ & I & & & isS & 20 \\
\hline SOBA & 62.31 & 111 & $P$ & 03 & 49.00 & $-3.7 x$ & 1 & & & isSS & 23 \\
\hline & & & e & 03 & 54.90 & & I EKA & 78.03 & 36 & Pd & 05 \\
\hline & & & e & 04 & 28.00 & & 1 & $1.3 \mathrm{~s}$ & 875 & $5.00 \mathrm{~nm}$ & \\
\hline SOB & 62.56 & 111 & $P$ & 03 & 54.10 & -0.2 & 1 LIS & 78.11 & 53 & $P d$ & 05 \\
\hline & & & e & 04 & 01.60 & & 1 & & & ipP & 05 \\
\hline & & & e & 84 & 09.20 & & 1 & & & $P P$ & 09 \\
\hline & & & epP & 04 & 14.00 & $77 \mathrm{kmx}$ & 1 & & & S & 15 \\
\hline & & & esP & 04 & 21.90 & & I AF I & 79.25 & 251 & iP & 05 \\
\hline & & & e & 04 & 28.10 & & 1 & & & PP & 08 \\
\hline so83 & 62.69 & 112 & $P$ & 03 & 52.70 & -2.5 & 1 & & & $S$ & 15 \\
\hline & & & e & 03 & 55.60 & & 1 & & & SS & 20 \\
\hline & & & e & 03 & 57.90 & & 1 & & & SSS & 24 \\
\hline & & & e & 04 & 02.80 & & 1 & & & LO & 26 \\
\hline & & & e & 04 & 08.50 & & 1 & & & LR & 29 \\
\hline ANM & 63.92 & 334 & eP & 04 & 02.00 & -0.6 & I KHE & 80.57 & 4 & Pd & 05 \\
\hline NK I & 64.03 & 321 & eP & 04 & 01.40 & -2.0 & 1 & & & $i s P$ & 06 \\
\hline BAA & 64.69 & 144 & $P$ & 84 & 04.80 & $-3.0 x$ & 1 & & & es & 15 \\
\hline & & & $P C P$ & 84 & 18.80 & & I BER & 80.83 & 29 & iP & 05 \\
\hline
\end{tabular}




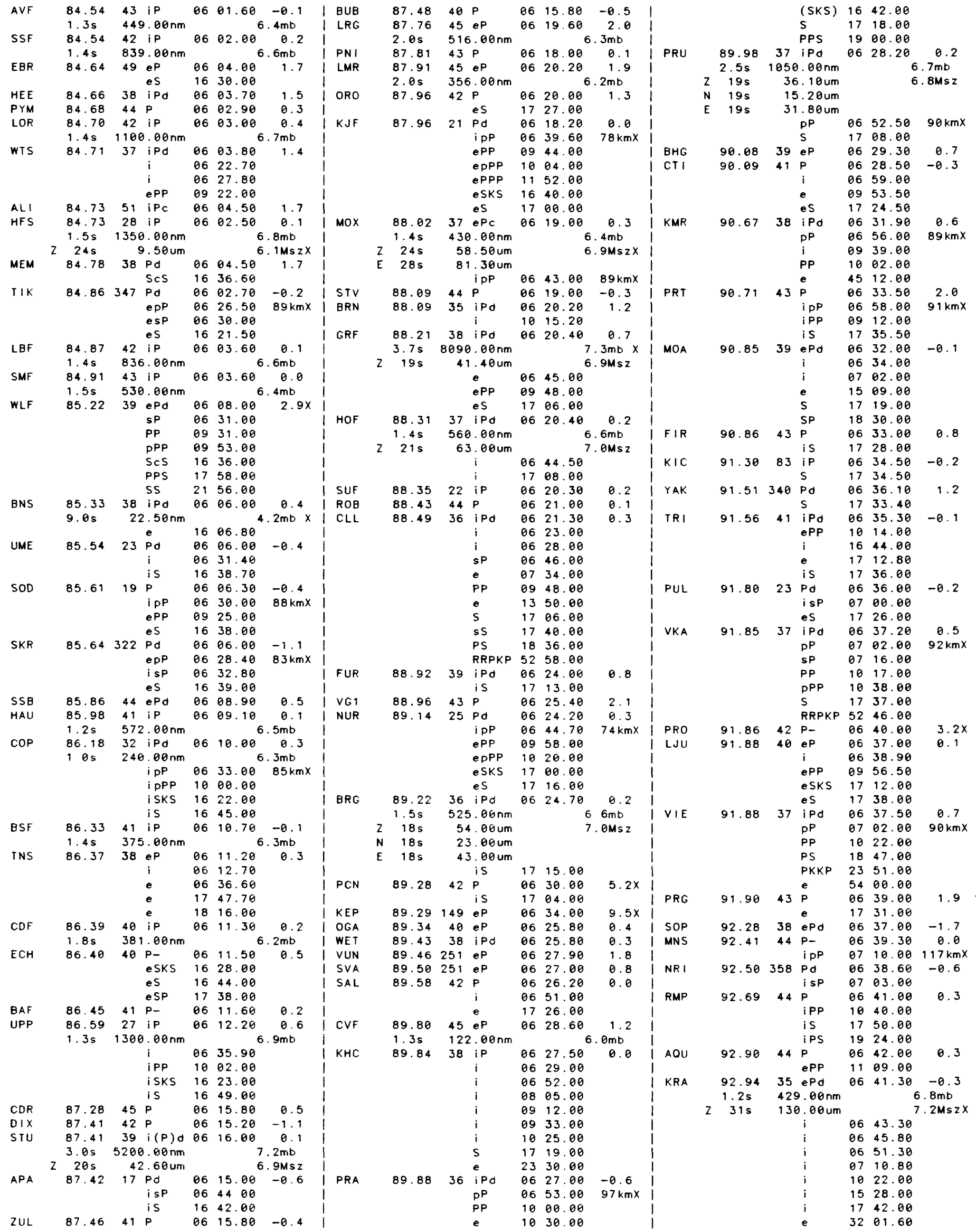




\begin{tabular}{|c|c|c|c|c|c|c|c|c|c|c|c|}
\hline & & & e & 32 & 07.30 & & I BUC & 99.61 & 37 & $e^{P}$ & 07 \\
\hline KUR & 93.01 & 320 & Pd & 06 & 42.00 & 0.0 & I WEL & 99.63 & 230 & $P$ & 07 \\
\hline & & & isP & 07 & 06.00 & & 1 & & & PP & 07 \\
\hline & & & es & 17 & 48.00 & & $i$ & & & e & 08 \\
\hline SRO & 93.23 & 37 & iP & 06 & 43.60 & 0.6 & 1 & & & PP & 11 \\
\hline & $26 s$ & 43 & $3.36 \mathrm{um}$ & & & & i & & & SKS & 17 \\
\hline & $26 s$ & & $3.30 \mathrm{um}$ & & & & 1 & & & ScS & 18 \\
\hline & & & $i$ & 07 & 11.70 & & 1 & & & SP & 20 \\
\hline & & & $i$ & 07 & 11.70 & & i & & & PPS & 20 \\
\hline & & & i (PP) & 10 & 37.00 & & 1 & & & PKKS & 25 \\
\hline & & & e & 17 & 21.00 & & I PVL & 99.83 & 38 & $\mathrm{Pd}$ & 87 \\
\hline & & & $i(s)$ & 17 & 46.80 & & $\mid M I Z$ & 100.47 & 317 & ePdif & 107 \\
\hline NIE & 93.49 & 35 & IPd & 06 & 46.30 & 2.1 & 1 & & & (SKS) & 10 \\
\hline$S P C$ & 93.58 & 35 & eP & 06 & 46.30 & 1.4 & I DIM & 100.73 & 39 & ePdif & 107 \\
\hline & $18 \mathrm{~s}$ & 124 & $4.00 \mathrm{um}$ & & & $.4 M S Z$ & $\mathrm{KDZ}$ & 100.83 & 39 & Pdifto & d07 \\
\hline & & & i & 06 & 48.80 & & I ATH & 101.92 & 43 & iPdif & 607 \\
\hline & & & $i(p P)$ & 07 & 09.70 & $86 \mathrm{kmX}$ & 1 & & & is & 11 \\
\hline & & & ePP & 10 & 33.70 & & 1 & & & iPp & 11 \\
\hline BUD & 93.81 & 37 & ePd & 06 & 47.00 & 1.3 & I EZN & 102.50 & 41 & Pdiff & 07 \\
\hline & & & epP & 07 & 10.00 & $84 \mathrm{kmX}$ & I EDC & 103.13 & 39 & Pdiff & 07 \\
\hline DUI & 93.92 & 44 & $\mathbf{P}$ & 06 & 45.00 & -1.3 & I ARU & 103.14 & 13 & Pdiffo & $d \theta 7$ \\
\hline & & & ePP & 10 & 14.50 & & I SVE & 103.17 & 12 & Pdiffo & $d \theta 7$ \\
\hline SGG & 94.00 & 44 & $P$ & 06 & 47.00 & 0.2 & I IST & 103.37 & 38 & Pdiff- & -07 \\
\hline Jos & 94.12 & 36 & $e^{P}$ & 06 & 48.00 & 0.9 & 1 & & & $\mathrm{~s}$ & 18 \\
\hline & $1.8 \mathrm{~s}$ & 448 & $0.00 \mathrm{~nm}$ & & & $.6 \mathrm{mb}$ & I ISK & 103.39 & 38 & Pdiff & 87 \\
\hline BLY & 4.14 & 40 & iP & 06 & 49.00 & 1.7 & I MAT & 103.84 & 316 & Pdift & +07 \\
\hline Ovo & 4.28 & 45 & $\mathrm{P}$ & 06 & 49.00 & 0.9 & $Z M$ & 103.94 & 41 & Pdiff & 07 \\
\hline UZH & 95.01 & 35 & Pd & 06 & 51.00 & -0.2 & INR & 104.08 & 265 & ePdit & 607 \\
\hline & & & es & 17 & 57.00 & & I ALT & 105.29 & 39 & Pdift & 07 \\
\hline YSS & 95.13 & 323 & $\mathrm{Pc}$ & 06 & 52.00 & 0.2 & I ANTO & 106.34 & 37 & ePdit & 107 \\
\hline & & & epP & 87 & 14.00 & $80 \mathrm{kmX}$ & 1 & $3.0 \mathrm{~s}$ & 256 & $6.00 \mathrm{~nm}$ & \\
\hline & & & esp & 07 & 18.00 & & I CN2 & 186.48 & 329 & Pditf & 07 \\
\hline & & & is & 17 & 54.00 & & 1 & & & e & 11 \\
\hline LVV & 95.25 & 33 & Pd & 86 & 52.70 & 0.4 & I & & & $P P$ & 12 \\
\hline & & & is & 18 & 00.00 & & I BCK & 106.59 & 40 & Pdiff & 07 \\
\hline GIB & 95.36 & 47 & $\mathbf{P}$ & 86 & 53.00 & -0.2 & I KVT & 167.33 & 35 & Pdiff & 07 \\
\hline & & & i & 10 & 24.00 & & I SPA & 108.11 & 180 & iPdift & 108 \\
\hline KUK & 5.47 & 82 & $\mathrm{P}$ & 86 & 54.00 & 0.1 & 1 & $0.5 \mathrm{~s}$ & 33 & $3.50 \mathrm{~nm}$ & \\
\hline CEI & 5.64 & 36 & $e P$ & 86 & 50.00 & $-4 \cdot 1 x$ & 1 & $20 s$ & 38 & 3. 20 um & \\
\hline TIM & 96.03 & 38 & eP & 86 & 58.00 & 2.1 & iHK & 108.65 & 317 & ePdiff & 607 \\
\hline BEO & 96.08 & 39 & $P$ & 06 & 56.60 & 0.4 & I SBA & 108.76 & 193 & Pditt & 68 \\
\hline & & & iPP & 10 & 38.20 & & 1 & & & e & 09 \\
\hline & & & eSKS & 17 & 37.00 & & 1 & & & e & 11 \\
\hline & & & $e$ & 18 & 05.00 & & 1 & & & PP & 12 \\
\hline BRT & 96.11 & 44 & $P+$ & 06 & 53.50 & -2.9 & 1 & & & SKS & 18 \\
\hline BMR & 96.22 & 35 & eP & 07 & 00.00 & $3.2 x$ & 1 & & & $e$ & 20 \\
\hline SSR & 96.82 & 38 & eP & 07 & 00.00 & 0.4 & 1 & & & PS & 21 \\
\hline DEV & 96.92 & 37 & eP & 07 & 00.00 & 0.0 & 1 & & & SP & 22 \\
\hline GZR & 97.13 & 38 & eP & 07 & 02.00 & 1.0 & 1 & & & SS & 27 \\
\hline MOS & 97.42 & 23 & $\mathrm{Pd}$ & 07 & 02.00 & 0.0 & 1 & & & e & 45 \\
\hline OBN & 97.45 & 24 & Pd & 07 & 02.00 & -0.1 & UER & 109.68 & 352 & ePditf & 607 \\
\hline TIR & 17.59 & 42 & $P$ & 07 & 05.20 & 2.1 & RAB & 109.94 & 272 & ePdiff & $f 08$ \\
\hline KMU & 97.68 & 319 & ep & 07 & 03.20 & 0.1 & 1 & $z 20 s$ & 35 & $5.50 \mathrm{um}$ & \\
\hline KRP & 98.13 & 233 & eP & 07 & 06.00 & 0.7 & GUA & 110.23 & 292 & $e(P d i f$ & $f 08$ \\
\hline & & & e & 07 & 24.00 & & UMO & 110.25 & 292 & e (Pdi) & 108 \\
\hline & & & $P P$ & 11 & 04.00 & & I GRO & 110.33 & 27 & ePdiff & 608 \\
\hline & & & PKKP & 23 & 34.00 & & I BKR & 110.62 & 30 & Pdiffo & $d 08$ \\
\hline & & & $e$ & 24 & 08.00 & & I LEN & 111.53 & 30 & ePdiff & 608 \\
\hline & & & e & 24 & 34.00 & & I SEM & 111.66 & 1 & ePdiff & $f \theta 8$ \\
\hline & & & PCPP. & 27 & 04.00 & & | HLW & 111.82 & 46 & iPdiff & $f 08$ \\
\hline & & & e & 27 & 26.00 & & I & $22 \mathrm{~s}$ & & $7.00 \mathrm{um}$ & \\
\hline SAP & 98.15 & 320 & $\in P$ & 07 & 05.00 & -0.5 & 1 & & & is & 13 \\
\hline SKO & 98.24 & 41 & ifd & 07 & 06.00 & 0.0 & I ERE & 112.30 & 30 & Pdiff & 08 \\
\hline & & & $\mathrm{i}$ & 07 & 08.30 & & I KRV & 112.78 & 29 & ePdif & 108 \\
\hline & & & i & 07 & 31.00 & & I NVL & 113.65 & 160 & ePdif & $t 68$ \\
\hline & & & iPP & 11 & 05.00 & & I GRS & 113.70 & 29 & Pdiffo & $d \theta 8$ \\
\hline & & & $i$ & 11 & 30.00 & & 1 BNG & 113.74 & 77 & iPdift & 108 \\
\hline & & & iPPP & 13 & 04.00 & & 1 & $1.2 \mathrm{~s}$ & 22 & 2. $10 \mathrm{~nm}$ & \\
\hline & & & $i$ & 15 & 07.00 & & | BJ I & 11377 & 332 & ePdift & $t 68$ \\
\hline & & & ISKKS & 18 & 01.00 & & 1 & $23 s$ & 50 & $0.00 \mathrm{um}$ & \\
\hline & & & $i(P S)$ & 20 & 27.00 & & I & $23 \mathrm{~s}$ & 37 & $7.00 \mathrm{um}$ & \\
\hline OHR & 98.31 & 42 & iP & 07 & 06.10 & -0.3 & 1 & & & EPP & 13 \\
\hline & & & $i$ & 07 & 31.50 & & I EIL & 114.44 & 44 & Pdiff & 08 \\
\hline CMP & 8.50 & 37 & ePC & 07 & 08.00 & 0.9 & I TAB & 114.84 & 30 & ePdiff & 608 \\
\hline IAS & 98.71 & 34 & eP & 67 & 09.00 & 1.0 & 1 & & & e & 12 \\
\hline$B A C$ & 8.74 & 35 & eP & 07 & 10.00 & 1.9 & 1 & & & e & 12 \\
\hline$A L R$ & 8.87 & 36 & eP & 07 & 10.00 & 1.0 & 1 & & & $i$ & 13 \\
\hline ING & 8.93 & 230 & eP & 07 & 12.00 & $3.0 x$ & ВTO & 115.86 & 336 & Pdiff & 68 \\
\hline SOF & 9.00 & 40 & $P d$ & 07 & 07.00 & -2.5 & 1 & & & $P P$ & 13 \\
\hline VRI & 9.08 & 35 & eP & 07 & 10.00 & 0.3 & I TIA & 116.38 & 329 & Pdiff & 08 \\
\hline VAY & 99.31 & 41 & eP & 07 & 11.00 & 0.2 & 1 & & & PP & 13 \\
\hline & & & $i$ & 07 & 36.00 & & I BHD & 117.56 & 35 & ePKP & 12 \\
\hline DDB & 99.32 & 35 & $e^{P}$ & 07 & 13.00 & 2.2 & I พMO & 118.03 & 355 & Pdiff & 08 \\
\hline$B \mid R$ & 99.32 & 35 & $e P$ & 07 & 08.00 & -2.8 & 1 & & & PP & 13 \\
\hline KIS & 99.51 & 34 & $P d$ & 87 & 11.00 & -0.6 & 1 & & & SKKS & 20 \\
\hline & & & isP & 07 & 35.00 & & I SSE & 118.06 & 322 & Pdiff & 88 \\
\hline 800 & 99.59 & 43 & Pd & 07 & 11.30 & -0.5 & 1 & & & e & 12 \\
\hline
\end{tabular}




\begin{tabular}{|c|c|c|c|c|c|c|c|c|c|c|c|}
\hline & & & e & 14 & 30.00 & & 1 & $1.0 \mathrm{~s}$ & 115 & $5.00 \mathrm{~nm}$ & \\
\hline GZH & 128.66 & 322 & PKP & 12 & 34.90 & -1.6 & i & & & es & 260 \\
\hline & & & PP & 14 & 41.40 & & I BDT & 140.90 & 333 & IPKPA & 12 \\
\hline & & & PKS & 15 & 58.90 & & i & $1.3 \mathrm{~s}$ & 716 & $5.00 \mathrm{~nm}$ & \\
\hline $\mathrm{HKC}$ & 128.77 & 321 & PKP & 12 & 36.00 & -0.7 & I MKS & 141.02 & 285 & ePKPd & 130 \\
\hline KSR & 128.84 & 109 & iPKPC & 12 & 37.00 & -0.1 & I KLG & 142.01 & 243 & ePKP & 12 \\
\hline MAW & 129.17 & 171 & ePKP & 12 & 35.00 & -1.1 & I $\mathrm{BOM}$ & 142.07 & 14 & PKP & 12 \\
\hline MAN & 129.34 & 308 & ePKP & 12 & 39.60 & 1.6 & I POO & 142.64 & 12 & PKP+ & 125 \\
\hline & & & es & 14 & 45.00 & & I MBL & 144.13 & 259 & PKPC & 13 \\
\hline QCP & 129.36 & 308 & PKP & 13 & 28.10 & $50.1 x$ & I VIS & 144.25 & 358 & PKP & 13 \\
\hline GYA & 129.45 & 331 & PKP & 12 & 35.60 & $-2.5 x$ & I HYB & 144.44 & 5 & PKP & 130 \\
\hline & & & $P P$ & 14 & 49.60 & & 1 & & & $p P$ & 16 \\
\hline & & & PKS & 15 & 59.60 & & 1 & & & SKS & 193 \\
\hline AAE & 129.62 & 61 & ePdift & $f 09$ & 30.00 & $3.1 x$ & I NWAO & 145.04 & 238 & PKPd & 130 \\
\hline QUE & 129.79 & 17 & PKP & 12 & 26.60 & $-12.2 x$ & MEK & 145.12 & 249 & ePKP & 130 \\
\hline BUL & 129.90 & 101 & ePKPC & 12 & 38.00 & -1.2 & I GOA & 145.59 & 14 & PKP & 130 \\
\hline & $20 \mathrm{~s}$ & 36 & 5.90 um & & & 7. $1 \mathrm{MsZ}$ & i & & & PP & 161 \\
\hline & $20 \mathrm{~s}$ & 16 & .30 um & & & & I MUN & 146.13 & 239 & ePKP & 130 \\
\hline & E $20 \mathrm{~s}$ & 19 & 3. 10 um & & & & I SWV & 146.27 & 240 & ePKP & 130 \\
\hline PRE & 130.00 & 109 & ePKP & 12 & 38.20 & -1.0 & I AVY & 147.72 & 97 & IPKPd & 131 \\
\hline & $2.3 \mathrm{~s}$ & 309 & $7.00 \mathrm{~nm}$ & & & & I GBA & 148.08 & 8 & PKP & 131 \\
\hline & & & $\mathrm{i}$ & 24 & 54.20 & & I NAU & 148.17 & 256 & PKPO & 131 \\
\hline DAV & 130.02 & 296 & PKPd & 12 & 38.00 & -1.4 & I TRT & 148.24 & 285 & IPKPC & 131 \\
\hline & & & es & 14 & 56.00 & & 1 & $1.2 \mathrm{~s}$ & 556 & $5.00 \mathrm{~nm}$ & \\
\hline KRI & 130.35 & 97 & ePKP & 12 & 38.00 & -2.1 & $1 \mathrm{PBA}$ & 148.41 & 339 & PKP & 131 \\
\hline BHK & 130.37 & 6 & PKP & 12 & 42.40 & $2.8 x$ & 1 & & & PP & 17 \\
\hline 102 & 130.48 & 259 & PKP & 12 & 21.20 & $-18.9 x$ & 1 & & & SKKS & 235 \\
\hline & & & $i$ & 12 & 39.30 & & I SNG & 148.67 & 322 & ePKP & 131 \\
\hline & & & $i$ & 12 & 59.50 & & i & $1.9 \mathrm{~s}$ & 61 & $.00 \mathrm{~nm}$ & \\
\hline & & & $\mathrm{i}$ & 15 & 12.70 & & I & & & es & 276 \\
\hline ASP & 131.26 & 254 & ePKP & 12 & 24.00 & $-17.6 x$ & I MDR & 148.93 & 3 & PKP & 131 \\
\hline & & & es & 16 & 04.00 & & I IPM & 150.46 & 318 & ePKPd & 131 \\
\hline ARO & 131.50 & 55 & ¡PKPO & 12 & 43.50 & 1.3 & 1 & $1.7 \mathrm{~s}$ & 360 & $.00 \mathrm{~nm}$ & \\
\hline & & & e & 14 & 52.10 & & I KGM & 150.79 & 311 & ePKPC & 131 \\
\hline & & & $i$ & 15 & 02.00 & & I & $1.6 \mathrm{~s}$ & 455 & $5.00 \mathrm{~nm}$ & \\
\hline & & & $\mathbf{i}$ & 16 & 36.50 & & I KOD & 151.46 & 9 & ePKP & 131 \\
\hline KKR & 131.85 & 6 & PKP & 12 & 44.00 & 1.6 & 1 & & & e & 133 \\
\hline MTN & 132.29 & 269 & ePKP & 12 & 44.00 & 0.4 & 1 & & & e & 133 \\
\hline KMI & 132.33 & 334 & PKP & 12 & 32.00 & $-11.8 x$ & I & & & PKS & 165 \\
\hline & & & e & 12 & 43.00 & & 1 & & & ePP & 170 \\
\hline & & & PKP & 12 & 46.00 & & 1 & & & SKS & 202 \\
\hline & & & PPKP & 13 & 02.00 & & 1 & & & PPP & 263 \\
\hline & & & SPKP & 13 & 09.00 & & i & & & SKSP & 271 \\
\hline & & & PP & 15 & 00.00 & & 1 & & & LO & 045 \\
\hline & & & PKS & 16 & 12.00 & & I LEM & 152.42 & 291 & IPKPC & 131 \\
\hline AA I & 132.38 & 282 & $e(P K P)$ & 12 & 31.50 & $-12.4 x$ & i & $2.0 \mathrm{~s}$ & 588 & $3.00 \mathrm{~nm}$ & \\
\hline NAI & 132.75 & 74 & ePKP & 12 & 47.00 & 2.1 & | TSI & 152.82 & 321 & ePKPo & 132 \\
\hline & $2.0 \mathrm{~s}$ & 588 & $.00 \mathrm{~nm}$ & & & & I TRD & 153.03 & 11 & PKP & 131 \\
\hline$C \mid R$ & 132.76 & 102 & PKP+ & 12 & 46.00 & 1.6 & | PS I & 153.23 & 319 & ePKPd & 131 \\
\hline NDI & 133.16 & 6 & ePKP & 12 & 45.00 & 0.0 & 1 & $1.5 \mathrm{~s}$ & 800 & $.60 \mathrm{~nm}$ & \\
\hline & $20 \mathrm{~s}$ & 21 & 36 um & & & $6.9 \mathrm{Msz}$ & $|\mathbf{P P}|$ & 154.59 & 312 & ePKP & 132 \\
\hline & $20 \mathrm{~s}$ & 23 & $.46 \mathrm{um}$ & & & & I & $1.2 \mathrm{~s}$ & 190 & $.68 \mathrm{~nm}$ & \\
\hline & $20 s$ & 17 & $.70 \mathrm{um}$ & & & & 1 & $S . D=1$ & 1.1 & on 457 & of \\
\hline & & & ePP & 15 & 12.00 & & i & & & & \\
\hline $\mathrm{N} 1$ & 133.37 & 290 & ePKP & 12 & 46.50 & 0.7 & 18 & 08. & 1980 & $10 \mathrm{~h} 2$ & $7 \mathrm{~m} \mathrm{3}^{3}$ \\
\hline TOC & 133.56 & 344 & PKP & 12 & 56.00 & $10.3 x$ & 41. & $.088 \mathrm{~N}$ & & 12 & 4.64 \\
\hline CNG & 133.61 & 109 & ePKP & 12 & 44.00 & -1.9 & DEP & PTH $=$ & $12.8 \mathrm{kr}$ & $\mathrm{km}$ (ge & ophy \\
\hline & $2.0 \mathrm{~s}$ & 199 & $.00 \mathrm{~nm}$ & & & & 6.2 & $2 \mathrm{mb}(52$ & 2 obs & 3) 7. & $3 \mathrm{Msz}$ \\
\hline & & & e & 15 & 17.00 & & NEAR & COAST & OF NOF & DRTHERN & CAL \\
\hline & & & e & 15 & 56.00 & & $i$ & $\langle G S\rangle$ & Sixp & seople & inju \\
\hline & & & e & 16 & 12.00 & & 1 & domage & (V) & ) in $t$ & he $F$ \\
\hline & & & e & 17 & 34.00 & & i & Londing & gore & o. One & per \\
\hline & & & e & 17 & 46.00 & & 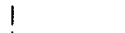 & at Fort & tuna. & Sligh & t do \\
\hline & & & e & 24 & 40.00 & & 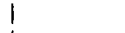 & ot many & y comr & muniti & eso \\
\hline TET & 133.98 & 95 & ePKP & 12 & 50.00 & $3.2 x$ & 1 & coost & from & Rio De & 11. \\
\hline & & & e & 13 & 12.00 & & 1 & to Broo & oking & s. Ores & gon. \\
\hline & & & e & 15 & 39.00 & & 1 & domage & est ir & moted & ot 1 \\
\hline PR & 134.15 & 304 & ePKP & 12 & 50.00 & $2.8 x$ & $i$ & dotlars & s. $S m$ & notl lor & nos I \\
\hline KNA & 135.11 & 266 & ePKP & 12 & 38.00 & $10.9 x$ & 1 & I ique fo & octior & on occu & ired \\
\hline A JM & 135.18 & 9 & PKP & 12 & 49.00 & 0.2 & 1 & Eel Riv & ver ar & no the & Big \\
\hline & & & PP & 15 & 24.00 & & $i$ & sond sp & pit. & Fett in & $n$ on \\
\hline CLK & 135.23 & 94 & PKP+ & 12 & 50.00 & 0.7 & 1 & approx & imote & ly 97 & 000 \\
\hline SHL & 135.38 & 347 & PKP & 12 & 35.00 & $-14.5 x$ & 1 & from no & or the & rn ore & gon \\
\hline VAR & 136.72 & 358 & PKP & 12 & 52.00 & 0.2 & 1 & Francis & sco B & 3oy ore & 0,0 \\
\hline & & & $P P$ & 15 & 34.00 & & 1 & in par & ts of & wester & $\ln N$ \\
\hline AGT & 137.15 & 347 & PKP & 12 & 54.00 & 1.4 & i & & & & \\
\hline & & & $P P$ & 15 & 39.00 & & $\mathrm{FHC}$ & 0.57 & 120 & iPc & 27 \\
\hline BOK & 138.02 & 355 & PKP & 12 & 54.90 & 0.6 & KBRM & 0.63 & 124 & iPc & 27 \\
\hline & & & PKS & 16 & 23.90 & & KCTM & .65 & 159 & iPd & 27 \\
\hline UPT & 138.77 & 275 & ePKP & 12 & 57.50 & 1.6 & WKC2 & 0.75 & 159 & iPd & 27 \\
\hline CAL & 138.99 & 351 & PKP & 13 & 07.00 & $11.0 x$ & KMPM & 0.78 & 149 & iPd & 27 \\
\hline & & & $P$ & 16 & 37.00 & & KGMM & .80 & 114 & iPc & 27 \\
\hline A & 139.18 & 92 & ePKP & 12 & 49.00 & $-7.6 x$ & KCRM & 0.91 & 136 & iPc & 27 \\
\hline & & & e & 13 & 01 & & KBBM & 1.08 & 146 & iPc & 27 \\
\hline & & & e & 15 & 53.00 & & KPPM & 1.22 & 127 & iPc & 27 \\
\hline n & 139.43 & 316 & PKP & 12 & 9.00 & 2.8 & KKPM & 1.37 & 133 & iPc & 27 \\
\hline $\mathrm{HG}$ & 139.52 & 335 & ePKP & 12 & 48.50 & $-8.7 x$ & I KBSM & 1.42 & 145 & iPc & 275 \\
\hline
\end{tabular}




\begin{tabular}{|c|c|c|c|}
\hline AARM & 3.31 & 122 & $i P_{C}$ \\
\hline AVRM & 3.31 & 127 & $i P C$ \\
\hline$M I X$ & 3.33 & 142 & ePC \\
\hline NPRM & 3.33 & 157 & ePd \\
\hline SNT & 3.36 & 149 & iPd \\
\hline ALNM & 3.36 & 129 & ePC \\
\hline NOLM & 3.36 & 154 & ePd \\
\hline GVR & 3.37 & 145 & $i P d$ \\
\hline AFRM & 3.42 & 131 & ePC \\
\hline ARWM & 3.42 & 127 & iPC \\
\hline APRM & 3.44 & 129 & $i P c$ \\
\hline VSBM & & 44 & iPd \\
\hline VMHM & 3.47 & 28 & iPd \\
\hline ARRM & 3.54 & 130 & $i P_{C}$ \\
\hline NLHM & 3.54 & 146 & iPd \\
\hline AHRM & 3.54 & 128 & $i P C$ \\
\hline$A D C$ & 3.54 & 126 & $i P c$ \\
\hline NTPM & 3.55 & 152 & iPd \\
\hline AFHM & 3.59 & 123 & $i P c$ \\
\hline$A G I M$ & 3.60 & 127 & $i P c$ \\
\hline NF IM & 3.62 & 159 & ¿Pd \\
\hline $\operatorname{CDSM}$ & 3.62 & 149 & ¿Pd \\
\hline VPMM & 3.63 & 53 & iPc \\
\hline VWEM & 3.63 & 38 & IPd \\
\hline COR & 3.63 & 15 & ¿Pnd \\
\hline$A G C$ & 3.65 & 151 & iPd \\
\hline HMR & 3.66 & 142 & ePd \\
\hline DUC & 3.67 & 145 & $\in P d$ \\
\hline JPRM & 3.69 & 152 & ePd \\
\hline BKS & 3.71 & 149 & iPnd \\
\hline AR JM & 3.71 & 129 & $i P_{c}$ \\
\hline VGPM & 3.72 & 23 & $i P d$ \\
\hline BKC & 3.72 & 147 & $i P_{c}$ \\
\hline ALAM & 3.79 & 130 & ePd \\
\hline CRPM & 3.81 & 145 & ePd \\
\hline CMCM & 3.81 & 149 & ePd \\
\hline$B G C$ & 3.83 & 148 & iPd \\
\hline MGA & 3.83 & 153 & ePd \\
\hline AODM & 3.90 & 128 & iPC \\
\hline LKC & 3.90 & 148 & iPd \\
\hline SAC & 3.90 & 153 & ePd \\
\hline CRAM & 3.92 & 147 & iPd \\
\hline MTC & 3.94 & 145 & ePd \\
\hline ADWM & 3.94 & 131 & EPC \\
\hline JEGM & 3.95 & 154 & ePd \\
\hline CSH & 3.97 & 149 & $\in P d$ \\
\hline PCC & 3.99 & 153 & iPnd \\
\hline VSMM & 3.99 & 16 & $\mathrm{iPd}$ \\
\hline DOO & 4.00 & 146 & ePd \\
\hline CBSM & 4.02 & 144 & ePd \\
\hline PLC & 4.03 & 148 & iPd \\
\hline CPNM & 4.05 & 147 & ePd \\
\hline JHPM & 4.07 & 153 & ePd \\
\hline CVLM & 4.08 & 147 & IPd \\
\hline CSAM & 4.10 & 145 & ePd \\
\hline CTLM & 4.14 & 145 & iPd \\
\hline WCN & 4. 14 & 114 & ePn \\
\hline SFT & 4.15 & 152 & ePd \\
\hline
\end{tabular}

\begin{tabular}{|c|c|c|c|c|c|c|c|}
\hline & & & & & & & \\
\hline$B G H$ & 4.15 & 154 & ePd & 28 & 33.58 & -3.8 & MTR \\
\hline VFBM & 4.15 & 50 & iPd & 28 & 35.98 & -1.7 & FRP \\
\hline MS J & 4.16 & 148 & IPd & 28 & 35.36 & -2.3 & SAO \\
\hline MNHM & 4.17 & 134 & ePc & 28 & 34.70 & -3.0 & HJSM \\
\hline VBPM & $\begin{array}{ll}418 \\
\text {. }\end{array}$ & 30 & iPd & 28 & 35.77 & -2.3 & BVYM \\
\hline LTW & 4.18 & 152 & ePd & 28 & 34.60 & -3.3 & BSLM \\
\hline VHOM & 4.18 & 9 & ePd & 28 & 34.65 & -3.3 & BSRM \\
\hline MNR & 4.20 & 145 & IPd & 28 & 36.15 & -2.0 & \\
\hline COVM & 4.20 & 146 & $\in P d$ & 28 & 36.13 & -2.1 & VTHM \\
\hline JEMM & 4.23 & 152 & iPd & 28 & 35.06 & -3.7 & BCGM \\
\hline SJH & 4.24 & 151 & ePd & 28 & 35.90 & -2.9 & VTDM \\
\hline CVR & 4.25 & 148 & $i P d$ & 28 & 36.30 & -2.6 & BPCM \\
\hline & & & es & 29 & 28.67 & & BLRM \\
\hline LT3 & 4.26 & 153 & ePd & 28 & 35.09 & -3.9 & EKH \\
\hline MRFM & 4.26 & 130 & $\in P C$ & 28 & 37.37 & -1.8 & $\mathrm{BSCM}$ \\
\hline PSD & 4.27 & 155 & ePd & 28 & 35.75 & -3.5 & \\
\hline SEC & 4.27 & 152 & $e P d$ & 28 & 35.60 & -3.6 & BRMM \\
\hline VHHM & 4.29 & 13 & ePd & 28 & 36.50 & -2.9 & JHC \\
\hline CMLM & 4.29 & 146 & iPd & 28 & 37.37 & -2.2 & EMT \\
\hline VRBM & 4.30 & 34 & iPd & 28 & 37.44 & -2.2 & VGB \\
\hline JSMM & 4.32 & 153 & ePd & 28 & 36.01 & -3.9 & BVL \\
\hline MHR & 4.35 & 148 & $i P d$ & 28 & 37.62 & -2.7 & BHSM \\
\hline CMMM & 4.37 & 145 & $i P d$ & 28 & 38.78 & -2.0 & LLA \\
\hline $\cos M$ & 4.38 & 144 & IPd & 28 & 38.63 & -2.2 & $B P \mid M$ \\
\hline LXR & 4.40 & 151 & ePd & 28 & 37.21 & -3.8 & BPFM \\
\hline JBLM & 4.40 & 153 & ePd & 28 & 37.15 & -3.9 & BMSM \\
\hline & & & & 29 & 28.40 & & SBT \\
\hline & & 34 & & & 38.30 & 1 & SHK \\
\hline
\end{tabular}

$\begin{array}{lllll}28 & 23.43 & -2.2 & \text { I VTCM }\end{array}$ $28 \quad 23.83 \quad-3.1$ | SVC \begin{tabular}{lll|l}
28 & 23.05 & -2.1 & -2.8 \\
$28 H C$
\end{tabular} \begin{tabular}{lll|l}
28 & 23.43 & -2.8 & LTI 15
\end{tabular} \begin{tabular}{lll|l}
28 & 23.43 & -2.8 & $\mathrm{CRH}$
\end{tabular} \begin{tabular}{lll|l|l}
28 & 23.55 & -2.7 & SOS
\end{tabular} \begin{tabular}{lll|l}
28 & 24.30 & -2.1 & ARN
\end{tabular} \begin{tabular}{lll|l}
28 & 23.73 & -3.3 & \\
28 & 24.04 & -3.0 & JSTM
\end{tabular} \begin{tabular}{lll|l}
28 & 24.27 & -3.0 & COE
\end{tabular} \begin{tabular}{lll|l}
28 & 26.50 & -1.2 & I MOYM
\end{tabular} \begin{tabular}{lll|l}
28 & 25.80 & -2.0 & । AMC
\end{tabular} $\begin{array}{llll}28 & 25.51 & -3.2\end{array}$ $\begin{array}{lll}28 & 26.58 & -2.2\end{array}$ $\begin{array}{llll}28 & 26.11 & -2.7\end{array}$ $\begin{array}{llll}28 & 25.96 & -2.9\end{array}$ $\begin{array}{lll}28 & 25.30 & -3.6\end{array}$ $\begin{array}{lll}28 & 27.42 & -2.3\end{array}$ $\begin{array}{lll}28 & 26.85 & -2.8\end{array}$ \begin{tabular}{lll|l}
28 & 26.80 & -3.1 & JUCM
\end{tabular} \begin{tabular}{lll|l}
28 & 28.55 & -1.5 & VBEM
\end{tabular} \begin{tabular}{lll|l}
28 & 28.81 & -1.4 & MSTM
\end{tabular} \begin{tabular}{lll|l}
28 & 28.95 & -1.3 & ADR
\end{tabular} \begin{tabular}{lll|l}
28 & 27.00 & -3.1 & MMWM \\
28 & 27.54 & -2.8 & CBO
\end{tabular} \begin{tabular}{lll|l}
28 & 27.54 & -2.8 & $\mathrm{CBO}$
\end{tabular} \begin{tabular}{lll|l}
28 & 29.53 & -1.0 & | JTGM
\end{tabular} \begin{tabular}{lll|l}
28 & 29.46 & -1.3 & EUC \\
28 & 27.98 & -3.6 & JRRM
\end{tabular} \begin{tabular}{lll|l|l}
28 & 27.98 & -3.0 & JRRM \\
28 & 28.30 & -3.0 & JBZM
\end{tabular} \begin{tabular}{lll|l}
28 & 28.57 & -2.8 & PEV
\end{tabular} \begin{tabular}{lll|l}
28 & 29.25 & -2.2 & I HSPM
\end{tabular} \begin{tabular}{lll|l|l}
28 & 29.53 & -1.9 & VJYM \\
28 & 29.65 & -2.8 & HGWM
\end{tabular} \begin{tabular}{lll|l}
28 & 29.88 & -2.8 & GHS
\end{tabular} \begin{tabular}{lll|l}
28 & 30.11 & -2.6 & HCZM
\end{tabular} \begin{tabular}{lll|l}
28 & 30.72 & -2.3 & PKC
\end{tabular} \begin{tabular}{lll|l}
28 & 29.79 & -3.2 & I HCPM
\end{tabular} \begin{tabular}{lll|l}
28 & 31.86 & -2.1 & CDC \\
28 & 31.45 & -2.4 & MBFM
\end{tabular}

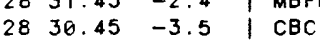
$\begin{array}{lllll}28 & 32.59 & -1.6 & \text { VHEM }\end{array}$ \begin{tabular}{lll|l|l}
28 & 32.43 & -2.1 & CSR \\
28 & 31.93 & -2.6 & HCOM
\end{tabular} $28 \quad 31.30-3.3$ PCL $\begin{array}{lll}28 & 32.60 & -2.3\end{array}$ \begin{tabular}{lll|l}
28 & 31.70 & -3.4 & SFL \\
28 & 33.90 & -1.3 & ANZ
\end{tabular} \begin{tabular}{lll|l}
28 & 33.90 & -1.3 & ANZ \\
28 & 33.41 & -1.9 & OCR
\end{tabular} \begin{tabular}{lll|l}
28 & 34.20 & -1.4 & VLMM
\end{tabular} \begin{tabular}{lll|l}
28 & 33.39 & -2.4 & $\mathrm{DII}$
\end{tabular} \begin{tabular}{lll|l}
28 & 34.66 & -1.4 & HSLM \\
28 & 32.38 & -3.9 & VMNM
\end{tabular} \begin{tabular}{llll|l}
28 & 32.38 & -3.9 & VMNM
\end{tabular} \begin{tabular}{lll|l|l}
28 & 35.39 & -1.3 & HBTM \\
28 & 35.42 & -1.9 & HJGM
\end{tabular} \begin{tabular}{lll|l}
28 & 35.50 & -2.0 & PKH
\end{tabular} \begin{tabular}{lll|l}
28 & 33.96 & -3.4 & HSFM
\end{tabular} 2925.23 ITR $\begin{array}{llll}28 & 35.98 & -1.7 & \text { IFRP }\end{array}$ \begin{tabular}{lll|l}
28 & 35.36 & -2.3 & SAO \\
28 & 34.70 & -3.0 & HJSM
\end{tabular} \begin{tabular}{lll|l}
28 & 35.77 & -2.3 & BVYM
\end{tabular} \begin{tabular}{lll|l}
28 & 34.65 & -3.3 & BSRM
\end{tabular} I VTHM $\checkmark T D M$ BLRM SCM JHC VGB
BVL

BHSM 8P IM
BPFM SBT
SHW \begin{tabular}{lll|l|l}
28 & 34.92 & -1.6 & HFHM \\
28 & 35.39 & -1.3 & HBTM
\end{tabular}

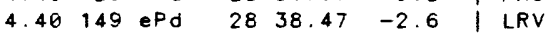
4.41147 iPnd $28 \quad 38.80 \quad-2.5$ L LHV $4.42 \quad 22$ iPd 4. 42152 ePd $4.43132 \mathrm{ePC}$ 4.44151 ePd

4. $45146 \mathrm{iPd}$

$4.46 \quad 149 \mathrm{ePd}$

$4.47148 \mathrm{ePd}$

$\begin{array}{lll}4.48 & 134 \mathrm{ePC} \\ 4.49 & 150 \mathrm{ePd}\end{array}$

$4.52 \quad 133 \mathrm{~S}$

$4.52 \quad 40 \mathrm{iPd}$

$4.53150 \mathrm{ePd}$

4.54144 iPd

4.55152 ifnd

4.55 152 iPd

$4.55153 \mathrm{ePO}$

$4.56 \quad 28 \mathrm{IPC}$

$4.57148 \mathrm{ePd}$

$4.58130 \mathrm{ePc}$

$4.59149 \mathrm{ePd}$

$4.59151 \mathrm{ePd}$

$4.59150 \mathrm{ePC}$

$4.62 \quad 150$ ePd

$4.63150 \mathrm{ePd}$

$4.65151 \mathrm{iPd}$

$4.65 \quad 147 \quad \mathrm{PC}$

$4.67 \quad 34$ iPd

$4.68 \quad 149 \mathrm{ePd}$

$4.70 \quad 147 \quad \mathrm{PO}$

$4.72 \quad 151 \mathrm{ePO}$

$4.72150 \mathrm{ePd}$

$4.73144 \mathrm{iPd}$

4.74148 iPd

$4.75134 \mathrm{ePC}$

$4.76 \quad 150$ ePd

$4.76 \quad 26 \quad \mathrm{PCC}$

$4.76 \quad 149$ ePd

$4.78 \quad 150$ ePd

$4.80146 \in P d$

$4.81147 \mathrm{ePd}$

$4.82 \quad 149 \mathrm{ePd}$

$4.83149 \mathrm{ePd}$

$4.84 \quad 22 \mathrm{iPd}$

$4.85150 \mathrm{ePO}$

$4.86 \quad 144 \mathrm{iPd}$

$4.87 \quad 31 \mathrm{iPd}$

4. $87148 \mathrm{ePd}$

$4.87149 \mathrm{ePO}$

$4.91150 \mathrm{ePd}$

$4.92 \quad 148 \in P d$

$4.92 \quad 149$ ePd

$4.94147 \mathrm{ePd}$

4. $95154 \mathrm{iPd}$

4. 98149 iPnd

$5.00147 \mathrm{EPd}$

5. 01149 iPd

5. $01148 \mathrm{iPd}$

$5.04 \quad 150 \mathrm{ePd}$

$5.07 \quad 35 \mathrm{iPd}$

$5.07 \quad 148 \mathrm{ePd}$

$5.07 \quad 27 \quad \mathrm{PAd}$

$5.09152 \mathrm{ePd}$

5. 14148 ePd

$5.18 \quad 147 \mathrm{ePd}$

5. $18148 \mathrm{ePC}$

5. 19144 i $\mathrm{Pd}$

$5.20150 \mathrm{iPd}$

$\begin{array}{lrl}5.21 & 147 & \mathrm{ePd} \\ 5.25 & 31 & \mathrm{iPd}\end{array}$

$\begin{array}{lrl}5.25 & 31 \mathrm{iPd} \\ 5.25 & 148 \mathrm{ePd}\end{array}$

$5.31 \quad 152 \mathrm{ePd}$

5.32 146 iPnd

$5.33148 \mathrm{ePd}$

$5.35154 \mathrm{ePd}$

$5.35145 \mathrm{ePd}$

$\begin{array}{lll}5.35 & 147 & \mathrm{iPd} \\ 5.39 & 18 \mathrm{eP}\end{array}$ $\begin{array}{lll}28 & 39.51 & -1.8\end{array}$

\begin{tabular}{lll|l}
28 & 37.57 & -3.8 & LMCR
\end{tabular}

\begin{tabular}{lll|l}
28 & 39.12 & -2.3 & $\mathrm{HCK}$
\end{tabular}

$\begin{array}{llllll}28 & 38.37 & -3.4 & \text { HVC }\end{array}$

\begin{tabular}{lll|l}
28 & 39.31 & -2.4 & BTW
\end{tabular}

\begin{tabular}{lll|l}
29 & 34.65 & & LRC \\
28 & 38.51 & -3.4 & $F R I$
\end{tabular}

\begin{tabular}{lll|l}
28 & 39.50 & -2.5 & MNV
\end{tabular}

\begin{tabular}{lll|l}
28 & 39.43 & -2.7 & BMN
\end{tabular}

\begin{tabular}{lll|l}
28 & 38.74 & -3.5 & PJLM
\end{tabular}

2932.82

\begin{tabular}{lll|l}
28 & 40.81 & -1.9 & CLKR
\end{tabular}

\begin{tabular}{lll|l}
28 & 41.18 & -1.7 & MOP
\end{tabular}

$\begin{array}{llll}28 & 39.52 & -3.5 & \text { PRCM }\end{array}$

$\begin{array}{lllll}28 & 41.17 & -1.8 & \text { I PAPM }\end{array}$

\begin{tabular}{lll|l}
28 & 39.60 & -3.6 & BONR
\end{tabular}

\begin{tabular}{lll|l}
28 & 39.44 & -3.7 & PRI
\end{tabular}

$\begin{array}{llll}28 & 41.54 & -1.8 & \text { I PTV }\end{array}$

$\begin{array}{llll}28 & 41.17 & -2.2 & \text { I PSAM }\end{array}$

\begin{tabular}{llll|l}
28 & 40.65 & -2.8 & I PARM \\
28 & 41.94 & -1.8 & I PSMM
\end{tabular}

\begin{tabular}{lll|l|l}
28 & 41.94 & -1.8 & PSMM \\
28 & 40.14 & -3.5 & PBYM
\end{tabular}

$\begin{array}{llll}28 & 40.23 & -3.5 & \text { | PCRM }\end{array}$

\begin{tabular}{lll|l}
28 & 40.44 & -3.4 & PIVM
\end{tabular}

\begin{tabular}{lll|l}
28 & 40.65 & -3.6 & I PHCM
\end{tabular}

\begin{tabular}{lll|l}
28 & 41.23 & -3.1 & PANM
\end{tabular}

\begin{tabular}{lll|l|l}
28 & 41.18 & -3.4 & TRC \\
28 & 41.91 & -2.8 & PHGM
\end{tabular}

$\begin{array}{llll}28 & 42.45 & -2.4\end{array}$

$\begin{array}{llll}28 & 41.42 & -3.7\end{array}$

$\begin{array}{llll}28 & 42.51 & -2.9\end{array}$

$\begin{array}{llll}28 & 42.15 & -3.5\end{array}$

$\begin{array}{llll}28 & 42.48 & -3.2\end{array}$

$28 \quad 44.10-1.6$

$\begin{array}{lll}28 & 42.94 & -3.6 \\ 28 & 43.52 & -2.5\end{array}$

$\begin{array}{lll}28 & 43.52 & -2.5 \\ 28 & 42.62 & -3.5\end{array}$

$\begin{array}{llll}28 & 44.44 & -1.9\end{array}$

$\begin{array}{llll}28 & 42.33 & -3.9\end{array}$

\begin{tabular}{lll|l}
28 & 42.84 & -3.6 & PMRM
\end{tabular}

$\begin{array}{llll}28 & 44.38 & -2.3 & \text { I PAGM }\end{array}$

\begin{tabular}{lll|l}
29 & 41.16 & & PSHM \\
28 & 43.96 & -3.0 & PCGM
\end{tabular}

$\begin{array}{llllll}28 & 43.28 & -3.8 & \text { | PTRM }\end{array}$

$\begin{array}{lllll}28 & 43.72 & -3.4 & \text { I TNP }\end{array}$

\begin{tabular}{lll|l}
28 & 45.47 & -1.8 & PMGM
\end{tabular}

\begin{tabular}{lll|l|l}
28 & 43.45 & -3.9 & GMW \\
28 & 46.71 & -0.9 & EUR
\end{tabular}

\begin{tabular}{lll|l}
28 & 46.71 & -0.9 & EUR \\
28 & 45.24 & -2.4 & | WASM
\end{tabular}

$2845.65-2.6$ | CFWM

\begin{tabular}{lll|l}
28 & 44.11 & -3.6 & I WKTM
\end{tabular}

\begin{tabular}{lll|l}
28 & 44.28 & -3.9 & CPTM
\end{tabular}

$\begin{array}{lllll}28 & 46.07 & -2.3 & \text { I WHFM }\end{array}$

\begin{tabular}{lll|l}
28 & 45.06 & -3.4 & WCHM \\
28 & 45.62 & -3.0 & RVCM
\end{tabular}

\begin{tabular}{lll|l|l}
28 & 45.62 & -3.6 & RVCM \\
28 & 44.54 & -4.2 & HWSM
\end{tabular}

$28 \quad 45.18 \quad-4.1$ । CGSM

\begin{tabular}{lll|l}
28 & 46.10 & -3.2 & WHVM
\end{tabular}

\begin{tabular}{lll|l}
28 & 46.11 & -3.4 & WORM
\end{tabular}

\begin{tabular}{lll|l}
28 & 46.04 & -3.7 & WBMM \\
28 & 46.67 & -3.0 & CSS
\end{tabular}

$\begin{array}{lllll}28 & 46.22 & -3.9 & \text { WWPM }\end{array}$

2944.93 WLPM

\begin{tabular}{lll|l}
28 & 48.25 & -2.2 & WNMM
\end{tabular}

\begin{tabular}{lll|l}
28 & 47.63 & -2.9 & WJPM \\
28 & 48.34 & -2.2 & RCWM
\end{tabular}

\begin{tabular}{lll|l}
28 & 48.34 & -2.2 & RCWM \\
28 & 46.42 & -4.4 & WBSM
\end{tabular}

$28 \quad 48.26-3.2$ WSCM

$\begin{array}{lll}28 & 49.26 & -3.2\end{array}$

2951.40

$\begin{array}{lll}28 & 50.32 & -1.9\end{array}$

WCW

I NEW

\begin{tabular}{lll|l}
28 & 48.23 & -4.1 & 1 \\
\hline & $4 A C$
\end{tabular}

$\begin{array}{llll}28 & 49.57 & -3.0 & \text { I MSO }\end{array}$

$\begin{array}{llll}28 & 51.06 & -2.0\end{array}$

\begin{tabular}{lll|l}
28 & 49.92 & -3.2 & $B E I$
\end{tabular}

\begin{tabular}{lll|l}
28 & 49.56 & -4.4 & PHC
\end{tabular}

$\begin{array}{lll}28 & 51.20 & -2.9\end{array}$

$\begin{array}{lll}28 & 50.39 & -3.9\end{array}$

$\begin{array}{lll}28 & 49.77 & -4.7\end{array}$

$\begin{array}{lll}28 & 52.29 & -2.2\end{array}$

I LDM

\begin{tabular}{lll|l}
28 & 51.57 & -3.0 & YKM
\end{tabular}

\begin{tabular}{lll|l}
28 & 52.30 & -2.9 & KNB
\end{tabular} $\begin{array}{lll}28 & 48.76 & -3.4\end{array}$

$\begin{array}{llllll}5.39 & 151 & \text { iPnd } 28 & 51.30 & -3.8\end{array}$

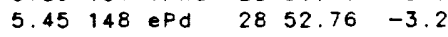
$\begin{array}{llllll}5.52 & 119 & \text { ePd } & 28 & 55.12 & -1.7\end{array}$

$\begin{array}{llllll}5.54 & 151 & \mathrm{ePd} & 28 & 52.94 & -4.4\end{array}$

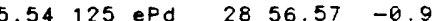
$\begin{array}{lllll}5.56 & 121 \mathrm{ePC} & 28 & 55.72 & -1.7\end{array}$ $\begin{array}{llllll}5.58 & 146 & \text { ePd } & 28 & 54.52 & -3.2\end{array}$

$\begin{array}{llllll}5.58 & 147 \mathrm{ePd} & 28 & 54.80 & -3.0\end{array}$ $\begin{array}{lllllll}5.59 & 149 & \text { ePd } & 28 & 54.17 & -3.8\end{array}$ $\begin{array}{llllll}5.61 & 135 & \text { iPC } & 28 & 56.11 & -2.1\end{array}$ $\begin{array}{llllll}5.66 & 116 & \text { ePd } & 28 & 56.23 & -2.8\end{array}$ $\begin{array}{llllll}5.67 & 94 & \text { ePnC } 28 & 55.50 & -3.7\end{array}$ $\begin{array}{llllll}5.69 & 150 & \mathrm{EPd} & 28 & 54.95 & -4.3\end{array}$

$5.70126 \mathrm{ePC}$ 5.72147 ePd

$5.76146 \mathrm{ePd}$

$5.77152 \mathrm{ePd}$

5.78125 EPC

$\begin{array}{ll}5.82 & 120 \mathrm{ePC} \\ 5.84 & 146 \mathrm{iPnd}\end{array}$

$5.84 \quad 147 \mathrm{ePd}$

$5.85 \quad 149$ ePd

$5.89144 \mathrm{ePd}$

$5.93 \quad 146$ ePd

5.96151 ePd

$.98145 \mathrm{ePd}$

6.03148 ePd

$6.05152 \mathrm{ePd}$

$6.06150 \mathrm{ePd}$

$6.09146 \mathrm{ePd}$

6.14147 ePd

$6.17146 \mathrm{ePd}$

$6.18 \quad 147 \mathrm{ePd}$

$6.20150 \mathrm{ePd}$

$6.21146 \mathrm{ePd}$

$6.22152 \mathrm{ePd}$

6.23146 ePd

$6.24145 \mathrm{ePd}$

$6.25149 \mathrm{ePd}$

$6.32 \quad 147 \mathrm{eP}$

es

$6.32145 \mathrm{ePd}$

$6.36146 \mathrm{ePd}$

$6.41 \quad 147 \mathrm{ePd}$

$6.43150 \mathrm{ePd}$

$6.44146 \mathrm{ePd}$

$6.47115 \mathrm{ePnc}$

6.51149 ePd

$6.5911 \mathrm{ePn}$

$6.82101 \mathrm{iPn}$

$7.16 \quad 136 \mathrm{ePc}$

$7.18 \quad 131 \mathrm{ePC}$

$7.18 \quad 135 \mathrm{ePC}$

$7.31131 \mathrm{ePC}$

7.31135 ePC

$7.32 \quad 133 \mathrm{ePC}$

$7.33 \quad 132 \mathrm{ePC}$

$7.33131 \mathrm{IPC}$

$7.35 \quad 129 \mathrm{ePC}$

$7.36137 \mathrm{ePC}$

$7.36 \quad 135 \mathrm{ePC}$

$7.37 \quad 138 \mathrm{ePC}$

$7.39131 \mathrm{ePc}$

$7.42 \quad 134 \mathrm{ePc}$

$7.42 \quad 137 \mathrm{ePC}$

$7.44133 \mathrm{ePc}$

$\begin{array}{llll}.46 & 137 & \mathrm{ePC}\end{array}$

$7.51131 \mathrm{ePC}$ 


\begin{tabular}{|c|c|c|c|c|c|c|c|c|c|c|c|}
\hline BUT & 10.05 & 57 & ePc & 29 & 58.50 & -1.8 & I & JSC & 34.72 & 87 & $e^{P}$ \\
\hline$R \times F$ & 10.29 & 38 & ip & 30 & 00.00 & -3.4 & 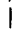 & HBF & 35.96 & 89 & eP \\
\hline I MW & 10.50 & 70 & ${ }_{i} P_{C}$ & 30 & 05.70 & -0.9 & & ADK & 36.68 & 305 & eP \\
\hline GCA & 10.95 & 108 & ePc & 30 & 11.90 & -0.6 & & PNY & 37.07 & 67 & $i P_{C}$ \\
\hline GLA & 11.21 & 133 & ePc & 30 & 13.50 & -2.5 & & GPD & 37.41 & 73 & eP \\
\hline & & & e & 30 & 16.80 & & t. & DVT & 38.00 & 66 & eP \\
\hline BDW & 11.36 & 76 & iPc & 30 & 16.20 & -2.0 & 1 & $M D_{4}$ & 38.67 & 72 & eP \\
\hline TUC & 14.14 & 124 & iPc & 30 & 54.90 & -0.3 & & MD2 & 38.72 & 72 & eP \\
\hline LD3 & 14.19 & 61 & IPC & 30 & 51.20 & -4.6 & 1 & BNH & 38.72 & 66 & eP \\
\hline GOL & 14.75 & 89 & $i P c$ & 31 & 00.70 & -2.7 & 1 & ILT & 39.29 & 331 & $P d$ \\
\hline GLD & 14.86 & 89 & eP & 31 & 01.10 & -3.6 & & & & & isP \\
\hline MSA & 5.09 & 100 & $i P c$ & 31 & 07.30 & -0.6 & & & & & $i p P$ \\
\hline SVM & 5.46 & 117 & ip & 31 & 12.90 & 0.3 & 1 & $\mathrm{SCH}$ & 39.95 & 50 & iPd \\
\hline ANMO & 5.58 & 107 & eP & 31 & 12.20 & -1.9 & | & $M \mid M$ & 40.09 & 65 & eP \\
\hline$A L O$ & 5.58 & 187 & ePc & 31 & 12.40 & -1.7 & & CBM & 40.31 & 62 & eP \\
\hline SIT & 17.41 & 340 & ePd & 31 & 37.10 & 0.2 & 1 & BPM & 40.42 & 66 & eP \\
\hline LUB & 19.57 & 105 & eP & 31 & 58.00 & -5.6 & 1 & POO & 41.23 & 65 & eP \\
\hline HON & 20.43 & 339 & eP & 32 & 12.10 & -0.3 & i & EMM & 41.28 & 65 & eP \\
\hline CMTX & 20.59 & 105 & eP & 32 & 09.60 & -4.8 & I & SMY & 42.20 & 307 & $e(P)$ \\
\hline YKA & 22.26 & 12 & $P$ & 32 & 31.60 & 0.6 & 1 & $z$ & $20 s$ & 145 & $5.0 \theta$ um \\
\hline JCT & 22.69 & 110 & ip & 32 & 31.90 & -3.6 & 1 & GDH & 45.66 & 28 & IPd \\
\hline S10 & 22.78 & 94 & $i P c$ & 32 & 31.50 & -4.8 & 1 & & $1.2 \mathrm{~s}$ & 937 & $.00 \mathrm{~nm}$ \\
\hline RLO & 23.59 & 92 & $i P c$ & 32 & 40.10 & -4.1 & 1 & & & & is \\
\hline KDC & 4.36 & 323 & ePd & 32 & 54.10 & 2.7 & 1 & PET & 51.19 & 311 & eP \\
\hline TOA & 4.70 & 336 & ePd & 32 & 57.20 & 2.4 & I & & & & es \\
\hline PMR & 25.32 & 332 & ePd & 33 & 02.00 & 1.5 & I & SEY & 51.36 & 324 & $P d$ \\
\hline & $18 \mathrm{~s}$ & 600 & $9.00 \mathrm{um}$ & & & $2 M s z$ & I & GAL & 52.75 & 110 & iP \\
\hline TYS & 6.21 & 84 & eP & 33 & 06.00 & -3.1 & 1 & MGD & 52.95 & 321 & ePd \\
\hline$F \vee M$ & 26.47 & 85 & iPc & 33 & 06.90 & -4.7 & I & & & & epP \\
\hline & $18 \mathrm{~s}$ & 100 & $9.00 \mathrm{um}$ & & & $4 \mathrm{Msz}$ & I & & & & esP \\
\hline POW & 26.50 & 90 & eP & 33 & 07.50 & -4.3 & 1 & & & & es \\
\hline DON & 27.10 & 87 & eP & 33 & 12.40 & -4.9 & 1 & SKR & 53.51 & 309 & eP \\
\hline JHP & 27.21 & 90 & eP & 33 & 20.60 & 2.3 & 1 & & & & es \\
\hline WLA & 27.21 & 91 & eP & 33 & 14.60 & -3.7 & 1 & DAG & 53.88 & 16 & iPd \\
\hline $\mathrm{COL}$ & 27.30 & 339 & eP & 33 & 19.00 & 0.1 & 1 & & $1.5 \mathrm{~s}$ & 861 & $1.08 \mathrm{~nm}$ \\
\hline & & & $e(S)$ & 37 & 50.00 & & 1 & SJG & 54.69 & 96 & ip \\
\hline FBA & 27.30 & 339 & ePd & 33 & 19.00 & 0.1 & 1 & & & & $\mathrm{i}$ \\
\hline SDN & 7.46 & 313 & eP & 33 & 24.00 & 3.7 & I & & & & is \\
\hline LST & 7.47 & 88 & eP & 33 & 17.00 & -3.6 & I & TIK & 56.43 & 339 & $P d$ \\
\hline SVW & 7.50 & 327 & $e P d$ & 33 & 21.20 & 0.5 & I & & & & es \\
\hline ELC & 27.59 & 86 & eP & 33 & 17.00 & -4.8 & 1 & TOV & 57.35 & 107 & $i P_{c}$ \\
\hline INK & 27.68 & 353 & iPc & 33 & 21.30 & -0.9 & 1 & & $0.6 \mathrm{~s}$ & 58 & 3. $80 \mathrm{~nm}$ \\
\hline & $0.5 \mathrm{~s}$ & 298 & $3.00 \mathrm{~nm}$ & & & $3 m b$ & 1 & SOV & 57.36 & 108 & IPO \\
\hline GR T & 27.79 & 88 & eP & 33 & 19.70 & -3.9 & 1 & & $0.9 \mathrm{~s}$ & 222 & $2.00 \mathrm{~nm}$ \\
\hline MPH & 27.83 & 91 & eP & 33 & 20.60 & $-3 \cdot 3$ & 1 & FUQ & 57.55 & 114 & ip \\
\hline MET & 27.83 & 91 & $i P_{c}$ & 33 & 20.60 & -3.4 & 1 & KBS & 57.58 & 9 & iPd \\
\hline & & & $\mathrm{s}$ & 38 & 14.00 & & 1 & $B O G$ & 57.93 & 115 & iP \\
\hline EBZ & 28.26 & 91 & eP & 33 & 24.50 & -3.4 & 1 & $B O C 0$ & 57.98 & 115 & iPc \\
\hline TTA & 28.67 & 330 & $e(P)$ & 33 & 30.10 & -1.2 & 1 & KHE & 58.54 & 359 & $P d$ \\
\hline ACM & 28.76 & 74 & eP & 33 & 29.50 & -2.8 & 1 & & & & es \\
\hline IMA & 9.85 & 337 & ePd & 33 & 42.00 & 0.0 & I & $O A Q$ & 58.58 & 122 & iPCt \\
\hline AN 11 & 0.06 & 78 & ePc & 33 & 39.80 & -4.2 & I & NCE & 58.92 & 123 & iP \\
\hline AN 10 & 0.24 & 78 & eP & 33 & 41.90 & -3.7 & 1 & CAR & 58.96 & 104 & iPc \\
\hline 110 & 0.32 & 127 & eP & 33 & 46.00 & -0.8 & 1 & & $0.5 \mathrm{~s}$ & & $3.10 \mathrm{~nm}$ \\
\hline AN 12 & 30.34 & 77 & ePc & 33 & 42.30 & -4.1 & 1 & REY & 58.98 & 30 & ip \\
\hline AAM & $\begin{array}{lll}30 & 42\end{array}$ & 74 & ePc & 33 & 43.70 & -3.4 & 1 & $A K U$ & 59.54 & 27 & iPd \\
\hline UTO & 30.62 & 75 & $i P d$ & 33 & 46.50 & $-2 \cdot 3$ & 1 & & $1.4 \mathrm{~s}$ & 1550 & $9.00 \mathrm{~nm}$ \\
\hline TAC & 30.63 & 127 & eP & 33 & 45.00 & -4.5 & 1 & TPT & 59.71 & 206 & ip \\
\hline & & & es & 38 & 58.50 & & 1 & & $1.2 \mathrm{~s}$ & & $5.00 \mathrm{~nm}$ \\
\hline $11 \mathrm{M}$ & 30.70 & 127 & iP & 33 & 51.00 & 0.9 & 1 & & & & $i p P$ \\
\hline AN3 & 30.71 & 77 & ePc & 33 & 45.80 & -3.9 & I & PMO & 59.82 & 206 & ip \\
\hline $11 P$ & 30.84 & 127 & eP & 33 & 51.00 & -0.4 & 1 & & $1.2 \mathrm{~s}$ & 35 & $5.00 \mathrm{~nm}$ \\
\hline 111 & 1.26 & 129 & ip & 33 & 53.60 & -1.4 & 1 & & & & $i p P$ \\
\hline BE TH & 1.33 & 82 & eP & 33 & 50.70 & -4.4 & 1 & RUV & 59.83 & 206 & iP \\
\hline I IT & 1446 & 126 & eP & 33 & 56.60 & -0.2 & 1 & & $1.2 \mathrm{~s}$ & 110 & $3.00 \mathrm{~nm}$ \\
\hline ORT & 1.79 & 86 & eP & 33 & 55.70 & -3.5 & 1 & & & & $i p P$ \\
\hline NK I & 1.89 & 307 & eP & 34 & 01.00 & 1.1 & 1 & VAH & 59.95 & 206 & iP \\
\hline CLE & 32.15 & 75 & iPd & 33 & 59.50 & -2.8 & 1 & & $1.2 \mathrm{~s}$ & & $9.00 \mathrm{~nm}$ \\
\hline & & & $S$ & 39 & 16.00 & & I & & & & ipp \\
\hline$T K L$ & 32.28 & 86 & $e^{P}$ & 33 & 59.10 & -4.4 & 1 & $A M N$ & 60.54 & 198 & iP \\
\hline ANM & 33.08 & 329 & eP & 34 & 10.40 & 0.2 & 1 & & $1.3 \mathrm{~s}$ & 345 & $5.00 \mathrm{~nm}$ \\
\hline HIL & 33.54 & 240 & $P$ & 34 & 14.00 & -0.6 & 1 & OTP & 60.84 & 198 & ip \\
\hline HKL & 33.60 & 242 & $P$ & 34 & 19.00 & 3.9 & 1 & & $1.3 \mathrm{~s}$ & 315 & $5.00 \mathrm{~nm}$ \\
\hline MKH & 33.71 & 240 & $P$ & 34 & 17.00 & 0.9 & 1 & KUR & 60.88 & 306 & $e^{P}$ \\
\hline MRG & 33.80 & 77 & $i P d$ & 34 & 14.90 & -1.8 & 1 & YAK & 61.24 & 329 & iPd \\
\hline & & & es & 39 & 41.00 & & 1 & & $2.0 \mathrm{~s}$ & 1620 & $0.00 \mathrm{~nm}$ \\
\hline HVO & 33.89 & $24 \theta$ & ip & 34 & 22.00 & 4.2 & 1 & PPN & 62.69 & 207 & iP \\
\hline & & & is & 39 & 04.00 & & 1 & & $1.2 \mathrm{~s}$ & 80 & $0.00 \mathrm{~nm}$ \\
\hline PRM & 3.97 & 88 & eP & 34 & 14.00 & -4.2 & & PPT & 62.78 & 207 & iP \\
\hline MOK & 4.11 & 245 & $P$ & 34 & 23.00 & 3.5 & 1 & & $1.2 \mathrm{~s}$ & 110 & $9.00 \mathrm{~nm}$ \\
\hline KKH & 34.18 & 241 & $P$ & 34 & 22.00 & 1.8 & 1 & AFR & 62.82 & 207 & ip \\
\hline$B L A$ & 34.24 & 82 & iPct & 34 & 17.60 & -3.0 & 1 & & $1.2 \mathrm{~s}$ & 910 & $0.60 \mathrm{~nm}$ \\
\hline & $1.2 \mathrm{~s}$ & 98 & 8.6 & & & $6 \mathrm{mb}$ & & PAE & 62.86 & 207 & ip \\
\hline & & & es & 39 & 42.80 & & 1 & & $1.2 \mathrm{~s}$ & 75 & $500 \mathrm{~nm}$ \\
\hline DHN & 34.25 & 71 & eP & 34 & 13.50 & -7.1 & 1 & TVO & 62.87 & 206 & iP \\
\hline IP & 34.32 & 245 & $e^{P}$ & 34 & 25.00 & 3.7 & 1 & & $1.2 \mathrm{~s}$ & 85 & $5.00 \mathrm{~nm}$ \\
\hline HON & 34.38 & 245 & 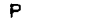 & 34 & 24.00 & 2.1 & 1 & YSS & 63.02 & 310 & \\
\hline
\end{tabular}


08d $10 h$

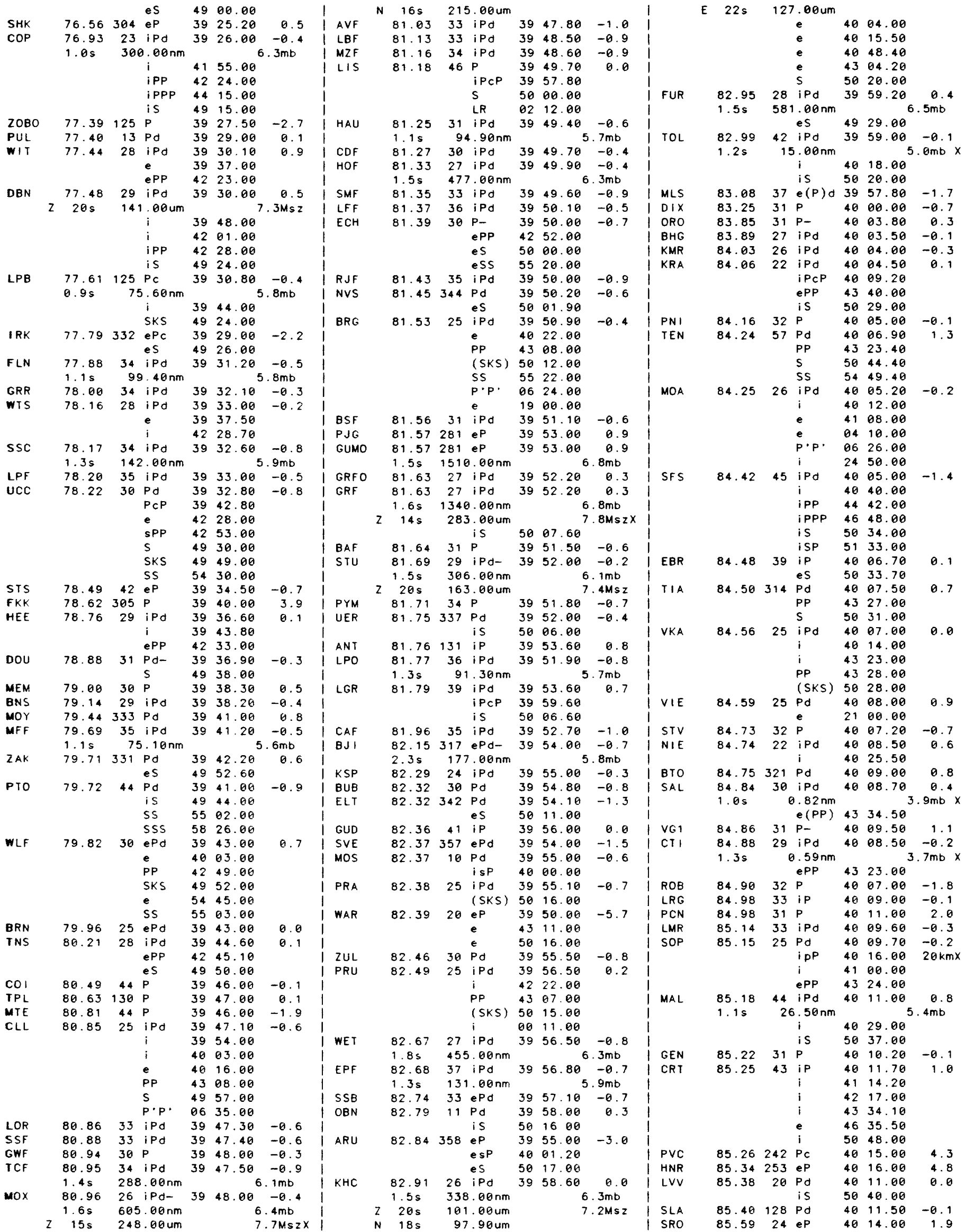




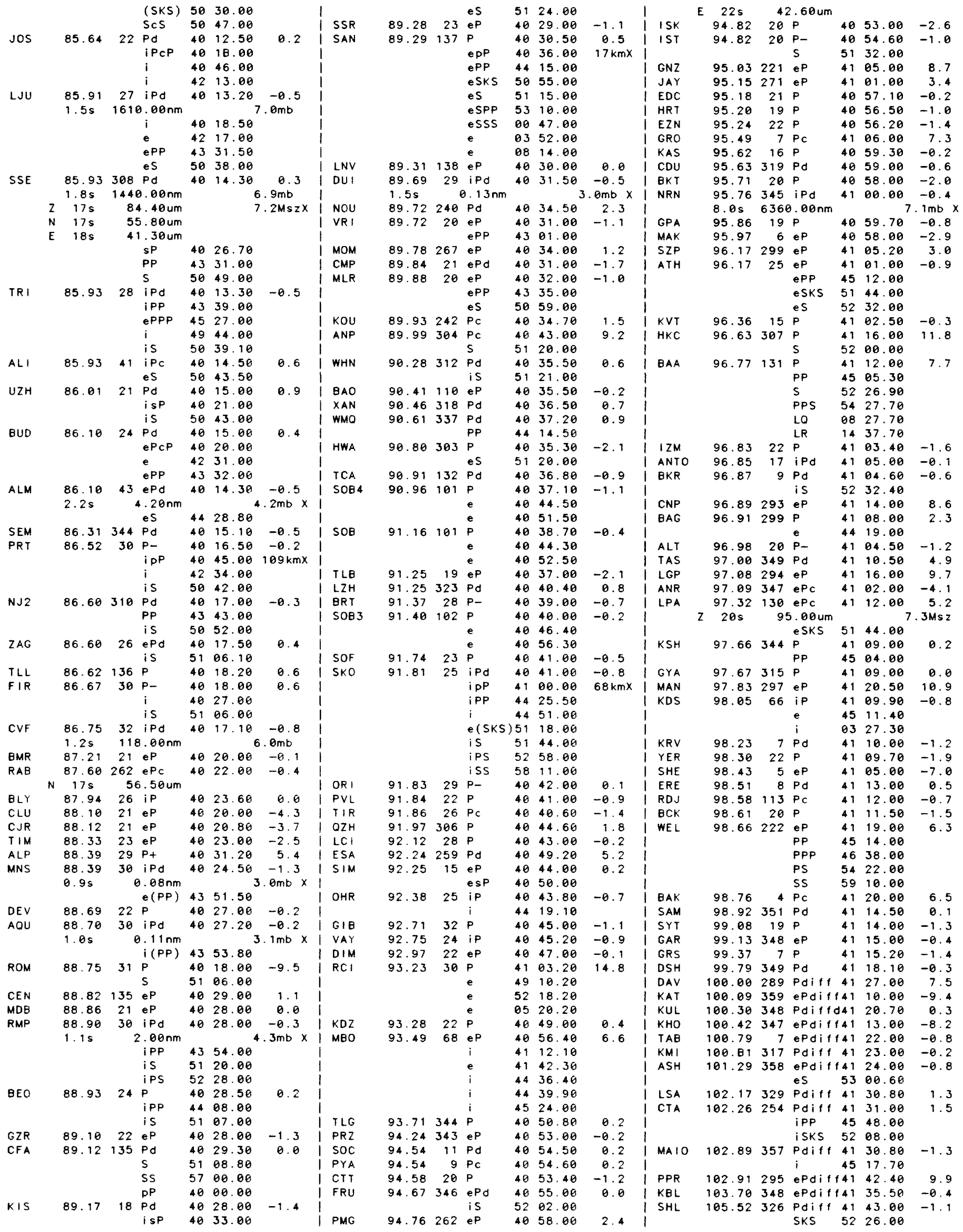


e8d $10 \mathrm{~h}$

DD1 $\quad 105.91340$ Pdiff 4148.60 eS 4528.00

EIL $107.22 \quad 19$ Pdiff 4156.50

NHA $\quad 107.36 \quad 304$ Pdiff $42 \quad 05.00$

ISO 107.56258 ePdiff42 03.00 4531.00

KIC $\quad 107.61 \quad 65$ ePdiff41 51.90

NDI $\quad 107.69 \quad 340$ Pdiff 4208.00 ePP 4528.00 ePPP $48 \quad 36.00$ eSS 0544.00 eSSS 1120.00

CHG $\quad 107.98317$ ePdif142 07.00 es $52 \quad 45.00$

MTN 109.02269 ePdiff42 06.00

$\begin{array}{lllll}\text { KUK } & 110.68 & 61 \text { PKP } 46 & 03.00\end{array}$

WB2 $110.96^{\prime} 261$ Pdiff 4215.00

WH2 $\quad 110.96 \quad 261$ PKP 4554.00 i $\quad 46 \quad 15.00$ \begin{tabular}{l}
$i \quad 4649.70$ \\
\hline
\end{tabular}$\quad 4917.10$

WRA 110.97261 Pdiff 4217.00 $1.0 \mathrm{~s} \quad 2.40 \mathrm{~nm}$

WRA 110.97261 PKPC $46 \quad 14.40$ $0.7 \mathrm{~s} \quad 3.40 \mathrm{~nm}$

MKS $112.82 \quad 284$ e(PKP) $46 \quad 18.90$

KUPT $112.98 \quad 277$ e(PKP) $46 \quad 26.00$

TOO $113.34 \quad 240$ ePKP $46 \quad 07.00$

ASP $\quad 113.53 \quad 259$ ePKP $46 \quad 19.00$

TAU $115.16 \quad 234$ ePdiff42 30.00 4720.00 5512.00 5532.00 0232.00 0700.00 e 1040.00

$\begin{array}{llllrr}A D E & 116.45 & 246 & \text { PKP } & 46 & 24.00 \\ \text { SNG } & 116.53 & 308 & \text { ePdiff } 42 & 44.00\end{array}$

SNG $\quad 116.53308$ ePdiff 4244.00 es 5316.00

HYB 117.94335 ePdiff $42 \quad 49.00$

HYB $\quad 117.94335$ ePKP $46 \quad 19.00$ eSKS 5528.00

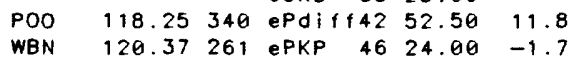

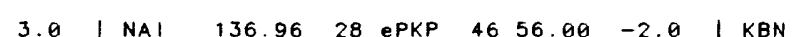
0.1

5.11 W

12.8
9.9

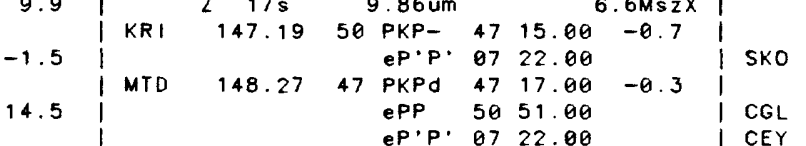

1 TET

$\begin{array}{llll}\text { TET } & 148.82 \quad 44 & \text { IPKPd } 47 \quad 20.00\end{array}$

BUL 149.1350 e 5052.00

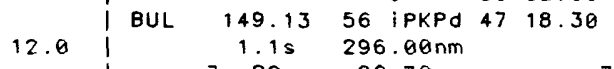

$2 \quad 22 \mathrm{~s} \quad 80.70 \mathrm{um}$

N $21 \mathrm{~s} \quad 55.20 \mathrm{um}$

E $22 \mathrm{~s} \quad 79.30 \mathrm{um}$

-4.5
6.8

CLK

-1.7 NPA

149.13 41 PKP-

0726.00

$\begin{array}{cccc}33 \text { ePKP } & 47 & 22.00 \\ e & 47 & 27.00\end{array}$

e
e $\quad 4727.00$

8.7 CIR $151.60 \quad 52$ PKP- 4722.00

\begin{tabular}{l|llllll}
6.5 & PRE & 152.80 & 64 & IPKPC & 51 & 21.00 \\
& & 25.20
\end{tabular}

$\begin{array}{cc}152.80 & 64 \\ 1.95 & 44.90 \mathrm{~nm}\end{array}$

$\begin{array}{lll}1.0 \mathrm{~s} & 44.00 \mathrm{~nm} & 47 \quad 46.50\end{array}$

$\begin{array}{lllll}153.11 & 186 & \text { ePKP } \quad 47 & 23.00\end{array}$

155.8781 iPKPd+47 37.50

$1.2 \mathrm{~s} \quad 46.90 \mathrm{~nm}$

-5.0 GRM

6.2

3.7

I AVY

$222 \mathrm{~s} \quad 57.00 \mathrm{um}$

5148.06

156.96 19 iPKPd $47 \quad 35.60$

\begin{tabular}{l|l}
828 obs. associoted & ZGN \\
& VG1
\end{tabular}

0.4

CEY

TRI

$\mathrm{CVF}$

0.3 LJU

1.2

BEO

$-0.31$

1.2 VAY

I MBZ

I SBS

$0.1 \mid \mathrm{CT}$

CTI
PCN

SAL

GEN

NOV 23, 1980 18h $34 \mathrm{~m} 53.28 \pm 0.08 \mathrm{~s}$ । SSR $40.884 \mathrm{~N} \pm 1.4 \mathrm{~km} 15.325 \mathrm{E} \pm 1.0 \mathrm{~km}$ । SOF DEPTH $=8.0 \mathrm{~km}$ ( 15 depth phases) / ROB $6.1 \mathrm{mb}(54$ obs.) $6.8 \mathrm{Msz}$ ( 19 obs.) । SOUTHERN I TALY

(390) I TIM

Over 3000 people killed, obout I OGA 1900 missing. 7750 injured,

250,000 homeless and extensive damage.

11.8

$\begin{array}{llllll}\text { BS! } & 120.96 & 312 & \text { ePKP } & 46 & 40.50 \\ \text { PSI } & 121.10 & 306 & \text { iPKPC } & 46 & 25.20\end{array}$ $1.0 \mathrm{~s} \quad 27.60 \mathrm{~nm}$

\begin{tabular}{llllll|l} 
MDR 121.49 & 331 & PKP & 46 & 35.00 & 6.9 & ORI
\end{tabular}

$\begin{array}{llllll}\text { GBA } & 121.87 & 335 & \text { PKPd } 46 & 25.20 & -3.6\end{array}$

LEM $\quad 122.46 \quad 291$ e (PKP) $46 \quad 18.50-11.7$

e(S) 5402.00

BCAO $123.58 \quad 46$ ePKP $46 \quad 30.90$

BNG $123.58 \quad 46$ ePKP

$K O D \quad 125.91333$ PKP 4635.00 $\begin{array}{lll}\text { EPP } & 48 & 20.00\end{array}$ SKS 5340.00 SKKS $54 \quad 16.00$

SKKKS 5521.00 PS 5808.00 SS 0532.00 SSS 0952.00

$\begin{array}{llllll}\text { SBA } & 125.55 & 194 & \text { IPKP } & 46 & 33.30\end{array}$ $\begin{array}{lll}\text { PP } & 48 & 37.00 \\ \text { PKS } & 59 & 99.00\end{array}$ PS 5833.00 - 0234.00 SS 0534.00 LO 2000.00

LR 2500.00

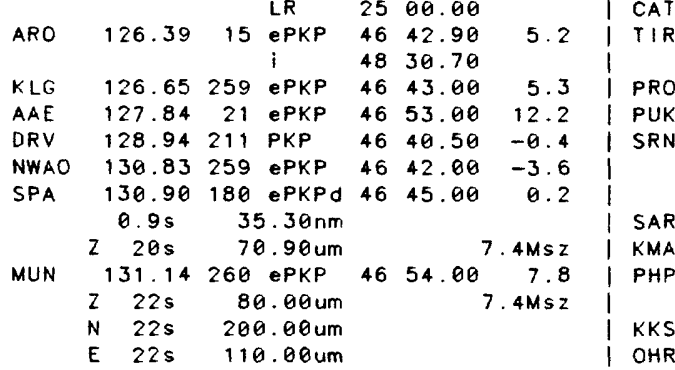

BRT

$|A C|$

\begin{tabular}{l|l}
-3.3 & AOU \\
-2.2 & RMP
\end{tabular}

16.3 LLI

\begin{tabular}{l|l}
-0.3 & $A C L$ \\
$M N S$
\end{tabular}

MNS

I MES

1011

I NOV

GIB

$-0.9 \mid P R G$

I VLO

TTG

TTG

I

CAT

PRO

PUK
SRN

SAR

KMA

KKS
OHR
$0.71266 \mathrm{Pg} \quad 3507.50$

$0.87305 \mathrm{Pg}$

$1.01320 \mathrm{ePg}$

$1978 \mathrm{Pg}$

1. $20134 P_{*}$

isg

$.4290 \mathrm{Pnt}$

$\begin{array}{lll}1.67 & 156 \mathrm{Pn} \\ 2.06 & 316 \mathrm{Pn}\end{array}$

$\begin{array}{lll}2.06 & 316 & \mathrm{Pn} \\ 2.18 & 296 \mathrm{Pn}\end{array}$

$2.45187 \mathrm{Pn}$

$2.46198 \mathrm{Pn}$

$2.49308 \mathrm{Pn}$

$2.52186 \mathrm{Pn}$

$2.69176 \mathrm{Pn}$

$2.74174 \mathrm{Pn}$

2.86 $183 \mathrm{Pn}$

$3.07200 \mathrm{Pn}-$

$3.11316 \mathrm{Pn}$

i $\mathrm{Sn}$

3.2096 Pnd

()

$3.3361 \mathrm{ePn}$

$3.3369 \mathrm{Pn}$

()

$3.37183 \mathrm{Pn}$

$3.4681 \mathrm{Pn}$

()

$3.6270 \mathrm{Pnc}$

$3.71104 \mathrm{Pn}$

()

$3.77 \quad 37$ ipn

3.9468 Pno

$3.9477 \mathrm{PnC}$

()

4. $8071 \mathrm{Pn}$

$\begin{array}{ll}4.90 & 71 \mathrm{Pn} \\ 4.15 & 85 \mathrm{Pn}\end{array}$
I FRF

\begin{tabular}{lll|l}
35 & 11.70 & 1.4 & MOA
\end{tabular}

3514.50

3516.00

$35 \quad 14.50$

3533.00

\begin{tabular}{lll|l}
35 & 17.00 & -2.4 & BUD
\end{tabular}

3521.00

3530.60

3530.50

$3533.5 \theta$

3531.00

3535.50

3534.06

3534.80

3535.40

3538.00

3542.20

$36 \quad 18.7 \theta$

3545.00

$36 \quad 33.00$

3545.40

$36 \quad 08.3 \theta$

$\begin{array}{ll}35 & 45.00 \\ 35 & 48.00\end{array}$

$36 \quad 28.80$

3547.50

3548.00

$36 \quad 66.50$

3550.30

3550.00

$\begin{array}{lll}35 & 53 & 70 \\ 36 & 42 & 70\end{array}$

$36 \quad 42.70$

$35540 \theta$

3555.30

$35 \quad 54.70$

$36 \quad 37.20$

$\begin{array}{ll}35 & 55.40 \\ 35 & 57.56\end{array}$

4. $1892 \mathrm{Pn}$

4. $18315 \mathrm{Pn}-$ isn

3559.60

$36 \quad 02.50$

$\begin{array}{ll}36 & 47.20\end{array}$

$36 \quad 03.50$

iP* 3612.00

iPg $36 \quad 18.30$

isn $36 \quad 54.50$

$\begin{array}{lllll}4.72 & 75 & \text { iPne } 36 & 06.00 & -0.5\end{array}$

isn 3702.60

$4.86254 \mathrm{Pn} \quad 3603.00$

$3603.00-5.6 x$

$\begin{array}{lllll}4.90 & 353 \mathrm{iPn} & 36 & 08.10 & -0.8\end{array}$

esn 3704.30

$4.95347 \mathrm{Pn} \quad 3608.00$ iPgPg $36 \quad 29.20$ isn 3707.00

$5.12291 \mathrm{Pn} \quad 36 \quad 10.50$ Sn 3708.50

5.19354 iPnc 3612.10

$\begin{array}{ll}36 & 15.30\end{array}$

$36 \quad 38.90$

es

3737.50

$5.4542 \mathrm{Pn} \quad 3616.60$

$-0.1$

iPg 3641.60

isg 3801.00

$5.4983 \mathrm{iPn}$

$5.56222 \mathrm{iPn}$

$36 \quad 15.00$

$3616.50-2.3$

$5.58226 \mathrm{iPn}$

$5.81334 \mathrm{Pnt}$

$\begin{array}{lll}36 & 18.00 & -0.6\end{array}$

$\begin{array}{rrr}36 & 25.00 & 2.3\end{array}$

$5.88325 \mathrm{Pnt}$

3736.28

$\begin{array}{lll}36 & 22.00 & -0.7\end{array}$

$5.89309 \mathrm{Pn}$

3625.60

3739.20

$\begin{array}{lll}36 & 23.3 \theta & -2.3\end{array}$

$6.08224 \mathrm{iPn}$

$6.10314 \mathrm{Pn}$

$36 \quad 29.50$

3625.09

$36 \quad 28.50$

3631.00

$6.2571 \mathrm{iPn}$

eSn

3751.00

$6.48 \quad 40 \mathrm{ePn} \quad 3630.00$ 


\begin{tabular}{|c|c|c|c|c|c|c|c|c|c|c|c|}
\hline CMP & 8.35 & 55 & eP & 37 & 00.00 & 2.5 & 1 & & & e & 40 \\
\hline BบB & 8.38 & 327 & $P$ & 36 & 55.00 & -2.9 & $i$ & & & $\mathrm{Sg}$ & 40 \\
\hline CLU & 8.40 & 43 & ePd & 36 & 57.00 & -1.1 & BGF & 10.66 & 306 & $\mathbf{P}$ & 37 \\
\hline MDB & 8.42 & 48 & eP & 37 & 00.00 & 1.6 & I MZF & 10.67 & 304 & $P$ & 37 \\
\hline WET & 8.44 & 349 & iPd & 36 & 55.30 & $-3.4 x$ & | YER & 10.75 & 106 & P- & 37 \\
\hline JOS & 8.47 & 24 & $i P c$ & 36 & 58.80 & -0.2 & I MLS & 10.82 & 286 & $\in P$ & 37 \\
\hline & $2.0 \mathrm{~s}$ & 57 & $0.00 \mathrm{~nm}$ & & 6 & $.5 \mathrm{mb}$ & I IAS & 10.83 & 50 & $E P$ & 37 \\
\hline$E Z N$ & 8.47 & 94 & $\mathbf{P}$ & 36 & 56.20 & -2.9 & I WLF & 10.89 & 327 & ePd & 37 \\
\hline CEI & 8.51 & 35 & $\mathrm{P}$ & 37 & 00.00 & 0.4 & 1 & & & $\mathrm{~s}$ & 39 \\
\hline BUC1 & 8.62 & 63 & ip & 37 & 04.00 & 2.8 & I TCF & 10.93 & 304 & $P$ & 37 \\
\hline$B \cup C$ & 8. 69 & 62 & eP & 37 & 00.00 & -2.1 & RJF & 11.02 & 298 & $P$ & 37 \\
\hline BMR & 8. 96 & 38 & eP & 37 & 08.00 & 2.1 & $1 \angle P O$ & 11.06 & 295 & $P$ & 37 \\
\hline SPC & 9.00 & 21 & eP & 37 & 09.30 & 2.7 & EBR & 11.24 & 275 & eP & 37 \\
\hline STU & 9.01 & 333 & ePc & 37 & 04.00 & -2.5 & 1 & & & eS & 39 \\
\hline & $1.0 \mathrm{~s}$ & 340 & $0.00 \mathrm{~nm}$ & & & $.7 \mathrm{mb}$ & I LSF & 11.35 & 303 & $P$ & 37 \\
\hline MLR & 9.02 & 56 & $\mathrm{Pc}$ & 37 & 05.80 & -1.0 & I EPF & 11.36 & 286 & $P$ & 37 \\
\hline PRU & 9.12 & 357 & $P$ & 37 & 05.20 & -2.9 & GPA & 11.41 & 88 & $P$ & 37 \\
\hline & $1.0 \mathrm{~s}$ & 93 & $2.00 \mathrm{~nm}$ & & & . $1 \mathrm{mb} \times$ & I LFF & 11.44 & 296 & $P$ & 37 \\
\hline & & & $s$ & 38 & 49.80 & & $A L T$ & 11.49 & 94 & $\mathbf{P}$ & 37 \\
\hline BAF & 9.15 & 322 & eP & 37 & 06.40 & -2.2 & I KIS & 11.52 & 53 & PC & 37 \\
\hline UZH & 9.20 & 30 & $P d$ & 37 & 09.40 & 0.3 & I BNS & 11.56 & 333 & $P$ & 37 \\
\hline & & & is & 38 & 49.00 & & 1 & $2.3 \mathrm{~s}$ & 19 & $9.20 \mathrm{~nm}$ & \\
\hline PRA & 9.21 & 356 & iPc & 37 & 07.00 & -2.2 & BRN & 11.63 & 354 & ePC & 37 \\
\hline BSF & 9.25 & 322 & $P$ & 37 & 07.70 & -2.2 & I MEM & 11.69 & 329 & $\mathbf{P}$ & 37 \\
\hline & & & Sn & 38 & 48.60 & & I DOU & 11.89 & 324 & $P$ & 37 \\
\hline ISR & 9.25 & 59 & eP & 37 & 09.00 & -1.0 & 1 & & & $\epsilon$ & 37 \\
\hline GRF & 9.27 & 343 & iPc & 37 & 07.20 & -2.9 & 1 & & & $S$ & 40 \\
\hline & & & eSn & 38 & 47.20 & & 1 & & & $P \subset P$ & 43 \\
\hline BUH & 9.28 & 329 & eP & 37 & 07.70 & -2.6 & I HEE & 11.92 & 330 & iPd & 37 \\
\hline ECH & 9.36 & 324 & eP & 37 & 08.90 & -2.5 & I WAR & 12.01 & 17 & ePc & 37 \\
\hline DMK & 9.40 & 80 & $P$ & 37 & 09.20 & -2.8 & 1 & & & $\boldsymbol{e}$ & 38 \\
\hline CDF & 9.46 & 325 & $P$ & 37 & 10.20 & -2.6 & 1 & & & $\epsilon$ & 38 \\
\hline & & & Sn & 38 & 52.40 & & 1 & & & $\epsilon$ & 38 \\
\hline $12 M$ & 9.54 & 101 & $P$ & 37 & 12.60 & -1.3 & 1 & & & $\epsilon$ & 40 \\
\hline EDC & 9.55 & 89 & $P$ & 37 & 12.20 & -1.9 & I ATE & 12.13 & 286 & $\in P$ & 37 \\
\hline HAU & 9.58 & 321 & $P$ & 37 & 12.10 & -2.4 & I BCK & 12.33 & 101 & $P-$ & 37 \\
\hline & & & Sn & 38 & 57.70 & & I ZGT & 12.40 & 82 & $P$ & 37 \\
\hline VR I & 9.68 & 55 & eP & 37 & 15.00 & -0.8 & $|A L|$ & 12.45 & 263 & $i P_{c}$ & 37 \\
\hline KRA & 9.73 & 18 & eP & 37 & 16.70 & 0.3 & 1 & & & $e$ & 40 \\
\hline & $2.0 \mathrm{~s}$ & 319 & $0.00 \mathrm{~nm}$ & & 7 & $.4 \mathrm{mb} \times$ & I UCC & 12.51 & 326 & Pct & 37 \\
\hline & & & $i$ & 37 & 18.20 & & 1 & & & e & 38 \\
\hline & & & i & 37 & 21.20 & & 1 & & & s & 40 \\
\hline & & & $i$ & 37 & 27.20 & & I MFF & 12.56 & 302 & P & 37 \\
\hline & & & $i$ & 37 & 32.30 & & I WTS & 12.56 & 335 & iPc & 37 \\
\hline & & & is & 39 & 17.00 & & $M G N$ & 12.76 & 84 & $P$ & 37 \\
\hline & & & e & 56 & 43.20 & & I HAM & 13.11 & 346 & iPd & 38 \\
\hline & & & $i$ & 56 & 47.70 & & I DBN & 13.20 & 332 & iPd & 38 \\
\hline BRD & 9.73 & 58 & eP & 37 & 17.00 & 0.4 & I & & & $\epsilon$ & 39 \\
\hline HOF & 9.73 & 347 & eP & 37 & 13.80 & -2.7 & 1 & & & es & 40 \\
\hline GWF & 9.76 & 329 & eP & 37 & 14.80 & -2.1 & I WIT & 13.31 & 337 & $e P$ & 38 \\
\hline ODB & 9.84 & 56 & eP & 37 & 22.00 & $3.9 x$ & I SYT & 13.34 & 100 & $\mathrm{Pt}$ & 38 \\
\hline KSP & 9.98 & 4 & eP & 37 & 18.50 & -1.5 & I ANTO & 13.36 & 89 & EP & 38 \\
\hline & & & $\mathrm{i}$ & 37 & 21.30 & & 1 & $0.9 \mathrm{~s}$ & & $5.00 \mathrm{~nm}$ & \\
\hline BRG & 10.04 & 355 & iPd & 37 & 17.90 & -2.8 & 1 & & & $i$ & 38 \\
\hline T LB & 10.05 & 64 & eP & 37 & 08.00 & $-13 \cdot 0 x$ & $1 \mathrm{SSC}$ & 13.39 & 310 & $P$ & 38 \\
\hline MOX & 10.10 & 346 & iPd & 37 & 19.30 & -2.3 & I LGR & 13.42 & 283 & iPc & 38 \\
\hline & $1.2 \mathrm{~s}$ & 95 & $5.00 \mathrm{~nm}$ & & & $.1 \mathrm{mb} x$ & 1 & $1.6 \mathrm{~s}$ & & $1.91 \mathrm{~nm}$ & \\
\hline & $15 \mathrm{~s}$ & 779 & $.00 \mathrm{um}$ & & & & 1 & & & iPP & 38 \\
\hline & $20 \mathrm{~s}$ & 140 & $9.00 \mathrm{um}$ & & & & 1 & & & is & 40 \\
\hline SMF & 10.10 & 309 & $P$ & 37 & 21.30 & -0.4 & I LPF & 3.68 & 307 & $P$ & 38 \\
\hline$B A C$ & 10.12 & 52 & $e^{P}$ & 37 & 23.00 & 1.2 & I FLN & 13.69 & 310 & $P$ & 38 \\
\hline LBF & 10.20 & 310 & $P$ & 37 & 21.30 & -1.7 & I GRR & 13.73 & 309 & $P$ & 38 \\
\hline PYM & 10.21 & 303 & $P$ & 37 & 23.50 & 0.3 & I KAS & 13.91 & 82 & P- & 38 \\
\hline & & & $\mathrm{Pg}$ & 38 & 10.30 & & I SIM & 14.35 & 67 & $P c$ & 38 \\
\hline & & & Sn & 39 & 26.50 & & 1 & & & is & 40 \\
\hline & & & $\mathrm{Sg}$ & 40 & 31.10 & & I $A L M$ & 14.43 & 260 & ¡Po & 38 \\
\hline CFR & 10.33 & 61 & eP & 37 & 22.00 & -2.7 & i & $12 \mathrm{~s}$ & & $3.00 \mathrm{um}$ & \\
\hline ISK & 10.39 & 85 & $P$ & 37 & 23.70 & -1.9 & 1 & & & iPP & 38 \\
\hline BIR & 10.42 & 55 & $e P$ & 37 & 29.00 & $3.0 x$ & 1 & & & is & 41 \\
\hline LOR & 10.42 & 312 & $P$ & 37 & 25.10 & -1.0 & I GUD & 14.77 & 275 & iPd & 38 \\
\hline CIN & 10.43 & 104 & eP & 37 & 27.00 & 0.9 & I TOL & 14.80 & 272 & Pc & 38 \\
\hline & & & $\mathrm{i}$ & 37 & 32.00 & & 1 & & & i & 38 \\
\hline & & & isg & 37 & 56.00 & & 1 & & & is & 41 \\
\hline AVF & 10.47 & 308 & $P$ & 37 & 27.20 & 0.6 & I COP & 14.93 & 354 & IPd & 38 \\
\hline TNS & 10.50 & 335 & eP & 37 & 24.60 & -2.6 & i & 1.45 & 945 & $9.00 \mathrm{~nm}$ & \\
\hline & & & es & 39 & 14.30 & & 1 & & & is & 41 \\
\hline SSF & 10.51 & 310 & $P$ & 37 & 27.60 & 0.3 & I CRT & 15.16 & 262 & iPC & 38 \\
\hline$C L L$ & 10.55 & 352 & iP & 37 & 24.90 & -2.8 & i & & & $\mathrm{i}$ & 41 \\
\hline & & & $\mathrm{i}$ & 37 & 28.00 & & 1 & & & $\epsilon$ & 46 \\
\hline & & & $i$ & 37 & 37.00 & & I KVT & 15.65 & 82 & $P$ & 38 \\
\hline & & & $i$ & 37 & 42.00 & & I MAL & 15.93 & 261 & iPo & 38 \\
\hline & & & $i$ & 37 & 47.00 & & 1 & & & is & 41 \\
\hline & & & $\mathrm{i}$ & 37 & 55.00 & & I HLW & 17.05 & 125 & iPc & 38 \\
\hline & & & $\mathrm{Pg}$ & 38 & 23.00 & & I & $3.0 \mathrm{~s}$ & 80 & $0.00 \mathrm{~nm}$ & \\
\hline & & & $S$ & 39 & 20.00 & & 1 & & & is & 43 \\
\hline & & & i & 39 & 38.00 & & I MTE & 17.35 & 276 & $P$ & 38 \\
\hline & & & $\mathrm{i}$ & 40 & 00 & & | & & & & 42 \\
\hline
\end{tabular}


$23 d \quad 18 h$

\begin{tabular}{|c|c|c|c|c|c|c|c|c|c|c|c|}
\hline APA & 28.48 & 14 & $P d$ & 40 & 52.00 & 1.5 & | DD I & 51.10 & 81 & $P$ & 44 \\
\hline & & & $i s p$ & 40 & 54.00 & & i & & & s & 51 \\
\hline & & & is & 45 & 33.00 & & | NDI & 51.30 & 83 & eP & 44 \\
\hline TEN & 28.61 & 254 & Pc & 40 & 52.70 & 0.7 & i & $20 \mathrm{~s}$ & 62 & $2.40 u \mathrm{~m}$ & \\
\hline & & & $s$ & 45 & 51.80 & & i & $20 \mathrm{~s}$ & 44 & $4.80 \mathrm{um}$ & \\
\hline TRO & 28.88 & 3 & ip & 40 & 54.20 & 0.2 & i & & & es & 51 \\
\hline KEV & 29.58 & 8 & $\mathrm{Pc}$ & 41 & 01.20 & 0.9 & 1 WMO & 51.97 & 61 & Pd & 44 \\
\hline & & & es & 45 & 52.00 & & i & & & PP & 46 \\
\hline SHD & 31.07 & 85 & eP & 41 & 16.70 & 2.6 & I UER & 53.01 & 50 & Pd & 44 \\
\hline$A K U$ & 31.19 & 334 & iP & 41 & 16.30 & 1.7 & I ВOM & 53.44 & 96 & $\mathrm{P}+$ & 44 \\
\hline & $1.2 \mathrm{~s}$ & 3400 & $0.00 \mathrm{~nm}$ & & & $.1 \mathrm{mb} X$ & i & & & PP & 46 \\
\hline & $z \quad 19 s$ & 100 & $0.00 \mathrm{um}$ & & & $.5 M s z$ & i & & & $S$ & 51 \\
\hline & & & $\mathrm{i}$ & 41 & 20.10 & $13 \mathrm{~km}$ & i & & & PPS & 52 \\
\hline KAT & 31.19 & 79 & Pd & 41 & 18.00 & $3.1 x$ & 1 & & & SS & 56 \\
\hline & & & es & 46 & 27.00 & & I POO & 54.44 & 96 & $\mathrm{P}+$ & 44 \\
\hline REY & 31.71 & 330 & iP & 41 & 20.20 & 1.0 & $i$ & & & PP & 46 \\
\hline ARU & 31.91 & 46 & $e P$ & 41 & 19.00 & -2.1 & i & & & PPP & 47 \\
\hline & & & es & 46 & 35.00 & & i & & & PS & 52 \\
\hline SHI & 32.16 & 99 & eP & 41 & 24.00 & 0.3 & i & & & ses & 54 \\
\hline SVE & 33.09 & 46 & ePd & 41 & 31.00 & -0.3 & I GOA & 56.29 & 99 & $P$ & 44 \\
\hline & & & isp & 41 & 36.00 & & I VAR & 57.48 & 83 & P- & 44 \\
\hline & & & is & 46 & 48.00 & & i & & & $P C P$ & 45 \\
\hline $\mathrm{KHI}$ & 34.73 & 87 & iPc & 41 & 45.80 & -0.3 & i & & & PP & 46 \\
\hline TGI & 35.65 & 88 & eP & 41 & 53.40 & -0.5 & 1 & & & PPP & 48 \\
\hline $\mathrm{BCAO}$ & 36.40 & 175 & eP & 41 & 56.50 & $-3.6 x$ & i & & & PS & 52 \\
\hline & $1.1 \mathrm{~s}$ & 66 & $5.00 \mathrm{~nm}$ & & & $4 \mathrm{mb}$ & i & & & PPS & 52 \\
\hline$B N G$ & 36.40 & 175 & iPd & 41 & 56.80 & $-3.3 x$ & i & & & PPS & 53 \\
\hline & $0.7 \mathrm{~s}$ & 24 & $4.90 \mathrm{~nm}$ & & & $.1 \mathrm{mb}$ & 1 & & & Scs & 54 \\
\hline KDS & 37.12 & 228 & iP & 42 & 05.40 & -0.7 & 1 & & & sS & 56 \\
\hline KUK & 37.29 & 206 & $P$ & 42 & 06.50 & -1.1 & I IRK & 58.49 & 46 & iPd & 44 \\
\hline$T D D$ & 37.76 & 132 & ep & 42 & 12.40 & 0.9 & I TIK & 58.51 & 20 & Pd & 44 \\
\hline AAE & 37.93 & 140 & $e(P)$ & 42 & 13.50 & 0.2 & i & & & epP & 44 \\
\hline ARO & 37.96 & 133 & IPd & 42 & 14.90 & 1.7 & 1 & & & esp & 44 \\
\hline & & & ePP & 43 & 52.00 & & I HYB & 58.67 & 94 & $e P$ & 44 \\
\hline & & & ess & 50 & 59.20 & & 1 & & & es & 52 \\
\hline KBS & 38.17 & 359 & iP & 42 & 15.70 & 1.4 & I & & & eP'P' & 14 \\
\hline ATA & 38.22 & 132 & eP & 42 & 17.60 & 2.3 & I ZAK & 58.79 & 48 & eP & 44 \\
\hline MBO & 38.54 & 236 & ip & 42 & 20.40 & 2.4 & i & & & es & 53 \\
\hline & & & $\mathrm{i}$ & 42 & 21.80 & $5 \mathrm{~km}$ & I KR I & 58.93 & 164 & P- & 44 \\
\hline & & & $\mathbf{i}$ & 42 & 24.00 & & I & & & eP'P. & 14 \\
\hline & & & $\mathrm{i}$ & 42 & 33.00 & & I CLK & 59.16 & 158 & P- & 44 \\
\hline & & & $\mathrm{i}$ & 42 & 45.00 & & i & & & $e P^{\prime} P^{\prime}$ & 14 \\
\hline & & & $\mathrm{i}$ & 43 & 33.00 & & I TET & 59.21 & 159 & eP & 44 \\
\hline KIC & 38.77 & 213 & eP & 42 & 18.30 & -1.7 & i & $1.5 \mathrm{~s}$ & & $0.62 \mathrm{~nm}$ & \\
\hline DAG & 38.82 & 348 & iPc & 42 & 19.00 & -0.8 & $i$ & & & e & 45 \\
\hline & $1.2 \mathrm{~s}$ & 453 & 3. $00 \mathrm{~nm}$ & & 6 & $\theta m b$ & $i$ & & & e & 47 \\
\hline & & & iPPP & 43 & 56.00 & & i & & & $e$ & 48 \\
\hline & & & is & 48 & 20.00 & & 1 & & & e & 10 \\
\hline SAM & 38.96 & 74 & ePc & 42 & 21.00 & -0.5 & I MTD & 59.32 & 162 & eP & 44 \\
\hline & & & $i s P$ & 42 & 24.00 & & I NPA & 59.95 & 153 & eP & 45 \\
\hline TAS & 40.11 & 71 & ePc & 42 & 31.00 & 0.1 & 1 & & & $e$ & 45 \\
\hline & & & es & 48 & 41.00 & & I GBA & 60.18 & 98 & Pd & 45 \\
\hline DSH & 40.65 & 75 & Pd & 42 & 37.00 & 1.6 & 1 & $1.4 \mathrm{~s}$ & 131 & $1.00 \mathrm{~nm}$ & \\
\hline GAR & 41.62 & 74 & eP & 42 & 43.00 & -0.5 & I $80 K$ & 60.39 & 83 & $\mathbf{P}$ & 45 \\
\hline & & & epp & 42 & 43.60 & $2 \mathrm{~km}$ & $1 B O D$ & 60.76 & 37 & eP & 45 \\
\hline & & & es & 49 & 07.60 & & I LSA & 61.12 & 74 & Pd & 45 \\
\hline KAAO & 42.41 & 81 & $e P$ & 42 & 50.30 & 0.2 & i & & & $s$ & 53 \\
\hline & $1.0 \mathrm{~s}$ & 42 & $2.00 \mathrm{~nm}$ & & & $1 \mathrm{mb}$ & 1 BUL & 61.96 & 166 & P- & 45 \\
\hline KBL & 42.41 & 81 & iPc & 42 & 50.00 & -0.1 & i & & & eP'P' & 14 \\
\hline ANR & 42.49 & 71 & ePd & 42 & 51.00 & 0.6 & I VIs & 62.08 & 90 & $P$ & 45 \\
\hline & & & es & 49 & 21.00 & & & & & $s$ & 53 \\
\hline $\mathrm{KHE}$ & 42.73 & 9 & ePd & 42 & 54.00 & 2.1 & I MDV & 62.25 & 305 & $\mathbf{P}$ & 45 \\
\hline & & & is & 49 & 17.00 & & I KOD & 62.37 & 101 & Pd & 45 \\
\hline OUE & 42.80 & 88 & $e P$ & 42 & 53.18 & -0.2 & I & & & $P C P$ & 45 \\
\hline & & & es & 49 & 22.00 & & 1 & & & PP & 47 \\
\hline $\mathrm{KHO}$ & 43.07 & 76 & $P$ & 42 & 55.70 & 0.2 & I & & & PPP & 49 \\
\hline & & & $s$ & 49 & 22.70 & & i & & & PS & 54 \\
\hline FRU & 43.41 & 67 & ePd & 42 & 58.00 & 0.1 & i & & & PPS & 54 \\
\hline & & & $i s p$ & 43 & 01.50 & & i & & & SSS & 00 \\
\hline & & & is & 49 & 33.60 & & 1 & & & LO & 01 \\
\hline GDH & 45.64 & 332 & IPd & 43 & 11.50 & 0.8 & i & & & LR & 03 \\
\hline & $0.8 \mathrm{~s}$ & 313 & $3.00 \mathrm{~nm}$ & & & $3 m b$ & I WNY & 62.47 & 306 & $P$ & 45 \\
\hline & & & IPCP & 45 & 00.00 & & MDR & 62.63 & 97 & $P$ & 45 \\
\hline & & & is & 49 & 45.00 & & i & & & $s$ & 53 \\
\hline TLG & 45.19 & 65 & $\mathrm{Pc}$ & 43 & 12.00 & -0.3 & I CAL & 63.07 & 83 & P- & 45 \\
\hline & & & epP & 43 & 13.00 & $3 \mathrm{~km}$ & i & & & $S$ & 53 \\
\hline & & & is & 49 & 58.00 & & I APH & 63.14 & 306 & $P$ & 45 \\
\hline KSH & 45.54 & 71 & Pd & 43 & 17.00 & 1.7 & I SHL & 63.96 & 78 & $P-$ & 45 \\
\hline & & & is & 49 & 59.00 & & i & & & $S$ & 54 \\
\hline NVS & 45.85 & 48 & $P$ & 43 & 16.60 & -0.7 & I GPD & 64.63 & 303 & $\mathbf{P}$ & 45 \\
\hline & & & is & 50 & 01.80 & & I YAK & 64.71 & 28 & Pd & 45 \\
\hline PRZ & 46.15 & 66 & eP & 43 & 21.00 & 0.9 & i & & & $i s P$ & 45 \\
\hline NA : & 46.29 & 150 & eP & 43 & 24.00 & 2.5 & 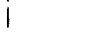 & & & is & 54 \\
\hline & $3.0 \mathrm{~s}$ & 1600 & $.00 \mathrm{~nm}$ & & & $5 \mathrm{mb}$ & I PRIN & 65.15 & 303 & $P$ & 45 \\
\hline BHK & 49.34 & 81 & $P$ & 43 & 47.00 & 2.0 & I LZH & 66.53 & 62 & Pd & 45 \\
\hline & & & 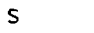 & 50 & 52.20 & & I AVY & 66.68 & 146 & ePd & 45 \\
\hline$J M$ & 50.43 & 88 & $P+$ & 43 & 52.00 & -1.3 & I KSR & 67.27 & 169 & iPd & 45 \\
\hline
\end{tabular}




\begin{tabular}{|c|c|c|c|c|c|c|c|c|c|c|c|}
\hline SVW & & 78.09 & 356 & eP & 46 & 55.00 & 1.2 & 1 & & & \\
\hline LD3 & & 78.69 & 323 & iPd & 46 & 58.80 & 1.4 & 1 & ANP & & 85.16 \\
\hline & & $1.5 \mathrm{~s}$ & 572 & $2.00 \mathrm{~nm}$ & & & $6.4 \mathrm{mb}$ & 1 & & & \\
\hline VLA & & 78.71 & 42 & $P d$ & 46 & 57.60 & 0.1 & 1 & TATO & & 85.28 \\
\hline NJ2 & & 78.83 & 57 & $P d$ & 46 & 59.00 & 0.7 & 1 & & & $1.5 \mathrm{~s}$ \\
\hline SIT & & 79.34 & 344 & eP & 47 & 03.10 & 2.5 & I & SHK & & 85.39 \\
\hline TOV & & 80.00 & 274 & eP & 47 & 07.00 & 2.0 & 1 & & & \\
\hline 8BJ & & 80.11 & 286 & $e P$ & 47 & 06.30 & 0.8 & I & MSA & & 85.81 \\
\hline RLO & & 80.23 & 310 & $e^{P}$ & 47 & 5.50 & -0.3 & 1 & LUB & & 86.00 \\
\hline & & & & $\mathrm{i}$ & 47 & 36 & $6 \mathrm{~km}$ & I & $M I Z$ & & 86.28 \\
\hline$\times F$ & & 80.51 & 329 & iP & 47 & 08.10 & 0.8 & 1 & & & \\
\hline YKM & & 80.74 & 330 & iP & 47 & 09.30 & 0.8 & I & BOG & & 86.55 \\
\hline YSS & & 80.77 & 33 & ePd & 47 & 96 & 0.6 & 1 & & & \\
\hline & & & & ipp & 47 & 0 & $3 \mathrm{~km}$ & 1 & PPI & & 86.56 \\
\hline & & & & is & 57 & 0 & & 1 & & & 1. \\
\hline LGN & & 80.88 & 275 & eP & 47 & 0 & -0.6 & 1 & $A B U$ & & 86.74 \\
\hline Lt & & 80 & 329 & ip & 7 & $\theta$ & 1.1 & 1 & HEN & & 86 \\
\hline SSE & & 80.95 & 57 & $P d$ & 47 & 0 & 0.3 & 1 & MAT & & 86.85 \\
\hline & & & & PP & 50 & 11.00 & & 1 & & & $1.9 \mathrm{~s}$ \\
\hline & & & & s & 57 & 13.00 & & 1 & & & \\
\hline GZH & & 80.98 & 67 & Pd & 47 & 11.00 & 1.1 & 1 & $\mathrm{JCT}$ & & 87.07 \\
\hline & & & & PP & 50 & 24.00 & & 1 & & z & $20 s$ \\
\hline BAO & & 81.17 & 241 & eP & 47 & 11.00 & -0.2 & 1 & & & \\
\hline$D C$ & & 81.20 & 353 & $\in P$ & 47 & 1.10 & 0.6 & 1 & COR & & 87.16 \\
\hline SDV & & 81.21 & 274 & eP & 47 & 1.30 & -0.3 & 1 & KGM & & 87.17 \\
\hline PET & & 81.25 & 21 & eP & 47 & 10.00 & -0.9 & 1 & & & $1.6 \mathrm{~s}$ \\
\hline & & & & esp & 47 & 14.80 & & 1 & $A L O$ & & 87.50 \\
\hline & & & & es & 57 & 10.00 & & 1 & & $z$ & $19 \mathrm{~s}$ \\
\hline OR & & 81.39 & 293 & $P d$ & 47 & 12.00 & -0.2 & 1 & $\mathrm{CHN}$ & & 87.50 \\
\hline MSO & & 81.69 & 328 & iPd & 47 & 14.60 & 1.1 & 1 & MSU & & 87.58 \\
\hline & & $1.6 \mathrm{~s}$ & 560 & $0.00 \mathrm{~nm}$ & & & $6.4 \mathrm{mb}$ & 1 & DDR & & 87.77 \\
\hline & $z$ & $20 \mathrm{~s}$ & 59 & 9.30 um & & & $6.9 M s z$ & 1 & TSK & & 88.07 \\
\hline & & & & $\mathrm{PCP}$ & 47 & 43.50 & & 1 & BMN & & 88.29 \\
\hline & & & & es & 57 & 29.60 & & 1 & & & $1.5 \mathrm{~s}$ \\
\hline NEW & & 81.77 & 330 & iPd & 47 & 15.10 & 1.3 & 1 & EUR & & 88.53 \\
\hline & Z & $18 \mathrm{~s}$ & 86 & $6.00 \mathrm{um}$ & & & 7. $2 M s z$ & 1 & & & $1.1 \mathrm{~s}$ \\
\hline & $\mathrm{N}$ & $18 \mathrm{~s}$ & & $5.00 \mathrm{um}$ & & & & 1 & $P \mid P$ & & 89.21 \\
\hline & $E$ & $18 \mathrm{~s}$ & & $5.00 \mathrm{um}$ & & & & 1 & $M I N$ & & 90.17 \\
\hline & & & & e & 47 & 29.00 & $48 \mathrm{kmX}$ & 1 & WCN & & 90.29 \\
\hline & & & & e & 47 & 34.00 & & 1 & WDC & & 90.33 \\
\hline UAV & & 81.77 & 274 & eP & 47 & 15.00 & 0.5 & 1 & & & \\
\hline & & $0.6 \mathrm{~s}$ & 28 & $8.60 \mathrm{~nm}$ & & & $5.5 \mathrm{mb}$ & 1 & & & \\
\hline SNG & & 81.80 & 88 & $\mathrm{iPC}$ & 47 & 16.00 & 1.6 & 1 & BAG & & 90.48 \\
\hline & & $1.4 \mathrm{~s}$ & & $5.00 \mathrm{~nm}$ & & & $4.9 \mathrm{mb} \times$ & 1 & & & \\
\hline & & & & es & 57 & 20.00 & & 1 & $\mathrm{FHC}$ & & 90.69 \\
\hline BCM & & 81.90 & 326 & $\mathrm{iPd}$ & 47 & 15.96 & 1.2 & 1 & ORV & & 90.80 \\
\hline & & & & $e(p P)$ & 47 & 54.80 & $156 \mathrm{kmx}$ & 1 & & & \\
\hline HKC & & 82.07 & 68 & $P$ & 47 & 18.06 & 2.4 & 1 & PSO & & 91.24 \\
\hline & & & & $\mathrm{pP}$ & 47 & 36.00 & $65 \mathrm{kmx}$ & 1 & LPS & & 91.37 \\
\hline & & & & PPP & 50 & 46.00 & & 1 & & & $1.2 \mathrm{~s}$ \\
\hline & & & & () & 52 & 18.06 & & 1 & & & \\
\hline & & & & $\mathrm{s}$ & 57 & 32.00 & & 1 & JAS & & 91.73 \\
\hline$Y M C$ & & 82.10 & 325 & $P$ & 47 & 17.46 & 1.6 & 1 & & & \\
\hline SKR & & 82.58 & 24 & $P c$ & 47 & 20.00 & 2.1 & I & & & \\
\hline 151 & & 82.61 & 92 & $\in P c$ & 47 & 10.80 & $-7.8 x$ & 1 & TUC & & 91.81 \\
\hline & & $0.9 \mathrm{~s}$ & 326 & $6.00 \mathrm{~nm}$ & & & $5.5 \mathrm{mb}$ & 1 & MAN & & 92.02 \\
\hline BDW & & 83.00 & 323 & $i \mathrm{Pd}$ & 47 & 21.00 & 0.5 & 1 & & & \\
\hline & & $1.5 \mathrm{~s}$ & 446 & $6.00 \mathrm{~nm}$ & & & $5.4 \mathrm{mb}$ & | & FRI & & 92.23 \\
\hline GLD & & 83.21 & 318 & $\mathrm{P}$ & 47 & 23.20 & 1.6 & 1 & & & \\
\hline & $z$ & $18 \mathrm{~s}$ & 73 & $3.00 \mathrm{um}$ & & & 7. $1 \mathrm{Msz}$ & 1 & & & \\
\hline SAP & & 83.29 & 37 & eP & 47 & 23.06 & 1.4 & 1 & BKS & & 92.53 \\
\hline & & & & es & 57 & 42.06 & & 1 & & & $2.0 \mathrm{~s}$ \\
\hline GOL & & 83.33 & 318 & iP & 47 & 23.20 & 0.9 & 1 & & 2 & $20 s$ \\
\hline & & $1.5 \mathrm{~s}$ & 402 & $2.00 \mathrm{~nm}$ & & & $5.4 \mathrm{mb}$ & 1 & & $N$ & $20 s$ \\
\hline & $z$ & $18 \mathrm{~s}$ & 48 & 3. $00 \mathrm{um}$ & & & 6. $9 \mathrm{Msz}$ & 1 & & $E$ & $20 \mathrm{~s}$ \\
\hline $2 \mathrm{ZH}$ & & 83.37 & 63 & $\mathrm{Pd}$ & 47 & 22.50 & 0.2 & ( & & & \\
\hline & & & & $P P$ & 50 & 43.00 & & I & ARN & & 92.69 \\
\hline & & & & es & 57 & 40.00 & & 1 & $M H C$ & & 92.75 \\
\hline SI & & 83.40 & 92 & ePc & 47 & 21.10 & -1.6 & 1 & & & \\
\hline & & $1.4 \mathrm{~s}$ & 491 & $1.00 \mathrm{~nm}$ & & & $5.5 \mathrm{mb}$ & 1 & & & \\
\hline & & & & $\mathrm{i}$ & 47 & 23.80 & $9 \mathrm{~km}$ & 1 & OAQ & & 93.04 \\
\hline$G A L$ & & 83.48 & 279 & iP & 47 & 10.50 & $-12.5 x$ & 1 & & & \\
\hline RDJ & & 83.53 & 232 & $P d$ & 47 & 24.40 & 1.4 & 1 & SAO & & 93.16 \\
\hline & & & & ePP & 50 & 38.00 & & 1 & GLA & & 93.25 \\
\hline & & & & es & 57 & 48.40 & & 1 & NCE & & 93.33 \\
\hline DGC & & 83.69 & 334 & eP & 47 & 24.40 & 0.8 & 1 & PR I & & 93.36 \\
\hline IPM & & 83.78 & 89 & ePd & 47 & 24.10 & -0.5 & 1 & & & \\
\hline & & $1.1 \mathrm{~s}$ & 91 & $1.80 \mathrm{~nm}$ & & & $.9 \mathrm{mb}$ & 1 & PPR & & 93.63 \\
\hline $50 \mathrm{~N}$ & & 84.08 & 358 & eP & 47 & 27.20 & 1.8 & 1 & & & $1.2 \mathrm{~s}$ \\
\hline KUR & & 84.31 & 32 & Pc & 47 & 30.40 & $3.6 x$ & 1 & PAS & & 93.93 \\
\hline & & & & is & 57 & 55.00 & & 1 & & & $2.0 \mathrm{~s}$ \\
\hline MW & & 84.42 & 333 & $P$ & 47 & 29.06 & 1.6 & 1 & & $z$ & $20 \mathrm{~s}$ \\
\hline LON & & 84.76 & 332 & iPd & 47 & 30.40 & 1.2 & ! & & $N$ & $20 \mathrm{~s}$ \\
\hline & & $1.8 \mathrm{~s}$ & 1300 & $3.00 \mathrm{~nm}$ & & & $5.9 \mathrm{mb}$ & 1 & & $E$ & $20 \mathrm{~s}$ \\
\hline MY & & 84.97 & 13 & $e(P)$ & 47 & 31.70 & 1.8 & & & & \\
\hline & Z & $18 \mathrm{~s}$ & 20 & $.00 \mathrm{um}$ & & & $.5 \mathrm{MsZ}$ & 1 & & & \\
\hline HA & & 85.02 & 78 & & 47 & 32.00 & 1 & & & & \\
\hline
\end{tabular}




\begin{tabular}{|c|c|c|c|c|c|}
\hline \multirow{3}{*}{$\begin{array}{l}\text { RKT } \\
\text { NOU }\end{array}$} & \multirow{3}{*}{$\begin{array}{c}1.5 \mathrm{~s} \\
149.42 \\
1.3 \mathrm{~s} \\
149.42\end{array}$} & \multicolumn{2}{|c|}{$1440.88 \mathrm{~nm}$} & \\
\hline & & $\begin{array}{c}296 \\
570\end{array}$ & $\begin{array}{l}\text { iPKP } \\
0.00 \mathrm{~nm}\end{array}$ & 54 & 46.50 \\
\hline & & 62 & PKPd & 54 & 40.50 \\
\hline & 149.49 & 311 & iPKP & 54 & 46.70 \\
\hline & $1.5 \mathrm{~s}$ & 920 & $0.00 \mathrm{~nm}$ & & \\
\hline NAE & $\begin{array}{c}149.58 \\
1.5 \mathrm{~s}\end{array}$ & $\begin{array}{l}310 \\
1040\end{array}$ & $\begin{array}{l}\text { ¡PKP } \\
\theta .00 \mathrm{~nm}\end{array}$ & 54 & 46.98 \\
\hline FRT & $\begin{array}{r}149.60 \\
1.5 \mathrm{~s}\end{array}$ & $\begin{array}{c}311 \\
688\end{array}$ & $\begin{array}{l}\text { ¿PKP } \\
\text { Q. OOBnm }\end{array}$ & 54 & 46.98 \\
\hline TPT & $\begin{array}{c}150.23 \\
1.5 \mathrm{~s}\end{array}$ & $\begin{array}{l}325 \\
1600\end{array}$ & $\begin{array}{l}\text { iPKP } \\
0.00 \mathrm{~nm}\end{array}$ & 54 & 48.80 \\
\hline RUV & $\begin{array}{c}150.31 \\
1.5 \mathrm{~s}\end{array}$ & $\begin{array}{c}325 \\
960\end{array}$ & $\begin{array}{l}\text { ¡PKP } \\
9.00 \mathrm{~nm}\end{array}$ & 54 & 48.80 \\
\hline PMO & $\begin{array}{c}150.37 \\
1.5 \mathrm{~s}\end{array}$ & $\begin{array}{c}326 \\
960\end{array}$ & $\begin{array}{l}\text { iPKP } \\
\theta .00 \mathrm{~nm}\end{array}$ & 54 & 49.88 \\
\hline VAH & $\begin{array}{c}150.46 \\
1.5 \mathrm{~s}\end{array}$ & $\begin{array}{c}325 \\
800\end{array}$ & $\begin{array}{l}\text { ¡PKP } \\
0.00 \mathrm{~nm}\end{array}$ & 54 & 49.00 \\
\hline$A F I$ & 152.42 & 15 & PKP & 54 & 44.00 \\
\hline & & & PP & 58 & 29.00 \\
\hline & & & PPP & 02 & 10.00 \\
\hline & & & SKSP & 08 & 36.00 \\
\hline & & & SKKS & 11 & 17.00 \\
\hline & & & SSP & 18 & 44.08 \\
\hline & & & SSS & 23 & 25.00 \\
\hline & & & LQ & 35 & 00.00 \\
\hline PPN & $\begin{array}{c}153.28 \\
1.5 \mathrm{~s}\end{array}$ & $\begin{array}{c}326 \\
330\end{array}$ & $\begin{array}{l}\text { ePKP } \\
0.00 \mathrm{~nm}\end{array}$ & 54 & 51.00 \\
\hline PPT & $\begin{array}{c}153.37 \\
1.5 \mathrm{~s}\end{array}$ & $\begin{array}{c}326 \\
52 \theta\end{array}$ & $\begin{array}{c}\text { ePKP } \\
9.00 \mathrm{~nm}\end{array}$ & 54 & 51.00 \\
\hline TVO & $\begin{array}{c}153.42 \\
1.5 \mathrm{~s}\end{array}$ & $\begin{array}{c}326 \\
720\end{array}$ & $\begin{array}{l}\text { iPKP } \\
.0 \theta \mathrm{nm}\end{array}$ & 54 & 50.80 \\
\hline AFR & $\begin{array}{c}153.43 \\
1.5 \mathrm{~s}\end{array}$ & $\begin{array}{r}327 \\
520\end{array}$ & $\begin{array}{l}\text { ePKP } \\
9.00 \mathrm{~nm}\end{array}$ & 54 & .00 \\
\hline PAE & $\begin{array}{c}153.46 \\
1.5 \mathrm{~s}\end{array}$ & $\begin{array}{c}326 \\
560\end{array}$ & $\begin{array}{c}\text { ePKP } \\
0.00 \mathrm{~nm}\end{array}$ & 54 & 51.00 \\
\hline MSZ & 159.57 & 110 & $\begin{array}{l}\text { ePKP } \\
\text { e }\end{array}$ & $\begin{array}{l}55 \\
55\end{array}$ & $\begin{array}{l}04.00 \\
37.00\end{array}$ \\
\hline$A R$ & 159.95 & 347 & ePKP & 55 & 01.00 \\
\hline HP & 161.13 & 108 & iPKP & 54 & 56.50 \\
\hline & & & $P P$ & 55 & \\
\hline & & & e & 56 & 19.00 \\
\hline & & & e & 59 & 31.00 \\
\hline $\mathrm{COE}$ & 62.94 & 98 & iPK & 54 & 50.80 \\
\hline KRP & 164.42 & 85 & iPKP & 54 & 54.80 \\
\hline VEL & 164.49 & 98 & PKP & 54 & 54.00 \\
\hline & & & $P P$ & 58 & 56.00 \\
\hline & & & PPP & 03 & 32.00 \\
\hline & & & PCPP. & 05 & 05.00 \\
\hline & & & SKKS & 07 & 34.00 \\
\hline & & & SKSP & 12 & 25.00 \\
\hline & & & SS & 20 & 30.00 \\
\hline & & & sS & 27 & 06.00 \\
\hline
\end{tabular}

\begin{tabular}{l|l}
$6.5 x$ & YAM \\
0.5 & FKS \\
$6.5 \times$ & HAC \\
$6.6 \times$ & $\begin{array}{l}\text { SKH } \\
\text { YAMJ }\end{array}$ \\
$6.6 \times$ & ONA \\
$7.5 \times$ & AKI \\
$7.3 \times$ & SHR \\
$7.5 \times$ & AOM \\
$7.3 \times$ & MOM \\
7.7 & UTS \\
7 & NIJ
\end{tabular}

$5.3 \times$ MAE

$5.1 \times$ TOK

$4.7 \times \quad$ DOR

TKD

5. $0 \times$ I MRRJ

$\mathrm{CHJJ}$

$5.0 \times \quad$ NGN MAT

\begin{tabular}{l|l}
$11.1 \times$ & MAT \\
$7.0 \times$ & TMS
\end{tabular}

\begin{tabular}{l|l}
$7.0 \times$ & TMS \\
1.8 & KUS
\end{tabular}

MTMJ KOF

$-5.7 \times$

$-3.2 x$ | SAP

$-4.0 x$

MTM

SUT

KUSJ

AJ I

MIS

SSS 2706.00

GNZ $\quad 166.50 \quad 86$ IPKP $5504.50 \quad 4.8 \times \quad$ KTJ

S.D. $=1.4$ on 432 of 584 obs. |OSH

JAN $18,1981,18 \mathrm{~h} 17 \mathrm{~m} 26.03 \pm 0.25 \mathrm{~s}$

$38.649 \mathrm{~N} \pm 1.4 \mathrm{~km} 142.836 \mathrm{E} \pm 2.0 \mathrm{~km}$ TOY

DEPTH $=48.2 \pm 2.1 \mathrm{~km} / 11 \mathrm{DJ}$

6. $2 \mathrm{mb}$ ( 66 ODS.) 7.0Msz ( 11 ODS.) / IID

NEAR EAST COAST OF HONSHU JAPAN (228)

Felt (IV JMA) ot Miyoko. Sendoi TKY

and Morioka; (111 JMA) at

Ofunoto, Ishinamaki, Fukushimo, SHZ

Hochinahe, Onohama, Akito.

Shirakawo, Sakato and

Utsunomiyo: ( 11 JMA) ot Urokowo.

Moeboshi, Tokyo, Toteyamo,

Yokohamo and Kofu. Locol tsunomi

generoted with maximum wove

heights (peak to trough) $20 \mathrm{~cm}$.

ot Urokawo and Ofunato, $15 \mathrm{~cm}$.

at Aikowo, $14 \mathrm{~cm}$. ot Hochinohe.

$13 \mathrm{~cm}$. at $\mathrm{Hir}$ ao and $12 \mathrm{~cm}$. ot

Miyako and Ofunato.

\begin{tabular}{|c|c|c|c|c|c|c|c|}
\hline OFU & 0.97 & 296 & P- & $\begin{array}{l}17 \\
17\end{array}$ & $\begin{array}{l}42.30 \\
56.60\end{array}$ & -1.1 & NAG \\
\hline OFUJ & 1.01 & 296 & p & 17 & 42.00 & -2.0 & FUK \\
\hline$M I Y$ & 1.20 & 326 & P- & 17 & 44.60 & -2.1 & \\
\hline & & & s & 18 & 02.90 & & HJJ \\
\hline ISN & 1.22 & 260 & P- & 17 & 46.00 & -1.0 & \\
\hline & & & is & 18 & 01.60 & & TKOZ \\
\hline SEN & 1.57 & 256 & $\mathrm{P}+$ & 17 & 51.20 & -0.7 & HIK \\
\hline & & & $s$ & 18 & 10.40 & & \\
\hline ARK & 1.67 & 309 & $\begin{array}{l}P \\
\text { eS }\end{array}$ & $\begin{array}{l}17 \\
18\end{array}$ & $\begin{array}{l}53.00 \\
18.00\end{array}$ & -0.3 & $\begin{array}{l}\text { TSRJ } \\
\text { TKO }\end{array}$ \\
\hline
\end{tabular}

$5.08233 \mathrm{P}-$

$6.06 \quad 205 \mathrm{P}$

$\begin{array}{lll}1.99 & 259 & \mathrm{P}- \\ & & \mathrm{S} \\ 2.06 & 245 & \mathrm{P} \\ & & \mathrm{eS} \\ 2.13 & 332 & \mathrm{P} \\ & & \mathrm{iS} \\ 2.25 & 276 & \mathrm{P} \\ 2.25 & 259 & \mathrm{P}+ \\ 2.29 & 223 & \mathrm{P} \\ & & \mathrm{iS} \\ 2.38 & 298 & \mathrm{P} \\ & & \mathrm{S} \\ 2.57 & 234 & \mathrm{P} \\ 2.68 & 324 & \mathrm{P} \\ 2.69 & 316 & \mathrm{P} \\ 2.94 & 228 & \mathrm{P}+ \\ & & \mathrm{eS} \\ 3.16 & 229 & \mathrm{P} \\ 3.34 & 246 & \mathrm{P}\end{array}$

$3.34246 P$

$3.51359 \mathrm{P}$

$3.592 \mathrm{eS}$

$3.67262 \mathrm{P}$

$3.71229 \mathrm{P}$

$3.74234 \mathrm{P}$

1759.70

1824.28

1758.70

1828.08

$18 \quad 01.60$

$\begin{array}{ll}18 & 26.60 \\ 18 & 01.00\end{array}$

\begin{tabular}{lll|l}
18 & 01.00 & -0.5 & \\
18 & 01.30 & -0.2 & OWA
\end{tabular}

18 Q1.00

1826.80

1804.00

1836.08

$18 \quad 05.00$

$18 \quad 10.00$

$18 \quad 10.00$

1812.90

1849.00

1814.00

$18 \quad 18.00$

$18 \quad 19.00$

$\begin{array}{ll}19 & 02.00\end{array}$

$\begin{array}{lll}18 & 17.90\end{array}$

$18 \quad 25.60$

1918.80

1822.00

1913.00

$\begin{array}{ll}18 & 23.00\end{array}$

1908.90

1822.00

$19 \quad 09.80$

1823.80

$3.93229 \mathrm{eP}$

$3.94248 \mathrm{P}$

$4.00341 \mathrm{P}$

$4.01231 \mathrm{P}$

$4.18243 \mathrm{P}$

$4.23242 P$

$4.44214 \mathrm{P}$

$4.48 \quad 15 \mathrm{P}$

$4.49244 \stackrel{\mathrm{P}}{\mathrm{P}}$

$4.53230 \mathrm{P}$

$4.55346 \mathrm{P}$

$4.55240 \stackrel{P}{P}$

$4.58335 \mathrm{P}$

$4.67 \quad 17 \mathrm{P}$

$4.68221 \mathrm{P}$

$4.72223 \mathrm{P}$

$4.77220 \mathrm{P}$

$4.77217 \mathrm{P}$

$4.85 \quad 257 \quad P$

$4.87248 \stackrel{P}{P}$

$5.05233 \mathrm{P}$

$5.09242 \mathrm{P}$

$5.11225 \mathrm{P}$

$5.1223 \stackrel{S}{P}$

$5.13356 \stackrel{\mathrm{P}}{\mathrm{P}}$

$5.34249 \stackrel{P}{P}$

$\begin{array}{lll}5.34 & 249 & P \\ 5.47 & 359 & P\end{array}$

$5.69228 \mathrm{P}$

$5.84225 P$

$5.84238 \mathrm{P}$

$5.85235 \mathrm{P}$

$5.87246 \mathrm{P}$

$6.23223 \stackrel{5}{P}$

$6.26239 \mathrm{P}$

$6.30243 \mathrm{P}$

$6.46223 \mathrm{P}$

$18 \quad 38.00$

1933.00

1951.00

1847.00

1846.00

1851.00

$20 \quad 04.20$

$\begin{array}{ll}18 & 52.00 \\ 18 & 52.00\end{array}$

2003.00

1853.00

2012.00

1853.00

$20 \quad 08.90$

1854.00

$\begin{array}{ll}20 & 04.88 \\ 18 & 56.80\end{array}$

1856.00

$20 \quad 22.48$

$\begin{array}{ll}20 & 22.40 \\ 18 & 58.00 \\ 18 & 59.00\end{array}$

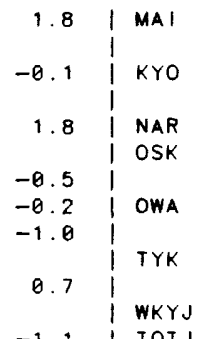

$6.75244 P \quad 1907.00$ $\begin{array}{ccc}6.75240 \mathrm{P} & 2023.10 \\ & 1907.50\end{array}$

$\begin{array}{lll}20 & 12.00 & 1.5\end{array}$
2021.80

1911.00

1911.00

$20 \quad 30.00$

1910.00

2020.90

1911.60

2036.60

1912.00

1917.00

1923.08

2100.98

$19 \quad 11.40$

2036.00

$\begin{array}{ll}19 & 16.70\end{array}$

2043.50

1917.90

1919.00

1919.00

$20 \quad 44.00$

1920.70

2057.50

1921.50

2103.50

1925.90

2059.40

$\begin{array}{lll}19 & 27.00 & 1.0\end{array}$

1925.50

2107.00

1924.00

1929.80

2103.10

1928.00

1935.00

2122.00

1941.00

2132.30

1938.40

1943.00

1941.00

2134.00

1941.00

2130.00

1947.00

1953.00

2142.00

1959.00

2001.00

$22 \quad 01.00$

1959.40

2211.90

2006.00

2211.00

$20 \quad 05.10$

$\begin{array}{ll}22 & 14.00 \\ 20 & 10.00\end{array}$

$\begin{array}{ll}22 & 19.00\end{array}$

2011.50

2224.00

2011.00

2238.00

$20 \quad 09.00$

2231.00

2.5

$4.2 x$

0.9

1.5

$-1.1$

$6.7 x$

$-5.8 x$

$-0.1$

1.1

0.4

$-0.9$

$-2.0$

0.9

$-0.8$

$-3.4 x$

0. 7

-1.1
0.9

$3.7 x$

0. 2

1.3
-0.9

$-1.1$

-2.0
$4.0 x$

2.6

$3.3 x$

$3.8 \mathrm{x}$

$-0.6$

2.8

2.0

0.9

$-1.2$

$11.52242 \stackrel{\mathrm{eS}}{\mathrm{P}}$

$11.54183 \mathrm{P}$

006.00

2212.00

$2015.00 \quad 1.0$

$\begin{array}{lll}20 & 13.50 & -0.8\end{array}$

$2018.00 \quad 0.0$

$\begin{array}{lll}20 & 20.00 & -0.7\end{array}$

2245.00

$\begin{array}{lll}20 & 20.00 & -1.1\end{array}$

2023.00

2253.00

$20 \quad 32.00$

$23 \quad 03.00$

$\begin{array}{lll}20 & 45.00 & 0.9\end{array}$

$\begin{array}{lll}20 & 55.00 & -2.8\end{array}$

2349.00

2059.00

2339.60

2123.20

2406.08 


\begin{tabular}{|c|c|c|c|c|c|c|c|c|c|c|c|}
\hline NGO & 17.33 & 230 & $p$ & 21 & 22.50 & $-3.5 x$ & I PRZ & 48.05 & 296 & ep & 26 \\
\hline & & & es & 24 & 34.50 & & i & & & es & 33 \\
\hline NAH & 17.78 & 230 & $P$ & 21 & 28.00 & $-3.6 x$ & I ESA & 48.71 & 170 & $P$ & 26 \\
\hline & & & es & 24 & 49.70 & & I MKS & 48.72 & 212 & ePC & 26 \\
\hline PET & 18.05 & 32 & eP & 21 & 38.00 & $3.3 x$ & I NRN & 50.09 & 296 & eP & 26 \\
\hline SSE & 19.27 & 254 & $p$ & 21 & 46.00 & $-3.5 x$ & $i$ & & & es & 33 \\
\hline$T \mid A$ & 20.54 & 271 & $P_{C}$ & 22 & 00.00 & $-2.9 x$ & I HNR & 50.42 & 158 & $e^{P}$ & 26 \\
\hline $\mathrm{N} J 2$ & 20.61 & 259 & $\mathrm{PC}$ & 22 & 00.00 & $-3.6 x$ & FRU & 50.58 & 298 & ePc & 26 \\
\hline BJI & 20.64 & 282 & $\mathrm{PC}$ & 22 & 01.00 & $-2.9 x$ & i & & & is & 33 \\
\hline MGD & 22.06 & 11 & ep & 22 & 03.00 & $-14.9 x$ & I KSH & 50.81 & 293 & $\mathrm{PC}$ & 26 \\
\hline & & & $i s P$ & 22 & 08.00 & & I IPM & 50.87 & 239 & iPd & 26 \\
\hline & & & es & 26 & 22.00 & & $i$ & $1.5 \mathrm{~s}$ & 46 & $6.00 \mathrm{~nm}$ & \\
\hline TATO & 22.62 & 239 & $e^{P}$ & 22 & 22.00 & -1.7 & $\mathrm{KGM}$ & 51.29 & 235 & ePd & 26 \\
\hline HWA & 23.22 & 237 & $p$ & 22 & 26.20 & $-3.4 x$ & $i$ & $1.4 \mathrm{~s}$ & 327 & $7.00 \mathrm{~nm}$ & \\
\hline & & & es & 26 & 36.50 & & KHE & 51.30 & 348 & $\mathrm{ePc}$ & 26 \\
\hline $\mathrm{TCU}$ & 23.72 & 239 & $p$ & 22 & 22.10 & $-12 \cdot 3 x$ & $i$ & & & es & 33 \\
\hline & & & s & 28 & 07.30 & & KUPT & 51.76 & 204 & ePd & 26 \\
\hline $\mathrm{QZH}$ & 24.63 & 243 & $\mathrm{Pc}$ & 22 & 41.00 & -2.3 & MTN & 52.38 & 194 & $e P$ & 26 \\
\hline WHN & 24.73 & 260 & $\mathrm{PC}$ & 22 & 43.00 & -1.2 & I INK & 52.45 & 28 & eP & 26 \\
\hline SEY & 24.97 & 10 & $P d$ & 22 & 44.40 & -1.8 & 1 & $1.0 \mathrm{~s}$ & 184 & $4.00 \mathrm{~nm}$ & \\
\hline GUA & 25.08 & 175 & $e(P)$ & 22 & 53.00 & $5.4 x$ & MUR & 52.70 & 293 & $P$ & 26 \\
\hline & $1.9 \mathrm{~s}$ & 3968 & $0.00 \mathrm{~nm}$ & & & $6.6 \mathrm{mb}$ & ANR & 52.90 & 296 & $\mathrm{Pc}$ & 26 \\
\hline & & & e (S) & 27 & 14.00 & & $i$ & & & is & 34 \\
\hline SMY & 25.74 & 47 & $e^{P}$ & 22 & 58.50 & $5.0 x$ & $\mid$ TS I & 53.32 & 240 & $e(P)$ & 26 \\
\hline XAN & 27.61 & 271 & $p$ & 23 & 09.00 & -1.8 & TRT & 53.99 & 218 & ePC & 26 \\
\hline$S Z P$ & 28.70 & 229 & $i P c$ & 23 & 22.00 & 1.4 & | BS | & 54.15 & 245 & $e(P)$ & 26 \\
\hline & $1.0 \mathrm{~s}$ & & $6.06 \mathrm{~nm}$ & & & $5.0 \mathrm{mb} \times$ & NDI & 54.48 & 280 & $p$ & 26 \\
\hline & & & is & 28 & 26.00 & & 1 & & & is & 34 \\
\hline HKC & 29.43 & 245 & $p$ & 23 & 28.00 & 0.8 & $\mathrm{MBC}$ & 54.66 & 17 & eP & 26 \\
\hline BAG & 29.52 & 228 & eP & 23 & 28.00 & -0.3 & $i$ & $1.0 \mathrm{~s}$ & 281 & $1.00 \mathrm{~nm}$ & \\
\hline & & & es & 28 & 21.00 & & SVE & 54.70 & 318 & PC & 26 \\
\hline $\mathrm{GZH}$ & 29.53 & 247 & Pc & 23 & 27.30 & -0.8 & 1 & & & $i p P$ & 26 \\
\hline IRK & 29.87 & 310 & $\mathrm{PC}$ & 23 & 29.00 & -2.0 & $i$ & & & is & 34 \\
\hline & & & es & 28 & 20.00 & & KHO & 54.79 & 293 & $P C$ & 26 \\
\hline ZAK & 30.19 & 306 & $\mathrm{Pc}$ & 23 & 33.00 & -0.7 & I TAS & 54.81 & 298 & $P C$ & 26 \\
\hline LGP & 30.52 & 219 & ePc & 23 & 39.80 & 2.8 & I MNL & 55.04 & 287 & eP & 26 \\
\hline MAN & 30.65 & 225 & ip & 23 & 38.00 & -0.1 & $|P P|$ & 55.04 & 235 & eP & 26 \\
\hline OCP & 30.67 & 225 & eP & 23 & 26.10 & $-12.1 x$ & NIL & 55.19 & 288 & eP & 26 \\
\hline$L Z H$ & 30.94 & 278 & $P C$ & 23 & 40.00 & -0.7 & I KNA & 55.69 & 196 & $e P$ & 26 \\
\hline & & & $s$ & 28 & 33.00 & & $\mathrm{KUL}$ & 55.90 & 294 & PC & 27 \\
\hline ADK & 30.99 & 51 & eP & 23 & 44.70 & $4.0 x$ & DSH & 56.31 & 295 & $P C$ & 27 \\
\hline$P L P$ & 31.67 & 215 & ePc & 23 & 45.50 & -1.6 & i & & & is & 34 \\
\hline GYA & 32.62 & 259 & $\mathrm{PC}$ & 23 & 55.00 & -0.4 & $\mathrm{PSH}$ & 56.41 & 289 & $e P$ & 27 \\
\hline & & & $\mathrm{s}$ & 29 & 05.00 & & I SAM & 57.09 & 297 & $\mathrm{PC}$ & 27 \\
\hline CDU & 32.73 & 268 & P & 23 & 56.00 & -0.2 & i & & & is & 35 \\
\hline & & & es & 29 & 06.00 & & I KAAO & 57.90 & 291 & $e^{P}$ & 27 \\
\hline TIK & 33.82 & 352 & ep & 24 & 03.00 & -2.2 & I ALE & 58.45 & 4 & $e P$ & 27 \\
\hline & & & es & 29 & 19.00 & & i & $1.3 \mathrm{~s}$ & 525 & $5.00 \mathrm{~nm}$ & \\
\hline CGP & 34.21 & 213 & $\mathbf{e P C}$ & 24 & 06.20 & $-2.9 x$ & I CTA & 58.51 & 176 & $P$ & 27 \\
\hline DAV & 35.08 & 211 & $P$ & 24 & 15.00 & -1.5 & I WB2 & 58.82 & 189 & $P$ & 27 \\
\hline$B G V$ & 35.83 & 252 & $P$ & 23 & 23.00 & $-59.9 x$ & $i$ & & & $i$ & 27 \\
\hline PPR & 35.96 & 223 & ePd & 25 & 24.50 & $60.5 x$ & $i$ & & & es & 35 \\
\hline ILT & 36.13 & 24 & $P_{c}$ & 24 & 21.60 & $-3.3 x$ & I WRA & 58.82 & 189 & Pd & 27 \\
\hline & & & es & 30 & 03.00 & & 1 & $0.9 \mathrm{~s}$ & 45 & $5.20 \mathrm{~nm}$ & \\
\hline KMI & 36.33 & 260 & $P C$ & 24 & 25.50 & -1.9 & KBS & 59.27 & 350 & $\mathrm{iPC}$ & 27 \\
\hline & & & $s$ & 30 & 02.00 & & HYB & 59.37 & 268 & $\mathrm{PCt}$ & 27 \\
\hline NHA & 39.81 & 238 & $P$ & 24 & 57.70 & 1.4 & 1 & & & e & 30 \\
\hline & & & es & 31 & 00.00 & & 1 & & & es & 35 \\
\hline MOM & 40.71 & 173 & eP & 25 & 03.00 & -0.6 & 1 & & & eSS & 39 \\
\hline ELT & 40.85 & 310 & $P C$ & 25 & 03.90 & -0.5 & RES & 60.76 & 15 & eP & 27 \\
\hline & & & es & 31 & 15.00 & & 1 & $0.7 \mathrm{~s}$ & 206 & $6.00 \mathrm{~nm}$ & \\
\hline JAY & 41.01 & 183 & $e P c$ & 25 & 06.70 & 0.6 & I PVC & 60.96 & 152 & $P C$ & 27 \\
\hline & $1.0 \mathrm{~s}$ & 62 & $2.30 \mathrm{~nm}$ & & & $5.3 \mathrm{mb}$ & I PHC & 61.16 & 47 & eP & 27 \\
\hline WMO & 41.14 & 295 & $\mathrm{PC}$ & 25 & 07.00 & 0.0 & I OUE & 61.54 & 287 & $i P c$ & 27 \\
\hline & & & is & 31 & 20.00 & & 1 & & & es & 35 \\
\hline NRI & 42.12 & 334 & $P C$ & 25 & 12.00 & -2.6 & $A P A$ & 61.71 & 336 & $P C$ & 27 \\
\hline & & & is & 31 & 35.00 & & 1 & & & is & 35 \\
\hline CHTO & 42.79 & 255 & eP & 25 & 20.00 & -0.7 & $Y K C$ & 61.91 & 31 & eP & 27 \\
\hline LSA & 43.20 & 274 & $\mathrm{PC}$ & 25 & 23.20 & $-1 \cdot 3$ & i & $1.1 \mathrm{~s}$ & 177 & $7.00 \mathrm{~nm}$ & \\
\hline & & & $\mathrm{s}$ & 31 & 48.00 & & $\mathrm{KOU}$ & 62.24 & 157 & $\mathrm{PC}$ & 27 \\
\hline TTA & 43.45 & 36 & $e(P)$ & 25 & 27.40 & 1.7 & $\mathrm{KEV}$ & 62.33 & 339 & $\mathrm{PC}$ & 27 \\
\hline RAB & 43.49 & 166 & $P$ & 25 & 25.00 & $-1,4$ & i & & & $i$ & 27 \\
\hline SVW & 43.54 & 38 & $e^{P}$ & 25 & 27.10 & 0.7 & i & & & es & 36 \\
\hline MDG & 43.76 & 176 & eP & 25 & 30.00 & 1.5 & i & & & eSs & 40 \\
\hline WAB & 43.93 & 179 & eP & 25 & 30.00 & -0.2 & i POO & 62.42 & 272 & iPC & 27 \\
\hline AAI & 44.25 & 201 & ePc & 25 & 34.20 & 1.7 & $i$ & & & IPCP & 28 \\
\hline & $0.8 \mathrm{~s}$ & 115 & $5.00 \mathrm{~nm}$ & & & $.7 \mathrm{mb}$ & i & & & IPP & 30 \\
\hline IMA & 44.73 & 31 & $e^{P}$ & 25 & 36.00 & 0.0 & i & & & is & 36 \\
\hline SEM & 44.87 & 306 & $P C$ & 25 & 35.90 & -1.3 & i & & & iPPS & 36 \\
\hline & & & es & 32 & 09.40 & & $i$ & & & iscs & 37 \\
\hline KDC & 45.20 & 43 & eP & 25 & 39.30 & -0.4 & GBA & 2.46 & 266 & Pd & 27 \\
\hline BKB & 46.39 & 217 & ePd & 25 & 52.70 & 3. $2 x$ & 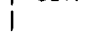 & $0.9 \mathrm{~s}$ & 253 & $3.00 \mathrm{~nm}$ & \\
\hline PMR & 46.66 & 38 & eP & 25 & 50.20 & -0.9 & I SOD & 63.92 & 337 & eP & 27 \\
\hline & $1.1 \mathrm{~s}$ & 281 & $1.00 \mathrm{~nm}$ & & & $6.1 \mathrm{mb}$ & KAR & 64.12 & 282 & $e(P)$ & 27 \\
\hline FBA & 47.14 & 33 & $e P$ & 25 & 54.80 & -0.1 & I DAG & 64.27 & 355 & $\mathrm{iPC}$ & 27 \\
\hline$P M G$ & 47.98 & 174 & eP & 26 & 02.00 & 0.0 & 1 & $1.0 \mathrm{~s}$ & 198 & $0.00 \mathrm{~nm}$ & \\
\hline & $20 s$ & & $5.50 \mathrm{um}$ & & & 5. $5 \mathrm{MszX}$ & 1 & & & is & 36 \\
\hline
\end{tabular}




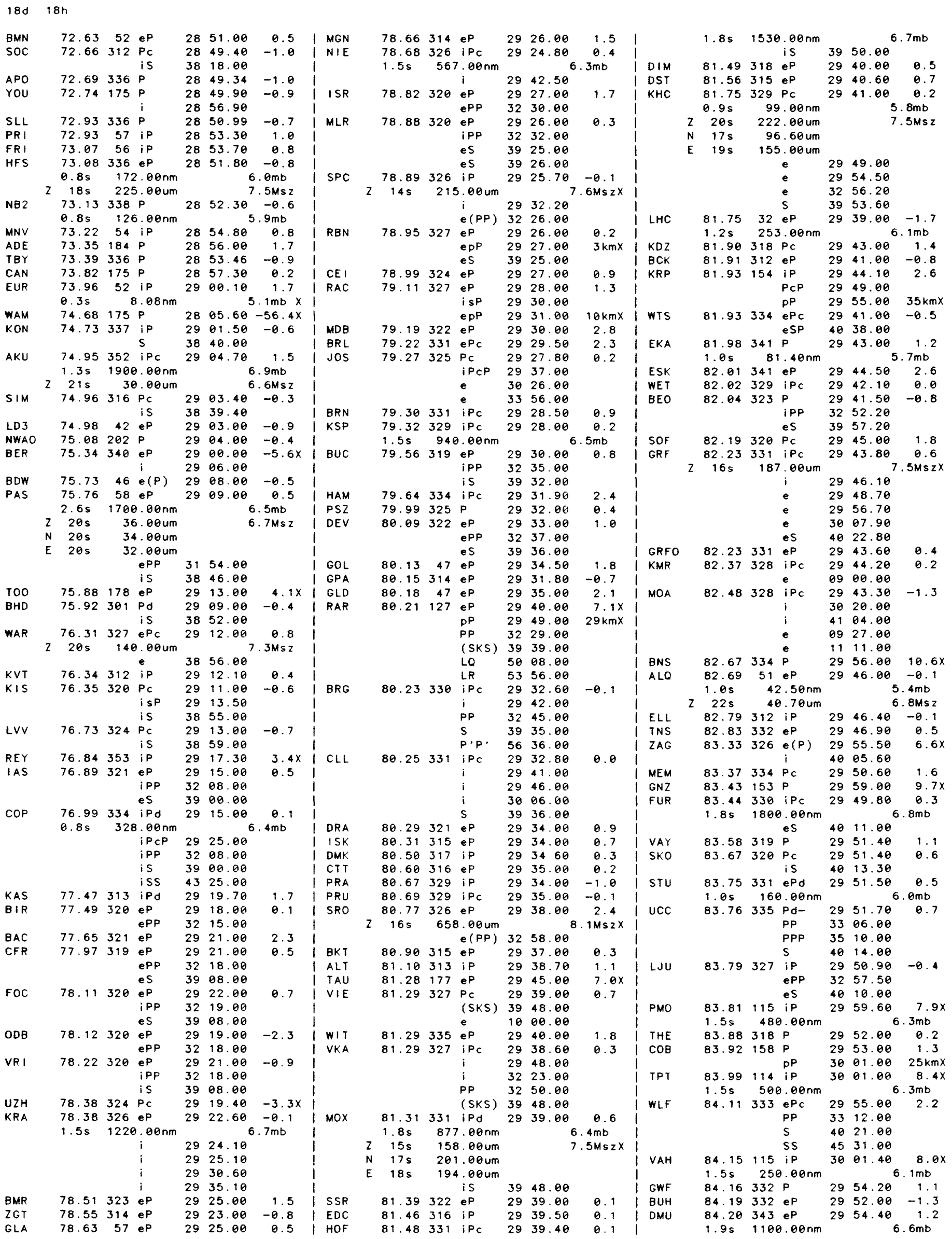


AFR $\quad 84.20118$ iP $\quad 2959.00 \quad 5.4 \times \quad$ AOU

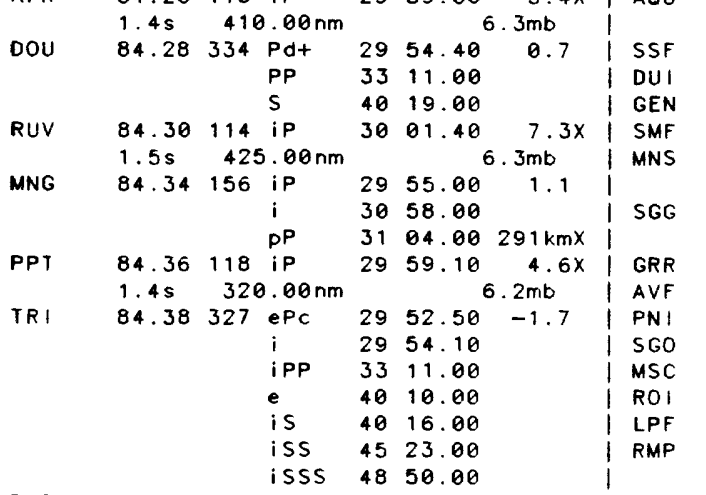

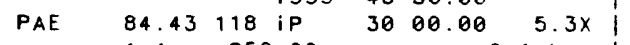

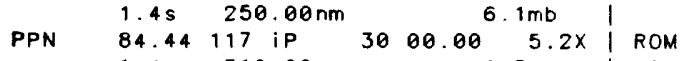

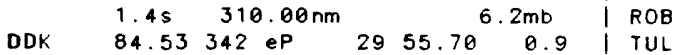

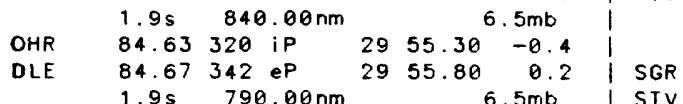

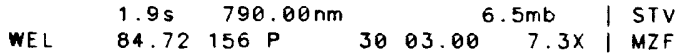

\begin{tabular}{llll|l}
$P P$ & 33 & 23.00 & & \\
(SKS) & 40 & 04.00 & I RLO
\end{tabular}

(SKS) 4004.00

SS 4556.00

SSS 4934.00

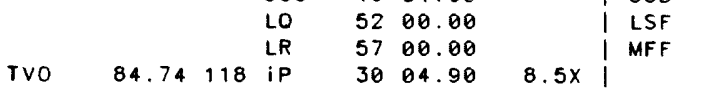

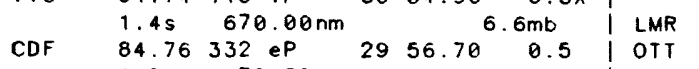

DCN $\quad 84.80343 \mathrm{eP}^{2} 2956.40$\begin{tabular}{lll|l}
$5.8 \mathrm{mb}$ & 0.2 & $\mathrm{RJF}$
\end{tabular} \begin{tabular}{cc|c|c}
$84.80343 \mathrm{eP}$ & $2956.40 \quad 0.2$ & $R J F$
\end{tabular}

$\begin{array}{lllllll} & & & \end{array}$

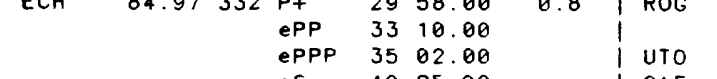

CTI $\quad 85.00 \quad 328$ P 4025.00

i 3010.00

$\begin{array}{llll} & & \mathrm{e} & 3341.00 \\ \text { ZUL } & 85.12331 \mathrm{P} & 2957.30\end{array}$

\begin{tabular}{lllll|l|l} 
BAF & 85.35 & $332 \mathrm{P}$ & 30 & 00.00 & 0.8 & MNT
\end{tabular}

BSF \begin{tabular}{cccc|c}
85.42 & $332 \mathrm{eP}$ & 29 & $59.30-0.3$ & -0 \\
& $1.0 \mathrm{~s}$ & $29.20 \mathrm{~nm}$ & $5.4 \mathrm{mb}$ & $\mathrm{Clz}$
\end{tabular}

HAU $85.44332 \mathrm{eP} 2959.60 \quad 0.0 \quad$ LFF

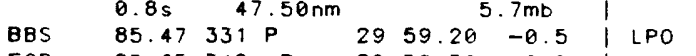

$\begin{array}{llllll}\text { ECP } & 85.65 & 342 \mathrm{eP} & 29 & 59.50 & -0.9 \\ & 2.0 \mathrm{~S} & 1260.00 \mathrm{~nm} & & 6.8 \mathrm{mb}\end{array}$

$\begin{array}{llllll}\text { SAL } & 85.82329 \mathrm{P} & 30 & 02.00 & 0.6 \\ & & i & 30 & 14.00 & \end{array}$

$\begin{array}{llll}\text { RHP } & 85.97161 \mathrm{P} \\ \text { NEC } & 86.06331 \mathrm{P}\end{array}$

CMZ 86.19159 iP

PCN $\quad 86.62329 \mathrm{P}$

HLW $86.63306 \mathrm{P}$

DIX $86.66331 \mathrm{P}$

VA

ORO

FO

\section{VOT}

LOR

86.96
86.98
327

$\begin{array}{rl}86.98 \quad 327 & P \\ & \text { ipP } \\ & \text { iPP }\end{array}$

3004.00

3002.00

$\begin{array}{ll}30 & 15.10 \\ 30 & 08.00\end{array}$

$\begin{array}{ll}30 & 08.00 \\ 40 & 48.00\end{array}$

3007.00

$\begin{array}{ll}30 & 05.90 \\ 30 & 05.00\end{array}$

$\begin{array}{ll}30 & 05.00 \\ 40 & 31.00\end{array}$

$86.82330 \stackrel{\text { is }}{P}$

$P$

3006.50

3447.00

$40 \quad 42.00$

3010.00

3009.50

3006.80

$30 \quad 17.00$

$30 \quad 41.00$

$\begin{array}{ll}33 & 03.50 \\ 40 & 46.00\end{array}$

is

FIR

PRG

$87.00327 \mathrm{P}$

$\begin{array}{ll}40 & 46.00 \\ 30 & 07.00\end{array}$

$87.05326 \mathrm{P}$

4030.00

3000.00

\begin{tabular}{lll|l}
40 & 10.00 & & ALI \\
30 & 07.40 & -0.2 & MTD
\end{tabular}

FLN

SSC

$87.09337 \quad \mathrm{i}$ $1.0 \mathrm{~s} \quad 57.60 \mathrm{~nm}$ 87.13336 iP

$1.1 \mathrm{~s} \quad 55.40 \mathrm{~nm}$ 87.16333 iP $5.8 \mathrm{mb}$ $3007.70 \quad-0.1$ $\begin{array}{lll}30 & 07.90^{5.7 m b} & -0.1\end{array}$
2.5 EBR

\begin{tabular}{c|c}
-0.2 & \\
$9.9 \times$ & ORT
\end{tabular}

$89 \mathrm{kmX}$ BLA
$87.20325 \mathrm{P}$

87.26334 iP

$87.27324 \mathrm{P}$

$87.44329 \mathrm{P}$

$87.50333 \mathrm{eP}$

$87.50325 \mathrm{P}$

$87.52324 \mathrm{P}$

$87.54337 \mathrm{iP}$

$87.54333 \mathrm{eP}$

$87.67330 \mathrm{P}$

$87.74323 \mathrm{P}$

$87.87324 \mathrm{P}$

$87.90321 P$

$87.91337 \mathrm{iP}$

$87.95325 \mathrm{Pt}$

ePP
iS
iPPS

$87.96325 \mathrm{P}$

$87.97329 \mathrm{P}$

$88.15 \quad 44$ iPd

$1.1 \mathrm{~s} \quad 109.00 \mathrm{~nm}$

$z 19 \mathrm{~s} \quad 36.30 \mathrm{um}$

$88.16336 \mathrm{eP}$

$88.23330 \mathrm{P}$

88.31334 iP

$88.3743 \mathrm{eP}$

$88.37334 \mathrm{iP}$

88.41332 iPd

88.64334 iP

88.89335 iP

$1.0 \mathrm{~s} \quad 65.70 \mathrm{~nm}$

$89.31330 \mathrm{eP}$

$89.4426 \mathrm{eP}$

$1.0 \mathrm{~s} \quad 154.00 \mathrm{~nm}$

89.47334 iP

$89.48331 \mathrm{ePd}$

$89.5833 \mathrm{ePd}$

89.61333 iP

89.8250 iP

$22 \mathrm{~s} \quad 53.70 \mathrm{um}$

$90.0425 \mathrm{eP}$

$1.0 \mathrm{~s} \quad 96.00 \mathrm{~nm}$

$90.05152 \mathrm{eP}$

90.06334 iP

$0.8 \mathrm{~s} \quad 62.60 \mathrm{~nm}$
$90.13334 \mathrm{iP}$

$\begin{array}{ll}90.13 & 334 \\ 0.9 \mathrm{~s} & 28.80 \mathrm{~nm}\end{array}$

$28.80 \mathrm{~nm} \quad 22.30 \quad 0.2$ XAN

$90.55285 \mathrm{iPc} \quad 3025.90^{5.6 \mathrm{mb}} 1.3$

$00.58 \quad$ eS $34 \quad 03.20$

$\begin{array}{rrrr}90.58 \quad 32 & \text { (SK } & 30 & 25.26 \\ & \text { (SKS) } & 40 & 58.00\end{array}$

$90.99 \quad 24 \mathrm{eP} \quad 3026.30$

$91.16 \quad 21$ eP 3027.20

91.67333 IPd 3032.10

$91.70 \quad 22 \mathrm{eP} \quad 3029.90$

$1.0 \mathrm{~s} \quad 48.50 \mathrm{~nm}$

$\begin{array}{ll}91.87 & 333 \mathrm{iP} \\ 0.9 \mathrm{~s} & 24.46 \mathrm{~nm}\end{array}$

$\begin{array}{ll}0.9 \mathrm{~s} & 24.46 \\ 91.99 & 25 \mathrm{eP}\end{array}$

$92.22 \quad 23 \mathrm{eP}$

93.34335 iPd

iPP

is

$93.75332 \mathrm{eP}$

$93.97 \quad 37 \mathrm{eP}$

$94.63 \quad 33 \mathrm{eP}$

$95.78327 \mathrm{P}$

$96.17335 \mathrm{eP}$

IS $\quad 3056.00$

is 4202.00

iSSS 5250.00

$96.28332 \mathrm{iPC} 3047.50$

116.77269 ePKP 3601.00

IPPP 3716.00

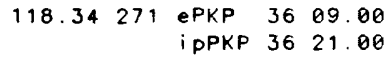

$\begin{array}{lll}\text { i PPKP } & 36 & 21.00 \\ \text { iPP } & 37 & 35.00\end{array}$

72 $\begin{array}{lllll}120.98 & 268 \text { PKP } \quad 36 \quad 14.80 & -0.4\end{array}$

$\begin{array}{llr}Z & 22 \mathrm{~s} & 34.80 \mathrm{um} \\ \mathrm{N} & 22 \mathrm{~s} & 20.70 \mathrm{um}\end{array}$

E $21 \mathrm{~s} 20.10 \mathrm{um}$

i PPKP $36 \quad 26.00$

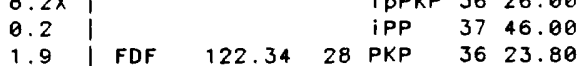

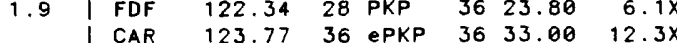

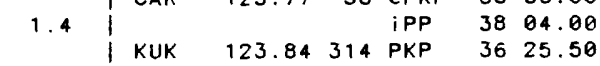

314 PKP $36 \quad 25.50 \quad 4.8 X$

\begin{tabular}{l|lllllll}
0.2 & MBO & 123.95 & 336 & PKP & 36 & 20.30 & -0.5 \\
0.1 & KIC & 126.05 & 319 ePKP & 36 & 22.40 & $-2.6 x$
\end{tabular}

\begin{tabular}{l|llllll}
$7.0 x$ & & & e & 38 & 19.00 & \\
0.2 & NNA & 135.89 & 64 ePKP & 36 & 45.60 & 1.9
\end{tabular}

0.4 1.0s $22.00 \mathrm{~nm}$

ES $39 \quad 18.00$

$\begin{array}{llllll}136.99 & 62 & \text { ePKP } & 36 & 46.90 & 0.6\end{array}$

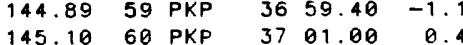

LPB $145.10 \quad 60$ PKP 3701.00

$\begin{array}{lllllll}\text { ANT } 148.09 & 72 \text { ePKP } & 37 & 08.00 & 3.3 \times\end{array}$

\begin{tabular}{l|lllll}
$-4.9 \times$ & SOB3 & 151.01 & 8 ePKP & 37 & 14.40 \\
-1.0 & & & & 37 & 16.40
\end{tabular}

$\begin{array}{lllll}2.5 & \text { e } & 37 & 17.90\end{array}$

$\begin{array}{llllll}152.11 & 91 & \text { ePKP } & 37 & 15.00 & 4.5 x\end{array}$

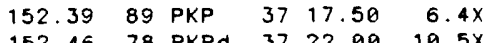

152.4678 PKPd 3722.00

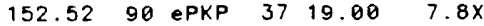

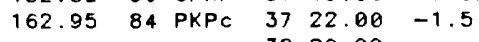

$\begin{array}{lll}\text { e } & 38 & 20.00 \\ P P & 42 & 04.00\end{array}$

SKKS $48 \quad 40.00$

SKSP $52 \quad 19.00$

SS 0224.00

$\begin{array}{lllll}163.46 & 20 & \text { PKP } \quad 37 & 23.00 & -1.3\end{array}$

S.D. $=1.2$ on 416 of 509 obs.

JAN $23,198121 \mathrm{~h} 13 \mathrm{~m} \mathrm{48.12 \pm 0.08 \textrm {s }}$ $30.967 \mathrm{~N}+1.8 \mathrm{~km} 101.134 \mathrm{E} \pm 1.6 \mathrm{~km}$ DEPTH $=10.0 \mathrm{~km}$ (geophysicist)

$5.8 \mathrm{mb}$ ( 71 obs.) 6.8Msz ( 9 obs.) SICHUAN PROVINCE, CHINA (307)

About 150 people killed, 300

injured and extensive domage in the Dowu oreo.

\begin{tabular}{|c|c|c|c|c|c|}
\hline \multirow{3}{*}{$\begin{array}{l}2.49 \\
5.58\end{array}$} & 0 & $P d$ & 14 & 28.60 & -6 \\
\hline & 23 & $\mathbf{P c}$ & 15 & 12.50 & -0.9 \\
\hline & & $\mathrm{Pg}$ & 5 & 33.50 & \\
\hline \multirow[t]{3}{*}{5.99} & 166 & $P c$ & 15 & 19.50 & 0.3 \\
\hline & & 8 & 5 & 45.00 & \\
\hline & & in & 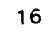 & 31.00 & \\
\hline \multirow[t]{2}{*}{6.61} & 131 & $P$ & 5 & 28.00 & 0.1 \\
\hline & & & 6 & 44.00 & \\
\hline .26 & 63 & $e^{P}$ & 5 & 26.00 & -10.8 \\
\hline \multirow[t]{2}{*}{8.72} & 264 & & & 57.00 & -0 \\
\hline & & 2 & 7 & 38.00 & \\
\hline \multirow[t]{2}{*}{9.77} & 239 & iP & & 10.00 & -1.8 \\
\hline & & es & 3 & 90 & \\
\hline 66 & 153 & $\mathbf{P}$ & 6 & 00 & -1.9 \\
\hline \multirow[t]{2}{*}{.38} & 89 & & 6 & 29.20 & \\
\hline & & & 8 & 31.00 & \\
\hline .26 & 190 & iPc & 16 & 46.90 & 1. \\
\hline \multirow[t]{2}{*}{$8 \mathrm{~s}$} & $75 e$ & 0.00 & & & \\
\hline & & es & 20 & 30.00 & \\
\hline \multirow[t]{2}{*}{13.41} & 123 & $P d$ & 6 & 56.00 & -4.9 \\
\hline & & es & 19 & 18.00 & \\
\hline .80 & 189 & iPc & 7 & 50 & 0.5 \\
\hline \multirow[t]{2}{*}{14.31} & 64 & $P$ & 7 & 11.60 & -1.2 \\
\hline & & es & 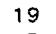 & & \\
\hline \multirow[t]{2}{*}{14.49} & 123 & $P$ & 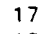 & 13.00 & -2.2 \\
\hline & & s & 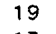 & & \\
\hline \multirow[t]{3}{*}{15.16} & 81 & $\mathrm{Pd}$ & 7 & 21.40 & -2.5 \\
\hline & & $s$ & 20 & 10.00 & \\
\hline & & i & & & \\
\hline \multirow[t]{2}{*}{15.21} & 49 & P & 7 & 26.00 & 1. \\
\hline & & 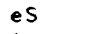 & $\theta$ & & \\
\hline 1 & 179 & iP & 7 & 38.00 & 0. \\
\hline & & 3.3 & & & \\
\hline \multirow[t]{2}{*}{16.54} & 107 & $P d$ & 17 & 38.00 & \\
\hline & & es & 0 & & \\
\hline \multirow[t]{2}{*}{6.66} & 324 & $\mathrm{Pc}$ & 17 & 41.50 & r \\
\hline & & es & 20 & & \\
\hline \multirow[t]{3}{*}{7.1} & 84 & $\mathrm{Pd}$ & 17 & 48.00 & -1 \\
\hline & & & 20 & 58 & \\
\hline & & & 21 & 15.00 & \\
\hline
\end{tabular}




\begin{tabular}{|c|c|c|c|c|c|c|c|c|c|c|c|}
\hline & & & $\mathrm{S}$ & 23 & 42.70 & & I DAV & 32.98 & 131 & eP & 20 \\
\hline ANP & 18.88 & 103 & Po & 18 & 11.00 & 0.1 & 1 & $1.6 \mathrm{~s}$ & 1070 & $0.00 \mathrm{~nm}$ & \\
\hline & & & $S$ & 21 & 49.80 & & 1 & & & es & 25 \\
\hline HWA & 19.45 & 106 & $P$ & 18 & 14.20 & $-3.6 x$ & I SAP & 33.98 & 58 & eP & 20 \\
\hline & & & es & 22 & 05.50 & & 1 & & & es & 25 \\
\hline TTN & 19.62 & 110 & $P$ & 18 & 23.60 & $3.9 x$ & I KMU & 35.10 & 59 & $e P$ & 20 \\
\hline HEN & 19.68 & 112 & eP & 18 & 22.00 & 1.7 & I $\mathrm{BKB}$ & 35.38 & 152 & ePd & 20 \\
\hline & & & Sn & 24 & 07.80 & & | TGI & 35.47 & 284 & eP & 20 \\
\hline NHA & 20.10 & 156 & $P$ & 18 & 23.00 & -1.9 & I YSS & 35.61 & 51 & Pd & 20 \\
\hline NDI & 20.87 & 270 & ¿PO & 18 & 29.80 & -3.0 & 1 & & & epp & 21 \\
\hline & $0.5 \mathrm{~s}$ & 352 & $2.00 \mathrm{~nm}$ & & & $.0 \mathrm{mb}$ & I & & & es & 26 \\
\hline & $18 s$ & 45 & $5.46 \mathrm{um}$ & & & & I ASH & 35.72 & 293 & eP & 20 \\
\hline & $18 s$ & & $3.20 u m$ & & & & I KHI & 35.77 & 287 & eP & 20 \\
\hline & & & is & 22 & 16.00 & & I YAK & 36.17 & 22 & $\mathrm{Pc}$ & 20 \\
\hline UER & 21.23 & 348 & $\mathrm{Pc}$ & 18 & 34.90 & -1.3 & 1 & & & is & 26 \\
\hline I RK & 21.42 & 5 & ePc & 18 & 36.00 & -2.1 & I KAT & 37.31 & 295 & $P$ & 21 \\
\hline & & & es & 22 & 30.00 & & 1 & & & is & 26 \\
\hline PRZ & 21.48 & 309 & eP & 18 & 39.00 & 0.0 & LEM & 38.09 & 170 & iPC & 21 \\
\hline & & & es & 22 & 29.00 & & 1 & $1.0 \mathrm{~s}$ & 20 & $0.00 \mathrm{~nm}$ & \\
\hline PIP & 21.68 & 121 & iPd & 18 & 41.00 & 0.0 & 1 & & & is & 27 \\
\hline & $1.0 \mathrm{~s}$ & 442 & $2.00 \mathrm{~nm}$ & & & $.8 \mathrm{mb}$ & I SVE & 38.11 & 325 & $P c$ & 21 \\
\hline SZP & 22.06 & 123 & ePd & 18 & 50.50 & $5.7 x$ & 1 & & & $i s P$ & 21 \\
\hline KSH & 22.18 & 299 & Pd & 18 & 48.00 & 2.0 & 1 & & & is & 27 \\
\hline & & & is & 22 & 53.00 & & I KUR & 38.89 & 55 & eP & 21 \\
\hline T LG & 22.48 & 310 & $P$ & 18 & 49.90 & 0.9 & I ARU & 38.97 & 323 & eP & 21 \\
\hline LAH & 22.90 & 278 & $e(P)$ & 18 & 49.00 & $-4.1 x$ & 1 & & & esp & 21 \\
\hline BAG & 22.90 & 125 & ePc & 18 & 55.00 & 1.6 & 1 & & & es & 27 \\
\hline & $2.1 \mathrm{~s}$ & 3330 & $3.00 \mathrm{~nm}$ & & & $.5 \mathrm{mb}$ & I NRI & 39.19 & 353 & Pd & 21 \\
\hline & & & is & 23 & 00.00 & & I & & & is & 27 \\
\hline $\mathrm{CN} 2$ & 23.08 & 49 & $P$ & 18 & 56.00 & 1.2 & I TRT & & 162 & iPd & 21 \\
\hline & & & is & 23 & 08.00 & & 1 & $0.4 \mathrm{~s}$ & 128 & 8. $00 \mathrm{~nm}$ & \\
\hline MNL & 23.29 & 283 & eP & 18 & 52.10 & $-4.8 x$ & I MKS & 40.02 & 151 & $e P c$ & 21 \\
\hline SNG & 23.67 & 181 & iPC & 19 & 02.00 & 1.3 & I TEH & 41.47 & 290 & eP & 21 \\
\hline & $1.8 \mathrm{~s}$ & & 3. $00 \mathrm{~nm}$ & & & $.1 \mathrm{mb}$ & I SHI & 41.72 & 281 & $P$ & 21 \\
\hline & & & es & 25 & 11.00 & & 1 & & & $S$ & 28 \\
\hline$N I L$ & 23.69 & 284 & eP & 19 & 00.20 & -0.6 & $|A A|$ & 43.06 & 138 & ePc & 21 \\
\hline FRU & 24.18 & 307 & $P C$ & 19 & 06.40 & 0.9 & I & $0.7 \mathrm{~s}$ & 299 & $9.00 \mathrm{~nm}$ & \\
\hline & & & es & 23 & 14.00 & & I TIK & 43.40 & 12 & ePd & 21 \\
\hline & & & iss & 23 & 30.00 & & 1 & & & es & 28 \\
\hline MAN & 4.46 & 127 & ip & 19 & 10.00 & 1.7 & 1 & & & ess & 28 \\
\hline OCP & 24.47 & 127 & $P$ & 19 & 14.06 & $5.6 x$ & GUMO & 43.69 & 103 & eP & 21 \\
\hline HYB & 24.56 & 242 & ePd & 19 & 09.00 & -0.3 & I GUA & 43.76 & 103 & $e(P)$ & 21 \\
\hline & & & e & 19 & 19.50 & & 1 & $0.9 \mathrm{~s}$ & 249 & $9.00 \mathrm{~nm}$ & \\
\hline & & & es & 23 & 24.00 & & I & & & $e(S)$ & 28 \\
\hline ELT & 24.75 & 338 & $\mathrm{Pc}$ & 19 & 10.30 & -0.5 & I MGD & 43.81 & 33 & Pc & 21 \\
\hline SEM & 24.91 & 327 & eP & 19 & 10.00 & -2.4 & 1 & & & $i p p$ & 22 \\
\hline & & & epP & 19 & 20.10 & $37 \mathrm{kmx}$ & 1 & & & is & 28 \\
\hline & & & es & 23 & 36.30 & & I MAK & 43.86 & 301 & Pd & 22 \\
\hline ANR & 5.19 & 301 & eP & 19 & 15.00 & -0.2 & I SKR & 44.87 & 48 & eP & 22 \\
\hline PSH & 5.20 & 285 & eP & 19 & 14.00 & -1.4 & 1 & & & es & 28 \\
\hline WRS & 5.21 & 285 & iPc & 19 & 14.20 & -1.2 & I GRS & 44.97 & 297 & $\mathrm{Pc}$ & 22 \\
\hline KHO & 5.26 & 293 & Pd & 19 & 15.90 & -0.2 & 1 & & & es & 28 \\
\hline BS I & 5.92 & 193 & ePd & 19 & 21.50 & -0.7 & I GRO & 45.12 & 362 & $P C$ & 22 \\
\hline & . Os & 250 & $.00 \mathrm{~nm}$ & & & $.9 \mathrm{mb}$ & 1 & & & is & 28 \\
\hline GAR & 26.40 & 296 & eP & 19 & 24.00 & -2.6 & SEY & 45.47 & 30 & ePd & 22 \\
\hline & & & es & 24 & 03.60 & & 1 & & & $s$ & 28 \\
\hline PPR & 26.69 & 138 & ePc & 19 & 27.00 & -2.3 & I KUPT & 46.22 & 149 & eP & 22 \\
\hline & $1.1 \mathrm{~s}$ & 208 & $3.00 \mathrm{~nm}$ & & & $.7 \mathrm{mb}$ & 1 & $0.9 \mathrm{~s}$ & 243 & $3.00 \mathrm{~nm}$ & \\
\hline KUL & 26.71 & 294 & eP & 19 & 29.00 & -0.4 & I ERE & 46.29 & 298 & Po & 22 \\
\hline NVS & 27.09 & 337 & $P$ & 19 & 32.20 & -0.4 & I PET & 46.63 & 45 & eP & 22 \\
\hline & & & es & 24 & 11.00 & & 1 & & & es & 29 \\
\hline KAAO & 27.16 & 286 & ip & 19 & 32.20 & -1.6 & I LEN & 46.71 & 299 & Po & 22 \\
\hline$V L A$ & 7.23 & 55 & P & 19 & 33.20 & -0.8 & I BKR & 46.85 & 300 & Pd & 22 \\
\hline LGP & 27.34 & 125 & ePd & 19 & 35.00 & -0.2 & I PYA & 46.98 & 303 & $\mathrm{PC}$ & 22 \\
\hline TS I & 3 & 186 & ePd & 19 & 35.50 & -0.5 & 1 & & & is & 29 \\
\hline & $.1 \mathrm{~s}$ & 970 & $.00 \mathrm{~nm}$ & & & $.4 \mathrm{mb}$ & BHD & 47.61 & 288 & Po & 22 \\
\hline DSH & 27.54 & 295 & Pd & 19 & 37.80 & 0.8 & 1 & & & es & 27 \\
\hline POO & 27.62 & 250 & IPC & 19 & 39.50 & 1.7 & ? & & & e & 46 \\
\hline & & & iPPP & 20 & 41.50 & & RAM & 49.14 & 295 & eP & 22 \\
\hline & & & is & 24 & 20.50 & & I SOC & 49.43 & 303 & eP & 22 \\
\hline & & & iss & 25 & 28.50 & & 1 & & & is & 29 \\
\hline GBA & 27.82 & 237 & $P C$ & 19 & 38.90 & -0.7 & I TBZ & 49.74 & 299 & iP & 22 \\
\hline & $1.4 \mathrm{~s}$ & 59 & $.60 \mathrm{~nm}$ & & & $.2 \mathrm{mb}$ & I JAY & 50.27 & 124 & $e(P)$ & 22 \\
\hline$B O D$ & 28.35 & 15 & eP & 19 & 44.00 & 0.0 & I MOS & 50.29 & 319 & $P_{c}$ & 22 \\
\hline KGM & 28.87 & 175 & ePc & 19 & 48.00 & -1.0 & ! & & & $i s P$ & 23 \\
\hline & $1.3 \mathrm{~s}$ & 438 & $.00 \mathrm{~nm}$ & & & . $1 \mathrm{mb}$ & 1 & & & es & 30 \\
\hline SAM & 29.04 & 297 & eP & 19 & 51.00 & 0.5 & I OBN & 50.86 & 318 & $\mathrm{PC}$ & 22 \\
\hline & & & $e s$ & 24 & 37.90 & & I & & & esp & 23 \\
\hline & & & ess & 24 & 52.70 & & 1 & & & is & 30 \\
\hline ABU & 29.09 & 73 & ePd & 19 & 51.00 & 0.1 & I MTN & 52.23 & 142 & eP & 23 \\
\hline PLP & 29.60 & 127 & ePd & 19 & 55.00 & -0.6 & I KVT & 52.51 & 300 & iP & 23 \\
\hline KAR & 30.54 & 267 & $e(P)$ & 20 & 05.00 & 1.0 & I KHE & 52.55 & 352 & ePc & 23 \\
\hline MAT & 31.19 & 70 & eP & 20 & 07.00 & -2.6 & 1 & & & es & 30 \\
\hline & $1.7 \mathrm{~s}$ & 431 & $.00 \mathrm{~nm}$ & & 6 & $.1 \mathrm{mb}$ & I SIM & 53.23 & 305 & eP & 23 \\
\hline & & & es & 25 & 16.00 & & I & & & esp & 23 \\
\hline PPI & 31.26 & 181 & eP & 20 & 09.00 & -1.3 & 1 & & & is & 36 \\
\hline PPH & 32.81 & 129 & eP & 20 & 21.00 & -2.9 & I APA & 53.32 & 334 & $P c$ & 23 \\
\hline
\end{tabular}




\begin{tabular}{|c|c|c|c|c|c|c|c|c|c|c|c|}
\hline & $25 \mathrm{~s}$ & & $1.80 \mathrm{um}$ & & & & | & & & e & 40 \\
\hline & $25 \mathrm{~s}$ & & $5.60 \mathrm{um}$ & & & & MOA & 65.79 & 312 & iPc & 24 \\
\hline & & & $i$ & 24 & 11.90 & & i & & & $P \subset P$ & 25 \\
\hline & & & $i$ & 24 & 17.70 & & I KMR & 65.81 & 313 & $e^{P}$ & 24 \\
\hline & & & $\mathrm{i}$ & 24 & 32.90 & & I WET & 66.21 & 314 & iPc & 24 \\
\hline & & & is & 32 & 33.00 & & i & $1.2 \mathrm{~s}$ & 96 & $6.00 \mathrm{~nm}$ & \\
\hline jos & 61.60 & 312 & P & 24 & 88.00 & 0.1 & i LJU & 66.24 & 311 & eP & 24 \\
\hline & & & $i$ & 24 & 12.00 & & I OAG & 66.45 & 347 & iPc & 24 \\
\hline & & & $i$ & 25 & 07.00 & & i & $1.1 \mathrm{~s}$ & 175 & $5.00 \mathrm{~nm}$ & \\
\hline & & & ePP & 26 & 32.00 & & i & & & is & 33 \\
\hline SPC & 61.60 & 313 & ep & 24 & 88.40 & 0.3 & i & & & iss & 37 \\
\hline AAE & 61.75 & 263 & eP & 24 & 12.00 & 2.2 & I MOX & 66.46 & 316 & ip & 24 \\
\hline WBN & 61.78 & 154 & $\mathrm{Pc}$ & 24 & 10.60 & 1.2 & i & $2.0 \mathrm{~s}$ & 177 & $7.00 \mathrm{~nm}$ & \\
\hline SSR & 61.81 & 308 & eP & 24 & 08.00 & -1.4 & i & $z \quad 15 \mathrm{~s}$ & 48 & $0.80 \mathrm{um}$ & \\
\hline$T I M$ & 61.91 & 309 & ep & 24 & 04.00 & $-6.0 x$ & i & $\mathrm{N} \quad 24 \mathrm{~s}$ & 177 & $7.00 \mathrm{um}$ & \\
\hline KNT & 62.10 & 303 & $e(P)$ & 24 & 12.40 & 1.0 & i & E $24 \mathrm{~s}$ & 119 & $9.00 \mathrm{um}$ & \\
\hline PSZ & 62.17 & 311 & $P$ & 24 & 13.20 & 1.3 & ALE & 66.48 & 358 & eP & 24 \\
\hline THE & 62.22 & 303 & $e(P)$ & 24 & 20.00 & $7.9 x$ & i & $1.2 \mathrm{~s}$ & 222 & $2.00 \mathrm{~nm}$ & \\
\hline & & & $e(s)$ & 32 & 41.60 & & I BHG & 66.71 & 313 & $\mathrm{iPc}$ & 24 \\
\hline APO & 62.24 & 326 & $P$ & 24 & 10.05 & -2.0 & I CTA & 66.74 & 134 & $P$ & 24 \\
\hline VAY & 62.29 & 303 & eP & 24 & 12.00 & -0.6 & i & & & is & 33 \\
\hline HFS & 62.45 & 326 & $\mathrm{P}$ & 24 & 12.00 & -1.5 & $|T R|$ & 66.87 & 310 & eP & 24 \\
\hline & $0.5 \mathrm{~s}$ & & $2.00 \mathrm{~nm}$ & & & $.6 \mathrm{mb}$ & i & & & e & 26 \\
\hline & & & e & 53 & 00.00 & & i & & & is & 33 \\
\hline$E S A$ & 62.49 & 122 & $P$ & 24 & 16.20 & 1.9 & i & & & iss & 38 \\
\hline GRG & 62.52 & 303 & $e(P)$ & 24 & 11.60 & -2.6 & i & & & $\mathrm{i}$ & 40 \\
\hline SLL & 62.55 & 326 & $\mathrm{P}$ & 24 & 12.54 & -1.6 & i & & & $\mathrm{i}$ & 44 \\
\hline BEO & 62.70 & 308 & $P$ & 24 & 14.20 & -1.1 & I GRF & 67.03 & 315 & $i P_{C}$ & 24 \\
\hline & & & es & 32 & 44.40 & & i & $1.1 \mathrm{~s}$ & 57 & $7.00 \mathrm{~nm}$ & \\
\hline ASP & 62.76 & 146 & eP & 24 & 15.00 & -1.0 & i & $214 \mathrm{~s}$ & 35 & $5.00 \mathrm{um}$ & \\
\hline SKO & 62.89 & 304 & iPc & 24 & 16.00 & -0.6 & is SGO & 67.79 & 305 & $P$ & 24 \\
\hline & & & is & 32 & 50.00 & & DUI & 67.89 & 306 & $p$ & 24 \\
\hline & & & iss & 37 & 08.00 & & I IMA & 67.94 & 25 & $e^{p}$ & 24 \\
\hline SRO & 63.22 & 311 & eP & 24 & 22.00 & $3.3 x$ & OGA & 68.20 & 312 & $i P c$ & 24 \\
\hline & $N \quad 20 \mathrm{~s}$ & & $8.40 \mathrm{um}$ & & & & $i$ & $1.0 \mathrm{~s}$ & 113 & $3.00 \mathrm{~nm}$ & \\
\hline & $20 \mathrm{~s}$ & & $3.20 \mathrm{um}$ & & & & I AOU & 68.35 & 307 & $P_{-}$ & 24 \\
\hline & & & is & 32 & 58.00 & & 1011 & 68.36 & 302 & $\mathrm{P}$ & 24 \\
\hline 150 & 63.26 & 139 & eP & 24 & 19.00 & -0.3 & I WIT & 68.51 & 319 & ePc & 24 \\
\hline NB2 2 & 63.39 & 327 & $\mathrm{P}$ & 24 & 19.20 & -0.6 & i & & & e & 25 \\
\hline & $0.9 \mathrm{~s}$ & & $8.90 \mathrm{~nm}$ & & & $3 \mathrm{mb}$ & | STU & 68.61 & 315 & ePc & 24 \\
\hline OHR & 63.62 & 304 & eP & 24 & 21.20 & -0.3 & i & $1.0 \mathrm{~s}$ & 60 & $0.00 \mathrm{~nm}$ & \\
\hline$T I R$ & 64.21 & 304 & $P$ & 24 & 23.80 & -1.5 & I WTS & 68.76 & 319 & $i P C$ & 24 \\
\hline MUN & 64.21 & 166 & eP & 24 & 24.00 & -1.3 & $i$ & & & e & 25 \\
\hline VIE & 64.33 & 312 & $P$ & 24 & 30.00 & $4.0 x$ & I MNS & 68.84 & 307 & P+ & 24 \\
\hline & & & $P P$ & 26 & 59.00 & & i & & & $P$ & 14 \\
\hline & & & $\mathrm{i}$ & 28 & 36.00 & & i & & & es & 20 \\
\hline & & & e & 33 & 25.00 & & |NAI & 68.86 & 255 & eP & 24 \\
\hline & & & e & 33 & 43.00 & & i & $2.0 \mathrm{~s}$ & 294 & $400 \mathrm{~nm}$ & \\
\hline & & & e & 54 & 00.00 & & I BNS & 68.95 & 317 & eP & 24 \\
\hline COP & 64.34 & 321 & eP & 24 & 26.00 & 0.1 & I SAL & 69.01 & 311 & $P$ & 24 \\
\hline r & $0.7 \mathrm{~s}$ & 76 & $6.70 \mathrm{~nm}$ & & 6 & $.0 \mathrm{mb}$ & RMP & 69.05 & 307 & $p$ & 25 \\
\hline & & & is & 33 & 07.00 & & i & & & es & 34 \\
\hline VKA & 64.36 & 312 & iPd & 24 & 26.20 & 0.0 & I ROM & 69.14 & 307 & $\mathrm{P}$ & 24 \\
\hline & & & e & 24 & 35.00 & & i & & & $s$ & 34 \\
\hline & & & PP & 26 & 51.00 & & I HNR & 69.17 & 115 & $e^{P}$ & 24 \\
\hline LG & 64.37 & 161 & eP & 24 & 25.08 & -1.4 & i & & & es & 34 \\
\hline KON & 64.57 & 326 & iP & 24 & 27.00 & -0.4 & I ВUн & 69.24 & 315 & EPC & 24 \\
\hline$B R L$ & 64.86 & 318 & eP & 24 & 31.00 & 1.7 & I FIR & 69.25 & 309 & $P$ & 24 \\
\hline PRU & 64.91 & 315 & $P$ & 24 & 29.80 & 0.0 & i & & & is & 34 \\
\hline & $19 \mathrm{~s}$ & 56 & $6.30 \mathrm{um}$ & & & $.8 \mathrm{Msz}$ & | PRT & 69.32 & 309 & $P$ & 25 \\
\hline & $20 \mathrm{~s}$ & 43 & $3.50 \mathrm{um}$ & & & & i & & & $i p p$ & 25 \\
\hline & E $20 \mathrm{~s}$ & 45 & $5.90 \mathrm{um}$ & & & & & & & iPP & 28 \\
\hline & & & $\mathrm{i}$ & 24 & 34.00 & & 1 & & & is & 34 \\
\hline & & & $s$ & 33 & 14.00 & & svw & 69.39 & 30 & ep & 24 \\
\hline$R A$ & 64.95 & 315 & ip & 24 & 30.00 & 0.0 & I GWF & 69.48 & 315 & $P$ & 24 \\
\hline & & & e & 24 & 35.00 & & I ZUL & 69.62 & 314 & $\mathrm{P}$ & 24 \\
\hline & & & $s$ & 33 & 14.00 & & I DBN & 69.64 & 319 & eP & 24 \\
\hline$R G$ & 64.99 & 316 & $i \mathrm{Pc}$ & 24 & 30.50 & 0.3 & i & $220 \mathrm{~s}$ & & $0.00 \mathrm{um}$ & \\
\hline & $1.6 \mathrm{~s}$ & 132 & $2.00 \mathrm{~nm}$ & & 5 & $.9 \mathrm{mb}$ & i & & & es & 34 \\
\hline & 245 & 150 & $0.80 \mathrm{um}$ & & & & i & & & e & 35 \\
\hline & $24 \mathrm{~s}$ & & $4.00 \mathrm{um}$ & & & & i & & & e & 36 \\
\hline & & & es & 33 & 13.00 & & $i$ & & & ess & 38 \\
\hline DY & 65.08 & 29 & eP & 24 & 31.50 & 0.8 & i & & & esss & 41 \\
\hline WAO & 65.35 & 165 & eP & 24 & 32.00 & -0.6 & MEM & 69.78 & 317 & $P$ & 25 \\
\hline LL & 65.41 & 316 & $i P c$ & 24 & 32.80 & -0.1 & $i$ & & & PP & 25 \\
\hline & & & $i$ & 24 & 37.00 & & I PCN & 69.79 & 311 & $P$ & 25 \\
\hline & & & $p P$ & 24 & 42.00 & $30 \mathrm{~km} x$ & $C D F$ & 69.92 & 315 & ep & 25 \\
\hline & & & PPP & 28 & 32.00 & & I BVT & 69.94 & 310 & $P$ & 25 \\
\hline & & & $\mathrm{s}$ & 33 & 18.00 & & $\mathrm{ECH}$ & 70.07 & 315 & $P$ & 25 \\
\hline & & & e & 33 & 29.00 & & i & & & is & 34 \\
\hline & & & PS & 33 & 43.00 & & MBC & 70.08 & 10 & eP & 25 \\
\hline & & & $S P$ & 33 & 51.00 & & i & $0.8 \mathrm{~s}$ & $11 e$ & $0.00 \mathrm{~nm}$ & \\
\hline & & & SS & 37 & 24.00 & & BB & 17 & 314 & $P$ & 25 \\
\hline & & & SSS & 40 & 37.00 & & BAF & 70.33 & 314 & $P$ & 25 \\
\hline$H C$ & 65.79 & 314 & ip & 24 & 35.70 & 0.2 & BSF & 70.46 & 315 & $e^{P}$ & 25 \\
\hline & & & $i$ & 24 & 40.00 & & 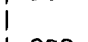 & $1.0 \mathrm{~s}$ & 34 & $4.60 \mathrm{~nm}$ & \\
\hline & & & $\mathrm{s}$ & 33 & 27.00 & & I ORO & 70.63 & 312 & $P$ & 25 \\
\hline
\end{tabular}

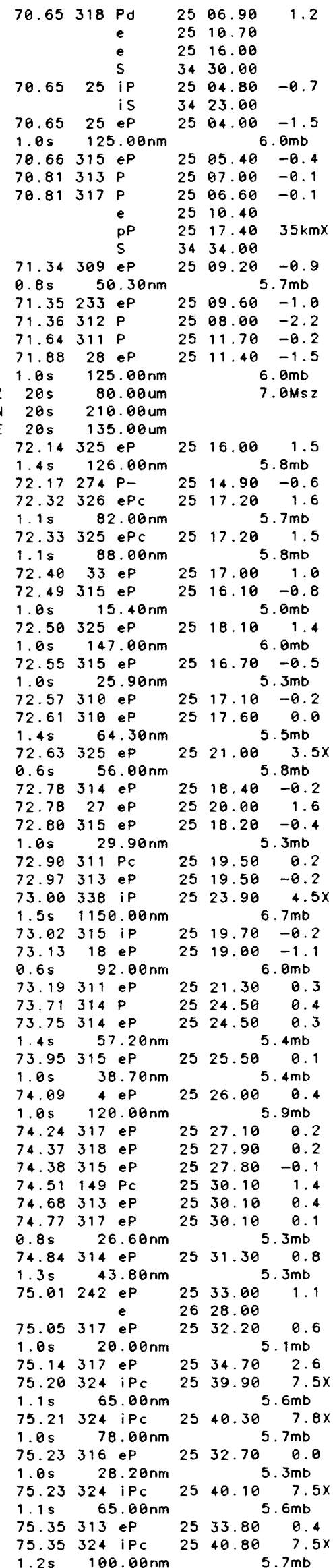




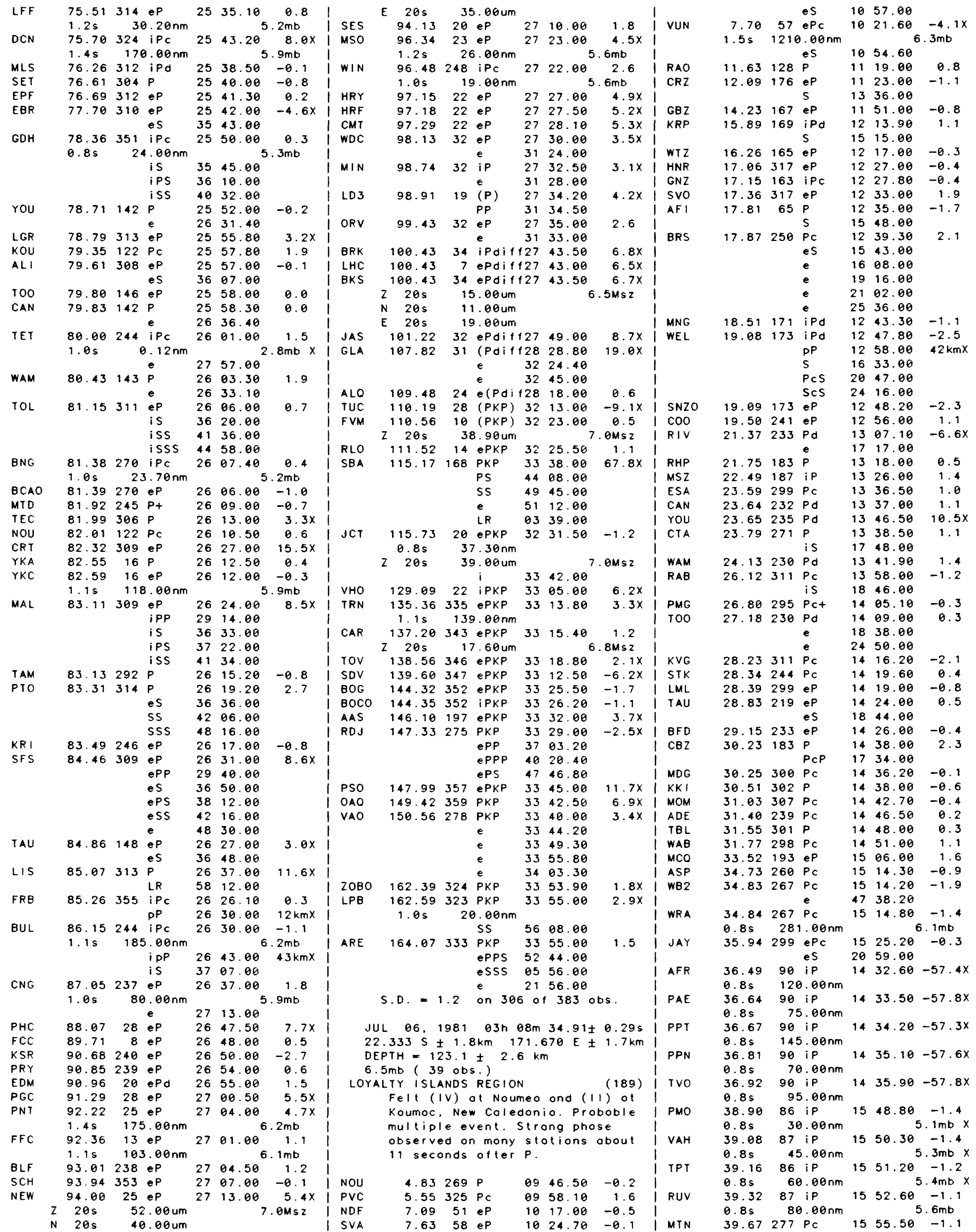




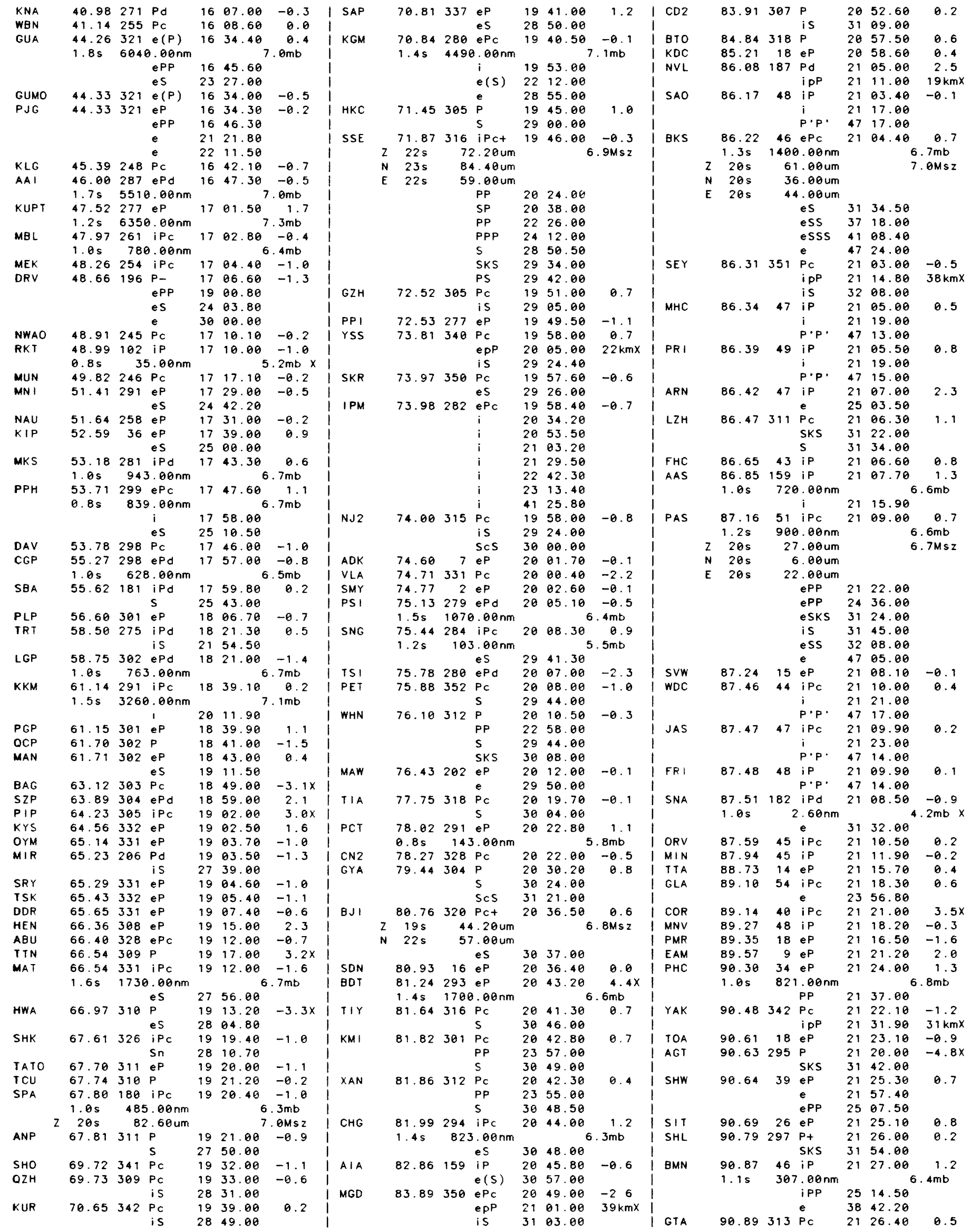




\begin{tabular}{|c|c|c|c|c|c|c|c|c|c|c|c|}
\hline & & & & iSKS & 31 & 47.00 & & 1 & & & ePS \\
\hline & & & & is & 31 & 58.00 & & 1 & & & ess \\
\hline PCA & & 90.97 & 22 & eP & 21 & 25.40 & -0.4 & 1 & & & eSSS \\
\hline LON & & 91.18 & 39 & iPd & 21 & 27.50 & 0.5 & I JCT & 99.80 & 61 & eP \\
\hline & & $1.2 \mathrm{~s}$ & 32 & $0.00 \mathrm{~nm}$ & & & $4 \mathrm{mb}$ & i & $20 \mathrm{~s}$ & 21 & $1.60 \mathrm{um}$ \\
\hline PGC & & 91.27 & 37 & $e^{P}$ & 21 & 26.60 & -0.6 & I UER & 99.84 & 322 & $\mathrm{PC}$ \\
\hline EUR & & 91.27 & 48 & ip & 21 & 27.80 & 0.0 & I $\mathrm{MDZ}$ & 100.33 & 132 & elPdif \\
\hline & & $0.5 \mathrm{~s}$ & & $1.20 \mathrm{~nm}$ & & & $.9 \mathrm{mb}$ & I TLL & 100.47 & 129 & Pdiff \\
\hline TUC & & 91.83 & 56 & iPd & 21 & 32.30 & 1.9 & I WMO & 100.97 & 313 & Pdiffc \\
\hline & $z$ & $18 s$ & & $2.92 \mathrm{um}$ & & & $.8 \mathrm{MszX}$ & I CFA & 101.48 & 131 & Pdiff \\
\hline & & & & ePP & 25 & 12.00 & & I YKA & 102.36 & 27 & Pdiff \\
\hline I MA & & 91.96 & 13 & eP & 21 & 21.20 & $-9.1 x$ & I YKC & 102.41 & 27 & ePdiff \\
\hline $\mathrm{COL}$ & & 92.38 & 16 & ip & 21 & 30.30 & -1.8 & i & $1.6 \mathrm{~s}$ & 113 & $3.00 \mathrm{~nm}$ \\
\hline & $z$ & $19 \mathrm{~s}$ & & $6.30 \mathrm{um}$ & & & $.0 M s z$ & I SEH & 102.47 & 290 & Pdifft \\
\hline & & & & es & 32 & 24.00 & & I GOA & 102.84 & 282 & Pdiff \\
\hline $\mathrm{FBA}$ & & 92.38 & 16 & eP & 21 & 30.00 & -2.1 & i & & & SKS \\
\hline CAL & & 92.53 & 293 & $P$ & 21 & 38.00 & $4.4 x$ & I VCA & 103.14 & 129 & Pdiffo \\
\hline & & & & PP & 25 & 30.00 & & I LPS & 103.63 & 80 & Pdiff \\
\hline & & & & SKS & 32 & 00.00 & & i & & & PP \\
\hline$B O D$ & & 93.05 & 333 & eP & 21 & 34.00 & -1.2 & i & & & SKS \\
\hline LSA & & 93.07 & 301 & $P c$ & 21 & 37.90 & 1.3 & i & & & SKKS \\
\hline & & & & SKS & 32 & 02.90 & & I POO & 103.82 & 285 & Pdifft \\
\hline PNT & & 93.77 & 38 & eP & 21 & 39.00 & 0.2 & I & & & SKS \\
\hline & & $1.2 \mathrm{~s}$ & 413 & $3.00 \mathrm{~nm}$ & & & $6 m b$ & I NDI & 104.10 & 295 & iPdiff \\
\hline & & & & $P P$ & 21 & 05.90 & & i & $21 \mathrm{~s}$ & & $6.90 \mathrm{um}$ \\
\hline ZAK & & 94.12 & 323 & $\mathrm{Pc}$ & 21 & 40.50 & 0.2 & I & & & is \\
\hline TMU & & 94.53 & 135 & eP & 21 & 39.00 & $-3.7 x$ & 1 & & & iSSS \\
\hline I RK & & 94.53 & 325 & ePc & 21 & 41.00 & -1.2 & I ANT & 104.26 & 123 & Pdiff \\
\hline & & & & epP & 21 & 53.00 & $39 \mathrm{kmX}$ & 1 & & & $\mathrm{pP}$ \\
\hline & & & & esp & 21 & 55.00 & & 1 & & & $P P$ \\
\hline & & & & es & 32 & 43.00 & & 1 & & & $\mathbf{i}$ \\
\hline NEW & & 94.69 & 39 & eP & 21 & 43.00 & -0.1 & 1 & & & PPP \\
\hline & $z$ & $19 \mathrm{~s}$ & & $0.00 \mathrm{um}$ & & & $.8 M s z$ & 1 & & & SKS \\
\hline & $N$ & $19 \mathrm{~s}$ & & $3.90 \mathrm{um}$ & & & & 1 & & & $S P$ \\
\hline & $E$ & $19 \mathrm{~s}$ & & $8.00 \mathrm{um}$ & & & & 1 & & & SSP \\
\hline CHA & & 95.19 & 297 & P- & 21 & 48.30 & 2.5 & 1 & & & SS \\
\hline BOK & & 95.21 & 293 & P & 21 & 45.70 & -0.2 & 1 & & & SSS \\
\hline & & & & PP & 25 & 30.70 & & 1510 & 104.26 & 57 & e (Pdif \\
\hline & & & & SKS & 32 & 10.00 & & I TUL & 104.71 & 57 & e (Pdif \\
\hline $\mathrm{CON}$ & & 95.27 & 133 & $P$ & 22 & 49.00 & $62.9 x$ & 1 & $1.5 \mathrm{~s}$ & & $8.40 \mathrm{~nm}$ \\
\hline YKM & & 95.80 & 39 & iP & 21 & 51.40 & $3.1 x$ & i & $18 \mathrm{~s}$ & & $7.40 \mathrm{um}$ \\
\hline LDM & & 95.84 & 40 & iP & 21 & 51.70 & $3.3 x$ & I BOM & 104.87 & 285 & Pdift \\
\hline MSO & & 95.95 & 42 & iPc & 21 & 50.30 & 1.3 & 1 & & & SKS \\
\hline & & $1.5 \mathrm{~s}$ & 143 & $3.00 \mathrm{~nm}$ & & & $.2 \mathrm{mb}$ & ELT & 104.92 & 322 & Pdiff \\
\hline MOY & & 96.03 & 324 & $\mathrm{Pc}$ & 21 & 48.30 & -0.7 & I CYA & 105.04 & 130 & Pdiff \\
\hline$R \times F$ & & 96.15 & 39 & iP & 21 & 51.20 & 1.4 & I RLO & 105.38 & 57 & ePdifi \\
\hline ALO & & 96.19 & 55 & eP & 21 & 49.20 & -1.3 & I BHK & 105.57 & 298 & Pdiff \\
\hline & & $1.0 \mathrm{~s}$ & 66 & $6.30 \mathrm{~nm}$ & & & $1 \mathrm{mb}$ & 1 & & & SKS \\
\hline & $z$ & $18 \mathrm{~s}$ & & 2. $60 \mathrm{um}$ & & & $.8 \mathrm{Msz}$ & I FFC & 105.88 & 37 & ePdiff \\
\hline & & & & e & 22 & 03.00 & & 1 & $1.1 \mathrm{~s}$ & & $8.00 \mathrm{~nm}$ \\
\hline MDR & & 96.19 & 281 & $\mathbf{P}$ & 21 & 51.80 & 1.3 & I LPA & 106.14 & 139 & Pdiffo \\
\hline & & & & SKS & 32 & 07.80 & & I LPA & 106.14 & 139 & PKP \\
\hline ANMO & & 96.19 & 55 & $e^{P}$ & 21 & 51.00 & 0.5 & i & & & PP \\
\hline AMM & & 96.24 & 43 & ePd & 21 & 50.90 & 0.5 & 1 & & & PPP \\
\hline BUT & & 96.45 & 43 & ePd & 21 & 54.30 & $3.6 x$ & 1 & & & SKS \\
\hline BDW & & 97.05 & 47 & iPc & 21 & 55.20 & 1.0 & 1 & & & SP \\
\hline & & $1.5 \mathrm{~s}$ & 162 & $2.00 \mathrm{~nm}$ & & & $3 \mathrm{mb}$ & I $M B C$ & 106.70 & 13 & ePdiff \\
\hline & & & & e & 24 & 16.50 & & 1 & $0.9 \mathrm{~s}$ & & $8.00 \mathrm{~nm}$ \\
\hline & & & & iPP & 25 & 53.60 & & I ARE & 107.14 & 116 & ePdiff \\
\hline YTC & & 97.23 & 45 & $(P)$ & 21 & 57.50 & 2.5 & 1 & & & ePP \\
\hline TRD & & 97.51 & 276 & $\mathrm{P}+$ & 21 & 58.50 & 2.0 & 1 & & & eSKS \\
\hline & & & & SKS & 32 & 32.50 & & 1 & & & ePS \\
\hline VHO & & 97.81 & 75 & iP & 22 & 02.00 & $4.0 x$ & I LAH & 107.32 & 298 & ePdiff \\
\hline$T I K$ & & 98.22 & 347 & eP & 21 & 57.00 & -1.5 & I SLA & 107.42 & 127 & Pdifto \\
\hline & & & & epP & 22 & 08.00 & $35 \mathrm{kmX}$ & I MNL & 108.22 & 299 & ePdiff \\
\hline & & & & es & 33 & 12.00 & & I NRN & 108.53 & 308 & ePdiff \\
\hline SAN & & 98.75 & 131 & eP & 22 & 04.00 & 2.0 & I NIL & 108.75 & 299 & ePdiff \\
\hline INK & & 98.76 & 18 & ePc & 22 & 00.30 & -0.7 & I FVM & 109.40 & 56 & (Pdiff \\
\hline & & $2.0 \mathrm{~s}$ & 724 & $4.00 \mathrm{~nm}$ & & & $.9 \mathrm{mb}$ & i & $18 \mathrm{~s}$ & & $6.50 \mathrm{um}$ \\
\hline & & & & $P P$ & 22 & 36.00 & & i & & & e \\
\hline GOL & & 98.79 & 51 & eP & 22 & 02.70 & 0.6 & I LPB & 109.90 & 118 & Pdiff \\
\hline & & $1.2 \mathrm{~s}$ & 52 & $2.60 \mathrm{~nm}$ & & & $0 m b$ & 1 & & & SKS \\
\hline & $z$ & $20 \mathrm{~s}$ & 65 & $5.20 \mathrm{um}$ & & & $1 \mathrm{Msz}$ & i & & & PS \\
\hline & & & & e & 22 & 14.40 & & i & & & e \\
\hline GBA & & 98.90 & 281 & $\mathrm{Pc}$ & 22 & 03.30 & 0.6 & 12080 & 110.01 & 118 & Pdiff \\
\hline & & $2.0 \mathrm{~s}$ & 874 & 4. $00 \mathrm{~nm}$ & & & $.0 m b$ & I PSH & 110.29 & 299 & ePditi \\
\hline PEL & & 98.91 & 131 & $P+$ & 22 & 03.00 & 0.3 & I WRS & 110.35 & 299 & ePdiff \\
\hline GLD & & 98.92 & 51 & eP & 22 & 04.00 & 1.4 & I KHO & 111.01 & 303 & Pdiff \\
\hline & $z$ & $18 \mathrm{~s}$ & & $0.90 \mathrm{um}$ & & & $.7 \mathrm{Msz}$ & I ANR & 111.07 & 307 & ePdifi \\
\hline & & & & e & 22 & 15.70 & & I AVY & 111.57 & 238 & ¿PKPC \\
\hline EDM & & 99.07 & 36 & eP & 22 & 02.50 & -0.3 & I GAR & 112.26 & 304 & ePdiff \\
\hline SES & & 99.20 & 39 & eP & 22 & 03.00 & -0.5 & I LHC & 112.27 & 45 & ePdi f 1 \\
\hline & & $1.4 \mathrm{~s}$ & 295 & $5.00 \mathrm{~nm}$ & & & $.7 \mathrm{mb}$ & I RES & 112.27 & 17 & ePdiff \\
\hline HYB & & 99.23 & 285 & eP & 22 & 04.00 & -0.3 & 1 & $1.2 \mathrm{~s}$ & & $2.60 \mathrm{~nm}$ \\
\hline & & & & ePP & 26 & 04.00 & & I KAAO & 112.35 & 299 & elPdif \\
\hline & & & & eSKS & 32 & 33.50 & & I KBL & 112.35 & 299 & elPdif \\
\hline & & & & es & 33 & 30.00 & & I KUL & 112.48 & 303 & ePdiff \\
\hline
\end{tabular}




\begin{tabular}{|c|c|c|c|c|c|c|c|c|c|c|c|}
\hline TRN & 128.04 & 94 & ePKP & 27 & 27.10 & -1.6 & KIS & 141.28 & 319 & PKP & 27 \\
\hline & 1.05 & 200 & $3.00 \mathrm{~nm}$ & & & & I CFR & 142.52 & 316 & ePKP & 27 \\
\hline BAK & 128.04 & 304 & PKPC & 27 & 32.00 & $4.1 x$ & I WAR & 142.58 & 330 & iPKP & 27 \\
\hline EV & 28.10 & 345 & ePdif & 24 & 12.00 & 0.6 & i & & & ePP & 31 \\
\hline EV & 128.10 & 345 & ePKP & 27 & 28.00 & 0.8 & I GPA & 142.80 & 308 & ePKP & 27 \\
\hline & & & $\mathrm{i}$ & 27 & 42.20 & & $1 O D B$ & 142.95 & 318 & EPKP & 27 \\
\hline & & & ePP & 29 & 25.00 & & 1 & & & $\mathbf{i}$ & 28 \\
\hline MAK & 129.71 & 308 & ePdif & 124 & 22.00 & $2.8 x$ & | VRI & 143.12 & 318 & ePKP & 27 \\
\hline TA & 29.73 & 266 & i PKP & 27 & 34.80 & $2.9 x$ & I COP & 143.29 & 340 & iPKP & 27 \\
\hline 80 & 29.82 & 267 & iPKP & 27 & 35.60 & $3.5 x$ & 1 & & & $\mathrm{i}$ & 28 \\
\hline SOD & 129.94 & 343 & ePKP & 27 & 28.00 & $-2.7 x$ & 1 & & & iPP & 31 \\
\hline & & & $\mathrm{i}$ & 27 & 41.30 & & I ALT & 143.30 & 306 & iPKPC & 27 \\
\hline & & & ePP & 29 & 40.00 & & I BCK & 143.33 & 303 & iPKP & 27 \\
\hline & & & ESKP & 30 & 42.00 & & I ISK & 143.46 & 310 & iPKP & 27 \\
\hline & & & e & 31 & 12.00 & & I ISR & 143.56 & 317 & ePKP & 27 \\
\hline RO & 29.95 & 348 & ePKP & 27 & 37.00 & $6.3 x$ & BKT & 143.70 & 308 & iPKP & 27 \\
\hline ARO & 30.08 & 266 & iPKP & 27 & 35.70 & $3.0 x$ & I MLR & 143.78 & 318 & ePKP & 27 \\
\hline NAI & 130.14 & 248 & ePKP & 27 & 35.00 & 2.0 & 1 & & & iPP & 31 \\
\hline & $2.0 \mathrm{~s}$ & 1410 & $0.00 \mathrm{~nm}$ & & & & 1 & & & eSKKS & 38 \\
\hline ER & 130.30 & 297 & ePKP & 27 & 32.00 & -0.7 & 1 & & & ePPS & 43 \\
\hline AF & 30.40 & 266 & iPKP & 27 & 36.60 & $3.4 x$ & | CTT & 143.89 & 310 & iPKP & 27 \\
\hline RV & 30.74 & 305 & ePKP & 27 & 19.00 & $-14 \cdot 1 x$ & IELL & 144.02 & 302 & IPKP & 27 \\
\hline RS & 30.82 & 303 & PKPC & 27 & 34.40 & 0.9 & I BMR & 144.11 & 323 & ePKPd & 27 \\
\hline TAB & 130.90 & 302 & ePKP & 27 & 32.00 & -1.7 & I DMK & 144.12 & 311 & iPKP & 27 \\
\hline & & & e & 27 & 34.00 & & I BUC & 144.18 & 316 & ePKP & 27 \\
\hline RO & 30.94 & 309 & PKPC & 27 & 34.00 & 0.6 & 1 & & & ePP & 31 \\
\hline$K I R$ & 131.11 & 346 & $P K P$ & 27 & 33.60 & 0.6 & I UZH & 144.22 & 325 & PKPC & 27 \\
\hline & & & iPP & 29 & 54.30 & & I DST & 144.24 & 307 & iPKP & 27 \\
\hline ITA & 31.79 & 307 & ePKP & 27 & 21.00 & $-14.0 x$ & I BUCl & 144.25 & 316 & EPKP & 27 \\
\hline KJF & 131.86 & 340 & IPKP & 27 & 31.70 & $-2.8 x$ & I MDE & 144.41 & 320 & ePKP & 28 \\
\hline & & & $i$ & 27 & 36.90 & & 1 & & & $\mathrm{i}$ & 28 \\
\hline & & & ePP & 29 & 52.00 & & I CMP & 144.44 & 318 & ePKPc & 27 \\
\hline & & & iPKS & 31 & 00.20 & & I KRA & 144.53 & 328 & IPKPC & 27 \\
\hline & & & $\mathrm{i}$ & 31 & 19.10 & & 1 & $1.5 \mathrm{~s}$ & 1100 & $9.00 \mathrm{~nm}$ & \\
\hline & & & PS & 39 & 54.00 & & 1 & & & e & 27 \\
\hline 105 & 32.50 & 327 & ePdiff & 24 & 34.00 & $2.7 x$ & 1 & & & i & 28 \\
\hline BHD & 132.55 & 295 & PKP & 27 & 28.00 & $-8.7 x$ & 1 & & & $\mathrm{i}$ & 28 \\
\hline & & & ePP & 27 & 39.50 & & 1 & & & $\mathrm{i}$ & 31 \\
\hline & & & ePPP & 27 & 51.00 & & 1 & & & e & 37 \\
\hline & & & e & 29 & 16.00 & & I EDC & 144.56 & 309 & iPKP & 27 \\
\hline & & & $\mathrm{i}$ & 30 & 09.00 & & I CEI & 144.68 & 323 & ePKP & 27 \\
\hline & & & iPCP & 30 & 56.00 & & 1 & & & $\mathrm{i}$ & 28 \\
\hline & & & is & 31 & 18.00 & & I NIE & 144.76 & 327 & iPKPC & 27 \\
\hline & & & e & 33 & 25.00 & & 1 & & & $\mathbf{i}$ & 28 \\
\hline & & & e LO & 35 & 49.00 & & I MFT & 144.83 & 310 & iPKP & 27 \\
\hline & & & eLR & 36 & 46.00 & & I GET & 144.85 & 309 & ePKP & 27 \\
\hline LEN & 32.61 & 305 & ePKP & 27 & 36.00 & -0.9 & $1 S P C$ & 144.94 & 327 & IPKP & 27 \\
\hline PYA & 32.72 & 310 & ePdif & 24 & 32.00 & -0.6 & 1 & $19 s$ & 57 & $7.10 u m$ & \\
\hline BKR & 32.74 & 307 & ePKP & 27 & 29.00 & $-8 \cdot 1 x$ & 1 & & & $i$ & 28 \\
\hline$A E$ & 32.96 & 261 & ePKP & 27 & 39.20 & 0.7 & 1 & & & $i$ & 28 \\
\hline $\mathrm{BN}$ & 33.28 & 326 & ePdif & 24 & 36.00 & 1.3 & I DRA & 145.22 & 318 & EPKP & 27 \\
\hline SUF & 33.39 & 339 & ePKP & 27 & 24.00 & $-13.4 x$ & $1 \mathrm{JOS}$ & 145.23 & 326 & PKPC & 27 \\
\hline PUL & 133.77 & 334 & ePdif & 24 & 38.00 & 1.2 & 1 & & & $\epsilon$ & 28 \\
\hline SOB3 & 134.61 & 131 & PKP & 27 & 38.00 & $-3 \cdot 2 x$ & 1 & & & $e$ & 29 \\
\hline & & & e & 27 & 42.90 & & i & & & $e$ & 35 \\
\hline SOB 4 & 34.76 & 131 & ePKP & 27 & 37.80 & $-3.7 x$ & I PVL & 145.34 & 315 & PKPC & 27 \\
\hline SOB2 & 34.96 & 131 & PKP & 27 & 34.60 & $-7.2 x$ & I DIM & 145.53 & 313 & ePKP & 27 \\
\hline Soc & 35.18 & 310 & ePdif & $f 24$ & 42.00 & -1.5 & $11 Z M$ & 145.62 & 306 & iPKPC & 27 \\
\hline NUR & 35.45 & 337 & ePdift & 24 & 44.00 & -0.2 & I BRL & 145.63 & 336 & ePKP & 28 \\
\hline NUR & 135.45 & 337 & iPKP & 27 & 40.90 & -0.5 & I KSP & 145.67 & 332 & iPKPc & 27 \\
\hline & & & $\mathrm{i}$ & 28 & 08.50 & & I BRN & 145.71 & 336 & iPKPc & 28 \\
\hline & & & ePP & 30 & 08.00 & & IEZN & 145.85 & 309 & iPKP & 28 \\
\hline & & & ePKS & 31 & 10.00 & & KDZ & 145.85 & 312 & EPKP & 28 \\
\hline$A K U$ & 136.19 & 6 & ePKP & 27 & 43.70 & 1.1 & I PSZ & 145.93 & 325 & PKP & 28 \\
\hline & $1.0 \mathrm{~s}$ & 500 & $0.00 \mathrm{~nm}$ & & & & I HAM & 145.95 & 340 & i PKPC & 28 \\
\hline & & & $\mathrm{i}$ & 27 & 54.10 & & I BRG & 146.63 & 334 & IPKPC & 28 \\
\hline & & & $\mathbf{i}$ & 30 & 21.30 & & 1 & & & PP & 31 \\
\hline $5 \mathrm{TJ}$ & 36.55 & 44 & ePKP & 27 & 49.80 & $5.9 x$ & 1 & & & e & 44 \\
\hline REY & 137.24 & 9 & ePKP & 27 & 46.80 & 2.1 & I BUD & 146.66 & 325 & PKP & 28 \\
\hline & & & $i$ & 30 & 30.70 & & 1 & & & $e$ & 28 \\
\hline UPP & 138.27 & 341 & PKP & 27 & 43.70 & $-3.0 x$ & 1 & & & e & 28 \\
\hline & & & i & 28 & 04.30 & & I & & & ePP & 31 \\
\hline & & & iPP & 30 & 38.00 & & I SSR & 146.66 & 320 & PKP & 28 \\
\hline VT & 38.38 & 307 & ePKP & 27 & 38.00 & $-9.6 x$ & 1 & & & iPP & 31 \\
\hline AFS & 139.17 & 343 & PKP & 27 & 39.10 & $-9.3 x$ & I CLL & 146.66 & 335 & iPKPC & 28 \\
\hline & $0.8 \mathrm{~s}$ & & $4.00 \mathrm{~nm}$ & & & & I & & & $\mathrm{i}$ & 28 \\
\hline & & & e & 26 & 00.00 & & 1 & & & PKP & 28 \\
\hline JNJ & 139.75 & 294 & PKP & 27 & 45.00 & $-5.4 x$ & 1 & & & $\mathrm{i}$ & 28 \\
\hline KAS & 139.98 & 308 & ePKP & 27 & 44.30 & $-6.2 x$ & 1 & & & $\mathrm{i}$ & 28 \\
\hline & & & $i$ & 27 & 52.30 & & 1 & & & $P P$ & 31 \\
\hline & & & $i$ & 28 & 04.80 & & 1 & & & PKS & 31 \\
\hline$O N$ & 140.61 & 346 & ePKP & 27 & 43.00 & $-7.9 x$ & 1 & & & (SKS) & 35 \\
\hline & & & $\mathbf{i}$ & 27 & 48.00 & & SRO & 146.81 & 326 & ePKP & 28 \\
\hline EF & 140.78 & 349 & iPKP & 27 & 46.70 & $-4.5 x$ & | & & & $\mathrm{i}$ & 28 \\
\hline NTO & 141.08 & 306 & ePKPd & 27 & 47.00 & $-5.6 x$ & 1 & & & $\mathrm{i}$ & 28 \\
\hline & $1.5 \mathrm{~s}$ & 56 & $0.80 \mathrm{~nm}$ & & & & l & & & $e$ & 29 \\
\hline & 141.25 & 308 & iPKP & 27 & 49.60 & $2 x$ & 1 EKA & 46 & 355 & PKPD & 28 \\
\hline
\end{tabular}


96d $93 \mathrm{~h}$

TTG $\quad 149.49 \quad 318$ PKP $27 \quad 05.00-61.3 \times$ PN

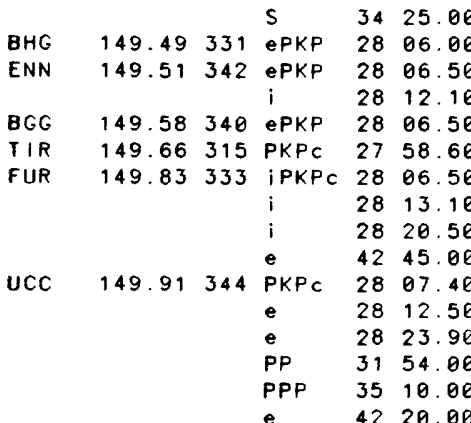

LJU $\quad 149.92 \quad 327$ iPKP 2807.30 2817.00 $\begin{array}{lll}28 & 43.10\end{array}$

STU $\quad 150.16 \quad 336$ ePKPC $28 \quad 07.00$ $2.0 \mathrm{~s} \quad 235.00 \mathrm{~nm}$

ECP $\quad 150.17 \quad 358$ iPKPC $28 \quad 08.40$ $2.0 \mathrm{~s} \quad 2760.00 \mathrm{~nm}$

VAL 150.412 PKPd+ 2806.00 PP $\quad 3148.00$ (SKS) 3922.00 SS 4507.00 $\begin{array}{llll}L O & 52 & 48.00 \\ \text { LR } & 57 & 1300\end{array}$

DOU $\quad 150.48 \quad 343$ ePKPC $28 \quad 08.00$ $\epsilon \quad 2813.00$ $\begin{array}{lll}\epsilon & 2827.00\end{array}$ PKS $3149 \quad 00$ PP $32 \quad 01.00$ PPP $35 \quad 14.00$ e 4225.00

IRI $\quad 150.54 \quad 328$ ePKP 2807.00 i 3156.00 SKKS 3922.00 PS 4222.00 (SS) 4722.00 SSS 5258.00 $\begin{array}{llllll}\text { GWF } & 150.55 & 338 & \text { PKP } & 28 & 07.00 \\ \text { BUH } & 150.59 & 337 & \text { PKPC } & 28 & 07.70\end{array}$ $\begin{array}{llllll}\text { BUH } & 150.59 & 337 \text { ePKPC } 28 & 07.70\end{array}$ $\begin{array}{llllll}\text { CDF } & 151.15 & 338 & \text { ePKP } 28 & 08.40\end{array}$ ECH $151.36338 \mathrm{PKP}+2808.00$ $\begin{array}{lll}\text { e } & 28 & 22.00 \\ \text { ePP } & 31 & 55.00\end{array}$ ESKS 3530.00

$\begin{array}{llllll}\text { ZUL } & 151.53 \quad 336 & \text { PKP } & 28 & 07.50\end{array}$ $\begin{array}{llllll}\text { BRT } & 151.62 & 317 & \text { PKP } & 28 & 12.00\end{array}$ $\begin{array}{llllll}\text { BAF } & 151.74 & 338 & \text { PKP } & 28 & 08.80\end{array}$ BSF 151.81338 IPKPC 2809.50 HAU 151.81339 iPKPC 2809.70 $\begin{array}{llllll}\text { BBS } & 151.87 & 337 & \text { PKP } & 28 & 09.00\end{array}$ NEC $\quad 152.46 \quad 337$ PKP $28 \quad 10.00$ ORI $\quad 152.55316$ PKP $28 \quad 11.00$ $\begin{array}{llllll} & & \text { EPP } & 32 & 01.00 \\ 152.90 & 349 & \text { iPKPC } & 28 & 11.20\end{array}$ $153.00 \quad 348$ IPKPC $28 \quad 11.4 \theta$

$\begin{array}{ccccc}A Q U & 153.01323 & \text { PKP } & 28 & 11.00 \\ & & \text { iPP } & 32 & 02.00 \\ & \epsilon & 35 & 43.00\end{array}$

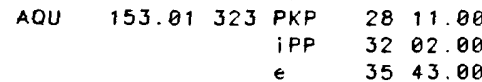

$\begin{array}{lllllll}\text { PRG } & 153.06 & 325 & \text { PKP } & 35 & 43.00 \\ & & & \end{array}$

$\begin{array}{llllll}\text { DIX } & 153.07 & 335 \text { PKP } & 28 & 10.50\end{array}$

FIR 153.17327 PKP- 2812.00

$\begin{array}{llll}i & 2838.08\end{array}$ $\begin{array}{lll}\text { PKP } & 28 & 13.00 \\ \text { i PPKP } & 28 & 22.50\end{array}$ i $\quad 2853.00$ iSKS 3605.08

$\begin{array}{lllllll}\text { LOR } & 153.25 & 341 \text { iPKPC } & 28 & 11.90\end{array}$

$\begin{array}{lllllll}\text { GRR } & 153.32 & 349 & \text { IPKPC } & 28 & 12.00\end{array}$

GRC $\quad 153.45 \quad 342$ iPKPC $28 \quad 11.20$ i $\quad 2819.40$ i $\quad \begin{array}{lll}\text { iPP } & 32 & 34.60 \\ \text { P } & 11.80\end{array}$ $\begin{array}{lllllll} & & & \text { IPP } & 32 & 11.80 \\ \text { SIE } & 153.47 & 327 & \text { PKP } & 28 & 24.60\end{array}$ LBF $\quad 153.47 \quad 341$ iPKPC $28 \quad 12.10$ $\begin{array}{llllll}\text { SSF } & 153.54 & 342 & \text { iPKPC } & 28 & 12.20\end{array}$ LPF $\quad 153.70 \quad 349$ iPKPC 2812.50 GEN $153.78 \quad 331$ PKP $28 \quad 11.50$ $\begin{array}{llllll}\text { SMF } & 153.82 & 341 & \text { IPKPC } & 28 & 12.40\end{array}$ $\begin{array}{llllll}\text { AVF } & 153.83 & 342 & \text { IPKPC } & 28 & 12.48\end{array}$ $\begin{array}{llllll}\text { SGR } & 153.99 & 349 & \text { ePKP } 28 & 14.00\end{array}$

CAF

I LPO

$-1.3 \quad$ MLS

I EPF

ESCF

STS
EBR

0.5

TOL

-0.7
-0.2 0.3 $-0.7$

0.1 |

0.3
-1.0

$-1.0$

-0.1
-0.1

-0.1
1.4

\begin{tabular}{c|l}
-0.2 & ROB \\
-0.5 & TCF \\
0.5 & STV \\
0.3 & LSF \\
$-8.0 \times$ & PYM \\
-0.2 & MFF \\
& CVF \\
& GIB \\
0.8 & LRG \\
& LMR \\
& RJF \\
& CDR \\
& ROG \\
0.4 & \\
&
\end{tabular}

$54.07 \quad 334$ PKP $\quad 28 \quad 11.50 \quad-1.4$ 154.34332 PKP $2812.00 \quad-1.4$ $154.58 \quad 342$ IPKPC $28 \quad 14.10 \quad 0.6$ $\begin{array}{llllll}154.61 & 343 & \text { iPKPC } 28 & 13.60 & 0.0\end{array}$ $\begin{array}{llllll}154.62 & 333 & \text { PKP } & 28 & 13.30 & -0.4\end{array}$ $\begin{array}{lllll}154.87 & 341 & \text { PKP } 28 & 13.50\end{array}$ $154.90 \quad 347 \quad$ IPKPC $28 \quad 14.20$ $\begin{array}{lllll}155.24 & 329 \text { IPKPC } 28 \quad 14.4 \theta\end{array}$ $\epsilon \quad 3621.00$

$155.66 \quad 333$ iPKPC $28 \quad 15.30$ $155.70 \quad 333$ IPKPC $28 \quad 15.2 \theta$ $\begin{array}{lllll}155.71 & 343 \text { IPKPC } 28 & 15.58\end{array}$

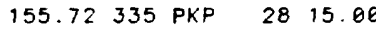
155.89335 ePKP $28 \quad 15.50$

$$
\begin{array}{lll}
i & 2840.00 \\
i & 2843.30 \\
i & 2934.00 \\
\text { ipP } & 3223.60
\end{array}
$$

155.90342 iPKPC $28 \quad 16.00$

$156.26 \quad 344$ iPKPC $28 \quad 16.20$

156.37343 IPKPC $28 \quad 16.58$

$\begin{array}{llll}157.99 & 341 & \text { ePKP } 28 & 19.50\end{array}$

158.13343 iPKPC $28 \quad 18.50$

$158.33 \quad 344$ ePKP $28 \quad 18.8 \theta$

$\begin{array}{llll}159.50 & \theta \text { ePKP } 28 \quad 22.0 \theta\end{array}$

160.12340 ePKP $28 \quad 21.00$ PP $32 \quad 39.00$ $\begin{array}{lll}i P K P c & 28 & 23.00 \\ \epsilon & 28 & 34.00\end{array}$ $\begin{array}{lll}\epsilon & 28 & 34.00 \\ \text { iPKKP } & 29 & 11.50\end{array}$ i 2921.50 iPKS 3150.00 IPP 3253.00 iSKS 3520.00 IPPP $36 \quad 20.00$ iSKKS 3902.00 SKKKS 4118.00 ESKSP 4306.00 i 4400.00 iPPS $46 \quad 55.0 \theta$ iSS 5355.00 iSSS 5950.00

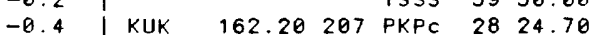

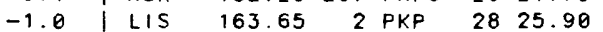
LR $31 \quad 12.00$ IPP $33 \quad 08.80$ SKS 3516.00

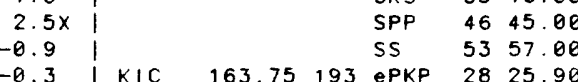

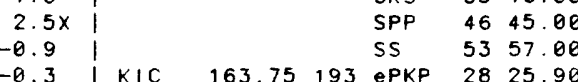

\begin{tabular}{l|llllll}
-0.3 & KIC & 163.75 & 193 ePKP & 28 & 25.90 \\
0.0 & & & & $i$ & 28 & 37.40
\end{tabular}

\begin{tabular}{r|r|rr}
0.0 & 1 & $i$ & 2837.40 \\
-0.8 & $i$ & 2930.00
\end{tabular}

-0.6 ALM 164.66342 iPKPO $28 \quad 25.20$

$0.1 \quad 2.0 \mathrm{~s} \quad 3.48 \mathrm{~nm}$ $\begin{array}{rrr}Z & 23 \mathrm{~s} & 0.60 \mathrm{um} \\ \mathrm{N} & 27 \mathrm{~s} & 2.10 \mathrm{um}\end{array}$

\begin{tabular}{r|ccc}
0.1 & $\mathrm{~N} 20 \mathrm{~s}$ & $4.00 \mathrm{um}$ \\
-0.6 & iPP 3311.90
\end{tabular}

$\begin{array}{rlll}-0.6 & \text { iPP } & 33 & 11.90 \\ & \text { is } & 3928.40\end{array}$ $10.4 \times$ MAL 165.26348 iPKPc 2825.00 iPP $\quad 3316.00$

\begin{tabular}{r|rr}
-1.3 & & iSKKS 3954.00 \\
0.4 & iSKSP 4346.00 \\
& SFS 165.80353 iPKP 2814.00
\end{tabular}

\begin{tabular}{r|rr}
-1.3 & & iSKKS 3954.00 \\
0.4 & iSKSP 4346.00 \\
& SFS 165.80353 iPKP 2814.00
\end{tabular}

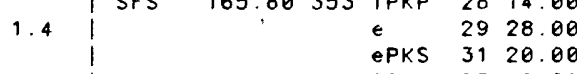
ISKS 3310.00

$\begin{array}{llllll}\text { TAM } & 167.19 & 275 & \text { PKP } & 28 & 31.00\end{array}$ 0.2 MBO 168.63133 iPKP 2834.70

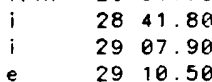

e $\quad 29 \quad 10.50$

$\begin{array}{lll}\mathrm{i} & 29 & 51.50 \\ \mathrm{e} & 30 & 11.70\end{array}$

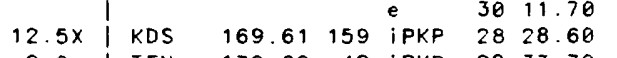

\begin{tabular}{l|llllll}
0.0 & TEN & 170.60 & 48 & IPKP & 28 & 33.70
\end{tabular}

-0.0
0.1

0.1
-0.5
0.3

-0.5
0.3

-0.1
-2.3

0.2

0.2

0.6

3. $3 x$

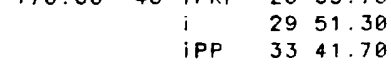

ePPP $37 \quad 41.70$

S.D. $=1.1$ on 402 of 515 obs.

$4 \times$

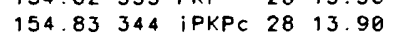
$\begin{array}{lllll}155.26 & 314 & \text { PKP } 28 & 12.50\end{array}$ i $\quad 2836.40$

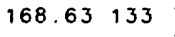

0.1 TLL

0.4 I CEN

$-0.21$

0.11

1

PTOS

$\begin{array}{lll}36.34 & 70 & \mathrm{eP} \\ 36.37 & 88 & \mathrm{P}\end{array}$

1 PP

$0.6 \quad$ NNA $36.68 \quad 66$ ip

\begin{tabular}{l|lll}
0.4 & VCA & 36.71 & $97 \mathrm{P}$
\end{tabular}

0.5 IVCA

$\begin{array}{lll}36.71 & 97 & P \\ 37.84 & 67 & P\end{array}$

$0.3 \quad 1$

2.31

eP
eP
eS

ARE

38.5677 (P)

7 eP

eLO

eLR

$39.14102 \mathrm{PC}$

$\begin{array}{ll}\text { LPB } & 41.37 \quad 79 \mathrm{PC}\end{array}$

ZOBO $41.47 \quad 79 \mathrm{P}$

$\begin{array}{ll}\text { BAA } & 43.52109 \mathrm{PC}\end{array}$

LPA

$S$

$9 \mathrm{PC}$

43. $91109 \mathrm{PC}$

1

$A \mid A$

is
SS

$44.11 \quad 154 \mathrm{eP}$

e (S)

1.9 RAR

44.84271

LO

PSO

PSO

BOCO

$45.29 \quad 51 \mathrm{eP}$

$49.24 \quad 49$ eP

$49.98 \quad 51$ iP

$B O G$

FUo

$50.00 \quad 51 \mathrm{eP}$

$50.84 \quad 51 \mathrm{eP}$

$51.49 \quad 15 \mathrm{eP}$

$51.66100 \mathrm{P}$

$51.67 \quad 14 \mathrm{eP}$

$52.01 \quad 14 \mathrm{eP}$

$54.88 \quad 50 \mathrm{eP}$

$55.42 \quad 51 \mathrm{eP}$

$55.95 \quad 49 \mathrm{eP}$

$56.6298 \mathrm{P}$

$56.64 \quad 51$ ерP

$\begin{array}{rrr}56.64 & 51 & \mathrm{e} \\ 582 & 194 \mathrm{P}\end{array}$

$4 \mathrm{P}$

LQ

LR

$58.31273 \mathrm{eP}$

SSS

$\begin{array}{rrr}58.85 & 239 & \text { IP } \\ 58.89 & 90 & \text { PP }\end{array}$

1

I MNG

BAO

CAR

I WEL

I WEL

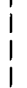

KRP

RDJ

$58.89 \quad 90$ eP

$0.6 \mathrm{~s} \quad 29.30 \mathrm{~nm}$

59.18238 iP

$\mathrm{iP}$

SS

LO

$59.60242 \mathrm{P}$

$59.83100 \mathrm{PC}$

$P c$

iPPP

$10.694 w \pm 3.2 \mathrm{~km}$

eophysicist)

obs.)

(685)

$\begin{array}{lll}41 & 00.50 & -0.2\end{array}$

$4100.30-0.6$

4632.00

4716.00

$\begin{array}{ll}48 & 45.00 \\ 49 & 24.00\end{array}$

4950.00

$4105.50 \quad 0.6$

$\begin{array}{lll}41 & 17.00 & 0.0\end{array}$

$\begin{array}{lll}4120.00 & -0.5\end{array}$

4252.00

4712.00

$\begin{array}{lll}4125.50 & 2.3\end{array}$

$\begin{array}{lll}41 & 22.00 & -1.3\end{array}$

4248.00

$46 \quad 40.00$

$\begin{array}{lll}4126.00 & 0.0\end{array}$

256.00

$4127.50 \quad 1.1$

$\begin{array}{lll}41 & 39.40 & 3.2 x\end{array}$

4325.40

443.90

$\begin{array}{lll}50 & 09.90\end{array}$

$\begin{array}{lll}41 & 44.00 & 1.8\end{array}$

4320.00

5206.00 


\begin{tabular}{|c|c|c|c|c|c|c|c|c|c|c|c|}
\hline & & & & is & 52 & 44.40 & & 1 & & & e \\
\hline RHP & & 61.68 & 234 & $P$ & 44 & 37.00 & -0.5 & I BLA & 73.86 & 25 & ¡Pd \\
\hline $\mathrm{JCT}$ & & 62.37 & 11 & iP & 44 & 42.00 & -0.1 & i & & & is \\
\hline & $z$ & $18 \mathrm{~s}$ & 44 & $4.70 \mathrm{um}$ & & & $.7 \mathrm{Msz}$ & I IMW & 74.90 & 360 & ePc \\
\hline & & & & is & 53 & 18.00 & & 1 & & & e \\
\hline BRZ & & 62.89 & 231 & eP & 44 & 48.00 & 2.6 & I KOU & 75.50 & 255 & Pc \\
\hline MSZ & & 62.98 & 232 & $P$ & 44 & 44.00 & -2.0 & I COR & 76.45 & 351 & iPd \\
\hline TRN & & 63.04 & 57 & eP & 44 & 45.60 & -1.2 & I UTO & 76.85 & 21 & epd \\
\hline TUC & & 63.33 & 360 & $e P c$ & 44 & 48.20 & -0.3 & I BUT & 77.03 & 359 & ePc \\
\hline & & & & $i$ & 44 & 54.30 & & I TAU & 77.24 & 227 & eP \\
\hline SVM & & 63.83 & 2 & ep & 44 & 52.00 & 0.0 & I CLE & 77.32 & 22 & iPd \\
\hline GLA & & 64.19 & 356 & $\mathrm{iPc}$ & 44 & 54.20 & 0.1 & i & & & $S$ \\
\hline & & & & $i$ & 45 & 00.20 & & i MSO & 77.88 & 358 & eP \\
\hline LUB & & 65.13 & 8 & eP & 45 & 05.00 & $4.7 x$ & $i$ & $2.0 \mathrm{~s}$ & 588 & $3.00 \mathrm{~nm}$ \\
\hline SJG & & 65.18 & 47 & eP & 45 & 02.00 & 1.2 & NEW & 79.47 & 356 & ePc \\
\hline & & & & is & 53 & 45.00 & & LOM & 79.56 & 357 & $i P_{c}$ \\
\hline PAS & & 65.54 & 353 & $e P$ & 45 & 02.00 & -0.7 & WAM & 79.67 & 234 & p \\
\hline & & $2.2 \mathrm{~s}$ & 900 & $0.00 \mathrm{~nm}$ & & & $.6 \mathrm{mb}$ & i & & & e \\
\hline & $z$ & $20 \mathrm{~s}$ & 28 & $8.00 \mathrm{um}$ & & & $.5 \mathrm{Msz}$ & RXF & 79.96 & 357 & iPc \\
\hline & 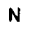 & $20 s$ & 36 & $6.00 \mathrm{um}$ & & & & I YKM & 79.99 & 357 & ¿PC \\
\hline & $E$ & $20 \mathrm{~s}$ & 13 & $3.00 u m$ & & & & CAN & 80.04 & 235 & $p$ \\
\hline & & & & e & 46 & 10.00 & & icoo & 80.39 & 240 & $e^{P}$ \\
\hline & & & & ePP & 47 & 19.00 & & I PNT & 80.71 & 354 & eP \\
\hline & & & & is & 53 & 58.00 & & i & $1.2 \mathrm{~s}$ & 131 & $.00 \mathrm{~nm}$ \\
\hline & & & & e & 14 & 07.00 & & I MIR & 80.73 & 189 & $P_{C}$ \\
\hline Sow & & 65.89 & 354 & eP & 45 & 05.50 & 0.4 & I YOU & 81.02 & 235 & $P$ \\
\hline & & & & $i$ & 45 & 10.10 & & I MAW & 81.22 & 178 & ep \\
\hline$A L O$ & & 66.07 & 4 & ePc & 45 & 05.80 & -0.6 & I TOO & 81.25 & 231 & eP \\
\hline & & $1.7 \mathrm{~s}$ & 135 & $5.00 \mathrm{~nm}$ & & & $.9 \mathrm{mb}$ & i & & & e \\
\hline & $z$ & $20 \mathrm{~s}$ & & 2. $50 \mathrm{um}$ & & & $4 \mathrm{Msz}$ & BRS & 81.34 & 243 & $p$ \\
\hline & & & & es & 54 & 00.00 & & i & & & eSKS \\
\hline PRI & & 67.80 & 351 & i Pc & 45 & 17.50 & 0.3 & $B F D$ & 83.42 & 230 & eP \\
\hline GCA & & 67.98 & 359 & $(P)$ & 45 & 19.80 & 1.4 & I EDM & 84.26 & 358 & iPc \\
\hline MSA & & 68.01 & 4 & eP & 45 & 18.80 & -0.1 & 1 & $1.2 \mathrm{~s}$ & 364 & $.00 \mathrm{~nm}$ \\
\hline & & & & e & 45 & 25.30 & & $M I M$ & 85.12 & 28 & (P) \\
\hline YMT 1 & & 68.08 & 355 & $e^{P}$ & 45 & 19.80 & 0.8 & I ADE & 87.23 & 230 & $\mathrm{PC}$ \\
\hline & & & & $i$ & 45 & 25.90 & & I CTA & 90.27 & 246 & $p$ \\
\hline RMU & & 68.88 & 360 & eP & 45 & 20.00 & 1.0 & $i$ & & & iSKS \\
\hline TUL & & 68.37 & 13 & $i P c-$ & 45 & 20.20 & -0.5 & $\mathrm{i}$ & & & is \\
\hline & & $1.0 \mathrm{~s}$ & 318 & $8.00 \mathrm{~nm}$ & & 6 & $.5 \mathrm{mb}$ & i & & & iSP \\
\hline & $z$ & $18 \mathrm{~s}$ & & $9.14 \mathrm{um}$ & & & $.1 \mathrm{Msz}$ & i & & & i \\
\hline & & & & es & 54 & 22.00 & & RAB & 3.92 & 263 & ep \\
\hline & & & & ess & 58 & 43.50 & & i 150 & 95.08 & 242 & eP \\
\hline SAO & & 68.52 & 351 & $\mathrm{iP}$ & 45 & 20.40 & -1.2 & PMG & 95.37 & 256 & eP \\
\hline FRI & & 68.52 & 352 & $\mathrm{i} P \mathrm{C}$ & 45 & 20.90 & -0.6 & ASP & 97.45 & 237 & ep \\
\hline DRV & & 68.75 & 204 & $P$ & 45 & 23.60 & 0.9 & PMR & 97.72 & 343 & eP \\
\hline RLO & & 68.76 & 14 & iP & 45 & 22.50 & -0.6 & i & & & $i$ \\
\hline ARN & & 69.10 & 351 & iPd & 45 & 31.50 & $6.3 x$ & I พB2 & 99.43 & 240 & $P$ \\
\hline MHC & & 69.11 & 351 & iP & 45 & 25.90 & 0.5 & 1 & & & e \\
\hline TNP & & 69.35 & 355 & IPC & 45 & 27.80 & 0.8 & i & & & e \\
\hline & & & & $i$ & 45 & 33.60 & & i & & & e \\
\hline JAS & & 69.55 & 352 & $I P C$ & 45 & 28.00 & 0.1 & I WRA & 99.44 & 240 & PC \\
\hline BKS & & 69.72 & 350 & $e(P)$ & 45 & 30.00 & 1.1 & $i$ & $0.7 \mathrm{~s}$ & & $.00 \mathrm{~nm}$ \\
\hline & 2 & $20 \mathrm{~s}$ & 37 & $7.00 \mathrm{um}$ & & & $.6 \mathrm{Msz}$ & I INK & 00.80 & 352 & ePdiff \\
\hline & $\mathrm{N}$ & $20 \mathrm{~s}$ & 34 & 4. $00 \mathrm{um}$ & & & & I SKR & 15.46 & 315 & ePKP \\
\hline & $E$ & $20 s$ & & $7.00 \mathrm{um}$ & & & & I BUL & 15.60 & 137 & EPKP \\
\hline & & & & es & 54 & 49.60 & & SEY & 20.50 & 328 & ePKP \\
\hline MNV & & 69.78 & 354 & $i P C$ & 45 & 30.00 & 0.5 & I TOL & 21.29 & 60 & ePKP \\
\hline NVL & & 70.35 & 163 & $P_{C}$ & 45 & 30.00 & -2.5 & i & & & iss \\
\hline & & & & is & 54 & 48.00 & & LPF & 25.24 & 51 & ePKP \\
\hline PRM & & 70.36 & 25 & $e^{P}$ & 45 & 33.10 & 0.1 & GRR & 25.42 & 50 & ePKP \\
\hline EUR & & 70.66 & 356 & ip & 45 & 34.70 & -0.2 & MFF & 25.60 & 53 & EPKP \\
\hline & & $0.5 \mathrm{~s}$ & & $6.00 \mathrm{~nm}$ & & 5 & $.4 \mathrm{mb}$ & FLN & 125.72 & 50 & EPKP \\
\hline GOL & & 70.87 & 4 & eP & 45 & 36.50 & 0.2 & LFF & 125.91 & 55 & ePKP \\
\hline & 2 & $18 \mathrm{~s}$ & & $0.30 \mathrm{um}$ & & 4 & $6 \mathrm{MszX}$ & SSC & 125.94 & 50 & ePKP \\
\hline & & & & e & 45 & 42.20 & & I LPO & 126.18 & 55 & EPKP \\
\hline DON & & 70.90 & 17 & $e^{P}$ & 45 & 33.20 & -2.9 & I RJF & 26.52 & 54 & ePKP \\
\hline & & & & i & 45 & 39.50 & & I LSF & 26.69 & 53 & ePKP \\
\hline GLD & & 70.93 & 4 & $e^{P}$ & 45 & 37.20 & 0.7 & I CAF & 26.84 & 55 & ePKP \\
\hline & $z$ & $18 \mathrm{~s}$ & & $0.80 u m$ & & & $.0 M s z X$ & TCF & 7.16 & 53 & EPKP \\
\hline JSC & & 70.94 & 26 & $e P$ & 45 & 36.30 & -0.1 & I MZF & 7.40 & 53 & ePKP \\
\hline ELC & & 71.18 & 18 & eP & 45 & .90 & -0.9 & $A V F$ & 3.01 & 53 & ePKP \\
\hline DUG & & 71.22 & 358 & ePc & 45 & 38.20 & 0.0 & SS & 8.15 & 52 & ep \\
\hline & & & & $i$ & 45 & 44.00 & & SMF & 28.32 & 53 & ePKP \\
\hline ORV & & 71.27 & 351 & iPc & 45 & 38.20 & -0.1 & I LOR & 28.41 & 52 & ePk \\
\hline ORT & & 71.36 & 22 & $e(P)$ & 45 & 38.80 & -0.2 & I LBF & 28.46 & 53 & ePKP \\
\hline FVM & & 71.54 & 17 & $i P_{C}$ & 45 & 39.60 & -0.4 & I TIK & 128.95 & 339 & ePKP \\
\hline & $z$ & $18 \mathrm{~s}$ & & $5.80 u m$ & & & $.9 M s z$ & UCC & 129.06 & 48 & PKPC \\
\hline & & & & $i$ & 45 & 46.00 & & i & & & e \\
\hline BMN & & 71.69 & 355 & $i P_{C}$ & 45 & 41.40 & 0.4 & I DOU & 129.16 & 49 & PKP \\
\hline & & $2.3 \mathrm{~s}$ & 971 & $1.00 \mathrm{~nm}$ & & & $.5 \mathrm{mb}$ & i & & & $e$ \\
\hline & & & & e & 45 & 46.30 & & i & & & SS \\
\hline$M I N$ & & 72.06 & 351 & ip & 45 & 42.30 & -0.9 & ENN & 130.05 & 48 & EPKP \\
\hline YOC & & 72.42 & 351 & IPC & 45 & 44.70 & -0.5 & i & & & e \\
\hline NOU & & 72.90 & 254 & $P C$ & 45 & 50.30 & 1.8 & 1 & & & $=$ \\
\hline BDW & & 73.78 & & IPC & 45 & 52.20 & -1.1 & & 10 & 48 & PKP \\
\hline & & s & 60 & 0.00 & & & . & J & 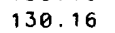 & 52 & ePKP \\
\hline
\end{tabular}


TSI 140.93231 (PKP) 5350.50 BJI 141.34296 PKP 53535 VAY

$K D Z$

DIM

AAE

VR

IAS

I ZM

KIS

YER

CTT

BT

$\begin{array}{llllll}\text { BKT } & 146.48 & 64 \text { PKP } & 53 & 59.30\end{array}$

$\begin{array}{llrlll}\text { XAN } & 146.70 & 285 & \text { EPKP } & 53 & 58.80 \\ \text { MOS } & 147.02 & 33 & \text { EPKP } & 54 & 00.00\end{array}$

GYA

IRK

ELL

GPA

BCK
ZAK

ZAK

MGN

MOY

KAS

CHG

147.04271 PKP $54 \quad 02.00$

147.05320 ePKP 5359.00

147.0870 PKP 5401.40

$\begin{array}{lllll}147.37 & 63 & \text { PKP } & 54 & 00.20 \\ 147.39 & 83 & \text { EPKPd } & 54 & 05.08\end{array}$

$\begin{array}{lllll}147.65 & 68 & \text { PKP } & 54 & 02.20\end{array}$

148.43317 PKPC 5403.10

$\begin{array}{lllll}148.59 & 123 & \text { IPKP } & 54 & 04.60\end{array}$

148.7462 PKP $54 \quad 09.00$

$\begin{array}{lllll}149.15 & 320 & \text { ePKP } & 53 & 59.00 \\ 149.62 & 249 & \text { iPKPC } & 54 & 05.60\end{array}$

149.62249 iPKPC
$1.05 \quad 188.00 \mathrm{~nm}$

$\begin{array}{llll}149.87 & 61 \text { PKP } 54 \quad 07.00\end{array}$

150.49252 iPKPC $54 \quad 07.80$ $1.6 \mathrm{~s} \quad 121.00 \mathrm{~nm}$ eS 0451.00

CHTO 150.49252 EPKP $54 \quad 07.20$

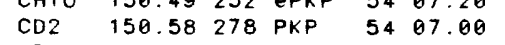

$\begin{array}{llllll}\text { LZH } & 150.99 & 289 & \text { ePKP } & 54 & 09.00\end{array}$

$\begin{array}{llllll}\text { KVT } & 151.62 & 61 & \text { PKP } & 54 & 10.20\end{array}$

$\begin{array}{lllllll}\text { UER } & 152.80 & 325 & \text { PKPd } & 54 & 06.10\end{array}$

$\begin{array}{llllll}\text { SVE } & 153.85 & 11 & \text { ePKPC } & 54 & 12.00\end{array}$

$\begin{array}{llllll}A R U & 153.87 & 14 & \text { PKPC } & 54 & 12.00\end{array}$

ELT $\quad 154.91336$ ePKP 5407.00

$\begin{array}{llllll}\text { PYA } & 155.80 & 51 & \text { PKP } & 54 & 16.60\end{array}$

$\begin{array}{llllll}\text { BKR } & 156.92 & 56 & \text { PKPC } & 54 & 17.60\end{array}$

$\begin{array}{llllll}\text { LEN } & 157.45 & 58 & \text { ePKP } & 54 & 22.00\end{array}$

$\begin{array}{llllll}\text { ERE } & 158.09 & 59 & \text { ePKP } & 54 & 14.00\end{array}$

$\begin{array}{llllll}\text { MAK } & 159.11 & 50 & \text { ePKP } & 54 & 34.00\end{array}$

$\begin{array}{llllll}\text { KRV } & 159.28 & 57 & \text { ePKP } & 54 & 15.00\end{array}$

SEM 159.29340 ePKP 5414.00

SHIO $159.34 \quad 259$ ePKP $54 \quad 15.00$

$\begin{array}{lrrrrr}\text { SHL } & 159.34 & 259 & \text { PKP } & 54 & 19.00 \\ \text { GRS } & 159.63 & 60 & \text { PKPC } & 54 & 21.00\end{array}$

$\begin{array}{llllll}\text { TAB } & 159.99 & 64 & \text { ePKP } & 54 & 22.00\end{array}$

GBA $\quad 160.81205$ PKP $54 \quad 28.00$

$1.2 \mathrm{~s} \quad 13.90 \mathrm{~nm}$

CAL
WMO
LSA
KER
BOK
HYB

$\begin{array}{llll}160.89 & 247 \text { PKP } 55 \quad 11.00\end{array}$

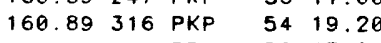

$\begin{array}{lllll}161.11 & 271 & \text { PP } & 58 & 47.00 \\ 161.12 & 75 & 54 & 23.20\end{array}$

161.1275 ePKP 5423.00

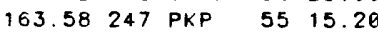

163.77213 ePKP 5426.00 $\begin{array}{lll}\text { EPP } & 59 & 00.00 \\ \text { ePPS } & 12 & 48.00\end{array}$ ESS 1926.00

$T L G$

POO 166.58199 PKP-

PRZ 166.74329 EPKP

KRD $\quad 167.17 \quad 341$ PKP+

BOM

NRN

ASH

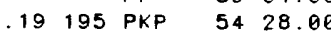

167.80341 ePKP 5424.00

168.60334 ePKP 5423.00

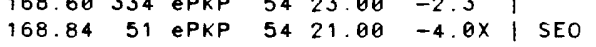

\begin{tabular}{rrrrrr|r}
168.84 & 51 & ePKP & 54 & 21.00 & $-4.0 \times$ & SEO \\
169.30 & 222 & PKP- & 54 & 20.00 & $-5.7 \times$ & CD2
\end{tabular}

\begin{tabular}{l|l}
-0.5 & ANR \\
\hline SAM
\end{tabular}

$11.9 x$ BHJ

$4.5 \times$

$-3.4 x \mid D D 1$

\begin{tabular}{l|l}
-0.7 & NDI \\
-1.6 &
\end{tabular}

-1.6
$-2.8 \times$

I DSH

\begin{tabular}{l|l}
-1.8 & $K K R$ \\
-2.4 & $K U L$
\end{tabular}

$-3.8 \times$ KHO

$11.5 \times \mid \mathrm{NIL}$

$4.0 \times$ I KAAO

-0.4 KBL

-1.5 | OUE

-1.9
-1.6

1.7

-1.0
0.0

0.5

-0.3
1.1

2.0

\begin{tabular}{r|l}
-0.1 & \\
1.6 & SZP
\end{tabular}

0.2 BAG

$4.7 \times \mid$ HEN

\begin{tabular}{l|l}
1.6 & \\
1.8 & TTN
\end{tabular}

1.9 TWM1

$6.8 \times \mid$ MAN

$-3.4 \times$

1.5 OCP

$3.1 X$ HWA

2.4 TWD

I TCU

\begin{tabular}{l|l}
1.8 & Two \\
1.7 & Tw
\end{tabular}

$3.1 \times$ I TWC

$3.7 \times$ LGP

$2.9 \times$ TATO

2.8X | ANP

$-3.6 \times \quad$

4. $4 X$ I HKC

$7.4 \mathrm{X}$ CNP

\begin{tabular}{l|l}
-1.3 & \\
$17.8 \times$ & PLP
\end{tabular}

\begin{tabular}{l|l}
-1.5 & \\
-2.1 & $P P R$
\end{tabular}

\begin{tabular}{r|l}
-2.2 & \\
1.8 & $C G P$
\end{tabular}

\begin{tabular}{l|l}
1.8 & $\mathrm{CGP}$ \\
$3.9 \mathrm{P}$ & $\mathrm{PPH}$
\end{tabular}

$4.5 \times$

$9.3 \times \quad$ SSE

$52.4 \times \quad$ WHN

1.0 NJ2

$3.9 x \mathrm{KKM}$

4. $2 x$

$53.9 x$ GYA

4. $3 x$ I MN I

TIA

\begin{tabular}{l|l}
-0.4 & \\
$-0.1 \times$ & $\mathrm{KMI}$
\end{tabular}

$-0.7$

0.1

$3.5 \times$ XAN

\begin{tabular}{l|l}
$3.5 \times$ & XHK \\
-0.3 & $5 H K$
\end{tabular} \begin{tabular}{l|lllllll}
0.7 & TAS & 170.06 & 0 & ePKP & 54 & 23.00 & $-2.7 X$
\end{tabular} \begin{tabular}{r|r}
$3.3 \times$ & $\mathrm{KSH}$ \\
$-14.8 \times$ &
\end{tabular}

$18.93 \quad 32 \mathrm{ePC}$

$19.50 \quad 15 \mathrm{iPd}$

$19.63311 \mathrm{Pd}$

$\begin{array}{ll}54 & 28.00 \\ 59 & 39.00\end{array}$

170.31346 ePKP 5428.00

$\begin{array}{llll}171.50 & 12 & \text { PKPC } 54 & 30.20\end{array}$

$\begin{array}{llll}171.91 & 182 \text { PKP } & 5431.00 \\ & & 34\end{array}$

172.41264 PKP

172.64251 PKP

i $\mathrm{i} P \mathrm{SP}$

5426.70

5425.00

5944.00

172.813 ePKP 5426.00

173.37260 PKP 5437.00

173.47357 ePKP 5430.00

$\begin{array}{lllll}173.62 & 344 & \text { ePKP } & 54 & 27.00\end{array}$

$175.96 \quad 305$ ePKP $54 \quad 26.30$

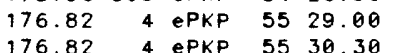

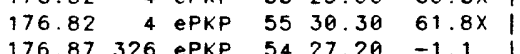

$177.66 \quad 119$ ePKP $54 \quad 29.00$

\begin{tabular}{l|l}
$-2.7 X$ & \\
2.0 & TIY \\
2.1 & \\
$3.7 x$ & BKB \\
$4.0 X$ & \\
-0.4 & CHU \\
-2.2 & \\
& CHTO \\
-1.1 &
\end{tabular}

$9.6 \times$

$2.7 x$ BDT

$-0.6 \quad$

$-1.8 \mid \mathrm{BJI}$

-1.1
0.3
0

LZH

OYM

ov $22,198,15 \mathrm{~h} 05 \mathrm{~m} \mathrm{22.28 \pm 0.24 \textrm {s }}$

$8.750 \mathrm{~N} \pm 1.2 \mathrm{~km} 120.829 \mathrm{E} \pm 1.6 \mathrm{~km}$ SRY

DEPTH $=36.0 \pm 2.1 \mathrm{~km}$

$A A I$

( 61 Obs.) 6.5Msz ( 17 ODS.) DDR (SLANDS

areo. Felt the Bacarro-Laoag Luzon.

$1.24 \quad 197 \mathrm{iPd}$

$2.34186 \mathrm{Pc}$

$3.994 \mathrm{P}$

$4.07355 \mathrm{eP}$

$4.07 \quad 177 \quad \mathrm{iPd}$

$4.10177 \mathrm{P}$

$4.50356 \mathrm{eP}$

$5.35 \quad 8 \mathrm{eP}$

$5.37359 \mathrm{P}$

$5.50 \quad 0 \mathrm{iPd}$

$5.91 \quad 9 \mathrm{eP}$

is

$\begin{array}{ll}6.22 & 6 \mathrm{eP} \\ 6.43 & 6 \mathrm{Pc}\end{array}$

$7.17301 \mathrm{P}$

$7.22149 \mathrm{ePc}$

$8.54151 \mathrm{ePC}$

$9.15193 \mathrm{eP}$

$10.90 \quad 159 \quad \mathrm{iPd}$

$12.02 \quad 154 \mathrm{ePC}$

$12.30 \quad 1 \mathrm{iPd}$

$12.48158 \mathrm{Pd}$

$13.13335 \mathrm{P}$

$13.36353 \mathrm{P}$

$13.41200 \quad \mathrm{iPC}$

$0.8 \mathrm{~s} 221.00 \mathrm{~nm}$

$15.16303 \mathrm{Pd}$

$17.64167 \mathrm{ePc}$

$17.70 \quad 350$ Pd

$17.93294 \mathrm{PO}$ SP PP

$18.57327 \mathrm{Pd}$

1249.00

1300.00

0940.00

1322.20

0949.50

0950.00

(249) I GUMO

BTO
GUA

GUA

$06 \quad 13.00$

0625.00

23.00
06.60

$\begin{array}{ll}07 & 13.00 \\ 06 & 24.00 \\ 06 & 29.00\end{array}$

$06 \quad 29.00$

0641.60

$06 \quad 41.90$

0803.00

$06 \quad 49.00$

$08 \quad 07.90$

0658.00

$08 \quad 04.00$

$08 \quad 19.00$

9818.00

26.50
99
01.00

34.00
09
16.00

$\begin{array}{ll}08 & 01.00 \\ 08 & 18.20\end{array}$

1031.00

$08 \quad 22.00$

$\begin{array}{ll}08 & 28.00 \\ 08 & 30.00\end{array}$

$08 \quad 37.50$

0855.00

0929.50

0928.70

1243.50

0931.00

.

0

0.1

KYS

\section{2}

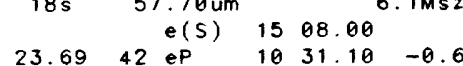

$\begin{array}{rrrrrr}23.69 & 42 & \mathrm{eP} & 10 & 31.10 & -0.6 \\ 23.85 & 183 \mathrm{iPc} & 10 & 33.70 & 0.3\end{array}$

$0.8 \mathrm{~s} \quad 418.00 \mathrm{~nm} \quad 6.0 \mathrm{mb}$

$23.90 \quad 228 \mathrm{ePd} \quad 10 \quad 34.50 \quad 0.7$

$1.5 \mathrm{~s} 1690.00 \mathrm{~nm} \quad 6.3 \mathrm{mb}$

23.93236 iPd $1033.20 \quad-0.9$

$1.3 \mathrm{~s} \quad 1190.00 \mathrm{~nm} \quad 6.3 \mathrm{mb}$

$\begin{array}{lll}i & 1102.20\end{array}$

$\begin{array}{llllll}24.32 & 40 \mathrm{e} & 14 & 16.90 & \\ & 10 & 33.00 & -4.7 x\end{array}$

$24.38 \quad 233$ ePd $1039.40 \quad 0.9$

$1.2 \mathrm{~s} 1350.00 \mathrm{~nm} 6.4 \mathrm{mb}$

$25.288 \mathrm{iPd} \quad 1045.80-1.0$

SP $\quad 1056.00$

$\begin{array}{lllll}26.53 & 238 \mathrm{ePd} & 15 & 09.00 & \\ 26.73 & 23600 & 1.3\end{array}$

$26.73236 \mathrm{ePd} \quad 1059.50 \quad-1.0$

$1.2 \mathrm{~s} \quad 516.00 \mathrm{~nm} \quad 6.0 \mathrm{mb}$

$\begin{array}{ccccc}27.46 & 323 \mathrm{iPd} & 1107.30 & 0.1 \\ & \mathrm{~s} & 1548.00 & \end{array}$

27.50198 iPd $11 \quad 07.50 \quad 0.0$

$0.9 \mathrm{~s} \quad 304.00 \mathrm{~nm} \quad 6.0 \mathrm{mb}$

27.61289 iPd $1108.70 \quad 0.0$

27.70229 EPd $11 \quad 09.50 \quad 0.2$ 


\begin{tabular}{|c|c|c|c|c|c|c|c|c|c|c|c|c|}
\hline WMO & 37.36 & 319 & $\begin{array}{l}i \mathrm{Pd} \\
S\end{array}$ & $\begin{array}{l}12 \\
18\end{array}$ & $\begin{array}{l}34.20 \\
17.50\end{array}$ & 0.8 & PSH & 46.35 & 299 & $\begin{array}{l}\text { iPd } \\
\text { eS }\end{array}$ & $\begin{array}{l}13 \\
20\end{array}$ & $\begin{array}{l}47.40 \\
32.20\end{array}$ \\
\hline PMG & 38.17 & 135 & ePd & 12 & 38.00 & -2.4 & I MGD & 46.54 & 20 & $P d$ & 13 & 47.00 \\
\hline RAB & 38.37 & 123 & Pd- & 12 & 42.00 & -0.1 & i & & & $i p P$ & 13 & 55.00 \\
\hline & & & is & 13 & 23.00 & & 1 & & & is & 20 & 29.00 \\
\hline LMG & 38.49 & 133 & $\mathrm{Pc}$ & 12 & 41.80 & -1.4 & I KHO & 46.75 & 304 & $\mathbf{P}$ & 13 & 51.00 \\
\hline UER & 38.94 & 333 & $P d$ & 12 & 47.50 & 1.1 & i & & & $S$ & 20 & 40.70 \\
\hline MBL & 39.68 & 181 & $e P$ & 12 & 50.00 & -2.8 & I ANR & 46.76 & 309 & Pd & 13 & 51.60 \\
\hline HYB & 0.14 & 275 & $P d-$ & 12 & 56.50 & -0.3 & I & & & esP & 14 & 09.10 \\
\hline & & & e & 13 & 01.80 & & 1 & & & is & 20 & 45.10 \\
\hline & & & e & 13 & 19.00 & & I HNR & 47.67 & 123 & ePd & 13 & 58.00 \\
\hline & & & ePP & 14 & 27.00 & & 1 & & & es & 20 & 24.00 \\
\hline & & & e & 14 & 52.00 & & I GAR & 47.95 & 306 & $\mathrm{P}$ & 14 & 00.00 \\
\hline & & & ePcP & 15 & 02.50 & & 1 & & & es & 20 & 49.30 \\
\hline & & & e & 15 & 20.00 & & 1 & & & es & 20 & 51.30 \\
\hline & & & ePcS & 18 & 49.00 & & $1 \mathrm{KUL}$ & 48.21 & 304 & Po & 14 & 02.00 \\
\hline & & & es & 18 & 56.00 & & 1 & & & eS & 20 & 59.60 \\
\hline & & & ePS & 19 & 24.00 & & I KAAO & 48.38 & 300 & iPd & 14 & 03.40 \\
\hline & & & ess & 21 & 52.00 & & I DSH & 49.07 & 305 & $P d$ & 14 & 08.30 \\
\hline & & & escs & 23 & 14.00 & & 1 & & & es & 21 & 04.60 \\
\hline WRA & 40.67 & 160 & $\mathrm{PC}$ & 12 & 58.90 & -2.1 & I TAS & 49.15 & 309 & $P d$ & 14 & 10.00 \\
\hline & $0.6 \mathrm{~s}$ & & $1.98 \mathrm{~nm}$ & & & $5.4 \mathrm{mb}$ & 1 & & & is & 21 & 18.00 \\
\hline WB 2 & 40.68 & 160 & Po & 12 & 57.80 & $-3.3 x$ & I SEY & 49.17 & 18 & $P d$ & 14 & 09.30 \\
\hline & & & es & 19 & 00.70 & & I & & & $i p P$ & 14 & 18.70 \\
\hline & & & $e^{\prime} P^{\prime}$ & 44 & 44.30 & & I & & & is & 21 & 13.30 \\
\hline SA & .94 & 131 & $e P$ & 12 & 08.00 & $-55.3 x$ & I KLG & 49.25 & 179 & $P d$ & 14 & 07.00 \\
\hline NDI & 4 & 292 & $i P d$ & 13 & 01.00 & -2.2 & I OUE & 49.95 & 294 & iPd- & 14 & 15.00 \\
\hline & $18 \mathrm{~s}$ & 39 & $9.50 \mathrm{um}$ & & & $5.3 M S Z$ & 1 & & & eS & 21 & 25.00 \\
\hline & & & ePP & 14 & 42.00 & & I KLB & 50.14 & 183 & $\mathrm{PC}$ & 14 & 15.00 \\
\hline & & & es & 19 & 05.00 & & I SAM & 50.60 & 306 & $P d$ & 14 & 20.40 \\
\hline & & & eSSS & 22 & 21.00 & & 1 & & & isP & 14 & 34.00 \\
\hline GBA & 41.91 & 270 & $P$ & 13 & 12.00 & 0.7 & 1 & & & is & 21 & 37.20 \\
\hline & $0.9 \mathrm{~s}$ & 167 & $7.00 \mathrm{~nm}$ & & & $5.8 \mathrm{mb}$ & I MUN & 50.63 & 185 & $e P$ & 14 & 18.00 \\
\hline PAA & 42.27 & 123 & eP & 13 & 12.00 & -2.4 & I NWAO & 51.50 & 184 & $\mathrm{PC}$ & 14 & 25.80 \\
\hline SKR & 42.37 & 33 & Pd & 13 & 15.00 & 0.4 & 1 & & & es & 21 & 47.00 \\
\hline & & & $i p P$ & 13 & 21.00 & $20 \mathrm{~km} x$ & I TIK & 53.10 & 3 & $P d$ & 14 & 36.00 \\
\hline & & & is & 19 & 34.00 & & 1 & & & $p P$ & 14 & 43.00 \\
\hline & & & ess & 19 & 50.20 & & 1 & & & SP & 14 & 51.00 \\
\hline KOD & 42.74 & 265 & Pd & 13 & 18.50 & 0.1 & 1 & & & es & 21 & 58.00 \\
\hline & & & PP & 15 & 80.00 & & I SMY & 53.26 & 37 & $\mathbf{P}$ & 14 & 39.30 \\
\hline & & & $P C P$ & 15 & 12.00 & & I STK & 54.14 & 158 & $e P$ & 14 & 43.00 \\
\hline & & & PPP & 15 & 32.00 & & | NR I & 54.48 & 346 & $P d$ & 14 & 46.20 \\
\hline & & & es & 19 & 42.00 & & 1 & & & is & 22 & 15.00 \\
\hline & & & SS & 22 & 40.00 & & I BRS & 55.34 & 145 & $P d$ & 14 & 52.80 \\
\hline & & & LO & 23 & 00.00 & & 1 & & & $i$ & 14 & 58.80 \\
\hline & & & Scs & 23 & 20.00 & & 1 & & & iPCP & 15 & 55.50 \\
\hline & & & SSS & 23 & 30.00 & & 1 & & & eSCP & 19 & 53.00 \\
\hline & & & LR & 25 & 13.00 & & I ADE & 56.06 & 162 & $P d$ & 14 & 58.60 \\
\hline$R Z$ & 42.91 & 313 & $\mathrm{Pc}$ & 13 & 1.68 & 2.2 & | MH I & 56.25 & 302 & $e^{P P}$ & 15 & 02.00 \\
\hline UKR & 42.95 & 327 & $\mathrm{Pd}$ & 13 & 20.00 & 0.6 & | KH I & 56.96 & 299 & eP & 15 & 06.40 \\
\hline 150 & 43.30 & 154 & $\mathrm{Pd}$ & 13 & 20.00 & -2.6 & I ASH & 57.19 & 303 & $P d$ & 15 & 09.30 \\
\hline YAK & 43.67 & 6 & $\mathrm{Pd}$ & 13 & 24.70 & -0.3 & 1 & & & $\mathrm{~s}$ & 23 & 05.80 \\
\hline & & & is & 19 & 48.80 & & I VAN & 57.39 & 303 & ePd & 15 & 09.00 \\
\hline LAH & 3.67 & 296 & eP & 13 & 25.00 & -0.5 & 1 & & & $\mathrm{~s}$ & 23 & 06.50 \\
\hline KSH & 43.75 & 308 & $i P d$ & 13 & 29.00 & 2.8 & 1000 & 57.43 & 148 & eP & 15 & 10.00 \\
\hline & & & SP & 13 & 38.00 & & I SVE & 58.27 & 326 & $P d$ & 15 & 15.50 \\
\hline & & & S & 19 & 58.00 & & 1 & & & is & 23 & 17.00 \\
\hline$T L G$ & 43.88 & 314 & Pd & 13 & 29.50 & 2.3 & I ADK & 58.53 & 40 & $P$ & 15 & 15.30 \\
\hline & & & is & 20 & 04.50 & & I KAT & 58.84 & 305 & $\mathrm{Pc}$ & 15 & 20.50 \\
\hline ASP & 44.06 & 163 & Pd & 13 & 26.30 & -2.4 & 1 & & & es & 23 & 28.00 \\
\hline & & & e & 19 & 55.00 & & 1 YOU & 58.90 & 153 & $P d$ & 15 & 18.40 \\
\hline NRN & 44.28 & 311 & Pd & 13 & 31.60 & 0.9 & 1 & & & $\mathrm{i}$ & 16 & 10.10 \\
\hline & & & es & 20 & 05.00 & & I PVC & 59.10 & 125 & $\mathrm{Pc}$ & 15 & 21.40 \\
\hline 00 & 44.41 & 278 & iPd & 13 & 31.50 & -0.2 & I ARU & 59.27 & 325 & Pd & 15 & 22.40 \\
\hline & & & iPP & 15 & 14.50 & & 1 & & & epp & 15 & 28.00 \\
\hline & & & iPPP & 15 & 40.50 & & 1 & & & esp & 15 & 37.50 \\
\hline & & & IPCS & 19 & 12.00 & & 1 & & & es & 23 & 29.00 \\
\hline & & & is & 20 & 00.00 & & I BFD & 59.27 & 160 & eP & 15 & 20.00 \\
\hline & & & iss & 23 & 06.00 & & I CAN & 60.05 & 154 & $P d$ & 15 & 26.50 \\
\hline & & & iscs & 23 & 24.50 & & 1 & & & $\mathrm{i}$ & 16 & 14.40 \\
\hline & & & isss & 23 & 57.00 & & I NOU & 0.45 & 130 & $\mathrm{Pc}$ & 15 & 29.50 \\
\hline $\mathrm{NIL}$ & 44.81 & 299 & iPd & 13 & 35.00 & 0.2 & I TOO & 60.64 & 158 & eP & 15 & 30.00 \\
\hline & & & es & 20 & 16.00 & & I WAM & 60.75 & 154 & $P$ & 15 & 32.00 \\
\hline $\mathrm{BN}$ & 4.97 & 173 & eP & 13 & 35.00 & -1.0 & 1 & & & $\mathrm{i}$ & 16 & 15.90 \\
\hline PET & 45.02 & 32 & Po & 13 & 35.50 & -0.5 & | ILT & 61.80 & 22 & $\mathrm{Pd}$ & $\begin{array}{l}15 \\
15\end{array}$ & 38.00 \\
\hline & & & es & 20 & 04.00 & & ! & & & is & 15 & 46.50 \\
\hline$S E M$ & 45.09 & 324 & $\mathrm{Pd}$ & 13 & 37.00 & 0.4 & 1 & & & is & 23 & 58.00 \\
\hline & & & es & 26 & 16.60 & & NKI & 3.41 & 40 & $P$ & 15 & .3 \\
\hline EK & 5 & 183 & eP & 13 & 36.00 & -1.4 & BAK & 63.72 & 306 & $e P$ & 15 & 53.00 \\
\hline FRU & 45.67 & 312 & $P d$ & 13 & 42.80 & 1.4 & 1 & & & $\mathrm{~S}$ & 24 & 31.00 \\
\hline & & & es & 20 & 21.80 & & MAK & 65.43 & 309 & $P$ & 16 & 02.40 \\
\hline ETA & 45.95 & 146 & $i P d-$ & 13 & 42.00 & -1.8 & I & & & is & 24 & 47.60 \\
\hline & $1.0 \mathrm{~s}$ & 121 & $.00 \mathrm{~nm}$ & & 5 & $.8 \mathrm{mb}$ & TAU & 66.02 & 159 & $e^{P}$ & 16 & 07.00 \\
\hline & & & $i(P \subset S)$ & 19 & 17.00 & & ANM & 66.27 & 27 & ePd & 16 & 09.30 \\
\hline & & & is & 20 & 23.00 & & KRV & 66.42 & 307 & Pd & 16 & 1000 \\
\hline VS & 45.98 & 331 & $P d$ & 13 & 43.20 & -0.4 & 1 & & & $\mathrm{~S}$ & 25 & 01.00 \\
\hline & & & is & 20 & 16.00 & & KER & 66.47 & 300 & ePd & 16 & 09.50 \\
\hline
\end{tabular}


$22 d \quad 15 h$

\begin{tabular}{|c|c|c|c|c|c|c|c|c|c|c|c|}
\hline KRP & 76.27 & 138 & Pd & 17 & 11.00 & 1.9 & 1 & $0.8 \mathrm{~s}$ & 120 & $0.00 \mathrm{~nm}$ & \\
\hline TNZ & 76.32 & 140 & ip & 17 & 11.80 & 2.4 & I KRA & 82.59 & 320 & iPd & 17 \\
\hline KIR & 76.66 & 337 & Po & 17 & 10.60 & -0.3 & 1 & $0.7 \mathrm{~s}$ & 198 & $8.00 \mathrm{~nm}$ & \\
\hline ANTO & 76.76 & 308 & iPd & 17 & 12.70 & 0.6 & 1 & $20 s$ & & $5.00 \mathrm{um}$ & \\
\hline NUR & 76.87 & 330 & ip & 17 & 11.90 & -0.2 & 1 & $20 s$ & & 3.00 um & \\
\hline & $1.5 \mathrm{~s}$ & 543 & $3.00 \mathrm{~nm}$ & & & $0.4 \mathrm{mb}$ & i & $20 s$ & & 8.00 um & \\
\hline$z$ & $16 \mathrm{~s}$ & 70 & $0.60 \mathrm{um}$ & & & 7. $1 M s z X$ & 1 & & & $i p P$ & 17 \\
\hline & & & ePP & 20 & 08.00 & & i & & & ePP & 20 \\
\hline & & & es & 26 & 56.00 & & I & & & es & 27 \\
\hline & & & escs & 27 & 26.00 & & 1 & & & eSS & 33 \\
\hline & & & ess & 32 & 08.00 & & I SPC & 82.71 & 319 & iPd & 17 \\
\hline BRZ & 76.91 & 148 & $e P$ & 17 & 13.00 & 0.5 & 1 & $15 s$ & & 7.70 um & \\
\hline MGN & 76.97 & 309 & $P$ & 17 & 14.20 & 1.0 & 1 & & & $i(P P)$ & 20 \\
\hline RHP & 77.86 & 146 & iP & 17 & 13.80 & 0.3 & $1 \mathrm{JOS}$ & 82.76 & 319 & Pd & 17 \\
\hline KIS & 77.83 & 316 & $\mathrm{Pd}$ & 17 & 17.00 & -0.7 & 1 & & & $\mathbf{i}$ & 18 \\
\hline & & & is & 27 & 06.00 & & 1 & & & $\mathbf{i}$ & 19 \\
\hline & & & is & 27 & 16.00 & & 1 & & & i & 21 \\
\hline SNZO & 77.83 & 142 & eP & 17 & 17.60 & -0.1 & 1 N82 & 82.86 & 332 & $\mathbf{P}$ & 17 \\
\hline WEL & 77.86 & 141 & iPd & 17 & 18.50 & 0.7 & 1 & $0.8 \mathrm{~s}$ & & $2.20 \mathrm{~nm}$ & \\
\hline & & & PP & 20 & 20.00 & & 1 & & & PP & 20 \\
\hline & & & $\mathrm{s}$ & 27 & 13.00 & & I VIS & 82.96 & 312 & $P d$ & 17 \\
\hline & & & SS & 33 & 16.00 & & 1 & & & is & 28 \\
\hline & & & LO & 40 & 00.00 & & I OUR & 83.13 & 310 & ePd & 17 \\
\hline & & & LR & 43 & 00.00 & & I SRS & 83.16 & 311 & ePd & 17 \\
\hline MNG & 77.87 & 141 & iPd & 17 & 17.50 & -0.5 & I SSR & 83.21 & 315 & $\mathbf{P}$ & 17 \\
\hline & & & i & 17 & 59.00 & & I TIM & 83.26 & 316 & $\mathrm{Pc}$ & 17 \\
\hline UME & 77.93 & 334 & Pd & 17 & 17.00 & -0.9 & I PSZ & 83.37 & 318 & $\mathrm{P}$ & 17 \\
\hline & & & is & 26 & 57.00 & & I SOH & 83.43 & 311 & eP & 17 \\
\hline & & & ISKS & 27 & 36.00 & & $\mid$ PAIG & 83.48 & 310 & ePd & 17 \\
\hline GNZ & 78.27 & 138 & iP & 17 & 21.90 & 1.7 & I KNT & 83.64 & 311 & ePd & 17 \\
\hline PCA & 78.29 & 30 & $P$ & 17 & 21.10 & 1.0 & I THE & 83.77 & 311 & iPc & 17 \\
\hline IAS & 78.65 & 316 & eP & 17 & 23.00 & 0.8 & I & & & iSn & 28 \\
\hline$B \backslash R$ & 78.85 & 315 & eP & 17 & 25.00 & 1.7 & I VAY & 83.82 & 311 & $i P d$ & 17 \\
\hline$A L T$ & 78.98 & 308 & P- & 17 & 24.00 & -0.3 & I GRG & 84.06 & 311 & eP & 17 \\
\hline$B C K$ & 79.03 & 306 & $P$ & 17 & 23.10 & -1.5 & I BUD & 84.07 & 318 & $P C$ & 17 \\
\hline ISK & 79.22 & 310 & $\mathbf{P}$ & 17 & 25.30 & -0.2 & 1 & & & $\mathrm{i}$ & 18 \\
\hline$B A C$ & 79.25 & 316 & $e P$ & 17 & 23.00 & -2.5 & 1 & & & $\mathrm{i}$ & 19 \\
\hline & & & es & 27 & 23.00 & & 1 & & & iPP & 21 \\
\hline IST & 79.28 & 310 & P- & 17 & 25.00 & -0.8 & I ATH & 84.09 & 308 & iPd & 17 \\
\hline & & & PKP & 20 & 28.00 & & 1 & & & is & 28 \\
\hline$B K T$ & 79.41 & 309 & $P$ & 17 & 25.40 & -1.4 & I BEO & 84.09 & 315 & $P$ & 17 \\
\hline VRI & 79.56 & 315 & $P$ & 17 & 26.10 & -1.1 & i & & & is & 28 \\
\hline & & & es & 27 & 27.00 & & $1 \mathrm{KON}$ & 84.20 & 332 & iP & 17 \\
\hline CTT & 79.66 & 310 & $\mathbf{P}$ & 17 & 26.40 & -1.5 & I LIT & 84.29 & 310 & eP & 17 \\
\hline AAE & 79.69 & 276 & $e^{P}$ & 17 & 30.50 & 1.6 & I SKO & 84.48 & 312 & iPd & 17 \\
\hline ELL & 79.74 & 305 & P- & 17 & 27.00 & -1.6 & 1 & & & iPP & 21 \\
\hline DST & 79.94 & 308 & $P$ & 17 & 28.80 & -0.7 & I & & & is & 28 \\
\hline LVV & 80.17 & 319 & $P d$ & 17 & 31.10 & 0.7 & I & & & iPS & 29 \\
\hline & & & is & 27 & 31.00 & & 1 & & & iss & 33 \\
\hline MLR & 80.19 & 315 & Pd & 17 & 31.50 & 0.7 & 1 & & & iSSS & 37 \\
\hline EDC & 80.30 & 309 & $\mathrm{P}$ & 17 & 30.40 & -0.9 & I SRO & 84.40 & 319 & eP & 17 \\
\hline$B \cup C$ & 80.40 & 314 & eP & 17 & 32.00 & 0.3 & 1 & $16 \mathrm{~s}$ & 19 & 9.70 um & \\
\hline & & & IPP & 17 & 33.00 & & I & $16 \mathrm{~s}$ & & $7.80 \mathrm{um}$ & \\
\hline & & & iPP & $2 \theta$ & 30.00 & & 1 & & & e & 19 \\
\hline & & & is & 27 & 38.00 & & i & & & i & 21 \\
\hline UPP & 80.41 & 330 & $P d$ & 17 & 30.70 & -0.7 & I & & & $e(S)$ & 28 \\
\hline & & & $\mathrm{i}$ & 27 & 34.00 & & 1 & & & e & 28 \\
\hline BuC? & 80.46 & 314 & iPd & 17 & 3000 & -2.0 & I KSP & 84.47 & 322 & eP & 17 \\
\hline$H L W$ & 80.47 & 298 & iPd & 17 & 33.00 & 0.6 & 1 & $1.3 \mathrm{~s}$ & 190 & $0.00 \mathrm{~nm}$ & \\
\hline & & & is & 27 & 18.50 & & 1 & & & $i$ & 17 \\
\hline CMP & 80.87 & 315 & $\mathrm{P}$ & 17 & 35.10 & 0.9 & 1 & & & $\mathrm{i}$ & 18 \\
\hline & & & eP & 17 & 36.00 & & 1 & & & $e$ & 28 \\
\hline & & & es & 27 & 38.00 & & I COP & 84.61 & 327 & iPC- & 17 \\
\hline YER & 80.89 & 306 & $\mathbf{P}$ & 17 & 3450 & 0.0 & 1 & $1.4 \mathrm{~s}$ & 744 & $4.00 \mathrm{~nm}$ & \\
\hline AVY & 80.92 & 247 & iPd & 17 & 34.00 & -1.1 & 1 & & & ePP & 21 \\
\hline WAR & 81.21 & 322 & ePd & 17 & 38.00 & 2.2 & 1 & & & iSKS & 28 \\
\hline$z$ & $18 \mathrm{~s}$ & 33 & 3.00 um & & & $5.7 \mathrm{Msz}$ & I OHR & 85.15 & 312 & eP & 17 \\
\hline & & & ePP & 20 & 42.00 & & 1 & & & es & 28 \\
\hline & & & es & 27 & 42.00 & & I VIE & 85.44 & 320 & Pd & 17 \\
\hline & & & ePS & 28 & 46.00 & & 1 & & & $i$ & 18 \\
\hline$P V L$ & 81.41 & 313 & $P d$ & 17 & 37.00 & -0.1 & 1 & & & $i$ & 19 \\
\hline$D \mid M$ & 81.45 & 311 & ep & 17 & 38.00 & 0.7 & i & & & $P P$ & 21 \\
\hline$U \mathrm{ZH}$ & 81.61 & 318 & $\mathrm{Pd}$ & 17 & 39.00 & $1 . \theta$ & I & & & $\mathrm{i}$ & 22 \\
\hline & & & pP & 17 & 50.00 & $35 \mathrm{kmx}$ & 1 & & & i & 29 \\
\hline & & & es & 27 & 48.00 & & 1 & & & e & 05 \\
\hline DAG & 81.68 & 351 & iPd & 17 & 37.10 & -0.7 & I BRL & 85.52 & 324 & ePd & 17 \\
\hline & & & is & 27 & 47.00 & & I SOP & 85.54 & 319 & Pd & 17 \\
\hline & & & iPS & 28 & 48.00 & & 1 & & & $i$ & 33 \\
\hline$\angle D Z$ & 81.74 & 311 & $P d$ & 17 & 39.00 & 0.2 & 1 & & & $\mathrm{i}$ & 33 \\
\hline SIT & 81.74 & 32 & $\mathbf{P}$ & 17 & 39.70 & 1.3 & 1 & & & e & 36 \\
\hline APO & 81.86 & 331 & $\mathbf{P}$ & 17 & 38.15 & -0.9 & BRN & 85.59 & 324 & iPd & 17 \\
\hline DEV & 82.11 & 316 & eP & 17 & 36.00 & $-4.6 x$ & 1 & & & es & 28 \\
\hline HFS & 82.13 & 331 & $P$ & 17 & 39.70 & -0.7 & BER & 85.72 & 333 & iP & 17 \\
\hline & $0.9 \mathrm{~s}$ & 114 & $1.00 \mathrm{~nm}$ & & 5 & $.9 \mathrm{mb}$ & | TIR & 85.72 & 312 & $P$ & 18 \\
\hline & & & $\epsilon$ & 55 & 00.00 & & I TTG & 85.75 & $3: 3$ & $P$ & 17 \\
\hline SLL & 82.16 & 332 & $\mathbf{P}$ & 17 & 39.97 & -0.7 & | BRG & 85.82 & 323 & iPd & 17 \\
\hline NIE & 82.58 & 320 & iPd & 17 & 42.00 & -1.1 & 1 & & & 1 & 18 \\
\hline
\end{tabular}




\begin{tabular}{|c|c|c|c|c|c|c|c|c|c|c|c|}
\hline & $20 s$ & & 3.50 um & & & $.4 M s z$ & | PN I & 92.51 & 319 & $\mathbf{P}$ & 18 \\
\hline BNS & 89.59 & 325 & iPd & 18 & 18.00 & 0.5 & CVF & 92.66 & 317 & eP & 18 \\
\hline$A O U$ & 89.76 & 315 & $P$ & 18 & 18.50 & -0.1 & I LOR & 93.37 & 323 & eP & 18 \\
\hline & & & $e$ & 24 & 49.50 & & I LBF & 93.45 & 322 & eP & 18 \\
\hline MES & 90.00 & 311 & $P$ & 18 & 18.70 & -0.9 & I FRF & 93.60 & 318 & eP & 18 \\
\hline DBN & 90.09 & 326 & IFo & 18 & 20.00 & 0.2 & I SSF & 93.68 & 323 & eP & 18 \\
\hline & $21 \mathrm{~s}$ & 15 & $5.80 u m$ & & & $.4 M s z$ & i & $1.6 \mathrm{~s}$ & 161 & $1.00 \mathrm{~nm}$ & \\
\hline & & & ePP & 21 & 56.00 & & SMF & 93.71 & 322 & eP & 18 \\
\hline & & & eSKS & 28 & 55.00 & & I LMR & 93.80 & 318 & eP & 18 \\
\hline & & & ePS & 30 & 16.00 & & I LRG & 93.83 & 318 & eP & 18 \\
\hline PRG & 90.11 & 316 & $P$ & 18 & 22.00 & 1.9 & AVF & 93.91 & 322 & eP & 18 \\
\hline & & & e & 22 & 14.00 & & MZF & 94.67 & 322 & $e P$ & 18 \\
\hline BUH & 90.12 & 322 & eP & 18 & 19.50 & -0.6 & I PYM & 94.68 & 322 & $P$ & 18 \\
\hline SAL & 90.19 & 319 & $\mathrm{P}-$ & 18 & 21.00 & 0.6 & TCF & 94.85 & 322 & eP & 18 \\
\hline MNS & 90.23 & 315 & $\mathrm{P}-$ & 18 & 19.70 & -0.9 & 1 & $1.6 \mathrm{~s}$ & 153 & $3.00 \mathrm{~nm}$ & \\
\hline & & & iPP & 21 & 57.00 & & I SSC & 94.85 & 325 & eP & 18 \\
\hline GWF & 90.33 & 323 & P- & 18 & 20.80 & -0.2 & I DMU & 94.92 & 332 & eP & 18 \\
\hline ENN & 90.38 & 325 & ePd & 18 & 21.00 & -0.2 & i & $1.7 \mathrm{~s}$ & 430 & $0.00 \mathrm{~nm}$ & \\
\hline & & & e & 18 & 33.00 & & FLN & 94.95 & 325 & eP & 18 \\
\hline & & & EPP & 21 & 33.00 & & $i$ & $1.6 \mathrm{~s}$ & 65 & $5.40 \mathrm{~nm}$ & \\
\hline MEM & 90.41 & 325 & $\mathrm{Pc}$ & 18 & 24.70 & $3.4 x$ & I DDK & 95.00 & 331 & eP & 18 \\
\hline RMP & 90.47 & 315 & P- & 18 & 21.00 & -0.8 & i & $1.5 \mathrm{~s}$ & 260 & $0.00 \mathrm{~nm}$ & \\
\hline & & & iPP & 21 & 59.00 & & I DKM & 95.05 & 331 & eP & 18 \\
\hline & & & is & 28 & 52.00 & & i & $1.7 \mathrm{~s}$ & 230 & $0.00 \mathrm{~nm}$ & \\
\hline & & & iPPS & 30 & 24.00 & & I DLE & 95.15 & 331 & eP & 18 \\
\hline LLS & 90.54 & 320 & $P$ & 18 & 22.00 & -0.3 & 1 & $2.0 \mathrm{~s}$ & 420 & $0.00 \mathrm{~nm}$ & \\
\hline$F \mid R$ & 90.55 & 317 & $P d$ & 18 & 23.50 & 1.4 & I LSF & 95.26 & 323 & eP & 18 \\
\hline & & & $P P$ & 21 & 46.00 & & $i$ & $1.6 \mathrm{~s}$ & 108 & $8.00 \mathrm{~nm}$ & \\
\hline & & & PPP & 23 & 36.00 & & GRR & 95.37 & 325 & eP & 18 \\
\hline & & & $s$ & 28 & 06.00 & & $D C N$ & 95.45 & 332 & eP & 18 \\
\hline ZUL & 90.61 & 321 & $P d$ & 18 & 21.50 & -0.9 & i & $1.5 \mathrm{~s}$ & 170 & $0.00 \mathrm{~nm}$ & \\
\hline PRT & 90.61 & 317 & $P$ & 18 & 23.00 & 0.6 & I FHC & 95.63 & 44 & iP & 18 \\
\hline & & & iPP & 22 & 00.00 & & I NEW & 95.64 & 35 & $e^{p}$ & 18 \\
\hline & & & is & 29 & 01.00 & & I LPF & 95.68 & 325 & eP & 18 \\
\hline & & & iPS & 31 & 29.50 & & I CAF & 95.69 & 321 & $e^{P}$ & 18 \\
\hline ME I & 90.72 & 310 & $\mathbf{P}$ & 18 & 22.50 & -0.5 & i & $1.6 \mathrm{~s}$ & 90 & $0.60 \mathrm{~nm}$ & \\
\hline CDF & 90.80 & 322 & eP & 18 & 23.00 & -0.3 & I ECP & 95.80 & 330 & eP & 18 \\
\hline & $1.6 \mathrm{~s}$ & 321 & $1.00 \mathrm{~nm}$ & & 6 & $4 \mathrm{mb}$ & i & $0.9 \mathrm{~s}$ & 40 & $0.00 \mathrm{~nm}$ & \\
\hline WLF & 90.81 & 324 & ePd & 18 & 28.00 & $4.8 x$ & I RJF & 95.80 & 322 & eP & 18 \\
\hline & & & $P P$ & 22 & 06.00 & & 1 & $1.4 \mathrm{~s}$ & 170 & $0.00 \mathrm{~nm}$ & \\
\hline $\mathrm{ECH}$ & 90.97 & 322 & $P-$ & 18 & 23.60 & -0.4 & I CIR & 95.87 & 250 & eP & 18 \\
\hline & & & ePP & 21 & 59.00 & & i & & & i PP & 22 \\
\hline & & & EPPP & 24 & 02.00 & & I MAW & 95.88 & 199 & $e^{P}$ & 18 \\
\hline & & & iSKS & 28 & 59.00 & & MFF & 96.02 & 324 & eP & 18 \\
\hline PCN & 90.98 & 319 & $P$ & 18 & 26.50 & 2.5 & i & $1.6 \mathrm{~s}$ & 175 & $5.00 \mathrm{~nm}$ & \\
\hline & & & es & 28 & 34.00 & & I YKM & 96.03 & 34 & $i P c$ & 18 \\
\hline BBS & 91.14 & 321 & $P d$ & 18 & 23.50 & -1.3 & I RXF & 96.34 & 33 & $i P c$ & 18 \\
\hline BVT & 91.18 & 318 & $P$ & 18 & 25.40 & 0.5 & I LPO & 96.34 & 321 & eP & 18 \\
\hline$G 18$ & 91.19 & 311 & $P$ & 18 & 24.00 & $-1 \cdot 3$ & $|K R|$ & 96.38 & 254 & eP & 18 \\
\hline UCC & 91.21 & 325 & $P d-$ & 18 & 24.80 & -0.2 & I LFF & 96.46 & 322 & eP & 18 \\
\hline & & & $P P$ & 22 & 03.06 & & I LDM & 96.49 & 34 & iPc & 18 \\
\hline & & & SKS & 29 & 01.00 & & I WDC & 96.69 & 43 & iPd & 18 \\
\hline & & & $\mathrm{s}$ & 29 & 26.00 & & I CNG & 96.93 & 245 & $i P C$ & 18 \\
\hline & & & $S P$ & 30 & 28.00 & & i & & & $\epsilon$ & 22 \\
\hline BAF & 91.25 & 322 & P- & 18 & 24.80 & -0.6 & ALS & 7.37 & 320 & $e P$ & 18 \\
\hline BSF & 91.37 & 322 & $e^{p}$ & 18 & 25.40 & -0.6 & I $M I N$ & 7.42 & 43 & iP & 18 \\
\hline & 165 & 273 & $3.06 \mathrm{~nm}$ & & 6 & $4 \mathrm{mb}$ & I VAL & 97.73 & 332 & Pd & 18 \\
\hline DOU & 9145 & 325 & ePd & 18 & 2600 & -0.1 & i & & & PPP & 22 \\
\hline & & & PP & 22 & 03.00 & & 1 & & & PPS & 31 \\
\hline & & & SKS & 29 & 02.00 & & i & & & SS & 36 \\
\hline EDU & 91.50 & 333 & $e^{P}$ & 18 & 24.80 & -1.5 & $i$ & & & LO & 49 \\
\hline & $1.5 \mathrm{~s}$ & 566 & $5.00 \mathrm{~nm}$ & & 6 & $.7 \mathrm{mb}$ & i & & & LR & 55 \\
\hline HAU & 91.54 & 322 & eP & 18 & 26.36 & -0.4 & EPF & 97.78 & 320 & eP & 18 \\
\hline NEC & 91.69 & 321 & Pd & 18 & 26.50 & -0.9 & i & $1.4 \mathrm{~s}$ & 18 & $8.90 \mathrm{~nm}$ & \\
\hline GEN & 91.74 & 318 & $P$ & 18 & 27.06 & -0.6 & I ORV & 97.91 & 44 & iPd & 18 \\
\hline & & & es & 43 & 20.00 & & SET & 98.12 & 312 & $P$ & 18 \\
\hline $1 x$ & 1.88 & 320 & $P d$ & 18 & 28.00 & -0.5 & I BUL & 98.27 & 252 & $\mathrm{P}+$ & 18 \\
\hline EBH & 91.91 & 333 & $e P$ & 18 & 27.40 & -0.7 & i & & & iPP & 23 \\
\hline & $1.5 \mathrm{~s}$ & 350 & 0.00 & & 6 & $6 m b$ & 1 & & & is & 29 \\
\hline PGC & 1.92 & 37 & $e P$ & 18 & 20.00 & $-8.3 x$ & EKS & 98.30 & 45 & eP & 18 \\
\hline EBL & 22.00 & 332 & $\mathrm{iPc}$ & 18 & 28.30 & -0.2 & 1 & $2.2 \mathrm{~s}$ & 390 & 0.0 & \\
\hline & $1.4 \mathrm{~s}$ & 291 & $1.00 \mathrm{~nm}$ & & 6 & $.5 \mathrm{mb}$ & 1 & $22 \mathrm{~s}$ & 23 & 3.0 & \\
\hline EAU & 92.13 & 332 & ePC & 18 & 28.70 & -0.5 & $i$ & $22 \mathrm{~s}$ & & 7.00 um & \\
\hline & $1.3 \mathrm{~s}$ & 353 & $3.00 \mathrm{~nm}$ & & 6 & $.6 \mathrm{mb}$ & i & $22 \mathrm{~s}$ & 26 & 6.00 um & \\
\hline $\mathrm{GDH}$ & 92.15 & 358 & iPc & 18 & 30.00 & 1.0 & i & & & es & 29 \\
\hline & $1.5 \mathrm{~s}$ & 211 & $1.00 \mathrm{~nm}$ & & & $.3 \mathrm{mb}$ & i & & & ePS & 31 \\
\hline & & & iPP & 22 & 10.00 & & i & & & ess & 36 \\
\hline & & & iSKS & 29 & 00.00 & & i & & & eSSS & 40 \\
\hline & & & iss & 35 & 34.00 & & $E B R$ & 98.93 & 318 & $e^{p}$ & 19 \\
\hline ERC & 2.19 & 311 & $P$ & 18 & 29.00 & -0.7 & $i$ & & & ePP & 23 \\
\hline$E A B$ & 92.31 & 333 & eP & 18 & 29.10 & -0.8 & 1 & & & eSKKS & 30 \\
\hline & $1.5 \mathrm{~s}$ & 312 & $2.00 \mathrm{~nm}$ & & 6 & $.5 \mathrm{mb}$ & MHC & 98.98 & 46 & ip & 19 \\
\hline EKA & & 332 & $P$ & 18 & 0.00 & 0.0 & ARN & 99.05 & 46 & $\mathrm{eP}^{\mathrm{P}}$ & 19 \\
\hline & $1.6 \mathrm{~s}$ & 417 & $7.00 \mathrm{~nm}$ & & & $6 \mathrm{mb}$ & AMM & 99.22 & 35 & eP & 19 \\
\hline ESK & 92.35 & 332 & eP & 18 & 31.56 & 1.3 & JAS & 99.47 & 45 & iPd & 19 \\
\hline ROB & 92.47 & 319 & $P$ & 18 & 30.00 & -1.0 & 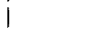 & & & $P^{\prime} P$. & 4 \\
\hline REY & 92.50 & 345 & iP & 18 & 32.20 & 1.5 & LRM & 99.66 & 35 & e & 1 \\
\hline
\end{tabular}


$22 d \quad 15 h$

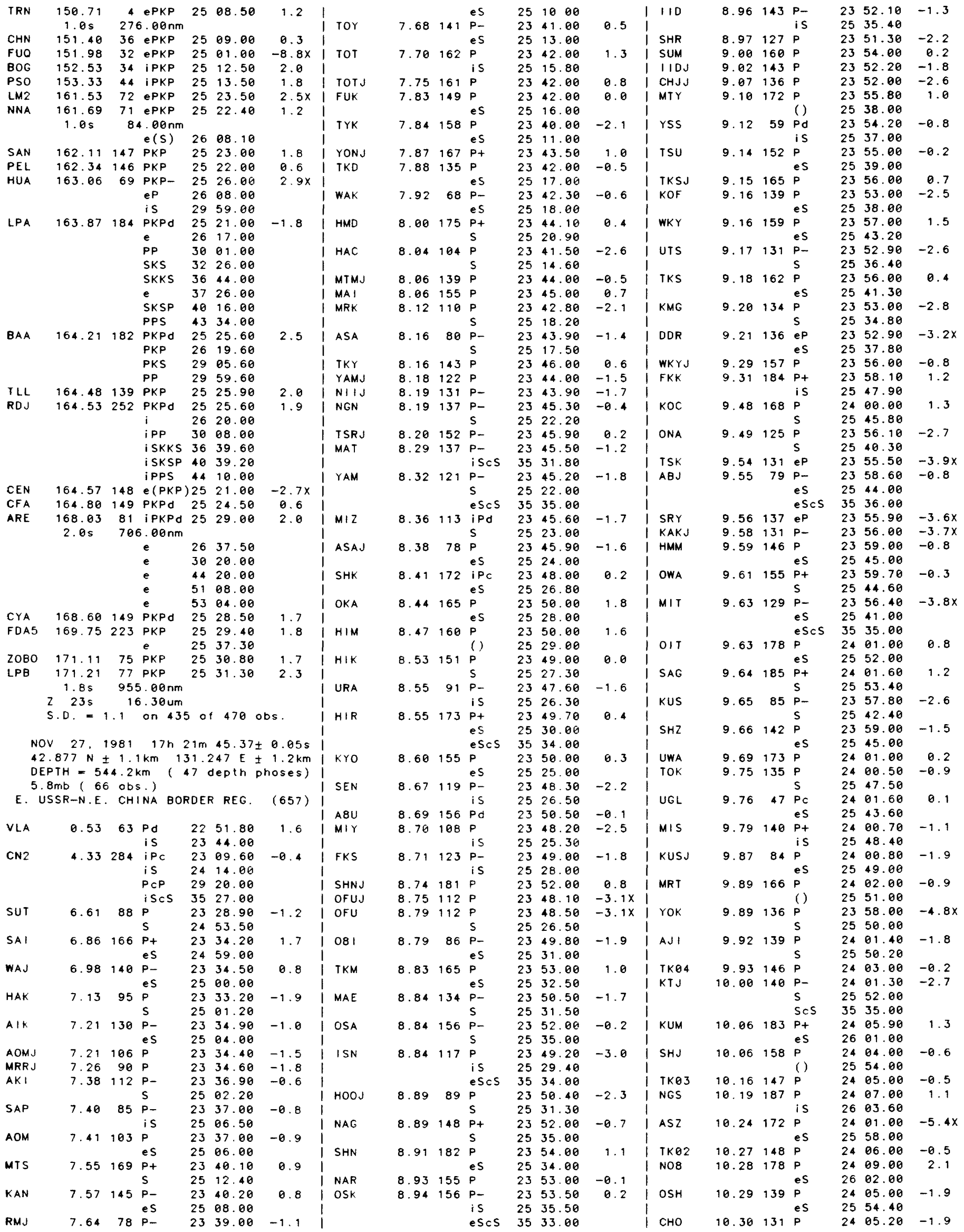




\begin{tabular}{|c|c|c|c|c|c|c|c|c|c|c|c|}
\hline & & & $\mathrm{s}$ & 25 & 54.20 & & ! & & & s & 30 \\
\hline KYS & 10.33 & 135 & eP & 24 & 04.00 & $-3.3 x$ & $1 \mathrm{CD} 2$ & 24.90 & 250 & Pd & 26 \\
\hline KUMJ & 0.33 & 182 & $P$ & 24 & 08.00 & 0.6 & 1 & & & SP & 29 \\
\hline FKJ & 10.34 & $19 t$ & $\mathbf{P}$ & 24 & 09.00 & 1.6 & 1 & & & $\mathrm{~s}$ & 30 \\
\hline & & & $s$ & 26 & 06.50 & & 1 & & & $\operatorname{ses}$ & 36 \\
\hline TKO1 & 10.37 & 149 & $\mathbf{P}$ & 24 & 06.00 & -1.4 & I HKC & 24.97 & 220 & P & 26 \\
\hline TMS & 10.38 & 137 & $P$ & 24 & 06.00 & -1.9 & I GYA & 25.88 & 239 & Pd & 26 \\
\hline & & & $\mathrm{s}$ & 25 & 57.70 & & 1 & & & SP & 29 \\
\hline NEM & 10.50 & 83 & $\mathbf{P}$ & 24 & 07.00 & -2.1 & 1 & & & s & 30 \\
\hline & & & es & 26 & 01.00 & & I CVP & 26.34 & 201 & ePc & 26 \\
\hline$M Y Z$ & 10.94 & 179 & $\mathbf{P}$ & 24 & 14.00 & 0.4 & I UER & 26.45 & 302 & Pd & 26 \\
\hline & & & eS & 26 & 14.00 & & 1 & & & is & 30 \\
\hline TYV & 11.15 & 40 & $\mathrm{P}$ & 24 & 16.00 & 0.4 & I SZP & 26.86 & 203 & eP & 26 \\
\hline & & & is & 26 & 16.80 & & 1 & $1.0 \mathrm{~s}$ & 228 & $.00 \mathrm{~nm}$ & \\
\hline KAG & 11.30 & 183 & $P$ & 24 & 20.50 & $3.3 x$ & 1 & & & e & 28 \\
\hline & & & $\mathrm{s}$ & 26 & 36.00 & & I BAG & 27.91 & 202 & $P$ & 26 \\
\hline $\mathrm{SHO}$ & 11.37 & 80 & Pd & 24 & 16.70 & -1.2 & I TIK & 28.86 & 358 & $\mathrm{PC}$ & 26 \\
\hline & & & is & 26 & 18.20 & & 1 & & & EsP & 29 \\
\hline BJI & 11.66 & 261 & iPd & 24 & 21.00 & 0.1 & 1 & & & es & 31 \\
\hline & & & is & 26 & 24.00 & & $|K M|$ & 29.33 & 242 & iPd & 27 \\
\hline & & & ScS & 35 & 37.00 & & 1 & & & PP & 28 \\
\hline KAGJ & 11.67 & 182 & $\mathbf{P}$ & 24 & 19.00 & -2.0 & 1 & & & SP & 29 \\
\hline HJJ & 11.85 & 143 & $P$ & 24 & 20.00 & -2.8 & 1 & & & is & 31 \\
\hline & & & $\mathrm{S}$ & 26 & 30.30 & & 1 & & & SS & 34 \\
\hline NKL & 12.06 & 28 & $\mathbf{P}$ & 24 & 24.50 & -0.3 & 1 & & & $\operatorname{ScS}$ & 36 \\
\hline & & & is & 26 & 31.50 & & I MAN & 29.45 & 200 & eP & 27 \\
\hline KUR & 12.19 & 73 & Pd & 24 & 27.00 & 0.8 & 1 & & & es & 31 \\
\hline & & & is & 26 & 38.00 & & I SMY & 30.01 & 56 & $i P c$ & 27 \\
\hline REI & 12.30 & 73 & $P$ & 24 & 27.70 & 0.4 & I CNP & 30.79 & 193 & ePc & 27 \\
\hline & & & is & 26 & 41.10 & & 1 & $0.9 \mathrm{~s}$ & 1070 & $.00 \mathrm{~nm}$ & \\
\hline $\mathrm{OKH}$ & 13.20 & 32 & $P C$ & 24 & 35.30 & -1.0 & I ELT & 31.31 & 305 & $P d$ & 27 \\
\hline & & & is & 26 & 55.30 & & 1 & & & es & 31 \\
\hline TUP & 13.76 & 331 & Pd & 24 & 41.70 & -0.3 & 1 WMO & 31.42 & 287 & iPd & 27 \\
\hline & & & $S$ & 27 & 07.26 & & 1 & & & PP & 28 \\
\hline SSE & 14.23 & 218 & $\mathrm{Pd}$ & 24 & 49.00 & 2.2 & 1 & & & $\mathrm{~s}$ & 31 \\
\hline & & & $\mathrm{s}$ & 27 & 17.00 & & 1 & & & SS & 34 \\
\hline $\mathrm{NJ} 2$ & 14.60 & 226 & iPd & 24 & 51.00 & 0.5 & 1 & & & iscs & 36 \\
\hline & & & SP & 26 & 44.00 & & I GUMO & 31.47 & 154 & $e(P)$ & 27 \\
\hline & & & is & 27 & 20.50 & & 1 & & & $e(S)$ & 31 \\
\hline & & & $\operatorname{SCP}$ & 32 & 06.00 & & I GUA & 31.53 & 154 & $e(P)$ & 27 \\
\hline & & & ScS & 35 & 46.50 & & 1 & 0.75 & 115 & $.00 \mathrm{~nm}$ & \\
\hline TIY & 15.25 & 257 & iPd & 24 & 58.00 & 1.0 & I PLP & 32.06 & 192 & eP & 27 \\
\hline & & & is & 27 & 36.00 & & 1 & 0.75 & 208 & $.00 \mathrm{~nm}$ & \\
\hline BTO & 16.01 & 269 & IPd & 25 & 05.00 & 0.5 & I UKR & 32.31 & 301 & eP & 27 \\
\hline & & & $S P$ & 27 & 13.50 & & 1 & & & is & 32 \\
\hline & & & $\mathrm{s}$ & 27 & 50.00 & & I NVS & 33.09 & 308 & $P d$ & 27 \\
\hline & & & $\operatorname{ses}$ & 35 & 48.00 & & 1 & & & is & 32 \\
\hline WHN & 18.26 & 233 & iPd & 25 & 27.50 & 1.3 & 1 & & & is & 32 \\
\hline & & & SP & 27 & 40.00 & & | NR I & 34.52 & 335 & $\mathrm{Pd}$ & 27 \\
\hline & & & is & 28 & 24.00 & & 1 & & & isP & 30 \\
\hline & & & iscs & 35 & 55.00 & & 1 & & & is & 32 \\
\hline$B O D$ & 18.44 & 330 & $\mathrm{Pd}$ & 25 & 26.90 & -0.8 & LSA & 34.57 & $26 ?$ & $i \mathrm{Pd}$ & 27 \\
\hline & & & $\mathrm{s}$ & 28 & 28.40 & & 1 & & & S & 32 \\
\hline SKR & 18.66 & 57 & $\mathrm{Pc}$ & 25 & 28.60 & -1.3 & I PPR & 34.74 & 202 & ePd & 27 \\
\hline & & & es & 28 & 37.50 & & 1 & $1.0 \mathrm{~s}$ & 758 & $.00 \mathrm{~nm}$ & \\
\hline YAK & 19.19 & 358 & $P C$ & 25 & 33.60 & -1.2 & CGP & 34.77 & 191 & ePc & 27 \\
\hline & & & is & 28 & 39.80 & & 1 & $1.0 \mathrm{~s}$ & 2170 & $.00 \mathrm{~nm}$ & \\
\hline ANP & 19.38 & 207 & $\mathrm{PC}$ & 25 & 41.00 & 4. $1 x$ & I PPH & 35.17 & 189 & ePd & 27 \\
\hline & & & $s$ & 28 & 48.00 & & 1 & $0.7 \mathrm{~s}$ & 215 & $.00 \mathrm{~nm}$ & \\
\hline XAN & 19.54 & 251 & iPd & 25 & 38.20 & -0.1 & I SEM & 35.21 & 300 & Po & 27 \\
\hline & & & SP & 28 & 00.50 & & 1 & & & $i p P$ & 29 \\
\hline & & & is & 28 & 46.00 & & 1 & & & $i s P$ & 30 \\
\hline & & & ScS & 35 & 58.00 & & 1 & & & es & 32 \\
\hline TATO & 19.58 & 207 & ip & 25 & 41.10 & 2.4 & I DAV & 35.99 & 190 & $\mathrm{P}$ & 28 \\
\hline I RK & 20.35 & 307 & $\mathrm{Pd}$ & 25 & 46.00 & 0.3 & 1 & & & es & 33 \\
\hline & & & esP & 28 & 06.00 & & $1 \mathrm{LT}$ & 36.28 & 29 & $P d$ & 28 \\
\hline & & & es & 29 & 00.00 & & 1 & & & esP & 30 \\
\hline ZAK & 20.53 & 301 & Pd & 25 & 47.60 & 0.3 & 1 & & & es & 33 \\
\hline & & & s & 29 & 02.00 & & 1 & & & ess & 35 \\
\hline $\mathrm{TCU}$ & 20.62 & 208 & $P$ & 25 & 51.40 & $3.1 x$ & I CHG & 36.31 & 239 & iPd & 28 \\
\hline & & & $S$ & 29 & 08.50 & & 1 & $1.2 \mathrm{~s}$ & 330 & $.00 \mathrm{~nm}$ & \\
\hline $\mathrm{OZH}$ & 20.70 & 214 & iPd & 25 & 48.50 & -0.5 & 1 & & & $i$ & 33 \\
\hline & & & is & 29 & 03.00 & & 1 & & & es & 36 \\
\hline PET & 20.85 & 51 & $\mathrm{PC}$ & 25 & 50.00 & -0.2 & I CHTO & 36.31 & 239 & iPd & 28 \\
\hline & & & $\mathbf{S P}$ & 28 & 12.00 & & $15 \mathrm{HIO}$ & 36.46 & 255 & iPd & 28 \\
\hline & & & eS & 29 & 04.00 & & I BDT & 37.45 & 237 & ¡Pd & 28 \\
\hline MGD & 20.97 & 28 & $\mathrm{PC}$ & 25 & 51.00 & -0.2 & 1 & $0.6 \mathrm{~s}$ & 372 & $.00 \mathrm{~nm}$ & \\
\hline & & & is & 29 & 10.00 & & I PRZ & 38.32 & 288 & $\mathrm{PC}$ & 28 \\
\hline $\mathrm{LZH}$ & 22.15 & 261 & IPd & 26 & 83.05 & 0.6 & 1 & & & is & 33 \\
\hline & & & SP & 28 & 29.00 & & I TLG & 38.77 & 290 & Po & 28 \\
\hline & & & 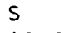 & 29 & 29.00 & & 1 & & & sP & 31 \\
\hline & & & ises & 36 & 11.00 & & 1 & & & is & 33 \\
\hline MOY & 22.21 & 304 & $P d$ & 26 & 02.40 & -0.1 & 1 & & & ess & 36 \\
\hline SEY & 23.56 & 24 & PC & 26 & 15.40 & e. 8 & I KKM & 39.02 & 204 & iPd & 28 \\
\hline & & & SP & 28 & 43.90 & & 1 & $0.9 \mathrm{~s}$ & 411 & $.00 \mathrm{~nm}$ & \\
\hline & & & is & 29 & 51.90 & & 1 & & & e & 33 \\
\hline GZH & 24.70 & 223 & iPd & 26 & 24.60 & -0.6 & I NRN & 40.36 & 287 & $e^{P}$ & 28 \\
\hline
\end{tabular}




\begin{tabular}{|c|c|c|c|c|c|c|c|c|c|c|c|}
\hline & $1.0 \mathrm{~s}$ & 366 & $5.00 \mathrm{~nm}$ & & & $5.9 \mathrm{mb}$ & 1 & & & is & 38 \\
\hline MDG & 49.68 & 161 & $P$ & 29 & 50.50 & 1.4 & I HNR & 58.28 & 146 & ePc & 30 \\
\hline RAB & 50.54 & 153 & $\mathrm{Pc}$ & 29 & 50.00 & $-5.4 x$ & $i$ & $0.9 \mathrm{~s}$ & 15 & $1.00 \mathrm{~nm}$ & \\
\hline HYB & 51.17 & 257 & ePd- & 29 & 59.00 & -1.2 & i & & & es & 32 \\
\hline & & & e & 30 & 16.50 & & I KNA & 58.38 & 183 & $P d$ & 30 \\
\hline & & & e & 30 & 54.00 & & I BAK & 58.53 & 298 & eP & 30 \\
\hline & & & ePCP & 31 & 02.00 & & i & & & $s$ & 38 \\
\hline & & & e & 31 & 28.50 & & I OBN & 58.60 & 318 & iPd- & 30 \\
\hline & & & epp & 31 & 44.00 & $557 \mathrm{~km} X$ & i & $1.0 \mathrm{~s}$ & 670 & $0.00 \mathrm{~nm}$ & \\
\hline & & & ePP & 32 & 01.00 & & i & & & ePP & 33 \\
\hline & & & esp & 32 & 45.00 & & i & & & ESP & 33 \\
\hline & & & eSCP & 34 & 08.00 & & i & & & ePPP & 34 \\
\hline & & & esPP & 34 & 44.00 & & i & & & is & 38 \\
\hline & & & es & 36 & 35.00 & & 1 & & & iscs & 39 \\
\hline & & & escs & 38 & 48.00 & & 1 & & & ess & 41 \\
\hline & & & ess & 39 & 40.00 & & 1 & & & ess & 42 \\
\hline & & & ess & 40 & 28.00 & & 1 & & & $\mathrm{i}$ & 43 \\
\hline PPI & 51.27 & 221 & eP & 30 & 00.70 & -0.1 & 1 & & & isss & 45 \\
\hline & $1.0 \mathrm{~s}$ & 179 & $9.00 \mathrm{~nm}$ & & & $5.4 \mathrm{mb}$ & I MAK & 58.74 & 301 & $P d$ & 30 \\
\hline LAT & 51.34 & 160 & eP & 30 & 02.00 & 0.7 & 1 & & & ipP & 32 \\
\hline QUE & 52.03 & 278 & iPd & 30 & 05.20 & -1.3 & 1 & & & is & 38 \\
\hline & & & ePcP & 31 & 04.40 & & I SUF & 59.10 & 329 & $\mathbf{P}$ & 30 \\
\hline & & & epP & 31 & 49.60 & $548 \mathrm{~km}$ & I DAG & 59.13 & 352 & iPc & 30 \\
\hline & & & es & 36 & 49.20 & & i & $0.5 \mathrm{~s}$ & 225 & $5000 \mathrm{~nm}$ & \\
\hline & & & escs & 38 & 55.00 & & i & & & $i$ & 31 \\
\hline TRT & 53.12 & 203 & iPc & 30 & 13.30 & -0.8 & 1 & & & $\mathrm{i}$ & 31 \\
\hline & $1.1 \mathrm{~s}$ & 150 & $0.00 \mathrm{~nm}$ & & & $5.2 \mathrm{mb}$ & i & & & is & 38 \\
\hline KUPT & 53.23 & 189 & eP & 30 & 14.00 & -0.9 & I SHE & 59.22 & 299 & $P d$ & 30 \\
\hline & $0.5 \mathrm{~s}$ & 300 & $.00 \mathrm{~nm}$ & & & $5.9 \mathrm{mb}$ & 1 & & & is & 38 \\
\hline & & & $e(S)$ & 36 & 55.00 & & I GRO & 59.66 & 303 & $P d$ & 30 \\
\hline PCA & 53.39 & 39 & $\mathrm{iPc}$ & 30 & 16.20 & 0.4 & 1 & & & is & 38 \\
\hline LMG & 53.80 & 159 & eP & 30 & 19.00 & -0.1 & I TEH & 60.03 & 292 & ePd & 31 \\
\hline POO & 53.84 & 262 & iPd & 30 & 18.50 & -0.9 & I LNK & 60.11 & 297 & $P d$ & 31 \\
\hline PMG & 54.02 & 160 & eP & 30 & 21.00 & 0.5 & 1 & & & es & 38 \\
\hline APA & 54.05 & 332 & $\mathrm{Pd}$ & 30 & 18.30 & -1.9 & I UME & 60.62 & 332 & $\mathbf{P}$ & 31 \\
\hline & & & isP & 33 & 02.00 & & i & & & is & 38 \\
\hline & & & is & 37 & 09.50 & & I KRV & 60.69 & 300 & eP & 31 \\
\hline ASH & 54.12 & 291 & $\mathbf{P}$ & 30 & 21.00 & -0.1 & 1 & & & $s$ & 38 \\
\hline & & & $S$ & 37 & 20.00 & & I PYA & 60.90 & 304 & $P d$ & 31 \\
\hline MHI & 54.16 & 289 & Pd- & 30 & 20.80 & -0.7 & i & & & i pP & 32 \\
\hline & & & $\mathrm{i}$ & 34 & 23.10 & & 1 & & & is & 38 \\
\hline & & & is & 37 & 15.80 & & I NUR & 60.97 & 327 & iPd & 31 \\
\hline VAN & 54.29 & 291 & ePd & 30 & 21.00 & -1.3 & 1 & $0.7 \mathrm{~s}$ & 302 & $2.00 \mathrm{~nm}$ & \\
\hline & & & $\mathrm{pP}$ & 32 & 07.20 & $551 \mathrm{~km}$ & 1 & $20 \mathrm{~s}$ & & $0.80 \mathrm{um}$ & \\
\hline & & & $s$ & 37 & 18.20 & & 1 & & & $\mathrm{i}$ & 31 \\
\hline GBA & 54.52 & 255 & $\mathrm{Pc}$ & 30 & 23.20 & -0.8 & 1 & & & ¿PCP & 31 \\
\hline & $0.6 \mathrm{~s}$ & 368 & $3.00 \mathrm{~nm}$ & & & 5. $9 \mathrm{mb}$ & 1 & & & iPP & 33 \\
\hline$A L E$ & 54.69 & 2 & eP & 30 & 24.00 & -0.5 & 1 & & & is & 38 \\
\hline & $0.6 \mathrm{~s}$ & 327 & $.00 \mathrm{~nm}$ & & & $5.8 \mathrm{mb}$ & I & & & iscs & 39 \\
\hline KAT & 54.92 & 293 & $P d$ & 30 & 26.50 & -0.1 & 1 & & & eSS & 41 \\
\hline & & & epP & 32 & 14.00 & $558 \mathrm{kmx}$ & 1 & & & e & 42 \\
\hline & & & esP & 33 & 15.00 & & 1 & & & eSSS & 46 \\
\hline & & & is & 37 & 28.06 & & I MTA & 61.09 & 301 & $P d$ & 31 \\
\hline & & & ess & $4 \theta$ & 40.00 & & $i$ & & & $i p p$ & 32 \\
\hline KEV & 55.07 & 336 & Pd & 30 & 26.00 & -1.3 & 1 & & & is & 38 \\
\hline & & & $\mathrm{i}$ & 30 & 27.90 & & I GRS & 61.32 & 299 & $P d$ & 31 \\
\hline & & & $\mathrm{i}$ & 30 & 38.40 & & I & & & $i p P$ & 32 \\
\hline & & & epP & 32 & 14.00 & $561 \mathrm{~km} x$ & I & & & is & 38 \\
\hline & & & isp & 33 & 12.20 & & I STE & 61.74 & 301 & $P d$ & 31 \\
\hline & & & iSCP & 34 & 25.00 & & I & & & ipp & 33 \\
\hline & & & is & 37 & 26.00 & & 1 & & & is & 38 \\
\hline & & & iscs & 39 & 13.50 & & I BKR & 61.88 & 302 & $\mathrm{Pd}$ & 31 \\
\hline & & & ess & 40 & 40.00 & & 1 & & & $i p P$ & 33 \\
\hline & & & ess & 41 & 12.00 & & 1 & & & is & 38 \\
\hline MIN & 55.45 & 180 & $P C$ & 30 & 29.70 & -0.7 & I AKH & 62.08 & 302 & $\mathrm{Pc}$ & 31 \\
\hline TGI & 56.11 & 286 & iPd & 30 & 34.50 & -0.8 & I NAK & 62.09 & 299 & $\mathrm{Pc}$ & 31 \\
\hline SOD & 56.40 & 333 & $P d$ & 30 & 35.60 & -1.0 & 1 & & & $S$ & 38 \\
\hline & & & iPCP & 31 & 26.60 & & | ERE & 62.12 & 300 & $\mathrm{Pd}$ & 31 \\
\hline & & & isP & 33 & 21.90 & & 1 & & & $i p P$ & 33 \\
\hline & & & i ScP & 34 & 31.00 & & 1 & & & is & 38 \\
\hline SIT & 7.06 & 41 & $i P c$ & 30 & 42.60 & 1.4 & I TAB & 62.12 & 297 & iPd- & 31 \\
\hline TRO & 57.50 & 337 & ip & 30 & 42.70 & -1.3 & I LEN & 62.20 & 301 & $P$ & 31 \\
\hline$K J F$ & 57.70 & 330 & Po & 30 & 44.50 & -1.0 & 1 & & & is & 39 \\
\hline & & & $\mathrm{i}$ & 30 & 46.30 & & 1 ABS & 62.29 & 302 & $P d$ & 31 \\
\hline & & & ipP & 32 & 32.00 & $545 \mathrm{~km}$ & 1 & & & $i p P$ & 33 \\
\hline & & & isP & 33 & 31.90 & & 1 & & & es & 39 \\
\hline & & & i SCP & 34 & 37.00 & & I ZUG & 62.45 & 304 & $e P$ & 31 \\
\hline & & & es & 38 & 00.00 & & 1 & & & is & 39 \\
\hline & & & escs & 39 & 32.00 & & I YKA & 62.56 & 29 & $P$ & 31 \\
\hline & & & ess & 41 & 08.00 & & I WRA. & 62.57 & 177 & $P d$ & 31 \\
\hline & & & e & 41 & 52.00 & & 1 & $0.9 \mathrm{~s}$ & & $8.10 \mathrm{~nm}$ & \\
\hline MOS & 57.80 & 318 & $\mathrm{Pd}$ & 30 & 44.00 & -2.3 & I WB2 & 62.57 & 177 & $P d$ & 31 \\
\hline & & & $i p P$ & 32 & 32.00 & $548 \mathrm{~km}$ & 1 & & & e & 33 \\
\hline & & & isP & 33 & 31.00 & & 1 & & & es & 39 \\
\hline & & & is & 38 & 00.00 & & 1 SOC & 63.16 & 305 & Pd & 31 \\
\hline$K I R$ & 58.12 & 335 & $P$ & 30 & 47.00 & -1.3 & I & & & epP & 33 \\
\hline & & & i & 30 & 48.70 & $6 \mathrm{~km} x$ & 1 & & & es & 39 \\
\hline
\end{tabular}




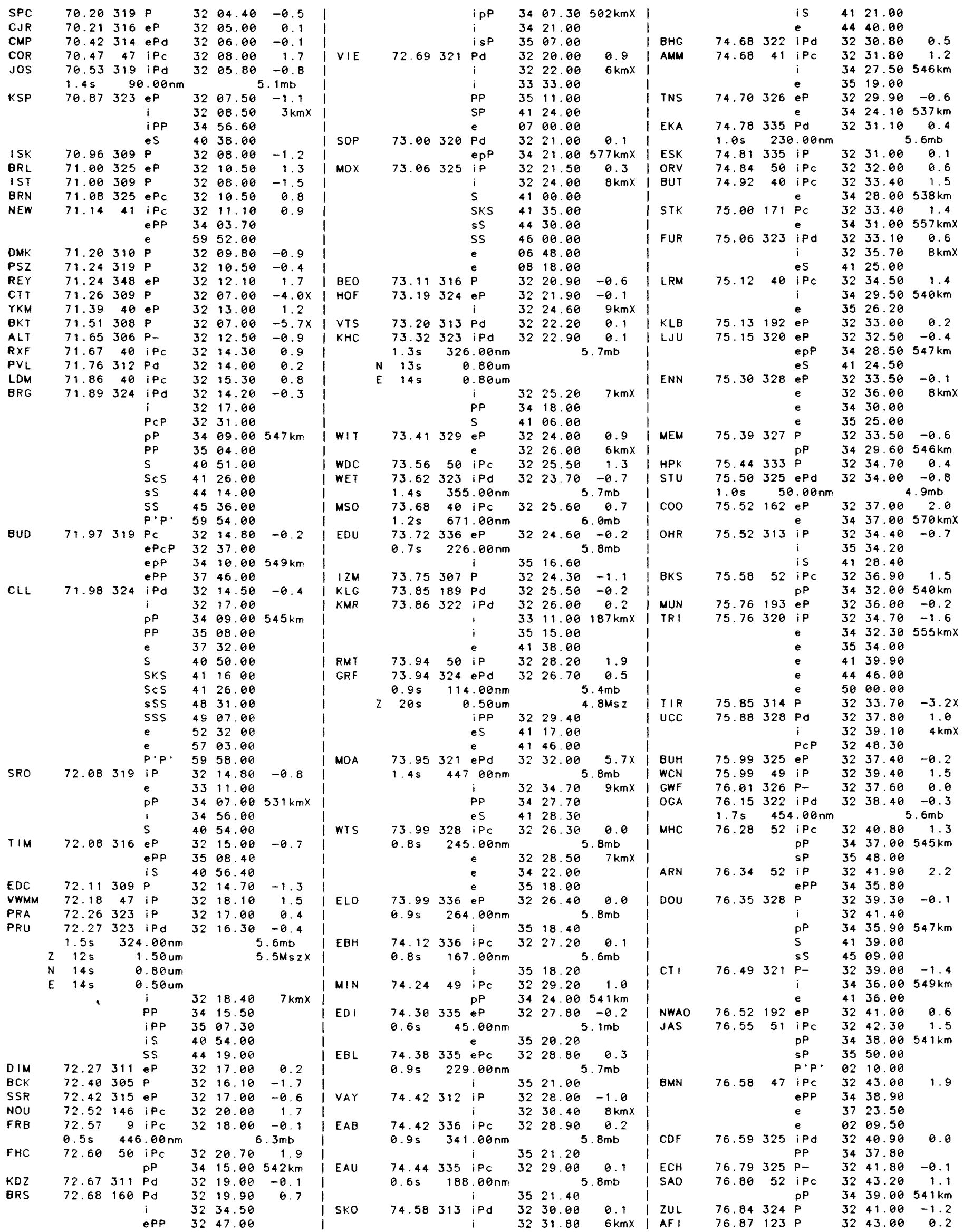




\begin{tabular}{|c|c|c|c|c|c|c|c|c|c|c|c|}
\hline & & & $p P$ & 34 & 45.00 & $579 \mathrm{kmx}$ & I ROB & 79.55 & 322 & $P$ & 32 \\
\hline HLW & 76.94 & 299 & iPd & 32 & 41.00 & -2.0 & | GLR & 79.56 & 48 & iP & 32 \\
\hline & & & is & 41 & 42.00 & & i & & & ePP & 34 \\
\hline DMU & 77.15 & 336 & iPC & 32 & 43.90 & 0.2 & I GRR & 79.81 & 330 & IPd & 32 \\
\hline & $0.9 \mathrm{~s}$ & 370 & $9.00 \mathrm{~nm}$ & & & $5.8 \mathrm{mb}$ & I VAL & 79.84 & 337 & iPd & 32 \\
\hline BAF & 77.16 & 325 & $P-$ & 32 & 43.90 & -0.1 & I PYR & 79.90 & 52 & ip & 33 \\
\hline BBS & 77.23 & 325 & $P$ & 32 & 43.00 & -1.3 & 1 & & & ePP & 34 \\
\hline BSF & 77.25 & 325 & $i P d$ & 32 & 44.20 & -0.3 & I LPF & 80.18 & 330 & iPd & 32 \\
\hline I MW & 77.26 & 41 & ip & 32 & 47.10 & 2.2 & I MZF & 80.27 & 327 & IPd & 33 \\
\hline HAU & 77.30 & 326 & IPd & 32 & 44.30 & -0.4 & I WAM & 80.32 & 166 & $\mathrm{PC}$ & 33 \\
\hline & $0.9 \mathrm{~s}$ & 103 & $3.00 \mathrm{~nm}$ & & & $5.3 \mathrm{mb}$ & 1 & & & ePCP & 35 \\
\hline SAL & 77.34 & 322 & $P$ & 32 & 44.00 & -0.8 & I TCF & 80.36 & 327 & iPd & 33 \\
\hline & & & $i$ & 34 & 50.00 & $602 \mathrm{kmx}$ & I CVF & 80.44 & 321 & iPd & 33 \\
\hline DDK & 77.42 & 336 & eP & 32 & 45.00 & -0.1 & 1 & $0.8 \mathrm{~s}$ & 91 & $1.30 \mathrm{~nm}$ & \\
\hline & $1.0 \mathrm{~s}$ & 310 & $9.00 \mathrm{~nm}$ & & & $5.7 \mathrm{mb}$ & 1080 & 80.47 & 277 & iPd & 33 \\
\hline MNV & 77.49 & 49 & iPC & 32 & 47.60 & 1.5 & I PAS & 80.53 & 52 & $P+$ & 33 \\
\hline & & & $p P$ & 34 & 43.00 & $538 \mathrm{~km}$ & I LSF & 80.67 & 327 & eP & 33 \\
\hline DKM & 77.51 & 335 & iPc & 32 & 45.60 & -0.1 & I FRF & 80.67 & 323 & iPd & 33 \\
\hline & $0.7 \mathrm{~s}$ & 240 & $9.00 \mathrm{~nm}$ & & & $5.7 \mathrm{mb}$ & $1 T D D$ & 80.87 & 278 & ¿Pd & 33 \\
\hline DLE & 77.57 & 336 & iPc & 32 & 46.00 & 0. 1 & I ATA & 80.88 & 277 & iPd & 33 \\
\hline & $0.8 \mathrm{~s}$ & 320 & $0.00 \mathrm{~nm}$ & & & $.8 \mathrm{mb}$ & I LRG & 80.88 & 323 & eP & 33 \\
\hline FRI & 77.64 & 51 & iPC & 32 & 47.90 & 1.3 & | LMR & 80.91 & 323 & iPd & 33 \\
\hline & & & $\mathrm{pP}$ & 34 & 44.00 & $541 \mathrm{~km}$ & I MFF & 81.03 & 328 & iPd & 33 \\
\hline & & & SP & 35 & 59.00 & & 1 & & & PP & 35 \\
\hline PRI & 77.68 & 52 & iPc & 32 & 49.10 & 2.1 & I ARO & 81.10 & 277 & iPd & 33 \\
\hline & & & $p P$ & 34 & 45.00 & $546 \mathrm{~km}$ & 1 & & & $\mathrm{i}$ & 35 \\
\hline & & & $\mathbf{S P}$ & 36 & 00.00 & & 1100 & 81.11 & 169 & eP & 33 \\
\hline$D C N$ & 77.74 & 336 & $i P c$ & 32 & 47.20 & 0.3 & 1 & & & e & 35 \\
\hline & $0.7 \mathrm{~s}$ & 370 & $0.00 \mathrm{~nm}$ & & & $5.9 \mathrm{mb}$ & I DAF & 81.28 & 278 & iPd & 33 \\
\hline$A D E$ & 77.77 & 174 & Pd & 32 & 48.70 & 1.5 & I SGH & 81.31 & 277 & iPd & 33 \\
\hline NEC & 77.83 & 325 & $\mathbf{P}$ & 32 & 46.50 & -1.0 & $1 \mathrm{KSU}$ & 81.40 & 278 & $i P d$ & 33 \\
\hline EUR & 77.93 & 47 & iP & 32 & 50.00 & 1.5 & I MEI & 81.40 & 314 & $P$ & 33 \\
\hline $\mathrm{PCN}$ & 78.15 & 322 & $P$ & 32 & 50.50 & 1.3 & I RJF & 81.45 & 327 & eP & 33 \\
\hline BEI & 78.17 & 43 & iP & 32 & 51.10 & 1.4 & I CAF & 81.53 & 326 & iPd & 33 \\
\hline TNP & 78.26 & 49 & iP & 32 & 52.00 & 1.8 & 1 & & & $P P$ & 35 \\
\hline$D \mid x$ & 78.36 & 324 & $\mathbf{P}$ & 32 & 50.00 & -0.6 & I NUE & 81.97 & 125 & $P$ & 33 \\
\hline PRG & 78.36 & 319 & $\mathbf{P}$ & 32 & 53.00 & 2.6 & 1 LFF & 82.06 & 327 & eP & 33 \\
\hline & & & is & 41 & 59.00 & & I ERC & 82.09 & 316 & $P$ & 33 \\
\hline YOU & 78.37 & 166 & $P C$ & 32 & 52.20 & 1.9 & 1 LPO & 82.09 & 327 & iPd & 33 \\
\hline & & & epP & 33 & 26.20 & $136 \mathrm{kmx}$ & 1 GCA & 82.11 & 46 & eP & 33 \\
\hline & & & ePP & 33 & 48.20 & & 1 & & & ePP & 35 \\
\hline & & & ePCP & 35 & 51.30 & & I RMU & 82.36 & 46 & ip & 33 \\
\hline PRT & 78.39 & 320 & $P$ & 32 & 50.00 & -0.5 & 1 & & & ePP & 35 \\
\hline & & & $i p P$ & 33 & 18.50 & $111 \mathrm{kmX}$ & 1 & & & $\epsilon$ & 02 \\
\hline & & & is & 42 & 02.50 & & I GOL & 83.15 & 40 & iPc & 33 \\
\hline & & & iss & 44 & 08.00 & & 1 & & & ePP & 35 \\
\hline$F \mid R$ & 78.39 & 320 & $P c$ & 32 & 50.50 & 0.0 & 1 & & & eSP & 36 \\
\hline & & & $s$ & 42 & 00.00 & & 1 & & & $\epsilon$ & 36 \\
\hline DU 1 & 78.42 & 317 & $P-$ & 32 & 51.20 & 0.5 & 1 & & & $\epsilon$ & 02 \\
\hline & & & e & 34 & 48.00 & $544 \mathrm{~km}$ & I GLD & 83.18 & 40 & ip & 33 \\
\hline & & & $e$ & 36 & 01.00 & & 1 & $1.0 \mathrm{~s}$ & 838 & $8.00 \mathrm{~nm}$ & \\
\hline & & & es & 42 & 10.00 & & 1 & & & ePP & 35 \\
\hline$A O U$ & 78.43 & 318 & $\mathrm{P}$ & 32 & 51.50 & 0.7 & I GLA & 83.24 & 51 & iP & 33 \\
\hline & & & $\mathrm{i}$ & 34 & 49.00 & $548 \mathrm{~km}$ & 1 & & & ePP & 35 \\
\hline & & & $\epsilon$ & 42 & 46.50 & & I MLS & 83.55 & 326 & eP & 33 \\
\hline EСB & 78.44 & 335 & $e^{P}$ & 32 & 50.80 & 0.2 & I & & & i & 33 \\
\hline & $1.0 \mathrm{~s}$ & 45 & $3.00 \mathrm{~nm}$ & & & $.9 \mathrm{mb}$ & I & & & es & 42 \\
\hline$E C P$ & 78.47 & 335 & eP & 32 & 50.00 & -0.7 & I EPF & 83.80 & 326 & iPd & 33 \\
\hline & $0.8 \mathrm{~s}$ & 25 & $.00 \mathrm{~nm}$ & & & $1.7 \mathrm{mb} \times$ & 1 & & & $P P$ & 35 \\
\hline$B \vee T$ & 78.54 & 321 & $\mathbf{P}$ & 32 & 50.50 & -0.7 & I MBZ & 84.11 & 316 & eP & 33 \\
\hline MNS & 78.76 & 318 & $P_{-}$ & 32 & 52.20 & -0.2 & I KCHT & 84.14 & 317 & eP & 33 \\
\hline & & & ePP & 35 & 46.00 & & I ZGN & 84.63 & 316 & eP & 33 \\
\hline & & & $e$ & 42 & 08.00 & & I LGR & 85.42 & 328 & iPc & 33 \\
\hline BDW & 78.77 & 41 & $i P c$ & 32 & 53.80 & 1.0 & I & & & is & 43 \\
\hline & & & ePP & 34 & 49.30 & & I EBR & 85.57 & 325 & eP & 33 \\
\hline & & & eSP & 35 & 45.60 & & I & & & e & 43 \\
\hline & & & $e$ & 37 & 35.60 & & I & & & es & 43 \\
\hline & & & e & 42 & 10.00 & & I TUC & 86.06 & 49 & iPc & 33 \\
\hline & & & e & 02 & 19.30 & & I & & & ePP & 35 \\
\hline CSI & 78.87 & 315 & $P$ & 32 & 53.80 & 0.7 & 1 & & & $\epsilon$ & 37 \\
\hline ROI & 78.88 & 314 & $P$ & 32 & 54.40 & 1.2 & I ALO & 86.34 & 44 & ePc & 33 \\
\hline LOR & 78.90 & 327 & iPd & 32 & 53.00 & -0.2 & I & $1.5 \mathrm{~s}$ & 375 & $5.00 \mathrm{~nm}$ & \\
\hline & & & $P P$ & 34 & 50.50 & & 1 & & & ePP & 35 \\
\hline DUG & 79.08 & 45 & iP & 32 & 56.00 & 1.6 & I TAL & 86.61 & 168 & $e^{P}$ & 33 \\
\hline LBF & 79.08 & 326 & eP & 32 & 53.80 & -0.3 & 1 & & & e & 35 \\
\hline RMP & 79.18 & 318 & $P+$ & 32 & 55.50 & 0.8 & 1 & & & $\epsilon$ & 35 \\
\hline & & & ePP & 35 & 51.00 & & $1 \mathrm{TOL}$ & 88.23 & 327 & i Pd & 33 \\
\hline & & & is & 42 & 12.00 & & 1 & $1.2 \mathrm{~s}$ & & $3.00 \mathrm{~nm}$ & \\
\hline SSF & 79.21 & 327 & iPd & 32 & 54.80 & 0.1 & 1 & & & $i(P P)$ & 34 \\
\hline PNI & 79.31 & 323 & $P$ & 32 & 54.50 & -0.8 & 1 & & & $\mathrm{i}$ & 35 \\
\hline$F L N$ & 79.36 & 330 & iPd & 32 & 55.20 & -0.3 & 1 & & & iSKS & 43 \\
\hline SSC & 79.37 & 330 & iPd & 32 & 55.00 & -0.5 & 1 & & & is & 43 \\
\hline SMF & 79.41 & 326 & iPd & 32 & 55.60 & -0.2 & 1 & & & $i(P S)$ & 47 \\
\hline$A \vee F$ & 79.49 & 327 & iPd & 32 & 56.20 & 0.0 & 1 & & & ess & 49 \\
\hline CAN & 79.50 & 165 & $\mathrm{Pc}$ & 32 & 58.20 & 2.0 & 1 & & & eSSS & 52 \\
\hline & & & ePP & 33 & 39.20 & & $\mathrm{ACO}$ & 88.47 & 38 & iPC & 33 \\
\hline & & & ¿PCP & 35 & 59.40 & & 1 & $1.8 \mathrm{~s}$ & 3440 & $9.00 \mathrm{~nm}$ & \\
\hline
\end{tabular}


IPKKP 5035.00

CIR 110.92260 ePdiff35 30.00

CIR $110.92 \quad 260$ ePKP 3920.00

$\begin{array}{lll}\text { EPP } & 40 & 03.00\end{array}$

IPKKP $50 \quad 30.00$

LPS $112.11 \quad 43$ ePKP 3855.00

BUL 112.53263 ePdiff35 32.00

BUL 112.53263 ePKP 3923.00 $\begin{array}{lll}\text { IPP } & 40 & 14.00 \\ \text { iPKKP } & 50 & 23.00\end{array}$

$\begin{array}{lllll} & & \text { IPKKP } & 50 & 23.00 \\ \text { KDS } \quad 115.47 \quad 320 \text { ePKP } 39 & 26.00\end{array}$

SLR $\quad 116.20 \quad 258 \quad$ IPKPC $39 \quad 27.80$ $0.7 \mathrm{~s} \quad 37.00 \mathrm{~nm}$

116.80309 PKP 4211.40

KSR $117.28 \quad 259$ ePKP $39 \quad 30.40$ $0.5 \mathrm{~s} \quad 16.90 \mathrm{~nm}$

S 5901.70

MAW 121.49205 ePKP $39 \quad 39.00$

SBA $122.40 \quad 172$ IPKP 3939.40

$\begin{array}{llllll} & & & \text { i } & 39 & 42.00 \\ \text { WIN } \quad 122.46 & 268 & \text { ePKP } & 39 & 41.50\end{array}$

TOV $124.18 \quad 25$ ePKP $39 \quad 43.30$

$\begin{array}{llllll}\text { CAR } & 124.34 & 22 & \text { EPKPC } & 39 & 49.00\end{array}$

$\begin{array}{cc}124.34 & 22 \text { ePKPC } \\ 0.7 \mathrm{~s} & 63.00 \mathrm{~nm}\end{array}$

SOV $124.75 \quad 27$ ePKPC $39 \quad 45.50$

$0.5 \mathrm{~s} \quad 115.00 \mathrm{~nm}$

IRN $\quad \begin{array}{llllll}0.5 .49 & 15 & \text { iPKPC } & 39 & 45.70\end{array}$

$0.8 \mathrm{~s} \quad 72.00 \mathrm{~nm}$

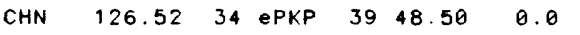

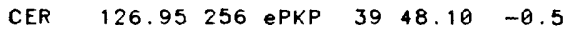

$0.6 \mathrm{~s} \quad 61.30 \mathrm{~nm}$

BOG $\quad 127.45 \quad 32$ ePKP $39 \begin{array}{lll}41.00 \\ \text { ePP }\end{array}$

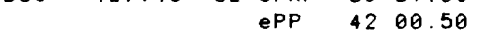

BOCO $127.49 \quad 32$ ePKP 3949.50

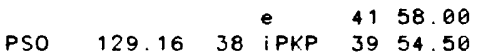

$\begin{array}{llllll}\text { SPA } & 132.68 & 180 & \text { ePKPd } 39 & 54.00\end{array}$

$1.0 \mathrm{~s} \quad 150.00 \mathrm{~nm}$

$\begin{array}{lll}e & 42 \quad 34.00\end{array}$

NVL $\quad 139.40206$ PKPd $40 \quad 03.00$

NNA $\quad 140.76 \quad 47$ iPKPd $40 \quad 09.30$ $0.8 \mathrm{~s} \quad 97.80 \mathrm{~nm}$ ES 4257.00

HUA $141.60 \quad 45$ PKP 4014.00 $\begin{array}{lll}\text { eP } & 40 & 44.00 \\ \text { eP } & 43 & 04.00\end{array}$

$8.3 \times$
1.8

TE

\begin{tabular}{l|l}
$3.0 \times$ & RDJ \\
1.6 & AVE
\end{tabular}

$-1.0$

IFR

-0.5 FDA5

FDA $\quad 37.93226 \mathrm{P}$

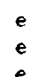

\begin{tabular}{r|ll}
-0.9 & SFS & $39.97 \quad 20$
\end{tabular}

$\begin{array}{lll}i & 18 & 11.00 \\ i P P & 19 & 13.00\end{array}$

i $\quad 2010.00$

is $23 \quad 32.00$

$\begin{array}{lll}\text { iSS } & 26 \quad 35.00 \\ \text { iSSS } & 29 & 10.00\end{array}$

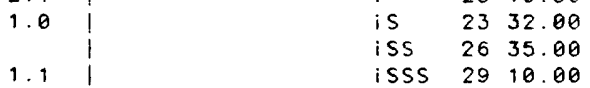

\begin{tabular}{r|l}
1.1 & \\
-0.5 & BNG
\end{tabular}

$\begin{array}{ll}40.74 & 82 \mathrm{iPc} \\ 0.9 \mathrm{~s} & 138.00 \mathrm{~nm}\end{array}$

1734.70

$\begin{array}{ll}0.9 \mathrm{~s} & 138.00 \mathrm{~nm} \\ 40.84 & 22 \mathrm{iPc}\end{array}$

$0.8 \mathrm{~s} \quad 6.50 \mathrm{~nm}$

$41.17 \quad 15 \mathrm{P}$

LIS

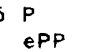

1734.50

$.00^{4}$

eS $\quad 2354.00$

eSS 2624.00

$\begin{array}{llll} & \text { LR } & 2900.00 \\ 41.55 & 22 \mathrm{iPC} & 1741.50\end{array}$

CRT

ALM

0.5

$-1.1$

$-4.6 x$

I

$7.8 \times \mid$ FDF

PRL

$-3.0 \times$ | COI

PAG
MTE

$\begin{array}{lllll} & & 141.77 \quad 47 & \text { IPKPO } 40 & 12.30\end{array}$ eS $40 \quad 42.80$

$\begin{array}{lllllll}\text { PTO3 } & 142.98 & 47 & \text { IPKPC } & 40 & 16.60\end{array}$

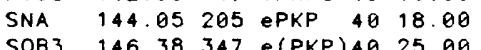

$\begin{array}{llllll}146.38 & 347 & \text { e(PKP) } 4 \theta \quad 25.00 \\ & & \end{array}$

ARE $\quad 147.29 \quad 43$ IPKPd $40 \quad 27.50$

$\begin{array}{llllll}2 O B O & 148.74 & 38 & \text { PKP } & 42 & 37.00 \\ & & 40 & 29.2 \theta\end{array}$

LPB $\quad 148.98 \quad 38$ PKPC $40 \quad 30.20$

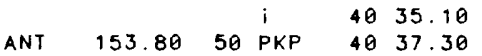

$\begin{array}{lll} & i & 40 \quad 45.20\end{array}$

$\begin{array}{lrrrrr}\text { RDJ } & 159.55 & 345 \text { PKP } & 4 \theta & 40.00 \\ \text { CYA } & 160.08 & 49 \text { PKPC } & 40 & 43.00\end{array}$

$\begin{array}{llllll}C Y A & 160.08 & 49 & \text { PKPC } & 40 & 43.00 \\ & & & 5 & 41 & 28.00\end{array}$

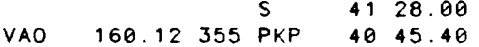

$\begin{array}{llllll} & & \text { e } & 41 & 28.50 \\ \text { PEL } & 160.22 & 68 & \text { PKP } & 40 & 43.00\end{array}$

$\begin{array}{ll}40 & 43.00 \\ 41 & 28.90\end{array}$

4238.50

$\begin{array}{llllll}\text { CFA } & 160.88 & 60 & \mathrm{PKPC} & 40 & 43.10 \\ \text { ICA } & 162.94 & 53 \mathrm{PKPC} & 40 & 45.80\end{array}$

FDA5 163.17 8 PKP $40 \quad 47.70$

4051.00

e $\quad 4141.60$

$-4.4 \times \mid$ CUM

PTO

-2.2 TOL

$-0.9$

\begin{tabular}{l|l}
0.5 & ALI \\
\hline CAR
\end{tabular}

$\begin{array}{l:l}1.3 & \text { CAR } \\ 0.4 & \text { EBR }\end{array}$

1.2 LGR

\begin{tabular}{l|l}
2.1 & SJG
\end{tabular}

\begin{tabular}{r|r}
-2.3 & \\
0.2 & ZGN
\end{tabular}

2.4

LPA

0.2 BAA

ZOBO

-0.4 LPB

\begin{tabular}{l|l}
0.2 \\
1.7
\end{tabular}

I EPF

MLS

Tov

S.D. $=1.2$ on 572 of 611 obs

JAN $03,1982 \quad 14 \mathrm{~h} 09 \mathrm{~m} 50.55 \pm 0.09 \mathrm{~s}$ SDV

$0.945 \mathrm{~S} \pm 2.2 \mathrm{~km} \quad 21.877 \mathrm{~W} \pm 1.8 \mathrm{~km}$

DEPTH $=10.0 \mathrm{~km}$ (geophysicist)

$5.8 \mathrm{mb}$ ( 64 obs.) 6.5Msz ( 12 obs.)

CENTRAL MID-ATLANTIC RIDGE

$\begin{array}{lrrlrrr:l} & & & & & & \\ \text { MBO } & 16.00 & 18 & \mathrm{P} & 13 & 32.40 & -5.0 \times & \text { UAV } \\ \text { KDS } & 16.50 & 35 & \text { iP } & 14 & 41.70 & -2.1 & \text { TUH } \\ \text { KIC } & 18.58 & 67 & \text { iP } & 1409.50 & -0.3 & \text { LGN } \\ \text { SOB3 } & 21.18 & 245 \mathrm{eP} & 1438.40 & -0.3 & \text { CAF }\end{array}$

1744.60 $\begin{array}{lll}41.70 & 24 \text { iPd } \\ 2.0 \mathrm{~s} & 2.20 \mathrm{~nm}\end{array}$

$2.20 \mathrm{~nm}$
$5.60 \mathrm{um}$

E $11 \mathrm{~s} 22.50 \mathrm{um}$

$\begin{array}{lll}i & 19 & 26.30\end{array}$

IPP $20 \quad 27.10$

IPPP $22 \quad 37.20$

i

$\begin{array}{ll}22 & 37.20 \\ 27 & 19.10 \\ 30 & 40.10\end{array}$

41.86293 is

3040.10

42.01283 iPd

$42.22 \quad 17 \mathrm{P}$

$42.41294 \mathrm{eP}$

$42.76 \quad 15 \mathrm{P}$

$42.76295 \mathrm{eP}$

$43.1816 \mathrm{Pt}$

43.57286 iPd

$43.59 \quad 15 \mathrm{Pt}$

$43.7820 \mathrm{PC}$

IPP

$43.79 \quad 24 \mathrm{iPO}$

$46.23 \quad 285 \mathrm{iPc}$

$0.8 \mathrm{~s} \quad 98.50 \mathrm{~nm}$

$\begin{array}{ll}17 & 44.70 \\ 17 & 45.20\end{array}$

1748.00

1751.30

1750.00

1755.10

1756.50

1807.60

1751.50

1956.00

$46.2924 \mathrm{eP}$

1809.00

$5.9 \mathrm{mb}$ । KSR

eS $\quad 251100$

(PS) $25 \quad 1700$

$46.6120 \mathrm{IPC} \quad 18 \quad 23.00$

47.48296 iP

$\begin{array}{lll}19 & 43.00\end{array}$

$\begin{array}{rl}47.48296 & 1827.50 \\ e & 2526.00\end{array}$

$47.57 \quad 35$ iP

$47.65221 \mathrm{PC}$

$\begin{array}{ll}25 & 26.00 \\ 18 & 28.70\end{array}$

1834.00

2033.00

$47.82222 \mathrm{PC}$

$48.03249 \mathrm{P}$

$48.07249 \mathrm{PC}$

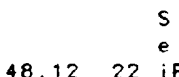

$\begin{array}{rrrr}48.34 & 23 & i P C \\ 48.87 & 284 & i P C\end{array}$

$0.9 \mathrm{~s} 100.00 \mathrm{~nm}$

49.54282 Pam $5.9 \mathrm{mb}$ | DUI

$0.7 \mathrm{~s} \quad 57.10 \mathrm{~nm}$

$49.8521 \mathrm{iPC}$

$1.1 \mathrm{~s} \quad 150.00 \mathrm{~nm}$

$\begin{array}{ll}49.91 & 21 \mathrm{iPC} \\ 1.1 \mathrm{~s} & 118 \mathrm{POnm}\end{array}$

$1.1 \mathrm{~s} \quad 118.00 \mathrm{~nm}$
$50.00282 \mathrm{eP}$

$1.0 \mathrm{~s} \quad 274.00 \mathrm{~nm}$

$50.16134 \mathrm{iPd}$

$\begin{array}{rrl}50.37 & 284 & \mathrm{eP} \\ 50.38 & 22 & \mathrm{iPC}\end{array}$

2516.00

1832.60

$2527.4 \theta$

$\begin{array}{llll}18 & 32.09 & -1.0\end{array}$

$18 \quad 33.50$

2534.00

$\begin{array}{ll}34 & 10.00\end{array}$

1834.90

1835.60

1840.0

\begin{tabular}{cc|c}
18 & $44.70 \quad 0.5$ & BLF \\
$5.7 \mathrm{mb}$ & PCN
\end{tabular} $1846.70^{5.7 \mathrm{mb}} 0.8$

$5.9 \mathrm{mb}$ | MMK

\begin{tabular}{lll|l}
18 & $47.40 \quad 9.1$ & CEN
\end{tabular} \begin{tabular}{ll|l|l}
18 & $48.90^{5.8 \mathrm{mb}}$ & $\mathrm{CHN}$ \\
1.1 & NEC
\end{tabular} $6.2 \mathrm{mb}$ | FOG \begin{tabular}{lll|l}
18 & 46.00 & -2.4 & H HUA
\end{tabular} \begin{tabular}{lll|l}
18 & 52.00 & 1.7 & SLR \\
18 & 50.50 & 0.5 &
\end{tabular}
I SLR

$\begin{array}{llllll}50.42 & 229 & \mathrm{ePC} & 18 & 49.80 & -0.8\end{array}$ $\begin{array}{cccccc}50.50 & 21 \mathrm{iPc} & 18 & 51.30 & 0.4 \\ 1.0 \mathrm{~s} & 56.60 \mathrm{~nm} & & 5.5 \mathrm{mb}\end{array}$

$\begin{array}{lllll}50.85 & 26 \mathrm{Pc} & 18 & 54.00 & 0.5\end{array}$

$\begin{array}{llllll}50.90 & 27 \quad \mathrm{PC} & 18 & 54.30 & 0.4\end{array}$

$0.8 \mathrm{~s} \quad 70.40 \mathrm{~nm} \quad 5.6 \mathrm{mb}$

$\begin{array}{llllll}50.93 \quad 26 \text { iPC } 18 \quad 54.70 & 0.6\end{array}$

$1.0 \mathrm{~s} \quad 80.00 \mathrm{~nm} \quad 5.6 \mathrm{mb}$

$\begin{array}{lllll}50.95 & 37 \mathrm{P} & 18 & 57.00 & 2.5\end{array}$

$\begin{array}{llllll}51.06 & 19 \mathrm{iPc} & 19 & 55.00 & \\ 0.48 & 0.4\end{array}$

$0 . \mathrm{Bs} \quad 81.80 \mathrm{~nm} \quad 5.7 \mathrm{mb}$

$\begin{array}{llllll}51.11 & 38 \mathrm{P} & 18 & 56.00 & 0.5 \\ 51.14 & 26 & \mathrm{iPC} & 18 & 56.00 & 0.3\end{array}$

$1.0 \mathrm{~s} \quad 31.00 \mathrm{~nm} \quad 5.2 \mathrm{mb}$

$\begin{array}{lllll}51.23 & 250 \text { eP } & 18 & 55.00 & -2.2\end{array}$

$\begin{array}{llllll}51.32 & 21 \mathrm{ES} & 26 & 20.00 & \\ \mathrm{iPC} & 18 & 57.50 & 0.4\end{array}$

$1.0 \mathrm{~s} \quad 114.00 \mathrm{~nm} \quad 5.8 \mathrm{mb}$

$\begin{array}{llllll}51.60 & 21 & \text { iPc } & 18 & 59.70 & 0.5\end{array}$

$\begin{array}{lll}0.8 \mathrm{~s} & 37.20 \mathrm{~nm} & 5.4 \mathrm{mb} \\ 51.64 & 24 \mathrm{PC} & 1900.00^{0.5}\end{array}$

$\begin{array}{llllll}51.67 & 22 & \text { iPc } & 19 & 00.40 & 0.7\end{array}$

$1.0 \mathrm{~s} \quad 80.40 \mathrm{~nm} \quad 5.6 \mathrm{mb}$

$\begin{array}{lllll}51.98 & 26 P & P & 02.70 & 0.5\end{array}$

$\begin{array}{lllll}52.04 \quad 18 \mathrm{iPc} & 19 & 02.50 & 0.0\end{array}$

$1.0 \mathrm{~s} \quad 40.00 \mathrm{~nm} \quad 5.3 \mathrm{mb}$

$\begin{array}{lllll}52.17 & 240 \mathrm{Pc} & 19 & 03.50 & -0.3\end{array}$

$52.27 \quad 27$ is $\quad 2628.00$

$\begin{array}{lll}19 & 04.50 & 0.2\end{array}$

$\begin{array}{lllll}52.42 & 18 \mathrm{eP} & 19 & 05.00 & -0.3\end{array}$

$1.0 \mathrm{~s} \quad 71.20 \mathrm{~nm} \quad 5.6 \mathrm{mb}$

$\begin{array}{llllll}52.43 & 277 & \text { iP } & 19 & 06.00 & -0.4\end{array}$

$\begin{array}{llllll}52.44 & 22 & \text { IPC } & 19 & 06.10 & 0.6\end{array}$

$1.0 \mathrm{~s} \quad 62.00 \mathrm{~nm} \quad 5.5 \mathrm{mb}$

$\begin{array}{llllll}52.50 & 22 & \mathrm{iPC} & 19 & 06.60 & 0.6\end{array}$

$1.0 \mathrm{~s} \quad 51.20 \mathrm{~nm} \quad 5.4 \mathrm{mb}$

$\begin{array}{lllll}52.51 & 26 P & 19 & 05.80 & -0.3\end{array}$

$\begin{array}{lllll}52.73 & 22 \mathrm{IPC} & 19 & 08.10 & 0.4\end{array}$

$\begin{array}{lllll}52.80 & 32 \mathrm{Pt} & 1909.00 & 0.7\end{array}$

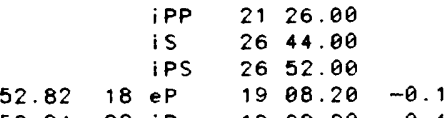

$\begin{array}{llllll}52.84 & 22 & \text { IPC } & 19 & 08.90 & 0.4\end{array}$

$1.0 \mathrm{~s} \quad 111.00 \mathrm{~nm} \quad 5.7 \mathrm{mb}$

$\begin{array}{llllll}52.86 & 18 & \mathrm{iPC} & 19 & 08.40 & -0.2\end{array}$

$1.0 \mathrm{~s} \quad 41.80 \mathrm{~nm} \quad 5.3 \mathrm{mb}$

$\begin{array}{lllll}52.89 & 115 \mathrm{P}- & 19 & 10.00 & 0.5\end{array}$

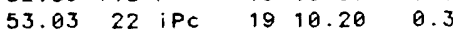

$1.0 \mathrm{~s} \quad 97.60 \mathrm{~nm} \quad 5.7 \mathrm{mb}$

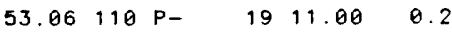

$\begin{array}{llllll}53.07 \quad 122 & \text { iPd } 19 & 09.40 & -1.4\end{array}$

$0.9 \mathrm{~s} \quad 84.00 \mathrm{~nm} \quad 5.7 \mathrm{mb}$

$\begin{array}{lllll}53.20 & 32 \mathrm{Pt} & 19 & 11.00 & -0.2\end{array}$

$\begin{array}{lllll}53.26 & 25 \mathrm{Pc} & 19 & 11.50 & -0.3\end{array}$

$\begin{array}{lllll}53.32 & 230 \mathrm{ePC} & 19 & 11.50 & -0.9\end{array}$

$\begin{array}{llllll}53.46 & 36 \mathrm{P} & 19 & 13.80 & 0.6\end{array}$

$\begin{array}{lllll}53.47 & 29 \mathrm{P} & 19 & 18.00 & 4.9 \mathrm{X}\end{array}$

ipP $\quad 1942.00$

IPP $\quad 21 \quad 35.00$

$\begin{array}{lllll} & e S & 27 & 28.50 & \\ 53.47 & 25 \mathrm{PC} & 19 & 13.50 & 0.0\end{array}$

$\begin{array}{lllll}53.4730 P & 19 & 13.00 & -0.1\end{array}$

PP 2118.00

$\begin{array}{llllll}53.50 & 37 \mathrm{P} & 26 & 46.00 & \\ & & 19 & 13.20 & -0.3\end{array}$

$\begin{array}{lllll}53.56 & 32 \mathrm{P}+ & 19 & 14.50 & 0.6\end{array}$

$\begin{array}{lllll}53.56 & 31 & \text { P- } & 1914.00 & 0.2\end{array}$

$\begin{array}{rrrrr}53.60 & 9 \text { IPP } & 21 & 00.00 & \\ & \text { PPd } & 19 & 32.00 & 18.1 x\end{array}$

$\begin{array}{lll}\text { S } & 26 & 49.00 \\ \text { SS } & 30 & 30.00\end{array}$

LO 3156.00

$\begin{array}{llllll}53.6533 P & 19 & 14.50 & 0.0\end{array}$

$\begin{array}{lllll}53.65 & 126 & \text { IPd } \quad 19 & 15.10\end{array}$

$\begin{array}{lllll}53.67 & 27 \mathrm{P} & 19 & 16.50 & 2.0\end{array}$

$\begin{array}{lllll}53.68 & 25 \mathrm{PC} & 27 & 00.00 & 19 \\ 53.00 & 0.0\end{array}$

$\begin{array}{lllll}53.71 & 231 \mathrm{eP} & 19 & 15.00 & -0.3\end{array}$

$\begin{array}{llllll}54.00 & 277 & \text { eP } & 19 & 17.00 & -0.7\end{array}$

$\begin{array}{llllll}54.02 & 24 \mathrm{P} & 19 & 16.50 & -0.7\end{array}$

$\begin{array}{llllll}54.12 & 34 \mathrm{P} & 19 & 29.50 & 11.6 \mathrm{X}\end{array}$

$\begin{array}{lllll}54.13 \quad 256 \text { eP } & 19 & 20.00 & 1.0\end{array}$ 
$63 d 14 h$

\begin{tabular}{|c|c|c|c|c|c|c|c|c|c|c|c|}
\hline SAL & 54.48 & 28 & $P+$ & 19 & 19.50 & -1.1 & 1 & $1.0 \mathrm{~s}$ & & $5.00 \mathrm{~nm}$ & \\
\hline HAU & 54.59 & 23 & $i P_{C}$ & 19 & 21.40 & 0.0 & $i$ & $18 \mathrm{~s}$ & & 1. $40 \mathrm{um}$ & \\
\hline & $0.8 \mathrm{~s}$ & 192 & $2.00 \mathrm{~nm}$ & & & $.2 \mathrm{mb}$ & 1 & & & e & 19 \\
\hline BES & 54.61 & 24 & $P$ & 19 & 21.00 & -0.6 & I GRG & 57.89 & 39 & $P$ & 19 \\
\hline BSF & 54.62 & 23 & $i P_{C}$ & 19 & 21.50 & -0.2 & I KMR & 57.96 & 28 & iPc & 20 \\
\hline & $0.8 \mathrm{~s}$ & 130 & $3.00 \mathrm{~nm}$ & & & $.0 \mathrm{mb}$ & i WTS & 58.03 & 20 & iPC & 19 \\
\hline BAF & 54.70 & 23 & iPc & 19 & 21.80 & -0.5 & $i$ & $1.0 \mathrm{~s}$ & 159 & $9.00 \mathrm{~nm}$ & \\
\hline TLL & 54.75 & 233 & $P$ & 19 & 22.20 & -1.0 & I THE & 58.07 & 39 & $P$ & 19 \\
\hline LLS & 54.77 & 26 & $P$ & 19 & 21.80 & -1.1 & I ESK & 58.13 & 12 & eP & 19 \\
\hline PTQ3 & 54.86 & 254 & eP & 19 & 10.90 & $-13.0 x$ & I EKA & 58.15 & 12 & $P$ & 19 \\
\hline RFA & 54.90 & 227 & ePc & 19 & 19.60 & $-4.4 x$ & i & $1.2 \mathrm{~s}$ & 70 & $0.60 \mathrm{~nm}$ & \\
\hline MTD & 54.90 & 110 & P- & 19 & 23.00 & -1.3 & | WET & 58.19 & 26 & $i P c$ & 19 \\
\hline ZUL & 54.99 & 25 & $\mathrm{PC}$ & 19 & 23.50 & -0.9 & 1 & $1.4 \mathrm{~s}$ & 207 & $7.00 \mathrm{~nm}$ & \\
\hline $\mathrm{ECH}$ & 55.07 & 23 & $P+$ & 19 & 24.40 & -0.6 & I VAY & 58.21 & 38 & eP & 19 \\
\hline & & & ePcP & 20 & 35.00 & & I SOH & 58.42 & 39 & $P$ & 19 \\
\hline & & & ePP & 21 & 26.00 & & i $\mathrm{KHC}$ & 58.49 & 27 & iPc & 19 \\
\hline & & & is & 27 & 05.00 & & $i$ & $1.0 \mathrm{~s}$ & & $8.00 \mathrm{~nm}$ & \\
\hline$C D F$ & 55.28 & 23 & $i P_{c}$ & 19 & 26.30 & -0.2 & i & $12 \mathrm{~s}$ & 14 & $4.40 u m$ & \\
\hline & $0.8 \mathrm{~s}$ & 92 & $.60 \mathrm{~nm}$ & & 5 & $.9 \mathrm{mb}$ & i & $13 s$ & 12 & $2.40 \mathrm{um}$ & \\
\hline ST J & 55.31 & 335 & eP & 19 & 31.00 & 4. $4 x$ & i & $12 \mathrm{~s}$ & & $8.50 \mathrm{um}$ & \\
\hline PTO2 & 55.33 & 255 & iPd & 19 & 25.20 & -2.1 & i & & & e & 19 \\
\hline CTI & 55.34 & 28 & $P+$ & 19 & 26.40 & -0.6 & i & & & S & 27 \\
\hline PTOG & 55.35 & 254 & eP & 19 & 26.40 & -1.0 & I OUR & 58.55 & 40 & $P$ & 19 \\
\hline PSO & 55.49 & 272 & iP & 19 & 28.50 & -0.5 & I HOF & 58.64 & 25 & $i P c$ & 19 \\
\hline STR & 55.61 & 23 & $P$ & 19 & 24.00 & $-4.8 x$ & i & & & es & 28 \\
\hline GRM & 55.65 & 131 & eP & 19 & 29.00 & -0.4 & I WIT & 58.66 & 20 & eP & 19 \\
\hline & $1.3 \mathrm{~s}$ & 539 & $.00 \mathrm{~nm}$ & & 6 & $.4 \mathrm{mb}$ & 1 & $1.0 \mathrm{~s}$ & 85 & $5.00 \mathrm{~nm}$ & \\
\hline$O G A$ & 55.66 & 27 & $i P_{c}$ & 19 & 28.70 & -0.7 & I NAI & 58.67 & 91 & eP & 20 \\
\hline & $1.5 \mathrm{~s}$ & 214 & $1.00 \mathrm{~nm}$ & & 6 & $.0 m b$ & i & $2.0 \mathrm{~s}$ & 294 & $4.00 \mathrm{~nm}$ & \\
\hline DOU & 55.67 & 20 & $\mathrm{Pc}$ & 19 & 28.40 & -0.8 & I SRS & 58.73 & 39 & $\mathbf{P}$ & 19 \\
\hline & & & $S$ & 27 & 22.00 & & I MOX & 58.79 & 24 & ¡P & 19 \\
\hline$C I R$ & 55.80 & 115 & $P-$ & 19 & 30.00 & -0.6 & i & & & $i$ & 20 \\
\hline LM2 & 55.82 & 256 & eP & 20 & 29.00 & $58.3 x$ & i & & & e & 23 \\
\hline PEL & 55.85 & 236 & $P+$ & 19 & 30.30 & -0.5 & i & & & $S$ & 28 \\
\hline BUH & 55.87 & 24 & $e P$ & 19 & 30.00 & -0.7 & $i$ & & & SS & 32 \\
\hline WLF & 55.87 & 22 & ePc & 19 & 39.00 & $8.4 x$ & 1 & & & LQ & 34 \\
\hline & & & $S$ & 27 & 26.00 & & i & & & $e$ & 44 \\
\hline SAN & 55.96 & 229 & $P$ & 19 & 32.00 & 0.4 & i & & & e & 47 \\
\hline TR I & 56.10 & 29 & iPc & 19 & 32.40 & 0.1 & I SOP & 58.86 & 29 & $\mathrm{Pc}$ & 19 \\
\hline & & & $e(P P P)$ & 22 & 44.00 & & 1 & & & ipP & 19 \\
\hline & & & $i(s)$ & 27 & 22.00 & & 1 & & & is $P$ & 20 \\
\hline & & & e & 33 & 12.00 & & 1 & & & iPCP & 20 \\
\hline & & & i (SSS) & 34 & 06.00 & & I BEO & 59.03 & 34 & $\mathbf{P}$ & 19 \\
\hline & & & i & 45 & 12.00 & & I CON & 59.15 & 227 & $P$ & 19 \\
\hline GAP & 56.14 & 26 & eP & 19 & 31.90 & -0.7 & IVIE & 59.15 & 29 & P & 20 \\
\hline ucc & 56.19 & 20 & $\mathrm{PC}+$ & 19 & 32.50 & -0.3 & 1 & & & $p P$ & 20 \\
\hline & & & e & 20 & 48.40 & & i & & & $i$ & 21 \\
\hline & & & $\mathrm{S}$ & 27 & 23.00 & & i & & & PPP & 22 \\
\hline STU & 56.34 & 24 & ePc & 19 & 33.00 & -1.0 & 1 & & & S & 27 \\
\hline & 105 & 48 & $3.00 \mathrm{~nm}$ & & & $.5 \mathrm{mb}$ & 1 & & & PS & 28 \\
\hline$z$ & $20 \mathrm{~s}$ & 21 & $1.30 \mathrm{um}$ & & & $.2 \mathrm{Msz}$ & $i$ & & & ScS & 29 \\
\hline$T I R$ & 56.57 & 37 & $P$ & 19 & 36.50 & 0.7 & $i$ & & & SS & 31 \\
\hline MEM & 56.60 & 21 & eP & 19 & 35.00 & -0.8 & i & & & SSS & 34 \\
\hline ENN & 56.70 & 21 & iPc & 19 & 36.80 & 0.2 & 1 & & & $\epsilon$ & 55 \\
\hline & $1.1 \mathrm{~s}$ & 186 & $5.00 \mathrm{~nm}$ & & & $.0 \mathrm{mb}$ & I HLW & 59.21 & 54 & eP & 19 \\
\hline LJU & 56.72 & 30 & iPc & 19 & 36.90 & 0.1 & $\mathrm{ISST}$ & 59.48 & 29 & IP & 19 \\
\hline FUR & 56.75 & 26 & iPc & 19 & 36.50 & -0.5 & I & & & SP & 20 \\
\hline & $1.2 \mathrm{~s}$ & 304 & $+.00 \mathrm{~nm}$ & & & $.2 \mathrm{mb}$ & i & & & i & $2 \theta$ \\
\hline & & & es & 27 & 34.00 & & I PRU & 59.55 & 26 & $\mathrm{Pc}$ & 19 \\
\hline TTG & 56.87 & 36 & $P$ & 19 & 38.30 & 0.4 & I & $2.0 \mathrm{~s}$ & 148 & $8.00 \mathrm{~nm}$ & \\
\hline & & & $S$ & 27 & 35.50 & & i & $13 s$ & & $0.80 \mathrm{um}$ & \\
\hline OHR & 56.99 & 38 & iP & 19 & 38.40 & -0.5 & 1 & $13 s$ & & $9.50 \mathrm{um}$ & \\
\hline & & & $i$ & 19 & 50.50 & & 1 & 145 & & $0.50 u m$ & \\
\hline & & & $\mathrm{i}$ & 20 & 06.80 & & i & & & i & 20 \\
\hline ATH & 57.12 & 42 & $e P_{c}$ & 19 & 40.00 & 0.2 & i & & & is & 28 \\
\hline & & & is & 27 & 40.00 & & I PRA & 59.56 & 26 & IPC & 19 \\
\hline BHG & 57.14 & 27 & $i P_{C}$ & 19 & 38.70 & -1.1 & 1 & & & e & 20 \\
\hline & $0.9 \mathrm{~s}$ & 137 & $7.00 \mathrm{~nm}$ & & & $.8 \mathrm{mb}$ & 1 & & & $S$ & 28 \\
\hline TNS & 57.19 & 23 & iPc & 19 & 39.70 & -0.5 & I SSR & 59.79 & 35 & $P$ & 19 \\
\hline SAR & 57.31 & 34 & $P$ & 19 & 41.20 & 0.1 & I SRO & 59.82 & 30 & eP & 19 \\
\hline & & & $S$ & 27 & 16.70 & & 1 & & & $S$ & 28 \\
\hline BNS & 57.31 & 21 & eP & 19 & 41.10 & 0.2 & 1 & & & e & 52 \\
\hline & & & is & 27 & 44.80 & & I CLL & 59.86 & 25 & $I P C$ & 19 \\
\hline LIT & $57 \quad 47$ & 40 & $P$ & 19 & 41.20 & -1.0 & 1 & & & $\mathrm{i}$ & 20 \\
\hline DBN & 57.56 & 19 & eP & 19 & 50.00 & $7.4 \times$ & i & & & $\mathrm{i}$ & 20 \\
\hline$z$ & $20 s$ & & $.00 \mathrm{um}$ & & & $5 \mathrm{Msz}$ & i & & & i & 21 \\
\hline & & & is & 27 & 44.00 & & i & & & $P P$ & 22 \\
\hline & & & eSS & 31 & 47.00 & & i & & & e & 22 \\
\hline & & & eSSS & 33 & 40.00 & & I & & & S & 28 \\
\hline SKO & 57.89 & 37 & i Pc & 19 & 45.00 & -0.1 & i & & & e & 30 \\
\hline & 1.35 & 190 & $.00 \mathrm{~nm}$ & & & $.0 \mathrm{mb}$ & I & & & $P \cdot P$. & 49 \\
\hline & & & e & 19 & 56.50 & & I YER & 59.86 & 45 & iP & 19 \\
\hline & & & $i$ & 20 & 11.50 & & I BRG & 59.93 & 25 & iPc & 19 \\
\hline & & & iPPP & 23 & 18.50 & & 1 & & & i & 20 \\
\hline & & & is & 27 & 51.00 & & 1 & & & e & 22 \\
\hline FO & 7.89 & 25 & eP & 19 & 44.10 & -0.9 & 1 & & & S & 28 \\
\hline$R F$ & 57.89 & 25 & iPc & 19 & 50 & 0 . & & & & $\mathbf{P}$ & 49 \\
\hline
\end{tabular}




\begin{tabular}{|c|c|c|c|c|c|c|c|c|c|c|c|}
\hline MRG & 66.55 & 314 & ip & 20 & 48.60 & $5.5 x$ & I RLO & & 77.00 & 307 & iPd \\
\hline NB2 2 & 66.83 & 17 & $P$ & 20 & 44.60 & 0.0 & I TEH & & 77.01 & 53 & eP \\
\hline & $1.1 \mathrm{~s}$ & & $5.48 \mathrm{~nm}$ & & & $5.5 \mathrm{mb}$ & GBO & & 77.07 & 307 & ePd \\
\hline HFS & 66.87 & 18 & $P$ & 20 & 44.20 & -0.5 & $111 T$ & & 77.49 & 290 & ip \\
\hline & $1.0 \mathrm{~s}$ & & $7.58 \mathrm{~nm}$ & & & $5.4 \mathrm{mb}$ & TUL & & 77.57 & 307 & iPd \\
\hline & & & e & 42 & 00.00 & & $i$ & $z$ & $22 \mathrm{~s}$ & 23 & 3.20 um \\
\hline$K V T$ & 67.00 & 44 & ip & 20 & 45.30 & -0.7 & i & & & & i \\
\hline$S L L$ & 67.02 & 18 & $P$ & 20 & 45.30 & -0.4 & I DAG & & 77.64 & 1 & iPc \\
\hline APO & 67.26 & 18 & $P$ & 20 & 46.41 & -0.8 & $i$ & & $0.8 \mathrm{~s}$ & & $3.00 \mathrm{~nm}$ \\
\hline SIM & 67.32 & 40 & eP & 20 & 49.00 & 1.1 & i & & & & iPP \\
\hline & & & es & 29 & 39.80 & & $i$ & & & & is \\
\hline UPP & 67.96 & 20 & $P C$ & 20 & 50.90 & -0.7 & i & & & & isSS \\
\hline & & & is & 29 & 54.00 & & I KEV & & 77.67 & 16 & eP \\
\hline MNK & 68.19 & 29 & $P C$ & 20 & 56.00 & 2.9 & i & & $1.1 \mathrm{~s}$ & & $5.28 \mathrm{~nm}$ \\
\hline & & & es & 30 & 00.00 & & 1 & & & & $i$ \\
\hline ORT & 68.52 & 369 & ip & 20 & 55.30 & -0.3 & $i$ & & & & ePPP \\
\hline ANN & 69.35 & 41 & $\mathrm{PC}$ & 28 & 59.00 & -1.4 & I & & & & es \\
\hline & & & es & 29 & 55.00 & & 1 & & & & ess \\
\hline UTO & 69.86 & 315 & ePc & 21 & 04.00 & 0.4 & $111 P$ & & 78.09 & 290 & iP \\
\hline AN 10 & 70.09 & 314 & eP & 21 & 04.60 & -0.5 & $111 \mathrm{M}$ & & 78.34 & 290 & iP \\
\hline AAM & 70.14 & 316 & ip & 21 & 05.50 & 0.2 & I TAC & & 78.36 & 290 & iP \\
\hline AVY & 70.42 & 110 & iPd & 21 & 09.70 & 1.9 & 1110 & & 78.45 & 290 & iP \\
\hline SNA & 70.45 & 173 & $e(P)$ & 21 & 14.00 & $7.3 x$ & 1111 & & 78.53 & 289 & iP \\
\hline SOC & 70.46 & 43 & eP & 21 & 06.00 & -1.3 & $1 \mathrm{JCT}$ & & 80.08 & 301 & iP \\
\hline & & & es & 30 & 18.00 & & i & $z$ & $18 \mathrm{~s}$ & & 2.70 um \\
\hline IN3 & 70.67 & 312 & $e P$ & 21 & 08.30 & -0.3 & I KAT & & 81.41 & 50 & $\mathrm{PC}$ \\
\hline & & & $i$ & 21 & 14.40 & & $F C C$ & & 81.65 & 330 & iPc \\
\hline BHD & 70.84 & 54 & $\mathrm{Pc}$ & 21 & 15.00 & $5.2 x$ & i & & $1.5 \mathrm{~s}$ & 612 & $2.00 \mathrm{~nm}$ \\
\hline & & & $e$ & 21 & 30.00 & & KBS & & 81.68 & 6 & eP \\
\hline & & & ePP & 24 & 00.00 & & LUB & & 82.18 & 304 & eP \\
\hline & & & eSKP & 24 & 41.00 & & I VAN & & 82.67 & 52 & eP \\
\hline & & & ePPP & 26 & 47.00 & & $|T G|$ & & 83.02 & 57 & $e(P)$ \\
\hline & & & eSKKS & 30 & 25.00 & & I MHI & & 83.60 & 53 & $\mathrm{PC}$ \\
\hline & & & ePS & 34 & 17.00 & & i & & & & e \\
\hline NUR & 70.97 & 22 & $\mathrm{PC}$ & 21 & 09.80 & -0.2 & i & & & & is \\
\hline & & & $i$ & 21 & 18.00 & & I FFC & & 85.05 & 325 & eP \\
\hline & & & e & 22 & 16.00 & & i & & $1.2 \mathrm{~s}$ & & $2.00 \mathrm{~nm}$ \\
\hline & & & ePP & 23 & 48.00 & & I ALE & & 85.19 & 355 & eP \\
\hline & & & ePPP & 25 & 40.00 & & i & & $1.5 \mathrm{~s}$ & 110 & $0.00 \mathrm{~nm}$ \\
\hline & & & es & 30 & 27.00 & & I GLD & & 85.47 & 310 & eP \\
\hline & & & eSS & 34 & 28.00 & & $i$ & $z$ & $21 s$ & & $6.80 \mathrm{um}$ \\
\hline$A \mid A$ & 70.99 & 197 & $e(P)$ & 21 & 10.00 & -6.1 & I ARU & & 85.51 & 33 & $\mathrm{PC}$ \\
\hline UME & 71.68 & 18 & $P C$ & 21 & 14.20 & 0.0 & I ANMO & & 86.09 & 305 & eP \\
\hline & & & is & 30 & 46.00 & & I $A<O$ & & 86.69 & 305 & eP \\
\hline LEN & 72.45 & 47 & $\mathrm{Pc}$ & 21 & 20.00 & 0.5 & i & & $1.5 \mathrm{~s}$ & 392 & $2.00 \mathrm{~nm}$ \\
\hline & & & es & 30 & 40.00 & & 1 & $z$ & $22 s$ & & 4. 40 um \\
\hline$B K R$ & 72.50 & 46 & $P C$ & 21 & 19.00 & -0.8 & I RES & & 86.46 & 345 & ePc \\
\hline GRT & 72.60 & 308 & $e(P)$ & 21 & 19.00 & -1.3 & i & & $1.3 \mathrm{~s}$ & 153 & $3.00 \mathrm{~nm}$ \\
\hline ELC & 72.71 & 310 & eP & 21 & 19.60 & -1.3 & I SVE & & 86.68 & 33 & $\mathrm{PC}$ \\
\hline ERE & 72.76 & 47 & $e P$ & 21 & 22.00 & 0.7 & I MAW & & 87.12 & 158 & $\mathrm{eP}$ \\
\hline & & & es & 30 & 38.00 & & I BDW & & 88.94 & 313 & $\mathrm{eP}$ \\
\hline $\mathrm{CHI}$ & 72.81 & 315 & $P$ & 21 & 21.00 & -0.4 & I SPA & & 89.06 & 180 & iPc \\
\hline PYA & 72.89 & 43 & $\mathrm{PC}$ & 21 & 22.30 & B. 4 & $i$ & & $0.9 \mathrm{~s}$ & & $6.80 \mathrm{~nm}$ \\
\hline & & & $\mathrm{S}$ & 30 & 46.40 & & I KHE & & 89.28 & 9 & eP \\
\hline SUF & 72.93 & 21 & $P C$ & 21 & 21.70 & 0.0 & I SAM & & 89.72 & 51 & $\mathrm{Pc}$ \\
\hline NVL & 73.68 & 169 & $\mathrm{PC}$ & 21 & 26.00 & $36 x$ & I & & & & is \\
\hline & & & is & 31 & 09.00 & & I SES & & 90.19 & 320 & $\mathrm{ePc}$ \\
\hline DON & 73.23 & 309 & $e P$ & 21 & 23.20 & -0.7 & $i$ & & $1.3 \mathrm{~s}$ & 111 & $1.00 \mathrm{~nm}$ \\
\hline GDH & 73.26 & 349 & iPd & 21 & 23.50 & 0.0 & I LRM & & 91.07 & 316 & ePc \\
\hline & $0.8 \mathrm{~s}$ & 15 & $5.00 \mathrm{~nm}$ & & 5 & $5.1 \mathrm{mb}$ & DSH & & 91.69 & 52 & $\mathrm{PC}$ \\
\hline & & & is & 30 & 55.00 & & I KAAO & & 91.29 & 56 & $e(P)$ \\
\hline$K E R$ & 73.29 & 54 & $e^{p}$ & 21 & 25.00 & 0.4 & I KBL & & 91.29 & 56 & iPc \\
\hline$O B N$ & 73.35 & 31 & $\mathrm{eP}$ & 21 & 22.00 & -2.2 & I AMM & & 91.42 & 316 & eP \\
\hline & & & $i s P$ & 21 & 24.00 & & I TAS & & 91.50 & 49 & ePc \\
\hline & & & is & 30 & 47.00 & & I EDM & & 91.64 & 323 & ePc \\
\hline TA & 73.41 & 46 & $P$ & 21 & 25.00 & D. 1 & $i$ & & $1.3 \mathrm{~s}$ & 328 & $3.00 \mathrm{~nm}$ \\
\hline$T A B$ & 73.56 & 50 & $\mathrm{eP}$ & 21 & 26.00 & -0.1 & I KUL & & 91.86 & 52 & eP \\
\hline FVM & $\begin{array}{ll}73 & 81\end{array}$ & 310 & ePd & 21 & 26.10 & -1.2 & I YKA & & 92.10 & 332 & $P$ \\
\hline GRS & 73.93 & 48 & $\mathrm{Pc}$ & 21 & 27.60 & -0.6 & I GAR & & 92.30 & 51 & ePc \\
\hline & & & $i s P$ & 21 & 33.60 & & $M B C$ & & 92.70 & 346 & $e P$ \\
\hline MOS & 74.13 & 30 & eP & 21 & 16.00 & $-12.7 x$ & i & & $1.1 \mathrm{~s}$ & 119 & $9.00 \mathrm{~nm}$ \\
\hline KRV & 74.23 & 47 & eP & 21 & 29.00 & -0.7 & I GLA & & 92.99 & 303 & $e(P)$ \\
\hline KJF & 74.40 & 20 & $P$ & 21 & 30.80 & 0.6 & i & & & & $\mathrm{i}$ \\
\hline & & & $i$ & 21 & 43.50 & & I KHO & & 93.28 & 53 & eP \\
\hline & & & es & 31 & 04.00 & & I EUR & & 93.76 & 309 & iP \\
\hline GRO & 74.50 & 44 & $P C$ & 21 & 32.00 & 0.8 & i & & $0.6 \mathrm{~s}$ & & $3.33 \mathrm{~nm}$ \\
\hline & & & is & 31 & 04.00 & & I ANR & & 93.83 & 49 & eP \\
\hline$K \backslash R$ & 74.61 & 15 & $P C$ & 21 & 31.50 & B. 1 & i & & & & es \\
\hline & & & is & 31 & 23.00 & & I GLR & & 93.87 & 307 & $e(P)$ \\
\hline MAK & 5.64 & 45 & eP & 21 & 38.00 & 0.2 & I NEW & & 94.20 & 318 & eP \\
\hline LHC & 5.88 & 321 & ipd & 21 & 41.40 & 2.4 & i & & $20 \mathrm{~s}$ & & $9.00 \mathrm{um}$ \\
\hline & $1.1 \mathrm{~s}$ & 175 & $5.00 \mathrm{~nm}$ & & 6 & $.1 \mathrm{mb}$ & i & $\mathbb{N}$ & $20 \mathrm{~s}$ & & $2.00 \mathrm{um}$ \\
\hline SHE & 75.92 & 48 & $e^{P}$ & 21 & 36.00 & $-3 \cdot 4 x$ & i & $E$ & $20 \mathrm{~s}$ & & 3. $00 \mathrm{um}$ \\
\hline & & & is & 31 & 23.00 & & $B M N$ & & 94.68 & 310 & eP \\
\hline SOD & 76.04 & 17 & $P C$ & 21 & 40.30 & 0.7 & 1 & & $1.0 \mathrm{~s}$ & & $800 \mathrm{~nm}$ \\
\hline & & & $i$ & 21 & 45.10 & & 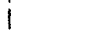 & & & & $e$ \\
\hline 41 & 76.94 & 59 & eP & 21 & 45.00 & -0.6 & FRU & & 95.41 & 47 & eP \\
\hline
\end{tabular}




\begin{tabular}{|c|c|c|c|c|c|c|c|}
\hline \multirow[t]{2}{*}{ WEL } & 135.33 & 198 & $\begin{array}{l}\text { PKP } \\
\text { PP }\end{array}$ & $\begin{array}{l}30 \\
31\end{array}$ & $\begin{array}{l}03.00 \\
51.00\end{array}$ & $50.3 x$ & $\begin{array}{l}\text { BPM } \\
\text { HKM }\end{array}$ \\
\hline & & & ss & & 03.00 & & I MNO \\
\hline ANP & 137.24 & 53 & PKP & 30 & 11.00 & $54.1 x$ & I GNT \\
\hline SHK & 138.99 & 33 & ePKP & 29 & 26.20 & $6.4 x$ & DVT \\
\hline PPR & 139.85 & 76 & ePKP & 29 & 37.50 & $15.7 x$ & WNH \\
\hline$A D E$ & 139.92 & 155 & PKP & 29 & 26.20 & $4.7 x$ & ONH \\
\hline BAG & 139.95 & 65 & $e_{e}^{\text {PKP }}$ & $\begin{array}{l}29 \\
32\end{array}$ & $\begin{array}{l}27.00 \\
18.00\end{array}$ & $4.8 x$ & $\begin{array}{l}\text { MNT } \\
\text { HNH }\end{array}$ \\
\hline \multirow[t]{2}{*}{ MAT } & 140.07 & 25 & EPKP & 29 & \multirow{2}{*}{\multicolumn{2}{|c|}{$\begin{array}{c}10.00-11.7 \mathrm{X} \\
6.6 \mathrm{Msz}\end{array}$}} & GLO \\
\hline & $222 \mathrm{~s}$ & 1 & 1.10 um & & & & PNY \\
\hline DDR & 140.95 & 25 & ePKP & 29 & 34.80 & $11.5 x$ & I MDV \\
\hline$O C P$ & 140.95 & 68 & PKP & 29 & 10.00 & $-13.7 x$ & PNH \\
\hline WAM & 142.06 & 168 & PKP & 29 & 33.50 & $8.3 x$ & I BLVT \\
\hline \multirow[t]{2}{*}{ CAN } & 142.93 & 168 & PKP & 29 & 32.10 & $5.3 x$ & I WFM \\
\hline & & & i & & 38.00 & & I WNY \\
\hline \multirow[t]{2}{*}{100} & 143.79 & 166 & PKP & 29 & 27.50 & -0.8 & WES \\
\hline & & & $i$ & 29 & 34.00 & & I IVT \\
\hline \multirow[t]{2}{*}{ STK } & 143.79 & 156 & ePKP & 29 & 26.00 & -2.3 & DUX \\
\hline & & & e & 29 & 39.00 & & I BGR \\
\hline KUPT & 143.93 & 109 & EPKP & 29 & 29.40 & 0.4 & I RSNY \\
\hline$C C P$ & 144.74 & 73 & EPKP & 29 & 35.70 & $5.4 x$ & OUA \\
\hline CNP & 144.90 & 69 & EPKP & 29 & 39.60 & $9.0 x$ & GAC \\
\hline PLP & 145.56 & 72 & ePKP & 29 & 39.20 & $7.5 x$ & I APH \\
\hline CGP & 145.88 & 76 & ePKPc & 29 & 32.50 & 0.2 & і отт \\
\hline$A S P$ & 146.03 & 138 & PKPC & 29 & 33.70 & 1.3 & I FHO \\
\hline KNA & 146.59 & 121 & ePKP & 29 & 35.00 & 1.6 & HDM \\
\hline MNI & 146.72 & 89 & ePKP & 29 & 35.50 & 1.8 & I ECT \\
\hline \multirow[t]{8}{*}{$A F I$} & 146.73 & 243 & ePKP & 29 & 47.00 & $13.3 x$ & $B C T$ \\
\hline & & & PP & 33 & 40.00 & & $\mathrm{SCH}$ \\
\hline & & & PPP & 36 & 33.00 & & I GPD \\
\hline & & & SKKS & 45 & 14.00 & & INY \\
\hline & & & ss & 52 & 16.00 & & I JAO \\
\hline & & & sss & 57 & 12.00 & & STJ \\
\hline & & & LO & 11 & 00.00 & & I EFO \\
\hline & & & $L R$ & 19 & 00.00 & & NA2 \\
\hline DAV & 146.98 & 78 & PKP & 29 & 43.00 & $8.9 x$ & GHV \\
\hline PPH & 147.45 & 77 & ePKP & 29 & 38.50 & $3.7 x$ & i CVL \\
\hline coo & 148.10 & 170 & ePKP & 29 & 40.00 & $4.5 x$ & BV 4 \\
\hline \multirow[t]{4}{*}{ WB2 } & 148.79 & 133 & PKP & 29 & 37.20 & 0.3 & I AAM \\
\hline & & & e & 29 & 40.00 & & $B L A$ \\
\hline & & & $i$ & 29 & 47.00 & & NAV \\
\hline & & & iPP & 31 & 15.80 & & AN 10 \\
\hline In & 149.92 & 118 & ePKP & 29 & 40.00 & 1.4 & I PUV \\
\hline \multirow[t]{4}{*}{ BRS } & 151.36 & 170 & PKPC & 29 & 59.80 & $19.2 x$ & I LHC \\
\hline & & & iPKP & 30 & 00.80 & & i \\
\hline & & & $i$ & 30 & 32.90 & & IN3 \\
\hline & & & e & 33 & 38.90 & & CGTN \\
\hline SVA & 152.45 & 226 & ePKP & 30 & 03.00 & $20.7 x$ & I JSC \\
\hline VUN & 152.52 & 226 & ePKP & 30 & 01.80 & $19.4 x$ & FRB \\
\hline NOU & 155.50 & 199 & PKPd & 29 & 49.00 & $2.6 x$ & ORT \\
\hline CTA & 156.88 & 152 & PKP & 29 & 45.30 & -2.0 & PRM \\
\hline KOU & 157.79 & 195 & PKPC & 30 & 89.60 & $20.2 x$ & $E L C$ \\
\hline PVC & 158.90 & 208 & PKPC & 30 & 29.80 & $39.1 x$ & $\mathrm{FVM}$ \\
\hline GUMO & 161.83 & 46 & $e(P K P)$ & 30 & 10.00 & $16.2 x$ & DON \\
\hline GUA & 161.90 & 46 & $e(P K P)$ & 30 & 14.00 & $20.1 x$ & I PWLA \\
\hline$T Z Z$ & 162.03 & 110 & $e(P K P)$ & 30 & 12.00 & $17.8 x$ & $\mathrm{FCC}$ \\
\hline PMG & 165.00 & 134 & ePKP & 30 & 02.00 & $5.1 x$ & POW \\
\hline LMG & 166.85 & 135 & $e(P K P)$ & 30 & 15.00 & $17.0 x$ & OLY \\
\hline LAT & 166.58 & 124 & ePKP & 30 & 10.00 & $11.8 x$ & LGAR \\
\hline ESA & 167.13 & 146 & PKP & 30 & 15.50 & $16.9 x$ & $\mathrm{GOH}$ \\
\hline \multirow[t]{2}{*}{ RAB } & 172.16 & 131 & ePKP & 30 & 08.00 & $6.6 x$ & \\
\hline & S.D. $=$ & & on 3 & & 4414 & obs. & \\
\hline
\end{tabular}
\& JAN $09,1982 \quad 12 \mathrm{~h} 53 \mathrm{~m} 51.80 \mathrm{~s}$ DEPTH $=10.0 \mathrm{~km}$ (geophysicist) $5.8 \mathrm{mb}$ ( 57 obs.) $5.4 \mathrm{Msz}$ ( 2 obs.) NEW BRUNSWICK <SPEC Slight domoge (VI) (451) ports of New Brunswick and a Bridgewoter, Caribou, Easton. Hoynesville. Monticella, Presque Isle, Ookfield and Stockhalm. Moine. Felt in the Maritime

Provinces, parts of Quebec ond throughout much of New England

UN $1.03179 \mathrm{Pg}$ $\begin{array}{lll}54 & 11.50 & 0.2\end{array}$

EBN $1.21298 \mathrm{Pg}$ $1.23229 P_{*}$ $1.71131 \mathrm{Pn}$ $1.95351 \mathrm{Pnc}$ $2.13193 \mathrm{Pn}$

$\begin{array}{lll}\text { LPO } & 2.32 & 280 \mathrm{PnC} \\ \text { EMM } & 2.32 & 195 \mathrm{Pn}\end{array}$ $\begin{array}{lll}54 & 14.30 & -0.1\end{array}$ $\begin{array}{llll}54 & 14.60 & -0.1\end{array}$ $\begin{array}{lll}54 & 21.09 & -0.8 \\ 54 & 24.50 & -0.8\end{array}$ $\begin{array}{llll}54 & 26.40 & -1.5\end{array}$ \begin{tabular}{lll|l}
54 & 30.00 & -0.5 & BDW
\end{tabular}
$2.40224 \mathrm{Pn}$ $2.79213 \mathrm{Pn}$ $3.13223 \mathrm{Pn}$

$3.82339 \mathrm{Pnc}$

$3.98263 \mathrm{Pnc}$

$4.34244 \mathrm{Pn}$

$4.57229 \mathrm{Pn}$

$5.05225 \mathrm{Pn}$

5.06255 Pnd

$5.15232 \mathrm{Pn}$

5.22 $215 \mathrm{Pn}$

$5.27248 \mathrm{Pn}$

$5.47239 \mathrm{Pn}$

$5.50227 \mathrm{Pn}$

$5.55231 \mathrm{Pn}$

$5.56220 \mathrm{Pn}$

$5.67245 \mathrm{Pn}$

$5.67218 \mathrm{Pn}$

$5.69235 \mathrm{Pn}$

$5.73212 \mathrm{Pn}$

$5.80251 \mathrm{Pn}$

6.02249 iPnd

$6.09224 \mathrm{Pn}$

$6.24261 \mathrm{Pn}$

$6.35243 \mathrm{Pn}$

$6.49259 \mathrm{Pn}$

$6.81261 \mathrm{Pn}$

$6.92220 \mathrm{Pn}$

$7.06226 \mathrm{Pn}$

$7.31224 \mathrm{Pn}$

$7.84359 \mathrm{Pn}$

8. $20226 \mathrm{ePd}$

$8.34241 \mathrm{P}$

$9.49 \quad 81 \mathrm{P}$

$9.77251 \mathrm{P}$

$12.04227 \mathrm{P}$

$12.48 \quad 227 \mathrm{P}$

$12.51228 \mathrm{P}$

$12.96233 \mathrm{P}$

$12.98255 \mathrm{eP}^{\mathrm{P}}$

$14.11231 \mathrm{P}$

$14.24232 \mathrm{iPC}$

$14.42258 \mathrm{eP}$

$14.46232 \mathrm{P}$

$5.28284 \mathrm{P}$

$15.93248 \mathrm{eP}$

$16.41237 \mathrm{P}$

$16.81226 \mathrm{eP}$

$16.83 \quad 357$

$17.21236 \mathrm{eP}$

17.52228 eP

19.28248 eP

$19.64251 \mathrm{eP}$

$19.80249 \mathrm{eP}$

$20.05241 \mathrm{P}$

20.16316P

$21.22248 \mathrm{eP}$

$21.81247 \mathrm{P}$

$21.85244 P$

$23.27 \quad 12 \mathrm{iPd}$

$1.1 \mathrm{~s} \quad 152.00 \mathrm{~nm}$

$23.40302 \mathrm{P}$

$23.69253 \mathrm{eP}$

Pd-

24.34254 iPd-

$1.0 \mathrm{~s} \quad 351.00 \mathrm{~nm}$

$18 \mathrm{~s}$
$\mathrm{eS}$

$\begin{array}{ll} & \mathrm{eS} \\ 24.68 & 256 \mathrm{eP} \\ 0.9 \mathrm{~s} & 225.00 \mathrm{~nm}\end{array}$

$25.33255 \mathrm{eP}^{\mathrm{P}}$

$1.0 \mathrm{~s} \quad 332.00 \mathrm{~nm}$

$\begin{array}{lc}26.13 & 259 \mathrm{e}(P) \\ 0.8 \mathrm{~s} & 214.00 \mathrm{~nm}\end{array}$

$\begin{array}{lll}26.17 & 278 \mathrm{eP} \\ 26.30 & 256 \mathrm{e}(\mathrm{P})\end{array}$

$\begin{array}{ll}26.30 & 256 \mathrm{e}(P) \\ 1.0 \mathrm{~s} & 126.00 \mathrm{~nm}\end{array}$

$27.25256 \mathrm{eP}^{\mathrm{P}}$

$28.85270 \stackrel{\mathrm{e}}{\mathrm{eP}}$

$0.7 \mathrm{~s} \quad 22.00 \mathrm{~nm}$

30.37249 iP

$\begin{array}{ll}30.41 & 278 \mathrm{eP} \\ 0.85 & 27.00 \mathrm{~nm}\end{array}$ $\begin{array}{lllll}54 & 30.40 & -1.4 & \text { I IMW }\end{array}$ \begin{tabular}{lll|l}
54 & 35.90 & -1.3 & LRM \\
54 & 40.60 & -1.4 & BUT
\end{tabular} $54 \quad 49.30-2.6$ \begin{tabular}{lll|l}
54 & 52.40 & -1.8 & AKU
\end{tabular} $\begin{array}{lll}54 & 57.40 & -2.0\end{array}$ \begin{tabular}{lll|l}
54 & 59.90 & -2.6 & ALO
\end{tabular} $\begin{array}{lll}507.80 & -1.5\end{array}$ $\begin{array}{lll}55 & 06.70 & -2.7\end{array}$ \begin{tabular}{lll|l}
55 & 07.80 & -2.9 & RXF
\end{tabular} \begin{tabular}{lll|l}
55 & 08.50 & -3.2 & LDM
\end{tabular} \begin{tabular}{lll|l}
55 & 09.90 & -2.6 & IKM
\end{tabular} \begin{tabular}{lll|l}
55 & 11.60 & -3.7 & LHD
\end{tabular} $\begin{array}{lll}55 & 13.60 & -2.1 \\ 55 & 13.50 & \text { I NEW }\end{array}$ \begin{tabular}{lll|l}
55 & 13.50 & -2.9 & DUG
\end{tabular} \begin{tabular}{lll|l}
55 & 13.58 & -3.1 & RMU
\end{tabular} \begin{tabular}{lll|l}
55 & 14.78 & -3.5 & PNT
\end{tabular}

\begin{tabular}{lll|l}
55 & 14.90 & -3.2 & I DAG
\end{tabular}

$14.80-3.6$

$\begin{array}{lll}15.80 & -3.1\end{array}$

$5516.90-3.0$ | ALE

\begin{tabular}{lll|l}
55 & 19.78 & -3.3 & I EUR
\end{tabular}

\begin{tabular}{lll|l}
5 & 21.20 & -2.7 & $\mathrm{MBC}$
\end{tabular}

\begin{tabular}{lll|l}
55 & 22.50 & -3.6 & I VAL
\end{tabular}

$5524.10-3.6$ | BMN

$\begin{array}{lll}55 & 26.00 & -3.6\end{array}$

$\begin{array}{llll}55 & 31.00 & -3.1\end{array}$

$\begin{array}{lll}5 & 31.50 & -4.2\end{array}$

$\begin{array}{lll}55 & 32.00 & -5.7\end{array}$

$\begin{array}{lll}55 & 34.00 & -7.1\end{array}$

$552.60-6.0$

$\begin{array}{lll}55 & 49.50 & -4.1\end{array}$

$\begin{array}{lll}55 & 53.50 & -2.1 \\ 55 & 57.50 & -6.4\end{array}$

$\begin{array}{llll}56 & 06.30 & -5.8\end{array}$

$\begin{array}{lll}56 & 09.20 & -6.8\end{array}$

$\begin{array}{llll}56 & 43.50 & -2.7\end{array}$

$\begin{array}{lll}56 & 50.00 & -2.1\end{array}$

$\begin{array}{lll}56 & 50.80 & -1.8\end{array}$

5650.58

5652.40

$57 \quad 12.00$

5713.10

5713.38

5717.08

22.00

$00 \quad 04.00$

$\begin{array}{ll}57 & 31.38 \\ 57 & 41.90\end{array}$

5748.40

5739.40

5752.00

5757.50

$58 \quad 16.60$

5820.70

5828.08

$\begin{array}{lll}58 & 27.40\end{array}$

5824.00

$\begin{array}{llll}58 & 38.20 & -1.6\end{array}$

$5848.10,1.9$ WCH

$5859.00 \quad-0.9$

$0312.00^{5}$

\begin{tabular}{lll|l}
58 & 59.00 & -2.3 & EDI
\end{tabular}

$\begin{array}{lll}59 & 03.30 & -1.8\end{array}$

$59 \quad 10.10$

$5.9 \mathrm{mb}$

0335.50

\begin{tabular}{lll|l}
59 & 13.00 & -0.9 & EKA
\end{tabular} $5918.40^{5.8 m b}-1.7$ SDW $6.0 \mathrm{mb}$ $\begin{array}{ccc}59.00 & -1.6 \\ 59.9 \mathrm{mb} \\ 5 & 27.20^{-0.9}\end{array}$ MIN ORV \begin{tabular}{lll|l}
59 & 30.50 & 1.3 & ILR
\end{tabular} $5935.40^{5.6 m b}-2.5$ 5938.20 $\begin{array}{lll}59 & 41.70\end{array}$ $\begin{array}{lll}59 & 50.00 & -2.6\end{array}$ $00 \quad 04.2 \theta^{5.1 \mathrm{mb}}$ $00 \quad 03.70 \quad-2.8$ $5.2 \mathrm{mb}$
$30.91281 \mathrm{eP}$

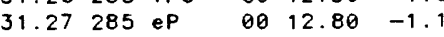
$31.50 \quad 286 \mathrm{ePC} \quad 00 \quad 14.00 \quad-2.0$ $\begin{array}{llllll}31.67 & 36 & \text { iP } \quad \theta 0 & 14.70 & -2.2\end{array}$ $1.0 \mathrm{~s} \quad 72.00 \mathrm{~nm} \quad 5.5 \mathrm{mb}$ $\begin{array}{lllll}32.04 & 262 \mathrm{ePc} & 00 & 19.50 & -1.3\end{array}$ $0.9 \mathrm{~s} \quad 15.80 \mathrm{~nm} \quad 4.9 \mathrm{mb}$

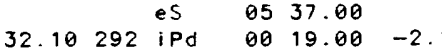
32.34291 iPd $\theta 021.00-2.1$ 32.48292 eP $\quad 0023.30-1.1$ $\begin{array}{lllll}32.54 & 291 \text { iP } \quad \theta 0 & 23.30 & -1.7\end{array}$ $33.54291 \mathrm{P} \quad 00 \quad 30.00 \quad-3.6$ $\begin{array}{llllll}33.71 & 276 & \text { IPC } & \theta 0 & 33.70 & -1.6\end{array}$ $\begin{array}{lllll}33.97 & 269 \mathrm{eP} & 00 & 36.10 & -1.4\end{array}$ $\begin{array}{llllll}34.81294 P & 80 & 42.50 & -2.0\end{array}$ $\begin{array}{lllllll}35.41 & 17 \mathrm{PPC} \quad 00 & 47.10 & -2.1\end{array}$ $0.8 \mathrm{~s} \quad 51.00 \mathrm{~nm} 0317.00$

$\begin{array}{llllll}35.67 \quad 1 P & 00 & 49.50 & -1.9\end{array}$ 36.19276 iP $\quad 0054.60-2 . \theta$ $\begin{array}{llllll}36.21 & 61 & \text { iPd } \quad 00 & 54.90 & -1.3\end{array}$ $36.60279 \mathrm{ePC} \quad 0858.80-1.1$ $1.0 \mathrm{~s} \quad 79.38 \mathrm{~nm}$

$37.16 \quad 185$ iPd $0105.70 \quad 1.0$ $1.05 \quad 114.00 \mathrm{~nm} \quad 0105.80^{5.6 \mathrm{mb}}$ $37.36 \quad 273 \mathrm{eP} \quad 0105.80$

EPCP 0325.80

$\begin{array}{llllll}37.59 & 58 \mathrm{iPC} & 01 & 05.40 & -2.4\end{array}$ $0.7 \mathrm{~s} \quad 220.00 \mathrm{~nm} \quad 6.0 \mathrm{mb}$ $\begin{array}{lllll}37.63 & 217 \mathrm{eP} & 01 & 09.80 & 0.4\end{array}$ $1.2 \mathrm{~s} \quad 180.00 \mathrm{~nm} \quad 5.7 \mathrm{mb}$ $\begin{array}{llllll}37.66 & 57 & \mathrm{IPC} & 01 & 05.80 & -2.6\end{array}$ $0.7 \mathrm{~s} \quad 170.00 \mathrm{~nm} \quad 5.9 \mathrm{mb}$

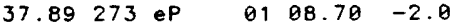

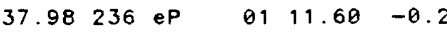

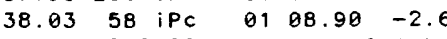
$0.7 \mathrm{~s} 250.08 \mathrm{~nm} \quad 6.1 \mathrm{mb}$ $\begin{array}{lllll}38.05 & 234 \mathrm{eP} & 01 & 12.30 & 0.0\end{array}$ $\begin{array}{llllll}38.12 & 186 & \text { iPd } & 01 & 13.00 & 0.2\end{array}$ $0.9 \mathrm{~s} \quad 111.00 \mathrm{~nm} \quad 5.6 \mathrm{mb}$ $\begin{array}{llllllll}38.12 & 57 \quad \mathrm{iPC} & 0108.30 & -4.0\end{array}$ $0.8 \mathrm{~s} 200.00 \mathrm{~nm} \quad 5.9 \mathrm{mb}$ $\begin{array}{lllll}38.12 & 235 \mathrm{eP} \quad 01 & 17.30 & 4.3\end{array}$ $\begin{array}{llllll}38.19 & 276 & \text { iP } & 01 & 12.70 & -0.6\end{array}$ $38.19276 \mathrm{eP} \quad 0111.50-1.8$ $\begin{array}{llllll}38.20 & 58 \quad \mathrm{iPC} & 01 & 10.10 & -2.8\end{array}$ $0.8 \mathrm{~s} 270.00 \mathrm{~nm} \quad 6.0 \mathrm{mb}$ $\begin{array}{llllll}38.34 & 285 & \text { e } & 01 & 13.70 & -0.8\end{array}$ $\begin{array}{llllll}38.54 & 53 \mathrm{ePc} & 01 & 13.20 & -2.6\end{array}$ $0.8 \mathrm{~s} \quad 220.00 \mathrm{~nm} \quad 5.9 \mathrm{mb}$ $\begin{array}{llllll}38.66 & 230 & \text { iP } & 01 & 17.00 & -0.4\end{array}$ $\begin{array}{llllll}38.82 & 52 \mathrm{iPC} & 01 & 15.60 & -2.5\end{array}$ $0.8 \mathrm{~s} 284.00 \mathrm{~nm} \quad 6.0 \mathrm{mb}$ $38.82267 \mathrm{eP} \quad 01 \quad 18.50 \quad 0.0$ $\begin{array}{lllll}38.85 & 278 \mathrm{eP} & 01 & 17.80 & -1.0\end{array}$ $\begin{array}{llllll}38.98 & 52 & \mathrm{iPC} & 01 & 16.88 & -2.7\end{array}$

$0.8 \mathrm{~s} \quad 464.00 \mathrm{~nm} \quad 6.2 \mathrm{mb}$ $\begin{array}{lllllllll}39.11 & 53 & \mathrm{IPC} & 01 & 17.80 & -2.7\end{array}$ $0.8 \mathrm{~s} \quad 176.00 \mathrm{~nm} \quad 5.8 \mathrm{mb}$ $\begin{array}{llllll}39.23 & 53 & \mathrm{iPC} & 01 & 19.70 & -1.9\end{array}$

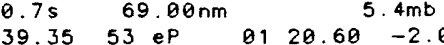
$\begin{array}{lllll}39.35 & 53 \mathrm{eP} & 01 & 20.60 & -2.0 \\ 0.9 \mathrm{~s} & 112.00 \mathrm{~nm} & & 5.5 \mathrm{mb}\end{array}$

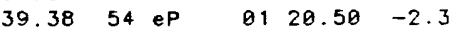
$1.0 \mathrm{~s} \quad 160.00 \mathrm{~nm} \quad 5.6 \mathrm{mb}$ $39.40 \quad 54 \mathrm{Pd} \quad 0121.30 \quad-1.7$ $0.8 \mathrm{~s} \quad 79.98 \mathrm{~nm} \quad 5.4 \mathrm{mb}$

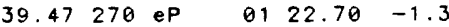

ePCP $03 \quad 31.40$

39.60281 iP $\quad 0123.20 \quad-1.8$

$\begin{array}{lllll}39.91280 P & 0125.00 & -2.4\end{array}$

PCP $03 \quad 31.00$

$39.94207 \mathrm{eP} \quad 0128.98 \quad 0.9$

$\begin{array}{lllll}39.99 & 275 \text { iP } & 01 & 25.70 & -2.4\end{array}$

$\begin{array}{llll} & \text { PCP } & 03 & 35.00\end{array}$

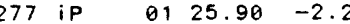

40.11282 iP $\quad 0125.50 \quad-3.6$

40.48271 PCP 0333.00 $\begin{array}{llll}\text { eP } & 01 & 33.00 & 0.8\end{array}$ ePP $\quad 03 \quad 10.00$ $\begin{array}{lll}\text { eS } & 0746.00 \\ \text { e } & 1435.00\end{array}$ 


\begin{tabular}{|c|c|c|c|c|c|c|c|c|c|c|c|}
\hline STS & 40.53 & 74 & $e^{P}$ & 01 & 31.00 & -1.4 & 1 & IFR & & 47.68 & 83 \\
\hline ARN & 41.04 & 277 & $e^{P}$ & 01 & 36.30 & -0.5 & $i$ & & & & \\
\hline & & & ePcP & 03 & 37.20 & & 1 & SSB & & 47.78 & 64 \\
\hline PR I & 41.09 & 275 & eP & 01 & 38.00 & 0.7 & i & TNS & & 47.80 & 57 \\
\hline MHC & 1.12 & 277 & $e^{P}$ & 01 & 35.00 & -2.5 & $i$ & GWF & & 7.89 & 59 \\
\hline BKS & 41.25 & 278 & eP & 01 & 39.00 & 0.6 & 1 & BSF & & 7.93 & 60 \\
\hline & & & e & 07 & 50.00 & & 1 & $C D F$ & & 7.95 & 60 \\
\hline & & & LO & 15 & 58.00 & & 1 & & & $0.6 \mathrm{~s}$ & 133 \\
\hline & & & LR & 18 & 42.00 & & 1 & UME & & 7.96 & 37 \\
\hline SAO & 41.29 & 276 & $e^{P}$ & 01 & 37.00 & -1.8 & 1 & $\mathrm{ECH}$ & & 47.97 & 60 \\
\hline PTO & 1.30 & 76 & iPd & 01 & 36.90 & -1.8 & 1 & & & & \\
\hline LIS & 42.12 & 80 & $P$ & 01 & 44.00 & -1.5 & 1 & & & & \\
\hline & & & ePP & 03 & 31.00 & & 1 & BAF & & 48.05 & 60 \\
\hline KBS & 42.16 & 16 & iPd & 01 & 45.30 & -0.1 & 1 & KEV & & 48.08 & 28 \\
\hline & $1.0 \mathrm{~s}$ & & $3.08 \mathrm{~nm}$ & & & $3 m b$ & 1 & & & & \\
\hline MTE & 42.36 & 77 & $P$ & 01 & 45.00 & -2.6 & 1 & & & & \\
\hline BOG & 42.68 & 191 & iP & 01 & 51.50 & 0.8 & 1 & $A L 1$ & & 48.09 & 75 \\
\hline & & & ePS & 08 & 20.00 & & 1 & BUH & & 8.39 & 59 \\
\hline $\mathrm{BOCO}$ & 2.72 & 191 & $e^{P}$ & 01 & 51.70 & 0.6 & 1 & NEC & & 48.42 & 61 \\
\hline GRR & 3.08 & 63 & iPc & 01 & 51.20 & -2.0 & 1 & UPP & & 48.42 & 42 \\
\hline LPF & 3.11 & 63 & $e^{P}$ & 01 & 51.30 & -2.2 & i & & & & \\
\hline TEN & 43.14 & 97 & ¿Pd & 01 & 53.10 & -0.8 & 1 & BBS & & 48.53 & 61 \\
\hline & & & ePP & 02 & 53.40 & & 1 & EMS & & .86 & 62 \\
\hline & & & es & 06 & 50.40 & & 1 & STU & & 91 & 58 \\
\hline$F L N$ & 43.16 & 62 & iPc & 01 & 52.10 & -1.8 & 1 & & & $1.0 \mathrm{~s}$ & 160 \\
\hline SSC & 43.46 & 62 & iPc & 01 & 54.50 & -1.8 & i & & $z$ & $20 \mathrm{~s}$ & \\
\hline & $1.0 \mathrm{~s}$ & 1620 & $0.00 \mathrm{~nm}$ & & & $.8 m b$ & 1 & $S O D$ & & 48.94 & 31 \\
\hline MFF & 44.27 & 65 & iPc & 02 & 00.70 & -2.2 & 1 & & & & \\
\hline KON & .72 & 45 & iP & 02 & 4.30 & -2.1 & 1 & SLE & & .97 & 60 \\
\hline TOL &. & 75 & $P d$ & 02 & 07.00 & -1.5 & 1 & ZUL & & 5 & 60 \\
\hline MB 2 & 45.03 & 42 & $\mathrm{P}$ & 02 & 07.60 & -1.4 & 1 & KHE & & 49.08 & 10 \\
\hline & - & & $1.80 \mathrm{~nm}$ & & & . Omb & I & & & & \\
\hline & 45.14 & & $P P$ & 03 & 49.20 & $559 \mathrm{kmx}$ & 1 & $D I X$ & & 49.15 & 62 \\
\hline DBN & 45.20 & $\begin{array}{l}56 \\
58\end{array}$ & $e^{P}$ & 02 & 09.00 & -0.8 & 1 & $C D R$ & & .34 & 66 \\
\hline UCC & & 58 & $P d-$ & 02 & 09.00 & -1.3 & 1 & T TA & & 49.34 & 323 \\
\hline & 45.23 & & e & 02 & 12.00 & & 1 & MOX & & 49.41 & 55 \\
\hline COL & 0.85 & 324 & eP & 02 & 09.00 & -1.5 & 1 & & & $1.5 \mathrm{~s}$ & 173 \\
\hline & & & $2.20 \mathrm{~nm}$ & & & $5 \mathrm{mb}$ & 1 & & & & \\
\hline FBA & .23 & 324 & $\mathrm{P}$ & 02 & 09.10 & -1.4 & 1 & & & & \\
\hline LSF & 5.46 & 65 & iPc & 02 & 10.10 & -2.4 & 1 & & & & \\
\hline LFF & 45.57 & 67 & $i P c$ & 02 & 11.00 & -2.4 & 1 & & & & \\
\hline DOU & 45.63 & 59 & $P d+$ & 02 & 11.40 & -2.4 & 1 & BRN & & .46 & 53 \\
\hline & & & $i$ & 02 & 15.50 & & 1 & MMK & & .50 & 62 \\
\hline$W \perp T$ & 45.69 & 54 & $e^{P}$ & 02 & 14.00 & -0.2 & 1 & GRFO & & 49.63 & 56 \\
\hline & $1.0 \mathrm{~s}$ & 88 & $300 \mathrm{~nm}$ & & & $.7 \mathrm{mb}$ & $i$ & GRF & & 49.64 & 56 \\
\hline TCF & 45.87 & 64 & $i P_{C}$ & 02 & 13.40 & -2.3 & 1 & & & $1.3 \mathrm{~s}$ & 221 \\
\hline & $1.0 \mathrm{~s}$ & 625 & $5.00 \mathrm{~nm}$ & & & $.5 \mathrm{mb}$ & 1 & & $z$ & $28 \mathrm{~s}$ & \\
\hline RJF & 5.89 & 66 & $i P_{C}$ & 02 & 13.30 & -2.6 & 1 & PNI & & 49.67 & 64 \\
\hline LPO & .97 & 67 & $e^{P}$ & 02 & 14.20 & -2.4 & 1 & LLS & & 49.71 & 61 \\
\hline WTS & .11 & 55 & $e^{P}$ & 02 & 15.50 & -2.0 & 1 & HOF & & .71 & 55 \\
\hline & $1.0 \mathrm{~s}$ & 232 & $2.00 \mathrm{~nm}$ & & & $1 \mathrm{mb}$ & i & LRG & & 49.82 & 66 \\
\hline TBY & 46.11 & 43 & $\mathbf{P}$ & 02 & 05.40 & -12.0 & 1 & $C L L$ & & 49.87 & 54 \\
\hline ENN & 46.12 & 57 & iPc & 02 & 15.20 & -2.4 & 1 & & & & \\
\hline & $1.0 \mathrm{~s}$ & 276 & $5.00 \mathrm{~nm}$ & & & $.2 m b$ & 1 & & & & \\
\hline$M Z F$ & 5.13 & 64 & iPc & 02 & 15.10 & -2.7 & 1 & FRF & & 49.95 & 65 \\
\hline SLL & 0.20 & 43 & $P$ & 02 & 06.23 & -11.9 & i & LMR & & 49.98 & 66 \\
\hline EPF & 6.23 & 69 & iPc & 02 & 17.00 & -1.7 & $i$ & $K D C$ & & 50.07 & 316 \\
\hline & $0.6 \mathrm{~s}$ & 28 & $3.60 \mathrm{~nm}$ & & & $.5 m b$ & 1 & ROB & & 50.33 & 64 \\
\hline AVE & 46.25 & 85 & iP & 02 & 16.50 & -2.4 & 1 & FUR & & 50.41 & 58 \\
\hline & & & $\mathrm{i}$ & 03 & 27.00 & & 1 & & & $1.5 \mathrm{~s}$ & 310 \\
\hline SSF & 31 & 63 & $\mathrm{iPc}$ & 02 & 16.20 & -3.0 & I & BRG & & 50.60 & 54 \\
\hline$V F$ & 6. 34 & 63 & iPc & 02 & 16.80 & -2.6 & i & & & & \\
\hline CAF & 1 & 66 & iPc & 02 & 17.50 & -2.6 & i & & & & \\
\hline LOR & .43 & 62 & iPc & 02 & 17.30 & -2.9 & 1 & & & & \\
\hline & $1.0 \mathrm{~s}$ & 437 & $7.00 \mathrm{~nm}$ & & & $.4 \mathrm{mb}$ & 1 & GAP & & 50.63 & 59 \\
\hline HFS & 46.49 & 43 & $\mathbf{P}$ & 02 & 08.30 & -12.2 & 1 & KJF & & 50.76 & 34 \\
\hline & $0.7 \mathrm{~s}$ & & $5.00 \mathrm{~nm}$ & & & & 1 & & & $1.2 \mathrm{~s}$ & 116 \\
\hline & & & e & 20 & 00.00 & & $i$ & & $z$ & $17 \mathrm{~s}$ & \\
\hline So & .55 & 195 & eP & 02 & 21.50 & -0.3 & 1 & & & & \\
\hline$K \mid R$ & 0.56 & 31 & $\mathbf{P}$ & 02 & 19.70 & -1.2 & 1 & & & & \\
\hline PYM & .61 & 65 & $\mathbf{P}$ & 02 & 19.90 & -1.8 & 1 & & & & \\
\hline LBF & .63 & 63 & iPc & 02 & 19.00 & -2.8 & 1 & & & & \\
\hline CRT & 46.65 & 78 & ip & 02 & 21.40 & -0.7 & 1 & SUF & & 84 & 36 \\
\hline SMF & 46.70 & 63 & iPc & 02 & 19.60 & -2.7 & I & WET & & .85 & 56 \\
\hline ALS & 4 & 69 & ePc & 02 & 20.00 & -2.7 & 1 & & & $1.2 \mathrm{~s}$ & 164 \\
\hline PMR & .92 & 320 & $\mathbf{P}$ & 02 & 22.70 & -1.1 & 1 & GEN & & 50.91 & 63 \\
\hline IMA & 7.16 & 326 & $P$ & 02 & 24.70 & -1.1 & 1 & OGA & & 50.92 & 60 \\
\hline EBR & 47.44 & 72 & eP & 02 & 26.00 & -2.1 & 1 & & & $1.5 \mathrm{~s}$ & 167 \\
\hline & & & e & 09 & 32.00 & & 1 & $P C N$ & & .03 & 62 \\
\hline & & & e & 12 & 58.00 & & 1 & APA & & .15 & 29 \\
\hline HAU & 47.59 & 60 & iPc & 02 & 27.00 & -2.3 & 1 & $\mathrm{KHC}$ & & 51.25 & 56 \\
\hline & & 275 & $.00 \mathrm{~nm}$ & & & $3 \mathrm{mb}$ & 1 & & & $1.0 \mathrm{~s}$ & \\
\hline COP & 47.62 & 49 & iPd & 02 & 28.30 & -1.0 & 1 & & $z$ & $14 \mathrm{~s}$ & \\
\hline & $0.9 \mathrm{~s}$ & 44 & $1.00 \mathrm{~nm}$ & & & $.5 \mathrm{mb}$ & 1 & & $\mathbf{N}$ & $14 \mathrm{~s}$ & \\
\hline & & & $\mathrm{i}$ & 02 & 32.00 & & 1 & & $E$ & $14 \mathrm{~s}$ & \\
\hline & & & is & 09 & 32.00 & & 1 & & & & \\
\hline & & & iss & 13 & 07.00 & & 1 & SAL & & 51.26 & 61 \\
\hline
\end{tabular}


$09 d \quad 13 h$

\begin{tabular}{|c|c|c|c|}
\hline CMP & 59.88 & 55 & $\mathrm{Pd}$ \\
\hline OHR & 59.97 & 61 & ip \\
\hline MLR & 60.25 & 54 & $P$ \\
\hline PTO2 & 60.29 & 191 & eP \\
\hline $\mathrm{SOB} 2$ & 68.78 & 151 & $\begin{array}{l}\text { eP } \\
\mathbf{e}\end{array}$ \\
\hline KIS & 60.89 & 51 & $\begin{array}{l}P c \\
\text { i sP }\end{array}$ \\
\hline VAY & 60.90 & 60 & eP \\
\hline$T I K$ & 61.17 & 354 & $\begin{array}{l}\text { EPC } \\
\text { esP }\end{array}$ \\
\hline & 6975 & & es \\
\hline PVL & 61.27 & $\begin{array}{r}196 \\
57\end{array}$ & ipd \\
\hline$D \mid M$ & 62.20 & 57 & $e^{P}$ \\
\hline KDZ & 62.32 & 58 & Pd \\
\hline NRI & 62.45 & 10 & $\begin{array}{l}\text { ePc } \\
\text { es }\end{array}$ \\
\hline ZOBO & 62.97 & 182 & $\mathbf{P}$ \\
\hline LPB & 63.23 & 182 & $\begin{array}{l}P_{c} \\
S \\
\text { e }\end{array}$ \\
\hline ARE & 63.30 & 185 & eP \\
\hline DMK & 63.59 & 56 & eP \\
\hline ATH & 63.70 & 62 & eP \\
\hline CTT & 64.41 & 57 & eP \\
\hline EDC & 64.61 & 57 & ip \\
\hline IST & 64.80 & 56 & $e(P)$ \\
\hline$S I M$ & 65.02 & 50 & eP \\
\hline DST & 65.54 & 58 & ip \\
\hline SEY & 66.14 & 342 & $\begin{array}{l}\text { ep } \\
\text { es }\end{array}$ \\
\hline $\mathrm{KIC}$ & 66.39 & 107 & iP \\
\hline
\end{tabular}

MGN $\quad 66.68 \quad 55$ iP

ALT $66.76 \quad 57$ iP

KAS $67.25 \quad 53 \mathrm{iPC}$

$67.2929 \mathrm{Pc}$

$$
\begin{aligned}
& \text { is } P \\
& \text { eS }
\end{aligned}
$$

SVE $\quad 67.63 \quad 28 \mathrm{eP}$

BCK $68.12 \quad 58$ iP

ELL $68.20 \quad 59$ iP

KVT 68.7052 iP

MGD $\quad 68.99341 \mathrm{ePC}$

PYA $\quad 70.23 \quad 46 \mathrm{P}$

ANT $\quad 70.44 \quad 184 \mathrm{P}$

YAK $70.56 \quad 352 \mathrm{Pd}$

$\begin{array}{llll}\text { GRO } & 72.02 & 45 \mathrm{Pd}\end{array}$

$\begin{array}{lrrr}\text { BKR } & 72.14 & 48 & \text { Po } \\ \text { PET } & 73.15 & 333 & \mathrm{eP}\end{array}$

FDA5 $74.98166 P$

KRV $\quad 74.37 \quad 47 \mathrm{PPP}$

GRS $75.23 \quad 47 \mathrm{PC}$

NVS $\quad 75.37 \quad 17 \mathrm{PC}$

BOD $75.53 \quad 360 \mathrm{PC}$

TAB $\quad 76.32 \quad 48 \mathrm{eP}$

$\begin{array}{llll}\text { ELT } & 77.51 & 16 \mathrm{PC} & \mathrm{PC} \\ & & \mathrm{eS}\end{array}$

ICA $\quad 77.98 \quad 178 \mathrm{ePd}$

SEM $\quad 78.87 \quad 21 \mathrm{PC}$

$\begin{array}{llll}\text { UKR } & 79.38 \quad 18 \mathrm{PC}\end{array}$

PEL $\quad 79.84 \quad 183 \mathrm{PL}$

IRK $\quad 80.82 \quad 6 \mathrm{eP}$

MOY $81.14 \quad 8 \mathrm{eP}$

ZAK $82.63 \quad 6 \mathrm{PC}$

YSS $82.92340 \mathrm{Pd}$

BNG

CON

TAS

FRU

SAM

TLG

TMU

PRZ

NRN

$\begin{array}{lll}84.21 & 28 & \\ 84.33 & 34 & \mathrm{eP} \\ 84.67 & 26 \mathrm{Pc}\end{array}$

$85.33 \quad 30$ eP

$85.6825 \mathrm{PO}$
$0358.00-1.9$ | DSH

\begin{tabular}{lll|l}
03 & 58.40 & -2.2 & SHI \\
04 & 02.00 & -0.5 & WMO
\end{tabular}

$0402.00-0.5$ | WMO

\begin{tabular}{lll|l}
04 & 01.10 & -1.7 & \\
04 & 04.60 & -1.5 & KUL
\end{tabular}

0405.10 I MDJ

\begin{tabular}{lll|l}
04 & 03.00 & -3.6 & \\
04 & 07.50 & & $\mathrm{KSH}$
\end{tabular}

\begin{tabular}{llll|l}
04 & 07.50 & & $\mathrm{KSH}$ \\
04 & 03.70 & -3.1 &
\end{tabular}

\begin{tabular}{lll|l}
04 & 06.00 & -2.2 & KHO
\end{tabular}

\begin{tabular}{ll|l}
0407.50 & CN2
\end{tabular}

\begin{tabular}{l|l}
1230.00 & $\mathrm{KBL}$
\end{tabular}

\begin{tabular}{lll|l}
0407.50 & -1.8 & BTO
\end{tabular}

\begin{tabular}{lll|l}
04 & 07.00 & -2.3 & I GTA
\end{tabular}

\begin{tabular}{lll|l}
04 & 12.00 & -3.6 & BJI
\end{tabular}

\begin{tabular}{lll|l}
04 & 14.00 & -2.4 & \\
04 & 15.00 & -1.8 & MAT
\end{tabular}

\begin{tabular}{ll|l}
12 & 29.00 & -1.8 \\
0 & 1 TIY
\end{tabular}

\begin{tabular}{lll|l}
04 & 19.30 & -2.1 & LZH
\end{tabular}

\begin{tabular}{lll|l}
04 & 21.00 & -2.0 & TIA \\
12 & 52.00 & & NDI
\end{tabular}

$2234.00 \quad$ XAN

\begin{tabular}{lll|l}
0420.00 & -3.3 & GBA
\end{tabular}

$\begin{array}{lll}04 & 21.60 & -3.2\end{array}$

$0423.00-2.5$ | CHG

$\begin{array}{lllll}04 & 26.50 & -3.7 & \text { I PSI }\end{array}$

\begin{tabular}{lll|l}
04 & 29.50 & -1.9 & NOU
\end{tabular}

$0430.00 \quad-2.6 \quad$ KOU

$\begin{array}{llll}04 & 33.00 & -1.0 & \text { PPI }\end{array}$

\begin{tabular}{lll|l}
04 & 35.10 & -2.4 & MNG \\
04 & 38.00 & -2.9 & SPA
\end{tabular}

0438.00

0440.90

05.20 I MTN

\begin{tabular}{lll|l}
04 & 44.50 & -0.3 & $\mathrm{SBA}$
\end{tabular}

\begin{tabular}{lll|l}
04 & 47.50 & -0.9 & COO
\end{tabular}

0446.00

1343.00

0448.00

0452.90

0453.90

0456.40

0456.00

0505.00

0507.00

0506.20

$05 \quad 17.00$

0517.50

0522.00

0529.70

0533.50

$0530.00-1.2$

$\begin{array}{lll}05 & 34.80 & -1.7\end{array}$

0540.60

0533.80

1518.10

0536.29

0543.00

$08 \quad 33.00$

0546.80

1541.00

0551.00

0554.60

1555.0

0556.90

1600.90

0601.80

$\begin{array}{ll}06 & 02.00 \\ 06 & 09.00\end{array}$

0609.00

$06 \quad 16.50$

0618.00

$06 \quad 22.60 \quad 1.4$ (

\begin{tabular}{lrr|l}
06 & 22.60 & 1.4 .00 & -1.1
\end{tabular}

$06 \quad 23.90-0.1$ MDG

$0624.20-0.3$ | AFI

0625.00

0626.00

0629.00

0630.00

\begin{tabular}{l|l}
-0.1 & \\
-0.8 & CoO
\end{tabular}

0632.00

0633.60

1710.00
1324.70

$0443.00-2.4$ | BRS

$85.98 \quad 33 \mathrm{PC}$

$86.10 \quad 50 \mathrm{eP}$

86. $78 \quad 18 \mathrm{PC}$

$86.93 \quad 33 \mathrm{ePd}$

$87.67348 \mathrm{P}$

$87.7128 \mathrm{P}$

$87.9532 \mathrm{P}$

$88.97351 \mathrm{P}$

$89.6135 \mathrm{e}(\mathrm{P})$

$92.75 \quad 3 \mathrm{P}$

$93.32358 \mathrm{eP}$

$1.5 \mathrm{~s} \quad 52.00 \mathrm{~nm}$

$93.91340 \mathrm{P}-$

$95.68 \quad 1 \mathrm{P}$

$97.11357 \mathrm{P}$

$97.93 \quad 32 \mathrm{P}$

$99.25 \quad 4 \mathrm{P}$

11.6438 PKPC

$0.7 \mathrm{~s} \quad 2.60 \mathrm{~nm}$

$113.24 \quad 15$ ePKP

$128.93 \quad 19$ ePKP

130.97281 PKPC

131.21285 PKPC

132.3018 ePKP

$\begin{array}{lll}13 & 05.50 & -2.4\end{array}$

$\begin{array}{cc}136.79 & 180 \text { iPKPC } \\ 1.0 \mathrm{~s} & 25.00 \mathrm{~nm}\end{array}$

142.10304 PKP

$142.83 \quad 330$ ePKP

$\begin{array}{lll}143.01 & 196 & \text { iPKP } \\ 143.49 & 288 & \text { PKPd }\end{array}$

$\begin{array}{lll}143.49 & 288 & \text { PKPd } \\ 146.17 & 285 & \text { ePKP }\end{array}$

146.32332 ePKP

148.04320 PKP

315.00

$13 \quad 19.60$

\begin{tabular}{llll|l}
13 & 21.00 & -6.4 & I SNZO
\end{tabular}

$\begin{array}{lll}13 & 22.80 & -3.1\end{array}$

\begin{tabular}{lll|l}
13 & 22.80 & -3.1 & \\
13 & 27.10 & -1.1 & WB2
\end{tabular}

$1333.00 \quad 0.3$

$\begin{array}{lll}13 & 32.00 & -1.2\end{array}$

1333.40

1336.00

$148.05320 \mathrm{PKPC}$

$0.9 \mathrm{~s} \quad 19.70 \mathrm{~nm}$

150.79283 PKP $13 \quad 39.30$

$150.98281 \quad 1345.08$

1339.30

1344.00

$\begin{array}{lllll}151.50 & 318 & \text { ePKP } & 13 & 39.00 \\ 151.54 & 279 & \text { PKP } & 13 & 45.50\end{array}$

1345.50

153.66295 PKP 1349.00

393 obs. os socioted

$-2.5$

RHP

G $05,1982 \quad 20 \mathrm{~h} 32 \mathrm{~m} 48.98 \pm 1.79 \mathrm{~s}$

$12.573 \mathrm{~S} \pm 2.7 \mathrm{~km} 165.968 \mathrm{E} \pm 2.4 \mathrm{~km}$ GUMO

DEPTH $=5.0 \pm 10.5 \mathrm{~km}$

$6.0 \mathrm{mb}$ ( 30 obs.) $7.1 \mathrm{Msz}$ ( 30 Obs.) / RAR SANTA CRUZ ISLANDS

$85.92 \quad 27 \mathrm{PC}$ 


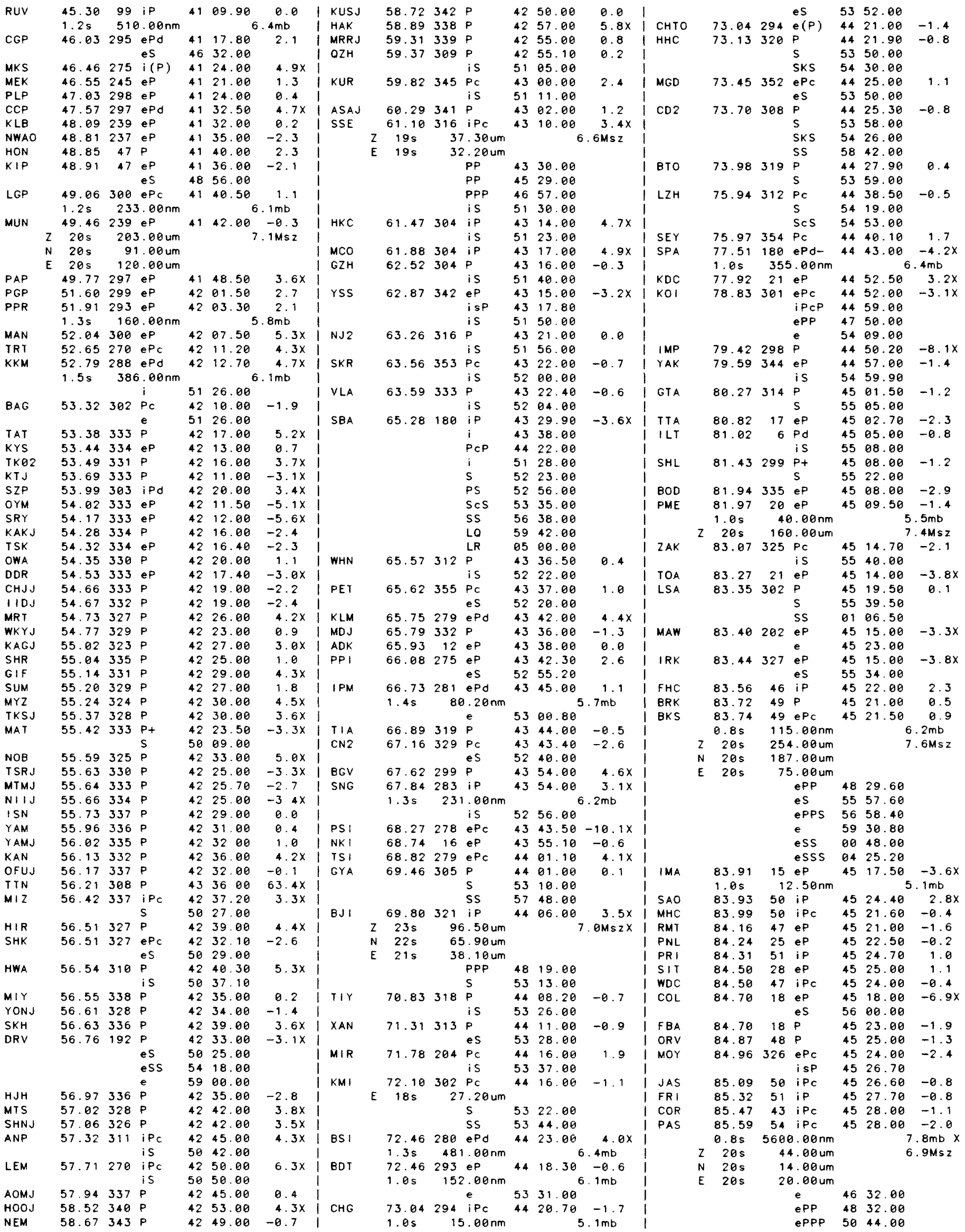




\begin{tabular}{|c|c|c|c|c|c|c|c|c|c|c|c|}
\hline & & & & es & 56 & 00.00 & & $1 P 00$ & 95.89 & 288 & $\mathrm{P}+$ \\
\hline & & & & ePPS & 57 & 05.00 & & I YKA & 96.22 & 27 & $\mathrm{P}$ \\
\hline & & & & ess & 01 & 00.00 & & I MOT & 96.32 & 60 & $P$ \\
\hline & & & & eSSS & 85 & 24.00 & & I PRZ & 96.60 & 312 & $\in P$ \\
\hline KTM & & 85.89 & 52 & eP & 45 & 31.70 & 0.1 & 1 & & & es \\
\hline BOK & & 86.23 & 295 & $\mathrm{P}$ & 45 & 36.00 & $2.7 x$ & AJM & 96.86 & 295 & $\mathrm{P}+$ \\
\hline & & & & $s$ & 56 & 09.00 & & i & & & $P P$ \\
\hline BFW & & 86.26 & 41 & $e^{P}$ & 45 & 36.68 & $3.5 x$ & I SEM & 96.87 & 320 & ePc \\
\hline SDW & & 6.59 & 54 & $e(P)$ & 45 & 35.50 & 0.4 & 1601 & 96.89 & 51 & $e(P)$ \\
\hline VPIM & & 86.76 & 44 & $e^{P}$ & 45 & 34.20 & -1.6 & I & $0.9 \mathrm{~s}$ & & $9.00 \mathrm{~nm}$ \\
\hline VHEM & & 86.82 & 42 & eP & 45 & 37.70 & 1.7 & $180 \mathrm{M}$ & 96.92 & 288 & $P$ \\
\hline VIS & & 86.94 & 289 & Pt & 45 & 43.00 & $6.1 x$ & I SNA & 96.92 & 184 & $e(P)$ \\
\hline MNA & & 86.95 & 50 & eP & 45 & 36.00 & -0.8 & | NR I & 97.45 & 340 & $\mathrm{PC}$ \\
\hline$T I K$ & & 87.61 & 349 & EPC & 45 & 40.00 & 1.0 & I TLG & 97.48 & 313 & $\mathrm{Pc}_{\mathrm{c}}$ \\
\hline & & & & es & 56 & 15.00 & & I KSH & 97.86 & 309 & $P$ \\
\hline YMT 1 & & 87.69 & 52 & $e^{P}$ & 45 & 41.20 & 0.9 & i & & & PP \\
\hline GLA & & 87.93 & 56 & eP & 45 & 38.80 & -2.7 & i & & & SKS \\
\hline BMN & & 88.26 & 48 & eP & 45 & 38.80 & $-4.2 x$ & $i$ & & & es \\
\hline & & $0.8 \mathrm{~s}$ & 15 & $5.00 \mathrm{~nm}$ & & 5 & $.4 \mathrm{mb}$ & I NRN & 98.17 & 311 & eP \\
\hline EUR & & 88.89 & 49 & ip & 45 & 46.80 & 0.6 & 1 & & & es \\
\hline & & $1.2 \mathrm{~s}$ & & $1.00 \mathrm{~nm}$ & & & $.0 \mathrm{mb}$ & I $M B C$ & 98.52 & 13 & $e^{P}$ \\
\hline VAR & & 89.05 & 296 & $p_{+}$ & 45 & 51.20 & $4.3 x$ & i & $1.5 \mathrm{~s}$ & & $0.00 \mathrm{~nm}$ \\
\hline WMO & & 90.32 & 315 & $\mathrm{Pc}$ & 45 & 50.00 & -2.6 & $11 \mathrm{M}$ & 98.58 & 72 & $\mathrm{Pd} 4$ \\
\hline & & & & PP & 49 & 30.50 & & I LUB & 98.62 & 58 & $e^{P}$ \\
\hline & & & & eSKS & 56 & 26.00 & & I KRD & 99.17 & 312 & $\mathrm{P}+$ \\
\hline & & & & $\mathrm{s}$ & 56 & 41.00 & & I FRU & 99.41 & 312 & ePc \\
\hline$K O D$ & & 90.75 & 280 & eP & 46 & 02.00 & $6.6 x$ & 1 & & & is \\
\hline & & & & $P \subset P$ & 46 & 07.30 & & I ANR & 100.80 & 309 & ePdiff 4 \\
\hline & & & & PP & 49 & 35.00 & & i & & & eS \\
\hline & & & & PPP & 51 & 33.00 & & I KHO & 101.02 & 306 & Pdiffc4 \\
\hline & & & & SKS & 56 & 31.00 & & i & & & $\mathrm{s}$ \\
\hline & & & & SKKS & 56 & 40.00 & & I GAR & 102.15 & 308 & ePdiff 4 \\
\hline & & & & es & 56 & 50.30 & & i & & & es \\
\hline & & & & PS & 57 & 57.00 & & I KUL & 102.47 & 306 & ePdiff 4 \\
\hline & & & & PPS & 58 & 28.00 & & I DSH & 103.38 & 307 & ePdiff4 \\
\hline & & & & ss & 02 & 48.00 & & I SAM & 104.78 & 308 & Pdiffc4 \\
\hline & & & & SSS & 06 & 21.00 & & I SVE & 108.82 & 326 & Pditfc4 \\
\hline & & & & LO & 10 & 02.00 & & I ARU & 109.99 & 326 & ePdiff 4 \\
\hline & & & & $L R$ & 15 & 44.00 & & I ARU & 109.99 & 326 & APKP 5 \\
\hline NEW & & 90.76 & 41 & $e^{P}$ & 45 & 54.80 & 0.4 & i & & & iPP \\
\hline & 2 & $20 \mathrm{~s}$ & 30 & $0.00 \mathrm{um}$ & & & $.7 \mathrm{Msz}$ & i & & & ePS \\
\hline & $\mathrm{N}$ & $20 \mathrm{~s}$ & & $2.00 \mathrm{um}$ & & & & i & & & ESPP $\quad 0$ \\
\hline & $E$ & $20 \mathrm{~s}$ & & $3.00 \mathrm{um}$ & & & & I MHI & 110.56 & 304 & Pdiffed \\
\hline I NK & & 91.27 & 19 & $e^{P}$ & 45 & 58.00 & 1.7 & $i$ & & & $e$ \\
\hline HYB & & 91.28 & 287 & $e^{P c}$ & 45 & 57.00 & -0.4 & I & & & i \\
\hline & & & & e & 46 & 01.50 & & I ASH & 111.47 & 306 & ePditf 4 \\
\hline & & & & e & 46 & 11.00 & & I VAN & 111.67 & 306 & ePdiff4 \\
\hline & & & & ePP & 49 & 38.00 & & I KAT & 113.07 & 307 & ePdiff \\
\hline & & & & e & 55 & 12.50 & & I UTO & 113.51 & 50 & iPdiff4 \\
\hline & & & & eSKS & 56 & 31.50 & & I CLE & 315.05 & 50 & Pdiffc4 \\
\hline & & & & es & 57 & 00.00 & & i & & & esks 5 \\
\hline & & & & ePS & 58 & 16.00 & & I DAG & 115.76 & 1 & ePKP 5 \\
\hline DUG & & 91.43 & 49 & $e^{P}$ & 45 & 59.10 & 1.3 & i & & & $\mathrm{iPP}$ \\
\hline MSU & & 91.47 & 51 & $e^{P}$ & 45 & 55.60 & -2.6 & & & & is \\
\hline GBA & & 91.50 & 284 & $P d$ & 45 & 59.70 & 1.3 & i & & & IPS \\
\hline & & $1.0 \mathrm{~s}$ & & $9.90 \mathrm{~nm}$ & & & $.7 \mathrm{mb}$ & BLA & 116.26 & 55 & ePdiff 4 \\
\hline MSO & & 92.40 & 43 & ePc & 46 & 04.00 & 1.9 & i & & & es \\
\hline & $z$ & $18 \mathrm{~s}$ & 18 & $8.50 \mathrm{um}$ & & & $6 \mathrm{Msz}$ & ARE & 116.30 & 116 & IPKP 5 \\
\hline BUT & & 93.11 & 44 & ePc & 6 & .00 & -0.5 & APA & 116.98 & 342 & ePdiff 4 \\
\hline LRM & & 93.13 & 44 & $e^{P}$ & 46 & 08.10 & 2.4 & APA & 116.98 & 342 & iPKP 5 \\
\hline I MW & & 93.70 & 46 & ePd & 46 & 09.20 & 0.8 & i & $z \quad 22 \mathrm{~s}$ & 88 & $8.00 \mathrm{um}$ \\
\hline HRY & & 93.77 & 43 & eP & 46 & 09.60 & 1.9 & $i$ & $23 \mathrm{~s}$ & 40 & $0.00 \mathrm{um}$ \\
\hline A|A & & 93.83 & 161 & $e(P)$ & 46 & 10.00 & 1.7 & 1 & E $20 \mathrm{~s}$ & 45 & $5.00 \mathrm{um}$ \\
\hline SEH & & 93.85 & 293 & $\mathrm{P}$ & 46 & 11.00 & 1.9 & t & & & iPP \\
\hline UKR & & 94.20 & 321 & $P_{C}$ & 46 & 07.80 & -2.3 & & & & iSP \\
\hline BDW & & 94.42 & 47 & $e^{P}$ & 46 & 11.80 & 0.1 & LPA & 117.06 & 140 & PKPd 5 \\
\hline & & $1.1 \mathrm{~s}$ & 25 & $5.88 \mathrm{~nm}$ & & & $.5 \mathrm{mb}$ & 1 & & & $\mathrm{PP}$ \\
\hline & $z$ & $20 \mathrm{~s}$ & 156 & $6.25 \mathrm{um}$ & & & $.5 \mathrm{MsZ}$ & i & & & PKS \\
\hline DDI & & 94.47 & 300 & $P$ & 46 & 14.00 & 2.1 & i & & & PPP \\
\hline & & & & SKS & 56 & 53.00 & & i & & & SKS \\
\hline ND 1 & & 94.85 & 298 & iPc & 46 & 15.40 & 1.8 & I KEV & 117.33 & 345 & ePdiff4 \\
\hline & $z$ & $18 \mathrm{~s}$ & & $8.20 u m$ & & & $.6 \mathrm{Msz}$ & I KEV & 117.33 & 345 & ePKP 5 \\
\hline & & & & iPP & 50 & 14.40 & & & $1.0 \mathrm{~s}$ & 52 & $2.00 \mathrm{~nm}$ \\
\hline & & & & iSKS & 56 & 56.40 & & i & $z 20 \mathrm{~s}$ & 61 & $1.20 \mathrm{um}$ \\
\hline & & & & is & 57 & 25.40 & & & & & $i$ \\
\hline & & & & iPS & 58 & 42.40 & & 1 & & & ePP \\
\hline & & & & iss & 03 & 50.40 & & 1 & & & ePS \\
\hline$N V L$ & & 95.00 & 188 & eP & 46 & 15.00 & 1.4 & I INY & 8.66 & 48 & ePKP \\
\hline & & $1.6 \mathrm{~s}$ & 190 & 0.08 & & & $3 \mathrm{mb}$ & OT & 118.67 & 45 & ePKP 5 \\
\hline & $z$ & $21 \mathrm{~s}$ & & $6.70 \mathrm{um}$ & & 7 & $.2 M s z$ & I YJA & 118.86 & 124 & $e(P K P) 5$ \\
\hline & & & & es & 57 & 22.00 & & SOD & 119.08 & 343 & ePKP \\
\hline ALO & & & & eP & 46 & & -2.1 & & & & 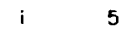 \\
\hline & & $1.0 \mathrm{~s}$ & 16 & $6.50 \mathrm{~nm}$ & & & $.4 \mathrm{mb}$ & i & & & ePKKP 0 \\
\hline & z & $19 \mathrm{~s}$ & 139 & $9.08 \mathrm{um}$ & & & $.4 \mathrm{Msz}$ & I LPB & 119.24 & 117 & Pdiffc4 \\
\hline 10 & & 95.13 & 55 & eP & 46 & 17.00 & 1.9 & I LPB & 119.24 & 117 & PKP \\
\hline OA & & 95.32 & 285 & $P$ & 46 & 19.10 & $3.1 x$ & & & & SKS \\
\hline & & & & SK & 56 & 49.50 & & $20 B O$ & 119.32 & 117 & PKPC \\
\hline
\end{tabular}


NUR $\begin{array}{cccc}2 & 17 \mathrm{~s} & 162.00 \mathrm{um} & \\ 124.41 & 338 \text { iPKP } 51 & 49.00^{7.8 M s z X} & -1.4\end{array}$

$\begin{array}{ccc}124.41 & 338 \text { iPKP } 51 \quad 49.00 \\ 0.9 \mathrm{~s} & 98.00 \mathrm{~nm}\end{array}$

$z 22 \mathrm{~s} \quad 54.10 \mathrm{um}$

i 5154.98

$\begin{array}{lll}\text { ePP } & 53 & 32.00\end{array}$

ESKP $54 \quad 44.00$

ePS $03 \quad 32.00$

ePPS 0504.00

eSS 1036.00

NUR 124.41338 ePdiff $48 \quad 28.00$

$\begin{array}{llllll}\text { POO } & 124.41 & 44 & \text { ePKP } 51 & 50.20\end{array}$

ARO $124.46 \quad 275$ iPKPC 5155.40

TUH 124.48214 ePKP 5152.00

124.62315 ePKP 5153.50

$\begin{array}{lll}\text { iPS } & 03 & 40.00 \\ \text { iSS } & 10 & 36.00\end{array}$

SOC $\quad 124.62315$ ePdiff $48 \quad 29.00$

KSR $\quad 124.80 \quad 226$ e(PKP) $51 \quad 55.00$

TET 124.95240 ePKP 5159.00 $\begin{array}{lll}e & 53 & 53.00 \\ e & 5523.00\end{array}$

ANN $\quad 125.86 \quad 317$ ePKP 5154.00

ANN $\quad 125.86 \quad 317$ ePdiff $48 \quad 37.00$

AKU $\quad 126.88 \quad 2$ ePKP 5151.40

$1.0 \mathrm{~s} \quad 52.00 \mathrm{~nm}$

$220 \mathrm{~s} 22.70 \mathrm{um} 5200.20$

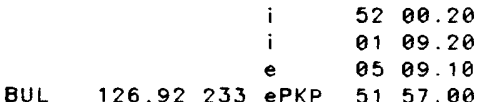
$\begin{array}{lll}\text { EPP } & 53 & 27.00\end{array}$ iPKKP 0110.00

UPP $\quad 127.31 \quad 341$ Pdiff $48 \quad 39.00$

UPP $\quad 127.31 \quad 341$ iPKP 5155.00 $\begin{array}{lll}\text { iPP } & 53 & 56.00 \\ \text { iPKS } & 55 & 12.00\end{array}$

NAI 127.71258 ePKP $52 \quad 04.00$ $\begin{array}{ccc}1.0 \mathrm{~s} & 30.00 \mathrm{~nm} & \\ \text { eS } 20 \quad 48.00\end{array}$

APO $\quad 127.90 \quad 343$ PKP $51 \quad 58.55$

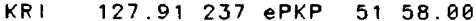
$\begin{array}{lll}\text { ISKP } & 55 \quad 33.00\end{array}$ iPKKP Q1 11.00

SIM $\quad 127.94318$ ePKP 5153.00 ePP 5404.00 SIM 127.94318 ePdiff48 43.00 KVT $\quad 127.97 \quad 313$ ePKP 5158.00 AAE $\quad 128.08 \quad 272$ ePKP $52 \quad 03.00$

$\begin{array}{llllll}\text { SLL } & 128.13 & 343 & \text { PKP } & 51 & 59.25\end{array}$

REY $128.21 \quad 4$ ePKP 5157.20

$\begin{array}{llllll} & & & i & 52 & 06.10 \\ & 128.22 & 345 & \text { PKP } & 51 & 51.60\end{array}$ HFS 128.30343 PKP 5159.10 $\begin{array}{cc}128.30 & 343 \text { PKP } \\ 1.0 \mathrm{~s} & 48.00 \mathrm{~nm}\end{array}$

$\begin{array}{ll}4 & 5100.00\end{array}$

TBY $\quad \begin{array}{llllll}128.59 & 343 & \text { PKP } & 51 & 59.65\end{array}$

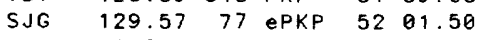
$1.0 \mathrm{~s} \quad 45.00 \mathrm{~nm}$

$z 20 \mathrm{~s} 23.40 \mathrm{um}$

$e^{5518.00}$

KON $\quad 129.83 \quad 345$ iPKP $52 \quad 02.50$

KON $129.83 \quad 345$ ePdiff48 52.00

BER $\quad 130.18 \quad 348$ ePdiff49 01.00

KIS 130.32322 iPKP $52 \quad 04.00$ $\begin{array}{lll}Z & 20 \mathrm{~s} & 30.00 \mathrm{um} \\ \mathrm{N} & 20 \mathrm{~s} & 24.00 \mathrm{um}\end{array}$

E $20 \mathrm{~s} \quad 18.00 \mathrm{um}$

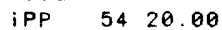

KIS 130.32322 ePdiff 4856.00

IAS 131.01323 ePKP 5204.00

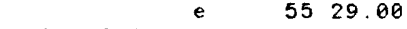

$\begin{array}{lllll}\text { WAR } \quad 131.45332 \text { ePKP } & 52 & 02.00 \\ & & \text { e } & 52 & 38.00\end{array}$

PPE $131.47 \quad 322$ EPKP 5208.00

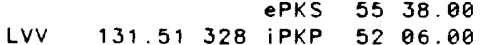
$\begin{array}{lll}\text { iPKP } & 52 & 06.00 \\ \text { iPP } & 54 & 28.00\end{array}$ IPPP $57 \quad 14.00$ iSKS $59 \quad 18.00$ iSS 1202.00

LVV 131.51328 ePdiff 4900.00

$\begin{array}{lllll}\text { CFR } & 131.65 & 321 & \text { ePKP } 52 & 12.00\end{array}$

$\begin{array}{lll}\text { ePP } & 54 & 36.50\end{array}$ ePKS $55 \quad 39.50$ eSKS 5540.00 $3.4 \times$ 0.5

$-3.7 \times$

$-0.2$

$2.9 \times$

$7.2 \times$
ESKKS 5851.00

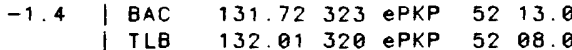
$\begin{array}{lll}\text { ePP } & 54 & 30.00\end{array}$

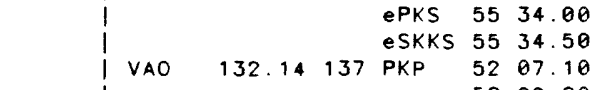
$\begin{array}{llllll} & & \text { ePKS } & 55 & 34.00 \\ \text { V VAO } & 132.14 & 137 & \text { PSKKS } & 55 & 34.50 \\ \text { I } & & 52 & 07.10\end{array}$ e 5209.80
VAOT $\begin{array}{llllll}\text { VRI } & 132.18 & 322 & \text { ePKP } & 52 & 07.00 \\ \text { ePP } & 54 & 32.00 \\ & & & & \end{array}$ \begin{tabular}{l|lllll}
$3.1 \times$ & COP $132.32 \quad 340$ iPKP 52 & 11.00
\end{tabular} 2. $1 \times$ I COP $Z 22 \mathrm{~s} \quad 44.00 \mathrm{um}$
$\mathrm{iPP}$ iPKS $55 \quad 40.00$ I GPA $132.33 \quad 314$ ePKP $52 \quad 07.00$ \begin{tabular}{l|lllll}
$2.6 \times$ & ISR & 132.66 & $321 \mathrm{ePKP}$ & 52 & 10.00
\end{tabular} $\begin{array}{llll}2.5 \times 1 & \text { ePP } & 54 & 32.00 \\ 2.2 \times & \text { ePKS } & 55 & 37.00\end{array}$ $6.2 \times \quad$ ESKKS 5537.50 I MUD 132.69343 ePKP 5209.00 I MLR $132.84 \quad 322$ ePKP $52 \quad 06.00$ $\begin{array}{lll}\text { EPP } & 54 & 34.00\end{array}$ ePKS 5536.00 eSKKS 0121.00

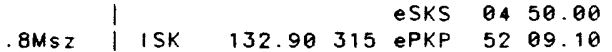

5. $2 x$

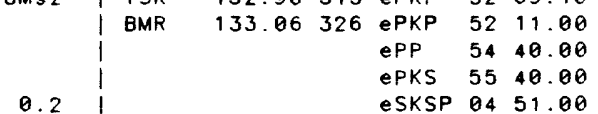
UZH 133.13327 iPKP $52 \quad 10.00$

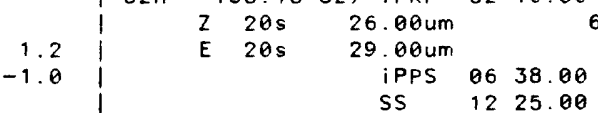
$\begin{array}{rll}5 S & 1225.00 \\ 5 S 5 & 1720.00\end{array}$ $\begin{array}{lllll}5.5 \times \mid \text { UZH } & 133.13 \quad 327 \text { ePdiff49 } 10.00\end{array}$ \begin{tabular}{r|rrrrr} 
& BUC & 133.30321 & ePKP & 52 & 08.00 \\
& & & ePP & 54 & 30.00 \\
1.3 & & & ePKS & 55 & 40.00 \\
0.7 & K & & &
\end{tabular} -0.7 I KRA 133.41330 ePKP $52 \quad 02.00$ $\begin{array}{lll}Z & 28 \mathrm{~s} & 67.00 \mathrm{um}\end{array}$

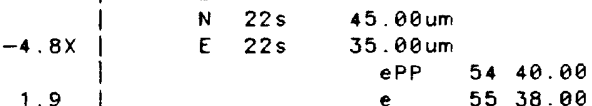

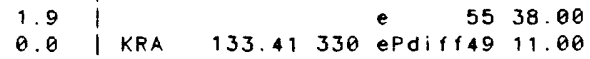
$3.7 X \mid \mathrm{MDB} \quad 133.42324$ ePKP 5213.00 1.6 CMP $\begin{array}{llll}1.6 & \text { CMP } 133.51322 \text { ePKP } 5204.00\end{array}$

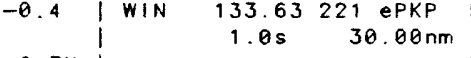
$-6.3 x$ NIE

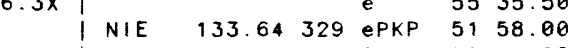
SPC

$.9 \mathrm{Msz}$ FDF

\begin{tabular}{l|l}
1.6 & DRA \\
\hline DEV
\end{tabular}

$10.4 \times$ PVL .OMsz

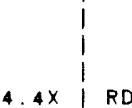

0.5 RDJ

\begin{tabular}{l|l|l} 
& BRN \\
-2.2 & HLW
\end{tabular}

\begin{tabular}{l|l}
-2.2 & HLW \\
$3.6 \times$ & DIM
\end{tabular}

$\mathrm{KDZ}$

\begin{tabular}{l|l|l}
1.6 & $1 Z M$ \\
EDU
\end{tabular}

EDU

EZN

I TIM

$3.2 X \mid E L O$

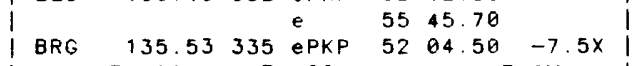

133.83329 ePKP

$\begin{array}{ll}52 & 14.00 \\ 01 & 23.00\end{array}$

$\begin{array}{llll}329 & \text { ePKP } 52 \quad 04.10\end{array}$ iPP

134.02315 ePKP $54 \quad 37.20$ 5537.00

$134.22 \quad 82$ ePKP

134.29322 ePKP

134.42324 ePKP

134.52320 ePKP

134.55333 ePKP 5208.00 5203.50 5206.00 5209.00 5430.00 5214.00 5202.00 $52 \quad 15.50$ $55 \quad 43.50$ 0123.50 0455.60

$134.61 \quad 141$ ePKP 134.61141 PKP 5208.00 134.64337 EPKP $52 \quad 11.00$ 134.66300 ePKPC $52 \quad 14.00$

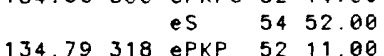
135.14318 ePKP 135.25313 ePKP 135.30351 ePKP \begin{tabular}{ll|l|} 
& \\
& &
\end{tabular} $5201.00-10.6 \times \mid$ SKO $5204.80-7.1 x$ $\begin{array}{lll}52 & 11.70 \quad 0.3\end{array}$

135.31315 ePKP 135.46325 ePKP 135.48352 ePKP 5544.80

8. $1 \times$ E 20 $35.00 \mathrm{um}$

$2.5 X$ | BUD 135.56328 ePKP 5213.80 0.6

1.2 EBH

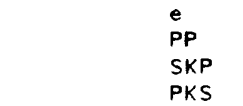
5450.00 SKP $55 \quad 46.00$ $S 5$
$P K S .00$

$\begin{array}{llllll}5.3 \times & \text { EBH } & 135.67 \quad 352 \text { ePKP } 52 & 12.80\end{array}$ IMSZ SSR 135.67323 ePKP 5546.00 I $\begin{array}{llllllll}1.8 & \text { SRO } 135.70 & 329 & \text { ePKP } & 52 & 11.60 & -0.8\end{array}$

\begin{tabular}{l|lll}
1.8 & $N$ & $18 s$ & $27.90 u m$
\end{tabular}

$3.2 \times \quad$ E $18 \mathrm{~s} \quad 26.00 \mathrm{um}$

$3.2 X$ IPA 5456.00

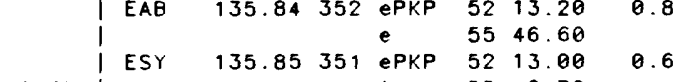
\begin{tabular}{l|lll}
$2.6 \mathrm{X}$ & i 5546.70
\end{tabular} 135.93334 iPKP 5205.00 e $\quad 5213.00$ $-7.8 x$

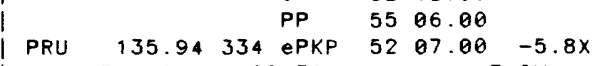
$\begin{array}{llll}2 & 28 \mathrm{~s} & 28.30 \mathrm{um} & \\ \mathrm{N} & & \end{array}$

\begin{tabular}{l|lll}
1.8 & $N$ & $18 \mathrm{~s}$ & $23.80 u m$
\end{tabular} $18 \mathrm{~s} \quad 16.70 \mathrm{um}$ $\begin{array}{lll}\text { e } & 52 \quad 15.50\end{array}$ PP $\quad 54 \quad 48.00$

$2.5 \times$ । VTS $6.9 \mathrm{MSZ}$ I ZST I

EAU

$5.9 \times \quad$ BAO

0.1 I VIE $-6.0 \times$

.4MSZ VKA BEO

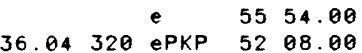

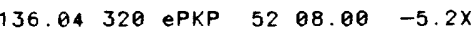
136.05330 ePKP $52 \quad 14.10$ 136.05351 IPP 5455.70 $136.06 \quad 351$ ePKP $52 \quad 13.80$ $\begin{array}{rllll} & e & 55 & 47.40 \\ 136.24 & 129 & \text { e(PKP) } 52 & 03.00 & -11.5 x\end{array}$ $\begin{array}{lllll}136.37 & 331 & \text { ePKPC } 52 & 06.00 & -7.7 x\end{array}$ $\begin{array}{lll}\text { i } & 5216.00 \\ \text { ePP } & 54 & 49.00 \\ \text { ESP } & 0456.00\end{array}$ $\begin{array}{lll}\text { i } & 52 & 16.00 \\ \text { ePP } & 54 & 49.00 \\ \text { eSP } & 04 & 56.00\end{array}$

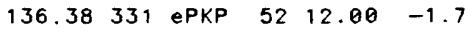
$\begin{array}{llll} & \text { e } & 54 & 57.00 \\ 136.44 & 324 & \text { PKP } & 5208.00\end{array}$ $\begin{array}{lll}\text { PKP } & 52 & 08.00 \\ \text { iPCP } & 52 & 22.60\end{array}$ $5.7 \times \quad$ iPP 5450.00 $4.8 \times$

$-4.4 x$

$5.6 \times$ ESK is $\quad 0117.20$

136.52351 ePKP $52 \quad 16.00$ $136.57 \quad 342$ ePKP $52 \quad 16.50$ e $\quad 52 \quad 48.50$

ePP 5458.50

e $\quad 5555.50$

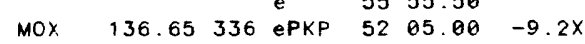
$Z 20 \mathrm{~s} 41.50 \mathrm{um} \quad 7.2 \mathrm{Msz}$

$\mathrm{N} 19 \mathrm{~s} \quad 30.40 \mathrm{um}$

e $\quad 5216.50$ ePP 5459.00 e(PKS) 5550.00 EPKKP 8128.80 (PKKS) $04 \quad 10.00$

$-3.8 \times$

$-1.0$

$3.7 \times$ I KHC

$-8.2 \times$

$5200.00-11.9 x$ $52 \quad 13.80$ $\begin{array}{ll}52 & 13.00 \\ 52 & 12.30\end{array}$

$\begin{array}{|llllll|} & & \text { e } & 55 & 45.70 & \\ \text { BRG } 135.53 & 335 \text { ePKP } & 52 & 04.50 & -7.5 \times\end{array}$

$\begin{array}{lll}Z & 22 \mathrm{~s} & 37.00 u m \\ N & 20 \mathrm{~s} & 22.00 \mathrm{um}\end{array}$ 
$05 d 20 h$

DBN $137.64 \quad 342$ ePKP $5204.00-11.9 \times \quad$ LSF 22

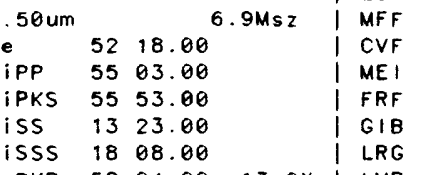

\begin{tabular}{lrlll|l} 
& ISSS 1808.00 & \\
ATH $137.96 \quad 314$ ePKP & 5204.00 & $-13.0 \times$ & LRG
\end{tabular}

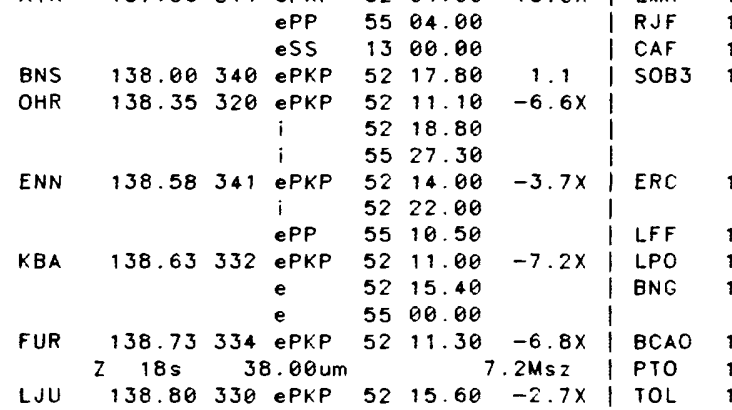
$\begin{array}{lll}\text { ePP } & 55 & 12.00 \\ \text { ePKS } & 55 & 50.50\end{array}$

$\begin{array}{lllllll} & & \text { EPPP } & 58 & 14.50 \\ \text { TIR } \quad 138.81 & 320 & \text { PKP } & 52 & 17.00\end{array}$

UCC $139.03 \quad 342$ ePKP $52 \quad 11.00$ $\begin{array}{lll}\text { PKP } & 52 & 19.50\end{array}$

$\begin{array}{lllll}\text { TRI } 139.42 & 330 \text { IPKP } 52 & 19.30\end{array}$ IPP $\quad 55 \quad 16.20$ i $\quad 5650.00$ ESKS 5922.00

WLF $\quad 139.45 \quad 340$ ePKP $52 \quad 13.00$ PP $\quad 5520.00$ PPS $07 \quad 38.00$ SS 1338.00

BUH $\quad 139.54337$ EPKP $52 \quad 15.50$

DOU $139.58 \quad 342$ PKP $52 \quad 13.00$ $\begin{array}{lll}\text { PP } & 55 & 18.00 \\ \text { SKP } & 56 & 00.00\end{array}$ $\begin{array}{lll}\text { SKP } & 56 & 00.00 \\ \text { PKKP } & 01 & 33.50\end{array}$ PPS 0740.00 SS 1340.00

CDF $\quad 140.11338$ ePKP 5213.80

CTI $140.18 \quad 332$ PKP $52 \quad 14.00$ e $\quad 5520.00$

SLE
ECH
BAF
LLS
BSF
HAL
SAL
PRO
TMA
NEC
MMK
DUI
PCN
AQU
PRG
DIX
FIR

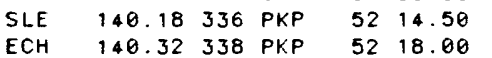

140.70338 PKKP 5217.00

LLS 140.70335 PKPC 5219.00

BSF $\quad 140.78 \quad 338$ ePKP $52 \quad 15.00$

HAU $\quad 140.79 \quad 338$ ePKP $52 \quad 15.20$

$\begin{array}{ccccc}\text { SAL } \quad 141.02332 \text { PKP } & 52 & 19.00 \\ & & 5 & 56 & 07.00\end{array}$

PRO

TMA

NEC

DUI

PRG

$F \mid R$

PRT 141.96328 PKP

ORO

SSC

LOR

MNS

LBF

GRR

RMP

SMF

AVF

LPF

$M Z F$

TCF
PYM
141.12329 PKP

$141.36 \quad 334$ PKPC 141.41337 PKP 141.78335 PKP 141.83325 PKP 141.84333 PKP 141.95326 PKP 141.98336 PKP

142.05330 PKP IPP 5540.00 iSKS 5903.00

$52 \quad 22.50$

5213.50 $52 \quad 24.50$ 5222.00 5230.00 5221.50 $52 \quad 24.00$ 5219.40 $\begin{array}{ll}59 & 03.00 \\ 52 & 24.00\end{array}$ 142.05330 PKP 5231.60 142.12335 PKP 5221.50 $\begin{array}{llll}142.28 & 345 & \text { ePKP } 52 & 18.00\end{array}$ 142.29340 ePKP $52 \quad 18.60$ 142.32327 PKP

142.50340 ePKP 142.59340 ePKP 142.65345 ePKP

iPP

142.84340 ePKP $142.88 \quad 340$ ePKP 143.02345 ePKP 143.64340 ePKP $\begin{array}{lll}143.69 & 341 & \text { ePKP } \\ 143.90 & 340 & \text { PKPd }\end{array}$

5218.00 $56 \quad 04.50$ 5219.70 $\begin{array}{lll}52 & 19.80\end{array}$ 5219.00 5220.00 5528.00 0548.00 5220.20 5220.20 5220.60 5221.30 5221.40 $142.70326 \quad$ IPKP

ALI
$-4.1 x$ ALR

$-6.5 x \mid T E$ $-6.9 \times$ TAM $-6.9 x$ $-6.3 \times$ I

$-4.8 x$
$-3.0 x$ $-6.9 x$

$-6.7 x$

$-3.3 x$

0.0
$-9.6 x$

$-9.6 x$
1.5

-10.6
-1.9

6. $3 x$

$-2.7 x$

-0.1
$-5.0 x$

$-6.2 x$

$-0.2$

$-2.9 \times$

$-6.4 \times$

-6 ex

$-6.7 x$

$-5.3 x$

$-5.2 \times$

$-5.4 x$

\begin{tabular}{l|l|l} 
& $H J J$ \\
$-5.3 x$ & $T K \theta$
\end{tabular}

$-5.1 x \mid T K \theta 3$

$-5.6 \times \mid T K \theta 4$

$-5.6 \times$ I OSH

$-2.4 \times$ 5213.40

$143.94 \quad 342$ ePKP $52 \quad 22.20 \quad-5.2 \times$ ।

$\begin{array}{lllllll}144.11 & 344 & \text { ePKP } & 52 & 22.40 & -5.2 \times & \text { I OMA }\end{array}$ 144.11331 ePKP $5221.30 \quad-6.5 \times$ । TMS 144.20318 PKP 5229.50 $144.35 \quad 334$ ePKP $52 \quad 22.40$ 144.43320 PKP- 5228.50 144.55334 ePKP $52 \quad 23.28$ 144.59334 ePKP $52 \quad 23.20$ 144.79341 ePKP $52 \quad 24.20$ 144.95340 ePKP $52 \quad 24.60$ 1.5 $-5.7 \times \mid$ TAT $-0.1$ $-5.2 x \mid K T J$ $-5.3 \times \mid \mathrm{SHJ}$ $-4.6 \times$ $-4.6 \times$

\begin{tabular}{l|lll}
-1.4 & ALI $151.63 \quad 337$ IPKPd 5242.00
\end{tabular}

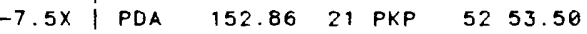

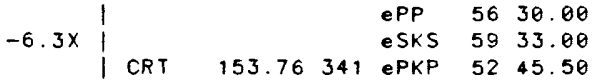
\begin{tabular}{llllll}
\hline SFS & 155.19 & 345 & iPKPC & 52 & 49.00
\end{tabular}

$-6.1 x \mid \mathrm{CB}$ ।

$-5.3 x \mid T K \theta 2$ $145.01 \quad 128$ ePKP 5228.00 $\begin{array}{ccc}145.01128 \text { ePKP } & 5228.00 \\ e & 5229.60\end{array}$ $\begin{array}{lll}\text { e } & 5231.80 \\ \text { e } & 5239.40\end{array}$ $\begin{array}{lllll}145.37 & 341 & \text { ePKP } & 51 & 42.50 \\ 145.45 & 341 & \text { ePKP } & 52 & 26.80\end{array}$ $\begin{array}{llll}145.45 & 341 & \text { ePKP } 52 \quad 26.20\end{array}$ 146.81259 iPKPC 5231.40 $1.05 \quad 31.60 \mathrm{~nm}$

$146.82 \quad 259$ e(PKP) $52 \quad 31.00$ 151.15351 IPKP 5241.00

151.41344 PKPC $52 \quad 45.00$ $\begin{array}{lll}\text { iPP } & 55 & 38.00 \\ \text { iPKS } & 57 & 10.00\end{array}$ isS 1630.00 153.35253 PKP+ 5247.90 153.60351 PKP 5246.00 $\begin{array}{lll}i & 53 & 07.60 \\ \text { LR } & 54 & 24.00\end{array}$ $\begin{array}{lll}\text { LR } & 54 & 24.00 \\ \text { ePP } & 56 & 30.00\end{array}$ 154.71339 IPKP 5247.00 $i \quad 5305.50$ $\begin{array}{lll}\text { i } & 53 & 16.00 \\ \text { i SP } & 55 & 21.00 \\ \text { iSP } & 0708.00\end{array}$ $\begin{array}{lll}\text { iSP } & 0708.00 \\ \text { iSS } & 1705.00\end{array}$ 158.80301 PKP $53 \quad 17.00$ 5302.60 e $\quad 54 \quad 15.80$

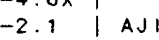

I HMM

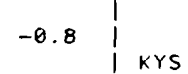

$-4.0 \times$ I SHZ

$-3.8 \times \mid \mathrm{MIS}$

$-1.7$

-2.2 OWA

2.0

5. $5 x$ YOK

OYM

$2.2 \times \mid$ TSU

$1.9 \times \mid$ FUN

$4.8 X$ SRY

$3.4 \times$ TOK

WKYJ

$2.5 \times \mid 110 J$

2. $9 x$ KOF

NAG

4. $2 \times$

NAR

WKY

$27.1 \times \mid$ MRT

$3.5 X$

$\begin{array}{lll}\text { PP } & 58 & 01.80 \\ \text { PPKP } & 53 & 03.80\end{array}$

$176.63 \quad 57$ ePKP 5303.80

e $\quad 5310.70$

$\begin{array}{lll}\text { e } & 5325.10 \\ \text { e } & 5408.90\end{array}$

5450.90

5838.90

$\begin{array}{lll}58 & 57.90\end{array}$

01 15.00

0134.90

0213.00

0310.90

S.D. $=1.4$ on 257 of 526 obs.

1.91

osk

DDR

OSA

GIF

I

$\mathrm{CHJJ}$

I KMG

I SUM

I KAKJ

TSK

SEP $06,198201 \mathrm{~h} 47 \mathrm{~m} 03.85 \pm 0.20 \mathrm{~s}$ TKS $29.414 \mathrm{~N} \pm 1.4 \mathrm{~km} \quad 140.397 \mathrm{E} \pm 1.5 \mathrm{~km}$

DEPTH $=185.1 \pm 1.7 \mathrm{~km}$

$6.5 \mathrm{mb}$ ( 97 Obs.)

SOUTH OF HONSHU, JAPAN

(211)

Fukushimo. Felt (1) JMA) on

Chichi-jima, Hachijo-jimo ond

Oshimo and ot Yokohoma, Choshi.

Tokyo, Kumagaya, Mito.

Utsunomiyo, Onahama, Kakioka,

Sendoi, Morioko, Miyoko ond

Ofunoto.

$2.80 \quad 145 \mathrm{P}$

$\begin{array}{llll}3.71 & 352 & \mathrm{~S}+\end{array}$

4. $95332 \mathrm{P}$

$5.05334 \mathrm{P}-$

$5.17337 \mathrm{P}-$

$5.40337 \mathrm{P}$

$5.40351 \mathrm{P}-$

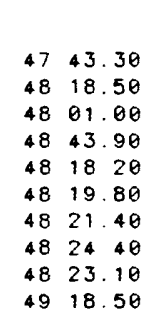

$-7.4 x$

HIK

i

KYO

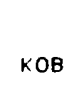

MIT

MAE

MAE

\begin{tabular}{l|l}
-1.0 & TKS \\
& KAZ
\end{tabular}

0.5 UTS

\begin{tabular}{l|}
0.8 \\
0.8
\end{tabular}

0.7 ASZ

\begin{tabular}{r|r}
0.7 & ASZ \\
-0.7 &
\end{tabular}

KOC \begin{tabular}{l|l}
$7.5 \times$ & \\
$4.2 \times$ & 110
\end{tabular}

5937.90

iscs $02 \quad 01.20$

$\begin{array}{lllll}5.50341 \mathrm{P} & 48 & 25.40 & 0.4\end{array}$

$5.51355 \mathrm{P} \quad 4825.10$

5.57356 is 4925.50

$\begin{array}{lll}48 & 24.50 & -1.5\end{array}$

$\begin{array}{ll}49 & 25.50\end{array}$ 


\begin{tabular}{|c|c|c|c|c|c|c|c|c|c|c|c|c|}
\hline$H I M$ & 7.25 & 320 & P- & 48 & 49.30 & 1.2 & SAI & 9.01 & 321 & $\begin{array}{l}\text { escs } \\
\text { p }\end{array}$ & $\begin{array}{l}02 \\
49\end{array}$ & $\begin{array}{l}08.00 \\
11.00\end{array}$ \\
\hline & & & is & 50 & 15.50 & & i & & & s & 50 & 57.28 \\
\hline TKM & 7.28 & 314 & P- & 48 & 50.48 & 1.9 & I ISN & 9.02 & 5 & P+ & 49 & 07.70 \\
\hline & & & is & 50 & 17.00 & & I & & & is & 50 & 39.00 \\
\hline & & & escs & 82 & 07.00 & & I & & & escs & 02 & 08.00 \\
\hline MAT & 7.34 & 346 & P- & 48 & 47.80 & -1.6 & I SHNJ & 9.19 & 303 & P- & 49 & 15.50 \\
\hline & & & $s$ & 50 & 06.00 & & I SHN & 9.24 & 302 & $P$ & 49 & 16.30 \\
\hline MAI & 7.38 & 326 & $\mathrm{P}$ & 48 & 50.60 & 0.8 & 1 & & & $\mathrm{~s}$ & 51 & 84.90 \\
\hline & & & es & 58 & 18.00 & & 1 & & & $\operatorname{ses}$ & 02 & 08.70 \\
\hline MZH & 7.39 & 326 & $P$ & 48 & 50.60 & 0.6 & I SAG & 9.44 & 296 & P- & 49 & 19.50 \\
\hline & & & es & 50 & 18.00 & & 1 & & & $s$ & 51 & 05.20 \\
\hline NGN & 7.46 & 346 & $\mathbf{P}_{-}$ & 48 & 49.70 & -1.2 & 1 & & & escs & 02 & 10.00 \\
\hline & & & es & 58 & 08.20 & & I FKK & 9.51 & 298 & P- & 49 & 20.30 \\
\hline FUK & 7.49 & 333 & P- & 48 & 52.50 & 1.1 & 1 & & & $s$ & 51 & 13.28 \\
\hline & & & s & 58 & 15.60 & & 1 & & & $\operatorname{ses}$ & 02 & 10.50 \\
\hline ONA & 7.52 & 3 & $\mathrm{P}$ & 48 & 48.60 & $-3.1 x$ & I NZJ & 9.61 & 266 & $P$ & 49 & 22.00 \\
\hline & & & is & 50 & 06.80 & & 1 & & & es & 51 & \\
\hline & & & $\operatorname{scs}$ & 82 & 04.00 & & I NGS & 9.61 & 293 & $P$ & 49 & 21.70 \\
\hline OKA & 7.60 & 315 & P & 48 & 54.46 & 1.6 & 1 & & & $s$ & 51 & 10.60 \\
\hline & & & $s$ & 50 & 25.28 & & I OFU & 9.69 & 6 & $P+$ & 49 & 16.00 \\
\hline & & & escs & 02 & 88.00 & & 1 & & & is & 50 & 55.60 \\
\hline TYK & 7.70 & 324 & P- & 48 & 55.10 & 0.9 & 1 & & & $\operatorname{ses}$ & 02 & 09.20 \\
\hline & & & is & 50 & 24.30 & & $I M I Z$ & 9.71 & 3 & iPd & 49 & 17.80 \\
\hline & & & escs & 02 & 88.00 & & I MRK & 18.28 & 3 & $P$ & 49 & 25.00 \\
\hline UWA & 7.71 & 302 & P- & 48 & 56.00 & 1.8 & 1 & & & $\mathrm{~s}$ & 51 & 10.48 \\
\hline TOY & 7.75 & 341 & $P$ & 48 & 55.80 & 0.3 & $|A K|$ & 18.28 & 359 & $P$ & 49 & 25.00 \\
\hline & & & (S) & 50 & 21.08 & & 1 & & & es & 51 & 13.00 \\
\hline KAN & 7.78 & 337 & P- & 48 & 55.38 & 0.2 & $\mid M I Y$ & 10.29 & 7 & P & 49 & 26.00 \\
\hline & & & es & 50 & 15.00 & & 1 & & & is & 51 & 08.80 \\
\hline MTY & 7.85 & 306 & P- & 48 & 58.70 & 2.6 & 1 & & & escs & 02 & 12.00 \\
\hline & & & is & 50 & 31.00 & & $1 \mathrm{FKJ}$ & 10.44 & 291 & P- & 49 & 31.78 \\
\hline & & & escs & 02 & 07.00 & & & & & es & 51 & 37.00 \\
\hline TKD & 7.88 & 347 & $P$ & 48 & 54.50 & -1.9 & 1120 & 10.58 & 300 & P- & 49 & 34.08 \\
\hline & & & s & 50 & 20.58 & & 1 & & & es & 51 & 29.00 \\
\hline$N \mid I J$ & 7.89 & 352 & $\mathbf{P}$ & 48 & 53.10 & $-3.5 x$ & 1 & & & Ses & 02 & 14.50 \\
\hline TOT J & 7.96 & 321 & $P$ & 48 & 59.50 & 2.0 & HAC & 11.12 & 4 & $\mathbf{P}$ & 49 & 35.00 \\
\hline TOT & 8.03 & 321 & $P$ & 48 & 58.50 & 0.0 & I & & & es & 51 & 28.08 \\
\hline & & & $s$ & 50 & 33.30 & & 1 & & & escs & 82 & 12.00 \\
\hline & & & escs & 02 & 09.00 & & NGO & 11.33 & 259 & $P$ & 49 & 43.50 \\
\hline MYZ & 8.12 & 290 & $\mathbf{P}$ & 49 & 02.28 & 2.6 & 1 & & & $s$ & 51 & 55.80 \\
\hline & & & es & 50 & 45.00 & & $A O M$ & 11.38 & 1 & $P$ & 49 & 41.30 \\
\hline & & & escs & 82 & 10.80 & & 1 & & & $s$ & 51 & 37.00 \\
\hline NOB & 8.12 & 295 & $P$ & 49 & 00.80 & 1.2 & 1 & & & $\operatorname{ScS}$ & 02 & 14.30 \\
\hline & & & $s$ & 50 & 44.80 & & NAH & 11.70 & 257 & $P$ & 49 & 47.60 \\
\hline TAJ & 8.25 & 281 & P- & 49 & 03.80 & 2.5 & 1 & & & is & 52 & 03.50 \\
\hline & & & $s$ & 50 & 44.40 & & HAK & 12.38 & 1 & $P$ & 49 & 51.60 \\
\hline & & & $S c S$ & 02 & 10.10 & & 1 & & & s & 52 & 02.90 \\
\hline SHK & 8.31 & 310 & iPd & 49 & 03.30 & 1.2 & 1 & & & escs & 92 & 15.00 \\
\hline FKS & 8.32 & 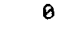 & $P$ & 48 & 58.00 & $-4.2 x$ & I KMJ & 12.41 & 259 & $P$ & 49 & 56.00 \\
\hline & & & is & 50 & 29.00 & & 1 & & & is & 52 & 19.00 \\
\hline & & & $\operatorname{ses}$ & 02 & 06.70 & & I URA & 12.86 & 8 & $\mathrm{P}$ & 49 & 59.80 \\
\hline HIR & 8.37 & 308 & $P$ & 49 & 04.70 & 1.8 & i & & & es & 52 & 13.00 \\
\hline & & & $\mathrm{s}$ & 50 & 45.00 & & 1 & & & escs & 82 & 19.00 \\
\hline & & & Ses & 02 & 07.00 & & I MRRJ & 3.00 & 2 & $P$ & 49 & 59.00 \\
\hline KAGJ & 8.41 & 284 & $\mathbf{P}$ & 49 & 05.50 & 2.1 & $1 \mathrm{HOOJ}$ & 13.15 & 9 & $P$ & 50 & 02.90 \\
\hline 011 & 8.41 & 299 & $\mathbf{P}$ & 49 & 06.40 & $3.0 x$ & I SUT & 13.35 & 359 & $P$ & 50 & 06.10 \\
\hline & & & es & 50 & 56.00 & & 1 & & & $s$ & 52 & 28.80 \\
\hline WAJ & 8.47 & 341 & $P$ & 49 & 03.30 & -0.9 & S SAP & 13.64 & 3 & $P$ & 50 & 07.80 \\
\hline & & & es & 50 & 38.00 & & & & & es & 52 & 33.00 \\
\hline$N I I$ & 8.55 & 353 & P- & 49 & 04.60 & -0.6 & 1 & & & escs & 02 & 17.00 \\
\hline & & & es & & 35.00 & & $|O B|$ & 13.67 & 9 & $P$ & 50 & 10.10 \\
\hline MTS & 8.64 & 316 & $\mathbf{P}$ & 49 & 08.10 & 1.8 & i & & & $s$ & 52 & 32.00 \\
\hline & & & is & 50 & 52.30 & & I KUS & 13.91 & 12 & $\mathrm{P}$ & 50 & 12.10 \\
\hline ASJ & 8.70 & 296 & P- & 49 & 09.50 & 2.1 & 1 & & & $s$ & 52 & 35.60 \\
\hline & & & $s$ & 50 & 54.10 & & I KUSJ & 14.09 & 13 & $P$ & 50 & 14.60 \\
\hline YAMJ & 8.74 & 358 & ePc & 49 & 06.00 & -1.7 & MYK & 14.23 & 255 & $P$ & 50 & 20.50 \\
\hline & & & s & 50 & 37.50 & & 1 & & & es & 53 & 06.20 \\
\hline & & & $\operatorname{scs}$ & 02 & 0650 & & ASA & 14.41 & 6 & P & 50 & 18.00 \\
\hline KAG & 8.76 & 287 & $P-$ & 49 & 10.50 & 2.5 & I & & & $s$ & 52 & 49.50 \\
\hline & & & $s$ & 58 & 50.40 & & NEM & 14.50 & 15 & $P$ & 50 & 19.80 \\
\hline & & & ScS & 02 & 10.00 & & & & & is & 52 & 50.00 \\
\hline AlK & 8.77 & 349 & $\mathbf{P}$ & 49 & 04.90 & $-3.2 x$ & I RMJ & 14.54 & 4 & $P$ & 50 & 20.00 \\
\hline & & & es & 50 & 37.00 & & 1 & & & $s$ & 52 & 52.50 \\
\hline YAM & 8.82 & 360 & $\mathrm{P}+$ & 49 & 06.00 & -2.7 & ASAJ & 14.78 & 6 & P & 50 & 22.70 \\
\hline & & & s & 50 & 37.50 & & $A B J$ & 14.90 & 11 & $P$ & 50 & 25.80 \\
\hline & & & $\operatorname{ses}$ & 02 & 06.50 & & I & & & es & 53 & 00.00 \\
\hline SEN & 8.83 & 3 & P- & 49 & 06.00 & -2.9 & I & & & escs & 02 & 21.00 \\
\hline & & & s & 50 & 36.20 & & 151 & 15.34 & 255 & $P$ & 50 & 35.70 \\
\hline & & & $\mathrm{ScS}$ & 82 & 07.00 & & & & & $\mathrm{~s}$ & 53 & 35.00 \\
\hline MVI & 8.88 & 248 & $P$ & 49 & 88.00 & -1.5 & WAK. & 16.01 & 3 & $P$ & 50 & 40.00 \\
\hline & & & s & 50 & 48.60 & & 1 & & & es & 53 & 31.00 \\
\hline HMD & 8.92 & 310 & $P$ & 49 & 12.60 & 2.5 & $P J G$ & 16.28 & 164 & eP & 50 & 42.20 \\
\hline & & & s & 50 & 52.00 & & GUA & 16.34 & 164 & eP & 50 & 42.80 \\
\hline & & & es & 02 & 06.00 & & ! & $0.8 \mathrm{~s}$ & 158 & $9.88 \mathrm{~nm}$ & & \\
\hline KUM & 8.97 & 295 & $\mathbf{P}$ & 49 & 13.50 & 2.8 & 1 & & & es & 53 & 36.00 \\
\hline
\end{tabular}


$06 \mathrm{~d} 01 \mathrm{~h}$

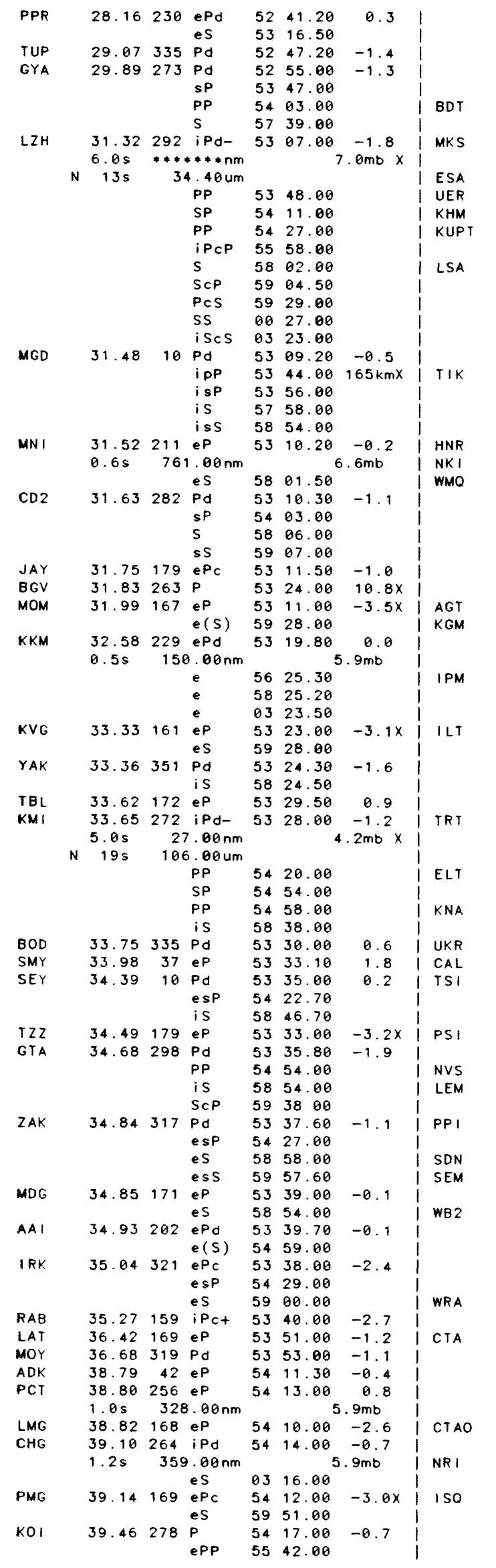

\begin{tabular}{|c|c|c|c|c|c|c|c|c|c|}
\hline & & $\mathrm{e}$ & 57 & 18.00 & & I VAR & 50.70 & 280 & P+ \\
\hline & & es & 59 & 54.00 & & I PRZ & 50.82 & 303 & $\mathrm{Pc}$ \\
\hline & & e & 01 & 23.00 & & $i$ & & & es \\
\hline & & e & 02 & 14.00 & & i TLG & 51.47 & 304 & Pd \\
\hline & & e & 04 & 03.50 & & I AAA & 51.79 & 304 & $\mathrm{Pd}$ \\
\hline 9.70 & 262 & iPd & 54 & 13.00 & $-6.6 x$ & I svw & 52.19 & 34 & $P$ \\
\hline $0.8 \mathrm{~s}$ & 51 & $4.00 \mathrm{~nm}$ & & 6 & $.2 m b$ & I TTA & 52.26 & 31 & ep \\
\hline 39.87 & 214 & IPC & 54 & 20.70 & -0.3 & I NRN & 52.73 & 302 & eP \\
\hline $0.6 \mathrm{~s}$ & 25 & $8.00 \mathrm{~nm}$ & & 6 & $.0 \mathrm{mb}$ & I KSH & 53.06 & 299 & $P d$ \\
\hline 40.20 & 164 & $i P c$ & 54 & 22.50 & -1.1 & i & & & sP \\
\hline 40.72 & 316 & $\mathrm{PC}$ & 54 & 26.50 & -1.1 & i & & & $P C P$ \\
\hline 41.04 & 276 & eP & 54 & 29.00 & -1.8 & I ASPA & 53.14 & 187 & Pd \\
\hline 42.54 & 205 & ep & 54 & 41.20 & -1.5 & i & & & es \\
\hline & & es & 55 & 33.70 & & I VIS & 53.16 & 271 & P- \\
\hline 42.57 & 283 & $P d$ & 54 & 43.20 & -0.4 & 1001 & 53.44 & 288 & P \\
\hline & & $S P$ & 55 & 35.20 & & I KDC & 53.53 & 38 & eP \\
\hline & & PP & 56 & 24.20 & & FRU & 53.54 & 303 & Pd \\
\hline & & PCS & 00 & 27.20 & & 1 & & & $i s P$ \\
\hline & & $s$ & 00 & 52.20 & & i & & & es \\
\hline & & ScS & 04 & 25.20 & & I IMA & 53.75 & 28 & eP \\
\hline
\end{tabular}

$42.74355 \mathrm{Pd} \quad 5425.20$

\begin{tabular}{rrrr|r}
42.74355 Pd & 5444.50 & 0.7 & MBL \\
SP & 5537.00 & & PVC \\
ES & 0056.00 & &
\end{tabular}

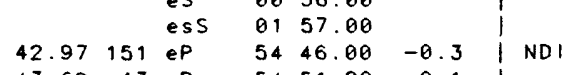

$43.69 \quad 43 \mathrm{eP}$

$43.96304 \mathrm{Pd}$

SP $\quad 5553.40$

$\begin{array}{lll}S P & 55 & 46.00 \\ P P & 56 & 37.00\end{array}$

PCP $56 \quad 40.00$

SCP 0013.50

$S \quad 0118.00$

SS 0420.00

$44.02275 \mathrm{P}$

5458.50

$\begin{array}{ll}44.54 & 239 \mathrm{ePd} \\ 1.3 \mathrm{~s} & 1530.00 \mathrm{~nm}\end{array}$

44.67244 iPd

$45.4320 \stackrel{e}{\mathrm{Pd}}$

ipP

is

iss

$45.56220 \mathrm{iPC}$

$0.9 \mathrm{~s} \quad 158.00 \mathrm{~nm}$

$45.75317 \stackrel{\mathrm{Pd}}{\mathrm{Pd}}$

$46.28 \quad 196 \quad$ is

$46.38314 \mathrm{P}$

$46.96274 \mathrm{P}$

$47.27245 \mathrm{ePd}$

$0.7 \mathrm{~s} \quad 947.00 \mathrm{~nm}$

$\begin{array}{lll}0.7 \mathrm{~s} & 92 \quad 05.00 \\ 4 & \text { es }\end{array}$

$\begin{array}{ll}47.48 & 244 \mathrm{ePd} \\ 0.8 \mathrm{~s} & 51.80 \mathrm{~nm}\end{array}$

$47.73319 \mathrm{Pd}$

$47.88226 \mathrm{iPd}$

$48.36 \quad 239 \mathrm{eP}$
$1.8 \mathrm{~s} \quad 827.00 \mathrm{~nm}$
$48.93 \quad 41 \mathrm{eP}$

$49.20313 \mathrm{Pd}$
$49.41187 \mathrm{PS}$

iScP
iS
iScS
SKKP

$\begin{array}{ll}49.42 & 188 \mathrm{PC} \\ 0.8 \mathrm{~s} & 298.00 \mathrm{~nm}\end{array}$

$\begin{array}{ll}0.8 \mathrm{~s} & 298 \\ 49.54 & 173\end{array}$

$\begin{aligned} & \text { ipP } \\ & \text { iSCP } \\ & \text { is } \\ & \text { ises } \\ 49.54173 & \text { eP } \\ & \text { e }\end{aligned}$

$49.77 \quad 338 \stackrel{\mathrm{Pd}}{\mathrm{Pd}}$

$49.84181 \mathrm{eP}$

e
$00 \quad 17.00$

$55 \quad 00.10$ $\begin{array}{ll}00 & 19.10 \\ 01 & 26.50\end{array}$ 5505.80

5553.00

0234.00

$\begin{array}{lll}55.90 & -1.0\end{array}$

$57 \quad 06.50$

$55 \quad 05.70$

0132.00

$\begin{array}{ll}55 & 11.00 \\ 01 & 41.00\end{array}$

5510.80

5521.00

5520.00

$\begin{array}{lll}55 & 21.20 & -0.9\end{array}$

$\begin{array}{ll}21.70 & -1.8\end{array}$

$\begin{array}{lll}55 & 28.00 & -0.8\end{array}$ $6.2 \mathrm{mb}$ | TAS

\begin{tabular}{ll|l|l|l}
52.60 & -0.1 & BRS
\end{tabular} $\begin{array}{lll}55 & 32.80 & -2.1\end{array}$ $02 \quad 24.20$ $55 \quad 34.20$ $00 \quad 35.00$ 0221.00 0515.00

$-2.6$
$5535.80^{5.9 m b}-1.9$ $56 \quad 15.00 \quad 175 \mathrm{kmX}$ MEK $00 \quad 33.00$ 0224.00 0511.00 5535.50 $56 \quad 09.00$ $55 \quad 37.50$ 0231.00 5538.00 5611.00 $00 \quad 37.00$ 0231.00

HON

FBA

KHO

NOU I

HYB
0131.00

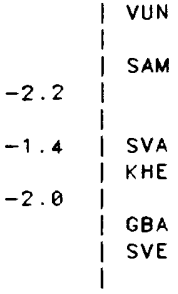

$54.07 \quad 147 \mathrm{PC}$

$54.61286 \mathrm{P}$ $i p P$

is iss

kou<smiles>CCCC[I-]I</smiles>

PMR

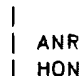




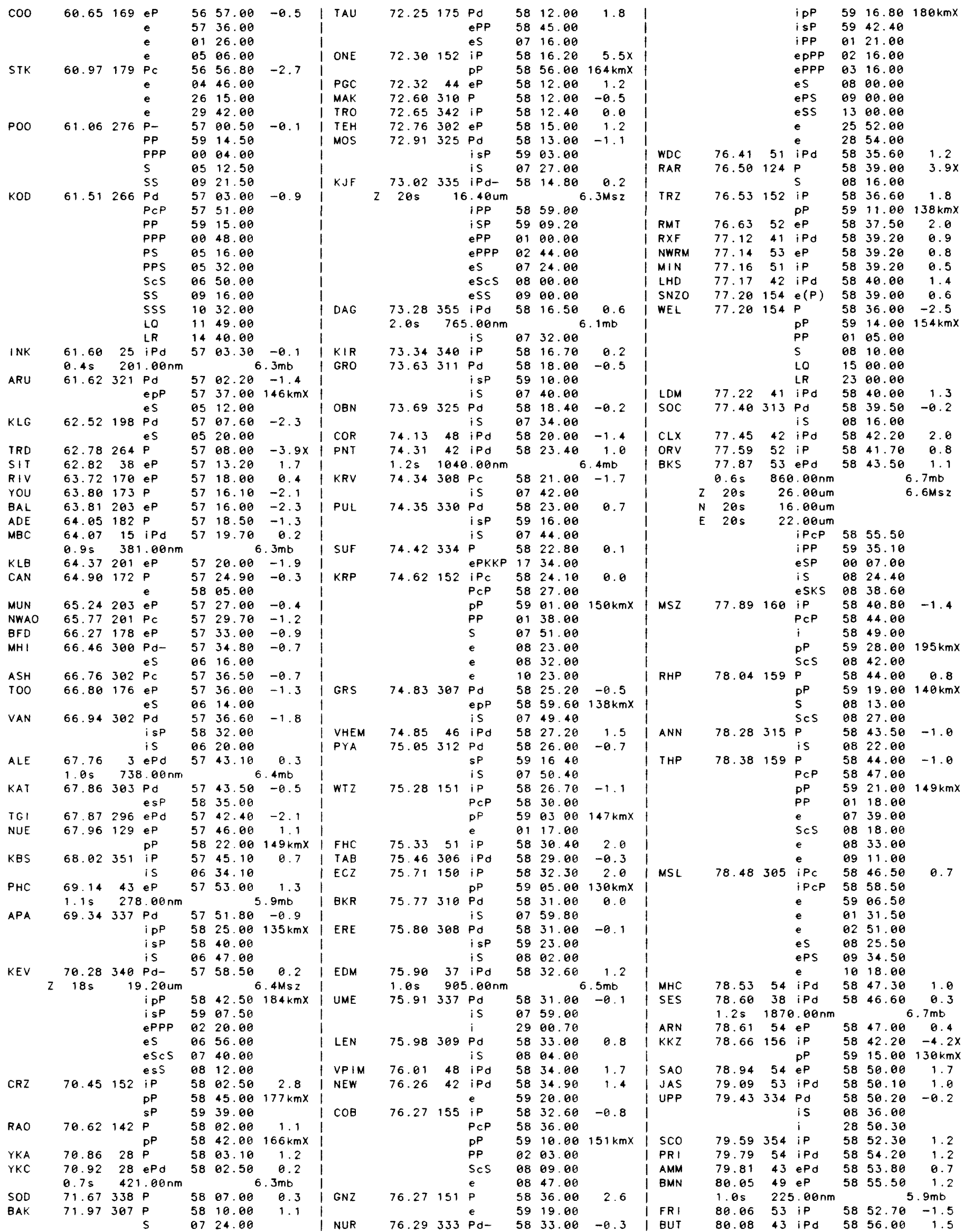


g6d 01h

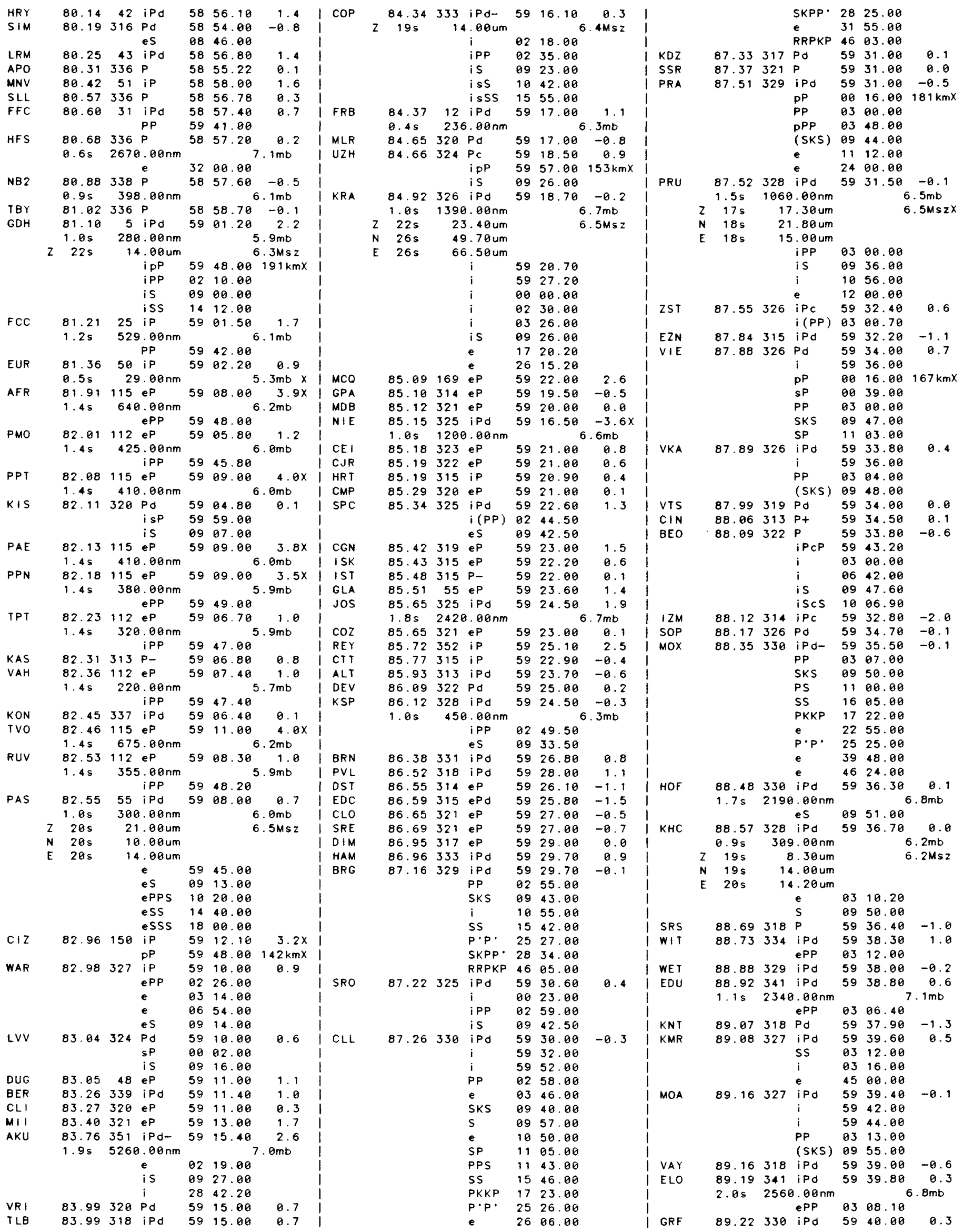




\begin{tabular}{|c|c|c|c|c|c|c|c|c|c|c|c|}
\hline$z$ & $20 s$ & & $7.70 \mathrm{um}$ & & & . $1 \mathrm{MSZ}$ & 1 & & & e & 17 \\
\hline & & & i & 59 & 42.20 & & I LHC & 90.68 & 31 & $e^{P}$ & 59 \\
\hline & & & ePP & 03 & 16.20 & & $|T| R$ & 90.70 & 320 & $\mathbf{P}$ & 59 \\
\hline & & & isKS & 09 & 57.00 & & I STU & 90.79 & 330 & iPd- & 59 \\
\hline & & & es & 10 & 19.30 & & i & 1. $1 \mathrm{~s}$ & 1060 & $0.00 \mathrm{~nm}$ & \\
\hline & & & iPS & 11 & 21.30 & & i & $20 s$ & 14 & $4.20 u m$ & \\
\hline WTS & 89.31 & 333 & iPd & 59 & 39.50 & -0.5 & I GAP & 90.93 & 328 & iPd & 59 \\
\hline & $1.0 \mathrm{~s}$ & & 4. $.00 \mathrm{~nm}$ & & & $.7 \mathrm{mb}$ & i & $1.2 \mathrm{~s}$ & 1740 & $0.00 \mathrm{~nm}$ & \\
\hline & & & ePP & 03 & 12.00 & & | TRI & 90.93 & 326 & eP & 59 \\
\hline & & & es & 09 & 55.00 & & i & & & e (PP) & 03 \\
\hline & & & e & 11 & 22.00 & & 1 & & & e (S) & 10 \\
\hline & & & e & 17 & 22.00 & & 1 & & & e & 11 \\
\hline EBH & 89.32 & 341 & iPd & 59 & 40.40 & 0.3 & 1 & & & $e(S S)$ & 16 \\
\hline & $2.0 \mathrm{~s}$ & 3080 & $0.00 \mathrm{~nm}$ & & & $.9 \mathrm{mb}$ & 1 & & & e & 23 \\
\hline & & & ePP & 03 & 08.00 & & I UCC & 91.20 & 334 & Pd- & 59 \\
\hline ESY & 89.34 & 340 & iPd & 59 & 40.60 & 0.4 & I & & & PP & 03 \\
\hline & $1.1 \mathrm{~s}$ & 1380 & $0.00 \mathrm{~nm}$ & & & $.8 \mathrm{mb}$ & 1 & & & SKS & 10 \\
\hline & & & ePP & 03 & 08.60 & & i & & & $s$ & 10 \\
\hline THE & 89.37 & 318 & $P d$ & 59 & 38.80 & -1.7 & $i$ & & & SP & 11 \\
\hline SKO & 89.40 & 319 & iPd & 59 & 40.70 & 0.0 & I & & & $p P$ & 59 \\
\hline & & & IPCP & 59 & 43.00 & & I BUH & 91.28 & 331 & ep & 59 \\
\hline & & & iPP & 00 & 35.50 & & I GWF & 91.31 & 331 & iPd & 59 \\
\hline & & & iPP & 03 & 16.00 & & I WLF & 91.39 & 332 & ePd & 59 \\
\hline & & & is & 09 & 56.00 & & i & & & PP & 03 \\
\hline & & & iscs & 10 & 24.00 & & 1 & & & SKS & 10 \\
\hline & & & iPS & 11 & 20.00 & & I & & & $s$ & 10 \\
\hline EDI & 89.51 & 340 & iPd & 59 & 41.20 & 0.3 & 1 & & & SP & 11 \\
\hline & $2.0 \mathrm{~s}$ & 1920 & $0.00 \mathrm{~nm}$ & & & $.7 \mathrm{mb}$ & 1 & & & e & 16 \\
\hline & & & ePP & 03 & 10.00 & & I OGA & 91.39 & 328 & iPd & 59 \\
\hline & & & isKS & 09 & 53.80 & & I STR & 91.55 & 331 & P- & 59 \\
\hline EBL & 89.59 & 340 & iPd & 59 & 41.90 & 0.5 & 1 & & & ePP & 03 \\
\hline & $2.0 \mathrm{~s}$ & 4046 & $0.00 \mathrm{~nm}$ & & & $.0 \mathrm{mb}$ & 1 & & & is & 10 \\
\hline & & & iPP & 03 & 11.00 & & 1 & & & $e$ & 35 \\
\hline EAU & 89.65 & 341 & i Pd & 59 & 42.20 & 0.6 & I DOU & 91.67 & 333 & $\mathrm{Pd}-$ & 59 \\
\hline & $1.0 \mathrm{~s}$ & 1100 & $0.00 \mathrm{~nm}$ & & & $.8 \mathrm{mb}$ & I & & & $P P$ & 00 \\
\hline & & & ePP & 03 & 11.00 & & 1 & & & $P P$ & 03 \\
\hline DBN & 89.85 & 334 & eP & 59 & 43.00 & 0.4 & 1 & & & SKS & 10 \\
\hline & & & ePP & 00 & 24.00 & & 1 & & & 5 & 10 \\
\hline & & & eSP & 00 & 42.00 & & 1 & & & SP & 11 \\
\hline & & & ePP & 03 & 20.00 & & | CTI & 91.70 & 327 & P- & 59 \\
\hline & & & ePPP & 04 & 10.00 & & 1 & & & iPP & 03 \\
\hline & & & es & 10 & 00.00 & & 1 & & & e & 10 \\
\hline & & & eSS & 16 & 28.00 & & 1 & & & e & 25 \\
\hline BHG & 89.91 & 328 & iPd & 59 & 43.10 & 0.1 & CDF & 91.89 & 331 & iPd & 59 \\
\hline BNS & 89.96 & 333 & iPd & 59 & 42.80 & -0.3 & I ECH & 92.09 & 331 & $P-$ & 59 \\
\hline & & & iPP & 03 & 17.30 & & 1 & & & epP & 00 \\
\hline & & & es & 09 & 59.50 & & 1 & & & iPP & 03 \\
\hline LIT & 89.99 & 317 & $P d$ & 59 & 41.60 & -1.9 & 1 & & & iSKS & 10 \\
\hline EKA & 90.00 & 340 & $P_{c}$ & 59 & 44.28 & 1.0 & I ZUL & 92.12 & 330 & $P d$ & 59 \\
\hline & $1.2 \mathrm{~s}$ & 588 & $8.00 \mathrm{~nm}$ & & & $.4 \mathrm{mb}$ & I DMU & 92.33 & 341 & $i P d$ & 59 \\
\hline TNS & 90.01 & 331 & iPd & 59 & 43.50 & 0.1 & 1 & $1.7 \mathrm{~s}$ & 2440 & $0.00 \mathrm{~nm}$ & \\
\hline & & & ePP & 03 & 19.00 & & I BSF & 92.55 & 331 & iPd & 59 \\
\hline & & & eSKS & 10 & 01.50 & & I & $0.9 \mathrm{~s}$ & 714 & $4.00 \mathrm{~nm}$ & \\
\hline & & & ePS & 11 & 30.50 & & I SAL & 92.56 & 327 & $P$ & 59 \\
\hline ESK & 90.03 & 340 & iPd & 59 & 43.50 & 0.1 & I HAU & 92.60 & 331 & iPd & 59 \\
\hline & $1.0 \mathrm{~s}$ & 1080 & $0.00 \mathrm{~nm}$ & & & $8 \mathrm{mb}$ & 1 & $0.7 \mathrm{~s}$ & 424 & $4.00 \mathrm{~nm}$ & \\
\hline KBA & 90.15 & 327 & iPd & 59 & 43.80 & -0.5 & I DDK & 92.62 & 341 & iPd & 59 \\
\hline & $1.1 \mathrm{~s}$ & 400 & $0.00 \mathrm{~nm}$ & & & $.3 m b$ & 1 & $2.0 \mathrm{~s}$ & 2400 & $0.00 \mathrm{~nm}$ & \\
\hline & & & i & 59 & 45.40 & & DKM & 92.72 & 341 & iPd & 59 \\
\hline & & & $\mathrm{i}$ & 59 & 48.00 & & ! & $2.0 \mathrm{~s}$ & 2700 & $0.00 \mathrm{~nm}$ & \\
\hline & & & iPP & 03 & 21.10 & & I DLE & 92.77 & 341 & IPd & 59 \\
\hline & & & i & 03 & 25.00 & & 1 & $2.0 \mathrm{~s}$ & 2700 & $0.00 \mathrm{~nm}$ & \\
\hline & & & $\mathrm{i}$ & 03 & 26.70 & & $\mathrm{I} \mathrm{SCH}$ & 92.91 & 15 & ePd & 59 \\
\hline ANMO & 90.18 & 49 & ePd & 59 & 46.00 & 1.3 & I DCN & 92.93 & 341 & iPd & 59 \\
\hline & & & e & 00 & 47.00 & & 1 & $1.7 \mathrm{~s}$ & 2540 & $0.00 \mathrm{~nm}$ & \\
\hline$A L O$ & 90.18 & 49 & eP & 59 & 45.50 & 0.8 & I ETA & 93.19 & 340 & iPC & 59 \\
\hline & $0.8 \mathrm{~s}$ & 98 & $8.90 \mathrm{~nm}$ & & & $.8 \mathrm{mb}$ & 1 & $1.2 \mathrm{~s}$ & 690 & $0.00 \mathrm{~nm}$ & \\
\hline$z$ & $20 \mathrm{~s}$ & & $3.20 \mathrm{um}$ & & & $.0 M s z$ & 1 DUI & 93.45 & 323 & $P-$ & 59 \\
\hline & & & e & 00 & 25.00 & & I & & & iPP & 03 \\
\hline LJU & 90.31 & 326 & ePd & 59 & 44.00 & -0.8 & 1 & & & e & 10 \\
\hline & & & epp & 00 & 32.50 & $195 \mathrm{kmx}$ & I PRG & 93.49 & 325 & $\mathbf{P}$ & 00 \\
\hline & & & es & 09 & 58.00 & & 1 & & & es & 10 \\
\hline EUR & 90.32 & 329 & iPd & 59 & 45.20 & 0.3 & I ORI & 93.49 & 320 & $P$ & 00 \\
\hline & $0.9 \mathrm{~s}$ & 2370 & $0.00 \mathrm{~nm}$ & & & $.2 m b$ & I AQU & 93.51 & 324 & $\mathbf{P}$ & 59 \\
\hline & & & $e$ & 83 & 24.00 & & I & & & $i$ & 03 \\
\hline & & & es & 10 & 02.50 & & I FIR & 93.56 & 326 & $\mathrm{P}+$ & 00 \\
\hline DHR & 90.32 & 319 & iPd & 59 & 44.00 & -1.0 & I & & & iPP & 03 \\
\hline & & & is & 09 & 56.20 & & I & & & is & 10 \\
\hline$A T H$ & 90.59 & 315 & iPd & 59 & 44.00 & -2.2 & I PRT & 93.56 & 326 & $P$ & 00 \\
\hline & & & i PP & 03 & 20.00 & & 1 & & & IPP & 00 \\
\hline & & & is & 10 & 00.00 & & 1 & & & iPP & 03 \\
\hline$E N N$ & 90.61 & 333 & i Pd & 59 & 46.00 & -0.1 & 1 & & & is & 10 \\
\hline & 1. & 721 & $1.00 \mathrm{~nm}$ & & & $.6 \mathrm{mb}$ & I DIX & 93.63 & 329 & $\mathrm{Pd}$ & $\theta \theta$ \\
\hline & & & ePP & 03 & 22.00 & & I ECB & 93.65 & 341 & iPc & 00 \\
\hline & & & e & 05 & 20.00 & & I & $1.7 \mathrm{~s}$ & 1950 & $9.00 \mathrm{~nm}$ & \\
\hline & & & es & 89 & 48.00 & & I ECP & 93.69 & 340 & $i P_{C}$ & $\theta 0$ \\
\hline & & & e & 11 & 36.00 & & I & $1.5 \mathrm{~s}$ & 1450 & $9.00 \mathrm{~nm}$ & \\
\hline
\end{tabular}


$06 \mathrm{~d} \quad 02 \mathrm{~h}$

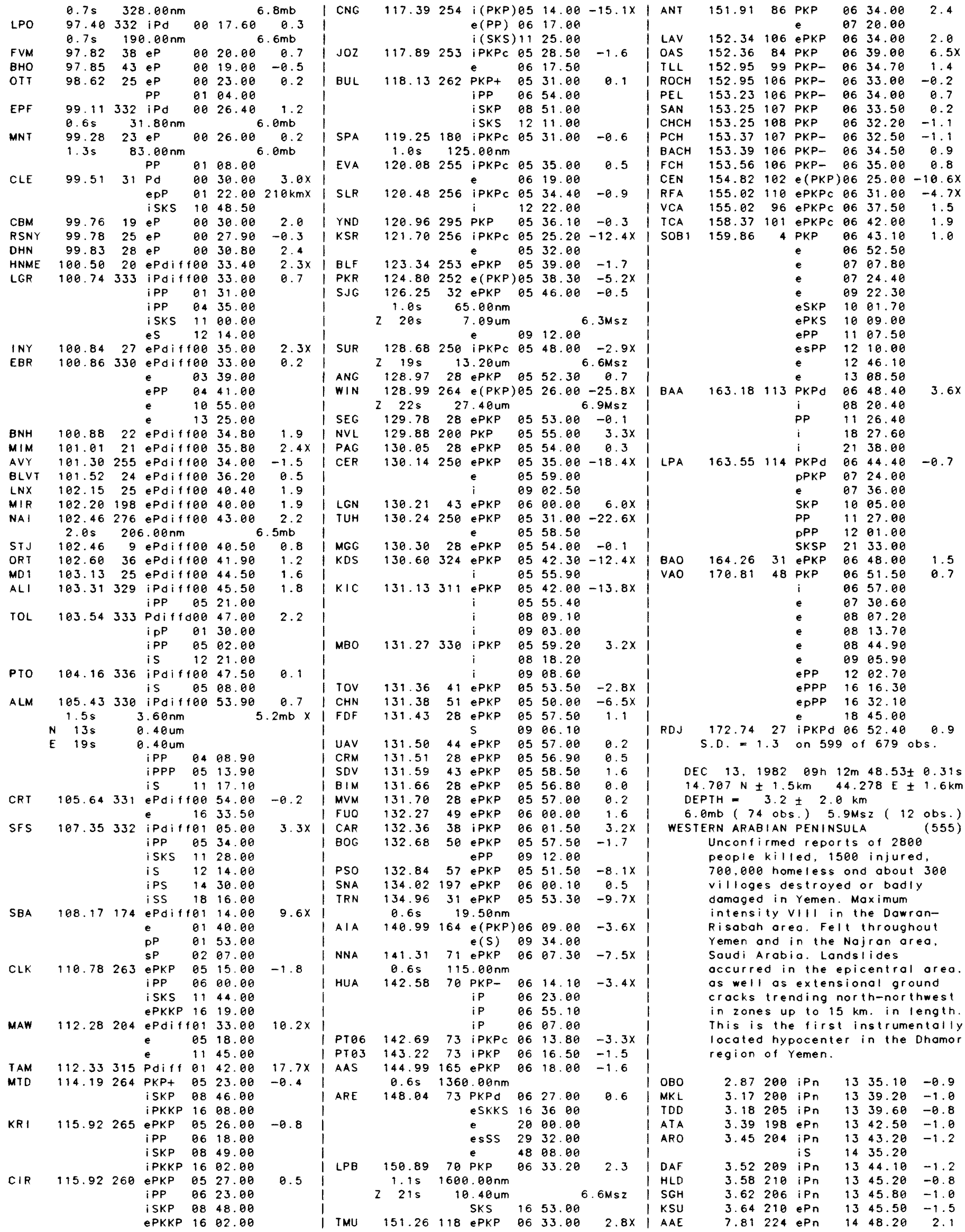




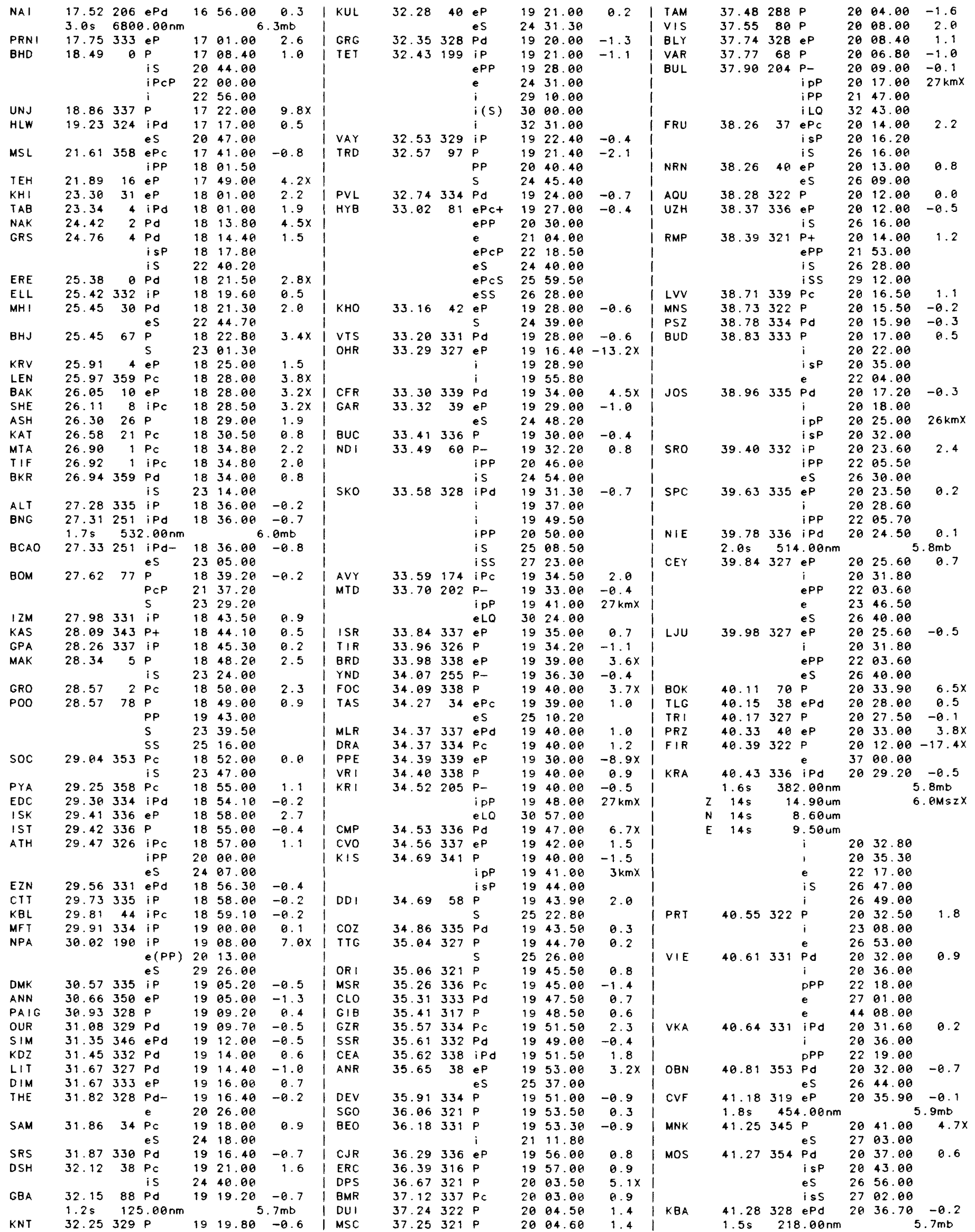




\begin{tabular}{|c|c|c|c|c|c|c|c|c|c|c|c|}
\hline & & & & $\mathbf{i}$ & 20 & 42.20 & & 1 & 2.05 & 952 & $2.00 \mathrm{~nm}$ \\
\hline & & & & $\mathbf{i}$ & 20 & 47.10 & & I EMS & 44.22 & 323 & $\mathrm{Pc}$ \\
\hline & & & & ePP & 22 & 27.30 & & I SLE & 44.29 & 326 & $\mathrm{Pc}$ \\
\hline & & & & $\mathrm{i}$ & 22 & 45.70 & & I CLL & 44.32 & 332 & iPd \\
\hline MOA & & 41.34 & 329 & iPd & 20 & 37.20 & 0.0 & i & & & $\mathrm{i}$ \\
\hline & & & & i & 20 & 42.00 & & I & & & PP \\
\hline CTI & & 41.53 & 326 & $\mathbf{P}$ & 20 & 39.00 & 0. 1 & 1 & & & s \\
\hline WAR & & 41.79 & 339 & eP & 20 & 43.00 & 2.3 & I MOX & 44.51 & 331 & iPd \\
\hline & & & & ePP & 22 & 36.00 & & 1 & $2.0 \mathrm{~s}$ & 853 & $3.00 \mathrm{~nm}$ \\
\hline & & & & ePcS & 26 & 28.00 & & 1 & $19 \mathrm{~s}$ & & $0.80 \mathrm{um}$ \\
\hline & & & & es & 27 & 08.00 & & 1 & $18 \mathrm{~s}$ & & $2.80 u m$ \\
\hline SAL & & 41.89 & 324 & $\mathbf{P}$ & 20 & 46.50 & $4.9 x$ & I & $19 \mathrm{~s}$ & & $5.60 \mathrm{um}$ \\
\hline BHG & & 41.95 & 328 & iPd & 20 & 41.60 & -0.6 & 1 & & & ePP \\
\hline & & $1.2 \mathrm{~s}$ & 179 & $9.00 \mathrm{~nm}$ & & & $.7 \mathrm{mb}$ & 1 & & & is \\
\hline PCN & & 42.06 & 323 & $\mathbf{P}$ & 20 & 43.58 & 0.5 & 1 & & & eSs \\
\hline VG1 & & $42 \cdot 32$ & 323 & $\mathbf{P}$ & 20 & 46.00 & 0.8 & I STU & 44.52 & 327 & $\mathrm{iPc}$ \\
\hline OGA & & 42.37 & 326 & iPd & 20 & 45.90 & 0.0 & 1 & $1.0 \mathrm{~s}$ & 100 & $0.00 \mathrm{~nm}$ \\
\hline & & $1.7 \mathrm{~s}$ & 786 & $6.00 \mathrm{~nm}$ & & & $.2 \mathrm{mb}$ & 1 & $20 s$ & & 0.60 um \\
\hline CAL & & $42 \cdot 38$ & 73 & $\mathbf{P}$ & 20 & 53.00 & $7.0 x$ & I BBS & 44.66 & 325 & $P d$ \\
\hline & & & & $\mathrm{ScP}$ & 26 & 20.00 & & I NEC & 44.72 & 324 & $P d$ \\
\hline & & & & $s$ & 27 & 21.00 & & I BUH & 44.97 & 327 & ePd \\
\hline KSP & & 42.54 & 334 & ip & 20 & 46.00 & -0.9 & $18 R L$ & 45.01 & 334 & eP \\
\hline & & $1.8 \mathrm{~s}$ & 1320 & $0.00 \mathrm{~nm}$ & & & $.4 \mathrm{mb}$ & I BRN & 45.01 & 333 & eP \\
\hline & & & & eS & 27 & 17.00 & & I BAF & 45.16 & 325 & iPd \\
\hline $\mathrm{KHC}$ & & 42.54 & 330 & iPd & 20 & 46.20 & -0.9 & I STR & 45.17 & 326 & $P$ \\
\hline & & $1.5 \mathrm{~s}$ & 366 & $6.00 \mathrm{~nm}$ & & & $.9 m b$ & 1 & & & ePP \\
\hline & $z$ & 135 & & $2.80 \mathrm{um}$ & & & $3 M s z X$ & 1 & & & isKS \\
\hline & $\mathbf{N}$ & $12 \mathrm{~s}$ & & 2.00 um & & & & $185 F$ & 15.27 & 325 & eP \\
\hline & $E$ & $12 \mathrm{~s}$ & & 3. 10 um & & & & I ECH & 45.28 & 325 & P- \\
\hline & & & & e & 21 & 10.80 & & 1 & & & ePP \\
\hline & & & & PP & 22 & 37.40 & & $\mathrm{COF}$ & 45.33 & 326 & eP \\
\hline & & & & $\mathrm{S}$ & 27 & 23.50 & & 1 & $1.8 \mathrm{~s}$ & 350 & $0.00 \mathrm{~nm}$ \\
\hline PRU & & 42.69 & 332 & iPd & 20 & 47.60 & -0.6 & I GWF & 45.47 & 327 & iPd \\
\hline & & $1.4 \mathrm{~s}$ & 140 & $0.00 \mathrm{~nm}$ & & & $.5 \mathrm{mb}$ & I SWZ & 45.52 & 204 & iPc \\
\hline & $\mathrm{z}$ & $17 \mathrm{~s}$ & & $0.10 \mathrm{um}$ & & & $.8 M s z X$ & I LSA & 45.58 & 63 & $\mathbf{P}$ \\
\hline & $\mathbf{N}$ & $16 s$ & & 5.20 um & & & & 1 & & & PP \\
\hline & $E$ & $18 \mathrm{~s}$ & & 7.50 um & & & & 1 & & & $\mathbf{s}$ \\
\hline & & & & e & 21 & 15.50 & & 1 & & & SS \\
\hline & & & & PP & 22 & 44.00 & & I HAU & 45.61 & 325 & eP \\
\hline & & & & es & 27 & 21.00 & & 1 & $1.8 \mathrm{~s}$ & 285 & $5.00 \mathrm{~nm}$ \\
\hline PRA & & 42.80 & 332 & iPd & 20 & 48.00 & -1.1 & I WIN & 45.62 & 216 & ePd \\
\hline & & & & e & 20 & 54.00 & & 1 & $.1 \mathrm{~s}$ & 80 & $0.00 \mathrm{~nm}$ \\
\hline & & & & e & 30 & 44.00 & & I VIR & 5.76 & 202 & eP \\
\hline WET & & 42.89 & 330 & iPd & $2 \theta$ & 48.70 & -1.2 & I SHL & 45.77 & 69 & $P+$ \\
\hline & & $1.8 \mathrm{~s}$ & 636 & $6.00 \mathrm{~nm}$ & & &.$\theta m b$ & 1 & & & PP \\
\hline & & & & es & 27 & 32.00 & & 1 & & & PPP \\
\hline ARU & & 43.03 & 12 & Pd & 20 & 51.20 & 0.3 & 1 & & & S \\
\hline & & & & $i s P$ & 20 & 54.20 & & 1 & & & SS \\
\hline & & & & $i p P$ & 20 & $56.4 \theta$ & $17 \mathrm{kmx}$ & 1 & & & SSS \\
\hline & & & & es & 27 & 22.70 & & I TNS & 45.79 & 328 & eP \\
\hline FUR & & 43.06 & 328 & iPd & 20 & 50.50 & -0.8 & I SEM & 45.98 & 32 & ePd \\
\hline & & $1.2 \mathrm{~s}$ & 340 & $\theta . \theta 0 \mathrm{~nm}$ & & &.$\theta m b$ & 1 & & & es \\
\hline LMR & & 43.06 & 319 & eP & 20 & 50.80 & -0.5 & E8R & 46.02 & 313 & eP \\
\hline & & $1.8 \mathrm{~s}$ & 298 & $3.00 \mathrm{~nm}$ & & & $.7 \mathrm{mb}$ & 1 & & & es \\
\hline FRF & & 43.09 & 319 & eP & 20 & 51.20 & -6.3 & I PUL & 46.15 & 350 & $P d$ \\
\hline & & $1.8 \mathrm{~s}$ & 479 & 9.00 & & & $9 \mathrm{mb}$ & $!$ & & & isp \\
\hline SLR & & 43.11 & 201 & IPd- & 20 & 52.50 & 0.4 & 1 & & & es \\
\hline & & $1.5 \mathrm{~s}$ & 417 & $7.00 \mathrm{~nm}$ & & & $\theta \mathrm{mb}$ & 1 & & & ess \\
\hline & $z$ & $17 \mathrm{~s}$ & & 9.90 um & & & $.6 M s z X$ & $1 B G G$ & 46.35 & 328 & eP \\
\hline & $\mathrm{N}$ & $19 \mathrm{~s}$ & & $5.60 \mathrm{um}$ & & & & 1 & $2.2 \mathrm{~s}$ & & $0.82 \mathrm{~nm}$ \\
\hline & $E$ & $19 \mathrm{~s}$ & & 0. 80 um & & & & SMF & 46.35 & 322 & eP \\
\hline & & & & IPCP & 22 & 41.26 & & 1 & $1.8 \mathrm{~s}$ & 218 & $0.00 \mathrm{~nm}$ \\
\hline & & & & es & 27 & 24.00 & & I PYM & 46.42 & 320 & Pc \\
\hline TMA & & 43.12 & 324 & $\mathrm{Pc}$ & 20 & 51.40 & -0.6 & $1 \mathrm{MLS}$ & 6.43 & 316 & iP \\
\hline PRE & & 43.16 & 201 & iPd & 20 & 54.50 & 2.0 & LBF & 46.44 & 322 & eP \\
\hline LRG & & 43.21 & 319 & eP & $2 \theta$ & 52.30 & -0.3 & CAF & 6.63 & 319 & eP \\
\hline & & $1.8 \mathrm{~s}$ & $\begin{array}{r}454 \\
\end{array}$ & $.00 \mathrm{~nm}$ & & & $9 \mathrm{mb}$ & I LOR & 6.65 & 323 & eP \\
\hline ORO & & 43.36 & 323 & P & 20 & 52.50 & -1.4 & AVF & 46.71 & 322 & eP \\
\hline EVA & & 43.55 & 200 & iPd & $2 \theta$ & 56.80 & 1.1 & 1 & $1.8 \mathrm{~s}$ & 253 & $3.00 \mathrm{~nm}$ \\
\hline & & $1.0 \mathrm{~s}$ & 208 & $3.00 \mathrm{~nm}$ & & & $.9 m b$ & I SSF & 46.76 & 322 & eP \\
\hline BRG & & 43.59 & 332 & iPd & $2 \theta$ & 54.80 & -0.7 & I BNS & 46.88 & 328 & iPc \\
\hline & & & & $\mathrm{i}$ & 21 & 21.00 & & 1 & $2.0 \mathrm{~s}$ & & $0.49 \mathrm{~nm}$ \\
\hline & & & & e & 21 & 45.00 & & 1 & & & $i$ \\
\hline & & & & PP & 22 & 45.00 & & T & & & ¿PP \\
\hline P $\mid$ & & 43.60 & 201 & iPc & 20 & 57.50 & 1.4 & $M Z F$ & 6.90 & 321 & eP \\
\hline MK & & 4 & 323 & $\mathrm{Pc}$ & 20 & 55.00 & -1.1 & $8 \mathrm{LF}$ & 46.93 & 202 & eP \\
\hline CDR & & 43.70 & 319 & $\mathrm{Pc}$ & $2 \theta$ & 55.50 & -1.0 & WMO & 46.94 & 43 & $P$ \\
\hline KSR & & 43. & 203 & iPd & $2 \theta$ & 55.70 & -1.5 & 1 & & & $P P$ \\
\hline & & $1.0 \mathrm{~s}$ & 148 & $3.00 \mathrm{~nm}$ & & & $.7 \mathrm{mb}$ & 1 & & & $\mathrm{SCP}$ \\
\hline SVE & & 43.83 & 13 & Pd & $2 \theta$ & 57.50 & 0. 1 & I & & & $\mathrm{s}$ \\
\hline & & & & es & 27 & 33.00 & & I ALM & 46.95 & 307 & iPc \\
\hline DIX & & 43. & 323 & Pc & $2 \theta$ & 58.30 & -0.5 & I & $1.1 \mathrm{~s}$ & & $8.40 \mathrm{~nm}$ \\
\hline GRF & & 44.07 & 329 & IPA & 20 & 58.30 & -1.1 & 1 & & & ¿PP \\
\hline & & $1.7 \mathrm{~s}$ & 261 & $1.00 \mathrm{~nm}$ & & & $.8 \mathrm{mb}$ & I & & & ¡PPP \\
\hline & $z$ & $18 \mathrm{~s}$ & & $\$ .50 \mathrm{um}$ & & & $.4 M S z$ & 1 & & & is \\
\hline & & & & e & 21 & 03.90 & & LPO & 47.09 & 318 & eP \\
\hline OF & & 44.16 & 330 & eP & $2 \theta$ & 58.90 & -1.3 & 1 & $1.8 \mathrm{~s}$ & 396 & $6.00 \mathrm{~nm}$ \\
\hline
\end{tabular}




\begin{tabular}{|c|c|c|c|c|c|c|c|c|c|c|c|}
\hline & & & eSCS & 31 & 52.00 & & 1 & & & epp & 23 \\
\hline APO & 50.80 & 341 & $P$ & 22 & 00.39 & $8.4 x$ & 1 & & & es & 31 \\
\hline SLL & 50.94 & 341 & $P$ & 22 & 01.14 & $8.1 x$ & I XAN & 61.01 & 59 & $P d$ & 23 \\
\hline BSI & 50.97 & 95 & ePd & 21 & 55.20 & 1.3 & i & & & $P P$ & 25 \\
\hline KON & 51.58 & 338 & $e^{P}$ & 21 & 56.00 & -1.9 & 1 & & & $\mathrm{~s}$ & 31 \\
\hline & & & e & 47 & 00.00 & & 1 & & & SS & 35 \\
\hline UME & 51.82 & 347 & $P$ & 21 & 57.80 & -1.8 & I NR I & 61.12 & 16 & $P d$ & 23 \\
\hline & & & $\mathrm{i}$ & 22 & 01.80 & & 1 & & & $i s P$ & 23 \\
\hline & & & is & 29 & 24.00 & & I ВТO & 62.21 & 52 & $P$ & 23 \\
\hline NAO & 52.01 & 340 & $P$ & 22 & 15.00 & $13.8 x$ & 1 & & & $P P$ & 25 \\
\hline & $1.5 \mathrm{~s}$ & 878 & $8.00 \mathrm{~nm}$ & & & & I HHC & 63.40 & 52 & $P$ & 23 \\
\hline $\mathrm{CHG}$ & 52.34 & 77 & iPc & 22 & 03.00 & -1.3 & I TIY & 63.95 & 55 & $P$ & 23 \\
\hline & $1.1 \mathrm{~s}$ & & $8.50 \mathrm{~nm}$ & & 5 & $.1 \mathrm{mb}$ & I GZH & 65.37 & 71 & PC & 23 \\
\hline & & & es & 29 & 44.00 & & I & & & $P P$ & 26 \\
\hline CHTO & 52.34 & 77 & ePct & 22 & 02.50 & -1.8 & I AKU & 65.46 & 336 & ip & 23 \\
\hline & 1.15 & 29 & $9.40 \mathrm{~nm}$ & & 5 & $.1 \mathrm{mb}$ & 1 & $1.8 \mathrm{~s}$ & 436 & $6.00 \mathrm{~nm}$ & \\
\hline & & & iSP & 22 & 09.50 & & I WHN & 65.66 & 63 & $P$ & 23 \\
\hline BDT & 52.53 & 79 & eP & 22 & 04.00 & -1.7 & I KBS & 6.07 & 353 & iP & 23 \\
\hline APA & 53.29 & 355 & $P d$ & 22 & 10.40 & -0.2 & I HKC & 66.22 & 72 & ip & 23 \\
\hline & & & isP & 22 & 12.80 & & 1 & & & is & 31 \\
\hline & & & es & 29 & 48.00 & & I KHE & 66.23 & 2 & $P d$ & 23 \\
\hline CER & 53.42 & 206 & $i P c$ & 22 & 13.50 & 1.5 & 1 & & & $i p P$ & 23 \\
\hline TUH & 3.42 & 206 & eP & 22 & 12.50 & 0.5 & 1 & & & is & 32 \\
\hline BER & 3.65 & 337 & eP & 22 & 11.90 & -1.4 & I REY & 66.54 & 334 & ip & 23 \\
\hline SOD & 3.88 & 352 & $\mathrm{P}$ & 22 & 13.80 & -1.1 & | BJI & 66.89 & 53 & $P$ & 23 \\
\hline UER & 54.06 & 36 & $\mathrm{PC}$ & 22 & 15.10 & -1.3 & 1 & & & es & 32 \\
\hline & & & is & 29 & 55.30 & & $1 B O D$ & 6.99 & 33 & eP & 23 \\
\hline GTA & 54.29 & 52 & $P d$ & 22 & 17.50 & -1.0 & I TIA & 67.72 & 57 & $\mathrm{P}$ & 23 \\
\hline & & & $\mathrm{S}$ & 29 & 50.50 & & 1 & & & $\mathbf{P P}$ & 26 \\
\hline & & & SS & 33 & 28.50 & & I & & & $\mathrm{s}$ & 32 \\
\hline EKA & 54.48 & 329 & $P d$ & 22 & 17.60 & -1.9 & 1 & & & SS & 37 \\
\hline & 1.45 & 106 & $6.80 \mathrm{~nm}$ & & & $.7 \mathrm{mb}$ & $15 c 0$ & 68.37 & 341 & ip & 23 \\
\hline ESK & 54.49 & 329 & $(\mathrm{P})$ & 22 & 18.00 & -1.6 & I & $0.9 \mathrm{~s}$ & 78 & $8.00 \mathrm{~nm}$ & \\
\hline ISI & 54.61 & 96 & $e(P)$ & 22 & 23.00 & 1.9 & NJ2 & 69.40 & 61 & $P$ & 23 \\
\hline ECP & 54.79 & 324 & iPc & 22 & 23.40 & 1.6 & 1 & & & $P P$ & 26 \\
\hline & $1.5 \mathrm{~s}$ & 405 & $5.00 \mathrm{~nm}$ & & & $.2 \mathrm{mb}$ & 1 & & & is & 33 \\
\hline ETA & 54.91 & 325 & $e P$ & 22 & 13.30 & $-9.4 x$ & 1 & & & SS & 37 \\
\hline & $1.9 \mathrm{~s}$ & 470 & $0.00 \mathrm{~nm}$ & & & $.2 \mathrm{mb}$ & I DAG & 69.73 & 347 & iPd & 23 \\
\hline ECB & 55.10 & 324 & $I P C$ & 22 & 21.90 & -2.2 & 1 & 0.95 & & $2.00 \mathrm{~nm}$ & \\
\hline & 1.3 & 250 & $000 \mathrm{~nm}$ & & 6 & $.1 \mathrm{mb}$ & I & & & is & 33 \\
\hline PCT & 55.13 & 82 & iP & 22 & 20.70 & $-4.1 x$ & I TUP & 69.81 & 37 & ePd & 24 \\
\hline & $1.2 \mathrm{~s}$ & 115 & $5.00 \mathrm{~nm}$ & & 5 & $.8 \mathrm{mb}$ & $\mathrm{O} O \mathrm{ZH}$ & 69.92 & 69 & Pd & 24 \\
\hline PSI & 55.17 & 97 & ePd & 22 & 23.30 & -1.9 & 1 & & & $P P$ & 26 \\
\hline & $1.5 \mathrm{~s}$ & 169 & $9.00 \mathrm{~nm}$ & & 5 & $.9 \mathrm{mb}$ & 1 & & & es & 33 \\
\hline DDK & 55.27 & 326 & eP & 22 & 23.90 & -1.4 & 1 & & & SS & 37 \\
\hline & $2.0 \mathrm{~s}$ & 728 & $0.00 \mathrm{~nm}$ & & 6 & $.4 \mathrm{mb}$ & I TRT & 71.33 & 103 & $i P_{C}$ & 24 \\
\hline KIR & 55.31 & 349 & $P$ & 22 & 23.20 & -2.3 & ! & $0.7 \mathrm{~s}$ & & $8.70 \mathrm{~nm}$ & \\
\hline & & & $\mathrm{i}$ & 22 & 27.40 & & I SSE & 71.48 & 62 & Pc & 24 \\
\hline DLE & 55.34 & 326 & eP & 22 & 25.00 & -0.8 & I & & & PP & 26 \\
\hline & $1.8 \mathrm{~s}$ & 910 & $0.00 \mathrm{~nm}$ & & & $.5 \mathrm{mb}$ & 1 & & & es & 33 \\
\hline KMI & 55.57 & 69 & $P C$ & 22 & 27.00 & -1.3 & 1 & & & SS & 38 \\
\hline & $17 \mathrm{~s}$ & & $6.40 u m$ & & & & I ANP & 72.52 & 68 & $P C$ & 24 \\
\hline & & & $S P$ & 23 & 15.00 & & 1 & & & $\mathrm{~s}$ & 33 \\
\hline & & & PP & 24 & 33.00 & & I TATO & 72.52 & 68 & ePc & 24 \\
\hline & & & $\mathrm{S}$ & 30 & 10.00 & & 1 & $2.0 \mathrm{~s}$ & 1498 & $0.00 \mathrm{~nm}$ & \\
\hline & & & SS & 34 & 10.00 & & I PPR & 72.65 & 84 & eP & 24 \\
\hline $\mathrm{DCN}$ & 55.76 & 325 & $I P C$ & 22 & 28.10 & -0.8 & I SZP & 72.77 & 76 & ePc & 24 \\
\hline & 1.85 & 910 & $0.00 \mathrm{~nm}$ & & & $.5 \mathrm{mb}$ & I BAG & 73.10 & 77 & $P$ & 24 \\
\hline DMU & 55.79 & 326 & IPC & 22 & 27.70 & -1.4 & 1 & & & eS & 33 \\
\hline & 1.7 & 350 & $0.00 \mathrm{~nm}$ & & 6 & $1 \mathrm{mb}$ & CN2 & 73.65 & 48 & $\mathrm{Pd}$ & 24 \\
\hline KEV & 56.07 & 353 & iP & 22 & 30.50 & -0.4 & 1 & & & PP & 27 \\
\hline & $1.1 \mathrm{~s}$ & 154 & $4.00 \mathrm{~nm}$ & & 5 & $.9 m b$ & I PGP & 74.01 & 80 & $e P c$ & 24 \\
\hline & $12 \mathrm{~s}$ & 18 & 0.20 um & & & $.1 M s z X$ & 1 & $1.0 \mathrm{~s}$ & 69 & $9.00 \mathrm{~nm}$ & \\
\hline & & & $\mathrm{i}$ & 22 & 37.80 & & I TIK & 74.44 & 19 & ePd & 24 \\
\hline & & & ePPP & 26 & 08.00 & & 1 & & & es $P$ & 24 \\
\hline & & & es & 30 & 24.00 & & 1 & & & es & 34 \\
\hline & & & escs & 32 & 24.00 & & I YAK & 75.00 & 29 & $\mathrm{Pd}$ & 24 \\
\hline CD2 & 56.54 & 63 & $\mathrm{P}$ & 22 & 33.00 & -2.0 & 1 & & & es & 34 \\
\hline & & & $P P$ & 26 & 04.00 & & 1 MDJ & 76.52 & 47 & $\mathrm{Pc}$ & 24 \\
\hline$L Z H$ & 56.98 & 56 & iPd- & 22 & 37.00 & -1.2 & 1 & & & $P P$ & 27 \\
\hline & 5 & 3510 & 0.0 & & & $.6 \mathrm{mb} x$ & 1 & & & $\mathbf{S}$ & 34 \\
\hline & $17 \mathrm{~s}$ & & $6.30 \mathrm{um}$ & & & & 1 & & & PS & 35 \\
\hline & $14 \mathrm{~s}$ & & $9.80 \mathrm{um}$ & & & & 1 & & & SS & 39 \\
\hline & & & PP & 22 & 44.00 & & I LGP & 6.73 & 80 & e & 24 \\
\hline & & & $S$ & 30 & 30.00 & & I MKS & 77.08 & 99 & $\in(F$ & 24 \\
\hline & & & SS & 30 & 40.00 & & I ALE & 77.67 & 353 & eP & 24 \\
\hline & & & eSS & 34 & 26.00 & & 1 & $1.7 \mathrm{~s}$ & 548 & $8.00 \mathrm{~nm}$ & \\
\hline PI & 7.49 & 100 & eP & 22 & 1.60 & -0.2 & PLP & 78.35 & 81 & eP & 24 \\
\hline OY & .12 & 38 & ePd & 22 & 4.00 & -1.6 & VLA & 78.39 & 48 & $\mathrm{PC}$ & 24 \\
\hline GV & 8.97 & 74 & $P$ & 22 & 51.00 & -1.1 & 1 & & & es & 34 \\
\hline GYA & 59.08 & 68 & $\mathrm{P}$ & 22 & 51.00 & -1.9 & CGP & 8.68 & 84 & $\in P$ & 24 \\
\hline & & & $\mathbf{P P}$ & 25 & 11.00 & & I GDH & 79.12 & 339 & eP & 24 \\
\hline & & & PPP & 26 & 33.00 & & 1 & $1.2 \mathrm{~s}$ & & $6.90 \mathrm{~nm}$ & \\
\hline & & & $\mathrm{S}$ & 30 & 54.00 & & 1 & & & $\mathrm{i}$ & 35 \\
\hline ZAK & 59.15 & 40 & $\in P$ & 22 & 51.00 & -1.8 & DAV & 79.85 & 85 & $P$ & 25 \\
\hline & & & es & 31 & 03.30 & & 1 & & & es & 35 \\
\hline $10 k$ & & 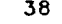 & er & 22 & 59.00 & -1.5 & 1 & 00.20 & of & Er & 25 \\
\hline
\end{tabular}


13d $09 \mathrm{~h}$

CTA $\quad 105.95 \quad 107$ iPdiff27 $07.40 \quad 3.7 \times$ । $1.2 \mathrm{~s} \quad 21.18 \mathrm{~nm} \quad 6.8 \mathrm{mb}$

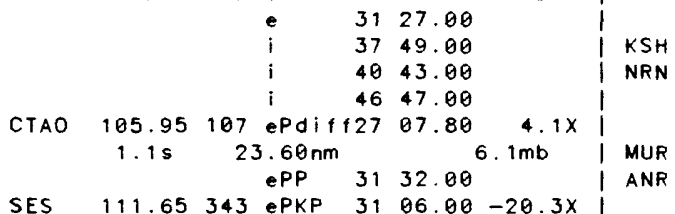

RSSD 114.71335 (PKP) $3131.50-1.1$ I SFK

\begin{tabular}{llllll|l} 
& & e & 42 & 10.80 & & \\
LPB & 115.13 & 258 ePKP & 31 & 35.00 & 0.6 & FRG
\end{tabular} $260 \mathrm{~s} 66.38 \mathrm{um} \quad 6.2 \mathrm{Msz}$

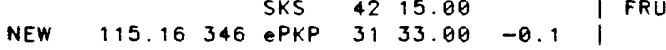

BOG 115.96283 ePKP $32 \quad 38.50$ 62.5X I NAM

LRM $\quad 116.19 \quad 342$ ePKP $30 \quad 35.40-60.0 \times \quad$

$\begin{array}{llllllll}\text { RLO } & 116.45 & 324 & \text { ePKP } & 31 & 35.20 & -0.7 & \text { PRZ }\end{array}$

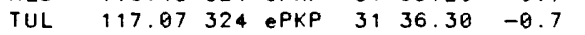

$1.2 \mathrm{~s} \quad 44.28 \mathrm{~nm}$

$Z 20 \mathrm{~s} \quad 1.86 \mathrm{um} \quad 5.7 \mathrm{Msz}$

e $\quad 3144.70$

e $\quad 3243.00$

BDW $\quad 117.96 \quad 338$ ePKPC 3138.00 e
e 44.00

ARE 118.34259 EPKP 3143.00 EPP 3300.00 ePS 4244.00 ELR $\quad 0550.00$ eLR $16 \quad 36.00$

GOL 118.93334 (PKP) 3139.80 $\begin{array}{rrrr}195 & 2.12 \mathrm{um} & -1.0 \\ 129.12 & 280 & 5.8 \mathrm{MsZ}\end{array}$

PSO 120.12280 ePKP $3144.50 \quad 0.5$

HUA 121.32265 PKP $3146.50 \quad 0.3 \quad 0 B G$

BMN $\quad 122.51343$ (PKP) 3147.300 .1

KOU 122.79105 PKP+ $3148.00-0.3$

EUR 123.03342 iPKP $3147.80-1.6$

$1.0 \mathrm{~s} \quad 3.46 \mathrm{~nm}$

123.31323 ePKP $3148.00 \quad-1.1$

$\begin{array}{rr}1.0 \mathrm{~s} & 42.50 \mathrm{~nm} \\ Z \quad 18 \mathrm{~s} & 3.44 \mathrm{um}\end{array}$

$e^{440 m} 3288.00^{6}$

ALO $\quad 123.34 \quad 331$ ePKP $31 \quad 47.00$ $2 \quad 20 \mathrm{~s} 3.90 \mathrm{um}$

WDC

123.65348 iPKPC 3149.00
124.41347 124.91107 PKP+ 3149.00

125.67300 ePKP 3154.20

JAS $\quad 125.70 \quad 345$ IPKPC 3153.80

BKS 126.18347 ePKP 3155.00

5620.00 e $\quad 1020.00$

PVC $\quad 126.34101$ PKP+ 3154.50

$\begin{array}{llllll}\text { FRI } & 126.41 & 344 & \text { IPKP } & 31 & 55.20 \\ \text { ARN } & 126.53 & 346 & \text { ePKP } & 31 & 55.80\end{array}$

$\begin{array}{llllll}\text { ARN } & 126.53 & 346 & \text { ePKP } & 31 & 55.80 \\ \text { MHC } & 126.56 & 346 & \text { iPKPC } & 31 & 56.20\end{array}$

SLD $126.72 \quad 346$ ePKP 3156.80

$127.46 \quad 345$ ePKPC 3158.40

GLA $\quad 128.48 \quad 338$ ePKP 3159.30

PAS $128.62 \quad 341$ ePKP 3201.00 $2 \quad 20 \mathrm{~s}$

I TAS

TAS -2.3
$1 \mathrm{Msz}$

-0.5 I URT $-0.4$

$-3.4 X \mid C G T$

0.0 | LNA

0.5 I DSH

DZE

I DZI

0.3 SAM

0.6

0.8

0.4

1.3 AGL

0.2 NUT

1.7 WMO e $\quad 3203.00$

(III) at Frunze and ( 11 ) at Tasnkent, USSR.

$\begin{array}{llllll}0.80 & 132 & \text { iPgd } 40 \quad 27.00 & -0.1\end{array}$

$\begin{array}{lll}1.56 & 22 \text { iPgc } 40 \quad 36.40\end{array}$

$0.8 \mathrm{~s} * * * * * * * \mathrm{~nm}$

esg $40 \quad 56.70$

$1.90212 \mathrm{ePn} \quad 40 \quad 46.90$

2.31290 iPnc 4051.30

isg 4124.30

2.41328 iPg 4035.40 isg 4052.00

2.65280 iPn 4055.60

2.87351 iPnd 4109.90 is* 4138.00

$2.88291 \mathrm{ePn} \quad 4058.40$ eS* 4141.80

3.4643 iPnd 4108.20

$1.2 \mathrm{~s} 1600.00 \mathrm{~nm}$

3.52 esn 4149.20

$2.0 \mathrm{~s} \quad 104.00 \mathrm{~nm}$

iP* 4111.30

iPg $41,17.80$

3.6526 iPnc 4109.20 iP* 4117.80

3.81230 iPnc 4115.40

3.91257 iPnc 4113.60 eSg 4220.60

$4.30 \quad 33 \mathrm{IPn} \quad 4118.50$ iP* 4127.90 es* 4219.50

$4.40 \quad 15 \mathrm{ePn} \quad 4115.60$

$\begin{array}{rrr}4.45255 \mathrm{ePn} & 4221.80 \\ \mathrm{ePn} & 4121.00\end{array}$ eP* 4131.60 isg 4236.30

4.69288 iPnc 4122.30

$1.8 \mathrm{~s} * * * * * * * \mathrm{~nm}$

E $14 \mathrm{~s} 2560.00 \mathrm{um}$

eP* 4136.30

esn 4222.30

4.74246 ePnc 4124.50

$0.5 \mathrm{~s} \quad 3200.00 \mathrm{~nm}$

$-3.5 \times \mid \mathrm{CHM}$

0.2 I KRU

ePP 3524.00

ESKKS 4230.00

CPS 4544.00

EPPS 4656.00

ESSS $57 \quad 14.00$

VUN $136.00 \quad 101$ ePKP $32 \quad 16.10 \quad 2.4 \quad$ NDI

AFI $\quad 145.08 \quad 93$ ePKP $3230.00 \quad-0.1$ OUE \begin{tabular}{lll} 
SSP & 5524.00 \\
\hline & $35 P$ & 32.09
\end{tabular}

NUE S.D. $=1.2$ on 372 of 435 obs

FEB 13,1983 01n $40 \mathrm{~m} 11.90 \pm 0.35 \mathrm{~s}$ | VAN $39.994 \mathrm{~N} \pm 2.2 \mathrm{~km} \quad 75.210 \mathrm{E} \pm 1.5 \mathrm{~km}$ DEPTH $=20.1 \pm 2.6 \mathrm{~km}$

$5.7 \mathrm{mb}$ ( 110 obs.) $6.3 \mathrm{Msz}$ ( 18 obs.) SOUTHERN XINJIANG, CHINA (321) $\mathrm{KHI}$

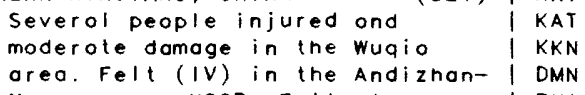
area. Felt (IV) in the Andizhan- I DMN

\section{iP* 4139.80 \\ $\begin{array}{rrr}\text { iP* } & 41 & 39.80 \\ \text { iSg } & 42 & 49.90\end{array}$}

4.76271 iPn 4124.80

$\begin{array}{rrr}4.82300 \mathrm{eSg} & 4247.30 \\ 4.8 n & 4124.80\end{array}$

4.88256 iPn 4126.10

$5.01251 \mathrm{ePn}$

$5.06255 \mathrm{iPn}$

5.20256 iPnc

4125.50

5.36258 esn

$5.69273 \mathrm{iPn}$

4130.50

4238.00

$\begin{array}{rll}5.69 & 41 & 36.30 \\ \text { eSn } & 4245.70\end{array}$

6.34270 iPnc

45.70

$2.8 \mathrm{~s} \quad * * * * * * * \mathrm{~nm}$ isg

$6.39272 \mathrm{iPn}$

7.31277 iP

$10.06 \quad 64 \mathrm{iPC}$ $s P$

4146.90

4157.60

4157.60
4236.80

4236.80
4249.00

$\mathrm{S} \quad 4434.00$

$\begin{array}{ll}11.00 & 17 \mathrm{ePd} \\ 3.4 \mathrm{~s} & 1890.00 \mathrm{~nm}\end{array}$

$0.5 \mathrm{~s} \quad 141.00 \mathrm{~nm}$

$11.89217 \mathrm{eP}$

$13.28267 \stackrel{P}{P}$

$2.0 \mathrm{~s} \quad * * * * * * * \mathrm{~nm}$ is

$13.47267 \mathrm{eP}$

$\begin{array}{rr}1.4 \mathrm{~s} & 730.00 \mathrm{~nm} \\ 28 \mathrm{~s} & 228.00 \mathrm{um}\end{array}$

$14.45251 \mathrm{ePt}$

$14.63273 \mathrm{iPc}$

$14.75143 \mathrm{iPc}$

$14.83 \quad 143 \mathrm{iPC}$

$15.00 \quad 143 \mathrm{iPc}$
4128.40 iP* 4209.50

$11.40171 \mathrm{iPd}$ 4335.70
$4248.80-2.3$ $4455.10^{6.8 \mathrm{mb} \times \text { । NAK }}$

$4254.00-2.7 \times$ । MTA $4300.60^{6.5 m b}-2.8 \times \mid$ STE 4505.20 $4318.00-3.8 x$ $4547.00^{8}$ $0.0 \mathrm{mb} x$ $4318.80-5.6 x$ 4550.30 4332.30 4334.50 4337.20 4339.40
6. $4 \mathrm{mb}$

$-5.1 x$

$-5.1 x$

$-5.7 \times$

$-5.2 x$ | ERE

$-5.4 \times$

ELT

NVS

LSA

| UER

GTA

TEH

BAK

.51

I SVE

I

ARU (18) IRT
SHL LNK I MAK

.21

$$
\text { E } 22 s
$$

$\begin{array}{lllll}5.25 & 26 \text { iPC } & 43 & 45.60 & -1.9\end{array}$ $2.5 \mathrm{~s} \quad 1760.00 \mathrm{~nm} \quad 5.9 \mathrm{mb}$ $\begin{array}{llllll}15.87 & 17 \text { iPd } 4351.70 & -3.8 x\end{array}$ $1.3 \mathrm{~s} \quad 800.00 \mathrm{~nm} \quad 5.7 \mathrm{mb}$ $\begin{array}{lllll}16.61 & 123 \mathrm{Pd} & 44 & 06.30 & 0.7\end{array}$ $\begin{aligned} 16.61123 \text { PP } & 4421.10\end{aligned}$

$\begin{array}{llllll}17.47 & 42 \mathrm{IPC} & 44 & 16.50 & 0.8\end{array}$

$1.6 \mathrm{~s} 1010.00 \mathrm{~nm}$

$18.9384 \mathrm{iPC} \quad 4433.50^{5.7 \mathrm{mb}}$

$\begin{array}{lllll}19.27 & \text { is } & 485 & 10.50 & \\ \mathrm{ePC} & 44 & 40.00 & 1.8\end{array}$

19.33279 iPd $4442.00 \quad 3.3 x$

$6.0 \mathrm{~s} 8100.00 \mathrm{~nm}$

$N 10 \mathrm{~s} \quad 92.40 \mathrm{um}$

E $10 \mathrm{~s} \quad 81.80 \mathrm{um}$

$\begin{array}{lllll}19.34 & 335 \mathrm{iPd} & 44 & 38.20 & -0.5\end{array}$

$2.7 \mathrm{~s} \quad 2900.00 \mathrm{~nm}$

$\begin{array}{cc}19.71 & 332 \mathrm{eP} \\ 3.0 \mathrm{~s} & 7500.00 \mathrm{~nm} \\ & \text { is }\end{array}$

$19.87265 \mathrm{eP}$

20.04131 iP

$20.41275 \mathrm{iPC}$

$2.0 \mathrm{~s} \quad 18.00 \mathrm{~nm}$

20.98287 iPC

$22 \mathrm{~s} \quad * * * * * \mathrm{~nm}$

$22 \mathrm{~s} \quad 24.30 \mathrm{um}$

IPP 4520.00

ePPP 4530.00

is $\quad 4849.00$

isS 4915.00

ESSS 4929.00

$\begin{array}{llllll}21.30 & 48 \text { ePSS } & 49 & 29.00 & \\ & & 44 & 59.20 & -0.1\end{array}$

i 4502.50

$\begin{array}{llllll}21.42 & 184 & \text { EPCP } & 49 & 03.60 & \\ 21 P d & 44 & 59.00 & -1.8\end{array}$

is 4848.00

21.99281 eP $\quad 45 \quad 02.00 \quad-4.3 x$ eS 4907.00

$\begin{array}{lllll}22.17 & 278 \text { iPC } 45 & 07.20 & -1.2\end{array}$

$2.0 \mathrm{~s} \quad 2500.00 \mathrm{~nm}$

iPP 4528.40

ePPP $45 \quad 49.60$

is $49 \quad 12.60$

$\begin{array}{llllll}22.22 \quad 53 \text { iPd } 45 \quad 08.90 & 0.4\end{array}$

$1.7 \mathrm{~s} \quad 1140.00 \mathrm{~nm}$

$Z \quad 14 \mathrm{~s} \quad 72.00 \mathrm{um}$

N $15 \mathrm{~s} \quad 49.90 \mathrm{um}$

E $13 \mathrm{~s} \quad 58.10 \mathrm{um}$

is 4917.00

22.26288 iPC $4508.00 \quad-0.9$

$2.0 \mathrm{~s} \quad 4200.00 \mathrm{~nm} \quad 6.5 \mathrm{mb}$

$7 \quad 18 \mathrm{~s} \quad 170.00 \mathrm{um}$

N $18 \mathrm{~s} 280.00 \mathrm{um}$

E $18 \mathrm{~s} 300.00 \mathrm{um}$

is 4913.80

$22.48274 \mathrm{iPCt} 4513.00 \quad 1.6$

$22.68172 \mathrm{ePd} \quad 4511.00 \quad-2.4$

$1.0 \mathrm{~s} 430.00 \mathrm{~nm} \quad 5.9 \mathrm{mb}$

$\begin{array}{lllll}22.84 & \text { eS } & 49 & 14.00 \\ \mathrm{PCt} & 45 & 16.50 & 1.5\end{array}$

$7.0 \mathrm{~s} \quad 3600.00 \mathrm{~nm} \quad 6.0 \mathrm{mb} x$

E $13 \mathrm{~s} \quad 95.30 \mathrm{um}$ 


\begin{tabular}{|c|c|c|c|c|c|c|c|c|c|c|c|}
\hline & $12 \mathrm{~s}$ & & $9.00 u m$ & & & & 1 & & $1.8 \mathrm{~s}$ & 280 & $0.00 \mathrm{~nm}$ \\
\hline & & & esp & 45 & 23.00 & & 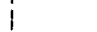 & $z$ & $18 \mathrm{~s}$ & & $8.00 \mathrm{um}$ \\
\hline & & & $I P P$ & 45 & 55.00 & & 1 & $\mathrm{~N}$ & $14 \mathrm{~s}$ & & $7.00 \mathrm{um}$ \\
\hline & & & is & 49 & 33.00 & & 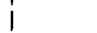 & $E$ & $12 \mathrm{~s}$ & & $2.00 \mathrm{um}$ \\
\hline & & & isSS & 50 & 29.00 & & i & & & & ePP \\
\hline LEN & 23.84 & 282 & iPc & 45 & 27.00 & 2.3 & i & & & & EPPP \\
\hline & $3.0 \mathrm{~s}$ & 130 & $90.00 \mathrm{~nm}$ & & & $.6 m b$ & i & & & & is \\
\hline BKR & 23.97 & 285 & iPc & 45 & 26.60 & 0.8 & $S I M$ & & 30.47 & 293 & eP \\
\hline & $1.4 \mathrm{~s}$ & 10 & $0.00 \mathrm{~nm}$ & & & $.2 \mathrm{mb}$ & $i$ & $z$ & $20 \mathrm{~s}$ & & 9.50 um \\
\hline & $16 \mathrm{~s}$ & & $5.00 \mathrm{um}$ & & & $.4 M S Z X$ & i & $N$ & $20 \mathrm{~s}$ & & $6.50 \mathrm{um}$ \\
\hline & $12 \mathrm{~s}$ & & $37.00 \mathrm{um}$ & & & & i & $E$ & $20 \mathrm{~s}$ & & 6.50 um \\
\hline & $16 \mathrm{~s}$ & & $4.90 \mathrm{um}$ & & & & $i$ & & & & EPP \\
\hline & & & iPP & 46 & 00.50 & & 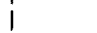 & & & & EPPP \\
\hline & & & is & 49 & 47.60 & & 1 & & & & es \\
\hline PYA & 24.14 & 290 & $I P C$ & 45 & 28.00 & 0.6 & $i$ & & & & eSS \\
\hline & $1.0 \mathrm{~s}$ & 15 & $0.00 \mathrm{~nm}$ & & & $.5 \mathrm{mb}$ & $B O D$ & & 30.65 & 41 & eP \\
\hline & $12 \mathrm{~s}$ & & 5.76 um & & & & $i$ & & $1.0 \mathrm{~s}$ & 230 & $0.00 \mathrm{~nm}$ \\
\hline & & & iPP & 45 & 59.00 & & | BJI & & 31.18 & 76 & eP \\
\hline & & & EPPP & 46 & 18.00 & & 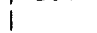 & & & & es \\
\hline & & & is & 49 & 45.00 & & $i$ & & & & SS \\
\hline$A B S$ & 24.47 & 285 & eP & 45 & 31.00 & 0.5 & | TIA & & 32.98 & 83 & eP \\
\hline & $2.5 \mathrm{~s}$ & 200 & $0.00 \mathrm{~nm}$ & & & $.3 m b$ & 1 & & & & pP \\
\hline & & & isP & 45 & 36.00 & & i & & & & ePP \\
\hline & & & is & 49 & 50.00 & & $i$ & & & & $\in S$ \\
\hline CD2 & 24.87 & 102 & eP & 45 & 35.80 & 1.2 & i TUP & & 33.00 & 49 & eP \\
\hline & & & es & 50 & 02.00 & & i & & $1.7 \mathrm{~s}$ & 310 & $0.00 \mathrm{~nm}$ \\
\hline ZUG & 25.09 & 287 & iP & 45 & 40.50 & $4.0 x$ & I WHN & & 33.10 & 94 & eP \\
\hline MSL & 25.38 & 272 & ePo & 45 & 39.00 & -0.3 & $i$ & & & & is \\
\hline & & & iPP & 46 & 22.50 & & 1 & & & & PCS \\
\hline & & & ¡PPP & 46 & 39.50 & & JER & & 33.22 & 268 & eP \\
\hline & & & es & 50 & 07.50 & & i & & & & es \\
\hline & & & iss & 51 & 13.50 & & I KIS & & 33.93 & 298 & iP \\
\hline & & & iss & 51 & 38.50 & & 1 & $z$ & $19 s$ & & $8.40 \mathrm{um}$ \\
\hline & & & eLO & 54 & 26.00 & & $i$ & $\mathrm{~N}$ & $14 \mathrm{~s}$ & & $0.00 \mathrm{um}$ \\
\hline & & & $\epsilon$ & 55 & 58.00 & & i & $E$ & $15 \mathrm{~s}$ & & $5.00 \mathrm{um}$ \\
\hline & & & $i$ & 58 & 14.50 & & i & & & & iPP \\
\hline BHD & 25.55 & 265 & ePd & 45 & 45.20 & $4.4 X$ & i & & & & iPPP \\
\hline & & & e & 50 & 15.00 & & i & & & & is \\
\hline GBA & 26.36 & 175 & $P$ & 45 & 47.00 & -1.5 & I GPA & & 34.05 & 285 & $\in P$ \\
\hline ВTO & 26.46 & 77 & ep & 45 & 50.80 & 1.4 & $\mathrm{PCT}$ & & 34.07 & 130 & $E P$ \\
\hline SOC & 26.58 & 289 & $\mathrm{iPC}$ & 45 & 52.00 & 1.7 & $i$ & & $1.2 \mathrm{~s}$ & & $6.30 \mathrm{~nm}$ \\
\hline & $2.5 \mathrm{~s}$ & 400 & $0.00 \mathrm{~nm}$ & & & $6 \mathrm{mb}$ & PUL & & 34.15 & 320 & $\mathrm{iPd}$ \\
\hline & $20 s$ & & 4.00 um & & & & 1 & & $1.2 \mathrm{~s}$ & 40 & $0.00 \mathrm{~nm}$ \\
\hline & $15 \mathrm{~s}$ & & 9.00 um & & & & 1 & $z$ & $12 \mathrm{~s}$ & & $0.00 \mathrm{um}$ \\
\hline & & & is & 50 & 27.00 & & 1 & $E$ & $12 \mathrm{~s}$ & & $0.00 \mathrm{um}$ \\
\hline KMI & 27.40 & 114 & Pd- & 45 & 59.00 & 0.8 & $i$ & & & & ePP \\
\hline & $10 \mathrm{~s}$ & & $6.10 \mathrm{um}$ & & & & $i$ & & & & EPCP \\
\hline & & & $P P$ & 47 & 40.00 & & 1 & & & & is \\
\hline & & & $S$ & 50 & 37.00 & & $A L T$ & & 34.52 & 283 & $E P$ \\
\hline XAN & 27.46 & 92 & $P$ & 45 & 57.80 & -0.7 & I BCK & & 34.63 & 280 & $e P$ \\
\hline HHC & 27.57 & 76 & eP & 46 & 01.00 & 1.5 & I IAS & & 34.80 & 298 & eP \\
\hline & & & es & 50 & 42.00 & & I PPE & & 34.87 & 296 & eP \\
\hline ANN & 28.22 & 293 & eP & 46 & 04.40 & -0.8 & ICLI & & 35.06 & 297 & iPc \\
\hline & $15 \mathrm{~s}$ & & $5.00 \mathrm{um}$ & & & $4 M s z X$ & FOC & & 35.24 & 295 & eP \\
\hline & $18 \mathrm{~s}$ & 128 & 0.00 um & & & & I BAC & & 35.32 & 297 & $e P$ \\
\hline & $15 \mathrm{~s}$ & & 6.50 um & & & & $E L L$ & & 35.38 & 280 & $i p$ \\
\hline & & & ePP & 46 & 48.40 & & DST & & 35.47 & 285 & eP \\
\hline & & & ePcP & 49 & 21.40 & & | VRI & & 35.54 & 296 & eP \\
\hline & & & es & 50 & 50.40 & & DMK & & 35.56 & 289 & eP \\
\hline$T I Y$ & 28.94 & 82 & $\mathrm{Pc}$ & 46 & 13.00 & 1.1 & ISR & & 35.77 & 295 & eP \\
\hline & & & $p P$ & 46 & 21.00 & $28 \mathrm{kmx}$ & I APA & & 35.83 & 334 & iPd \\
\hline & & & PP & 47 & 02.00 & & 1 & & $0.9 \mathrm{~s}$ & & $0.00 \mathrm{~nm}$ \\
\hline & & & PPP & 47 & 16.50 & & 1 & $z$ & $11 \mathrm{~s}$ & & $0.00 \mathrm{um}$ \\
\hline & & & $P \subset P$ & 49 & 24.50 & & 1 & & & & $i s p$ \\
\hline & & & s & 50 & 59.00 & & i & & & & is \\
\hline & & & sS & 51 & 16.00 & & $E D C$ & & 35.84 & 286 & ep \\
\hline MOS & 29.28 & 315 & iPd & 46 & 15.00 & 0.4 & I N 2 & & 35.89 & 89 & $\mathrm{Pc}$ \\
\hline & $2.0 \mathrm{~s}$ & 960 & $0.00 \mathrm{~nm}$ & & & $.2 \mathrm{mb}$ & 1 & & & & es \\
\hline & $16 \mathrm{~s}$ & 318 & 0.00 um & & & & $M L R$ & & 36.13 & 295 & $i P_{c}$ \\
\hline & $18 \mathrm{~s}$ & & 4. 90 um & & & & $B \cup C_{1}$ & & 36.27 & 293 & eP \\
\hline & & & iPP & 47 & 04 & & $\mathrm{GZH}$ & & 36.29 & 106 & eP \\
\hline & & & iPPP & 47 & 25.00 & & $K J F$ & & 36.61 & 327 & iP \\
\hline & & & es & 51 & 08.00 & & 1 & & $0.8 \mathrm{~s}$ & & $9.60 \mathrm{~nm}$ \\
\hline $\mathrm{CHG}$ & 29.38 & 129 & $i P d$ & 46 & 13.90 & -2.1 & $i$ & & & & es \\
\hline & $1.7 \mathrm{~s}$ & & $5.80 \mathrm{~nm}$ & & & $1 \mathrm{mb}$ & I CMP & & 36.80 & 295 & $I P C$ \\
\hline & & & es & 51 & 38.00 & & SUF & & 36.86 & 325 & $i p$ \\
\hline СHTO & 29.38 & 129 & eP- & 46 & 13.00 & $-3.0 x$ & 1 & & $0.5 \mathrm{~s}$ & & $9.10 \mathrm{~nm}$ \\
\hline & $2.0 \mathrm{~s}$ & 58 & $8.10 \mathrm{~nm}$ & & & $.0 \mathrm{mb}$ & i LVV & & 36.90 & 303 & $e^{P}$ \\
\hline & & & i & 46 & 19.50 & & & $z$ & $15 \mathrm{~s}$ & & $5.00 \mathrm{um}$ \\
\hline GYA & 29.40 & 108 & $P$ & 46 & 17.00 & 0.8 & i & $\mathrm{N}$ & $16 \mathrm{~s}$ & 148 & $8.00 \mathrm{um}$ \\
\hline & & & $p P$ & 46 & 27.00 & $35 \mathrm{kmx}$ & $i$ & $E$ & $14 \mathrm{~s}$ & & $0.00 \mathrm{um}$ \\
\hline KVT & 29.62 & 285 & eP & 46 & 19.00 & 1.1 & $i$ & & & & ePP \\
\hline OBN & 29.69 & 314 & ePc & 46 & 18.30 & 0.0 & i & & & & ePPP \\
\hline & $1.4 \mathrm{~s}$ & 420 & $0.00 \mathrm{~nm}$ & & & $1 \mathrm{mb}$ & 1 & & & & is \\
\hline & & & EPP & 47 & 10 & & 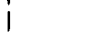 & & & & iss \\
\hline & & & is & 51 & 14 & & & & & & isS \\
\hline $\mathbf{R} \mathbf{I}$ & 30.26 & 9 & $i P_{C}$ & 46 & 23.60 & 0.4 & $i$ & & & & iSSS \\
\hline
\end{tabular}


$13 d \quad 01 \mathrm{~h}$

\begin{tabular}{|c|c|c|c|c|c|c|c|c|c|c|c|c|}
\hline $\mathrm{OHR}$ & & 40.79 & 290 & iP & 47 & 55.00 & 1.7 & 1 & & & & ! \\
\hline & & & & $\mathbf{i}$ & 49 & 29.10 & & i & & & & i \\
\hline SRO & & 40.91 & 300 & $e(P)$ & 48 & 02.00 & $7.8 x$ & 1 & & & & $\mathrm{i}$ \\
\hline & & & & $e(P P)$ & 49 & 31.20 & & i & & & & i \\
\hline & & & & $e(S)$ & 53 & 55.00 & & i & & & & (S) \\
\hline & & & & $i$ & 57 & 05.00 & & | TRI & & 44.44 & 298 & eP \\
\hline KHE & & 41.24 & 356 & ep & 47 & 59.00 & 2.5 & $i$ & & & & $i$ \\
\hline & & $1.3 \mathrm{~s}$ & 410 & $0.00 \mathrm{~nm}$ & & & . Amb & $i$ & & & & ePP \\
\hline & $z$ & 145 & 78 & 8.00 um & & & $.7 M s z X$ & i & & & & iPPP \\
\hline & $N$ & $14 \mathrm{~s}$ & 58 & 8.00um & & & & i & & & & is \\
\hline & $E$ & $14 \mathrm{~s}$ & 35 & $5.00 \mathrm{um}$ & & & & 1 & & & & iss \\
\hline & & & & iPP & 49 & 40.00 & & I KON & & 44.56 & 319 & iPt \\
\hline & & & & is & 54 & 16.00 & & $|P| P$ & & 44.57 & 185 & eP \\
\hline & & & & is & 57 & 06.00 & & I MOX & & 44.67 & 306 & $e^{P}$ \\
\hline ANP & & 41.26 & 97 & ePt & 48 & 02.00 & $4.6 x$ & 1 & & 2.25 & 438 & $8.00 \mathrm{~nm}$ \\
\hline & & & & is & 54 & 25.00 & & i & $z$ & $13 \mathrm{~s}$ & 61 & $1.90 \mathrm{um}$ \\
\hline TIK & & 41.28 & 23 & ePc & 47 & 57.80 & 0.1 & 1 & $\mathrm{~N}$ & $16 \mathrm{~s}$ & 132 & $2.00 \mathrm{um}$ \\
\hline & & 0.95 & 210 & $0.00 \mathrm{~nm}$ & & & $.9 m b$ & 1 & $E$ & $17 \mathrm{~s}$ & 65 & $5.50 \mathrm{um}$ \\
\hline & $z$ & $15 \mathrm{~s}$ & 90 & 0.00 um & & & $8 M s z X$ & i & & & & i \\
\hline & $N$ & 115 & 35 & $5.00 \mathrm{um}$ & & & & 1 & & & & iPP \\
\hline & $E$ & $15 \mathrm{~s}$ & 87 & 7.00 um & & & & 1 & & & & is \\
\hline & & & & ePP & 49 & 37.00 & & i & & & & iss \\
\hline & & & & EPCP & 49 & 59.00 & & I $A A E$ & & 44.75 & 236 & eP \\
\hline & & & & ePPP & 50 & 05.00 & & I SZP & & 44.94 & 106 & eP \\
\hline & & & & es & 54 & 12.00 & & I GRF & & 45.12 & 305 & eP \\
\hline TATO & & 41.35 & 97 & $\mathrm{P}+$ & 48 & 05.00 & $7.0 x$ & 1 & & $1.1 \mathrm{~s}$ & 313 & $3.00 \mathrm{~nm}$ \\
\hline ZST & & 41.64 & 301 & iP & 48 & 02.30 & 2.1 & I & $z$ & $16 \mathrm{~s}$ & 78 & 3. $50 \mathrm{um}$ \\
\hline & & & & $e$ & 49 & 25.80 & & 1 & & & & e \\
\hline & & & & $i(P P)$ & 49 & 40.50 & & HAM & & 45.16 & 310 & eP \\
\hline$K S P$ & & 41.71 & 305 & $e P$ & 47 & $59.5 \theta$ & -1.2 & I SHK & & 45.39 & 78 & $e P$ \\
\hline & & $1.5 \mathrm{~s}$ & 147 & $7.00 \mathrm{~nm}$ & & & $.5 \mathrm{mb}$ & I FUR & & 45.45 & 302 & $e P$ \\
\hline & & & & iPP & 49 & 44.00 & & I & & $1.2 \mathrm{~s}$ & 396 & $5.00 \mathrm{~nm}$ \\
\hline & & & & e & 54 & 18.00 & & i & & & & es \\
\hline & & & & is & 57 & 10.00 & & I KGM & & 45.61 & 139 & ePc \\
\hline VLA & & 41.84 & 67 & $e P$ & 48 & 02.00 & 0.1 & i & & & & e \\
\hline & & & & ePP & 49 & 46.00 & & I BAG & & 45.75 & 108 & $e P$ \\
\hline TSI & & 42.03 & 144 & $e(P)$ & 48 & 06.00 & 2.4 & i & & $1.7 \mathrm{~s}$ & 308 & $3.00 \mathrm{~nm}$ \\
\hline VIE & & 42.12 & 301 & eP & 48 & 05.50 & 1.4 & i & & & & es \\
\hline & & & & e & 49 & 43.00 & & I OGA & & 45.95 & 301 & $e^{P}$ \\
\hline & & & & $e$ & 57 & 05.00 & & I KBS & & 45.98 & 346 & ip \\
\hline & & & & $\mathbf{i}$ & 03 & 37.00 & & I & & & & is \\
\hline VKA & & 42.14 & 301 & $e(P)$ & 48 & 06.00 & 1.7 & $|P P|$ & & 46.36 & 144 & ePc \\
\hline & & & & e & 50 & 02.80 & & 1 & & $0.8 \mathrm{~s}$ & 70 & 0. $10 \mathrm{~nm}$ \\
\hline IPM & & $42 \cdot 25$ & 140 & ePd & 48 & 05.00 & -0.5 & 1 & & & & $e(S)$ \\
\hline HFS & & 42.47 & 319 & $e P$ & 48 & 05.80 & -1.0 & $\mathrm{I} \mathrm{OKH}$ & & 46.57 & 49 & iPc \\
\hline & & $0.8 \mathrm{~s}$ & 128 & $8.00 \mathrm{~nm}$ & & & $.7 \mathrm{mb}$ & I & & $6.0 \mathrm{~s}$ & 2600 & $0.00 \mathrm{~nm}$ \\
\hline & $z$ & $16 \mathrm{~s}$ & 71 & $1.70 \mathrm{um}$ & & & $.7 M \leq z X$ & i & $z$ & $12 \mathrm{~s}$ & 49 & $9.90 \mathrm{um}$ \\
\hline PSI & & 42.90 & 144 & ePc & 48 & 08.20 & -2.6 & 1 & $\mathrm{~N}$ & $12 \mathrm{~s}$ & 20 & $0.00 \mathrm{um}$ \\
\hline & & $0.6 \mathrm{~s}$ & 24 & $4.90 \mathrm{~nm}$ & & & . $1 \mathrm{mb}$ & 1 & $E$ & $12 \mathrm{~s}$ & 46 & $5.80 u m$ \\
\hline PRU & & 42.96 & 304 & $e P$ & 48 & 10.20 & -0.7 & 1 & & & & is \\
\hline & & $1.5 \mathrm{~s}$ & 134 & $4.00 \mathrm{~nm}$ & & & $.5 m b$ & i & & & & ESPP \\
\hline & $\mathrm{N}$ & $17 \mathrm{~s}$ & 219 & $9.00 \mathrm{um}$ & & & & I BER & & 46.62 & 320 & iPd \\
\hline & E & $17 \mathrm{~s}$ & 68 & 8.40 um & & & & I $F \mid R$ & & 46.71 & 297 & $e P$ \\
\hline & & & & $P P$ & 49 & 56.00 & & 1 & & & & ePP \\
\hline & & & & $S$ & 54 & 2700 & & 1 & & & & IPPP \\
\hline BRG & & 43.17 & 306 & $e^{P}$ & 48 & 11.90 & -0.8 & 1 & & & & is \\
\hline & & $1.4 \mathrm{~s}$ & 190 & $0.00 \mathrm{~nm}$ & & & $.6 \mathrm{mb}$ & I TNS & & 46.74 & 306 & $e P$ \\
\hline & & & & e & 48 & 17.90 & & 1 & & & & ePP \\
\hline & & & & e & 49 & 59.00 & & 1 & & & & e \\
\hline & & & & es & 54 & 4400 & & I WIT & & 47.21 & 310 & $e P$ \\
\hline & & & & $\operatorname{scs}$ & 58 & 13.10 & & 1 & & $1.0 \mathrm{~s}$ & 206 & $6.00 \mathrm{~nm}$ \\
\hline$B R L$ & & 43.31 & 368 & $e P$ & 48 & 14.56 & 0.8 & I WTS & & 47.31 & 309 & $e P$ \\
\hline COP & & 43.40 & 313 & IPc & 48 & 19.80 & $5.4 X$ & 1 & & $0.8 \mathrm{~s}$ & & $5.00 \mathrm{~nm}$ \\
\hline & & 0.75 & 148 & B. $00 \mathrm{~nm}$ & & & $.9 m b$ & 1 & & & & e \\
\hline & & & & is & 54 & 45.00 & & 1 & & & & e \\
\hline KMR & & 43.62 & 302 & eP & 48 & 30.00 & $13.7 x$ & BGG & & 47.43 & 306 & eP \\
\hline CLL & & 43.68 & 306 & eP & 48 & 15.00 & -1.8 & I ZUL & & 47.51 & 302 & ePd \\
\hline & & $1.3 \mathrm{~s}$ & 135 & $5.00 \mathrm{~nm}$ & & & $.6 \mathrm{mb}$ & I GWF & & 47.58 & 304 & $i P_{C}$ \\
\hline & & & & es & 54 & 46.00 & & CDF & & 47.96 & 304 & eP \\
\hline NB2 & & 43.68 & 321 & $P$ & 48 & 14.30 & -2.5 & i & & $0.9 \mathrm{~s}$ & 48 & $3.20 \mathrm{~nm}$ \\
\hline & & $0.8 \mathrm{~s}$ & 106 & $6.00 \mathrm{~nm}$ & & 5 & $.7 \mathrm{mb}$ & | YSS & & 48.09 & 58 & ePc \\
\hline $\mathrm{KHC}$ & & 43.74 & 303 & iPd & 48 & 16.90 & -0.5 & $i$ & & $0.8 \mathrm{~s}$ & 30 & $0.00 \mathrm{~nm}$ \\
\hline & & $1.2 \mathrm{~s}$ & 90 & $0.00 \mathrm{~nm}$ & & & $.4 \mathrm{mb}$ & 1 & $z$ & $13 \mathrm{~s}$ & 40 & 0.00 um \\
\hline & $z$ & $10 \mathrm{~s}$ & 18 & 8. 50 um & & & $.3 M \leq z X$ & 1 & $\mathrm{~N}$ & $13 \mathrm{~s}$ & 39 & $9.20 \mathrm{um}$ \\
\hline & $E$ & $10 \mathrm{~s}$ & 19 & $9.00 \mathrm{um}$ & & & & i & $E$ & 135 & & 1.80 um \\
\hline & & & & $i$ & 48 & $2 \theta .00$ & & i & & & & EPCP \\
\hline & & & & $S$ & 54 & 50.00 & & i & & & & ePP \\
\hline LJU & & 43.83 & 299 & $e P$ & 48 & 17.90 & -0.2 & 1 & & & & es \\
\hline & & & & es & 54 & 52.40 & & I ENN & & 48.16 & 307 & $e P$ \\
\hline & & & & eSS & 58 & 12.00 & & i & & $1.0 \mathrm{~s}$ & 87 & $7.00 \mathrm{~nm}$ \\
\hline$K L M$ & & 43.84 & 140 & ePc & $4 \varepsilon$ & 19.50 & 1.1 & i & & & & e \\
\hline CEY & & 43.98 & 298 & $e P d$ & 48 & 21.70 & 2.4 & 1 & & & & e \\
\hline WET & & 44.19 & 303 & $\mathrm{iPC}$ & 48 & 20.60 & -0.4 & I DBN & & 48.27 & 309 & eP \\
\hline & & $1.4 \mathrm{~s}$ & 216 & $6.00 \mathrm{~nm}$ & & & $.8 \mathrm{mb}$ & 1 & $z$ & $20 s$ & 23 & $3.00 \mathrm{um}$ \\
\hline$K B A$ & & 44.37 & 300 & $e^{P}$ & 48 & 22.00 & -0.6 & 1 & & & & ePP \\
\hline & & $0.9 \mathrm{~s}$ & 56 & $6.80 \mathrm{~nm}$ & 48 & $29.30^{5}$ & $.4 \mathrm{mb}$ & 1 & & & & $\begin{array}{l}\text { es } \\
\text { ess }\end{array}$ \\
\hline
\end{tabular}




\begin{tabular}{|c|c|c|c|c|c|c|c|c|c|c|c|}
\hline & $0.8 \mathrm{~s}$ & & $5.30 \mathrm{~nm}$ & & & $.6 \mathrm{mb}$ & I GUA & 65.91 & 92 & $e(P)$ & 50 \\
\hline NAI & 54.00 & $23 \theta$ & $E P$ & 49 & 39.00 & 1.8 & i & $18 \mathrm{~s}$ & & 3. $25 \mathrm{um}$ & \\
\hline & $1.5 \mathrm{~s}$ & & $7.20 \mathrm{~nm}$ & & & $6 \mathrm{mb}$ & I CLK & 66.86 & 223 & eP & 51 \\
\hline CGP & 54.04 & 112 & eP & 49 & 34.50 & -2.7 & 1 & & & es & 59 \\
\hline EPF & 54.39 & 299 & iPc & 49 & $37.3 \theta$ & -2.3 & I KUPT & 67.04 & 127 & eP & 51 \\
\hline & $0.8 \mathrm{~s}$ & & $9.80 \mathrm{~nm}$ & & & $2 \mathrm{mb}$ & I IMA & 67.76 & 19 & ePc & 51 \\
\hline DDK & 54.53 & 313 & i Pc & 49 & 38.10 & -2.3 & I TET & 67.98 & 224 & iP & 51 \\
\hline & $0.7 \mathrm{~s}$ & 15 & D. $\theta 00 \mathrm{~nm}$ & & 6 & $1 \mathrm{mb}$ & 1 & $1.0 \mathrm{~s}$ & & d. $11 \mathrm{~nm}$ & \\
\hline DMU & 54.67 & 314 & iPc & 49 & 41.00 & -0.4 & 1 & & & $e(S)$ & 00 \\
\hline & $1.2 \mathrm{~s}$ & 22 & $0.00 \mathrm{~nm}$ & & 6 & $1 \mathrm{mb}$ & I TTA & 69.56 & 22 & ePd & 51 \\
\hline DLE & 54.68 & 313 & iPc & 49 & 39.70 & -1.8 & I MTD & 69.57 & 225 & eP & 51 \\
\hline & $0.7 \mathrm{~s}$ & 9 & $0.00 \mathrm{~nm}$ & & & $.9 \mathrm{mb}$ & I INK & 69.91 & 11 & eP & 51 \\
\hline$D C N$ & 55.07 & 314 & iPC & 49 & 42.00 & -2.4 & 1 & $1.2 \mathrm{~s}$ & 167 & $.00 \mathrm{~nm}$ & \\
\hline & $0.7 \mathrm{~s}$ & 12 & $0.00 \mathrm{~nm}$ & & & $\theta m b$ & $1 \mathrm{COL}$ & 70.16 & 18 & eP & 51 \\
\hline SKR & 55.17 & 50 & eP & 49 & 44.20 & -0.9 & 1 & $19 \mathrm{~s}$ & & $2.00 \mathrm{um}$ & \\
\hline & $0.6 \mathrm{~s}$ & & $0.00 \mathrm{~nm}$ & & & $.7 \mathrm{mb}$ & I FBA & 70.16 & 18 & $P$ & 51 \\
\hline$z$ & $14 \mathrm{~s}$ & & $1.60 \mathrm{um}$ & & & $5 M s z X$ & 1 & $.9 \mathrm{~s}$ & & $0.90 \mathrm{~nm}$ & \\
\hline $\mathrm{N}$ & 145 & 3 & 1. $60 \mathrm{um}$ & & & & | KR | & 70.69 & 226 & eP & 51 \\
\hline$E$ & $14 \mathrm{~s}$ & & $5.30 \mathrm{um}$ & & & & | PMR & 72.58 & 21 & ePc & 51 \\
\hline & & & ePCP & 50 & $42.4 \theta$ & & i & $0.8 \mathrm{~s}$ & & $5.78 \mathrm{~nm}$ & \\
\hline & & & es & 57 & 31.60 & & 1 & $18 \mathrm{~s}$ & & $.00 \mathrm{um}$ & \\
\hline & & & eSS & 01 & 05.60 & & | FRB & 72.67 & 344 & eP & 51 \\
\hline$A B A$ & 55.21 & 291 & eP & 49 & $45.5 \theta$ & -0.1 & I TOA & 72.91 & 19 & $P$ & 51 \\
\hline EBR & 55.21 & 297 & $e P$ & 49 & 47.00 & 1.5 & I JAY & 73.12 & 108 & ePd & 51 \\
\hline & & & es & 57 & 26.00 & & 1 BUL & 73.93 & 225 & eP & 51 \\
\hline LEM & 55.36 & 140 & ePd & 49 & 48.50 & 1.5 & 1 & & & is & 01 \\
\hline DAV & 55.61 & 112 & eP & 49 & 50.60 & 1.3 & $K D C$ & 4.75 & 24 & $P$ & 51 \\
\hline & & & eS & 57 & 40.00 & & $P C A$ & 76.08 & 17 & $(P)$ & 51 \\
\hline$A K U$ & 55.65 & 330 & eP & 49 & 48.80 & 0.5 & I CNG & 77.16 & 219 & iP & 52 \\
\hline & $1.2 \mathrm{~s}$ & & $5.00 \mathrm{~nm}$ & & & $6 \mathrm{mb}$ & I YKA & 7.56 & 5 & eP & 52 \\
\hline DET & 55.73 & 47 & eP & 49 & 44.00 & $-5.1 x$ & I RSNT & 77.57 & 5 & $P$ & 52 \\
\hline$z$ & $14 \mathrm{~s}$ & 1 & $7.00 \mathrm{um}$ & & & $3 M s=X$ & I YKC & 77.58 & 5 & eP & 52 \\
\hline $\mathbf{N}$ & $14 \mathrm{~s}$ & & $3.00 \mathrm{um}$ & & & & 1 & $0.9 \mathrm{~s}$ & 114 & $4.00 \mathrm{~nm}$ & \\
\hline$E$ & $14 \mathrm{~s}$ & & $5.40 u m$ & & & & I MEK & 77.58 & 141 & eP & 52 \\
\hline & & & es & 57 & 34.00 & & I KIC & 78.25 & 268 & ep & 52 \\
\hline & & & escs & 59 & 40.00 & & I SLR & 78.65 & 222 & iPc & 52 \\
\hline LGR & 56.53 & 300 & iPc & 49 & 57.30 & 2.2 & 1 & $1.2 \mathrm{~s}$ & & $2.80 \mathrm{~nm}$ & \\
\hline & & & $i$ & 50 & 48.30 & & 1 & $18 \mathrm{~s}$ & & $5.02 \mathrm{um}$ & \\
\hline VAL & 57.29 & 313 & ip & 50 & 02.70 & 2.5 & I PRE & 78.72 & 222 & $e(P)$ & 52 \\
\hline & & & is & 57 & 54.00 & & $3 P 1$ & 9.14 & 222 & eP & 52 \\
\hline TRT & 58.71 & 135 & iPC & 50 & 09.00 & -1.5 & | PRY & 80.04 & 222 & eP & 52 \\
\hline & $0.4 \mathrm{~s}$ & & $5.20 \mathrm{~nm}$ & & 5 & $9 \mathrm{mb}$ & I MUN & 81.06 & 146 & eP & 52 \\
\hline TOL & 58.73 & 298 & $e P$ & 50 & 11.00 & 0.4 & I WRA & 81.16 & 125 & $P C$ & 52 \\
\hline & & & ePP & 52 & 28.00 & & 1 & $1.3 \mathrm{~s}$ & & $5.40 \mathrm{~nm}$ & \\
\hline & & & iPPP & 53 & 53.00 & & 1 พB2 & 81.16 & 125 & eP & 52 \\
\hline & & & is & 58 & 21.00 & & $1 F C C$ & 81.20 & 354 & eP & 52 \\
\hline & & & isS & 02 & 08.00 & & $|V| R$ & 81.29 & 222 & eP & 52 \\
\hline I LT & 59.20 & 25 & iPC & 50 & 12.80 & -0.6 & I WBN & 81.34 & 135 & eP & 52 \\
\hline & $1.0 \mathrm{~s}$ & & $0.00 \mathrm{~nm}$ & & & $.7 \mathrm{mb}$ & 1 & $0.5 \mathrm{~s}$ & & $4.00 \mathrm{~nm}$ & \\
\hline$z$ & $18 \mathrm{~s}$ & & $0.00 u m$ & & & $7 \mathrm{Msz}$ & I SWZ & 81.37 & 224 & iPC & 52 \\
\hline $\mathrm{N}$ & $20 \mathrm{~s}$ & & $2.00 \mathrm{um}$ & & & & 1 & $0.6 \mathrm{~s}$ & & $.00 \mathrm{~nm}$ & \\
\hline E & $20 s$ & & $5.00 \mathrm{um}$ & & & & I NWAO & 82.32 & 145 & eP & 52 \\
\hline & & & IPCP & 51 & 10.00 & & 1 & $20 \mathrm{~s}$ & & 2. $40 \mathrm{um}$ & \\
\hline & & & iPP & 52 & 25.00 & & 1 & $20 \mathrm{~s}$ & & 2. 00 um & \\
\hline & & & is & 58 & 16.00 & & 1 & 205 & & 1.30 um & \\
\hline & & & ESP & 58 & 29.00 & & I KLG & 82.46 & 141 & eP & 52 \\
\hline & & & eSSS & 04 & 38.00 & & I BLF & 82.47 & 222 & eP & 52 \\
\hline CRT & 59.71 & 295 & eP & 50 & 1.00 & $3.5 x$ & I PMG & 82.47 & 109 & eP & 52 \\
\hline TAM & 60.48 & 276 & ip & 50 & 18.50 & $-4.5 x$ & I MBO & 82.53 & 282 & eP & 52 \\
\hline MAL & 60.50 & 295 & iP & 50 & 22.50 & -0.3 & i & & & e & 52 \\
\hline & & & iPPP & 54 & 23.00 & & 1 & & & e & 02 \\
\hline & & & is & 58 & 28.00 & & 1 & & & e & 03 \\
\hline & & & iss & 02 & 38.00 & & I KIM & 2.91 & 223 & eP & 52 \\
\hline $\mathrm{KS}$ & 60.65 & 127 & $e(P)$ & 50 & 22.00 & -2.0 & 1 & $.9 \mathrm{~s}$ & & $.00 \mathrm{~nm}$ & \\
\hline PTO & 61.18 & 301 & eP & 50 & 28.00 & 0.7 & I HVD & 84.03 & 221 & iPd & 52 \\
\hline & & & es & 54 & 22.00 & & 1 & $0.9 \mathrm{~s}$ & & $3.00 \mathrm{~nm}$ & \\
\hline SFS & 61.89 & 295 & eP & 50 & 30.00 & -2.2 & I FFC & 85.62 & 358 & $e^{P}$ & 52 \\
\hline & & & ePP & 53 & 22.00 & & 1 & 9 & & $1.00 \mathrm{~nm}$ & \\
\hline & & & es & 57 & 41.00 & & I EDM & 6.86 & 5 & ePC & 52 \\
\hline & & & ess & 82 & 01.00 & & 1 & $0.6 \mathrm{~s}$ & & $7.00 \mathrm{~nm}$ & \\
\hline BNG & 61.96 & 251 & IPd & 50 & 31.00 & -1.9 & I CBM & 87.48 & 336 & eP & 53 \\
\hline & $1.0 \mathrm{~s}$ & & $0.00 \mathrm{~nm}$ & & & $2 \mathrm{mb}$ & I SUR & 87.87 & 224 & IPd & 53 \\
\hline BCAO & 61.97 & 251 & iPdt & 50 & 30.00 & -3.0 & 1 & 0.95 & & $.00 \mathrm{~nm}$ & \\
\hline & $1.0 \mathrm{~s}$ & 11 & $1.00 \mathrm{~nm}$ & & & $\theta \mathrm{mb}$ & I & $19 \mathrm{~s}$ & & 3. $80 \mathrm{um}$ & \\
\hline & & & es & 58 & 52.00 & & I RSON & 89.01 & 353 & eP & 53 \\
\hline IFR & 62.64 & 292 & iP & 50 & 39.50 & 2.0 & 1 & & & $\mathrm{i}$ & 53 \\
\hline$M B C$ & 63.67 & & eP & 50 & 41.50 & -1.9 & I CTA & 89.08 & 117 & iPc & 53 \\
\hline & $0.8 \mathrm{~s}$ & 195 & $5.00 \mathrm{~nm}$ & & 6 & $3 \mathrm{mb}$ & I & & & iSKS & 03 \\
\hline$M$ & 63.97 & 209 & ePd & 50 & 45.10 & -1.1 & 1 & & & $\mathrm{i}$ & 09 \\
\hline PA & 64.21 & 279 & $e^{P}$ & 50 & 50.00 & 2. & I CTAO & 89.08 & 117 & $e(P)$ & 53 \\
\hline$A V E$ & 64.38 & 293 & iP & 50 & 50.50 & 1. 8 & 1 & $1 \mathrm{~s}$ & & $9.60 \mathrm{~nm}$ & \\
\hline GDH & 64.74 & 342 & iPC & 50 & 50.20 & -0.3 & I EMM & 89.22 & 334 & eP & 53 \\
\hline & $1.0 \mathrm{~s}$ & & $0.00 \mathrm{~nm}$ & & 5 & $2 \mathrm{mb}$ & I SES & 89.82 & & eP & 53 \\
\hline & & & $\mathrm{i}$ & 59 & 42.00 & & 1 & $0.8 \mathrm{~s}$ & & $5.00 \mathrm{~nm}$ & \\
\hline ANM & 65.52 & 24 & $P$ & 50 & 55.00 & -0.5 & I PNT & 90.11 & 10 & eP & 53 \\
\hline GUMO & 65.85 & 92 & $P+$ & 50 & 58.00 & -0.3 & 1 & $0.6 \mathrm{~s}$ & 38 & $3.00 \mathrm{~nm}$ & \\
\hline & & & es & 59 & 44.00 & & $1 P G C$ & 90.20 & 12 & eP & 53 \\
\hline
\end{tabular}


$03 d \quad 02 \mathrm{~h}$

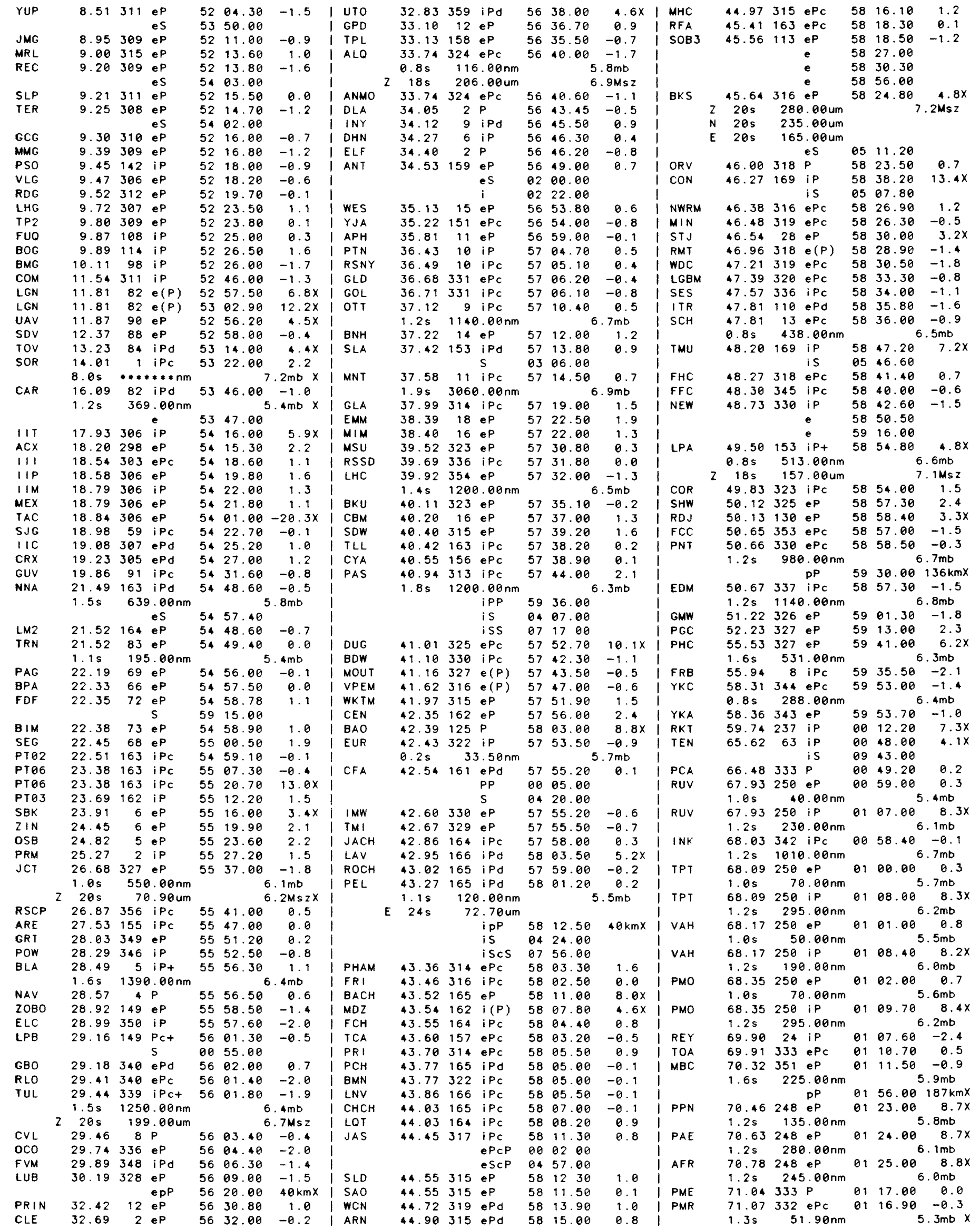




\begin{tabular}{|c|c|c|c|c|c|c|c|c|c|c|c|}
\hline & $18 \mathrm{~s}$ & $34 e$ & $0.00 \mathrm{um}$ & & & $.7 \mathrm{MsZ}$ & 1 & & $1.7 \mathrm{~s}$ & 685 & $5.00 \mathrm{~nm}$ \\
\hline $\mathrm{COL}$ & 71.55 & 336 & IPC & 01 & 19.70 & -0.4 & $M Z F$ & & 80.84 & 45 & iPc \\
\hline & $1.0 \mathrm{~s}$ & 26 & $4.00 \mathrm{~nm}$ & & & $.1 \mathrm{mb}$ & 1 & & $1.2 \mathrm{~s}$ & 513 & $3.00 \mathrm{~nm}$ \\
\hline & & & es & 10 & 47.00 & & $A V F$ & & 81.34 & 44 & $I P C$ \\
\hline FBA & 71.55 & 336 & $P$ & 01 & 19.00 & -1.1 & $i$ & & $1.6 \mathrm{~s}$ & 524 & $4.00 \mathrm{~nm}$ \\
\hline$K D C$ & 71.71 & 328 & ePc & 01 & 21.30 & 0.2 & SSF & & 81.43 & 44 & $i P_{C}$ \\
\hline AKU & 71.97 & 23 & ip & 01 & 21.40 & $-1 \cdot 1$ & i & & $1.5 \mathrm{~s}$ & 515 & $5.00 \mathrm{~nm}$ \\
\hline & $1.4 \mathrm{~s}$ & 1108 & $0.00 \mathrm{~nm}$ & & & $.6 \mathrm{mb}$ & i LOR & & 81.65 & 43 & iPc \\
\hline & $22 \mathrm{~s}$ & 203 & $3.00 \mathrm{um}$ & & & $.3 M s z$ & I SMF & & 81.68 & 44 & iPC \\
\hline & & & $\mathrm{i}$ & 25 & 02.60 & & 1 & & $1.6 \mathrm{~s}$ & 537 & $7.00 \mathrm{~nm}$ \\
\hline LIS & 72.13 & 52 & IPC & 81 & 24.40 & 0.4 & LBF & & 81.75 & 44 & iPC \\
\hline VAL & 72.67 & 38 & iP & 01 & 26.36 & -0.6 & $i$ & & $1.8 \mathrm{~s}$ & 549 & $9.00 \mathrm{~nm}$ \\
\hline & & & $\mathrm{s}$ & 10 & 56.00 & & UCC & & 81.76 & 40 & Pot \\
\hline PTO & 72.70 & 50 & $i P_{C}$ & 01 & 27.10 & -0.2 & i & & & & $P P$ \\
\hline & & & is & 10 & 48.00 & & 1 & & & & SKS \\
\hline HON & 72.84 & 290 & eP & 01 & 30.00 & 1.6 & 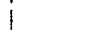 & & & & ss \\
\hline & & & es & 11 & 00.00 & & i Dou & & 81.95 & 41 & Pct \\
\hline KIP & 72.84 & 290 & $e^{P}$ & 01 & 30.00 & 1.6 & 1 & & $1.5 \mathrm{~s}$ & 1070 & $0.00 \mathrm{~nm}$ \\
\hline & & & e & 01 & 44.70 & & $i$ & & & & e \\
\hline AVE & 73.37 & 58 & iP & 01 & 32.00 & 0.6 & 1 & & & & PP \\
\hline SVW & 73.98 & 331 & ePc & 01 & 33.90 & -0.5 & $i$ & & & & SKS \\
\hline IMA & 74.24 & 336 & $P$ & 01 & 35.80 & -0.2 & i & & & & e \\
\hline$A L E$ & 74.29 & 3 & eP & 01 & 34.00 & -1.9 & i & & & & PS \\
\hline & $1.2 \mathrm{~s}$ & 41 & $1.00 \mathrm{~nm}$ & & 6 & $.3 m b$ & DBN & & 82.18 & 38 & iPt \\
\hline $\mathrm{OHC}$ & 74.37 & 168 & IPd & 01 & 44.10 & $7.6 x$ & $i$ & $z$ & $20 \mathrm{~s}$ & 112 & $2.00 \mathrm{um}$ \\
\hline SFS & 74.40 & 55 & $I P_{C}$ & 01 & 40.00 & 2.7 & $i$ & & & & is \\
\hline & & & ePP & 04 & 05.00 & & I & & & & ePS \\
\hline & & & es & 11 & 20.00 & & 1 & & & & eSS \\
\hline & & & eSS & 14 & 30.00 & & 1 & & & & e \\
\hline & & & eSSS & 16 & 10.00 & & i & & & & e \\
\hline TTA & 74.53 & 333 & ePc & 01 & 37.10 & -0.5 & SSB & & 82.27 & 45 & $i P c$ \\
\hline IFR & 75.29 & 58 & iP & 01 & 43.00 & 0.3 & I ENN & & 82.75 & 40 & ePc \\
\hline SDN & 75.70 & 325 & ePc & 01 & 45.20 & 0.9 & 1 & & $1.1 \mathrm{~s}$ & 417 & $7.00 \mathrm{~nm}$ \\
\hline$D A G$ & 75.84 & 12 & iPc & 01 & 43.10 & -1.7 & 1 & & & & e \\
\hline & $1.3 \mathrm{~s}$ & 769 & $9.00 \mathrm{~nm}$ & & 6 & $.5 m b$ & i & & & & e \\
\hline & & & is & 11 & 27.00 & & I WLF & & 82.99 & 41 & $\mathrm{P}+$ \\
\hline MAL & 75.84 & 54 & iPc & 01 & 47.00 & 1.5 & i & & & & SKS \\
\hline & $1.2 \mathrm{~s}$ & 13 & $3.50 \mathrm{~nm}$ & & & $.8 \mathrm{mb} x$ & i & & & & e \\
\hline & & & iPP & 04 & 12.00 & & I & & & & PS \\
\hline & & & is & 11 & 55.00 & & I & & 83.03 & 38 & ePd \\
\hline TOL & 76.12 & 51 & iPt & 01 & 47.50 & 0.4 & i & & $1.0 \mathrm{~s}$ & 813 & $3.00 \mathrm{~nm}$ \\
\hline & & & iPP & 04 & 55.00 & & 1 & & & & e \\
\hline & & & iPPP & 06 & 28.00 & & i & & & & e \\
\hline & & & is & 11 & 39.00 & & I WTS & & 83.19 & 39 & IPC \\
\hline & & & $\mathrm{i}(\mathrm{PS})$ & 12 & 25.00 & & i & & $1.0 \mathrm{~s}$ & 549 & $9.00 \mathrm{~nm}$ \\
\hline & & & iss & 16 & 15.00 & & 1 & & & & e \\
\hline & & & iSSS & 20 & 00.00 & & 1 & & & & e \\
\hline & & & i & 21 & 50.00 & & I HAU & & 83.27 & 43 & eP \\
\hline CRT & 76.49 & 54 & ip & 01 & 51.60 & 2.3 & BSF & & 83.59 & 43 & $e^{P}$ \\
\hline & & & is & 11 & 46.10 & & I BAF & & 83.72 & 43 & iPc \\
\hline ALR & 76.96 & 55 & $e^{P}$ & 01 & 50.00 & -1.8 & I LRG & & 83.73 & 47 & eP \\
\hline & & & $i$ & 02 & 04.00 & & 1 & & $1.6 \mathrm{~s}$ & 496 & $6.00 \mathrm{~nm}$ \\
\hline ESK & 77.18 & 35 & eP & 01 & 52.00 & -0.6 & $\mathrm{ECH}$ & & 83.79 & 42 & $i P_{C}$ \\
\hline & $1.5 \mathrm{~s}$ & 1400 & $0.00 \mathrm{~nm}$ & & & $.8 \mathrm{mb}$ & I CDF & & 83.85 & 42 & eP \\
\hline EKA & 77.20 & 35 & $\mathrm{Pc}$ & 01 & 51.70 & -1.1 & L LMR & & 83.85 & 47 & eP \\
\hline & $1.2 \mathrm{~s}$ & 118 & $8.00 \mathrm{~nm}$ & & & $.8 \mathrm{mb}$ & i & & $1.8 \mathrm{~s}$ & 648 & $8.00 \mathrm{~nm}$ \\
\hline LGR & 77.29 & 48 & iPC & 01 & 55.00 & 1.4 & FRF & & 83.93 & 47 & $I P C$ \\
\hline & & & ePP & 04 & 58.00 & & $i$ & & $1.6 \mathrm{~s}$ & 578 & $8.00 \mathrm{~nm}$ \\
\hline & & & es & 11 & 54.00 & & I GWF & & 84.01 & 41 & $I P C$ \\
\hline ALM & 77.41 & 54 & $i P c$ & 01 & 53.70 & -0.6 & i ILT & & 84.09 & 338 & $\mathbf{P}$ \\
\hline & $1.3 \mathrm{~s}$ & & $4.10 \mathrm{~nm}$ & & 4 & $.3 \mathrm{mb} x$ & $D$ DIX & & 84.19 & 44 & ePt \\
\hline & $30 \mathrm{~s}$ & & $2.10 \mathrm{um}$ & & & & TNS & & 84.41 & 40 & ePc \\
\hline & $20 s$ & & 6.00 um & & & & BUH & & 84.45 & 42 & ePc \\
\hline & & & iPP & 05 & 12.40 & & I NB2 & & 84.57 & 29 & $\mathrm{P}$ \\
\hline & & & is & 12 & 38.70 & & i & & $1.6 \mathrm{~s}$ & 2240 & $0.00 \mathrm{~nm}$ \\
\hline KIC & 77.64 & 85 & iPc & 01 & 56.20 & 0.2 & I MMK & & 84.58 & 44 & ePt \\
\hline & & & is & 12 & 37.20 & & ZUL & & 84.70 & 43 & ePt \\
\hline LPF & 78.32 & 43 & eP & 01 & 58.70 & -0.3 & SLE & & 84.73 & 43 & ePt \\
\hline GRR & 78.44 & 42 & $i P_{C}$ & 01 & 59.70 & 0.0 & HAM & & 84.91 & 37 & IPC \\
\hline & $1.5 \mathrm{~s}$ & 1220 & $0.00 \mathrm{~nm}$ & & & $.7 \mathrm{mb}$ & STU & & 85.07 & 42 & iPct \\
\hline$F L N$ & 78.69 & 42 & $i P C$ & 02 & 01.00 & -0.1 & 1 & & $1.3 \mathrm{~s}$ & 577 & $7.00 \mathrm{~nm}$ \\
\hline & $1.5 \mathrm{~s}$ & 1050 & $9.00 \mathrm{~nm}$ & & & $.6 \mathrm{mb}$ & i & $z$ & $20 s$ & 199 & $9.00 \mathrm{um}$ \\
\hline ANM & 78.86 & 334 & ePC & 02 & 02.50 & 0.8 & LLS & & 85.19 & 43 & ePt \\
\hline$A L I$ & 78.92 & 53 & $i P_{-}$ & 02 & 04.00 & 1.5 & TMA & & 85.20 & 44 & ePt \\
\hline & & & es & 12 & 21.00 & & $A D K$ & & 85.40 & 322 & $i P c$ \\
\hline LDF & 78.93 & 42 & $i P_{C}$ & 02 & 02.10 & -0.3 & CVF & & 85.67 & 48 & $I P C$ \\
\hline & $1.5 \mathrm{~s}$ & 1150 & $0.00 \mathrm{~nm}$ & & & $.6 \mathrm{mb}$ & i & & $1.5 \mathrm{~s}$ & 653 & $3.00 \mathrm{~nm}$ \\
\hline$M F F$ & 78.94 & 44 & $i P c$ & 02 & 02.40 & -0.1 & HFS & & 85.92 & 30 & ip \\
\hline & $1.6 \mathrm{~s}$ & 800 & $0.00 \mathrm{~nm}$ & & & $.4 \mathrm{mb}$ & 1 & $z$ & $30 \mathrm{~s}$ & & 3. $50 \mathrm{um}$ \\
\hline EPF & 79.38 & 48 & iPc & 02 & 05.30 & 0.3 & COP & & 86.02 & 34 & iPd \\
\hline LFF & 79.60 & 46 & IPC & 02 & 06.30 & 0.2 & 1 & & $1.2 \mathrm{~s}$ & 1130 & $0.00 \mathrm{~nm}$ \\
\hline LPO & 79.93 & 46 & $I P C$ & 02 & 08.00 & 0.1 & 1 & & & & is \\
\hline LSF & 80.11 & 45 & iPc & 02 & 08.60 & -0.2 & GRFO & & 86.25 & 40 & $P$ \\
\hline RJF & 80.14 & 45 & iPc & 02 & 09.10 & 0.1 & GRF & & 86.25 & 40 & $i P c$ \\
\hline NKI & 80.54 & 322 & ePc & 02 & 11.70 & 0.8 & . & $z$ & $21 \mathrm{~s}$ & 336 & 6.00um \\
\hline CAF & 80.54 & 46 & $i P_{C}$ & 02 & 11.20 & 0.0 & 1 & & & & e \\
\hline TCF & 80.58 & 44 & iPC & 02 & 10.90 & -0.4 & & & & & eSKS \\
\hline
\end{tabular}




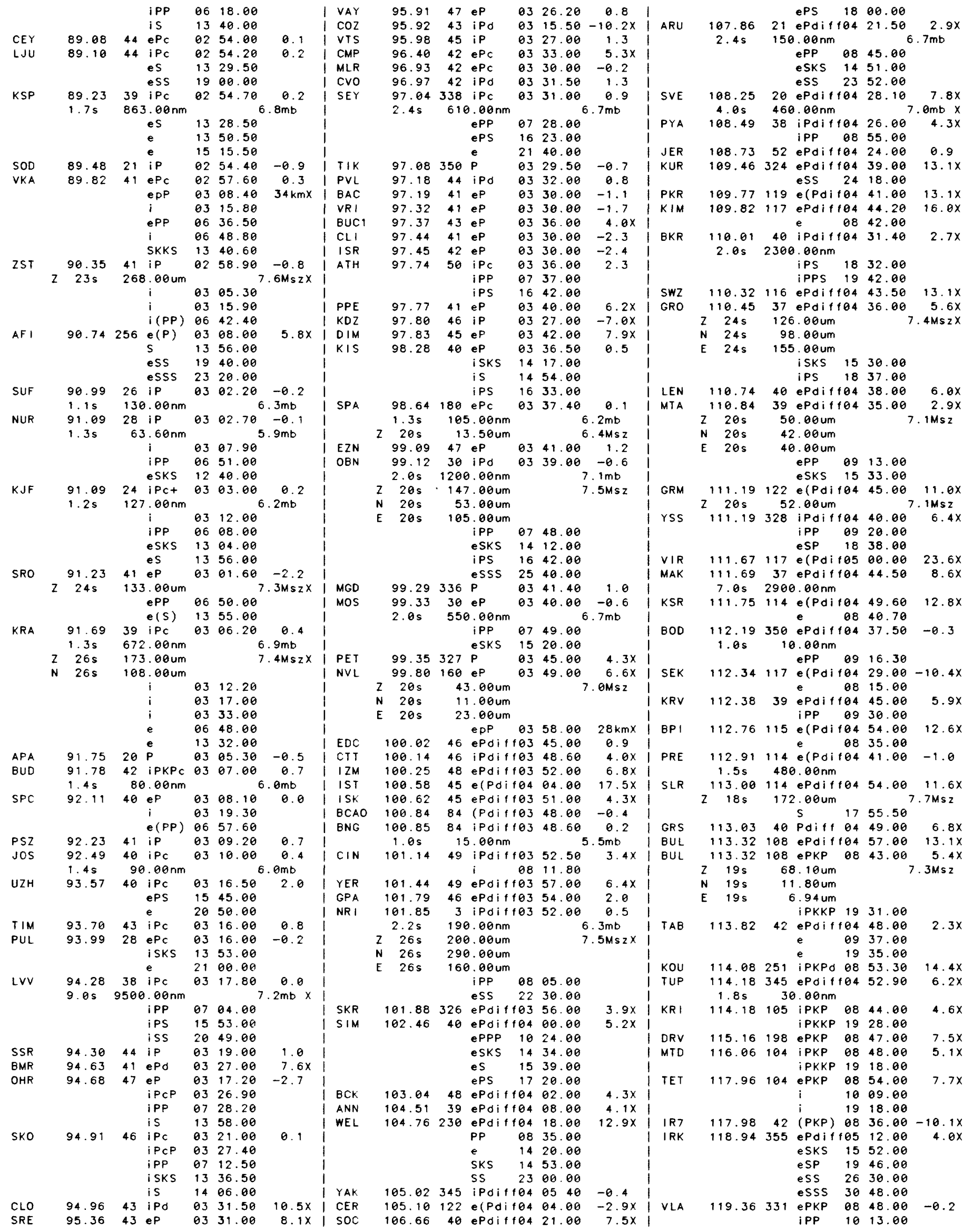




\begin{tabular}{|c|c|c|c|c|c|c|c|c|c|c|c|}
\hline$V L A$ & 119.36 & 331 & ipdif & 165 & 15.00 & $4.9 x$ & 1 & & & $P P$ & 11 \\
\hline SEM & 119.39 & 12 & ePdif & $f 65$ & 23.20 & 13. $2 x$ & 1 & & & PKS & 12 \\
\hline NAI & 119.79 & 86 & IPKPC & 68 & 53.20 & $3.0 x$ & BTO & 129.47 & 347 & ePKP & 09 \\
\hline & $1.0 \mathrm{~s}$ & 20 & $0.60 \mathrm{~nm}$ & & & & 1 & & & PKS & 12 \\
\hline DDR & 120.31 & 321 & EPKP & 08 & 56.00 & $5.6 x$ & $K B L$ & 129.57 & 30 & $e(P K P)$ & 09 \\
\hline$Y M$ & 120.64 & 320 & ePKP & 08 & 56.50 & $5.5 x$ & LAT & 130.29 & 269 & ePKP & 09 \\
\hline ZAK & 120.90 & 355 & EPKP & 08 & 50.40 & -0.5 & PMG & 130.35 & 265 & e (PKP) & 09 \\
\hline & & & ePP & 10 & 22.00 & & 1 & $18 \mathrm{~s}$ & 40 & 0.50 um & \\
\hline & & & eSKS & 16 & 04.00 & & I CTA & 130.99 & 251 & IPKP & 09 \\
\hline & & & ess & 26 & 45.00 & & I AVY & 131.37 & 107 & EPKPC & 09 \\
\hline ZAK & 120.90 & 355 & ePdif & $f 05$ & 21.50 & $4.8 x$ & I GTA & 132.06 & 357 & ePKP & 09 \\
\hline & $2.0 \mathrm{~s}$ & & $0.68 \mathrm{~nm}$ & & & & 1 & & & PKS & 12 \\
\hline VAN & 121.17 & 35 & ePdif & 165 & 19.00 & 0.8 & I MVI & 132.09 & 317 & ePKP & 09 \\
\hline & & & eSKS & 16 & 00.00 & & 1 & & & e & 12 \\
\hline & & & esss & 31 & 30.00 & & I STK & 132.56 & 234 & ePKP & 09 \\
\hline$A S H$ & 121.31 & 35 & ePKP & 68 & 53.00 & 0.9 & I ADE & 133.67 & 229 & e (PKP) & 09 \\
\hline & & & ePP & 10 & 25.00 & & I SSE & 134.07 & 331 & PKP- & 08 \\
\hline & & & ePPP & 13 & 07.00 & & 1 & $18 \mathrm{~s}$ & 62 & $2.90 u m$ & \\
\hline & & & eSP & 20 & 11.60 & & 1 & $N \quad 18 s$ & & $8.70 u m$ & \\
\hline & & & eSPP & 21 & 30.00 & & 1 & $18 \mathrm{~s}$ & & $4.10 u m$ & \\
\hline & & & iPPS & 21 & 45.50 & & i & & & $P P$ & 11 \\
\hline ARO & 122.66 & 70 & ePKP & 09 & 02.00 & $6.6 x$ & 1 & & & PKS & 12 \\
\hline SHI & 122.80 & 46 & e (PKP) & 08 & 50.00 & $-5.5 x$ & 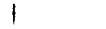 & & & $P P P$ & 14 \\
\hline NPA & 123.41 & 102 & ePKP & 09 & 03.00 & $6.2 x$ & & & & SKS & 15 \\
\hline & & & e & 10 & 42.00 & & 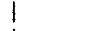 & & & PS & 22 \\
\hline HI & 124.08 & 38 & $e(P K P)$ & $\longdiv { 6 8 }$ & 56.00 & -1.9 & 1 & & & PPS & 23 \\
\hline TAS & 124.15 & 25 & ePKP & 08 & 58.00 & 0.4 & 1 & & & SS & 29 \\
\hline & & & ePP & 10 & 44.80 & & 1 & & & SSP & 30 \\
\hline & & & eSKS & 16 & 00.00 & & 1 & & & SSS & 35 \\
\hline & & & eSP & 20 & 35.00 & & $1 \mathrm{TZZ}$ & 135.85 & 271 & ePKP & 09 \\
\hline TAS & 124.15 & 25 & ePdiff & $f 05$ & 34.00 & $2.5 x$ & I JAY & 135.96 & 275 & iPKPd & 09 \\
\hline $\mathrm{COO}$ & 124.38 & 239 & ePKP & 08 & 58.00 & -0.4 & 1150 & 137.14 & 249 & ePKP & 09 \\
\hline SAM & 124.49 & 28 & PKP & 09 & 01.00 & $2.7 x$ & I WHN & 137.52 & 337 & PKP & 09 \\
\hline SAM & 124.49 & 28 & ePdif & 105 & 40.00 & $6.9 x$ & 1 & & & $P P$ & 12 \\
\hline FRU & 124.85 & 20 & ePKP & 09 & 02.00 & $3.1 x$ & 1 & & & PKS & 12 \\
\hline & $22 \mathrm{~s}$ & 117 & 7.00 um & & & $.5 M s z$ & I ANP & 138.63 & 325 & ePKP+ & 09 \\
\hline & $20 s$ & 88 & $0.00 \mathrm{um}$ & & & & $1 \mathrm{CD} 2$ & 140.07 & 351 & EPKP & 89 \\
\hline & $22 \mathrm{~s}$ & 105 & $5.00 \mathrm{um}$ & & & & 1 & & & ePP & 12 \\
\hline & & & eSPKP & 09 & 16.00 & & 1 & & & SKS & 16 \\
\hline & & & iPP & 10 & 47.00 & & $\mathrm{I} O Z \mathrm{H}$ & 140.41 & 328 & PKPd & 09 \\
\hline & & & iPPP & 13 & 30.00 & & 1 & & & $P P$ & 12 \\
\hline & & & eSP & 20 & 38.00 & & 1 & & & SKS & 16 \\
\hline & & & $\mathrm{i}$ & 28 & 00.00 & & I LSA & 141.43 & 8 & ePKP & 09 \\
\hline & & & eSS & 32 & 18.00 & & 1 & & & $P P$ & 12 \\
\hline FRU & 124.85 & 20 & ePdif & $f 05$ & 46.00 & $11.4 x$ & I ASPA & 141.58 & 243 & ePKP & 09 \\
\hline RAB & 124.89 & 271 & ePKP & 08 & 58.00 & -1.7 & I KKN & 142.04 & 17 & iPKPC & 09 \\
\hline AAA & 125.19 & 18 & IPKPC & 89 & 04.00 & $4.4 X$ & 1 & $1.2 \mathrm{~s}$ & 89 & $9.00 \mathrm{~nm}$ & \\
\hline & & & i sPKP & 09 & 18.00 & & WB2 & 142.08 & 249 & ePKP & 09 \\
\hline & & & iPP & 10 & 50.00 & & 1 & & & $\mathrm{i}$ & 09 \\
\hline SHK & 125.24 & 324 & ePKP & 09 & 10.50 & 0.7 & 1 & & & $\mathrm{i}$ & 09 \\
\hline TLG & 125.33 & 17 & iPKP & 08 & 9.80 & 0.0 & 1 & & & ePP & 14 \\
\hline & & & ¿PP & 10 & 55.00 & & 1 & & & e & 20 \\
\hline ILG & 125.33 & 17 & ePdif & $f 05$ & 42.50 & $5.7 x$ & NRA & 142.09 & 249 & PKPd & 09 \\
\hline MAN & 125.48 & 232 & ePKP & 09 & 04.60 & 4. $4 x$ & i & $1.1 \mathrm{~s}$ & $7 e$ & $0.70 \mathrm{~nm}$ & \\
\hline CAN & 125.63 & 233 & ePKP & 09 & 3.40 & $2.7 x$ & $M N$ & 142.16 & 17 & IPKPC & 09 \\
\hline ANR & 125.86 & 23 & ePKP & $\theta$ & 12.40 & 1.5 & I & $.1 \mathrm{~s}$ & 94 & $4.00 \mathrm{~nm}$ & \\
\hline & & & eSKS & 16 & 03.40 & & PK I & 142.28 & 17 & IPKPC & 09 \\
\hline ANR & 125.86 & 23 & ePdif & $f 05$ & 58.60 & $19.5 x$ & 1 & $1.2 \mathrm{~s}$ & $8 e$ & $0.00 \mathrm{~nm}$ & \\
\hline & $6.0 \mathrm{~s}$ & 600 & $0.08 \mathrm{~nm}$ & & & & I GYA & 143.78 & 345 & PKP & 09 \\
\hline KVG & 126.02 & 273 & ePKP & 69 & 1.00 & -1.0 & CVP & 143.99 & 317 & iPKPd & 09 \\
\hline DSH & 126.20 & 27 & PKP & 09 & 01.50 & -0.2 & PIP & 144.34 & 319 & iPKPd & 09 \\
\hline asi & 126.20 & 27 & ePdif & 105 & 46.00 & $5.2 x$ & $G Z H$ & 144.52 & 333 & PKPC & 09 \\
\hline$P R Z$ & 126.34 & 17 & ePKP & 09 & 02.50 & 0.5 & 1 & & & $\mathrm{i}$ & 09 \\
\hline & $3.0 \mathrm{~s}$ & 100 & $0.00 \mathrm{~nm}$ & & & & i & & & $P P$ & 12 \\
\hline & & & ePP & 11 & 3.00 & & POO & 144.68 & 40 & IPKPC & 09 \\
\hline & & & ePPP & 13 & 1.50 & & 1 & $1.6 \mathrm{~s}$ & 1000 & $0.00 \mathrm{~nm}$ & \\
\hline & & & eSKS & 16 & 02.50 & & I HKC & 144.82 & 331 & iPKP & 89 \\
\hline ou & 126.41 & 234 & ePKP & 09 & 5.50 & $3.3 x$ & i & & & is & 12 \\
\hline GAR & 126.52 & 26 & ePKP & 0 & 00.10 & -2.3 & NP & 45.25 & 307 & iPKPd & 89 \\
\hline & & & i SPP & 2 & 30.40 & & I MCO & 5.26 & 332 & iPKP & 69 \\
\hline & & & isSS & 32 & 40.40 & & $B A G$ & 145.74 & 317 & ePKP & 09 \\
\hline RI & 126.56 & 19 & PKP & $\theta$ & 02.60 & -0.1 & 1 & s & $106 e$ & $0.00 \mathrm{~nm}$ & \\
\hline KUL & 127.18 & 27 & ePKP & 09 & 03.30 & -0.3 & | KMI & 145.90 & 350 & IPKPC & 09 \\
\hline & & & iPP & 11 & 01.80 & & 1 & & & PP & 13 \\
\hline UL & 127.18 & 27 & ePdiff & $f 05$ & 7.80 & $2.7 x$ & 1 & & & PKS & 13 \\
\hline UA & 127.42 & 294 & PKP & 0 & $\theta 0$ & $4.4 x$ & $N$ & 09 & 260 & ePKP & 09 \\
\hline GUMO & 127.44 & 294 & PKP & 0 & 11.60 & $7.0 x$ & MAN & 5.60 & 314 & EPKPC & 09 \\
\hline TOO & 127.61 & 229 & ePKP & 09 & 03.00 & -1.4 & $1 O C P$ & 16.61 & 314 & ePKP & 09 \\
\hline KHO & 128.31 & 26 & ePKP & 09 & 04.40 & -1.5 & I WBN & 146.88 & 235 & IPKPA & 69 \\
\hline & $18 \mathrm{~s}$ & & 6.40 um & & & $3 \mathrm{Msz}$ & $1 \mathrm{CCP}$ & 147.10 & 305 & ePKP & 09 \\
\hline & $18 \mathrm{~s}$ & & $9.00 \mathrm{um}$ & & & & I DAV & 147.36 & 298 & ePKP & 09 \\
\hline & $18 \mathrm{~s}$ & & $6.40 \mathrm{um}$ & & & & 1 & $1.5 \mathrm{~s}$ & 867 & $7.00 \mathrm{~nm}$ & \\
\hline & & & ePP & 11 & 16.00 & & I PGP & 147.46 & 312 & iPKPd & 09 \\
\hline & & & eSP & 2 & 12.00 & & 1 & $1.4 \mathrm{~s}$ & 448 & $8.00 \mathrm{~nm}$ & \\
\hline KSH & 128.32 & 21 & ePKP & 09 & 07.00 & 1.2 & I KLG & 148.22 & 223 & IPKPC & 09 \\
\hline & & & $P P$ & 1 & 20 & & I HYB & .37 & 35 & ePKPC & 69 \\
\hline IHC & 128.87 & 346 & PKPC & 09 & 07.60 & 0.8 & 1 & $1.2 \mathrm{~s}$ & 557 & $7.00 \mathrm{~nm}$ & \\
\hline
\end{tabular}


$04 \mathrm{~d} \quad 02 \mathrm{~h}$

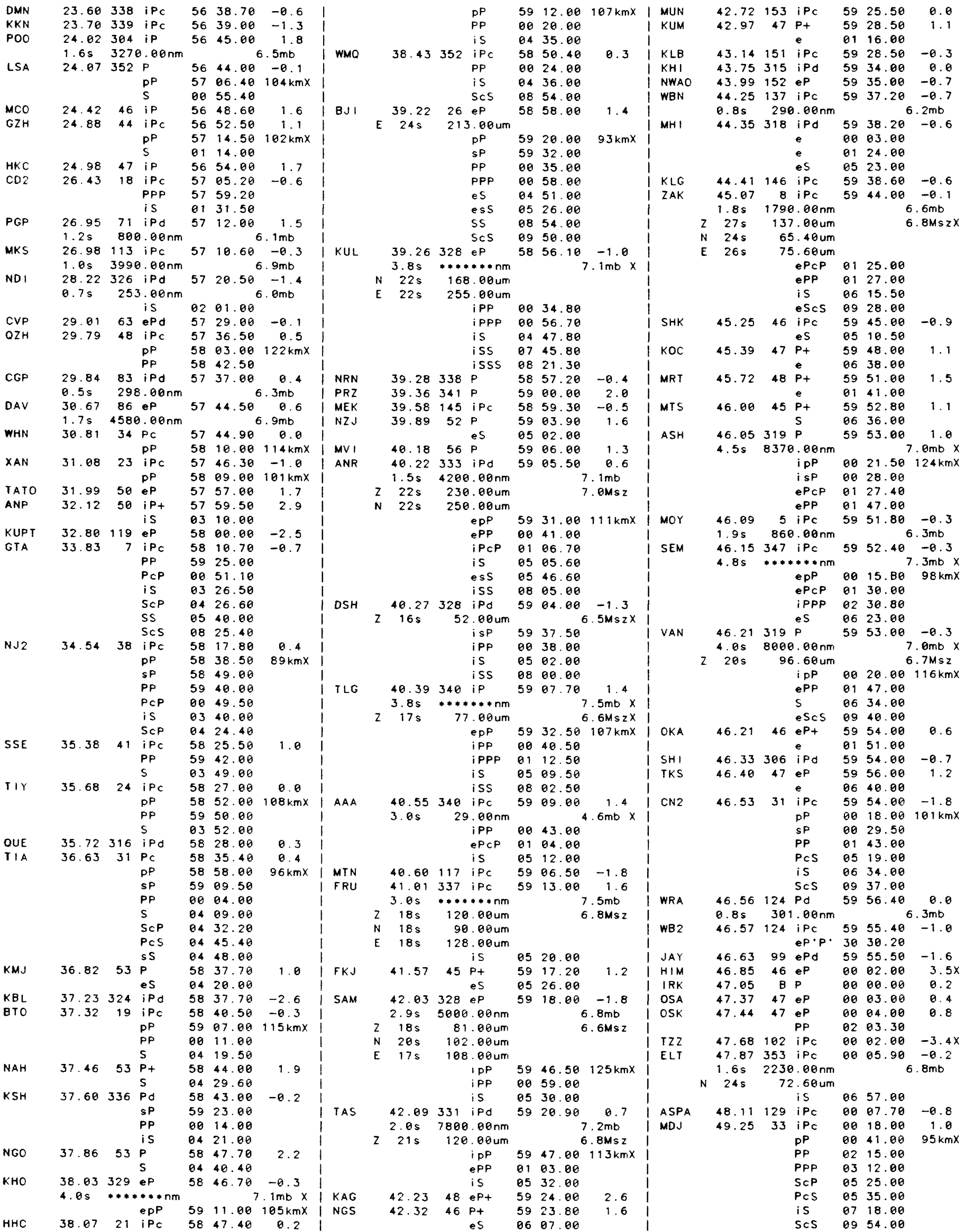




\begin{tabular}{|c|c|c|c|c|c|c|c|c|c|c|c|}
\hline VLA & & 49.58 & 36 & iPd & 00 & 19.00 & -0.5 & 1 & & & \\
\hline & $z$ & $16 \mathrm{~s}$ & 15 & $7.00 \mathrm{um}$ & & & $.1 M s z X$ & $i$ & & & \\
\hline & $N$ & $16 s$ & & $4.00 \mathrm{um}$ & & & & i & & & \\
\hline & $E$ & $16 \mathrm{~s}$ & 5 & 7.00 um & & & & I ASA & & 56.34 & 40 \\
\hline & & & & iPP & 02 & 19.00 & & $i$ & & & \\
\hline & & & & is & 07 & 19.00 & & $|\mathrm{OB}|$ & & 56.50 & 41 \\
\hline & & & & iss & 07 & 59.00 & & $i$ & & & \\
\hline & & & & iscs & 10 & 03.00 & & I KVG & & 56.59 & 97 \\
\hline TEH & & 49.73 & 313 & ePc & 00 & 22.00 & 1.0 & I WAK & & 56.70 & 38 \\
\hline NVS & & 49.88 & 351 & iPc & 00 & 20.00 & -1.5 & $i$ & & & \\
\hline & & $1.2 \mathrm{~s}$ & $* * *$ & $* * * * \mathrm{~nm}$ & & 8 & $.0 \mathrm{mb} \times$ & I CTA & & 56.76 & 118 \\
\hline & & & & is & 07 & 17.30 & & $i$ & & $1.2 \mathrm{~s}$ & 844 \\
\hline GUMO & & 49.95 & 77 & ePt & 00 & 21.50 & -1.2 & $i$ & & & \\
\hline & & $1.0 \mathrm{~s}$ & 146 & $0.00 \mathrm{~nm}$ & & 7 & $.0 \mathrm{mb}$ & I MTA & & 56.95 & 317 \\
\hline PJG & & 49.95 & 77 & eP & $\theta 0$ & 21.20 & -1.5 & i & $z$ & $25 \mathrm{~s}$ & 25 \\
\hline GUA & & 49.99 & 77 & ePt & 00 & 21.50 & -1.5 & $i$ & $N$ & $25 \mathrm{~s}$ & 30 \\
\hline & & $1.0 \mathrm{~s}$ & 149 & $0.00 \mathrm{~nm}$ & & 7 & $.0 \mathrm{mb}$ & i & $E$ & $25 \mathrm{~s}$ & 24 \\
\hline & & & & pP & 00 & 25.70 & $14 \mathrm{kmX}$ & 1 & & & \\
\hline & & & & es & 07 & 26.50 & & 1 & & & \\
\hline MAT & & 50.17 & 46 & iPc & 00 & 23.40 & -0.8 & 1 & & & \\
\hline & & $1.5 \mathrm{~s}$ & 181 & $0.00 \mathrm{~nm}$ & & 6 & $.9 \mathrm{mb}$ & I GRO & & 57.07 & 319 \\
\hline & & & & es & 07 & 28.00 & & I LEN & & 57.22 & 315 \\
\hline OSH & & 50.26 & 49 & $P$ & 00 & 24.20 & -0.6 & I SVE & & 57.58 & 339 \\
\hline OYM & & 50.43 & 48 & eP & 00 & 25.80 & -0.4 & $i$ & & $9.0 \mathrm{~s}$ & 8500 \\
\hline DDR & & 50.65 & 47 & eP & 00 & 25.30 & -2.5 & $i$ & $z$ & $24 \mathrm{~s}$ & 130 \\
\hline KYS & & 51.01 & 49 & eP & 00 & 28.10 & -2.4 & $i$ & $\mathrm{~N}$ & $24 \mathrm{~s}$ & 59 \\
\hline ATA & & 51.21 & 280 & eP & 00 & 33.50 & 1.2 & $i$ & $E$ & $24 \mathrm{~s}$ & 127 \\
\hline iso & & 51.25 & 122 & iPd & 00 & 32.20 & -0.4 & $i$ & & & \\
\hline MKL & & 51.28 & 280 & eP & $\theta 0$ & 35.50 & 2.7 & $i$ & & & \\
\hline TSK & & 51.41 & 47 & eP & 00 & 31.80 & -1.7 & 1 & & & \\
\hline TDD & & 51.52 & 280 & $e^{P}$ & 00 & 36.50 & 1.8 & I BKR & & 57.84 & 316 \\
\hline ARO & & 51.57 & 280 & iPd & 00 & 36.80 & 1.7 & 1 & & $5.0 \mathrm{~s}$ & 8600 \\
\hline & & & & i & 07 & 55.90 & & 1 & & & \\
\hline SGH & & 51.77 & 280 & eP & 00 & 38.50 & 1.8 & $i$ & & & \\
\hline DAF & & 51.88 & 280 & eP & 00 & 39.70 & 2.3 & 1 & & & \\
\hline KSU & & 51.96 & 280 & eP & 00 & 40.50 & 2.5 & I ADE & & 57.89 & 138 \\
\hline MDG & & 52.12 & 101 & eP & $\theta 0$ & 37.00 & -2.2 & i & & $1.0 \mathrm{~s}$ & 760 \\
\hline AVY & & 52.39 & 241 & ePc & 00 & 42.20 & 0.8 & I YSS & & 58.11 & 37 \\
\hline YAM & & 52.46 & 45 & $P_{+}$ & 00 & 41.70 & 0.3 & 1 & $z$ & $19 \mathrm{~s}$ & 50 \\
\hline TUP & & 52.64 & 18 & $I P C$ & 00 & 41.90 & -0.5 & $i$ & $\mathrm{~N}$ & $18 \mathrm{~s}$ & 32 \\
\hline & & $1.4 \mathrm{~s}$ & 104 & $0.00 \mathrm{~nm}$ & & & $.7 \mathrm{mb}$ & 1 & $E$ & $18 \mathrm{~s}$ & 40 \\
\hline$A K I$ & & 52.95 & 43 & eP & 00 & 46.00 & 1.0 & $i$ & & & \\
\hline & & & & e & 08 & 16.00 & & 1 & & & \\
\hline MOM & & 53.16 & 97 & eP & 00 & 45.00 & -1.9 & i & & & \\
\hline ISN & & 53.21 & 45 & eP & 00 & 46.00 & -0.9 & 1 & & & \\
\hline LAT & & 53.59 & 103 & IPd & 00 & 49.00 & -1.0 & 1 & & & \\
\hline BHD & & 54.12 & 307 & ePd & 00 & 56.00 & 2.4 & RAB & & 58.19 & 98 \\
\hline & & & & $e$ & 03 & 23.50 & & I STK & & 58.22 & 133 \\
\hline & & & & es & 08 & 22.00 & & I NEM & & 58.24 & 42 \\
\hline & & & & ess & 12 & 13.00 & & i & & & \\
\hline & & & & eSSS & 14 & 44.00 & & I NAI & & 58.27 & 265 \\
\hline$B O D$ & & 54.15 & 13 & IPC & 00 & 52.40 & -1.1 & $i$ & & $1.0 \mathrm{~s}$ & 290 \\
\hline & & $1.0 \mathrm{~s}$ & 125 & $0.00 \mathrm{~nm}$ & & 6 & $.9 \mathrm{mb}$ & I NPA & & 58.74 & 249 \\
\hline$M I Y$ & & 54.21 & 44 & $\mathrm{P}+$ & 00 & 56.70 & 2.5 & I & & & \\
\hline & & & & $s$ & 08 & 26.80 & & $i$ & & & \\
\hline PMG & & 54.36 & 106 & iPC+ & $\theta \theta$ & 54.10 & -1.6 & i & & & \\
\hline HAK & & 54.37 & 41 & $e^{P}$ & 00 & 55.00 & -0.4 & 1 & & & \\
\hline & & & & $e$ & 08 & 31.00 & & I PYA & & 59.08 & 319 \\
\hline TAB & & 54.39 & 313 & eP & $0 \theta$ & 55.00 & -0.8 & I & & $1.0 \mathrm{~s}$ & 1800 \\
\hline & & & & $\mathrm{i}$ & 00 & 57.80 & & i & & & \\
\hline SUT & & 54.50 & 40 & $P$ & 00 & 56.40 & 0.1 & i & & & \\
\hline & & & & s & 08 & 32.50 & & i & & & \\
\hline GRS & & 54.97 & 315 & $\mathrm{P}$ & 01 & 00.00 & 0.0 & i & & & \\
\hline LMG & & 55.19 & 105 & iPd & 01 & 00.90 & -1.1 & i & & & \\
\hline & & & & $e$ & 08 & 35.00 & & I KUR & & 60.52 & 40 \\
\hline SAP & & 55.34 & 40 & eP & 01 & 02.00 & -0.3 & I & & $1.0 \mathrm{~s}$ & 1980 \\
\hline & & & & es & 08 & 43.00 & & 1 & & & \\
\hline KRV & & 55.45 & 316 & iPd & 01 & 01.00 & $-2 \cdot 3$ & $i$ & & & \\
\hline & & & & EPCP & 02 & 02.00 & & i & & & \\
\hline & & & & $\mathrm{S}$ & 08 & 34.00 & & soc & & 61.13 & 317 \\
\hline AAE & & 55.56 & 277 & eP & 01 & 07.70 & 2.8 & 1 & & $2.0 \mathrm{~s}$ & 2500 \\
\hline MAK & & 55.78 & 319 & $\mathrm{IPC}$ & 01 & 06.00 & 0.5 & i & & & \\
\hline & & $3.6 \mathrm{~s}$ & $* * *$ & $* * * * \mathrm{~nm}$ & & & $.3 m b \times$ & i & & & \\
\hline & $z$ & $24 s$ & & $4.00 \mathrm{um}$ & & & $.6 \mathrm{Ms} z \mathrm{X}$ & I CMS & & 61.16 & 131 \\
\hline & $N$ & $24 \mathrm{~s}$ & & $9.00 \mathrm{um}$ & & & & I JER & & 61.21 & 303 \\
\hline & $\mathrm{E}$ & $18 \mathrm{~s}$ & & $4.00 \mathrm{um}$ & & & & 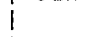 & & & \\
\hline & & & & ePP & 03 & 05.40 & & BFD & & 61.70 & 138 \\
\hline & & & & is & 08 & 33.00 & & I PAA & & 61.81 & 100 \\
\hline URA & & 55.87 & 42 & eP & 01 & 07.00 & 0.8 & I YAK & & 61.82 & 18 \\
\hline & & & & es & 08 & 53.00 & & 1 & & $1.0 \mathrm{~s}$ & 3040 \\
\hline RMJ & & 55.96 & 40 & $\mathrm{P}$ & 01 & 07.40 & 0.6 & 1 & & & \\
\hline & & & & es & 08 & 51.00 & & 1 & & & \\
\hline MSL & & 56.11 & 310 & ePd & 01 & 06.00 & -2.1 & i & & & \\
\hline & & & & $i P C P$ & 02 & 09.50 & & $K V T$ & & 62.88 & 313 \\
\hline & & & & IPPP & 84 & 24.00 & & ANN & & 63.22 & 318 \\
\hline & & & & IPCS & 06 & 07.50 & & $i$ & $z$ & $22 \mathrm{~s}$ & 35 \\
\hline & & & & is & 08 & 42.00 & & $i$ & $N$ & $22 s$ & \\
\hline
\end{tabular}


$04 d \quad 03 h$

\begin{tabular}{|c|c|c|c|c|c|c|c|c|c|c|c|}
\hline & $\mathrm{E}$ & $25 \mathrm{~s}$ & & 0.00 um & & & & 1 & & & \\
\hline & & & & iPCP & 03 & 06.00 & & 1 & & & \\
\hline & & & & iPPP & 06 & 56.00 & & 1 & & & \\
\hline & & & & is & 11 & 32.00 & & 1 & & & \\
\hline CFR & & 69.52 & 316 & iPc & 02 & 36.00 & -0.7 & 1 & & & \\
\hline BUL & & 69.86 & 246 & $i P c$ & 02 & 40.00 & 0.7 & 1 & & & \\
\hline & & $0.7 \mathrm{~s}$ & 259 & $9.00 \mathrm{~nm}$ & & & $.3 \mathrm{mb}$ & I APA & & 74.06 & 339 \\
\hline & $z$ & $18 \mathrm{~s}$ & & 9.90 um & & 6 & $.6 M s z$ & 1 & & $1.5 \mathrm{~s}$ & 3700 \\
\hline & $N$ & $18 \mathrm{~s}$ & & .50 um & & & & 1 & $z$ & $22 \mathrm{~s}$ & 135 \\
\hline & $E$ & $18 s$ & & 2.70 um & & & & I & $N$ & $20 \mathrm{~s}$ & 11 \\
\hline & & & & ipP & 02 & 59.00 & $71 \mathrm{kmx}$ & 1 & $E$ & $22 \mathrm{~s}$ & 50 \\
\hline & & & & is & 11 & 44.00 & & 1 & & & \\
\hline PET & & 69.91 & 35 & $\mathbf{P}$ & 02 & 38.00 & -0.8 & 1 & & & \\
\hline PPE & & 70.12 & 317 & ePc & 02 & 39.00 & -1.3 & 1 & & & \\
\hline BIR & & 70.12 & 317 & eP & 02 & 39.00 & $-1 \cdot 3$ & 1 & & & \\
\hline BRD & & 70.35 & 316 & eP & 02 & 43.00 & 1.2 & I UZH & & 74.13 & 319 \\
\hline IAS & & 70.37 & 318 & eP & 02 & 41.00 & -0.8 & 1 & & $1.6 \mathrm{~s}$ & 4500 \\
\hline$C L I$ & & 70.42 & 317 & iPc & 02 & 41.50 & -0.7 & 1 & 2 & $18 s$ & 21 \\
\hline SEY & & 70.60 & 24 & $i P c$ & 02 & 42.30 & -0.7 & 1 & $N$ & $18 \mathrm{~s}$ & 12 \\
\hline & & $1.4 \mathrm{~s}$ & 1180 & $0.00 \mathrm{~nm}$ & & & $.6 \mathrm{mb}$ & 1 & $\mathbf{E}$ & $18 \mathrm{~s}$ & 15 \\
\hline & $Z$ & $22 \mathrm{~s}$ & 44 & $.00 \mathrm{um}$ & & & $.7 \mathrm{Msz}$ & 1 & & & \\
\hline & $N$ & $20 \mathrm{~s}$ & 44 & $4.50 \mathrm{um}$ & & & & 1 & & & \\
\hline & $E$ & $23 \mathrm{~s}$ & 44 & 4.50 um & & & & 1 & & & \\
\hline & & & & iPCP & 03 & 02.80 & & 1 & & & \\
\hline & & & & ipp & 03 & 05.30 & $88 \mathrm{kmX}$ & I OHR & & 74.15 & 311 \\
\hline & & & & IPP & 05 & 15.30 & & 1 & & & \\
\hline & & & & iPPP & 07 & 09.30 & & 1 & & & \\
\hline & & & & is & 11 & 45.30 & & I BLF & & 74.32 & 238 \\
\hline & & & & iscs & 12 & 31.30 & & I SWZ & & 74.54 & 240 \\
\hline ISR & & 70.62 & 316 & ip & 02 & 44.50 & 1.0 & I KJF & & 74.97 & 335 \\
\hline VR I & & 70.65 & 317 & iPc & 02 & 43.00 & -0.6 & I & & $0.7 \mathrm{~s}$ & 130 \\
\hline$B A C$ & & 70.68 & 317 & eP & 02 & 42.00 & -1.7 & I & & & \\
\hline DIM & & 70.73 & 312 & eP & 02 & 43.00 & -1.1 & 1 & & & \\
\hline CGN & & 70.81 & 315 & ePd & 02 & 44.50 & -0.1 & I & & & \\
\hline $8 \cup C 1$ & & 70.83 & 315 & $e^{P}$ & 02 & 43.50 & -1.1 & 1 & & & \\
\hline$K D Z$ & & 70.83 & 312 & $i P c$ & 02 & 44.00 & -0.8 & 1 & & & \\
\hline cvo & & 71.02 & 316 & $\mathrm{iPc}$ & 02 & 45.00 & -0.8 & 1 & & & \\
\hline MLR & & 71.11 & 316 & iPc & 02 & 46.00 & -0.5 & I GRM & & 75.19 & 234 \\
\hline EVA & & 71.13 & $24 \theta$ & iPc & 02 & 47.50 & 0.4 & 1 & & $1.9 \mathrm{~s}$ & 720 \\
\hline & & & & $i$ & 14 & 49.70 & & 1 & $z$ & $20 \mathrm{~s}$ & 53 \\
\hline PVL & & 71.22 & 314 & iPd & 02 & 47.00 & -0.1 & I & & & \\
\hline ATH & & 71.54 & 308 & iPc & 02 & 48.00 & -1.1 & I suF & & 75.19 & 334 \\
\hline & & & & iPP & 05 & 40.00 & & 1 & & $0.6 \mathrm{~s}$ & 182 \\
\hline & & & & iPPP & 07 & 20.00 & & I Jos & & 75.26 & 319 \\
\hline & & & & is & 11 & 5800 & & 1 & & $0.9 \mathrm{~s}$ & 270 \\
\hline SLR & & 71.60 & 241 & iPct & 02 & 50.00 & 0. 1 & I NUR & & 75.29 & 331 \\
\hline & & $0.9 \mathrm{~s}$ & 975 & $5.00 \mathrm{~nm}$ & & & $.7 \mathrm{mb}$ & I & & $1.5 \mathrm{~s}$ & 1540 \\
\hline & 2 & $18 s$ & 330 & 3.00 um & & 7 & $6 \mathrm{Msz}$ & 1 & & & \\
\hline & & & & $\mathrm{i}$ & 03 & 12.50 & & 1 & & & \\
\hline PRE & & 71.69 & 241 & iPC & 02 & 50.40 & 0.0 & 1 & & & \\
\hline & & $0.8 \mathrm{~s}$ & 615 & $5.00 \mathrm{~nm}$ & & & $.6 \mathrm{mb}$ & 1 & & & \\
\hline CMP & & 71.69 & 316 & iPC & 02 & 48.00 & -1.9 & 1 & & & \\
\hline$M I R$ & & 72.12 & 181 & ip & 02 & 52.00 & 0.2 & 1 & & & \\
\hline & & $3.0 \mathrm{~s}$ & 260 & $0.00 \mathrm{~nm}$ & & 5 & $6 \mathrm{mb} \times$ & I HVD & & 75.30 & 236 \\
\hline & & & & iPCP & 03 & 12.00 & & 1 & & $0.9 \mathrm{~s}$ & 287 \\
\hline & & & & $i p P$ & 03 & 21.00 & $115 \mathrm{kmx}$ & $!$ & & & \\
\hline & & & & ePPP & 07 & 18.00 & & I NOU & & 75.42 & 115 \\
\hline & & & & is & 12 & 10.00 & & I & & & \\
\hline & & & & ess & 16 & 42.00 & & 1 & & & \\
\hline RA & & 72.14 & 315 & eP & 02 & 52.00 & -0.5 & I SPC & & 75.55 & 319 \\
\hline $\mathrm{COZ}$ & & 72.19 & 316 & iPC & 02 & 51.00 & -2.0 & 1 & & & \\
\hline PUL & & 72.36 & 331 & $P$ & 82 & 52.00 & -1.4 & 1 & & & \\
\hline THE & & 72.47 & 311 & $\mathrm{PC}$ & 02 & 53.50 & -1.0 & I & & & \\
\hline & & & & $s$ & 11 & 28.00 & & 1 & & & \\
\hline VTS & & 72.56 & 313 & iP & 82 & 54.00 & -1.0 & I PSZ & & 75.61 & 318 \\
\hline KSR & & 72.84 & 241 & iPC & 02 & 57.20 & 0.0 & BNG & & 75.85 & 273 \\
\hline & & & & $i$ & 83 & 11.00 & & 1 & & $1.0 \mathrm{~s}$ & 295 \\
\hline VAY & & 72.85 & 311 & $i P c$ & 02 & 55.20 & -1.6 & I BCAO & & 75.87 & 273 \\
\hline & & & & $i$ & 83 & 15.80 & & I KRA & & 75.87 & 320 \\
\hline SRE & & 72.87 & 315 & eP & 02 & 57.00 & 0. 2 & 1 & & $1.6 \mathrm{~s}$ & 3480 \\
\hline SEK & & 72.89 & 238 & iPc & 02 & 57.60 & 0.2 & ! & $z$ & $24 s$ & 43 \\
\hline KOU & & 73.07 & 113 & $i P c$ & 02 & 59.10 & b. 8 & 1 & $N$ & $20 \mathrm{~s}$ & 31 \\
\hline & & & & is & 12 & 22.50 & & 1 & E & $20 s$ & 46 \\
\hline & & & & $S C P$ & 15 & 00.30 & & 1 & & & \\
\hline LO & & 73.22 & 315 & $\mathrm{iPc}$ & 02 & 57.50 & -1.3 & i & & & \\
\hline LVV & & 73.22 & 320 & iPc & 02 & 58.00 & -0.7 & 1 & & & \\
\hline & & $9.0 \mathrm{~s}$ & $* * *$ & $* * * n \mathrm{~nm}$ & & & $.8 \mathrm{mb} \times$ & 1 & & & \\
\hline & $z$ & $20 s$ & 37 & $7.70 \mathrm{um}$ & & & $.7 M s Z$ & 1 & & & \\
\hline & $N$ & $18 \mathrm{~s}$ & 15 & $5.00 \mathrm{um}$ & & & & I BUD & & 76.14 & 317 \\
\hline & $E$ & $21 \mathrm{~s}$ & 21 & $1.40 \mathrm{um}$ & & & & 1 & & $1.1 \mathrm{~s}$ & 118 \\
\hline & & & & IPCP & 03 & 16.00 & & I PVC & & 76.22 & 110 \\
\hline & & & & is & 12 & 15.00 & & 1 & & & \\
\hline & & & & ess & 17 & 03.00 & & 1 & & & \\
\hline EV & & 73.28 & 316 & iPc & 82 & 59.50 & 0.4 & ! SOD & & 76.48 & 338 \\
\hline VIR & & 73.46 & 239 & iPc & 03 & 01.00 & 0.3 & I & & & \\
\hline SKO & & 73.78 & 312 & iPc & $\begin{array}{l}03 \\
03\end{array}$ & $\begin{array}{l}00.10 \\
01.80\end{array}$ & -2.0 & I MAW & & 76.58 & 192 \\
\hline & & & & $\mathrm{i}$ & 03 & 01.80 & & 1 & & $1.8 \mathrm{~s}$ & 580 \\
\hline
\end{tabular}




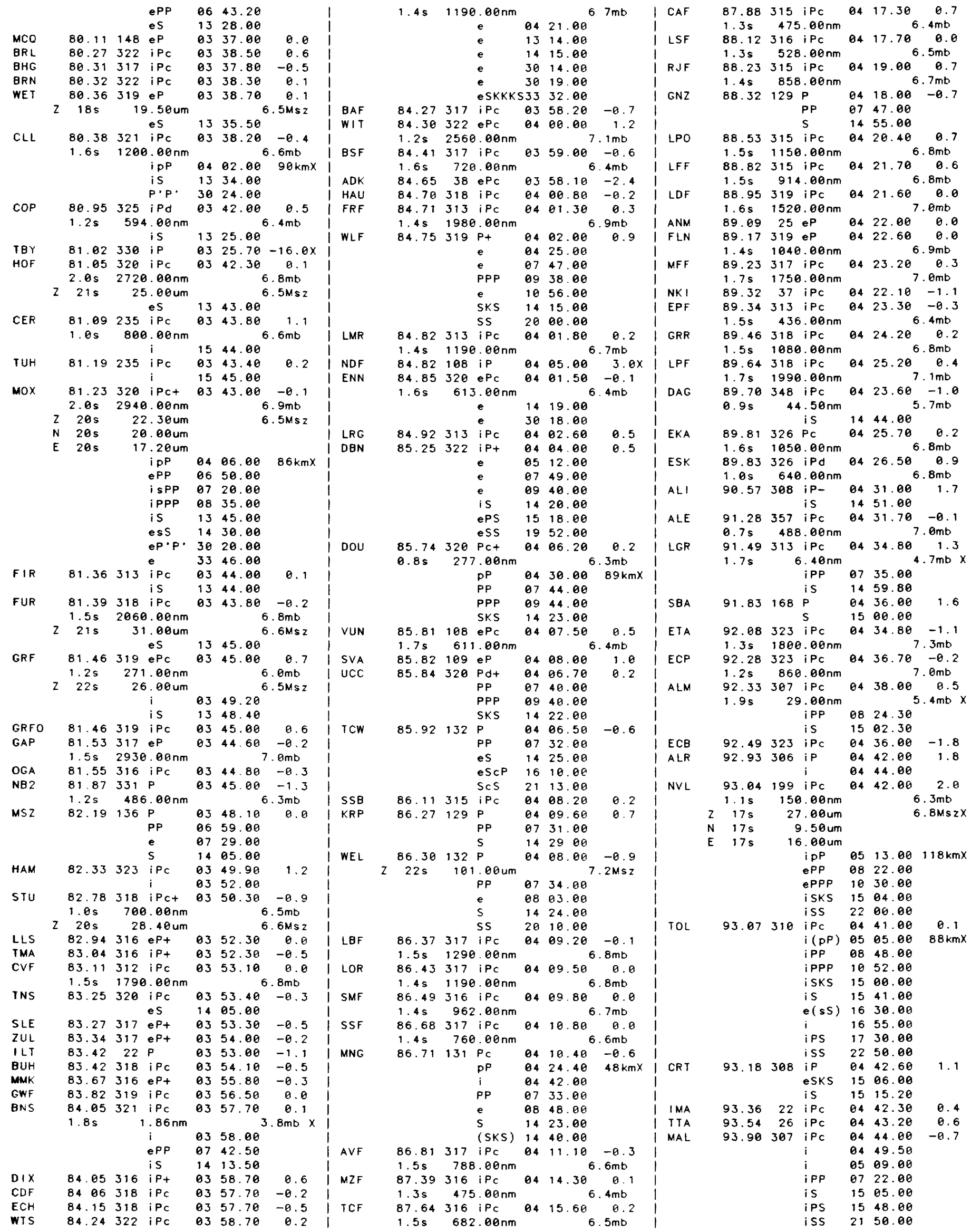


$04 \mathrm{~d} \quad 03 \mathrm{~h}$

\begin{tabular}{|c|c|c|c|c|c|c|c|c|c|c|c|}
\hline$A K U$ & 93.99 & 338 & iP & 04 & 46.30 & 1.8 & $\mid M I N$ & 123.27 & 33 & EPKP & 10 \\
\hline & $1.3 \mathrm{~s}$ & 592 & $2.00 \mathrm{~nm}$ & & & $6.9 \mathrm{mb}$ & NWRM & 123.86 & 35 & EPKP & 10 \\
\hline & & & e & 08 & 15.70 & & I BKS & 124.63 & 35 & ePKP & 10 \\
\hline & & & $\mathrm{i}$ & 17 & 04.60 & & 1 & $1.3 \mathrm{~s}$ & 990 & $0.00 \mathrm{~nm}$ & \\
\hline VAL & 94.66 & 323 & iP & 04 & 49.00 & 1.2 & $i$ & & & epPKP & 10 \\
\hline & & & $\mathrm{s}$ & 15 & 55.00 & & $i$ & & & ePP & 12 \\
\hline IFR & 95.04 & 304 & iP & 84 & 51.50 & 1.2 & i & & & e & 23 \\
\hline SFS & 95.37 & 307 & IPC & 04 & 50.00 & -1.4 & I WCN & 125.00 & 32 & IPKP & 10 \\
\hline & & & iPP & 68 & 12.00 & & I MHC & 125.34 & 35 & ePKP & 10 \\
\hline & & & ePPP & 09 & 51.00 & & CBM & 125.40 & 346 & ePKP & 10 \\
\hline & & & is & 15 & 20.00 & & I IMW & 125.43 & 23 & ePKP & 10 \\
\hline & & & IPS & 16 & 34.00 & & i & & & i & 10 \\
\hline & & & eSS & 20 & 00.00 & & BMN & 125.47 & 30 & ePKP & 10 \\
\hline SPA & 95.70 & 180 & ePc & 04 & $52.4 \theta$ & -0.1 & TMI & 125.54 & 24 & ePKP & 10 \\
\hline & $1.1 \mathrm{~s}$ & 101 & $1.00 \mathrm{~nm}$ & & & $6.2 \mathrm{mb}$ & I JAS & 125.60 & 34 & EPKPC & 10 \\
\hline & $22 \mathrm{~s}$ & 48 & $8.00 \mathrm{um}$ & & & $6.9 \mathrm{Msz}$ & i & & & ePPKP & 10 \\
\hline & & & e & 08 & 50.00 & & $i$ & & & ePPP & 12 \\
\hline$M B C$ & 95.78 & 8 & iPc & 04 & 52.30 & -0.3 & i & & & epPPP & 15 \\
\hline $\mathrm{COL}$ & 96.08 & 22 & iP & 04 & 53.50 & -0.7 & i & & & ePKKP & 20 \\
\hline & $20 s$ & 31 & 1. $90 \mathrm{um}$ & & & $6.8 \mathrm{Msz}$ & i & & & $e$ & 23 \\
\hline & & & e & 08 & 47.00 & & 1 & & & eSS & 27 \\
\hline & & & es & 15 & 53.00 & & I SAO & 125.85 & 36 & EPKP & 10 \\
\hline FBA & 96.08 & 22 & iPc & 84 & 53.00 & -1.2 & I LHC & 125.96 & 3 & ePKP & 10 \\
\hline & $1.5 \mathrm{~s}$ & 148 & $8.00 \mathrm{~nm}$ & & & $6.3 \mathrm{mb}$ & i & $0.8 \mathrm{~s}$ & 397 & $7.00 \mathrm{~nm}$ & \\
\hline REY & 96.11 & 337 & iP & 04 & 56.50 & 2.2 & $|B E|$ & 126.59 & 24 & ePKP & 10 \\
\hline & & & $i$ & 08 & 55.20 & & I FRI & 126.69 & 34 & ePKP & 10 \\
\hline AVE & 96.96 & 304 & ip & 05 & 01.50 & 2.8 & | PR I & 126.74 & 36 & ePKP & 10 \\
\hline PMR & 97.03 & 26 & iPc & 04 & 57.90 & -0.6 & I & & & epPKP & 10 \\
\hline$K D C$ & 97.05 & 30 & $e^{P}$ & 04 & 59.00 & 0.4 & 30W & 126.90 & 22 & ePKP & 10 \\
\hline PME & 97.05 & 26 & eP & 04 & 59.60 & 1.0 & I MIM & 127.21 & 346 & ePKP & 10 \\
\hline & $1.2 \mathrm{~s}$ & 326 & $6.00 \mathrm{~nm}$ & & & $0.7 \mathrm{mb}$ & I EMM & 127.28 & 344 & ePKP & 10 \\
\hline & $20 \mathrm{~s}$ & 47 & 7.50 um & & 7 & $7.0 \mathrm{Msz}$ & I MOUT & 127.32 & 25 & ePKP & 10 \\
\hline LIS & 97.18 & 310 & iPd & 05 & 04.50 & $4.9 x$ & i RSSD & 127.56 & 17 & $(P K P)$ & 10 \\
\hline KIC & 98.74 & 277 & iP & 05 & 08.30 & 1.2 & I MNT & 127.94 & 350 & IPKPC & 10 \\
\hline & & & es & 15 & 39.40 & & $i$ & $1.5 \mathrm{~s}$ & 560 & $0.00 \mathrm{~nm}$ & \\
\hline INK & 98.88 & 16 & ePC & 05 & 11.10 & $4.4 x$ & I WKTM & 128.26 & 35 & ePKP & 10 \\
\hline & $1.0 \mathrm{~s}$ & 562 & $2.00 \mathrm{~nm}$ & & & $7.1 \mathrm{mb}$ & BNH & 128.34 & 347 & EPKP & 10 \\
\hline PCA & 101.54 & 25 & ePdiff & 105 & 20.00 & 1.1 & I OTT & 128.39 & 351 & EPKP & 10 \\
\hline HON & 103.84 & 67 & Pdiff & 05 & 36.00 & $6.1 x$ & i & $1.3 \mathrm{~s}$ & 480 & $0.00 \mathrm{~nm}$ & \\
\hline YKA & 108.35 & 14 & ePdiff & 105 & 49.90 & 0.9 & i & & & PP & 10 \\
\hline YKC & 108.39 & 14 & ePdiff & 105 & 49.00 & -0.2 & I PTN & 129.07 & 351 & ¿PKP & 10 \\
\hline & $1.1 \mathrm{~s}$ & 84 & $4.00 \mathrm{~nm}$ & & & $6.8 \mathrm{mb}$ & I RKT & 129.13 & 115 & iPKP & 10 \\
\hline MBO & 109.38 & 287 & ePdiff & 105 & 54.30 & -0.3 & 1 PAS & 129.59 & 36 & iPKP & 10 \\
\hline & & & e & 06 & 02.80 & & 1 & & & ipP & 10 \\
\hline & & & $e$ & 06 & 20.70 & & 1 & & & iPP & 12 \\
\hline & & & $e$ & 09 & 58.10 & & 1 & & & iSKP & 13 \\
\hline & & & e & 10 & 02.80 & & 1 & & & iPKS & 14 \\
\hline & & & e & 10 & 32.10 & & 1 & & & eSKS & 17 \\
\hline FRB & 109.57 & 352 & ePdiff & 105 & 54.00 & -0.3 & 1 & & & iPS & 22 \\
\hline FCC & 115.32 & 5 & EPKPd & 10 & 07.90 & -0.4 & i & & & ePPS & 24 \\
\hline & $0.8 \mathrm{~s}$ & 86 & $6.00 \mathrm{~nm}$ & & & & i & & & iss & 30 \\
\hline$P G C$ & 116.38 & 27 & EPKP & 10 & 10.80 & 0.2 & $i$ & & & esss & 35 \\
\hline$M C W$ & 116.64 & 27 & PKP & 10 & 11.90 & 0.7 & I APH & 129.70 & 350 & ePKP & 10 \\
\hline EDM & 116.65 & 18 & ePdiff & 106 & 27.90 & 1.7 & 1 & & & eSKP & 13 \\
\hline EDM & 116.65 & 18 & iPKPd & 10 & 11.20 & 0.1 & I SDW & 129.89 & 35 & ePKP & 10 \\
\hline & $1.2 \mathrm{~s}$ & 516 & $5.00 \mathrm{~nm}$ & & & & i WES & 130.45 & 346 & ePKP & 10 \\
\hline GMW & 117.49 & 28 & PKP & 10 & 13.10 & 0.3 & I GOL & 131.12 & 21 & ePKP & 10 \\
\hline PNT & 117.55 & 25 & EPKP & 10 & 13.00 & 0.1 & I ELF & 131.17 & 356 & PKP & 10 \\
\hline & $0.8 \mathrm{~s}$ & 122 & $2.00 \mathrm{~nm}$ & & & & I INY & 131.39 & 351 & EPKP & 10 \\
\hline & & & $p P$ & 10 & 36.00 & & I DLA & 131.52 & 356 & PKP & 10 \\
\hline $\mathrm{SCH}$ & 117.73 & 348 & ePdiff & 106 & 33.50 & $2.5 x$ & I GLA & 132.30 & 34 & ePKP & 10 \\
\hline & $1.1 \mathrm{~s}$ & 183 & $3.00 \mathrm{~nm}$ & & & & I GPD & 132.43 & 349 & IPKP & 10 \\
\hline TPT & 118.14 & 104 & ePKP & 10 & 16.00 & 1.1 & I UTO & 132.80 & 358 & ePKPC & 10 \\
\hline & $1.1 \mathrm{~s}$ & & $2.50 \mathrm{~nm}$ & & & & I CLE & 132.88 & 356 & IPKP & 10 \\
\hline FFC & 118.16 & 11 & ePKP & 10 & 13.00 & -0.9 & I ITR & 133.46 & 263 & $e(P K P)$ & 10 \\
\hline & $0.5 \mathrm{~s}$ & 114 & $4.00 \mathrm{~nm}$ & & & & I ALO & 134.87 & 25 & EPKP & 10 \\
\hline & & & $P P$ & 11 & 26.00 & & i & $22 \mathrm{~s}$ & 31 & 1.50 um & \\
\hline LON & 118.53 & 28 & (PKP) & 10 & 14.00 & -0.9 & $i$ & & & e & 10 \\
\hline COR & 119.37 & 30 & IPKPC & 10 & 14.00 & -2.5 & IACO & 135.83 & 16 & ePKP & 10 \\
\hline NEW & 119.39 & 24 & iPKP & 10 & 15.20 & -1.3 & 1 & $1.1 \mathrm{~s}$ & 530 & $0.00 \mathrm{~nm}$ & \\
\hline STJ & 119.67 & 335 & ePKP & 10 & 17.00 & 0.1 & i & & & $e$ & 10 \\
\hline$R \times F$ & 119.69 & 22 & IPKP- & 10 & 17.70 & 0.6 & i & & & $e$ & 10 \\
\hline SES & 119.81 & 19 & iPKPd & 10 & 17.00 & -0.2 & i & & & e & 12 \\
\hline & & & $\mathrm{pP}$ & 11 & 39.00 & & I & & & e & 14 \\
\hline LDM & 19.95 & 23 & iPKP- & 10 & 18.00 & 0.5 & I NA12 & 135.96 & 352 & ePKP & 10 \\
\hline LHD & 20.02 & 23 & iPKP- & 10 & 18.00 & 0.3 & $i$ & & & 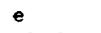 & 10 \\
\hline$C L X$ & 20.22 & 23 & iPKP- & 10 & 18.50 & 0.2 & I CVL & 136.05 & 352 & ePKP & 10 \\
\hline PNO & 120.77 & 27 & PKP & 10 & 18.40 & -0.7 & I RDJ & 136.05 & 243 & ePKP & 10 \\
\hline $\mathrm{FHC}$ & 121.65 & 34 & ePKPC & 10 & 21.40 & 0.4 & I FVM & 136.24 & 6 & ePKP & 10 \\
\hline LGBM & 122.24 & 32 & ePKP & 10 & 21.90 & -0.5 & i & & & ESKP & 14 \\
\hline NOC & 122.59 & 33 & EPKPC & 10 & 22.00 & -0.7 & i sob3 & 136.25 & 262 & ePKP & 10 \\
\hline & & & epPKP & 10 & 47.00 & & & & & e & 10 \\
\hline & & & ePP & 12 & 02.00 & & 1 & & & e & 10 \\
\hline & & & ePKKP & 20 & 19.00 & & I BLA & 137.84 & 354 & ePKP & 10 \\
\hline & & & e & 24 & 05.00 & & I ELC & 137.04 & 5 & EPKP & 10 \\
\hline MT & 122.98 & 34 & iPKP & 10 & 24.50 & 1. 1 & & & & iSKP & 14 \\
\hline & 123 & 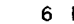 & PKP & $\theta$ & 0 & -93 & & & & ePKP & 10 \\
\hline
\end{tabular}


GA

$\begin{array}{llllll}\text { GAL } & 160.80 & 329 & \text { IPKP } & 11 & 32.50 \\ \text { ARE } & 162.81 & 231 & \text { iPKPC } & 11 & 30.50 \\ \text { FUO } & 163.99 & 314 & \text { iPKP } & 11 & 32.00\end{array}$

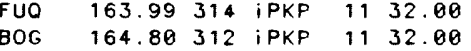

CHN $\quad 165.65318$ iPKP 1132.00

$\begin{array}{llllll}\text { PTO6 } & 168.09 & 227 & \text { IPKPd } & 11 & 34.80\end{array}$

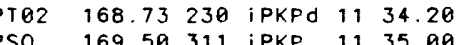

S.D. $=1.1$ on 494 of 543 obs.

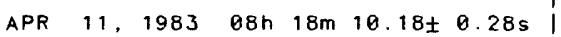

$10.426 \mathrm{~N} \pm 1.4 \mathrm{~km} \quad 62.775 \mathrm{~W} \pm 1.3 \mathrm{~km} \mathrm{|} \mathrm{IN4}$

DEPTH $=40.1 \pm 2.4 \mathrm{~km}$

$6.1 \mathrm{mb}(102$ obs.) $5.9 \mathrm{MsZ}$ ( 40 obs.) I GRT NEAR COAST OF VENEZUELA (97) IN3

Felt ( $V$ ) on Trinidad. Felt in the nor theostern venezuelo stotes of Sucre, Monogos. Anzoategui and Bolivor. Also

Carocos.

TCE

$1.0475 \mathrm{P}$

$1.31 \quad 95 \mathrm{P}$

$1.37 \quad 81 \mathrm{P}$

$2.0432 \mathrm{P}$

$4.00 \quad 27 \mathrm{eP}$

4.09271 iPnC

$4.39 \quad 22 \quad \mathrm{iPd}$

4.4924 iPd

$4.67 \quad 23 \quad \mathrm{iPd}$

$5.99 \quad 15 \mathrm{ePd}$

$6.07 \quad 12 \mathrm{ePd}$

$6.285 \mathrm{P}$

$6.64 \quad 8 \mathrm{ePd}$

$6.672 \mathrm{P}$

$\begin{array}{ll}6.94 & 265 \mathrm{iPc} \\ 0.65 & 70.60 \mathrm{~nm}\end{array}$

$0.65 \quad 70.60 \mathrm{~nm}$

$7.23 \quad 7 \mathrm{P}$
$7.90260 \mathrm{PC}$

$0.8 \mathrm{~s} \quad 320.00 \mathrm{~nm}$

$8.31337 \mathrm{eP}$

$e(S)$

3.37269

8.45258 iPnd 2013.70

$0.5 \mathrm{~s} \quad 133.00 \mathrm{~nm}$

12.28273 iP

BOG

12.59244 i

$\begin{array}{rllll}13.83248 & \text { is } & 2218.00 & \\ \text { if } & 2124.00 & -2.1\end{array}$

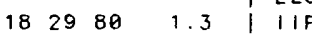

$\begin{array}{lll}18 & 31.70 & -0.6\end{array}$

\begin{tabular}{lll|l}
18 & 30.90 & -2.2 & I UTO
\end{tabular}

\begin{tabular}{rrr|r}
19 & 42.90 & 0.0 & IN 1 \\
19 & 10.15 & -0.5 & IPOW
\end{tabular}

$1954.90 \quad 111$

\begin{tabular}{rrr|l}
19 & 14.00 & 2.1 & IIC \\
19 & 15.50 & -0.8 &
\end{tabular}

$\begin{array}{lll}19 & 16.82 & -0.9\end{array}$

$\begin{array}{llll}9 & 17.92 & -0.9\end{array}$

\begin{tabular}{lll|l}
20 & 07.80 & -0.8 & OTT
\end{tabular}

$\begin{array}{lll}19.48 & -0.6\end{array}$

$\begin{array}{lllll}19 & 39.75 & -0.1 & \text { IAM }\end{array}$

$1943.00 \quad 0.2$ | VAO

$\begin{array}{ll}47.10 & -0.8\end{array}$

$953.80 \quad 1.7$

$1955.00^{5.7 m b}$

$.3 \mathrm{mb}$

\begin{tabular}{c|c}
0.4 & RDJ \\
$2 \mathrm{mb}$ & GBO
\end{tabular}

\begin{tabular}{ccc|c|c}
21 & 10.50 & $5.1 \times$ & RLO \\
21 & 08.50 & -1.3 & TUL
\end{tabular}

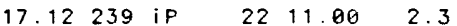

$\begin{array}{lllll}22.92305 & \text { iPd } 23 \quad 1400 \quad 2.2\end{array}$

$Z 18 \mathrm{~s} \quad 32.60 u m$

\begin{tabular}{ll|l}
14.00 & 2.2 & VCA
\end{tabular}

LPS

NNA

T02

LPB

ZIN

$A R E$

COM

SOB 3

$29.60326 \mathrm{ePd}$

$30.83127 \mathrm{e}(\mathrm{P})$

$30.84335 \mathrm{eP}$

2339.30

5. $8 \mathrm{MsZ}$

26.05281 is 2806.00

$1.0 \mathrm{~s} \quad 105.00 \mathrm{~nm}$

26.29212 iPd $23 \quad 47.00$

$0.6 \mathrm{~s} 240.00 \mathrm{~mm}$ es

2818.00 $5.4 \mathrm{mb}$

GFM

$30.85329 \mathrm{e}(\mathrm{P})$

$31.06332 \mathrm{iPt}$
$1.95 \quad 1090.00 \mathrm{~nm}$

\begin{tabular}{lll|l}
23 & 50.10 & 0.6 & OZO
\end{tabular}

$6.0 \mathrm{mb}$

$27.05191 \mathrm{iPd}$

1 . Os $940.00 \mathrm{~nm}$

2835.00

2.3 ACO

$\begin{array}{ll}835.00 \\ 23 & 57.70\end{array}$

$\begin{array}{lll}23 & 57.70 & 1.3\end{array}$

$30 \quad 49.50$

2358.00

$2411.00 \quad 1.1$

\begin{tabular}{lll|l}
24 & 11.80 & 0.1 & LUB
\end{tabular}

2414.800 .9 । MDZ

\begin{tabular}{lll|l}
24 & 23.20 & 0.5 & $\mathrm{JACH}$
\end{tabular}

\begin{tabular}{rrr|l}
24 & 22.00 & -3.0 & ROCH \\
24 & 25.50 & 0.7 & LHC
\end{tabular}

$\begin{array}{rrr}24 & 25.50 & 0.7 \\ 24 & 24.40 & -0.7\end{array}$

\begin{tabular}{lll|l}
24 & 28.10 & 1.4 & $\mathrm{PEL}$
\end{tabular}

31.31332 is 3129.60

$31.54326 \mathrm{eP}$

$32.11346 \mathrm{eP}$

2430.30

2431.90

$M D 3$

$32.17343 \mathrm{eP}$
2436.50

2452.80
$5.4 \times$ | WES

1.0 | PWLA

1.2 EMM

0.4 I AN

0.0

1.2 APH

0.2 $\mathrm{BNH}$

DHN
SLA $11 \mathrm{~T}$

I AN10

I LST

CBM

CRX

FVM

ACM

STJ

$3.8 \mathrm{X}$ ATX

S10

i

ATO

RRO

ACO

$32.70348 e^{P}$

$33.59321 \mathrm{eP}$

$34.43354 \mathrm{eP}$

34.73192 iP

$34.81345 \quad \mathrm{iPd}$

$34.82349 \mathrm{eP}$

$34.97340 \mathrm{eP}$

$35.04184 \mathrm{eP}$

$35.87330 \mathrm{ePd}$

$35.11352 \mathrm{eP}$

$35.27321 \mathrm{ePC}$

$35.29328 \mathrm{ePd}$

$35.35288 \mathrm{eP}$

$35.48345 \mathrm{eP}$

$35.56331 \mathrm{ePd}$

$35.62321 \mathrm{ePc}$

$35.73331 \mathrm{ePd}$

$35.77332 \mathrm{ePd}$

$35.82323 \mathrm{iPd}$

$35.97289 \mathrm{ePC}$

$36.09 \quad 333 \mathrm{iPd}$

$36.30 \quad 330 \mathrm{ePd}$

$36.31319 \mathrm{ePc}$

$36.35287 \mathrm{eP}$

$\begin{array}{ll}36.36 \quad 289 \mathrm{ePC} & \mathrm{PPP}\end{array}$

$36.40307 \quad \mathrm{PP}$

$36.42337 \mathrm{P}$

$36.57345 \mathrm{eP}^{\mathrm{P}}$

$0.9 \mathrm{~s} \quad 81.00 \mathrm{~nm}$

$36.64334 \mathrm{ePd}$

$36.65155 \mathrm{eP}^{\mathrm{P}}$

$36.66 \quad 354 \stackrel{\mathrm{e}}{\mathrm{eP}}$

$36.69288 \mathrm{ePC}$

$36.99322 \mathrm{iPC}$

$37.90 \quad 332 \mathrm{ePd}$

$37.9811 \mathrm{eP}$

38.16306 iP

$38.25150 \mathrm{eP}$

$38.78 \quad 316 \quad \mathrm{iPd}$

38.85316 iPd

39.23316 iPd-

$1.7 \mathrm{~s} \quad 408.00 \mathrm{~nm}$

$2 \quad 18 \mathrm{~s} \quad 25.00 \mathrm{um}$

$39.29188 \mathrm{ePd}$

$39.50 \quad 315 \quad \mathrm{iPd}$

$39.76 \quad 306$ iPd

$.6 \mathrm{~s} 200.00 \mathrm{~nm}$

$40.80314 \mathrm{i}$

$40.80313 \mathrm{ePd}$

$1.5 \mathrm{~s} \quad 691.00 \mathrm{~nm}$

41.09191 iPC

$41.23312 \mathrm{eP}$

$41.56182 \mathrm{ePd}$

$\begin{array}{ll}41.96 & 315 \mathrm{iPd} \\ 1.7 \mathrm{~s} & 1770.00 \mathrm{~nm}\end{array}$

$42.12 \quad 187 \stackrel{\mathrm{e}}{\mathrm{ePd}}$

$42.15188 \mathrm{~S}$

$42.58309 \mathrm{eP}$

$43.45187 \mathrm{i}(P)$

$43.51 \quad 190 \quad \mathrm{iPd}$

43.86190 iPd

$\begin{array}{ll}43.89 & 335 \mathrm{iPd} \\ 1.0 \mathrm{~s} & 291.00 \mathrm{~nm}\end{array}$

43.98190 iPd

$44.09189 \mathrm{iPd}$

$44.15189 \mathrm{ePC}$
$44.39357 \mathrm{eP}$

$\begin{array}{ll}44.39 & 357 \mathrm{eP} \\ 0.5 \mathrm{~s} & 147.00 \mathrm{~nm}\end{array}$

$44.42189 \mathrm{iPC}$

0.9 SCH

$3.6 \times \mid \mathrm{PCH}$

0.1 T TACH

$44.52190 \mathrm{iPC}$

44.74189 iPd

2442.80

2458.50

2457.50

$30 \quad 22.00$

2500.18

$\begin{array}{lll}25 & 02.28\end{array}$

$\begin{array}{lll}25 & 14.50\end{array}$

2501.90

2500.00

3040.00

502.20

$\begin{array}{lll}25 & 03.78\end{array}$

2504.08

$\begin{array}{lll}25 & 04.50\end{array}$

2505.50

2506.00

2506.50

2507.00

2508.28

2508.48

2508.40

2511.50

2526.18

$25 \quad 12.00$

2512.70

2513.00

2514.50

$25 \quad 14.20$

$\begin{array}{ll}25 & 29.50\end{array}$

2515.00

2514.10

2515.00

$2529.00^{\circ}$

2516.00

2515.60

$25 \quad 19.60$

$26 \quad 05.40$

$\begin{array}{ll}25 & 17.00\end{array}$

2530.80

2517.10

2518.28

3120.00

2526.20

$25 \quad 25.50$

2528.00

2528.88

2532.98

533.20

-0.1
56.90

5. $9 \mathrm{mb}$

2710.00

$\begin{array}{ll}25 & 37.90\end{array}$

2538.78

25.00

$27 \quad 25.00$

\begin{tabular}{l|l}
.8 & M \\
.1 & \\
.6 & \\
.2 & LNV \\
0.8 & RFA
\end{tabular}

MBO $\quad 44.87 \quad 80 \quad$ iP 


\begin{tabular}{|c|c|c|c|c|c|c|c|c|c|c|c|}
\hline WCN & 58.04 & 310 & ePd & 28 & 02.70 & 0.8 & I MZF & & 65.53 & 44 & iPd \\
\hline PRI & 58.07 & 306 & ep & 28 & 01.30 & -0.8 & I VDM & & 65.87 & 41 & iP- \\
\hline JAS & 38.26 & 308 & ePd & 28 & 02.80 & -0.5 & I PHC & & 66.01 & 321 & ePd \\
\hline MAL & 58.56 & 53 & iP- & 28 & 06.00 & 0.7 & I AVF & & 66.16 & 43 & iPd \\
\hline & & & is & 36 & 10.00 & & I SSF & & 66.31 & 43 & IPd \\
\hline SLD & 58.69 & 307 & ePc & 28 & 06.20 & -0.1 & I SMF & & 66.47 & 44 & iPd \\
\hline EDM & 58.71 & 327 & iPd & 28 & 04.90 & -1.3 & I LOR & & 66.59 & 43 & iPd \\
\hline & $0.6 \mathrm{~s}$ & 479 & $9.00 \mathrm{~nm}$ & & 6 & $.8 m b$ & I LBF & & 66.62 & 43 & IPd \\
\hline NEW & 58.87 & 321 & iPd & 28 & 06.10 & -1.3 & I UCC & & 67.57 & 39 & iPc- \\
\hline & & & e & 28 & 26.00 & & 1 & & & & $s$ \\
\hline ARN & 58.99 & 307 & ePc & 28 & 08.60 & 0.2 & I DOU & & 67.58 & 40 & $\mathrm{PC}-$ \\
\hline PNO & 9.05 & 317 & eP & 28 & 07.00 & -1.7 & 1 & & $1.5 \mathrm{~s}$ & 1340 & $0.00 \mathrm{~nm}$ \\
\hline MHC & 59.07 & 307 & ePd & 28 & 09.20 & 0.1 & 1 & & & & $s$ \\
\hline CRT & 59.29 & 53 & iPd & 28 & 12.50 & 2.0 & | LRG & & 67.77 & 47 & eP \\
\hline ORV & 59.41 & 310 & $\mathbf{P}$ & 28 & 10.90 & -0.4 & 1 & & $1.4 \mathrm{~s}$ & 81 & $1.50 \mathrm{~nm}$ \\
\hline TOL & 59.48 & 50 & iPd & 28 & 12.00 & 0.2 & I LMR & & 67.86 & 47 & eP \\
\hline & $1.6 \mathrm{~s}$ & & $9.00 \mathrm{~nm}$ & & 4 & $.7 \mathrm{mb} \times$ & 1 & & $1.4 \mathrm{~s}$ & 125 & $5.00 \mathrm{~nm}$ \\
\hline & & & $\mathrm{i}$ & 28 & 19.00 & & I FRF & & 67.99 & 47 & eP \\
\hline & & & ePCP & 28 & 55.00 & & 1 & & $1.0 \mathrm{~s}$ & & $9.60 \mathrm{~nm}$ \\
\hline & & & ePP & 30 & 18.00 & & I DBN & & 68.33 & 38 & iP- \\
\hline & & & is & 36 & 23.00 & & 1 & $z$ & $20 \mathrm{~s}$ & & 4. 30 um \\
\hline & & & $\mathrm{i}$ & 36 & 42.00 & & 1 & & & & es \\
\hline & & & iss & 39 & 10.00 & & 1 & & & & ess \\
\hline BKS & 59.64 & 308 & IPd & 28 & 13.00 & 0.2 & I HAU & & 68.37 & 42 & iPd \\
\hline & $1.1 \mathrm{~s}$ & $11 e$ & $0.00 \mathrm{~nm}$ & & & $.9 \mathrm{mb}$ & 1 & & $1.2 \mathrm{~s}$ & & $7.50 \mathrm{~nm}$ \\
\hline 2 & $20 s$ & & $6.00 \mathrm{um}$ & & & $.7 \mathrm{MsZ}$ & I WLF & & 68.50 & 41 & P- \\
\hline 1 & $20 \mathrm{~s}$ & $1 e$ & $0.00 \mathrm{um}$ & & & & 1 & & & & $s$ \\
\hline$E$ & $20 \mathrm{~s}$ & & $0.00 \mathrm{um}$ & & & & I ENN & & 68.54 & 39 & iPc \\
\hline & & & e & 28 & 37.00 & & 1 & & $1.0 \mathrm{~s}$ & 135 & $5.00 \mathrm{~nm}$ \\
\hline & & & ePP & 30 & 30.00 & & I BSF & & 68.64 & 43 & IPd \\
\hline & & & ePPP & 32 & 00.00 & & 1 & & $0.8 \mathrm{~s}$ & & $8.40 \mathrm{~nm}$ \\
\hline & & & es & 36 & 15.00 & & I DIX & & 68.83 & 45 & ifd \\
\hline & & & eSs & $4 \theta$ & 30.00 & & I CDF & & 69.04 & 42 & iPd \\
\hline LGBM & 60.32 & 312 & IPd & 28 & 16.00 & -1.8 & 1 & & $1.2 \mathrm{~s}$ & 138 & $8.00 \mathrm{~nm}$ \\
\hline RMT & 60.37 & 310 & eP & 28 & 15.70 & -2.0 & I STB & & 69.09 & 40 & IPd \\
\hline WDC & 60.41 & 311 & ePd & 28 & 15.30 & -2.8 & I MMK & & 69.21 & 45 & IPd \\
\hline PNT & 60.79 & 321 & eP & 28 & 19.00 & -1.5 & I WTS & & 69.30 & 38 & IPC \\
\hline & $1.1 \mathrm{~s}$ & 11 & $1.00 \mathrm{~nm}$ & & 5 & $.9 \mathrm{mb}$ & 1 & & $0.9 \mathrm{~s}$ & 188 & $8.00 \mathrm{~nm}$ \\
\hline REY & 60.80 & 19 & iP & 28 & 14.50 & $-5.8 x$ & I GWF & & 69.35 & 42 & IPd \\
\hline & & & $\mathrm{i}$ & 28 & 21.00 & & I BNS & & 69.36 & 39 & iPd \\
\hline ECB & 61.35 & 35 & IPd & 28 & 23.80 & -0.4 & 1 & & $2.0 \mathrm{~s}$ & & $0.43 \mathrm{~nm}$ \\
\hline LON & 61.41 & 318 & ePc & 28 & 23.50 & -1.3 & 1 & & & & es \\
\hline$D C N$ & 61.45 & 34 & iPd & 28 & 25.60 & 0.8 & I WIT & & 69.36 & 37 & ePd \\
\hline & $1.0 \mathrm{~s}$ & $55 e$ & $0.00 \mathrm{~nm}$ & & & $.6 \mathrm{mb}$ & 1 & & $1.0 \mathrm{~s}$ & 625 & $5.00 \mathrm{~nm}$ \\
\hline$E C P$ & 61.52 & 36 & iPd & 28 & 24.80 & -0.6 & I CVF & & 69.51 & 48 & ep \\
\hline & $1.7 \mathrm{~s}$ & 828 & $0.00 \mathrm{~nm}$ & & 6 & $.6 \mathrm{mb}$ & 1 & & $0.9 \mathrm{~s}$ & & $2.60 \mathrm{~nm}$ \\
\hline $\mathrm{FHC}$ & 61.53 & 311 & ePd & 28 & 25.80 & 0.1 & I ZUL & & 69.66 & 43 & iPd \\
\hline ETA & 61.80 & 35 & iPd & 28 & 26.90 & -0.3 & I BUH & & 69.70 & 42 & ePd \\
\hline & $1.7 \mathrm{~s}$ & $125 e$ & $0.00 \mathrm{~nm}$ & & 6 & $.8 \mathrm{mb}$ & I SLE & & 69.77 & 43 & iPd \\
\hline DLE & 61.83 & 34 & iPd & 28 & 27.90 & 0.5 & I TMA & & 69.84 & 45 & iPd \\
\hline & $1.7 \mathrm{~s}$ & 900 & $0.00 \mathrm{~nm}$ & & 6 & $.6 m b$ & 1 LLS & & 70.01 & 44 & iPd \\
\hline COR & 61.86 & 315 & iPd & 28 & 26.00 & -1.8 & TNS & & 70.05 & 40 & iPc \\
\hline DMU & 61.86 & 34 & iPd & 28 & 28.20 & 0.5 & I DAG & & 70.25 & 10 & iPd \\
\hline & $1.0 \mathrm{~s}$ & 368 & $0.00 \mathrm{~nm}$ & & & $.5 \mathrm{mb}$ & 1 & & $1.1 \mathrm{~s}$ & & $8.50 \mathrm{~nm}$ \\
\hline$A L I$ & 61.92 & 52 & $\mathrm{iP+}$ & 28 & 28.00 & -0.3 & FIR & & 71.34 & 47 & eP \\
\hline & & & $\mathrm{i}$ & 36 & 56.00 & & 1 & & & & es \\
\hline DDK & 61.98 & 34 & iPd & 28 & 28.80 & 0.4 & I OGA & & 71.39 & 44 & iPd \\
\hline & $1.5 \mathrm{~s}$ & 500 & $0.00 \mathrm{~nm}$ & & & $.4 \mathrm{mb}$ & 1 & & $2.0 \mathrm{~s}$ & 435 & $5.00 \mathrm{~nm}$ \\
\hline GMW & 62.27 & 318 & $e P$ & 28 & 28.20 & -2.3 & I HAM & & 71.40 & 37 & iPd \\
\hline BFW & 62.27 & 317 & ep & 28 & 29.40 & -1.2 & I MUD & & 71.41 & 34 & IPd \\
\hline MCW & 62.61 & 320 & $e^{P}$ & 28 & 31.00 & -1.8 & I FUR & & 71.67 & 43 & iPd \\
\hline$P G C$ & 63.00 & 320 & eP & 28 & 32.90 & -2.3 & 1 & & $2.2 \mathrm{~s}$ & 1030 & $0.00 \mathrm{~nm}$ \\
\hline AKU & 63.04 & 19 & iP & 28 & 35.30 & 0.0 & 1 & Z & $19 \mathrm{~s}$ & & $3.28 \mathrm{um}$ \\
\hline & $1.9 \mathrm{~s}$ & 463 & $3.00 \mathrm{~nm}$ & & 6 & $.3 \mathrm{mb}$ & I GRF & & 71.77 & 41 & iPd \\
\hline EBR & 63.07 & 49 & $e^{P}$ & 28 & 38.00 & 2.1 & 1 & & $1.9 \mathrm{~s}$ & 616 & $6.00 \mathrm{~nm}$ \\
\hline & & & es & 37 & 08.00 & & 1 & $z$ & $22 s$ & & $3.60 \mathrm{um}$ \\
\hline EPF & 3.36 & 47 & iPd & 28 & 38.50 & 0.7 & $151 T$ & & 71.83 & 327 & eP \\
\hline LPF & 3.52 & 41 & iPd & 28 & 38.60 & -0.1 & I ALE & & 72.08 & 0 & ePd \\
\hline GRR & 63.72 & 41 & iPd & 28 & 40.00 & -0.1 & 1 & & $0.7 \mathrm{~s}$ & & $4.00 \mathrm{~nm}$ \\
\hline & 1.05 & 104 & $4.00 \mathrm{~nm}$ & & 5 & $.9 \mathrm{mb}$ & $1 \operatorname{MOX}$ & & 72.11 & 40 & iPd- \\
\hline MFF & 63.76 & 43 & iPd & 28 & 40.40 & 0.0 & 1 & & $2.2 \mathrm{~s}$ & 438 & $8.00 \mathrm{~nm}$ \\
\hline & $1.5 \mathrm{~s}$ & 225 & $5.00 \mathrm{~nm}$ & & 6 & $.0 \mathrm{mb}$ & 1 & $z$ & $19 \mathrm{~s}$ & & 3. $60 \mathrm{um}$ \\
\hline LFF & 64.01 & 45 & eP & 28 & 42.00 & 0.0 & 1 & $E$ & $19 \mathrm{~s}$ & & $2.80 u m$ \\
\hline & 1 & 199 & $9.00 \mathrm{~nm}$ & & 6 & $.0 \mathrm{mb}$ & 1 & & & & $\mathrm{i}$ \\
\hline$F L N$ & 64.06 & 41 & iPd & 28 & 42.10 & -0.2 & I & & & & es \\
\hline LDF & 64.25 & 41 & iPd & 28 & 43.50 & 0.0 & 1 & & & & e \\
\hline LPO & 64.28 & 45 & iPd & 28 & 43.70 & -0.1 & 1 & & & & e \\
\hline & $1.2 \mathrm{~s}$ & 157 & $7.00 \mathrm{~nm}$ & & 6 &.$\theta m b$ & 1 & & & & ess \\
\hline ESK & 64.38 & 33 & ePd & 28 & 43.00 & -1.2 & 1 & & & & i LO \\
\hline & $1.0 \mathrm{~s}$ & 200 & $0.00 \mathrm{~nm}$ & & 6 & $.1 \mathrm{mb}$ & I HOF & & 72.25 & 40 & eP \\
\hline EKA & 64.41 & 33 & $P d$ & 28 & 43.50 & -0.9 & 1 & & $2.4 \mathrm{~s}$ & 764 & $4.00 \mathrm{~nm}$ \\
\hline$R J F$ & 64.63 & 45 & $e^{P}$ & 28 & 46.00 & 0.0 & 1 & $z$ & $18 \mathrm{~s}$ & & $3.90 \mathrm{um}$ \\
\hline & $1.5 \mathrm{~s}$ & 158 & $8.00 \mathrm{~nm}$ & & & $.9 m b$ & I $M B C$ & & 72.29 & 348 & ePd \\
\hline LSF & 64.82 & 44 & iPd & 28 & 47.20 & -0.1 & 1 & & $0.4 \mathrm{~s}$ & & $4.00 \mathrm{~nm}$ \\
\hline CAF & 64.93 & 45 & iPd & 28 & 47.90 & -0.2 & 1 & & & & PP \\
\hline TCF & 65.29 & 44 & iPd & 28 & 50.40 & 0.1 & I WET & & 72.80 & 42 & iPd \\
\hline & $1.2 \mathrm{~s}$ & 86 & $6.40 \mathrm{~nm}$ & & & $.7 \mathrm{mb}$ & 1 & & 2. & 667 & 7.0 \\
\hline
\end{tabular}




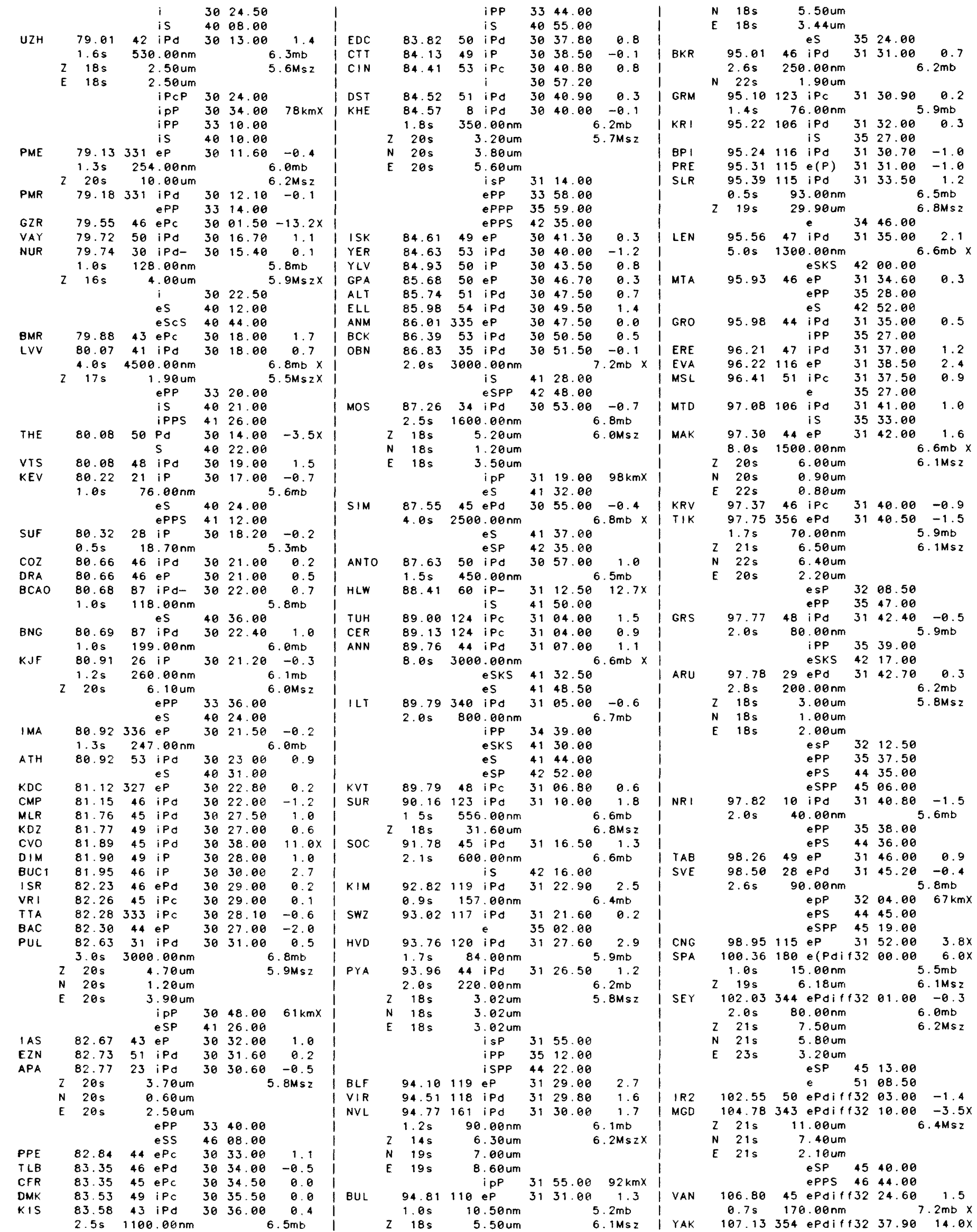


$11 \mathrm{~d} \theta \mathrm{Bh}$

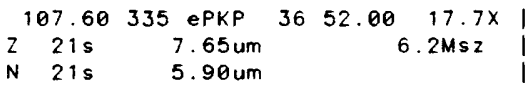

E $21 \mathrm{~s} \quad 4.45 \mathrm{um}$

SEM $111.39 \quad 24$ ePKP $36 \quad 37.70 \quad-3.9 \times \quad$ PCT EPP $\quad 37 \quad 19.00$ ESP $\quad 46 \quad 40 \quad 40$

\section{BO}

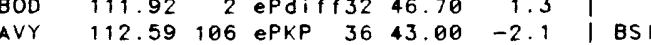

DSH $113.57 \quad 40$ iPdiff32 $39.00-14.3 \times$ | BAG

ANR

$\begin{array}{llllll}114.38 & 36 \text { ePdiff33 } 15.60 & 18.8 \times & \text { OCP }\end{array}$

$1.0 \mathrm{~s} \quad 150.00 \mathrm{~nm}$

$\begin{array}{llllll}114.58 & 46 & \text { iPdiff32 } & 52.20 & -5.6 \times & \text { SNG }\end{array}$

$115.10 \quad 16$ ePKPd $3656.80 \quad 8.1 \times \quad$ TS

$0.9 \mathrm{~s} \quad 50.00 \mathrm{~nm}$

iPP 3750.00

TUP

115.35358 ePKP $36 \quad 48.10$

$\begin{array}{llllll}116.57 & 11 & \text { EPKP } & 36 & 49.40 & -2.1\end{array}$

$\begin{array}{llllll}118.24 & 10 & \text { ePKP } & 36 & 51.50 & -3.2 x\end{array}$

$0.9 \mathrm{~s}$

$10.00 \mathrm{~nm}$

$220 \mathrm{~s} \quad 5.18 \mathrm{um}$

N $20 \mathrm{~s} \quad 3.13 \mathrm{um}$

E $20 \mathrm{~s} \quad 0.69 \mathrm{um}$

$\begin{array}{llll}\text { ePP } & 38 & 07.00\end{array}$

$\begin{array}{lllllll}\text { RHP } & 123.55 & 224 & \text { PKP } & 37 & 05.40 & 0.2\end{array}$

NDI $\quad \begin{array}{llllll}25.18 & 44 & \text { EPKP } & 37 & 08.00 & -0.7\end{array}$

MAT $\quad 129.28 \quad 338$ IPKPC $37 \quad 15.00 \quad-1.4$ $0.8 \mathrm{~s} \quad 12.70 \mathrm{~nm}$

$2 \quad 21 \mathrm{~s}$

BJ $129.78,1$ ePKP $3715.50^{6.2 M s z}$

$\mathrm{LZH} \quad 132.05 \quad 15 \mathrm{PKPd} \quad 3721.00 \quad-0.9$

$2.0 \mathrm{~s} \quad 151.00 \mathrm{~nm}$

E $20 \mathrm{~s} \quad 4.20 \mathrm{um}$

PPP 3938.00

eS $44 \quad 40.50$

eSS 4756.00

HYB $132.82 \quad 54$ EPKPC $3707.50-16.1 \times$

e 3722.50

$\begin{array}{lllllll}\text { TIA } & 133.61 & 0 & \text { ePKP } & 37 & 23.20 & -1.4\end{array}$

GBA 133.86 60 PKP $3720.00 \quad-5.6 \mathrm{X}$

$0.8 \mathrm{~s} \quad 10.00 \mathrm{~nm}$

$133.86 \quad 60$ PKPd $37 \quad 24.90$

$1.1 \mathrm{~s} \quad 31.50 \mathrm{~nm}$

$\begin{array}{llllll}\text { XAN } 135.08 & 10 \text { PKP } 37 & 26.20 & -1.3\end{array}$

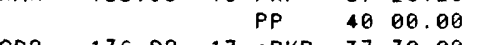

$\begin{array}{lllllll}\mathrm{CD} 2 & 136.92 & 17 & \text { ePKP } & 37 & 30.00 & -1.1\end{array}$ EPP $\quad 40 \quad 11.00$

$\begin{array}{lllll}\mathrm{NJ} 2 & 137.74 & 358 \mathrm{PKP} & 37 & 30.00\end{array}$ PP $\quad 40 \quad 16.00$

PKS 4106.00

$\begin{array}{lllll}\text { SSE } \quad 138.54355 \text { PKPd } & 37 & 32.00 \\ & & \text { PP } & 40 & 18.00\end{array}$ $\begin{array}{lll}\text { PP } & 40 & 18.00 \\ \text { PKS } & 41 & 06.00\end{array}$

WHN $139.17 \quad 4$ EPKP 3731.50 $\begin{array}{lll}\text { EPP } & 40 & 20.06 \\ \text { PKS } & 41 & 06.20\end{array}$

$\begin{array}{rrlll}141.54 & & \text { PKS } & 41 & 06.20 \\ \text { EPKP } & 37 & 36.00\end{array}$

CAN 141.94224 ePKP $37 \quad 39.40$

KM) $141.95 \quad 22$ ePKP $37 \quad 33.00$ $\begin{array}{cc}141.95 & 22 \text { ePKP } \\ 7.0 \mathrm{~s} & 0.50 \mathrm{~nm}\end{array}$

E $20 \mathrm{~s} \quad 2.90 \mathrm{um}$

$\begin{array}{lll}P P & 40 \quad 45.00\end{array}$

YOU $\quad 142.93225$ ePKP $37 \quad 39.10$

ANP $\quad 144.36353$ ePKP 3739.00

RAB 144.81278 IPKP 3743.00

$\mathrm{OZH} \quad 144.82358$ iPKPd $37 \quad 44.00$ $\begin{array}{lll}P P & 40 & 56.00\end{array}$

144.93216 IPKPd 3744.90

$145.78 \quad 281$ ePKP $37 \quad 46.06$

145.8132 iPKPd-37 46.30

$2.0 \mathrm{~s} \quad 765.00 \mathrm{~nm}$

eS 4156.00

CMS $146.23 \quad 227$ EPKP 3748.00

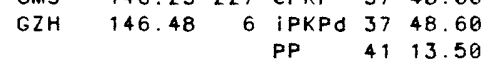

BDT $147.13 \quad 33$ ePKP $37 \quad 48.00$

$0.8 \mathrm{~s} \quad 130.00 \mathrm{~nm}$

HKC $\quad 147.34 \quad 5$ IPKP 3750.00

MCO $147.46 \quad 6$ ePKP 3751.50

ADE 148.69215 IPKPd $37 \quad 55.50$

$0.5 \mathrm{~s} \quad 63.40 \mathrm{~nm}$

MOM 148.90283 ePKP 3753.00

STK 148.96223 ePKP 3751.00

LAT $150.33 \quad 275$ ePKP $37 \quad 57.00$

$-2.5$

QUE

KHI

$\mathrm{ASH}$

$-2.7 \times \quad \mathrm{DSH}$

$-5.6 \times$

$-2.6$

-1.2 | IR

\begin{tabular}{l|l}
-0.2 & SAM \\
-1.2 & GAR
\end{tabular}

$-1.2$

$-0.8$

\begin{tabular}{l|l}
0.5 & NDI \\
0.6 & POO \\
& KER
\end{tabular}

-1.2 I TAS

0.6 A

$\begin{array}{ll}\text { 1. } 9 & 1 \times \\ 4\end{array}$

0.8

\begin{tabular}{r|r}
-0.8 & BAK
\end{tabular}

$2.7 \times \mid \mathrm{KSH}$

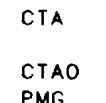

$\begin{array}{ll}50.44 & 248 \text { iPKP } \\ 1.7 \mathrm{~s} & 144.00 \mathrm{~nm}\end{array}$

150.44248 ePKPd $3754.00-0.3 \quad$ TAB

$\begin{array}{llllll}150.51 & 269 \text { e (PKP) } 37 & 48.00 & -6.6 \times \text { | GRS }\end{array}$

150.5432 ePKP 3755.60

$$
\text { e } \quad 4134.90
$$

1.1

$152.02 \quad 353$ ePKP 37

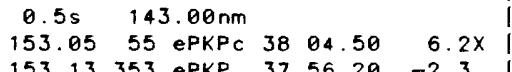

$\begin{array}{lllll}153.13 & 353 & \text { ePKP } 37 & 56.20 & -2.3\end{array}$

$1.7 \mathrm{~s} \quad 185.00 \mathrm{~nm}$

$\begin{array}{lllll}154.81 & 351 & \text { ePKP } 38 \quad 04.00\end{array}$

$\begin{array}{llll}155.40 & 287 & \text { EPKPC } 38 \quad 01.00\end{array}$

$\begin{array}{lllll}155.93 & 44 & \text { ePKP } 38 \quad 02.00\end{array}$

$\begin{array}{llll}156.83 & 54 & \text { e(PKP) } 38 \quad 15.00\end{array}$

$157.60 \quad 55$ ePKPd $38 \quad 04.60$

$1.5 \mathrm{~s} \quad 80.00 \mathrm{~nm}$

$158.03 \quad 48$ ePKPd $38 \quad 05.00$

$$
\text { e } \quad 3838.60
$$

$42 \quad 18.20$

ASPA 159.35228 ePKP $38 \quad 06.00$

$\begin{array}{rrrrr}159.35 & 228 & \text { EPKP } & 38 & 06.00 \\ 160.56 & 60 & \text { ePKP } & 38 & 07.50\end{array}$

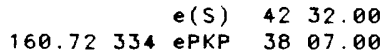

$\begin{array}{llllll}\text { WB2 } & 160.97 & 238 & \text { i PKPd } & 38 & 07.20 \\ & & & i & 38 & 51.30\end{array}$

$\begin{array}{llllll} & & & \text { e } & 42 & 29.80 \\ \text { WRA } & 160.98 & 238 & \text { PKP } & 38 & 08.00\end{array}$

$2.2 \mathrm{~s} \quad 266.00 \mathrm{~nm}$

$\begin{array}{llllll}\text { I KGM } & 161.44 \quad 49 & \text { ePKPC } & 38 & 09.10\end{array}$

$$
\begin{array}{lll}
\text { e } & 38 & 53.00 \\
\text { e } & 42 & 36.50
\end{array}
$$

WBN 162.04208 ePKP 3808.00

163.614 ePKPd 3809.20 eS 3902.00

MEK $\quad 163.85184$ EPKP 3810.00

\begin{tabular}{l|l|l}
$3.4 \times$ & FRU \\
\hline & KRV
\end{tabular}

\begin{tabular}{c|c}
$3.4 \times$ & KRV \\
-0.6 & HYB
\end{tabular}

$-0.2$

$1.6 \mathrm{x}$

0.2

0.1

MAK

NRN

NAK

MSL

RU
RV
YB

$-0.1$

-0.1 I AAA

$-0.7$

\begin{tabular}{l|l}
-0.7 & PRZ
\end{tabular}

LEN

167.22301 e(PKP) 3811.00

S.D. $=1.1$ on 414 of 449 obs

APR 18,1983 ion $58 \mathrm{~m} 48.45 \pm 0.33 \mathrm{~s}$ $27.748 \mathrm{~N} \pm 1.7 \mathrm{~km} \quad 62.051 \mathrm{E} \pm 1.2 \mathrm{~km}$

DEPTH $=39.5 \pm 2.8 \mathrm{~km}$

$6.6 \mathrm{mb}$ ( 113 obs.) $6.4 \mathrm{Msz}$ ( 25 obs.) SOUTHERN IRAN

Felt strongly in southeastern

Iran. Also felt at Karachi.

Pakiston.

$4.9359 \mathrm{iPd}$

$7.01336 \mathrm{iPt}$

8.58285 iPc 0155.00

$8.80346 \mathrm{iPct}$

$9155.0061 .8 \times$ JER

$1.5 \mathrm{~s} 4800.00 \mathrm{~nm}$

$10.64344 \mathrm{eP}$

0259.00

02.5

22.501 .1 SOC

$0.7 \mathrm{~s} \quad 170.00 \mathrm{~nm} \quad 6.3 \mathrm{mb}$ I ARO

\begin{tabular}{cccc|c|c} 
& $\mathrm{eS}$ & 03 & 24.00 & & \\
$10.70343 \mathrm{eP}$ & 01 & 22.70 & 0.5 & KVT
\end{tabular}

$\begin{array}{rlll}\text { eS } 0320.20 & \text { I LSA }\end{array}$

\begin{tabular}{cccc|c|c}
12.01 & $31 \mathrm{iPc}$ & 01 & 41.80 & 1.8 \\
$4.6 \mathrm{~s}$ & $* * * * * * * \mathrm{~nm}$ & & $7.5 \mathrm{mb} \times$ & ANN
\end{tabular}

N $12 \mathrm{~s} \quad 242.00 \mathrm{um}$

1 is 0359.30

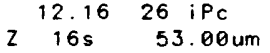

eS $03 \quad 59.20$

12.34313 iPc 0147.70

$\begin{array}{ccc}13.17 & 29 \mathrm{iPc} \\ 2 & 15 \mathrm{~s} & 272.00 \mathrm{~m}\end{array}$

$155.20-0.3$

is 0420.40

$\begin{array}{lllll}13.41 & 82 \mathrm{iPd} \quad 01 & 55.00 & -3.5 \mathrm{X}\end{array}$

0.7s $596.00 \mathrm{~nm}$

$\begin{array}{clll}\text { is } 0411.00 & \text { IS SM }\end{array}$

\begin{tabular}{llll|l}
$401 \mathrm{eP}$ & 02 & 13.50 & 1.9 & $\mathrm{KDE}$
\end{tabular}

\begin{tabular}{lllll|l}
14.79 & $22 \mathrm{P}$ & 02 & 16.00 & -0.6 & $\mathrm{ELL}$
\end{tabular}

$4.0 \mathrm{~s} \quad 8100.00 \mathrm{~nm}$

$211 \mathrm{~s} 105.00 \mathrm{um}$

$\mathrm{N}$ iाs $130.00 \mathrm{um}$

$115 \quad 230.00 \mathrm{um}$

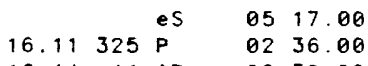

$16.44 \quad 41 \mathrm{iPC} \quad 02 \quad 39.00$

2.5 $\begin{array}{llllllll}15.51 & 31 & \mathrm{iPC} & 02 & 25.70 & -0.2 & \mathrm{ARU}\end{array}$

$\begin{array}{lll} & & \text { PP } \\ & \text { eS } \\ 16.72 & 312 & \mathrm{iPt} \\ 17.54 & 316 \text { iPc } \\ 2.0 \mathrm{~s} & 700.00 \mathrm{~nm} \\ 17.81 & 36 \mathrm{P} \\ 17.95 & 314 \text { iPd } \\ 18.17303 \text { iPc } & \\ & & \text { iS } \\ & & \text { iPcP }\end{array}$

$18.18 \quad 31 \mathrm{P}$

$18.26319 \mathrm{eP}$

18.36121 iPd

$1.0 \mathrm{~s} \quad 1440.00 \mathrm{~nm}$

$\begin{array}{lll}\text { eS } & 03 & 18.0 \\ \text { eS } & 21.0\end{array}$

19.30326 IPC $\quad 03 \quad 13.00 \quad 0.2$

$6.0 \mathrm{~s} \quad * * * * * * * \mathrm{~nm}$

$25 \mathrm{~s} \quad 302.00 \mathrm{um}$

$\begin{array}{lll}N & 26 s & 272.00 u m \\ E & 25 s & 894.00 u m\end{array}$

$\begin{array}{llllll}19.62 & 34 & \text { iPC } & 03 & 16.00 & -0.5\end{array}$

$6.0 \mathrm{~s}-4.60 \mathrm{~nm}$

is 0651.00

$\begin{array}{llllll}19.82 & 38 \mathrm{P} & 0318.00 & -0.8\end{array}$

$19.82316 \mathrm{iPc} \quad 0316.00 \quad-2.8$

$2.0 \mathrm{~s} 2800.00 \mathrm{~nm}$

$Z 24 \mathrm{~s} \quad 84.00 \mathrm{um}$

E $24 \mathrm{~s} \quad 60.00 \mathrm{um}$

$\begin{array}{cc}19.84 & 35 \mathrm{iPc} \\ 16 \mathrm{~s} & 80.00 \mathrm{um}\end{array}$

$20.10131 \mathrm{P}$

$20.42 \quad 85 \mathrm{iPO}$

$20.43324 \mathrm{P}$

$20.5584 \mathrm{IPO}$

$20.60317 \mathrm{iPc}$

$2.8 \mathrm{~s} * * * * * * \mathrm{~nm}$

E $20 \mathrm{~s} \quad 466.00 \mathrm{um}$

ePPP

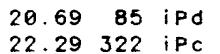

$\begin{array}{ll}22.29 & 322 \mathrm{iPc} \\ 1.5 \mathrm{~s} & 1880.00 \mathrm{~nm}\end{array}$

N $13 \mathrm{~s} \quad 27.50 \mathrm{um}$

$\begin{array}{lll}\text { eS } & 07 & 46.5 \\ \text { iss } & 08 & 14.00\end{array}$

iSSS 0833.00

$22.67 \quad 137$ iPd

$1.0 \mathrm{~s} \quad 400.00 \mathrm{~nm}$

23.55232 iP

$23.63286 \mathrm{eP}$

$23.84 \quad 232 \quad$ if

23.95232 iP

$23.95317 \mathrm{P}$

$24.18 \quad 232$ iPd

25.13309 iP

$25.57 \quad 79$ IPd

$\begin{array}{lll}03 & 49.20 & 1.2\end{array}$

$0402.5 .8 \mathrm{mb}$

$0359.00 \quad 2.0$

0814.00

$0403.40 \quad 4.5 x$

$0403.80 \quad 3.8 x$

$03 \quad 59.00 \quad-0.8$

$0405.80 \quad 3.4 x$

0835.00

$\begin{array}{lll}04 & 12.40 & 1.1\end{array}$

26.12317 is

$04 \quad 17.78$

1. 7

$7.0 \mathrm{~s} 7000.00 \mathrm{~nm}$

$Z 28 \mathrm{~s} \quad 76.00 \mathrm{um}$

N 28s 160.00um

E $28 \mathrm{~s} 60.00 \mathrm{um}$

$26.12 \quad 45 \mathrm{iPC}$

84

0845.00

$0-0.3$

6. $1 M s z x$

7 PCS

1120.00

0423.90

0511.80

0845.40

$04 \quad 29.50$

0908.00

$0428.90 \quad 0.0$

$27.03304 \mathrm{eP}$

$28.10315 \mathrm{P}$

$28.11298 \mathrm{eP}$ 


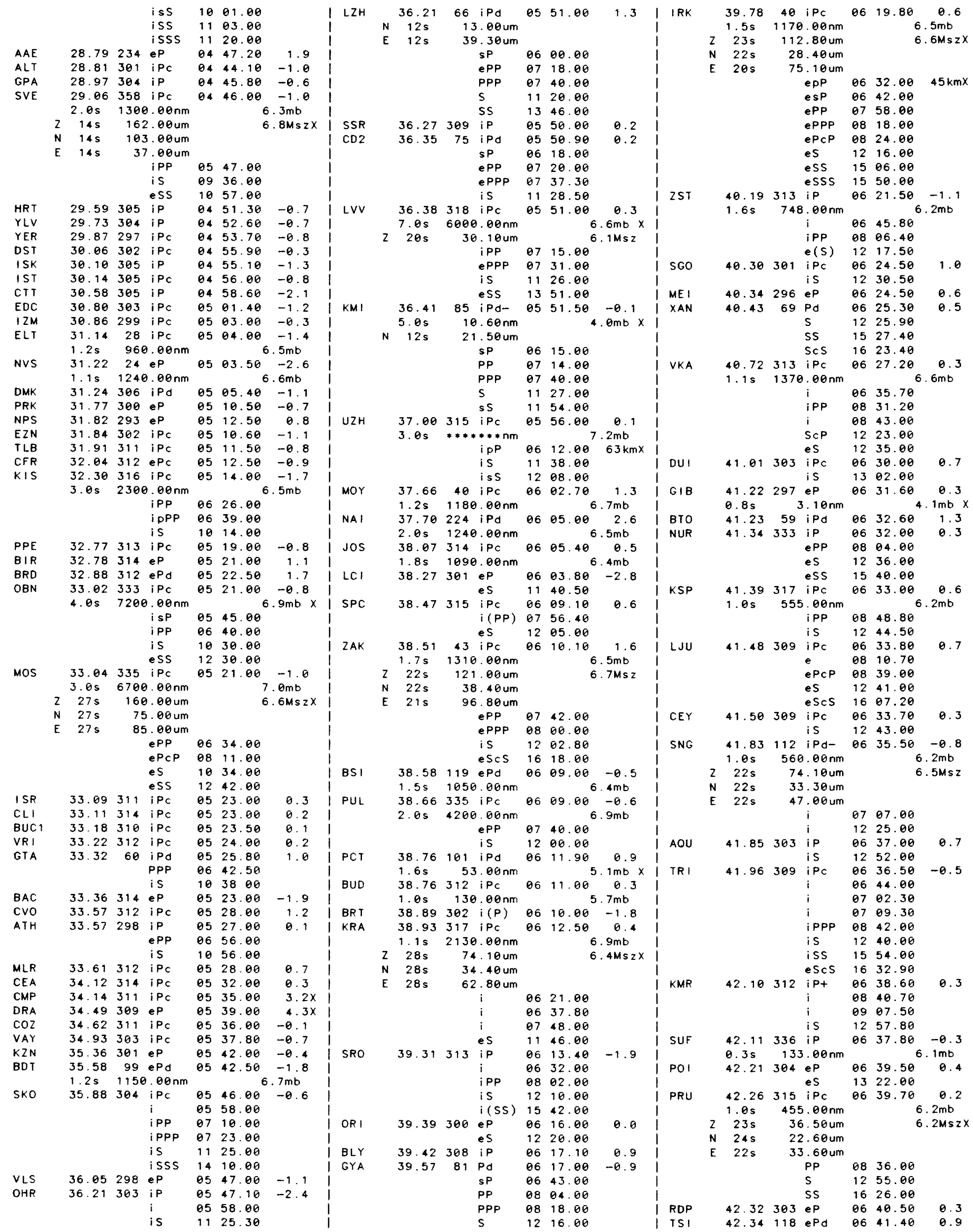




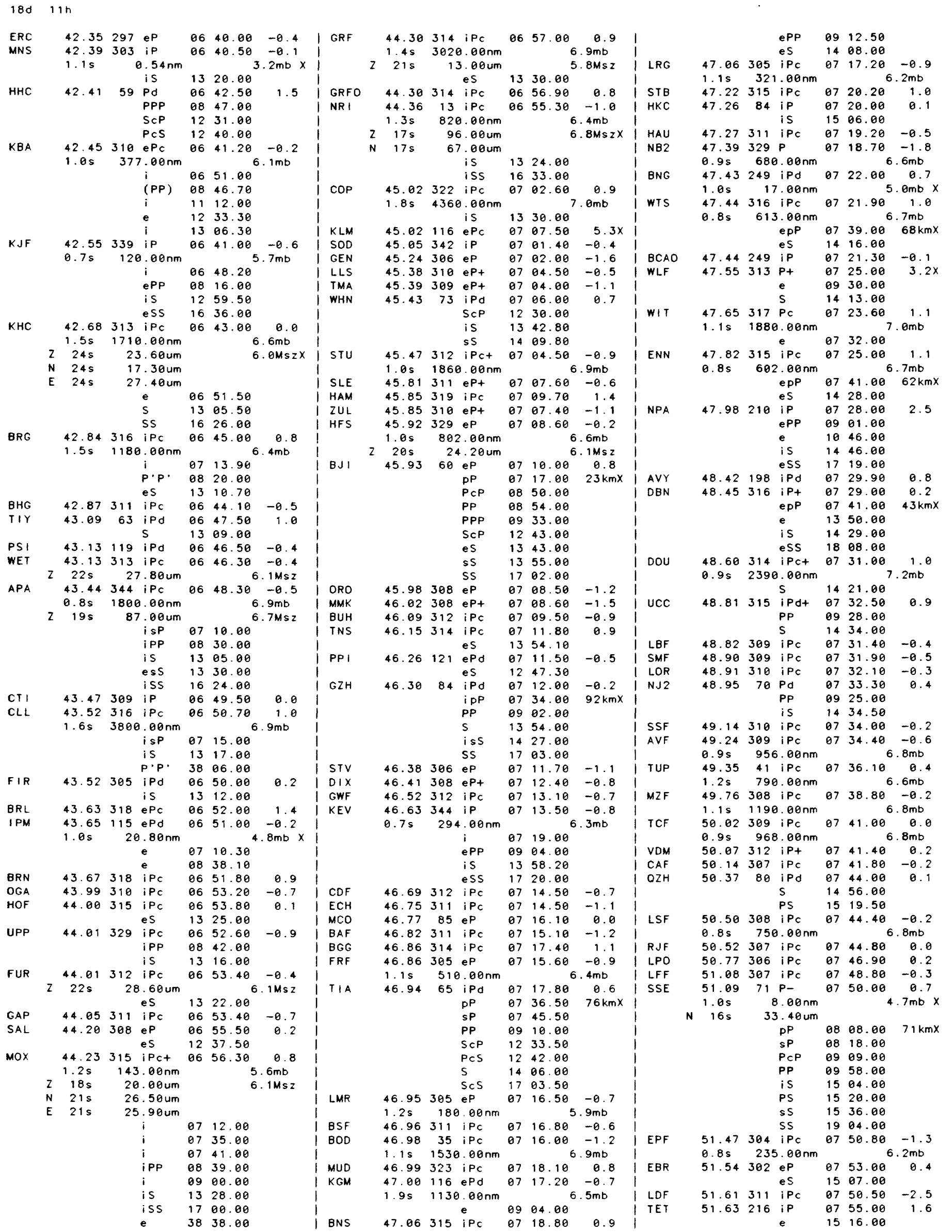


MFF $\quad 51.65309 \quad \mathrm{PC} \quad 07 \quad 50.80 \quad-2.5 \quad$ OCP

$1.0 \mathrm{~s} \quad 4200.00 \mathrm{~nm}$

$\begin{array}{llll}\text { LPR } & 52.10 & 311 & \mathrm{PPC} \\ \text { LPF } & 52.23 & 311 & \mathrm{iPC}\end{array}$

AL

CN2

52.59299 eP

$52.64 \quad 55$ iPd

CN2

$S P$
$P P$
$P C S$

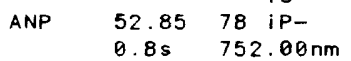

TATO $52.88 \quad 79 \mathrm{eP}$

$52.99359 \mathrm{IPC}$

$1.3 \mathrm{~s} \quad 920.00 \mathrm{~nm}$

$\begin{array}{ll}\text { ePPP } & 1108.00^{6.6 \mathrm{mb}} \\ \text { is } & 1528.00\end{array}$

ESS 1858.00

ipP

LGR

$53.61304 \quad \mathrm{iPc}$

$1.3 \mathrm{~s} \quad 5.03 \mathrm{~nm}$

iPP

$\begin{array}{lll}\text { EKA } & 53.67 \quad 320 \mathrm{Pc} \\ & 1.7 \mathrm{~s} \quad 1350.08 \mathrm{~nm}\end{array}$

$53.70320 \mathrm{ePc}$

$1.0 \mathrm{~s} \quad 760.00 \mathrm{~nm}$

8 $32.00113 \mathrm{kmx}$

$08 \quad 08.30 \quad 0.3$

$10 \quad 07.00$

1541.00

ESK

KR

ALM

54.33219 iPd

ipP

$\begin{array}{lllll}08 & 13.00 & -0.6 & \text { PTO }\end{array}$

$08 \quad 42.00122 \mathrm{kmX}$ | KAG

$\begin{array}{ll}54.37 & 297 \\ 1.1 \mathrm{~s} & 7.00 \mathrm{~nm}\end{array}$

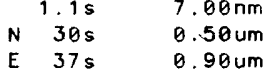

0.90 um

iPP

BAG $\quad 54.96 \quad 89$ iPd-

ALR $\quad 55.00 \quad 296 \quad$ iP

GUD

TOL

\section{$55.07 \quad 302 \quad \mathrm{iP}$}

i

$\begin{array}{ll}55.10 & 301 \mathrm{iPc} \\ 0.8 \mathrm{~s} & 20.00 \mathrm{~nm}\end{array}$

i $\quad 0828.50$

$\begin{array}{lll}i & 08 & 46.00 \\ \text { ePCP } & 09 & 24.00\end{array}$

$\begin{array}{lll}\text { iPP } & 10 \quad 24.00\end{array}$

(PPP) 1158.00

is 1557.00

iPS $16 \quad 38.00$

i(SS) 2027.00

CRT
YAK

$55.21298 \quad \mathrm{PP}$

0819.89

$\begin{array}{llllll}55.44 & 32 & \text { iPc } & 08 & 18.70 & -2.4\end{array}$

$1.5 \mathrm{~s} 2230.00 \mathrm{~nm}$

$Z 14 \mathrm{~s} 63.30 \mathrm{um}$

$N \quad 14 \mathrm{~s} \quad 17.18 \mathrm{um}$

E $15 \mathrm{~s} \quad 48$. Doum

IPCP 0920.70

is $\quad 1556.70$

iSCS 1803.10

OKM

$\begin{array}{llllll}55.46 & 317 \quad \mathrm{iPC} & 08 & 21.80 & 0.5\end{array}$

$1.0 \mathrm{~s} \quad 670.00 \mathrm{~nm}$

$55.50317 \mathrm{eP}$

$1.0 \mathrm{~s} \quad 335.00 \mathrm{~nm}$

$08 \quad 21.70^{6.6 \mathrm{mb}}$

$3 \mathrm{Pd}$

$P P$

PCS

S $\quad 1557.00$

SCS 1806.00

$\begin{array}{lllllll}\text { CVP } & 55.58 & 87 & \mathrm{ePC} & 08 & 22.00 & -0.6\end{array}$

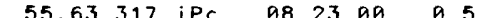

$1.0 \mathrm{~s} 560.00 \mathrm{~nm} \quad 6.5 \mathrm{mb}$

$\begin{array}{lllll}55.63 & 102 \mathrm{ePd} \quad 08 \quad 23.00 & -0.2\end{array}$

$1.0 \mathrm{~s} \quad 396.00 \mathrm{~nm} \quad 6.4 \mathrm{mb}$

$\begin{array}{lllllll}55.83 & 318 \quad \mathrm{iPC} & 08 & 24.60 & 0.6\end{array}$

$1.3 \mathrm{~s} 4200.00 \mathrm{~nm}$

$\begin{array}{llllll}55.94 & 297 \quad \text { IPC } & 08 & 24.00 & -0.9\end{array}$

iPP $10 \quad 36.00$

DCN is $16 \quad 03.00$

\begin{tabular}{lllll|l}
56.07 & $317 \mathrm{iPC}$ & 08 & 26.50 & 0.8 & MKS
\end{tabular}

$1.0 \mathrm{~s} 1560.00 \mathrm{~nm}$
$56.1091 \mathrm{eP} \quad 0828.50 \quad 2.1$

$\begin{array}{llllll}56.64 & 20 \mathrm{iPC} & 88 & 27.58 & -2.1 & \mathrm{DOR}\end{array}$

$1.1 \mathrm{~s} \quad 1510.00 \mathrm{~nm}$

$216 \mathrm{~s} \quad 52.00 \mathrm{um}$

N $15 \mathrm{~s} \quad 17.00 \mathrm{um}$

E $17 \mathrm{~s} \quad 49.00 \mathrm{um}$

$\begin{array}{lll}\text { esP } & 08 \quad 54.00\end{array}$

EPCP $Q 925.00$

$\begin{array}{lll}\text { EPP } & 10 \quad 38.00\end{array}$

EPPP 1154.00

ES $\quad 16 \quad 13.00$

\begin{tabular}{cc|c|c} 
eS & 16 & 13.00 & KYS \\
ePS & 1638.00 & MIY
\end{tabular}

$\begin{array}{llllll}57.38 & 55 & \mathrm{iPC} & 08 & 34.00 & 0.2\end{array}$

$Z 14 \mathrm{~s} \quad 41.70 \mathrm{um}$

N $14 \mathrm{~s} \quad 30.50 \mathrm{um}$

E $14 \mathrm{~s} \quad 11.80 \mathrm{um}$

isP 0858.00

is $\quad 16 \quad 28.00$

ises $18 \quad 12.00$

$57.40297 \mathrm{iPC}$

$\begin{array}{lll}\text { IPC } & 08 & 35.00 \\ \text { ePP } & 10 & 42.00\end{array}$

is $16 \quad 30.00$

iSS 1912.00

iSSS 2100.00

BUL

$57.56 \quad 218 \quad \mathrm{iPd}$

$0.8 \mathrm{~s} \quad 75.80 \mathrm{~nm}$

$\begin{array}{lll}08 & 37.00 & 0.2\end{array}$

$$
\text { i PP }
$$

$09 \quad 05.00116 \mathrm{kmx}$

$57.96316 \quad$ iP

1633.00

is

$58.31303 \mathrm{iPc}$

$\begin{array}{ll}08 & 39.20 \\ 16 & 32.00\end{array}$

$58.77 \quad 68 \mathrm{Pd}$

$59.11294 \mathrm{iP}$

$59.21300 \mathrm{iPc}$

$59.73 \quad 64$ iPd

$59.95 \quad 109 \mathrm{ePC}$

$60.26118 \mathrm{iPC}$

$1.3 \mathrm{~s} \quad 321.00 \mathrm{~nm}$

$08 \quad 42.30$

1640.00

$08 \quad 45.30$

\begin{tabular}{lll|l}
16 & 49.00 & & MAG \\
08 & 47.00 & -0.3 & BLF
\end{tabular}

$0848.10 \quad 0.2$ KIC

$\begin{array}{llll}08 & 51.50 & -0.1 & 1\end{array}$

$0856.503 .1 \times \mid \mathrm{KIM}$

$68 \quad 52.90$

- -2.6

$0.99 \quad 92 \mathrm{ePc}$

$61.03332 \mathrm{iPC}$

$0.9 \mathrm{~s} 1410.00 \mathrm{~nm}$

$61.10345 \mathrm{iPc}$

$0.7 \mathrm{~s} \quad 411.00 \mathrm{~nm}$

61.9395 i Pd

$62.32 \quad 214 \mathrm{iPc}$

$1.4 \mathrm{~s} \quad 735.00 \mathrm{~nm}$

$223 \mathrm{~s} \quad 27.30 \mathrm{um}$

$0858.00^{6.3 m b}$

$0900.80 \quad-2.5$

7.1mb HVD

$0858.70 \quad-1.8$

$\begin{array}{lll}62.57 & 213 \mathrm{iPd} \\ 62.80 & 331 \mathrm{eP}\end{array}$

EVA
REY

BPI

$62.80331 \mathrm{eP}$

$62.81214 \quad \mathrm{PPd}$

$63.13216 \mathrm{iPd}$

$63.3396 \mathrm{iPd}-$

$1.8 \mathrm{~s} \quad 7270.00 \mathrm{~nm}$

$\begin{array}{llll}63.56 & 61 \text { is } & 1800.00 \\ 1.85 & 1110.00 n m & 16.40\end{array}$

$1.8 \mathrm{~s} 1110.00 \mathrm{~nm}$

$z 20 \mathrm{~s}, 1.60 \mathrm{um}$

63.71214 iPo

$64.13 \quad 57 \mathrm{eP}$

$\begin{array}{ll}17 & 46.00 \\ 09 & 27.00\end{array}$

$\begin{array}{ll}09 & 27.00 \\ 09 & 28.00\end{array}$

0922.00

$\begin{array}{lll}e & 1858.00\end{array}$

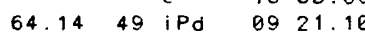

$0.8 \mathrm{~s} \quad 2050.00 \mathrm{~nm}$

$\begin{array}{lll}Z & 18 \mathrm{~s} & 19.60 \mathrm{um}\end{array}$

$\mathrm{N} 15 \mathrm{~s} \quad 14.00 \mathrm{um}$

E $18 \mathrm{~s} \quad 18.20 \mathrm{um}$

epP $0940.00 \quad 72 \mathrm{kmx}$

ePCP 0948.00

$\begin{array}{lll}\text { EPPP } & 13 & 17.00\end{array}$

is 1754.00

ESPP $18 \quad 20.00$

ESCS 1908.00

ESS 2205.00

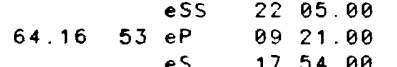

es

$64.31111 \mathrm{iPd}$

$1.3 \mathrm{~s} \quad 2940.00 \mathrm{~nm}$

1754.00

64.3456 eP $\begin{array}{cccc}09 & 20 & 80 & -1.8 \\ 7.2 \mathrm{mb} \\ 09 & 24.00 & 1.6\end{array}$
SEY

MaV

eS $18 \quad 04.00$

$\begin{array}{llllll}64.68 & 62 \mathrm{eP} & 99 & 24.10 & -0.6\end{array}$

64.96214 iPd $9926.50-0.2$

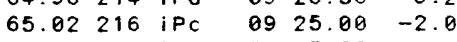

3813.00

$\begin{array}{llllll}65.12 & 61 & \text { iPd } & 09 & 26.10 & -1.4\end{array}$

$\begin{array}{llllll}65.37 & 102 \text { eP } \quad 09 \quad 28.20 & -1.2\end{array}$

$1.5 \mathrm{~s} \quad 805.00 \mathrm{~nm} \quad 6.6 \mathrm{mb}$

$\begin{array}{lllll}65.45 & 62 \text { eP } & 09 & 22.40 & -7.3 x\end{array}$

$\begin{array}{llllll}65.54 & 57 \mathrm{eP} & 09 & 30.00 & -0.1\end{array}$ eS $18 \quad 10.00$

$\begin{array}{llllll}65.76 & 33 & \mathrm{iPc} & 09 & 30.50 & -0.8\end{array}$

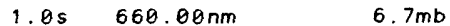

$218 \mathrm{~s} \quad 47.00 \mathrm{um}$

$N 185 \quad 13.00 \mathrm{um}$

E $18 \mathrm{~s} 40.00 \mathrm{um}$

epP $09 \quad 45.00 \quad 52 \mathrm{kmx}$

ESP $\quad 9952.00$

PPCP 1004.00

EPP $11 \quad 52.00$

is $18 \quad 10.00$

ESPP $18 \quad 48.00$

EPPS $18 \quad 52.00$

eSCS 1910.00

$65.84 \quad 30 \mathrm{iPc} \quad 09 \quad 32.40 \quad 0.7$

$\begin{array}{lll}1.2 \mathrm{~s} \quad 1580.00 \mathrm{~nm} & 7.0 \mathrm{mb}\end{array}$

$220 \mathrm{~s} \quad 77.40 \mathrm{um} \quad 6.9 \mathrm{Msz}$

$\mathrm{N} 20 \mathrm{~s} \quad 28.10 \mathrm{um}$

E $20 \mathrm{~s} \quad 58.00 \mathrm{Um}$

PPCP $Q 958.40$

is $18 \quad 13.40$

eSCS 1922.40

$\begin{array}{llllll}65.89 & 34 P & 09 & 30.50 & -1.6\end{array}$

$66.15214 \mathrm{iPc} \quad 0934.00 \quad-0.3$

$\begin{array}{llllll}66.54 & 265 \text { iP } & 09 & 38.10 & 1.1\end{array}$

\begin{tabular}{llllll}
66.57 & 215 & $\mathrm{e}$ & 38 & 09.60 & \\
\hline $\mathrm{Pd}$ & 09 & 36.90 & -0.1
\end{tabular}

$0.9 \mathrm{~s} \quad 241.00 \mathrm{~nm} \quad 6.3 \mathrm{mb}$

$\begin{array}{lllll}66.85 & 353 \mathrm{ePC} & 09 & 36.90 & -1.1\end{array}$

$\begin{array}{llll}1.0 \mathrm{~s} & 849.00 \mathrm{~nm} & 6.8 \mathrm{mb}\end{array}$

$\begin{array}{lllll}67.71 & 214 & \text { iP } 09 \quad 44.00 & -0.2\end{array}$

$1.0 \mathrm{~s} \quad 210.00 \mathrm{~nm} \quad 6.2 \mathrm{mb}$

67.80291 i $\quad 0952.00$

$\begin{array}{lll}\text { IPCP } & 109 & 48.00 \\ \text { CPP } & 12 & 28.00\end{array}$

EPP $12 \quad 28.60$

is $18 \quad 42.00$

ePPS $19 \quad 16.60$

$\begin{array}{llllll}68.07 & 49 & \mathrm{IPd} & 09 & 46.50 & 0.4\end{array}$

$2.0 \mathrm{~s} \quad 5400.00 \mathrm{~nm} \quad 7.3 \mathrm{mb}$

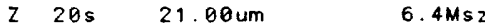

N 205

E $20 \mathrm{~s} \quad 20.40 \mathrm{um}$

$\begin{array}{lll}\text { IPCP } 10 \quad 12.00 & 0\end{array}$

iPP $\quad 12 \quad 23.00$

is 1841.00

EPPS 1921.00

iSSS 2603.00

GRM $\quad 69.44211$ iPd $0955.20 \quad 0.5$

$1.3 \mathrm{~s} \quad 392.00 \mathrm{~nm}$

$\begin{array}{lll}2 & 18 \mathrm{~s} & 8.81 \mathrm{um}\end{array}$

$70.45113 \mathrm{eP}$

$70.84 \quad 105$ ePd

$2.0 \mathrm{~s} 1660.00 \mathrm{~nm}$

$6.0 \mathrm{Msz}$

$\begin{array}{lllll}71.49 & 42 \text { eP } 1006.00 & -1.0\end{array}$

$Z 20 \mathrm{~s}$

$56.10 \mathrm{um}$

$6.8 \mathrm{Msz}$

N $20 \mathrm{~s}$

46. $40 \mathrm{um}$

$\begin{array}{lll}\text { EPCP } & 10 \quad 26.00\end{array}$

esP 1031.50

is 1917.40

ePS 1954.00

$\begin{array}{lllll}71.85 & 129 & \text { iPd } 10 & 08.90 & -0.6\end{array}$ 
$18 d \quad 11 h$

\begin{tabular}{|c|c|c|c|c|c|c|c|c|c|c|c|c|}
\hline CER & 73.07 & 216 & $i^{i P c}$ & $\begin{array}{l}10 \\
10\end{array}$ & $\begin{array}{l}18.40 \\
28.50\end{array}$ & 1.9 & $\sum_{\text {I MAW }}$ & $\begin{array}{l}95.06 \\
1.1 \mathrm{~s}\end{array}$ & $\begin{array}{r}180 \\
71\end{array}$ & $\begin{array}{l}\mathrm{iPc} \\
1.00 \mathrm{~nm}\end{array}$ & 12 & 10.20 \\
\hline TUH & 73.10 & 217 & iPd & 10 & 17.00 & 0.4 & I ADE & 95.52 & 127 & $e^{P}$ & 12 & 11.00 \\
\hline & & & i & 10 & 26.00 & & $i$ & $1.0 \mathrm{~s}$ & 84 & $4.00 \mathrm{~nm}$ & & 6 \\
\hline MBO & 73.85 & 278 & ip & 10 & 23.00 & 1.7 & i STK & 96.12 & 123 & eP & 12 & 12.00 \\
\hline & & & $\mathrm{i}$ & 10 & 40.00 & & I BNH & 96.34 & 328 & eP & 12 & 15.80 \\
\hline & & & i & 10 & 45.00 & & i & & & ePP & 12 & 24.50 \\
\hline & & & $\mathrm{i}$ & 19 & 48.20 & & I MNT & 96.70 & 330 & $i P C_{C}$ & 12 & 17.00 \\
\hline MBL & 74.05 & 125 & $i P c$ & 10 & 21.10 & -1.2 & 1 & $1.0 \mathrm{~s}$ & 64 & $4.00 \mathrm{~nm}$ & & 6 \\
\hline $1 \mathrm{LT}$ & 74.64 & 20 & iPc & 10 & 25.00 & -0.1 & $I F F C$ & 96.72 & 351 & eP & 12 & 16.00 \\
\hline & $1.3 \mathrm{~s}$ & 1400 & $0.00 \mathrm{~nm}$ & & & $6.8 \mathrm{mb}$ & i & $1.0 \mathrm{~s}$ & 416 & $6.00 \mathrm{~nm}$ & & \\
\hline & $18 \mathrm{~s}$ & 38 & $3.00 \mathrm{um}$ & & & $6.7 \mathrm{Msz}$ & I MIR & 96.90 & 168 & ip & 12 & 17.00 \\
\hline & $22 \mathrm{~s}$ & 32 & $2.00 \mathrm{um}$ & & & & i & $2.0 \mathrm{~s}$ & 10 & $0.00 \mathrm{~nm}$ & & 5 \\
\hline & $22 \mathrm{~s}$ & 38 & $3.40 u m$ & & & & 1 & & & epP & 12 & 37.00 \\
\hline & & & IPCP & 10 & 42.00 & & i & & & esP & 12 & 45.00 \\
\hline & & & isP & 10 & 52.00 & & i & & & iPP & 16 & 12.00 \\
\hline & & & iPPP & 15 & 00.00 & & i & & & iSKS & 22 & 52.00 \\
\hline & & & is & 19 & 54.00 & & 1 & & & $\mathrm{i}$ & 30 & 18.00 \\
\hline & & & iSP & 20 & 30.00 & & I OTT & 97.69 & 331 & eP & 12 & 25.00 \\
\hline & & & iPPS & 20 & 52.00 & & 1 & $0.5 \mathrm{~s}$ & 16 & $5.00 \mathrm{~nm}$ & & \\
\hline & & & iss & 24 & 45.00 & & I RSNY & 97.85 & 330 & eP & 12 & 22.30 \\
\hline MBC & 76.25 & 0 & iPc & 10 & 33.80 & -0.3 & I PTN & 98.03 & 331 & $e(P)$ & 12 & 22.70 \\
\hline & $0.8 \mathrm{~s}$ & 678 & $3.00 \mathrm{~nm}$ & & & $6.7 \mathrm{mb}$ & $i$ & & & $i p p$ & 12 & 32.50 \\
\hline & & & PP & 11 & 01.50 & $108 \mathrm{kmx}$ & I APH & 98.39 & 330 & $e P$ & 12 & 24.00 \\
\hline MEK & 6.60 & 130 & iPc & 10 & 35.40 & -1.5 & 1 & & & epP & 12 & 34.00 \\
\hline & $0.6 \mathrm{~s}$ & 30 & $.00 \mathrm{~nm}$ & & & $5.5 \mathrm{mb} \times$ & 1 & & & ePP & 16 & 19.00 \\
\hline GUMO & 77.53 & 81 & $e(P)$ & 10 & 42.00 & -0.2 & I RSON & 98.86 & 345 & $P$ & 12 & 26.50 \\
\hline & & & $e(S)$ & 20 & 15.80 & & I EDM & 99.28 & 357 & ePc & 12 & 28.00 \\
\hline$B A L$ & 78.03 & 134 & iPc & 10 & 43.10 & -1.5 & 1 & $1.2 \mathrm{~s}$ & 298 & $8.00 \mathrm{~nm}$ & & \\
\hline MTN & 78.09 & 111 & eP & 10 & 44.00 & -1.2 & i LHC & 99.94 & 341 & ep & 12 & 31.50 \\
\hline MUN & 78.64 & 135 & iPd & 10 & 46.10 & -1.9 & i & $1.2 \mathrm{~s}$ & 156 & $5.00 \mathrm{~nm}$ & & 6 \\
\hline NWAO & 79.92 & 135 & ¿Pd & 10 & 53.80 & -1.1 & 1100 & 101.58 & 126 & ePdiff & $\uparrow 12$ & 39.00 \\
\hline & $0.7 \mathrm{~s}$ & 125 & $5.00 \mathrm{~nm}$ & & & $6.0 \mathrm{mb}$ & 1 YOU & 102.28 & 122 & iPdiff & $\uparrow 12$ & 49.00 \\
\hline SMY & 80.49 & 35 & eP & 10 & 59.00 & 1.4 & I CAN & 103.18 & 123 & iPdiff & $f 12$ & 46.50 \\
\hline ANM & 30.87 & 19 & iPc & 11 & 00.30 & 0.9 & I ITR & 103.26 & 267 & ePdiff & +12 & 38.20 \\
\hline FRB & 10.89 & 340 & iPc & 10 & 59.50 & 0.0 & 1 & & & e & 12 & 50.20 \\
\hline JAY & 81.14 & 98 & ePd & 11 & 01.00 & -0.8 & I PNT & 103.27 & 1 & ePdiff & $f 12$ & 45.00 \\
\hline & $.9 \mathrm{~s}$ & 2190 & $9.00 \mathrm{~nm}$ & & & $6.8 \mathrm{mb}$ & I WAM & 103.45 & 124 & ePdiff & $\uparrow 12$ & 52.00 \\
\hline$K L G$ & 81.24 & 131 & iPd & 11 & 00.90 & -1.0 & I PGC & 103.79 & 4 & ePdiff & $\uparrow 12$ & 52.00 \\
\hline & $1.2 \mathrm{~s}$ & 420 & $0.00 \mathrm{~nm}$ & & & $6.3 \mathrm{mb}$ & I NEW & 104.34 & 359 & ePdiff & $\$ 12$ & 51.00 \\
\hline WBN & 81.97 & 125 & ¿Pd & 11 & 05.40 & -0.4 & i & & & $\mathrm{e}$ & 17 & 07.00 \\
\hline IMA & 82.57 & 14 & eP & 11 & 08.70 & 0.2 & I GMW & 104.93 & 3 & e(Pdif & $\$ 12$ & 55.00 \\
\hline INK & 83.56 & 6 & ePc & 11 & 13.50 & 0.2 & I SOB 1 & 105.64 & 268 & ePKP & 17 & 08.10 \\
\hline & 1. & 510 & $0.00 \mathrm{~nm}$ & & & $6.5 \mathrm{mb}$ & 1 & & & e & 17 & 21.90 \\
\hline & & & PP & 11 & 32.00 & $67 \mathrm{kmX}$ & I LON & 105.77 & 3 & ePdiff & +12 & 58.00 \\
\hline NWW & 4.23 & 97 & ep & 11 & 18.50 & 0.9 & I BLA & 106.40 & 330 & elPdif & $f 12$ & 50.00 \\
\hline WRA. & 4.46 & 116 & Pc & 11 & 18.80 & 0.2 & I LRM & 106.61 & 356 & ePdiff & $\uparrow 13$ & 01.40 \\
\hline & $.9 \mathrm{~s}$ & 305 & $5.00 \mathrm{~nm}$ & & & $6.4 \mathrm{mb}$ & I RSSD & 107.36 & 350 & elPdif & $\uparrow 13$ & 03.00 \\
\hline$T T A$ & 34.69 & 16 & iPd & 11 & 19.80 & 0.6 & I COR & 107.85 & 4 & iPdiff & $\uparrow 13$ & 22.00 \\
\hline $\mathrm{COL}$ & 84.78 & 12 & iPc & 11 & 20.00 & 0.4 & I BDW & 109.40 & 353 & e(Pdif & 113 & 09.00 \\
\hline & $1.3 \mathrm{~s}$ & 903 & $3.00 \mathrm{~nm}$ & & & $5.8 \mathrm{mb}$ & I FVM & 109.71 & 337 & e(Pdif & $f 13$ & 10.00 \\
\hline & $23 \mathrm{~s}$ & 43 & $3.90 \mathrm{um}$ & & & $6.8 \mathrm{MszX}$ & I & $20 \mathrm{~s}$ & & $6.39 u m$ & & 6 \\
\hline & & & es & 21 & 37.00 & & I FHC & 111.57 & 5 & ePKP & 17 & 23.00 \\
\hline FBA & 84.78 & 12 & $i P c$ & 11 & 20.00 & 04 & 1 & & & ePP & 18 & 03.00 \\
\hline STJ & 84.88 & 322 & eP & 11 & 21.50 & 1.2 & I GLD & 111.81 & 349 & e (PKP) & ) 17 & 38.00 \\
\hline ADK & 85.51 & 32 & eP & 11 & 21.90 & -1.5 & 1 & $20 \mathrm{~s}$ & 20 & 0. $00 \mathrm{um}$ & & 6 \\
\hline A SPA & 86.09 & 119 & $\mathrm{iPd}$ & 11 & 26.60 & -0.1 & 1 WDC & 111.89 & 4 & ePKP & 17 & 25.00 \\
\hline SVW & 86.32 & 17 & eP & 11 & 28.40 & 10 & 1 & & & ePP & 18 & 05.00 \\
\hline $\mathrm{SCH}$ & 86.79 & 333 & ePd & 11 & 30.30 & 0.6 & 1 & & & ePKKP & 28 & 14.00 \\
\hline & $.1 \mathrm{~s}$ & 474 & $.00 \mathrm{~nm}$ & & & $6.6 \mathrm{mb}$ & I BMN & 112.17 & 359 & ePdiff & $f 13$ & 33.80 \\
\hline $10 \mathrm{M}$ & 86.82 & 94 & eP & 11 & 30.00 & -0.5 & 1 & & & eSKS & 25 & 07.50 \\
\hline ADG & 86.89 & 98 & eP & 11 & 28.00 & -2.8 & 1 & & & ePKKP & 28 & 16.40 \\
\hline PMR & 87.47 & 14 & iPc & 11 & 33.20 & 0.4 & $M I N$ & 112.17 & 3 & PKP & 17 & 29.00 \\
\hline & $20 \mathrm{~s}$ & 23 & $3.50 \mathrm{um}$ & & & $5.6 \mathrm{Msz}$ & I SJG & 112.22 & 306 & ePKP & 17 & 42.00 \\
\hline TOA & 87.63 & 13 & eP & 11 & 35.20 & 1.5 & $i$ & $22 \mathrm{~s}$ & & $7.41 \mathrm{um}$ & & 6 \\
\hline NKI & 88.25 & 28 & eP & 11 & 36.00 & -0.7 & NOU & 112.27 & 104 & iPKPC & 17 & 13.00 \\
\hline LAT & 88.61 & 98 & eP & 11 & 39.00 & -0.1 & RLO & 112.86 & 340 & ePdiff & $\{13$ & 40.00 \\
\hline SDN & 89.59 & 23 & eP & 11 & 43.50 & 0.4 & I ORV & 112.96 & 3 & elPdif & $\{13$ & 34.00 \\
\hline KDC & 90.03 & 18 & IPd & 11 & 46.20 & 1.1 & I EUR & 113.09 & 358 & i (Pdif & $\{13$ & 39.00 \\
\hline PMG & 0.03 & 101 & eP- & 19 & 42.00 & $-3.7 x$ & 1 & $0.2 \mathrm{~s}$ & 14 & $4.00 \mathrm{~nm}$ & & \\
\hline YKA & 90.04 & 358 & eP & 11 & 46.10 & 1.1 & WCN & 113.27 & 2 & ePKP & 17 & 25.80 \\
\hline$Y K C$ & 90.05 & 358 & ePc & 11 & 45.50 & 0.5 & TUL & 113.32 & 341 & ePdiff & $f 13$ & 38.40 \\
\hline & $1.1 \mathrm{~s}$ & 1110 & $.00 \mathrm{~nm}$ & & 7 & $7.1 \mathrm{mb}$ & 1 & $0.8 \mathrm{~s}$ & 15 & $5.40 \mathrm{~nm}$ & & \\
\hline KVG & 90.07 & 93 & eP & 11 & 45.00 & -1.0 & I & $22 \mathrm{~s}$ & & $9.60 \mathrm{um}$ & & \\
\hline PCA & 90.58 & 11 & eP & 11 & 49.20 & 1.5 & 1 & & & e & 24 & 00.00 \\
\hline LMG & 90.67 & 100 & $e P$ & 11 & 49.00 & 0.1 & 1 & & & e & 27 & 41.00 \\
\hline$F C C$ & 91.56 & 348 & iPc & 11 & 52.60 & 0.5 & 1 & & & e & 45 & 00.00 \\
\hline & $1.2 \mathrm{~s}$ & 1120 & $.00 \mathrm{~nm}$ & & & $.2 \mathrm{mb}$ & MNA & 114.17 & 0 & ePKP & 17 & 26.20 \\
\hline RAB & 92.03 & 94 & iP- & 11 & 44.70 & $-10.3 x$ & 1 & & & ePKKP & 28 & 10.50 \\
\hline CBM & 93.13 & 328 & eP & 12 & 01.10 & 1.6 & I JAS & 114.61 & 2 & ePdiff & $\uparrow 13$ & 41.50 \\
\hline CTA & 94.24 & 110 & iPd- & 12 & 04.40 & -0.6 & I MHC & 115.16 & 3 & ePKP & 17 & 36.00 \\
\hline & 1 & 275 & $.00 \mathrm{~nm}$ & & & $5.5 \mathrm{mb}$ & 1 & & & EPP & 18 & 25.00 \\
\hline & & & iSKS & 22 & 28.00 & & RMU & 115.19 & 354 & e(Pdif & $\uparrow 13$ & 51.20 \\
\hline CTAO & 94.24 & 110 & eP & 12 & 04.60 & -0.4 & 1 SLD & 115.45 & 3 & ePKP & 17 & 30.00 \\
\hline & & & pl & 12 & 13 & $26 \mathrm{kmX}$ & $|F R|$ & 115.58 & 2 & ePKP & 17 & 36.50 \\
\hline $51 T$ & 94.26 & 9 & eP & 12 & 06.00 & 1.4 & PR I & 116.40 & 2 & EPKP & 17 & 42.00 \\
\hline$M I M$ & 94.81 & 328 & $e(P)$ & 12 & 07.20 & -0.1 & 1 & & & ePP & 18 & 37.00 \\
\hline
\end{tabular}


PPT $\quad 149.19 \quad 78 \quad$ iPKP $18 \quad 34.00$

\begin{tabular}{|c|c|c|c|c|c|}
\hline & $1.2 \mathrm{~s}$ & $\begin{array}{l}18 \\
185\end{array}$ & $.00 \mathrm{~nm}$ & (1) & - \\
\hline & & & $i$ & 18 & 38.30 \\
\hline PAE & $\begin{array}{c}149.22 \\
1.25\end{array}$ & $\begin{array}{l}78 \\
145\end{array}$ & $\begin{array}{l}\text { iPKP } \\
.00 \mathrm{~nm}\end{array}$ & 18 & 34.00 \\
\hline & & & $i$ & 18 & 38.20 \\
\hline PMO & 149.46 & 72 & iPKP & 18 & 33.30 \\
\hline & & & $\mathrm{i}$ & 18 & 38.40 \\
\hline TPT & $\begin{array}{c}149.68 \\
1.2 \mathrm{~s}\end{array}$ & $\begin{array}{l}71 \\
90\end{array}$ & $\begin{array}{l}\text { ¿PKP } \\
.00 \mathrm{~nm}\end{array}$ & 18 & 34.20 \\
\hline & & & i & 18 & 38.80 \\
\hline VAH & 149.80 & 72 & iPKP & 18 & 34.20 \\
\hline & & & & 18 & 38.80 \\
\hline RUV & $\begin{array}{c}149.98 \\
1.2 \mathrm{~s}\end{array}$ & $\begin{array}{l}72 \\
30\end{array}$ & $\begin{array}{l}\text { iPKP } \\
.00 \mathrm{~nm}\end{array}$ & 18 & 35.00 \\
\hline
\end{tabular}

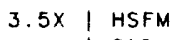

$3.5 \times\left\{\begin{array}{l}\text { HSFM } \\ \text { SLD } \\ \text { HKRM }\end{array}\right.$

\begin{tabular}{l|l}
$3.5 \times$ & $\mathrm{PCL}$ \\
$\mathrm{HFHM}$
\end{tabular} SFL

$2.4 \times$ HJGM

BPFM
HBTM

\begin{tabular}{l|l}
$3.0 \times$ & HBTM \\
OCR \\
HCPM
\end{tabular}

$2.8 \times \mid$ ANZ

O OIL

$3.3 \times 1$ CSR

S.D. $=1.1$ on $420^{18}$ of 48.40 obs

8. MAY 02, $1983 \quad 23 \mathrm{~h} 42 \mathrm{~m} 37.76 \mathrm{~s}$ $36.219 \mathrm{~N} \quad 120.317 \mathrm{~W}$ DEPTH $=10.2 \mathrm{~km}$ (geophysicist)

CENTRAL CALIFORNIA KCSP. Forty-five people injured ond estimated 31 million dollors $C B O$ domoge in the Coolingo oreo. EUC Moximum intensity VIII. Some damage of Avenol and other surrounding communities. Felt from Los Angeles to Socramento ond from Son Francisco to Reno.

\begin{tabular}{|c|c|c|c|}
\hline PARM & 0.04 & 326 & i $P_{C}$ \\
\hline PCRM & 0.16 & 217 & iPd \\
\hline PRCM & 0.25 & 279 & $\mathrm{iPd}$ \\
\hline CTM & 0.29 & 183 & $\mathrm{iPd}$ \\
\hline PRI & 0.29 & 255 & $i P d$ \\
\hline PKF & 0.35 & 193 & iPd \\
\hline PTV & 0.35 & 251 & $i P d$ \\
\hline PHGM & 0.37 & 202 & iPd \\
\hline MOP & 0.39 & 270 & $i P d$ \\
\hline GHC & 0.39 & 184 & $\mathrm{iPd}$ \\
\hline PHAM & 0.39 & 190 & $\mathrm{iPd}$ \\
\hline PIVM & 0.43 & 224 & iP \\
\hline WKR & 0.43 & 201 & $i P d$ \\
\hline HVC & 0.43 & 291 & iPd \\
\hline PMRM & 0.44 & 171 & $i P_{C}$ \\
\hline PAGM & 0.49 & 174 & $i P_{c}$ \\
\hline PMCM & 0.50 & 185 & $i P_{c}$ \\
\hline PSAM & 0.50 & 247 & IPd \\
\hline BTW & 0.50 & 281 & ePc \\
\hline PTRM & 0.57 & 171 & $i \mathrm{Pc}$ \\
\hline BMSM & 0.58 & 319 & $i \mathrm{PC}$ \\
\hline LRC & 0.59 & 273 & iPc \\
\hline LRV & 0.60 & 290 & $i \mathrm{Pc}$ \\
\hline PSHM & 0.63 & 187 & iPc \\
\hline LLA & 0.64 & 308 & $i P_{c}$ \\
\hline PANM & 0.65 & 228 & $i P_{C}$ \\
\hline SBT & 0.68 & 296 & $i P_{c}$ \\
\hline PJLM & 0.69 & 259 & $i P C$ \\
\hline BPIM & 0.74 & 292 & iPc \\
\hline PBYM & 0.74 & 237 & $i P_{C}$ \\
\hline EMT & 0.77 & 305 & iPc \\
\hline SHG & 0.78 & 285 & $i P c$ \\
\hline BVL & 0.79 & 297 & $i P C$ \\
\hline PMGM & 0.80 & 192 & $i P_{c}$ \\
\hline EKH & 0.82 & 303 & $i P_{c}$ \\
\hline BSCM & 0.85 & 299 & IPC \\
\hline PRS & 0.86 & 278 & IPC \\
\hline PCGN & 0.86 & 204 & iPC \\
\hline PBRM & 0.87 & 220 & $i P_{C}$ \\
\hline BLRM & 0.89 & 300 & $i P_{C}$ \\
\hline PAPI & 0.90 & 250 & $i P_{c}$ \\
\hline BJOM & 0.90 & 297 & iPc \\
\hline FRI & 0.91 & 32 & IPd \\
\hline $\mathrm{JHC}$ & 0.93 & 291 & iPc \\
\hline$B C G$ & 0.96 & 301 & iPc \\
\hline HJSM & 0.99 & 307 & IPC \\
\hline BHSM & 1.00 & 278 & $i P_{c}$ \\
\hline BVYM & 1.03 & 301 & $\mathrm{IPC}$ \\
\hline LTR & 1.04 & 310 & iPc \\
\hline PSEM & 1.04 & 201 & $i P_{c}$ \\
\hline SAO & 1.06 & 301 & IPC \\
\hline HSL & 1.06 & 324 & $i P_{C}$ \\
\hline BSF & 1.07 & 295 & iPc \\
\hline PKF & 08 & 306 & $\therefore$ \\
\hline & 1.12 & 288 & IPC \\
\hline
\end{tabular}

4240.83

$42 \quad 42.23$

4244.01

$42 \quad 44.98$

4245.40

$42 \quad 46.19$

4246.05

4246.65

$42 \quad 46.87$

4246.70

4246.97

4247.82

$42 \quad 47.85$

$42 \quad 47.45$

4247.81

$42 \quad 48.55$

4248.81

$42 \quad 48.59$

$\begin{array}{ll}42 & 48.57 \\ 42 & 49.91\end{array}$

4250.32

$42 \quad 49.98$

4250.51

4251.15

4251.00

$\begin{array}{ll}42 & 51.44 \\ 42 & 51.72\end{array}$

4251.72
4252.00

4252.46

$42 \quad 52.86$

4253.51

4253.14

4253.66

$\begin{array}{ll}42 & 53.66 \\ 42 & 54.22\end{array}$

4254.94

4254.80

4255.02

4255.35

$42 \quad 55.59$

$42 \quad 55.59$

4255.12

$\begin{array}{ll}42 & 54.50 \\ 42 & 55.71\end{array}$

4255.71

4256.73

$\begin{array}{ll}42 & 56.77 \\ 42 & 57.07\end{array}$

4257.49

4257.57

$\begin{array}{ll}42 & 57.44 \\ 42 & 57.80\end{array}$

4256.91

4257.91

4259.00
4258.66

\section{0}

0.8 SYP

0.9 JAS

1.1 CWC

1.5 SBC

.3 TIN

1.1 VPEM

1.2

1.0 NWRM

1.2 SDW

1.3 RMT

1.2 WDC

0.8 I LGBM

1.1 FHC

0.8 | GLA

$1.0 \mid$ BKU

0.7 MSU

0.6 I DUG

0.6 MOUT

0.3 BEI

0.6 VGB

0.7 PNO

\begin{tabular}{l|l}
0.7 & TMI \\
0.7 & SHW
\end{tabular}

0.5 IMW

0.2 I LON

0.6 | LRM

0.8 ALQ

0.2 ANMO

0.5 GMW

0.3 NEW

0.6
0.8

$0.5 \quad 50 L$

0.7 GLD

0.8 LHD

$0.5 \mid \mathrm{MCW}$

0.1 PGC

0.7
0.3

0.7 PNT

0.2 RSSD

0.2 PHC

0.1

0.1 SES

-0.8 | ACO

0.9 EDM
0.6 BDW

0.3 RXF
$1.12302 \mathrm{iPC}$ 1. $12320 \mathrm{iP}$

$1.14317 \mathrm{PPC}$

$1.14306 \quad \mathrm{Pc}$

$1.16311 \mathrm{iPC}$

$1.17300 \mathrm{iPC}$

$1.18271 \mathrm{iPc}$

$1.18303 \mathrm{ePc}$

$1.19306 \mathrm{IPC}$

$1.20325 \mathrm{eP}$

$1.22303 \mathrm{iPC}$

$1.23300 \mathrm{iPC}$

$1.24311 \mathrm{iPC}$

$1.26306 \mathrm{iPc}$

$1.26314 \mathrm{iPC}$

$1.29304 \mathrm{iPC}$

1.31313 iPC

$1.33304 \mathrm{iPC}$

$1.34307 \mathrm{iPc}$

$1.35287 \mathrm{iPC}$

$1.39325 \mathrm{ePc}$

$1.41307 \quad \mathrm{PPC}$

$1.41312 \mathrm{iPc}$

$1.42309 \mathrm{IPC}$

$1.46305 \mathrm{iPc}$

$1.49319 \mathrm{iPc}$

$1.50314 \quad \mathrm{PC}$

$1.54317 \quad \mathrm{PCC}$

$1.58301 \mathrm{iPc}$

$1.58 \quad 105 \quad \mathrm{PPd}$

$1.60110 \mathrm{eP}$
$\mathrm{eS}$

$\begin{array}{llll}1.71 & 171 & \mathrm{eS} \\ \mathrm{iP}\end{array}$

$1.73357 \mathrm{ePC}$

$1.8282 \mathrm{iP}$

$\begin{array}{llll}1.84 & 164 & \text { iP }\end{array}$

$\begin{array}{lll}1.88 & 63 & \mathrm{iP} \\ 2.04 & 97 & \mathrm{iPd}\end{array}$

$2.26318 \mathrm{ePC}$

3.03318 eS

$3.10120 \mathrm{iPC}$

4.13334 iPd

$4.69339 \mathrm{ePd}$

$5.32345 \mathrm{ePd}$

$5.40329 \mathrm{ePd}$

$5.52 \quad 123 \mathrm{iPc}$

$6.17 \quad 66 \mathrm{ePd}$

$6.88 \quad 68$ eP

$7.1254 \mathrm{eP}$

8. $25 \quad 51 \mathrm{ePn}$

8. $8646 \mathrm{ePn}$

$9.29358 \mathrm{eP}$

$9.467 \mathrm{ePnc}$

0.07352 e(P)

$10.50 \quad 40 \mathrm{ePd}$

$10.57 \quad 48 \mathrm{ePd}$

$10.58354 \mathrm{ePc}$

$11.28 \quad 29 \mathrm{ePd}$

$11.36 \quad 92 \mathrm{ePc}$

$11.36 \quad 92 \mathrm{eP}$

$11.47352 \mathrm{eP}$

$12.27 \quad 10 \mathrm{eP}$

$12.3069 \mathrm{ePd}$

$12.42 \quad 69 \mathrm{eP}$

$12.53 \quad 15 \mathrm{iPC}$

$12.59352 \mathrm{eP}$

$12.64350 \mathrm{eP}$

$12.77 \quad 15 \mathrm{iPc}$

$13.10 \quad$ eS

$13.20 \quad 15 \mathrm{iPC}$

$14.72 \quad 53 \mathrm{eS}$

$15.36343 \mathrm{eP}$

$1.4 \mathrm{~s} \quad 219.00 \mathrm{~nm}$

$15.68 \quad 22 \mathrm{eP}$

$\begin{array}{ll}17.04 & 82 \mathrm{eP} \\ 1.0 \mathrm{~s} & 1220.00 \mathrm{~nm}\end{array}$

$\begin{array}{ll}1.0 \mathrm{~s} & 1220.00 \mathrm{~nm} \\ 17.69 & 14 \mathrm{eP}\end{array}$

$17.82 \quad 86 \mathrm{eP}$
4259.04

4259.22

4259.06

4259.50

4259.43

4259.10

4259.23

4259.68

4259.86
4259.40

$42 \quad 59.94$

4300.46

4300.54

4300.82

4300.88

4301.31

$43 \quad 01.54$

4301.37

$\begin{array}{lll}43 & 01.75 \\ 43 & 02.21 & -1\end{array}$

$\begin{array}{lll}43 & 02.21\end{array}$

4302.41

4302.68

4302.66

$\begin{array}{lll}43 & 03.23\end{array}$

$\begin{array}{lll}43 & 03.93\end{array}$

$\begin{array}{lll}43 & 04.21\end{array}$

4304.70

$\begin{array}{ll}43 & 04.80\end{array}$

4305.40

4305.80

4403.90

$\begin{array}{lll}43 & 07.70\end{array}$

$43 \quad 07.50$

4309.30

$\begin{array}{ll}43 & 09.70\end{array}$

4310.60

4312.70

4314.40

$43 \quad 42.00$

4325.00

4325.90

$\begin{array}{ll}43 & 41.60 \\ 43 & 48.40\end{array}$

4403.00

4400.60

4358.50

4410.50

4420.50

4424.80
4441.80

4441.80
4448.08

4450.70

4457.50

4459.30

4500.60

4508.50

$45 \quad 13.50$

4513.30

$45 \quad 14.00$

4523.00

4524.00

4525.00

4523.30

4536.00

4540.50

4907.00

4537.50

$45 \quad 39.50$

4541.40
4918.70

4541.40

4541.68

4544.70

4912.70

4548.00

$45 \quad 49.40$

4923.30

$\begin{array}{lll}46 & 07.40 & -0.6\end{array}$ $46 \quad 18.50$

$4621.00^{5} 3 \mathrm{mb}$ $\begin{array}{llll}46 & 38 & 50 & 0.9\end{array}$

$4644.50^{6.0 m b}$

$\begin{array}{rrr}4644.50 & -1.2 \\ 4650.00 & 2.6\end{array}$

I ATO

\begin{tabular}{l|l}
\hline JCT \\
OCO
\end{tabular}

.4 I PCO 


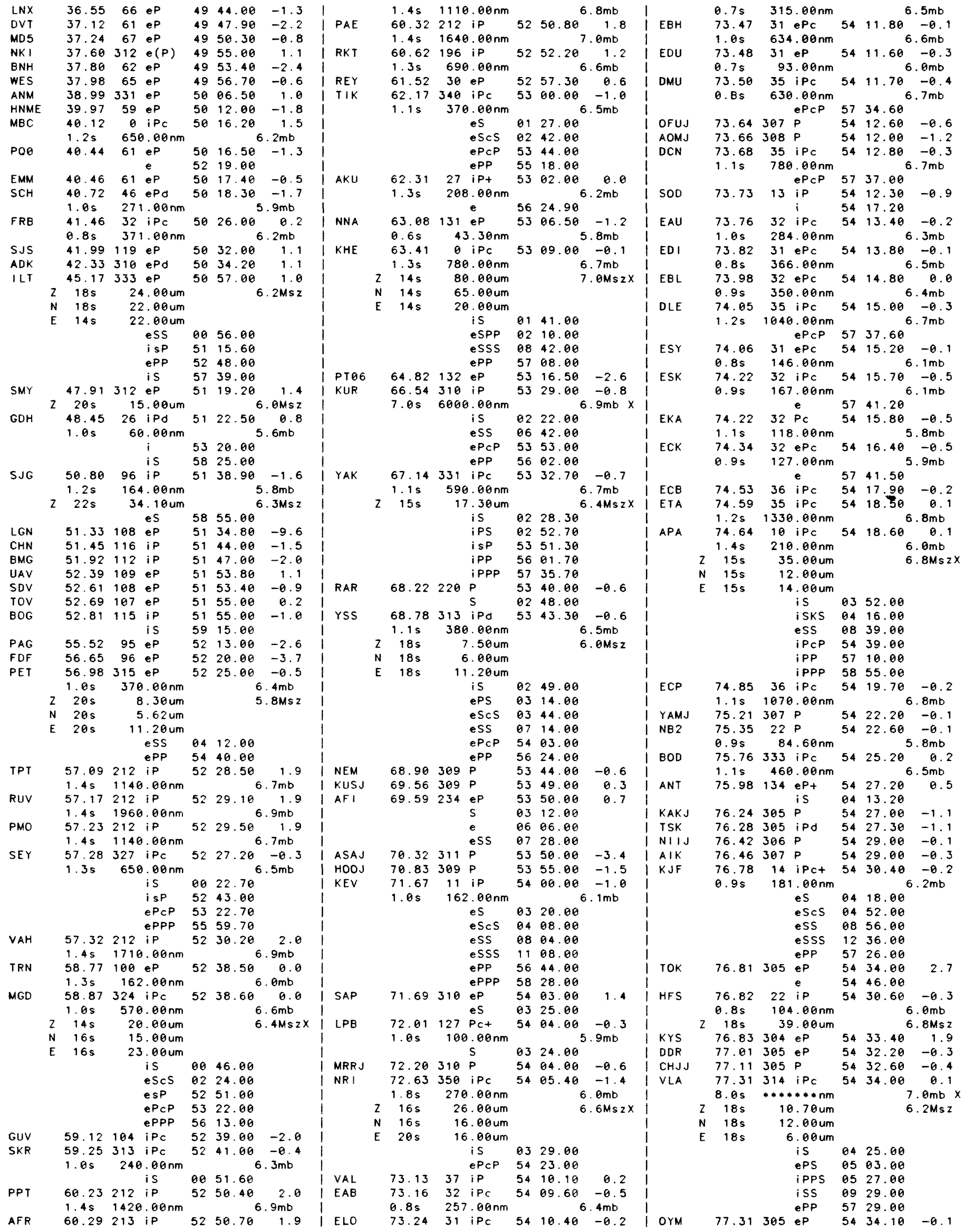




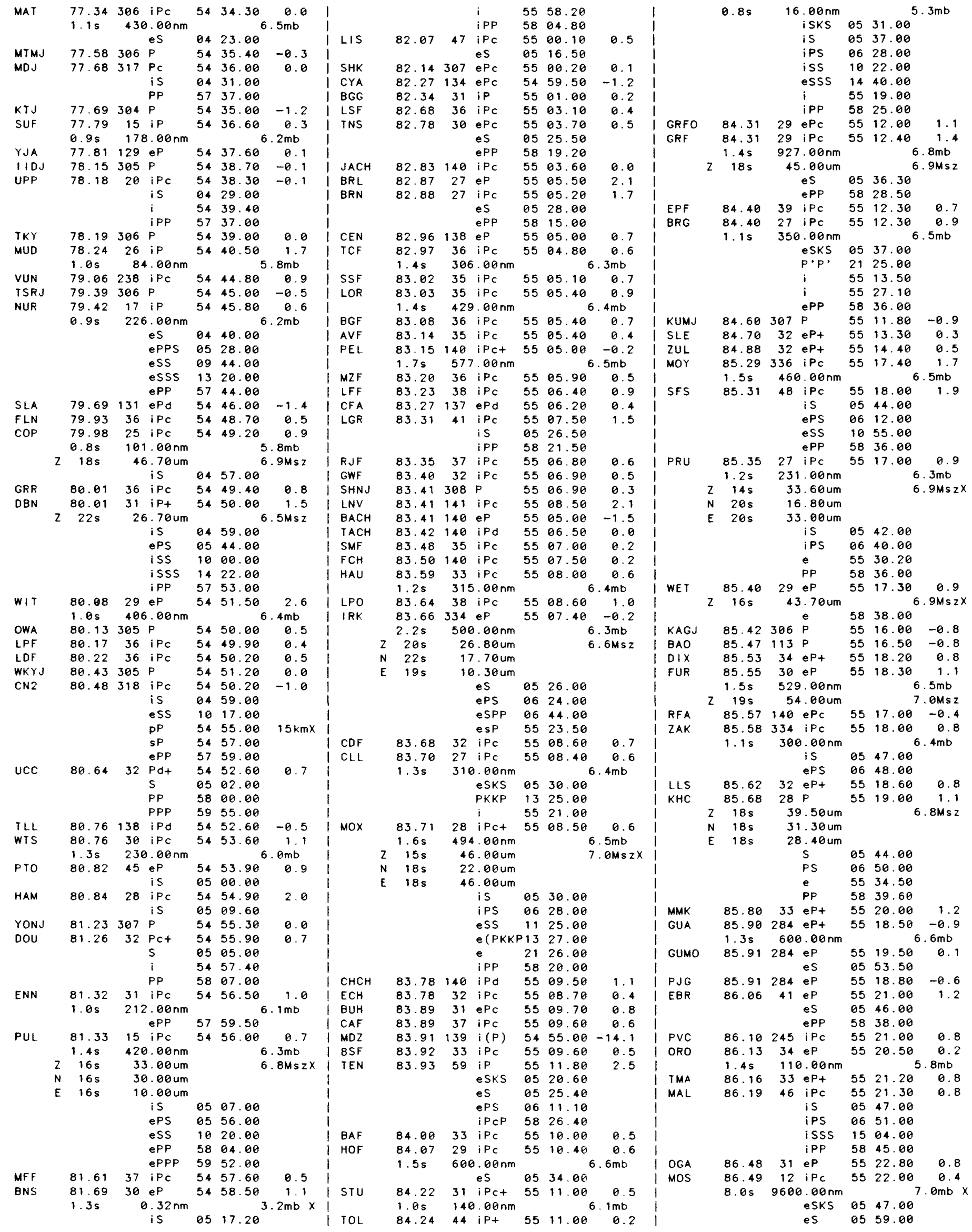




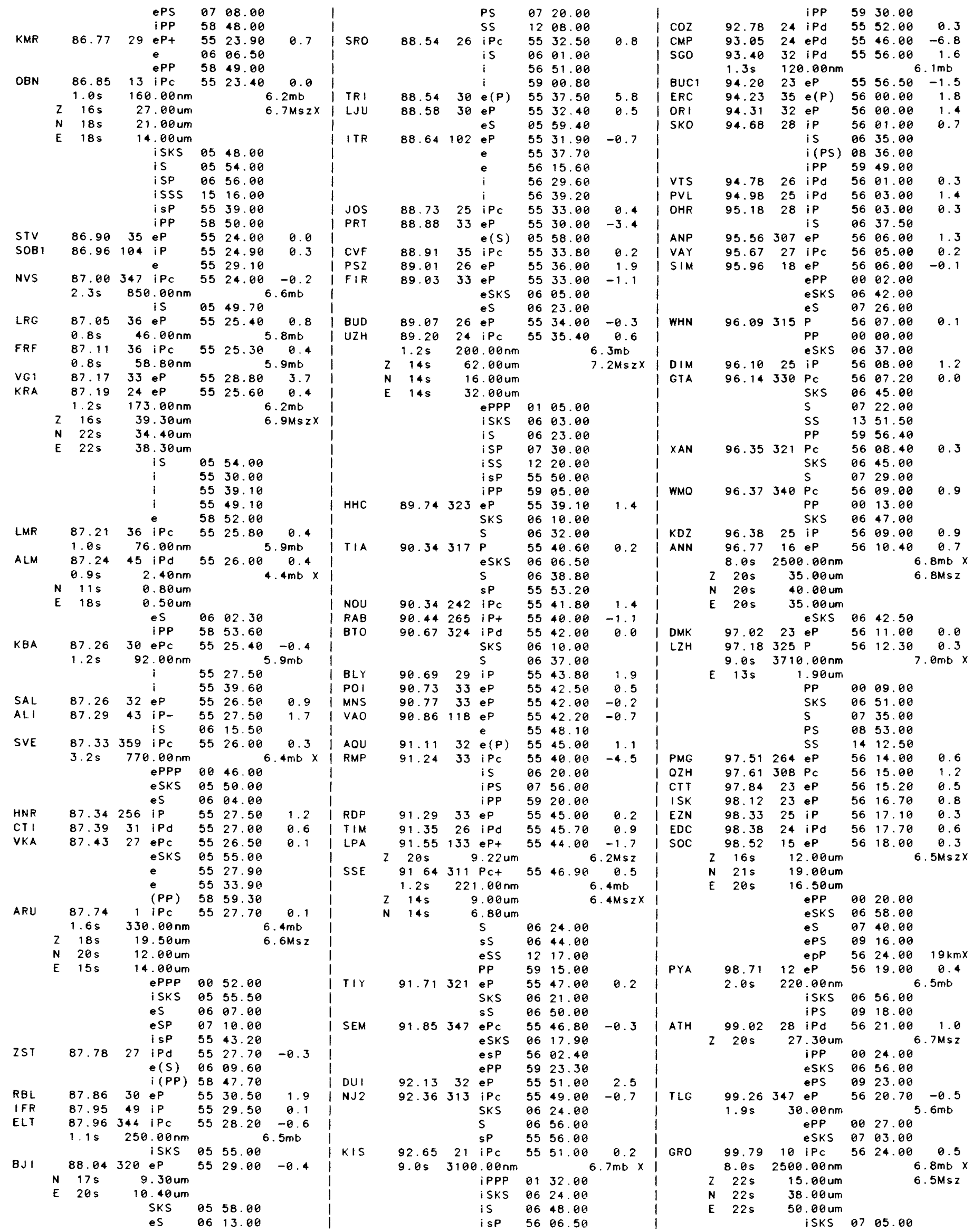


PRZ

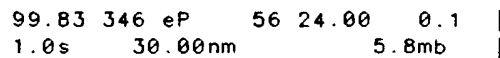
EPP $00 \quad 33.00$ EPPP $02 \quad 45.00$ ESKS $07 \quad 04.00$ ePS 0927.00 eSS 1452.00

FRU $\quad 100.15 \quad 349$ ePdiff56 $25.20 \quad 0.2$ $2.5 \mathrm{~s} \quad 240.00 \mathrm{~nm}$ E $\quad 22 \mathrm{~s} \quad 19.80 \mathrm{um}$ EPP $\quad 00 \quad 34.00$ EPPP $02 \quad 42.00$ eSKS 0704.00 eSKS 0800.00 ESP 0922.00

ANTO $100.34 \quad 21$ Pdiff $56 \quad 27.00$

MAK $100.35 \quad 9$ ePdiff 5629.00 $8.0 \mathrm{~s} 1500.00 \mathrm{~nm}$

EPP $\quad 00 \quad 34.00$ iSKS 0708.50 ESP 0931.00

BKR $101.02 \quad 12$ ePdiff56 $27.00 \quad-2.0$ $1.4 \mathrm{~s} \quad 30.00 \mathrm{~nm} \quad 5.7 \mathrm{mb}$ EPP $00 \quad 40.00$ iPS 0942.00

MTA $\begin{array}{lllll}101.27 & 11 \text { ePdiff56 } 31.60 & 1.8\end{array}$ $\mathrm{N} 20 \mathrm{~s} \quad 8.00 \mathrm{um}$ E $20 \mathrm{~s} \quad 13.00 \mathrm{um}$ EPP $00 \quad 40.60$ EPPP 0252.60 ISKS $07 \quad 11.60$ ESP 0941.60

NRN $\quad 101.29348$ ePdiff56 30.20 ESKS $07 \quad 10.00$

$\begin{array}{lrrlr}\text { CD2 } & 101.46 & 322 & \text { Pdiff } 56 & 31.00 \\ \text { LEN } & 102.02 & 12 \text { ePdiff56 } & 34.00\end{array}$ $\begin{array}{ccc}102.02 & 12 \text { ePdiff56 } 34.00 \\ 7.0 \mathrm{~s} & 1400.00 \mathrm{~nm}\end{array}$ $102.23 \quad 311$ Pdiff $56 \quad 35.00$

$\begin{array}{lllll}\text { GZH } & 102.25 & 311 & \text { Pdirf } & \\ \text { BAG } & 102.26 & 301 \text { ePdift56 } & 34.00\end{array}$

IAS $102.33 \quad 353$ ePdit\$56 35.00 $3.0 \mathrm{~s} \quad 280.00 \mathrm{~nm}$

$z \quad 20$

$$
\begin{array}{ccc}
15.40 \text { Um } & & \\
\text { EPP } & 00 & 50.00 \\
\text { ESKS } & 07 & 13.00 \\
\text { ES } & 08 & 21.00
\end{array}
$$$$
\text { EPS } 9958.50
$$

ANR $\quad 102.52 \quad 350$ ePdif $\$ 56 \quad 36.20$ $3.0 \mathrm{~s} \quad 240.00 \mathrm{~nm}$ EPP $00 \quad 56.40$ ESKS $07 \quad 16.20$ eS 0820.60 iPS 1002.20

ERE $\quad 102.70 \quad 12$ ePdiff56 38.00

SHE 102.859 ePdiff56 40.00 iSKS 0720.00

KSH 103.21347 ePdiff56 40.00 SKS $07 \quad 19.00$

GYA $103.53 \quad 318$ Pdiff $56 \quad 41.00$ SKS $07 \quad 17.00$

GRS 103.6611 iPdiff56 43.00 iPP $\quad 00 \quad 59.40$ iPS $\quad 10 \quad 08.00$

SAM $\quad 104.18 \quad 354$ ePdiff56 44.00 $7.0 \mathrm{~s} \quad 1000.00 \mathrm{~nm} 0105.40$ ISKS 0726.00 iPS $10 \quad 15.00$

CTA $\quad 104.26 \quad 255$ iPdiff56 45.00 $00 \quad 01.00$ 0726.00 0852.00 $09 \quad 17.00$ $\begin{array}{lll}10 & 12.00\end{array}$ 1456.00 5934.00

GAR $\quad 104.51351$ ePdiff56 47.40 SKS $07 \quad 26.40$

DAV $104.82 \quad 290$ ePdiff56 46.00

TAB $105.06 \quad 11$ ePdif $\$ 5650.00$

e 0109.00 diff56 50.40 EPP $\quad 0108.50$ ESKS 0722.50 ESP $18 \quad 18.50$

KUL $\quad 105.67 \quad 352$ ePdiff56 49.20 $\begin{array}{lll}\text { EPP } & 01 & 17.20 \\ \text { ESKS } & 07 & 29.50\end{array}$ $6 \mathrm{mb}^{3} \mathrm{x}$

$-0.2$

8.7

0.7
$.4 \mathrm{mb}$

1.3

$-0.31$ $\begin{array}{rlll}\text { eS } & 08 \quad 46.70\end{array}$ $\begin{array}{rrr}105.85 & 350 \text { ePdiff56 } & 52.40 \\ \text { ESKS } 07 & 32.40\end{array}$ ePS 1032.00

106.19 ASH ASH KM I

I MHI

I LSA

MTN 1.1 NDI

I WRA SHI BDT

PCT

ASPA

POO

IPM
BNG

SPA

-0.1 DRV $0.5 \mid$ PSI $.7 \mathrm{mb} \times$ I GBA

PPI \begin{tabular}{r|r}
-1.1 & PPI \\
0.3 & ARO
\end{tabular} $6.4 \mathrm{mb}$ KOD 6Ms I AAE

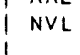

(

1

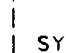

I SYO

| KR I

SUR

$1.6 \quad$ SUR

$\begin{array}{llll}147.81 & 95 & \text { IPKPC } 02 \quad 24.30\end{array}$

$0.6 \mathrm{~s} \quad 200.00 \mathrm{~nm}$

$219 \mathrm{~s} \quad 19.90 \mathrm{um}$

1.2 MTD 148.4460 IPKPC 0225.00 ISKP $05 \quad 49.00$

0.5 BUL 148.4568 iPKPC $02 \quad 27.00$

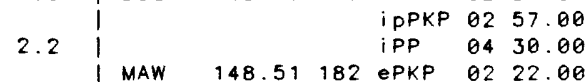

$2.2\left\{\begin{array}{lllll} & \text { IPPKP } & 02 & 57.00 \\ \text { MAW } & 148.51182 & \text { EPK } & 04 & 30.00 \\ & & 14.35 & 02 & 22.00\end{array}\right.$

$1.3 \mathrm{~s} \quad 110.00 \mathrm{~nm}$

$149.54 \quad 83$ iPKPC $02 \quad 24.00$

1.0 swz

$8 \mathrm{mb} \times 1$

KIM

$\begin{array}{lllll}149.75 \quad 86 \quad \text { iPKPC } 02 & 26.70\end{array}$

$1.2 \mathrm{~s} \quad 200.00 \mathrm{~nm}$

$\begin{array}{lllll}\text { KSR } & 150.20 \quad 79 \text { IPKPC } 02 \quad 25.80\end{array}$

BFS $\quad 150.58 \quad 81$ IPKPO $02 \quad 27.00$

$1.0 \mathrm{~s} \quad 600.00 \mathrm{~nm}$

HVD $\quad 151.02 \quad 89$ IPKPC $02 \quad 29.10$

$1.0 \mathrm{~s} \quad 302.00 \mathrm{~nm}$

$i \quad 02 \quad 34.20$

BLF $151.03 \quad 86$ ePKP $02 \quad 30.00$

\begin{tabular}{l|llllll}
2.8 & & & i & 02 & 37.00 \\
PRY & 151.15 & 80 & iPKPC & 02 & 27.00
\end{tabular}

$\begin{array}{lllllll}2.9 & & & & \\ 1 & \text { PRE } & 151.16 & 83 & \text { EPKP } & 02 & 28.50 \\ & 151.17 & 78 & \text { EPKP } & 02 & 29.00\end{array}$

$1.2 \mathrm{~s}-1.04 \mathrm{~nm}$

i $\quad 0234.50$

SLR

$151.23 \quad 78$ iPKPC $02 \quad 28.90$

$0.9 \mathrm{~s} \quad 750.00 \mathrm{~nm}$

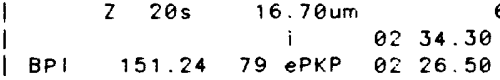

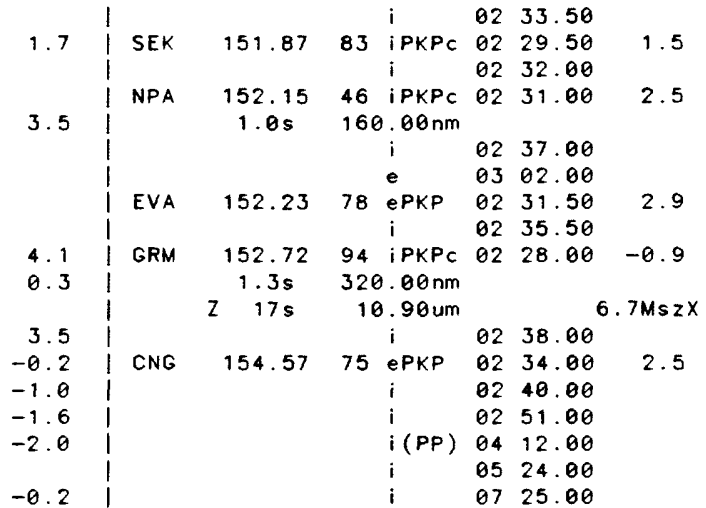
569 obs. associated

MAY 26,1983 B2h $59 \mathrm{~m} 59.45 \pm 0.21 \mathrm{~s}$ $40.465 \mathrm{~N} \pm 1.0 \mathrm{~km} 139.133 \mathrm{E} \pm 1.2 \mathrm{~km}$ DEPTH $=23.0 \pm 1.6 \mathrm{~km}$

$6.8 \mathrm{mb}$ ( 110 Obs.) $7.8 \mathrm{Msz}$ ( 11 obs.) NEAR WEST COAST OF HONSHU, JAPAN (226) One hundred four people killed, 163 injured and extensive damoge to dwellings, roods and vessels coused by eorthquoke ond 0 tsunomi olong the Japon Seo coost from southern Hokkoido to the Ni igato areo, Honshu. Mony of the cosuolties ond much of the domage occurred on the ogo Peninsulo. Tsunomi damoge occurred os for owoy os Yomoguchi Prefecture in southwestern Honshu, olong the Jopon Sea coost of USSR, ond olong the eostern ond southern coosts of South Koreo, where 3 additionol people were killed Felt (V JMA) of Akito, Fukauro and Mutsu. Felt on Hokkoido ond throughout northern and centrol Honshu. Estimoted tsunomi heights were $14 \mathrm{~m}$. ot Minehamo, Honshu, 2-6 m. olong southern Hokkoido ond northern Honshu, up to $8 \mathrm{~m}$. olong the coost of USSR, and $4 \mathrm{~m}$. alang the coost of

South Koreo.

\section{$9 M \leq z$}

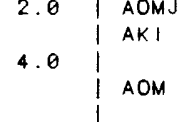

$0.95 \quad 84$ iP

$1.05135 \mathrm{iPt}$

$1.3074 \mathrm{iPt}$

1.38144 iP

$1.65160 \mathrm{eP}^{\mathrm{P}}$

$S$

1.73158 iP

$1.74116 \mathrm{ipt}$

ipt

$\begin{array}{lll}1.83 & 87 \mathrm{iPt}\end{array}$

$.83 \mathrm{~S}$
$\mathrm{~S}$

ep

$32 \quad 36 \mathrm{eP}$

2. $32110 \mathrm{Pt}$ $S$

2.39124 iP

2. $39163 \quad$ iP

2. $40156 \mathrm{Pt}$

2. $44124 \mathrm{Pt}$

$2.4436 \mathrm{iP}$

2. $47 \quad 19 \mathrm{iPt}$ es

$2.54196 \mathrm{eS}$ es

$00 \quad 16.50 \quad-0.6$

$\begin{array}{llll}00 & 17.80 & -0.9\end{array}$

$00 \quad 36.50$

$\begin{array}{lll}00 & 21.80 & -0.5\end{array}$

$00 \quad 46.00$

$00 \quad 21.30 \quad-2.1$

$00 \quad 31.00 \quad 3.7 x$

$00 \quad 53.80$

$00 \quad 26.80 \quad-1.7$

$00 \quad 28.00 \quad-0.6$

0056.00

$00 \quad 30.80 \quad 1.0$

0101.00

0029.50

$00 \quad 56.00$

0032.00

0059.00

$0038.00 \quad 1.2$

$00 \quad 37.30 \quad 0.3$

0116.00

$00 \quad 36.50-1.5$

$00 \quad 35.90 \quad-2.1$

$00 \quad 36.20-1.9$

$00 \quad 37.00 \quad-1.6$

0051.00

$\begin{array}{lll}00 & 38.20 & -0.4\end{array}$

$0040.10 \quad 1.1$

0115.00

$\begin{array}{lll}00 & 38.00 & -2.0\end{array}$

$\begin{array}{ccc}2.55 & 181 & \mathrm{P}+ \\ \mathrm{S}\end{array}$

$00 \quad 39.50 \quad-0.7$

$\begin{array}{ll}00 & 39.50 \\ 01 & 03.00\end{array}$ 
$260 \quad 63 h$

\begin{tabular}{|c|c|c|c|c|c|c|c|c|c|c|c|}
\hline SEN & 2.59 & 148 & $P+$ & 00 & 40.60 & -0.2 & 1 & & & es & 62 \\
\hline & & & es & 01 & 13.00 & & I HMM & 5.85 & 191 & eP & 01 \\
\hline ISN & 2.64 & 140 & $P+$ & 00 & 40.00 & -1.5 & I NGT & 5.86 & 182 & eP & 01 \\
\hline & & & e & 00 & 46.00 & & I OMA & 5.90 & 187 & eP & 01 \\
\hline FKS & 2.90 & 158 & $P+$ & 00 & 43.60 & -1.5 & I TYK & 5.98 & 216 & eP & 01 \\
\hline & & & e & 01 & 06.20 & & VLA & 6.03 & 298 & iP & 01 \\
\hline SAP & 3.07 & 32 & P+ & 00 & 49.70 & 2.2 & I KYO & 6.07 & 207 & eP & 01 \\
\hline & & & es & 01 & 34.00 & & I TSU & 6.12 & 201 & eP & 01 \\
\hline$N \mid$ I & 3.22 & 182 & iP & 00 & 47.30 & -2.4 & 1 & & & $\mathrm{~s}$ & 62 \\
\hline URA & 3.23 & 57 & P+ & 00 & 51.40 & 1.6 & I TKO4 & 6.15 & 190 & eP & 01 \\
\hline & & & es & 01 & 36.00 & & $\mid$ SA & 6.23 & 229 & eP & 01 \\
\hline TKD & 3.43 & 192 & eP & 00 & 51.00 & -1.6 & I TOTJ & 6.29 & 219 & $P$ & 01 \\
\hline & & & es & 01 & 31.00 & & | TOT & 6.30 & 220 & ePd & 01 \\
\hline SHR & 3.45 & 165 & eP & 00 & 52.00 & -1.0 & 1 & & & is & 02 \\
\hline & & & e & 01 & 48.00 & & I NAR & 6.34 & 205 & eP & 01 \\
\hline WAJ & 3.53 & 210 & $P+$ & 00 & 53.00 & -1.1 & I TKO3 & 6.36 & 189 & eP & 01 \\
\hline & & & es & 01 & 26.00 & & I OSA & 6.45 & 208 & ePt & 01 \\
\hline HOOJ & 3.66 & 57 & $\mathrm{P}$ & 00 & 55 & -0.1 & 1 & & & e & 63 \\
\hline ONA & 3.78 & 158 & $\mathrm{P}$ & 00 & 59.80 & 2.2 & I OSK & 6.46 & 206 & ept & 01 \\
\hline & & & e & 01 & 35.10 & & KOB & 6.56 & 210 & iPd & 01 \\
\hline NGN & 3.87 & 191 & eP & 01 & 01.00 & 2.1 & I & & & e & 63 \\
\hline & & & es & 01 & 46.06 & & TKO2 & 6.60 & 190 & $P$ & 01 \\
\hline 081 & 3.92 & 50 & $P+$ & 01 & 01.80 & 2.2 & I HIM & 6.63 & 213 & eP & 01 \\
\hline & & & es & 02 & 05.00 & & I & & & es & 63 \\
\hline RMJ & 3.95 & 27 & eP & 01 & 02.00 & 2.1 & I SHO & 6.63 & 57 & iPc & 01 \\
\hline & & & $S$ & 01 & 57.60 & & ! & $12 \mathrm{~s}$ & 9615 & .00 um & \\
\hline UTS & 3.96 & 171 & Pd & 01 & 01.60 & 1.4 & ! & $15 s$ & 9552 & 2.00 um & \\
\hline & & & es & 01 & 46.00 & & ! & & & es & 02 \\
\hline MAT & 3.98 & 191 & iPc & 60 & 58.80 & $-1 \cdot 8$ & I TKO1 & 6.80 & 191 & $P$ & 01 \\
\hline MTMJ & 4.01 & 195 & iP & 00 & 59.00 & -2.0 & I OWA & 6.80 & 201 & eP & 01 \\
\hline TOY & 4.04 & 203 & eP & 01 & 04.00 & 2.6 & 1 & & & e & 03 \\
\hline & & & es & 01 & 42.00 & & I WKYJ & 6.84 & 205 & $P$ & 01 \\
\hline MAE & 4.06 & 181 & eP & 01 & 02.00 & 0.4 & YONJ & 6.92 & 222 & $P$ & 01 \\
\hline & & & $\mathrm{s}$ & 01 & 50.10 & & I MTS & 6.92 & 226 & Pd & 01 \\
\hline ASA & 4.09 & 35 & $P+$ & 01 & 04.40 & 2.4 & I & & & is & 02 \\
\hline & & & e & 02 & 18.00 & & I SUM & 6.98 & 210 & $P$ & 01 \\
\hline$K A Z$ & 4.14 & 187 & eP & 01 & 03.00 & 0.0 & I WKY & 6.98 & 208 & ePd & 01 \\
\hline$M ! T$ & 4.21 & 165 & Pd & 61 & 65.70 & 1.9 & $!$ & & & is & 02 \\
\hline & & & es & 01 & 52.00 & & Y YSS & 7.04 & 20 & iPc & 01 \\
\hline MTM & 4.31 & 193 & eP & 01 & 15.60 & $9.7 x$ & ! & $1.0 \mathrm{~s}$ & $* * * *$ & $* * * n m$ & \\
\hline KMG & 4.32 & 177 & ep & 01 & 07.00 & 1.8 & OKA & 7.10 & 217 & ept & 01 \\
\hline & & & e & 02 & 14.00 & & 1 & & & e & 03 \\
\hline KAKJ & 4.33 & 169 & iP & 01 & 03.40 & -2.1 & I TKM & 7.35 & 215 & eP & 01 \\
\hline KAN & 4.37 & 207 & eP & 61 & 05.00 & -1.0 & 1 & & & is & 03 \\
\hline $\mathrm{CHJJ}$ & 4.41 & 181 & iP & 01 & 02.60 & $-4.0 x$ & TKS & 7.35 & 211 & ePt & 01 \\
\hline $\mathrm{CHJ}$ & 4.47 & 181 & eP & 01 & 20.00 & $12.6 x$ & I & & & es & 03 \\
\hline & & & e & 62 & 16.00 & & I HJJ & 7.37 & 176 & Pd & 01 \\
\hline TKY & 4.55 & 200 & iP & 01 & 07.80 & -0.9 & 1 & & & $\mathrm{~s}$ & 03 \\
\hline KUS & 4.67 & 56 & eP & 01 & 10.00 & -0.3 & I SHJ & 7.50 & 202 & ePd & 01 \\
\hline & & & e & 62 & 04.00 & & 1 & & & e & 03 \\
\hline TOK & 4.80 & 174 & eP & 01 & 14.00 & 1.9 & TKS J & 7.63 & 214 & $P$ & 01 \\
\hline & & & e & 62 & 30.60 & & SHK & 7.83 & 223 & ePd & 01 \\
\hline KOF & 4.81 & 186 & eP & 01 & 13.00 & 0.7 & HMD & 7.89 & 227 & eP & 01 \\
\hline & & & es & 62 & 01.00 & & ! & & & e & 03 \\
\hline $\mathrm{CHO}$ & 4.92 & 164 & eP & 01 & 13.00 & -0.8 & $H I R$ & 8.69 & 223 & eP & 02 \\
\hline & & & es & 62 & 11.00 & & 1 & & & e & 03 \\
\hline FUK & 4.96 & 208 & ePd & 61 & 14.00 & -0.4 & $1 \mathrm{MDJ}$ & 8. 17 & 304 & Po & 01 \\
\hline & & & es & 02 & 03.50 & & $\mathrm{KOC}$ & 8.22 & 215 & eP & 02 \\
\hline FUN & 4.97 & 183 & eP & 01 & 19.00 & $4.4 x$ & 1 & & & is & 03 \\
\hline & & & es & 02 & 49.00 & & MRT & 8. 22 & 210 & eP & 02 \\
\hline YOK & 5.04 & 175 & eP & 01 & 15.00 & -0.5 & 1 & & & es & 03 \\
\hline & & & es & 02 & 09.00 & & I MTY & 8.33 & 219 & eP & 82 \\
\hline 110 & 5.05 & 192 & Pd & 01 & 18.80 & 2.3 & 1 & & & e & 03 \\
\hline & & & is & 02 & 16.50 & & I UGL & 8.87 & 13 & iPc & 02 \\
\hline $110 \mathrm{~J}$ & 5.07 & 191 & $P$ & 01 & 14.60 & -1.4 & $!$ & $1.0 \mathrm{~s}$ & 11 & $.00 \mathrm{~nm}$ & \\
\hline$A B J$ & 5.22 & 45 & $\mathrm{Pt}$ & 01 & 20.10 & 2.1 & I UWA & 8.94 & 218 & eP & 62 \\
\hline & & & es & 02 & 27.00 & & SHNJ & 8. 99 & 228 & $P$ & 02 \\
\hline WAK & 5.29 & 20 & eP & 01 & 22.00 & $3.6 x$ & ASZ & 9.16 & 214 & $P$ & 02 \\
\hline & & & es & 02 & 32.00 & & 1 & & & $s$ & 03 \\
\hline MIS & .35 & 182 & epd & 01 & 22.00 & 2.1 & I SHN & 9.21 & 228 & ePd & 02 \\
\hline TSR & 5.38 & 208 & eP & 01 & 40.30 & $199 x$ & 1 & & & es & 03 \\
\hline & & & es & 02 & 47.40 & & 1011 & 9.39 & 222 & Pd & 02 \\
\hline GIF & 5.39 & 201 & ePd & 01 & 21.00 & 0.5 & 1 & & & e & 04 \\
\hline & & & eS & 02 & 12.00 & & I FKK & 9.80 & 228 & IPO & 62 \\
\hline AJI & .41 & 180 & ep & 01 & 19.00 & -1.8 & ! & & & es & 04 \\
\hline TAT & .50 & 174 & P & 01 & 24.40 & 2.3 & NOB & 9.89 & 220 & Po & 02 \\
\hline & & & es & 02 & 21.00 & & ! & & & es & 04 \\
\hline KTJ & .51 & 180 & eP & 01 & 22.00 & -0.2 & I ASJ & 9.96 & 223 & ePd & 02 \\
\hline $\mathrm{SHZ}$ & .51 & 186 & eP & 01 & 24.00 & 1.8 & 120 & 10.02 & 235 & eP & 02 \\
\hline TSRJ & .51 & 208 & iP & 01 & 21.40 & -0.8 & ! & & & $S$ & 04 \\
\hline NAG & 5.56 & 199 & eP & 01 & 23.00 & 0.1 & I SAG & 10.09 & 227 & eP & 62 \\
\hline & & & S & 02 & 12.60 & & I & & & S & 04 \\
\hline NEM & .60 & 57 & $P+$ & 01 & 22.40 & -1.0 & I KUM & 10.20 & 224 & Po & 02 \\
\hline$H I k^{\prime}$ & 5.66 & 205 & eP & 01 & 25.00 & 0.7 & 1 & & & S & 64 \\
\hline & & & is & 02 & 31.50 & & I KUMJ & 10.35 & 223 & $\mathbf{P}$ & 02 \\
\hline SH & .70 & 178 & ePd & 01 & 26.00 & 1.2 & I MYZ & 10.56 & 219 & eP & 02 \\
\hline$M Z H$ & 5.84 & 212 & $P$ & 01 & 26.30 & -0.5 & 1 & & & e & 05 \\
\hline
\end{tabular}




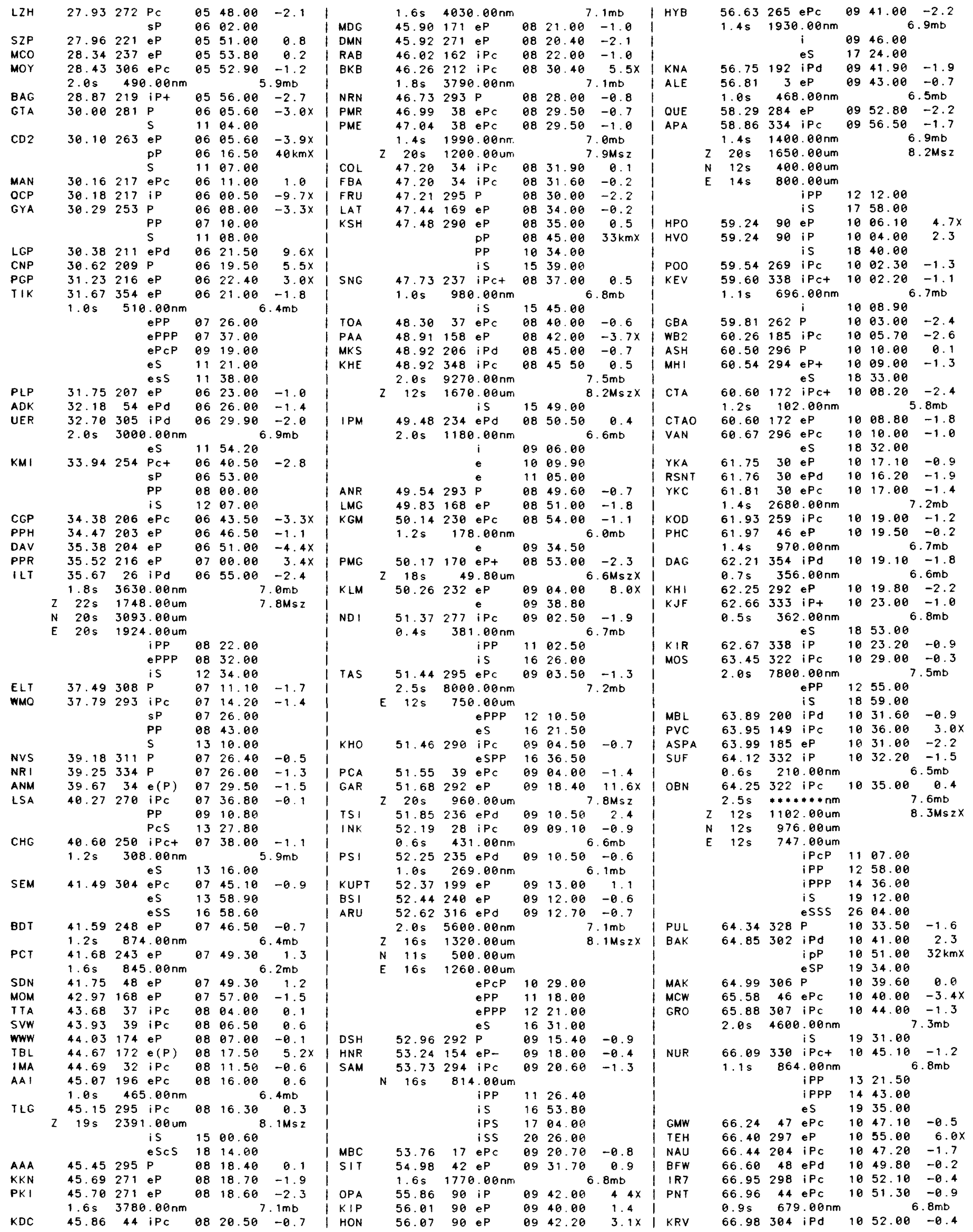




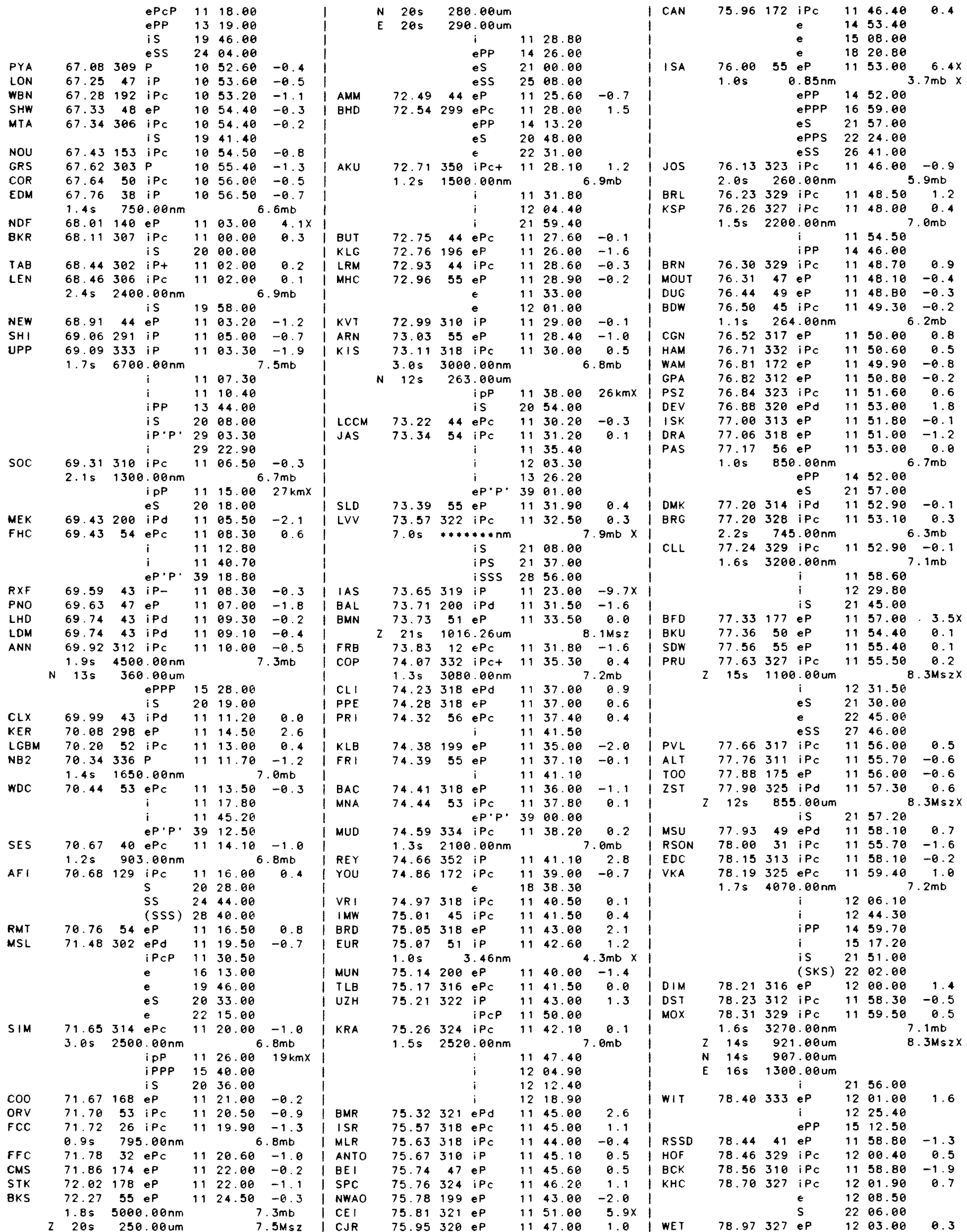




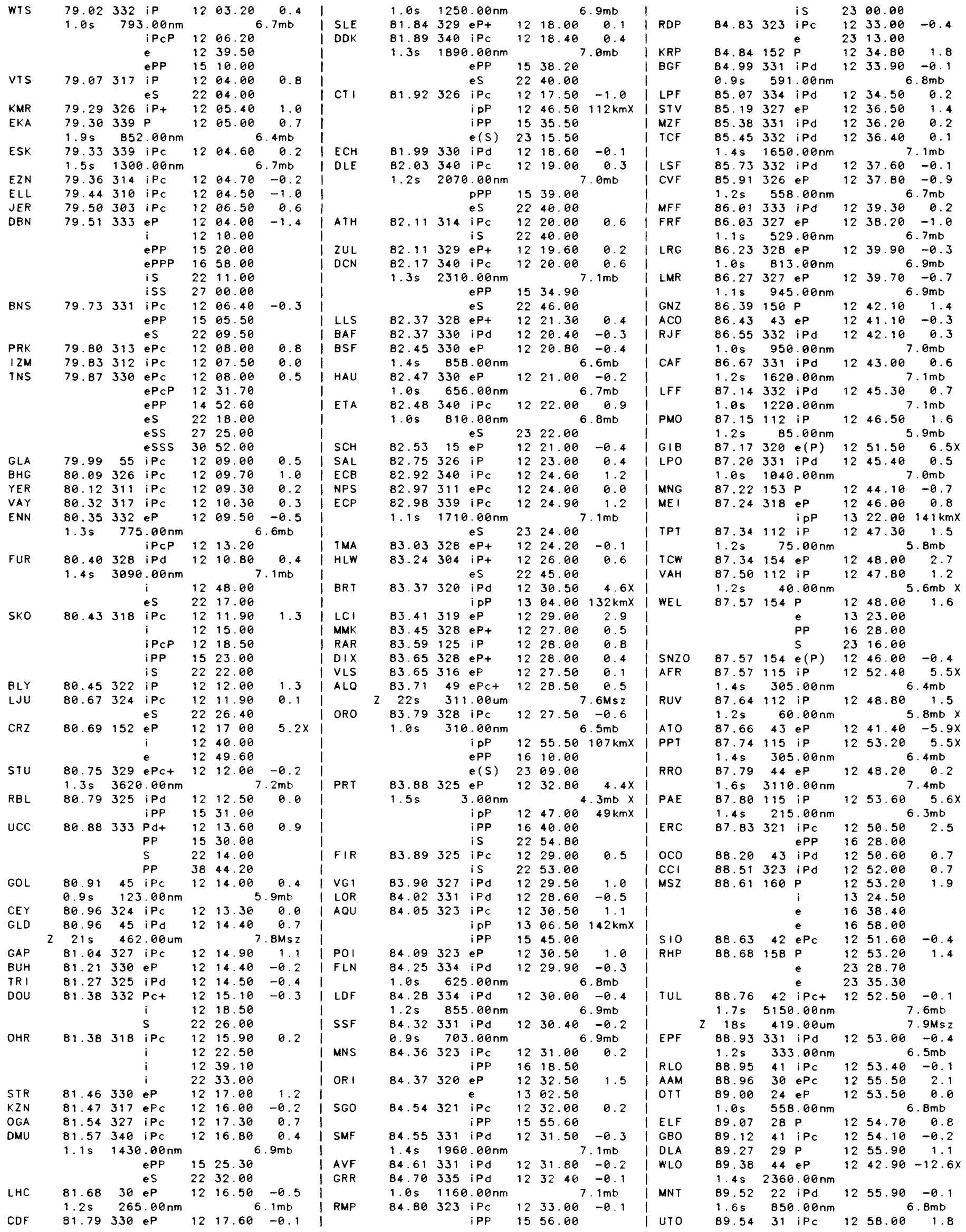




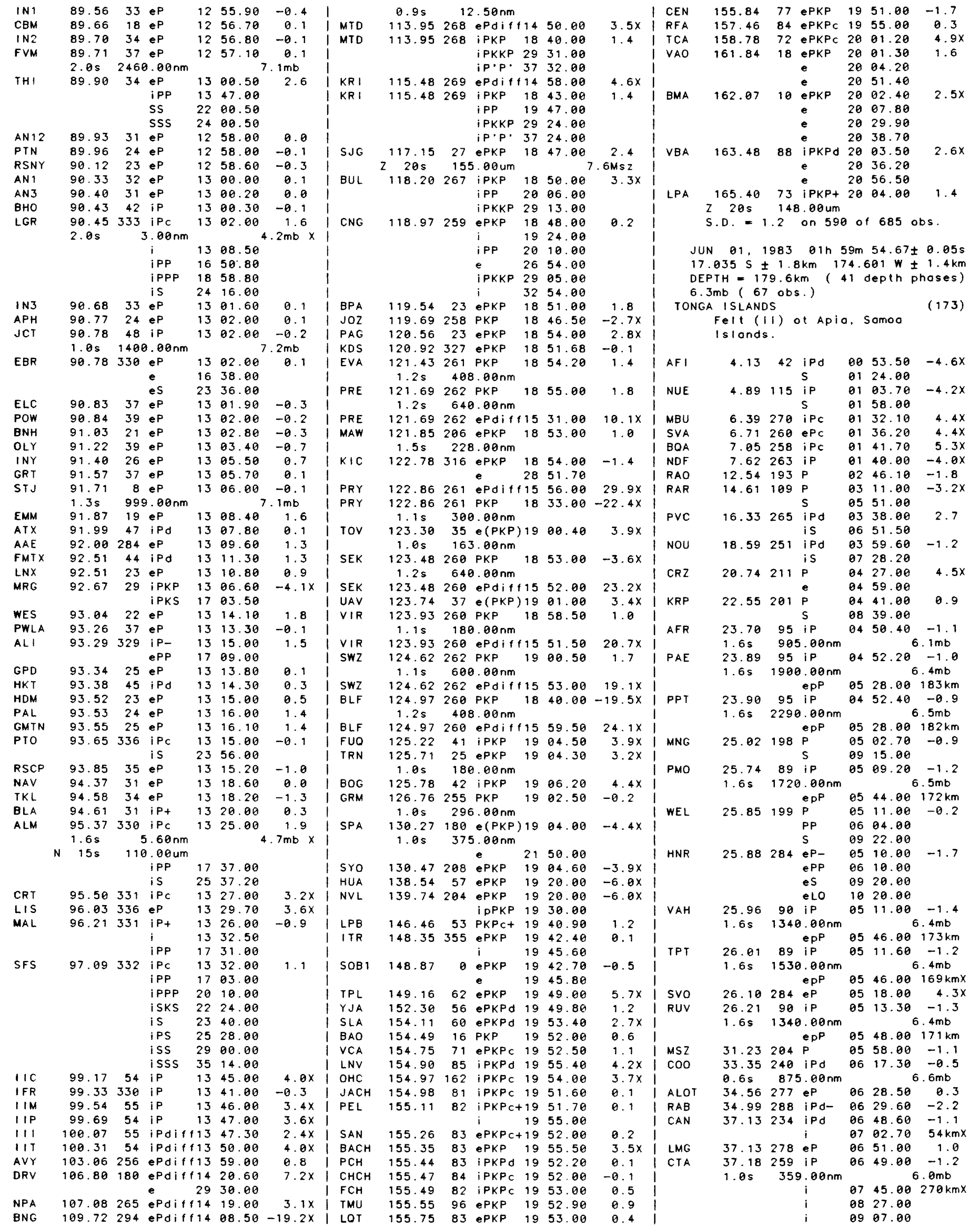




\begin{tabular}{|c|c|c|c|c|c|c|c|c|c|c|c|}
\hline & & & is & 12 & 19.00 & & I TKSJ & 70.46 & 316 & $P$ & 10 \\
\hline & & & $i$ & 13 & 30.00 & & I HOOJ & 70.71 & 328 & $P$ & 10 \\
\hline YOU & 37.27 & 235 & iPd & 86 & 49.90 & -1.0 & I KUR & 70.73 & 333 & iPd & 10 \\
\hline WAM & 7.52 & 232 & iPd & 06 & 52.40 & -0.5 & 1 & $1.0 \mathrm{~s}$ & 1150 & $0.00 \mathrm{~nm}$ & \\
\hline RKT & 7.62 & 106 & iP & 06 & 54.40 & 0.6 & i & & & epp & 11 \\
\hline PMG & 7.94 & 277 & iPd & 86 & 52.00 & $-4.6 x$ & 1 & & & esP & 11 \\
\hline CMS & 8.62 & 241 & IPd & 07 & 01.50 & -0.6 & i & & & escs & 20 \\
\hline LAT & 8.90 & 281 & $i P_{c}$ & 07 & 06.00 & 1.5 & I WAJ & 70.80 & 321 & $P$ & 10 \\
\hline OM & 0.21 & 288 & $i P c$ & 07 & 16.10 & 0.8 & OMJ & 70.94 & 325 & $P$ & 10 \\
\hline HPO & 8.49 & 28 & ePd & 07 & 17.20 & -0.2 & AGJ & 70.98 & 312 & $P$ & 10 \\
\hline MOG & 0.51 & 282 & eP & 07 & 18.00 & a. 3 & I TRT & 71.35 & 267 & iPd & 10 \\
\hline TOO & 10.55 & 232 & iPd & 07 & 17.10 & -0.8 & i & $1.2 \mathrm{~s}$ & 2010 & $0.00 \mathrm{~nm}$ & \\
\hline & $0.4 \mathrm{~s}$ & 345 & $5.00 \mathrm{~nm}$ & & & 5. $3 \mathrm{mb}$ & I YONJ & 71.57 & 317 & $P$ & 10 \\
\hline HON & 1.44 & 24 & eP & 07 & 25.40 & 0.1 & I HAK & 71.61 & 326 & $P$ & 10 \\
\hline$K I P$ & 1.53 & 24 & ep & 07 & 25.60 & -8.4 & I SHK & 71.69 & 316 & $i P d$ & 10 \\
\hline OPA & 1.78 & 23 & eP & 07 & 23.60 & $-4.4 x$ & I HIR & 1.75 & 316 & $P$ & 10 \\
\hline STK & 2.24 & 241 & iPd & 07 & 31.80 & 0.1 & I MRRJ & 1.88 & 327 & $P$ & 10 \\
\hline $\mathrm{MCO}$ & 2.60 & 203 & eP & 07 & 35.00 & 0.7 & I BAG & 72.81 & 294 & iPd- & 11 \\
\hline BFD & 2.66 & 233 & iPc & 07 & 34.60 & -0.5 & i & & & e & 12 \\
\hline$w w w$ & 3.08 & 283 & eP & 07 & 38.08 & -0.8 & i & & & es & 20 \\
\hline$A D E$ & 5.17 & 237 & iPd & 07 & 55.20 & 0.0 & I KKM & 2.08 & 282 & $e^{P}$ & 11 \\
\hline & $1.2 \mathrm{~s}$ & 247 & $7.00 \mathrm{~nm}$ & & & $5.6 \mathrm{mb}$ & I & $0.9 \mathrm{~s}$ & 325 & $5.00 \mathrm{~nm}$ & \\
\hline GUA & 50.21 & 305 & eP- & 08 & 34.50 & 0.0 & I SKR & 72.16 & 341 & eP & 11 \\
\hline & $1.3 \mathrm{~s}$ & $125 t$ & $0.00 \mathrm{~nm}$ & & & $5,4 \mathrm{mb}$ & & $1.5 \mathrm{~s}$ & 4060 & $0.00 \mathrm{~nm}$ & \\
\hline & & & es & 15 & 42.00 & & i & & & ePCP & 11 \\
\hline GUMO & 0.28 & 305 & eP- & 08 & 35.00 & 0.1 & i & & & epp & 11 \\
\hline PJG & 0.28 & 305 & $e(P)$ & 08 & 35.30 & 0.3 & i & & & esP & 12 \\
\hline MTN & 2.47 & 267 & eP & 08 & 50.00 & -1.4 & I & & & ePP & 13 \\
\hline KNA & 4.18 & 263 & eP & 89 & 03.00 & -1.0 & I & & & es & 20 \\
\hline WBN & .06 & 250 & iPd & $\theta 9$ & 09.00 & -1.2 & $A P$ & 2.20 & 328 & $P$ & 1 \\
\hline AA I & 57.61 & 277 & eP & 89 & 27.60 & -0.8 & I ASAJ & 72.31 & 329 & $P$ & 11 \\
\hline & $1.0 \mathrm{~s}$ & 796 & $0.00 \mathrm{~nm}$ & & & $.5 \mathrm{mb}$ & I SHNJ & 72.50 & 315 & $P$ & 11 \\
\hline DRV & 57.68 & 200 & iPd & 89 & 27.50 & -0.6 & I SUT & 72.57 & 327 & $P$ & 11 \\
\hline$K L G$ & 9.34 & 244 & iPd & 89 & 38.30 & -1.9 & I SPA & 73.07 & 180 & $i P c$ & 11 \\
\hline & $1.0 \mathrm{~s}$ & 105 & $5.00 \mathrm{~nm}$ & & & $.6 \mathrm{mb}$ & i & $1.0 \mathrm{~s}$ & 195 & $5.00 \mathrm{~nm}$ & \\
\hline$S B A$ & 61.54 & 184 & iP & $\theta 9$ & 56.08 & 1.6 & i & $18 \mathrm{~s}$ & & $2.61 \mathrm{um}$ & \\
\hline$M B L$ & 61.72 & 255 & iPc & 09 & 45.20 & $-11.2 x$ & I SDN & 73.08 & 8 & $i P d$ & 11 \\
\hline & $1.0 \mathrm{~s}$ & $148 e$ & $0.00 \mathrm{~nm}$ & & & & I NWRM & 73.44 & 40 & ePd & 11 \\
\hline MEK & 62.20 & 249 & eP & 09 & 58.00 & -1.6 & I PHAM & 73.44 & 43 & iP & 11 \\
\hline & $0.6 \mathrm{~s}$ & 77 & $7.00 \mathrm{~nm}$ & & & $5.7 \mathrm{mb}$ & I PRI & 73.45 & 43 & ePd & 11 \\
\hline$K L B$ & 62.46 & 243 & $i P c$ & 10 & 00.00 & -1.2 & i & & & epP & 11 \\
\hline & $1.0 \mathrm{~s}$ & 396 & $0.00 \mathrm{~nm}$ & & & $.2 \mathrm{mb}$ & BKS & 73.48 & 41 & ePd & 11 \\
\hline NWAO & 62.82 & 241 & iPd & 10 & 02.80 & -0.8 & i & $0.8 \mathrm{~s}$ & 50 & $0.00 \mathrm{~nm}$ & \\
\hline BAL & 63.43 & 244 & iPd & 10 & 06.40 & -1.2 & i & & & epp & 11 \\
\hline MUN & 3.75 & 242 & eP & 10 & 08.00 & -1.7 & 1 & & & esP & 12 \\
\hline DAV & 3.80 & 287 & eP & 10 & 10.00 & -0.1 & i & & & is & 20 \\
\hline NAU & 65.48 & 253 & iPd & 10 & 20.20 & -0.7 & I & & & eSKS & 20 \\
\hline & $0.4 \mathrm{~s}$ & $16 e$ & $0.00 \mathrm{~nm}$ & & & $6.2 \mathrm{mb}$ & i & & & e & 21 \\
\hline MKS & 65.49 & 272 & iPd & 10 & 21.00 & -0.1 & i & & & e & 21 \\
\hline & $1.2 \mathrm{~s}$ & 457 & $7.00 \mathrm{~nm}$ & & & $6.2 \mathrm{mb}$ & i & & & ePKKP & 28 \\
\hline HJJ & 66.20 & 319 & $P$ & 10 & 25.00 & -0.2 & I PET & 73.49 & 344 & iPd & 11 \\
\hline KYS & .39 & 321 & iPd & 10 & 32.60 & a. e & i & $2.0 \mathrm{~s}$ & 2470 & a. $00 \mathrm{~nm}$ & \\
\hline KTJ & .86 & 320 & $\mathrm{P}$ & 10 & 34.80 & -0.8 & i & & & esP & 12 \\
\hline A Jl & .92 & 320 & $P$ & 10 & 34.00 & -1.9 & I & & & es & 20 \\
\hline TKO & .03 & 318 & $P$ & 10 & 36.60 & 0.4 & I MHC & 73.53 & 42 & ePd & 1 \\
\hline TKO2 & 68.04 & 319 & $P$ & 10 & 37.00 & 0.6 & i & & & epP & 11 \\
\hline TKO3 & 68.05 & 319 & $P$ & 10 & 36.60 & a. a & I ARN & 73.61 & 42 & $i P_{c}$ & 11 \\
\hline KAKJ & 3.05 & 322 & 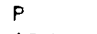 & 10 & 36.40 & -0.3 & & & & epp & 11 \\
\hline OYM & 3.08 & 321 & IPd & 10 & 35.30 & -1.7 & I SLD & 73.64 & 42 & $i P c$ & 1 \\
\hline TSK & .09 & 322 & iPd & 10 & 36.40 & -0.6 & I PAS & 73.98 & 46 & $i P d$ & 1 \\
\hline OMA & .18 & 319 & P & 10 & 37.00 & -0.5 & I FHC & 74.26 & 38 & eP & 1 \\
\hline$S R$ & 19 & 321 & $i P c$ & 10 & 30 & -1.3 & I YSS & 74.40 & 331 & $i P d$ & 11 \\
\hline$T K \theta$ & .25 & 319 & $P$ & 10 & 38.60 & 0.8 & 1 & $1.7 \mathrm{~s}$ & 680 & $0.00 \mathrm{~nm}$ & \\
\hline $\mathrm{SH}$ & 30 & 320 & $P$ & 10 & .00 & -0.3 & 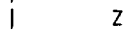 & $17 \mathrm{~s}$ & & $1.50 \mathrm{um}$ & \\
\hline DDR & .50 & 321 & t & 10 & 3 & -0.4 & & & & epP & 12 \\
\hline A & .65 & 359 & $\mathrm{i}$ & 10 & 8 & -1 & & & & esP & 12 \\
\hline $\mathrm{CH}$ & .65 & 321 & 1 & 10 & 10 & -0.3 & & & & ePP & 14 \\
\hline$t$ & .94 & 320 & $F$ & & $\theta$ & -0 & & & & es & 20 \\
\hline $\mathrm{SHJ}$ & .00 & 317 & P & 10 & $\theta$ & 0 & & & & $P P$ & 21 \\
\hline ow & .11 & 318 & 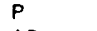 & & 4 & -0 & & & & & 21 \\
\hline BKB & 15 & 275 & $\mathrm{iPC}$ & 10 & 10 & 2 & i & & 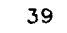 & & 1 \\
\hline$M 1$ & .43 & 325 & $P$ & 10 & 45.00 & a & I FRI & 77 & 43 & e & 1 \\
\hline 11 & .44 & 322 & $P$ & 10 & 45.00 & -0. & I JAS & 74.66 & 42 & ef & 1 \\
\hline MAT & 45 & 321 & iPd & 10 & 45.00 & -0.3 & & & & ep & 11 \\
\hline & & & 0.00 & & & $.5 \mathrm{mb}$ & & & & eP & 14 \\
\hline & & & es & 19 & 40.00 & & KTM & 2 & 44 & iPc & 1 \\
\hline$A M$ & & 323 & $P$ & 10 & 45.10 & -0.3 & ORV & & 40 & $\mathrm{i}$ & 11 \\
\hline WKY & 0 & 318 & P & 10 & 0 & 0.4 & i & & & $D P$ & 12 \\
\hline & & 321 & $P$ & 10 & 46.00 & -1 & WDC & & 38 & & 11 \\
\hline$K Y$ & 78 & 320 & $P$ & 10 & 47.08 & -0.4 & 1 & & & epp & 12 \\
\hline NK & & & eP & 10 & & -1 & & & & iP & 1 \\
\hline MRt & $\theta$ & 325 & $\theta$ & 10 & 48.00 & 0 & ANP & 3 & 303 & eP & 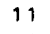 \\
\hline su & & 317 & P & 10 & 50.30 & 0 & $M I R$ & & 804 & iPd & 11 \\
\hline SM & & 353 & ePd & $\theta$ & 48.00 & -1.2 & & $1.5 \mathrm{~s}$ & & $0.00 \mathrm{~nm}$ & \\
\hline & & 319 & 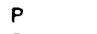 & a & 51.00 & & & & & ePCP & 11 \\
\hline HJ & & 324 & $P$ & $\theta$ & 49.00 & -0 & & & & e & 12 \\
\hline AIK & 0.41 & 322 & P & 10 & 51.00 & $-\theta$. & & & & ef & 14 \\
\hline
\end{tabular}




\begin{tabular}{|c|c|c|c|c|c|c|c|c|c|c|c|c|}
\hline & & & $\begin{array}{l}\text { S } \\
\text { sS }\end{array}$ & $\begin{array}{l}22 \\
23\end{array}$ & $\begin{array}{l}10.00 \\
35.00\end{array}$ & & INK & $\begin{array}{l}90.12 \\
1.3 \mathrm{~s}\end{array}$ & $\begin{array}{l}14 \\
80\end{array}$ & $\begin{array}{l}\mathrm{eP} \\
1.00 \mathrm{~nm}\end{array}$ & 12 & 34.00 \\
\hline LHD & 83.55 & 35 & ipd & 12 & 03.30 & -0.4 & i & & & $p P$ & 13 & 18.00 \\
\hline$C L X$ & 83.76 & 35 & iP- & 12 & 04.50 & -0.4 & I YAK & 90.13 & 337 & iPd & 12 & 33.60 \\
\hline SEY & 83.76 & 345 & iPd & 12 & 04.40 & 0.1 & i & $2.0 \mathrm{~s}$ & 108 & $0.00 \mathrm{~nm}$ & & \\
\hline & & 316 & $0.00 \mathrm{~nm}$ & & & $6.7 \mathrm{mb}$ & i & & & iSKS & 22 & 48.50 \\
\hline & & & epP & 12 & 49.10 & $181 \mathrm{~km}$ & i & & & is & 23 & 03.90 \\
\hline & & & esP & 13 & 10.50 & & I TUP & 90.26 & 328 & iPd & 12 & 36.70 \\
\hline & & & es & 22 & 10.00 & & i & $2.2 \mathrm{~s}$ & 940 & $0.00 \mathrm{~nm}$ & & \\
\hline & & & eSP & 23 & 09.50 & & I ВTO & 90.28 & 313 & $e P$ & 12 & 37.20 \\
\hline & & & ESPP & 23 & 26.50 & & I FMTX & 90.47 & 57 & iP & 12 & 37.60 \\
\hline LDM & 83.80 & 35 & iPd & 12 & 04.50 & -0.4 & 1510 & 90.65 & 53 & iPd & 12 & 38.00 \\
\hline VHO & 83.89 & 70 & iP & 12 & 07.00 & 0.9 & I KMI & 90.69 & 296 & iP- & 12 & 42.00 \\
\hline I MW & 83.93 & 41 & IPd & 12 & 06.40 & 0.4 & 1 & 3.05 & & $4.50 \mathrm{~nm}$ & & \\
\hline LRM & 84.01 & 38 & ePd & 12 & 06.40 & 0.2 & 1 & & & sP & 13 & 40.00 \\
\hline$R \times F$ & 84.13 & 35 & iPd & 12 & 06.30 & -0.3 & 1 & & & $P P$ & 16 & 18.00 \\
\hline $\mathrm{COL}$ & 84.21 & 11 & iPd & 12 & 06.20 & -0.3 & 1 & & & SKS & 23 & 10.00 \\
\hline & $21 \mathrm{~s}$ & & $2.87 \mathrm{um}$ & & & $5.6 \mathrm{Msz}$ & 1 & & & $s$ & 23 & 22.00 \\
\hline & & & is & 22 & 16.00 & & I SYO & 90.80 & 192 & iP & 12 & 38.10 \\
\hline BDW & 84.24 & 42 & iPd & 12 & 06.70 & -0.8 & I TUL & 31.10 & 53 & iPc- & 12 & 40.20 \\
\hline & & & $p P$ & 12 & 52.20 & $184 \mathrm{~km}$ & 1 & 1.35 & 21 & $4.00 \mathrm{~nm}$ & & \\
\hline & & & ePP & 15 & 21.40 & & 1 & $18 \mathrm{~s}$ & & $3.47 \mathrm{um}$ & & \\
\hline$I L T$ & 84.74 & 358 & iPd & 12 & 09.00 & 0.0 & i & & & eSKS & 23 & 00.00 \\
\hline & $2.0 \mathrm{~s}$ & 2000 & $0.00 \mathrm{~nm}$ & & & $6.5 \mathrm{mb}$ & i LNV & 91.12 & 126 & iPd & 12 & 41.80 \\
\hline & & & isp & 13 & 15.00 & & I $\mathrm{BHO}$ & 91.30 & 54 & ip & 12 & 40.80 \\
\hline & & & is & 22 & 14.00 & & 1 & $1.2 \mathrm{~s}$ & 116 & $6.00 \mathrm{~nm}$ & & \\
\hline & & & IPS & 23 & 36.00 & & BDT & 91.62 & 287 & iPd & 12 & 44.00 \\
\hline$K L M$ & 84.94 & 275 & ePc & 12 & 12.00 & 0.8 & 1 & 1.05 & 578 & $8.00 \mathrm{~nm}$ & & \\
\hline LUB & 85.60 & 53 & $e^{P}$ & 12 & 14.00 & -0.2 & $1 \mathrm{CD} 2$ & 91.68 & 302 & $P$ & 12 & 44.80 \\
\hline GOL & 85.61 & 46 & iPd & 12 & 14.60 & 0.2 & 1 & & & $\mathbf{S P}$ & 13 & 51.00 \\
\hline & $1.0 \mathrm{~s}$ & 153 & $3.00 \mathrm{~nm}$ & & & $5.8 \mathrm{mb}$ & 1 & & & eSKS & 23 & 00.00 \\
\hline & & & PP & 12 & 55.83 & $165 \mathrm{kmX}$ & $\mathrm{CHCH}$ & 1.72 & 126 & iPc & 12 & 46.00 \\
\hline GLD & 85.74 & 46 & iPC & 12 & 15.10 & 0.2 & I RLO & 1.78 & 53 & iPd & 12 & 43.30 \\
\hline & $1.0 \mathrm{~s}$ & $14 e$ & $0.00 \mathrm{~nm}$ & & & $5.7 \mathrm{mb}$ & I $\mathrm{PCH}$ & 1.94 & 126 & iPc & 12 & 45.50 \\
\hline & & & $p P$ & 12 & 59.00 & $176 \mathrm{~km}$ & I PEL & 91.98 & 125 & iPd & 12 & 46.00 \\
\hline & & & e & 15 & 34.00 & & I RSNT & 92.05 & 24 & $i P c$ & 12 & 43.70 \\
\hline BJI & 85.76 & 314 & $P d$ & 12 & 15.00 & 0.3 & 1 & & & ipp & 13 & 31.00 \\
\hline & & & $p P$ & 12 & 56.00 & $164 \mathrm{kmx}$ & i & & & IPP & 16 & 24.70 \\
\hline & & & SP & 13 & 14.00 & & I YKA & 92.05 & 24 & eP & 12 & 44.80 \\
\hline & & & es & 22 & 24.00 & & I $\mathrm{BACH}$ & 92.06 & 126 & iP & 12 & 46.50 \\
\hline & & & sS & 23 & 43.00 & & I YKC & 92.09 & 24 & iPd & 12 & 43.90 \\
\hline MAW & 85.88 & 199 & IPC & 12 & 16.00 & 1.1 & 1 & $1.0 \mathrm{~s}$ & 135 & $5.00 \mathrm{~nm}$ & & \\
\hline & $0.9 \mathrm{~s}$ & & $2.00 \mathrm{~nm}$ & & & $5.0 \mathrm{mb} \times$ & 1 & & & $\mathrm{pP}$ & 16 & 25.50 \\
\hline$I P M$ & 85.96 & 276 & ePd & 12 & 17.60 & 1.3 & I LOT & 92.10 & 126 & iP & 12 & 48.20 \\
\hline & $0.9 \mathrm{~s}$ & 444 & $4.00 \mathrm{~nm}$ & & & $6.3 \mathrm{mb}$ & I СНTO & 92.14 & 289 & $P$ & 12 & 47.00 \\
\hline & & & e & 13 & 03.90 & $187 \mathrm{~km}$ & I FCH & 92.22 & 126 & iPd & 12 & 48.00 \\
\hline JCT & 86.01 & 57 & $i P d$ & 12 & 16.10 & -0.1 & 1 & & & $\mathbf{i}$ & 13 & 33.00 \\
\hline & $1.0 \mathrm{~s}$ & 300 & $0.00 \mathrm{~nm}$ & & & $6.1 \mathrm{mb}$ & I JACH & 92.23 & 125 & iPd & 12 & 47.40 \\
\hline & $18 \mathrm{~s}$ & & $2.41 \mathrm{um}$ & & & $5.6 \mathrm{Msz}$ & I NVL & 92.31 & 182 & ip & 12 & 45.00 \\
\hline & & & $i$ & 13 & 02.00 & $185 \mathrm{~km}$ & 1 & & & epP & 13 & 27.00 \\
\hline SNG & 87.12 & 278 & ip & 12 & 24.00 & 2.1 & 1 & & & esP & 13 & 45.00 \\
\hline & $1.0 \mathrm{~s}$ & 628 & $0.00 \mathrm{~nm}$ & & & $6.4 \mathrm{mb}$ & $i$ & & & eSKS & 23 & 00.00 \\
\hline & & & is & 22 & 44.00 & & 1 & & & es & 23 & 29.00 \\
\hline SES & 87.19 & 35 & eP & 12 & 21.00 & -0.5 & I RFA & 93.07 & 128 & $e P d$ & 12 & 50.80 \\
\hline & $1.0 \mathrm{~s}$ & 688 & $8.00 \mathrm{~nm}$ & & & $6.5 \mathrm{mb}$ & I LZH & 93.26 & 307 & $P d$ & 12 & 51.50 \\
\hline TIY & 87.43 & 311 & $\mathrm{Pd}$ & 12 & 24.70 & 1.7 & 1 & & & SKS & 23 & 09.00 \\
\hline & & & $P P$ & 15 & 45.50 & & 1 & & & S & 23 & 41.00 \\
\hline EDM & 87.47 & 32 & IPd & 12 & 22.20 & -0.6 & I NNA & 3.80 & 104 & eP & 12 & 55.70 \\
\hline & $1.0 \mathrm{~s}$ & 537 & $7.00 \mathrm{~nm}$ & & & $6.4 \mathrm{mb}$ & 1 & $1.7 \mathrm{~s}$ & 115 & $5.00 \mathrm{~nm}$ & & \\
\hline & & & PP & 13 & 0800 & $184 \mathrm{~km}$ & I FFC & 94.09 & 34 & ePd & 12 & 52.50 \\
\hline ATX & 87.50 & 57 & iP & 12 & 24.00 & 0.6 & i & $2.0 \mathrm{~s}$ & $42 e$ & $0.00 \mathrm{~nm}$ & & \\
\hline GYA & 87.79 & 298 & $P d$ & 12 & 27.00 & 2.0 & 1 & & & $p P$ & 13 & 37.00 \\
\hline & & & $p P$ & 13 & 11.00 & $176 \mathrm{~km}$ & $O L Y$ & 94.30 & 54 & ePd & 12 & 54.50 \\
\hline & & & SP & 13 & 30.50 & & $1 B O D$ & 94.66 & 329 & iPd & 12 & 56.40 \\
\hline & & & s & 22 & 40.00 & & 1 & $1.3 \mathrm{~s}$ & $13 e$ & $0.00 \mathrm{~nm}$ & & \\
\hline TSI & 87.99 & 274 & ePd & 12 & 28.20 & 2.1 & I POW & 94.72 & 54 & ePC & 12 & 56.70 \\
\hline RSSD & 88.44 & 43 & ePc & 12 & 27.50 & -0.4 & I ANT & 95.65 & 117 & eP & 13 & 10.00 \\
\hline & & & $\mathrm{pP}$ & 13 & 11.10 & $174 \mathrm{~km}$ & 1 & & & epPp & 13 & 45.00 \\
\hline PCT & 88.65 & 286 & iPd & 12 & 32.20 & $3.1 x$ & I & & & es & 24 & 10.00 \\
\hline & $1.6 \mathrm{~s}$ & 173 & $3.00 \mathrm{~nm}$ & & & $5.8 \mathrm{mb}$ & I FVM & 95.84 & 52 & iPC & 13 & 01.60 \\
\hline XAN & 88.65 & 306 & iPd & 12 & 30.10 & 1.2 & 1 & $1.2 \mathrm{~s}$ & 229 & $9.78 \mathrm{~nm}$ & & \\
\hline & & & SP & 13 & 36.00 & & 1 & & & epp & 13 & 46.80 \\
\hline & & & isKS & 22 & 42.50 & & i & & & ePP & 16 & 53.78 \\
\hline $\mathrm{ACO}$ & 88.86 & 51 & ePd & 12 & 29.00 & -0.8 & $1 \mathrm{LST}$ & 95.95 & 54 & $e(P)$ & 13 & 02.50 \\
\hline & $1.1 \mathrm{~s}$ & 484 & $4.00 \mathrm{~nm}$ & & & $6.4 \mathrm{mb}$ & I GRT & 96.11 & 54 & eP & 13 & 03.70 \\
\hline & & & e & 13 & 15.00 & $184 \mathrm{~km}$ & I TIK & 96.25 & 345 & ePd & 13 & 02.50 \\
\hline RRO & 88.99 & 52 & eP & 12 & 30.60 & 0.2 & 1 & $1.0 \mathrm{~s}$ & 190 & $0.00 \mathrm{~nm}$ & & 6 \\
\hline & $0.8 \mathrm{~s}$ & & $5.40 \mathrm{~nm}$ & & & $5.6 \mathrm{mb}$ & I ELC & 96.55 & 53 & ePd & 13 & 05.10 \\
\hline & & & e & 13 & 17.40 & $188 \mathrm{~km}$ & 1 & & & epp & 13 & 50.20 \\
\hline HKT & 39.02 & 58 & ip & 12 & 31.50 & 0.9 & I PWLA & 96.82 & 56 & ePd & 13 & 06.10 \\
\hline $\mathrm{HHC}$ & 89.29 & 313 & $P d$ & 12 & 33.40 & 1.6 & 1 & & & epp & 13 & 50.90 \\
\hline ATO & 89.30 & 52 & eP & 12 & 31.20 & -0.6 & I ARE & 97.25 & 110 & eP & 13 & 11.00 \\
\hline & & & e & 13 & 15.80 & $178 \mathrm{~km}$ & I GTA & 97.30 & 309 & iPd & 13 & 09.40 \\
\hline OCO & 89.69 & 53 & ePd & 12 & 32.80 & -0.8 & 1 & & & $p P$ & 13 & 5800 \\
\hline & & & e & 13 & 18.90 & $185 \mathrm{~km}$ & 1 & & & sP & 14 & 15.00 \\
\hline LPS & 89.87 & 75 & eP & 12 & 36.00 & 1.1 & 1 & & & $P P$ & 17 & 05.00 \\
\hline & & & es & 22 & 53.00 & & 1 & & & SKS & 23 & 30.50 \\
\hline
\end{tabular}


TAS $120.57 \quad 30 \mathrm{~B}$ ePKPd $18 \quad 26.50 \quad-0.1 \quad$ $2.5 \mathrm{~s} \quad 1000.00 \mathrm{~nm}$ 1.30 um $1950.50^{5}$ $\begin{array}{lll}\text { EPP } & 19 & 50.50 \\ \text { ESKS } & 25 & 10.50\end{array}$ ePS $29 \quad 40.00$ ESPP $30 \quad 46.50$

DSH $\quad 121.12305$ IPKPd $18 \quad 28.10$ eSKS 2504.50

SAM 122.44306 ePKP $18 \quad 29.70$ $2.0 \mathrm{~s} \quad 1600.00 \mathrm{~nm}$ $\begin{array}{lll}\text { IPP } 2008.80 & 08\end{array}$ $\begin{array}{llll}\text { ePPP } & 22 & 42.00\end{array}$ eSKS $25 \quad 09.00$ ESP $29 \quad 36.00$

QUE $\quad 122.70 \quad 295$ IPKPd $18 \quad 31.70$ $1.2 \mathrm{~s} \quad 2200.00 \mathrm{~nm}$

SVE $\quad 122.90 \quad 327 \quad$ ePKP $18 \quad 30.00$ $2.2 \mathrm{~s} \quad 260.00 \mathrm{~nm}$

STJ $123.80 \quad 44$ EPKP $18 \quad 31.00$

ARU $124.10 \quad 327$ iPKPd $18 \quad 32.78$

$\begin{array}{lll}\text { i PPKP } 1922.30 & 20\end{array}$

$\begin{array}{lll}\text { iPP } \quad 20 \quad 25.00 & 0\end{array}$

$\begin{array}{lll}\text { EPPP } & 23 & 02.00\end{array}$

eSKS 2522.00

AVY $125.15 \quad 231$ ePKPC $18 \quad 35.00$

KEV $\quad 125.63 \quad 351$ ePKP $18 \quad 29.08$ $0.8 \mathrm{~s} \quad 82.10 \mathrm{~nm}$

i $18 \quad 35.00$

IPPKP $19 \quad 25.00$

ePP $20 \quad 28.00$

esPP $21 \quad 40.00$

e 3300.00

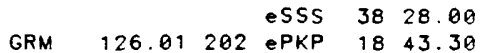
3 MszX BPI CAI

iswz

0.3 I PRE -0.5 I SUF

I KSR I PUL

0.

0.5 I MOS

-0.5 NUR
$-1.5$ $-0.1$ I

APA $\quad 126.40 \quad 347 \quad$ IPKP $18 \quad 35.80$ $1.0 \mathrm{~s} 100.00 \mathrm{~nm}$

EPPKP 1922.90

$\begin{array}{lll} & \text { IPP } 20 \quad 30.80\end{array}$

SOB1 $127.32 \quad 116$ iPKP 1839.80

$$
\begin{array}{lll}
\text { e } & 1842.10 \\
\text { e } & 1850.30
\end{array}
$$$$
\text { e } \quad 1906.30
$$
i $\quad 1929.70$

i

-1.3 OBN $-6.4 \times$ OBN

BAK

$5.9 \times \quad$ NB2 $-1.2$

$\mid \begin{aligned} & \text { SHE } \\ & \text { UPP }\end{aligned}$
$-0.6$

UPP<smiles>I[I-]</smiles>

HFS
BUL

$\begin{array}{lllllllll}\text { CER } & 128.16 & 195 & \text { IPKPC } & 18 & 41.00 & -0.5 & \text { B }\end{array}$

$\begin{array}{lllllll}\text { KIR } & 128.31 & 353 & \text { ePKP } 18 & 26.00 & -14.6 \times\end{array}$

MHI $128.67 \quad 303$ iPKPd 1842.40 $1.4 \mathrm{~s} \quad 298.00 \mathrm{~nm}$

e $\quad 20 \quad 47.00$

$\begin{array}{lllll}\text { SUR } & 128.70 & 197 \text { EPKP } & 18 & 42.80\end{array}$ $0.8 \mathrm{~s} 169.00 \mathrm{~nm}$

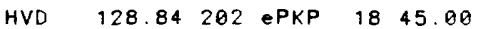

AKU $\quad 128.92 \quad 12$ IPKP $18 \quad 42.80$ $1.9 \mathrm{~s} \quad 400.00 \mathrm{~nm}$

129.34305 i 1932.30 IPKPC $18 \quad 44.00$

$\begin{array}{llllll} & & & \text { EPP } & 20 & 53.50 \\ \text { REY } & 129.38 & 15 & \text { ePKP } & 18 & 44.20\end{array}$

0.0 | GRO i

KRV \begin{tabular}{l|l}
0.1 & GRS \\
& GRS
\end{tabular}

$2.0 \quad 1$

GRS

1.8

I MTA

I PYA

0.5 TAB

I ERE

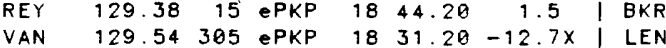

VAN $129.54 \quad 305$ ePKP $1843.20 \quad-0.7 \quad$ MUD

$$
\begin{array}{ccc}
2.0 \mathrm{~S} & 360.00 \mathrm{~nm} & \\
\text { i PPKP } 19 & 32.00 \\
\text { ePP } 20 & 53.00
\end{array}
$$

EPS 3104.00

BLF 129.92204 IPKPC $18 \quad 45.40$

$\begin{array}{lllllll}\text { SEK } & 130.04 & 206 & \text { ePKP } & 18 & 45.00 & -0.4 \\ \text { KJF } & 130.47 & 347 & \text { ePKP } & 18 & 29.00 & -15.8 x\end{array}$ $0.7 \mathrm{~s} \quad 54.70 \mathrm{~nm}$

i $18 \quad 43.00$

i PPKP 1935.00

$\begin{array}{lll}\text { EPP } & 2100.00\end{array}$

ISKP 2154.00

iPKS 2207.50

IPPKS 2256.00

ePS 3052.00

$$
\text { e } 3456.00
$$

$\begin{array}{lllll}\text { VIR } & 130.57 \quad 205 \text { e(PKP) } 18 & 47.00\end{array}$

KIM $130.78 \quad 203$ ePKP $18 \quad 45.90$

EVA $\quad 130.99208$ ePKP 1847.90

PRY

$$
131.33207 \text { ePKP }
$$

$18 \quad 34.50-13.3 \times$ VAL

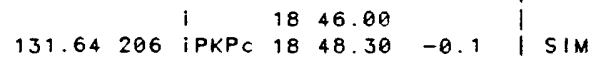

BFS

DMU

$D C N$

DCN

DDK

0.7 EPPKP 1921.08 \begin{tabular}{lll} 
ePPKP $19 \quad 20.00$ \\
\hline
\end{tabular} $2.4 \mathrm{~s} \quad 700.00 \mathrm{~nm}$

ePPS $31 \quad 32.00$

$0.5 \mathrm{~s} \quad 52.00 \mathrm{~nm}$

\begin{tabular}{lllll|l}
131.74 & 208 EPKP & 18 & 46.50 & -2.2 & ETA
\end{tabular} 131.88116 iPKPd $18 \quad 48.50 \quad-0.5$

1850.40

\begin{tabular}{ll|lll|l|l} 
& & & 19 & 36.80 & & ECB \\
131.98204 & PKP & 18 & 48.00 & -1.0 & HAM
\end{tabular}

\begin{tabular}{llll|l}
132.01 & 208 ePKP $18 \quad 49.50$ & 0.4 & GBR
\end{tabular}

1932.00 DAF

\begin{tabular}{lllll|l}
132.03 & 208 ePKP 18 & 49.50 & 0.3 & ECP
\end{tabular}

$\begin{array}{llllll}132.12 & 347 & \text { IPKP } & 18 & 33.40 & -14.6 \mathrm{X}\end{array}$

$0.5 \mathrm{~s}$ $4.70 \mathrm{~nm}$

HLD

$\begin{array}{lllll}132.51207 & \text { e(PKP) } 18 & 46.10 & -4.0 \times & \text { I LVV }\end{array}$

133.64343 ePKP $18 \quad 51.00$ EPPKP $19 \quad 38.00$

$\begin{array}{llll}\text { EPP } & 21 & 18.00\end{array}$

eSKS $25 \quad 35.00$ eSP 3122.00

134.25335 IPKPd 1853.00

$2.0 \mathrm{~s} \quad 550.00 \mathrm{~nm}$

$\begin{array}{llllll}134.42 & 347 & \text { IPKP } & 18 & 37.00 & -15.4 X\end{array}$

$0.8 \mathrm{~s} \quad 156.00 \mathrm{~nm}$

i 1852.00

iPPKP $19 \quad 42.20$

$\begin{array}{lll}\text { ePP } & 21 & 24.00\end{array}$

ISKP $22 \quad 09.00$

IPKS $22 \quad 22.00$

IPPKS $23 \quad 11.00$

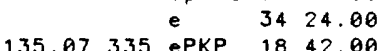

$\begin{array}{lllll}135.07 & 335 & \text { ePKP } 18 \quad 42.00 & -11.8 \times & \text { DBN }\end{array}$

$\begin{array}{llllll}135.07 & 335 & \text { IPKP } & 18 & 52.00 & -1.8\end{array}$

$1.0 \mathrm{~s} \quad 150.00 \mathrm{~nm}$

$\begin{array}{lll}Z & 22 \mathrm{~s} & 2.20 \mathrm{um} \\ \mathrm{N} & 22 \mathrm{~s} & 1.60 \mathrm{um}\end{array}$

E $20 \mathrm{~s} \quad 0.80 \mathrm{~mm}$

ESP 3130.00 $\begin{array}{ccccc}135.16311 & \text { IPP } & 31 & 30.00 \\ & \text { IPKC } & 18 & 56.00 \\ & \text { iPP } & 21 & 32.00\end{array}$

135.23295 ePKP $18 \quad 44.00-11.2 x$

135.85356 PKP $18 \quad 39.40-15.8 x$

$1.3 \mathrm{~s} \quad 117.00 \mathrm{~nm}$

$\begin{array}{llllll}136.00 & 311 & \text { IPKP } & 18 & 57.00 & 1.0\end{array}$

$\begin{array}{llllll}136.32 & 351 & \text { IPKP } & 18 & 42.00 & -14.0 \times\end{array}$

136.32351 1 $\quad 1856.20$
$1 \quad 2159.30$

$\begin{array}{ll}18 & 56.20 \\ 21 & 29.30\end{array}$

$22 \quad 27.20$

$\begin{array}{lll}23 & 17.30\end{array}$

136.54354 (PKP) $1842.80-14.5 x$

136.59213 PKP $1846.80-12.0 \times$

136.59213 PKPd 1900.00 ISKP $22 \quad 33.00$

137.01316 iPKPd $18 \quad 58.00$ $1.0 \mathrm{~s} \quad 300.00 \mathrm{~nm}$

$$
\begin{array}{lll}
\text { iPP } 2140.00 & 000
\end{array}
$$

137.63312 iPKP $18 \quad 49.00-10.2 x$

138.04311 iPKPC $1847.00-13.2 x$

138.04311 ePKP 1901.00

IPPKP 1951.80

$\begin{array}{lll}1 P P & 2153.00\end{array}$

138.29314 iPKPC $18 \quad 55.80$

138.41318 ePKP $18 \quad 51.00$

$138.53 \quad 309$ ePKP 1854.00

139.10 312 iPKPd $18 \quad 54.00$

139.15315 iPKP $18 \quad 55.00$

$139.33 \quad 314$ EPKP 1902.00

$140.54 \quad 357 \quad$ iPKPC $18 \quad 56.30$

$1.1 \mathrm{~s} \quad 100.00 \mathrm{~nm}$

$140.73 \quad 320$ ePKP $18 \quad 58.00$

$2.0 \mathrm{~s} \quad 400.00 \mathrm{~nm}$

141.05354 IPKPC $18 \quad 58.00$

$0.9 \mathrm{~s} \quad 128.00 \mathrm{~nm}$

$141.21 \quad 8$ PKPC 1858.60

$1.2 \mathrm{~s} \quad 47.00 \mathrm{~nm}$

$141.46 \quad 323$ ePKP 1856.00

$9.0 \mathrm{~s} 2700.00 \mathrm{~nm}$ $\begin{array}{lll}\text { ePP } 22 \quad 08.00 & 0\end{array}$
ESKS 2554.50

$\begin{array}{llllll}141.98 & 12 & \text { IPKPC } & 19 & 01.50 & -5.1 x\end{array}$

$1.0 \mathrm{~s} \quad 200.00 \mathrm{~nm}$

$\begin{array}{llllll}142.43 & 12 & \text { IPKPC } & 19 & 02.28 & -5.1 x\end{array}$

$1.7 \mathrm{~s} \quad 660.00 \mathrm{~nm}$

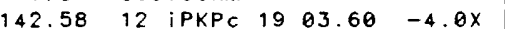

$1.3 \mathrm{~s} \quad 230.00 \mathrm{~nm}$

\begin{tabular}{lllll|l}
142.63 & 12 ePKP 19 & 03.60 & $-4.1 \times$ &
\end{tabular}

$1.2 \mathrm{~s} \quad 310.00 \mathrm{~nm}$

$\begin{array}{llllll}143.03 & 16 \text { PKP } 1902.00 & -6.4 \times & V R I\end{array}$ PPS $34 \quad 40.00$

\begin{tabular}{lllll|l}
143.20 & 325 ePKPd 19 & 05.00 & $-3.9 \times$ & UOS
\end{tabular}
KIS

$\begin{array}{lll}\text { ePP } \quad 22 & 21.00\end{array}$

$\begin{array}{llllll}143.26 & 12 & \text { IPKPC } & 19 & 07.70 & -1.1\end{array}$ $2.0 \mathrm{~s} \quad 720.00 \mathrm{~nm}$

$\begin{array}{lllll}143.34 & 266 & \text { ePKPd } 19 & 07.00 & -3.1 \mathrm{X}\end{array}$ $\begin{array}{llllll}143.46 & 13 & \text { iPKPC } & 19 & 06.90 & -2.2\end{array}$ $\begin{array}{lllll}143.47 & 355 & \text { iPKPd } 19 & 06.30 & -2.8 \mathrm{x}\end{array}$ 143.62266 ePKP+ $19 \quad 10.00-0.6$ $\begin{array}{lllll}143.66 & 266 \text { ePKP+ } 19 & 10.60 & 0.0\end{array}$ $\begin{array}{lllll}143.72 & 12 & \text { iPKPC } 19 & 07.70 & -1.8\end{array}$ $2.4 \mathrm{~s} 2200.00 \mathrm{~nm}$

143.75266 iPKP+ $19 \quad 10.80 \quad 0.1$ 144.00339 ePKPd $19 \quad 08.00 \quad-2.1$

$Z 20 \mathrm{~s} 2.20 \mathrm{um} \quad 5.9 \mathrm{Msz}$

$\mathrm{N} 20 \mathrm{~s} \quad 1.50 \mathrm{um}$

ePP $22 \quad 30.00$

ESKS $26 \quad 01.00$

$\begin{array}{lllllll}\text { BRL } & 144.10 & 352 & \text { ePKPd } 19 & 08.50 & -1.6\end{array}$

144.16352 iPKPd $19 \quad 08.20 \quad-2.1$

144.29359 iPKPd $1909.90 \quad-0.6$ e PPKP 2000.00

144.37332 IPKPd 1908.00

$3.0 \mathrm{~s} 2800.00 \mathrm{~nm}$

$Z 22 \mathrm{~s} \quad 1.90 \mathrm{um}$

I PPKP 1958.00

iPP $22 \quad 32.00$

$\begin{array}{llllllll}\text { KVT } & 144.37 & 319 & \text { iPKP } & 19 & 10.40 & -0.7\end{array}$

$\begin{array}{llllll}144.37 & 319 & \text { IPRP } & 19 & 10.40 & -0.7 \\ 144.80 & 333 & \text { ePKP } & 19 & 11.00 & -0.6\end{array}$

145.010 IPKP- $19 \quad 11.00-0.7$

i $19 \quad 15.00$

ePPKP 1956.00

$\begin{array}{lll}\text { EPP } \quad 22 & 23.00\end{array}$

ISKKS $30 \quad 32.00$

IPKKS 3245.00

e $\quad 3505.00$

iss 4256.00

$\begin{array}{lllllll}\text { KRA } & 145.03 & 344 & \text { IPKPd } & 19 & 11.30 & -0.6\end{array}$

$1.1 \mathrm{~s} 1560.00 \mathrm{~nm}$

$Z 22 \mathrm{~s} \quad 3.50 \mathrm{um}$

$\mathrm{N} 22 \mathrm{~s} \quad 2.30 \mathrm{um}$

E $22 \mathrm{~s} \quad 2.80 \mathrm{um}$

1914.20

$\begin{array}{ll}19 & 21.50\end{array}$

1925.20

1933.40

1952.08

2104.00

$22 \quad 28.00$

$\begin{array}{lllll}145.10 & 358 & \text { iPKPd } 19 & 11.40 & -0.5\end{array}$

$\begin{array}{lllll}145.16 & 348 & \text { iPKPd } 19 & 12.00 & -0.1\end{array}$

$1.2 \mathrm{~s} \quad 588.00 \mathrm{~nm}$

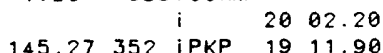

$1.3 \mathrm{~s} 2600.00 \mathrm{~nm}$

PPKP $20 \quad 01.00$

SPKP 2018.00

145.42333 iPKPd 1913.00

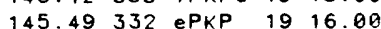

$\begin{array}{llll}145.54 & 332 & \text { IPKPd } 19 & 13.50\end{array}$

$\begin{array}{llll}145.55 & 350 & \text { IPKPd } 19 & 13.00\end{array}$

$2.2 \mathrm{~s} 2100.00 \mathrm{~nm}$

i 2803.50

$145.60 \quad 340$ ePKPd $19 \quad 13.00$ 


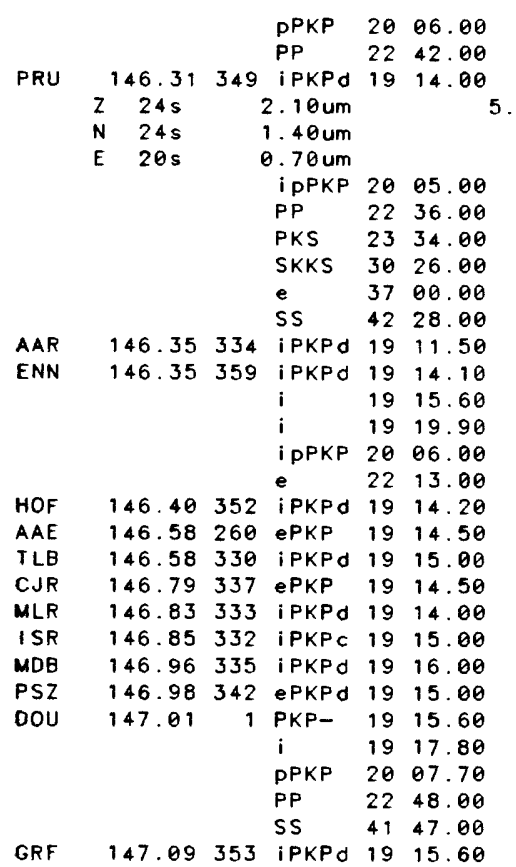

\begin{tabular}{ccccc}
147.09 & 353 & iPKPd & 19 & 15.60 \\
\hline & $24 \mathrm{~s}$ & $1.30 \mathrm{um}$ & &
\end{tabular} e $\quad 1922.90$

$\begin{array}{llllll} & & & e & 20 & 07.20 \\ \text { GRFO } & 147.09 & 353 & \text { ePKPO } & 19 & 15.00\end{array}$

$\begin{array}{llllll}\text { ANTO } & 147.10 & 319 & \text { PKP } & 19 & 16.00\end{array}$

$\begin{array}{lllllll}\text { KHC } \quad 147.30 \quad 350 & \text { iPKPd } & 19 & 15.80\end{array}$

$\begin{array}{lll}Z & 23 \mathrm{~s} & 2.50 \mathrm{um} \\ \mathrm{N} & 21 \mathrm{~s} & 2.10 \mathrm{um} \\ \mathrm{E} & 22 \mathrm{~s} & 1.90 \mathrm{~mm}\end{array}$

e $\quad 19 \quad 32.50$

PPKP 2005.00

$\begin{array}{lllllll}\text { CMP } \quad 147.40 & 334 & \text { IPKPC } & 19 & 16.00\end{array}$

$\begin{array}{llllll}\text { WET } & 147.40 & 351 & \text { IPKPO } 19 & 15.90\end{array}$

WLF $\quad 147.45359$ PKP- 1918.00

$$
\begin{array}{lll}
\text { PPKP } & 20 & 10.00 \\
P P & 22 & 48.00
\end{array}
$$$$
\text { PP } \quad 22 \quad 48.00
$$

$\begin{array}{llllll} & & \text { i } & 43 & 40.00 \\ \text { ZST } \quad 147.48 & 345 & \text { iPKPC } & 19 & 15.40\end{array}$

$\begin{array}{rll}i & 2006.90 \\ & i & 2046.90\end{array}$

SRO $\quad 147.52 \quad 344$ IPKPd $19 \quad 14.20$

$$
\text { i } \quad 2003.10
$$

(PP) $22 \quad 48.20$

VKA $\quad 147.59 \quad 346$ IPKPd $19 \quad 16.40$ $2.3 \mathrm{~s} \quad 3260.00 \mathrm{~nm}$

1922.20 2005.80 2009.30 $\begin{array}{ll}26 & 32.30\end{array}$ 2650.80

BUD $147.63 \quad 343$ iPKPd $19 \quad 15.90$

$\begin{array}{llllll}\mathrm{BOZ} & 147.64335 \text { IPKPC } 19 & 17.00\end{array}$

$\begin{array}{llllll}\text { BUC1 } 147.69 & 332 & \text { ePKP } 19 & 18.00\end{array}$

$\begin{array}{llllll}\text { DEV } & 147.74337 & \text { IPKPC } & 19 & 29.00\end{array}$

$\begin{array}{lllllll}\text { CGN } & 147.84 & 332 & \text { iPKPd } 19 & 21.00\end{array}$

$\begin{array}{llllll}\text { FLN } & 147.99 \quad 7 & \text { iPKPd } 19 & 17.00\end{array}$

$\begin{array}{llllll}\text { GWF } & 148.09 & 357 & \text { iPKP } 19 & 16.60\end{array}$

STU 148.20355 IPKPO 1916.90

$\begin{array}{llllll}\text { DRA } & 148.20 & 334 & \text { ePKP } & 19 & 17.00\end{array}$

$\begin{array}{llllll}\text { LDF } & 148.20 \quad 7 & \text { iPKPd } 19 & 17.40\end{array}$

KMR $\quad 148.24349$ iPKP- $19 \quad 17.20$

$\begin{array}{llllll}\text { GRR } & 148.31 & 8 & \text { iPKPd } & 19 & 17.80\end{array}$

$\begin{array}{llllll}\text { GPA } & 148.35 & 322 & \text { iPKP } & 19 & 17.10\end{array}$

BUH 148.36356 ePKPd 1917.50

$\begin{array}{llllll}\text { HRT } & 148.37 & 323 & \text { ePKP } & 19 & 21.80\end{array}$

$\begin{array}{lllllll}\text { STR } & 148.47 & 357 & \text { iPKPO } & 19 & 18.00\end{array}$

$\begin{array}{llllll}\text { ISK } & 148.55 & 324 & \text { ePKP } 19 & 17.50\end{array}$

FUR $148.58 \quad 352$ iPKPd 1917.70 1922.20 1925.00

LPF $\quad 148.64 \quad 8$ iPKPJ 1918.20

$\begin{array}{llllll}\text { CDF } & 148.67 & 358 & \text { ePKP } 19 & 18.30\end{array}$

BHG $148.78 \quad 350$ IPKPO $19 \quad 18.10$ $\begin{array}{llllll}\text { CTT } & 148.84 & 325 & \text { IPKP } & 19 & 18.00\end{array}$ $\begin{array}{lllllll}\text { ECH } & 148.87 & 358 & \text { iPKPO } 19 & 17.40\end{array}$ 0.0 | PVL $8 M S Z X \mid S S R$ I HAU I ALT SLE I BAF BSF I KBA $-2.8 x$ 0 . 149.02331 IPKPd $19 \quad 19.00$ $149.04 \quad 337$ ePKP $19 \quad 19.00$ 149.10359 IPKPO 1918.80 149.25320 ePKP $19 \quad 19.10$ 149.25356 ePKPd $19 \quad 18.50$ $149.26 \quad 358$ iPKPd $19 \quad 18.50$ $149.27 \quad 358$ ePKP $19 \quad 19.20$ 149.33349 PKPd $19 \quad 18.00$ $0.7 \mathrm{~s} \quad 280.00 \mathrm{~nm}$

1922.70 1926.30 2013.90 2237.60 2308.30

ZUL

I EDC

0.0 MFT

-1.4 DST

0.4 LOR

$-0.5 \mid$ OGA

-1.2 RBL

-0.1 SSF

$0.8 \mid K D Z$

$-0.3 \mid$ LJU 0.5

LLS MFF
AVF \begin{tabular}{c|c}
0.3 & AVF \\
$6 M S Z X$ & VTS
\end{tabular}

CEY
SMF -0.3 BGF $0.3|T R|$ 0.1
$9 \operatorname{Ms} 2 x$ ins $2 x$

0.3 DIX

I SAL

I

PRK I I ZM

$-0.3$

\begin{tabular}{l|l}
0.5 & \\
1.6 & YER
\end{tabular} $12.6 \mathrm{X}$ | SKO

4. $4 \times$

0.31

-0.3
-0.2

-0.2 RJF 0.3 I VAY 0.3
0.0

0.6 LFF $-0.5 \mid$ 0.1 I 0.5 LPO -0.4 OHR 0.0

0.4 PRT 0.3 $0.0 \quad K Z N$

149.54356 ePKPd $19 \quad 18.80$ 149.71325 iPKPd $19 \quad 19.00$ $149.72 \quad 326$ iPKP $19 \quad 19.50$ $149.77 \quad 323$ iPKPO $19 \quad 19.70$
149.82 $\begin{array}{llllll}149.82 & 2 & \text { iPKPd } 19 & 20.30\end{array}$ 149.89352 IPKPO 1920.38 $149.90 \quad 349$ PKP 1920.00 150.023 IPKPd 1920.60 $150.08 \quad 329$ IPKPd $19 \quad 19.00$ 150.09347 iPKPd 1920.20 i $\quad 1925.60$ (PP) $23 \quad 44.10$

$150.10 \quad 355$ ePKPO 1920.30 $150.16 \quad 8$ iPKPO 1920.70 150.283 IPKPO 1920.50 $150.33 \quad 333$ iPKPd 1920.00 $150.40 \quad 347$ iPKPd 1920.50 $\begin{array}{llll}150.45 \quad 2 \text { ePKP } 19 & 21.10\end{array}$ 150.494 IPKPd 1921.10 150.57348 iPKP 1920.50 $\begin{array}{lll}i & 20 & 12.10 \\ \text { eSKP } & 22 & 55.40\end{array}$ e 2404.00 ESKS $33 \quad 16.00$ e 3416.00 ESPP 3728.00 e $\quad 3822.00$ ESS 4324.00 ESSS $44 \quad 10.00$ ESSS 4936.00

150.61351 PKP 1920.50 $+\quad 1926.50$ 2305.00 $\begin{array}{llll}150.695 & 5 \text { IPKPO } 19 & 21.10\end{array}$ $\begin{array}{lllll}150.71 \quad 5 & \text { iPKPd } 19 & 21.40\end{array}$ $150.76 \quad 317$ iPKPd 1921.00 $\begin{array}{lllll}150.814 & 4 & \text { iPKPd } 19 & 21.90\end{array}$ 150.87355 EPKPd 1921.10 150.99356 ePKPd 1922.20 150.99357 ePKPd 1922.50 $\begin{array}{llll}151.19353 \text { PKP } 1921.50 & 19\end{array}$ e $\quad 1928.50$ $\begin{array}{lll}e & 22 & 38.00 \\ \text { EPKP } & 19 & 22.00\end{array}$ $\begin{array}{lllll}151.35 & 325 & \text { EPKP } & 19 & 22.00 \\ 151.37 & 322 & \text { iPKPd } & 19 & 22.10\end{array}$ $\begin{array}{lllll}151.37 & 322 & \text { iPKPd } & 19 & 22.10 \\ 151.41 & 356 & \text { PKP } & 19 & 21.50\end{array}$ $\epsilon \quad 1928.50$ e $\quad 2307.50$ $151.57 \quad 319$ ePKP 1922.50 151.60334 iPKPd $19 \quad 22.20$ 1929.20 1939.00 iPP 2311.00 iSKSP 3324.00 $151.63 \quad 6$ iPKPd 1923.10 151.64332 iPKP 1921.50 1927.30

$\begin{array}{llll}151.91 \quad 7 & \text { iPKPd } 19 & 23.60\end{array}$

152.02354 PKP 1923.50 1930.50 $\begin{array}{lll}152.05 \quad 5 & 2316.00\end{array}$

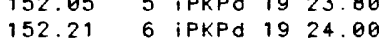
152.58334 iPKPd 1923.80 $\begin{array}{ccccc}152.58334 & \text { iPKPd } & 19 & 23.80 \\ & i & 19 & 31.80\end{array}$ 1942.30 $\begin{array}{lllll}152.81 \quad 351 & \text { PKP } & 19 & 26.50\end{array}$ PPKP 2001.00 PP 2356.00

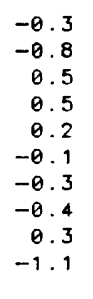

I STV

I FIR

FRF

LRG

ATH

POI

LMR

AOU

MNS

FOG

DU -0.5
-0.7 -0.3
-0.2 0.6

0.3

0.1

0.7

0. 1

0.0
0.6
0.2
-0.5
-0.1
0.5
0.5
-0.3

0.6 EBR

GIB SFS

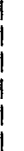

I ALI

I ERC I ME ।

ALM $-0.5$

CVF

NPS
RMP

RDP

sGo

VLS

R

$152.83 \quad 357$ PKP

$1924.00 \quad-0.3$ $\begin{array}{lllll}19 & 351 \text { PKP } 1924.00 & -0.2\end{array}$ PP 2315.00 $\begin{array}{llll}153.54 & 358 & \text { ePKP } 19 & 25.30\end{array}$ 153.65358 iPKPd 1926.20 153.66326 IPKPd 1925.00 $\begin{array}{lllll}153.73 \quad 8 \text { ePKP } 19 & 26.30\end{array}$ 153.74347 PKP 1925.00 153.77358 ePKP 1925.80 153.85346 PKP 1926.00 $\begin{array}{lllll} & & \in & 23 & 12.00 \\ 153.98 & 348 & \text { PKP } & 19 & 25.00\end{array}$ $\begin{array}{lll}1 & 23 \quad 14.00\end{array}$ 154.15342 PKP $19 \quad 39.50$ 154.25344 PKP 1926.50 154.36337 PKP 1927.00 2327.00

154.38354 iPKPd 1926.70 $154.42 \quad 319$ ePKP 1927.50 154.52347 PKPd 1926.00 $\begin{array}{rll}e & 2320.00 \\ e & 2608.00\end{array}$ 154.57347 PKP 1927.00 $\begin{array}{ll}19 & 27.00 \\ 19 & 50.50\end{array}$ 2326.50

155.04342 PKP 1927.00 2322.00

155.07331 ePKP 1927.50

155.14340 PKP 1927.00 $\begin{array}{ccccc}155.92 & 9 & \text { ePKP } & 19 & 24.00 \\ & \text { ePP } & 23 & 30.00\end{array}$ $157.80 \quad 342$ PKP 1934.00 158.0926 IPKPd 1934.00 $\begin{array}{lll}\text { ePP } & 20 & 10.00 \\ \text { eP } & 44.00\end{array}$ e $\quad 2708.00$ eSKS $34 \quad 15.00$ $\begin{array}{lll}3 & 3820.00\end{array}$ $158.14 \quad 13$ iPKP+ 1933.50 iPP 2347.50

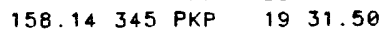
$\epsilon \quad 2345.00$ 158.17339 PKP 1931.20 $159.07 \quad 18$ IPKPO $19 \quad 32.70$ $1.5 \mathrm{~s} \quad 6.10 \mathrm{~nm}$ $20 \quad 10.90$ 2101.50

$165.48 \quad 136$ IPKPd $1939.0 \theta$ S.D. $=0.9$ on 461 of 529 obs

0. 2 0.9

$-0.5$ 


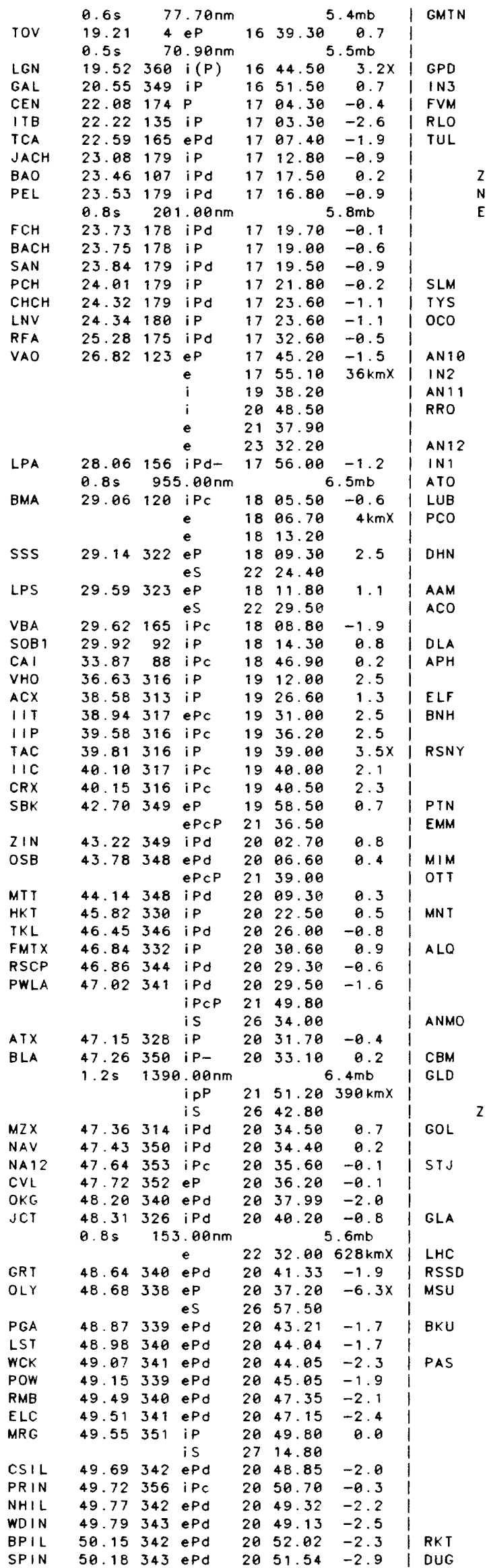

50.20357 IP $50.35357 i$ $50.37 \quad 345 \mathrm{iPd}$ $50.54340 \mathrm{ePd}$ $50.66335 \mathrm{iPd}$ 50.76334 iPd$0.7 \mathrm{~s} \quad 14.00 \mathrm{~nm}$ $z 20 \mathrm{~s} \quad 1.02 \mathrm{um}$ $N 22 \mathrm{~s}$ $0.48 \mathrm{um}$ 215 D. 69 um

$$
\text { e }
$$

$51.06341 \mathrm{ePd}$ $51.07340 \mathrm{ePd}$

$51.19332 \mathrm{iPd}$

(Lg)

$\begin{array}{lll}51.21 & 347 & \mathrm{iPd} \\ 51.26 & 345 & \mathrm{iPd}\end{array}$

$51.34 \quad 347 \quad \mathrm{PPd}$

51.54332 iPd

$0.5 \mathrm{~s} \quad 595.00 \mathrm{~nm}$

$51.58 \quad 347$ iPd

$51.61346 \mathrm{Pd}$

51.70332 iP

$51.82327 \mathrm{eP}$

$51.94 \quad 334$ iPd

$52.46 \quad 354 \quad \mathrm{iPd}$

$52.79348 \quad \mathrm{iPd}$

52.92332 iPd

$0.6 \mathrm{~s} \quad 324.00 \mathrm{~nm}$

$52.96350 \mathrm{P}$

$53.17 \quad 357$ iPd

$53.24351 \stackrel{\mathrm{ePP}}{\mathrm{P}}$

$53.84 \quad 360 \quad \mathrm{iPd}$

53.88357 iPCP

$\begin{array}{lll}53.88 & 357 & \text { IPd } \\ & & \text { iPCP } \\ & & \text { iPP }\end{array}$

$\begin{array}{lll}53.93 & 357 & \text { iPP } \\ 54.89 & 3 & \text { IPd }\end{array}$

$\begin{array}{rrr}54.093 & 3 \text { IPd } \\ \text { ePP } & \end{array}$

$54.522 \mathrm{iPd}$

$\begin{array}{ll}54.79 & 356 \mathrm{eP} \\ 1.45 & 628.00 \mathrm{~nm}\end{array}$

$\begin{array}{lll}1.45 & 628.00 \mathrm{~nm} \\ 54.79 & 358 \mathrm{iPd}\end{array}$

$0.8 \mathrm{~s} 1400.00 \mathrm{~nm}$

$\begin{array}{ll}55.36 & 325 \mathrm{iPd} \\ 0.9 \mathrm{~s} & 149.00 \mathrm{~nm}\end{array}$

$$
\begin{aligned}
& \text { ePCP } \\
& \text { ePP }
\end{aligned}
$$

$55.36325 \mathrm{iPd}$

$56.25 \quad 3 \quad \mathrm{iPd}$

$58.26330 \mathrm{iPC}$

$\begin{array}{rr}1.5 \mathrm{~s} & 2500.00 \mathrm{~nm} \\ 18 \mathrm{~s} & 2.76 \mathrm{um}\end{array}$

58.30329 iPd
eS

$\begin{array}{ll}59.15 & 14 \mathrm{eS} \\ 0.5 \mathrm{~s} & 590.00 \mathrm{~nm}\end{array}$

$\begin{array}{cc}0.5 \mathrm{~s} & 590.00 \mathrm{~nm} \\ & \mathrm{PP} \\ 59.28 \quad 318 \mathrm{iPd} & \end{array}$

$\begin{array}{lll}59.83 & 346 \quad \mathrm{iPd}\end{array}$

$\begin{array}{lll}59.83 & 346 & \mathrm{iPd} \\ 61.10 & 334 & \mathrm{iPc}\end{array}$

$61.13324 \mathrm{iPC}$

$61.70323 \quad \mathrm{iP}$

iPd

7 IPd

$62.17317 \mathrm{iPd}$

PPCP 22

$\begin{array}{lll}\text { ePP } & 24 & 16.00 \\ & 25 & 05.00\end{array}$

is 3000.00

eSP $30 \quad 26.00$

eSCS 3107.00

ess 3326.00

eSS $34 \quad 30.00$

eSSS $38 \quad 01.00$

$\begin{array}{lllll}62.25 & 249 & \text { iP } & 22 & 12.00\end{array}$

$62.63325 \mathrm{iPd}$ $\begin{array}{llll}20 & 54.4 \theta & -0.2 & \text { | BOW }\end{array}$ $2 \theta 329 \mathrm{kmX}$ I MOUT \begin{tabular}{ll|l}
55.60 & -0.1 & RSON
\end{tabular} $\begin{array}{lll}54.30 & -1.6\end{array}$ \begin{tabular}{ll|l}
55.21 & $-2 . \theta$ & WKTM
\end{tabular}

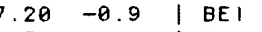
$30-0.6$ SCCM 4.8Msz I $\left\{\begin{array}{l}I M W \\ T M I\end{array}\right.$ \begin{tabular}{l|l}
\hline & 43.00
\end{tabular} \begin{tabular}{ll|l}
58.50 & \\
58.63 & -2.4 & MNA
\end{tabular} \begin{tabular}{ll|l}
$58.8 \theta$ & -2.3 & | FRI
\end{tabular} \begin{tabular}{ll|l}
26.50 & -0.6 & I PRI
\end{tabular} 00.50

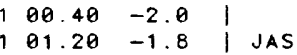
$6.3 \mathrm{mb}$ $2.80-2.0$ \begin{tabular}{ll|l}
.48 & -1.3 & $\mathrm{MHC}$
\end{tabular} \begin{tabular}{lll|l|l}
21 & 07.10 & -0.2 & I LRM
\end{tabular} $09.50291 \mathrm{kmX}$ | BKS 210.70

$11.80-1.6$ $5.9 \mathrm{mb}$ $7.80601 \mathrm{~km}$ \begin{tabular}{lll|l}
$20.5 \theta$ & -0.2 & ORV \\
& 20.2 & NWRM
\end{tabular} \begin{tabular}{l|l}
20.78 & $\mathrm{KIC}$
\end{tabular} $2 \theta .4 \theta-\theta .9$ WDC $\begin{array}{lll}22.40 & 0.0\end{array}$ $26.30-1.3$ $5.8 \mathrm{mb}$ $6.3 \mathrm{mb}$

3
SES FFC PNO PNO \begin{tabular}{l|l|l}
23 & $25.00600 \mathrm{~km}$ & LOM \\
& FHC
\end{tabular} $2137.30 \quad 0.0 \quad$ NEW $6.2 \mathrm{mb}$ FCC (a) $.50-0.3$ FOR
SHW LON $2352.00602 \mathrm{~km}$ | 2922.00 2210.10 $\begin{array}{lll}29 & 48.28\end{array}$ $\begin{array}{ll}22 & 14.60\end{array}$ $\begin{array}{ll}22 & 14.60 \\ 29 & 53.00\end{array}$ $\begin{array}{ll}22 & 12.00 \\ 22 & 20.30\end{array}$
62.70329 iPd 62.79326 iPd $62.99319 \mathrm{iPd}$ 63.20344 iPd eS

$63.31318 \mathrm{iPC}$ $63.38327 \mathrm{ePd}$ $63.98316 \mathrm{ePC}$ 64.00323 iP $64.18 \quad 3 \mathrm{iPd}$ PP

$64.28329 \mathrm{iPO}$ 64.29328 iPd $64.63317 \mathrm{ePd}$

$64.69321 \mathrm{PPO}$ $64.84319 \mathrm{iPd}$ $64.98 \quad 317 \mathrm{iPd}$ $65.35 \quad 323 \quad \mathrm{iPd}$

$\begin{aligned} & \text { epP } \\ & e S \\ 65.86319 & \text { iPd }\end{aligned}$

$\begin{array}{ccc} & \mathrm{e} \\ 66.24318 & \stackrel{\mathrm{iPC}}{\mathrm{eS}} \\ & & \mathrm{es}\end{array}$

$66.31318 \mathrm{iPd}$

$66.32330 \mathrm{iPd}$

66.99318 iPd

$0.8 \mathrm{~s} \quad 70.00 \mathrm{~nm}$

e
e
is
e
e

$67.46 \quad 320 \quad \mathrm{iPd}$

67.74318 iPd

$68.08 \quad 79 \mathrm{iPd}$

$\begin{array}{lll} & & \text { S } \\ 68.41 & 320 & \text { iP } \\ 68.70 & 320 & \text { iPd }\end{array}$

$68.70320 \mathrm{iPd}$

$\begin{aligned} 68.91321 & \text { iPd } \\ & \text { ePCP }\end{aligned}$

PP

$\begin{array}{ll}68.97 & 334 \text { iPd } \\ 0.5 \mathrm{~s} & 386.00 \mathrm{~nm}\end{array}$

$69.01341 \mathrm{iPd}$ $P P$

$69.48 \quad 326 \mathrm{ePd}$

$69.56330 \mathrm{iP}-$

69.73320 iPd

$70.32 \quad 329$ iPd i

70.50348 iPd

$0.6 \mathrm{~s} \quad 502.00 \mathrm{~nm}$

$71.43 \quad 324$ iPd

$71.75325 \mathrm{iPc}$

$71.85326 \mathrm{iPd}$

72.01335 iPd $P P$

$\begin{array}{ll}72.26 & 329 \mathrm{iPd} \\ 1.0 \mathrm{~S} & 552.00 \mathrm{~nm}\end{array}$

$552.00 \mathrm{~nm}$

$\begin{array}{rlr}72.47325 & \mathrm{PP} \\ & \mathrm{iPC} \\ & \mathrm{epP}\end{array}$

$72.86326 \quad \mathrm{PPO}$

iPd

$73.06 \quad 1 \mathrm{eP}$ ePP $\quad 25 \quad 28.80591 \mathrm{~km}$ eS $32 \quad 10.50$

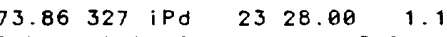
$0.7 \mathrm{~s} \quad 292.00 \mathrm{~nm} \quad 5.9 \mathrm{mb}$ $\begin{array}{lllllll}74.31 & 257 & \text { iP } & 23 & 30.80 & 0.8\end{array}$ $1.1 \mathrm{~s} \quad 175.00 \mathrm{~nm} \quad 5.5 \mathrm{mb}$ $\begin{array}{lllllllllll}74.54 & 257 & \text { iP } & 23 & 32.00 & 0.7\end{array}$ $1.1 \mathrm{~s} \quad 185.00 \mathrm{~nm} \quad 5.5 \mathrm{mb}$ $\begin{array}{llllll}74.56 & 257 \text { iP } & 23 & 32.48 & 1.8\end{array}$ $1.1 \mathrm{~s} 200.00 \mathrm{~nm} \quad 5.6 \mathrm{mb}$ $\begin{array}{llllll}74.82 & 257 & \text { iP } & 23 & 33.80 & 0.9\end{array}$ 


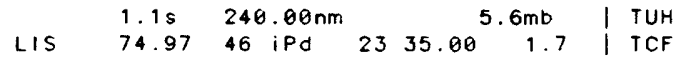

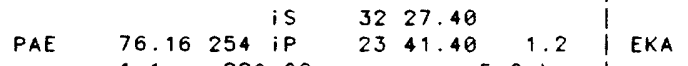

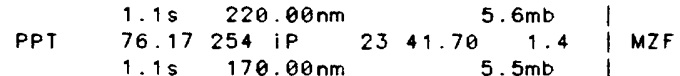

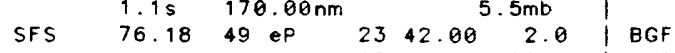

\begin{tabular}{cccccc|c} 
& e & 25 & 46.00 & $593 \mathrm{~km}$ & AVF \\
AFR & 76.36254 & is & 32 & 44.00 & & \\
& iP & 23 & 42.40 & 1.1 & SSF
\end{tabular}

\begin{tabular}{lllll|l|l} 
& $1.1 \mathrm{~s}$ & $480.00 \mathrm{~nm}$ & $5.9 \mathrm{mb}$ & \\
PTO & 76.40 & $44 \mathrm{iPd}$ & 23 & 42.00 & 1.0 & SMF
\end{tabular} IPP $25 \quad 45.00$

is 3248.80 LOR

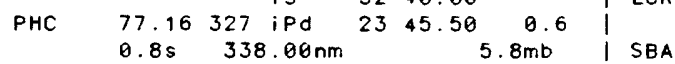

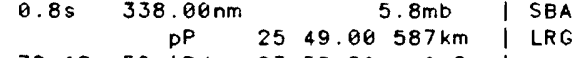
\begin{tabular}{cccc|c}
79.12 & $50 \mathrm{iPd}$ & 23 & 55.80 & 0.2 \\
$1.1 \mathrm{~s}$ & $6.20 \mathrm{~nm}$ & $4.0 \mathrm{mb} \times$ & LMR
\end{tabular} $\begin{array}{lll}\text { iPP } 26 \quad 03.30 & \end{array}$

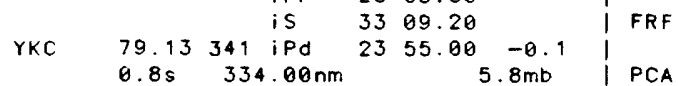

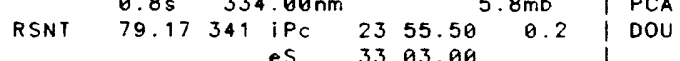

$\begin{array}{llllll}\text { YKA } & 79.19341 \mathrm{eS} & 33 & 03.00 & \\ \text { YP } & 23 & 56.20 & 0.8\end{array}$

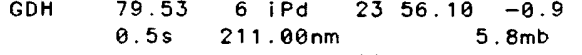

$\begin{array}{lllllll} & \text { SPA } & 80.56 & 180 & \text { iPC } & 24 & 05.00 \\ & 1.05 & 395 & 0.1\end{array}$

\begin{tabular}{ccccc|c} 
& $1.0 \mathrm{~s}$ & $395.00 \mathrm{~nm}$ & $5.9 \mathrm{mb}$ & \\
LGR & 81.10 & $44 \mathrm{iPd}$ & 26 & $10.00601 \mathrm{~km}$ & UCC
\end{tabular} $1.7 \mathrm{~s} \quad 1.28 \mathrm{~nm} 2407.803 .1 \mathrm{mbx}$ ipP $26 \quad 13.80593 \mathrm{~km}$

$\begin{array}{lll}\text { i (PP) } & 26 & 22.50 \\ \text { is } & 33 & 26.50\end{array}$

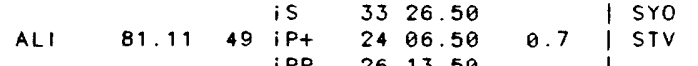

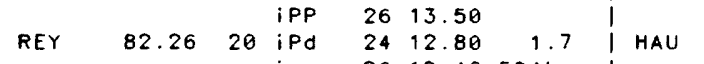

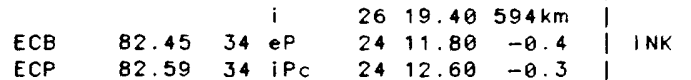

\begin{tabular}{llllll|l} 
ECP & 82.59 & $34 \mathrm{iPc}$ & 24 & 12.60 & -0.3 & \\
& $1.6 \mathrm{~s}$ & $870.00 \mathrm{~nm}$ & & $6.0 \mathrm{mb}$ & $\mathrm{DIX}$
\end{tabular}

$\begin{array}{lllllllll}D C N & 82.61 & 33 & \mathrm{iPC} & 24 & 13.28 & 0.2 & \mathrm{BSF}\end{array}$

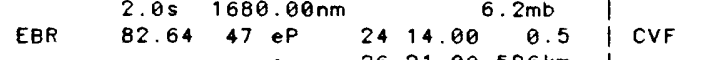
e $\quad 2621.00596 \mathrm{~km}$ eS 3338.00

\begin{tabular}{llllll|l} 
& & $e$ & 37 & 31.00 & & DAF \\
ETA & 82.90 & $34 \mathrm{iPC}$ & 24 & 14.50 & 0.0 &
\end{tabular}

$\begin{array}{llllll} & 1.4 \mathrm{~s} & 860.00 \mathrm{~nm} & & 6.1 \mathrm{mb} \\ \text { DLE } & 82.98 & 33 \mathrm{iPC} & 24 & 14.90^{0.0}\end{array}$

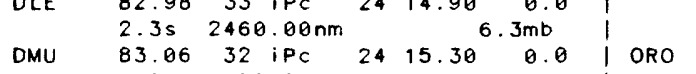

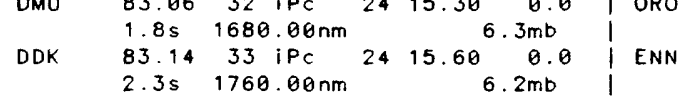

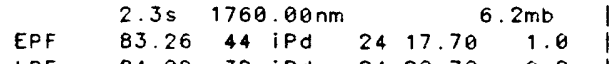

LPF $\quad 84.09 \quad 39$ iPd $2428.78 \quad 0.2$

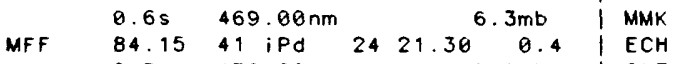

LFF \begin{tabular}{llll|l}
$0.5 \mathrm{~s}$ & $172.80 \mathrm{~nm}$ & \multicolumn{1}{c}{$5.9 \mathrm{mb}$} & $\mathrm{CDF}$
\end{tabular}

\begin{tabular}{ccc|c}
84.17 & $43.1 P d$ & 24 & 21.50 \\
$0.8 \mathrm{~s}$ & $275.08 \mathrm{~nm}$ & $5.9 \mathrm{mb}$ & $V G 1$
\end{tabular}

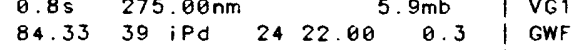
\begin{tabular}{lll|l}
$0.7 \mathrm{~s}$ & $350.00 \mathrm{~nm}$ & $6.1 \mathrm{mb}$ & TMA
\end{tabular}

\begin{tabular}{llllll|l|l} 
SIT & 84.37 & 330 & $\mathrm{ePd}$ & 24 & 22.50 & 0.8 & ZUL \\
LPO & 84.39 & 43 & $\mathrm{iPd}$ & 24 & 22.50 & 0.4 & $\mathrm{MBC}$
\end{tabular}

\begin{tabular}{llllll|l} 
LPO & 84.39 & $43 \mathrm{iPd}$ & 24 & 22.50 & 0.4 & MBC \\
& $1.0 \mathrm{~s}$ & $144.00 \mathrm{~nm}$ & & $5.6 \mathrm{mb}$ & \\
AKU & 84.50 & $19 \mathrm{iPd}$ & 24 & 24.00 & 1.9 & WTS
\end{tabular}

OKP $\quad 84.67 \quad 119 \quad \mathrm{PPd} \quad 26 \quad 30.90592 \mathrm{~km}$ $\begin{array}{ccccc}84.67 & 119 \mathrm{iPd} & 24 & 23.30-0.6 \\ 0.5 \mathrm{~s} & 25.00 \mathrm{~nm} & 5.1 \mathrm{mb}\end{array}$

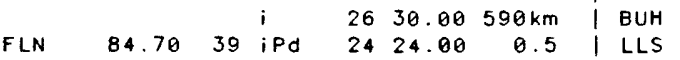

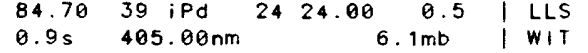

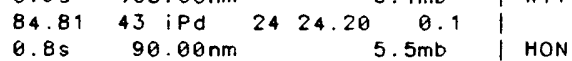
\begin{tabular}{lllll|l}
$0.8 \mathrm{~s}$ & $90.00 \mathrm{~nm}$ & & $5.5 \mathrm{mb}$ & HON \\
84.86 & $39 \mathrm{iPd}$ & 24 & $24.70^{0.4}$ & $\mathrm{KIP}$
\end{tabular}

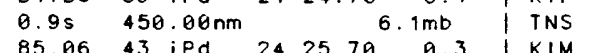

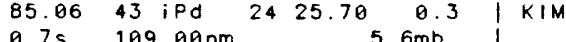

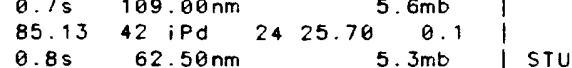

$\begin{array}{llllll}85.17 & 123 & \text { eP } & 24 & 24.50 & -1.6\end{array}$

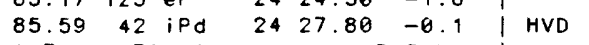
\begin{tabular}{llll|l}
$0.7 \mathrm{~s}$ & $70.00 \mathrm{~nm}$ & $5.5 \mathrm{mb}$ & \\
85.64 & $32 \mathrm{Pd}$ & 24 & $27.50^{5}-0.3$ & ERC
\end{tabular}

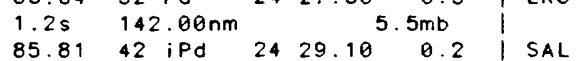
\begin{tabular}{lllll|l}
85.81 & $42 \mathrm{iPd}$ & 24 & 29.10 & 0.2 & $\mathrm{SAL}$
\end{tabular} $\begin{array}{llllll}1.1 \mathrm{~s} & 113.00 \mathrm{~nm} & & & 5.5 \mathrm{mb} & \\ 86.09 & 42 \mathrm{iPd} & 24 & 30.40^{0} & 0.2 & \text { PRT }\end{array}$

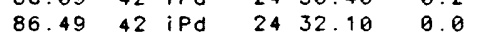
$0.8 \mathrm{~s} \quad 55.00 \mathrm{~nm} \quad 5.3 \mathrm{mb}$ $\begin{array}{lllllll}86.68 & 41 & \mathrm{iPd} & 24 & 32.80 & -0.2\end{array}$ $\begin{array}{lll}1.1 \mathrm{~s} & 102.00 \mathrm{~nm} \quad 5.5 \mathrm{mb}\end{array}$ $\begin{array}{llllll}86.77 \quad 42 \text { iPd } 24 & 33.70 & 0.2 & \text { DAG }\end{array}$ $\begin{array}{lll}1.2 \mathrm{~s} & 203.00 \mathrm{~nm} & 5.7 \mathrm{mb}\end{array}$ $\begin{array}{llllll}86.97 & 41 & \text { iPd } & 24 & 34.10 & -0.3\end{array}$ $1.0 \mathrm{~s} \quad 131.00 \mathrm{~nm} \quad 5.6 \mathrm{mb}$ $\begin{array}{llllllll}87.16 & 190 & \text { iP } & 24 & 35.50 & 0.6\end{array}$

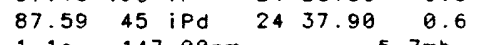
$\begin{array}{lll}1.1 \mathrm{~s} & 147.00 \mathrm{~nm} & 5.7 \mathrm{mb}\end{array}$ $\begin{array}{llllll}87.66 & 46 & \text { IPd } & 24 & 38.10 & 0.4\end{array}$ $\begin{array}{lll}1.1 \mathrm{~s} & 160.00 \mathrm{~nm} \quad 5.7 \mathrm{mb}\end{array}$ $\begin{array}{lllll}87.82 & 45 & \mathrm{iPd} & 24 & 38.70 \quad 0.3\end{array}$ $0.9 \mathrm{~s} \quad 118.00 \mathrm{~nm} \quad 5.7 \mathrm{mb}$ $\begin{array}{lllllll}88.00 & 332 \quad \mathrm{iPC} & 24 & 39.50 & 0.5\end{array}$

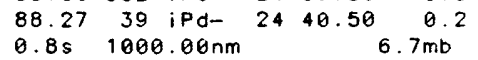

$$
\begin{array}{llll}
\text { PP } & 26 & 49.00 & 594 \mathrm{~km} \\
\text { PPPP } & 31 & 10.00
\end{array}
$$$$
\begin{array}{llll}
\text { SKS } & 34 & 12.50
\end{array}
$$$$
\text { S } \quad 34 \quad 33.80
$$$$
\text { SP } \quad 35 \quad 45.00
$$$$
\text { SSKS } 38 \quad 16.00
$$

$88.33 \quad 38$ PC- $2441.08 \quad 0.4$

PP $26 \quad 49.30592 \mathrm{~km}$

SKS $34 \quad 14.00$

$\begin{array}{lll}S & 34 & 36.00 \\ \text { SP } & 35 & 42.00\end{array}$

88.44160 iP $\quad 2441.40$

$\begin{array}{llll}88.46 & 45 P & 2441.50 & 0.0\end{array}$

$\begin{array}{lll}P P & 26 & 51.50\end{array}$

$\begin{array}{llllll}88.79 & 41 & \mathrm{iPd} & 24 & 42.80 & -0.1\end{array}$

$\begin{array}{lll}1.5 \mathrm{~s} & 144.00 \mathrm{~nm} & 5.7 \mathrm{mb}\end{array}$

88.94341 iPd $24 \quad 43.70 \quad 0.6$

\begin{tabular}{lll}
$0.3 \mathrm{~s}$ & $241.00 \mathrm{~nm}$ & \multicolumn{1}{c}{$6.6 \mathrm{mb}$} \\
88.99 & $43 \mathrm{ePd}$ & $2444.6 \theta^{\theta .5}$
\end{tabular}

$89.03 \quad 41$ iPd $24 \quad 43.90 \quad-0.2$

$\begin{array}{lll}1.5 \mathrm{~s} & 207.00 \mathrm{~nm} & 5.8 \mathrm{mb}\end{array}$

$89.15 \quad 47$ iPd $24 \quad 44.90 \quad 0.3$

$1.3 \mathrm{~s} \quad 87.80 \mathrm{~nm} \quad 5.5 \mathrm{mb}$

$89.17 \quad 41$ iPd $24 \quad 44.50 \quad-0.2$

$\begin{array}{rrrrr}89.20 & 37 \text { iP- } & 24 & 46.00 & 1.5 \\ & \text { ipP } & 26 & 53.00 & 584 \mathrm{~km}\end{array}$

$\begin{array}{llll}\text { ipP } & 26 & 53.00 & 584 \mathrm{~km} \\ \text { iSKS } & 34 & 18.00\end{array}$

is 3443.00

esSS 3822.00

$\begin{array}{llllll}89.26 & 44 \mathrm{Pd} & 38 & 22.00 & \\ & & 245.00 & -0.2\end{array}$

$\begin{array}{lllll} & \mathrm{PP} & 26 & 54.20 & \\ 89.27 & 38 \mathrm{iPd} & 24 & 45.30 & 0.4\end{array}$

$0.9 \mathrm{~s} \quad 191.00 \mathrm{~nm} \quad 2653.50^{6.0 \mathrm{mb}}$ $\begin{array}{lll}\text { epP } & 26 & 53.50 \\ \text { eS } & 34 & 39.50\end{array}$

$\begin{array}{llllll}89.36 & 43 \mathrm{iPd} & 24 & 46.60 & 0.8\end{array}$

89.3741 iPd $2445.60 \quad 0.1$

$\begin{array}{ccccc}89.50 & 41 \mathrm{iPd} & 24 & 46.20 & 0.0 \\ 1.1 \mathrm{~s} & 152.00 \mathrm{~nm} & & 5.8 \mathrm{mb}\end{array}$

$\begin{array}{lllll}89.86 & 44 P & 24 & 48.50 & 0.8\end{array}$

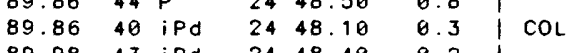

$\begin{array}{llllll}89.98 & 43 & \mathrm{iPd} & 24 & 48.40 & -0.2\end{array}$

$89.99 \quad 42$ ePd $24 \quad 48.50 \quad 0.1$

\begin{tabular}{cccc|c|c}
90.10 & 350 iPd & 24 & 49.00 & 0.7 & FBA \\
$0.5 \mathrm{~s}$ & $288.00 \mathrm{~nm}$ & $6.5 \mathrm{mb}$ & BHC
\end{tabular}

\begin{tabular}{llll|l}
$0.5 \mathrm{~s}$ & $288.00 \mathrm{~nm}$ & & $6.5 \mathrm{mb}$ & BHG \\
90.14 & $37 \mathrm{iPd}$ & 24 & $49.60^{0.7}$ & DUI
\end{tabular}

$1.1 \mathrm{~s} \quad 171.00 \mathrm{~nm} \quad 5.9 \mathrm{mb}$

i 2452.10

ipP $\quad 2658.90596 \mathrm{~km}$

$\begin{array}{rrrrrr} & & \mathrm{eS} & 34 & 43.50 & \\ 90.17 \quad 41 \mathrm{eP} & 24 & 48.90 & -0.3\end{array}$

$\begin{array}{rrrrr}90.25 & 43 \mathrm{ePd} & 24 & 50.00 & 0.2\end{array}$

$\begin{array}{rrrrr}90.27 & 36 \mathrm{ePd} & 24 & 51.00 & 1.6 \\ & \mathrm{iPP} & 26 & 58.60 & 586 \mathrm{~km}\end{array}$

$90.44291 \mathrm{ePd} 2451.80 \quad 0.9$

90.47292 iPC $2452.00 \quad 1.0 \quad$ SEK

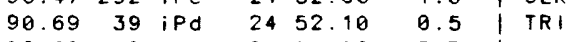

$90.69 \quad 119$ iPd $24 \quad 54.98 \quad 2.7$

$0.9 \mathrm{~s} \quad 106.00 \mathrm{~nm} \quad 5.8 \mathrm{mb}$

WET

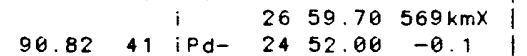

$\begin{array}{lll}1.0 \mathrm{~s} & 120.00 \mathrm{~nm} \quad 5.9 \mathrm{mb}\end{array}$

$90.94 \quad 121$ iPd $24 \quad 53.70 \quad 0.3$

$\begin{array}{rrrrrr} & i & 27 & 01.90 & 588 \mathrm{~km} \\ 90.95 & 52 \mathrm{p} & 24 & 53.50 & 0.6\end{array}$

$\begin{array}{llllll}91.00 & 44 & P P & 27 & 03.60 & \\ & & 24 & 53.50 & 0.5 \\ & & 27 & 02.50 & \end{array}$

$\begin{array}{llllll}91.02 & 46 P P & 27 & 02.50 & \\ & & 24 & 54.50 & 1.4\end{array}$

$\begin{array}{cll}P P & 27 & 09.00626 \mathrm{kmX}\end{array}$

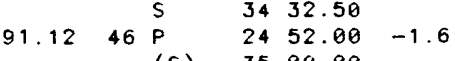

$\begin{array}{llllll}91.21 & 11 \mathrm{iPd} & 24 & 53.70 & 0.3\end{array}$

$0.9 \mathrm{~s} \quad 134.00 \mathrm{~nm}$

$6.0 \mathrm{mb}$

$91.28 \quad 124$ iPd $24 \quad 53.30 \quad-1.4$

$0.4 \mathrm{~s} \quad 1150.00 \mathrm{~nm} \quad 7.3 \mathrm{mb} x$

$91.47 \quad 118$ iPd $2455.00 \quad-0.8$

$91.60 \quad 43$ e $\quad 2705.00598 \mathrm{~km}$

$91.77 \quad 2705.40593 \mathrm{~km}$

$\begin{array}{lllll}91.77 & 48 \mathrm{Pd} & 24 & 57.50 & 0.8\end{array}$

$\begin{array}{llllll}91.77 & 48 & \mathrm{Pd} & 24 & 57.50 & 0.8\end{array}$

$\begin{array}{lll}9.5 \mathrm{~s} & 0.80 \mathrm{~nm}\end{array}$

$3.5 \mathrm{mb} \times$

PP $2707.00595 \mathrm{~km}$

PP $\quad 28 \quad 04.00$

$\begin{array}{llllll} & & & \mathrm{S} & 33 & 32.00 \\ & 120 \mathrm{eP} & 24 & 56.50 & -1.0\end{array}$

$0.5 \mathrm{~s} \quad 41.00 \mathrm{~nm} \quad 2456.50 \quad 5.7 \mathrm{mb}$

e $2706.60599 \mathrm{~km}$

$\begin{array}{llllll}91.86 & 47 \mathrm{P} & 24 & 57.70 & 0.7 \\ & \text { PP } & 27 & 07.00 & \end{array}$

$91.96 \quad 1$ iPd $2456.70 \quad 0.0$

$0.4 \mathrm{~s} \quad 49.00 \mathrm{~nm} \quad 5.9 \mathrm{mb}$

$\begin{array}{llllll}92.84 & 42 & \text { ifd } & 24 & 58.30 & 0.5\end{array}$

$1.0 \mathrm{~s} \quad 145.00 \mathrm{~nm} \quad 6.8 \mathrm{mb}$

$92.05 \quad 52 \mathrm{Pd} \quad 2458.50 \quad 0.3$

$\begin{array}{llllll}92.09 & 47 \mathrm{PP} & 27 & 08.00 & \\ 9 & 24 & 58.50 & 0.4\end{array}$

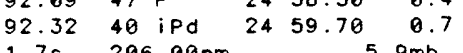

$1.7 \mathrm{~s} \quad 206.00 \mathrm{~nm} \quad 5.9 \mathrm{mb}$

$\begin{array}{llllll}92.33 & 36 & \text { iPd } & 25 & 00.00 & 1.1 \\ 92.38 & 48 & P & 25 & 00.50 & 1.0\end{array}$

$\begin{array}{lllll}92.38 & 48 \mathrm{P} & 25 & 00.50 \\ & & \mathrm{PP} & 27 & 09.00\end{array}$

$\begin{array}{llllll}92.56 & 332 & \text { iPd } & 24 & 59.50 & -0.3\end{array}$

$1.0 \mathrm{~s} \quad 130.00 \mathrm{~nm} \quad 5.9 \mathrm{mb}$

$\begin{array}{llllll}92.58 & 33 \mathrm{iPd} & 27 & 08.08 & 589 \mathrm{~km} \\ 9 & 01.10 & 1.1\end{array}$

$1.0 \mathrm{~s} \quad 126.00 \mathrm{~nm} \quad 5.9 \mathrm{mb}$

$\begin{array}{llllll}92.59 & 332 \text { iPd } 24 \quad 59.70 & -0.3\end{array}$ EPP $\quad 27 \quad 07.30584 \mathrm{~km}$

$92.62119 \mathrm{eP} \quad 25 \quad 00.00 \quad-1.1$

$92.76 \quad 39$ iPd- $25 \quad 01.50 \quad 0.5$

$2.1 \mathrm{~s} \quad 400.00 \mathrm{~nm} \quad 6.1 \mathrm{mb}$

ipP $27 \quad 11.00 \quad 595 \mathrm{~km}$

$\begin{array}{lll}\text { ePP } 28 \quad 54.00 & 0\end{array}$

iSKS 3440.00

eSP $36 \quad 30.00$

$\begin{array}{llllll}92.78 & 53 & \mathrm{P} P & 24 & 57.00 & -4.4 \mathrm{X}\end{array}$

92.80118 IPd $2500.70-1.3$

$0.7 \mathrm{~s} \quad 111.00 \mathrm{~nm} \quad 6.0 \mathrm{mb}$

$\begin{array}{llllll}92.86 & 40 \text { i Pd } & 27 & 09.20 & 589 \mathrm{~km} \\ 92.10 & 0.6\end{array}$

$2.5 \mathrm{~s} \quad 692.00 \mathrm{~nm} \quad 6.3 \mathrm{mb}$

e $\quad 3633.00$

92.90335 ip $\quad 25 \quad 01.50 \quad 0.1$

e $\quad 2708.00578 \mathrm{~km}$

$\begin{array}{lll}e & 34 \quad 37.00\end{array}$

92.90335 iPd $25 \quad 01.00 \quad-0.4$

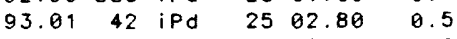

$\begin{array}{lllll}93.03 & 48 P & 25 & 03.00 & 0.6\end{array}$

$\begin{array}{llllll}93.11 & 117 & \text { PP } & 27 & 13.00 & \\ & & & 02.50 & -1.0\end{array}$

$\begin{array}{ll}i & 27 \\ 0 & 12.00595 \mathrm{~km}\end{array}$

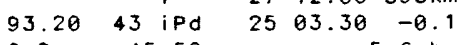

$0.9 \mathrm{~s} \quad 45.50 \mathrm{~nm} \quad 5.6 \mathrm{mb}$

$$
\begin{array}{llll}
i & 25 & 06.80 & \\
\text { eDP } & 27 & 12.40 & 592 \mathrm{~km} \\
\mathrm{e} & 34 & 32.50 &
\end{array}
$$

$34 \quad 39.30$

$93.23119 \mathrm{IPd} 2504.00 \quad 0.0$

$\begin{array}{llllll}93.24 & 44 & \text { iPC } & 25 & 03.50 & 0.2\end{array}$

$\begin{array}{llllll}93.27 & 41 & \mathrm{iPd} & 25 & 03.90 & 0.4\end{array}$

$1.2 \mathrm{~s} \quad 134.00 \mathrm{~nm} \quad 5.9 \mathrm{mb}$ 


\begin{tabular}{|c|c|c|c|c|c|c|c|c|c|c|c|}
\hline & & & es & 34 & 42.00 & & 1 & $\mathrm{~N} 16 \mathrm{~s}$ & & $1.40 \mathrm{um}$ & \\
\hline KDC & 93.33 & 328 & iPd & 25 & 03.40 & 0.0 & 1 & $16 \mathrm{~s}$ & & $2.60 \mathrm{um}$ & \\
\hline PRY & 93.40 & 118 & $i P_{c}$ & 25 & 02.50 & -2.2 & 1 & & & $i$ & 25 \\
\hline & & & e & 27 & 06.60 & $564 \mathrm{kmX}$ & 1 & & & $\mathrm{i}$ & 25 \\
\hline$S G 0$ & 93.48 & 50 & $P d$ & 25 & 06.00 & 1.5 & i & & & epP & 27 \\
\hline & & & PP & 27 & 15.00 & & 1 & & & e & 29 \\
\hline CEY & 93.70 & 44 & $i P d$ & 25 & 05.80 & 0.3 & I & & & e & 35 \\
\hline & & & $i(p P)$ & 27 & 14.80 & $592 \mathrm{~km}$ & I SPC & 98.07 & 41 & ip & 25 \\
\hline & & & es & 34 & 43.60 & & 1 & & & e & 27 \\
\hline & & & $e(s s)$ & 38 & 07.50 & & 1 Jos & 98.20 & 42 & $i P d$ & 25 \\
\hline KHC & 93.72 & 41 & $i P d$ & 25 & 06.00 & 0.5 & I SKO & 98.24 & 49 & iPd & 25 \\
\hline & & & $P P$ & 27 & 15.00 & $592 \mathrm{~km}$ & 1 & $z 20 \mathrm{~s}$ & & $1.00 \mathrm{um}$ & \\
\hline & & & e & 28 & 03.00 & & I & E $24 \mathrm{~s}$ & & $2.70 u m$ & \\
\hline & & & $e$ & 34 & 42.00 & & 1 & & & $\mathrm{i}$ & 27 \\
\hline & & & e.p. & 36 & 40.00 & & 1 & & & $\vdots$ & 35 \\
\hline & & & $P \cdot P \cdot$ & 50 & 11.50 & & 1 & & & $i$ & 36 \\
\hline CLL & 93.75 & 39 & iPd & 25 & 06.00 & 0.5 & 1 & & & $i$ & 37 \\
\hline & $2.4 \mathrm{~s}$ & 430 & $0.00 \mathrm{~nm}$ & & & $5.2 \mathrm{mb}$ & I VAY & 99.03 & 50 & eP & 25 \\
\hline & & & epp & 27 & 15.00 & $592 \mathrm{~km}$ & I ATH & 99.65 & 53 & $e^{P}$ & 25 \\
\hline & & & eSKS & 34 & 44.00 & & 1 & & & es & 35 \\
\hline & & & es & 35 & 22.00 & & I ANM & 100.30 & 334 & ePdifi & \\
\hline LJU & 93.83 & 44 & iPd & 25 & 06.50 & 0.5 & I $\mathrm{coz}$ & 100.58 & 46 & iPdiff & 25 \\
\hline & & & e & 27 & 15.50 & $591 \mathrm{~km}$ & I CMP & 101.07 & 46 & iPdiff & \\
\hline & & & es & 34 & 43.00 & & I NUR & 101.09 & 30 & iPdiff & 25 \\
\hline KMR & 93.90 & 42 & iP- & 25 & 07.00 & 0.7 & 1 & $0.7 \mathrm{~s}$ & & $8.70 \mathrm{~nm}$ & \\
\hline & & & e & 27 & 15.40 & $588 \mathrm{~km}$ & 1 & & & $i p P$ & 27 \\
\hline & & & e & 31 & 52.00 & & 1 & & & ePP & 29 \\
\hline BPI & 94.04 & 117 & iPd & 25 & 05.20 & -2.5 & I & & & epPP & 31 \\
\hline BRN & 94.07 & 38 & $i P d$ & 25 & 08.20 & 1.3 & i & & & eSKS & 35 \\
\hline & & & $i$ & 27 & 16.80 & $589 \mathrm{~km}$ & 1 & & & es & 36 \\
\hline $\mathrm{COP}$ & 94.21 & 34 & iPd- & 25 & 88.30 & 0.8 & I & & & eSP & 37 \\
\hline & $0.9 \mathrm{~s}$ & 145 & $5.00 \mathrm{~nm}$ & & & $.2 \mathrm{mb}$ & 1 & & & ePKKP & 41 \\
\hline & & & $i$ & 27 & 17.00 & $590 \mathrm{~km}$ & 1 & & & ess & 43 \\
\hline & & & is & 34 & 49.00 & & $1 \mathrm{KDZ}$ & 101.14 & 49 & iPdiff & 25 \\
\hline BRG & 94.26 & 39 & iPd & 25 & 88.50 & 0.7 & I KEV & 101.70 & 21 & iPdiff & 25 \\
\hline & $1.8 \mathrm{~s}$ & 167 & $7.00 \mathrm{~nm}$ & & & $.0 \mathrm{mb}$ & 1 & $0.6 \mathrm{~s}$ & 17 & $7.00 \mathrm{~nm}$ & \\
\hline & & & epp & 27 & 17.20 & $589 \mathrm{~km}$ & $i$ & & & $i p^{p}$ & 27 \\
\hline & & & eSKS & 34 & 48.00 & & i & & & ePP & 29 \\
\hline & & & es & 35 & 28.00 & & i & & & ePPP & 32 \\
\hline & & & $P \cdot P \cdot$ & 50 & 17.00 & & 1 & & & eSKS & 35 \\
\hline PRE & 94.28 & 117 & $e^{P}$ & 25 & 07.70 & -1.0 & i & & & eSP & 38 \\
\hline SLR & 94.36 & 117 & iPd & 25 & 07.90 & -1.2 & i & & & ePS & 39 \\
\hline & $1.2 \mathrm{~s}$ & 444 & $4.80 \mathrm{~nm}$ & & & $.6 \mathrm{mb}$ & 1 & & & IPKKP & 41 \\
\hline PRU & 94.48 & 40 & $P d$ & 25 & 09.70 & 0.8 & 1 & & & esps & 41 \\
\hline & $2.4 \mathrm{~s}$ & 208 & $8.00 \mathrm{~nm}$ & & & $5.9 \mathrm{mb}$ & i & & & ess & 43 \\
\hline & & & $p P$ & 27 & 19.00 & $593 \mathrm{~km}$ & I MLR & 101.72 & 45 & iPdiff & 25 \\
\hline & & & $P P$ & 29 & 06.50 & & 1500 & 101.73 & 23 & iPdiff & 25 \\
\hline & & & s & 36 & 12.00 & & I SUF & 101.76 & 28 & iPdiff & 25 \\
\hline & & & $p \cdot p \cdot$ & 50 & 12.00 & & ! & $0.7 \mathrm{~s}$ & & $2.40 \mathrm{~nm}$ & \\
\hline NB2 & & 29 & $\mathbf{P}$ & 25 & 09.20 & 0.0 & I VRI & 102.27 & 45 & ePdiff & 25 \\
\hline & $1.0 \mathrm{~s}$ & 97 & $7.00 \mathrm{~nm}$ & & & $0.0 \mathrm{mb}$ & I KJF & 102.39 & 26 & iPdiff & 25 \\
\hline EVA & 94.89 & 118 & $e^{P}$ & 25 & 11.00 & -0.5 & 1 & $0.7 \mathrm{~s}$ & 18 & $8.10 \mathrm{~nm}$ & \\
\hline & & & e & 27 & 20.70 & $595 \mathrm{~km}$ & 1 & & & ipp & 27 \\
\hline VKA & 95.38 & 42 & $i \mathrm{Pd}$ & 25 & 13.50 & 0.5 & I & & & iPP & 30 \\
\hline & $2.6 \mathrm{~s}$ & 524 & $4.00 \mathrm{~nm}$ & & & 5. $3 \mathrm{mb}$ & & & & ePPP & 32 \\
\hline & & & $p^{P}$ & 27 & 22.10 & $588 \mathrm{~km}$ & I & & & eSKS & 35 \\
\hline LCI & 95.44 & 50 & $\mathrm{P}$ & 25 & 14.58 & 1.1 & 1 & & & es & 36 \\
\hline I MA & 95.56 & 336 & $i \mathrm{Pd}$ & 25 & 13.00 & -0.6 & 1 & & & eSP & 38 \\
\hline & & & e & 27 & 20.00 & $579 \mathrm{kmX}$ & 1 & & & ePKKP & 41 \\
\hline HFS & 95.64 & 30 & eP & 25 & 14.00 & 0.2 & 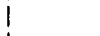 & & & $\mathrm{i}$ & 42 \\
\hline & $0.9 \mathrm{~s}$ & & $5.00 \mathrm{~nm}$ & & & $.4 \mathrm{mb}$ & i & & & ess & 43 \\
\hline$K S P$ & 95.72 & 40 & $\mathrm{iPd}$ & 25 & 15.50 & 1.1 & I TLE & 103.18 & 46 & ePdiff & 25 \\
\hline & $0.8 \mathrm{~s}$ & 170 & $0.00 \mathrm{~nm}$ & & & $5.3 \mathrm{mb}$ & I KVT & 109.19 & 50 & iPKP & 30 \\
\hline & & & $i$ & 27 & 24.60 & $591 \mathrm{~km}$ & I AVY & 113.53 & 115 & ePKPd & 30 \\
\hline & & & e & 29 & 16.50 & & I TAB & 117.44 & 52 & ePKP & 30 \\
\hline & & & e & 31 & 49.50 & & KER & 118.64 & 56 & ePKP & 30 \\
\hline BUL & 6 & 111 & $\mathrm{iPd}$ & 25 & 16.10 & 0.2 & WAI & 120.93 & 217 & iPKPd & 30 \\
\hline & $0.8 \mathrm{~s}$ & & $5.10 \mathrm{~nm}$ & & & $5.5 \mathrm{mb}$ & CAN & 121.44 & 218 & iPKPd & 30 \\
\hline & & & $i p P$ & 27 & 27.00 & $601 \mathrm{~km}$ & TOO & 121.92 & 214 & iPKPd & 30 \\
\hline & & & is & 34 & 57.90 & & & $0.6 \mathrm{~s}$ & 33 & $3.00 \mathrm{~nm}$ & \\
\hline TTA & 2 & 3 & $e^{P C}$ & 25 & 14.90 & -0.7 & YOU & 122.51 & 219 & iPKPd & 30 \\
\hline MAW & $\theta$ & 164 & e & 2 & 17.00 & -0.3 & $\mathrm{coO}$ & 122.55 & 224 & iPKPd & 30 \\
\hline BUD & 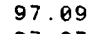 & 43 & ePd & 2 & 21.00 & 0.3 & i & $0.7 \mathrm{~s}$ & 250 & $.00 \mathrm{~nm}$ & \\
\hline$J 0 z$ & 27 & 119 & eP & 25 & 24.00 & 2.0 & B & 123.80 & 212 & iPKPd & 30 \\
\hline SDN & 97.32 & 325 & ep & 25 & 21.00 & -0.6 & 1 CMS & 125.97 & 219 & iPKPd & 30 \\
\hline UPP & 97.56 & 31 & iP & 25 & 22.00 & -0.5 & $A D E$ & 127.49 & 211 & iPKPd & 30 \\
\hline & & & $i$ & 27 & 32.20 & $599 \mathrm{~km}$ & ! & $0.6 \mathrm{~s}$ & 97 & $.30 \mathrm{~nm}$ & \\
\hline & & & is & 34 & 59.00 & & $\mathrm{KHI}$ & 128.02 & 54 & iPKPd & 30 \\
\hline & & & $i$ & 35 & 54.00 & & | MHI & 128.06 & 51 & iPKPd- & -30 \\
\hline & & & $i$ & 37 & 17.00 & & 1 & & & $e$ & 32 \\
\hline $\mathrm{OHR}$ & 97.68 & 50 & ip & 25 & 24.50 & & & 8.25 & & & 30 \\
\hline & & & $i$ & 25 & 42.00 & $62 \mathrm{kmx}$ & CTA & 132.83 & 231 & ePKP & 30 \\
\hline & & & $\mathrm{i}$ & 27 & 34.90 & & I NEM & 133.51 & 323 & PKP & 30 \\
\hline & & & $i$ & 35 & 03.60 & & ALOT & 134.10 & 246 & ePKP & 31 \\
\hline KRA & 5 & 41 & $i \mathrm{Pd}$ & 25 & 25.20 & 6 & KUSJ & 134.17 & 323 & PKP & 30 \\
\hline & $1.1 \mathrm{~s}$ & 103 & $.00 \mathrm{~nm}$ & & & $1 \mathrm{mb}$ & I ASAJ & 134.73 & 326 & PKP & 30 \\
\hline & $16 \mathrm{~s}$ & & $.00 \mathrm{um}$ & & & Mszx & I HOOJ & 135.43 & 323 & PKP & 30 \\
\hline
\end{tabular}


$02 d 20 h$

\begin{tabular}{|c|c|c|c|c|c|c|c|c|c|c|c|c|}
\hline & $1.0 \mathrm{~s}$ & 126 & $\begin{array}{l}6.00 \mathrm{~nm} \\
\text { ePP }\end{array}$ & 33 & 47.00 & & & BDT & $\begin{array}{c}0.6 \mathrm{~s} \\
167.77\end{array}$ & $50^{3}$ & $\begin{array}{l}3.60 \mathrm{~nm} \\
\text { iPKPd }\end{array}$ & 31 \\
\hline GTA & 149.16 & 14 & iPKP & 31 & 29.60 & 0.3 & 1 & BUI & $\begin{array}{c}0.9 \mathrm{~s} \\
0.17\end{array}$ & & $1.46 \mathrm{~nm}$ & 31 \\
\hline & & & i PPKP & 33 & 49.00 & & i & PPR & 170.18 & 272 & ePKPc & 31 \\
\hline & & & PP & 35 & 11.50 & & I & KGM & 170.79 & 144 & ePKPd & 31 \\
\hline & & & SKKS & 41 & 00.00 & & i & & & & e & 33 \\
\hline KUMJ & 149.33 & 322 & PKP & 31 & 29.10 & -0.4 & $i$ & IPM & 170.88 & 122 & ePKPd & 31 \\
\hline HYB & 149.60 & 72 & iPKPd & 31 & 30.00 & -0.4 & i & & $0.9 \mathrm{~s}$ & & $2.90 \mathrm{~nm}$ & \\
\hline & $0.8 \mathrm{~s}$ & 492 & 2. $00 \mathrm{~nm}$ & & & & i & & & & $\epsilon$ & 33 \\
\hline & & & e & 33 & 46.00 & & 1 & & & & e & 35 \\
\hline NGS & 149.77 & 323 & PKP & 31 & 30.90 & 0.7 & i & $P C T$ & 171.14 & 54 & iPKPd & 31 \\
\hline KAGJ & 150.20 & 320 & PKP & 31 & 30.90 & 0.0 & i & & $1.0 \mathrm{~s}$ & & $5.60 \mathrm{~nm}$ & \\
\hline$D M N$ & 151.32 & 48 & iPKPd & 31 & 33.00 & 0.0 & 1 & & & & e & 33 \\
\hline$K K N$ & 151.35 & 47 & iPKPd & 31 & 33.00 & 0.0 & 1 & & & & e & 35 \\
\hline & $0.8 \mathrm{~s}$ & 340 & $0.00 \mathrm{~nm}$ & & & & i & SNG & 171.60 & 105 & ePKP & 31 \\
\hline PKI & 151.56 & 48 & iPKPd & 31 & 33.20 & -0.3 & i & KKM & 171.87 & 246 & ePKPd & 31 \\
\hline & $1.0 \mathrm{~s}$ & 250 & $0.00 \mathrm{~nm}$ & & & & i & & $1.1 \mathrm{~s}$ & 107 & $7.00 \mathrm{~nm}$ & \\
\hline TIY & 151.72 & 354 & PKPd & 31 & 33.40 & 0.4 & i & & D. $=1$ & 1.0 & on 41 & 7 of \\
\hline & & & e & 31 & 52.00 & & i & & & & & \\
\hline & & & PPKP & 33 & 49.00 & & i & JUN & 09,1 & 1983 & $18 \mathrm{~h}$ & $46 \mathrm{~m}$ \\
\hline & & & PP & 35 & 25.50 & & 1 & 51.4 & $441 \mathrm{~N} \pm$ & \pm 1.8 & $8 \mathrm{~km} \quad 1$ & 74.1 \\
\hline & & & SKKS & 41 & 34.00 & & i & & $T H=3$ & 34.3 & \pm 2 & $6 \mathrm{~km}$ \\
\hline & & & ss & 53 & 57.00 & & i & $6.2 n$ & $2 \mathrm{mb} \quad(153$ & 3 obs & s.) & $0 \mathrm{Ms}$ \\
\hline TIA & 152.35 & 345 & PKPd & 31 & 33.90 & 0.0 & $i$ & ANDREA & EANOF IS & SLAND & $D S, \quad A L$ & EUT I \\
\hline & & & e & 31 & 53.80 & & i & & Felt & $111)$ & on Ad & ok. \\
\hline & & & PPKP & 33 & 50.50 & & i & & & & & \\
\hline & & & PPKP & 34 & 07.50 & & i & $A D 2$ & 1. 27 & 292 & IPd & 46 \\
\hline & & & PKS & 35 & 07.20 & & 1 & $A D 8$ & 1.64 & 290 & iPd & 46 \\
\hline & & & PP & 35 & 27.60 & & i & $A D K$ & 1.66 & 287 & iPd & 46 \\
\hline & & & SKKS & 41 & 16.00 & & i & AK5 & 1.77 & 283 & iPd & 46 \\
\hline LZH & 153.16 & 9 & PKPd & 31 & 36.50 & 1.3 & $i$ & $A D 7$ & 1.91 & 285 & ePd & 46 \\
\hline & & & e & 31 & 58.00 & & $i$ & $A D 6$ & 1.97 & 279 & eP & 46 \\
\hline & & & PPKP & 33 & 52.00 & & i & AK 1 & 2.21 & 277 & IPC & 46 \\
\hline & & & PP & 35 & 34.00 & & i & NKI & 3.57 & 63 & eP & 46 \\
\hline & & & SKKS & 41 & 26.50 & & I & SMY & 7.37 & 285 & eP & 47 \\
\hline LSA & 154.00 & 37 & IPKPd & 31 & 37.90 & 1.0 & i & & & & e & 47 \\
\hline & & & e & 32 & 03.10 & & i & SDN & 9.03 & 59 & $\mathbf{P}$ & 48 \\
\hline & & & SKKS & 41 & 27.10 & & $i$ & $K D C$ & 14.00 & 55 & eP & 49 \\
\hline XAN & 155.58 & 360 & IPKP & 31 & 38.00 & -0.4 & i & svw & 14.06 & 39 & $\mathbf{P}$ & 49 \\
\hline & & & e & 32 & 07.00 & & $i$ & TTA & 15.05 & 33 & $\mathbf{P}$ & 49 \\
\hline & & & PPKP & 33 & 56.50 & & i & I LT & 16.66 & 354 & iPc & 49 \\
\hline & & & $i$ & 34 & 17.00 & & 1 & & $1.4 \mathrm{~s}$ & 1530 & $0.00 \mathrm{~nm}$ & \\
\hline & & & PP & 35 & 45.50 & & i & $z$ & $18 \mathrm{~s}$ & 122 & $2.00 \mathrm{um}$ & \\
\hline SSE & 155.61 & 333 & PKPC & 31 & 38.00 & -0.4 & i & $\mathbf{N}$ & $18 \mathrm{~s}$ & 93 & 3. 00 um & \\
\hline & & & e & 32 & 08.00 & & i & $E$ & $18 \mathrm{~s}$ & 42 & $2.00 \mathrm{um}$ & \\
\hline & & & $p P$ & 33 & 54.00 & & i & PET & 16.72 & 286 & $i P_{c}$ & 49 \\
\hline NJ2 & 155.70 & 339 & IPKPd & 31 & 38.00 & -0.5 & i & $z$ & $18 \mathrm{~s}$ & 23 & $3.30 \mathrm{um}$ & \\
\hline & & & $i$ & 32 & 07.00 & & i & $N$ & $14 \mathrm{~s}$ & 14 & $4.00 \mathrm{um}$ & \\
\hline & & & PPKP & 33 & 56.00 & & i & $E$ & $18 \mathrm{~s}$ & 25 & 5. $30 \mathrm{um}$ & \\
\hline & & & PP & 35 & 45.00 & & 1 & & & & es & 52 \\
\hline$A A_{1}$ & 156.64 & 237 & ePKP & 31 & 39.50 & -0.8 & | & PMR & 17.01 & 44 & $e(P)$ & 49 \\
\hline & $0.6 \mathrm{~s}$ & 144 & $4.00 \mathrm{~nm}$ & & & & 1 & PME & 17.07 & 44 & eP & 49 \\
\hline $\mathrm{CD} 2$ & 158.19 & 12 & PKP & 31 & 42.50 & 0.8 & i & & $1.0 \mathrm{~s}$ & 42 & $2.50 \mathrm{~nm}$ & \\
\hline & & & $e$ & 32 & 17.50 & & 1 & IMA & 17.95 & 28 & eP & 50 \\
\hline & & & epP & 33 & 59.00 & & 1 & SKR & 18.68 & 279 & $i P_{C}$ & 50 \\
\hline & & & $i p P$ & 34 & 28.00 & & i & & $2.0 \mathrm{~s}$ & 3910 & $0.00 \mathrm{~nm}$ & \\
\hline & & & PKS & 35 & 16.00 & & i & $z$ & $20 \mathrm{~s}$ & 45 & 5. 50 um & \\
\hline & & & $P P$ & 36 & 00.00 & & | & $N$ & $20 \mathrm{~s}$ & 26 & 6. 00 um & \\
\hline & & & SKS & 38 & 04.00 & & 1 & $E$ & $20 \mathrm{~s}$ & 45 & $5.50 \mathrm{um}$ & \\
\hline WHN & 158.43 & 347 & ePKP & 31 & 40.00 & -1.8 & i & $\mathrm{COL}$ & 19.15 & 35 & eP & 50 \\
\hline & & & $i$ & 32 & 19.50 & & i & & $0.9 \mathrm{~s}$ & 276 & $6.00 \mathrm{~nm}$ & \\
\hline & & & PPKP & 33 & 58.00 & & | & & & & es & 54 \\
\hline & & & PP & 36 & 00.00 & & | & FBA & 19.15 & 35 & eP & 50 \\
\hline ANP & 160.25 & 324 & ePKP & 31 & 46.00 & 2.0 & 1 & PCA & 20.71 & 52 & eP & 50 \\
\hline MKS & 161.91 & 217 & ePKPC & 31 & 45.00 & -0.9 & 1 & SEY & 21.19 & 316 & $i P c$ & 50 \\
\hline $\mathrm{OZH}$ & 162.03 & 330 & PKPd & 31 & 46.00 & 0.3 & $i$ & & $1.6 \mathrm{~s}$ & 800 & $0.00 \mathrm{~nm}$ & \\
\hline & & & e & 32 & 36.00 & & i & $z$ & ? $18 \mathrm{~s}$ & 68 & 8.60 um & \\
\hline & & & PP & 36 & 20.00 & & 1 & $N$ & $18 \mathrm{~s}$ & 31 & $1.80 \mathrm{um}$ & \\
\hline TRT & 162.48 & 193 & IPKPd & 31 & 47.20 & 0.8 & 1 & $E$ & $18 \mathrm{~s}$ & 42 & 2. 40 um & \\
\hline & $0.6 \mathrm{~s}$ & 77 & $7.40 \mathrm{~nm}$ & & & & 1 & & & & ePP & 51 \\
\hline GYA & 163.02 & 7 & PKP & 31 & 47.00 & 0.2 & 1 & & & & es & 54 \\
\hline & & & e & 32 & 40.00 & & i & & & & IPCP & 54 \\
\hline & & & epP & 34 & 04.00 & & 1 & MGD & 21.32 & 308 & $i P c$ & 50 \\
\hline & & & ipP & 34 & 49.00 & & i & & $2.4 \mathrm{~s}$ & 930 & $0.00 \mathrm{~nm}$ & \\
\hline & & & PP & 36 & 24.00 & & | & $z$ & $19 \mathrm{~s}$ & & $7.00 \mathrm{um}$ & \\
\hline DAV & 163.21 & 263 & ePKP & 31 & 47.00 & -0.2 & 1 & $N$ & $19 \mathrm{~s}$ & & $0.00 \mathrm{um}$ & \\
\hline KM! & 163.43 & 20 & IPKPD- & -31 & 48.00 & 0.6 & 1 & $E$ & $19 \mathrm{~s}$ & & $3.00 \mathrm{um}$ & \\
\hline & $4.0 \mathrm{~s}$ & & $.80 \mathrm{~nm}$ & & & & 1 & & & & ePP & 51 \\
\hline GZH & 165.80 & 343 & iPKPd & 31 & 50.00 & 0.8 & i & & & & ePPP & 51 \\
\hline & & & e & 32 & 51.00 & & i & & & & ePcP & 54 \\
\hline & & & $P P$ & 36 & 39.20 & & i & SIT & 23.11 & 61 & ePc & 51 \\
\hline $\mathrm{HKC}$ & 166.26 & 339 & ePKP & 31 & 50.00 & 0.5 & I & INK & 25.75 & 34 & ePc & 51 \\
\hline $\mathrm{CHG}$ & 166.72 & 45 & iPKPd & 31 & 49.00 & -1.0 & 1 & & $1.1 \mathrm{~s}$ & 569 & $9.00 \mathrm{~nm}$ & \\
\hline & $0.9 \mathrm{~s}$ & & $4.60 \mathrm{~nm}$ & & & & & KUR & 25.80 & 271 & iPc & 51 \\
\hline НTO & 166.72 & 45 & PKP & 31 & 50.10 & . 1 & I & & $1.0 \mathrm{~s}$ & 1700 & $0.00 \mathrm{~nm}$ & \\
\hline OCP & 166.94 & 294 & ePKP & 32 & 08.50 & $18 \cdot 3 x$ & 1 & & & & iPP & 52 \\
\hline PPI & 167.06 & 139 & ePKPd & 31 & 49.80 & -0.6 & & 55 & 28.22 & 278 & iPc & 51 \\
\hline
\end{tabular}




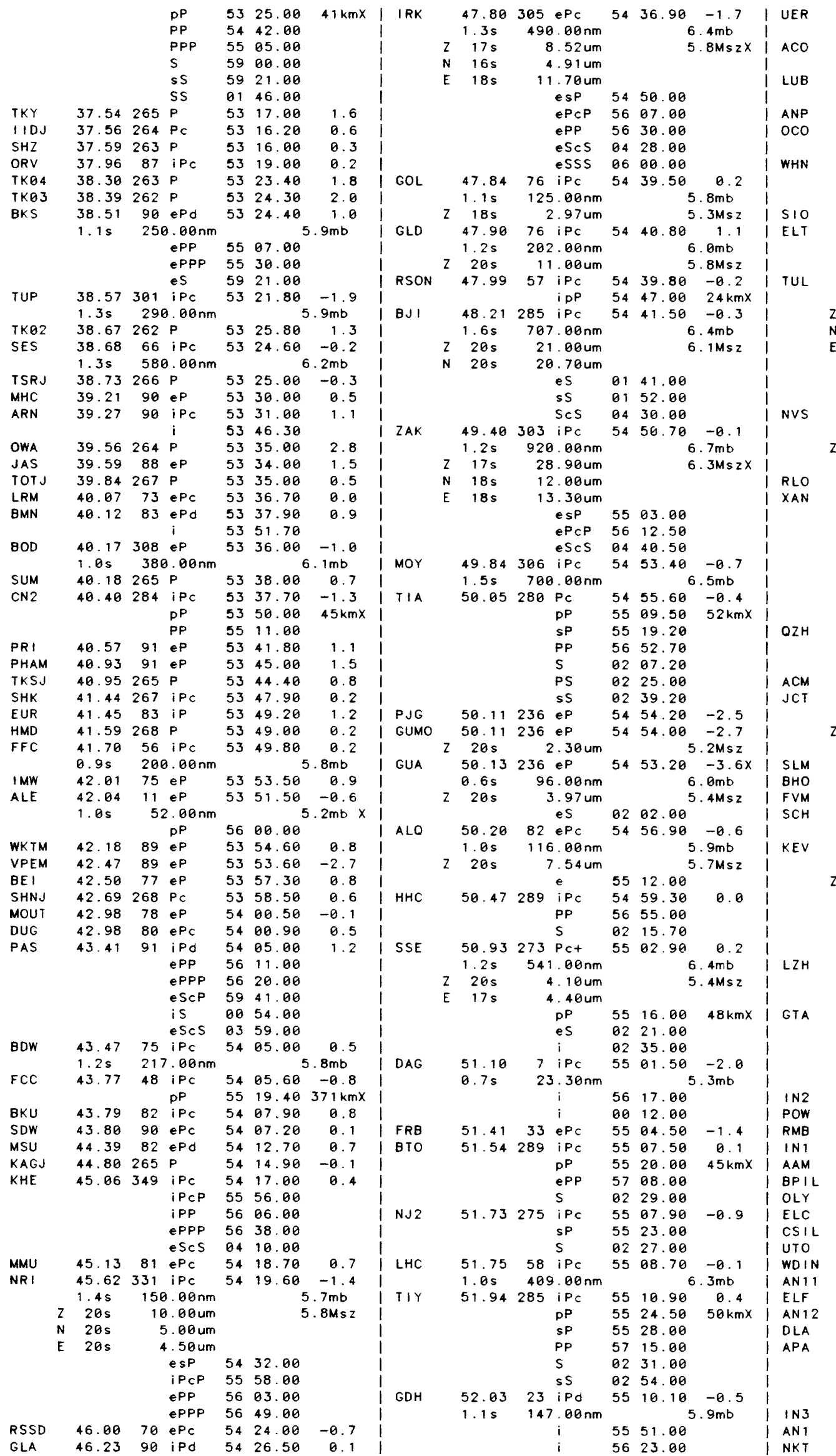

$\begin{array}{llllll}53.34 & 309 & \text { iPd } & 55 & 19.40 & -1.1\end{array}$ $2.0 \mathrm{~s} \quad 300.00 \mathrm{~nm} \quad 5.9 \mathrm{mb}$

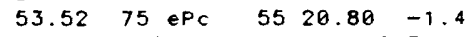
$\begin{array}{lll}1.6 \mathrm{~s} & 952.00 \mathrm{~nm} & 6.5 \mathrm{mb}\end{array}$

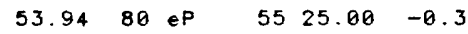

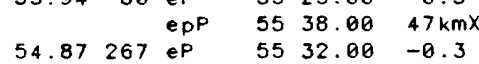

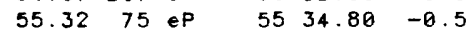
$\begin{array}{llllll}55.57 & 277 \mathrm{iPc} & 55 & 49.40 & & \\ & & S & 5.80 & -1.3\end{array}$ S $\quad 03 \quad 22.50$ SS $03 \quad 39.50$

$\begin{array}{llllll}55.87 & 74 & \mathrm{IPC} & 55 & 38.10 & -1.2\end{array}$ $\begin{array}{llllll}55.90 & 314 & \mathrm{iPC} & 55 & 36.00 & -3.2 \mathrm{x}\end{array}$ $2.3 \mathrm{~s} \quad 440.00 \mathrm{~nm} \quad 6.1 \mathrm{mb}$ eScS 0523.00

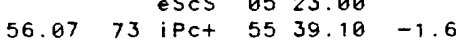
$\begin{array}{lll}1.6 \mathrm{~s} & 976.00 \mathrm{~nm} & 6.6 \mathrm{mb}\end{array}$

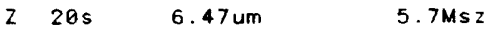

$\mathrm{N} 21 \mathrm{~s} \quad 4.41 \mathrm{um}$ E $21 \mathrm{~s} \quad 5.10 \mathrm{um}$ - $\quad 5549.70$ $\begin{array}{lll}\text { e } & 5533.80 \\ \text { is } & 0327.50\end{array}$ $\begin{array}{llllll}56.09 & 317 \mathrm{iPC} & 55 & 38.70 & -1.8\end{array}$ $3.0 \mathrm{~s} \quad 500.00 \mathrm{~nm} \quad 6.0 \mathrm{mb}$

$\begin{array}{lll}20 \mathrm{~s} & 31.00 \mathrm{um} & 6.4 \mathrm{Msz}\end{array}$ ISP $03 \quad 34.00$

$\begin{array}{llllll}56.35 & 73 \mathrm{iPC} & 55 & 41.00 & -1.8\end{array}$ $\begin{array}{llllll}56.51 & 284 & \mathrm{iPC} & 55 & 42.80 & -1.2\end{array}$ PP $\quad 5556.00 \quad 47 \mathrm{kmx}$ PCP 5725.00 PP $\quad 57 \quad 48.50$ PPP $58 \quad 05.50$ S $\quad 03 \quad 32.50$ SCS $05 \quad 28.00$ SS 0731.00

$\begin{array}{lllll}56.88 & 269 \mathrm{iPC} & 55 & 46.00 & -0.6\end{array}$ SP 5602.00 $\begin{array}{lllll}57.29 & 61 \mathrm{eP} & 55 & 48.10 & -1.2\end{array}$ $\begin{array}{llllll}57.34 & 81 \text { iP } & 55 & 48.00 & -1.9\end{array}$ $\begin{array}{lll}1.0 \mathrm{~s} & 125.00 \mathrm{~nm} & 5.9 \mathrm{mb}\end{array}$ $\begin{array}{lll}20 \mathrm{~s} & 8.16 \mathrm{um} & 5.8 \mathrm{Msz}\end{array}$

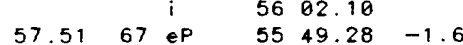
$\begin{array}{llllll}57.66 & 74 & e(P) & 55 & 51.40 & -0.6\end{array}$ $\begin{array}{lllll}57.84 & 68 \mathrm{ePc} & 55 & 51.20 & -2.0\end{array}$ $\begin{array}{llllll}58.13 & 41 \mathrm{ePC} & 55 & 53.50 & -1.6\end{array}$ $1.0 \mathrm{~s} \quad 82.00 \mathrm{~nm} \quad 5.8 \mathrm{mb}$ 58.14352 iPC+ $5553.00-1.9$ $1.0 \mathrm{~s} 200.00 \mathrm{~nm} \quad 6.1 \mathrm{mb}$

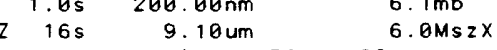
$i \quad 56 \quad 44.00$ $\begin{array}{llll}\text { ePP } & 58 \quad 04.00\end{array}$ eS 0408.00 eSS 0752.00

$\begin{array}{llllll}58.16 & 289 & \text { iPc } & 55 & 55.56 & -0.2\end{array}$

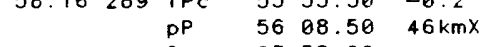
$58.24295 \mathrm{IPC} \quad 0358.00$

$\begin{array}{llllll}58.24 & 295 & \mathrm{iPC} & 55 & 54.60 & -1.6\end{array}$ pP $\quad 56 \quad 06.00 \quad 39 \mathrm{kmx}$ PPP $59 \quad 33.50$ S $\quad 0358.50$ SCS $05 \quad 39.30$

$\begin{array}{llllll}58.60 & 64 & \mathrm{iPC} & 55 & 57.40 & -1.1\end{array}$ $\begin{array}{lllll}58.64 & 70 \mathrm{ePC} & 55 & 55.79 & -3.1 x\end{array}$ $\begin{array}{llllll}58.67 & 69 & \text { ePC } & 55 & 56.58 & -2.5\end{array}$ $\begin{array}{llllll}58.69 & 63 & \text { iPc } & 55 & 57.40 & -1.7\end{array}$

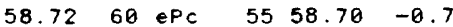
$\begin{array}{llllll}58.75 & 67 & \text { ePC } & 55 & 57.73 & -1.9\end{array}$ $\begin{array}{llllll}58.92 & 71 \in P C & 55 & 58.10 & -2.7\end{array}$ $\begin{array}{llllll}59.01 & 68 & \text { ePC } & 55 & 59.19 & -2.2\end{array}$ $\begin{array}{lllll}59.03 & 67 \mathrm{ePC} & 55 & 59.29 & -2.2\end{array}$

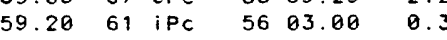

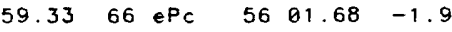
$\begin{array}{lllll}59.35 & 62 \mathrm{ePC} & 56 & 02.00 & -1.7\end{array}$ $\begin{array}{llllll}59.37 & 58 \mathrm{P} & 56 & 03.70 & -0.1\end{array}$ $59.38 \quad 62$ ePC $56 \quad 02.60 \quad-1.3$ $\begin{array}{llllll}59.46 & 59 \mathrm{P} & 56 & 04.80 & 0.3\end{array}$ $\begin{array}{llllll}59.55 & 348 \text { iPc } & 56 & 03.00 & -1.8\end{array}$ $1.5 \mathrm{~s} 250.08 \mathrm{~nm} \quad 6.1 \mathrm{mb}$ $\begin{array}{lll}E P & 58 & 18.00\end{array}$ EPS 0428.00

$\begin{array}{llllll}59.62 & 64 & \mathrm{eP} & 56 & 04.10 & -1.5\end{array}$ $\begin{array}{lllll}59.71 & 62 \mathrm{ePc} & 56 & 05.10 & -1.1\end{array}$ $\begin{array}{llllll}59.81 & 69 & \text { eP } & 56 & 05.23 & -1.7\end{array}$ 
$09 d \quad 18 \mathrm{~h}$

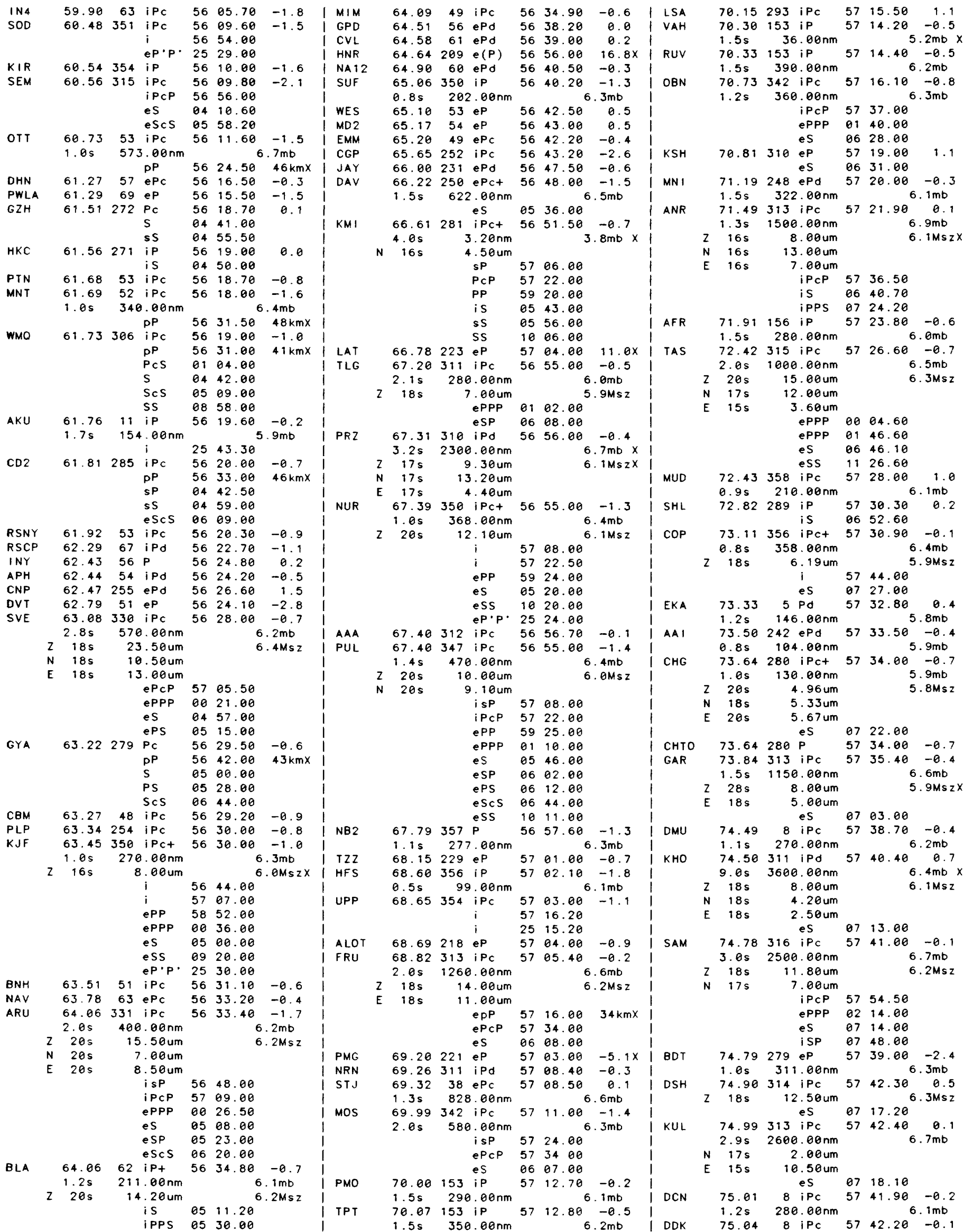




\begin{tabular}{|c|c|c|c|c|c|c|c|c|c|c|c|c|}
\hline & & $1.2 \mathrm{~s}$ & & $0.00 \mathrm{~nm}$ & & & $.0 m b$ & 1 & & 2 & $18 \mathrm{~s}$ & \\
\hline PKI & & 75.04 & 295 & iPc & 57 & 43.30 & 0.1 & 1 & & & & \\
\hline DLE & & 75.12 & 8 & $i P c$ & 57 & 42.60 & -0.2 & 1 & & & & \\
\hline & & $1.0 \mathrm{~s}$ & 14 & $5.00 \mathrm{~nm}$ & & & $.9 \mathrm{mb}$ & 1 & GRFO & & 79.14 & 356 \\
\hline DMN & & 75.19 & 296 & $i P c$ & 57 & 44.40 & 0.5 & i & CTA & & 79.23 & 218 \\
\hline PCT & & 75.24 & 275 & eP & 57 & 45.50 & 1.5 & 1 & & & $1.4 \mathrm{~s}$ & \\
\hline & & $1.0 \mathrm{~s}$ & 8 & $39.70 \mathrm{~nm}$ & & & $.7 \mathrm{mb}$ & 1 & & & & \\
\hline NOU & & 75.39 & 199 & iPd & 57 & 47.50 & 2.9 & 1 & & & & \\
\hline MAM & & 75.41 & 358 & $3 \mathrm{iPC}$ & 57 & 45.00 & a. 6 & & & & & \\
\hline ETA & & 75.74 & 8 & iPc & 57 & 46.40 & 0.1 & & MAK & & 79.24 & 330 \\
\hline & & $1.2 \mathrm{~s}$ & 43 & $0.00 \mathrm{~nm}$ & & & $.3 \mathrm{mb}$ & 1 & & & $3.0 \mathrm{~s}$ & 3000 \\
\hline ECB & & 76.02 & 8 & iPc & 57 & 47.50 & -0.3 & 1 & & & & \\
\hline VAL & & 76.10 & 10 & ip & 57 & 48.20 & -0.1 & 1 & WLF & & 79.27 & 360 \\
\hline & & & & is & 07 & 28.00 & & 1 & & & & \\
\hline WIT & & 76.12 & 360 & iPc & 57 & 49.90 & 1.5 & 1 & & & & \\
\hline ECP & & 76.24 & 8 & iPc & 57 & 49.00 & -0.1 & 1 & UZH & & 79.32 & 349 \\
\hline & & $1.3 \mathrm{~s}$ & 46 & $0.00 \mathrm{~nm}$ & & & $.3 m b$ & 1 & & & $1.2 \mathrm{~s}$ & 206 \\
\hline BRL & & 76.27 & 355 & $\mathrm{iPc}$ & 57 & 50.00 & 0.8 & 1 & & & & \\
\hline BRN & & 76.33 & 355 & iPc & 57 & 50.20 & 0.6 & 1 & & & & \\
\hline DBN & & 76.83 & $\theta$ & ipt & 57 & 52.50 & 0.1 & 1 & GRO & & 79.47 & 332 \\
\hline & 2 & $19 \mathrm{~s}$ & & 3.60 um & & & $.7 \mathrm{Msz}$ & 1 & & & $2.0 \mathrm{~s}$ & 668 \\
\hline & & & & ePPP & 02 & 37.00 & & 1 & & & & \\
\hline & & & & es & 08 & 00.00 & & 11 & $\mathrm{KHC}$ & & 79.59 & 355 \\
\hline WTS & & 76.94 & 359 & iPc & 57 & 53.30 & 0.3 & 1 & & $z$ & $18 \mathrm{~s}$ & \\
\hline & & $1.0 \mathrm{~s}$ & 317 & $7.00 \mathrm{~nm}$ & & & $.3 \mathrm{mb}$ & 1 & & $\mathrm{~N}$ & $18 \mathrm{~s}$ & \\
\hline CLL & & 77.45 & 355 & iPc & 57 & 55.70 & -0.1 & 1 & & $E$ & $18 \mathrm{~s}$ & \\
\hline & & $1.5 \mathrm{~s}$ & 66 & $0.00 \mathrm{~nm}$ & & 6 & $.4 \mathrm{mb}$ & 1 & & & & \\
\hline & & & & i & 58 & 08.40 & & 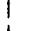 & & & & \\
\hline KSP & & 77.71 & 353 & iPc & $\begin{array}{l}07 \\
57\end{array}$ & $\begin{array}{l}42.00 \\
56.80\end{array}$ & -0.5 & 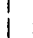 & PYA & & 79.60 & 334 \\
\hline BRG & & 77.83 & 355 & iPct & 57 & 58.00 & 0.0 & $i$ & & & $1.5 \mathrm{~s}$ & $45 e$ \\
\hline & & $1.2 \mathrm{~s}$ & 50 & $5.00 \mathrm{~nm}$ & & & $.4 \mathrm{mb}$ & 1 & & $z$ & $22 \mathrm{~s}$ & \\
\hline & $z$ & $19 \mathrm{~s}$ & & $4.50 u m$ & & & $.8 \mathrm{Msz}$ & I & & & & \\
\hline & $\mathrm{N}$ & $19 \mathrm{~s}$ & & $5.50 u m$ & & & & 1 & & & & \\
\hline & $E$ & $19 \mathrm{~s}$ & & $9.00 \mathrm{um}$ & & & & 1 & & & & \\
\hline & & & & $i$ & 58 & 11.20 & & 1 & & & & \\
\hline & & & & es & 07 & 48.00 & & 1 & WET & & 79.62 & 355 \\
\hline LVV & & 77.94 & 348 & iPc & 57 & 58.40 & -0.2 & 1 & & & $1.2 \mathrm{~s}$ & 261 \\
\hline & $z$ & $19 \mathrm{~s}$ & & $9.80 u m$ & & & $2 \mathrm{Msz}$ & 1 & & $z$ & $19 \mathrm{~s}$ & \\
\hline & $E$ & $19 \mathrm{~s}$ & & 5. $30 \mathrm{um}$ & & & & 1 & MTN & & 79.63 & 234 \\
\hline & & & & IPCP & 58 & 11.50 & & 1 & JOS & & 79.66 & 350 \\
\hline & & & & ePP & 01 & 00.00 & & 1 & & & $0.8 \mathrm{~s}$ & \\
\hline & & & & ePPP & 02 & 48.00 & & 1 & MKS & & 79.69 & 248 \\
\hline & & & & iscs & 88 & 10.00 & & 1 & ASH & & 79.85 & 320 \\
\hline & & & & ESPP & 98 & 46.00 & & 1 & & $\mathrm{~N}$ & $16 \mathrm{~s}$ & \\
\hline ucc & & 78.13 & 1 & $\mathrm{Pc}^{-}$ & 57 & 59.70 & 0.2 & 1 & & E & $16 \mathrm{~s}$ & \\
\hline & & & & e & 08 & 10.00 & & I & & & & \\
\hline ENN & & 78.17 & 360 & IPC & 58 & 00.30 & 0.5 & 1 & & & & \\
\hline & & $1.0 \mathrm{~s}$ & 452 & $2.00 \mathrm{~nm}$ & & & $.4 \mathrm{mb}$ & 1 & & & & \\
\hline MOX & & 78.17 & 356 & $i P c$ & 58 & 00.00 & 0.1 & 1 & GWF & & 79.95 & 359 \\
\hline & & $2.2 \mathrm{~s}$ & $75 e$ & $0.00 \mathrm{~nm}$ & & & $.3 \mathrm{mb}$ & I & KIS & & 79.95 & 344 \\
\hline & 2 & $16 \mathrm{~s}$ & & 3.80 um & & & $.8 M s 2 X$ & 1 & & & $3.0 \mathrm{~s}$ & 2200 \\
\hline & 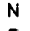 & $17 \mathrm{~s}$ & & $3.40 \mathrm{um}$ & & & & I & & & & \\
\hline & $E$ & $18 \mathrm{~s}$ & & $1.80 u m$ & & & & 1 & & & & \\
\hline & & & & $\mathbf{i}$ & 58 & 13.50 & & 1 & & & & \\
\hline & & & & es & 07 & 52.00 & & 1 & & & & \\
\hline & & & & ess & 13 & 15.00 & & I & & & & \\
\hline KRA & & 78.18 & 351 & iPC & 57 & 59.30 & -0.5 & I & VAN & & 79.95 & 321 \\
\hline & & $1.0 \mathrm{~s}$ & 102 & $2.00 \mathrm{~nm}$ & & & $.8 \mathrm{mb}$ & I & & & $1.5 \mathrm{~s}$ & 420 \\
\hline & & & & e & 58 & 09.70 & & I & & $z$ & $18 \mathrm{~s}$ & \\
\hline & & & & $\mathbf{i}$ & 58 & 15.30 & & I & & & & \\
\hline & & & & e & 08 & 48.00 & & 1 & & & & \\
\hline HOF & & 78.50 & 356 & iPC & 58 & 01.90 & 0.2 & 1 & IAS & & 79.99 & 345 \\
\hline & & $1.3 \mathrm{~s}$ & & $8.00 \mathrm{~nm}$ & & & $.3 \mathrm{mb}$ & I & $F L N$ & & 80 & 4 \\
\hline & $z$ & $17 \mathrm{~s}$ & & $2.80 \mathrm{um}$ & & & $7 \mathrm{Ms} z \mathrm{X}$ & 1 & & & $1.1 \mathrm{~s}$ & 329 \\
\hline NDI & & 78.67 & 302 & iPc & 58 & 02.50 & -0.5 & 1 & STU & & 80.13 & 358 \\
\hline & & $1.0 \mathrm{~s}$ & 28 & $0.00 \mathrm{~nm}$ & & 6 & $.2 \mathrm{mb}$ & 1 & & & $1.0 \mathrm{~s}$ & $36 e$ \\
\hline & z & $20 \mathrm{~s}$ & & $7.45 \mathrm{um}$ & & & $.0 \mathrm{Msz}$ & 1 & & $z$ & $20 \mathrm{~s}$ & \\
\hline & 1 & $20 \mathrm{~s}$ & & $6.03 \mathrm{um}$ & & & & I & LDF & & 80.21 & \\
\hline & $E$ & $20 \mathrm{~s}$ & & 3. 19 um & & & & I & & & $1.1 \mathrm{~s}$ & 348 \\
\hline & & & & is & 08 & 11.50 & & & UH & & 80.24 & 358 \\
\hline$R U$ & & 78.68 & 354 & $\mathrm{iPC}$ & 58 & 02.70 & 0.1 & 1 & VKA & & 80.27 & 353 \\
\hline & & $1.2 \mathrm{~s}$ & 248 & $8.00 \mathrm{~nm}$ & & & $.1 \mathrm{mb}$ & 1 & & & $3.0 \mathrm{~s}$ & 873 \\
\hline & 2 & $19 \mathrm{~s}$ & & $6.40 \mathrm{um}$ & & & $\theta M s z$ & 1 & & $z$ & 185 & \\
\hline & 1 & $19 \mathrm{~s}$ & & $4.60 u m$ & & & & 1 & ZST & & 80.28 & 352 \\
\hline & 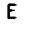 & $19 \mathrm{~s}$ & & $1.00 \mathrm{um}$ & & & & 1 & ANN & & 80.28 & 338 \\
\hline & & & & e & 58 & 16.50 & & 1 & & & 9.05 & 1800 \\
\hline & & & & $P P$ & 00 & 49.50 & & 1 & & & & \\
\hline & & & & e & 01 & 26.40 & & 1 & PSZ & & 80.29 & 350 \\
\hline & & & & es & 07 & 58.00 & & 1 & GRR & & 80.38 & \\
\hline TNS & & 78.69 & 358 & ePc & 58 & 03.00 & 0.2 & I & & & 1.15 & 386 \\
\hline Dou & & 78.84 & 1 & $\mathrm{Pct}$ & 58 & 03.50 & 0.0 & 1 & CDF & & 80.52 & 359 \\
\hline & & $1.2 \mathrm{~s}$ & 1908 & $0.00 \mathrm{~nm}$ & & &.$\theta \mathrm{mb}$ & 1 & SRO & & 80.55 & 352 \\
\hline & & & & $\mathrm{S}$ & 08 & 03.00 & & I & & & $20 \mathrm{~s}$ & \\
\hline$S P C$ & & 79.00 & 350 & iPc & 58 & 04.50 & -0.2 & 1 & & E & $20 \mathrm{~s}$ & \\
\hline GRF & & 79.14 & 356 & $i P C$ & 58 & 06.10 & 0.9 & 1 & & & & \\
\hline & & $1.1 \mathrm{~s}$ & 996 & $\mathrm{~m}$ & & & $.7 \mathrm{mb}$ & & KMR & & 80.63 & 34 \\
\hline
\end{tabular}




\begin{tabular}{|c|c|c|c|c|c|c|c|}
\hline & $1.1 \mathrm{~s}$ & 180 & $0.00 \mathrm{~nm}$ & & & $.1 \mathrm{mb}$ & 1 \\
\hline & & & (s) & 08 & 42.00 & & I HYB \\
\hline GRS & 82.78 & 330 & IPC & 58 & 25.20 & 0.5 & i \\
\hline & $1.8 \mathrm{~s}$ & 450 & $0.00 \mathrm{~nm}$ & & & $.2 \mathrm{mb}$ & I OHR \\
\hline & & & ipp & 58 & 32.60 & $23 \mathrm{kmX}$ & 1 \\
\hline & & & $i s P$ & 58 & 38.00 & & i \\
\hline & & & is & 08 & 43.00 & & I RMP \\
\hline TMA & 82.80 & 358 & i $P+$ & 58 & 25.00 & 0.3 & I \\
\hline DIX & 82.85 & 359 & iP+ & 58 & 26.00 & 0.9 & i \\
\hline MMK & 82.87 & 359 & iP+ & 58 & 26.30 & 1.1 & I РTO \\
\hline CEY & 82.92 & 354 & iPc & 58 & 25.00 & -0.2 & I \\
\hline DRA & 82.95 & 347 & eP & 58 & 26.00 & 0.7 & i $c 00$ \\
\hline $8 \cup C_{1}$ & 83.01 & 346 & iPc & 58 & 24.50 & -1.1 & I DUI \\
\hline $\mathrm{KHI}$ & 83.02 & 318 & iP & 58 & 26.50 & 0.4 & I RDP \\
\hline SSR & 83.10 & 349 & $e^{P}$ & 58 & 27.00 & 0.9 & FOG \\
\hline IPM & 83.23 & 269 & ePC & 58 & 27.40 & 0.2 & I DST \\
\hline & $1.0 \mathrm{~s}$ & 56 & $6.80 \mathrm{~nm}$ & & & $.6 \mathrm{mb}$ & I KER \\
\hline SAL & 83.25 & 357 & $\mathrm{PC}$ & 58 & 27.00 & 0.2 & I EZN \\
\hline ORO & 83.30 & 359 & $P C$ & 58 & 28.00 & 0.8 & I BRT \\
\hline NAK & 83.35 & 330 & iPd & 58 & 27.90 & 0.5 & I ASPA \\
\hline RJF & 83.56 & 3 & iPC & 58 & 29.00 & 0.6 & $|P A| G$ \\
\hline & $1.2 \mathrm{~s}$ & 315 & $5.00 \mathrm{~nm}$ & & & $.3 m b$ & | EBR \\
\hline$K G M$ & 83.79 & 265 & $\mathrm{ePc}$ & 58 & 30.50 & 0.5 & i \\
\hline LFF & 83.90 & 4 & iPc & 58 & 31.00 & 0.9 & $\mid \mathrm{LCl}$ \\
\hline & $1.2 \mathrm{~s}$ & 828 & $3.00 \mathrm{~nm}$ & & & $.8 \mathrm{mb}$ & i \\
\hline CAF & 83.96 & 3 & $I P C$ & 58 & 31.40 & 0.9 & i sGo \\
\hline & $1.2 \mathrm{~s}$ & 563 & $3.00 \mathrm{~nm}$ & & & $.6 \mathrm{mb}$ & i \\
\hline VGI & 83.97 & 358 & $\mathrm{Pd}$ & 58 & 32.00 & 1.5 & i \\
\hline$T A B$ & 84.09 & 329 & eP+ & 58 & 32.00 & 0.6 & | ORI \\
\hline WB2 & 84.15 & 228 & iPC & 58 & 30.20 & -1.4 & $1 P O O$ \\
\hline & & & $\mathrm{i}$ & 58 & 43.80 & & I KRP \\
\hline WRA & 84.16 & 228 & $P C$ & 58 & 30.50 & -1.2 & i \\
\hline & $1.0 \mathrm{~s}$ & 30 & D. $10 \mathrm{~nm}$ & & 5 & $.4 \mathrm{mb}$ & I ATH \\
\hline LPO & 84.17 & 3 & iPC & 58 & 32.30 & 0.8 & I BPA \\
\hline & $1.2 \mathrm{~s}$ & 765 & $5.00 \mathrm{~nm}$ & & & $.7 \mathrm{mb}$ & I LGN \\
\hline$K \vee T$ & 84.18 & 338 & iP & 58 & 32.60 & 0.9 & $|A L|$ \\
\hline PVL & 84.31 & 346 & iPd & 58 & 32.00 & -0.3 & I GBA \\
\hline STV & 84.69 & 359 & $\mathrm{PC}$ & 58 & 34.00 & -0.2 & $i$ \\
\hline PRT & 84.95 & 356 & $P$ & 58 & 38.50 & $3.1 x$ & I GIB \\
\hline & & & $P P$ & 58 & 55.00 & $58 \mathrm{kmx}$ & I ERC \\
\hline & & & $\mathrm{s}$ & 08 & 57.00 & & PAG \\
\hline$F \mid R$ & 85.05 & 356 & $i P c$ & 58 & 37.00 & 1.1 & I MGG \\
\hline & & & is & 08 & 58.00 & & I TOV \\
\hline VTS & 85.14 & 347 & iPc & 58 & 37.00 & 0.7 & i \\
\hline DMK & 85.18 & 344 & iPd & 58 & 36.60 & 0.0 & I UAV \\
\hline$D I M$ & 85.33 & 345 & $i P c$ & 58 & 39.00 & 1.7 & | ME I \\
\hline FRF & 85.38 & 359 & iPc & 58 & 38.50 & 0.9 & I SDV \\
\hline & $1.1 \mathrm{~s}$ & 545 & $5.00 \mathrm{~nm}$ & & & $.7 \mathrm{mb}$ & 1 \\
\hline LRG & 85.48 & 360 & iPc & 58 & 39.40 & 1.3 & $\mathrm{CHN}$ \\
\hline & $1.1 \mathrm{~s}$ & 545 & $5.00 \mathrm{~nm}$ & & & $.7 \mathrm{mb}$ & I YOU \\
\hline LMR & 85.60 & 360 & IPC & 58 & 39.80 & 1.1 & 1 \\
\hline & $1.1 \mathrm{~s}$ & 567 & $.00 \mathrm{~nm}$ & & & $.7 \mathrm{mb}$ & I STK \\
\hline TSI & 85.61 & 270 & $e(P)$ & 58 & 39.00 & -0.1 & I FUO \\
\hline ISK & 85.68 & 343 & eP & 58 & 39.20 & 0.1 & I CAN \\
\hline TRT & 85.70 & 252 & iPC & 58 & 38.50 & -1.0 & 1 \\
\hline & $1.0 \mathrm{~s}$ & 101 & $.00 \mathrm{~nm}$ & & & $.0 \mathrm{mb}$ & I CAR \\
\hline CTT & 85.72 & 343 & ip & 58 & 38.30 & -1.0 & 1 \\
\hline$K D Z$ & 85.76 & 345 & iPc & 58 & 40.00 & 0.5 & i \\
\hline EPF & 85.79 & 4 & iPc & 58 & 39.70 & 0.0 & i \\
\hline & $1.2 \mathrm{~s}$ & 324 & $4.00 \mathrm{~nm}$ & & & $4 \mathrm{mb}$ & 1 \\
\hline
\end{tabular}

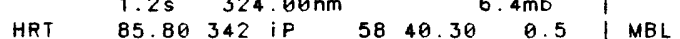
SKO
$1.3 \mathrm{~s} 1300.00 \mathrm{~nm}$

5855.00

IS 0904.00

\begin{tabular}{lllll|l} 
& & ES & 09 & 04.00 & JER \\
& & iSS & 1504.00 & & PSO \\
YLV & $86.10342 \mathrm{eP}$ & 5841.30 & -0.1 & NAU
\end{tabular}

PDI $\quad 86.16 \quad 355 \quad \mathrm{PC}$

GPA

LGR

86.19

$86.20 \quad 6$ iPc

$1.4 \mathrm{~s} \quad 1.27 \mathrm{~nm}$

iPP $\quad 02 \quad 04.20$

MFT
CVF

\begin{tabular}{llllll|l} 
& eS & 09 & 14.70 & & \\
86.27344 & iP & 58 & 42.30 & 0.1 & NAI
\end{tabular}

AOU

MNS

$86.34358 \mathrm{iPC}$

\begin{tabular}{lll|l|l}
$86.36354 \mathrm{PC}$ & 5843.50 & $1.8 \mathrm{mb}$ & ITR \\
BAO
\end{tabular}

86. $37355 \mathrm{PC}$

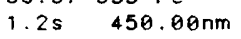
$(\mathrm{S})$
$\mathrm{i} \mathrm{PC}$

VAY

SRS

$\begin{array}{llll}\text { KNT } & 86.59 & 347 \mathrm{eP}\end{array}$

86.64331 iP

86.7165 iP

$1.2 \mathrm{~s} \quad 195.00 \mathrm{~nm}$

$220 \mathrm{~s} 12.10 \mathrm{um}$ \begin{tabular}{lll|l}
58 & 42.00 & 0.5 & $K I C$
\end{tabular}

\begin{tabular}{lll|l}
58 & 42.30 & 0.6
\end{tabular} SOB 1 BNG

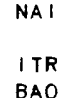
\begin{tabular}{lll|l}
58 & 42.50 & 0.0 & VAO
\end{tabular} 0846.00 $6.6 \mathrm{mb}$

I BMA \begin{tabular}{llc|l}
58 & 44.30 & 1.2 & \\
58 & 53.70 & $10.3 \times$ & RDJ
\end{tabular} \begin{tabular}{lll|l}
58 & 44.50 & 0.6 & NPA
\end{tabular} $58 \quad 45.28$ 0.6
$6.2 \mathrm{mb}$
$6.3 \mathrm{Msz}$

wo \begin{tabular}{lll|l}
58 & 54.50 & $10.8 X$ & AVY
\end{tabular}

\begin{tabular}{|c|c|c|c|c|c|c|}
\hline & & i & 58 & 58.50 & & \\
\hline g & 294 & $\mathrm{ePc}$ & 58 & 45.00 & -0.5 & \\
\hline Os & 368 & $0.00 \mathrm{~nm}$ & & 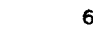 & $6 m b$ & KRI \\
\hline \multirow[t]{3}{*}{86.91} & 349 & iPc & 58 & 45.90 & 0.6 & \\
\hline & & i & 58 & 58.60 & & \\
\hline & & is & 09 & 09.20 & & SPA \\
\hline 86.94 & 355 & $P_{c}$ & 58 & 45.50 & 0.2 & \\
\hline \multirow[t]{2}{*}{$1.4 \mathrm{~s}$} & \multicolumn{3}{|c|}{$1.60 \mathrm{~nm}$} & \multicolumn{3}{|c|}{ 4. $1 \mathrm{mb} x$} \\
\hline & \multirow{3}{*}{11} & e & 02 & 20.00 & & BUL \\
\hline \multirow[t]{2}{*}{86.94} & & ip & 58 & 45.20 & -0.1 & \\
\hline & & es & 09 & 10.00 & & CNG \\
\hline 36.97 & 209 & eP & 58 & 47.00 & 1.6 & \\
\hline 86.98 & 354 & $\mathrm{PC}$ & & 46.20 & 0.7 & \\
\hline 86.99 & 355 & $\mathrm{Pc}$ & 3 & 45.80 & 0.1 & \\
\hline 87.11 & 353 & $\mathbf{P}$ & 8 & 47.50 & 1.4 & MAW \\
\hline 87.18 & 343 & iPc & 8 & 45.90 & -0.7 & \\
\hline 87.21 & 327 & eP & 8 & 48.00 & 1.0 & JOZ \\
\hline 87.37 & 344 & iPc & 8 & 47.10 & -0.3 & \\
\hline 87.53 & 351 & Pd & 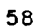 & 48.50 & 0.4 & SLR \\
\hline 87.58 & 226 & eP & 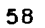 & 48.00 & -0.5 & \\
\hline 87.69 & 346 & eP & 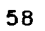 & 60 & -0.4 & \\
\hline \multirow[t]{2}{*}{88.00} & 4 & eP & 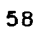 & 00 & -0.4 & PRE \\
\hline & & es & & 00 & & \\
\hline 8.00 & 351 & $\mathrm{Pc}$ & & 51.00 & 0.6 & EVA \\
\hline $.4 \mathrm{~s}$ & \multicolumn{3}{|c|}{$1700.00 \mathrm{~nm}$} & \multicolumn{2}{|c|}{$7.1 \mathrm{mb}$} & \\
\hline 88.01 & 353 & $\mathrm{Pc}$ & 58 & 50.20 & -0.2 & BPI \\
\hline \multirow[t]{2}{*}{$1.7 \mathrm{~s}$} & \multicolumn{3}{|c|}{$500.00 \mathrm{~nm}$} & \multirow{2}{*}{\multicolumn{2}{|c|}{$50^{6.5 m b}$}} & \\
\hline & & (S) & 09 & & & PRY \\
\hline 88.42 & 352 & $P C$ & 58 & 53.50 & 1.1 & BFS \\
\hline 88.64 & 298 & iPc & 58 & 54.00 & 0.1 & \\
\hline \multirow[t]{2}{*}{89.45} & 188 & $P$ & 58 & 58.00 & 1.0 & SWZ \\
\hline & & pP & 59 & 09.00 & $35 \mathrm{kmx}$ & \\
\hline 9.60 & 346 & eP & 58 & 58.00 & -0.1 & SEK \\
\hline 89.98 & 62 & eP & 59 & 00.00 & -0.2 & VIR \\
\hline 90.03 & 74 & eP & 59 & 16.50 & $16.1 x$ & $B L F$ \\
\hline 90.41 & 5 & iP- & 59 & 03.00 & 1.2 & $\mathrm{~K} I \mathrm{M}$ \\
\hline 90.59 & 293 & $P$ & 59 & 02.40 & -0.5 & \\
\hline $1.3 \mathrm{~s}$ & \multicolumn{3}{|c|}{$23.00 \mathrm{~nm}$} & \multicolumn{2}{|c|}{ 5. $3 \mathrm{mb}$} & 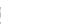 \\
\hline 90.67 & 354 & $(P)$ & 59 & 02.00 & -1.2 & HVD \\
\hline 90 & 355 & $(P)$ & 9 & 50 & $-\theta$ & \\
\hline 90 & 63 & eP & $\theta$ & 20 & 0 & \\
\hline & 63 & eP & 59 & 00 & 2.3 & SUR \\
\hline 91.20 & 73 & ePc & & 80 & 1.9 & \\
\hline $1.0 \mathrm{~s}$ & \multicolumn{3}{|c|}{$163.00 \mathrm{~nm}$} & \multicolumn{2}{|c|}{$6.4 \mathrm{mb}$} & \\
\hline 91.32 & 75 & eP & 59 & 00 & -2.6 & NVL \\
\hline 91.38 & 353 & $P$ & 59 & 05.50 & -0.8 & \\
\hline 91.41 & \multirow{2}{*}{\multicolumn{3}{|c|}{$103.00 \mathrm{~nm}$}} & \multirow{2}{*}{\multicolumn{2}{|c|}{$6.2 \mathrm{mb}$}} & \\
\hline & & & & & & \\
\hline 91.44 & 80 & eP & & 00 & 1.9 & \\
\hline
\end{tabular}

$\begin{array}{lllll}91.44 & 80 \mathrm{eP} & 59 & 09.00 & 1.9\end{array}$

$\begin{array}{ll}i & 5920.60\end{array}$

$\begin{array}{lllll}91.65216 \mathrm{eP} & 5907.00 & -0.3\end{array}$

$92.20 \quad 79 \mathrm{eP} \quad 59 \quad 12.00 \quad 1.2$

$\begin{array}{lllll}92.26 & 209 \text { eP } & 59 & 09.50 & -0.6\end{array}$

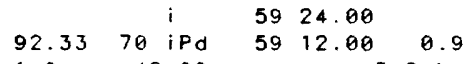

$\begin{array}{lll}1.0 \mathrm{~s} & 48.00 \mathrm{~nm} & 5.9 \mathrm{mb}\end{array}$

$Z 20 \mathrm{~s} 2.13 \mathrm{um}$

$\mathrm{N} 20 \mathrm{~s} \quad 2.84 \mathrm{um}$

$\begin{array}{llllll}92.45 & 239 & \text { iPd } 59 & 11.00 & -0.3\end{array}$

$92.66 \quad 79$ ePKP $59 \quad 14.00 \quad 1.0$

$\begin{array}{lllll}93.10209 \mathrm{eP} & 59 & 16.70 & 2.8\end{array}$

$93.13231 \mathrm{eP} \quad 59 \quad 14.00 \quad-0.3$

$93.22335 \mathrm{ePc} \quad 59 \quad 15.00 \quad 0.0$

$\begin{array}{llllll}93.32 & 84 \mathrm{eP} & 59 & 16.50 & 0.5\end{array}$

$\begin{array}{rrlrrr}95.91 & 241 & \text { еP } & 59 & 27.00 & -0.1 \\ 121.71 & 12 & \text { ePKP } & 04 & 54.30 & -0.5\end{array}$

0509.90

$\begin{array}{ccccc}123.17 & 59 \text { ePKP } & 04 & 57.40 \\ & e & 05 & 11.20\end{array}$

$123.29 \quad 345$ IPKPC $04 \quad 57.00$

$1.2 \mathrm{~s} \quad 55.00 \mathrm{~nm}$

123.64322 ePKP 0501.00

$1.0 \mathrm{~s} \quad 35.00 \mathrm{~nm}$

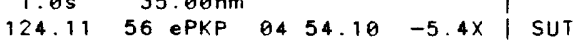

124.3671 PKP 0500.00

130.6475 ePKP $05 \quad 11.90$

$\begin{array}{cccc} & \text { e } & 05 & 25.60 \\ 132.10 & 73 \text { ePKP } & 05 & 15.10\end{array}$

$\begin{array}{lll}132.10 & 73 \text { e } 0529.10\end{array}$

132.8172 ePKP 0523.60

133.84299 ePKPC 0517.00

134.96311 ePKP $05 \quad 20.00$ $\begin{array}{rlr} & \text { EPPKP } 05 & 35.00 \\ & \text { e(PKS) } 08 & 52.00 \\ 139.82320 & \text { ePKP } 05 & 21.00\end{array}$

$\begin{array}{rrrr} & \text { EPPKP } 05 & 35.00 \\ & \text { e(PKS) } 08 & 52.00 \\ 139.82 \quad 320 & \text { ePKP } 05 & 21.00\end{array}$

151

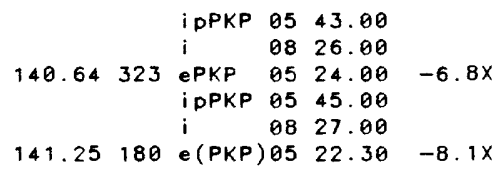

$\begin{array}{cc}14.25 & 180 \text { e(PKP) } \\ 1.0 \mathrm{~s} & 11.50 \mathrm{~nm}\end{array}$

$Z 18 \mathrm{~s} \quad 2.06 \mathrm{um} \quad 5.9 \mathrm{MsZ}$

$\begin{array}{llllll}144.03 & 322 & \text { iPKPd } 05 & 34.00 & -2.6\end{array}$

147.92311 iPKPd 0544.00

$1.0 \mathrm{~s} 900.00 \mathrm{~nm}$

i PPKP 0557.00

i (PKS) 0811.00

$\begin{array}{llllll}148.32 & 218 \text { IPKPO } 05 \quad 46.10 \quad 4.1 X\end{array}$

$1.2 \mathrm{~s} \quad 172.00 \mathrm{~nm}$

148.94310 iPKPC $05 \quad 46.50$

0600.20

$\begin{array}{lllll}149.17 \quad 318 & \text { iPKPC } 05 \quad 44.20 & -0.7\end{array}$

$1.0 \mathrm{~s} \quad 198.00 \mathrm{~nm}$

$218 \mathrm{~s} \quad 4.88 \mathrm{um} \quad 6.3 \mathrm{Msz}$

$\begin{array}{llllll}149.23 \quad 318 & \text { iPKPC } 05 \quad 49.50 \quad 4.5 X\end{array}$

$1.2 \mathrm{~s} \quad 992.00 \mathrm{~nm}$

149.51316 iPKPC 0550.20 0555.10

149.67318 iPKPd 0544.00

0549.00

150.56318 iPKPC 0552.00

$150.82 \quad 319$ IPKPC 0552.10

$0.8 \mathrm{~s} \quad 340.00 \mathrm{~nm}$

151.66321 iPKPC 0548.50 0555.00

151.72316 IPKPC 0554.00

151.83318 IPKPO 0555.00

153.01318 iPKPC 0558.50

153.27321 iPKP 0558.00

$0.9 \mathrm{~s} \quad 91.00 \mathrm{~nm}$

0611.60

154.61318 iPKPd $0502.00-50.6 x$ $1.1 \mathrm{~s} 328.00 \mathrm{~nm}$

$05 \quad 14.90$

03324 iPKPC $05 \quad 44.20-12.8 x$ $1.0 \mathrm{~s} \quad 792.00 \mathrm{~nm}$

$218 \mathrm{~s} 9.76 \mathrm{um}$

$\begin{array}{lllll}160.41 & 186 \text { ePKP } 06 \quad 11.00 & 12.9 \times\end{array}$

$2.5 \mathrm{~s} \quad 140.00 \mathrm{~nm}$ ePPKP $06 \quad 20.00$ ESPKP 0626.00

S.D. $=1.0$ on 477 of 509 obs.

JUN 21,1983 06h $25 \mathrm{~m} 26.43 \pm 0.05 \mathrm{~s}$ $41.339 \mathrm{~N} \pm 0.8 \mathrm{~km} 139.085 \mathrm{E} \pm 1.1 \mathrm{~km}$ DEPTH $=5.0 \mathrm{~km}$ (geophysicist)

$6.7 \mathrm{mb}$ ( 118 obs.) $7.0 \mathrm{Msz}$ ( 22 obs.) HOKKAIDO, JAPAN REGION

(224)

Some domoge in northern Honshu.

Felt (IV JMA) ot Aomori. Mori.

Fukaura and Esoshi; ( 11 I JMA) ot Hokodote, Akito, Muraron,

Hochinohe, Morioko and Mutsu;

(I) JMA) ot Suttsu, Kutchon,

otaru, Miyoko, Urokawo, Sokata,

Onahama and Tamakomai. One meter

tsunami ot Akita, Noshiro and

Wokami. Fifty $\mathrm{cm}$. tsunami

reported in many areas olong the

west coost of northern Honshu.

$\begin{array}{rrlrrr}1.25 & 128 & \text { iP } & 25 & 48.60 & -1.5 \\ 1.34 & 69 & \text { iPd } & 25 & 51.60 & 0.0 \\ & & \mathrm{eS} & 26 & 06.00 & \\ 1.35 & 55 \mathrm{eP} & 25 & 53.00 & 1.2 \\ & & \mathrm{~S} & 26 & 10.00 & \\ 1.39 & 112 & \mathrm{iPd} & 25 & 51.20 & -1.2 \\ & & \mathrm{~S} & 26 & 12.20 & \\ 1.69 & 30 & \mathrm{iPt} & 25 & 56.60 & 0.0 \\ & & \mathrm{~S} & 26 & 24.80 & \\ 1.72 & 55 & \mathrm{eP} & 25 & 58.00 & 0.8 \\ & & \mathrm{eS} & 26 & 19.00 & \\ 1.79 & 154 & \mathrm{eP} & 25 & 57.00 & -1.2 \\ & & \mathrm{eS} & 26 & 24.00 & \\ 1.84 & 53 & \mathrm{P} & 25 & 58.60 & -0.3 \\ 2.02 & 113 & \mathrm{iPd} & 25 & 59.80 & -1.7 \\ & & \mathrm{~S} & 26 & 28.50 & \\ 2.16 & 157 & \mathrm{iP} & 26 & 01.10 & -2.5 \\ 2.28 & 135 & \mathrm{Pd} & 26 & 04.30 & -1.0 \\ & & \mathrm{eS} & 26 & 36.00 & \end{array}$




\begin{tabular}{|c|c|c|c|c|c|c|c|c|c|c|c|c|}
\hline SAP & 2.40 & 43 & $\begin{array}{l}i^{P+}+ \\
S\end{array}$ & $\begin{array}{l}26 \\
26\end{array}$ & $\begin{array}{l}07.40 \\
40.28\end{array}$ & 0.5 & $\begin{array}{l}\text { KYS } \\
\text { GIF }\end{array}$ & 6.19 & $\begin{array}{l}172 \\
198\end{array}$ & $\begin{array}{l}e P \\
e P\end{array}$ & $\begin{array}{l}27 \\
27\end{array}$ & $\begin{array}{l}01.90 \\
04.00\end{array}$ \\
\hline SAK & 2.50 & 166 & $\mathbf{e P}$ & 26 & 15.00 & $6.6 x$ & 1 & & & e & 28 & 01.00 \\
\hline & & & $\mathrm{s}$ & 26 & 48.40 & & MIS & 6.22 & 181 & ePd & 27 & 05.00 \\
\hline SKH & 2.57 & 164 & $i P$ & 26 & 07.30 & -2.1 & $i$ & & & es & 28 & 16.00 \\
\hline$M I Y$ & 2.77 & 127 & $i P d$ & 26 & 10.90 & -1.4 & I SHO & 6.23 & 64 & $i \mathrm{Pc}$ & 27 & 00.00 \\
\hline & & & $\mathrm{s}$ & 26 & 57.50 & & 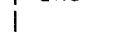 & $1.0 \mathrm{~s}$ & 8 & $8.40 \mathrm{~nm}$ & & \\
\hline URA & 2.88 & 72 & Pd & 26 & 14.60 & 0.7 & i & $213 \mathrm{~s}$ & 2674 & $4.00 \mathrm{um}$ & & \\
\hline & & & is & 26 & 54.80 & & i & $13 \mathrm{~s}$ & 2480 & $0.00 \mathrm{um}$ & & \\
\hline OFUJ & 3.00 & 138 & $\mathrm{P}$ & 26 & 13.70 & -1.8 & i & $13 \mathrm{~s}$ & 1000 & $0.00 \mathrm{um}$ & & \\
\hline OFU & 3.04 & 138 & Pd & 26 & 15.70 & -0.3 & i & & & is & 28 & 08.00 \\
\hline & & & is & 26 & 55.18 & & I YSS & 6.25 & 23 & $i P_{c}$ & 27 & 03.00 \\
\hline RMJ & 3.22 & 35 & $\mathrm{P}+$ & 26 & 19.50 & 1.0 & I TSRJ & 6.28 & 204 & $P$ & 27 & 01.40 \\
\hline & & & $\mathrm{s}$ & 27 & 06.80 & & | AJI & 6.29 & 180 & eP & 27 & 05.00 \\
\hline YAM & 3.23 & 162 & eP & 26 & 16.00 & -2.8 & TAT & 6.37 & 174 & $e^{P}$ & 27 & 06.00 \\
\hline YAMJ & 3.25 & 167 & iP & 26 & 16.50 & -2.5 & i & & & is & 28 & 17.50 \\
\hline HOOJ & 3.31 & 70 & iP & 26 & 21.00 & 1.1 & $\mathrm{SHZ}$ & 6.38 & 185 & eP & 27 & 04.00 \\
\hline I SN & 3.37 & 149 & eP & 26 & 21.00 & 0.2 & i & & & es & 28 & 20.00 \\
\hline & & & es & 27 & 07.00 & & NAG & 6.38 & 196 & $e^{P}$ & 27 & 04.00 \\
\hline SEN & 3.38 & 155 & $\mathrm{P}+$ & 26 & 21.10 & 0.2 & i & & & es & 28 & 25.00 \\
\hline & & & es & 27 & 02.00 & & $K T J$ & 6.38 & 180 & $\mathrm{P}$ & 27 & 04.20 \\
\hline AIK & 3.38 & 191 & eP & 26 & 18.00 & -2.9 & I HIK & 6.45 & 201 & $e^{P}$ & 27 & 04.00 \\
\hline & & & is & 26 & 58.50 & & OSH & 6.57 & 178 & $\mathrm{Pd}$ & 27 & 09.20 \\
\hline$N \mid$ I & 3.42 & 180 & $e^{P}$ & 26 & 18.00 & $-3.5 x$ & $\mathrm{MZH}$ & 6.58 & 208 & $\mathrm{P}$ & 27 & 04.90 \\
\hline & & & es & 27 & 04.00 & & i & & & es & 28 & 18.00 \\
\hline ASA & 3.44 & 44 & $\mathrm{iPt}$ & 26 & 23.00 & 1.3 & I TYK & 6.69 & 211 & eP & 27 & 07.00 \\
\hline & & & is & 27 & 10.50 & & $i$ & & & is & 28 & 35.10 \\
\hline OBI & 3.46 & 61 & $\mathrm{P+}$ & 26 & 23.60 & 1.6 & I HMM & 6.71 & 190 & Pd & 27 & 13.50 \\
\hline & & & es & 27 & 14.00 & & i & & & e & 29 & 04.60 \\
\hline FKS & 3.73 & 163 & eP & 26 & 26.00 & 0.0 & I NGT & 6.73 & 182 & $e^{P}$ & 27 & 16.00 \\
\hline & 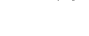 & & e & 27 & 24.30 & & I OMA & 6.76 & 186 & $e^{P}$ & 27 & 10.00 \\
\hline NIIJ & 4.09 & 181 & iP & 26 & 28.30 & -2.7 & SAI & 6.82 & 223 & eP & 27 & 08.00 \\
\hline & & & $\mathrm{s}$ & 27 & 14.40 & & KYO & 6.84 & 204 & eP & 27 & 10.00 \\
\hline KUS & 4.27 & 66 & eP & 26 & 34.00 & 0.4 & T TSU & 6.93 & 198 & eP & 27 & 12.00 \\
\hline & & & is & 27 & 22.50 & & $i$ & & & es & 28 & 19.00 \\
\hline TKD & 4.28 & 189 & eP & 26 & 31.00 & -2.6 & | TOTJ & 6.96 & 214 & $P$ & 27 & 10.30 \\
\hline & & & es & 27 & 21.00 & & TOT & 6.98 & 215 & eP & 27 & 09.00 \\
\hline WAJ & 4.29 & 204 & $e^{P+}$ & 26 & 32.00 & -1.8 & $i$ & & & e & 28 & 36.00 \\
\hline & & & es & 27 & 23.00 & & TKO4 & 7.01 & 188 & $P$ & 27 & 14.00 \\
\hline SHR & 4.31 & 168 & $\mathrm{P}$ & 26 & 32.70 & -1.4 & TKO3 & 7.22 & 187 & $\mathrm{P}$ & 27 & 15.80 \\
\hline WAK & 4.49 & 24 & $\mathrm{P}+$ & 26 & 39.10 & 2.5 & O OSA & 7.22 & 204 & eP & 27 & 15.00 \\
\hline & & & es & 27 & 52.00 & & 1 & & & i & 29 & 05.20 \\
\hline ONA & 4.61 & 162 & $\mathrm{P}$ & 26 & 39.60 & 1.3 & osk & 7.24 & 203 & eP & 27 & 16.00 \\
\hline & & & is & 27 & 41.30 & & $i$ & & & $i$ & 28 & 51.00 \\
\hline$A B J$ & 4.67 & 53 & $e^{P+}$ & 26 & 40.00 & 0.7 & Ков & 7.32 & 206 & $P+$ & 27 & 16.80 \\
\hline & & & is & 27 & 36.30 & & 1 & & & es & 29 & 00.00 \\
\hline NGN & 4.72 & 189 & $e^{P}$ & 26 & 40.10 & 0.1 & I HIM & 7.35 & 209 & $e^{P}$ & 27 & 18.00 \\
\hline & & . & $\mathrm{s}$ & 27 & 36.80 & & 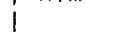 & 1.00 & & es & 28 & 53.00 \\
\hline UTS & 4.83 & 172 & ePt & 26 & 41.00 & -0.5 & TKOZ & 7.45 & 189 & $e^{P}$ & 27 & 19.00 \\
\hline & & & e & 27 & 40.00 & & KUR & 7.50 & 56 & iPc & 27 & 21.00 \\
\hline MAT & 4.84 & 188 & $P$ & 26 & 40.00 & -1.7 & MTS & 7.53 & 221 & eP & 27 & 21.00 \\
\hline MTMJ & 4.85 & 192 & $P$ & 26 & 39.60 & -2.3 & $i$ & & & is & 28 & 44.50 \\
\hline TOY & 4.85 & 198 & $e^{P}$ & 26 & 43.00 & 1.2 & YONJ & 7.57 & 218 & P & 27 & 19.10 \\
\hline & & & es & 27 & 38.00 & & OWA & 7.61 & 198 & eP & 27 & 20.00 \\
\hline MAE & 4.93 & 180 & $e^{P}$ & 26 & 42.00 & -0.9 & WKYJ & 7.62 & 202 & $P$ & 27 & 21.40 \\
\hline$M I T$ & 5.07 & 167 & eP & 26 & 47.00 & 2.1 & TKOI & 7.65 & 189 & $P$ & 27 & 24.10 \\
\hline & & & $\mathrm{s}$ & 27 & 47.20 & & MOJ & 7.69 & 298 & $i P d$ & 27 & 20.00 \\
\hline KAN & 5.15 & 202 & eP & 26 & 45.00 & -1.0 & SUM & 7.73 & 207 & eP & 27 & 23.00 \\
\hline & & & $\mathrm{s}$ & 27 & 45.80 & & I WKY & 7.75 & 205 & iPd & 27 & 25.00 \\
\hline TSK & 5.18 & 171 & eP & 26 & 44.20 & -2.3 & $i$ & & & is & 28 & 55.50 \\
\hline KMG & 5.19 & 177 & $P$ & 26 & 51.30 & $4.7 x$ & OKA & 7.80 & 213 & ePt & 27 & 23.00 \\
\hline KAKJ & 5.20 & 170 & ip & 26 & 44.60 & -2.1 & $i$ & & & es & 28 & 51.00 \\
\hline NEM & 5.21 & 65 & $P+$ & 26 & 46.70 & -0.2 & TKM & 8.06 & 211 & eP & 27 & 27.00 \\
\hline & & & $s$ & 27 & 43.40 & & $i$ & & & $\mathrm{~s}$ & 29 & 05.80 \\
\hline CHJJ & 5.28 & 181 & iP & 26 & 46.50 & -1.5 & TKS & 8.09 & 208 & $\mathrm{P}+$ & 27 & 27.50 \\
\hline DDR & 5.33 & 179 & eP & 26 & 47.50 & -1.2 & i & & & e & 29 & 41.00 \\
\hline TKY & 5.37 & 196 & $P$ & 26 & 48.50 & -0.8 & HJJ & 8.24 & 176 & $\mathrm{Pd}$ & 27 & 32.90 \\
\hline$V L A$ & 5.63 & 291 & iP & 26 & 50.00 & -2.8 & i & & & $\mathrm{s}$ & 29 & 19.30 \\
\hline YUK & 5.64 & 59 & $i P_{c}$ & 26 & 53.20 & 0.3 & SHJ & 8.31 & 200 & $P$ & 27 & 29.00 \\
\hline & $2.0 \mathrm{~s}$ & & $9.00 r$ & & & $2 \mathrm{mb} \times$ & & & & s & 29 & 09.70 \\
\hline & & & es & 27 & 57.60 & & TKSJ & 8.36 & 210 & $\mathrm{P}$ & 27 & 31.60 \\
\hline roK & 5.67 & 174 & $P$ & 27 & 00.00 & $6.6 x$ & I SHK & 8.47 & 219 & ip & 27 & 31.60 \\
\hline & & & es & 28 & 06.00 & & $i$ & & & es & 28 & 59.50 \\
\hline KOF & 5.68 & 184 & eP & 26 & 54.00 & 0.4 & HMD & 8.48 & 223 & eP & 27 & 34.00 \\
\hline & & & es & 27 & 59.00 & & i & & & $s$ & 29 & 10.10 \\
\hline SRY & 72 & 178 & $e^{P}$ & 26 & 55.10 & 0.9 & $H \mid R$ & 8.72 & 219 & $P$ & 27 & 40.00 \\
\hline FUK & & 204 & eP & 26 & 57.00 & 2.8 & & & & e & 28 & 21.50 \\
\hline & & & 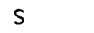 & 28 & 01.00 & & KOC & 8.93 & 211 & $P$ & 27 & 39.60 \\
\hline $\mathrm{CHO}$ & 5.77 & 166 & $P$ & 26 & 56.10 & 1.3 & i & & & $s$ & 29 & 50.90 \\
\hline & & & e & 28 & 10.00 & & MRT & 8.97 & 207 & $e^{P}$ & 27 & 41.00 \\
\hline FUN & 84 & 183 & $e^{P}$ & 27 & 02.00 & $6.2 x$ & $i$ & & & e & 29 & 28.00 \\
\hline 110 & 5.90 & 190 & ePt & 26 & 55.00 & -1.7 & MTY & 9.00 & 216 & eP & 27 & 4100 \\
\hline & & & is & 27 & 58.60 & & 1 & & & e & 29 & 37.00 \\
\hline OYM & & 179 & $e^{P}$ & 26 & 56.90 & 0.1 & SHNJ & 9.58 & 224 & $\mathrm{P}$ & 27 & 4840 \\
\hline YOK & 5.91 & 175 & $P d$ & 27 & 01.60 & $4.9 x$ & UWA & 9.62 & 215 & eP & 27 & 51.00 \\
\hline & & & es & 28 & 17.00 & & SHN & 9.80 & 224 & $P d$ & 27 & 53.40 \\
\hline $10 \mathrm{~J}$ & 5.92 & 189 & $\mathrm{P}$ & 26 & 55.40 & -1.6 & i & & & es & 29 & 40.00 \\
\hline
\end{tabular}


$210 \quad 06 \mathrm{~h}$ \begin{tabular}{lcccccc|c} 
GUMO & 28.10 & 168 & $\mathrm{ePt}$ & 31 & 19.50 & -1.7 & $\mathrm{COL}$ \\
PJG & 28.10 & $168 \mathrm{e}(\mathrm{P})$ & 31 & 19.50 & -1.7 & \\
& & & $(\mathrm{PP})$ & 31 & 26.60 & $25 \mathrm{kmX}$ & FBA \\
GUA & 28.16 & $168 \mathrm{ePt}$ & 31 & 19.50 & -2.2 & MDG \\
& $0.8 \mathrm{~s}$ & $269.00 \mathrm{~nm}$ & & $6.1 \mathrm{mb}$ & FRU
\end{tabular} $\begin{array}{cccc}2 & 22 \mathrm{~s} & 176.00 \mathrm{um} \\ \mathrm{GZH} & 28.22 & 238 \mathrm{PC}\end{array}$ HKC 28.27235 iP

MCO $28.78236 \mathrm{eP}$

BAG $29.53218 \mathrm{iPt}$

GTA $29.81279 \mathrm{iPd}$

PP

CD
GYA
TIK
MAN
OCP
CNP
ADK
PLP
KM

$\begin{array}{llll}\text { TIK } & 30.52 & 251 \mathrm{PC} \\ & 30.80 & 354 \mathrm{iPC}\end{array}$

$30.84216 \mathrm{iPC}$

$30.86216 \mathrm{eP}$

31.37208 ePd

$31.70 \quad 55 \quad \mathrm{iPc}$

$32.51206 \mathrm{ePd}$

$34.14253 \mathrm{Pt}$

$9.0 \mathrm{~s} \quad 19.30 \mathrm{~nm}$

3620.00 6. $6 \mathrm{Msz}$

3123.00

3124.80

3625.00

$3131.00 \quad-3.2 \times \mid$ RAB \begin{tabular}{ll|l|l}
31 & $34.70^{6.4 m b}$ & -1.9 & $K S H$ \\
& & KHE
\end{tabular}

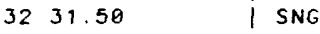
$\begin{array}{ll}36 & 31.20\end{array}$ $\begin{array}{lll}31 & 36.60 & -3.3 x\end{array}$ $\begin{array}{lll}31 & 43.00 & 0.0\end{array}$ $\begin{array}{llll}31 & 41.50 & -3.2 \times\end{array}$ $\begin{array}{llll}31 & 47.00 & 1.4 & \text { । }\end{array}$ $3134.00-11.8 \times$ I LAT \begin{tabular}{lll|l}
31 & 52.00 & 1.7 & | ANR
\end{tabular}

$3151.40-1.5$ । $\begin{array}{lll}31 & 57.30 & -3.0 \times\end{array}$

E $14 \mathrm{~s} 220.00 \mathrm{um}$ $\begin{array}{lll}32 & 14.00 & -0.7\end{array}$

$\begin{array}{lll}\text { PP } & 32 & 33.00 \\ \text { SP } & 32 & 41.00 \\ \text { PP } & 33 & 29.00 \\ \text { PCP } & 34 & 58.00 \\ \text { S } & 37 & 31.00 \\ \text { SS } & 37 & 54.00 \\ \text { iSCS } & 42 & 22.00\end{array}$

ILT $34.90 \quad 26 \mathrm{Pd}$

$\begin{array}{rrr}34.90 & 26 & \mathrm{PO} \\ 35.15 & 205 \mathrm{eP}\end{array}$

4222.00

36.17203 iPct

$36.93 \quad 307 \mathrm{iPc}$

37.43291 iPC is $\quad 3832.00$

WM

NR

$$
38.45334 \mathrm{iPC}
$$

$1.6 \mathrm{~s} \quad 750 \mathrm{PC}$ esP 3303.00 is 3838.00

ANM

LSA

KKM

SDN

BDT

PCT

TTA

JAY

IMA

PRZ

$\begin{array}{lll}38.97 & 34 & \text { iS } \\ \text { iPc }\end{array}$

$40.24269 \mathrm{PC}$

$40.64216 \mathrm{ePd}$

$41.20 \quad 49 \mathrm{eP}$

$41.89247 \mathrm{eP}$

$0.9 \mathrm{~s} \quad 147.00 \mathrm{~nm}$

$42.05242 \mathrm{iPc}$

$43.0137 \mathrm{iPc}$

$43.67 \quad 178 \mathrm{ePd}$

$43.83168 \mathrm{eP}$

$43.98 \quad 33 \mathrm{iPC}$

$\begin{array}{ll}44.32 & 293 \mathrm{eP} \\ 1.4 \mathrm{~s} & 750.00 \mathrm{~nm}\end{array}$

$218 \mathrm{~s} 960.00 \mathrm{um}$

E $18 \mathrm{~s} 1128.00 u m$

EPCP $35 \quad 22.00$

is $40 \quad 13.00$

TL

\subsection{0}

$33 \quad 07.20$

3310.00

3309.90

$\begin{array}{lll}33 & 12.70-0.5\end{array}$

$3316.00-3.3 x$

$5.7 \mathrm{mb}$

$23.006 .3 \mathrm{mb}$

$\begin{array}{lll}33 & 34.00 & -1.2\end{array}$

$\begin{array}{lll}33 & 34.70 & -1.3\end{array}$

$3338.00-1.1$
$6.4 \mathrm{mb}$

7. $8 \mathrm{Msz}$

$\begin{array}{cc}44.75 & 294 \text { iPc } \\ 18 \mathrm{~s} & 580.06 \mathrm{um}\end{array}$

EPP $35 \quad 34.5$

is 4023.50

iscs 4338.00

WWW

$K \vee G$

$44.90174 \mathrm{eP}$

$44.99163 \mathrm{eP}$

45.05294 iPc 3344.00 3343.00

3346.00 ePP 3529.00 is 4025.00

$K D C$
$K K N$

PKI

DMN

AAI

PMR

$220 \mathrm{~s} \quad 150.00 \mathrm{um}$

NRN
$45.26 \quad 45 \mathrm{iPc}$

$45.64270 \mathrm{eP}$

$45.66270 \mathrm{eP}$

$45.87270 \mathrm{eP}$

$45.90 \quad 195$ iPd

$0.7 \mathrm{~s} \quad 186.00 \mathrm{~nm}$

$\begin{array}{ll}46.33 & 39 \mathrm{iPc} \\ 2.05 & 446.00 \mathrm{~nm}\end{array}$

$\begin{array}{lll}33 & 45.70 & -0.5\end{array}$

$3350.40 \quad 0.4$

$\begin{array}{lll}33 & 47.90 & -2.4\end{array}$

$\begin{array}{lll}33 & 49.70 & -2.1\end{array}$

$3352.806 \frac{1.1}{6.2 \mathrm{mb}}$

$3354.30^{6}-0.4$

6. $2 \mathrm{mb}$

46. $36 \quad 292 \mathrm{eP}$
eP $\quad 3355.20^{-0.4}$ 4050.00

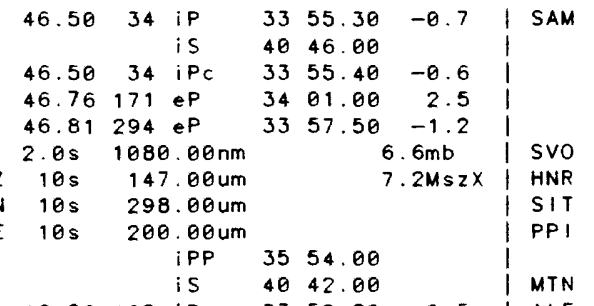
$46.86162 \mathrm{iPC+} 3359.80 \quad 0.5 \quad$ ALE $47.14290 \mathrm{eP} \quad 3403.00 \quad 1.5$ \begin{tabular}{llll|l}
48.06 & $347 \mathrm{iPc}$ & 3408.00 & -0.1 & HON
\end{tabular} 48.19236 iPCt $3409.00-0.8$ HYB $1.2 \mathrm{~s} \quad 1060.00 \mathrm{~nm}$

$\begin{array}{lll}Z & 22 \mathrm{~s} & 55.60 \mathrm{um} \\ \mathrm{N} & 20 \mathrm{~s} & 56.40 \mathrm{um}\end{array}$

E $20 \mathrm{~s} \quad 39.00 \mathrm{um}$

$\begin{array}{rrrrrr}48.31 & & \text { is } & 40 & 15.50 & \\ \text { eP } & 34 & 11.50 & 0.9\end{array}$

$49.16293 \mathrm{eP} \quad 3416.70 \quad-0.4$

$1.6 \mathrm{~s} \quad 650.00 \mathrm{~nm}$

Z $12 \mathrm{~s} \quad 240.00 \mathrm{um}$

$\mathrm{N} 12 \mathrm{~s} \quad 285.00 \mathrm{um}$

E $12 \mathrm{~s} \quad 455.00 \mathrm{um}$

$49.69 \quad 4124.50$

$49.73158 \mathrm{eP} \quad 3420.00 \quad-1.8$

$49.97233 \mathrm{ePd} \quad 3424.10 \quad 0.6$

$1.0 \mathrm{~s} \quad 159.00 \mathrm{~nm}$

$e^{34} 44.90$

KGM

$\begin{array}{lllll}50.68 & 229 \mathrm{ePd} & 3428.00 & -0.9\end{array}$

0.9 s $255.00 \mathrm{~nm}$

$$
\text { e }
$$

$55 \quad 47.50$

$\begin{array}{llllll}50.69 & 168 & \text { iPc } & 34 & 29.00 & -0.1\end{array}$

50.77316 IPC $3429.00 \quad-0.1$

$\begin{array}{rrr}9.0 \mathrm{~s} & * * * * * * * \mathrm{~nm} & 7.4 \mathrm{mb} x \\ 218 \mathrm{~s} & 531.00 \mathrm{um} & 7.6 \mathrm{Msz}\end{array}$

N $18 \mathrm{~s} \quad 167.00 \mathrm{um}$

E $18 \mathrm{~s} \quad 345$.

iPP $36 \quad 23.00$

is 4145.00

isS 4520.00

$\begin{array}{rrrrr}50.90 & 40 & \text { iPc } & 34 & 30.30 \\ 51.04 & 170 & \text { ePt } & 34 & 30.00\end{array}$

3430.30

51.04295 iP

3431.60

$3.0 \mathrm{~s} \quad 9500.00 \mathrm{~nm}$

$\begin{array}{lll}\text { iS } & 41 & 48.60 \\ \text { eSS } & 4520.00\end{array}$ eSSS 4658.60

ND I

$\begin{array}{llllll}51.23 & 276 & \text { iP } & 34 & 32.00 & -1.1\end{array}$

$\begin{array}{lll}\text { PCP } & 35 \quad 46.00\end{array}$

i $\quad 3612.00$

isS 4524.00

GAR

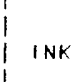

INK

ARU

$\begin{array}{ll}51.33 & 292 \mathrm{eP} \\ 2.5 \mathrm{~s} & 250.00 \mathrm{~nm}\end{array}$

$\begin{array}{lll} & \text { iPPP } 3732.50 \\ 51.44 \quad 28 & \end{array}$

$0.9 \mathrm{~s} \quad 650.00 \mathrm{~nm}$

51.97316 iPc $3438.00 \quad-0.3$

$2.0 \mathrm{~s} 3800.00 \mathrm{~nm}$

$217 \mathrm{~s} \quad 525.00 \mathrm{um}$

N $12 \mathrm{~s} 280.00 \mathrm{um}$

E $20 \mathrm{~s} 345.00 \mathrm{um}$

EPCP $35 \quad 45.00$

EPP $36 \quad 35.00$

is 4201.00

escs 4429.00

1

KUL

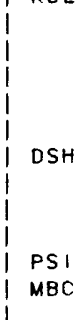

iSS 4536.00

$\begin{array}{rrrrr}52.21 & 291 \text { iPc } & 34 & 40.50 & 0.1 \\ 17 \mathrm{~s} & 7931.00 \mathrm{um} & & 8.8 \mathrm{Ms} 2 \mathrm{X}\end{array}$

N $16 \mathrm{~s} 461.00 \mathrm{um}$

is 4205.50

iSP 4209.50

iscs 4425.90

$\begin{array}{lllll}52.60 & 292 \mathrm{IPC} & 34 & 43.40\end{array}$

IPP $36 \quad 50.00$

iPPP 3750.00

eS 4210.00

52.73234 PO $343750 \quad-6.9 \times$ । MAK

$52.93 \quad 17$ Pc $3443.20-2.1$

$0.8 \mathrm{~s} \quad 546.00 \mathrm{~nm}$

53. $18199 \mathrm{eP}$
I KUPT
I VAN

$\begin{array}{llllll}53.34 & 294 & \text { iPc } & 34 & 47.60 & -1.2\end{array}$

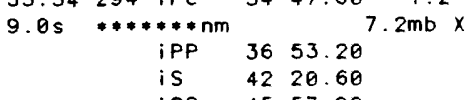

\begin{tabular}{llllll}
53.74 & iSS & 45 & 57.00 & \\
\hline$P$ & 34 & 55.00 & $3.2 x$
\end{tabular}

54.05154 e(P) $34 \quad 54.00 \quad 0.0$

$\begin{array}{llllll}54.36 & 42 \mathrm{ePc} & 34 & 56.70 & 0.8\end{array}$

54.37230 eP $3456.30 \quad-0.2$

$1.4 \mathrm{~s} \quad 224.00 \mathrm{~nm} \quad 6.0 \mathrm{mb}$

$54.41 \quad 190$ eP $\quad 34 \quad 53.00 \quad-3.7 x$

$\begin{array}{llllll}55.94 & 3 \text { iP } & 35 & 05.80 & -1.4\end{array}$

$0.7 \mathrm{~s} \quad 268.00 \mathrm{~nm} \quad 6.4 \mathrm{mb}$

$\begin{array}{lllll}56.11 & 90 \mathrm{P} & 35 & 11.00 & 1.9\end{array}$

$\begin{array}{lllll}56.67 & 264 \mathrm{ePc} & 35 & 12.00 & -1.3\end{array}$

$1.6 \mathrm{~s} \quad 1540.00 \mathrm{~nm} \quad 6.8 \mathrm{mb}$ eS 4302.00

$\begin{array}{llllll}58.06 & 334 & \text { iPc } & 35 & 21.20 & -1.2\end{array}$

$\begin{array}{llllll}58.60 & 91 & \mathrm{eP} & 35 & 26.00 & -0.7\end{array}$

58.78338 iPct $3526.00 \quad-1.4$

$0.6 \mathrm{~s} \quad 509.00 \mathrm{~nm} \quad 6.8 \mathrm{mb}$

EPPP $39 \quad 32.50$

QS 4324.00

$\begin{array}{lllll}59.53 & 268 \text { iPc } & 35 & 33.20 & -0.1\end{array}$

$\begin{array}{lllll}59.90 & 262 \mathrm{Pc} & 35 & 33.20 & -2.6\end{array}$

$0.8 \mathrm{~s} \quad 188.00 \mathrm{~nm} \quad 6.3 \mathrm{mb}$

60.08296 eP $\quad 3536.00 \quad-0.8$

$2.0 \mathrm{~s} \quad 2380.00 \mathrm{~nm} \quad 7.0 \mathrm{mb}$

ePCP $36 \quad 26.00$

EPP $37 \quad 46.00$

EPPP 3924.00

MHI $60.15294 \mathrm{iPC+} 3538.40 \quad 0.9$ $2.0 \mathrm{~s} \quad 988.00 \mathrm{~nm} \quad 6.6 \mathrm{mb}$

$\begin{array}{rlrr} & e & 39 & 24.00 \\ & \text { es } & 43 & 56.00 \\ 60.25296 & \text { ePC } & 35 & 36.50\end{array}$

$400.00 \mathrm{~nm} 35.50-1.5$

ePP 3752.00

ESP 4356.00

60.30336 iP $\quad 3536.70 \quad-1.2$

$\begin{array}{llllll}61.02 & 31 & \mathrm{P} & 35 & 41.60 & -1.2\end{array}$

$\begin{array}{llllll}61.03 & 31 & \text { iPC } & 35 & 42.00 & -0.9\end{array}$

$61.07 \quad 31 \mathrm{ePc} \quad 35 \quad 42.00 \quad-1.2$

$1.6 \mathrm{~s} \quad 1220.00 \mathrm{~nm} \quad 6.8 \mathrm{mb}$

$\begin{array}{llllll}61.13 & 185 \mathrm{iPc} & 35 & 40.70 & -3.2 x\end{array}$ $\begin{array}{lll}\text { eS } & 44 & 17.50 \\ \text { EP.P. } & 05 & 01.70\end{array}$

$\begin{array}{llllll}61.34 & 354 \mathrm{iPC} & 35 & 43.00 & -1.8\end{array}$

$1.1 \mathrm{~s} \quad 84.80 \mathrm{~nm} \quad 5.8 \mathrm{mb}$ is 4407.00

$\begin{array}{lllll}61.40 & 46 \mathrm{ePc} & 35 & 44.50 & -1.1\end{array}$

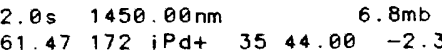

$1.6 \mathrm{~s} \quad 530.00 \mathrm{~nm} \quad 6.5 \mathrm{mb}$

is 4404.00

$61.47172 \mathrm{eP} \quad 3544.70 \quad-1.6$

$2.4 \mathrm{~s} \quad 1010.00 \mathrm{~nm} \quad 6.6 \mathrm{mb}$

$\begin{array}{llllll}61.85 & 338 & \text { ip } & 35 & 51.50 & \\ 65 & 47.30 & -1.2\end{array}$

$61.86332 \mathrm{ipt} 3547.00-1.6$

$1.6 \mathrm{~s} \quad 2530.00 \mathrm{~nm} \quad 7.2 \mathrm{mb}$

$\begin{array}{lll}\text { APP } & 37 & 44.00\end{array}$

ePPP 3924.00

eS 4412.00

$\begin{array}{lllll}61.88 & 292 \mathrm{eP} & 3549.50 & 0.1\end{array}$

$62.07259 \mathrm{iPC} \quad 3551.00 \quad 0.1$

$\begin{array}{llllll}63.34 & 332 & \text { iP } & 35 & 56.50 & -1.8\end{array}$

$\begin{array}{lllll}63.55 & 321 \mathrm{iPC} & 35 & 59.80 & 0.0\end{array}$

$\begin{array}{llllll}63.58 & 328 \text { iPc } & 35 & 59.50 & -0.5\end{array}$ 


\begin{tabular}{|c|c|c|c|c|c|c|c|c|c|c|c|}
\hline & & & is & 44 & 47.00 & & I LGBM & & 69.69 & 53 & ePc \\
\hline PGC & 64.68 & 47 & eP & 36 & 07.00 & -0.3 & I WDC & & 69.95 & 53 & iPc \\
\hline$M B L$ & 64.70 & 200 & eP & 36 & 07.00 & -0.6 & 1 & & & & e \\
\hline PVC & 64.72 & 149 & $i P c$ & 36 & 06.70 & -1.1 & 1 & & & & e \\
\hline ASPA & 64.85 & 185 & eP & 36 & 06.00 & -2.6 & I MEK & & 70.24 & 199 & eP \\
\hline $\mathrm{MCW}$ & 65.00 & 46 & eP & 36 & 09.30 & -0.2 & I RMT & & 70.28 & 54 & eP \\
\hline NUR & 65.31 & 330 & iPct & 36 & 10.20 & -1.0 & I MIN & & 70.66 & 53 & iPc \\
\hline & & & $\mathbf{i}$ & 36 & 20.00 & & FCC & & 70.95 & 26 & ePc \\
\hline & & & ePP & 38 & 32.00 & & i & & $2.2 \mathrm{~s}$ & 5050 & $9.00 \mathrm{~nm}$ \\
\hline & & & ePPP & 40 & 20.00 & & I MSL & & 70.99 & 302 & ePd \\
\hline & & & es & 44 & 56.00 & & SIM & & 71.02 & 313 & $i P c$ \\
\hline & & & ess & 48 & 28.00 & & i & & & & ePcP \\
\hline GRO & 65.32 & 307 & iPc & 36 & 11.00 & -0.6 & 1 & & & & ePP \\
\hline & 8.05 & $* * * *$ & $* * * n \mathrm{~nm}$ & & & $.8 \mathrm{mb} \times$ & 1 & & & & is \\
\hline & & & is & 44 & 55.00 & & 1 & & & & $\in S \subset S$ \\
\hline GMW & 65.68 & 47 & ep & 36 & 14.00 & 0. 2 & I FFC & & 71.06 & 32 & eP \\
\hline $\mathrm{KOU}$ & 65.87 & 154 & iPc & 36 & 15.10 & 0.0 & 1 & & $1.2 \mathrm{~s}$ & 840 & $0.00 \mathrm{~nm}$ \\
\hline TEH & 65.97 & 297 & eP & 36 & 16.00 & 0.0 & I ORV & & 71.21 & 54 & iPc \\
\hline BFW & 66.05 & 49 & eP & 36 & 16.90 & -0.3 & I BKS & & 71.80 & 55 & eP \\
\hline PNT & 66.36 & 44 & iPc & 36 & 17.60 & -0.5 & 1 & & $1.9 \mathrm{~s}$ & 3100 & $900 \mathrm{~nm}$ \\
\hline & $1.0 \mathrm{~s}$ & 159 & $.00 \mathrm{~nm}$ & & & $.2 \mathrm{mb}$ & $i$ & $z$ & $20 s$ & 34 & $4.00 \mathrm{um}$ \\
\hline KRV & 66.46 & 304 & iPd & 36 & 17.00 & -1.9 & I & $N$ & $20 s$ & 15 & $.00 \mathrm{um}$ \\
\hline$z$ & $12 \mathrm{~s}$ & 20 & .00 um & & & $.5 M s z X$ & $i$ & $E$ & $20 \mathrm{~s}$ & 18 & $3.00 \mathrm{um}$ \\
\hline $\mathbf{N}$ & $12 \mathrm{~s}$ & & $5.30 \mathrm{um}$ & & & & 1 & & & & es \\
\hline E & $12 \mathrm{~s}$ & 100 & $.00 \mathrm{um}$ & & & & i & & & & escs \\
\hline & & & EPPP & 40 & 26.00 & & i & & & & ess \\
\hline & & & is & 45 & 08.00 & & i & & & & esss \\
\hline & & & escs & 46 & 12.00 & & I AKU & & 71.85 & 350 & iPct \\
\hline & & & eSs & 49 & 18.00 & & 1 & & 1.95 & 2380 & $9.00 \mathrm{~nm}$ \\
\hline & & & esSS & 52 & 17.00 & & 1 & $z$ & $17 \mathrm{~s}$ & 72 & 2. $10 \mathrm{um}$ \\
\hline PYA & 66.50 & 309 & $\mathrm{iPc}$ & 36 & 18.00 & -1.1 & 1 & & & & $\mathrm{i}$ \\
\hline & $2.0 \mathrm{~s}$ & 1320 & $0.00 \mathrm{~nm}$ & & & $.8 \mathrm{mb}$ & I LRM & & 72.33 & 44 & ePc \\
\hline & & & iPP & 38 & 50.00 & & I KIS & & 72.44 & 318 & iPc \\
\hline & & & es & 45 & 07.00 & & i & & $2.0 \mathrm{~s}$ & 2200 & $.00 \mathrm{~nm}$ \\
\hline & & & is & 45 & 08.00 & & 1 & $z$ & $17 \mathrm{~s}$ & 308 & $3.00 \mathrm{um}$ \\
\hline & & & EPPS & 45 & 49.00 & & 1 & $N$ & $16 \mathrm{~s}$ & 179 & $9.00 \mathrm{um}$ \\
\hline LON & 66.68 & 48 & ip & 36 & 20.00 & -0.3 & i & E & $14 \mathrm{~s}$ & 341 & $.00 \mathrm{um}$ \\
\hline EDM & 67.10 & 38 & ePc & 36 & 20.80 & -2.0 & $i$ & & & & iPPP \\
\hline & $2.0 \mathrm{~s}$ & 2920 & $.00 \mathrm{~nm}$ & & & $.1 \mathrm{mb}$ & $i$ & & & & is \\
\hline COR & 67.11 & 50 & iPc & 36 & 23.00 & 0.1 & 1 & & & & iSP \\
\hline GRS & 67.12 & 303 & iPc & 36 & 22.20 & -1.1 & I $M H C$ & & 72.50 & 56 & eP \\
\hline & $2.0 \mathrm{~s}$ & 830 & $.00 \mathrm{~nm}$ & & 6 & $.6 \mathrm{mb}$ & 1000 & & 72.53 & 168 & iPd \\
\hline$z$ & $12 \mathrm{~s}$ & 32 & $.00 \mathrm{um}$ & & & $.8 \mathrm{MszX}$ & I ARN & & 72.56 & 56 & eP \\
\hline $\mathrm{N}$ & $12 \mathrm{~s}$ & & $.00 \mathrm{um}$ & & & & i CMS & & 72.73 & 174 & eP \\
\hline$E$ & $12 \mathrm{~s}$ & & $0.00 \mathrm{um}$ & & & & I LVV & & 72.86 & 322 & iPc \\
\hline & & & iPCP & 36 & 51.00 & & i & & $9.0 \mathrm{~s}$ & $* * * *$ & $* * * n \mathrm{~nm}$ \\
\hline & & & iPP & 38 & 59.00 & & i & $z$ & $12 \mathrm{~s}$ & 129 & $3.00 \mathrm{um}$ \\
\hline & & & is & 45 & 15.00 & & $i$ & & & & iPPP \\
\hline NAU & 67.22 & 204 & eP & 36 & 22.00 & -1.7 & 1 & & & & is \\
\hline ERE & 67.87 & 304 & iPc & 36 & 28.00 & 0.1 & 1 & & & & iscS \\
\hline & & & is & 45 & 28.00 & & I JAS & & 72.86 & 54 & iPc \\
\hline LEN & 67.93 & 305 & $i P_{c}$ & 36 & 29.00 & 0.6 & I STK & & 72.89 & 178 & eP \\
\hline & $7.0 \mathrm{~s}$ & $* * * *$ & $* * * \mathrm{~nm}$ & & 7 & $.3 \mathrm{mb} \times$ & I SLD & & 72.93 & 56 & ip \\
\hline & & & IPCP & 36 & 48.00 & & I FRB & & 72.98 & 12 & eP \\
\hline & & & iPP & 39 & 04.00 & & I BMN & & 73.20 & 51 & eP \\
\hline & & & EPPP & 40 & 40.00 & & 1 & & & & es \\
\hline$T A B$ & 67.95 & 302 & ipt & 36 & 29.00 & 0.4 & I COP & & 73.28 & 332 & iPc \\
\hline NOU & 68.23 & 153 & iPc & 36 & $29 \quad 40$ & -6.7 & 1 & & $1.5 \mathrm{~s}$ & 2220 & $9.00 \mathrm{~nm}$ \\
\hline UPP & 68.30 & 332 & ip & 36 & 18.50 & $-11.6 x$ & i & & & & is \\
\hline & & & is & 45 & 30.00 & & I CLI & & 73.55 & 318 & ePC \\
\hline NEW & 68.31 & 44 & IPO & 36 & 29.50 & -1.0 & I KLG & & 73.59 & 196 & iPC \\
\hline NDF & 68.70 & 141 & ePC & 36 & 40.20 & $7.1 x$ & I BAC & & 73.73 & 318 & $e P$ \\
\hline $\mathrm{SHI}$ & 68.71 & 291 & eP & 36 & 32.00 & -1.4 & I MUD & & 73.79 & 334 & iPc \\
\hline SOC & 68.72 & 310 & iPc & 36 & 32.00 & -1.0 & 1 & & $1.1 \mathrm{~s}$ & 230 & $.00 \mathrm{~nm}$ \\
\hline & $2.0 \mathrm{~s}$ & 1200 & $.00 \mathrm{~nm}$ & & & $.8 \mathrm{mb}$ & I REY & & 73.79 & 351 & ip \\
\hline & & & ePP & 39 & 04.00 & & | PR | & & 73.86 & 56 & eP \\
\hline & & & ePPP & 40 & 50.00 & & | FR I & & 73.92 & 55 & eP \\
\hline & & & is & 45 & 40.00 & & I CFR & & 74.04 & 317 & ePd \\
\hline $\mathrm{FHC}$ & 68.94 & 54 & eP & 36 & 35.40 & 0.9 & I VRI & & 74.30 & 318 & iPc \\
\hline PNO & 69.06 & 47 & eP & 36 & 35.00 & -0.1 & I IMW & & 74.42 & 45 & eP \\
\hline LDM & 69.13 & 43 & iPc & 36 & 35.40 & -0.2 & I UZH & & 74.50 & 322 & $i P c$ \\
\hline ANN & 69.30 & 312 & iPc & 36 & 36.00 & -0.6 & i & & $2.2 \mathrm{~s}$ & 420 & $.00 \mathrm{~nm}$ \\
\hline & $1.5 \mathrm{~s}$ & 1450 & $.00 \mathrm{~nm}$ & & & $9 m b$ & 1 & $z$ & $11 \mathrm{~s}$ & 250 & $900 \mathrm{~m}$ \\
\hline & & & ePCP & 37 & 06.50 & & 1 & & & & IPCP \\
\hline & & & ePP & 39 & 16.00 & & 1 & & & & IPP \\
\hline & & & ePPP & 40 & 48.00 & & 1 & & & & is \\
\hline & & & is & 45 & 43.06 & & i & & & & iSP \\
\hline & & & EPPS & 46 & 19.00 & & I TLB & & 74.51 & 316 & iPc \\
\hline GDH & 69.34 & 5 & iPc & 36 & 33.50 & -2.8 & I BAL & & 74.51 & 200 & eP \\
\hline & $1.5 \mathrm{~s}$ & 1560 & $.00 \mathrm{~nm}$ & & & $.0 \mathrm{mb}$ & KRA & & 74.53 & 324 & iPc \\
\hline VUN & 69.41 & 140 & iPd & 36 & 25.60 & $-11.8 x$ & i & & $1.5 \mathrm{~s}$ & 2320 & $.00 \mathrm{~nm}$ \\
\hline & $1.7 \mathrm{~s}$ & & $.90 \mathrm{~nm}$ & & & & i & $N$ & $20 \mathrm{~s}$ & 311 & $.00 \mathrm{um}$ \\
\hline HFS & 69.43 & 334 & eP & 36 & 36.50 & -0.6 & 1 & $E$ & $20 s$ & 339 & $.90 \mathrm{um}$ \\
\hline & $1.7 \mathrm{~s}$ & 2310 & $.00 \mathrm{~nm}$ & & & $.1 \mathrm{mb}$ & 1 & & & & $\mathrm{i}$ \\
\hline SVA & 69.50 & $14 \theta$ & i Pd & 36 & 39.50 & 1.5 & 1 & & & & $\mathrm{i}$ \\
\hline NB2 & 69.52 & 336 & $P$ & 36 & 36.00 & -1.8 & i & & & & $\mathrm{i}$ \\
\hline KER & 69.63 & 298 & $e^{p}$ & 36 & 40.50 & 1.5 & I & & & & $\mathrm{i}$ \\
\hline
\end{tabular}


$21006 \mathrm{~h}$

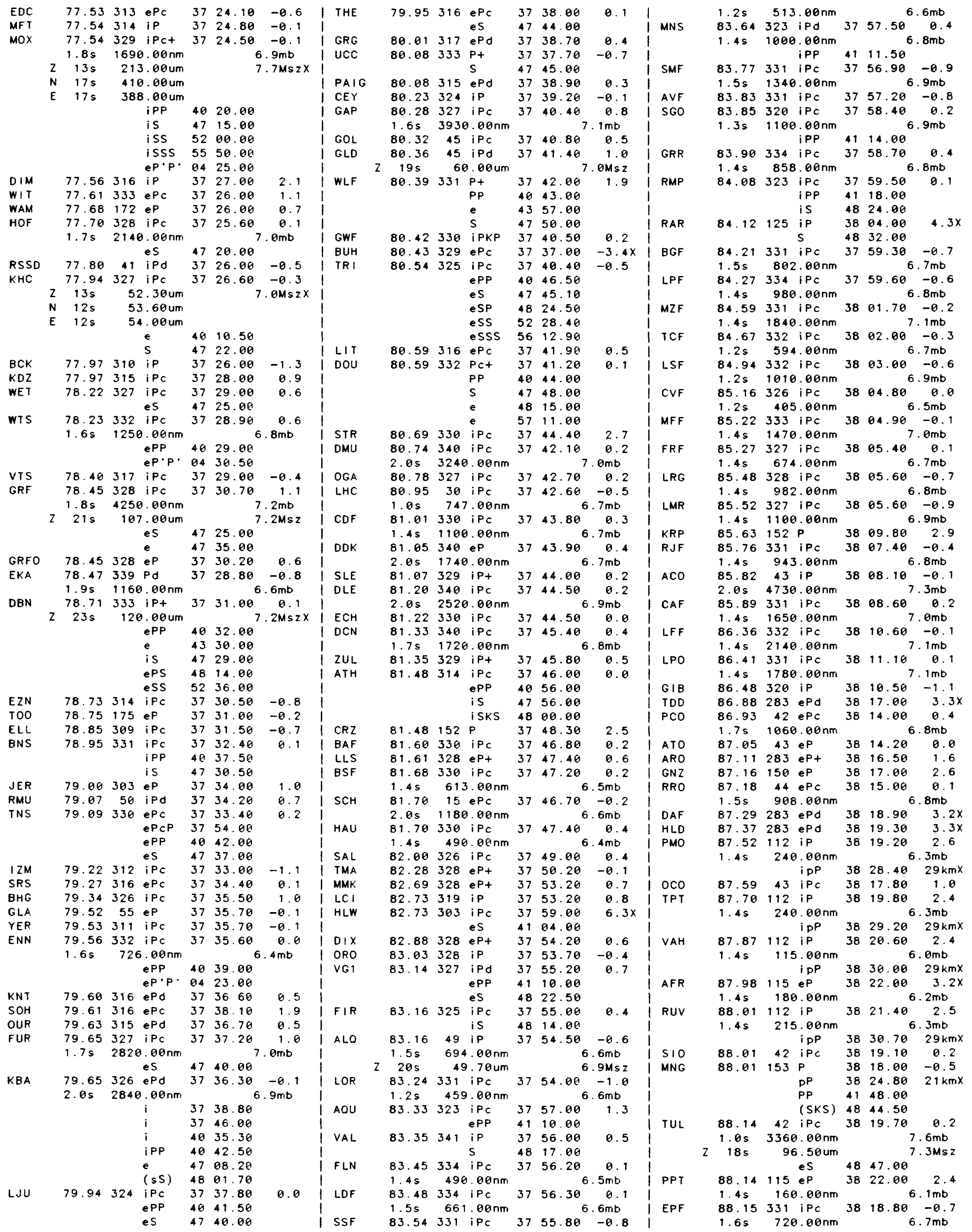




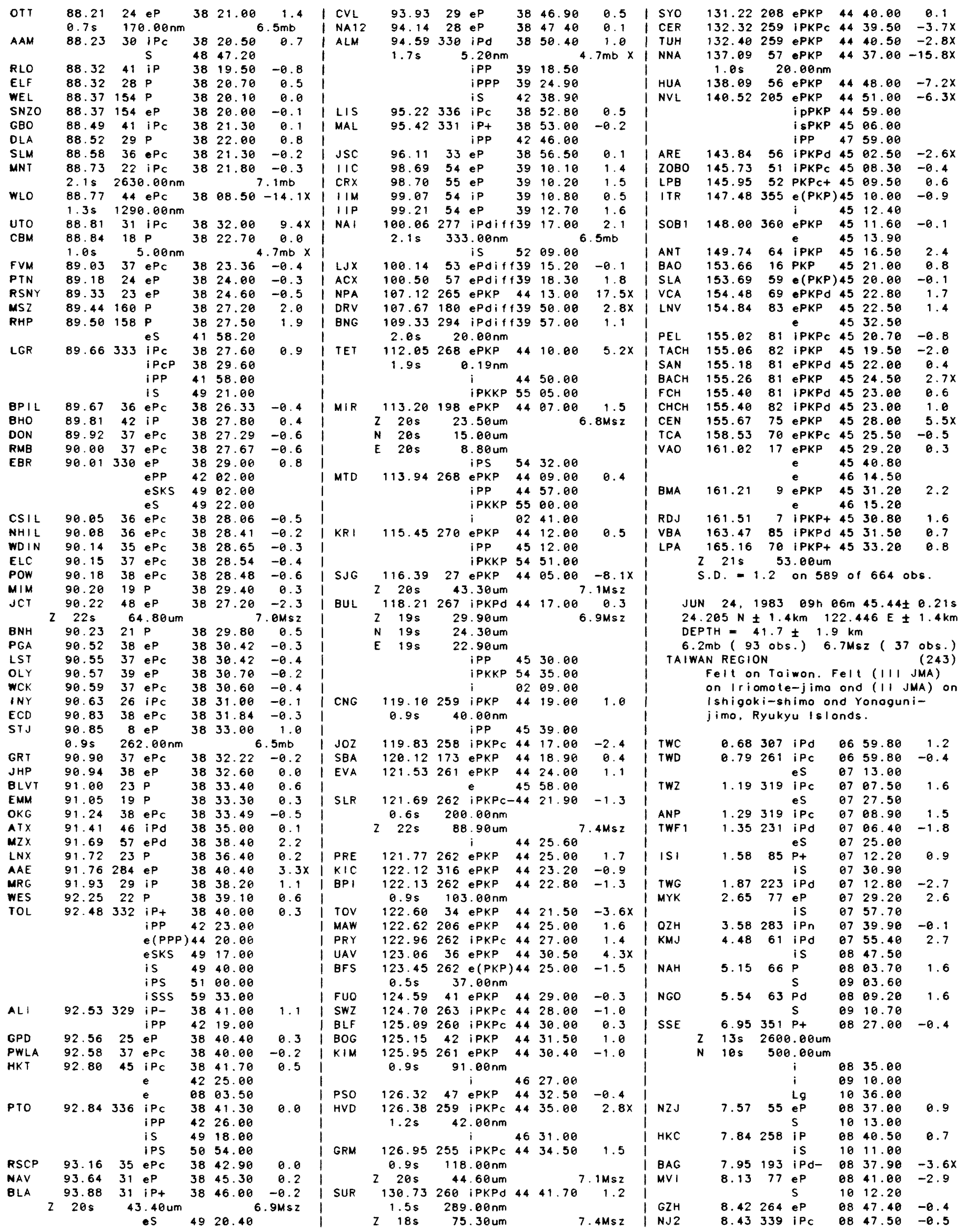




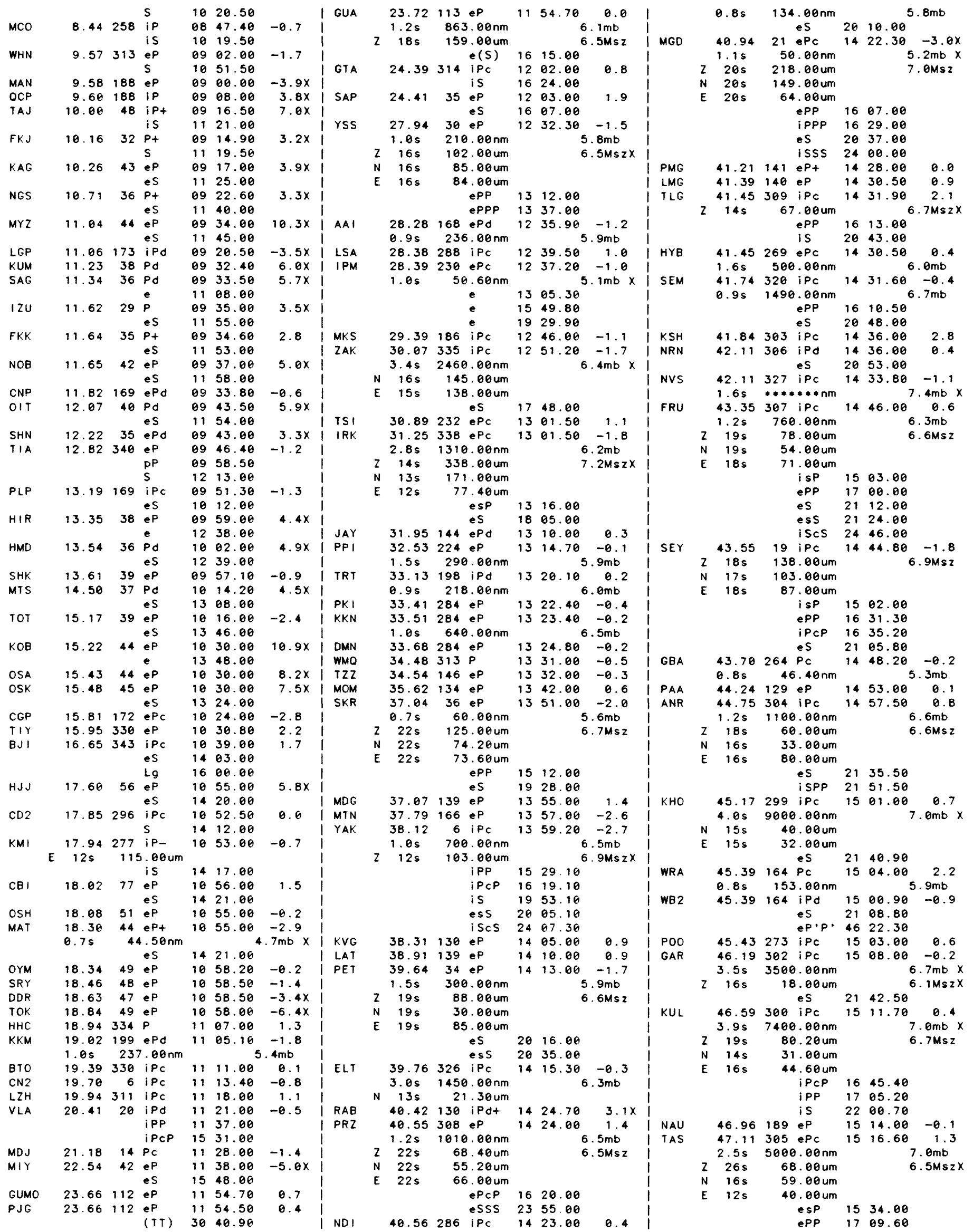




\begin{tabular}{|c|c|c|c|c|c|c|c|c|c|c|c|}
\hline & & & & ePPP & 17 & 48.60 & & 1 & $\mathrm{KHI}$ & & 55.80 \\
\hline & & & & es & 22 & 06.60 & & $i$ & MUN & & 56.18 \\
\hline & & & & eses & 25 & 08.60 & & $i$ & ILT & & 56.21 \\
\hline DSH & & 47.38 & 301 & iPc & 15 & 18.50 & 1.0 & 1 & & & $1.0 \mathrm{~s}$ \\
\hline & & $2.5 \mathrm{~s}$ & 1500 & $.00 \mathrm{~nm}$ & & & $.5 \mathrm{mb}$ & 1 & & $z$ & $16 s$ \\
\hline & $z$ & $18 s$ & 55 & .00 um & & & $.6 \mathrm{Msz}$ & 1 & & N & $16 s$ \\
\hline & $N$ & $18 \mathrm{~s}$ & & $.00 \mathrm{um}$ & & & & 1 & & E & $16 \mathrm{~s}$ \\
\hline & $E$ & $18 \mathrm{~s}$ & & $.00 \mathrm{um}$ & & & & 1 & & & \\
\hline & & & & iPP & 17 & 12.00 & & 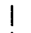 & & & \\
\hline & & & & es & 22 & 12.50 & & 1 & & & \\
\hline$T I K$ & & 47.60 & 3 & ePc & 15 & 16.00 & -2.7 & 1 & & & \\
\hline & $z$ & $17 \mathrm{~s}$ & 390 & $.00 \mathrm{um}$ & & & $.4 M \leqslant Z X$ & 1 & & & \\
\hline & $\mathrm{N}$ & $17 \mathrm{~s}$ & 250 & $.00 \mathrm{um}$ & & & & 1 & NWAO & & 57.03 \\
\hline & $\mathbf{E}$ & $14 \mathrm{~s}$ & & .00 um & & & & 1 & NKI & & 58.31 \\
\hline & & & & epp & 15 & 23.00 & $23 \mathrm{kmx}$ & 1 & STK & & 58.70 \\
\hline & & & & esP & 15 & 31.00 & & 1 & KOU & & 60.32 \\
\hline & & & & ePCP & 16 & 47.00 & & 1 & ANM & & 60.77 \\
\hline & & & & es & 22 & 02.00 & & 1 & $A D E$ & & 60.83 \\
\hline 150 & & 47.65 & 158 & eP & 15 & 19.00 & -0.8 & 1 & & & $1.2 \mathrm{~s}$ \\
\hline SAM & & 48.79 & 302 & iPc & 15 & 29.00 & 0.6 & 1 & PVC & & 61.15 \\
\hline & & $3.5 \mathrm{~s}$ & 8500 & $.00 \mathrm{~nm}$ & & & $.2 m b x$ & 1 & $\mathrm{COO}$ & & 61.35 \\
\hline & $N$ & $19 \mathrm{~s}$ & 67 & $.00 \mathrm{um}$ & & & & 1 & SHI & & 61.73 \\
\hline & $E$ & $19 \mathrm{~s}$ & 59 & .00 um & & & & 1 & BAK & & 61.81 \\
\hline & & & & IPP & 17 & 26.80 & & 1 & & & 4.05 \\
\hline & & & & is & 22 & 32.20 & & 1 & & & \\
\hline & & & & is S & 22 & 50.60 & & 1 & KHE & & 62.16 \\
\hline & & & & ess & 25 & 54.20 & & 1 & & & $1.6 \mathrm{~s}$ \\
\hline ASPA & & 48.87 & 166 & iPd & 15 & 28.50 & -0.6 & 1 & & $z$ & $14 \mathrm{~s}$ \\
\hline SVO & & 49.29 & 128 & $P$ & 15 & 38.00 & $5.5 x$ & 1 & & $\mathrm{~N}$ & 145 \\
\hline HNR & & 49.58 & 128 & eP & 15 & 36.00 & 1.3 & 1 & & $E$ & 145 \\
\hline & & & & e & 22 & 40.00 & & 1 & & & \\
\hline NRI & & 49.60 & 345 & eP & 15 & 30.30 & $-3.9 x$ & i & & & \\
\hline & & $3.4 \mathrm{~s}$ & 980 & $.00 \mathrm{~nm}$ & & & $.3 m b x$ & 1 & & & \\
\hline & $z$ & $17 \mathrm{~s}$ & 140 & $.00 \mathrm{um}$ & & &.$\theta M s z X$ & 1 & & & \\
\hline & $N$ & $17 \mathrm{~s}$ & 65 & .00 um & & & & i & & & \\
\hline & $E$ & $17 \mathrm{~s}$ & 119 & $.00 \mathrm{um}$ & & & & I & NOU & & 62.98 \\
\hline & & & & esP & 15 & 45.00 & & 1 & YOU & & 63.17 \\
\hline & & & & ePCP & 16 & 55.00 & & 1 & & & \\
\hline & & & & ePP & 17 & 25.00 & & 1 & RIV & & 63.85 \\
\hline & & & & ePPP & 18 & 18.00 & & 1 & BFD & & 63.92 \\
\hline & & & & es & 22 & 36.00 & & 1 & CAN & & 64.32 \\
\hline CTA & & 49.76 & 150 & IPd & 15 & 35.60 & -0.4 & 1 & & & \\
\hline & & $1.7 \mathrm{~s}$ & 506 & $.00 \mathrm{~nm}$ & & & $3 m b$ & 1 & GRO & & 64.44 \\
\hline & & & & is & 22 & 44.00 & & 1 & & & $1.5 \mathrm{~s}$ \\
\hline & & & & $i$ & 26 & 28.00 & & 1 & & $z$ & $16 s$ \\
\hline & & & & i & 31 & 18.00 & & 1 & KRV & & 64.45 \\
\hline CTAO & & 49.76 & 150 & eP & 15 & 35.50 & -0.5 & 1 & & & $1.0 \mathrm{~s}$ \\
\hline WBN & & 50.21 & 175 & $i P c$ & 15 & 39.40 & 0.1 & 1 & GRS & & 64.67 \\
\hline MEK & & 50.66 & 185 & iPc & 15 & 41.20 & -1.6 & 1 & & & $6.0 \mathrm{~s}$ \\
\hline ADK & & 53.46 & 42 & eP & 16 & 02.00 & -1.4 & 1 & & $z$ & $17 \mathrm{~s}$ \\
\hline$K L G$ & & 54.68 & 181 & eP & 16 & 12.00 & -0.6 & 1 & & $\mathrm{~N}$ & $17 \mathrm{~s}$ \\
\hline SVE & & 54.71 & 324 & $\mathrm{IPC}$ & 16 & 11.50 & -1.1 & 1 & & $E$ & $17 \mathrm{~s}$ \\
\hline & & $3.7 \mathrm{~s}$ & 3540 & $.00 \mathrm{~nm}$ & & & $.8 \mathrm{mb} \times$ & 1 & & & \\
\hline & $z$ & $17 \mathrm{~s}$ & 84 & .00 um & & & $.9 M \leq z X$ & 1 & & & \\
\hline & $N$ & $17 \mathrm{~s}$ & 22 & $.00 \mathrm{um}$ & & & & 1 & & & \\
\hline & $E$ & $17 \mathrm{~s}$ & 70 & $1.00 \mathrm{um}$ & & & & 1 & & & \\
\hline & & & & ePcP & 17 & 14.70 & & 1 & & & \\
\hline & & & & es & 23 & 53.00 & & 1 & $T A B$ & & 64.96 \\
\hline & & & & eSP & 23 & 57.00 & & 1 & WAM & & 65.06 \\
\hline BAL & & 54.78 & 186 & eP & 16 & 13.00 & -0.3 & 1 & & & \\
\hline MH I & & 54.85 & 298 & iPc & 16 & 15.00 & 0.9 & 1 & KER & & 65.15 \\
\hline & & $1.0 \mathrm{~s}$ & 420 & $.00 \mathrm{~nm}$ & & & $.4 \mathrm{mb}$ & 1 & TOO & & 65.16 \\
\hline & & & & es & 23 & 56.00 & & 1 & MTA & & 65.39 \\
\hline ASH & & 55.60 & 300 & P & 16 & 20.00 & 0.7 & 1 & & $z$ & $18 \mathrm{~s}$ \\
\hline & & $1.5 \mathrm{~s}$ & 2200 & $.00 \mathrm{~nm}$ & & & $.0 \mathrm{mb}$ & 1 & & $N$ & $18 \mathrm{~s}$ \\
\hline & & & & esP & 16 & 36.00 & & 1 & & $E$ & $18 \mathrm{~s}$ \\
\hline & & & & ePcP & 17 & 20.00 & & 1 & & & \\
\hline & & & & ePP & 18 & 30.00 & & 1 & & & \\
\hline & & & & ePPP & 19 & 44.00 & & 1 & IMA & & 65.67 \\
\hline & & & & es & 24 & 06.00 & & 1 & & & $1.1 \mathrm{~s}$ \\
\hline & & & & escs & 26 & 07.50 & & 1 & & & \\
\hline ARU & & 55.76 & 323 & eP & 16 & 18.70 & -1.5 & 1 & ERE & & 65.91 \\
\hline & & $1.5 \mathrm{~s}$ & 500 & $.00 \mathrm{~nm}$ & & & $.3 \mathrm{mb}$ & 1 & & & \\
\hline & $z$ & $16 s$ & 34 & $.50 u m$ & & & $.5 M s z X$ & 1 & PYA & & 66.19 \\
\hline & $\mathrm{N}$ & $16 s$ & 29 & $.80 u m$ & & & & I & & & $3.0 \mathrm{~s}$ \\
\hline & $\mathrm{E}$ & $16 s$ & 38 & $.00 \mathrm{um}$ & & & & 1 & & $z$ & $22 \mathrm{~s}$ \\
\hline & & & & ePcP & 17 & 17.00 & & 1 & & $N$ & $22 \mathrm{~s}$ \\
\hline & & & & iPP & 18 & 32.70 & & 1 & & $E$ & $22 \mathrm{~s}$ \\
\hline & & & & ePPP & 19 & 44.00 & & 1 & & & \\
\hline & & & & eSP & 24 & 13.00 & & 1 & & & \\
\hline & & & & escs & 26 & 03.00 & & 1 & & & \\
\hline VAN & & 55.79 & 300 & ePc & 16 & 21.00 & 0.3 & 1 & & & \\
\hline & $Z$ & $20 \mathrm{~s}$ & 49 & $.00 \mathrm{um}$ & & & $.6 \mathrm{Msz}$ & | & LEN & & 66.28 \\
\hline & $E$ & $19 \mathrm{~s}$ & 52 & $.00 \mathrm{um}$ & & & & | & & & $4.2 \mathrm{~s}$ \\
\hline & & & & $i p P$ & 16 & 32.50 & $39 \mathrm{kmX}$ & 1 & & $z$ & $20 \mathrm{~s}$ \\
\hline & & & & es & 24 & 10.00 & & I & & $\mathrm{N}$ & $20 s$ \\
\hline & & & & escs & 26 & 10.00 & & 1 & & $E$ & $20 \mathrm{~s}$ \\
\hline
\end{tabular}




\begin{tabular}{|c|c|c|c|c|c|c|c|c|c|c|c|c|c|}
\hline & $22 \mathrm{~s}$ & & $\begin{array}{l}6.30 \mathrm{um} \\
\text { i PcP }\end{array}$ & 18 & 28.00 & & $\left\{\begin{array}{l}I Z M \\
E Z N\end{array}\right.$ & & $\begin{array}{l}79.20 \\
79.33\end{array}$ & $\begin{array}{l}307 \\
309\end{array}$ & $\begin{array}{l}\text { iP } \\
\text { iPc }\end{array}$ & $\begin{array}{l}18 \\
18\end{array}$ & $\begin{array}{l}46.50 \\
47.00\end{array}$ \\
\hline & & & ePPP & 22 & 36.00 & & I KRA & & 79.38 & 320 & ePc & 8 & 47.70 \\
\hline & & & escs & 28 & 08.00 & & i & & $0.8 \mathrm{~s}$ & 130 & $0.00 \mathrm{~nm}$ & & 5 \\
\hline$K \mid R$ & 72.24 & 337 & i P & 18 & 02.20 & $-5.7 x$ & I & 2 & $16 s$ & 48 & b. 10 um & & \\
\hline HON & 72.43 & 74 & $\mathbf{P}$ & 18 & 16.00 & $6.2 x$ & I & $N$ & $16 s$ & 31 & $1.50 u m$ & & \\
\hline INK & 72.79 & 22 & eP & 18 & 09.00 & -2.1 & I & E & $16 \mathrm{~s}$ & & $2.30 \mathrm{um}$ & & \\
\hline & $0.9 \mathrm{~s}$ & 113 & $3.00 \mathrm{~nm}$ & & & $5.8 \mathrm{mb}$ & I & & & & $i$ & 18 & 50.80 \\
\hline PCA & 72.86 & 31 & eP & 18 & 12.30 & 0.5 & 1 & & & & $i$ & 18 & 55.70 \\
\hline NUR & 72.96 & 329 & iP & 18 & 11.20 & -1.0 & 1 & & & & e & 20 & 20.00 \\
\hline & $1.1 \mathrm{~s}$ & 187 & $7.00 \mathrm{~nm}$ & & & $.0 \mathrm{mb}$ & 1 & & & & e & 21 & 50.00 \\
\hline & $18 \mathrm{~s}$ & & $5.90 \mathrm{um}$ & & & $.1 M S Z$ & 1 & & & & is & 28 & 58.00 \\
\hline & & & epPP & 22 & 36.00 & & I KRP & & 79.39 & 140 & eP & 18 & 49.08 \\
\hline & & & epPPP & 24 & 00.00 & & I SPC & & 79.57 & 319 & iP & 18 & 50 \\
\hline & & & es & 27 & 28.00 & & 1 & & & & $i$ & 19 & 07.08 \\
\hline & & & eSP & 28 & 20.00 & & 1 & & & & e(PP) & 21 & 49.40 \\
\hline & & & ess & 33 & 00.00 & & 1 & & & & $e(S)$ & 29 & 20.70 \\
\hline$M B C$ & 72.99 & 13 & $e^{P}$ & 18 & 10.00 & -2.2 & LO & & 79.67 & 315 & ePc & 18 & 51.00 \\
\hline & $0.5 \mathrm{~s}$ & & $8.00 \mathrm{~nm}$ & & & $5 \mathrm{mb}$ & OS & & 79.68 & 319 & $e(P) c$ & 18 & 00 \\
\hline KIS & 75.03 & 315 & iPc & 18 & 24.00 & -8.5 & I MSZ & & 80.14 & 149 & eP & 18 & 2.28 \\
\hline & $5.0 \mathrm{~s}$ & 6000 & $0.00 \mathrm{~nm}$ & & & $.8 \mathrm{mb} \times$ & SZ & & 80.32 & 318 & ePc & 18 & $\theta$ \\
\hline & 2 & 27 & 7.70 um & & & $3.6 M s z X$ & TS & & 80.41 & 312 & $\mathrm{iPc}$ & 8 & 8 \\
\hline & $16 \mathrm{~s}$ & & $8.60 \mathrm{um}$ & & & & SR & & 80.44 & 315 & iP & 8 & 55.80 \\
\hline & $18 s$ & & $0.50 \mathrm{um}$ & & & & I AAE & & 80.63 & 276 & eP & 8 & 56.70 \\
\hline & & & iPCP & 18 & 38.00 & & I SRS & & 80.74 & 311 & ePd & 18 & 55.60 \\
\hline & & & is & 28 & 08.00 & & HP & & 80.76 & 147 & $\mathbf{P}$ & 8 & 58.10 \\
\hline JER & 75.47 & 98 & ePC & 18 & 28.00 & 0.6 & UR & & 80.79 & 310 & ePd & 8 & 57.00 \\
\hline TDD & 75.81 & 77 & $e^{P}$ & 18 & 31.00 & 1.6 & I COP & & 80.85 & 327 & $\mathrm{iPc}$ & 18 & 56.00 \\
\hline ARO & 5.96 & 276 & eP & 18 & 20.20 & $-10.2 x$ & 1 & & $0.8 \mathrm{~s}$ & 89 & $3.60 \mathrm{~nm}$ & & 5 \\
\hline EFR & .12 & 13 & ePd & 18 & 30.00 & -0.7 & 1 & & & & is & 29 & 15.00 \\
\hline CLI & i. & 15 & ePc & 18 & 31.00 & -0.2 & $\mathrm{OH}$ & & 81.03 & 311 & $e P c$ & 8 & $\theta$ \\
\hline DAF & 0.22 & 277 & eP & 18 & 33.50 & 1.7 & D & & 4 & 318 & ePc & B & 6 \\
\hline GPA & .32 & 308 & i P & 18 & 1.70 & -0.3 & KSP & & 13 & 322 & $i P c$ & 8 & 57.50 \\
\hline SIT & 76.36 & 33 & eP & 18 & 1.50 & -0.3 & 1 & & $1.5 \mathrm{~s}$ & 295 & $3.00 \mathrm{~nm}$ & & 6 \\
\hline TLB & 76.41 & 313 & ePc & 18 & 32.00 & -0.3 & $A \mid G$ & & 81.16 & 310 & ePd & 8 & $\theta$ \\
\hline UPP & 76.46 & $\begin{array}{l}330 \\
5399\end{array}$ & iPc & 18 & 31.20 & -1.1 & MNG & & 81.17 & 142 & eP & 8 & 50 \\
\hline & $1.0 \mathrm{~s}$ & 5260 & $\begin{array}{l}0.00 \mathrm{~nm} \\
\text { iPP }\end{array}$ & & $25.00^{7}$ & $.5 \mathrm{mb} \times$ & 1 & & & & PP & 9 & 10 \\
\hline & & & is & 28 & 01.00 & & 1 & & & & ePP & 2 & 0 \\
\hline & & & i & 28 & 04.00 & & 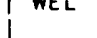 & 2 & $\begin{array}{l}81.22 \\
185\end{array}$ & $\begin{array}{r}143 \\
31\end{array}$ & $60 u m$ & 8 & \\
\hline DAG & 76.54 & 351 & iPd & 18 & 31.70 & -0.8 & 1 & $N$ & $18 s$ & 20 & $60 \mathrm{um}$ & & \\
\hline & $1.1 \mathrm{~s}$ & & $1.06 \mathrm{~nm}$ & & & $.6 \mathrm{mb}$ & I & $E$ & $20 s$ & 19 & 7. 90 um & & \\
\hline & & & i & 21 & 27.00 & & 1 & & & & PP & 22 & 20.00 \\
\hline & & & $i$ & 29 & 15.00 & & I & & & & SKS & 9 & 06 \\
\hline IRT & 76.59 & 309 & iP & 18 & 33.48 & -0.1 & 1 & & & & (S) & 30 & 04.00 \\
\hline VRI & 76.82 & 314 & EPC & 18 & 4.08 & -0.6 & 1 & & & & PS & 4 & 00.00 \\
\hline$A L T$ & 76.89 & 307 & iPc & 18 & 4.28 & -1.1 & 1 & & & & SS & 35 & 02.00 \\
\hline YLV & 76.89 & 309 & iP & 18 & 35.40 & 0.2 & 1 & & & & SSS & 40 & 42.00 \\
\hline I SK & 76.94 & 309 & ip & 18 & 35.90 & 0.5 & I SRO & & 81.32 & 319 & ip & 18 & 59.10 \\
\hline LVV & 77.05 & 319 & $i P c$ & 18 & 35.40 & -0.4 & 1 & $z$ & $18 \mathrm{~s}$ & 27 & 7. $40 u m$ & & \\
\hline & $6.0 \mathrm{~s}$ & 8700 & $0.00 \mathrm{~nm}$ & & & $.0 \mathrm{mb} \times$ & 1 & & & & $e(s)$ & 29 & 08.50 \\
\hline & $18 \mathrm{~s}$ & & $6.86 \mathrm{um}$ & & & $.7 \mathrm{MsZ}$ & I GNZ & & 81.33 & 139 & eP & 18 & 59 \\
\hline & $18 \mathrm{~s}$ & & $6.00 \mathrm{um}$ & & & & AY & & 81.36 & 311 & eP & 8 & \\
\hline & $18 \mathrm{~s}$ & & $6.00 \mathrm{um}$ & & & & I THE & & 81.37 & 311 & eP & 19 & 00.00 \\
\hline & & & iPcP & 18 & 41.00 & & ! & & & & es & 29 & 18.00 \\
\hline & & & iPP & 21 & 29.00 & & RG & & 81.63 & 311 & ePc & 19 & 00 \\
\hline & & & IPPP & 23 & 19.00 & & I SKO & & 81.86 & 312 & iPc & 19 & 02.00 \\
\hline & & & is & 28 & 21.00 & & I & & & & is & 29 & 28 \\
\hline & & & iSKS & 28 & 35.00 & & & & & & iss & 34 & 50.00 \\
\hline & & & iPS & 29 & 07.00 & & IST & & 81.87 & 319 & eP & 19 & 01.50 \\
\hline & & & iss & 33 & 20.00 & & 1 & & & & $i$ & 19 & 19 \\
\hline CK & .09 & 85 & eP & 18 & 5.36 & -1.1 & I & & & & e & 21 & 47 \\
\hline ISR & 20 & 14 & ePd & 18 & 00 & 1.2 & 1 & & & & el & 29 & 42 \\
\hline LR & .46 & 14 & iPd & 18 & 00 & 2.6 & I T & & .92 & 310 & ePd & 9 & 30 \\
\hline MK & 58 & 10 & eP & 18 & 00 & -0.9 & $\mathrm{RN}$ & & 82.07 & 324 & ePc & 9 & 50 \\
\hline ST & 7.77 & 308 & $e^{P}$ & 18 & 70 & $-1 \cdot 3$ & VKA & & 82.31 & 320 & ePc & 19 & 04.50 \\
\hline 3uc 1 & 83 & 313 & iPc & 18 & 0.20 & $-4.0 x$ & 1 & & $4.1 \mathrm{~s}$ & 5230 & $.00 \mathrm{~nm}$ & & \\
\hline ELL & 77.85 & 305 & ip & 18 & 3.10 & -1.6 & 1 & & & & ePP & 22 & 04.70 \\
\hline EDC & 4 & 89 & eP & 18 & 1.00 & -0.5 & | & & & & (s & 29 & 20.00 \\
\hline MFS & 78.11 & 331 & $(P)$ & 18 & 40.60 & -0.8 & BR G & & 82.42 & 323 & iPct & 19 & 04.40 \\
\hline & $1.2 \mathrm{~s}$ & 191 & $1.00 \mathrm{~nm}$ & & 6 & $5.0 \mathrm{mb}$ & 1 & & 3.05 & 1020 & .08 & & \\
\hline$M P$ & 78.14 & 314 & ePC & 18 & 41.00 & -0.9 & I & & $18 \mathrm{~s}$ & 63 & $3.00 \mathrm{um}$ & & \\
\hline F T & 29 & 09 & ip & 18 & 46 & -0.6 & 1 & & 18 & 20 & 0.0 & & \\
\hline$J R$ & 78.51 & 316 & eP & 18 & 5.10 & 1.1 & 1 & E & $18 \mathrm{~s}$ & 43 & $3.00 \mathrm{um}$ & & \\
\hline $\mathrm{IZH}$ & 78.56 & 318 & ePc & 18 & 44.50 & 0.4 & & & & & eSKS & 29 & 21 \\
\hline & $2.0 \mathrm{~s}$ & 1200 & $0.00 \mathrm{~nm}$ & & & $5.5 \mathrm{mb}$ & YKA & & 82.50 & 23 & eP & 19 & 06.50 \\
\hline & & & isp & 19 & 01.00 & & RSNT & & 82.52 & 23 & eP & 19 & 03.70 \\
\hline & & & iPP & 21 & 40.00 & & I PRU & & 82.52 & 322 & iPc & 19 & 05.00 \\
\hline & & & iPPP & 23 & 40.00 & & 1 & & $2.7 \mathrm{~s}$ & 772 & $.00 \mathrm{~nm}$ & & 6 \\
\hline & & & iSKS & 28 & 51.00 & & | & $z$ & $19 \mathrm{~s}$ & 37 & $.50 \mathrm{um}$ & & \\
\hline & & & iscs & 29 & 00.00 & & 1 & $\mathbf{N}$ & $24 \mathrm{~s}$ & 34 & 1.3 & & \\
\hline $0 z$ & 78.57 & 315 & iPd & 18 & 45.50 & 1.0 & 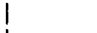 & $E$ & $17 \mathrm{~s}$ & & .0 & & \\
\hline IB2 & 4 & 332 & $\mathbf{P}$ & 18 & 45.00 & .1 & I & & & & e & 19 & 34 \\
\hline & 1. & 141 & $1.00 \mathrm{~nm}$ & & & 5. $9 \mathrm{mb}$ & I & & & & $\mathrm{S}$ & 29 & 80 \\
\hline$V L$ & 78.86 & 312 & $\mathrm{iPc}$ & 18 & 46.00 & 0.1 & YKC & & 82.56 & 23 & eP & 19 & 04.00 \\
\hline ER & 32 & 306 & $i P_{c}$ & 18 & 45 & -1.0 & 1 & & $1.0 \mathrm{~s}$ & 131 & $.00 \mathrm{~nm}$ & & 5 \\
\hline $1 \mathrm{M}$ & 79.00 & 11 & eP & 18 & 30 & 0.3 & OHR & & 82.66 & 312 & & 19 & 05.10 \\
\hline
\end{tabular}




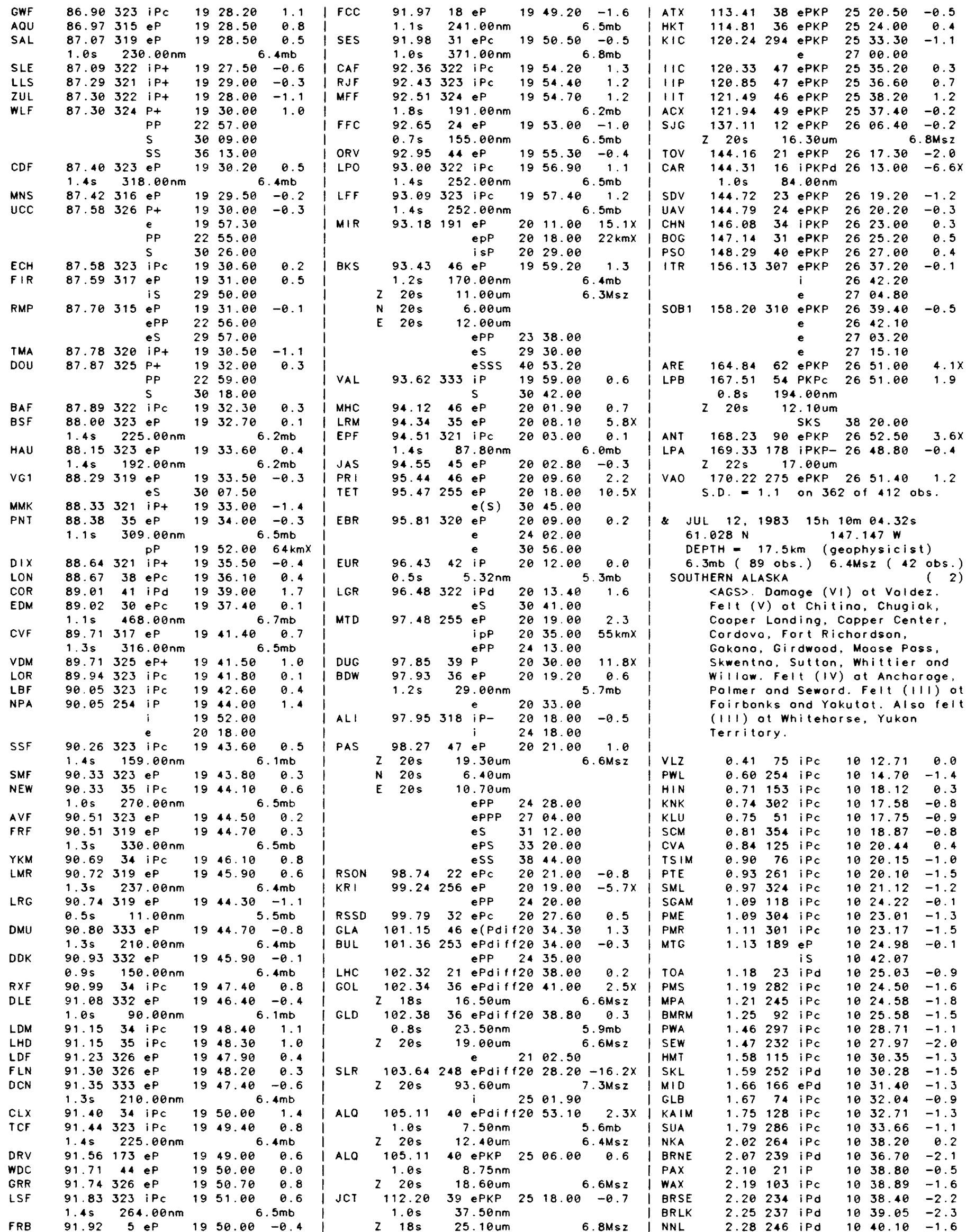


$12 \mathrm{~d} \quad 15 \mathrm{~h}$

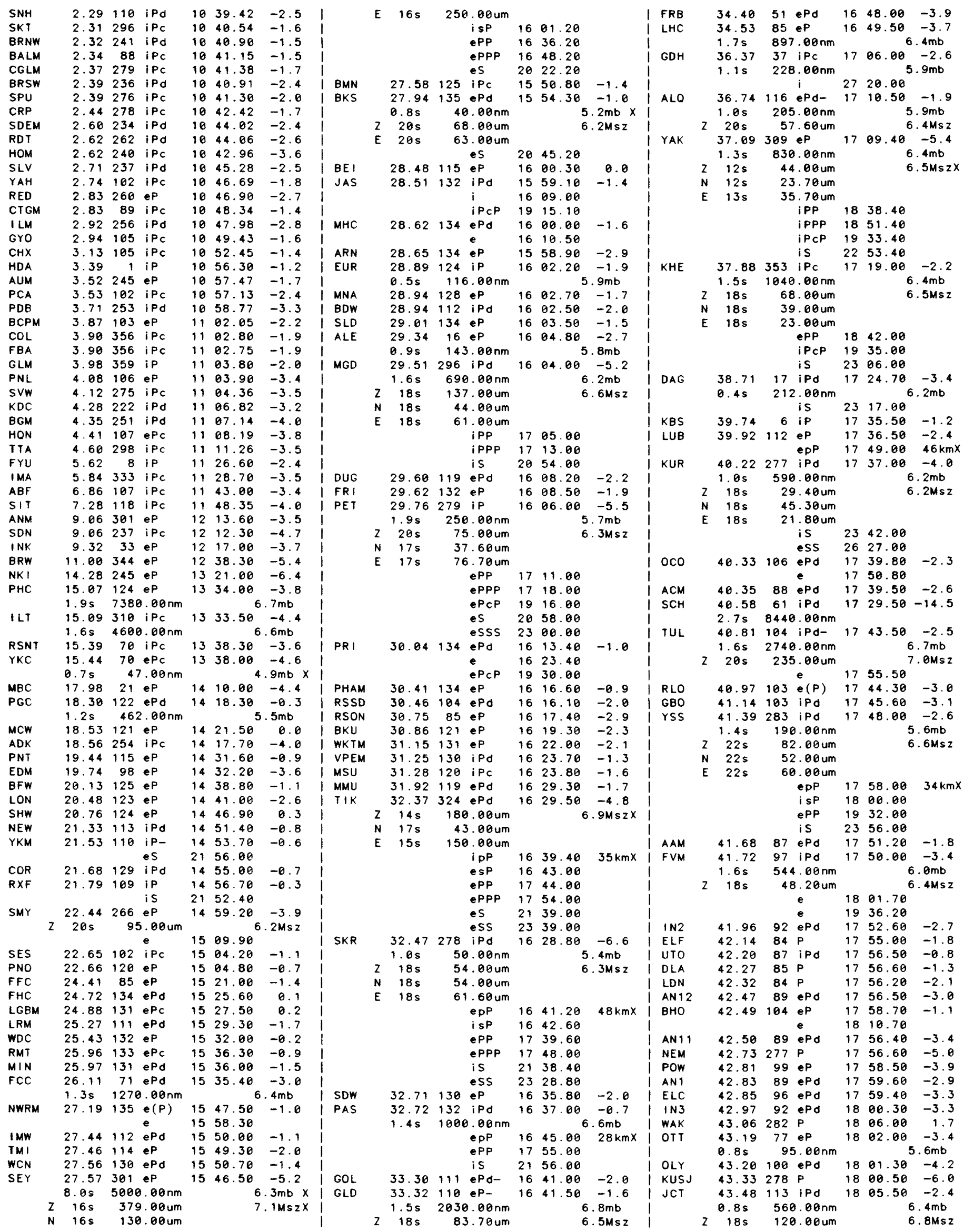




\begin{tabular}{|c|c|c|c|c|c|c|c|c|c|c|c|}
\hline & & & & es & 24 & 32.00 & & 1 & SOD & & 51.82 \\
\hline ASAJ & & 43.56 & 280 & $P$ & 18 & 03.70 & -4.7 & $i$ & & & \\
\hline $\mathrm{DHN}$ & & 43.93 & 82 & eP & 18 & 08.50 & -2.9 & $i$ & STJ & & 51.88 \\
\hline ASA & & 43.95 & 280 & $\mathbf{P}$ & 18 & 08.00 & -3.5 & i & & & $1.1 \mathrm{~s}$ \\
\hline NR I & & 44.05 & 335 & iPc & 18 & 07.50 & -4.5 & i & KTJ & & 52.42 \\
\hline & & $1.6 \mathrm{~s}$ & 140 & $0.00 \mathrm{~nm}$ & & & $.5 \mathrm{mb}$ & 1 & $110 \mathrm{~J}$ & & 52.56 \\
\hline & $N$ & $25 s$ & 108 & B. 00 um & & & & i & $\mathrm{SHZ}$ & & 52.73 \\
\hline & $E$ & $25 s$ & & 9.00 um & & & & $i$ & $11 \mathrm{C}$ & & 53.05 \\
\hline & & & & epp & 18 & 14.00 & $22 \mathrm{kmX}$ & i & TAC & & 53.40 \\
\hline & & & & $\mathrm{ePcP}$ & 19 & 58.00 & & 1 & TSRJ & & 53.44 \\
\hline & & & & es & 24 & 35.00 & & 1 & IIM & & 53.47 \\
\hline & & & & eScS & 27 & 56.00 & & 1 & HIK & & 53.53 \\
\hline MNT & & 44.10 & 76 & eP & 18 & 09.00 & -3.7 & 1 & IRK & & 53.56 \\
\hline & & $1.0 \mathrm{~s}$ & 140 & $0.00 \mathrm{~nm}$ & & & $.8 \mathrm{mb}$ & 1 & & & $2.0 \mathrm{~s}$ \\
\hline PTN & & 44.15 & 78 & eP & 18 & 10.00 & -3.2 & $i$ & & $z$ & $16 s$ \\
\hline RSNY & & 44.39 & 77 & eP & 18 & 11.70 & -3.4 & i & & $N$ & $16 \mathrm{~s}$ \\
\hline HOOJ & & 44.56 & 278 & $P$ & 18 & 19.70 & -4.7 & i & & $E$ & $16 \mathrm{~s}$ \\
\hline APH & & 44.93 & 78 & eP & 18 & 16.00 & -3.5 & $i$ & & & \\
\hline URA & & 44.97 & 278 & $\mathbf{P}$ & 18 & 16.00 & -3.8 & 1 & & & \\
\hline SAP & & 44.98 & 280 & eP & 18 & 15.00 & -4.9 & 1 & & & \\
\hline & & & & es & 24 & 59.00 & & $i$ & & & \\
\hline PWLA & & 45.26 & 97 & iPd & 18 & 19.10 & -3.1 & i & & & \\
\hline CBM & & 45.60 & 70 & eP & 18 & 26.30 & 1.5 & $i$ & IIP & & 53.57 \\
\hline$B O D$ & & 45.66 & 312 & iPd & 18 & 29.00 & -4.1 & 1 & $11 T$ & & 54.12 \\
\hline BNH & & 45.90 & 75 & ePc & 18 & 24.50 & -2.7 & i & 111 & & 54.20 \\
\hline & & & & epp & 18 & 34.70 & $34 \mathrm{kmx}$ & 1 & WKYJ & & 54.70 \\
\hline RSCP & & 45.96 & 95 & iPd & 18 & 24.50 & -3.3 & 1 & YONJ & & 54.93 \\
\hline TUP & & 46.03 & 306 & ePd & 18 & 24.00 & -4.0 & $i$ & KJF & & 55.03 \\
\hline & & $1.8 \mathrm{~s}$ & 260 & $0.00 \mathrm{~nm}$ & & & $.9 \mathrm{mb}$ & i & & & $0.7 \mathrm{~s}$ \\
\hline HAK & & 46.21 & 280 & $\mathbf{P}$ & 18 & 25.00 & -4.5 & i & & $z$ & $24 s$ \\
\hline$M I M$ & & 46.44 & 73 & eP & 18 & 28.40 & -3.0 & i & & & \\
\hline NAV & & 46.93 & 89 & ePd & 18 & 32.10 & -3.3 & 1 & & & \\
\hline$M Z X$ & & 47.03 & 125 & ePc & 18 & 35.70 & -0.5 & 1 & & & \\
\hline GPD & & 47.10 & 80 & eP & 18 & 33.50 & -3.2 & i & $A C X$ & & 55.38 \\
\hline$B L A$ & & 47.19 & 89 & iP- & 18 & 34.00 & -3.5 & 1 & ZAK & & 55.49 \\
\hline & & $1.4 \mathrm{~s}$ & 360 & $0.00 \mathrm{~nm}$ & & & $.2 \mathrm{mb}$ & 1 & & & $2.2 \mathrm{~s}$ \\
\hline & & & & i pp & 18 & 45.20 & $39 \mathrm{kmx}$ & i & & $z$ & $16 \mathrm{~s}$ \\
\hline & & & & is & 25 & 27.20 & & i & & $N$ & $16 \mathrm{~s}$ \\
\hline MIY & & 47.30 & 277 & $\mathbf{P}$ & 18 & 35.00 & -3.2 & 1 & & $E$ & $16 \mathrm{~s}$ \\
\hline AOMJ & & 47.36 & 279 & $\mathbf{P}$ & 18 & 33.00 & -5.7 & 1 & & & \\
\hline PRIN & & 47.48 & 81 & iPd & 18 & 37.00 & -2.7 & i & & & \\
\hline CVL & & 47.54 & 86 & ePc & 18 & 37.00 & -3.1 & i & TKSJ & & 55.63 \\
\hline EMM & & 47.54 & 72 & eP & 18 & 37.20 & -2.9 & $i$ & SHK & & 55.84 \\
\hline MRK & & 47.65 & 278 & $\mathbf{P}$ & 18 & 38.00 & -3.0 & i & VHO & & 56.38 \\
\hline NA12 & & 47.82 & 86 & ePc & 18 & 39.40 & -2.9 & 1 & SUF & & 56.47 \\
\hline OFU & & 47.88 & 277 & $\mathbf{P}$ & 18 & 39.00 & -3.8 & 1 & & & $0.7 \mathrm{~s}$ \\
\hline OFUJ & & 47.89 & 277 & $\mathbf{P}$ & 18 & 38.80 & -4.1 & $\mathrm{i}$ & SHNJ & & 56.92 \\
\hline$A K U$ & & 48.08 & 26 & ip & 18 & 41.70 & -2.3 & 1 & BER & & 57.07 \\
\hline & & $1.1 \mathrm{~s}$ & 324 & $4.00 \mathrm{~nm}$ & & & $3 \mathrm{mb}$ & i & NB2 & & 57.13 \\
\hline HJH & & 48.40 & 278 & $\mathbf{P}$ & 18 & 42.70 & -4.2 & $i$ & NVS & & 57.73 \\
\hline REY & & 48.57 & 29 & ip & 18 & 46.20 & -1.5 & i & & & $2.4 \mathrm{~s}$ \\
\hline ISN & & 48.58 & 276 & $\mathbf{P}$ & 18 & 44.00 & -4.2 & 1 & KONO & & 58.31 \\
\hline PRM & & 48.75 & 93 & iPd & 18 & 46.60 & -3.0 & 1 & KUMJ & & 58.36 \\
\hline JSC & & 49.13 & 92 & iPd & 18 & 49.10 & -3.4 & $i$ & $E L T$ & & 58.41 \\
\hline YAM & & 49.17 & 277 & $\mathbf{P}$ & 18 & 49.00 & -3.8 & 1 & & & $6.4 \mathrm{~s}$ \\
\hline TRO & & 49.25 & 6 & iP+ & 18 & 49.00 & -4.0 & 1 & NUR & & 58.63 \\
\hline YAMJ & & 49.39 & 277 & $\mathbf{P}$ & 18 & 51.00 & -3.5 & 1 & & & $0.9 \mathrm{~s}$ \\
\hline$V L A$ & & 49.39 & 288 & iPc & 18 & 50.00 & -4.4 & $i$ & & $z$ & $25 s$ \\
\hline & $z$ & $17 \mathrm{~s}$ & 117 & $.00 \mathrm{um}$ & & & $.9 M s z X$ & 1 & & & \\
\hline & $\mathbf{N}$ & $17 \mathrm{~s}$ & 119 & $9.00 \mathrm{um}$ & & & & $i$ & & & \\
\hline & $E$ & $17 \mathrm{~s}$ & 91 & $1.30 u m$ & & & & 1 & & & \\
\hline & & & & ePP & 20 & 51.00 & & 1 & & & \\
\hline & & & & is & 25 & 49.00 & & $i$ & NGS & & 58.66 \\
\hline & & & & iSP & 25 & 55.00 & & 1 & UPP & & 58.87 \\
\hline & & & & ises & 28 & 39.00 & & 1 & & & \\
\hline KEV & & 49.44 & 3 & iP & 18 & 50.70 & -3.7 & 1 & & & \\
\hline & & $0.7 \mathrm{~s}$ & 69 & $9.40 \mathrm{~nm}$ & & & $.8 \mathrm{mb}$ & $i$ & & & \\
\hline & $z$ & $24 \mathrm{~s}$ & 47 & $.10 \mathrm{um}$ & & & $.4 M s z X$ & 1 & BJ I & & 58.92 \\
\hline & & & & $\mathrm{i}$ & 18 & 59.90 & & 1 & & & \\
\hline & & & & es & 25 & 56.00 & & 1 & & & \\
\hline$O S B$ & & 49.92 & 92 & eP & 18 & 56.20 & -2.4 & 1 & & & \\
\hline SHR & & 50.13 & 276 & $\mathbf{P}$ & 18 & 57.00 & -3.2 & 1 & & & \\
\hline$A \mid K$ & & 50.37 & 279 & $\mathbf{P}$ & 18 & 57.80 & -4.2 & I & & & \\
\hline NIIJ & & 50.62 & 277 & $\mathbf{P}$ & 18 & 59.40 & -4.5 & 11 & KAGJ & & 59.44 \\
\hline KAKJ & & 50.88 & 276 & $\mathbf{P}$ & 19 & 01.70 & -4.2 & 1 & PUL & & 59.52 \\
\hline$K I R$ & & 51.11 & 6 & iP & 19 & 03.40 & -3.9 & 1 & & & $1.5 \mathrm{~s}$ \\
\hline MAT & & 51.56 & 278 & iPd & 19 & 06.60 & -4.5 & 1 & & & \\
\hline & & $1.6 \mathrm{~s}$ & 400 & $.00 \mathrm{~nm}$ & & & $.1 \mathrm{mb}$ & 1 & & & \\
\hline & $z$ & $20 \mathrm{~s}$ & & $3.50 \mathrm{um}$ & & & $.0 \mathrm{Msz}$ & i & & & \\
\hline & & & & es & 26 & 26.00 & & . & & & \\
\hline $\mathrm{CHJJ}$ & & 51.58 & 276 & P & 19 & 07.00 & -4.2 & 1 & & & \\
\hline APA & & 51.72 & 360 & iPc & 19 & 08.40 & -3.4 & 1 & & & \\
\hline & & $1.6 \mathrm{~s}$ & 1000 & $.00 \mathrm{~nm}$ & & & $.5 \mathrm{mb}$ & 1 & & & \\
\hline & & & & is & 26 & 26.00 & & 1 & SVE & & 60.49 \\
\hline & & & & iSPP & 26 & 41.00 & & 1 & & & $2.6 \mathrm{~s}$ \\
\hline & & & & eSS & 30 & 06.00 & & 1 & & z & $20 \mathrm{~s}$ \\
\hline MTMJ & & 51.7 & 278 & $\mathbf{P}$ & 19 & 08.10 & -4.3 & 1 & & $\mathrm{~N}$ & $20 s$ \\
\hline
\end{tabular}




\begin{tabular}{|c|c|c|c|c|c|c|c|c|c|c|c|}
\hline & & & & ePP & 23 & 30.00 & & SSF & & 69.68 & 21 \\
\hline & & & & e & 25 & 36.00 & & I FUR & & 69.77 & 15 \\
\hline & & & & es & 29 & 48.00 & & 1 & & & \\
\hline & & & & eP'P' & 49 & 15.00 & & I SLE & & 69.79 & 17 \\
\hline TNS & & 67.38 & 17 & ePd & 20 & 56.60 & -3.5 & I LBF & & 69.83 & 21 \\
\hline & & & & ePcP & 21 & 06.40 & & AVF & & 69.91 & 21 \\
\hline & & & & ePP & 23 & 33.00 & & I TLG & & 69.94 & 327 \\
\hline & & & & es & 29 & 52.70 & & 1 & & $2.3 \mathrm{~s}$ & 450 \\
\hline BRG & & 67.42 & 13 & IPd & 20 & 56.40 & -3.9 & 1 & & & \\
\hline & $z$ & $18 \mathrm{~s}$ & 10 & 0.00 um & & & $1 \mathrm{Msz}$ & 1 & & & \\
\hline & $\mathbf{N}$ & $18 s$ & & 1.06 um & & & & 1 & & & \\
\hline & $E$ & $18 \mathrm{~s}$ & & $9.00 \mathrm{um}$ & & & & 1 & & & \\
\hline & & & & es & 29 & 52.80 & & I SJG & & 69.98 & 89 \\
\hline LDF & & 67.54 & 23 & eP & 20 & 57.60 & $-3 \cdot 5$ & 1 & & $1.5 \mathrm{~s}$ & 375 \\
\hline GUMO & & 67.58 & 257 & eP- & 20 & 56.00 & -5.7 & I & $z$ & $20 s$ & 55 \\
\hline & & & & PP & 21 & 04.80 & $28 \mathrm{~km} x$ & I ZUL & & 70.05 & 17 \\
\hline PJG & & 67.58 & 257 & $e(P)$ & 20 & 55.20 & -6.5 & AAA & & 70.05 & 327 \\
\hline WLF & & 67.59 & 18 & P- & 21 & 00.00 & -1.3 & 1 & & & \\
\hline & & & & PP & 23 & 39.00 & & 1 & & & \\
\hline & & & & $S$ & 30 & 00.00 & & 1 & & & \\
\hline GRR & & 67.60 & 24 & eP & 20 & 58.10 & $-3 \cdot 3$ & I LSF & & 70.11 & 23 \\
\hline GUA & & 67.60 & 257 & eP- & 20 & 55.00 & -6.9 & SMF & & 70.14 & 21 \\
\hline & & $0.8 \mathrm{~s}$ & 143 & $3.00 \mathrm{~nm}$ & & & $.2 \mathrm{mb}$ & I TCF & & 70.19 & 22 \\
\hline & & & & es & 29 & 47.00 & & 1 & & $1.5 \mathrm{~s}$ & 563 \\
\hline KSP & & 67.69 & 11 & iPd & 20 & 58.00 & -4.0 & I KMR & & 70.22 & 13 \\
\hline & & & & $i$ & 21 & 08.50 & & I VIE & & 27 & 12 \\
\hline & & & & es & 29 & 57.00 & & I MZF & & 70.33 & 22 \\
\hline LPF & & 67.90 & 24 & eP & 21 & 00.20 & $-3 \cdot 1$ & I UZH & & 70.36 & 7 \\
\hline GRF & & 68.27 & 15 & ePd & 21 & 02.00 & -3.6 & 1 & & $1.7 \mathrm{~s}$ & 500 \\
\hline & & $2.1 \mathrm{~s}$ & 529 & $9.00 \mathrm{~nm}$ & & & $3 m b$ & 1 & $z$ & $20 s$ & 22 \\
\hline & $z$ & & & 8.00 um & & & $.9 M s z$ & 1 & $\mathbf{N}$ & $20 \mathrm{~s}$ & 16 \\
\hline & & & & epP & 21 & 12.20 & $33 \mathrm{kmx}$ & 1 & $E$ & $20 \mathrm{~s}$ & 11 \\
\hline & & & & ePP & 23 & 40.30 & & 1 & & & \\
\hline & & & & es & 30 & 03.30 & & I & & & \\
\hline PRU & & 68.36 & 13 & iPd & 21 & 02.50 & -3.7 & 1 & & & \\
\hline & $z$ & $16 \mathrm{~s}$ & & $3.20 \mathrm{um}$ & & & . $3 M s z X$ & 1 & & & \\
\hline & $\mathbf{N}$ & $18 \mathrm{~s}$ & & 9.30 um & & & & 1 & & & \\
\hline & $\mathbf{E}$ & $19 \mathrm{~s}$ & & 0.20 um & & & & I PRZ & & 70.40 & 326 \\
\hline & & & & $i$ & 21 & 12.50 & & 1 & & $1.0 \mathrm{~s}$ & 130 \\
\hline & & & & iPP & 23 & 40.00 & & 1 & 2 & $17 \mathrm{~s}$ & 90 \\
\hline & & & & is & 30 & 02.00 & & 1 & $\mathrm{~N}$ & $17 \mathrm{~s}$ & 110 \\
\hline & & & & e & 31 & 00.00 & & 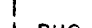 & & & \\
\hline GWF & & 68.48 & 18 & IPd & 21 & 03.40 & -3.6 & I BHG & & 70.41 & 14 \\
\hline KRA & & 68.78 & 9 & iPd & 21 & 04.90 & -3.9 & 1 & & & \\
\hline & & $1.9 \mathrm{~s}$ & 1520 & $0.00 \mathrm{~nm}$ & & & $.8 \mathrm{mb}$ & I SAX & & 70.41 & 17 \\
\hline & $z$ & $20 \mathrm{~s}$ & & $2.20 \mathrm{um}$ & & & $.6 \mathrm{Msz}$ & LLS & & 70.74 & 17 \\
\hline & $N$ & $28 \mathrm{~s}$ & & 8.50 um & & & & I SRO & & 70.88 & 10 \\
\hline & $E$ & $28 \mathrm{~s}$ & & 7.10 um & & & & 1 & $N$ & $20 \mathrm{~s}$ & 13 \\
\hline & & & & $i$ & 21 & 14.40 & & 1 & $E$ & $20 \mathrm{~s}$ & 12 \\
\hline & & & & i & 21 & 23.30 & & 1 & & & \\
\hline & & & & $\mathrm{i}$ & 21 & 28.90 & & I RJF & & 71.01 & 23 \\
\hline & & & & $\mathrm{i}$ & 21 & 37.90 & & I OGA & & 71.02 & 16 \\
\hline & & & & e & 23 & 54.00 & & I FRU & & 71.06 & 329 \\
\hline & & & & is & 30 & 09.00 & & 1 & & $2.5 \mathrm{~s}$ & 4200 \\
\hline BUH & & 68.86 & 17 & ePd & 21 & 06.10 & -3.3 & 1 & $z$ & $18 \mathrm{~s}$ & 113 \\
\hline ANP & & 68.94 & 284 & iP- & 21 & 08.00 & -2.2 & 1 & $\mathbf{N}$ & $18 \mathrm{~s}$ & 80 \\
\hline & & & & is & 30 & 12.00 & & I & $E$ & $18 s$ & 56 \\
\hline$D F$ & & $68 \cdot 98$ & 18 & $e P$ & 21 & 06.90 & -3.2 & 1 & & & \\
\hline WET & & 69.01 & 14 & eP & 21 & 06.70 & -3.5 & 1 & & & \\
\hline & $z$ & $22 \mathrm{~s}$ & & 4.00 um & & & $.4 \mathrm{Msz}$ & 1 & & & \\
\hline & & & & es & 30 & 13.50 & & 1 & & & \\
\hline $\mathrm{KHC}$ & & 69.10 & 13 & iPd & 21 & 07.50 & -3.3 & $1 \mathrm{KBA}$ & & 71.10 & 14 \\
\hline & $z$ & $22 \mathrm{~s}$ & & 3.80 um & & & $.2 \mathrm{Msz}$ & 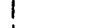 & & & \\
\hline & $N$ & $22 \mathrm{~s}$ & & $8.10 \mathrm{um}$ & & & & 1 & & & \\
\hline & $\mathbf{E}$ & $21 \mathrm{~s}$ & & 5.90 um & & & & 1 & & & \\
\hline & & & & i & 21 & 17.60 & & LFF & & 71.22 & 24 \\
\hline & & & & PP & 23 & 48.10 & & I DIX & & 71.27 & 18 \\
\hline & & & & 5 & 30 & 14.00 & & I MMK & & 71.38 & 18 \\
\hline $\mathrm{CH}$ & & 69.15 & 18 & iPd & 21 & 07.60 & -3.5 & 1 TMA & & 71.47 & 17 \\
\hline HAU & & 69.23 & 19 & eP & 21 & 08.30 & -3.3 & I CAF & & 71.48 & 23 \\
\hline LVV & & 69.28 & 6 & iPd & 21 & 08.00 & -3.8 & I LPO & & 71.55 & 23 \\
\hline & & $4.0 \mathrm{~s}$ & 3400 & $0.00 \mathrm{~nm}$ & & & $.9 \mathrm{mb} \times$ & I SAL & & 72.19 & 16 \\
\hline & $z$ & $22 \mathrm{~s}$ & & 4.80 um & & & $.4 \mathrm{Msz}$ & I LJU & & 72.25 & 13 \\
\hline & $\mathbf{N}$ & $20 \mathrm{~s}$ & & 4.90 um & & & & : & & & \\
\hline & E & $20 \mathrm{~s}$ & & $2.70 \mathrm{um}$ & & & & 1 & & & \\
\hline & & & & epp & 21 & 20.00 & $41 \mathrm{kmx}$ & KIS & & 72.26 & 3 \\
\hline & & & & is & 30 & 14.00 & & 1 & & $2.0 \mathrm{~s}$ & 600 \\
\hline & & & & eSPP & 30 & 45.00 & & 1 & $z$ & $19 \mathrm{~s}$ & 20 \\
\hline IFF & & 69.45 & 24 & eP & 21 & 10.60 & -2.9 & ! & 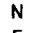 & $20 \mathrm{~s}$ & 19 \\
\hline BSF & & 69.47 & 18 & eP & 21 & 09.80 & -3.4 & 1 & $E$ & $20 \mathrm{~s}$ & \\
\hline BAF & & 69.50 & 18 & iPd & 21 & 10.00 & -3.3 & 1 & & & \\
\hline LOR & & 69.54 & 21 & eP & 21 & 10.20 & -3.3 & 1 & & & \\
\hline & & $1.4 \mathrm{~s}$ & 502 & $2.00 \mathrm{~nm}$ & & & $.5 \mathrm{mb}$ & I TRI & & 72.50 & 14 \\
\hline SPC & & 69.67 & 9 & eP & 21 & 11.90 & -2.6 & 1 & & & \\
\hline & & & & $\mathrm{i}$ & 21 & 21.50 & & 1 & & & \\
\hline & & & & $\mathrm{i}$ & 21 & 31.80 & & 1 & & & \\
\hline & & & & $i(P P)$ & 23 & 50.00 & & 1 & & & \\
\hline
\end{tabular}




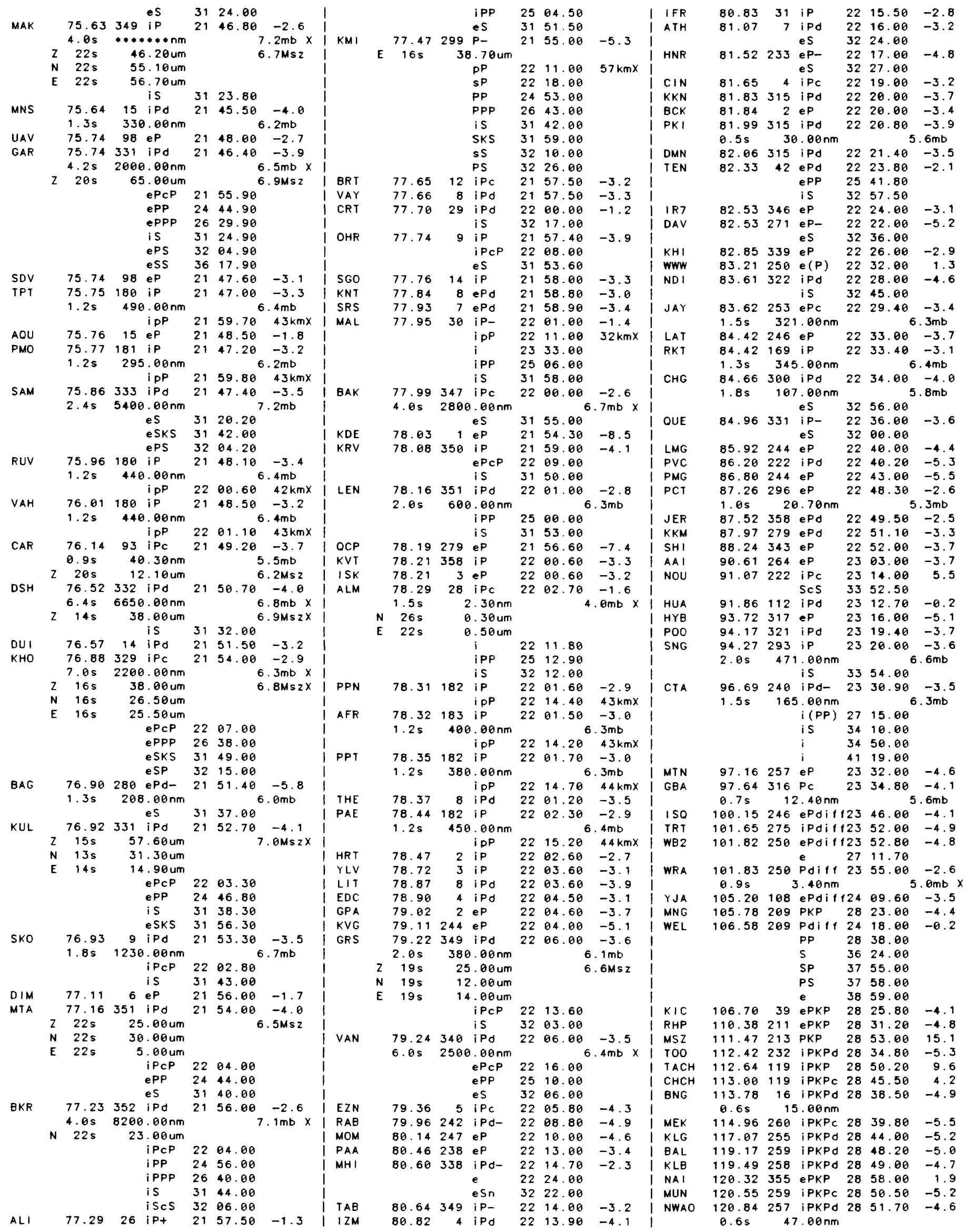


$12 d \quad 15 h$

\begin{tabular}{|c|c|c|c|c|c|c|}
\hline NPA & 133.89 & 351 & $\begin{array}{l}\text { ePKP } \\
\text { ePPKP }\end{array}$ & $\begin{array}{l}29 \\
32\end{array}$ & $\begin{array}{l}18.00 \\
01.00\end{array}$ & -3.7 \\
\hline \multirow[t]{3}{*}{ TET } & 135.18 & 359 & IPKP & 29 & 20.00 & -4.1 \\
\hline & $1.3 \mathrm{~s}$ & & $0.26 \mathrm{~nm}$ & & & \\
\hline & & & IPPKP & 34 & 04.00 & \\
\hline \multirow[t]{3}{*}{ KRI } & 135.80 & 4 & ePKP & 29 & 22.00 & -3.5 \\
\hline & & & ipPKP & 29 & 36.00 & \\
\hline & & & iSKP & 32 & 07.00 & \\
\hline \multirow[t]{2}{*}{ MTD } & 135.80 & 2 & EPKP & 29 & 22.00 & -3.4 \\
\hline & & & ISKP & 32 & 05.00 & \\
\hline VY & 136.63 & 339 & ePKP & 29 & 20.00 & -7.1 \\
\hline DRV & 138.07 & 215 & ePKP & 29 & 20.00 & -8.1 \\
\hline \multirow[t]{5}{*}{ BUL } & 139.05 & 6 & IPKPd & 29 & 31.00 & -0.5 \\
\hline & $22 \mathrm{~s}$ & & $2.60 \mathrm{um}$ & & & $.6 \mathrm{Msz}$ \\
\hline & $21 \mathrm{~s}$ & & $0.80 u m$ & & & \\
\hline & $21 \mathrm{~s}$ & & $7.17 \mathrm{um}$ & & & \\
\hline & & & iSKP & 32 & 19.00 & \\
\hline & 141.46 & 194 & iPKP & 29 & 25.90 & -8.1 \\
\hline \multirow[t]{2}{*}{ SLR } & 144.58 & 7 & ePKP & 29 & 34.20 & -6.8 \\
\hline & $z 21 \mathrm{~s}$ & 24 & $4.40 \mathrm{um}$ & & 7 & $.0 \mathrm{Msz}$ \\
\hline \multirow[t]{2}{*}{ PRE } & 144.59 & 7 & iPKPd & 29 & 35.50 & -5.6 \\
\hline & $2.0 \mathrm{~s}$ & $* * * *$ & $* * * * n \mathrm{~nm}$ & & & \\
\hline$S R$ & 144.61 & 9 & ¡PKPd & 29 & 35.50 & -5.6 \\
\hline PI & 145.00 & 8 & iPKPd & 29 & 36.60 & -5.2 \\
\hline EVA & 145.39 & 6 & IPKPd & 29 & 38.10 & -4.4 \\
\hline \multirow[t]{2}{*}{ BFS } & 145.63 & 10 & iPKPd & 29 & 38.90 & -3.9 \\
\hline & $1.5 \mathrm{~s}$ & 500 & $0.00 \mathrm{~nm}$ & & & \\
\hline$R Y$ & 145.71 & 9 & IPKPd & 29 & 40.00 & -3.0 \\
\hline NZ & 145.78 & 12 & IPKPC & 29 & 39.50 & -3.5 \\
\hline 7 & 146.42 & 1 & iPKPd & 29 & 39.70 & -4.2 \\
\hline IR & 146.81 & 10 & ePKP & 29 & 41.00 & -3.7 \\
\hline SEK & 147.11 & 9 & iPKPC & 29 & 41.50 & -3.7 \\
\hline \multirow[t]{2}{*}{ HVD } & 149.18 & 12 & IPKPd & 29 & 44.20 & -4.2 \\
\hline & $1.6 \mathrm{~s}$ & & $0.00 \mathrm{~nm}$ & & & \\
\hline \multirow[t]{3}{*}{ SUR } & 150.28 & 21 & ¿PKPc & 29 & 46.70 & -3.4 \\
\hline & $0.5 \mathrm{~s}$ & & $6.30 \mathrm{~nm}$ & & & \\
\hline & $z \quad 21 \mathrm{~s}$ & 40 & $0.60 u m$ & & & $.2 \mathrm{Msz}$ \\
\hline TUH & 150.86 & 24 & ePKP & 29 & 48.50 & -2.1 \\
\hline \multirow[t]{3}{*}{ SPA } & 150.86 & 180 & ePKPC & 29 & 43.50 & -6.3 \\
\hline & $1.0 \mathrm{~s}$ & 465 & $5.00 \mathrm{~nm}$ & & & \\
\hline & $z 20 \mathrm{~s}$ & & $1.50 u m$ & & & $.7 \mathrm{Msz}$ \\
\hline CER & 150.95 & 24 & ePKP & 29 & 49.00 & -1.8 \\
\hline \multirow[t]{3}{*}{ GRM } & 151.97 & 11 & ePKP & 29 & 44.00 & -8 \\
\hline & $1.4 \mathrm{~s}$ & 560 & $0.00 \mathrm{~nm}$ & & & \\
\hline & $z \quad 20 \mathrm{~s}$ & 12 & $2.60 u m$ & & & $.7 \mathrm{M}$ \\
\hline & 165.54 & 230 & ePKP & 30 & 03.00 & -3.8 \\
\hline & 171.52 & 196 & EPKP & 30 & 05.00 & -5.5 \\
\hline & $\therefore 0$ & & & & & \\
\hline
\end{tabular}

AUG $06,1983 \quad 15 \mathrm{~h} 43 \mathrm{~m} 52.59 \pm 0.08 \mathrm{~s}$ | CEI $40.130 \mathrm{~N} \pm 1.4 \mathrm{~km} 24.721 \mathrm{E} \pm 1.0 \mathrm{~km}$ DUI DEPTH $=10.0 \mathrm{~km}$ (geophysicist) 6. $2 \mathrm{mb}$ (120 obs.) $7.0 \mathrm{Msz}$ ( 16 obs.) / BUD AEGEAN SEA Slight domoge ( $V I$ ) on Limnos and to four monasteries in the Mount Athos oreo. Also felt (Vi) on Thosos and Lesvos. Felt (v) throughout much of northern Greece. Felt strongly in southern Bulgorio and nor thwestern Turkey.

OU
$P A$
$P L$
SOH
SR
$T H$
THE
$P R$
$K D$
$L I$
$K N$
GR
$P L D$
$D I M$
VAY
$P T$
$K Z$
$A T$
AT
ED
IZM
VTS
DM
JAM

0.60290 iPgd $44 \quad 05.80$

0.60290 iPgd

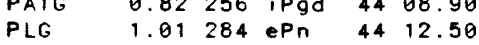

SOH $\quad 1.25304$ iPgC 4416.60

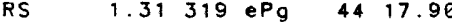

$\begin{array}{lllll}\text { THE } & 1.43291 & \text { IPbd } & 44 & 19.30 \\ \text { eSb } & 44 & 38.40\end{array}$

PRK $\quad 1.49126$ EPKP $44 \quad 18.50$

$\begin{array}{lllllll}K D Z & 1.58 & 17 & \mathrm{iPC} & 44 & 20.00 & -0 . \\ \text { LIT } & 1.71 & 270 & \mathrm{iPnd} & 44 & 22.20 & \end{array}$

1.73307 ePnd

1.95296 iPnc

$\begin{array}{lll}1.97 & 360 \mathrm{iPc} \\ 2.02 & 19 \mathrm{iP}\end{array}$

$2.02307 \mathrm{iPn}$

2.18198 iPnc

$\begin{array}{lll}2.27 & 275 & \text { iPn } \\ 2.29 & 200 & \text { iPn }\end{array}$

2.29200 iPn
isn

$2.4284 \mathrm{iPn}$

$2.62130 \mathrm{iPn}$

$2.72336 \mathrm{iPc}$

$2.8553 \mathrm{iPn}$

4423.60

4426.80

$4426.00-0.4$

$4426.00-1.1$

$\begin{array}{lll}44 & 28.30 & 1.2\end{array}$

$\begin{array}{lll}44 & 27.70 & -1.8\end{array}$

$\begin{array}{rrr}44 & 31.00 & 0.3 \\ 44 & 30.00 & -1.0\end{array}$

4456.50

$\begin{array}{lll}44 & 31.40 & -1.4\end{array}$

$\begin{array}{lll}44 & 34.50 & -1.3\end{array}$

$4438.00 \quad 0.9$

$\begin{array}{lll}44 & 36.90 & -2.1\end{array}$

3.01262 iPnc

I PVL

SKO

APE

RLS
IST

ORI

SGO

POI

I RMP

MNS

I CEY

I

SPC

ERC

L LVV

ZST

ZST
OHR

ISK

YLV

ITM

HRT
YER

CGN

GPA

BUC1

PSN

SRE

VAM

CVD

NPS
CLO

CMP

LOZ

SSR

BCK

$M L R$

BRT

$B I R$
$B A C$

CJR KAS BLY Kis UI (a)

\section{?}

ing

$3.036 \mathrm{iPc}$

$\begin{array}{lll}4 P_{n} & 440.00 & -1.8\end{array}$

$\begin{array}{lll} & \\ & \text { iPnd } 44 \quad 43.00 & 0.7\end{array}$

iPg 4456.00

isn 4526.30

$3.12168 \mathrm{ePn}$

3.14289 iPnd

3.27232 iPne

$3.3873 \mathrm{iPd}$

$3.4373 \mathrm{iPn}$

$\begin{array}{lll}3.58 & 82 & \text { iPno } \\ 3.64 & 133 & \mathrm{eP}\end{array}$

$\begin{array}{lll}3.64 & 133 \mathrm{eP} \\ 3.67 & 217 \mathrm{ePn}\end{array}$

3.76240 iPn

$3.8478 \mathrm{iPn}$

$4.09136 \mathrm{iPn}$

$4.14 \quad 13 \mathrm{iPd}$

$4.28 \quad 86 \mathrm{iPn}$

4.30103 iPn

4. $32 \quad 13 \mathrm{iPd}$

$4.39 \quad 35 \quad \mathrm{Pd}$

$4.56356 \mathrm{iPd}$

$4.67347 \mathrm{iPC}$

$4.73185 \mathrm{ePn}$

$4.74144 \mathrm{ePn}$

4.8729 iP

4.91171 iPnd

$5.14345 \mathrm{eP}$

$5.143 \mathrm{iPc}$

5.18274 ipnd

$5.20357 \quad \mathrm{PC}$

$5.22336 \mathrm{iP}$

5.29128 iPn

$5.30118 \mathrm{iPn}$

$5.439 \mathrm{iPC}$

$5.78280 \mathrm{ePn}$

$5.90348 \mathrm{iPc}$

6.34272 iPnd

6.4918 iP

$6.63 \quad 13 \mathrm{iPd}$

$6.63353 \mathrm{iPc}$

6.9877 i Pnd

20276 ipnd 4

$7.24312 \mathrm{Pn}$

$7.5122 \mathrm{iPc}$

$7.73349 \mathrm{e}$

7.92284 iPnc

8.06252 ifnd

8.42333 ePnc

$8.4452 \mathrm{iPc}$

$9.0 \mathrm{~s} * * * * * * * \mathrm{~nm}$

$z 24 \mathrm{~s} \quad 320.00 \mathrm{um}$

$24 \mathrm{~s} 620.00 \mathrm{um}$

$24 \mathrm{~s} 520.00 \mathrm{um}$

$8.53338 \mathrm{ePnc}$

8.60259 iPnd

$8.6780 \mathrm{iP}$

8.81288 iPnd

8.88 342 iPnc

8.96331 iP

$9.05331 \mathrm{is}$

E $10 \mathrm{~s} 1800.00 \mathrm{um}$

$\begin{array}{lll}440.70 & -2.1\end{array}$

$\begin{array}{lll}4443.80 & 0.7\end{array}$

4444.50

4444.00

4444.90

$448.50-1.7$

$\begin{array}{lll}44 & 49.50 & -1.1\end{array}$

$4453.50 \quad 1.6$

$4451.40-1.6$

$4455.20-1.3$

$\begin{array}{lll}44 & 56.50 & -0.7\end{array}$

$457.40-1.9$

$\begin{array}{lll}4 & 58.20 & -1.4\end{array}$

$\begin{array}{lll}45 & 02.30 \quad 2.5\end{array}$

$\begin{array}{lll}44 & 59.00 & -1.7\end{array}$

$\begin{array}{lll}45 & 03.00 & -0.1\end{array}$

4505.00

4503.10

4504.60

$46 \quad 05.00$

4505.80

4510.00

$45 \quad 10.00$

4510.00

4507.00

4512.00

$\begin{array}{ll}45 & 13.80\end{array}$

$\begin{array}{ll}45 & 12.68\end{array}$

$45 \quad 15.20$

4520.00

4522.00

4528.80

$\begin{array}{lll}45 & 31.50 & -0.9 \\ 45 & 32.10 & -0.4\end{array}$

4534.50

4538.08

4544.50

453.50

$45 \quad 42.70$

$47 \quad 15.20$

4544.00

4702.00

4550.00
4549.00

4548.08

4555.00

4556.00

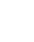

47

4727.00

$4557.00-2.0$ LLS

$4557.00-3.0$ GRF

$4559.00-2.0$

$\begin{array}{lll}46 & 01.20 & -1.8\end{array}$

4602.10

4603.40

$46 \quad 49.80$

4628.70

4722.30

4747.10

4800.00

I VIE
$46 \quad 11.20$

$46 \quad 07.50$

4608.50

4611.00

$9.35288 \mathrm{ePn}$

$9.40310 \mathrm{ePd}$

$9.51312 \mathrm{ePC}$

es

$9.60342 \mathrm{eP}$

9.66261 iPnd

$9.70357 \mathrm{iPC}$

9.74328 ip

$9.78308 \mathrm{ePn}$

10.09326 iP- $\begin{array}{ll}46 & 12.50\end{array}$

$46 \quad 16.2 \theta$

$46 \quad 13.00$

$\begin{array}{ll}46 & 12.50\end{array}$

$46 \quad 15.50$

$48 \quad 03.00$

$\begin{array}{ll}46 & 15.20\end{array}$

4616.00

$46 \quad 20.70$
48

$48 \quad 22.20$

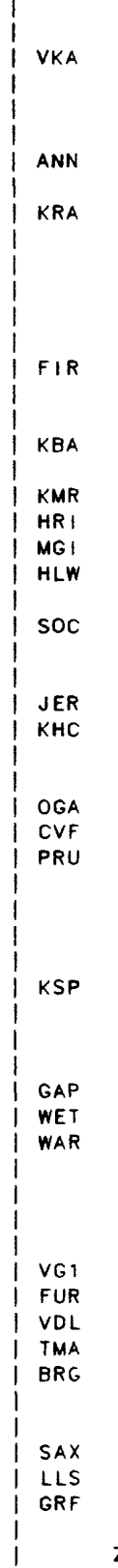

$-1.7$

I MMK

HOF

2.1 CLL

zU

$3.1 \times$

-1.4 SLE

-1.8 / MOX

\begin{tabular}{r|r}
-0.1 & \\
0.0 & $01 x$
\end{tabular}

I LMR

-0.9 STU

\begin{tabular}{r|l}
-2.1 & \\
0.4 & LRG
\end{tabular}

-0.5 PYA

$-0.2$

0.3

BKR

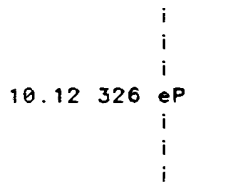

4901.60

$49 \quad 10.00$

4925.10

$\begin{array}{lll}46 & 19.00 & -1.9\end{array}$

4652.00

$47 \quad 10.70$

$\begin{array}{lll}46 & 21.00 & -4.2 x\end{array}$

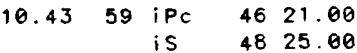

$\begin{array}{lllll}10.48 & 343 \mathrm{ePC} & 46 & 25.10 & -0.7\end{array}$

$2.2 \mathrm{~s} \quad 6210.00 \mathrm{~nm}$

4630.30

4634.40
$48 \quad 26.00$

4945.80 


\begin{tabular}{|c|c|c|c|c|c|c|c|c|c|c|c|}
\hline & $1.3 \mathrm{~s}$ & 330 & $0.00 \mathrm{~nm}$ & & & $.9 \mathrm{mb}$ & I UCC & 17.77 & 314 & $P C-$ & 48 \\
\hline & $16 \mathrm{~s}$ & 156 & $6.00 \mathrm{um}$ & & & & I RJF & 17.79 & 295 & iPd & 48 \\
\hline & $16 s$ & 370 & $0.00 \mathrm{um}$ & & & & 1 & $2.6 \mathrm{~s}$ & 6340 & $\theta 00 \mathrm{~nm}$ & \\
\hline BUH & 14.54 & 311 & eP & 47 & 18.80 & -1.4 & 1 MOS & 17.80 & 24 & iP & 47 \\
\hline LEN & 14.58 & 81 & iPd & 47 & 23.00 & 2.0 & 1 & $3.0 \mathrm{~s}$ & $* * * *$ & $* * * n m$ & \\
\hline BRN & 14.62 & 331 & iPc & 47 & 21.00 & -0.1 & I & $16 \mathrm{~s}$ & 228 & 3. 00 um & \\
\hline & & & es & & 10.00 & & & & & is & 51 \\
\hline $3 A F$ & 14.88 & 307 & iPd & 47 & 23.60 & $-1 \cdot 2$ & I LPO & 17.94 & 292 & iPd & 48 \\
\hline $\mathrm{ECH}$ & 14.95 & 309 & iPd & 47 & 4.00 & -1.6 & 1 & $1.8 \mathrm{~s}$ & 1720 & $0.00 \mathrm{~nm}$ & \\
\hline MSL & 14.95 & 99 & iPc & 47 & 28.06 & 2.4 & I LSF & 17.95 & 298 & iPd & 48 \\
\hline & & & iPP & 47 & 42.50 & & 1 & $1.3 \mathrm{~s}$ & 1110 & $0.00 \mathrm{~nm}$ & \\
\hline & & & is & 50 & 31.60 & & I DBN & 18.01 & 318 & iPt & 48 \\
\hline & & & ePcP & 52 & 12.00 & & 1 & & & is & 51 \\
\hline & & & eLO & 53 & 09.50 & & I SHE & 18.22 & 81 & $i P c$ & 48 \\
\hline & & & e & 53 & 39.00 & & I LFF & 18.29 & 293 & iPd & 48 \\
\hline $\mathrm{CDF}$ & 14.97 & 309 & ePd & 47 & 24.30 & -1.6 & 1 & $2.0 \mathrm{~s}$ & 3250 & $0.00 \mathrm{~nm}$ & \\
\hline & $1.5 \mathrm{~s}$ & 1650 & $0.00 \mathrm{~nm}$ & & & $.3 \mathrm{mb}$ & I EPF & 18.45 & 287 & iPd & 48 \\
\hline BSF & 15.01 & 307 & ePd & 47 & 25.10 & -1.3 & 1 & $1.8 \mathrm{~s}$ & 1260 & $3.00 \mathrm{~nm}$ & \\
\hline & $.9 \mathrm{~s}$ & 422 & $2.00 \mathrm{~nm}$ & & 5 & $.9 \mathrm{mb}$ & I KER & 18.71 & 101 & eP & 48 \\
\hline GWF & 5.03 & 312 & iPd & 47 & 26 . & -0.3 & MFF & 16 & 298 & IPd & 48 \\
\hline ERE & 15.13 & 83 & IPd & 47 & 28.50 & 0.5 & 1 & s & 1580 & $0.00 \mathrm{~nm}$ & \\
\hline & & & es & 50 & 17.00 & & I BAK & 19.21 & 81 & iPc & 48 \\
\hline INS & 15.25 & 317 & ePc & 47 & 29.40 & -0.1 & ! & 4.05 & $* * * *$ & $* * * * n m$ & \\
\hline & & & is & 50 & 35.00 & & 1 & & & is & 51 \\
\hline & & & e & 52 & 26.00 & & MUD & 19.26 & 333 & iPc & 48 \\
\hline $1 n$ & 15.26 & 78 & IPd & 47 & 29.40 & -0.3 & 1 & $1.0 \mathrm{~s}$ & 780 & $3.00 \mathrm{~nm}$ & \\
\hline & & & is & 50 & 22.20 & & I ALI & 19.59 & 273 & iP & 48 \\
\hline AU & 15.35 & 307 & ePd & 47 & 29.60 & -1.2 & 1 & & & es & 52 \\
\hline & $1.0 \mathrm{~s}$ & 606 & $6.00 \mathrm{~nm}$ & & & $.9 \mathrm{mb}$ & LDF & 9.61 & 304 & iPd & 48 \\
\hline GRO & 15.97 & 72 & iPd & 47 & 40.00 & 1.2 & I & $.9 \mathrm{~s}$ & 4518 & $3.00 \mathrm{~nm}$ & \\
\hline & $1.5 \mathrm{~s}$ & 4500 & $0.00 \mathrm{~nm}$ & & & $.4 \mathrm{mb}$ & $F L N$ & 9.88 & 304 & iPd & 48 \\
\hline & $12 \mathrm{~s}$ & 133 & 3.0 & & & $.5 M \leq z X$ & I & $0 s$ & 4420 & $9.00 \mathrm{~nm}$ & \\
\hline & $12 \mathrm{~s}$ & 670 & $0.00 \mathrm{um}$ & & & & PUL & .97 & 8 & iPc & 48 \\
\hline & $12 \mathrm{~s}$ & 230 & $0.00 \mathrm{um}$ & & & & 1 & $8.0 \mathrm{~s}$ & $* * * *$ & $* * * n m$ & \\
\hline WLF & 16.21 & 312 & P- & 47 & 46.00 & $4.2 x$ & ! & $14 \mathrm{~s}$ & 500 & 0.00 um & \\
\hline & & & $\mathrm{s}$ & 50 & 39.00 & & 1 & $14 \mathrm{~s}$ & 160 & 0.00 um & \\
\hline BNS & 16.34 & 317 & $\mathrm{iPC}$ & 47 & 45.30 & 1.9 & 1 & & & is & 52 \\
\hline & & & es & 51 & 02. & & GRR & .02 & 303 & IPd & 48 \\
\hline RV & 6.47 & 81 & iPd & 47 & 47.00 & 1.7 & LPF & 0.06 & 302 & iPd & 48 \\
\hline SMF & 16.49 & 300 & $\mathrm{iPd}$ & 47 & 44.50 & -1.0 & I UPP & 0.25 & 350 & $i P_{c}$ & 48 \\
\hline & $.7 \mathrm{~s}$ & 2350 & $0.00 \mathrm{~nm}$ & & & $.0 m b$ & 1 & $.5 \mathrm{~s}$ & 800 & $0.00 \mathrm{~nm}$ & \\
\hline LBF & 6.50 & 301 & iPd & 47 & 45.40 & -0.2 & I NUR & 0.40 & 360 & iP+ & 48 \\
\hline & $.7 \mathrm{~s}$ & 1636 & $0.00 \mathrm{~nm}$ & & & $.9 \mathrm{mb}$ & ! & $.5 \mathrm{~s}$ & 994 & $4.00 \mathrm{~nm}$ & \\
\hline GRS & 16.62 & 85 & iPd & 47 & 48.20 & 0.9 & 1 & & & es & 52 \\
\hline & $2.0 \mathrm{~s}$ & 270 & $0.00 \mathrm{~nm}$ & & & $.0 \mathrm{mb} \times$ & I IR7 & 20.87 & 94 & ePd & 48 \\
\hline & & & es & 50 & 51.00 & & I TEH & 1.45 & 93 & eP & 48 \\
\hline LOR & 16.66 & 302 & iPd & 47 & 47.40 & -0.2 & I $A L M$ & 1.49 & 270 & iPgd & 48 \\
\hline & $1.4 \mathrm{~s}$ & 980 & $0.00 \mathrm{~nm}$ & & & $.7 \mathrm{mb}$ & 1 & $1.8 \mathrm{~s}$ & & 5. $10 \mathrm{~nm}$ & \\
\hline IAM & 16.70 & 328 & iPc & 47 & 48.50 & 0.5 & 1 & $14 \mathrm{~s}$ & & $3.00 \mathrm{um}$ & \\
\hline SSF & 16.83 & 301 & iPd & 47 & 49.30 & -0.4 & 1 & $11 \mathrm{~s}$ & & 9.60 um & \\
\hline & $.0 \mathrm{~s}$ & 1930 & $0.00 \mathrm{~nm}$ & & 5 & $.9 \mathrm{mb}$ & 1 & & & ¡PP & 49 \\
\hline VF & 16.86 & 300 & ePd & 47 & 49.30 & -0.7 & 1 & & & ¿PPP & 49 \\
\hline & $.0 \mathrm{~s}$ & 3930 & $0.00 \mathrm{~nm}$ & & & $.2 \mathrm{mb}$ & 1 & & & is & 52 \\
\hline ENN & 16.89 & 315 & eP & 47 & 53.50 & $3.0 x$ & I CRT & 22.27 & 272 & iPd & 48 \\
\hline & $1.8 \mathrm{~s}$ & 1300 & $0.00 \mathrm{~nm}$ & & 5 & $.8 \mathrm{mb}$ & SUF & 22.64 & 2 & ip & 48 \\
\hline & & & e & 47 & 56.50 & & 1 & 0.45 & 38 & $3.80 \mathrm{~nm}$ & \\
\hline & & & $\mathbf{i}$ & 47 & 59.20 & & I MAL & 23.03 & 271 & iPd & 48 \\
\hline & & & es & 51 & 11.00 & & 1 & $1.5 \mathrm{~s}$ & & $3.00 \mathrm{~nm}$ & \\
\hline AB & 6.90 & 90 & iP- & 47 & 52.00 & 1.2 & 1 & & & $\mathrm{i}$ & 49 \\
\hline OBN & 17.00 & 24 & $i P_{c}$ & 47 & 46.80 & $-4 \cdot 9 x$ & 1 & & & is & 53 \\
\hline & $2.0 \mathrm{~s}$ & 9200 & $0.00 \mathrm{~nm}$ & & & $.6 \mathrm{mb}$ & 1 EKA & 3.92 & 319 & $\mathrm{Pc}$ & 49 \\
\hline & & & is & 50 & 45.00 & & 1 & $7 \mathrm{~s}$ & 90 & $0.30 \mathrm{~nm}$ & \\
\hline VTS & 17.13 & 320 & $e^{P}$ & 47 & 56.00 & 2.6 & 1 ESY & 23.93 & 320 & ePc & 49 \\
\hline & $1.7 \mathrm{~s}$ & 2080 & $0.00 \mathrm{~nm}$ & & & $.0 \mathrm{mb}$ & 1 & 45 & 1520 & $0.00 \mathrm{~nm}$ & \\
\hline & & & $\mathrm{i}$ & 47 & 58.90 & & EBL & 24.07 & 320 & $i P_{c}$ & 49 \\
\hline & & & es & 51 & 17. & & 1 & $4 \mathrm{~s}$ & 1810 & $0.00 \mathrm{~nm}$ & \\
\hline IHD & 17.17 & 107 & ePd & 47 & 56.00 & 1.9 & I KJF & 24.17 & 3 & iPc & 49 \\
\hline & & & iPP & 48 & 14.00 & & I & $.9 \mathrm{~s}$ & 892 & 2.00 & \\
\hline & & & ePPP & 48 & 31.00 & & 1 & & & es & 53 \\
\hline & & & es & 51 & 39.00 & & | ED I & 24.22 & 320 & iPc & 4 \\
\hline & & & e & 53 & 35.00 & & 1 & & & es & 53 \\
\hline & & & eLO & 55 & 16.50 & & EAU & 4.31 & 320 & iPc & 49 \\
\hline AAK & 17.23 & 73 & iPd & 47 & 57.40 & 2.7 & 1 & $.4 \mathrm{~s}$ & 2020 & $9.00 \mathrm{~nm}$ & \\
\hline & $1.5 \mathrm{~s}$ & 500 & $0.00 \mathrm{~nm}$ & & & $.4 \mathrm{mb}$ & 1 EDU & 4.44 & 321 & $i P_{C}$ & 49 \\
\hline & & & is & 51 & 1 & & 1 & $4 \mathrm{~s}$ & 3690 & $.00 \mathrm{~nm}$ & \\
\hline $\mathrm{ZF}$ & 17.23 & 8 & ePd & 4 & & -0 & I ECP & .48 & 310 & iPc & 49 \\
\hline OU & 17.30 & 312 & $\mathrm{Pc}-$ & 47 & 58 & $2.9 x$ & 1 & & 230 & $9.00 \mathrm{~nm}$ & \\
\hline & & & e & 48 & 03 & & SFS & 24.48 & 271 & iPd & 49 \\
\hline & & & $\mathrm{s}$ & 51 & 02 & & I & & & iPP & 5 \\
\hline & & & $P C P$ & 52 & 52 & & I & & & is & 53 \\
\hline Af & 7.36 & 293 & IPd & 47 & 56.50 & 0.1 & 1 & & & iss & 54 \\
\hline TCF & 17.50 & 298 & IPd & 47 & $58 \cdot 10$ & -0.1 & I EBH & 24.53 & 321 & iPc & 49 \\
\hline & $2.5 \mathrm{~s}$ & 6250 & $0.00 \mathrm{~nm}$ & & & $.3 \mathrm{mb}$ & 1 & $1.4 \mathrm{~s}$ & 4900 & $0.00 \mathrm{~nm}$ & \\
\hline COP & 17.56 & 336 & iPct & 47 & 57.30 & -1.5 & I ETA & 24.54 & 311 & $i P_{c}$ & 49 \\
\hline & $1.1 \mathrm{~s}$ & 1270 & $0.00 \mathrm{~nm}$ & & & $.0 \mathrm{mb}$ & 1 & $1.0 \mathrm{~s}$ & 110 & $.00 \mathrm{~nm}$ & \\
\hline & 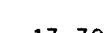 & 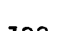 & is & 51 & 09.00 & & I ELO & 24.74 & 321 & ePc & 49 \\
\hline NIT & 17.70 & 322 & $e^{P}$ & 48 & 04.50 & $4.0 x$ & 1 & $1.3 \mathrm{~s}$ & 2060 & $.00 \mathrm{~nm}$ & \\
\hline
\end{tabular}




\begin{tabular}{|c|c|c|c|c|c|c|c|c|c|c|c|}
\hline & & $2.0 \mathrm{~s}$ & 260 & $0.00 \mathrm{~nm}$ & & & $6.8 \mathrm{mb}$ & 1 & & $1.7 \mathrm{~s}$ & 56 \\
\hline & Z & $14 \mathrm{~s}$ & & $8.00 \mathrm{um}$ & & & $6.5 \mathrm{Ms} z \mathrm{X}$ & $i$ & $z$ & $15 \mathrm{~s}$ & \\
\hline & N & $14 \mathrm{~s}$ & & $5.00 \mathrm{um}$ & & & & 1 & $N$ & $12 \mathrm{~s}$ & \\
\hline & $E$ & $14 \mathrm{~s}$ & & $1.00 \mathrm{um}$ & & & & 1 & $E$ & $13 \mathrm{~s}$ & \\
\hline & & & & ePP & 52 & 24.20 & & 1 & & & \\
\hline REY & & 36.11 & 327 & iP & 50 & 56.10 & 0.0 & 1 & & & \\
\hline & & & & $\mathrm{i}$ & 51 & 00.70 & & 1 & & & \\
\hline $\mathrm{KHO}$ & & 36.25 & 79 & eP & 51 & 00.00 & 2.1 & 1 & & & \\
\hline & z & $15 \mathrm{~s}$ & & $0.00 \mathrm{um}$ & & & 6. $2 \mathrm{MszX}$ & STJ & & 54.18 & 305 \\
\hline & $\mathrm{N}$ & $15 \mathrm{~s}$ & & $0.00 \mathrm{um}$ & & & & 1 & & $1.0 \mathrm{~s}$ & 55 \\
\hline & $E$ & $15 \mathrm{~s}$ & & $6.00 \mathrm{um}$ & & & & I LSA & & 54.32 & 79 \\
\hline & & & & ePP & 52 & 28.00 & & 1 & & & \\
\hline FRU & & 37.04 & 69 & iPd & 51 & 05.80 & 1.5 & I KOD & & 55.24 & 108 \\
\hline & & $1.8 \mathrm{~s}$ & 198 & $0.00 \mathrm{~nm}$ & & & $6.6 \mathrm{mb}$ & I GTA & & 56.03 & 65 \\
\hline & E & $28 s$ & 208 & $8.00 \mathrm{um}$ & & & & 1 & & & \\
\hline & & & & iPP & 52 & 33.00 & & I FRB & & 56.25 & 328 \\
\hline & & & & eSs & 59 & 16.00 & & $1 \mathrm{NPA}$ & & 56.57 & 163 \\
\hline NRN & & 38.39 & 71 & IPd & 51 & 17.60 & 1.6 & 1 & & $1.0 \mathrm{~s}$ & 398 \\
\hline & & & & es & 57 & 09.00 & & 1 & & & \\
\hline AAA & & 38.60 & 68 & $e P$ & 51 & 19.50 & 2.1 & 1 & & & \\
\hline & & $8.0 \mathrm{~s}$ & & $1.50 \mathrm{~nm}$ & & & $3.6 \mathrm{mb} \times$ & | TET & & 56.59 & 170 \\
\hline & & & & IPP & 52 & 47.10 & & 1 & & $4.0 \mathrm{~s}$ & \\
\hline & & & & is & 57 & 17.90 & & I & & & \\
\hline & & & & ess & 59 & 55.90 & & I TIK & & 56.67 & 22 \\
\hline & & & & eSSS & 00 & 29.80 & & I & & $1.5 \mathrm{~s}$ & $104 E$ \\
\hline TLG & & 38.91 & 68 & iPd & 51 & 20.30 & 0.3 & I & Z & $16 \mathrm{~s}$ & 145 \\
\hline & & $1.8 \mathrm{~s}$ & 83 & $0.00 \mathrm{~nm}$ & & & $6.1 \mathrm{mb}$ & 1 & $\mathrm{~N}$ & $17 \mathrm{~s}$ & \\
\hline & & & & ePP & 52 & 54.00 & & 1 & $E$ & $16 \mathrm{~s}$ & 135 \\
\hline & & & & is & 57 & 21.00 & & 1 & & & \\
\hline KSH & & 38.94 & 74 & $P d$ & 51 & 21.00 & 0.6 & 1 & & & \\
\hline & & & & es & 57 & 25.00 & & 1 & & & \\
\hline SEM & & 39.55 & 56 & iPc & 51 & 26.70 & 1.5 & ? & & & \\
\hline & & $2.0 \mathrm{~s}$ & 1538 & $0.00 \mathrm{~nm}$ & & & $6.3 \mathrm{mb}$ & 1 & & & \\
\hline & & & & esp & 51 & 43.20 & & 1 & & & \\
\hline & & & & iPP & 53 & 05.60 & & 1 & & & \\
\hline & & & & es & 57 & 30.10 & & | KR I & & 56.85 & 174 \\
\hline DAG & & 41.16 & 346 & $I P C$ & 51 & 37.70 & -0.4 & 1 & & & \\
\hline & & $1.4 \mathrm{~s}$ & & $2.60 \mathrm{~nm}$ & & & $4.9 \mathrm{mb} \times$ & I BOD & & 56.88 & 40 \\
\hline & & & & $\mathrm{i}$ & 51 & 51.00 & & I & & $1.0 \mathrm{~s}$ & $19 e$ \\
\hline & & & & $\mathrm{i}$ & 57 & 29.00 & & I MTD & & 56.97 & 172 \\
\hline KHE & & 42.40 & 8 & $i P c$ & 51 & 50.00 & 1.8 & 1 & & & \\
\hline & & $2.0 \mathrm{~s}$ & 1908 & $0.00 \mathrm{~nm}$ & & & $6.5 \mathrm{mb}$ & I SHL & & 57.02 & 83 \\
\hline & & & & iPP & 53 & 22.00 & & 1 & & & \\
\hline & & & & is & 58 & 12.00 & & $\mathrm{SCH}$ & & 59.21 & 318 \\
\hline NAI & & 42.66 & 162 & $\mathrm{IPC}$ & 51 & 53.00 & 1.7 & 1 & & $1.3 \mathrm{~s}$ & 633 \\
\hline & & $2.0 s$ & 529 & $9.00 \mathrm{~nm}$ & & & $5.9 \mathrm{mb}$ & I BUL & & 60.07 & 176 \\
\hline $\mathrm{KIC}$ & & 42.76 & 226 & iP & 51 & 52.00 & 0. 1 & 1 & & $1.2 \mathrm{~s}$ & 334 \\
\hline$E L T$ & & 42.89 & 51 & iPd & 51 & 51.60 & -0.9 & $!$ & $z$ & $20 \mathrm{~s}$ & 118 \\
\hline & & $2.0 \mathrm{~s}$ & 1880 & $0.00 \mathrm{~nm}$ & & & $6.5 \mathrm{mb}$ & 1 & N & $20 \mathrm{~s}$ & 136 \\
\hline & & & & es & 58 & 16.00 & & 1 & $E$ & $20 \mathrm{~s}$ & 152 \\
\hline NR I & & 43.78 & & $i P c$ & 52 & 01.00 & 1.5 & 1 & & & \\
\hline & & $1.5 \mathrm{~s}$ & 320 & $0.00 \mathrm{~nm}$ & & & $5.9 \mathrm{mb}$ & 1 & & & \\
\hline & & & & ePP & 53 & 41.00 & & I LZH & & 60.42 & 66 \\
\hline & & & & IPCP & 53 & 52.00 & & 1 & & $8.5 \mathrm{~s}$ & 157 \\
\hline & & & & ess & 01 & 37.00 & & 1 & $\mathbf{N}$ & $15 \mathrm{~s}$ & 73 \\
\hline NDI & & 44.22 & & iPd & 52 & 03.20 & -0.4 & 1 & $E$ & $10 \mathrm{~s}$ & \\
\hline & & $0.7 \mathrm{~s}$ & 103 & $3.00 \mathrm{~nm}$ & & & $5.8 \mathrm{mb}$ & 1 & & & \\
\hline & $z$ & $20 \mathrm{~s}$ & 37 & $7.60 \mathrm{um}$ & & & $6.3 \mathrm{MsZ}$ & I & & & \\
\hline & $E$ & $22 \mathrm{~s}$ & & $7.40 \mathrm{um}$ & & & & I & & & \\
\hline & & & & iPP & 53 & 38.00 & & I & & & \\
\hline & & & & is & 58 & 34.50 & & I TUP & & 61.28 & 42 \\
\hline & & & & iss & 01 & 43.00 & & 1 & & $1.3 \mathrm{~s}$ & 240 \\
\hline POO & & 47.25 & 102 & iPd & 52 & 27.80 & 0.0 & $M B C$ & & 61.66 & 351 \\
\hline & & $1.3 \mathrm{~s}$ & 423 & $3.00 \mathrm{~nm}$ & & & $6.4 \mathrm{mb}$ & I YAK & & 61.80 & 31 \\
\hline ALE & & 50.10 & 350 & $e P$ & 52 & 42.10 & $-7.0 x$ & 1 & & $1.4 \mathrm{~s}$ & 490 \\
\hline & & $0.9 \mathrm{~s}$ & 130 & $0.00 \mathrm{~nm}$ & & & $5.9 \mathrm{mb}$ & 1 & $z$ & $17 \mathrm{~s}$ & \\
\hline DMN & & 50.79 & 85 & iPd & 52 & 54.80 & $-e 6$ & 1 & $N$ & $13 \mathrm{~s}$ & \\
\hline$K K N$ & & 50.83 & 84 & IPd & 52 & 54.80 & -0.8 & 1 & $\mathrm{E}$ & $11 \mathrm{~s}$ & \\
\hline & & $1.2 \mathrm{~s}$ & 1146 & $0.00 \mathrm{~nm}$ & & & $6.7 \mathrm{mb}$ & I & & & \\
\hline PKI & & 51.04 & & iPd & 52 & 56.40 & -1.0 & 1 & & & \\
\hline & & $1.2 \mathrm{~s}$ & 810 & $0.00 \mathrm{~nm}$ & & & $6.5 \mathrm{mb}$ & I & & & \\
\hline HYB & & 51.47 & 100 & ePd & 52 & 58.00 & -2.3 & I & & & \\
\hline & & $1.0 \mathrm{~s}$ & 280 & $0.00 \mathrm{~nm}$ & & & 6. $1 \mathrm{mb}$ & 1 & & & \\
\hline & & & & eS & 00 & 16.00 & & 1 & & & \\
\hline MOY & & 51.99 & 50 & EPC & 53 & 05.00 & 1.2 & ВTO & & 62.35 & 59 \\
\hline & & $1.7 \mathrm{~s}$ & 210 & $0.00 \mathrm{~nm}$ & & & $5.8 \mathrm{mb}$ & 1 & & & \\
\hline GBA & & 53.01 & 104 & & 53 & 11.00 & -0.9 & I AVY & & 62.54 & 155 \\
\hline & & $1.8 \mathrm{~s}$ & 393 & $3.00 \mathrm{~nm}$ & & 6 & $6.0 \mathrm{mb}$ & $1 \mathrm{CD} 2$ & & 63.03 & 71 \\
\hline I RK & & 53.73 & 49 & $\mathrm{iPc}$ & 53 & 16.00 & -0.8 & 1 & & & \\
\hline & & $1.8 \mathrm{~s}$ & 420 & $0.00 \mathrm{~nm}$ & & & $6.1 \mathrm{mb}$ & I HHC & & 63.24 & 58 \\
\hline & $z$ & $20 s$ & & $7.70 \mathrm{um}$ & & & $6.9 \mathrm{Msz}$ & I XAN & & 65.04 & 66 \\
\hline & N & $16 s$ & & $5.60 \mathrm{um}$ & & & & I KMI & & 65.46 & 77 \\
\hline & $E$ & $22 s$ & & 1.70 um & & & & 1 & $E$ & $20 s$ & \\
\hline & & & & esp & 53 & 32.00 & & 1 & & & \\
\hline & & & & EPPP & 56 & 30.00 & & 1 & & & \\
\hline & & & & es & 00 & 50.00 & & & & & \\
\hline ZAK & & 53.81 & 51 & $\mathrm{IPC}$ & 53 & 18.00 & 0.7 & & & & \\
\hline
\end{tabular}




\begin{tabular}{|c|c|c|c|c|c|c|c|c|}
\hline$Y K C$ & & 72.61 & 341 & eP & 55 & 21.00 & -0.6 & I \\
\hline YKA & & 72.63 & 342 & eP & 55 & 25.30 & $3.6 x$ & i \\
\hline RSNT & & 72.64 & 342 & $i P c$ & 55 & 22.70 & 1.0 & ANP \\
\hline \multirow[t]{2}{*}{ NJ2 } & & 73.02 & 62 & $\mathrm{Pd}$ & 55 & 23.00 & -1.4 & \\
\hline & & & & 5 & 04 & 51.00 & & SVW \\
\hline \multirow[t]{3}{*}{ GRM } & & 73.10 & 178 & $i P c$ & 55 & 27.00 & 2.3 & SAP \\
\hline & & $0.9 \mathrm{~s}$ & 437 & $7.00 \mathrm{~nm}$ & & & $.5 \mathrm{mb}$ & \\
\hline & z & $20 \mathrm{~s}$ & 56 & $5.70 \mathrm{um}$ & & 6 & $.8 \mathrm{MSZ}$ & $\mid$ PP I \\
\hline CAI & & 73.33 & 246 & ePd & 55 & 20.50 & $-6.0 x$ & PCA \\
\hline$E L F$ & & 73.56 & 313 & $\mathbf{P}$ & 55 & 30.25 & 2.8 & SHNJ \\
\hline LON & & 73.59 & 313 & $P$ & 55 & 30.25 & 2.6 & NGS \\
\hline DLA & & 73.92 & 313 & $P$ & 55 & 31.50 & 1.9 & i \\
\hline IMA & & 74.13 & 359 & (P) & 55 & 31.80 & 1.3 & I KGM \\
\hline VLA & & 74.25 & 47 & $i \mathrm{Pd}$ & 55 & 30.00 & -1.4 & SKR \\
\hline & & & & ePP & 58 & 17.00 & & I \\
\hline & & & & ePPP & $\theta 0$ & 07.00 & & \\
\hline & & & & is & 85 & 03.00 & & \\
\hline & & & & iss & 89 & 47.00 & & \\
\hline LHC & & 74.27 & 321 & $e^{P}$ & 55 & 35.00 & $3.5 x$ & i \\
\hline GZH & & 74.50 & 73 & $\mathrm{Pc}$ & 55 & 33.00 & -0.1 & 1 \\
\hline & & & & PPP & 00 & 13.00 & & EDM \\
\hline & & & & $s$ & 05 & 08.00 & & AOMJ \\
\hline RSON & & 74.62 & 325 & eP & 55 & 34.60 & 1.2 & KUR \\
\hline SNG & & $\begin{array}{l}74.63 \\
1.5 \mathrm{~s}\end{array}$ & $\begin{array}{l}94 \\
722\end{array}$ & $\begin{array}{l}\text { iPd- } \\
2.00 \mathrm{~nm}\end{array}$ & 55 & $28.00_{6}$ & $\begin{array}{l}-6.0 \mathrm{x} \\
.5 \mathrm{mb}\end{array}$ & \\
\hline & & & & is & 04 & 47.00 & & | \\
\hline MRG & & 75.06 & 309 & iPd & 55 & 39.30 & $3.1 x$ & i \\
\hline & & & & e & 05 & 25.00 & & \\
\hline FFC & & 75.10 & 331 & ep & 55 & 36.00 & -0.2 & RSCP \\
\hline & & $1.0 \mathrm{~s}$ & 126 & $6.00 \mathrm{~nm}$ & & & $.9 \mathrm{mb}$ & HJH \\
\hline $\mathrm{COL}$ & & 75.14 & 357 & eP & 55 & 37.00 & 0.8 & KAGJ \\
\hline & & & & is & 05 & 18.00 & & TKS J \\
\hline F8A & & 75.14 & 357 & $P$ & 55 & 36.90 & 0.7 & SKH \\
\hline SSE & & 75.18 & 62 & iPct & 55 & 36.00 & -1.0 & I TSRJ \\
\hline & & $8.0 \mathrm{~s}$ & & $4.80 \mathrm{~nm}$ & & & $.6 \mathrm{mb} \times$ & SIT \\
\hline & 2 & $16 \mathrm{~s}$ & & $5.50 \mathrm{um}$ & & & $.9 M s z X$ & SES \\
\hline & $\mathrm{N}$ & $13 \mathrm{~s}$ & & $0.80 \mathrm{um}$ & & & & 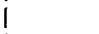 \\
\hline & $E$ & $14 \mathrm{~s}$ & & $6.00 \mathrm{um}$ & & & & MTMJ \\
\hline & & & & ePP & 58 & 26.00 & & OSK \\
\hline & & & & ePPP & 00 & 14.00 & & \\
\hline & & & & $s$ & 05 & 12.00 & & MIY \\
\hline & & & & $\mathrm{i}$ & 05 & 18.00 & & \\
\hline ANM & & 75.34 & 4 & $P$ & 55 & 40.00 & 2.7 & $M I Y$ \\
\hline TSI & & 75.41 & 98 & ePd & 55 & 39.50 & 1.0 & I YAMJ \\
\hline AAM & & 75.46 & 313 & eP & 55 & 41.20 & $2.8 x$ & I FVM \\
\hline & & & & $\mathrm{s}$ & 05 & 21.20 & & \\
\hline ITR & & 75.80 & 245 & eP & 55 & 40.10 & -0.6 & $\mid N \| 1 J$ \\
\hline & & & & $\mathrm{i}$ & 56 & 04.50 & & YAM \\
\hline & & & & $i$ & 58 & 37.00 & & I MAT \\
\hline ITO & & 75.83 & 313 & ePd & 55 & 44.00 & $3.5 x$ & \\
\hline PSI & & 76.20 & 98 & ePc & 55 & 41.50 & -1.5 & 1 \\
\hline & & $1.2 \mathrm{~s}$ & 192 & $2.00 \mathrm{~nm}$ & & & $1 \mathrm{mb}$ & \\
\hline IPM & & 76.59 & 95 & ePd & 55 & 44.90 & -0.4 & WKYJ \\
\hline & & $1.4 \mathrm{~s}$ & & $5.00 \mathrm{~nm}$ & & & $.6 \mathrm{mb}$ & OFUJ \\
\hline & & & & e & 56 & 17.80 & & KDC \\
\hline & & & & e & 58 & 22.30 & & PIP \\
\hline PA & & 76.65 & 281 & eP & 55 & 46.00 & 0.4 & $110 \mathrm{~J}$ \\
\hline SEG & & 76.79 & 280 & eP & 55 & 49.00 & 2.8 & $\mathrm{CH} J J$ \\
\hline$B \subset A$ & & 76.90 & 308 & iP- & 55 & 49.60 & $2.9 x$ & DDR \\
\hline & $z$ & $22 \mathrm{~s}$ & & $7.60 \mathrm{um}$ & & &.$\theta \mathrm{Msz}$ & $\mathrm{SHZ}$ \\
\hline & & & & is & 05 & 37.60 & & ONA \\
\hline GG & & 76.94 & 280 & eP & 55 & 50.50 & $3.4 x$ & SRY \\
\hline PAG & & 77.15 & 280 & eP & 55 & 52.50 & $4.2 x$ & I TSK \\
\hline $\mathrm{OZH}$ & & 77.16 & 68 & $P c$ & 55 & 48.00 & -0.2 & I KAKJ \\
\hline & & & & s & 85 & 32.00 & & OYM \\
\hline rSS & & 77.18 & 38 & $i P c$ & 55 & 50.20 & 2.2 & TOK \\
\hline & & 1.0 & 248 & $0.00 \mathrm{~nm}$ & & & $2 \mathrm{mb}$ & AJI \\
\hline & & & & IP CP & 55 & 56.80 & & KT J \\
\hline & & & & ePP & 58 & 44.00 & & $B A G$ \\
\hline & & & & iSKS & 05 & 48.00 & & \\
\hline & & & & ESPP & 06 & 23.00 & & CVP \\
\hline$T T$ & & 77.29 & $\theta$ & $P$ & 55 & 49.60 & 1.3 & 1 \\
\hline MDN & & 77.38 & 279 & eP & 55 & 54.50 & $5.8 x$ & RSSD \\
\hline WAK & & 77.82 & 40 & eP & 55 & 52.00 & 0.5 & KYS \\
\hline & & & & 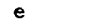 & 05 & 46.00 & & RXF \\
\hline TOA & & 77.85 & 356 & $\mathbf{P}$ & 55 & 53.50 & 2.0 & CAR \\
\hline SOB 1 & & 77.85 & 247 & eP & 55 & 51.90 & -0.2 & YKM \\
\hline & & & & $i$ & 58 & 42.70 & & SON \\
\hline & & & & $\mathrm{i}$ & 58 & 52.80 & & CLX \\
\hline AE & & 78.47 & 357 & eP & 55 & 55.50 & 0.7 & LHD \\
\hline PMR & & 78. & 357 & $\mathbf{P}$ & 55 & 55.40 & 0.4 & MVI \\
\hline & z & $20 \mathrm{~s}$ & 46 & $6.50 \mathrm{um}$ & & & $8 \mathrm{Msz}$ & \\
\hline ET & & 79.04 & 26 & $i P c$ & 55 & 57.00 & -1.0 & MAN \\
\hline & & $1.8 \mathrm{~s}$ & 420 & $0.00 \mathrm{~nm}$ & & & $.2 \mathrm{mb}$ & OCP \\
\hline & & & & ePCF & 56 & 03.00 & & PNT \\
\hline & & & & ePP & 59 & 00.00 & & \\
\hline & & & & ePPF & 00 & 48.00 & & NEW \\
\hline
\end{tabular}

\begin{tabular}{|c|c|c|c|c|c|c|c|c|c|c|c|}
\hline & & es & 05 & 55.00 & & 1 & & & & 57 & \\
\hline & & ePS & 06 & 40.00 & & I RLO & 86.02 & 315 & eP & 56 & 34.70 \\
\hline 79.08 & 66 & iPt & 56 & 03.00 & $4.1 x$ & I LRM & 86.23 & 332 & ePc & 56 & 36.80 \\
\hline & & is & 05 & 59.06 & & PHC & 86.31 & 343 & eP & 56 & 39.00 \\
\hline 79.12 & $\theta$ & $P$ & 55 & 59.80 & 1.4 & I GBO & 86.34 & 315 & eP & 56 & \\
\hline 79.34 & 42 & eP & 56 & 02.00 & 2.1 & I ADK & 86.48 & 13 & $e(P)$ & 56 & 43.50 \\
\hline & & es & 05 & 57.00 & & I NKI & 86.56 & 8 & (P) & 6 & 37.40 \\
\hline 79.36 & 100 & eP & 56 & 00.00 & -0.4 & I TUL & 86.63 & 316 & eP & 56 & 38.50 \\
\hline 79.37 & 352 & (P) & 56 & 02.40 & 2.6 & i & $1.0 \mathrm{~s}$ & 236 & $6.00 \mathrm{~nm}$ & & \\
\hline 79.69 & 54 & P & 56 & 04.00 & 2.1 & i & $19 \mathrm{~s}$ & & $2.70 \mathrm{um}$ & & \\
\hline 79.83 & 56 & $e^{P}$ & 56 & 06.00 & $3.3 x$ & i & & & e & 56 & 54.80 \\
\hline & & es & 06 & 16.00 & & I PGC & 87.22 & 340 & eP & 56 & 43.70 \\
\hline 9.98 & 96 & ePc & 56 & 03.40 & -0.4 & I BOW & 87.70 & 328 & eP & 6 & 43.00 \\
\hline 80.06 & 29 & $e^{P}$ & 56 & 04.60 & 1.0 & $\triangle A C O$ & 87.79 & 318 & $e(P)$ & 6 & 44.00 \\
\hline $6.0 \mathrm{~s}$ & 2000 & $0.00 \mathrm{~nm}$ & & & $.3 \mathrm{mb} \times$ & & 1.35 & 88 & $8.60 \mathrm{~nm}$ & & \\
\hline $18 \mathrm{~s}$ & 75 & $5.60 \mathrm{um}$ & & 7 & $.1 M S Z$ & $10 c 0$ & 87.82 & 316 & ePd & 56 & 47.20 \\
\hline $18 \mathrm{~s}$ & 64 & $4.80 \mathrm{um}$ & & & & ATO & 87.95 & 317 & $e(P)$ & 56 & 47.30 \\
\hline $18 \mathrm{~s}$ & 97 & $.00 \mathrm{um}$ & & & & I GLD & 88.32 & 324 & $e(P)$ & 56 & 47.00 \\
\hline & & iPP & 59 & 09.80 & & $i$ & $1.5 \mathrm{~s}$ & 259 & $9.00 \mathrm{~nm}$ & & \\
\hline & & is & 06 & 06.00 & & $i$ & $20 \mathrm{~s}$ & 150 & $0.00 \mathrm{um}$ & & \\
\hline 80.21 & 336 & ePc & 56 & 05.00 & 0.5 & LGP & 88.38 & 74 & iP & 56 & 45.00 \\
\hline 80.62 & 44 & $\mathbf{P}$ & 56 & 08.00 & 1.2 & SOV & .41 & 280 & eP & 6 & 49.00 \\
\hline 80.91 & 37 & iPc & 56 & 08.00 & -0.2 & I GOL & 88.43 & 324 & ePt & 56 & 46.80 \\
\hline $1.2 \mathrm{~s}$ & 480 & $0.00 \mathrm{~nm}$ & & & $.4 \mathrm{mb}$ & 1 & $1.3 \mathrm{~s}$ & 70 & $0.80 \mathrm{~nm}$ & & \\
\hline & & ePP & 59 & 19.00 & & LEM & 88.95 & 100 & eP & 56 & 50.00 \\
\hline & & is & 06 & 17.00 & & $i$ & $1.8 \mathrm{~s}$ & 364 & 4.00 & & 6 \\
\hline & & ePPS & 07 & 25.00 & & $i$ & & & es & 07 & 24.00 \\
\hline & & ess & 11 & 24.00 & & I UAV & 3.97 & 280 & eP & 56 & 54.20 \\
\hline 07 & 309 & ePd- & 56 & 13.00 & $3.7 x$ & RDJ & 9.02 & 239 & eP & 6 & 40 \\
\hline 81 & 45 & P & 56 & 10.70 & -0.1 & BMA & 49 & 239 & eP & 6 & 53.80 \\
\hline 81.50 & 56 & $\mathbf{P}$ & 56 & 11.00 & -0.6 & $i$ & & & e & 6 & 58.20 \\
\hline 81.59 & 52 & $P$ & 56 & 12.50 & 0 & $i$ & & & $\mathrm{i}$ & 7 & 30 \\
\hline 81.62 & 46 & $P$ & 56 & 16.00 & & NP & 9.49 & 74 & ePd & 6 & \\
\hline 81.68 & 50 & $\mathbf{P}$ & 56 & 11.00 & -1 & PLP & 60 & 75 & $e^{P}$ & 7 & 80 \\
\hline 81.73 & 349 & $P$ & 56 & 14.40 & & COR & 90 & 338 & eP & 7 & \\
\hline 81.85 & 333 & ePc & 56 & 14.00 & 0.8 & I HKT & 1.11 & 312 & eP & 7 & 03.00 \\
\hline $1.2 \mathrm{~s}$ & 353 & $.00 \mathrm{~nm}$ & & & $.3 m b$ & $i$ & & & es & $\theta$ & 49.00 \\
\hline 82.01 & 48 & $\mathbf{P}$ & 56 & 14.30 & 0.0 & I VAO & 1.57 & 241 & eP & 57 & 01.90 \\
\hline 82.13 & 51 & eP & 56 & 18.00 & $3.2)$ & $i$ & & & e & 7 & 30 \\
\hline & & s & 06 & 39.50 & & 1 & & & e & 7 & \\
\hline 82.13 & 44 & $e^{P}$ & 56 & 22.00 & $7.3 x$ & i LUB & 91.61 & 318 & eP & 7 & 00 \\
\hline & & es & 06 & 28.00 & & ATX & 91.99 & 313 & eP & 7 & \\
\hline 3 & 44 & $P$ & 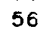 & 15.00 & 0 . & I ALO & 92.79 & 322 & eP & 7 & 06.00 \\
\hline 82 & 46 & $\mathbf{P}$ & 56 & 15.30 & 0. & $i$ & $1.0 \mathrm{~s}$ & 37 & $7.50 \mathrm{~nm}$ & & \\
\hline 82.18 & 314 & ePc & 56 & 15.70 & 0.7 & $i$ & $18 \mathrm{~s}$ & 85 & $5.90 \mathrm{um}$ & & \\
\hline $2.0 \mathrm{~s}$ & 774 & $1.00 \mathrm{~nm}$ & & & $.5 \mathrm{mb}$ & : & & & e & 7 & 00 \\
\hline 82.23 & 47 & $P$ & 56 & 15.00 & -0.2 & JCT & 92.93 & 315 & eP & 7 & 05.20 \\
\hline 82.26 & 46 & $\mathbf{P}$ & 56 & 18.80 & & $i$ & & 94 & 4.96 & & \\
\hline 82.27 & 48 & $e^{P}$ & 56 & 15.00 & -0.5 & $i$ & $22 \mathrm{~s}$ & 100 & 0.004 & & \\
\hline $1.4 \mathrm{~s}$ & 76 & $0.70 n$ & & 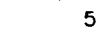 & $.6 \mathrm{mb}$ & 1 & & & es & 08 & 20.00 \\
\hline $21 \mathrm{~s}$ & 36 & $5.90 \mathrm{um}$ & & 6 & $.7 \mathrm{MSZ}$ & EUR & 93.01 & 331 & iP & 7 & 11.00 \\
\hline & & es & 06 & 30.00 & & $i$ & $1.0 \mathrm{~s}$ & 38 & $8.10 r$ & & \\
\hline 82.36 & 51 & $P$ & 56 & 18.20 & 2. & DAV & 93.70 & 78 & eP & 7 & 10.00 \\
\hline 82.38 & 44 & $\mathbf{P}$ & & 17.60 & 1 & $1 B O G$ & 75 & 279 & eP & 7 & 50 \\
\hline 82 & 358 & $\mathbf{P}$ & $\hat{\hat{o}}$ & 18.10 & 2.1 & 1 & & & es & 7 & 00 \\
\hline 82.77 & 72 & ePd & 5 & 08.50 & $-9.8 x$ & MI & 94 & 335 & $i P c$ & 7 & 70 \\
\hline & 49 & $P$ & & $\theta$ & & WDC & & 336 & eP & 7 & \\
\hline & 48 & $P$ & 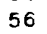 & 19.40 & $-\theta$ & & & & ePP & 01 & 13.00 \\
\hline 83 & 48 & eP & & 21.80 & & 1 & & 337 & eP & 7 & 70 \\
\hline & 49 & $\mathbf{P}$ & s & 24.00 & 2 & $\mathrm{CH}$ & 94.70 & 280 & iP & t & 22.00 \\
\hline 83.50 & 46 & P & & 29.00 & & I MN & & 332 & eP & & 80 \\
\hline & 48 & ep & 3 & 23.00 & 0. & JAS & 95.99 & 333 & iPc & 7 & 25.40 \\
\hline 83 & 47 & eP & & 21.20 & -1 & 1 & & & ePP & 1 & 22.00 \\
\hline 83.63 & 47 & $\mathbf{P}$ & 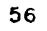 & 22.00 & -0 & $F R$ & & 332 & eP & 7 & 27.90 \\
\hline 83.66 & 48 & eP & 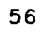 & 23.40 & & BKS & & 334 & eP & 57 & 29.00 \\
\hline & 48 & eP & & 28.00 & & - & 3. & 1160 & 0.08 & & \\
\hline & 49 & $P$ & & 24.00 & 0 & & 212 & & 8.0 & & \\
\hline 83 & 49 & P & & 24.80 & & & & 102 & & & \\
\hline 83. & 74 & eP & 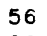 & 24.40 & $-\theta$ & & $2 E$ & & $7.00 \mathrm{u}$ & & \\
\hline & & es & 06 & 46.00 & & & & & ePP & 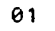 & \\
\hline & 72 & ePd & 56 & .20 & -0.6 & & & & es & 08 & \\
\hline & 307 & 7.00 & & & $.4 m$ & & & & ePS & 10 & 22.00 \\
\hline & 326 & eP & 56 & .50 & & & & & . & 12 & \\
\hline & 48 & $e^{P}$ & & 27.30 & & & & & ess & 15 & 00 \\
\hline & 335 & iP- & 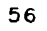 & 30.70 & & i & & & esss & 18 & \\
\hline & 279 & eP & ; & 53.00 & 25. & MH & & 334 & eP & 7 & \\
\hline & 335 & iP- & j & 31.50 & 3. & PR & & 332 & eP & & \\
\hline & 3 & $e^{P}$ & 5 & 32.00 & 4.5 & GLA & 98.14 & 327 & $e(P)$ & 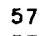 & 00 \\
\hline & 334 & ip & 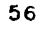 & 34.00 & & 1 & & & 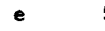 & 57 & \\
\hline & 335 & iP- & 56 & 32.70 & & PSO & & 279 & eP & . & 50 \\
\hline & 60 & ep & 56 & 35.00 & & PAS & & 330 & eP & 57 & 38.00 \\
\hline & & es & 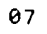 & 05.00 & & & 1.0 & 88 & & & \\
\hline & 75 & $e^{P}$ & & 34.00 & & & & & ePP & 01 & \\
\hline & 75 & eP & 56 & 40.00 & & & & & & 19 & \\
\hline & 337 & ep & 56 & 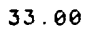 & 1 & LPB & 102.58 & 259 & Pdif & 7 & 00 \\
\hline & 195 & & & & & & & & & 28 & 00 \\
\hline & & et & 56 & 80 & 1.2 & MO & 98 & & & & 57.00 \\
\hline
\end{tabular}


$06015 \mathrm{~h}$

\begin{tabular}{|c|c|c|c|c|c|c|c|c|c|c|c|c|}
\hline PJG & 102.98 & 60 & e (Pdif & $f 57$ & 53.00 & 0.2 & 1 & $17 \mathrm{~s}$ & 170 & 0.00 um & & \\
\hline YJA & 104.10 & 253 & e (Pdif & $f 58$ & 03.00 & $4.7 x$ & i & $17 \mathrm{~s}$ & 250 & .00 um & & \\
\hline ARE & 105.09 & 261 & $e$ (Pdif & $f 58$ & 07.00 & $4.4 x$ & & $17 \mathrm{~s}$ & 230 & 0.00 um & & \\
\hline LPA & 106.57 & 238 & EPKP- & 02 & 36.00 & $16.8 x$ & I & & & es & 02 & 04.00 \\
\hline & $19 \mathrm{~s}$ & 31 & $1.90 \mathrm{um}$ & & & $.9 \mathrm{Msz}$ & I ILT & 15.22 & 30 & IPC & 59 & 30.00 \\
\hline ANT & 108.64 & 254 & $e(P K P)$ & 02 & 55.00 & $31.6 x$ & i & $1.2 \mathrm{~s}$ & 5600 & $0.00 \mathrm{~nm}$ & & \\
\hline MAW & 111.22 & 165 & ePKP & 02 & 29.00 & 2.0 & $A B J$ & 16.08 & 230 & eP & 59 & 41.00 \\
\hline WB2 & 117.39 & 94 & ePKP & 02 & 38.90 & -1.4 & I & & & $S$ & 03 & 06.90 \\
\hline & & & e & 02 & 46.70 & & NEM & 16.10 & 225 & $\mathbf{P}$ & 59 & 36.00 \\
\hline & & & e & 03 & 49.80 & & I WAK & 16.19 & 238 & eP & 59 & 45.00 \\
\hline & & & e & 13 & 11.30 & & 1 & & & es & 03 & 13.00 \\
\hline & & & e & 17 & 07.00 & & KUSJ & 16.65 & 227 & $P$ & 59 & 39.00 \\
\hline ASPA & 119.16 & 97 & ePKP & 02 & 44.00 & 0.4 & I ASAJ & 16.72 & 233 & $P$ & 59 & 47.50 \\
\hline ISO & 121.82 & 91 & ePKP & 02 & 48.00 & -0.7 & I KUS & 16.88 & 227 & $e P$ & 59 & 45.00 \\
\hline CTA & 126.59 & 86 & IPKP & 03 & 00.70 & $2.7 x$ & $i$ & & & es & 02 & 52.00 \\
\hline & $1.1 \mathrm{~s}$ & 101 & $1.00 \mathrm{~nm}$ & & & & I ASA & 17.11 & 233 & eP & 59 & 52.00 \\
\hline & & & $\mathrm{i}$ & 05 & 00.00 & & 1 & & & e & 80 & 09.50 \\
\hline & & & $\mathrm{i}$ & 05 & 30.00 & & I YAK & 17.30 & 304 & iPc & 59 & 54.10 \\
\hline$A D E$ & 128.48 & 107 & ePKP & 03 & 03.80 & $2.6 x$ & 1 & $1.6 \mathrm{~s}$ & $* * * *$ & $* * * * n m$ & & \\
\hline SPA & 129.94 & 180 & ePKPC & 03 & 04.30 & 1.2 & i & & & is & 03 & 10.90 \\
\hline & $1.2 \mathrm{~s}$ & 164 & $4.00 \mathrm{~nm}$ & & & & I RMJ & 17.31 & 235 & eP & 59 & 54.00 \\
\hline & $20 \mathrm{~s}$ & 58 & $8.30 \mathrm{um}$ & & & $.3 M s z$ & 1 & & & es & 03 & 15.00 \\
\hline & & & $\boldsymbol{e}$ & 05 & 03.00 & & IOB & 17.42 & 230 & $e^{P}$ & 59 & 56.00 \\
\hline CMS & 132.23 & 99 & ePKP & 03 & 10.00 & 1.6 & | NK | & 17.55 & 87 & $I P C$ & 59 & 57.70 \\
\hline 700 & 134.54 & 107 & ePKP & 03 & 08.00 & $-4.6 x$ & I HOOJ & 17.83 & 228 & $\mathbf{P}$ & 59 & 59.00 \\
\hline YOU & 135.38 & 101 & ePKP & 03 & 10.80 & $-3.5 x$ & I SAP & 18.14 & 233 & eP & 80 & 02.00 \\
\hline & & & $i$ & 03 & 17.50 & & i & & & e & 80 & 20.00 \\
\hline DRV & 136.00 & 149 & iPKP & 03 & 15.50 & 1.1 & I URA & 18.23 & 229 & eP & 00 & 05.00 \\
\hline CAN & 136.27 & 102 & iPKPC & 03 & 17.50 & 1.5 & 1 & & & e & 03 & 20.00 \\
\hline & & & $i$ & 03 & 22.00 & & ANM & 18.50 & 48 & eP & $B O$ & 0 \\
\hline WAM & 136.53 & 103 & ePKP & 03 & 12.70 & $-3.7 x$ & I MRRJ & 18.76 & 233 & $P$ & 00 & 12.80 \\
\hline & & & $\epsilon$ & 03 & 22.90 & & I SUT & 18.85 & 235 & eP & 00 & 10.00 \\
\hline SBA & 139.07 & 169 & iPKP & 03 & 20.90 & 1.0 & 1 & & & $\epsilon$ & 00 & 29.00 \\
\hline & & & is & 06 & 17.60 & & I HAK & 19.39 & 232 & $P$ & 80 & 17.00 \\
\hline KOU & 140.44 & 73 & IPKPC & 03 & 34.90 & $10.9 x$ & I HAC & 20.11 & 229 & eP & 00 & 25.00 \\
\hline NOU & 143.10 & 73 & IPKPC & 03 & 15.80 & $-12.8 x$ & 1 & & & $s$ & 04 & 11.70 \\
\hline$M C O$ & 146.45 & 131 & ePKP & 03 & 38.00 & $4.7 x$ & I AOMJ & 20.57 & 231 & $P$ & 00 & 29.00 \\
\hline NDF & 147.57 & 55 & ePKP & 03 & 42.50 & $6.4 x$ & I MIY & 20.66 & 226 & $\mathbf{P}$ & 00 & 32.00 \\
\hline SVA & 148.47 & 54 & ePKP & 03 & 43.00 & $5.4 x$ & I TIK & 20.91 & 332 & ePC & 00 & 35.00 \\
\hline MSZ & 152.60 & 112 & PKP & 03 & 51.70 & $8.7 x$ & i & $0.9 \mathrm{~s}$ & 1800 & $0.00 \mathrm{~nm}$ & & \\
\hline CRZ & 153.97 & 88 & PKP & 04 & 14.90 & $29.6 x$ & i & $15 \mathrm{~s}$ & 38 & $3.00 \mathrm{um}$ & & \\
\hline PPN & 156.92 & 346 & ePKP & 03 & 58.00 & $8.4 x$ & i & $14 \mathrm{~s}$ & 128 & $3.00 \mathrm{um}$ & & \\
\hline & 1.35 & & $0.00 \mathrm{~nm}$ & & & & 1 & $16 s$ & 247 & $.00 \mathrm{um}$ & & \\
\hline TVO & 157.13 & 345 & ePKP & 83 & 56.00 & $6.0 x$ & 1 & & & ePP & 01 & 08.00 \\
\hline & $1.3 \mathrm{~s}$ & 185 & $5.00 \mathrm{~nm}$ & & & & 1 & & & es & 84 & 23.00 \\
\hline KRP & 157.26 & 94 & ePKP & 04 & 07.00 & $17.5 x$ & 1 & & & ePCP & 04 & 42.00 \\
\hline & & & $(P \subset P)$ & 04 & 24.00 & & i & & & eSS & 04 & 51.00 \\
\hline GNZ & 159.33 & 95 & ePKP & 64 & 00.00 & $8.2 x$ & I MRK & 20.95 & 228 & eP & 00 & 34.00 \\
\hline & S.O. & 3 & on 385 & 5 of & f $492 \circ$ & bs. & i & & & eS & 04 & 19.00 \\
\hline & & & & & & & I OFU & 21.26 & 226 & ep & 00 & 38.00 \\
\hline$A U$ & 17, & 1983 & $10 h 5$ & $55 \mathrm{~m}$ & $54.53 \pm$ & $0.45 \mathrm{~s}$ & 1 & & & es & 84 & 20.00 \\
\hline 55 & $.851 \mathrm{~N}=$ & \pm 1.5 & $5 \mathrm{~km} \quad 16$ & 61.2 & $297 \mathrm{E} \pm$ & $1.4 \mathrm{~km}$ & I OFUJ & 21.27 & 226 & $P$ & 00 & 38.00 \\
\hline$D E$ & PTH = & 66.3 & $\pm \quad 4.1$ & $1 \mathrm{~km}$ & & & $|A K|$ & 21.39 & 230 & eP & 00 & 39.00 \\
\hline & $6 \mathrm{mb}(14$ & o obs & s.) & & & & 1 & & & es & 05 & 06.00 \\
\hline NEAR & EAST $C$ & OAST & OF KAM & $\mathrm{MCHA}$ & ATKA & $(218)$ & I SDN & 21.42 & 75 & $\mathbf{P}$ & $\theta \theta$ & 36.90 \\
\hline & $\mathrm{Felt}$ ( & $(v-v)$ & ) ot $\mathrm{Pe}$ & etro & oparlov & sk- & I HJH & 21.67 & 229 & $P$ & $B 0$ & 41.00 \\
\hline & Kameho & tskiy & & & & & I ISN & 21.97 & 226 & $P$ & 00 & 44.00 \\
\hline & & & & & & & I SEN & 22.28 & 226 & $P$ & 00 & 50.00 \\
\hline PET & 3.23 & 210 & iPn & 56 & 45.00 & 1.1 & I & & & es & 04 & 46.00 \\
\hline & & & $E S n$ & 57 & 20.00 & & I TTA & 22.48 & 54 & iPc & 00 & 50.10 \\
\hline SKR & 6.06 & 213 & $e^{P n}$ & 57 & 22.60 & -0.9 & I YAM & 22.51 & 227 & $P$ & $\theta 0$ & 50.00 \\
\hline & $2.0 \mathrm{~s}$ & $* * * *$ & $* * * * \mathrm{~nm}$ & & & $.7 \mathrm{mb} x$ & YAMJ & 22.71 & 228 & $P$ & 00 & 52.00 \\
\hline & $24 \mathrm{~s}$ & 1192 & 2. $00 \mathrm{um}$ & & & & SVW & 22.79 & 59 & $P$ & 00 & 53.60 \\
\hline MGD & 7.02 & 312 & ipnd & 57 & 41.30 & $4.5 x$ & I FKS & 22.88 & 226 & eP & 00 & 55.00 \\
\hline & $20 \mathrm{~s}$ & 794 & 4.00 um & & & & 1 & & & e & 05 & 40.00 \\
\hline & 145 & 418 & $8.00 u m$ & & & & I MDJ & 22.97 & 254 & $\mathrm{PC}$ & 00 & 54.00 \\
\hline & $17 \mathrm{~s}$ & 390 & 0.00 um & & & & 1 & & & sP & 01 & 15.00 \\
\hline SMY & 8.12 & 107 & eP & 57 & 49.90 & -2.1 & ONA & 23.41 & 225 & $P$ & 01 & 00.00 \\
\hline SEY & 8.40 & 331 & iPnd & 58 & 01.80 & $6.0 x$ & I TUP & 23.46 & 284 & eP & 00 & 59.20 \\
\hline & $15 \mathrm{~s}$ & 655 & $5.00 \mathrm{um}$ & & & & 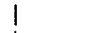 & $1.1 \mathrm{~s}$ & 910 & $0.00 \mathrm{~nm}$ & & \\
\hline & $14 \mathrm{~s}$ & 553 & 3.00 um & & & & 1 & & & epP & 01 & 21.00 \\
\hline & $16 \mathrm{~s}$ & 414 & $4.00 \mathrm{um}$ & & & & SHR & 23.53 & 226 & $P$ & 01 & .00 \\
\hline NKL & 12.25 & 266 & iPnd & 58 & 55.00 & $7.1 x$ & I IMA & 23.57 & 46 & $\mathbf{P}$ & 01 & 00.60 \\
\hline & 8.05 & $* * * *$ & $* * * * n m$ & & & $.2 \mathrm{mb} \times$ & $|A| K$ & 23.60 & 231 & $\mathbf{P}$ & 01 & 00.00 \\
\hline UGL & 13.50 & 248 & iPnc & 59 & 10.80 & $6.4 x$ & $|\mathrm{~N}| \mid J$ & 23.93 & 228 & $\mathbf{P}$ & 01 & 01.80 \\
\hline & 8.05 & 30 & $0.70 \mathrm{~nm}$ & & & $\theta m b x$ & I MIT & 24.07 & 225 & eP & 01 & 00 \\
\hline ADK & 13.57 & 98 & $P$ & 59 & 04.00 & -1.2 & I & & & es & 05 & 28.00 \\
\hline KUR & 13.61 & 224 & i Pnd & 59 & 06.50 & 0.8 & I UTS & 24.17 & 226 & $P$ & 01 & 05.30 \\
\hline & $1.2 \mathrm{~s}$ & 6840 & $0.00 \mathrm{~nm}$ & & & $.1 \mathrm{mb}$ & I KAKJ & 24.34 & 225 & $P$ & 01 & 05.00 \\
\hline YSS & 14.54 & 240 & iPC & 59 & 24.00 & $6.1 x$ & I TSK & 24.36 & 225 & eP & 01 & 05.50 \\
\hline & $1.0 \mathrm{~s}$ & 840 & $0.00 \mathrm{~nm}$ & & & $.0 \mathrm{mb}$ & I TKD & 24.37 & 229 & ePd & 01 & 10.00 \\
\hline & $14 \mathrm{~s}$ & 381 & 1.00 um & & & & i & & & i & 01 & 29.00 \\
\hline & $15 \mathrm{~s}$ & 264 & 4.00 um & & & & I RDT & 24.42 & 60 & $P$ & 01 & 08.60 \\
\hline & $16 \mathrm{~s}$ & 161 & $1.00 \mathrm{um}$ & & & & $\mathrm{I} \mathrm{CHO}$ & 24.50 & 223 & $\mathbf{P}$ & 01 & 10.00 \\
\hline & & & is & 82 & 04.00 & & MAE & 24.62 & 227 & eP & 01 & 12.00 \\
\hline SHO & $\begin{array}{l}15.17 \\
0.4 s\end{array}$ & 224 & eP & 59 & 20.30 & $-5.7 x$ & KMG & $\begin{array}{l}24.71 \\
24.72\end{array}$ & $\begin{array}{l}226 \\
232\end{array}$ & $\begin{array}{l}P \\
e P\end{array}$ & $\begin{array}{l}01 \\
01\end{array}$ & $\begin{array}{l}12.60 \\
12.00\end{array}$ \\
\hline
\end{tabular}




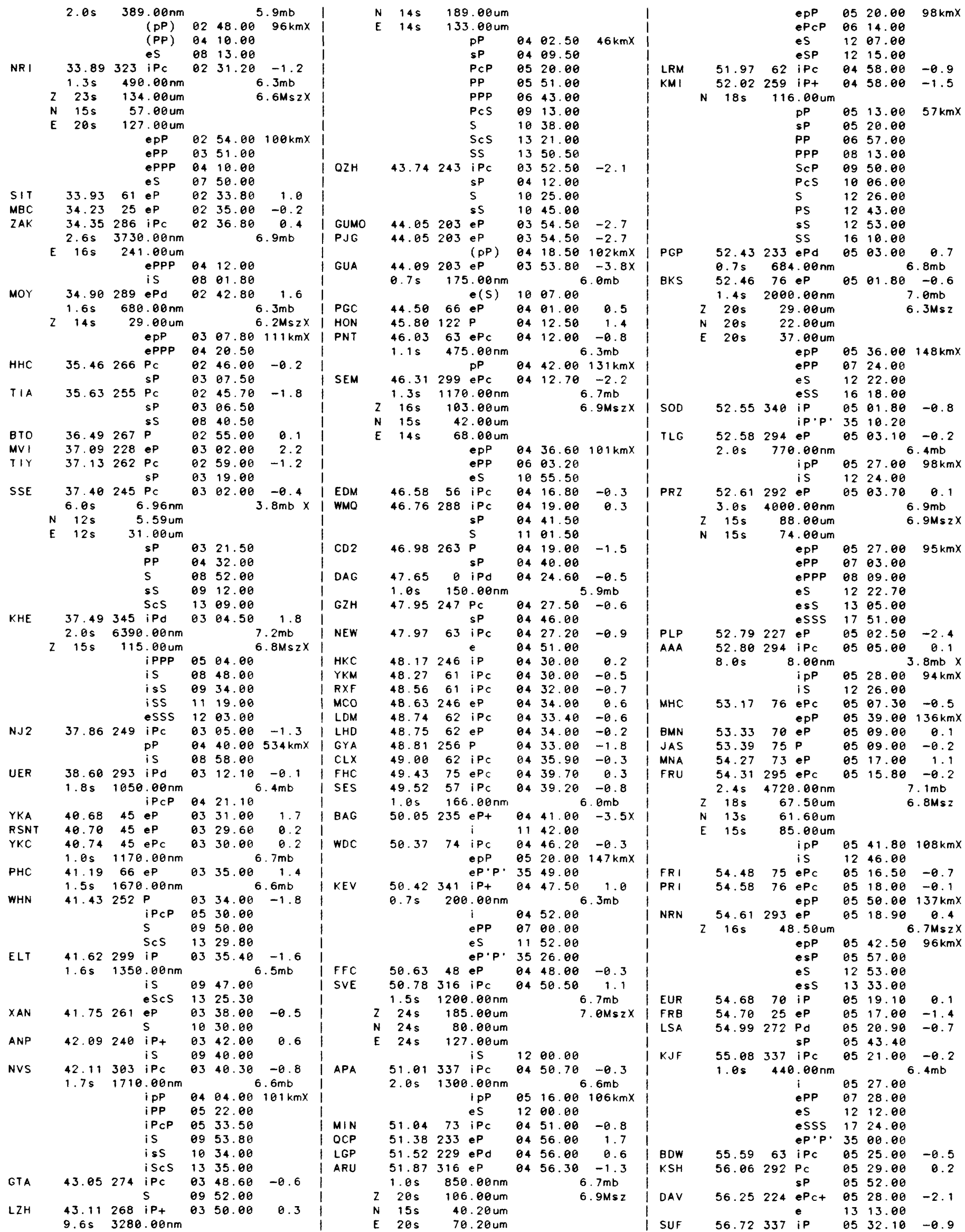




\begin{tabular}{|c|c|c|c|c|c|c|c|c|}
\hline & & 0.45 & & $1.00 \mathrm{~nm}$ & & & $6.5 \mathrm{mb}$ & 1 \\
\hline RSON & & 56.89 & 47 & eP & 05 & 33.30 & -1.1 & 1 \\
\hline ANR & & 56.98 & 295 & iPc & 05 & 35.20 & 0.0 & BDT \\
\hline & & 2.05 & 2500 & $0.00 \mathrm{~nm}$ & & & $7.0 \mathrm{mb}$ & \\
\hline & $z$ & $11 \mathrm{~s}$ & 37 & $7.00 \mathrm{um}$ & & & $6.7 M s z X$ & DSH \\
\hline & $\mathrm{N}$ & $11 \mathrm{~s}$ & & $3.00 \mathrm{um}$ & & & & 1 \\
\hline & E & 115 & & $8.00 \mathrm{um}$ & & & & i \\
\hline & & & & ipp & 05 & 57.60 & $90 \mathrm{kmX}$ & I KUL \\
\hline & & & & is & 13 & 23.00 & & \\
\hline RSSD & & 57.33 & 59 & iP & 05 & 37.10 & -0.7 & i \\
\hline PAS & & 57.43 & 76 & ip & 05 & 38.00 & -0.4 & i \\
\hline & & $0.6 \mathrm{~s}$ & & $0.00 \mathrm{~nm}$ & & & $6.1 \mathrm{mb}$ & $i$ \\
\hline & & & & es & 14 & 10.00 & & I SAM \\
\hline FRG & & 57.55 & 295 & $i P_{c}$ & 05 & 38.70 & -0.5 & i \\
\hline & & $2.7 \mathrm{~s}$ & & $5.40 \mathrm{~nm}$ & & & $4.2 \mathrm{mb} \times$ & i \\
\hline & $z$ & $13 \mathrm{~s}$ & & $1.00 \mathrm{um}$ & & & $6.7 \mathrm{Ms} z \mathrm{X}$ & i \\
\hline & $\mathrm{N}$ & $13 \mathrm{~s}$ & & $1.00 \mathrm{um}$ & & & & $i$ \\
\hline & & & & epp & 06 & 02.70 & $97 \mathrm{kmX}$ & 1 \\
\hline & & & & es & 13 & 17.20 & & \\
\hline SHL & & 57.76 & 269 & ip & 05 & 39.00 & -2.0 & I \\
\hline & & & & is & 13 & 34.00 & & i \\
\hline MUR & & 57.94 & 292 & eP & 05 & 41.80 & -0.7 & I OBN \\
\hline & & & & is & 13 & 38.70 & & \\
\hline TAS & & 58.10 & 298 & ePc & 05 & 43.00 & -0.1 & i \\
\hline & & $3.0 \mathrm{~s}$ & 8000 & $0.00 \mathrm{~nm}$ & & & $7.3 \mathrm{mb}$ & i \\
\hline & $z$ & $18 \mathrm{~s}$ & & $8.00 \mathrm{um}$ & & & 7. OMsZ & i \\
\hline & $\mathrm{N}$ & $17 \mathrm{~s}$ & & $9.00 \mathrm{um}$ & & & & \\
\hline & E & $12 \mathrm{~s}$ & & $0.00 \mathrm{um}$ & & & & i \\
\hline & & & & $i p p$ & 06 & 06.00 & $92 \mathrm{~km} x$ & i \\
\hline & & & & ePcP & 06 & 40.00 & & I \\
\hline & & & & es & 13 & 36.00 & & I $w w w$ \\
\hline & & & & ess & 17 & 34.00 & & NST \\
\hline PUL & & 58.32 & 333 & eP & 05 & 44.00 & -0.3 & UPP \\
\hline & & $3.0 \mathrm{~s}$ & 3700 & $0.00 \mathrm{~nm}$ & & & $7.0 \mathrm{mb}$ & \\
\hline & $z$ & $19 \mathrm{~s}$ & & $0.00 \mathrm{um}$ & & & 6. $9 M s z$ & $i$ \\
\hline & $\mathrm{N}$ & $19 \mathrm{~s}$ & & $0.00 \mathrm{um}$ & & & & $i$ \\
\hline & E & $20 \mathrm{~s}$ & & $8.00 \mathrm{um}$ & & & & $i$ \\
\hline & & & & $i p p$ & 06 & 12.00 & $115 \mathrm{kmX}$ & 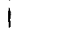 \\
\hline & & & & iPCP & 06 & 40.00 & & $\mathrm{KKM}$ \\
\hline & & & & iPP & 08 & 04.00 & & i \\
\hline & & & & is & 13 & 40.00 & & I PCT \\
\hline & & & & iSP & 13 & 50.00 & & i \\
\hline AKU & & 58.79 & 360 & eP & 05 & 48.80 & 1.4 & i \\
\hline MOM & & 58.81 & 196 & eP & 05 & 44.00 & $-4.1 x$ & I HFS \\
\hline GCA & & 58.85 & 69 & ep & 05 & 47.60 & -0.8 & I PAA \\
\hline NUR & & 59.02 & 337 & iPt & 05 & 49.00 & -0.1 & I KHT \\
\hline & & $1.3 \mathrm{~s}$ & 1200 & $0.00 \mathrm{~nm}$ & & & $6.9 \mathrm{mb}$ & i \\
\hline & $z$ & $24 \mathrm{~s}$ & 192 & $2.00 \mathrm{um}$ & & & 7. $1 \mathrm{MszX}$ & $\mathrm{ISCH}$ \\
\hline & & & & $i$ & 05 & 55.00 & & \\
\hline & & & & ePP & 08 & 16.00 & & I ALO \\
\hline & & & & ePPP & 09 & 40.00 & & \\
\hline & & & & es & 13 & 48.00 & & 1 \\
\hline & & & & $e^{\prime} \cdot P^{\prime}$ & 35 & 00.00 & & I TZZ \\
\hline RMU & & 59.10 & 69 & eP & 05 & 49.30 & -0.9 & I LAT \\
\hline CHTO & & 59.16 & 258 & eP & 05 & 49.80 & -0.8 & I NDI \\
\hline GAR & & 59.33 & 295 & eP & 05 & 51.20 & -0.6 & \\
\hline & 2 & $16 \mathrm{~s}$ & & $5.00 \mathrm{um}$ & & & 6. $6 M s z X$ & 1 \\
\hline & & & & epP & 06 & 14.90 & $95 \mathrm{kmX}$ & \\
\hline & & & & es & 13 & 49.90 & & i \\
\hline MOS & & 59.72 & 327 & iPc & 05 & 55.00 & 1.0 & i \\
\hline & & $2.0 \mathrm{~s}$ & 1400 & $0.00 \mathrm{~nm}$ & & & $6.7 \mathrm{mb}$ & NNT \\
\hline & $z$ & $26 \mathrm{~s}$ & 180 & $0.00 \mathrm{um}$ & & & 7. $1 M s 2 X$ & \\
\hline & N & 245 & 132 & $2.00 \mathrm{um}$ & & & & HNR \\
\hline & E & $24 \mathrm{~s}$ & & $3.00 \mathrm{um}$ & & & & AA I \\
\hline & & & & $i p p$ & 06 & 25.00 & $124 \mathrm{kmX}$ & \\
\hline & & & & esp & 06 & 32.00 & & LMG \\
\hline & & & & ePP & 08 & 05.00 & & MUD \\
\hline & & & & ePPP & 09 & 34.00 & & \\
\hline & & & & is & 13 & 59.00 & & 1 \\
\hline KKN & & 59.75 & 276 & $i \mathrm{Pc}$ & 05 & 52.60 & -2.3 & COP \\
\hline & & $0.5 \mathrm{~s}$ & 190 & $0.00 \mathrm{~nm}$ & & & $6.5 \mathrm{mb}$ & \\
\hline PKI & & 59.85 & 275 & iPc & 05 & 53.40 & -2.3 & KAT \\
\hline & & $0.8 \mathrm{~s}$ & 670 & $0.00 \mathrm{~nm}$ & & & $0.8 \mathrm{mb}$ & \\
\hline KHO & & 59.85 & 293 & iPc & 05 & 55.60 & 0.2 & \\
\hline & & $9.2 \mathrm{~s}$ & 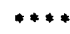 & $* * * * n m$ & & & & i \\
\hline & 2 & $17 \mathrm{~s}$ & & $2.60 \mathrm{um}$ & & & 7. $0 \mathrm{Msz} \mathrm{X}$ & \\
\hline & $\mathrm{N}$ & $17 \mathrm{~s}$ & & $2.10 \mathrm{um}$ & & & & i \\
\hline & E & $17 \mathrm{~s}$ & & $0.00 \mathrm{um}$ & & & & \\
\hline & & & & ePP & 08 & 03.00 & & i \\
\hline & & & & es & 13 & 58.00 & & $i$ \\
\hline DMN & & 59.99 & 276 & iPc & 05 & 55.28 & -1.4 & ASH \\
\hline GOL & & 59.99 & 63 & $i P_{C}$ & 05 & 56.40 & -0.1 & \\
\hline GLD & & 60.03 & 63 & ep & 05 & 57.00 & 0.4 & i \\
\hline & $z$ & $19 \mathrm{~s}$ & & $5.60 \mathrm{um}$ & & & $5.6 \mathrm{MSZ}$ & PMG \\
\hline GLA & & 60.08 & 74 & ip & 05 & 56.20 & -0.6 & \\
\hline RAB & & 60.31 & 190 & $e(P)$ & 05 & 58.20 & -0.2 & VAN \\
\hline REY & & 60.32 & 2 & ip & 05 & 58.70 & 0.8 & \\
\hline
\end{tabular}

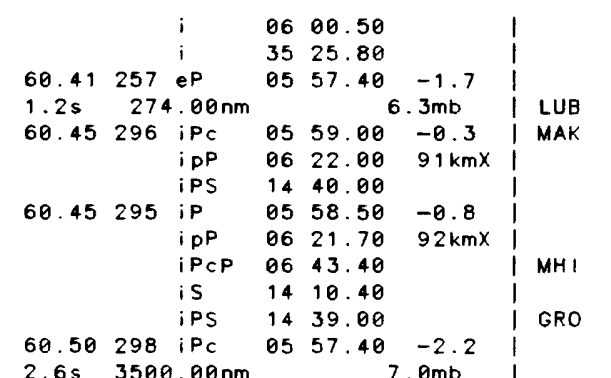

$2.6 \mathrm{~s} 3500.00 \mathrm{~nm}$

$Z 16 \mathrm{~s} \quad 92.70 \mathrm{um}$

$\mathrm{N} 14 \mathrm{~s} \quad 69.80 \mathrm{um}$

E $16 \mathrm{~s} \quad 72.40 \mathrm{um}$

ipP $06 \quad 22.40100 \mathrm{kmX}$

$\begin{array}{llll}\text { ip } & 06 & 22.40 & 100 \mathrm{kmX} \\ \text { is } & 14 & 07.00 & \end{array}$

$\begin{array}{lll}\text { is } & 1407.00 \\ \text { iPS } & 1443.00\end{array}$

iSCS 1533.20

$60.52327 \mathrm{iPC}$

$1.2 \mathrm{~s} \quad 680.00 \mathrm{~nm}$

$Z 26 \mathrm{~s} \quad 150.00 \mathrm{um}$

N $26 \mathrm{~s} \quad 110.00 \mathrm{um}$

E $26 \mathrm{~s} 68.00 \mathrm{um}$

IPCP 0642.00

IPPP 0946.00

is 1409.00

isS 1810.00

$60.99200 \mathrm{eP}$

$61.07255 \mathrm{eP}$

61.08340 iP

$1.3 \mathrm{~s} 3300.00 \mathrm{~nm}$

$$
\text { i }
$$

$06 \quad 04.00$

1.1.

0603.10

1008.00

S 1415.00

61.16234 iPd

$1.5 \mathrm{~s} \quad 1250.00 \mathrm{~nm}$

$\begin{array}{ll}61.20 & 253 \mathrm{iPc} \\ 1.6 \mathrm{~s} & 328.00 \mathrm{~mm}\end{array}$

$1.6 \mathrm{~s} \quad 328.00 \mathrm{~nm}$

61.54342 iP

$62.12187 \mathrm{eP}$

$62.64255 \mathrm{ePd}$

$62.9129 \mathrm{ePc}$

$1.5 \mathrm{~s} \quad 2480.00 \mathrm{~nm}$

$\begin{array}{ll}63.08 & 67 \mathrm{iPCt} \\ 1.05 & 265.00 \mathrm{~nm}\end{array}$

$\begin{array}{rr}1.0 \mathrm{~s} & 265.00 \mathrm{~nm} \\ 18 \mathrm{~s} & 46.40 \mathrm{um}\end{array}$

$63.12203 \mathrm{eP}$

$63.40196 \mathrm{eP}$

$63.55283 \mathrm{iPc}$

$0.8 \mathrm{~s} \quad 101.00 \mathrm{~nm}$

$Z 20 \mathrm{~s} \quad 48.90 \mathrm{um}$

$\begin{array}{lll}N & 20 \mathrm{~s} & 31.26 \mathrm{um} \\ \mathrm{E} & 20 \mathrm{~s} & 36.20 \mathrm{um}\end{array}$

is

$63.83253 \mathrm{eP}$

$65.05 \quad 181 \mathrm{eP}$

$65.25217 \mathrm{ePC}$

$1.9 \mathrm{~s} 4020.00 \mathrm{~nm}$

$65.44194 \mathrm{eP}$

$65.78343 \mathrm{iPc}$

$1.2 \mathrm{~s} \quad 990.00 \mathrm{~nm}$

$65.97341 \mathrm{iPct}$ is

$66.03305 \mathrm{iPC}$

$z \quad 15 \mathrm{~s} \quad 68.80 \mathrm{um}$

$\begin{array}{lll}N & 15 \mathrm{~s} & 50.00 \mathrm{um}\end{array}$

E $14 \mathrm{~s}$

54.00 um

epP $\quad 07 \quad 00.50 \quad 90 \mathrm{kmX}$

$\begin{array}{lll}\text { EPCP } & 07 & 06.00\end{array}$

ePPP $10 \quad 38.00$

ES $\quad 1518.00$

$66.06303 \mathrm{eP} \quad 0636.60$

IPCP $\quad 07 \quad 01.20$

$66.08195 \mathrm{ePt}$

$Z 18 \mathrm{~s} \quad 24.10 \mathrm{um}$

$66.18303 \mathrm{ePc}$

$1.5 \mathrm{~s} \quad 480.00 \mathrm{~nm}$
$216 \mathrm{~s} \quad 110.00 \mathrm{um}$

$\begin{array}{ccc}\text { ePCP } & 07 & 00.00 \\ \text { eS } & 15 & 10.00\end{array}$

$\begin{array}{lrllll}66.51 & 65 & \mathrm{eP} & 06 & 30.00 & -9.2 x \\ 66.80 & 313 \mathrm{iPc} & 06 & 41.00 & 0.3\end{array}$

$5.0 \mathrm{~s} \ldots \ldots \ldots+\mathrm{nm} \quad 7.0 \mathrm{mb} x$

IPCP $07 \quad 05.00$

IPP $09 \quad 16.00$

iSPP 1556.00

66.82301 iPct $06 \quad 41.80 \quad 0.6$

eS $\quad 1523.00$

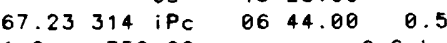

$\begin{array}{ll}1.0 \mathrm{~s} & 750.00 \mathrm{~nm}\end{array}$

$\begin{array}{llll}Z & 18 \mathrm{~s} & 72.00 \mathrm{um} & 6.9 \mathrm{MsZ}\end{array}$

$N 18 \mathrm{~s}-210.00 \mathrm{um}$

E $18 \mathrm{~s} \quad 75.00 \mathrm{um}$

IPCP $07 \quad 07.00$

iPP $\quad 09 \quad 17.00$

$\begin{array}{llllll}67.24 & 351 \mathrm{eP} & 06 & 42.10 & -1.2\end{array}$

$0.9 \mathrm{~s} \quad 509.00 \mathrm{~nm} \quad 6.5 \mathrm{mb}$

$67.38 \quad 351 \mathrm{ePC} \quad 06 \quad 43.20-1.0$

$0.6 \mathrm{~s} \quad 440.00 \mathrm{~nm} \quad 6.6 \mathrm{mb}$

$67.39335 \mathrm{eP} \quad 06 \quad 45.00 \quad 0.7$ \begin{tabular}{lll} 
e & 0700.00 \\
\hline & 1530.00
\end{tabular}

$\begin{array}{lllll} & \text { eS } & 15 & 30.00 & \\ 67.55 & 59 \mathrm{ePC} & 06 & 43.50 & -2.1\end{array}$

$\begin{array}{lllll}67.58 & 351 \mathrm{ePC} & 06 & 44.60 & -0.9\end{array}$

$0.8 \mathrm{~s} \quad 978.00 \mathrm{~nm} \quad 6.8 \mathrm{mb}$

67.6759 iPct $06 \quad 44.50 \quad-1.9$

$\begin{array}{lll}1.3 \mathrm{~s} & 4060.00 \mathrm{~nm} & 7.2 \mathrm{mb}\end{array}$

$Z 18 \mathrm{~s} \quad 48.10 \mathrm{um} \quad 6.8 \mathrm{Msz}$ e(S) $16 \quad 16.00$

$\begin{array}{llllll}67.69 & 317 \quad \mathrm{iPc} \quad 06 & 46.00 & -0.4\end{array}$

$\begin{array}{ll}1.0 \mathrm{~s} \quad 800.00 \mathrm{~nm} & 6.6 \mathrm{mb}\end{array}$

$z 28 \mathrm{~s} \quad 171.00 \mathrm{um} \quad 7.1 \mathrm{MszX}$ eS $\quad 1534.00$

ePS 1620.00

eSSS 2253.00

$\begin{array}{lllll}67.71 & 351 \mathrm{iPC} & 06 & 45.40 & -0.9\end{array}$ $0.7 \mathrm{~s} \quad 732.00 \mathrm{~nm} \quad 6.8 \mathrm{mb}$

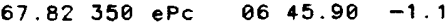

$0.7 \mathrm{~s} \quad 830.00 \mathrm{~nm} \quad 6.8 \mathrm{mb}$

67.8358 iPc $06 \quad 45.40-2.0$

67.88249 iPCt $06 \quad 50.80 \quad 2.9 \times$

$1.7 \mathrm{~s} \quad 2630.00 \mathrm{~nm} \quad 6.9 \mathrm{mb}$

$\begin{array}{lllll}67.93 & 310 \mathrm{iPd} \text { O6 } & 48.00 & 0.2\end{array}$

$\begin{array}{llll}Z & 18 \mathrm{~s} & 75.00 \mathrm{um} & \end{array}$

$N$ 18s $140.00 u m$

E $18 \mathrm{~s} \quad 78.00 \mathrm{um}$

is 1546.00

$\begin{array}{lllll}67.97 & 351 \mathrm{ePc} & 06 & 47.20 & -0.7\end{array}$

$1.1 \mathrm{~s} \quad 1460.00 \mathrm{~nm} \quad 6.9 \mathrm{mb}$

$68.01351 \mathrm{ePc} \quad 06 \quad 47.10 \quad-1.0$

$1.3 \mathrm{~s} \quad 1130.00 \mathrm{~nm} \quad 6.7 \mathrm{mb}$

$68.0158 \mathrm{ePC} \quad 06 \quad 46.50 \quad-2.0$

$\begin{array}{llllll}68.07 & 44 \mathrm{P} & 06 & 46.95 & -1.8\end{array}$

$\begin{array}{llllll}68.25 & 45 P & 06 & 48.30 & -1.5\end{array}$

$\begin{array}{llllll}68.25 & 44 \mathrm{P} & 06 & 47.70 & -2.1\end{array}$

$\begin{array}{llllll}68.31 & 40 & \text { eP } & 06 & 48.00 & -2.1\end{array}$

$\begin{array}{lll}1.3 \mathrm{~s} & 1710.00 \mathrm{~nm} \quad 6.8 \mathrm{mb}\end{array}$

$\begin{array}{llllll}68.44 & 47 \mathrm{PP} & 07 & 22.00 & 139 \mathrm{kmx} \\ & \mathrm{iPc} & 06 & 50.00 & -1.0\end{array}$

$68.45351 \mathrm{PC} \quad 0650.20 \quad-0.7$

$1.6 \mathrm{~s} \quad 3210.00 \mathrm{~nm} \quad 7.0 \mathrm{mb}$

$\begin{array}{llllll}68.47 & 351 & \mathrm{iPc} & 06 & 50.30 & -0.7\end{array}$

$0.9 \mathrm{~s} \quad 822.00 \mathrm{~nm} \quad 6.7 \mathrm{mb}$

$\begin{array}{llllll}68.51 & 54 & \text { eS } & 15 & 48.20 & \\ 68 & 06 & 49.70 & -1.8\end{array}$

68.53342 iPC $0653.00 \quad 1.6$

68.60350 iPC $06 \quad 50.90 \quad-0.9$

$0.8 \mathrm{~s} \quad 507.00 \mathrm{~nm} \quad 6.5 \mathrm{mb}$

eS $\quad 15 \quad 51.50$

$68.85332 \mathrm{iPC} \quad 06 \quad 52.00 \quad-1.4$

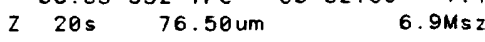

$\mathrm{N} 18 \mathrm{~s} \quad 83.60 \mathrm{um}$

$20 \mathrm{~s} \quad 47.60 \mathrm{um}$

is $\quad 15 \quad 52.00$

iSPP $16 \quad 21.00$

iPS $16 \quad 29.00$

iSS 2018.00

$68.93 \quad 340 \mathrm{iPC} \quad 0651.50 \quad-2.3$ $68.95 \quad 38$ iPd $06 \quad 53.00 \quad-1.1$ $\begin{array}{lll}1.2 \mathrm{~s} & 1600.00 \mathrm{~nm} & 6.8 \mathrm{mb}\end{array}$ $\begin{array}{lllll}68.97300 \mathrm{ePC} & 06 & 55.00 & 0.4\end{array}$ 68.97314 iP $0655.00 \quad 0.8$ $\begin{array}{lllll}\text { EPCP } & 07 \quad 1200\end{array}$

$\begin{array}{lllll}69.11 & 321 \text { iPC } & 06 & 55.00 & 0.0\end{array}$ 


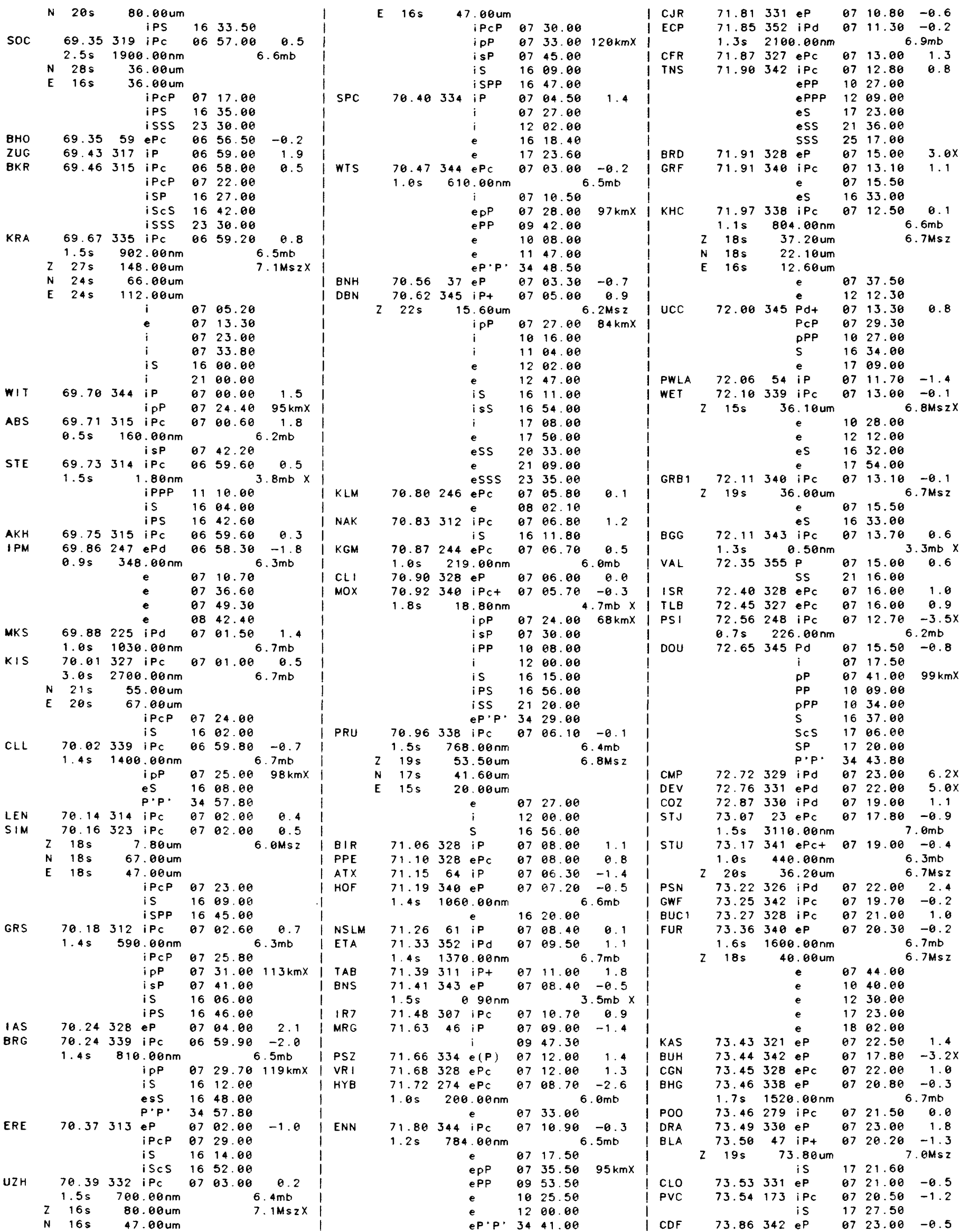




\begin{tabular}{|c|c|c|c|c|c|c|c|}
\hline & $1.2 \mathrm{~s}$ & 945 & $5.00 \mathrm{~nm}$ & & & $.6 \mathrm{mb}$ & MFF \\
\hline GAP & 74.06 & 339 & eP & 87 & 24.50 & -0.1 & i \\
\hline & $1.0 \mathrm{~s}$ & 59 & $4.80 \mathrm{~nm}$ & & & $.5 \mathrm{mb}$ & $M Z F$ \\
\hline $\mathrm{ECH}$ & 74.87 & 342 & iPc & 07 & 24.08 & -0.6 & 1 \\
\hline MSL & 74.16 & 312 & iPc & 87 & 26.00 & 0.7 & DST \\
\hline & & & ¿PCP & 87 & 47.00 & & ALT \\
\hline & & & iPPP & 10 & 49.00 & & I SKO \\
\hline & & & $i$ & 12 & 31.00 & & i \\
\hline & & & is & 16 & 54.50 & & I LSF \\
\hline & & & iPS & 17 & 29.50 & & 1 \\
\hline & & & iss & 21 & 26.50 & & I VAY \\
\hline KER & 74.18 & 308 & $e^{P}$ & 07 & 27.80 & 1.4 & I EZN \\
\hline SLE & 74.27 & 341 & ePd & 07 & 24.40 & -1.4 & | PRT \\
\hline HAL & 74.41 & 343 & $e^{P}$ & 07 & 26.00 & -0.6 & 1 \\
\hline & $1.2 \mathrm{~s}$ & 765 & $5.00 \mathrm{~nm}$ & & 6 & $.5 \mathrm{mb}$ & I \\
\hline$B A F$ & 74.46 & 342 & iPc & 07 & 26.50 & -0.5 & I FIR \\
\hline PP I & 74.47 & 245 & ePc & 67 & 27.80 & -0.3 & I \\
\hline BSF & 74.50 & 342 & $e^{P}$ & 07 & 26.60 & -0.6 & I \\
\hline ZUL & 74.56 & 341 & ePd & 67 & 26.80 & -0.7 & I RJF \\
\hline NDF & 74.58 & 164 & $\mathrm{eP}^{\mathrm{P}}$ & 67 & 29.80 & 1.2 & I \\
\hline SAX & 74.61 & 341 & $e^{P d}$ & 67 & 27.50 & -8.6 & I PRK \\
\hline$O G A$ & 74.65 & 339 & iPc & 07 & 28.30 & 0.1 & I NOU \\
\hline$F L N$ & 74.65 & 348 & iPc & 07 & 27.00 & -8.9 & I \\
\hline & $1.2 \mathrm{~s}$ & 693 & $3.00 \mathrm{~nm}$ & & & $.5 \mathrm{mb}$ & I BCK \\
\hline LJU & 74.69 & 337 & $e^{P}$ & 07 & 29.40 & 1.2 & I CAF \\
\hline & & & e & 10 & 44,00 & & 1 \\
\hline & & & es & 17 & 42.00 & & I KZN \\
\hline LDF & 74.77 & 347 & $e^{P}$ & 87 & 27.50 & -1.1 & I LFF \\
\hline & $1.3 \mathrm{~s}$ & 634 & $4.00 \mathrm{~nm}$ & & & $.4 \mathrm{mb}$ & I \\
\hline JMB & 74.82 & 327 & $e^{P}$ & 87 & 32.00 & $3.0 x$ & I IZM \\
\hline TRT & 74.98 & 230 & iPd & 87 & 27.80 & -2.4 & I POI \\
\hline & $0.8 \mathrm{~s}$ & 944 & $4.00 \mathrm{~nm}$ & & 6 & $.8 \mathrm{mb}$ & I LPO \\
\hline VUN & 74.99 & 163 & ePc & 07 & 30.80 & 0.7 & 1 \\
\hline & $1.4 \mathrm{~s}$ & 735 & $5.00 \mathrm{~nm}$ & & & $.4 \mathrm{mb}$ & $1 \mathrm{AOU}$ \\
\hline CEY & 75.01 & 337 & $e^{P}$ & 07 & 30.20 & 0.1 & I \\
\hline & & & $i$ & 87 & 39.80 & & 1150 \\
\hline & & & es & 17 & 43.40 & & 1 MNS \\
\hline DMK & 75.03 & 326 & $e^{P}$ & 07 & 30.30 & 0.1 & 1 \\
\hline LLS & 75.04 & 341 & ePd & 87 & 36.00 & -0.5 & I FRF \\
\hline GRR & 75.66 & 348 & $i P c$ & 07 & 29.60 & -0.7 & 1 \\
\hline & $1.2 \mathrm{~s}$ & 1050 & $0.00 \mathrm{~nm}$ & & & $6 \mathrm{mb}$ & I CIN \\
\hline SVA & 75.09 & 163 & ePc & 87 & 31.90 & 1.2 & I FOG \\
\hline TRI & 75.18 & 337 & iPc & 87 & 31.10 & 0.1 & | LRG \\
\hline & & & e & 11 & 39.60 & & 1 \\
\hline & & & e & 12 & 33.10 & & I DUI \\
\hline & & & is & 17 & 04.00 & & 1 WB2 \\
\hline & & & iSP & 17 & 40.00 & & ! \\
\hline & & & iSS & 22 & 32.00 & & I \\
\hline & & & iSSS & 25 & 47.80 & & 1 \\
\hline ISK & 75.30 & 325 & iP & 07 & 32.40 & 0.7 & ELL \\
\hline VDL & 75.32 & 340 & ePd & 07 & 32.00 & -0.1 & I LMR \\
\hline HRT & 75.32 & 324 & iP & 67 & 33.00 & 1.1 & 1 \\
\hline IST & 75.34 & 325 & $i P c$ & 07 & 32.20 & 0.3 & I BRT \\
\hline GBA & 75.43 & 273 & $\mathrm{Pc}$ & 07 & 29.40 & $-3.4 x$ & 1110 \\
\hline & $1.2 \mathrm{~s}$ & 259 & $9.08 \mathrm{~nm}$ & & 6 & $.8 \mathrm{mb}$ & I YER \\
\hline LPF & 75.44 & 348 & iPC & 07 & 31.70 & -0.7 & | RMP \\
\hline & $1.3 \mathrm{~s}$ & 972 & $2.80 \mathrm{~nm}$ & & 6 & $.6 \mathrm{mb}$ & I \\
\hline$D \mid M$ & 75.50 & 328 & iPd & 07 & 34.00 & 1.1 & I \\
\hline LOR & 75.52 & 344 & iPc & 67 & 32.10 & -0.9 & I \\
\hline & $1.2 \mathrm{~s}$ & 693 & $3.86 \mathrm{~nm}$ & & 6 & $.5 \mathrm{mb}$ & | RDP \\
\hline $\mathrm{SHI}$ & 75.62 & 302 & $e^{P}$ & 87 & 32.00 & -2.0 & I CVF \\
\hline VTS & 75.70 & 329 & ip & 07 & 33.00 & -0.9 & 1 \\
\hline PLD & 75.71 & 328 & $\mathrm{iPc}$ & 67 & 34.00 & -0.1 & I LCI \\
\hline SSF & 75.78 & 345 & $i P C$ & 07 & 33.80 & -0.6 & I TAC \\
\hline & 1.35 & 396 & $6.00 \mathrm{~nm}$ & & 6 & $2 \mathrm{mb}$ & ! \\
\hline LBF & 75.78 & 344 & eP & 07 & 33.60 & -0.9 & I IIM \\
\hline & $1.4 \mathrm{~s}$ & 472 & $2.00 \mathrm{~nm}$ & & 6 & $.2 \mathrm{mb}$ & $|I| P$ \\
\hline TMA & 75.80 & 341 & ePd & 87 & 34.00 & -0.7 & I SGO \\
\hline KDZ & 75.94 & 328 & iPd & 07 & 35.00 & -0.4 & ATH \\
\hline SAL & 75.96 & 339 & eP & 67 & 35.00 & -6.4 & I \\
\hline & $1.2 \mathrm{~s}$ & 1400 & $0.00 \mathrm{~nm}$ & & 6 & $.8 m b$ & | HRI \\
\hline MMK & 76.02 & 341 & ePd & 07 & 35.80 & -0.3 & | ORI \\
\hline AVF & 76.06 & 345 & eP & 67 & 35.30 & -0.7 & 1111 \\
\hline & $1.3 \mathrm{~s}$ & 803 & $3.80 \mathrm{~nm}$ & & 6 & $5 m b$ & $111 T$ \\
\hline DIX & 76.09 & 342 & ePd & 07 & 36.40 & -6.1 & I MMN \\
\hline SMF & 76.13 & 344 & $i P_{c}$ & 67 & 35.60 & -0.8 & I EPF \\
\hline & $1.4 \mathrm{~s}$ & 735 & $5.60 \mathrm{~nm}$ & & 6 & $.4 \mathrm{mb}$ & 1 \\
\hline KOU & 76.15 & 177 & $i P_{C}$ & 07 & 38.00 & 1.4 & $\mid \mathrm{CSI}$ \\
\hline EDC & 76.34 & 325 & $e^{P}$ & 67 & 37.90 & 0.3 & | RO! \\
\hline KNA & 76.40 & 212 & iPd & 67 & 38.10 & -0.1 & I VLS \\
\hline ORO & 76.42 & 341 & $\mathrm{iPc}$ & 67 & 40.00 & 1.8 & $A C I$ \\
\hline MMB & 76.48 & 329 & $e^{P}$ & 67 & 38.00 & -0.4 & $|c Z|$ \\
\hline CTA & 76.71 & 195 & iPc & 67 & 36.90 & -2.9 & JER \\
\hline & $0.8 \mathrm{~s}$ & 19 & $9.50 \mathrm{~nm}$ & & 5 & $.7 \mathrm{mb}$ & I NPS \\
\hline & & & is & 17 & 19.00 & & DOR \\
\hline TCF & & $\begin{array}{r}345 \\
47\end{array}$ & iPc & 07 & 39.40 & -0.3 & PMO \\
\hline
\end{tabular}

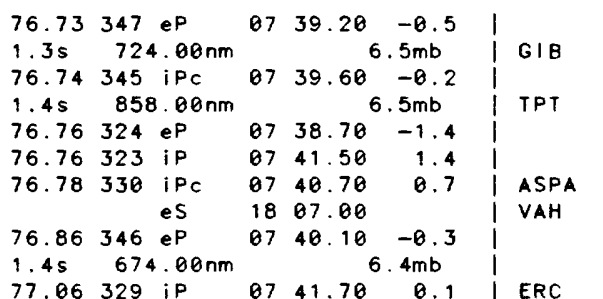

\begin{tabular}{rrlllr|l}
77.06 & 329 & iP & 07 & 41.70 & 0.1 & ERC \\
77.30 & 326 iPc & 07 & 42.60 & -0.3 & RUV
\end{tabular}

$77.50338 \mathrm{eP} \quad 0747.00 \quad 3.1 \times$

ipP $6801.00 \quad 49 \mathrm{kmX}$ ।

$\begin{array}{cllllll} & \text { is } & 17 & 32.50 & & \text { I MEI } \\ & \text { eP } & 97 & 46.50 & 2.2 & \text { I PTO }\end{array}$

iSKS 1732.00

is $18,10.00$

$77.78346 \mathrm{iPc} \quad 0745.30-0.3$ |

$1.4 \mathrm{~s} \quad 472.00 \mathrm{~nm}$

77.85326 (AFR

00.9

\begin{tabular}{ccccc|c}
77.97175 & is & 18 & 18.50 & 1.6 & \\
& is & PPT
\end{tabular}

\begin{tabular}{lllll|}
78.03 & $322 \mathrm{iP}$ & 07 & 47.56 & 0.4
\end{tabular}

\begin{tabular}{ccc|c}
$1.3 \mathrm{~s}$ & $739.80 \mathrm{~nm}$ & $6.5 \mathrm{mb}$ & PAE
\end{tabular}

$78.23 \quad 336 \mathrm{eP}^{\mathrm{P}} \quad 07 \quad 45.50^{0}-2.6$

$\begin{array}{rrlrr}78.23 & 336 \mathrm{eP} & 07 & 45.56 & -2.6 \\ 78.25 & 346 \mathrm{eP} & 07 & 48.28 & 0.1\end{array}$

\begin{tabular}{cc|c|c|c|}
$1.2 \mathrm{~s}$ & $783.00 \mathrm{~nm}$ & $6.5 \mathrm{mb}$ & TVO
\end{tabular}

$\begin{array}{lllll}78.30 & 325 & \text { iP } \quad 07 \quad 49.08 & 0.5\end{array}$

\begin{tabular}{llllll|l}
78.34 & $337 \mathrm{eP}^{\mathrm{P}}$ & 07 & 58.08 & 1.4 & \\
78.44 & $346 \mathrm{eP}$ & 07 & 49.26 & 0.6 & $\mathrm{MBL}$
\end{tabular}

$1.2 \mathrm{~s} \quad 873.88 \mathrm{~nm} \quad 6.6 \mathrm{mb}$ HLW

$\begin{array}{lllll}78.45 & 336 \mathrm{eP} \quad 07 & 50.20 & 0.9 & \text { । }\end{array}$

$\begin{array}{lll}1.0 \mathrm{~s} & 0.36 \mathrm{~nm} & 3.3 \mathrm{mb} \times\end{array}$

$78.46201 \mathrm{eP} \quad 0747.60 \quad-2.5$

$\begin{array}{lllll}78.58 & 337 \mathrm{eP} & 07 & 49.58 & -0.4\end{array}$

\begin{tabular}{ll|l|l|}
$1.2 \mathrm{~s}$ & $670.00 \mathrm{~nm}$ & $6.5 \mathrm{mb}$ & $\mathrm{ALI}$
\end{tabular}

$78.66341 \mathrm{eP} \quad 0756.26 \quad-6.2$

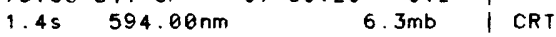

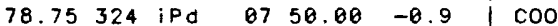

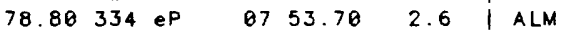

$78.82342 \mathrm{eP} \quad 0751.36 \quad 0.1$

$1.2 \mathrm{~s} \quad 720.00 \mathrm{~nm} \quad 6.5 \mathrm{mb}$

$\begin{array}{lllll}78.86 & 335 \mathrm{eP} & 07 & 51.50 & 0.0\end{array}$

$\begin{array}{llllll}78.88 & 266 & \text { iPC } & 67 & 49.10 & -2.7\end{array}$

$i \quad 0752.20$

IPCP $\quad 09 \quad 14.60$

\begin{tabular}{lllll|l}
78.90322 & $\mathrm{i}$ & 17 & 41.70 & WBN \\
& 87 & 51.30 & -0.7 & NAU
\end{tabular}

$78.91342 \mathrm{eP} \quad 0751.70 \quad 0.0 \quad$ SFS

$1.3 \mathrm{~s} \quad 761.00 \mathrm{~nm}$

$78.93333 \mathrm{eP}$

79.6271 iP

$79.11323 \mathrm{iPC}$

$79.13 \quad 337 \quad \mathrm{PPO}$$$
\text { is }
$$

is

$79.17337 \mathrm{eP}$

$\begin{array}{ll}79.21 & 340 \mathrm{iPc} \\ 1.2 \mathrm{~s} & 720.00 \mathrm{~nm}\end{array}$

$1.2 \mathrm{~s} \quad 720.00 \mathrm{~nm}$

79.3571 iP

79.4271 iP

$79.55 \quad 71$ iP

$79.71334 \mathrm{eP}$

$79.81327 \mathrm{iPc}$

$79.85316 \mathrm{eP}$

$79.90333 \mathrm{eP}^{\mathrm{P}}$

$80.0572 \mathrm{iP}$

80.1471 iP

$80.17334 \mathrm{eP}$

$80.18346 \mathrm{eP}$

$1.4 \mathrm{~s} \quad 764.00 \mathrm{~nm}$

$86.26333 \mathrm{eP}$

$80.32 \quad 333 \mathrm{eP}$

$80.54330 \mathrm{eP}^{\mathrm{P}}$

$80.62333 \mathrm{eP}$

$80.77333 \mathrm{eP}$

$81.39316 \mathrm{eP}$

$81.70325 \mathrm{eP}$

$81.82316 \mathrm{eP}$

$82.42131 \mathrm{iP}$

$1.3 \mathrm{~s} \quad 1500.00 \mathrm{~nm}$
$6.5 \mathrm{mb}$

$\begin{array}{rrr}07 & 51.00 & -0.8 \\ 07 & 54.80 & 1.7\end{array}$

$\begin{array}{rrr}87 & 54.80 & 1.7 \\ 67 & 52.70 & -0.3\end{array}$

b7 $53.50 \quad 0.5$

1853.60

1756.00

1846.00

87 $54.50 \quad 1.2$

$\begin{array}{rrr}67.00 & -0.4\end{array}$

\begin{tabular}{lc|c} 
& \multicolumn{1}{c}{$6.5 \mathrm{mb}$} & CMS
\end{tabular}

6809.00 14.2X I MEK

1843.00 | YOU

$0756.40 \quad 1.2$ | CRZ

6756.86

0756.00 0.0 CAN

\begin{tabular}{lll|l}
07 & 57.08 & 0.4 & WAM
\end{tabular}

\begin{tabular}{ll|l|l}
1742.80 & TOO \\
0757.00 & -0.1 & TOP
\end{tabular}

$\begin{array}{llr}080.80 & 2.9\end{array}$

$6757.20 \quad-1.3$

\begin{tabular}{llr|l}
880.00 & 1.0 & RKT
\end{tabular}

$\begin{array}{lll}08 & 01.08 & 2.5\end{array}$

\begin{tabular}{lll|l}
07 & 58.10 & -0.5 & MUN
\end{tabular}

\begin{tabular}{ll|l|l}
68 & $01.50^{6.4 \mathrm{mb}}$ & 2.7 & ARO
\end{tabular}

\begin{tabular}{ll|l|l|}
681.80 & 2.4 & NWAO
\end{tabular}

$8759.50-1.1$ SJG

08.02

$\begin{array}{lll}08.48 & 1.4\end{array}$

$\begin{array}{lll}08 & 04.00 & -2.7\end{array}$

$6809.60 \quad 1.6 \quad$ GBR

\begin{tabular}{lll|l}
08 & 12.60 & 2.1 & MNG
\end{tabular} $6.8 \mathrm{mb}$ WEL $\begin{array}{lll}08 & 02.50 & 0.8\end{array}$ ipp $\quad 88 \quad 35.40 \quad 85 \mathrm{kmX}$ $\begin{array}{lllll}82.46 & 334 \mathrm{eP} & 08 & 11.00 & 0.3\end{array}$

$130.00 \mathrm{~nm} \quad 5.8 \mathrm{mb}$

$\begin{array}{ccccc}82.52 & 131 \mathrm{iP} & 08 & 13.30 & 2.3 \\ 1.3 \mathrm{~s} & 2420.00 \mathrm{~nm} & & 7.0 \mathrm{mb}\end{array}$

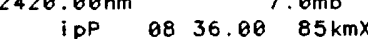

$82.56205 \mathrm{eP} \quad 08 \quad 09.00 \quad-2.2$

82.74131 iP $\quad 08 \quad 14.20 \quad 2.0$

$1.3 \mathrm{~s} \quad 1610.08 \mathrm{~nm} \quad 6.8 \mathrm{mb}$

ipP $\quad 08 \quad 37.00 \quad 85 \mathrm{kmx}$

$\begin{array}{llllll}82.75 & 336 \mathrm{eP} & 08 & 12.50 & 0.5\end{array}$

82.80131 iP $\quad 0814.70 \quad 2.2$

$1.3 \mathrm{~s} \quad 2100.00 \mathrm{~nm} \quad 6.9 \mathrm{mb}$

IPP $\quad 8837.60 \quad 86 \mathrm{kmX}$

$\begin{array}{lllll}333 & \mathrm{eP} & 88 & 13.58 & 0.4\end{array}$

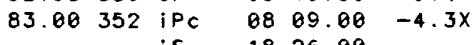

83.55144 is 1826.00

$\begin{array}{lll}08 & 17.00 & 0.8\end{array}$

83.90134 IP $\quad 8820.00 \quad 2.0$

$1.3 \mathrm{~s} 4580.88 \mathrm{~nm} \quad 7.3 \mathrm{mb}$

$\begin{array}{llll}\text { ipP } \quad 08 & 42.90 \quad 85 \mathrm{kmx}\end{array}$

84.81134 iP $\quad 8820.60 \quad 2.0$

$1.3 \mathrm{~s} 2070.08 \mathrm{~nm} \quad 7.0 \mathrm{mb}$

$\begin{array}{lllll} & \text { ipP } & 08 & 43.50 & 85 \mathrm{kmX}\end{array}$

$1.3 \mathrm{~s} 2270.08 \mathrm{~nm} \quad 7.0 \mathrm{mb}$

$\begin{array}{llllll} & \text { ipP } & 88 & 43.90 & 85 \mathrm{kmX}\end{array}$

$1.3 \mathrm{~s} \quad 1100.08 \mathrm{~nm} \quad 6.7 \mathrm{mb}$

$\begin{array}{llll}\text { ipP } 88 & 45.50 & 85 \mathrm{kmx}\end{array}$

$\begin{array}{llllll}218 \mathrm{eP} & 0820.08 & -0.3\end{array}$

es 1930.00

$84.70 \quad 57 \mathrm{iPC} \quad 0822.00 \quad-6.2$

$6.0 \mathrm{~s} * * * * * * \mathrm{~nm} \quad 7.2 \mathrm{mb} x$

84.88346 iP-

$\begin{array}{llll}\text { iP- } & 0824.00 & 1.2\end{array}$

$86.43 \quad 348$ iPc $\quad 0833.60 \quad 2.9 x$

$\begin{array}{llllll}86.46 & 188 \mathrm{eP} & 08 & 30.00 & -0.6\end{array}$

$\begin{array}{lllll}86.63 & 347 \mathrm{iPc} & 68 & 32.50 & 1.0\end{array}$

$\begin{array}{lll}2.1 \mathrm{~s} & 5.50 \mathrm{~nm} & 4.3 \mathrm{mb} \times\end{array}$

$N 26 \mathrm{~s} \quad 1.00 \mathrm{um}$

$\begin{array}{llll}185 & 0.90 u m \\ & \text { iPP } 1228.68\end{array}$

$\begin{array}{lll}\text { iPP } & 12 & 28.60 \\ \text { iPPP } & 13 & 09.70\end{array}$

is $\quad 1930.00$

$86.86211 \mathrm{eP} \quad 08 \quad 31.08 \quad-1.6$

87.21222 iPd $8834.20 \quad-0.1$

$87.43350 \mathrm{iPC} \quad 0837.00 \quad 1.6$

$i \quad 0846.08$

0921.00

iPP 1118.08

IPPP 1430.00

ISKS $19 \quad 10.00$

is $\quad 1936.00$

iPS 2024.00

iSP 2230.00

iSS 2750.00

iSSS 3700.00

$\begin{array}{llllll}87.99 & 193 \mathrm{iPd} \quad 08 & 39.08 & 1.1\end{array}$

$89.74188 \mathrm{eP} \quad 08 \quad 48.06 \quad 1.9$

89.84217 eP $\quad 0844.00 \quad-2.8$

$90.45 \quad 191$ iPd $\quad 8850.40 \quad 1.0$

$90.45171 \mathrm{eP} \quad 0852.06 \quad 2.6$

$91.42190 \mathrm{PC} \quad 0855.20 \quad 1.3$

$92.29 \quad 190 \mathrm{iPc} \quad 08 \quad 59.58 \quad 1.7$

$94.63193 \mathrm{eP}^{\mathrm{P}} \quad 0906.06 \quad 0.2$

$\begin{array}{lllll}94.20169 \mathrm{P} & 09 & 08.38 & 1.8\end{array}$

$S \quad 28 \quad 18.00$

sS 2056.00

$95.31124 \mathrm{iP} \quad 8914.00 \quad 2.0$

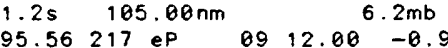

$95.70300 \mathrm{ePt} \theta 9 \quad 15.30 \quad 1.0$

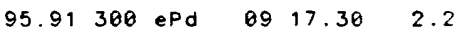

$96.08216 \mathrm{eP} \quad \theta 914.08 \quad-1.3$

$\begin{array}{llllll}96.17 & 45 & \mathrm{e}^{P} & 09 & 15.50 & -0.8\end{array}$

$\begin{array}{lll}1.0 \mathrm{~s} & 125.00 \mathrm{~nm} & 6.4 \mathrm{mb}\end{array}$

$z 20 \mathrm{~s} \quad 44.00 \mathrm{um} \quad 6.9 \mathrm{Msz}$

e $\quad 1308.00$

$\begin{array}{lrllll}96.21300 \text { eP } & 09 & 18.90 & 2.3\end{array}$

$96.85169 \mathrm{P} \quad 0917.00 \quad-1.6$

$97.44170 \mathrm{P} \quad 0920.20 \quad-1.0$ 


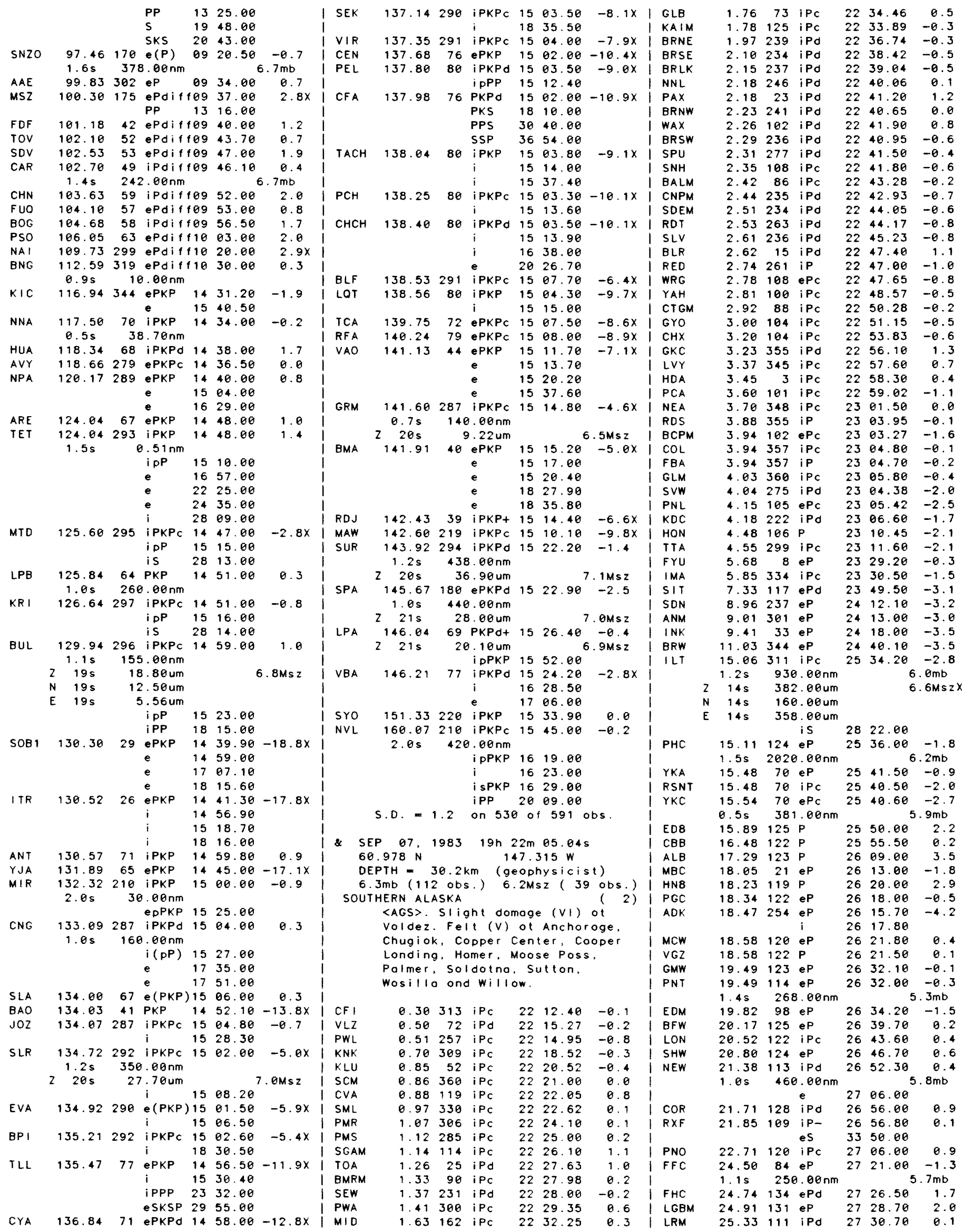


07d 19h

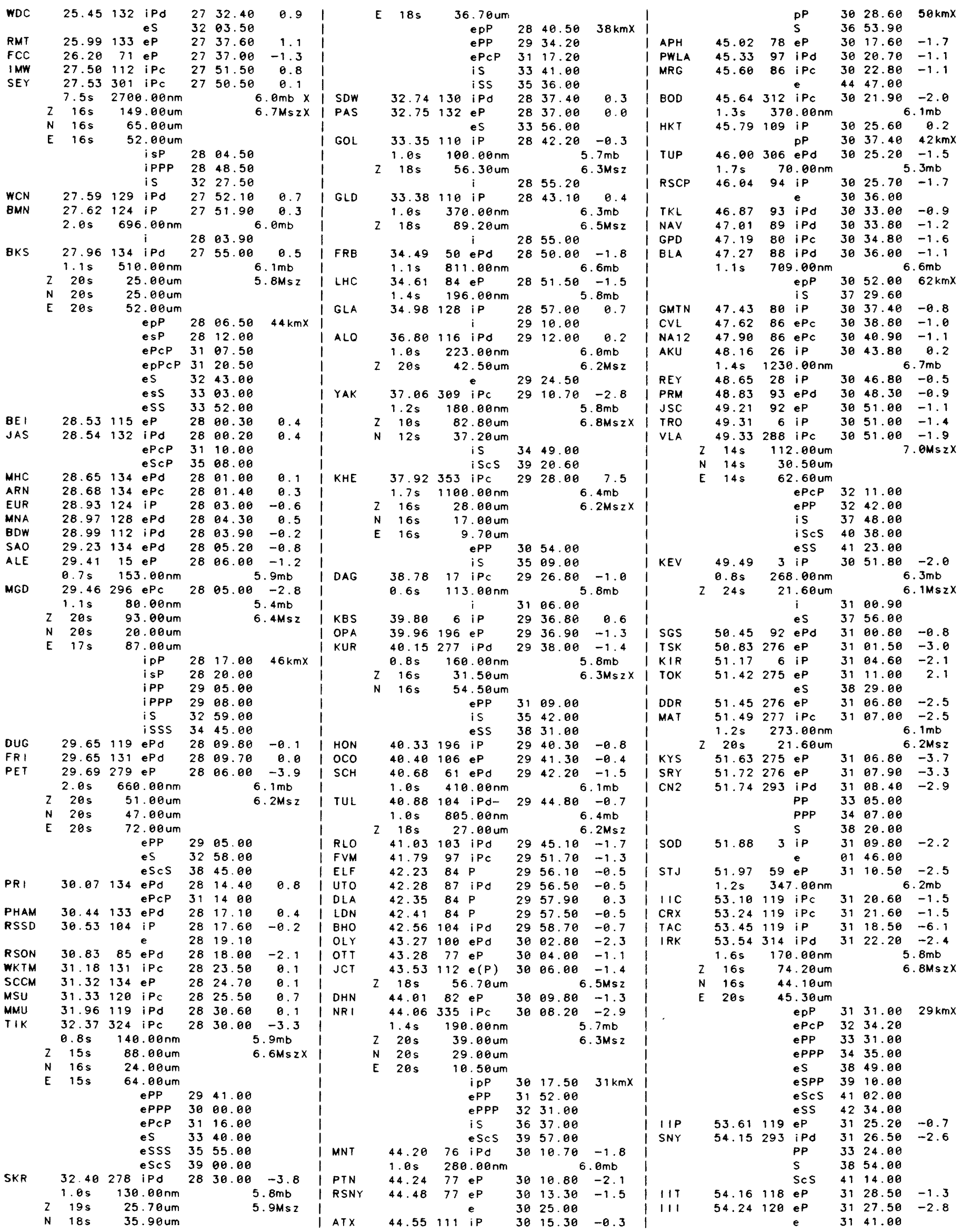




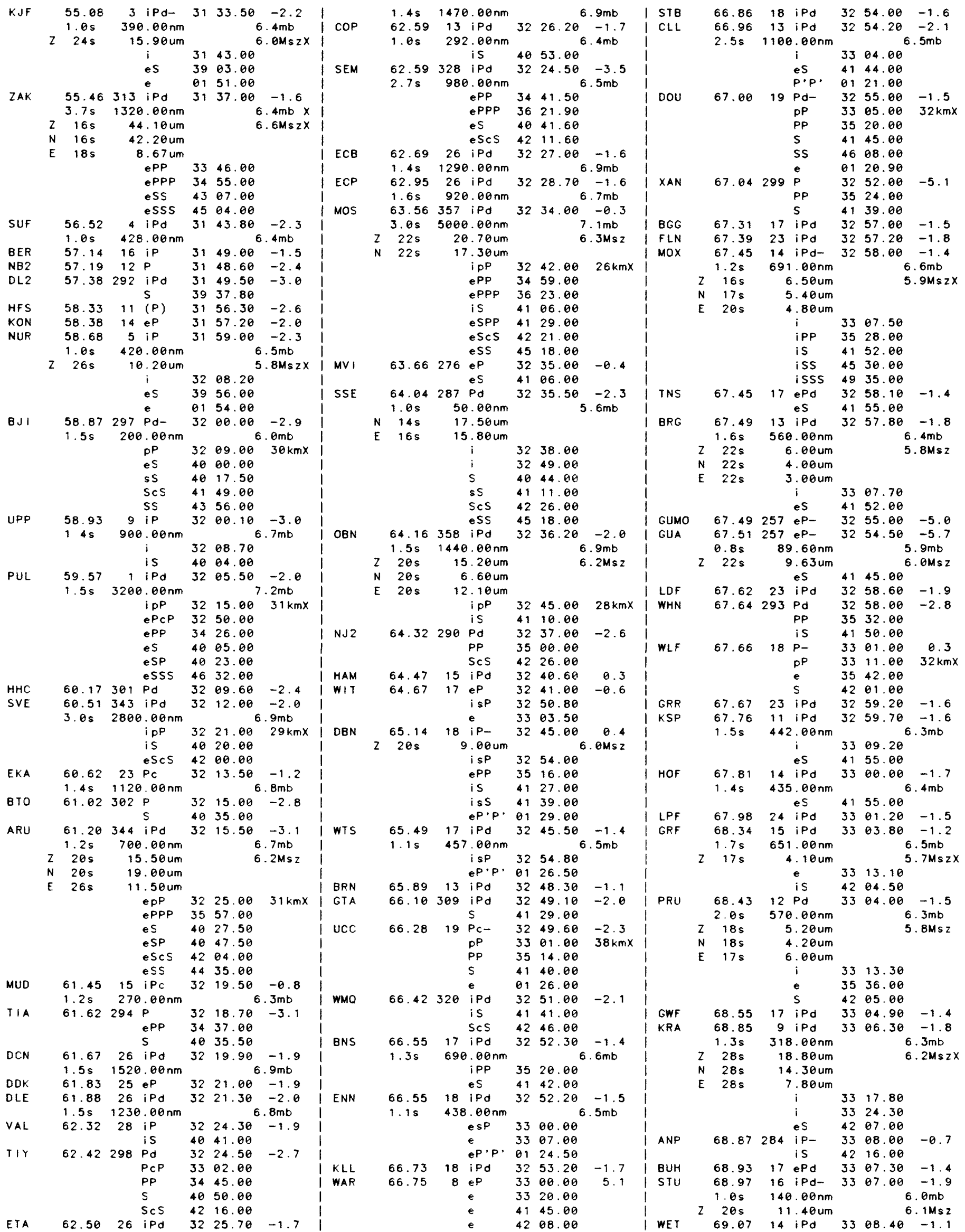


$07 d 19 n$

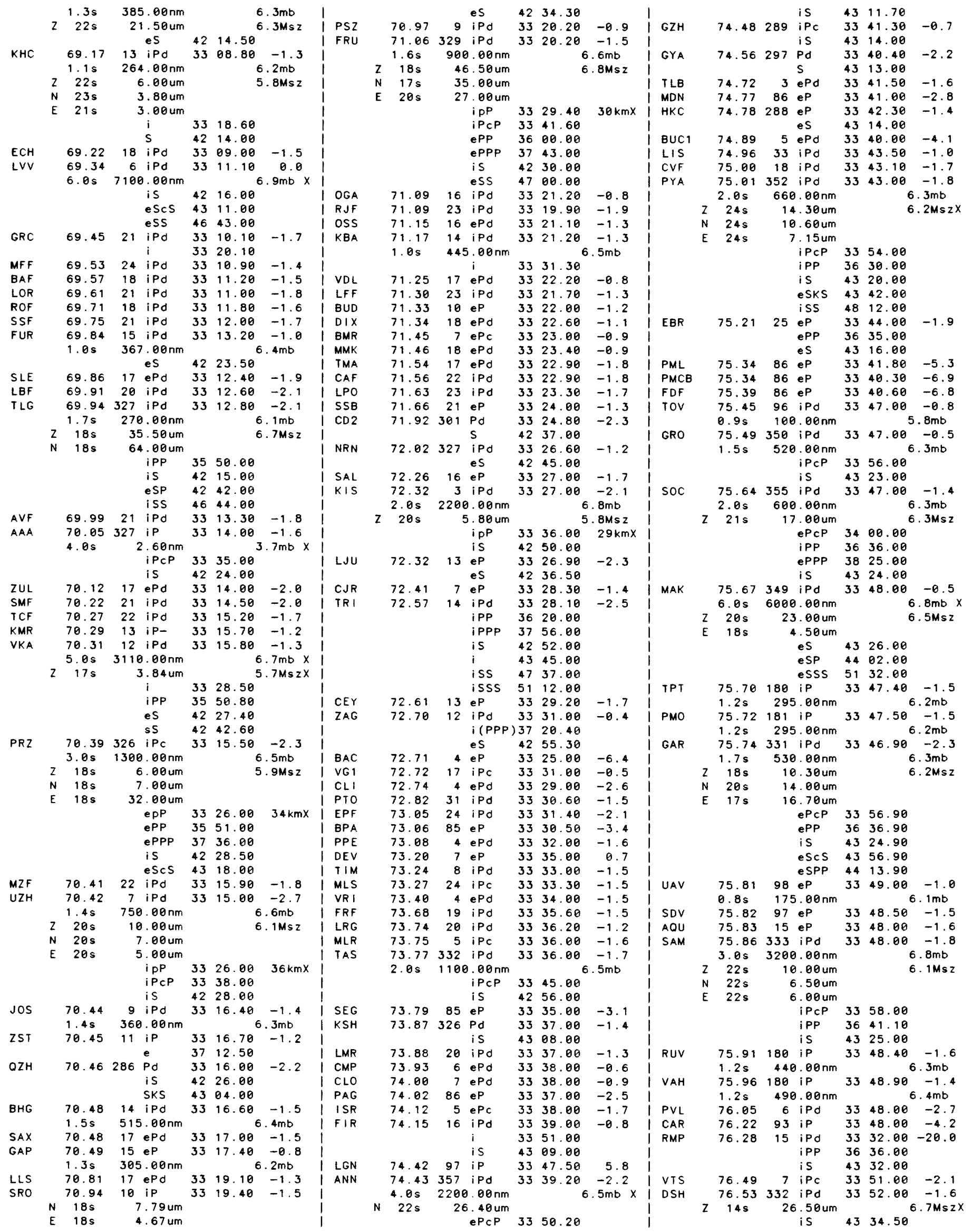


DU

JMB

$76.64 \quad 14 \mathrm{eP}$

$\begin{array}{lll}33 & 52.50 & -1.5\end{array}$

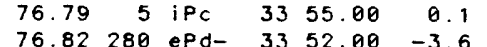
$1.3 \mathrm{~s} \quad 288.00 \mathrm{~nm}$ eS $43 \quad 36.00$

Кно

$\begin{array}{llllll}76.89 & 329 \text { iPd } & 33 & 36.00 & & \\ 73.60 & -2.1\end{array}$

$6.8 \mathrm{~s} 2600.00 \mathrm{~nm}$

$\begin{array}{lll}Z & 18 \mathrm{~s} & 27.70 \mathrm{um} \\ \mathrm{N} & 18 \mathrm{~s} & 13.90 \mathrm{um}\end{array}$

E $18 \mathrm{~s} \quad 12.20 \mathrm{um}$

$\begin{array}{lll}\text { ePCP } & 3406.60\end{array}$

ePP $36 \quad 44.60$

ePPP $38 \quad 37.20$

eS 4338.00

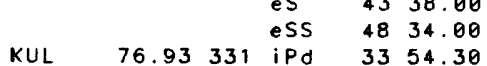

$3.2 \mathrm{~s} 2100.00 \mathrm{~nm}$

$Z 14 \mathrm{~s} \quad 11.20 \mathrm{um}$

$\begin{array}{lrr}\mathrm{N} & 14 \mathrm{~s} & 22.90 \mathrm{um} \\ \mathrm{E} & 14 \mathrm{~s} & 6.70 \mathrm{um}\end{array}$

iPP $\quad 36 \quad 46.60$

$\begin{array}{llllll} & & \text { is } & 43 & 38.60 & \\ \text { SKO } \quad 77.00 & 9 & \text { iPd } & 33 & 54.60 & -1.4\end{array}$

is $43 \quad 38.60$

$\begin{array}{lll}1.2 \mathrm{~s} & 450.00 \mathrm{~nm} & 33.60-1.4 \\ & 6.4 \mathrm{mb}\end{array}$

$\begin{array}{llll}\text { IPCP } & 34 & 03.80\end{array}$

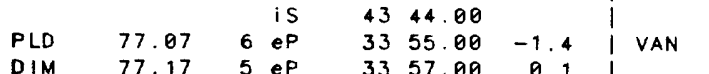

DIM

BKR

$77.17,5$ eP

$$
\text { ePCP }
$$

57.08

$\begin{array}{lll}33 & 56.00 & -1.1\end{array}$

es

4341.40

$\begin{array}{lll}77.26 & 352 \mathrm{iPd} \\ 1.4 \mathrm{~s} & 370.00 \mathrm{~nm}\end{array}$

$Z \begin{array}{ll}1.4 \mathrm{~s} & 370.00 \mathrm{~nm} \\ \mathrm{~N} & 11.90 \mathrm{um}\end{array}$

$\mathrm{N} 21 \mathrm{~s} \quad 7.50 \mathrm{um}$

E $21 \mathrm{~s} \quad 7.10 \mathrm{um}$

FUO $\quad$\begin{tabular}{rrrrr|l} 
& is & 43 & 44.00 & & PSO \\
KMI & $102 \mathrm{eP}$ & 33 & 58.00 & -0.4 & IEZN
\end{tabular}

KMI $\quad 77.42299$ iPd- $3356.50-2.4$ ABA $\begin{array}{ll}5.0 \mathrm{~s} & 1.70 \mathrm{~nm}\end{array}$

N $16 \mathrm{~s} 22.70 \mathrm{um}$

PP $\quad 34 \quad 06.00 \quad 30 \mathrm{kmX}$

PPP

is

$38 \quad 43.00$

4343.00

SKS $44 \quad 02.00$

DMK

KD

KDZ
BRT
VAY

VAY

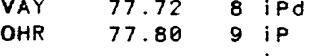

is

$\begin{array}{lll}\text { SGO } & 77.83 & 13 \mathrm{iPd} \\ & 1.5 \mathrm{~s} & 590.00 \mathrm{~nm}\end{array}$

SFS

$77.84 \quad 31 \mathrm{iPd}$

$i$ iPP
is
eSS

BOC eSS

BOCO $77.92 \quad 103 \stackrel{\mathrm{eS}}{\mathrm{P}}$

KAS $\quad 78.00 \quad 359 \quad \mathrm{iPC}$

BAK

\section{$78.02347 \mathrm{iPC}$}

$4.0 \mathrm{~s} 3100.00 \mathrm{~nm}$

IPCP $34 \quad 12.00$

ISKS 4404.00

MA

LEN

$$
\begin{array}{cccc}
78.03 \quad 30 & \text { iPd } & 34 & 02.00 \\
& \text { iPP } & 37 & 00.00 \\
& \text { is } & 43 & 56.00
\end{array}
$$

78.20351 iPd

$1.5 \mathrm{~s} \quad 700.00 \mathrm{~nm}$

$N 20 \mathrm{~s} 6.10 \mathrm{um}$

E $20 \mathrm{~s} \quad 2.30 \mathrm{um}$

$\begin{array}{lll}\text { EPP } & 37 \quad 05.00\end{array}$

EPPP $38 \quad 51.00$ es

PPN

$78.25 \quad 182 \quad$ iP

4354.00

KVT
AFR

ISK $\quad 1.2 \mathrm{~s} \quad 390.00 \mathrm{~nm}$

3401.60

$\begin{array}{ll}34 & 54.00-1.5\end{array}$

ist

$$
\begin{array}{ll}
78.27 & 3 \text { iP } \\
78.29 & 3
\end{array}
$$

3401.89

$80-1$.

$6.3 \mathrm{mb}$

RAB
PRK

PRK
MOM

ALT

MH I

PPT
I

1

SET

I TAB

VLS
IZM

I I M

ATH

ME I
SVO

HNR

I

I

I CGP

I CIN KKN BCK PK | 0.2

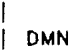

TEH DAV I

ELL MSL

i

$$
\begin{array}{lll}
\text { iPd } & 34 & 01.50 \\
\text { is } & 43 & 56.00 \\
\text { ip } & 34 & 02.00
\end{array}
$$

$\begin{array}{llllll}78.29 & 182 \text { if } & 34 & 02.00 & -1.3\end{array}$

$1.2 \mathrm{~s} \quad 475.00 \mathrm{~nm}$

$$
6.4 \mathrm{mb}
$$

$\begin{array}{llllll}78.33 & 341 \text { IPd } & 34 & 03.00 & -0.4\end{array}$ $7.0 \mathrm{~s} \quad 3100.00 \mathrm{~nm}$ $Z 16 \mathrm{~s} \quad 34.40 \mathrm{um}$ N $16 \mathrm{~s} \quad 43.00 \mathrm{um}$ $16 \mathrm{~s} \quad 19.50 \mathrm{um}$

$\begin{array}{ll}78.39 & 182 \mathrm{iP} \\ 1.2 \mathrm{~s} & 490.00 \mathrm{~nm} \\ 78.50 & 182 \mathrm{iP}\end{array}$

$\begin{array}{lll}78.53 & 2 & \text { iP } \\ 78.68 & 8 & \text { iP }\end{array}$

$78.73 \quad 351$ iPd

$\begin{array}{ll}78.77 & 3 \text { i } \mathrm{iP} \\ 78.95 & 4 \mathrm{iPc}\end{array}$

$78.95 \quad 4 \quad \mathrm{iPc}$

$79.26349 \mathrm{iPd}$

$4.0 \mathrm{~s} 2600.00 \mathrm{~nm}$

$\begin{array}{llr}Z & 18 \mathrm{~s} & 9.00 \mathrm{um} \\ \mathrm{N} & 18 \mathrm{~s} & 6.90 \mathrm{um}\end{array}$

E $18 \mathrm{~s} \quad 6.40 \mathrm{sm}$

iPCP $34 \quad 17.80$

$\begin{array}{lll}\text { ePP } & 37 & 12.40\end{array}$

is 4405.00

eSCS $44 \quad 19.60$

iSP $44 \quad 48.00$

$\begin{array}{llllll}79.25 & 340 \text { iPd } 34 & 07.20 & -1.3\end{array}$

$Z 20 \mathrm{~s} 20.30 \mathrm{um}$

$\begin{array}{lll}N & 20 \mathrm{~s} & 23.80 \mathrm{um} \\ \mathrm{E} & 20 \mathrm{~s} & 14.20 \mathrm{um}\end{array}$

ePCP $34 \quad 16.40$

$\begin{array}{lll}\text { ePP } & 37 & 14.00\end{array}$

eS 4405.00

eScS $44 \quad 22.00$

ePS 4454.00

ESS $49 \quad 12.00$

\begin{tabular}{lllll|l}
79.42 & $5 \mathrm{iPC}$ & 34 & 07.00 & -2.3 & SNG
\end{tabular}

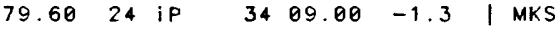

$\begin{array}{lllllll}79.72 & 3 & \text { iPd } & 34 & 09.40 & -1.6 & \text { IPM }\end{array}$

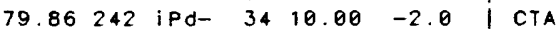

$\begin{array}{llllll}79.99 & 5 & \text { ip } & 34 & 11.00 & -1.4\end{array}$

80.04247 eP $\quad 34 \quad 11.00 \quad-2.0$

$80.30 \quad 2$ iPd $34 \quad 13.20 \quad-1.0$

$80.41 \quad 91$ iPd $34 \quad 12.90 \quad-2.1$

80.62338 iPd- $34+5.00 \quad-0.9$

$1.3 \mathrm{~s} \quad 246.00 \mathrm{~nm}$

$6.1 \mathrm{mb}$

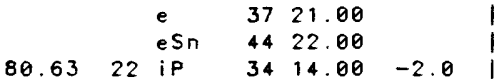

80.68349 iPd- $3416.00-0.3$

$80.70 \quad 10$ iP $\quad 34 \quad 14.50 \quad-1.7$

$\begin{array}{lllllll}80.88 & 4 \mathrm{eP} & 34 & 13.10 & -4.1 & \text { ISO }\end{array}$

81.137 iPd $3416.00-2.4$ |TRT

is 4423.50

$\begin{array}{llllll}81.14 & 14 \mathrm{eP} & 34 & 16.00 & -2.5\end{array}$

ASPA

$81.21233 \mathrm{eP}$

$81.42233 \mathrm{eP}$ -

$\begin{array}{lll}34 & 16.00 & -2.5 \\ 34 & 17.00 & -2.1\end{array}$

4233
eS

3418.00

eSS

4428.00

CLO 5244.00

$81.70272 \mathrm{eP}$

$52 \quad 14.00$

$81.70 \quad 4 \mathrm{iPc} \quad 34 \quad 19.80$

3430.00

81.81315 iPd $34 \quad 21.20 \quad-1.2$

$0.8 \mathrm{~s} \quad 150.00 \mathrm{~nm}$

$6.1 \mathrm{mb}$

$\begin{array}{llllll}90 & 2 & \text { ipd } & 34 & 20.40 & -2.1\end{array}$

$81.97 \quad 314$ iPd $34 \quad 21.70 \quad-1.7$

$1.0 \mathrm{~s} 260.00 \mathrm{~nm}$

82.04315 iPd $3422.3 \theta^{-1.4}$

82.42345 eP $\quad 3423.00 \quad-2.4$

82.45271 ePd- $3424.00-1.6$

$1.0 \mathrm{~s} \quad 320.00 \mathrm{~nm}$

es

$82.61352 \mathrm{ePd}$

$$
\text { e }
$$

$6.3 \mathrm{mb}$

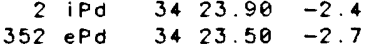

3434.50
e $\quad 4439.50$

eSP 4527.50

$83.40214 \mathrm{eP}$

$83.48213 \mathrm{eP}$

$83.53253 \mathrm{ePd} 3429.80$

1. Os $200.00 \mathrm{~m}$ $200.00 \mathrm{~nm}$

3429.00

$22 \mathrm{~s}$

$34.40 \mathrm{um}$

E $22 \mathrm{~s}$

$16.70 \mathrm{um}$

ITR

I ARO

BAO

CAN

TOO

BNG

BNG

I MEK

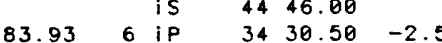

84.28348 eP $3435.00 \quad 0.1$

$84.32 \quad 245 \mathrm{eP} \quad 3433.00-2.1$

$84.39 \quad 169$ iP $\quad 34 \quad 33.60 \quad-1.6$

$1.2 \mathrm{~s} \quad 240.00 \mathrm{~nm} \quad 6.3 \mathrm{mb}$

84.61299 iPd $34 \quad 34.00 \quad-2.7$

$1.0 \mathrm{~s} \quad 60.00 \mathrm{~nm} \quad 5.7 \mathrm{mb}$

$\begin{array}{llllll}84.61299 & \mathrm{eS} & 4456.00 & \\ \mathrm{P} & 3434.20 & -2.4\end{array}$

$85.60350 \mathrm{eP} \quad 3441.50 \quad 0.2$

$\begin{array}{llllll}85.71 & 251 & \text { iPd } & 34 & 40.00 & -2.2\end{array}$

85.82243 eP $\quad 3440.00 \quad-2.8$

$\begin{array}{llllll}85.98 & 299 \text { iPd } 34 \quad 42.80 & -0.6\end{array}$

$1.5 \mathrm{~s} \quad 380.00 \mathrm{~nm} \quad 6.4 \mathrm{mb}$

$\begin{array}{llllllll}86.06 & 357 & \text { iP } & 34 & 43.50 & -0.3\end{array}$

$86.70244 \mathrm{eP}-\quad 3443.00-3.9$

86.89297 iPC $34 \quad 46.20 \quad-1.7$

87.21295 iPd $34 \quad 48.70 \quad-0.8$

$1.0 \mathrm{~s} \quad 42.40 \mathrm{~nm} \quad 5.6 \mathrm{mb}$

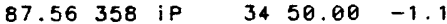
eS $45 \quad 14.00$

$87.90279 \mathrm{ePd} \quad 34 \quad 49.00 \quad-3.9$

88.26343 eP $\quad 3453.00 \quad-1.6$

88.35298 eP $3458.50 \quad 3.5$

88.99358 eP $\quad 3457.50-0.4$

$89.49 \quad 1$ iP- 3514.0013 .8

89.80296 eP $\quad 3457.00 \quad-4.9$

90.08224 iPd $3500.60 \quad-2.2$

90.52263 ePC $3502.00-3.1$

90.98222 iPd $35 \quad 05.30 \quad-1.6$

$93.70 \quad 317 \mathrm{ePd} \quad 35 \quad 17.00 \quad-2.8$

$1.6 \mathrm{~s} \quad 308.00 \mathrm{~nm} \quad 6.5 \mathrm{mb}$

$94.16 \quad 321$ iPd $35 \quad 20.00 \quad-1.9$

$1.3 \mathrm{~s} \quad 279.00 \mathrm{~nm} \quad 6.5 \mathrm{mb}$

$\begin{array}{lllll}94.21 & 293 \text { eP } & 35 & 21.00 & -1.1\end{array}$

96.09270 ePC $\quad 3528.80 \quad-1.9$

96.33291 ePd $3529.00 \quad-2.9$

96.59240 iPd $35 \quad 31.00 \quad-1.8$

$1.2 \mathrm{~s} \quad 79.70 \mathrm{~nm} \quad 6.1 \mathrm{mb}$

is $\quad 4624.00$

$\begin{array}{llllll} & & \text { i } & 5324.00 & \\ 97.07256 & \text { eP } & 3532.00 & -3.0\end{array}$ $97.62 \quad 316 \mathrm{Pd} \quad 35 \quad 34.70 \quad-3.0$ $0.9 \mathrm{~s} \quad 25.20 \mathrm{~nm} \quad 5.7 \mathrm{mb}$ 98.95292 ePd $3541.10 \quad-2.5$ $1.0 \mathrm{~s} \quad 37.30 \mathrm{~nm} \quad 5.9 \mathrm{mb}$ $\begin{array}{lllll}99.17 & 107 \text { eP } & 35 & 42.00 & -3.0\end{array}$ SKS $46 \quad 22.00$

$100.05 \quad 245$ ePdiff35 $45.00 \quad-3.6$ 101.57275 iPdiff35 $54.20-1.2$ $105.16 \quad 249$ ePdiff36 $13.00 \quad 1.8$ $\begin{array}{lllll}105.96 & 80 & \text { ePdiff36 } & 12.90 & -2.1\end{array}$ $\begin{array}{lll}\text { e } & 4012.80 \\ \text { e } & 4036.30\end{array}$ e 4042.80 
07d 19h

\begin{tabular}{|c|c|c|c|c|c|c|c|c|c|c|c|c|}
\hline & $22 \mathrm{~s}$ & & $2.40 \mathrm{um}$ & & & $.6 \mathrm{Msz}$ & I MUR & & 2.89 & 50 & iPn & 42 \\
\hline PRE & 144.65 & 7 & iPKPd & 41 & 37.00 & -2.9 & 1 & & $0.4 \mathrm{~s}$ & & $2.30 \mathrm{~nm}$ & \\
\hline & $1.3 \mathrm{~s}$ & 1200 & $0.00 \mathrm{~nm}$ & & & & & & & & eSn & 43 \\
\hline KSR & 144.68 & 9 & IPKPd & 41 & 33.50 & -6.5 & I URT & & 3.76 & 334 & iPn & 43 \\
\hline$|\mathbf{P}|$ & 145.06 & 7 & iPKPd & 41 & 32.70 & -8.0 & I FRG & & 3.88 & 7 & iPnd & 43 \\
\hline CNG & 145.32 & 1 & iPKPd & 41 & 37.00 & -3.8 & 1 & & $4.0 \mathrm{~s}$ & & $0.00 \mathrm{~nm}$ & \\
\hline & $0.9 \mathrm{~s}$ & 590 & $0.00 \mathrm{~nm}$ & & & & 1 & & & & i $S n$ & 43 \\
\hline & & & $i p P$ & 41 & 48.00 & & ANR & & 4.32 & 13 & iPnd & 43 \\
\hline EVA & 145.45 & 6 & iPKPd & 41 & 39.30 & -2.0 & 1 & & $4.0 \mathrm{~s}$ & $* * * *$ & $* * * n \mathrm{~nm}$ & \\
\hline FS & 145.69 & 9 & ¿PKPO & 41 & 39.30 & -2.4 & I NAM & & 4.46 & & ePn & 43 \\
\hline PRY & $\begin{array}{c}1.25 \\
145.78\end{array}$ & $\begin{array}{c}706 \\
8\end{array}$ & $\begin{array}{l}6.00 \mathrm{~nm} \\
\text { iPKPC }\end{array}$ & 41 & 39.50 & -2.3 & SAM & & $\begin{array}{c}2.2 s \\
4.52\end{array}$ & 315 & $\begin{array}{l}5.00 \mathrm{~nm} \\
\text { iPnd }\end{array}$ & 43 \\
\hline & $1.0 \mathrm{~s}$ & 220 & $0.00 \mathrm{~nm}$ & & & & I KSH & & 4.82 & 51 & iPd & 43 \\
\hline SWZ & 145.84 & 12 & iPKPc & 41 & 39.00 & -2.9 & 1 & & & & $\mathrm{~s}$ & 44 \\
\hline $\mathrm{JOZ}$ & 146.47 & 1 & IPKPd & 41 & 48.70 & 5.9 & I TAS & & 4. 99 & 344 & iPnd & 43 \\
\hline VIR & 146.87 & 9 & IPKPd & 41 & 43.00 & -0.6 & 1 & & $1.2 \mathrm{~s}$ & $* * * *$ & $* * * * n m$ & \\
\hline OKP & 147.16 & 24 & IPKPd & 41 & 43.30 & -0.6 & I NRN & & 6.19 & 36 & iPnd & 43 \\
\hline & $1.0 \mathrm{~s}$ & 320 & $0.00 \mathrm{~nm}$ & & & & I FRU & & 6.84 & 22 & iPnd & 43 \\
\hline SEK & 147.17 & 8 & iPKPd & 41 & 42.40 & -1.7 & 1 & & 3.25 & $* * * *$ & $* * * * \mathrm{~nm}$ & \\
\hline IM & 147.34 & 13 & iPKPC & 41 & 40.90 & -3.4 & 1 & & & & isn & 45 \\
\hline & $0.9 \mathrm{~s}$ & 226 & $6.00 \mathrm{~nm}$ & & & & I OUE & & 7.23 & 210 & iPd & 43 \\
\hline & & & i & 41 & 52.30 & & 1 & & & & es & 44 \\
\hline LF & 147.83 & 11 & ePKP & 41 & 39.00 & -6.1 & I AAA & & 8.08 & 32 & iPnd & 44 \\
\hline HVD & 149.25 & 12 & iPKPd & 41 & 46.00 & -1.3 & 1 & & $3.0 \mathrm{~s}$ & & $4.30 \mathrm{~nm}$ & \\
\hline & $1.2 \mathrm{~s}$ & 240 & $0.00 \mathrm{~nm}$ & & & & I PRZ & & 8.18 & 41 & iPnc & 44 \\
\hline SUR & 150.35 & 21 & iPKPd & 41 & 46.90 & -2.0 & 1 & & $2.0 \mathrm{~s}$ & 6900 & $0.00 \mathrm{~nm}$ & \\
\hline & $0.8 \mathrm{~s}$ & 53 & $3.70 \mathrm{~nm}$ & & & & I TLG & & 8.26 & 34 & $i P_{n}$ & 44 \\
\hline & $20 \mathrm{~s}$ & & 4. $61 \mathrm{um}$ & & & $.3 M S Z$ & | NDI & & 9.36 & 145 & iPnd & 44 \\
\hline & & & $\mathrm{i}$ & 41 & 52.70 & & 1 & & $0.7 \mathrm{~s}$ & 801 & $1.80 \mathrm{~nm}$ & \\
\hline SPA & 150.81 & 180 & ePKPc & 41 & 43.80 & -4.7 & ! & & & & is & 45 \\
\hline & $1.0 \mathrm{~s}$ & 23 & 3.0 & & & & I MHI & & 9.38 & 272 & iPnd- & 44 \\
\hline & $19 \mathrm{~s}$ & & $7.09 \mathrm{um}$ & & & $.5 \mathrm{Msz}$ & 1 & & $0.5 \mathrm{~s}$ & 1190 & $0.00 \mathrm{~nm}$ & \\
\hline & & & e & 41 & 53.00 & & 1 & & & & esn & 45 \\
\hline TUH & 150.94 & 24 & IPKPC & 41 & 47.50 & -2.0 & I ASH & & 10.28 & 282 & iPc & 44 \\
\hline & $0.6 \mathrm{~s}$ & 23 & $3.00 \mathrm{~nm}$ & & & & 1 & & $1.4 \mathrm{~s}$ & $* * * *$ & $* * * * \mathrm{~nm}$ & \\
\hline$B L E$ & 151.42 & 25 & iPKPC & 41 & 50.00 & -0.2 & I KHI & & 10.47 & 260 & iPd & 44 \\
\hline & $1.2 \mathrm{~s}$ & 196 & $0.00 \mathrm{~nm}$ & & & & I VAN & & 10.48 & 282 & iP & 44 \\
\hline GRM & 152.04 & 11 & iPKPd & 41 & 48.00 & -3.2 & 1 & & $4.0 \mathrm{~s}$ & $* * *$ & $* * * * n m$ & \\
\hline & $0.6 \mathrm{~s}$ & 535 & $5.80 \mathrm{~nm}$ & & & & I KAT & & 12.84 & 287 & iPd & 44 \\
\hline & $20 s$ & 10 & $9.30 \mathrm{um}$ & & & $.6 \mathrm{Msz}$ & 1 WMO & & 14.60 & 55 & $P c$ & 45 \\
\hline IAW & 165.45 & 230 & iPKPd & 42 & 03.90 & -1.6 & 1 & & & & sP & 46 \\
\hline & $0.9 \mathrm{~s}$ & 71 & 1.0 & & & & 1 & & & & $\mathrm{~S}$ & 48 \\
\hline NVL & 167.09 & 148 & iPKP & 42 & 00.00 & -6.7 & 1 & & & & $\mathbf{i}$ & 50 \\
\hline & & & epPKP & 42 & 10.00 & & 1 & & & & $S C P$ & 53 \\
\hline & & & $\mathrm{i}$ & 43 & 09.00 & & 1 & & & & $\mathrm{PcS}$ & 54 \\
\hline & & & iPP & 46 & 59.00 & & 1 & & & & Scs & 57 \\
\hline & 534 obs & $\therefore$ as & ssociat & ted & & & I DMN & & 14.81 & 123 & iPd & 45 \\
\hline & & & & & & & I KKN & & 14.81 & 122 & IPd & 45 \\
\hline st & $P \quad 12,1$ & 1983 & $15 h$ & $42 m$ & $08.57 \pm$ & $0.06 \mathrm{~s}$ & | PK I & & 15.04 & 122 & iPd & 45 \\
\hline & $.536 \mathrm{~N} \pm$ & 1.4 & $\mathrm{~km}$ & 71.1 & $122 E \pm$ & $1.0 \mathrm{~km}$ & I SEM & & 15.35 & 23 & iP & 45 \\
\hline & $P T H=20$ & $38.9 k$ & $\mathrm{~km} \quad(9$ & 910 & depth $p$ & hoses) & 1 & & $1.9 \mathrm{~s}$ & 4740 & $0.00 \mathrm{~nm}$ & \\
\hline & $3 \mathrm{mb}(130$ & obs & 5.) & & & & 1 & & & & is & 48 \\
\hline AFGI & IAN ISTAN- & -USSR & BORDE & $E R \quad R$ & REGION & $(717)$ & $E H$ & & 15.97 & 273 & ePc & 45 \\
\hline & Slight & dama & age in & the & $\Rightarrow$ Koshm & & SHI & & 17.02 & 252 & iPd & 45 \\
\hline & Volley. & $\mathrm{Fel}$ & $t(v)$ & at & Khorog & & I BAK & & 17.05 & 289 & $i P c$ & 46 \\
\hline & Pyondzh & , Sh & hoortuz & Z. D & Dust i or & nd & I LNK & & 17.79 & 284 & iPd & 46 \\
\hline & Gissor: & $11 \mathrm{~V}$ & $(-v)$ at & $t \mathrm{Ku}$ & diyob, & Nurek, & 1 & & & & es & 49 \\
\hline & Dushonb & se, 0 & Drdzhon & $n i k i$ & dzeoba & d, & I LSA & & 18.09 & 106 & $i P c$ & 46 \\
\hline & Toshken & t. G & Form, U & Uro- & - Tyube. & & 1 & & & & $\mathbf{s P}$ & 47 \\
\hline & Pendzhi & $k e n t$ & $t$ and $L$ & Leni & nabod: & (IV) & 1 & & & & $\mathrm{~S}$ & 49 \\
\hline & at Ishk & $\operatorname{coshi}$ & $\mathrm{m}$, Rus & shon & & & I POO & & 18.09 & 172 & $\mathrm{iPc}$ & 46 \\
\hline & Kalinin & rabad & d, Kurg & gan- & -Tyube, & & I MAK & & 19.29 & 297 & iP & 46 \\
\hline & Somarko & and 0 & and And & dizh & lan: (1) & $(1-\mid V)$ & 1 & & 2.05 & 8500 & 0.0 & \\
\hline & at Obig & gorm & and $\mathrm{Ch}$ & himk & cent: ( & $(11)$ & 1 & & $11 \mathrm{~s}$ & 11 & $1.70 \mathrm{um}$ & \\
\hline & ot Dzhi & rgot & 101 and & d $\mathrm{Na}$ & ryn; & $11-$ & 1 & 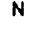 & $11 \mathrm{~s}$ & & $5.40 \mathrm{um}$ & \\
\hline & $111)$ at & $D z h$ & rombul & and & Talgo & $r$; and & 1 & & & & is & 49 \\
\hline & $(11)$ at & Fru & enze, U & USSR & 2. A $\mid \leq 0$ & felt & I KER & & 19.68 & 271 & eP & 46 \\
\hline & in the & Pesh & lawor $-L$ & Laha & dre are & $a$, & I GRS & & 19.74 & 286 & IPo & 46 \\
\hline & Pakisto & & & & & & 1 & & $3.0 \mathrm{~s}$ & 6100 & $0.00 \mathrm{~nm}$ & \\
\hline & & & & & & & I & & & & is & 49 \\
\hline KHO & 1.00 & 19 & iP & 42 & 39.90 & 0.1 & I TAE & & 19.77 & 282 & iP- & 46 \\
\hline & & & $e$ & 43 & 01.00 & & I KRV & & 19.79 & 290 & iPd & 46 \\
\hline KUL & 1.75 & 322 & iPnc & 42 & 45.30 & -0.3 & I & & & & es & 49 \\
\hline & & & is & 43 & 11.60 & & $E T$ & & 19.79 & 28 & i Pc & 46 \\
\hline LNA & 2.26 & 318 & ip & 42 & 50.90 & 0.2 & 1 & & $1.5 \mathrm{~s}$ & 2560 & $0.00 \mathrm{~nm}$ & \\
\hline & & & is & 43 & 20.90 & & 1 & & & & is & 49 \\
\hline$O B G$ & 2.45 & 333 & iP & 42 & $52.2 \theta$ & -0.7 & I HYB & & 20.15 & 159 & ePc & 46 \\
\hline & & & is & 43 & 24.80 & & 1 & & $0.8 \mathrm{~s}$ & 1570 & $0.00 \mathrm{~nm}$ & \\
\hline GAR & 2.54 & 346 & iP & 42 & 53.00 & -1.0 & 1 & & & & $\mathbf{i}$ & 46 \\
\hline & & & es & 43 & 25.50 & & 1 & & & & e & 47 \\
\hline$R U$ & 2.58 & 319 & iP & 42 & 53.60 & -0.7 & 1 & & & & es & 49 \\
\hline CGT & 2.62 & 324 & iPn & 42 & 53.40 & -1.3 & I NVS & & 20.18 & 21 & $\mathrm{iPC}$ & 46 \\
\hline & & & eSn & 43 & 26.40 & & 1 & & $0.8 \mathrm{~s}$ & 2090 & $0.00 \mathrm{~nm}$ & \\
\hline$D Z T$ & 2.68 & 2 & $i P_{n}$ & 42 & 55.30 & 0.0 & 1 & & $12 \mathrm{~s}$ & 21 & $1.78 \mathrm{um}$ & \\
\hline & & & esn & 43 & 29.30 & & 1 & & $12 \mathrm{~s}$ & 15 & 580 um & \\
\hline SH & 2.75 & 318 & iPnd & 42 & 55.30 & -1.0 & 1 & $E$ & $12 \mathrm{~s}$ & & $0.10 u m$ & \\
\hline & & & isn & 43 & 29.30 & & 1 & & & & i sP & 47 \\
\hline
\end{tabular}


$12 d \quad 15 h$

\begin{tabular}{|c|c|c|c|c|c|c|c|c|c|c|c|}
\hline & $10 \mathrm{~s}$ & & $0.96 \mathrm{um}$ & & & & 1 & & & iss & 54 \\
\hline & & & $\lg$ & 51 & 27.00 & & I ISK & 32.86 & 291 & ip & 48 \\
\hline & & & Lg & 51 & 50.00 & & I ELL & 32.89 & 283 & $\mathrm{IPC}$ & 48 \\
\hline ANN & 26.81 & 299 & iPc & 47 & 30.00 & -1.0 & $I \quad I S T$ & 32.91 & 291 & iPc & 48 \\
\hline & $0.8 \mathrm{~s}$ & 80 & $0.00 \mathrm{~nm}$ & & & $6.5 \mathrm{mb}$ & 1 & & & is & 53 \\
\hline & & & es & 51 & 50.40 & & I NST & 33.11 & 121 & IPd & 48 \\
\hline ZAK & 26.89 & 49 & iPc & 47 & 33.20 & 1.4 & I PSN & 33.31 & 296 & $\mathrm{i} \mathrm{Pc}_{\mathrm{c}}$ & 48 \\
\hline & $1.4 \mathrm{~s}$ & 109 & $10.00 \mathrm{~nm}$ & & & $6.4 \mathrm{mb}$ & 1 DST & 33.37 & 288 & iPc & 48 \\
\hline & & & epp & 48 & 17.00 & $221 \mathrm{kmX}$ & I TLB & 33.39 & 298 & iPc & 48 \\
\hline & & & esP & 48 & 38.68 & & I PPE & 33.68 & 300 & iPc & 48 \\
\hline & & & ePcP & 50 & 48.00 & & I DMK & 33.76 & 293 & IPd & 48 \\
\hline & & & es & 51 & 57.80 & & I EDC & 33.85 & 290 & IPd & 48 \\
\hline & & & ess & 53 & 11.60 & & I CLI & 33.91 & 301 & $i P C$ & 48 \\
\hline & & & ess & 53 & 23.00 & & I CIN & 34.14 & 285 & iPd & 48 \\
\hline KVT & 27.59 & 290 & iPd & 47 & 39.80 & 0.8 & 1 & & & $i$ & 51 \\
\hline $\mathrm{CD} 2$ & 27.64 & 92 & iPc & 47 & 46.70 & 2.0 & I BAC & 34.18 & 301 & ePd & 48 \\
\hline & & & $\mathrm{PP}$ & 48 & 24.00 & $217 \mathrm{~km}$ & | NR I & 34.21 & 11 & iPc & 48 \\
\hline & & & PP & 48 & 34.86 & & 1 & $2.0 \mathrm{~s}$ & 7400 & $0.00 \mathrm{~nm}$ & \\
\hline & & & is & 52 & 09.80 & & 1 & & & epp & 49 \\
\hline IRK & 28.14 & 45 & IPC & 47 & 43.60 & 0.0 & 1 & & & esP & 49 \\
\hline & $1.3 \mathrm{~s}$ & 86 & $0.00 \mathrm{~nm}$ & & & $6.3 \mathrm{mb}$ & 1 & & & es & 53 \\
\hline & & & $i p P$ & 48 & 27.00 & $220 \mathrm{kmX}$ & 1 & & & ess & 54 \\
\hline & & & esp & 48 & 51.00 & & | VRI & 34.38 & 300 & iPc & 48 \\
\hline & & & ePCP & 50 & 45.00 & & I JMB & 34.56 & 294 & $I P C$ & 48 \\
\hline & & & es & 52 & 11.00 & & $112 M$ & 34.62 & 287 & iPc & 48 \\
\hline$S I M$ & 29.07 & 298 & iPc & 47 & 51.00 & -0.3 & I PCT & 34.68 & 121 & $i P_{C}$ & 48 \\
\hline & $20 \mathrm{~s}$ & & 7.50 um & & & $5.3 M s z$ & 1 & $1.1 \mathrm{~s}$ & 430 & $3.00 \mathrm{~nm}$ & \\
\hline & $N \quad 20 \mathrm{~s}$ & & 0.00 um & & & & 1 & & & e & 49 \\
\hline & $20 \mathrm{~s}$ & & 5.28 um & & & & BUC1 & 34.83 & 297 & ip & 48 \\
\hline & & & ipP & 48 & 34.00 & $214 \mathrm{~km}$ & I MLR & 34.85 & 299 & $i P C$ & 48 \\
\hline & & & iPP & 48 & 50.00 & & I CGN & 34.86 & 297 & ePc & 48 \\
\hline & & & is & 52 & 26.00 & & I PUL & 34.94 & 325 & iPc & 48 \\
\hline$H R I$ & 29.10 & 274 & eP & 47 & 52.50 & 0.7 & 1 & $1.6 \mathrm{~s}$ & 4000 & $0.00 \mathrm{~nm}$ & \\
\hline KAS & 29.29 & 291 & IPC & 47 & 52.80 & -0.6 & 1 & $12 \mathrm{~s}$ & & $3.00 u m$ & \\
\hline KMI & 29.31 & 104 & iPct & 47 & 54.00 & 0.1 & 1 & $12 \mathrm{~s}$ & & 5.48 um & \\
\hline & $3.0 \mathrm{~s}$ & & $4.20 \mathrm{~nm}$ & & & $3.6 \mathrm{mb} x$ & 1 & $12 \mathrm{~s}$ & & $0.00 \mathrm{um}$ & \\
\hline & & & PP & 48 & 41.00 & $236 \mathrm{kmx}$ & 1 & & & $i p P$ & 49 \\
\hline & & & SP & 48 & 58.00 & & 1 & & & esP & 49 \\
\hline & & & $P C P$ & 50 & 54.00 & & $!$ & & & is & 53 \\
\hline & & & is & 52 & 34.00 & & 1 & & & ess & 55 \\
\hline & & & sS & 53 & 52.08 & & 1 & & & ess & 56 \\
\hline & & & SS & 54 & 27.00 & & I OBO & 34.99 & 233 & iPt & 48 \\
\hline MOS & 29.69 & 321 & IPd & 47 & 57.80 & 0. 4 & NNT & 35.05 & 126 & iPc & 48 \\
\hline & $2.0 \mathrm{~s}$ & 1200 & $0.00 \mathrm{~nm}$ & & & 6. $3 \mathrm{mb}$ & I EZN & 35.09 & 289 & iPd & 48 \\
\hline & $16 \mathrm{~s}$ & & $2.80 u m$ & & & 5. $6 \mathrm{MszX}$ & | BJ I & 35.25 & 70 & iPct & 48 \\
\hline & & & i pP & 48 & 46.00 & $213 \mathrm{~km}$ & 1 & $2.0 \mathrm{~s}$ & 1850 & $0.00 \mathrm{~nm}$ & \\
\hline & & & ePcP & 51 & 03.00 & & 1 & & & $\mathrm{pP}$ & 49 \\
\hline & & & es & 52 & 36.00 & & 1 & & & sP & 49 \\
\hline & & & ess & 53 & 44.00 & & 1 & & & $P \subset P$ & 51 \\
\hline OBN & 29.98 & 319 & iPd & 47 & 59.50 & 0.3 & I & & & $\mathrm{s}$ & 54 \\
\hline & $2.0 \mathrm{~s}$ & 2300 & $0.00 \mathrm{~nm}$ & & & $5.5 \mathrm{mb}$ & 1 & & & SCP & 54 \\
\hline & $20 \mathrm{~s}$ & & 9.00 um & & & $5.4 M s Z$ & 1 & & & $\mathrm{PCS}$ & 54 \\
\hline & $20 \mathrm{~s}$ & & 6.70 um & & & & 1 & & & sS & 55 \\
\hline & $20 \mathrm{~s}$ & & 7.80 um & & & & 1 & & & (SS) & 56 \\
\hline & & & is & 52 & 38.00 & & 1 & & & Ses & 58 \\
\hline JER & 29.99 & 272 & eP & 48 & 00.00 & 0.3 & I MKL & 35.29 & 233 & ePt & 48 \\
\hline & & & es & 52 & 40.00 & & $1 \mathrm{DIM}$ & 35.35 & 293 & IPd & 48 \\
\hline CHG & 30.15 & 118 & iPc & 48 & 01.00 & -0.1 & 1800 & 35.36 & 39 & iPc & 48 \\
\hline & $1.0 \mathrm{~s}$ & 198 & $0.00 \mathrm{~nm}$ & & & $5.8 \mathrm{mb}$ & 1 & $1.1 \mathrm{~s}$ & 1358 & $3.00 \mathrm{~nm}$ & \\
\hline & & & es & 52 & 52.00 & & I TDD & 35.38 & 233 & iPt & 48 \\
\hline RN I & 30.65 & 269 & eP & 48 & 06.00 & 0.6 & I ATA & 35.45 & 232 & iPt & 48 \\
\hline$B D T$ & 31.25 & 121 & eP & 48 & 13.80 & $3.2 x$ & I CMP & 35.58 & 299 & ePc & 48 \\
\hline & $1.0 \mathrm{~s}$ & 745 & $5.80 \mathrm{~nm}$ & & & 5. $3 \mathrm{mb}$ & I PVL & 35.54 & 295 & iPC & 48 \\
\hline $\mathrm{HHC}$ & 31.66 & 70 & iPc & 48 & 15.60 & 1.5 & I KDZ & 35.57 & 293 & IPd & 48 \\
\hline & & & S & 53 & 12.00 & & I ARO & 35.62 & 233 & iPt & 48 \\
\hline GYA & 31.77 & 98 & $\mathrm{Pc}$ & 48 & 16.00 & 0.7 & I DAF & 35.77 & 233 & iPt & 48 \\
\hline & & & SP & 49 & 23.00 & & $1 \mathrm{coz}$ & 35.98 & 299 & IPD & 48 \\
\hline & & & 5 & 53 & 09.00 & & $1 \quad P L D$ & 35.99 & 293 & iPc & 48 \\
\hline & & & PCS & 54 & 43.00 & & I LVV & 36.22 & 307 & $i P c$ & 48 \\
\hline GPA & 32.00 & 289 & iPc & 48 & 17.30 & 0.3 & 1 & $16 \mathrm{~s}$ & & 3.00 um & \\
\hline BCK & 32.21 & 284 & $e P$ & 48 & 17.60 & -1.4 & 1 & $10 \mathrm{~s}$ & & 5.26 um & \\
\hline ALT & 32.31 & 287 & iPC & 48 & 19.30 & -0.6 & 1 & & & $i p p$ & 49 \\
\hline HRT & 32.43 & 290 & iP & 48 & 21.10 & 0. 4 & 1 & & & $i s P$ & 49 \\
\hline YLV & 32.68 & 290 & iP & 48 & 23.20 & 0.2 & 1 & & & iPP & 50 \\
\hline KHT & 32.70 & 124 & iPd & 48 & 25.00 & 1.8 & 1 & & & iPCP & 51 \\
\hline TIY & 32.77 & 75 & $\mathrm{IPC}$ & 48 & 24.80 & 1.0 & 1 & & & is & 54 \\
\hline & & & $\mathrm{PP}$ & 49 & 08.50 & $212 \mathrm{~km}$ & WHN & 36.29 & 87 & $\mathrm{iPc}$ & 48 \\
\hline & & & $\mathbf{s P}$ & 49 & 31.00 & & 1 & & & PP & 49 \\
\hline & & & $\mathrm{s}$ & 53 & 28.60 & & 1 & & & $\mathbf{S P}$ & 50 \\
\hline & & & sS & 54 & 39.50 & & 1 & & & $\mathrm{PCP}$ & 51 \\
\hline & & & SS & 55 & 48.06 & & 1 & & & s & 54 \\
\hline & & & SCS & 58 & 25.00 & & 1 & & & sS & 55 \\
\hline 15 & 32.84 & 302 & iPc & 48 & 24.00 & -0.2 & 1 & & & SS & 56 \\
\hline & $1.0 \mathrm{~s}$ & 1700 & $0.00 \mathrm{~nm}$ & & & $.6 \mathrm{mb}$ & I CJR & 36.45 & 361 & eP & 48 \\
\hline & & & ipp & 49 & 07.00 & $268 \mathrm{~km}$ & I TIA & 36.77 & 76 & iPc & 48 \\
\hline & & & i sP & 49 & 28.00 & & 1 & & & sP & 50 \\
\hline & & & is & 53 & 26.06 & & & & & $\mathrm{PCP}$ & 51 \\
\hline
\end{tabular}




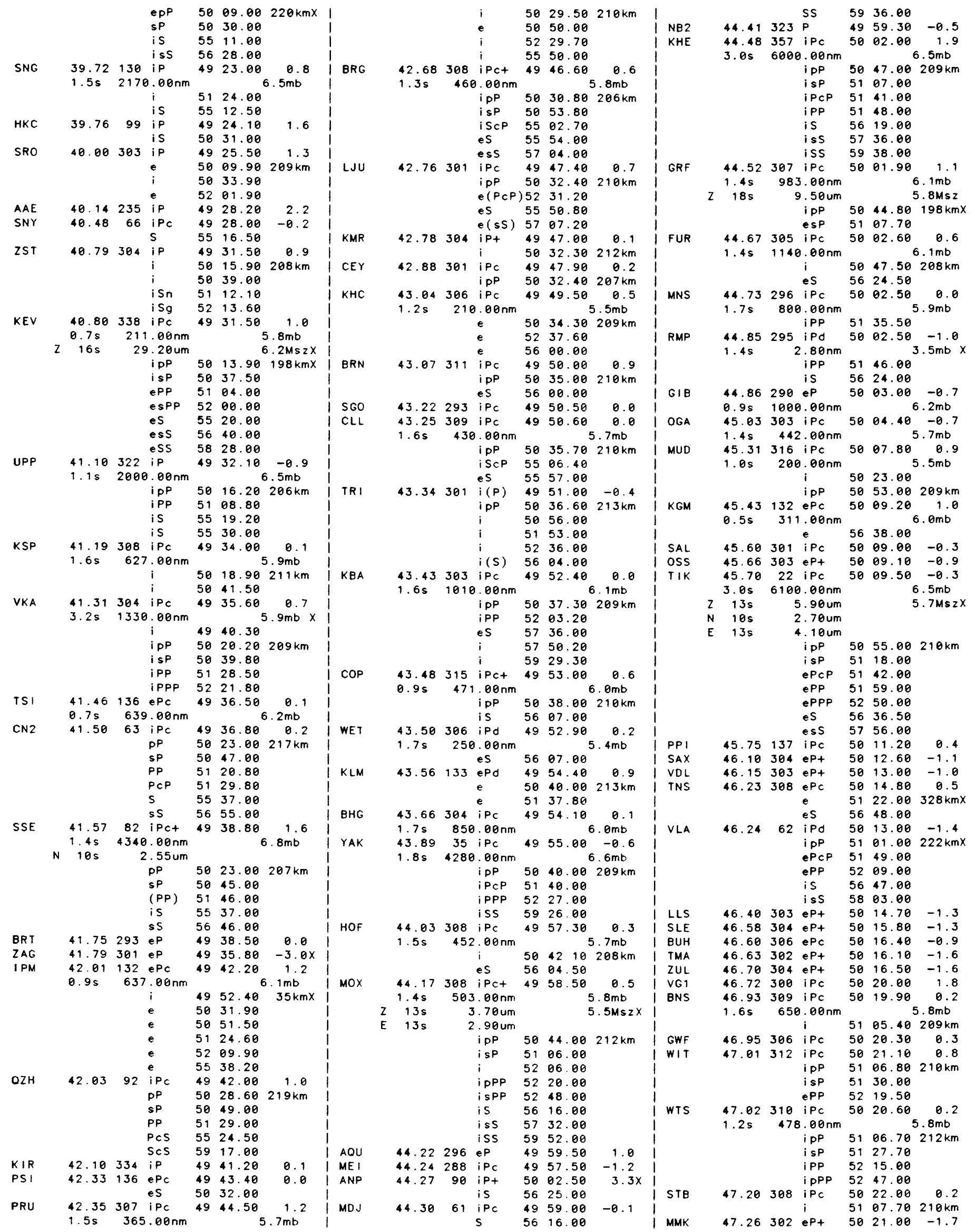




\begin{tabular}{|c|c|c|c|c|c|c|c|c|c|c|c|c|}
\hline$O F$ & $\begin{array}{l}47.27 \\
1.5 \mathrm{~s}\end{array}$ & $\begin{array}{c}305 \\
207\end{array}$ & $\begin{array}{c}\text { iPc } \\
7.00 \mathrm{~nm}\end{array}$ & 50 & 22.00 & $5.3 \mathrm{mo}$ & $\begin{array}{l}\text { I LOF } \\
\end{array}$ & 52.03 & 307 & $\begin{array}{l}\text { iPc } \\
\text { PP }\end{array}$ & $\begin{array}{l}50 \\
51\end{array}$ & $\begin{array}{l}57.60 \\
43.20\end{array}$ \\
\hline & & & PP & 51 & 07.40 & $208 \mathrm{~km}$ & $F L N$ & 52.22 & 307 & $i P_{C}$ & 50 & \\
\hline $\mathrm{ECH}$ & 7.38 & 305 & $\mathrm{iPC}$ & 50 & 22.80 & -0.5 & 1 & & & PP & 51 & 44.30 \\
\hline CVF & 47.43 & 297 & $\mathrm{iPC}$ & 50 & 23.00 & -0.7 & LPO & 52.23 & 302 & iPc & 50 & 59.70 \\
\hline & & & PP & 51 & 08.00 & $206 \mathrm{~km}$ & 1 & & & PP & 51 & 45.30 \\
\hline KLL & 47.52 & 309 & $\mathrm{IPC}$ & 50 & 24.20 & -0.1 & EKA & 52.33 & 316 & $\mathrm{Pd}$ & 51 & 00.40 \\
\hline & & & $i$ & 51 & 09.80 & $209 \mathrm{~km}$ & I & $1.2 \mathrm{~s}$ & 434 & $4.00 \mathrm{~nm}$ & & \\
\hline$A F$ & 7.56 & 305 & $i P c$ & 50 & 24.20 & -0.6 & LGP & 52.38 & 102 & eP & 51 & 03.00 \\
\hline $1 x$ & .63 & 302 & eP+ & 50 & 1.50 & -1.1 & I & $0 \mathrm{~s}$ & 83 & $3.00 \mathrm{~nm}$ & & \\
\hline$B S F$ & 47.70 & 305 & $i P C$ & 50 & 25.30 & -0.5 & LFF & 52.46 & 302 & iPc & 51 & 01.40 \\
\hline ENN & 7.75 & 309 & iPc & 50 & 25.20 & -0.8 & I & & & pP & 51 & 47.00 \\
\hline & $1.1 \mathrm{~s}$ & & $5.00 \mathrm{~nm}$ & & & $5.3 \mathrm{mb}$ & I GRR & 52.56 & 307 & iPc & 51 & 01.30 \\
\hline & & & ipP & 51 & 12.00 & $214 \mathrm{~km}$ & i & & & $p P$ & 51 & 46.90 \\
\hline & & & esp & 51 & 33.00 & & I MFF & 52.58 & 305 & $i P c$ & 51 & 01.50 \\
\hline & & & $\mathrm{IPCP}$ & 51 & 51.50 & & I LPF & 2.77 & 307 & $i P c$ & 51 & 02.80 \\
\hline & & & IPPP & 52 & 43.50 & & I & $0.5 \mathrm{~s}$ & & $5.00 \mathrm{~nm}$ & & \\
\hline LF & 47.78 & 307 & $P$ & 50 & 7.00 & 0.7 & 1 & & & PP & 51 & 48.80 \\
\hline & & & $P P$ & 51 & 14.00 & $215 \mathrm{~km}$ & I MLS & 2.82 & 300 & $i P_{C}$ & 51 & 03.00 \\
\hline & & & e & 51 & 39.00 & & I MAT & 52.85 & 69 & iPct & 51 & 03.40 \\
\hline & & & $P P$ & 52 & 27.00 & & i & 1.45 & 1220 & $0.00 \mathrm{~nm}$ & & \\
\hline & & & PPP & 53 & 02.00 & & I & $z \quad 20 \mathrm{~s}$ & & $1.42 \mathrm{um}$ & & \\
\hline & & & $\mathrm{s}$ & 57 & 09.00 & & I & & & es & 58 & 14.00 \\
\hline & & & ss & 58 & 17.00 & & I EPF & 3.32 & 300 & $i P c$ & 51 & 06.50 \\
\hline & & & e & 58 & 39.00 & & $A B A$ & $\theta$ & 292 & iP & 51 & 08.00 \\
\hline$A U$ & .96 & 305 & iPc & 50 & 27.30 & -0.4 & DDR & 9 & 69 & iPc & 51 & 10.70 \\
\hline DEN & 48.01 & 311 & $e(P)$ & 50 & 28.00 & 0.0 & I EBR & 53.93 & 298 & $e^{P}$ & 51 & 12.00 \\
\hline & $20 \mathrm{~s}$ & & $3.30 \mathrm{um}$ & & & 5. 3MsZ & 1 & & & es & 58 & 22.00 \\
\hline & & & epP & 51 & 13.00 & $205 \mathrm{~km}$ & i & & & ePS & 58 & 30.00 \\
\hline & & & esp & 51 & 36.00 & & I SRY & 4.00 & 69 & $i P c$ & 51 & 13.30 \\
\hline & & & IPPP & 53 & 23.00 & & I OrM & 5 & 69 & eP & 51 & 12.40 \\
\hline & & & is & 57 & 14.00 & & I MGD & .18 & 37 & $i P_{c}$ & 51 & 13.00 \\
\hline & & & iss & 58 & 42.00 & & 1 & $1.2 \mathrm{~s}$ & 1050 & $0.00 \mathrm{~nm}$ & & \\
\hline & & & ess & 00 & 48.00 & & i & $14 \mathrm{~s}$ & & $2.90 u m$ & & \\
\hline BAG & 48.02 & 101 & iPCt & 50 & 9.50 & 0.8 & I & E $14 \mathrm{~s}$ & & $2.70 \mathrm{um}$ & & \\
\hline & & & i & 51 & 38.00 & $332 \mathrm{kmX}$ & 1 & & & epp & 52 & 00.00 \\
\hline & & & es & 57 & 14.00 & & I & & & ePcP & 52 & 19.00 \\
\hline Dou & 48.70 & 308 & $\mathrm{Pc}+$ & 50 & 32.70 & -0.6 & I & & & ePP & 53 & 15.00 \\
\hline & & & $p P$ & 51 & 18.80 & $210 \mathrm{~km}$ & & & & ePPP & 54 & 37.00 \\
\hline & & & e & 51 & 27.20 & & 1 & & & es & 58 & 31.00 \\
\hline & & & SP & 51 & 39.50 & & i & & & ess & 59 & 53.00 \\
\hline & & & $\mathbf{P} \subset \boldsymbol{P}$ & 52 & 02.00 & & I SEY & 4.35 & 34 & $i P c$ & 51 & 15.30 \\
\hline & & & PPP & 53 & 14.00 & & 1 & $2.5 \mathrm{~s}$ & 3400 & $0.00 \mathrm{~nm}$ & & \\
\hline & & & PPPP & 53 & 41.30 & & i & & & $i p P$ & 52 & 02.80 \\
\hline & & & $\mathrm{s}$ & 57 & 21.00 & & $i$ & & & iPCP & 52 & 15.80 \\
\hline UCC & 48.72 & 309 & Pct & 50 & 32.80 & -0.7 & 1 & & & $i s \mathrm{P}$ & 52 & 24.80 \\
\hline & & & PP & 51 & 19.00 & $210 \mathrm{~km}$ & 1 & & & is & 8 & 38.00 \\
\hline & & & PPP & 53 & 14.00 & & I TSK & 4.40 & 68 & iPc & 1 & 14.30 \\
\hline & & & $\mathrm{s}$ & 57 & 24.00 & & I ETA & .70 & 313 & $i P c$ & 51 & 16.80 \\
\hline RF & 48.75 & 299 & iPc & 50 & 33.00 & -0.9 & 1 & $1 \mathrm{~s}$ & 355 & $5.00 \mathrm{~nm}$ & & \\
\hline & & & $p P$ & 51 & 18.50 & $207 \mathrm{~km}$ & DLE & .74 & 314 & $i P c$ & 51 & 17.30 \\
\hline LMR & 48.91 & 299 & $i P_{c}$ & 50 & 34.00 & -1.0 & I & $2 \mathrm{~s}$ & 2280 & $0.00 \mathrm{~nm}$ & & 6 \\
\hline & 2.05 & 512 & $2.00 \mathrm{~nm}$ & & & $5.6 \mathrm{mb}$ & I DAG & .75 & 344 & $i P_{c}$ & 51 & 17.20 \\
\hline & & & PP & 51 & 19.80 & $208 \mathrm{~km}$ & 1 & $1.0 \mathrm{~s}$ & 870 & $0.00 \mathrm{~nm}$ & & 6 \\
\hline LRG & 48.98 & 299 & iPc & 50 & 35.00 & -0.6 & & & & $i$ & 58 & 42.00 \\
\hline & & & $p P$ & 51 & 20.50 & $207 \mathrm{~km}$ & KYS & 1 & 69 & $i P c$ & 51 & 18.60 \\
\hline Ai & .31 & 228 & $i P_{c}$ & 50 & 40.00 & 1.4 & I ECP & .94 & 313 & iPc & 51 & 18.40 \\
\hline & $3 \mathrm{~s}$ & 577 & $7.00 \mathrm{~nm}$ & & & $5.9 \mathrm{mb}$ & I & $1.2 \mathrm{~s}$ & 820 & $0.00 \mathrm{~nm}$ & & 6 \\
\hline$H K$ & 37 & 73 & $i P c$ & 50 & 3 & & I LEM & 04 & 134 & ePd & 51 & 17.00 \\
\hline AN & .41 & 103 & $i$ & 50 & 80 & 3 & & Os & 60 & $0.00 \mathrm{~nm}$ & & \\
\hline LBF & 9.74 & 304 & $i P_{c}$ & 50 & 40.70 & -0.7 & i & & & es & 52 & 48.00 \\
\hline & & & p & 51 & 20 & $206 \mathrm{~km}$ & ECB & 3 & 313 & $i P_{c}$ & 51 & 20.00 \\
\hline$O R$ & 49.76 & 305 & iPc & 50 & 30 & -1.2 & i & & 1100 & $0.00 \mathrm{~nm}$ & & \\
\hline & & & PP & 1 & & $206 \mathrm{~km}$ & $I O C N$ & & 314 & iPc & 51 & 20.40 \\
\hline 58 & 8 & & $i P c$ & 0 & 00 & -0.7 & I & & 1770 & $0.00 \mathrm{~nm}$ & & \\
\hline SMF & 2 & 4 & $i P c$ & 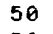 & 30 & -0.7 & I LGR & 18 & 300 & $i P_{c}$ & 51 & 23.30 \\
\hline GGP & 9 & 104 & if & 50 & 41.80 & -1.8 & & .6 & & $1.70 \mathrm{~nm}$ & & \\
\hline & Os & 186 & $6.00 \mathrm{~nm}$ & & & $5.6 \mathrm{mb}$ & & & & ipp & 52 & 10.80 \\
\hline SF & .04 & 304 & $i P_{C}$ & 0 & $\theta 0$ & -0.6 & & & & iPP & 53 & 43.50 \\
\hline & & & $p$ & 51 & 28.60 & 206 & & & & es & 58 & 50 \\
\hline / $\mathbf{F}$ & & 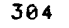 & iPc & 50 & 00 & -0 & SHO & & 57 & $i P_{c}$ & 51 & .00 \\
\hline RC & 7 & 305 & $i P c$ & 50 & 44.50 & -0.8 & & & & $0.40 n$ & & \\
\hline & & & $\mathrm{i}$ & 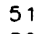 & 30.40 & $207 \mathrm{~km}$ & & 14 & & $1.10 \mathrm{um}$ & & \\
\hline $2 \mathrm{~F}$ & & 304 & & & 4 & $-\theta$ & & & & & & \\
\hline KM & .14 & 115 & $i P_{C}$ & 50 & 54.20 & 1.8 & 1 & $14 \mathrm{~s}$ & & $2.40 \mathrm{um}$ & & \\
\hline & & 128 & 0.0 & & & $6.4 \mathrm{mt}$ & i & & & eP & 52 & $\theta 0$ \\
\hline & & & e & 51 & 33.00 & $171 \mathrm{kmx}$ & 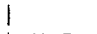 & & & es & 58 & 56.00 \\
\hline $\mathrm{KH}$ & 7 & 47 & iPc & 50 & 0 & & I KUR & & 55 & $i P c$ & 51 & 00 \\
\hline & & $* * * *$ & $* * * * \mathrm{~nm}$ & & & $7.0 \mathrm{mb}$ & & $1.0 \mathrm{~s}$ & 350 & $0.00 \mathrm{~nm}$ & & \\
\hline & & & epP & 51 & $\partial \theta$ & $212 \mathrm{~km}$ & 1 & & & $i p P$ & 52 & $\theta 0$ \\
\hline & & & ePPP & 54 & & & & & & ePCP & 52 & 00 \\
\hline & & & $i$ & 57 & & & & & & esP & 52 & $\theta$ \\
\hline IF & 6 & 302 & $\mathbf{i}$ & 50 & 55 & & & & & is & 59 & 00 \\
\hline & & & pP & 51 & 40.50 & 204 & $A K L$ & 4 & 330 & iP & 51 & .90 \\
\hline JF & 83 & 303 & iP & & & -8 & & 00 & 1080 & 0.0 & & \\
\hline & & & pf & 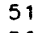 & & 204 & & & & & 52 & 70 \\
\hline & 5 & 291 & if & 50 & 56.00 & -2.2 & iv & 50 & 7 & & 51 & 00 \\
\hline
\end{tabular}


$12 d \quad 15 n$

BPI $\quad 74.43220 \mathrm{iPC}$

MTN $74.83119 \mathrm{iPC}$

KIC $\quad 74.89267$ iPC

PRY

SEK

VIR

SW

PME

PMR

MEK

BLF

$K$ IM

KDC

HAL

MUN

MOM

PCA

KLE

$\begin{array}{lll}\text { GRM } & 80.99216 \mathrm{iPC} \\ & 0.8 \mathrm{~s} 1890.00 \mathrm{~nm}\end{array}$

RSNT $81.22 \quad 3$ IPd $5401.70 \quad 1.7$

$\begin{array}{rrrlrrr} & & \text { i } & 54 & 51.60 & 205 \mathrm{~km} \\ \text { YKC } & 81.23 & 3 \mathrm{iPC} & 54 & 02.00 & 0.2\end{array}$

WEN $81.33131 \mathrm{PP} \quad 5452.00206 \mathrm{~km}$

$0.8 \mathrm{~s} \quad 130.00 \mathrm{~nm} \quad 5.7 \mathrm{mb}$

NWAO $\quad 81 \quad 44 \quad 142$ IPd $5402.80 \quad-0.5$

$\begin{array}{lllllll}\text { KLG } & 81.92 & 138 \mathrm{iPC} & 54 & 05.50 & -0.3\end{array}$

WB2 $81.94122 \mathrm{iPc} \quad 54 \quad 05.50 \quad-0.7$

$\begin{array}{llllll}\mathrm{SCH} & 82.10 & 337 \mathrm{ePC} & 54 & 06.20 & -0.4\end{array}$ $1.5 \mathrm{~s} \quad 954.00 \mathrm{~nm} \quad 6.3 \mathrm{mb}$

RKG $\quad 82.25 \quad 143$ iPC $\quad 5409.00 \quad 1.5$

STJ $\quad 82.40325 \mathrm{eP} \quad 5408.50 \quad 0.4$

$1.7 \mathrm{~s} \quad 1200.00 \mathrm{~nm} \quad 6.3 \mathrm{mb}$

82.70104 eP $\quad 5410.00 \quad-0.2$

SUR

$83.15221 \quad$ iPC $\quad 54 \quad 13.20 \quad 0.9$

$0.7 \mathrm{~s} \quad 1380.00 \mathrm{~nm} \quad 6.8 \mathrm{mb}$

$\begin{array}{llllll}83.23 & 98 \text { IPC } & 54 & 13.50 & 0.6\end{array}$

ASPA

FCC

PMG

$84.19125 \mathrm{iPC} \quad 5417.00 \quad-0.6$

$84.26 \quad 352$ iPc $54 \quad 18.20 \quad 0.9$

$1.2 \mathrm{~s} 1050.00 \mathrm{~nm}$

$84.52106 \mathrm{iPc} 5419.00-0.3$

$1.954000 .00 \mathrm{~nm} \quad 6.8 \mathrm{mb}$

$\begin{array}{lllll}84.74221 & \mathrm{iPc} & 54 & 21.50 & 1.5\end{array}$

$84.98105 \mathrm{eP} \quad 5421.00 \quad-0.8$

$\begin{array}{lllll}85.30 & 99 & \text { iPct } 54 & 23.10 & -0.2\end{array}$

$85.47221 \mathrm{iPc}$

$1.0 \mathrm{~s} \quad 400.00 \mathrm{~nm}$

$86.06119 \mathrm{PC}$

FFO \begin{tabular}{lll|l} 
PP & $5532.00209 \mathrm{~km}$ & NOU \\
\end{tabular}

CBM $\quad 89.22333 \mathrm{P} \quad 5445.00 \quad 3.3 \times$ ISA

$5540.00215 \mathrm{~km}$

5600.00

5934.00

0630.00

(PKKP12 15.80

$\begin{array}{cccccc}\text { EDM } \quad 90.53 & 3 \mathrm{iPc} & 54 & 48.70 & 1.1 \\ & & \mathrm{pP} & 55 & 40.00 & 208 \mathrm{~km}\end{array}$

PHC $\quad 91.61 \quad 12 \mathrm{ePC} \quad 5453.50 \quad 0.9$ $0.7 \mathrm{~s} \quad 160.00 \mathrm{~nm} \quad 6.2 \mathrm{mb}$ PP $\quad 55 \quad 46.00 \quad 213 \mathrm{~km}$

RSON $\quad 91.96 \quad 350$ iPd $54 \quad 54.30 \quad 0.1$

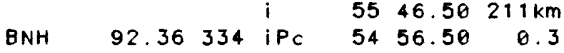

MNT $\quad 92.36336 \mathrm{eP} \quad 5456.50 \quad 0.4$

$1.6 \mathrm{~s} \quad 346.00 \mathrm{~nm} \quad 6.2 \mathrm{mb}$

OTT

$\begin{array}{lllll}93.12 & 337 \mathrm{ePc} & 5500.50 & 0.8\end{array}$

$1.8 \mathrm{~s} \quad 778.00 \mathrm{~nm} \quad 6.5 \mathrm{mb}$

RSNY

NO
NO
ISA
ISA
1 PTN

PNT

APH

HNR

STK

RXF

I $A D E$

LDM

I NEW

LHD

CLX

I ELF

LDN

CMS

BFD

RSSD

TOO
COO

BDW

CAN

BLA

WDC

FVM

I MAW

GLD

GOL 2

EUR
MIR
KOU

JAS

PVC

GBO
TUL

BHO

ALO

GLA

ITR

HKT

I JCT

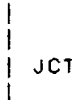

$\mathrm{JCT}$

1

I SOB

NVL
$93.63336 \mathrm{P}$

93.64347 iP

$1.4 \mathrm{~s} \quad 835.00 \mathrm{~nm}$

$\begin{array}{ll}93.99 & 7 \mathrm{iPC} \\ 1.0 \mathrm{~s} & 408.00 \mathrm{~nm}\end{array}$

$94.12336 \mathrm{P}$

$94.2010 \mathrm{eP}$

$94.60 \quad 98 \mathrm{eP}$

$94.79 \quad 4 \quad i P C$

$94.93130 \mathrm{iPC}$

\begin{tabular}{lccc}
$1.0 \mathrm{~s}$ & $280.00 \mathrm{~nm}$ & \multicolumn{2}{c}{$6.4 \mathrm{mb}$} \\
95.19 & $4 \mathrm{iPc}$ & 5509.30 & 0.1
\end{tabular}

$\begin{array}{lllll}95.26 & 6 \text { iPc } & 55 & 09.30 & -0.2\end{array}$

$1.1 \mathrm{~s} \quad 145.00 \mathrm{~nm}$

$05.37 \quad 5602.00213 \mathrm{~km}$

$\begin{array}{lllllll}95.45 \quad 4 \text { iPC } & 55 & 11.30 & 0.7 & 11 C\end{array}$

$96.77340 \mathrm{P}$

$96.88340 \mathrm{P}$

$97.15340 \mathrm{P}$

$\begin{array}{llllll}97.27 & 124 \mathrm{eP} & 55 & 18.00 & -0.6 \\ 98.72 & 130 \mathrm{eP} & 55 & 24.00 & -1.0\end{array}$

5517.10

5517.30

0.7
0.4

0. 4

$\begin{array}{lllll}99.60 & 356 & \text { iPd } 55 & 29.80 & 0.3\end{array}$

$100.80 \quad 129$ ePdiff55 $34.00 \quad-0.5$

$100.87 \quad 120$ ePdi $\$ 55 \quad 34.00-1.0$

$101.06 \quad 1$ iPdi $\$ 5535.60 \quad-0.2$

$1.3 \mathrm{~s} \quad 33.60 \mathrm{~nm} \quad 5.7 \mathrm{mb}$

$101.70 \quad 125$ ePdiff55 $37.80 \quad-0.8$

$$
\begin{array}{lll}
i & 56 & 30.70 \\
\text { e } & 57 & 19.80
\end{array}
$$

102.03337 Pdi $\$ 5540.00$

$1.7 \mathrm{~s} \quad 127.00 \mathrm{~nm}$

$102.24 \quad 11$ ePdif $\$ 5541.10$

$\begin{array}{r}41.10 \\ \hline 5631.80\end{array}$

103.91345 ePdiff55 48.90

$1.6 \mathrm{~s} \quad 147.00 \mathrm{~nm}$

$104.01 \quad 183$ iPdif $\$ 5549.20$

$1.3 \mathrm{~s} \quad 57.00 \mathrm{~nm}$

$1.4 \mathrm{~s} \quad 124.00 \mathrm{~nm}$

$\begin{array}{rr}1.4 \mathrm{~s} & 124.00 \mathrm{~nm} \\ 22 \mathrm{~s} & 3.71 \mathrm{um}\end{array}$

04.07357 iPdiff55 $50.00 \quad 0.7$

$1.4 \mathrm{~s} \quad 44.40 \mathrm{~nm}$

$7 \quad 22 \mathrm{~s} \quad 3.53 \mathrm{um} \quad 6.2 \mathrm{mb}$

$104.07 \quad 6$ iPditf55 $50.40^{5.9 M s} 1.1$

$1.0 \mathrm{~s} \quad 24.60 \mathrm{~nm} \quad 6.1 \mathrm{mb}$

$104.13 \quad 171$ ePdift55 $49.00 \quad 0.5$

104.39105 iPdift55 $50.10-0.6$

105.03341 ePdift55 53.20

105.129 ePdiff55 54.50

$56 \quad 45.80$
00

106.00100 iPKPC 0012.00

$106.53 \quad 348$ ePdif $\$ 56 \quad 00.60$

$106.86 \quad 348$ ePdif $f 56 \quad 02.10$

106.90349 ePdiff56 01.80

$1.3 \mathrm{~s} \quad 71.70 \mathrm{~nm}$

$222 \mathrm{~s} \quad 2.52 \mathrm{um}$

$\mathrm{N} 23 \mathrm{~s} \quad 1.06 \mathrm{um}$

E $20 \mathrm{~s}$

0. 30 um

e $\quad 0825.50$

$107.04 \quad 105 \quad \mathrm{iPdi}+\$ 56 \quad 02.20$

$107.04 \quad 105$ iPKPC $00 \quad 11.30$

$107.62 \quad 8$ ePdiff $56 \quad 05.00$

$107.62 \quad 8$ ePKP 0009.00

108.25348 ePdiff56 08.90

$1.5 \mathrm{~s} \quad 127.00 \mathrm{~nm}$

$108.85 \quad 358$ ePdiff56 12.00

$222 \mathrm{~s} \quad 3.70 \mathrm{um}$

.70um

110.5555

$\begin{array}{cccc}110.86275 \text { ePKP } & 00 & 17.70 \\ \text { e } & 00 & 58.60\end{array}$

112.74348 PKP 0022.10

112.80352 ePdiff56 28.50

$1.0 \mathrm{~s} \quad 9.50 \mathrm{~nm}$

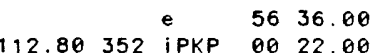

$1.0 \mathrm{~s} \quad 50.00 \mathrm{~nm}$

$Z 20 \mathrm{~s} \quad 2.31 \mathrm{um}$

-0.6 I CFA

Q. 2 I CEN

I RFA

$2.7 \times \mid \mathrm{BACH}$

$0.6 \quad$

\begin{tabular}{l|l}
0.7 & $\mathrm{PCH}$ \\
0.2 & $\mathrm{PEL}$
\end{tabular}

\begin{tabular}{l|l}
$7 \mathrm{mb}$ & $\mathrm{SAN}$ \\
$\mathrm{MSZ}$ & $\mathrm{CHCH}$
\end{tabular}

TACH

RKT

$-0.3$

0. 1

0.1
$-3.0 x$

113.10276 ePKP $0022.50^{5.8 M s z}$

$\begin{array}{ll}11.24 \quad 95 \text { iPKPd } 00 \quad 24.69 & -0.4\end{array}$

$1.0 \mathrm{~s} \quad 62.50 \mathrm{~nm}$

115.02198 IPKP 0025.00

$1.2 \mathrm{~s} \quad 160.00 \mathrm{~nm}$

iPP $01 \quad 32.00$

183

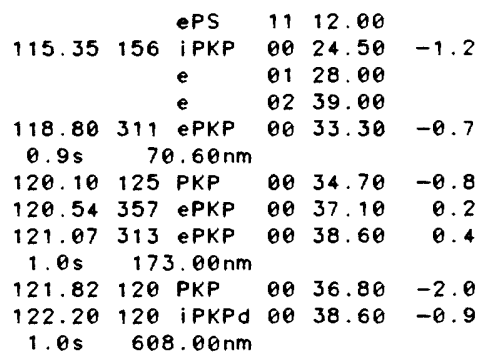
.

PP $\quad 00 \quad 50.70$

$\begin{array}{llllll}122.43 & 274 & \text { IPKP } & 00 & 40.00 & -0.8\end{array}$

122.80314 IPKPd $00 \quad 41.50 \quad-0.2$

$1.0 \mathrm{~s} \quad 333.00 \mathrm{~nm}$

$\begin{array}{llllll}123.02 & 265 & \text { IPKP } & 00 & 42.80 & 1.1\end{array}$

$\begin{array}{lllll}123.27 & 349 & \text { ePKPO } 00 & 42.30 & -0.3\end{array}$

123.63349 ePKP $0043.40 \quad 0.0$

$\begin{array}{llllll}123.69 & 350 & \text { ePKP } & 00 & 43.10 & -0.4\end{array}$

$\begin{array}{lllll}123.85 & 348 & \text { ePKPd } 00 & 43.30 & -0.4\end{array}$

$\begin{array}{lllll}124.67 & 349 & \text { ePKPd } 00 & 45.60 & 0.4\end{array}$

125.30346 IPKP $0041.00 \quad-5.5 x$

$\begin{array}{llllll}126.21 & 349 & \text { ePKP } & 00 & 48.10 & 0.1\end{array}$

$\begin{array}{lllll}126.35 & 180 & \text { ePKPd } 00 & 45.80 & -1.2\end{array}$

$1.0 \mathrm{~s} \quad 465.00 \mathrm{~nm}$

$\begin{array}{llllll}126.59 & 165 & \text { IPKP } 00 & 46.60 & -0.4\end{array}$

$S \quad 0246.00$

$\begin{array}{lllll}126.84 & 314 & \text { ePKP } \quad 00 & 49.50 & -0.2\end{array}$

127.71314 еPKP $0050.50 \quad-0.9$

128.32316 ePKP $00 \quad 49.50 \quad-2.8 x$

132.11268 PKP $\theta 0 \quad 52.50 \quad-6.5 x$

$\begin{array}{lllll}132.18 & 268 \text { PKP } 00 & 55.20 & -3.9 x\end{array}$

$\begin{array}{lllll}132.23 & 268 \text { PKP } \theta 0 & 50.10 & -9.2 X\end{array}$

132.37315 ePKP $\theta 0 \quad 46.00-14.4 X$

$\begin{array}{llllll}138.82 & 288 & \text { PKP } & 01 & 03.00 & -9.4 X\end{array}$

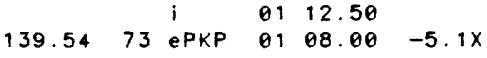

$1.1 \mathrm{~s} 230.00 \mathrm{~nm}$

$\begin{array}{lllll}139.91279 & \text { e(PKP) } 01 & 03.60 & -10.7 X\end{array}$

$\begin{array}{lllll}141.16 & 275 & \text { ePKPd } 01 & 07.00 & -9.1 \times\end{array}$

141.35291 ePKP $0110.00-6.8 x$

142.44302 ePKP $01 \quad 14.00 \quad-4.3 x$

143.02270 ePKPd $01 \quad 13.50 \quad-5.6 x$

$\begin{array}{llllll}143.14 & 265 & \text { ePKPC } 01 & 14.50 & -4.7 x\end{array}$

143.60282 ePKP $0117.50 \quad-2.6$

144.59280 iPKP $01 \quad 20.30 \quad-1.5$

$\begin{array}{llllll}145.08 & 271 & \text { ePKPC } & 01 & 22.40 & -0.3\end{array}$

$\begin{array}{lllll}146.16 & 266 \text { ePKPd } 0123.00 & -1.3\end{array}$

146.56267 ePKP $0126.00 \quad 1.0$

$\begin{array}{llllll}147.06 & 264 & \text { IPKP } 01 & 25.50 & -0.2\end{array}$

147.28261 ePKPC $0126.00 \quad 0.0$

148.51264 IPKP 0129.40

148.61264 iPKPd $0128.50 \quad 0.3$

148.61265 ePKP $0127.50-0.7$

$\begin{array}{llllll}148.68 & 264 \text { ePKPC } 01 \quad 28.00 & -0.2\end{array}$

148.80263 iPKPC $0128.10 \quad-0.3$

148.95264 IPKPd $0128.50 \quad-0.1$
153.81

$\begin{array}{rrrr}153.81 & 67 \text { ePKP } 0137.00 & 1.1\end{array}$

1.2s $\quad 185.00 \mathrm{~nm}$

S.D. $=1.0$ on 455 of 491 obs.

OCT $04,1983 \quad 18 \mathrm{~h} 52 \mathrm{~m} \quad 12.18 \pm 0.33 \mathrm{~s}$ $26.523 \mathrm{~S} \pm 1.8 \mathrm{~km} \quad 70.571 \mathrm{~W} \pm 2.4 \mathrm{~km}$ DEPTH $=8.8 \pm 1.9 \mathrm{~km}$

$6.3 \mathrm{mb}$ ( 57 obs.) $7.3 \mathrm{Msz}$ ( 17 obs.) NEAR COAST OF NORTHERN CHILE (122)

At least 5 people killed, 24

injured and extensive damage

(VIII) in the Copiopo-Chanaral

area. A 1.5 meter uplift near

Chanaral was reparted and same

raods were blocked by

landslides. Minar tsunami

recarded at Valparaisa. Felt in 


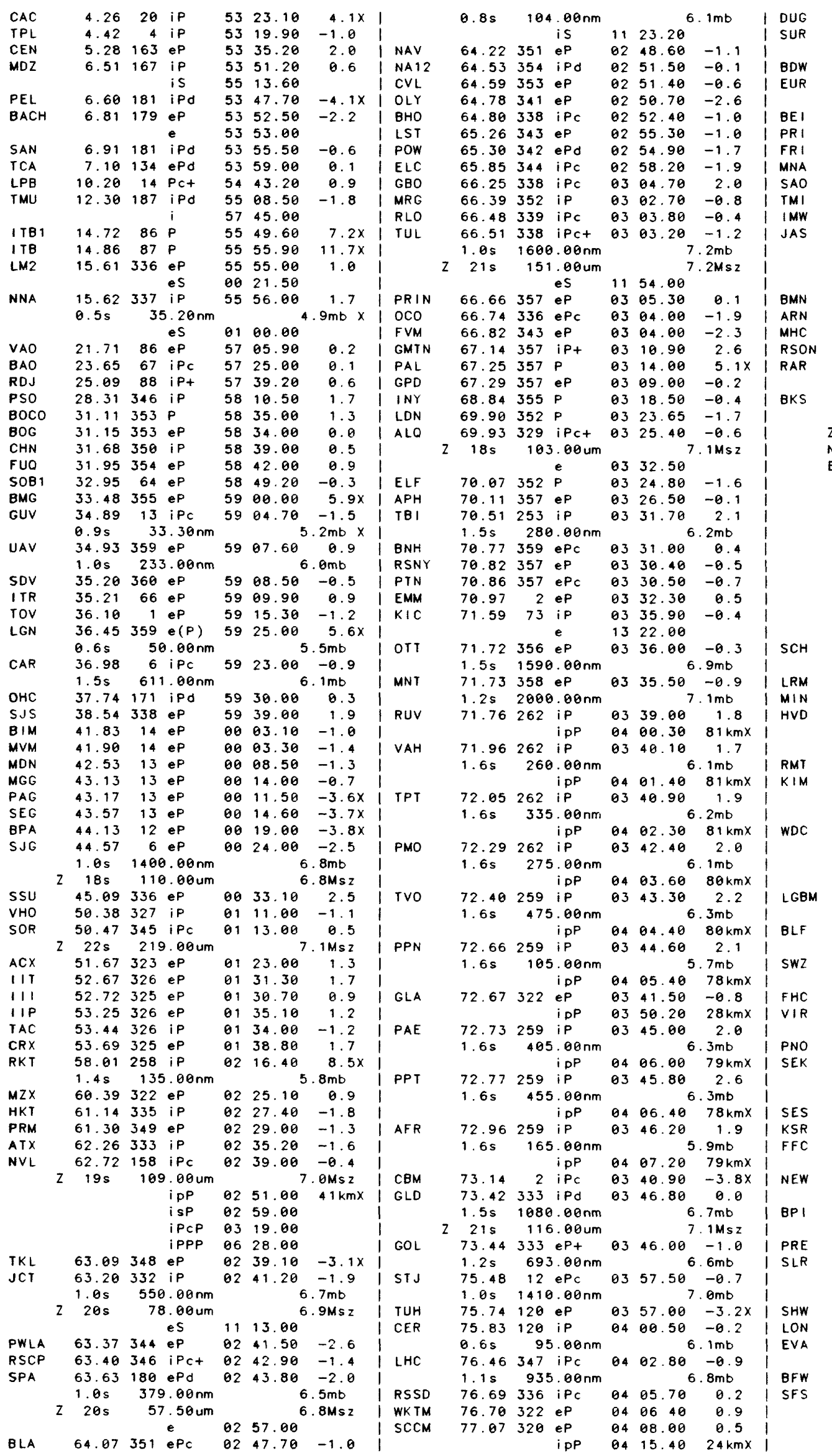

$\begin{array}{llllll}77.14 & 328 & \text { iPc } & 04 & 08.10 & 0.2\end{array}$ $\begin{array}{llllll}77.38 & 120 & \text { iPc } & 04 & 10.20 & 0.5\end{array}$ $1.1 \mathrm{~s} \quad 139.00 \mathrm{~nm} \quad 6.8 \mathrm{mb}$ $\begin{array}{lllllll}77.75 & 332 & \text { iPc } & 04 & 10.10 & -1.3\end{array}$ $\begin{array}{lllllll}78.14 & 326 & \text { iP } & 04 & 14.00 & 0.4\end{array}$ $1.0 \mathrm{~s} \quad 56.20 \mathrm{~nm} \quad 5.6 \mathrm{mb}$ $\begin{array}{lllll}78.19 & 330 \mathrm{eP} & 04 & 13.50 & -0.2\end{array}$ $\begin{array}{lllll}78.20 & 321 \mathrm{ePC} & 04 & 14.00 & 0.2\end{array}$ $\begin{array}{llllll}78.27 & 322 & \text { eP } & 04 & 14.20 & 0.2\end{array}$ $\begin{array}{llllll}78.48 & 324 & \mathrm{eP} & 04 & 15.20 & -0.2\end{array}$ $\begin{array}{llllll}79.09 & 321 \mathrm{eP} & 04 & 18.00 & -0.5\end{array}$ $\begin{array}{lllll}79.20 & 331 \mathrm{eP} & 04 & 19.20 & -0.1\end{array}$

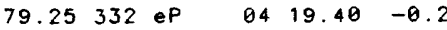

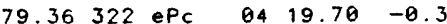
EPKKP 2329.00 eP'P' 3142.50 e $\quad 3500.00$ $\begin{array}{lllll}79.48 & 326 \mathrm{eP} & 04 & 20.00 & -0.8\end{array}$ $\begin{array}{lllll}79.55 & 321 \mathrm{eP} & 04 & 21.50 & 0.4\end{array}$ $\begin{array}{llllll}79.61 & 321 \mathrm{eP} & 04 & 21.60 & 0.1\end{array}$ $\begin{array}{lllll}79.71 & 345 \text { ePC } & 04 & 20.50 & -1.1\end{array}$ $\begin{array}{lllll}80.13251 P & 0424.00 & -0.5\end{array}$ $\begin{array}{lllll}80.32 & 321 \text { eP } & 14 & 59.00 & \\ 04 & 25.40 & 0.3\end{array}$ 2.0s $1670.00 \mathrm{~nm} \quad 6.7 \mathrm{mb}$ $\begin{array}{lll}2.0 \mathrm{~s} & 30.00 \mathrm{um} & 6.6 \mathrm{Msz}\end{array}$ N $20 \mathrm{~s} \quad 70.00 \mathrm{um}$ $\begin{array}{lll}i & 04 & 32.80 \\ i & 04 & 52.00 \\ \text { e (PP) } & 08 & 06.00 \\ \text { iS } & 14 & 34.00 \\ \text { e(PPS) } & 16 & 34.00 \\ \text { e } & 18 & 55.00 \\ \text { eSS } & 20 & 11.00 \\ \text { e } & 22 & 25.00 \\ \text { e } & 23 & 17.80 \\ \text { eSSS } & 23 & 29.80\end{array}$ $\begin{array}{lllll}81.07 \quad 2 \text { EPC } & 04 & 27.50 & -1.2\end{array}$ $\begin{array}{llllll}0.7 \mathrm{~s} & 310.00 \mathrm{~nm} & 04 & 31.20 & 0.1\end{array}$ 81.71323 eP $\quad 0431.20 \quad-1.4$

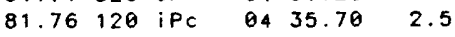
$1.2 \mathrm{~s} \quad 324.00 \mathrm{~nm} \quad 6.3 \mathrm{mb}$

$\begin{array}{llllll}81.99 & 322 \text { eP } & 04 & 59.40 & 35.00 & 1.3\end{array}$ $82.00 \quad 118$ iPc $04 \quad 34.50 \quad 0.0$ $\begin{array}{lll}1.2 \mathrm{~s} & 360.00 \mathrm{~nm} & 6.3 \mathrm{mb}\end{array}$ $\begin{array}{llll}i & 04 \quad 48.80\end{array}$ $82.38 \quad 323 \mathrm{ePc} \quad 04 \quad 34.20 \quad-1.6$ EPKKP 2322.00 EP'P' 3137.00 $\begin{array}{lllll}82.73323 \mathrm{eP} & 04 & 56.50 & \\ & 0.40 & -0.6\end{array}$ $\begin{array}{lllll} & i p P & 04 & 45.50 & 26 \mathrm{~km} x\end{array}$ $\begin{array}{llllll}83.12 & 117 & i & 05 & 19.00 & \\ 0 . P C & 04 & 38.50 & -1.8\end{array}$ $0.3 \mathrm{~s} \quad 40.00 \mathrm{~nm} \quad 6.1 \mathrm{mb}$ 83.32322 eP $04 \quad 41.20 \quad 0.4$ 83.94118 iPC $04 \quad 44.20 \quad-0.3$ 84.09328 eP $\quad 0444.00 \quad-0.5$ $\begin{array}{llllll}84.43 & 119 & \text { iPc } & 04 & 47.50 & 0.5\end{array}$ $0.9 \mathrm{~s} \quad 516.00 \mathrm{~nm} \quad 6.8 \mathrm{mb}$

84.55335 eP $\quad 0405.50$

$-9.3 x$ $84.96 \quad 116$ IPC $\quad 04 \quad 46.50 \quad-3.2 x$ 85.31342 eP $\quad 04 \quad 49.00 \quad-1.4$ $1.0 \mathrm{~s} \quad 76.00 \mathrm{~nm} \quad 5.9 \mathrm{mb}$ 85.34331 eP $\quad 0450.30 \quad-0.4$ $\begin{array}{llll}e & 05 \quad 12.00\end{array}$ $85.73 \quad 117$ iPd $0451.70 \quad-1.8$ $86.05117 \mathrm{eP} \quad 0454.00-1.1$ $86.13 \quad 117$ iPc $04 \quad 54.20 \quad-1.3$ $1.3 \mathrm{~s} \quad 258.00 \mathrm{~nm}$

$86.18327 \mathrm{e}(P)$ 0508.40 6. $2 \mathrm{mb}$

$\begin{array}{lllll}327 & e(P) & 04 & 55.30 & 0.2\end{array}$ 86.37328 ePd $0455.00 \quad-0.9$ 86.41118 eP $0456.50-0.4$ 86.87327 eP 0505.00 $04 \quad 58.10-0.3$ $\begin{array}{lllll}87.15 & 47 \mathrm{iPc} & 05 & 04.00\end{array}$ 0524.00 IPP $\quad 08 \quad 30.00$ iPPP 1050.00 


\begin{tabular}{|c|c|c|c|c|c|c|c|c|c|c|c|}
\hline & & & iSKS & 15 & 20.00 & & I DCN & 96.55 & 33 & $e P$ & 48.86 \\
\hline & & & is & 15 & 57.00 & & i & $1.2 \mathrm{~s}$ & 345 & $5.00 \mathrm{~nm}$ & \\
\hline & & & iss & 22 & 00.00 & & I MFF & 96.59 & 41 & iPc & 43.50 \\
\hline $\mathrm{FCC}$ & 87.19 & 348 & ePc & 84 & 59.10 & -0.4 & I ETA & 96.69 & 34 & eP & 47.50 \\
\hline & $1.2 \mathrm{~s}$ & 778 & $8.00 \mathrm{~nm}$ & & & $.8 m b$ & I & $2.3 \mathrm{~s}$ & 1760 & $0.00 \mathrm{~nm}$ & \\
\hline PNT & 87.22 & 336 & eP & 05 & 00.00 & 0. 1 & I LPF & 96.83 & 39 & iPc & 44.10 \\
\hline & $0.9 \mathrm{~s}$ & 388 & $8.00 \mathrm{~nm}$ & & & $.7 \mathrm{mb}$ & I DLE & 96.87 & 33 & eP & 50.00 \\
\hline GMW & 87.41 & 328 & eP & 05 & 00.20 & -0.7 & 1 & $1.3 \mathrm{~s}$ & 630 & $0.00 \mathrm{~nm}$ & \\
\hline MAL & 88.48 & 47 & ipt & 05 & 07.00 & 0.8 & I RJF & 96.90 & 43 & iPc & 45.00 \\
\hline & & & iPP & 09 & 00.00 & & I CAF & 97.04 & 43 & iPc & 46.00 \\
\hline & & & is & 16 & 16.00 & & I DMU & 97.05 & 32 & eP & 47.90 \\
\hline PGC & 88.50 & 328 & eP & 05 & 06.40 & 0.4 & I HON & 97.08 & 290 & 05 & 56.00 \\
\hline GNZ & 88.85 & 227 & $P$ & 05 & 17.00 & $8.9 x$ & I GRR & 97.12 & 39 & iPc & 45.40 \\
\hline & & & PP & 05 & 32.00 & $51 \mathrm{kmx}$ & I KIP & 97.13 & 290 & eP & 57.00 \\
\hline & & & $\mathrm{i}$ & 05 & 48.00 & & I LSF & 97.39 & 42 & iPc & 47.30 \\
\hline & & & $P P$ & 09 & 09.80 & & I FLN & 97.53 & 39 & $e P$ & 47.60 \\
\hline ALR & 88.95 & 49 & ip & 05 & 06.00 & -2.5 & 1 LDF & 97.65 & 39 & iPc & 48.00 \\
\hline & & & i & 05 & 15.00 & & I TCF & 97.82 & 42 & eP & 48.90 \\
\hline & & & i & 05 & 19.00 & & I & $1.2 \mathrm{~s}$ & 90 & B. $40 \mathrm{~nm}$ & 6 \\
\hline & & & $\mathrm{i}$ & 65 & 29.00 & & I MZF & 98.01 & 42 & $i P c$ & 50.00 \\
\hline BUL & 88.99 & 112 & iPct & 65 & 09.00 & -0.3 & I REY & 98.03 & 19 & $e P$ & 52.20 \\
\hline & $0.8 \mathrm{~s}$ & $27 \theta$ & $0.00 \mathrm{~nm}$ & & 6 & $.6 \mathrm{mb}$ & 1 & & & 06 & 05.80 \\
\hline & $21 \mathrm{~s}$ & 229 & $9.00 \mathrm{um}$ & & 7 & $.6 \mathrm{Msz}$ & I SSB & 98.74 & 44 & 05 & 53.80 \\
\hline i & $21 \mathrm{~s}$ & 131 & $1.00 \mathrm{um}$ & & & & I CDR & 98.75 & 46 & ePc & 54.00 \\
\hline & $21 \mathrm{~s}$ & 168 & $8.00 \mathrm{um}$ & & & & I & & & 89 & 33.30 \\
\hline & & & i PP & 68 & 43.00 & & I AVF & 98.76 & 42 & 05 & 53.10 \\
\hline & & & is & 15 & $5 B .00$ & & I GRC & 98.84 & 41 & iPc & 53.20 \\
\hline CRT & 89.28 & 47 & iPc & 05 & 10.20 & 0.1 & 1 & & & 05 & 55.30 \\
\hline MNG & 89.36 & 224 & $P$ & 85 & 11.00 & 0. 4 & I SMF & 98.98 & 42 & $\mathrm{iPc}$ & 54.30 \\
\hline & & & e & 85 & 35.00 & & I SSF & 98.98 & 42 & iPc & 54.00 \\
\hline WEL & 89.41 & 223 & $\mathbf{P}$ & 05 & 12.00 & 1.2 & I LRG & 99.02 & 46 & iPc & $55.4 \theta$ \\
\hline & & & $P P$ & 08 & 34.00 & & I LMR & 99.06 & 46 & iPc & 55.40 \\
\hline & & & SKS & 15 & 39.00 & & I LBF & 99.22 & 42 & iPc & 55.20 \\
\hline & & & $\mathrm{S}$ & 16 & 03.00 & & I FRF & 99.26 & 46 & iPc & 56.30 \\
\hline TCW & 89.76 & 223 & $P$ & 05 & 13.60 & 1.2 & I LOR & 99.29 & 42 & $i P c$ & 55.40 \\
\hline FRB & 89.98 & 1 & eP & 05 & 12.00 & -0.6 & I EKA & 99.66 & 32 & 06 & 02.30 \\
\hline RHP & 90.48 & 219 & $e^{P}$ & 05 & 17.00 & 1.2 & 1 & $1.4 \mathrm{~s}$ & 102 & $2.00 \mathrm{~nm}$ & \\
\hline & & & i & 05 & 39.30 & & I CVF & 100.26 & 48 & iPdiff06 & 00.70 \\
\hline & & & e & 05 & 55.20 & & 1 & $0.8 \mathrm{~s}$ & 52 & $2.40 \mathrm{~nm}$ & \\
\hline & & & ePKKP & 22 & 45.10 & & I AKU & 100.27 & 19 & ePdiff06 & 19.00 \\
\hline & & & ePKKP & 22 & 54.10 & & I & $2.1 \mathrm{~s}$ & 587 & $.00 \mathrm{~nm}$ & \\
\hline & & & e & 30 & 51.00 & & 1 & $18 s$ & 275 & $5.00 \mathrm{um}$ & \\
\hline & & & e & 31 & 06.00 & & 1 & & & 10 & 35.40 \\
\hline TOL & 90.52 & 45 & iPc & 05 & 16.00 & 0.2 & I NPA & 160.29 & 113 & ePditfo6 & 09.00 \\
\hline & & & $i$ & 05 & 37.00 & & 1 & & & 06 & 19.00 \\
\hline & & & $i$ & 05 & 43.00 & & 1 & & & ePP & 47.00 \\
\hline & & & iPP & 09 & 11.00 & & 1 & & & 12 & 41.00 \\
\hline & & & iPPP & 11 & 08.00 & & I ERC & 100.85 & 53 & ePdifte6 & 10.00 \\
\hline & & & iSKS & 15 & 38.00 & & I D IX & 100.90 & 44 & ePdiff06 & 64.20 \\
\hline & & & is & 16 & 05.00 & & I ORO & 101.05 & 44 & ePdiff06 & 04.00 \\
\hline & & & $i$ & 17 & 50.00 & & 1 & & & 10 & 13.60 \\
\hline & & & iss & 22 & 20.00 & & 1000 & 101.07 & 39 & Pdiff 66 & 63.40 \\
\hline & & & i & 23 & 10.00 & & 1 & & & 06 & 12.80 \\
\hline & & & isSS & 26 & 05.00 & & 1 & & & 10 & 19.00 \\
\hline & & & i & 29 & 31.00 & & 1 & & & SKKS & 02.00 \\
\hline KRP & 90.87 & 226 & $\mathbf{P}$ & 05 & 18.00 & 0.4 & 1 & & & 19 & 11.60 \\
\hline & & & $\mathrm{pP}$ & 05 & 33.00 & $51 \mathrm{kmx}$ & 1 & & & SPP & 38.00 \\
\hline & & & i & 85 & 41.70 & & I HAU & 101.12 & 42 & ePdiff06 & 03.80 \\
\hline & & & $\mathrm{S}$ & 16 & 16.00 & & 1 & $1.0 \mathrm{~s}$ & 45 & $.60 \mathrm{~nm}$ & 6 \\
\hline$B C A O$ & 91.16 & 86 & $i P c$ & 05 & 19.00 & -0.3 & I MMK & 101.23 & 44 & ePdiff06 & 65.50 \\
\hline BNG & 91.17 & 86 & $i P c$ & 05 & 19.50 & 0.1 & I UCC & 101.27 & 39 & Pdiffco6 & 05.20 \\
\hline & $1.2 \mathrm{~s}$ & 100 & $0.00 \mathrm{~nm}$ & & 6 & $.0 \mathrm{mb}$ & I & & & 09 & 56.80 \\
\hline KRI & 91.37 & 109 & $i P_{c}$ & 05 & 20.00 & -0.4 & I & & & 10 & 21.00 \\
\hline & & & iPP & 08 & 59.00 & & I & & & 17 & 12.00 \\
\hline PHC & 91.80 & 328 & eP & 05 & 29.00 & $7.7 x$ & I BSF & 101.31 & 42 & ePdifte6 & 04.50 \\
\hline LGR & 92.93 & 43 & $\mathrm{ePc}$ & 05 & 29.00 & 2.2 & I & $1.5 \mathrm{~s}$ & 148 & $3.00 \mathrm{~nm}$ & 6 \\
\hline & & & IPP & 09 & 00.00 & & I VG1 & 101.45 & 45 & ePdiff06 & 06.00 \\
\hline & & & eSKS & 15 & 58.00 & & I CDF & 101.86 & 42 & ePdiffo6 & 07.00 \\
\hline & & & es & 16 & 17.00 & & I & $0.9 \mathrm{~s}$ & 37 & $.40 \mathrm{~nm}$ & 6 \\
\hline & & & iPS & 17 & 24.00 & & I ZUL & 102.12 & 43 & ePdif $f 06$ & 08.50 \\
\hline MTD & 93.05 & 110 & $\mathrm{iPc}$ & 65 & 28.00 & -0.1 & I ENN & 102.14 & 39 & ePdif $\$ 06$ & 09.50 \\
\hline & & & iPP & 69 & 25.00 & & 1 & $1.0 \mathrm{~s}$ & 48 & $.00 \mathrm{~nm}$ & 6 \\
\hline$A B A$ & 93.55 & 50 & ip & 05 & 36.50 & $6.7 x$ & 1 & & & 06 & 25.50 \\
\hline VAL & 94.26 & 33 & ip & 65 & 32.00 & -0.6 & 1 & & & 09 & 34.50 \\
\hline & & & is & 17 & 15.00 & & LLS & 102.23 & 44 & ePdiff06 & 09.50 \\
\hline EPF & 95.00 & 44 & iPc & 05 & 37.20 & 0.8 & I SLE & 102.31 & 43 & ePdiff06 & 09.00 \\
\hline YKC & 95.36 & 341 & ePc & 65 & 36.70 & -0.8 & I GWF & 102.32 & 41 & ePdiff06 & 09.50 \\
\hline & $0.8 \mathrm{~s}$ & & $2.00 \mathrm{~nm}$ & & & $.2 \mathrm{mb}$ & I DBN & 102.33 & 38 & ePdiffo6 & 11.00 \\
\hline RSNT & 95.40 & 341 & ePc & 85 & 37.20 & -0.4 & 1 & $19 \mathrm{~s}$ & 256 & $.00 \mathrm{um}$ & 7 \\
\hline YKA & 95.41 & 341 & eP & 05 & 38.40 & 0.7 & 1 & & & 06 & 30.00 \\
\hline MLS & 95.43 & 44 & $\mathrm{iPc}$ & 05 & 38.60 & 0.3 & 1 & & & ePP & 58.00 \\
\hline LFF & 96.24 & 43 & $e P$ & 05 & 42.10 & 0.2 & I & & & eSKS & 5000 \\
\hline GDH & 96.30 & 6 & IPd & 05 & 43.60 & $2 . \theta$ & 1 & & & ePPS & 37.00 \\
\hline & $1.0 \mathrm{~s}$ & 40 & $0.00 \mathrm{~nm}$ & & & $9 m b$ & $F \mid R$ & 102.35 & 47 & ePdiff06 & 15.00 \\
\hline & & & is & 17 & 03.00 & & I & & & 10 & 29.00 \\
\hline PO & $\begin{array}{l}96.38 \\
1.4 \mathrm{~s}\end{array}$ & $\begin{array}{l}43 \\
228\end{array}$ & eP & 05 & 43.00 & 0.4 & VDL & 102.36 & $\begin{array}{l}44 \\
50\end{array}$ & ePdift06 & 10.00 \\
\hline & & 228 & 3.06 & & & $\mathrm{mb}$ & RDP & 102.49 & 50 & ePdiff06 & 17.00 \\
\hline
\end{tabular}



$25 \mathrm{~s} \quad 375.00 \mathrm{um}$ e $\quad 1005.00$ e $\quad 1105.00$ is 1728.00

BRN $\quad 106.95 \quad 40$ ePdiff06 31.00

TTG $107.25 \quad 51$ ePdift06 32.00 e $\quad 1016.00$

$\begin{array}{llllll}\text { VKA } & 107.34 & 44 & \text { iPKP } & 10 & 37.70\end{array}$ $6.5 \mathrm{~s} \quad 5510.00 \mathrm{~nm}$

iPP $10 \quad 56.80$

e $\quad 1107.00$

SKKS $17 \quad 26.50$

KON $\quad 107.75 \quad 32$ iPdiff06 34.00

PMR $\quad 107.76 \quad 331$ ePdiff06 $31.00 \quad-2.2$ $z 20 \mathrm{~s} 160.00 \mathrm{um}$

ZST $\quad 107.80 \quad 45$ e(Pdif06 41.00 $\begin{array}{lll}\text { e } & 0741.00 \\ \mathrm{e} & 0820.00 \\ \mathrm{e} & 0957.00 \\ \mathrm{e} & 1016.00\end{array}$ $\begin{array}{lll}e & 10 & 16.00 \\ \text { e (PP) } & 11 & 15.00\end{array}$

OHR $\quad 107.88 \quad 53$ ePdiff06 32.00

KSP $\quad 108.20 \quad 42$ ePdif $106 \quad 38.00$ IPP 1103.00

SRO $\quad 108.44 \quad 45$ ePdiff06 36.50 e (PP) 1050.50 e(SKS) $17 \quad 38.50$

COL 108.49334 ePdit\$06 36.00

FBA $\quad 108.49334$ ePdiff06 36.40

SKO $\quad 108.62 \quad 52$ iPdiff06 39.00

$\begin{array}{llllll}\text { SKO } & 108.62 & 52 & \text { iPKP } & 10 & 38.00 \\ & & & \text { iPP } & 11 & 15.00\end{array}$

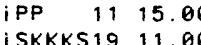
iPS 2108.00

ATH $\quad 108.97 \quad 56$ ePdiff06 41.00

GRG $108.98 \quad 53$ iPdiff06 42.50 ePP 1106.00 eS 1704.00 eS 1719.80 is 1740.00

N82 $109.01 \quad 31$ Pdiff $06 \quad 46.80$

$$
0.9 \mathrm{~s} \quad 7.50 \mathrm{~nm}
$$

SSR $\quad 109.75 \quad 49$ ePdiff06 55.00

HFS $109.86 \quad 32$ (Pdi $1106 \quad 54.80$

SPC $\quad 110.09 \quad 44$ ePdift06 44.80

MMB $\quad 110.13 \quad 53$ ePdiff06 54.00

KRA $\quad 110.16 \quad 43$ ePditto6 38.00 e $\quad 0702.00$ i $\quad 1120.00$ is 1742.00

CLO $\quad 110.53 \quad 49$ ePdit $106 \quad 50.00$

IMA 111.20334 ePKP 1040.00

UZH $\quad 111.23 \quad 45$ ePdiff06 50.00

UZH $\quad 111.23 \quad 45$ iPKP 1049.00

$$
Z 19 \mathrm{~s} \text { 150.00um } 1104.00
$$
ESKS 1720.00

AAE $\quad 111.2490$ ePdiff06 54.00

WAR $\quad 111.44 \quad 41$ elPdif06 52.00

$$
\text { e } \quad 07 \quad 12.00
$$

1120.00

\begin{tabular}{l} 
e \\
e $\quad 1120.00$ \\
\hline
\end{tabular}

$\begin{array}{llll}\text { EZN } & 111.48 & 55 \text { ePKP } 10 & 29.00\end{array}$

UPP $111.67 \quad 33$ iPdift06 51.08 $\begin{array}{lll}\text { i } & 07 & 12.00 \\ \text { iPP } & 11 & 40.00\end{array}$ iSKS 1745.00 i 2114.00

IZM $111.76 \quad 57$ EPKP $10 \quad 30.00-19.2 \mathrm{X}$

BUC1 $112.54 \quad 51$ ePdiff06 $55.00 \quad 0.2$

LVV $\quad 112.64 \quad 44$ ePdiff06 56.00 iPP $11 \quad 40.00$

VRI $\quad 113.41 \quad 49$ ePdiff87 15.50

0.8

$$
\text { iSKS } 1726.00
$$

$113.70 \quad 48$ ePKP $10 \quad 55.00$

113.8355 ePdif $f 07 \quad 05.00$

\begin{tabular}{l|l}
2.4 \\
$4.3 x$ & TIK \\
$2.9 x$ & MOM
\end{tabular}

114.5556 ePKP $10 \quad 41.00-13.6 \mathrm{X}$ SEY

GPA 114.55 56 ePKP $1041.00-13.6 \times$

$$
z \quad 23 \mathrm{~s}
$$

$299.00 \mathrm{um}$ iPP 1153.00
ISKS

iSKS 1801.00
$115.23 \quad 33$ ePdift07 96.00

NUR
$7.8 \mathrm{Ms} 2 \mathrm{X}$
I APA

WBN

8.0X I SMY

\begin{tabular}{l|l}
$2.5 X$ & GRO
\end{tabular}

\begin{tabular}{l|l}
0.7 & KER \\
$0.7 X$ & MEK
\end{tabular}

$9.7 X \mid \mathrm{MEK}$

$-6.1 \times$

$4 \times$

1.2
Msz

$3.8 \times$

$\begin{array}{l:l}3.8 \times & \\ 2.2 \times & \text { LMG }\end{array}$ BAK

$6.8 X \mid T E H$

5 I TIK

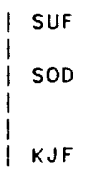

$\begin{array}{lllllll}116.26 & 31 & \text { iPKP } 10 \quad 52.00 & -5.0 \times & \text { I SVE }\end{array}$

$0.9 \mathrm{~s} \quad 8.20 \mathrm{~nm}$

26 IPKP $10 \quad 58.50$ $\begin{array}{lll}\text { i } & 11 & 07.00 \\ \text { EPKKP } & 21 & 32.00\end{array}$

$117.12 \quad 29$ ePdiff07 16.00

$117.23 \quad 23$ ePdiff07 16.00

118.3051 ePdiff07 24.00

$Z 24 \mathrm{~s} 325.00 \mathrm{um}$

N $24 \mathrm{~s} 261.00 \mathrm{um}$

E $24 \mathrm{~s} \quad 388.00 \mathrm{um}$

ESKS $18 \quad 02.00$

eSSS $32 \quad 48.00$

APA $119.52 \quad 25$ PKP 1103.00

$119.52 \quad 25$ ePdiff07 28.00

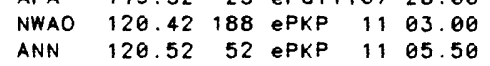

$N 20 \mathrm{~s} \quad 160.00 \mathrm{um}$

E 20

$160.00 \mathrm{um}$

$\begin{array}{lll}\text { EPPKP } & 11 & 18.80\end{array}$

ANN $\quad 120.52 \quad 52$ ePdiff07 26.00

OBN $121.03 \quad 40$ IPKP 1105.00 $\begin{array}{llll}\text { iPP } & 12 & 35.00\end{array}$ ISKS 1804.00 IPPS $23 \quad 50.00$

$2.6 \mathrm{X}$ OBN $121.03 \quad 40$ iPdiff07 34.00 $\begin{array}{lrlll}121.03 & 40 & \text { iPdiff } 107 & 34.00 \\ 121.11 & 335 & \text { PKP } 11 & 04.00\end{array}$ 121.47187 ePKP 1106.00

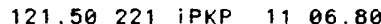

121.6439 PKP 1107.00

121.87192 ePKP 1110.00

$122.06 \quad 54$ еPKP 1106.00

$122.06 \quad 54$ ePdift07 40.00

$123.58 \quad 62$ ePKP $11 \quad 12.00$ i 1130.50 \begin{tabular}{l}
1251.50 \\
\hline$\quad 1543.50$
\end{tabular}

1.9 1 150

$3.3 \times$ LEN

GRS

GRS

4. 1X I KRV

I PMG

$$
\begin{aligned}
& Z \\
& N
\end{aligned}
$$$$
\text { E } 25
$$

$\begin{array}{llll}124.70 & 215 \text { ePKP } & 15 & 43.50 \\ 124.77 & 57 & 12.00\end{array}$

124.7757 ePKP

ESKS $18 \quad 15.00$
$3.05 \quad 800.00 \mathrm{~nm}$

$\begin{array}{lllll}125.10 & 199 & \text { ePKP } & 11 & 14.00\end{array}$

$\begin{array}{rrr}125.19 & 58 \text { iPKPd } 11 & 14.00\end{array}$

125.85317 ePKP

$220 \mathrm{~s} \quad 95.00 \mathrm{um}$

110.50

126.3654 iPKP $11 \quad 16.00$

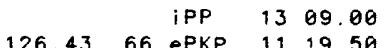

126.45190 ePKP $11 \quad 14.00$

$0.7 \mathrm{~s} \quad 24.00 \mathrm{~nm}$

$\begin{array}{lllll}126.52 & 59 & \text { iPKP } & 11 & 15.60\end{array}$

iPPP $16 \quad 03.20$

126.5259 ePdiff08 07.00

$126.63 \quad 58$ iPKP 1121.00

$\begin{array}{llll}\text { IPP } & 13 \quad 23.00\end{array}$

127.86210 iPKP $11 \quad 19.00$

1142.20

$\begin{array}{ll}11 & 42.20 \\ 16 & 04.70\end{array}$

2423.00

12787210 PKPd $11 \quad 17.60$

$0.7 \mathrm{~s} \quad 29.40 \mathrm{~nm}$

\begin{tabular}{lllll|l}
128.49 & 232 ePKP & 11 & 21.00 & -1.1 & KHO
\end{tabular}

$\begin{array}{llllll}128.84 & 231 & \text { ePKP } & 11 & 23.00 & 0.4\end{array}$

$20544.30 \mathrm{um} \quad 7.1 \mathrm{Msz}$

$129.32 \quad 58$ iPKP 1124.00

\begin{tabular}{rlll}
130.09 & 64 IPP & 13 & 36.00 \\
\hline
\end{tabular}

13350352 ePKP $1124.00 \quad-0.6$

$\begin{array}{ccccc}133.56 & 352 \text { ePKP } & 11 & 20.00 & -9.8 X \\ 26 s & 230.00 u m & & 7.8 M s z X\end{array}$

$27 \mathrm{~s} \quad 295.00 \mathrm{um}$

$150.00 \mathrm{um}$

e $\quad 1126.00$

EPPKP $11 \quad 39.00$

ESPKP $11 \quad 42.00$

ePP 1355.00

ESKS $18 \quad 39.00$

ePS $24 \quad 22.00$

ePPS $26 \quad 00.00$

eSSS $36 \quad 41.00$

$\begin{array}{lllll}133.56 & 352 & \text { PKP } & 11 & 26.00 \\ 133.64 & 238 & \text { PPKP } & 11 & 30.00\end{array}$

133.64238 ePKP 1130.00

$Z 24 \mathrm{~s} 203.00 \mathrm{um}$

\begin{tabular}{ll|l}
127.00 & -1.7 & $\mathrm{NRN}$ \\
\hline 27.00 & $-4.0 \times$ & $\mathrm{KSH}$
\end{tabular}

$245 \quad 203.004 m$

$7.8 \operatorname{MszX}$

E 245

$94.00 \mathrm{um}$

ePP $13 \quad 59.50$

eSS 3147.50

134.0735 ePKP $11 \quad 16.00-15.3 x$ $\begin{array}{lll}11 & 32.10\end{array}$

isPKP 1150.20

$134.18 \quad 60$ IPKPC $11 \quad 32.40$ ESPKP 1154.00

$\begin{array}{lll}\text { ePP } & 14 \quad 05.00\end{array}$

ESKS $18 \quad 41.00$

$\begin{array}{lll}\text { ESP } & 2408.00\end{array}$

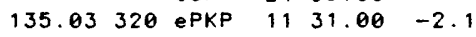

ePS 2428.00

$\begin{array}{lllll}135.03 & 320 & \text { PKP } & 32 & 20.00 \\ 11 & 35.00 & 1.9\end{array}$

$\begin{array}{llll}135.03 & 320 \text { ePdiff08 } 37.00 & 2.4 x\end{array}$

$135.30 \quad 11$ ePKP $11 \quad 15.00-18.2 x$ $\begin{array}{lll}i & 11 & 33.00 \\ \text { iPP } & 1409.00\end{array}$

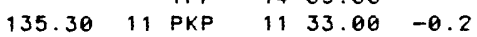

135.54211 ePKP $1125.00-10.3 x$

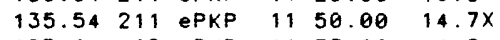

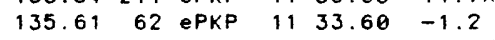

$z 24 \mathrm{~s} 20.30 \mathrm{um} \quad 6.8 \mathrm{MszX}$

ESPKP 1155.00

PPP $\quad 14 \quad 20.00$

ASH $\quad 135.81 \quad 62$ ePKP $11 \quad 35.00 \quad-0.2$

$4.0 \mathrm{~s} \quad 2310.00 \mathrm{~nm}$

$Z 24 \mathrm{~s} 210.00 \mathrm{um}$

N $20 \mathrm{~s} \quad 52.90 \mathrm{um}$

EPPKP $11 \quad 48.00$

IPP $14 \quad 14.00$

EPPP $17 \quad 15.00$

$\begin{array}{llllll}135.88 & 229 & \text { ePKP } & 11 & 36.00 & -0.1\end{array}$

136.16331 ePKP 1125.40

e $\quad 1132.00$

ePp $14 \quad 14.00$

$\begin{array}{llllll}137.21 & 317 \text { PKP } 11 & 34.00 & -3.4 X\end{array}$

137.21317 ePKP 1138.00

$1.0 \mathrm{~s} \quad 220.00 \mathrm{~nm}$

EPPKP 1154.50

E SPKP 1155.60

$\begin{array}{lll}\text { EPP } & 14 \quad 24.80\end{array}$

$\begin{array}{lllllll}\text { YAK } & 142.00 & 345 & \text { iPKPd } 11 & 37.20 & -8.5 x\end{array}$

$0.9 \mathrm{~s} \quad 100.00 \mathrm{~nm}$

$\begin{array}{llllll}142.44 & 59 & \text { iPKPC } & 11 & 39.00 & -8.3 x\end{array}$

$7.0 \mathrm{~s} \quad * * * * * * \mathrm{~nm}$

IPP 1450.50

IPPP $18 \quad 09.00$

143.9156 PKP $1147.70-2.0$

$\begin{array}{llllll}143.96 & 60 & \text { iPKPC } & 11 & 46.20 & -3.8 x\end{array}$

$z 27 \mathrm{~s} 90.00 \mathrm{um} \quad 7.4 \mathrm{MszX}$ PPP 1458.00 eSS $33 \quad 38.00$

144.11311 PKP $1147.00 \quad-2.8 x$

144.38256 e(PKP) 1147.50

$1.0 \mathrm{~s} 736.00 \mathrm{~nm}$

144.44256 e(PKP) $1148.00 \quad-3.3 x$

144.8061 iPKPC $11 \quad 49.40$ i SPKP 1209.50

145.0959 iPKP 1150.00 IPP $15 \quad 10.00$

145.75112 ePKP 1154.80

145.8127 PKP 1152.80

$6.0 \mathrm{~s} * * * * * * * \mathrm{~nm}$

$146.30 \quad 56$ iPKPc $1154.20 \quad 0.4$

$2.0 \mathrm{~s} 3100.00 \mathrm{~nm}$

$Z 25 \mathrm{~s} \quad 230.00 \mathrm{um}$

E $25 \mathrm{~s}$

$\begin{array}{lll}\text { ePP } 15 \quad 22.00 & 0\end{array}$

YSS 146.76317 iPKPC 1155.00

$0.8 \mathrm{~s} \quad 120.00 \mathrm{~nm}$

eSSS 3946.00

$\begin{array}{lllll}146.98 & 199 & \text { ESSS } & 39 & 46.00 \\ \text { iPKPd } & 11 & 56.50\end{array}$

$\begin{array}{llllll}147.34 & 35 & \text { iPKPC } & 11 & 54.90 & -0.1\end{array}$

$2.2 \mathrm{~s} \quad 2470.00 \mathrm{~nm}$ $\begin{array}{lll}\text { ESPKP } 12 \quad 13.00 & 0\end{array}$

$147.35 \quad 51$ PKP 1156.00

147.42107 PKP $1155.00 \quad 0.6$

148.51355 iPKPC $1155.80-0.9$

$1.1 \mathrm{~s} \quad 1970.00 \mathrm{~nm}$

$\begin{array}{lllll}148.76 & 53 \text { PKP } 11 & 57.00 & -1.1\end{array}$

$149.30 \quad 57$ PKPC $1200.00 \quad 1.3$

$149.93 \quad 304$ ePKP $12 \quad 01.00 \quad 1.6$

$\begin{array}{lllll}150.03 & 101 & \text { ePKP } 12 & 00.00 & -0.3\end{array}$

$1.0 \mathrm{~s} \quad 1120.00 \mathrm{~nm}$ 


\begin{tabular}{|c|c|c|c|c|c|c|c|c|c|c|c|}
\hline & & & esPKP & 12 & 20.00 & & 1 & & & SPKP & 12 \\
\hline & & & ePPP & 19 & 07.00 & & 1 & & & e & 13 \\
\hline CBI & 150.81 & 279 & ePKP & 12 & 06.00 & $4.8 x$ & 1 & & & e & 13 \\
\hline TUP & 151.07 & 347 & ePKPc & 12 & 00.90 & 0.3 & 1 & & & $P P$ & 17 \\
\hline & $1.2 \mathrm{~s}$ & 1070 & $0.00 \mathrm{~nm}$ & & & & I IIY & 168.56 & 348 & IPKPC & 12 \\
\hline NDI & 151.42 & 78 & ePKP & 12 & 02.00 & 0.0 & 1 & & & SPKP & 12 \\
\hline & $0.8 \mathrm{~s}$ & 418 & $8.00 \mathrm{~nm}$ & & & & 1 & & & e & 13 \\
\hline BKB & 151.45 & 196 & EPKPC & 12 & 16.30 & $13.8 x$ & 1 & & & $P P$ & 17 \\
\hline PPI & 151.80 & 161 & ePKP & 12 & 02.40 & -0.6 & 1 & & & SKS & 19 \\
\hline UER & 152.41 & 21 & iPKP & 12 & 02.00 & -0.7 & 1 NJ2 & 170.10 & 306 & PKPC & 12 \\
\hline & $2 \mathrm{~s}$ & 1060 & $0.00 \mathrm{~nm}$ & & & & 1 & & & $P P$ & 17 \\
\hline NII & 152.60 & 302 & ePKP & 12 & 06.00 & 2.6 & 1 & & & SKS & 19 \\
\hline MAT & 53.60 & 299 & (PKP) & 12 & 04.00 & -1.0 & I OZH & 171.59 & 261 & IPKPC & 12 \\
\hline IRK & 154.00 & 7 & PKP & 12 & 05.00 & 0.0 & 1 & & & PPKP & 12 \\
\hline PSI & 154.25 & 155 & EPKPC & 12 & 05.80 & -0.6 & 1 & & & e & 13 \\
\hline & $1.0 \mathrm{~s}$ & & B. $60 \mathrm{~nm}$ & & & & 1 & & & PKS & 15 \\
\hline TSI & 154.85 & 154 & ePKPd & 12 & 07.90 & 0.7 & 1 & & & iPP & 17 \\
\hline KGM & 154.94 & 165 & EPKPC & 12 & 09.30 & 2.0 & 1 & & & SKS & 19 \\
\hline & & & e & 16 & 09.00 & & I XAN & 172.50 & 3 & IPKPC & 12 \\
\hline VLA & 155.33 & 318 & IPKPd & 12 & 08.00 & 1.0 & 1 & & & e & 13 \\
\hline WMO & 155.35 & 40 & iPKPC & 12 & 07.00 & -0.1 & 1 & & & PP & 17 \\
\hline & & & e & 12 & 36.00 & & 1 & & & SS & 38 \\
\hline ZAK & 155.71 & 10 & IPKPC & 12 & 07.60 & 0.3 & $\mathrm{CD} 2$ & 173.37 & 47 & IPKPC & 12 \\
\hline & 35 & 3150 & $0.00 \mathrm{~nm}$ & & & & 1 & & & SPKP & 12 \\
\hline & & & e & 12 & 28.00 & & I & & & e & 13 \\
\hline & & & eSKS & 19 & 03.60 & & 1 & & & ePP & 17 \\
\hline MDJ & 155.72 & 323 & PKPC & 12 & 06.00 & -1.5 & I HKC & 173.97 & 227 & IPKP & 12 \\
\hline & & & SPKP & 12 & 21.00 & & I WHN & 174.10 & 314 & PKPC & 12 \\
\hline & & & PP & 16 & 26.00 & & 1 & & & PP & 17 \\
\hline & & & SKS & 19 & 05.00 & & I GZH & 175.06 & 227 & IPKPC & 12 \\
\hline IPM & 156.75 & 158 & ePKPd & 12 & 09.40 & -0.4 & I GYA & 177.52 & 91 & PKPC & 12 \\
\hline & & & e & 12 & 53.20 & & 1 & & & SPKP & 12 \\
\hline & & & e & 13 & 39.10 & & 1 & & & e & 14 \\
\hline & & & e & 14 & 52.10 & & 1 & & & PP & 18 \\
\hline & & & e & 17 & 08.00 & & 1 & & & SKS & 19 \\
\hline DMN & 158.32 & 82 & PKP & 12 & 10.40 & -1.3 & 1 & & & SKKS & 24 \\
\hline & $1.0 \mathrm{~s}$ & 94 & $4.00 \mathrm{~nm}$ & & & & I & & & SS & 39 \\
\hline CN2 & 158.42 & 327 & IPKPC & 12 & 09.30 & -1.6 & I & D. $=1$ & 1.1 & on 348 & 3 of \\
\hline & & & SPKP & 12 & 24.00 & & 1 & & & & \\
\hline & & & e & 12 & 46.00 & & OCT & 09,1 & 1983 & $11 \mathrm{~h} 2$ & $25 \mathrm{~m}$ \\
\hline & & & PP & 16 & 26.00 & & 26. & $141 \$ \pm$ & \pm 1.6 & $5 \mathrm{~km}$ & 70.5 \\
\hline & & & SPP & 16 & 40.00 & & DEP & 'TH $=$ & 6.5 & $\pm \quad 2.2$ & $2 \mathrm{~km}$ \\
\hline & & & ss & 36 & 25.00 & & 5.9 & $\mathrm{mb}(73$ & 3 obs & s.) & $3 \mathrm{Ms}$ \\
\hline SHK & 158.47 & 297 & EPKPC & 12 & 11.10 & -0.2 & NEAR & COAST O & OF NO & RTHERN & $\mathrm{N} \mathrm{CH}$ \\
\hline KKN & 158.48 & 81 & PKP & 12 & 10.60 & -1.2 & 1 & Feit (V & v) ot & Copia & apo, \\
\hline PKI & 158.58 & 82 & PKP & 12 & 10.80 & -1.2 & 1 & Chanaro & at an & id $(11)$ & 1) $i$ \\
\hline & $1.0 \mathrm{~s}$ & 100 & $0.00 \mathrm{~nm}$ & & & & 1 & Antofog & gasta & area. & \\
\hline$P L P$ & 158.83 & 227 & ePKPC & 12 & 16.00 & $3.9 x$ & I & & & & \\
\hline & $1.0 \mathrm{~s}$ & 145 & $5.00 \mathrm{~nm}$ & & & & ANT & 2.43 & 2 & iPct & 26 \\
\hline SNG & 159.01 & 155 & IPKPC & 12 & 12.00 & -0.4 & 1 & & & $i(s)$ & 26 \\
\hline SNY & 160.80 & 326 & IPKPC & 12 & 12.50 & -1.0 & I TLL & 4.02 & 184 & iPd & 26 \\
\hline & & & PPKP & 12 & 28.00 & & 1 & & & i & 26 \\
\hline & & & e & 12 & 59.00 & & I TPL & 4.03 & 4 & ip & 26 \\
\hline & & & PP & 16 & 40.00 & & I FSA & 4.05 & 90 & ePc & 26 \\
\hline & & & SS & 36 & 52.00 & & I SLA & 4.76 & 74 & $i P c$ & 27 \\
\hline NNT & 163.41 & 145 & ePKP & 12 & 15.68 & -1.3 & 1 & & & (S) & 27 \\
\hline LSA & 163.56 & 75 & PKPC & 12 & 18.20 & 0.9 & I CEN & 5.63 & 164 & eP & 27 \\
\hline & & & e & 13 & 11.20 & & I CFA & 5.80 & 160 & ePc & 27 \\
\hline & & & PP & 16 & 59.20 & & 1 & & & $\mathrm{~s}$ & 27 \\
\hline CP & 63.93 & 225 & ePKP & 12 & 26.00 & $8.7 x$ & I YJA & 6.05 & 50 & ePc & 27 \\
\hline$D L 2$ & 163.96 & 323 & IPKPC & 12 & 16.50 & -0.2 & $\mathrm{JACH}$ & 6.52 & 181 & ip & 27 \\
\hline & & & SPKP & 12 & 30.00 & & I $\mathrm{ROCH}$ & 6.82 & 183 & iPd & 27 \\
\hline & & & e & 13 & 28.00 & & $1 \mathrm{PEL}$ & 6.98 & 181 & iP & 27 \\
\hline & & & PP & 17 & 17.00 & & $18 \mathrm{ACH}$ & 7.19 & 180 & eP & 27 \\
\hline HT & 64.54 & 137 & ePKP & 12 & 22.80 & $4.9 x$ & I SAN & 7.29 & 181 & eP & 27 \\
\hline GTA & 164.84 & 30 & IPKPC & 12 & 18.50 & 0.8 & I TCA & 7.34 & 136 & $e P c$ & 27 \\
\hline & & & PP & 17 & 00.00 & & I $\mathrm{PCH}$ & 7.45 & 180 & iPd & 27 \\
\hline & & & SKKS & 23 & 39.00 & & I TACH & 7.50 & 183 & iPc & 27 \\
\hline & & & SS & 37 & 25.00 & & $\mathrm{CHCH}$ & 7.77 & 181 & eP & 27 \\
\hline BAG & 65.55 & 228 & IPKPCt & +12 & 18.00 & -1.0 & LNV & 7.83 & 185 & iPd & 27 \\
\hline CVP & 165.56 & 235 & ePKP & 12 & 19.00 & 0.3 & I ARE & 9.67 & 354 & ip & 27 \\
\hline $\mathrm{HHC}$ & 165.60 & 353 & PKPC & 12 & 19.50 & 1.2 & I LPB & 9.82 & 14 & $\mathbf{P} c+$ & 28 \\
\hline & & & PP & 17 & 02.50 & & 1 & & & is & 30 \\
\hline BTo & 165.95 & 358 & PKPC & 12 & 19.00 & 0.4 & 12080 & 10.07 & 13 & iPc & 28 \\
\hline & & & SPKP & 12 & 34.00 & & I CON & 10.87 & 191 & eP & 28 \\
\hline & & & e & 13 & 20.00 & & 1 & & & 1 & 28 \\
\hline & & & $P P$ & 17 & 04.50 & & | ITB 1 & 14.66 & 88 & ip & 29 \\
\hline & & & SS & 37 & 36.00 & & I HUA & 14.74 & 341 & eP & 29 \\
\hline $\mathrm{PCT}$ & 166.04 & 146 & ePKP & 12 & 20.70 & 1.6 & 1 & & & is & 32 \\
\hline & $0.8 \mathrm{~s}$ & & $0.70 \mathrm{~nm}$ & & & & I 1T87 & 14.78 & 90 & ip & 29 \\
\hline & & & e & 17 & 11.70 & & I ITB & 14.80 & 88 & $P$ & 29 \\
\hline NST & 166.16 & 139 & IPKPd & 12 & 19.00 & -0.2 & I LM2 & 15.28 & 335 & eP & 29 \\
\hline BDT & 166.63 & 132 & EPKP & 12 & 19.00 & -0.6 & I & & & es & 33 \\
\hline & $1.1 \mathrm{~s}$ & 274 & $4.00 \mathrm{~nm}$ & & & & NNA & 15.29 & 336 & ip & 29 \\
\hline $\mathrm{CHG}$ & 167.65 & 126 & ePKPC & 12 & 20.50 & 0.2 & 1 & $0.1 \mathrm{~s}$ & 2390 & .00 & \\
\hline & $1.1 \mathrm{~s}$ & 69 & $7.60 \mathrm{~nm}$ & & & & VAO & 21.64 & 87 & eP & 30 \\
\hline IA & 168.32 & 328 & PKP & 12 & 19.60 & 0.7 & BMA & 24.24 & 88 & eP & 30 \\
\hline
\end{tabular}




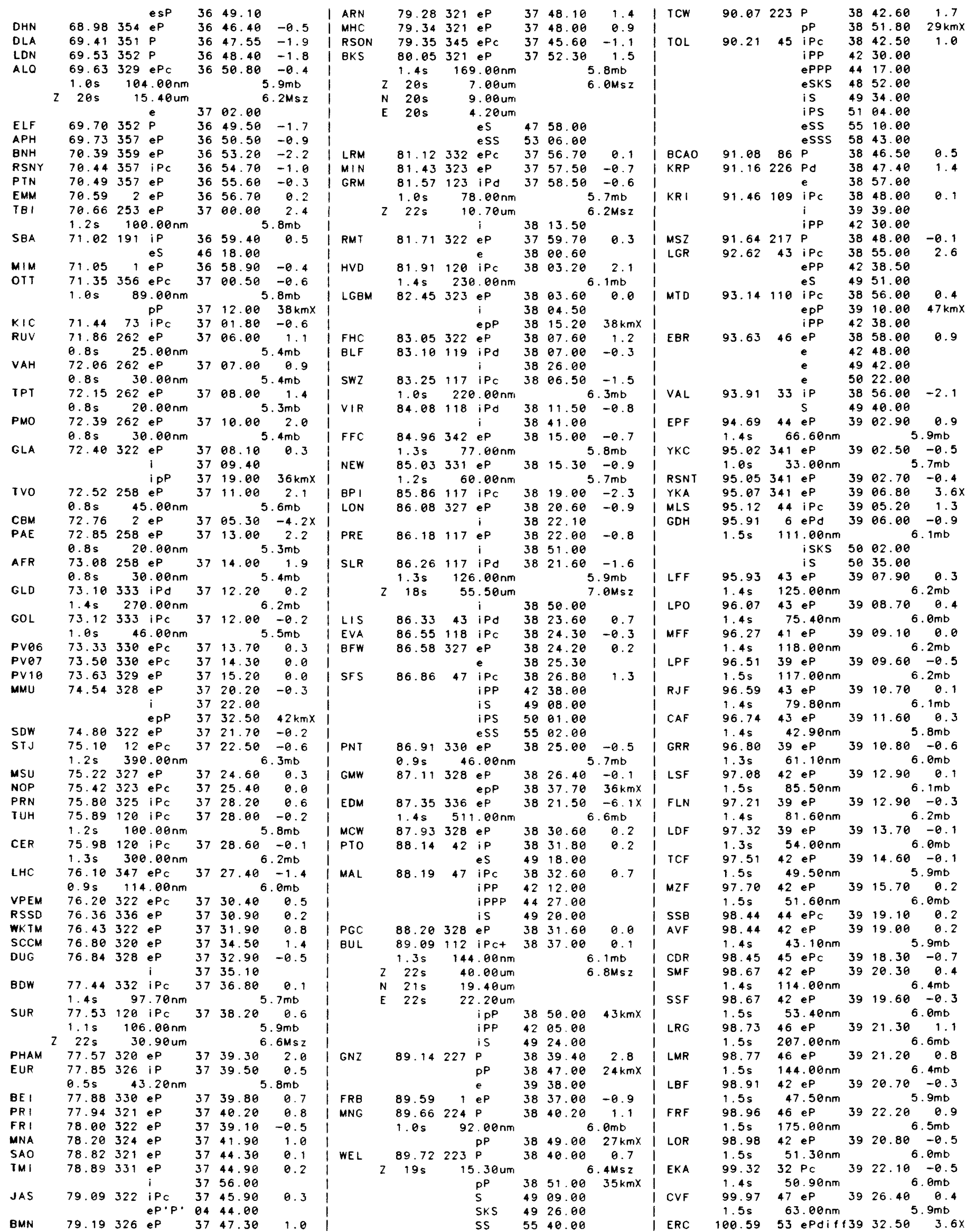


09d 11h

$\begin{array}{llllll}\text { DIX } \quad 100.59 & 44 \text { ePdiff39 } & 29.50 & 0.4\end{array}$ DOU 100.75 39 Pdiff $3929.00-0.3$ SKS $50 \quad 27.00$ S $\quad 5127.00$ SP $\quad 5257.00$

HAU 100.8142 ePOif $13929.40 \quad-0.3$ $1.4 \mathrm{~s} \quad 23.50 \mathrm{~nm} \quad 5.6 \mathrm{mb}$ $100.92 \quad 44$ ePditf $39 \quad 30.50 \quad 0.0$

ROF

\section{BSF}

VEI

$$
\text { WLF }
$$

TMA

$\begin{array}{lllll} & & \text { SP } & 52 & 57.00 \\ \text { TMA } \quad 101.52 & 44 & \text { ePdiff } 39 & 32.50\end{array}$

$\begin{array}{ccccc}\text { CDF } & 101.55 & 42 \text { ePditf39 } & 32.70 & -0.4 \\ & 1.4 \mathrm{~s} & 31.30 \mathrm{~nm} & 5.7 \mathrm{mb} \\ \text { ENN } & 101.81 & 39 \mathrm{ePdit}+39 & 32.50 & -1.5 \\ & 1.2 \mathrm{~s} & 20.00 \mathrm{~nm} & 5.6 \mathrm{mb}\end{array}$

$\begin{array}{lllllll}\text { ZUL } & 101.81 & 43 \mathrm{ePdi}+\mathrm{f} 39 & 34.00 & -0.2\end{array}$

$\begin{array}{llllll}\text { LLS } & 101.92 & 44 & \text { ePdift39 } & 35.00 & 0.1\end{array}$

$\begin{array}{llllll}\text { SLE } & 102.00 & 43 & \text { ePditf39 } & 34.50 & -0.5\end{array}$

DBN $102.00 \quad 38$ ePdiff39 35.00 $220 \mathrm{~s} 6.00 \mathrm{um}$ 6. $1 \mathrm{MsZ}$

e 4403.00 ESKS $50 \quad 37.00$ EPS 5318.00

VDL $\quad 102.05 \quad 44$ ePdiff39 35.50

FIR $\quad 102.06 \quad 47$ ePdift39 38.00 ePP 4400.00

RMP $\quad 102.22 \quad 49$ iPdiff39 36.00 EPP $43 \quad 46.00$ ESKS 5024.00 EPPS 5312.00

SAX $102.30 \quad 43$ ePdift39 36.50

$102.44 \quad 49$ ePdiff39 38.00

$102.56 \quad 44$ ePditf39 37.80

$102.70 \quad 49$ ePdiff39 39.50

$102.86 \quad 38$ ePditt39 39.00

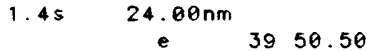
$\begin{array}{lrr}39 & 50.50 \\ \text { (Poit t39 } 39.10\end{array}$ 102.90331 (Pditt39 39.10 $\begin{array}{llll}102.92 & 49 \text { ePditf39 } & 41.00 \\ 103.19 & 44 & \text { iPditf39 } & 41.20\end{array}$ $\begin{array}{ccc}103.19 & 44 & \text { ipditt39 } \\ 1.5 \mathrm{~s} & 94.00 \mathrm{~nm} & 41.20\end{array}$ $103.25 \quad 45$ ePditt3941.00 ePP $43 \quad 04.50$ $\begin{array}{llll}103.53 & 51 \text { ePditt } 39 & 42.50 \\ 103.68 & 44 & \text { iPdit } 39 & 42.80\end{array}$ $\begin{array}{ccc}103.68 & 44 \text { iPdift } 39 & 42.80 \\ 1.25 & 33.00 \mathrm{~nm} & 6\end{array}$

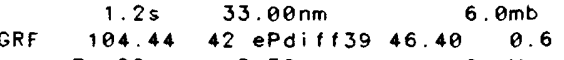

$\begin{array}{ccccc} & 20 s & 5.30 u m & 6.1 M s z \\ \text { TRI } & 104.47 & 46 \text { iPdiff39 } & 40.00^{6} & -6.0 x\end{array}$ iPP 4400.00 ESKS 5024.00 eSKKS $50 \quad 45.00$ e 5200.00 ESP $53 \quad 12.00$ eSS $58 \quad 50.00$ eSSS 0418.00

RBL $\quad 104.63 \quad 45$ ePoiff39 4400

CEY $104.91 \quad 46$ ePditi39 49.00

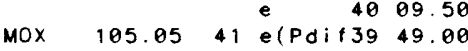
$1.5 \mathrm{~s} 22.00 \mathrm{~nm}$ $\begin{array}{lll}\text { EPP } & 44 & 04.00 \\ \text { ESKS } & 50 & 45.00\end{array}$ eS 5200.00 ePS 5325.00

LJU $105.10 \quad 46$ ePdiff39 49.50 $\begin{array}{lrr}\text { e } & 43 & 52.50 \\ \text { eS } & 50 & 21.50 \\ \text { ePditt } 39 & 55.00\end{array}$

$\begin{array}{llll}\text { LCI } & 105.32 & 52 \text { ePdiff } 3955.00\end{array}$

$\begin{array}{lllll}\text { BER } & 105.73 & 30 \text { ePdiff39 } & 58.00 \\ \text { BRG } & 106.50 & 41 \text { ePdiff } 39 & 56.50\end{array}$

$\begin{array}{cc}1.4 \mathrm{~s} & 12.00 \mathrm{~nm}\end{array}$ e 4320.00 ESKS $50 \quad 36.00$ ePKKP 5527.00

DAG $107.35 \quad 11$ ePOit 3958.00 COP

0.0
$2.7 x$

1.41

0.4

$7 \mathrm{mb}$

1. 7

0.7

$3 \mathrm{mb}$
0.3

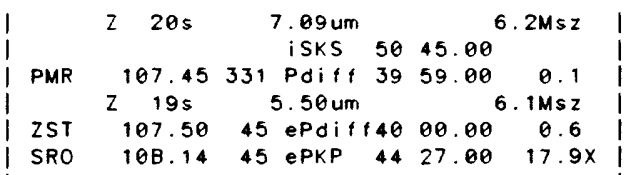

$\begin{array}{cc}108.14 & 45 \text { ePKP } \\ N 20 \mathrm{~s} & 2.10 \mathrm{um}\end{array}$

$\begin{array}{llllll} & \text { COL } 205 & 4.96 \mathrm{um} & & \\ 108.17 & 334 \mathrm{ePdiff40} & 03.00 & 1.0\end{array}$

$\begin{array}{lllllll}\text { FBA } & 108.17 & 334 & \text { Poiff } 40 & 01.50 & -0.5\end{array}$

I SKO 108.3552 ePOiff40 $03.00 \quad-0.4$

$z 21 \mathrm{~s} \quad 17.50 \mathrm{um}$

E $23 \mathrm{~s} 20.30 \mathrm{um}$

i 4429.00

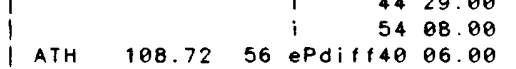

THE 109.0253 iPdiff40 08.00

$\begin{array}{lllll}\text { UZH } & 110.93 \quad 45 \text { ePdiff } 40 \quad 13.00\end{array}$

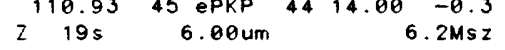

N $195 \quad 5.00 u m$

$\begin{array}{lll}\text { ESP } 54 & 23.00\end{array}$

WAR $111.13 \quad 41$ E(POit40 21.00 $224 \mathrm{~s} 13.00 \mathrm{um}$

e 4446.00

e $\quad 5110.00$

e $\quad 5216.00$

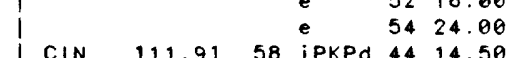

58 IPKPO $44 \quad 14.50-2.0$

$\mid$\begin{tabular}{lccccc|} 
LVV & 112.34 & 44 & ePoiff 40 & 16.00 & $-4.9 \times$ \\
LVV & 112.34 & 44 ePKP 44 & 16.00 & -1.0 \\
& 2 & 225 & $12.00 \mathrm{sm}$ & & $6.4 \mathrm{Msz}$
\end{tabular}

$2 \quad 225$

$6.4 \mathrm{Msz}$

E $215 \quad 12.00 \mathrm{um}$

\begin{tabular}{r|llll} 
& & & ePPS 56 & 00.00 \\
-0.1 & KIS $\quad 114.83$ & 48 ePdiff & 23.00
\end{tabular}

$\begin{array}{llllll}K I S & 114.83 \quad 48 & \text { IPKP } 44 & 20.50\end{array}$

iPP 4529.00

ISP 5501.00

\begin{tabular}{l|llllll}
-0.2 & SOD & 116.57 & 25 & iPKP & 44 & 24.50 \\
1.0 & SIM & 118.02 & 51 & ePKP & 44 & 27.00
\end{tabular}

\begin{tabular}{l|lllll}
1.0 & SIM 118.02 & 51 EPKP & 44 & 27.00 \\
0.1 & & ESKS & 51 & 31.00
\end{tabular}

$\begin{array}{llll}\text { ESKS } & 51 & 31.00 \\ \text { ESPP } & 56 & 51.00\end{array}$ ESS 0203.00

SIM $118.02 \quad 51$ ePOif 44049.00

APA $119.16 \quad 25$ ePOiff40 53.00

eSP 5536.00

$\begin{array}{lllll}120.25 & 52 \text { ePS } & 02 & 13.00 \\ 1 & \text { ePKP } & 44 & 31.50\end{array}$

ANN

(1)

OBN

$1.3 \mathrm{~s} \quad 160.00 \mathrm{~nm}$

EPS 5552.50

120.7140 iPKPd 4432.00

$1.3 \mathrm{~s} \quad 230.00 \mathrm{~nm}$

i sPKP $44 \quad 43.00$

eSKS 5128.00

ESKS 5142.00

iPS 5556.00

\begin{tabular}{|llllll} 
ILT & 120.78 & 335 & ePKP & 44 & 33.00 \\
& & & eSS & 02 & 36.00
\end{tabular}

MOS $121.31 \quad 39$ iPKPO 4434.00

$$
1.5 \mathrm{~s} \quad 210.00 \mathrm{~nm}
$$

$Z 20 \mathrm{~s} \quad 12.50 \mathrm{um}$

E $19 \mathrm{~s} 9.10 \mathrm{um}$

EPPKP 4444.00

EPS 5600.00

121.629 ePdit 41107.00

121.8053 iPKP 4435.00 ESP $56 \quad 00.00$

$121.80 \quad 53$ ePdift41 10.00 $\begin{array}{llll}121.81221 \text { iPKPC } 4435.60 & 35\end{array}$ $1.2 \mathrm{~s} \quad 39.10 \mathrm{~nm}$

IPP $46 \quad 11.00$

iSKP $47 \quad 46.00$

ISKS 5128.00

IPKKP $54 \quad 30.00$

i (SS) 1202.40

MS

i 1708.00

$5.1 \times$ MSL 123.3762 ePKPO 4438.50 e $\quad 46 \quad 17.50$

\begin{tabular}{c|c}
1.6 & P \\
$.7 \mathrm{mb}$ & PYA
\end{tabular}

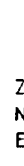

4858.00

$\begin{array}{ll}124.25 & 53 \text { iPKPd } 44 \quad 40.00 \\ 1.5 \mathrm{~s} & 190.00 \mathrm{~nm}\end{array}$

$226 \mathrm{~s} \quad 11.20 \mathrm{um}$

N $26 s \quad 480 u m$

E $26 \mathrm{~s} \quad 6.40 \mathrm{um}$

ESKS 5149.00

iPS 5631.00

$\begin{array}{ll}2.4 \times 1 & \text { ips } \\ & 189\end{array}$ $\begin{array}{lllll}124.52 \quad 57 \text { ePKP } 44 \quad 41.80 & 0.2\end{array}$ B. $0 \mathrm{~s} 1400.00 \mathrm{~nm}$

$\begin{array}{lllll}124.95 & 58 & \text { ePKP } 44 & 39.00 & -2.6 x\end{array}$ ESKS 5146.00 ePS $56 \quad 39.00$

$\begin{array}{lllll}125.04 & 215 & \text { ePKP } 44 & 41.00 & -1.2\end{array}$

125.14208 ePKP $4440.00 \quad-2.4$

125.3356 IPKPC $4442.20 \quad 0.1$

$\begin{array}{llllll}125.47 & 199 & \text { ePK } & 56 & 41.40 & \\ 124 & 41.00 & -1.9\end{array}$

126.1154 IPKPC $44 \quad 44.00 \quad 0.4$ IPP $46 \quad 37.00$ EPPP 4928.00

126.2365 e(PKP) $44 \quad 48.00 \quad 3.6 \times$

$\begin{array}{llllll}126.28 & 59 & \text { IPKPC } 44 & 42.68 & -1.8\end{array}$ $7.0 \mathrm{~s} \quad 600.00 \mathrm{~nm}$

$Z 205 \quad 4.30 \mathrm{um}$

E $205 \quad 6.78$ um

iPPP 4927.00

iSP $56 \quad 43.80$

$\begin{array}{lllllll}\text { KRV } & 126.39 & 57 & \text { iPKP } & 44 & 44.80 & -0.3\end{array}$

$\begin{array}{llllll} & \text { MAK } 127.37 \quad 55 \text { IPKP } 44 & 47.00 & 1.0\end{array}$

$8.0 \mathrm{~s} 1000.00 \mathrm{~nm}$

Z $24 \mathrm{~s} \quad 18.48 \mathrm{um}$

$\mathrm{N} 23 \mathrm{~s} \quad 11.78 \mathrm{um}$

E $225 \quad 4.60 \mathrm{um}$

iSP 5650.00

128.22210 PKPd $44 \quad 46.70 \quad-1.7$

$1.1 \mathrm{~s} \quad 79.30 \mathrm{~nm}$

$129.08 \quad 58$ iPKP $4452.00 \quad 2.6 X$

EPP 4700.00

129.11231 ePKP $4439.00-11.2 \times$

$\begin{array}{llllll}129.97 & 72 & \text { ePKP } 4451.00 & -0.7\end{array}$

132.8136 ePKPC $4456.00 \quad 0.0$

$1.6 \mathrm{~s} 280.00 \mathrm{~nm}$

IPPKP 4504.00

EPP $50 \quad 19.00$

eSP $57 \quad 34.00$

\begin{tabular}{l|llllll}
-0.1 & TIK 133.19 & 352 ePKP 44 & 39.50 & $-16.7 X$
\end{tabular}

$1.0 \mathrm{~s} \quad 10.00 \mathrm{~nm}$

$\begin{array}{lll}Z & 26 \mathrm{~s} & 6.40 \mathrm{um} \\ \mathrm{N} & 27 \mathrm{~s} & 6.90 \mathrm{um}\end{array}$

E $25 \mathrm{~s} \quad 4.90 \mathrm{um}$

e 4455.00

EPPKP 4505.00

EPPP $50 \quad 15.00$

ESKS 5200.00

133.71334 ePKP 4457.80

$1.5 \mathrm{~s} \quad 410.00 \mathrm{~nm}$

ESPKP 4509.80

EPP 4726.00

ESS 0513.08

SVE $133.74 \quad 35$ iPKPC 4458.00

$\begin{array}{cc}133.74 & 35 \text { iPKPC } \\ 2.1 \mathrm{~s} & 230.00 \mathrm{~nm}\end{array}$

ePPS 5933.00

ESS 0525.00

\begin{tabular}{l|llllll}
0.5 & KAT 133.96 & 60 & IPKPC 44 & 59.50 & 0.8
\end{tabular}

$21 \mathrm{~s} \quad 5.30 \mathrm{um}$

4. $80 \mathrm{um}$

EPPP $50 \quad 25.00$

$6.6 \mathrm{Msz}$

isp

5025.00

PET $\quad 134.77 \quad 320$ ePKP $44 \quad 46.00-13.7 \times$

ePP 4736.00

ESP $57 \quad 48.00$

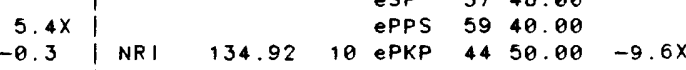

$1.5 \mathrm{~s} \quad 120.00 \mathrm{~nm}$

\begin{tabular}{c|ccc}
$6.8 \times$ & $i$ & 4459.00 \\
-0.4 & eSS & 0523.00
\end{tabular}

VAN 135.3962 ePKP $4451.00-10.5 \mathrm{X}$

$Z 22 \mathrm{~s} \quad 9.03 \mathrm{um} \quad 6.5 \mathrm{MsZ}$

\begin{tabular}{lllllll} 
& \multicolumn{1}{c}{ e } & 45 & 00.00 & \\
ASH $\quad 135.59$ & 62 ePKP & 44 & 53.00 & $-8.8 \times$
\end{tabular}

$6.0 \mathrm{~s} \quad 1600.00 \mathrm{~nm}$

e $\quad 4503.00$

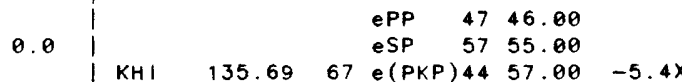

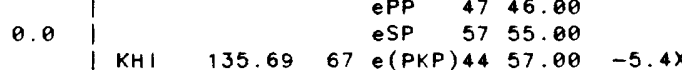

MGD $\quad 135.84 \quad 332$ ePKP $44 \quad 45.00-16.6 x$ $\begin{array}{lll}\text { e } & 4500.00 \\ \text { eSS } & 05 & 35.00\end{array}$

4MsZX MTN 135.89211 ePKP $4448.00-15.0 X$

MHI $136.48 \quad 64$ ePKP $44 \quad 49.00-14.8 \times$

$\begin{array}{lll}\text { e } & 4504.00 \\ \text { e } & 4744.00\end{array}$

es 5950.00 
$09011 \mathrm{~h}$

SKR $\quad 136.96 \quad 317$ ePKP $45 \quad 01.40 \quad-2.6 \times \quad$ KYS EPPP $5054.00 \quad$ I DDR \begin{tabular}{ll|l} 
ePS $58 \quad 10.00$ & SRY
\end{tabular}

YAK $141.65 \quad 345$ iPKPd $450505.50 \quad-6.7 \times \quad$ MAT $1.4 \mathrm{~s} \quad 220.00 \mathrm{~nm}$

$\begin{array}{lll}\text { iPP } 48 \quad 13.10 & 48\end{array}$

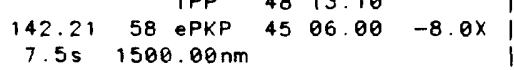
EPPS 9055.00

TAS $\begin{array}{lllll}143.66 & 55 & \text { IPKPC } & 45 & 11.50\end{array}$ $\begin{array}{rrr}1.2 \mathrm{~s} & 400.00 \mathrm{~nm} \\ 2 & 24 \mathrm{~s} & 10.50 \mathrm{um}\end{array}$ N $24 \mathrm{~s} \quad 5.60 \mathrm{um}$ E $24 \mathrm{~s} \quad 7.00 \mathrm{um}$ $\begin{array}{lll}\text { PPP } & 48 \quad 29.00\end{array}$

$\begin{array}{lllllll} & & \text { ePPS } & 01 & 10.00 & \\ \text { DSH } \quad 143.73 & 60 & \text { iPKPC } & 45 & 13.10 & -3.6 x\end{array}$ $\begin{array}{cccc}Z & 22 \mathrm{~s} & 10.00 \mathrm{um} & 6.5 \mathrm{Msz} \\ \mathrm{N} & 24 \mathrm{~s} & 4.80 \mathrm{um} & 6.50 \mathrm{~m}\end{array}$ E $24 \mathrm{~s} \quad 9.50 \mathrm{um}$

iPP $48 \quad 30.00$ ESKS $52 \quad 14.00$

KUR 143.89312 IPKP $45 \quad 13.00 \quad-3.6 x$ $1.0 \mathrm{~s} \quad 330.00 \mathrm{~nm}$

$\begin{array}{lllllll} & & & \text { ePP } & 48 & 30.00 & \\ \text { KUL } \quad 144.58 & 61 & \text { IPKPC } & 45 & 16.50 & -1.6 x\end{array}$ $2.0 \mathrm{~s} \quad 2400.00 \mathrm{~nm}$ $\begin{array}{lll}\text { EPP } \quad 48 \quad 35.80 & 30\end{array}$ EPPP 5154.10

GAR $\begin{array}{lllll}144.86 & 59 \quad \mathrm{iPKPC} & 45 & 16.50\end{array}$ $1.3 \mathrm{~s} \quad 1100.00 \mathrm{~nm}$

$\begin{array}{llllll}145.25 & 214 & \text { ePKPC } & 45 & 16.70 & -3.1 x\end{array}$ $1.2 \mathrm{~s} \quad 1390.00 \mathrm{~nm}$

$\begin{array}{lllllll}\text { NVS } & 145.45 & 27 & \text { ePKPC } & 45 & 18.20 & -0.7\end{array}$ $1.5 \mathrm{~s} \quad 1360.00 \mathrm{~nm}$

$\begin{array}{lllllll}145.85 & 112 & \text { P PKPC } 45 & 21.89 & 0.6 & \text { DMN }\end{array}$

$1,1 \mathrm{~s}$ $\begin{array}{lll}\text { ePP } 48 \quad 44.00 & 0\end{array}$

KHO

$\begin{array}{llllll}146.04 & 61 & \text { iPKPC } & 45 & 20.70 & -0.1\end{array}$ $9.0 \mathrm{~s} \quad * * * * * * \mathrm{~nm}$

$\begin{array}{lll}Z & 24 \mathrm{~s} & 5.70 \mathrm{um} \\ \mathrm{N} & 24 \mathrm{~s} & 2.70 \mathrm{um}\end{array}$

E $245 \quad 6.384 m$

ANR $\quad 146.04 \quad 55$ iPKP $45 \quad 21.50$

$1.4 \mathrm{~s} \quad 1700.00 \mathrm{~nm}$ $\begin{array}{lll}i P P & 48 & 44.00\end{array}$

YSS 146.51317 ePKPC $45 \quad 22.00$ $1.0 \mathrm{~s} \quad 170.00 \mathrm{~nm}$ $\begin{array}{lll}\text { ePP } 48 \quad 53.00 & 0\end{array}$ ESKS $52 \quad 28.00$

SEM $147.00 \quad 35$ iPKP $45 \quad 22.20$ $1.5 \mathrm{~s} \quad 810.00 \mathrm{~nm}$ ePPKP $45 \quad 32.40$ $\begin{array}{lll}\text { EPP } & 48 & 54.50\end{array}$

$\begin{array}{llllll} & & & & & \\ F R U & 147.08 & 51 & \text { iPKP } & 45 & 23.40\end{array}$ $1.4 \mathrm{~s} \quad 1760.00 \mathrm{~nm}$ ESPKP $45 \quad 37.20$ ESPP $01 \quad 32.00$ $\begin{array}{lll}0 & 42.00\end{array}$

LEM $147.18 \quad 177$ EPKPC $45 \quad 22.50$

MKS $\quad 147.36 \quad 199$ ePKPd $45 \quad 24.68$

$\begin{array}{llllll}\text { GBA } & 147.48 & 106 & \text { PKP } & 45 & 23.00\end{array}$

$\begin{array}{lllllll}\text { ELT } & 147.85 & 26 & \text { IPKPC } & 45 & 22.38\end{array}$ $2.0 \mathrm{~s} \quad 450.00 \mathrm{~nm}$

BOD $148.14 \quad 355$ iPKPC $45 \quad 22.90$ $1.3 \mathrm{~s} \quad 300.00 \mathrm{~nm}$

$\begin{array}{llllll}\text { NRN } & 148.50 & 53 & \text { ePKP } & 45 & 25.20\end{array}$

$\begin{array}{llllll}\text { TLG } & 148.82 & 49 & \text { iPKP } & 45 & 24.80\end{array}$

$\begin{array}{cclll}148.82 & 49 & \text { iPKP } & 4524.80 \\ & & i & 45 & 29.60 \\ & & i & 46 & 25.80\end{array}$ i 4625.80

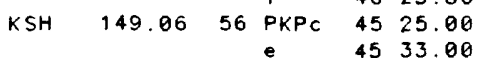

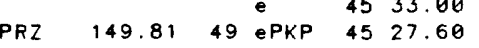
$1.4 \mathrm{~s} \quad 1600.00 \mathrm{~nm}$

ePP 4906.00 $\begin{array}{lllll}150.06 & 101 & \text { IPKPC } & 45 \quad 27.20\end{array}$ $1.25 \quad 729.00 \mathrm{~nm}$

NDI $\quad \begin{array}{llllll}151.29 & 78 & \text { IPKPC } & 45 & 29.50\end{array}$ $1.4 \mathrm{~s} \quad 209.00 \mathrm{~nm}$

ND1 $\quad 151.29 \quad 78$ iPKP $45 \quad 35.00$ $\begin{array}{lll}\text { iPP } 49 \quad 15.00 & 0\end{array}$ EPPP $\quad 52 \quad 46.00$
4

MNI $\quad 151.34 \quad 214$ ePKPc $45 \quad 30.90$ $1.3 \mathrm{~s} 1020.00 \mathrm{~nm}$

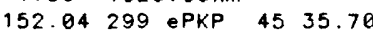

PPI OYM

$\begin{array}{lllll}152.21 & 296 & \text { ePKP } & 45 & 36.80\end{array}$ 152.81298 ePKP

152.83298 ePKP

152.89297 ePKP $45 \quad 36.50$

153.45300 (PKP) 4525.00

$1.5 \mathrm{~s} \quad 120.00 \mathrm{~nm}$

EPPKP 4539.00

e 4553.00

ePP 4924.00

ePPS $02 \quad 36.00$

PSI $154.58 \quad 155$ ePKPd $45 \quad 36.00$

$155.03 \quad 40$ PKPC 4533.50

PPKP $45 \quad 43.80$ $\begin{array}{lll}\text { e } & 4600.00 \\ P P & 4938.00\end{array}$ $\begin{array}{llllll}\text { VLA } \quad 155.08 & 319 & \text { iPKPd } 4530.08\end{array}$

155.08319 iPKPd 4530.00 EPPP 5302.00 ESSS 1502.08

155.29165 EPKPC

4536.00 $\begin{array}{lllll}155.33 & 10 & \text { iPKP } & 46 & 00.00 \\ 45 & 34.80\end{array}$ $9.0 \mathrm{~s} 2620.00 \mathrm{~nm}$

e 4600.00 ePP $\quad 49 \quad 36.00$ ePPS 0248.00 MDJ $155.44 \quad 324$ ePKP $45 \quad 34.30$ $\begin{array}{lllll}155.44 & 324 & \text { P PPP } & 45 & 34.30 \\ 157.08 & 158 \text { ePKPC } & 45 & 36.40\end{array}$ $1.3 \mathrm{~s} \quad 69.40 \mathrm{~nm}$

$\begin{array}{lll}69.40 \mathrm{~nm} & 46 & 05.30\end{array}$ CN2 $\quad 158.12 \quad 328$ iPKPC $45 \quad 36.50$ PPKP 4547.00 \begin{tabular}{lll} 
PP & 46 & 12.00 \\
\hline & 49 & 52.00
\end{tabular} $\begin{array}{ccccc} & & \text { PP } & 49 & 52.00 \\ 158.22 & 81 \text { PKP } & 45 & 38.50\end{array}$ 158.34298 ePKP $45 \quad 38.80$ $\begin{array}{lllll}158.48 & 81 & \text { PKP } & 45 & 38.30\end{array}$ $1.4 \mathrm{~s} \quad 280.00 \mathrm{~nm}$

$159.12 \quad 227$ ePKP $45 \quad 37.00$ $\begin{array}{llll}159.34 & 154 & \text { ePKP } & 45 \quad 39.00\end{array}$ $\begin{array}{lllll}160.51 & 327 & \text { i PKPc } & 46 & 17.00 \\ 160.00\end{array}$ e $\quad 4622.00$ $\begin{array}{lll}\text { PP } & 50 & 07.00\end{array}$ $163.42 \quad 73$ iPKPC $45 \quad 45.40$ $\begin{array}{lll}\text { P } & 46 \quad 35.40 \\ \text { iPP } & 50 & 23.90\end{array}$ 1.1

$\begin{aligned} 163.68324 & \text { PKP } \\ \text { e } & \text { PP }\end{aligned}$

4541.50 $46 \quad 36.00$
$P P \quad 50 \quad 20.00$

$\begin{array}{llll}163.70 & 144 & \text { ePKP } & 45 \quad 42.50\end{array}$ $\begin{array}{llll}164.49 & 29 & \text { iPKP } \quad 45 \quad 45.50\end{array}$ $i \quad 4640.20$ PP $\quad 50 \quad 22.00$

$\begin{array}{llll}\text { SKKS } & 57 & 12.00\end{array}$ 1.3

KHT BJI $\begin{array}{cccc}164.79136 \text { ePKP } & 45 & 46.20 \\ & \text { e } & 50 & 26.50\end{array}$ 165.05340 ePKP $45 \quad 45.00$ PPKP $45 \quad 56.00$ - $\quad 4638.00$ PKS 4921.00 PP $\quad 50 \quad 25.00$ (SKS) $52 \quad 46.00$ e $\quad 5404.00$ $\begin{array}{lll}\text { PPP } & 54 \quad 24.00\end{array}$ SKKS $57 \quad 17.00$ SKKKS $00 \quad 51.00$ SS 1041.00 HHC 165.23354 PKP 4546.80 C $\quad 4644.00$ \begin{tabular}{|lllll} 
& & PP & 50 & 29.00 \\
BTO & $165.57 \quad 358 \quad \mathrm{IPKPC}$ & 45 & 46.40
\end{tabular} P $\quad 46 \quad 45.00$ BAG 165.84229 ePKPC+45 45.00 $\begin{array}{lllll}\text { BAG } & 165.84 & 229 & \text { ePKPC+45 } & 45.00 \\ \text { PCT } & 166.33 & 145 & \text { PKP } 45 & 48.00\end{array}$

\begin{tabular}{c|ccccc}
-0.2 & PCT & 166.33 & $145 \mathrm{ePKP}$ & 45 & 48.00 \\
0.6 & & $1.3 \mathrm{~s}$ & $24.90 \mathrm{~nm}$ & & \\
0.1 & & $\mathrm{e}$ & 46 & 49.50
\end{tabular}

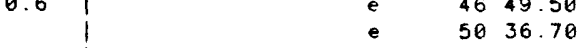
$6.1 \times$ NST 166.42138 iPKPd 4547.00 $\left\{\begin{array}{ccc}\text { BDT } & 166.85 & 130 \mathrm{iPKPC} \\ & 1.3 \mathrm{~s} & 293.00 \mathrm{~nm}\end{array}\right.$

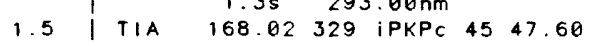
$5.9 \times$ 0.41 $\begin{array}{lll}\text { e } & 46 & 55.00 \\ \text { PKS } & 49 & 19.00\end{array}$ PP 5044.00

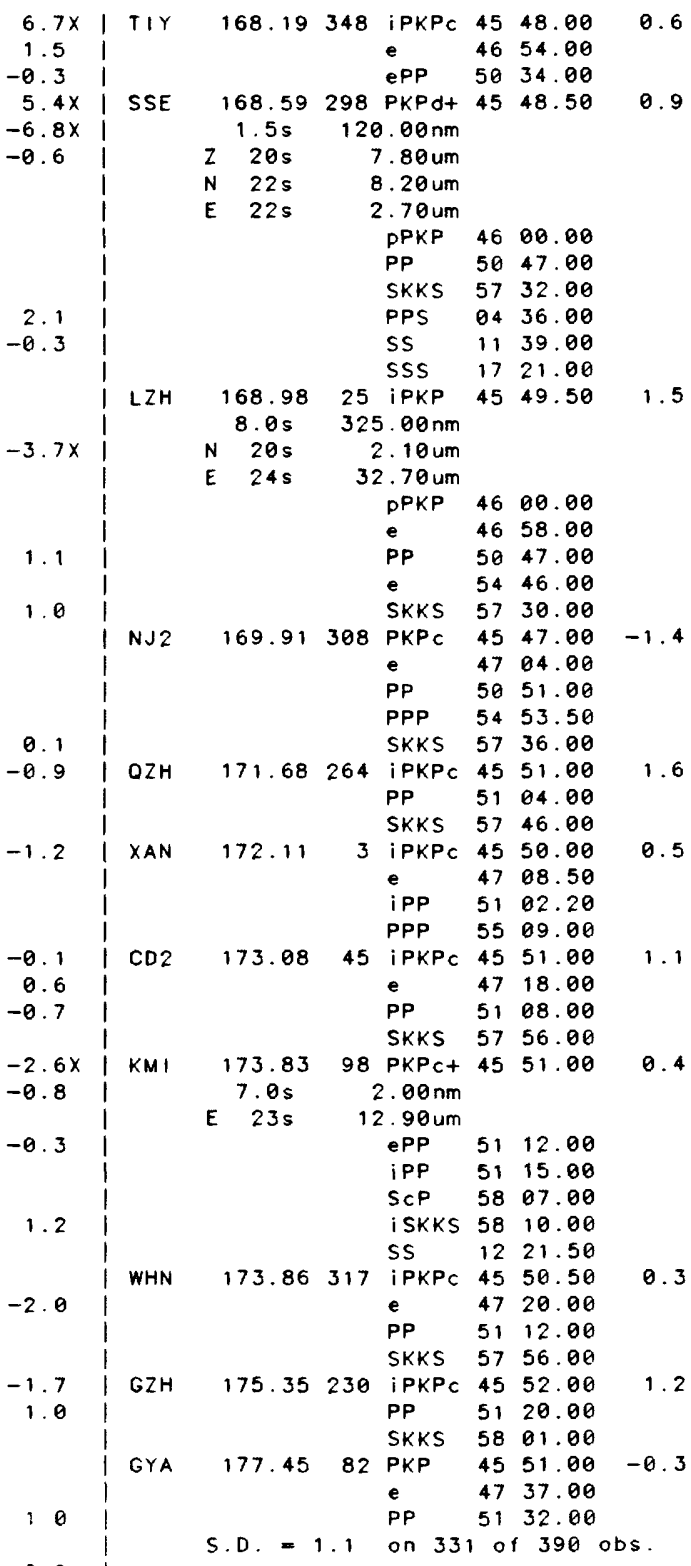

OCT $17,1983 \quad 19 \mathrm{n} 36 \mathrm{~m} 22.46 \pm 0.08 \mathrm{~s}$ $37.602 \mathrm{~N} \pm 2.0 \mathrm{~km} \quad 17.502 \mathrm{~W} \pm 1.1 \mathrm{~km}$ DEPTH $=16.0 \mathrm{~km}$ ( 17 depth phases $)$ 6. $1 \mathrm{mb}$ ( 107 obs.) 6.4Msz ( 22 obs.) NORTH ATLANTIC OCEAN

(402)

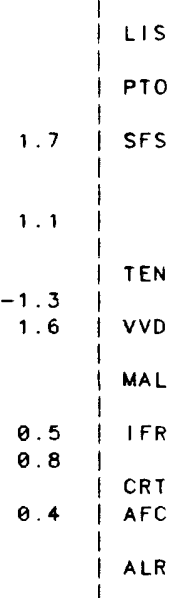

$$
6.6878 \mathrm{ePnC}
$$

3757.40

$-4.8 x$

7.74 isn 3903.50 $\begin{array}{lll}\text { isn } & 38 & 11.60 \\ \text { isn } & 39 & 31.20\end{array}$

9.1194 IPO $38 \quad 36.00$ i $\quad 3944.00$ is 4008.00 iss $40 \quad 38.00$

$9.17 \quad 173 \mathrm{iPC} \quad 38 \quad 32.00$

is 4004.00 $\begin{array}{cccc}9.77182 \mathrm{P} & 3841.4 \theta \\ & \mathrm{S} & 40 & 19.4 \theta\end{array}$

$10.4991 \mathrm{iPC} \quad 3851.00$ 10.87408 is 4113.00 $\begin{array}{rrrr}10.87 & 108 \text { iPd } & 3854.00 \\ & \text { iSn } & 40 & 46.00\end{array}$ $\begin{array}{llllll}11.07 & 88 & \mathrm{iPd} & 38 & 59.30 & -3.9 x\end{array}$ $\begin{array}{llllll}11.11 & 88 & \mathrm{iPg} & 38 & 59.40 & -4.4 \mathrm{X}\end{array}$ $11.72 \quad 94 \mathrm{e}(\mathrm{P}) \quad 40 \quad 45.50$ e(S) 4115.00
$-5.6 x$ $-0.1$ $-5.0 x$ $-4.8 x$ $-4 \cdot 1 x$ $-6.6 x$ $-3.9 x$ 
$17 d \quad 19 h$

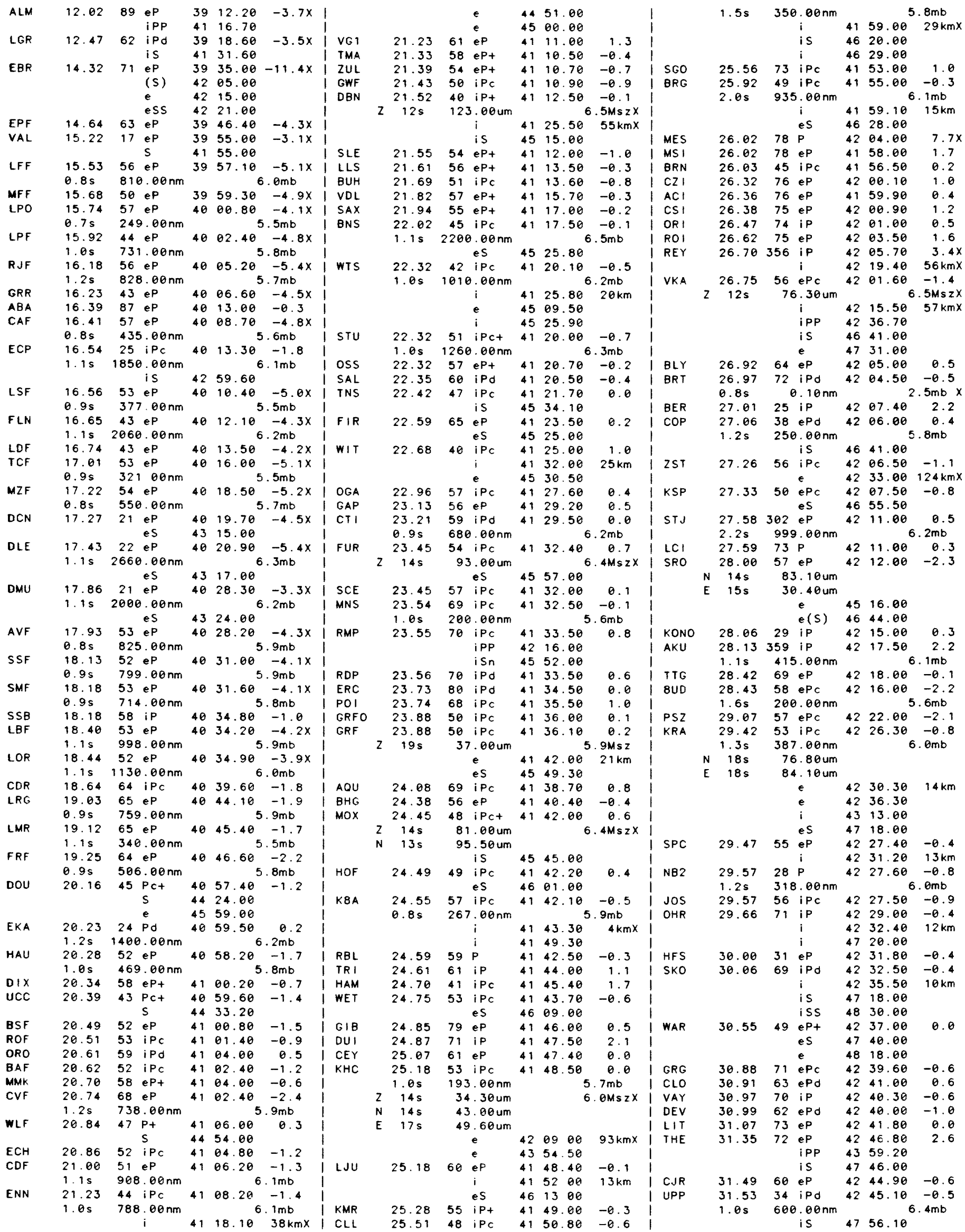


$17 \mathrm{~d} 19 \mathrm{~h}$

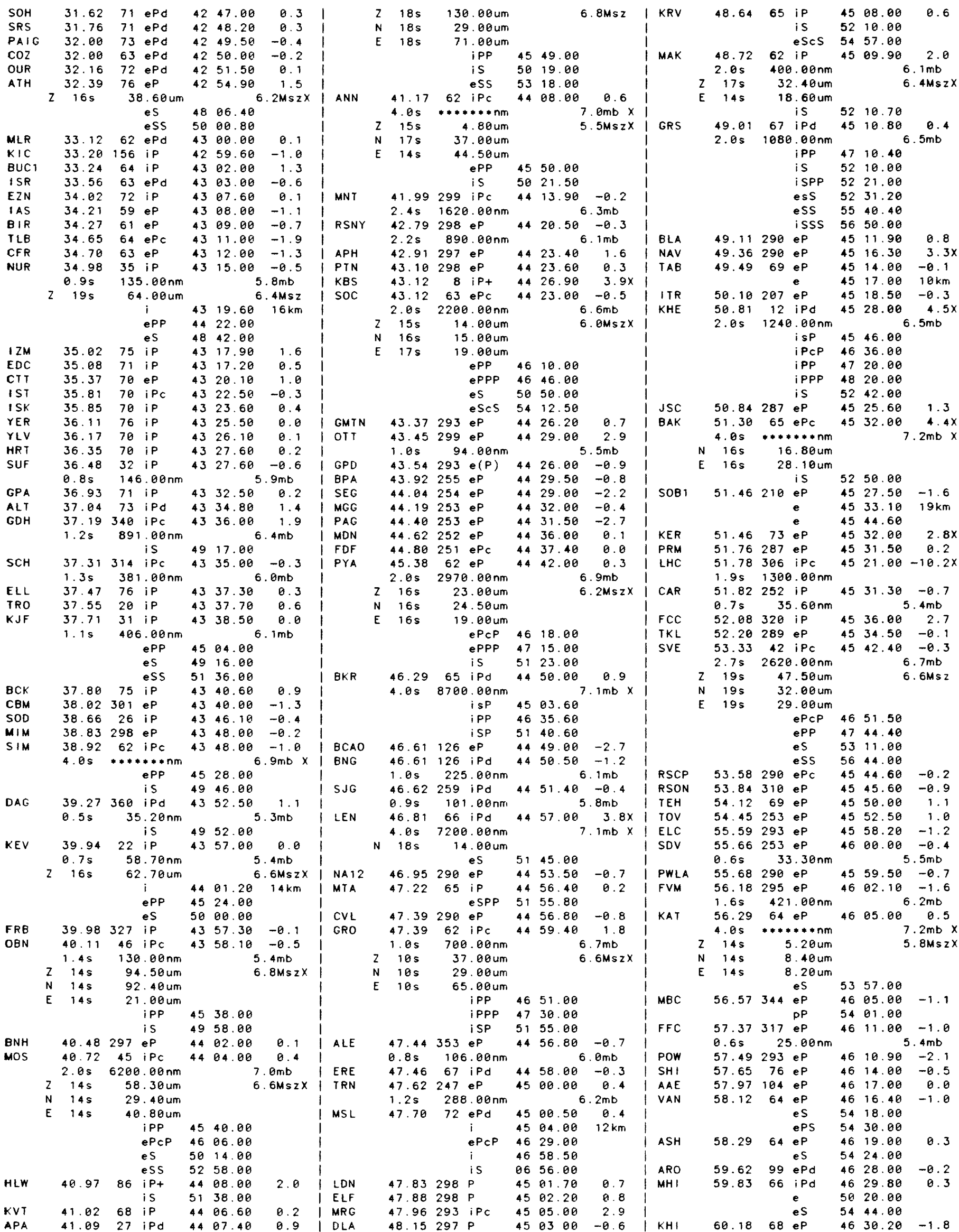


$17 d 19 n$

BA

YKC

NR I

$60.28214 P$ $\begin{array}{lll}60.28 & 295 \mathrm{iPc} \\ 60.44 & 328 \mathrm{eP}\end{array}$

$0.95 \quad 70.00 \mathrm{~nm}$ $60.48 \quad 23 \mathrm{iPc} \quad 46 \quad 34.00^{5.8 \mathrm{mb}} 0.6$ $2.5 \mathrm{~s} \quad 1500.00 \mathrm{~nm}$ $Z 20 \mathrm{~s} \quad 30.00 \mathrm{um}$ $\mathrm{N} 17 \mathrm{~s} \quad 31.00 \mathrm{um}$ E $20 \mathrm{~s} \quad 29.00 \mathrm{um}$ $\begin{array}{lll}\text { CPCP } 47 & 19.00\end{array}$ ePPP $50 \quad 14.00$ eS 5451.00 eSCS $56 \quad 19.00$ eSS 5844.00

RSNT $\quad 60.49328 \mathrm{eP} \quad 4632.60$

$\begin{array}{llcllll} & & \mathrm{i} & 46 & 36.40 & 12 \mathrm{~km} \\ \text { YKA } & 60.49 & 328 \mathrm{eP} & 46 & 34.00 & 0.5\end{array}$

$\begin{array}{lllllll}\text { GBO } & 60.53 & 294 & \mathrm{e}(\mathrm{P}) & 46 & 33.16 & -1.6\end{array}$

TUL $60.95295 \mathrm{iPc}-4635.20-1.7$

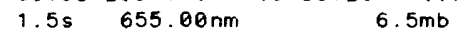
14. 70 um

$\mathrm{BHO}$

$\begin{array}{llllll}60.97 & 293 & \text { iPc } & 46 & 35.70 & -1.4\end{array}$ 60.99252 iP

$\begin{array}{llll}\text { CHN } & 61.95 & 253 \text { iP } \\ \text { NSLM } & 62.17 & 290 & \text { iP }\end{array}$

NSLM $\quad 62.17290$ eP $46 \quad 45.00-0.3$

SAM $63.26115 \mathrm{eP}$ $1.0 \mathrm{~s} \quad 110.00 \mathrm{~nm}$ 63.5759 eP $2.5 \mathrm{~s} \quad 2000.00 \mathrm{~nm}$ $4654.00^{6.0 m b}-0.5$ $214 \mathrm{~s} \quad 14.90 \mathrm{um}$ E $13 \mathrm{~s}$ 15. $30 \mathrm{um}$ $\begin{array}{lll}\text { ePP } \quad 49 \quad 15.0 & 0\end{array}$ ePPP $50 \quad 45.50$ eS 5528.00 eScS 5641.90

HKT

EDM 63.82289 eP 4655.00

INK

TAS $\begin{array}{ll}-1.1 \\ 40 & -0.4\end{array}$ $1.3 \mathrm{~s} \quad 167.00 \mathrm{~nm}$ $64.3457 \mathrm{eP}^{\mathrm{P}}$

$2.8 \mathrm{~s} \quad 6000.00 \mathrm{~nm}$ $\begin{array}{lrr}Z & 16 \mathrm{~s} & 24.00 \mathrm{um} \\ \mathrm{N} & 13 \mathrm{~s} & 16.90 \mathrm{um}\end{array}$ E $14 \mathrm{~s} \quad 8.00 \mathrm{um}$

$\begin{array}{lll}\text { ePP } & 49 & 18.00 \\ \text { EPPP } & 50 & 56.00\end{array}$

\begin{tabular}{lll} 
ePPP & 50 & 56.00 \\
\hline$S$ & 55 & 37.80
\end{tabular} ePPS 5607.00

BMA $\quad 65.01207 \mathrm{eP} \quad 4703.30$

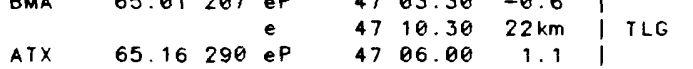

DSH eS 5555.00

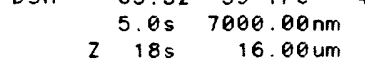

PSO $\quad 65.69252$ iP $\quad 47 \quad 13.50$

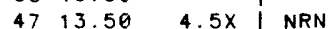
$65.71302 \mathrm{eP} \quad 4708.10-0.5$ I NEW $2.4 \mathrm{~s} 2400.00 \mathrm{~nm}$

2195 $6.9 \mathrm{mb}$ | BE ।

NVS

GOL $\quad \begin{array}{llllll} & 65.84302 \mathrm{eP} & 56 & 02.80 & \\ & 157.68 & 08.60 & -0.9\end{array}$ $1.2 \mathrm{~s} 50.80 \mathrm{~mm}$ $Z 19 \mathrm{~s} \quad 28.80 \mathrm{um}$ $5.6 \mathrm{mb}$

$7.0 \mathrm{~s} 3000.00 \mathrm{~nm} \quad 6.6 \mathrm{mb} \times$ is 5602.00

KUL $\quad \begin{array}{llllll}66.31 & 60 \mathrm{eP} & 56 & 02.00 & \\ & 3.1 \mathrm{~s} & 4600.00 \mathrm{~nm} & 11.90 & -0.4 \\ & & & 7.1 \mathrm{mb} \times\end{array}$

IPCP $47 \quad 42.30$ $66.35210 \mathrm{eP} \quad 5547.20$ $66.35210 \mathrm{eP} \quad 5547.20$

VAO 66.51291 iP $1.0 \mathrm{~s} \quad 215.00 \mathrm{~nm}$ $7.12 .60-0.2$ $66.52 \quad 44$ iPc $4713.00^{6.3 m b}$ $2.8 \mathrm{~s} \quad 3480.00 \mathrm{~nm} \quad 7.0 \mathrm{mb}$ EPP $\quad 4939.50$ ePPP $51 \quad 19.50$ ES $56 \quad 03.60$ ePPS $56 \quad 36.70$

BDW

FRU

LRM

I MW

ELT

TMI

| KR I

DUG

I COL

FBA

I RMU

\begin{tabular}{lllll|l}
66.64 & $56 \mathrm{ePC}$ & 47 & 14.40 & 0.1 & ITB
\end{tabular} 64.25338 P $4657.00-1.5$ $\begin{array}{ll}65.79 & 38 \mathrm{eP} \\ 2.0 \mathrm{~s} & 1700.00 \mathrm{~nm}\end{array}$ EPP 4939.40 EPCP $47 \quad 41.90$

Z $12 \mathrm{~s}$

N $12 \mathrm{~s} \quad 13.00 \mathrm{um}$

E $12 \mathrm{~s} \quad 17.00 \mathrm{um}$

13.00um

$$
\begin{array}{lll}
\text { iPP } & 4945.60 \\
\text { eS } & 5607.70
\end{array}
$$

$67.04307 \mathrm{eP} \quad 4607.70$

$0.7 \mathrm{~s} \quad 109.00 \mathrm{~nm}$

\begin{tabular}{ll|l}
15.50 & -1.6 I VHO
\end{tabular}

$\begin{array}{llllllll}67.05 & 53 \mathrm{eP} & 47 & 33.90 & 69 \mathrm{kmx} & \text { I ZOBO }\end{array}$

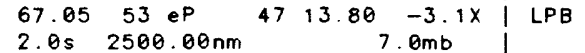

$\begin{array}{llll}Z & 18 \mathrm{~s} & 30.00 \mathrm{um} & 6.6 \mathrm{Msz}\end{array}$

$N \quad 19 s \quad 35.50 u m$

E $19 \mathrm{~s}$

31. 00 um
iPcP

ePP 4945.00

eS 5606.00

ESS 0028.00

$67.27311 \mathrm{eP}$

$47 \quad 17.90$

$67.33 \quad 309$ eP

-0.6 PGC

$67.7459 \mathrm{ePC}$

$5.6 \mathrm{~s} \quad 4000.00 \mathrm{~nm}$

$Z 16 \mathrm{~s} \quad 10.00 \mathrm{um}$

$4718.40-0.6$

$\begin{array}{llr}Z & 16 \mathrm{~s} & 10.00 \mathrm{um} \\ \mathrm{N} & 16 \mathrm{~s} & 2.50 \mathrm{um}\end{array}$

E $16 \mathrm{~s} \quad 15.80 \mathrm{um}$

$$
\text { EPP }
$$

EPS
ESS

$21.20-0.4 \quad$

$6.8 \mathrm{mb} \times 1$

I GMW

I PHC
I 111

$\begin{aligned} \text { EPS } & 5642.00 \\ \text { ESS } & 9030.00\end{aligned}$

4950.00

$2.2 \mathrm{~s}, 1340.00 \mathrm{~nm}$

N $16 \mathrm{~s} \quad 7.40 \mathrm{um}$

E $16 \mathrm{~s} \quad 32.00 \mathrm{um}$

68.25308 is

$68.2952 \quad \mathrm{iP}$

$3.8 \mathrm{~s} \quad 6.80 \mathrm{~nm}$

$Z 16 \mathrm{~s} \quad 21.50 \mathrm{um}$

$N$ 16s $19.50 \mathrm{um}$

E $16 \mathrm{~s} 18.50 \mathrm{um}$

iPP

$\begin{array}{ll}68.49 & 11 \mathrm{ePc} \\ 3.0 \mathrm{~s} & 1900.00 \mathrm{~nm}\end{array}$

$\begin{array}{rr}3.0 \mathrm{~s} & 1900.00 \mathrm{~nm} \\ 218 \mathrm{~s} & 11.00 \mathrm{um}\end{array}$

$\mathrm{N} 19 \mathrm{~s} \quad 9.00 \mathrm{sm}$

E $16 \mathrm{~s} \quad 31.80 \mathrm{um}$

$\begin{array}{lll}\text { EPCP } & 47 & 50.00 \\ \text { ePP } & 4958.00\end{array}$

$\begin{array}{lll}\text { ePP } & 49 & 58.00 \\ \text { ES } & 56 & 27.00\end{array}$

eSP 5642.00

EPPS 5710.00

\begin{tabular}{llllll|l}
68.56 & $51 \mathrm{eP}$ & 47 & 26.10 & -0.3 & LGBM
\end{tabular}

$2.7 \mathrm{~s} \quad 1030.00 \mathrm{~nm}$

$Z 16 \mathrm{~s} \quad 16.00 \mathrm{um}$

$N$ 15s $14.70 \mathrm{sm}$

E $15 \mathrm{~s} \quad 16.00 \mathrm{um}$

ipP

$68.65 \quad 54$ ep

$68.69315 \mathrm{eP}$

$68.79307 \mathrm{eP}$

$69.00298 \mathrm{eP}$

$2.0 \mathrm{~s} \quad 735.00 \mathrm{~mm}$

4958.00

$6.3 \mathrm{Ms} z \mathrm{X}$

MIN

BMN

I PME

$2.05 \quad 735.00 \mathrm{~nm}$

$\begin{array}{cc}18 \mathrm{~s} & 17.20 \mathrm{um} \\ 69.49 & 317 \mathrm{eP}\end{array}$

$1.2 \mathrm{~s} \quad 131.00 \mathrm{~nm}$

$69.6152 \mathrm{eP}$

$2.2 \mathrm{~s} \quad 1500.80 \mathrm{~nm}$

$N \quad 16 \mathrm{~s} \quad 38.00 \mathrm{um}$

E $16 \mathrm{~s} \quad 16.00 \mathrm{um}$

EPP 5011.00

EPPP 5152.00

$\begin{array}{rrrr}69.68 & 55 \mathrm{eP} & 5640.00 \\ & \mathrm{ePP} & 4736.00 \\ & & 5007.00\end{array}$

$\begin{array}{lll}69.95 & 132 & \text { SS } \\ & \text { IPo }\end{array}$

0110.00

ipP

4733.00

I RMT

$70.47306 \mathrm{eP}$

$70.56304 \mathrm{eP}$

$70.70340 \mathrm{eP}$

$222 \mathrm{~s} \quad 13.70 \mathrm{um}$ es

$70.70340 \mathrm{eP}$

$70.96302 \mathrm{eP}$

$\begin{array}{lll}47 & 39.00 & -0.1\end{array}$

5655.00

6. $2 \mathrm{MsZ}$

$\begin{array}{lll}47 & 39.50 & 0.4\end{array}$

$2.4 \mathrm{~s} 3000.00 \mathrm{~nm}$

I MSU

$70.97304 \mathrm{eP}$

$4741.10-0.2$

$4741.00-0.1$

71.01215 e(P)

$71.10 \quad 130 \quad \mathrm{iPd}$

$71.10313 \mathrm{eP}$

$71.28343 \mathrm{eP}$

71.29278 iP

$71.43303 \mathrm{eP}$

71.65231 iP

$71.83231 \mathrm{P}$

$1.0 \mathrm{~s} \quad 80.00 \mathrm{~nm}$

$z 22 \mathrm{~s} \quad 28.10 \mathrm{um}$

$\begin{array}{lc} & \mathrm{S} \\ 71.84 & 128 \mathrm{eP} \\ 0.9 \mathrm{~s} & 0.10 \mathrm{~nm}\end{array}$

$71.85281 \mathrm{eP}$

$71.91334 \mathrm{eP}$

$71.94318 \mathrm{eP}$

$71.98 \quad 135$ iPd

ipP

$72.17316 \mathrm{e}(\mathrm{P})$

$72.24317 \mathrm{eP}$

$72.73 \quad 321 \mathrm{eP}$

$72.79280 \mathrm{eP}$

72.89307 iP

$0.8 \mathrm{~s} \quad 14.80 \mathrm{~nm}$

$\begin{array}{lllll}1.61338 \mathrm{eP} & 47 & 56.60 & 0.3\end{array}$

$2.1 \mathrm{~s} \quad 539.00 \mathrm{~nm}$

$20 \mathrm{~s} 6.00 \mathrm{um} 4757.00 .9 \mathrm{Ms} 2$

$\begin{array}{lllll}73.66 & 338 \mathrm{eP} & 47 & 57.00 & 0.4 \\ 73.87 & 353 \mathrm{eP} & 47 & 58.00 & 0.4\end{array}$

$1.0 \mathrm{~s} \quad 170.00 \mathrm{~nm} \quad 6.0 \mathrm{mb}$

\begin{tabular}{lll} 
iPCP $48 \quad 18.60$ \\
\hline
\end{tabular}

$\begin{array}{lll}\text { EPP } & 50 & 47.00 \\ \text { iS } & 57 & 30.00\end{array}$

iss 0212.00

$73.92 \quad 234 \mathrm{eP} \quad 4757.50$

5728.00

5800.00

0220.00

0650.00

0810.00

1114.00

$73.96 \quad 279 \quad \mathrm{eP}$

$47 \quad 59.80$

$74.42 \quad 46$ iPc $48 \quad 02.00$

$74.73 \quad 123 \mathrm{eP}$

$75.42 \quad 288$ eP

$75.63311 \mathrm{eP}$

$75.83301 \mathrm{eP}$

$75.88310 \mathrm{eP}$

$75.95 \quad 141$ iPd

$76.13305 \mathrm{eP}$

$76.1434 \mathrm{iPC}$

$2.5 \mathrm{~s} \quad 3290.00 \mathrm{~nm}$

4804.00

$4809.80 \quad 2.4$

$\begin{array}{lll}48 & 09.20 \quad 0.5\end{array}$

$4803.20-6.5 x$

$48 \quad 10.00 \quad 0.0$

$\begin{array}{lll}48 & 07.00 & -3.3 x\end{array}$

$\begin{array}{lll}48 & 12.40 & 0.9\end{array}$

$\begin{array}{llllll}76.24 & 138 \mathrm{eP} & 48 & 12.00 & -0.1\end{array}$ 


\begin{tabular}{|c|c|c|c|c|c|c|c|c|c|c|c|c|c|c|c|c|c|c|c|c|c|}
\hline & & & & eScS & 58 & 33.00 & & 1 & & & SS & 05 & 57.00 & & I MTN & 142.72 & 57 & ePKP & 55 & 52.00 & $-5.0 x$ \\
\hline & & & & ess & 02 & 56.00 & & I HHC & 89.07 & 36 & $e^{P}$ & 49 & 21.50 & $3.3 x$ & KLB & 142.96 & 94 & ePKP & 55 & 53.00 & $-4.1 x$ \\
\hline \multirow[t]{6}{*}{ YAK } & & 77.25 & 15 & ePc & 48 & 16.60 & -0.4 & I PET & 89.69 & 2 & eP & 49 & 22.40 & 1.7 & NWAO & 143.02 & 97 & ePKP & 55 & 55.00 & -2.1 \\
\hline & & $0.7 \mathrm{~s}$ & & $0.00 \mathrm{~nm}$ & & & $6.8 \mathrm{mb}$ & 1 & & & is & 00 & 12.00 & & I KNA & 143.13 & 63 & ePKP & 55 & 54.00 & $-3.7 x$ \\
\hline & $z$ & $13 \mathrm{~s}$ & & $8.70 u m$ & & & $6.3 M s z X$ & $i$ & & & ess & 06 & 00.00 & & RAB & 145.43 & 18 & iPKP & 56 & 05.20 & $3.5 x$ \\
\hline & E & $15 \mathrm{~s}$ & & $9.50 \mathrm{um}$ & & & & | BJI & 91.82 & 34 & $e^{P}$ & 49 & 31.00 & 0.2 & $\mathrm{Kl}$ & 145.59 & 91 & iPKPd & 56 & 02.90 & 1.3 \\
\hline & & & & IPCP & 48 & 26.70 & & i & $1.5 \mathrm{~s}$ & & $1.90 \mathrm{~nm}$ & & - & 4. $3 \mathrm{mb} \times$ & Af & 147.14 & 309 & $e(P K P)$ & 56 & 12.00 & $7.5 x$ \\
\hline & & & & isks & 58 & 23.40 & & $i$ & N $19 \mathrm{~s}$ & & $0.20 \mathrm{um}$ & & & & wI & 147.61 & 80 & ePKP & 5 & 08.00 & $.0 x$ \\
\hline VA & & 77.32 & 138 & eP & 48 & 17.70 & -0.4 & $i$ & $18 \mathrm{~s}$ & 1 & $7.30 \mathrm{um}$ & & & & DF & 148.17 & 163 & i PKP & 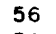 & .00 & 4 \\
\hline IR & & 77.45 & 141 & iPc & 48 & 18.50 & -0.2 & & & & $\mathrm{pP}$ & 49 & 39.00 & $25 \mathrm{~km}$ & $\mathrm{P}$ & 148.20 & 13 & ePKP & & & \\
\hline RN & & 77.67 & 308 & $e^{P}$ & 48 & 21.80 & 2.0 & i & & & $\mathrm{PP}$ & 53 & 11.00 & & WB2 & 149.87 & 62 & EPKP & 5 & 20 & 0.6 \\
\hline AC & & 77.74 & 308 & $e^{P}$ & 48 & 21.90 & 1.6 & & & & $e(S)$ & $\theta 0$ & 25.00 & & & & & ; & & 80 & \\
\hline DC & & 77.78 & 337 & $e^{P}$ & 48 & 21.00 & 1.1 & & & & ss & 00 & 40 & & $i$ & & & $i$ & & 70 & \\
\hline KS & & 77.78 & 308 & eP & 48 & 24 & $4.4 x$ & i & & & $\mathrm{s}$ & 96 & 42. & & & & & eSKP & & 30 & \\
\hline & $z$ & $20 \mathrm{~s}$ & & $0.00 \mathrm{um}$ & & & $6.4 \mathrm{Msz}$ & |TI & 91.96 & 37 & ef & 49 & 32.80 & 1.2 & i & & & eSKS & 3 & 14.00 & \\
\hline & $\mathrm{N}$ & $20 \mathrm{~s}$ & & $0.00 \mathrm{um}$ & & & & CN2 & 92. & 26 & $e(P)$ & 49 & 31.80 & -1.1 & $1 \mathrm{is}$ & 153.94 & 56 & EPKP & & 14.00 & -0.6 \\
\hline & E & $20 \mathrm{~s}$ & & $8.00 \mathrm{um}$ & & & & i & & & $\mathrm{PP}$ & 53 & 14.00 & & CTA & 157.54 & 43 & IPKP & 56 & 20.90 & 1.6 \\
\hline & & & & e & 49 & 05.20 & $164 \mathrm{kmX}$ & i & & & es & 00 & 30.00 & & $i$ & $1.3 \mathrm{~s}$ & & $1.10 \mathrm{~nm}$ & & & \\
\hline & & & & ePP & 51 & 22.00 & & & & & ss & 0 & 48. & & & & & $\mathrm{i}$ & 56 & 55.00 & \\
\hline & & & & es & 58 & 16.00 & & i & & & PS & 1 & 47 & & & & & $\mathrm{i}$ & & & \\
\hline & & & & escs & 58 & 48.00 & & C $\operatorname{co2}$ & 9 & 47 & $e^{P}$ & 19 & 35 & 1 & CTAO & 157.54 & $4 \quad 43$ & ePKP & 56 & 21.40 & 2.1 \\
\hline & & & & ess & 03 & 20.00 & & MD & & 23 & eP & 9 & 37. & 1 & & a 98 & & $0.40 \mathrm{nr}$ & & & \\
\hline 10 & & 77.97 & 307 & $e^{p}$ & 48 & 25.00 & $3.5 x$ & i & & & $\mathrm{s}$ & 0 & 42 & & 1 & & & e & 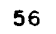 & & \\
\hline AK & & 78.08 & 34 & $i P c$ & 48 & 22.90 & 1.2 & XAN & 93.04 & 42 & e & 49 & 39.00 & 2.4 & $\mathrm{KC}$ & .95 & 5354 & $\mathrm{IPKPC}$ & & 90 & $11.0 x$ \\
\hline & & $3.0 \mathrm{~s}$ & 4750 & $0.00 \mathrm{~nm}$ & & & $7.0 \mathrm{mb}$ & & & & $P P$ & 53 & 20.00 & & YC & 168.09 & 78 & ePKP & 6 & 31.10 & 2.2 \\
\hline & $z$ & $20 \mathrm{~s}$ & 32 & $2.40 \mathrm{um}$ & & & $6.7 \mathrm{Msz}$ & & & & si & 00 & 13 & & $\mathrm{ct}$ & 168.89 & 82 & ePKP & 56 & & 1.8 \\
\hline & N & $16 s$ & & $6.10 \mathrm{um}$ & & & & SNY & 93.33 & 28 & IPd & 49 & 40.00 & 2.3 & WEL & 169.82 & 245 & $e(P K P)$ & 56 & 25.00 & $-4.7 x$ \\
\hline & & & & ePP & 51 & 20 & & & & & $P P$ & 53 & & & & & & $e(P P)^{\prime}$ & & & \\
\hline & & & & ePF & 53 & 14.00 & & & & & SKS & 00 & 17 & & & & & & 01 & 37.00 & \\
\hline & & & & es & 58 & 17.60 & & YSS & 93.92 & 13 & eP & 49 & 42.10 & 1.8 & & S.D. $=$ & 1.1 & on 372 & 2 of & $f 447$ & abs. \\
\hline
\end{tabular}

$7.40 \mathrm{um}$
SUR $\quad \begin{array}{lllll} & & \text { ESSS } & 06 & 24.00 \\ & 0.26 & 148 \mathrm{iPC} & 48 & 24.00\end{array}$

$0.8 \mathrm{~s} \quad 77.60 \mathrm{~nm} \quad 5.8 \mathrm{mb}$

$7 \quad 17 \mathrm{~s} \quad 32.20 \mathrm{~nm}$

$78.36149 \mathrm{iPc}$

$78.48149 \mathrm{iPC}$

$78.69228 \mathrm{eP}$

$79.97 \quad 74 \mathrm{eP}$

$81.29 \quad 24 \mathrm{eP}$

$2.5 \mathrm{~s} \quad 740.00 \mathrm{~nm}$

$6.3 \mathrm{mb}$

$81.33213 \mathrm{iPt} 4840.00 \quad 0.8$

$\begin{array}{rrr}80 \mathrm{~s} & 18.40 \mathrm{um} & 48.00 \\ & 62.4 \mathrm{Msz}\end{array}$

MGD $\quad \begin{array}{lll}2.17 & 6 \mathrm{eP} \\ 1.3 \mathrm{~s} & 160.00 \mathrm{~nm}\end{array}$

$216 \mathrm{~s} \quad 8.20 \mathrm{um}$

N $16 \mathrm{~s} \quad 8.50 \mathrm{um}$

E $16 \mathrm{~s} \quad 5.30 \mathrm{um}$

eS 5851.00

eSS 0420.00

DMN $\quad 82.75 \quad 61 \mathrm{iPC} \quad 4847.60 \quad 0.2$

KKN $\quad 82.76 \quad 61 \mathrm{iPC} \quad 4847.40 \quad 0.0$

$\begin{array}{lll}0.8 \mathrm{~s} & 190.00 \mathrm{~nm} & 6.3 \mathrm{mb} \\ 82.98 & 61 & \end{array}$

$\begin{array}{lllll}82.98 & 61 \mathrm{iPc} & 48 & 48.50 \quad-0.2 \\ 0.8 \mathrm{~s} & 280.00 \mathrm{~nm} & 6.5 \mathrm{mb}\end{array}$

$.82222 \mathrm{eP}$

TLL $\quad 83.84224 \mathrm{iPC}$

GTA

HYB

84. $16 \quad 44 \mathrm{PC}$

$S$

$\begin{array}{ll}84.22 & 73 \mathrm{eP} \\ 1.0 \mathrm{~s} & 220.00 \mathrm{~nm}\end{array}$

53.00

4853.00

4854.60

5906.00

$1.0 \mathrm{~s} 220.00 \mathrm{~nm}$

$54.50-0.2$

LSA

$85.5056 \mathrm{P}$

PP

4903.00

$52 \quad 18.80$

$\begin{array}{lll}59 & 31.80\end{array}$

iP

$\begin{array}{lll} & 85.66 & 223 \\ \text { GBA } & 85.67 & 76 \mathrm{P}\end{array}$

PEL 86.06222 iPC

BACH 86.12222 iP

SAN $86.29222 \mathrm{eP}$

$\mathrm{CHCH} \quad 86.65222$ iP

LNV 87.07222 iP

KOD $\quad 87.73 \quad 79 \mathrm{eP}$

BTO $88.54 \quad 37 \mathrm{eP}$

SHL $88.70 \quad 58$ iP

LZH

1.7

1.4
0.6

0.6
1.2

ค. 1

0.7
0.3
4902.50

4902.40

4904.00

4905.50

4906.00

4907.00

4909.50

4912.50

4916.40

4917.10

5959.80

$88.7644 \mathrm{Pct}$

$3.0 \mathrm{~s} \quad 4770.00 \mathrm{~nm}$

E $11 \mathrm{~s} \quad 13.70 \mathrm{sm}$

$\begin{array}{lll}\text { SP } & 49 & 55.00 \\ \text { PP } & 52 & 49.00 \\ i & 53 & 08.50 \\ \text { S } & 59 & 50.50 \\ \text { i } & 00 & 14.00 \\ \text { PS } & 01 & 10.00\end{array}$

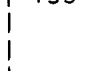

$\begin{array}{ll}2 & 18 \\ N & 18\end{array}$

E $17 \mathrm{~s}$

5. $60 \mathrm{um}$

5. 50 um

ePS

0212.00
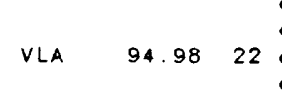

ep

EPP 5341.00

ES 0059.00

iPS 0219.00

$\begin{array}{llllll} & & \text { eSS } & 07 & 17.00 \\ \text { DL2 } & 95.08 & 31 & P & 49 & 51.00\end{array}$

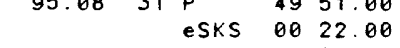

$\begin{array}{llllll} & & \text { eS } & 00 & 56.00 \\ \text { TIA } & 95.39 & 35 \mathrm{eP} & 49 & 49.70\end{array}$

$24.0 \mathrm{~km}$ ( 19 depth

$6.5 \mathrm{mb}$ ( 21 abs.) $6.8 \mathrm{Msz}$ ( 27 abs.)

$3.7 \times$ SOUTH SANDWICH ISLANDS REGION (153)

OHC $\quad 15.37246$ iP $\quad 2512.00 \quad 0.3$

$\begin{array}{lllllll}\text { OHC } & 15.37 & 246 & \mathrm{IP} & 25 & 12.00 & 0.3 \\ \mathrm{NVL} & 18.02 & 140 \mathrm{eP} & 25 & 40.00 & -5.0 \mathrm{X}\end{array}$

$\begin{array}{llllll}1 \text { SPA } & 29.50 & 180 \text { ePC } & 27 & 38.80 & -0.5\end{array}$

$5.2 \times 1.3 \mathrm{~s} 1140.00 \mathrm{~nm} \quad 6.5 \mathrm{mb}$

$1220 \mathrm{~s} 60.30 \mathrm{um} \quad 6.2 \mathrm{Msz}$

PP $53 \quad 39.00$

\begin{tabular}{|ccccc} 
& eSKS & 00 & 20.00 \\
KMI & eS & 00 & 54.70 \\
& 96.09 & $52 \mathrm{eP}$ & 4953.00
\end{tabular}

$7.0 \mathrm{~s} \quad 1.70 \mathrm{~nm}$

E $20 \mathrm{~s} \quad 5.60 \mathrm{um}$

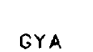

SKS $00 \quad 05.00$

is 0034.00

$97.5048 \mathrm{P} \quad 5001.00$ $\begin{array}{lll}\text { PP } & 53 & 56.00 \\ \text { SKS } & 00 & 41.00\end{array}$

$\begin{array}{llllll}\text { CHTO } & 98.07 & 59 \mathrm{eP} & 49 & 54.80\end{array}$

$98.6241 \mathrm{P}$

5005.00

$\begin{array}{lll}\text { iPP } & 54 & 04.00 \\ \text { SKS } & 00 & 45.00\end{array}$

PS 0254.00

BDT $99.19 \quad 60$ eP 5019.50

$\begin{array}{cclll}99.19 & 60 & \text { eP } & 50 & 19.50 \\ & & i & 54 & 11.90 \\ 99.62 & 36 & P d & 50 & 11.00\end{array}$

$\begin{array}{lll}\text { PP } & 54 & 14.00 \\ \text { SKS } & 00 & 46.00\end{array}$

$\begin{array}{lll}\text { SKS } & 00 & 46.00 \\ \text { SS } & 01 & 46.00\end{array}$

PS 0307.00

0.5 SSE

101.5135 Pdifft50 19.00

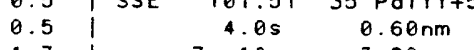

$3.20 \mathrm{um}$
$5.50 \mathrm{um}$

7. $90 \mathrm{um}$

$\begin{array}{lll}\text { EPP } 54 \quad 23.00 & 00\end{array}$

SKS 5720.50

PPP 5801.50

SKKS $00 \quad 59.00$

SS 1354.00

1 MAT

102.8820 ePdift50 24.00

dift50 $24.00 \quad 3.1 x$

7. $3 \mathrm{mb} x$

GZH
ANP
MAW
AAI
MEK
BAL
MUN

2205

$6.74 \mathrm{um}$

$2 \mathrm{MSZ}$

$106.63 \quad 38$ ePdiff50 $44.00 \quad 6.1 \times \quad$ ITB

\begin{tabular}{lllll|l}
120.68 & 154 & ePKP 55 & 16.00 & 2.0 & ITB1
\end{tabular}

\begin{tabular}{llllll|l}
133.91 & 51 & ePKP 55 & 33.00 & $-7.9 \times$ & SUR
\end{tabular}

$0.5 \mathrm{~s} \quad 50.40 \mathrm{~nm}$

$\begin{array}{llllll}141.50 & 87 \text { iPKPC } 55 & 53.10 & -1.6\end{array}$

141.7593 ePKP $5554.00-1.0 \quad$ SBA

$141.87 \quad 96$ EPKP $5551.00 \quad-4.2 \times \mid T L L$ $\begin{array}{lllll}33.14 & 306 \text { iPd+ } 28 & 16.80 \quad 5.6 x\end{array}$

$720 \mathrm{~s} 284.00 \mathrm{um} \quad 7.0 \mathrm{Msz}$

$\begin{array}{llllll}35.97 & 139 & \mathrm{iPC} & 28 & 35.80 & 0.7\end{array}$

$\begin{array}{lllll}38.19 & 287 \text { IPC } & 28 & 54.50 & 0.4\end{array}$

PP 2900.00

PcS 3443.20

3744.00

$\begin{array}{lllll}39.45292 \text { iPc } & 2904.50 & -0.3\end{array}$

$2911.10 \quad 22 \mathrm{~km}$

$\begin{array}{llllll}39.52 & 295 & \text { i (P) } & 29 & 05.20 & -0.1\end{array}$

$\begin{array}{lllll}39.65 & 292 \text { iP } \quad 2905.90 & -0.6\end{array}$

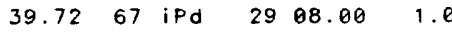

39.7367 IPC $\quad 2907.00 \quad-0.1$

$2907.50 \quad 0.1$

$29 \quad 14.00 \quad 22 \mathrm{~km}$

2931.50

$\begin{array}{cccccc}39.81292 \text { ip } & 29 & 06.80 & -0.9 \\ & \text { i } & 29 & 14.50 & 26 \mathrm{~km}\end{array}$

$39.86293 \mathrm{eP} \quad 2908.50 \quad 0.3$

$2916.20 \quad 26 \mathrm{~km}$

$\begin{array}{ccccc}40.12333 \mathrm{eP} & 2909.90 & -0.5 \\ & \mathrm{i} & 2911.40 & 5 \mathrm{~km}\end{array}$

$\begin{array}{cll}i & 29 & 11.40 \\ e & 29 & 16.80\end{array}$

$\begin{array}{lll}29 & 09.50 & -0.8\end{array}$

$40.13292 \mathrm{IPC} \quad 3056.80$

e $\quad 3724.00$

4017.00

$40.33296 \mathrm{ePd} \quad 29 \quad 10.50 \quad-1.5$

40.42292 iP $\quad 29 \quad 12.80 \quad-0.2$

40.47293 eP $\quad 2912.50 \quad-0.7$

i $2921.00 \quad 29 \mathrm{~km}$

40.51329 eP $\quad 2912.70 \quad-0.9$

$\begin{array}{cllll} & \text { i } & 29 & 20.60 & \\ 40.62318 \text { ip } & 29 & 14.70 & 0.3\end{array}$

40.94318 eP $\quad 2915.90 \quad-1.1$ $\begin{array}{llllll}41.13318 \text { IP } & 2918.00 & -0.6\end{array}$

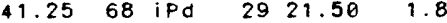
$0.5 \mathrm{~s} \quad 70.40 \mathrm{~nm} \quad 5.6 \mathrm{mb}$ 42.70295 iPd $2932.00 \quad 0.3$ $\begin{array}{lllll}i & 29 & 16.50 & 32 \mathrm{~km}\end{array}$

is $\quad 3423.00$

$\begin{array}{lllll}41.53184 \mathrm{eP} & 29 & 22.30 & 1.0\end{array}$ 


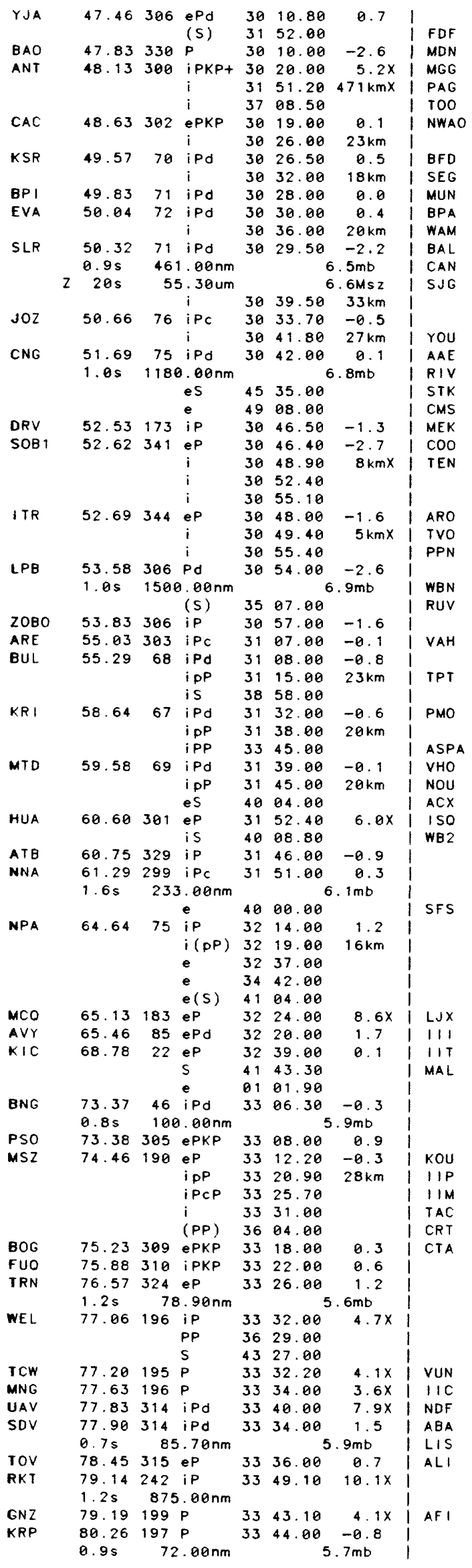

\begin{tabular}{|c|c|c|c|c|c|c|}
\hline & & $\mathrm{i}$ & 33 & 52.30 & $26 \mathrm{~km}$ & 1 \\
\hline 80.38 & 325 & eP & 33 & 47.25 & 1.6 & i \\
\hline 81.01 & 325 & ep & 33 & 50.60 & 1.7 & i \\
\hline 81.56 & 325 & eP & 33 & 53.60 & 1.9 & i \\
\hline 81.76 & 325 & eP & 33 & 55.00 & 2.1 & \\
\hline 81.84 & 173 & eP & 33 & 52.00 & -1.1 & PVC \\
\hline 81.87 & 149 & eP & 33 & 54.00 & 0.7 & KNA \\
\hline $0.9 \mathrm{~s}$ & 286 & $.00 \mathrm{~nm}$ & & & $3 \mathrm{mb}$ & PTO \\
\hline 82.02 & 170 & eP & 33 & 53.00 & -1.0 & i \\
\hline 82.07 & 325 & ep & 33 & 56.00 & 1.6 & | PRNI \\
\hline 82.51 & 148 & ep & 33 & 56.00 & -0.6 & EBR \\
\hline 82.78 & 325 & eP & 33 & 59.00 & 0.9 & 1 \\
\hline 83.38 & 175 & ep & 34 & 00.60 & -0.5 & \\
\hline 83.94 & 148 & eP & 34 & 03.00 & -1.0 & i \\
\hline 84.26 & 175 & eP & 34 & 05.20 & -0.4 & i \\
\hline 85.05 & 322 & eP & 34 & 06.00 & $-3.6 x$ & i \\
\hline $1.0 \mathrm{~s}$ & 178 & $0.00 \mathrm{~nm}$ & & & $.2 \mathrm{mb}$ & I LGR \\
\hline $22 \mathrm{~s}$ & 48 & $3.20 \mathrm{um}$ & & 6 & $.8 \mathrm{MsZ}$ & i \\
\hline 85.27 & 175 & eP & 34 & 89.28 & -1.5 & i \\
\hline 85.65 & 63 & eP & 34 & 17.50 & $4.4 x$ & i \\
\hline 85.81 & 177 & eP & 34 & 17.00 & $3.7 x$ & I KOD \\
\hline 87.18 & 169 & eP & 34 & 19.00 & -1.1 & i \\
\hline 87.91 & 173 & eP & 34 & 25.00 & 1.4 & I EPF \\
\hline 88.22 & 148 & iPc & 34 & 24.10 & -1.1 & i \\
\hline 89.07 & 178 & ep & 34 & 32.00 & 2.8 & I ATH \\
\hline \multirow[t]{3}{*}{89.12} & 8 & $\mathrm{PC}$ & 34 & 38.70 & $9.6 x$ & \\
\hline & & PP & 38 & 10.90 & & SGO \\
\hline & & $\mathrm{s}$ & 45 & 36.90 & & i \\
\hline 89.68 & 66 & $e^{P}$ & 34 & 36.00 & $3.8 x$ & I RDP \\
\hline 89.81 & 232 & iP & 34 & 40.80 & $7.9 x$ & \\
\hline 90.11 & 232 & iP & 34 & 42.00 & $7.8 x$ & RMP \\
\hline $1.3 \mathrm{~s}$ & 238 & $0.00 \mathrm{~nm}$ & & 6 & $.3 \mathrm{mb}$ & 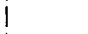 \\
\hline 90.55 & 155 & eP & 34 & 36.00 & -0.2 & i \\
\hline 91.42 & 235 & iP & 34 & 46.50 & $6.3 x$ & i \\
\hline $1.3 \mathrm{~s}$ & 1038 & $0.00 \mathrm{~nm}$ & & & $.0 \mathrm{mb}$ & I COR \\
\hline 91.47 & 235 & iP & 34 & 46.70 & $6.2 x$ & i \\
\hline $1.3 \mathrm{~s}$ & 915 & $5.00 \mathrm{~nm}$ & & 7 & $.0 \mathrm{mb}$ & i \\
\hline 91.70 & 235 & iP & 34 & 48.00 & $6.5 x$ & CAF \\
\hline $1.3 \mathrm{~s}$ & 1398 & $.80 \mathrm{~nm}$ & & & $.2 \mathrm{mb}$ & \\
\hline 91.79 & 235 & iP & 34 & 48.40 & $6.5 x$ & $12 M$ \\
\hline $1.3 \mathrm{~s}$ & 804 & $4.00 \mathrm{~nm}$ & & 7 & $.0 \mathrm{mb}$ & I BCK \\
\hline 94.31 & 161 & $e^{P}$ & 34 & 52.00 & -1.6 & GBA \\
\hline 96.04 & 294 & ip & 35 & 04.00 & 2.5 & $i$ \\
\hline 96.76 & 191 & $i P c$ & 35 & 09.00 & $4.3 x$ & THE \\
\hline 97.13 & 292 & eP & 35 & 14.80 & $8.6 x$ & i \\
\hline 98.01 & 166 & eP & 35 & 10.00 & -0.4 & LSF \\
\hline \multirow[t]{4}{*}{98.83} & 161 & $e^{P}$ & 35 & 06.00 & $-4.5 x$ & \\
\hline & & $i$ & 35 & 13.60 & $24 \mathrm{~km}$ & I JCT \\
\hline & & i & 35 & 18.20 & & \\
\hline & & es & 39 & 08.30 & & \\
\hline \multirow[t]{6}{*}{98.06} & 16 & iPd & 35 & 21.00 & $11.1 x$ & \\
\hline & & iPP & 39 & 20.00 & & I VAY \\
\hline & & ePPP & 41 & 30.00 & & I MZF \\
\hline & & iSKS & 45 & 59.00 & & \\
\hline & & is & 47 & 00.00 & & TCF \\
\hline & & & & & & TG \\
\hline
\end{tabular}

98.24295

$\begin{array}{lll}98.24 & 295 & \mathrm{eP} \\ 98.27 & 293 & \mathrm{iP}\end{array}$

$98.31294 \mathrm{eP}$

$98.57 \quad 17$ iP-

iPP
is
iPS
iss

iSS

$98.68 \quad 189 \mathrm{iPC}$

98.87294 iP

98.97293 iP

99.04293 iP

$99.14 \quad 18 \mathrm{eP}$

$99.26 \quad 172 \mathrm{IPC}$

$1.5 \mathrm{~s} \quad 340.00 \mathrm{~nm}$

5324.00

3521.18

3515.00

3523.58

3520.00

3924.00

4656.00

4812.00

5412.00

\begin{tabular}{llc|l}
35 & 14.60 & 1.2 & I \\
35 & 25.00 & $10.6 \times$ & ORO
\end{tabular}

3518.50

$3526.00 \quad 10.9 x$ | ORO

3528.00

3519.16

iPP $39 \quad 20.00$

ISKS 4600.00

i $\quad 4652.00$

IPS $48 \quad 22.00$

iSS 532200

$99.26203 \mathrm{eP} \quad 3523.60$

99.39294 iP 3519.00

$99.70202 \mathrm{eP} \quad 3527.50$

$99.89 \quad 23 \mathrm{eP} \quad 3538.00$

$99.93 \quad 13$ iPC $35 \quad 29.90$ 100.7720 iPdiff35 32.00

IPP $39 \quad 38 \quad 00$

IPS $48 \quad 42.00$

100.95213 IPdiff35 28.00

\begin{tabular}{l} 
e $\quad 3932.00$ \\
e \\
\hline
\end{tabular}

$9.5 \times 1$

\begin{tabular}{r|r}
$3.5 x$ & \\
$11.7 x$ & TSI
\end{tabular}

$7.9 \times$ I SKO

i

$10.6 x$ ORO

\begin{tabular}{r|l}
$13.2 X$ & \\
$3.1 X$ & $A V F$
\end{tabular}

6. $7 \mathrm{mb}$

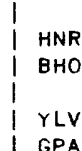

7. $6 \times$ POO

2.2 i CT I

$9.5 \times$

$19.8 \times$ | IST

$11.6 \times 1$

$9.8 \times \mid 15 K$

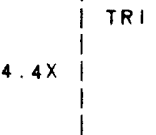

$\begin{array}{lll}\text { e } & 4732.00 \\ \text { e } & 4844.00 \\ \text { e } & 5000.00 \\ \text { e } & 5420.00 \\ \text { eLO } & 0503.00\end{array}$

101.08193 iPdif $\$ 3532.50$

101.09155 ePdiff35 32.00

$102.38 \quad 13$ iPdiff35 40.60

is 4707.00

$103.16 \quad 51$ ePdiff35 50.20

103.3420 ePdiff35 44.00

e $\quad 3945.00$

eSKS 4623.00

eS 4735.00

ePS 4907.00

$104.47 \quad 17$ ePdiff35 $50.50 \quad 11.9 \times$

i(PP) 4909.50

(PP) $40 \quad 07.50$

eS 4847.00

$105.20 \quad 96$ ePdiff $3550.00 \quad 7.1 \times$

$105.47 \quad$ ePP 4011.00

$1.2 \mathrm{~s} \quad 34.50 \mathrm{~nm}$

106.1939 ePdiff35 56.00

$13.9 x$

9. $6 x$

106.23

$17.5 x$

106.7329 ePdiff $36 \quad 02.50$

13. $7 x$

106.7829 ipdiff35 58.00

$9.1 X$ iPP $\quad 3944.00$ eS $\quad 4706.00$ iPS 4848.00

$\begin{array}{lllll}107.06 \quad 23 \text { ePdiff } 36 \quad 09.40 & 19.3 \mathrm{X}\end{array}$ e $\quad 3957.00$

4023.70

$107.60 \quad 2 \theta$ ePdiff35 $58.4 \theta \quad 5.9 X$

$1.2 \mathrm{~s} \quad 27.60 \mathrm{~nm} \quad 6.2 \mathrm{mb}$

$\begin{array}{llll}107.70 \quad 41 \text { ePdiff36 } 10.00 \quad 16.8 x & 0\end{array}$

$107.96 \quad 44$ ePdiff36 $13.00 \quad 18.6 x$

$108.08 \quad 94$ Pdiffd36 06.00 10.8X

$1.1 \mathrm{~s} \quad 9.10 \mathrm{~nm}$

$108.41 \quad 37$ ePdiff36 $07.40 \quad 11.2 x$

$\begin{array}{lll} & e & 40 \quad 32.80\end{array}$

$15.6 x$

$1.2 \mathrm{~s} \quad 23.00 \mathrm{~nm}$

$108.93 \quad 299$ ePdiff36 $05.50 \quad 6.7 x$

$1.0 \mathrm{~s} \quad 65.00 \mathrm{~nm} \quad 6.8 \mathrm{mb}$

$20 \mathrm{~s} 11.00 \mathrm{um}$ 6.4Msz

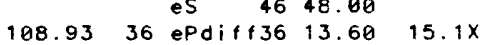

$\begin{array}{lllll}108.94 & 20 & \text { ePdiff } 36 & 16.20 & 17.8 X\end{array}$

$1.2 \mathrm{~s} \quad 24.00 \mathrm{~nm}$

$\begin{array}{llll}108.94 & 20 \text { ePdiff36 } & 09.20 & 10.7 x\end{array}$

$109.03 \quad 33$ elPdif36 $12.50 \quad 13.6 x$ $\begin{array}{lll}\mathrm{e} & 4037.00 \\ \mathrm{e} & 5007.00\end{array}$

$109.14 \quad 119$ e(PKP) $40 \quad 12.00 \quad 7.8 x$

$109.21 \quad 35$ ePdif $\$ 36 \quad 17.00 \quad 17.3 x$ iPP $40 \quad 38.2 \theta$ iSKS $46 \quad 48.00$ iSKKS 4827.00 iSP 4957.00 iSS 5602.00

109.3724 elPdif $35 \quad 45.00-15.5 x$ $109.37 \quad 24$ e 3918.00 if $f 3618.00$

109.6321 ePdiff36 $14.60 \quad 13.1 \mathrm{x}$ $1.5 \mathrm{~s} \quad 33.30 \mathrm{~nm}$

$110.00 \quad 186$ ePdiff36 $10.00 \quad 6.2 \times$ $\begin{array}{lllll}110.24 \quad 304 & \text { ePdiff36 } & 14.70 & 10.2 x\end{array}$ $1.1 \mathrm{~s} \quad 16.10 \mathrm{~nm}$

$110.35 \quad 42$ ePdiff36 $21.00 \quad 16.8 \mathrm{X}$ $\begin{array}{lllll}110.41 & 42 \text { ePdiff } 36 \quad 25.00 & 19.8 \times\end{array}$ $110.52 \quad 89$ ePdiff36 $18.00 \quad 11.8 x$ $110.66 \quad 41$ ipdiff $36 \quad 18.00 \quad 11.8 \times$ i 5120.00 $\begin{array}{lllll}110.70 \quad 41 & \text { ePdiff36 } 06.00 & -0.4\end{array}$ $\begin{array}{llll}110.7128 \text { iPdiff36 } 16.00 \quad 9.7 X & \end{array}$ IPP $40 \quad 47.00$ iPPP 4316.00 ISKKS 4756.00 $\begin{array}{llll}110.55 & 27 \text { ePdiff36 } 22.00 & 16.2 x\end{array}$ 


\begin{tabular}{|c|c|c|c|c|c|c|c|c|c|c|c|}
\hline & & & is & 48 & 36.00 & & 1 & $19 \mathrm{~s}$ & & $0.10 \mathrm{um}$ & \\
\hline & & & iSP & 50 & 14.00 & & 1 & $19 \mathrm{~s}$ & & $3.80 \mathrm{um}$ & \\
\hline & & & iSPP & 51 & 12.00 & & $i$ & & & e & 40 \\
\hline & & & iSS & 56 & 00.00 & & 1 & & & $\mathrm{i}$ & 41 \\
\hline & & & isss & 00 & 08.00 & & i & & & e & 49 \\
\hline I PM & 111.16 & 121 & ePKPd & 40 & 12.20 & 4. $1 x$ & 1 & & & e & 52 \\
\hline & & & e & 40 & 18.50 & & I PRU & 114.91 & 27 & PKP & 40 \\
\hline & & & e & 40 & 37.00 & & 1 & & & $\mathbf{i}$ & 40 \\
\hline$O G A$ & 111.19 & 26 & ePdiff & 36 & 28.00 & $19.3 x$ & WTS & 115.25 & 21 & ePKP & 40 \\
\hline LJU & 111.21 & 29 & epdiff & 36 & 16.80 & $8.3 x$ & 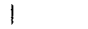 & $1.3 \mathrm{~s}$ & & $0.00 \mathrm{~nm}$ & \\
\hline & & & e & 40 & 52.00 & & 1 & & & e & 40 \\
\hline & & & e & 50 & 31.00 & & 1 & & & ePP & 41 \\
\hline BNH & 111.32 & 327 & $e(P K P)$ & 40 & 14.20 & $6.7 x$ & 1 & & & e & 41 \\
\hline RBL & 111.36 & 28 & ePKP & 40 & 20.00 & $12.4 x$ & I ERE & 115.47 & 53 & ePKP & 40 \\
\hline MSL & 111.66 & 54 & ePditf & 36 & 21.00 & $10.2 x$ & f & & & iSP & 51 \\
\hline & & & $\mathrm{i}$ & 40 & 21.00 & & I ERE & 115.47 & 53 & ePdift & 136 \\
\hline & & & iPPP & 42 & 04.50 & & I SPC & 115.54 & 31 & ePKP & 40 \\
\hline & & & is & 47 & 18.00 & & 1 & & & i & 41 \\
\hline & & & $\mathrm{i}$ & 50 & 26.00 & & I UZH & 115.57 & 33 & ePditf & 36 \\
\hline & & & $i$ & 50 & 46.00 & & 1 & $16 \mathrm{~s}$ & & $5.00 \mathrm{um}$ & \\
\hline RLO & 111.85 & 305 & e(Pdif & 36 & 27.00 & $15.3 x$ & 1 & $16 \mathrm{~s}$ & & $9.00 \mathrm{um}$ & \\
\hline HYB & 111.89 & 93 & ePdiff & 36 & 18.50 & $6.3 x$ & 1 & $16 \mathrm{~s}$ & & $5.00 \mathrm{um}$ & \\
\hline & & & e & 40 & 12.00 & & 1 & & & eSP & 51 \\
\hline TUL & 111.94 & 305 & Pdiff & 36 & 27.50 & $15.5 x$ & 1 & & & eSPP & 52 \\
\hline & $z 22 \mathrm{~s}$ & 27 & $7.40 u m$ & & & $5.8 \mathrm{MsZ}$ & 1 & & & iss & 57 \\
\hline RSNY & 112.19 & 324 & $e(P K P)$ & 40 & 14.30 & $5.2 x$ & I BRG & 115.61 & 26 & ePKP & 40 \\
\hline CLO & 112.45 & 35 & ePdiff & 36 & 34.00 & $19.9 x$ & 1 & $18 \mathrm{~s}$ & & $1.00 \mathrm{um}$ & \\
\hline AA I & 112.63 & 151 & EPKPd & 40 & 17.90 & $7.1 x$ & 1 & $18 \mathrm{~s}$ & & $1.00 \mathrm{um}$ & \\
\hline & $0.7 \mathrm{~s}$ & 51 & $1.70 \mathrm{~nm}$ & & & & 1 & $18 \mathrm{~s}$ & & $3.00 \mathrm{um}$ & \\
\hline BUC 1 & 112.72 & 37 & ePdift & 36 & 24.00 & $8.7 x$ & 1 & & & $i$ & 40 \\
\hline MNT & 112.81 & 325 & ePKP & 39 & 56.50 & $-13.7 x$ & 1 & & & ePKKP & 50 \\
\hline & & & $\mathrm{pP}$ & 40 & 18.00 & & I BRG & 115.61 & 26 & eP & 36 \\
\hline$W L F$ & 112.90 & 22 & Pdiff & 36 & 31.00 & $15.1 x$ & I GRS & 115.62 & 55 & IPKP & 40 \\
\hline & & & e & 41 & 05.00 & & I & & & iSP & 51 \\
\hline & & & SKS & 47 & 07.00 & & I GRS & 115.62 & 55 & iPdiff & 36 \\
\hline & & & SKKS & 48 & 12.00 & & I ALQ & 115.65 & 296 & ePKP & 40 \\
\hline & & & e & 49 & 09.00 & & I & $z 18 \mathrm{~s}$ & 25 & $5.80 \mathrm{um}$ & \\
\hline & & & PS & 50 & 37.00 & & I ALQ & 115.65 & 296 & ePdiff & 36 \\
\hline & & & SS & 56 & 52.00 & & I LEN & 115.72 & 52 & ePKP & 40 \\
\hline VAL & 112.91 & 10 & Pdiff & 36 & 31.00 & $15.1 x$ & 1 & $6.0 \mathrm{~s}$ & 2300 & $0.00 \mathrm{~nm}$ & \\
\hline & & & PS & 49 & 00.00 & & I & & & ePP & 41 \\
\hline$M R$ & 113.01 & 28 & e (Pdif & 36 & 27.00 & $10.5 x$ & I & & & ePS & 51 \\
\hline DOU & 113.03 & 21 & Pdiff- & -36 & 29.00 & $12.5 x$ & I & & & eSPP & 52 \\
\hline & & & $\epsilon$ & 39 & 50.50 & & CLL & 115.82 & 26 & ePKP & 40 \\
\hline & & & e & 40 & 21.50 & & 1 & & & iPP & 41 \\
\hline & & & PP & 41 & 09.50 & & I & & & eSKS & 47 \\
\hline & & & SKKKS & 48 & 13.00 & & SIM & 115.83 & 43 & ePdiff & 36 \\
\hline & & & PS & 50 & 38.00 & & 1 & & & eSKS & 47 \\
\hline $\mathrm{COZ}$ & 113.11 & 36 & ePKP & 40 & 21.00 & $9.9 x$ & I & & & ePS & 51 \\
\hline OTT & 113.31 & 324 & ePKP & 40 & 19.00 & $7.8 x$ & $1 \mathrm{~K} / \mathrm{S}$ & 115.99 & 38 & IPKP & 40 \\
\hline & $0.8 \mathrm{~s}$ & 36 & $0.00 \mathrm{~nm}$ & & & & 1 & $20 \mathrm{~s}$ & & $7.00 \mathrm{um}$ & \\
\hline ISR & 113.60 & 37 & ePdiff & 36 & 03.00 & $-16 \cdot 3 x$ & 1 & $19 \mathrm{~s}$ & & $3.60 \mathrm{um}$ & \\
\hline UCC & 113.67 & 20 & PKPC & 40 & 20.70 & $8.9 x$ & I & $18 \mathrm{~s}$ & & $3.60 \mathrm{um}$ & \\
\hline & & & e & 40 & 26.50 & & 1 & & & iSKS & 47 \\
\hline & & & PS & 51 & 01.00 & & 1 & & & iSP & 51 \\
\hline VKA & 113.71 & 29 & (PKP) & 39 & 56.50 & $-15 \cdot 5 x$ & $1 \mathrm{KIS}$ & 115.99 & 38 & ipdiff & 136 \\
\hline & $6.5 s$ & 8570 & $.00 \mathrm{~nm}$ & & & & I WIT & 116.01 & 21 & ePKP & 40 \\
\hline & $20 \mathrm{~s}$ & 17 & $7.90 \mathrm{um}$ & & & $5.7 \mathrm{MsZ}$ & I & & & e & 40 \\
\hline & & & e & 40 & 19.50 & & 1 & & & ePP & 41 \\
\hline & & & ePP & 41 & 11.00 & & I JAY & 116.09 & 165 & ePKPd & 40 \\
\hline & & & e & 50 & 01.50 & & I KSP & 116.11 & 28 & ePKP & 40 \\
\hline & & & $\mathrm{i}$ & 50 & 57.00 & & I & $2.3 \mathrm{~s}$ & 230 & $3.00 \mathrm{~nm}$ & \\
\hline WET & 113.75 & 26 & ePdiff & 36 & 36.20 & $16.4 x$ & 1 & & & e & 40 \\
\hline & $20 \mathrm{~s}$ & 35 & $5.20 \mathrm{um}$ & & & $.0 \mathrm{Msz}$ & 1 & & & e & 41 \\
\hline & & & e & 41 & 12.00 & & 1 & & & i & 41 \\
\hline TNS & 113.86 & 23 & ePKP & 40 & 19.90 & $7.6 x$ & | & & & e & 50 \\
\hline & & & e & 41 & 10.60 & & KRA & 116.27 & 31 & EPKPC & 40 \\
\hline ENN & 113.91 & 21 & ePKP & 40 & 15.50 & $3.3 x$ & I & $1.9 \mathrm{~s}$ & 289 & $9.00 \mathrm{~nm}$ & \\
\hline & $1.3 \mathrm{~s}$ & & $5.00 \mathrm{~nm}$ & & & & 1 & $18 \mathrm{~s}$ & 28 & $3.70 \mathrm{um}$ & \\
\hline & & & e & 40 & 23.50 & & 1 & $18 \mathrm{~s}$ & & $.20 \mathrm{um}$ & \\
\hline & & & ePP & 41 & 10.00 & & 1 & $18 \mathrm{~s}$ & & $.80 u m$ & \\
\hline CJR & 114.17 & 35 & ePKP & 40 & 05.80 & $-7.2 x$ & 1 & & & e & 40 \\
\hline VRI & 114.31 & 37 & ePKP & 40 & 15.50 & 2.3 & I & & & e & 40 \\
\hline BNS & 114.33 & 22 & iPdiff & 36 & 33.00 & $10.7 x$ & 1 & & & $\mathbf{i}$ & 41 \\
\hline & & & $\in P P$ & 40 & 20.70 & & 1 & & & is & 51 \\
\hline & & & e & 41 & 17.00 & & | KH I & 116.38 & 67 & $\in(P K P)$ & 140 \\
\hline & & & e & 50 & 59.00 & & I BKR & 116.42 & 51 & iPKP & 40 \\
\hline TAB & 114.39 & 55 & ePdiff & 36 & 33.00 & $9.9 x$ & I & & & ePPP & 44 \\
\hline & & & e & 40 & 24.00 & & I & & & eSP & 51 \\
\hline$o x$ & 114.9 & 25 & ePdiff & 36 & 35.00 & $10.1 x$ & 1 & & & $\in S S$ & 57 \\
\hline MOX & 114 & 25 & ePKP & 40 & 22.50 & $8.3 x$ & BKR & 116.42 & 51 & iPdiff & 36 \\
\hline & 2. & 810 & $.00 \mathrm{~nm}$ & & & & 1 & $1.2 \mathrm{~s}$ & 2800 & $m$ & \\
\hline & & & iPP & 41 & 20.00 & & SOC & 116.60 & 48 & IPKP & 40 \\
\hline & & & EPKKP & 51 & 00.00 & & 1 & & & iSP & 51 \\
\hline & & & iss & 57 & 20.00 & & SOC & 116.60 & $4 B$ & iPdiff & 36 \\
\hline PRU & 114.91 & 27 & Pdiff & 36 & 30.00 & $1 x$ & KRV & 116.60 & 54 & ePKP & 40 \\
\hline & 18 & 18 & $3.90 \mathrm{um}$ & & & $M s z$ & 1 & & & ePS & 51 \\
\hline
\end{tabular}




\begin{tabular}{|c|c|c|c|c|c|c|c|c|c|c|c|}
\hline & $1.2 \mathrm{~s}$ & 145 & $5.00 \mathrm{~nm}$ & & & & $\mid$ & & & $\epsilon$ & 40 \\
\hline KKN & 123.93 & 93 & EPKP & 40 & 31.40 & -1.0 & | & & & PP & 42 \\
\hline SAO & 124.05 & 285 & ePKP & 40 & 32.50 & 0.4 & I FHC & 128.45 & 287 & ePKP & 40 \\
\hline HFS & 124.22 & 22 & (PKP) & 40 & 31.70 & 0.0 & I KSH & 128.79 & 77 & PKP & 40 \\
\hline REY & 124.51 & 2 & ePKP & 40 & 35.30 & $3.3 x$ & 1 & & & PP & 42 \\
\hline & & & $\mathrm{i}$ & 40 & 42.60 & & I SUF & 129.16 & 28 & IPKP & 40 \\
\hline JAS & 124.52 & 287 & ePKP & 40 & 33.90 & 0.9 & i & $1.2 \mathrm{~s}$ & 195 & $5.00 \mathrm{~nm}$ & \\
\hline & & & ePP & 42 & 41.00 & & $B A G$ & 129.53 & 136 & ePKP & 40 \\
\hline & & & ePKKP & 50 & 26.30 & & I FFC & 129.96 & 313 & ePKP & 40 \\
\hline & & & ePKKS & 53 & 59.00 & & I KMI & 130.06 & 111 & ePKP & 40 \\
\hline KUL & 124.60 & 73 & ePKP & 40 & 33.80 & 0.6 & 1 & $7.0 \mathrm{~s}$ & & $4.40 \mathrm{~nm}$ & \\
\hline & $4.2 \mathrm{~s}$ & 2000 & $.00 \mathrm{~nm}$ & & & & I & E $19 \mathrm{~s}$ & 27 & $7.60 \mathrm{um}$ & \\
\hline & & & iPP & 42 & 31.30 & & I & & & $i$ & 40 \\
\hline MHC & 124.60 & 286 & ePKP & 40 & 34.50 & 1.2 & I & & & PP & 42 \\
\hline SAM & 124.73 & 70 & iPKP & 40 & 37.00 & $3.6 x$ & I & & & PKS & 44 \\
\hline & $z \quad 17 \mathrm{~s}$ & 45 & $5.00 \mathrm{um}$ & & & $.2 M s z X$ & I & & & SS & $\theta 0$ \\
\hline & $\mathrm{N} 17 \mathrm{~s}$ & 34 & $.00 \mathrm{um}$ & & & & | KMI & 130.06 & 111 & ePKP & 40 \\
\hline & & & iPP & 42 & 29.00 & & I & & & e & 40 \\
\hline SAM & 124.73 & 70 & ePdiff & f37 & 21.50 & $12.7 x$ & 1 & & & PP & 42 \\
\hline UPP & 124.74 & 25 & iPKP & 40 & 31.50 & -1.1 & I SES & 130.12 & 303 & EPKP & $4 \theta$ \\
\hline & & & $\mathrm{i}$ & 40 & 33.50 & & I & $1.4 \mathrm{~s}$ & 404 & $4.00 \mathrm{~nm}$ & \\
\hline & & & $\mathrm{i}$ & 40 & 41.50 & & I NRN & 130.34 & 75 & PKPd & 40 \\
\hline KHO & 125.11 & 75 & ePKP & 40 & 34.50 & 0.1 & I FCC & 130.48 & 320 & EPKP & $4 \theta$ \\
\hline & & & ePP & 42 & 33.00 & & I FRU & 130.77 & 73 & iPKP & 40 \\
\hline & & & ESPP & 54 & 00.00 & & I & $18 \mathrm{~s}$ & 29 & $9.00 \mathrm{um}$ & \\
\hline & & & isss & 04 & 21.00 & & I & $20 \mathrm{~s}$ & 29 & $9.00 \mathrm{um}$ & \\
\hline BKS & 125.31 & 286 & EPKP & 40 & 36.20 & 1.7 & 1 & & & iPP & 43 \\
\hline & $z 20 \mathrm{~s}$ & 16 & $3.00 \mathrm{um}$ & & & $.7 \mathrm{Msz}$ & I FRU & 130.77 & 73 & ePdiff & 37 \\
\hline & $N \quad 20 s$ & 11 & $.00 u m$ & & & & I KJF & 136.80 & 28 & IPKP & 40 \\
\hline & E $20 \mathrm{~s}$ & & 5.00 um & & & & I & $1.3 \mathrm{~s}$ & 508 & $3.00 \mathrm{~nm}$ & \\
\hline & & & ePP & 42 & 33.60 & & I & & & $\mathrm{i}$ & 40 \\
\hline & & & ePKKS & 54 & 04.00 & & I & & & iPP & 43 \\
\hline & & & eSS & 59 & 44.00 & & 1 & & & i SKP & 44 \\
\hline OBN & 125.48 & 39 & iPKP & 40 & 37.00 & $2.8 x$ & | & & & e & 50 \\
\hline & $z 20 \mathrm{~s}$ & 25 & $5.00 \mathrm{um}$ & & & $.9 M s z$ & I & & & es & 51 \\
\hline & $N \quad 20 \mathrm{~s}$ & 11 & $.00 u m$ & & & & 1 & & & ePS & 53 \\
\hline & E $20 \mathrm{~s}$ & 21 & $.00 \mathrm{um}$ & & & & I & & & eSs & 00 \\
\hline & & & iSPP & 54 & 06.00 & & I COR & 131.06 & 290 & IPKPC & 40 \\
\hline DBN & 125.48 & 39 & ePdiff & 37 & 32.00 & $20.4 x$ & I NEW & 131.07 & 298 & ePKP & 40 \\
\hline GAR & 125.74 & 73 & ePKP & 40 & 35.50 & 0.0 & 1 & $20 \mathrm{~s}$ & 48 & $3.00 \mathrm{um}$ & \\
\hline & $1.5 \mathrm{~s}$ & 450 & $.00 \mathrm{~nm}$ & & & & I & & & e & 40 \\
\hline & & & iPP & 42 & 34.00 & & ! & & & e & 44 \\
\hline AKU & 126.16 & 4 & ¿PKP & 40 & 32.70 & $-2.5 x$ & CVP & 131.15 & 137 & ePKPc & 40 \\
\hline & $1.0 s$ & 32 & $.00 \mathrm{~nm}$ & & & & I AAA & 132.20 & 74 & iPKPd & 40 \\
\hline & & & $\mathrm{i}$ & 40 & 42.90 & & I & & & iPP & 43 \\
\hline & & & $\mathrm{i}$ & 42 & 38.40 & & I PRZ & 132.30 & 76 & EPKP & 40 \\
\hline MOS & 126.28 & 39 & ePKPd & 40 & 38.00 & 2.2 & 1 & $1.0 \mathrm{~s}$ & 60 & $0.00 \mathrm{~nm}$ & \\
\hline & $z \quad 18 \mathrm{~s}$ & 17 & .90 um & & & $.8 \mathrm{Msz}$ & I & & & EPP & 43 \\
\hline & $19 \mathrm{~s}$ & 21 & $.30 \mathrm{um}$ & & & & I & & & eSKS & 48 \\
\hline & & & IPP & 42 & 46.00 & & I TLG & 132.41 & 75 & iPKP & 40 \\
\hline & & & ePP & 45 & 30.00 & & I & $7.0 \mathrm{~s}$ & 7000 & $9.00 \mathrm{~nm}$ & \\
\hline & & & eSKS & 47 & 40.00 & & I & $18 \mathrm{~s}$ & 22 & $2.00 \mathrm{um}$ & \\
\hline & & & ESP & 52 & 42.00 & & 1 & & & ePP & 43 \\
\hline & & & ESPP & 54 & 14.00 & & ! & & & ESPP & 55 \\
\hline NUR & 126.88 & 28 & EPKP & 40 & 33.00 & $-3.8 x$ & $\mathrm{HKC}$ & 132.52 & 126 & ePKP & 40 \\
\hline & $1.3 \mathrm{~s}$ & 571 & $.00 \mathrm{~nm}$ & & & & I & & & $\in S$ & 43 \\
\hline & & & $i$ & 40 & 46.80 & & GZH & 132.89 & 124 & iPKPd & 40 \\
\hline & & & ePP & 42 & 39.00 & & 1 & & & $P P$ & 43 \\
\hline & & & ESKP & 43 & 58.00 & & I & & & PKS & 44 \\
\hline & & & e & 49 & 04.00 & & I PNT & 132.95 & 297 & EPKP & 40 \\
\hline & & & es & 51 & 04.00 & & I & $1.5 \mathrm{~s}$ & 485 & $5.00 \mathrm{~nm}$ & \\
\hline & & & ePS & 52 & 46.00 & & I & & & $\mathrm{pP}$ & 40 \\
\hline & & & ess & 59 & 32.00 & & I GYA & 132.99 & 115 & PKP & 40 \\
\hline$M I N$ & 126.98 & 288 & ePKP & 40 & 37.70 & -0.2 & i & & & PP & 43 \\
\hline TAS & 127.11 & 70 & iPKP & 40 & 41.00 & 3. $1 x$ & I & & & PKS & 44 \\
\hline & $z \quad 22 \mathrm{~s}$ & & .50 um & & & $.1 \mathrm{MsZ}$ & EDM & 133.19 & 304 & EPKP & 40 \\
\hline & & & ePP & 42 & 46.00 & & I & $1.5 \mathrm{~s}$ & 1280 & $0.00 \mathrm{~nm}$ & \\
\hline & & & ESPP & 54 & 21.00 & & SOD & 133.27 & 25 & iPKP & 40 \\
\hline TAS & 127.11 & 70 & ePdiff & 37 & 28.00 & $8.7 x$ & I & & & $\mathbf{i}$ & 40 \\
\hline & $8.0 \mathrm{~s}$ & 800 & $.00 \mathrm{~nm}$ & & & & 1 & & & iPP & 43 \\
\hline LRM & 127.16 & 299 & ePKP & 40 & 27.80 & $-10.4 x$ & 1 & & & $\mathbf{i}$ & 44 \\
\hline WDC & 127.60 & 287 & ePKP & 40 & 38.90 & 0.1 & TRO & 133.79 & 20 & ePKP & 40 \\
\hline & & & ePP & 42 & 48.00 & & PGC & 134.17 & 294 & ePKP & 41 \\
\hline & & & ePKKS & 53 & 56.00 & & I APA & 134.88 & 28 & iPKPd & 40 \\
\hline PUL & 127.63 & 32 & ePKP & 40 & 41.00 & $2.8 x$ & 1 & & & iPP & 43 \\
\hline & $z \quad 19 s$ & 35 & .00 um & & & $.1 \mathrm{Msz}$ & 1 & & & iSPP & 55 \\
\hline & $\mathrm{N} \quad 18 \mathrm{~s}$ & & $.00 u m$ & & & & KEV & 135.36 & 23 & iPKP & 40 \\
\hline & $18 \mathrm{~s}$ & & .00 um & & & & I & $1.0 \mathrm{~s}$ & 100 & $0.00 \mathrm{~nm}$ & \\
\hline & & & ePPP & 45 & 47.00 & & ! & & & $i$ & 41 \\
\hline & & & ePS & 53 & 00.00 & & l & & & iPP & 43 \\
\hline PUL & 127.63 & 32 & ePdiff & 37 & 32.00 & $11.0 x$ & 1 & & & $\mathrm{i}$ & 44 \\
\hline OCP & 128.04 & 137 & iPKP & 40 & 47.00 & $6.7 x$ & 1 & & & EPPS & 55 \\
\hline ANR & 128.09 & 73 & ePKP & 40 & 38.00 & -1.8 & | & & & ess & 01 \\
\hline & $1.6 \mathrm{~s}$ & 430 & $.00 \mathrm{~nm}$ & & & & $\mathrm{CD} 2$ & 135.44 & 108 & PKP & 40 \\
\hline FRB & 128.28 & 337 & ePKP & 40 & 31.50 & $-7.8 x$ & 1 & & & $e$ & 40 \\
\hline & $1.3 \mathrm{~s}$ & 602 & $.00 \mathrm{~nm}$ & & & & I & & & PP & 43 \\
\hline LSA & 128.39 & 97 & PKP & 40 & 41.10 & -0.2 & I & & & PKS & 44 \\
\hline
\end{tabular}




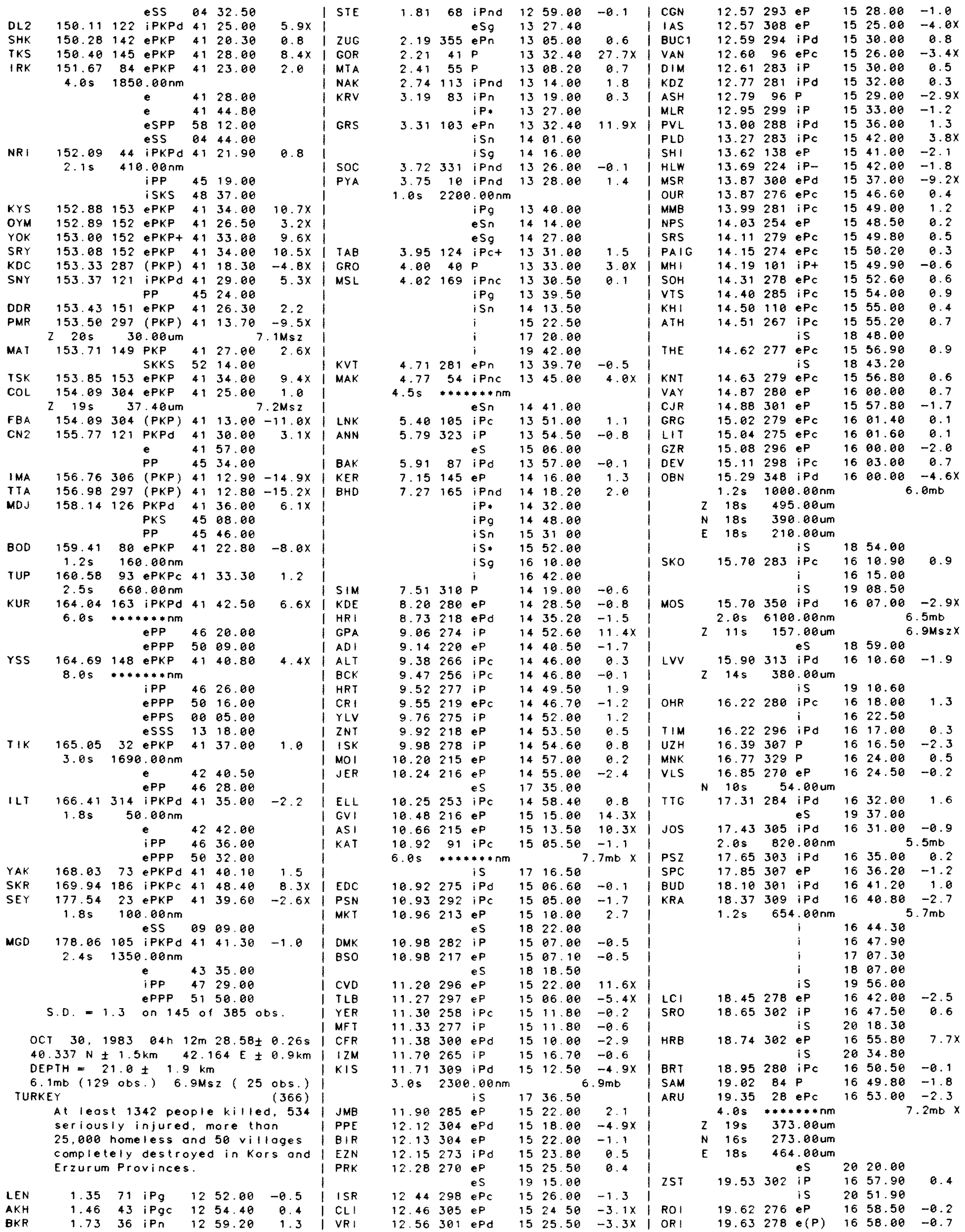


$30 d \quad 04 h$

CSI $\quad 19.80277 \mathrm{eP}$

$A C I \quad 19.94 \quad 276 \mathrm{eP}$

MMN $20.01277 \quad \mathrm{PPC}$

CZI $\quad 20.02 \quad 275 \mathrm{eP}$

VIE $\quad 20.02302 \mathrm{eP-}$

VKA $20.06302 \mathrm{ePd}$

$4.0 \mathrm{~s} \quad 806.00 \mathrm{~nm}$

$20.4130 \mathrm{iPC}$

$5.0 \mathrm{~s} \quad * * * * * * * \mathrm{~nm}$

$Z \quad 18 \mathrm{~s} \quad 618.00 \mathrm{um}$

N $18 \mathrm{~s} \quad 298.00 \mathrm{um}$

E $18 \mathrm{~s} \quad 262.00 \mathrm{um}$

$S G O$

TAS

$20.42279 \stackrel{\mathrm{eP}}{\mathrm{eP}}$

$20.5378 \mathrm{iPc}$

E $14 \mathrm{~s} \quad 240.00 \mathrm{um}$

DSH

MSH
PUL

$$
20.6086 \stackrel{\mathrm{PS}}{\mathrm{P}}
$$

$2.0 \mathrm{~s} 7600.00 \mathrm{~nm}$

$7 \quad 19 \mathrm{~s} \quad 420.00 \mathrm{um}$

$\mathrm{N} 20 \mathrm{~s} \quad 320.00 \mathrm{um}$

E $19 \mathrm{~s} 190.00 \mathrm{um}$

IPP $17 \quad 30.00$

ES 2100.00

iSSS 2142.00

$\begin{array}{lllllll}\text { KSP } & 20.83 & 309 & \mathrm{ePd} & 17 & 10.30 & -0.8\end{array}$

$2.0 \mathrm{~s} \quad 1540.00 \mathrm{~nm}$

$17 \quad 11.50^{\circ}$

2058.30

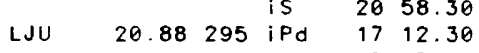

1715.90

1723.00

i(S) 2113.10

DUI $20.92 \quad 283 \mathrm{eP}^{\mathrm{P}} \quad 17 \quad 12.00$

$\begin{array}{llllll}\text { CEY } & 20.93 & 294 & \mathrm{iPd} & 17 & 12.80\end{array}$

$\begin{array}{lllll}21.39 & 294 & \text { C (S) } & 21 & 09.00 \\ & & \mathrm{PPC} & 17 & 16.30 \\ & \mathrm{PPP} & 17 & 49.60\end{array}$

IPP 1749.60

is $21 \quad 13.00$

KMR

KU

$21.44380 \mathrm{iP}-\quad 1717.80$

$21.5188 \mathrm{iPc} \quad 1719.20$

$3.0 \mathrm{~s} \quad * * * * * * \mathrm{~nm} 2128.00$

iSSS 2205.70

$\begin{array}{llllll} & & & & & \end{array}$

PR

$\begin{array}{ll}21.63 & 306 \mathrm{POd} \\ 1.6 \mathrm{~s} & 1040.00 \mathrm{~nm}\end{array}$

$z \quad 16 \mathrm{~s} \quad 95.00 \mathrm{um}$

$16 \mathrm{~s} 95.00 \mathrm{um} 2120.00$

$17 \quad 19.10$

$A O$

21.82298 iPd

$1.1 \mathrm{~s} \quad 298.00 \mathrm{~nm}$

$21.92273 \mathrm{eP}$

$21.93289 \mathrm{eP}$

$22.02303 \mathrm{iPd}$
$1.2 \mathrm{~s} \quad 1100.00 \mathrm{~nm}$

$215 \mathrm{~s} \quad 82.00 \mathrm{um}$

N $145 \quad 57.20 u m$

E $14 \mathrm{~s} \quad 57.00 \mathrm{um}$

MNS $\quad 22.18285 \stackrel{\mathrm{S}}{\mathrm{eP}}$

RDP $22.21283 \mathrm{eP}$

RMP $22.22 \quad 283 \quad \mathrm{iPC}$

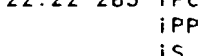

$1800 \quad 00$

2128.00

22.25308 i Pd

$1.5 \mathrm{~s} \quad 690.00 \mathrm{~nm}$

WET $\quad 22.47303$ iPd 1727.70

$\begin{array}{lll} & \text { ES } & 2138.00\end{array}$

PKK 22.56337 iP

CT) $\quad 22.88295 \mathrm{eP}$

ANR $22.9079 \mathrm{P}$

1728.10

1732.50

22.93338 iP

$\begin{array}{ll}17 & 34.00 \\ 17 & 32.20\end{array}$

$1.1 \mathrm{~s} \quad 93.60 \mathrm{~nm}$

z $19 \mathrm{~s} \quad 628.00 \mathrm{um}$

i $\quad 17 \quad 37.50$

es $\quad 2132.00$

$\mathrm{CLL} \quad 22.94308 \quad \mathrm{PPd} \quad 17 \quad 32.90 \quad 0.7$

$1.7 \mathrm{~s} 2200.00 \mathrm{~nm}$

is 2144.00

ERC

1731.50

\section{SUF}

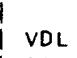

I SAX

VG1

ITS

UPP

UPP

COP

\begin{tabular}{l|l}
0.4 & \\
1.0 & $\mathrm{CVF}$
\end{tabular}

SLE

ZUL

$-0.1$

MszX

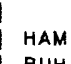

BUH

I ORO

TNS

NRN

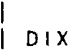

KSH

AAA

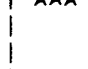

CDF

TLG

BSF

BNS

BNS

FRF

HFS

0.7

0.2

LMR

HAU

LRG

wTS

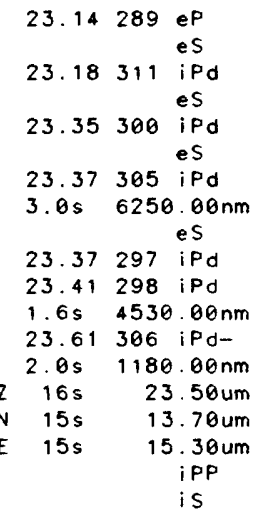

$23.64304 \quad \mathrm{Pd}$

$23.65293 \mathrm{eP}$

23.83341 iP

$23.96296 \mathrm{ePd}$

24.23340 iP

$24.32 \quad 73$ iPc

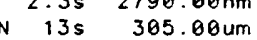

E $20 \mathrm{~s} 215.00 \mathrm{um}$

24.39342 is 2207.00

$0.9 \mathrm{~s} \quad 470.00 \mathrm{~nm}$

$24.42295 \mathrm{ePd}$

$24.55297 \mathrm{ePd}$

$24.65292 \mathrm{eP}$

$24.76296 \mathrm{ePd}$

24.80301 iPd-

$1.0 \mathrm{~s} 1120.00 \mathrm{~nm}$

24.83330 iP

24.86318 iPd

$24.97286 \mathrm{eP}$

$2.0 \mathrm{~s} 3180.00 \mathrm{~nm}$

$25.16298 \mathrm{ePd} \quad 1753.50^{6.6 \mathrm{mb}}$

$25.21298 \mathrm{ePd} \quad 1753.90 \quad-0.3$

25.36345 iP- $1755.00-0.3$

$0.5 \mathrm{~s} \quad 828.00 \mathrm{~nm}$ es

$25.39312 \mathrm{iPd}$

$25.43300 \mathrm{eP}$

$25.43293 \mathrm{eP}$

$25.45294 \mathrm{ePd}$

$25.50304 \mathrm{iPd}$

$25.5276 \mathrm{eP}$

es

$25.83294 \mathrm{ePd}$

$25.87 \quad 81 \mathrm{PC}$

$\begin{array}{rl}25.98 & 72 \text { is } \\ \text { i } P C\end{array}$

$8.0 \mathrm{~s} 21.00 \mathrm{~nm}$

26.03300 is 2237.00

\begin{tabular}{llll}
\hline$P$ & 18 & 00.80 & -1.1
\end{tabular}

$1.1 \mathrm{~s} 253.00 \mathrm{~nm}$

$26.30 \quad 72 \quad \mathrm{PCC} \quad 18 \quad 04.10 \quad-0.3$

$2.4 \mathrm{~s} \quad 1030.00 \mathrm{~nm} \quad 6.0 \mathrm{mb}$

$\begin{array}{lllll}26.31 & 298 e^{P} \quad 18 & 03.50 & -1.1\end{array}$

$0.7 \mathrm{~s} 295.00 \mathrm{~nm} \quad 6.0 \mathrm{mb}$

$26.43 \quad 305 \mathrm{iPd} \quad 1806.50 \quad 1.1$

$1.5 \mathrm{~s} \quad 730.00 \mathrm{~nm} \quad 6.1 \mathrm{mb}$

$26.49289 \mathrm{eS} \quad 2247.00$

$\begin{array}{llllll}1.3 \mathrm{~s} & 1160 & 00 \mathrm{~nm} & 18 & 05.60 & -0.5\end{array}$

$\begin{array}{lllll}26.56 & 328 \mathrm{eP} & 18 & 06.20 & -0.3\end{array}$

$0.8 \mathrm{~s} \quad 272.00 \mathrm{~nm} \quad 5.9 \mathrm{mb}$

$\begin{array}{lllll}26.61288 & \mathrm{eP}^{\mathrm{P}} \quad 1806.60 & -0.6\end{array}$

$1.4 \mathrm{~s} \quad 625.00 \mathrm{~nm} \quad 6.1 \mathrm{mb}$

$\begin{array}{llllll}26.62 & 299 & \text { eP } & 18 & 06.20 & -1.1\end{array}$

$1.3 \mathrm{~s} \quad 555.00 \mathrm{~nm}$

$\begin{array}{llllll}26.71 & 289 & \mathrm{eP} & 18 & 07.60 & -0.4\end{array}$

25.85308 Pd $1810.50 \quad 1.3$

$1.2 \mathrm{~s} \quad 750.00 \mathrm{~nm}$ 1825.00 MUD

e $\quad 1903.00$

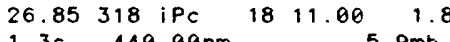

$26.89302 P \quad 1808.00^{\circ}-1.7$

WLF

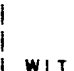

CDR

$\begin{array}{ccc}e & 1815.00 \\ 5 & 2253.00\end{array}$

$\begin{array}{rl}27.11309 & e P \\ e & 1859.00\end{array}$

$\begin{array}{ccc}27.11289 \mathrm{eP}^{\mathrm{P}} & 1818.00 \\ & \mathrm{e} & 1813.00\end{array}$

2.4

1813.00

0.1

2134.80

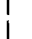

I PRZ

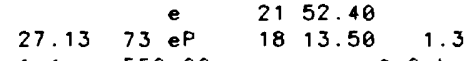

$\begin{array}{lll}1.4 \mathrm{~s} & 550.00 \mathrm{~nm} & 6.0 \mathrm{mb}\end{array}$

Z $16 \mathrm{~s} 150.00 \mathrm{um} \quad 6.7 \mathrm{MszX}$

E $16 \mathrm{~s} \quad 248.00 \mathrm{um}$

es 2252.00

ENN $\quad 27.18 \quad 305$ ePd $18 \quad 13.50 \quad 1.2$

$\begin{array}{lll}1.1 \mathrm{~s} & 469.00 \mathrm{~nm} & 6.1 \mathrm{mb}\end{array}$

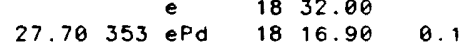

$\begin{array}{lll}1.5 \mathrm{~s} & 320.00 \mathrm{~nm} & 5.8 \mathrm{mb}\end{array}$

$z 19 \mathrm{~s} 198.00 \mathrm{um} \quad 6.7 \mathrm{Msz}$

27.86307 is 2253.00

$\begin{array}{lllll}145 & 18 & 19.00 & 0.6\end{array}$

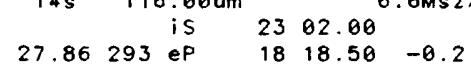

$\begin{array}{lllll}27.94303 \mathrm{PC} & 18 & 20.50 & 1.3\end{array}$

e $\quad 1826.00$

$\begin{array}{lll}P C P & 21 & 36.70 \\ S & 23 & 07.00\end{array}$

$\begin{array}{llllll}28.05 & 324 & \text { iP } & 18 & 19.20 & -0.9\end{array}$

$\begin{array}{llllll}28.08 & 328 & P & 18 & 19.00 & -1.4\end{array}$

$1.0 \mathrm{~s} \quad 576.00 \mathrm{~nm}$

$28.17305 \mathrm{Pd}$

1821.50

6. $3 \mathrm{mb}$

1828.50

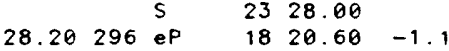

$1.1 \mathrm{~s} \quad 329.00 \mathrm{~nm} \quad 6.0 \mathrm{mb}$

$\begin{array}{llllll}28.28 & 297 \quad \mathrm{eP}^{\mathrm{P}} \quad 18 & 21.30 & -1.1\end{array}$

$1.1 \mathrm{~s} \quad 272.00 \mathrm{~nm} \quad 5.9 \mathrm{mb}$

28.30296 eP $18 \quad 22.00 \quad-0.5$

$1.1 \mathrm{~s} \quad 575.00 \mathrm{~nm} \quad 6.2 \mathrm{mb}$

$\begin{array}{lllll}28.30 & 56 \mathrm{ePc} & 18 & 23.20 & 0.7\end{array}$

$2.0 \mathrm{~s} 1350.00 \mathrm{~nm} \quad 6.3 \mathrm{mb}$

$\begin{array}{lll}28.42347 \text { es } & 2313.50\end{array}$

$\begin{array}{lllll}28.42 & 347 \text { iP } & 18 & 23.40 & 0.1\end{array}$

$.00 \mathrm{~nm} \quad 5.8 \mathrm{mb}$

$\begin{array}{llllll}28.63 & 296 & \text { eP } & 18 & 24.60 & -0.9\end{array}$

$1.3 \mathrm{~s} \quad 460.00 \mathrm{~nm} \quad 6.1 \mathrm{mb}$

$28.70 \quad 179$ iPd $18 \quad 27.58 \quad 1.1$

$\begin{array}{lllll}29.18 & 295 \mathrm{eP} & 18 & 29.78 & -0.8\end{array}$

$1.2 \mathrm{~s} \quad 572.00 \mathrm{~nm} \quad 6.2 \mathrm{mb}$

$\begin{array}{lllll}29.44 & 295 & \text { eP } \quad 18 \quad 32.20 & -0.6\end{array}$

$1.2 \mathrm{~s} \quad 369.00 \mathrm{~nm} \quad 6.0 \mathrm{mb}$

$\begin{array}{lllll}29.64 & 292 \mathrm{eP} \quad 18 \quad 33.90 & -0.7\end{array}$

$1.0 \mathrm{~s} \quad 209.00 \mathrm{~nm} \quad 5.9 \mathrm{mb}$

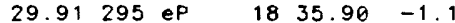

$1.3 \mathrm{~s} \quad 275.00 \mathrm{~nm} \quad 5.9 \mathrm{mb}$

$29.99293 \mathrm{eP} \quad 18 \quad 37.40 \quad-0.3$

$1.8 \mathrm{~s} \quad 911.00 \mathrm{~nm} \quad 6.3 \mathrm{mb}$

$\begin{array}{lllll}30.29 & 292 \text { eP } \quad 18 \quad 39.60 & -0.7\end{array}$

$1.1 \mathrm{~s} \quad 265.00 \mathrm{~nm} \quad 6.0 \mathrm{mb}$

30.29324 iP $1841.00 \quad 0.8$

$\begin{array}{llllll}30.54 & 350 & \text { iP } & 18 & 42.40 & 0.1\end{array}$

$1.2 \mathrm{~s} \quad 197.00 \mathrm{~nm} \quad 5.8 \mathrm{mb}$

i $\quad 1847.00$

$\begin{array}{rrrrrr}30.58 & 293 \mathrm{eS} & 23 & 40.00 & \\ \mathrm{eP} & 18 & 42.20 & -0.7\end{array}$

$1.1 \mathrm{~s} 284.00 \mathrm{~nm} \quad 6.0 \mathrm{mb}$

30.60289 iP $1841.30-1.8$

30.63276 iP $\quad 1844.50 \quad 1.1$

$\begin{array}{llllll}30.81 & 48 & \text { iPc } & 18 & 44.28 & -0.6\end{array}$ $2.8 \mathrm{~s} * * * * * * n \mathrm{~nm} 2349.80^{7.5 \mathrm{mb} X}$

$\begin{array}{llllll}30.94 & 101 \mathrm{iPc} & 18 & 46.00 & -0.3\end{array}$

$1.5 \mathrm{~s} \quad 1060.00 \mathrm{~nm} \quad 6.5 \mathrm{mb}$

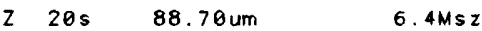

$\mathrm{N} 20 \mathrm{~s} \quad 83.30 \mathrm{um}$

E $20 \mathrm{~s} \quad 85.10 \mathrm{um}$

30.95300 is 2356.00

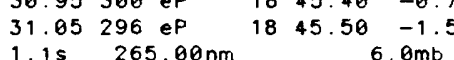




\begin{tabular}{|c|c|c|c|c|c|c|c|c|c|c|c|}
\hline EPF & 31.12 & 289 & eP & 18 & 45.60 & -2.1 & 1 & & & & e \\
\hline & $0.9 \mathrm{~s}$ & 118 & $8.00 \mathrm{~nm}$ & & & $.7 \mathrm{mb}$ & 1 & & & & es \\
\hline FLN & 31.19 & 300 & eP & 18 & 47.20 & -1.0 & 1 LIS & & 39.16 & 285 & eP \\
\hline$A A E$ & 31.32 & 186 & eP & 18 & 50.70 & 0.7 & I AVE & & 39.88 & 276 & ¡P \\
\hline EBR & 31.44 & 285 & ep & 18 & 53.00 & 2.5 & I KBS & & 40.51 & 351 & iP \\
\hline & & & $e$ & 22 & 56.00 & & 1 & & & & es \\
\hline & & & e & 23 & 32.00 & & I GBA & & 40.76 & 121 & $P$ \\
\hline & & & es & 24 & 04.00 & & I KHE & & 40.83 & 4 & eP \\
\hline GRR & 31.45 & 299 & $e P$ & 18 & 49.20 & -1.3 & i & & $2.0 \mathrm{~s}$ & 810 & $.00 \mathrm{~nm}$ \\
\hline & $1.2 \mathrm{~s}$ & 720 & $0.00 \mathrm{~nm}$ & & & $4 \mathrm{mb}$ & i & & & & iPP \\
\hline LPF & 31.58 & 299 & eP & 18 & 50.20 & -1.4 & i & & & & ePPP \\
\hline & $1.1 \mathrm{~s}$ & 265 & $5.00 \mathrm{~nm}$ & & & $\theta m b$ & i & & & & is \\
\hline JAU & 31.63 & 289 & $(P)$ & 18 & 52.70 & 0.3 & I LSA & & 41.84 & 89 & eP \\
\hline ATE & 31.87 & 289 & $(P)$ & 18 & 53.78 & -0.5 & I MOY & & 41.33 & 54 & $e P$ \\
\hline ELT & 32.31 & 51 & IPc & 18 & 56.68 & -1.3 & i & & $2.0 \mathrm{~s}$ & 1120 & $.08 \mathrm{~nm}$ \\
\hline & $2.1 \mathrm{~s}$ & 1336 & $0.00 \mathrm{~nm}$ & & & $.5 m b$ & I BNG & & 41.60 & 217 & iPd \\
\hline & & & is & 24 & 14.00 & & I & & $1.2 \mathrm{~s}$ & 45 & $3.00 \mathrm{~nm}$ \\
\hline ALI & 32.81 & 281 & ipt & 19 & 03.00 & 0.5 & I NAI & & 41.70 & 188 & iPd \\
\hline & & & iPP & 20 & 09.00 & & 1 & & $1.0 \mathrm{~s}$ & 100 & $.00 \mathrm{~nm}$ \\
\hline & & & is & 24 & 07.00 & & I AKU & & 41.94 & 327 & iPd \\
\hline RLA & 32.91 & 275 & iP & 19 & 05.00 & 1.7 & 1 & & $1.9 \mathrm{~s}$ & 1410 & $.00 \mathrm{~nm}$ \\
\hline EKA & 33.26 & 312 & $\mathrm{Pc}$ & 19 & 86.50 & 0.3 & 1 & & & & $\mathrm{i}$ \\
\hline & $0.8 \mathrm{~s}$ & 124 & $4.00 \mathrm{~nm}$ & & & $9 \mathrm{mb}$ & I ZAK & & 42.97 & 55 & $i \mathrm{Pc}$ \\
\hline LGR & 33.29 & 289 & IPd & 19 & 07.50 & 0.9 & 1 & & $2.2 \mathrm{~s}$ & 960 & $.00 \mathrm{~nm}$ \\
\hline & & & i PP & 20 & 18.20 & & 1 & $N$ & $16 \mathrm{~s}$ & 122 & .00 um \\
\hline & & & is & 24 & 35.00 & & 1 & $E$ & $18 \mathrm{~s}$ & 235 & $3.00 \mathrm{um}$ \\
\hline WMO & 33.65 & 69 & iPc & 19 & 10.50 & 0.7 & I & & & & ePP \\
\hline & & & $\mathrm{s}$ & 24 & 36.00 & & 1 & & & & es \\
\hline & & & PCS & 25 & 33.00 & & 1 & & & & eSS \\
\hline ALM & 34.77 & 279 & iPd & 19 & 19.30 & -0.2 & I IRK & & 43.27 & 52 & $i P c$ \\
\hline & $2.2 \mathrm{~s}$ & & $2.20 \mathrm{~nm}$ & & & $9 m b \times$ & 1 & & $2.0 \mathrm{~s}$ & 600 & $9.00 \mathrm{~nm}$ \\
\hline & & . & is & 24 & 47.10 & 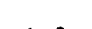 & 1 & $z$ & $16 s$ & 175 & $3.00 \mathrm{um}$ \\
\hline POO & 34.83 & 119 & $I P C$ & 19 & 21.80 & 1.6 & 1 & $N$ & $16 \mathrm{~s}$ & 101 & $1.00 \mathrm{um}$ \\
\hline & $1.0 \mathrm{~s}$ & 200 & $0.00 \mathrm{~nm}$ & & & $\theta m b$ & 1 & $E$ & $17 s$ & 115 & $3.00 \mathrm{um}$ \\
\hline ETA & 34.85 & 307 & eP & 19 & 19.50 & -0.5 & I & & & & ePCP \\
\hline & $1.1 \mathrm{~s}$ & 200 & $0.00 \mathrm{~nm}$ & & & $9 \mathrm{mb}$ & i & & & & ePPP \\
\hline$E C P$ & 34.95 & 306 & eP & 19 & 20.10 & -0.7 & 1 & & & & es \\
\hline & $0.7 \mathrm{~s}$ & 125 & $5.00 \mathrm{~nm}$ & & 5 & $9 m b$ & $1 \mathrm{KOD}$ & & 43.29 & 124 & $\in P$ \\
\hline TOL & 35.04 & 285 & iPd & 19 & 20.50 & -1.2 & I REY & & 43.45 & 325 & ip \\
\hline & $6.0 \mathrm{~s}$ & & $3.00 \mathrm{~nm}$ & & & $8 \mathrm{mb} x$ & 1 & & & & $i$ \\
\hline & & & $\mathrm{i}$ & 19 & 24.00 & & I GTA & & 43.57 & 72 & $i P c$ \\
\hline & & & iPP & 20 & 31.00 & & I & & & & $\mathbf{s P}$ \\
\hline & & & ePPP & 28 & 58.00 & & 1 & & & & PP \\
\hline & & & iPCP & 21 & 54.00 & & 1 & & & & S \\
\hline & & & $\mathrm{i}$ & 23 & 14.00 & & 1 & & & & SS \\
\hline & & & is & 24 & 55.00 & & I DAG & & 44.53 & 343 & $i P c$ \\
\hline & & & $(P \subset S)$ & 25 & 27.00 & & 1 & & $1.1 \mathrm{~s}$ & 109 & $.00 \mathrm{~nm}$ \\
\hline & & & iss & 27 & 32.00 & & 1 & & & & $\mathrm{i}$ \\
\hline OLE & 35.07 & 308 & eP & 19 & 20.80 & -1.0 & 1 & & & & $\mathrm{i}$ \\
\hline ECB & 35.20 & 307 & eP & 19 & 22.70 & -0.2 & I LZH & & 47.79 & 74 & iPct \\
\hline & $1.1 \mathrm{~s}$ & 85 & $5.80 \mathrm{~nm}$ & & 5 & $6 \mathrm{mb}$ & 1 & & $2.5 \mathrm{~s}$ & 3120 & $.00 \mathrm{~nm}$ \\
\hline DMU & 35.31 & 389 & eP & 19 & 23.10 & -0.8 & 1 & $\mathrm{~N}$ & $11 \mathrm{~s}$ & 69 & $.78 u m$ \\
\hline & $1.0 \mathrm{~s}$ & 180 & $0.00 \mathrm{~nm}$ & & 5 & $9 \mathrm{mb}$ & 1 & & & & $p P$ \\
\hline DCN & 35.51 & 308 & iPC & 19 & 26.30 & 0.7 & 1 & & & & SP \\
\hline & $1.3 \mathrm{~s}$ & 500 & $3.00 \mathrm{~nm}$ & & & $3 \mathrm{mb}$ & 1 & & & & PP \\
\hline & & & $i \mathrm{Pg}$ & 19 & 30.70 & & 1 & & & & EPPP \\
\hline MAL & 36.29 & 280 & iPd & 19 & 31.50 & -0.8 & 1 & & & & PCS \\
\hline & & & $i$ & 20 & 19.00 & & 1 & & & & is \\
\hline & & & iPP & 21 & 00.00 & & 1 & & & & SS \\
\hline & & & is & 24 & 56.00 & & $1 B O D$ & & 47.86 & 43 & eP \\
\hline UER & 37.86 & 55 & iPc & 19 & 39.00 & 0.4 & 1 & & $1.5 \mathrm{~s}$ & 730 & $.00 \mathrm{~nm}$ \\
\hline & $2.0 \mathrm{~s}$ & 1890 & $0.00 \mathrm{~nm}$ & & & $3 m b$ & I TEN & & 48.83 & 275 & iPc \\
\hline & & & is & 25 & 19.50 & & 1 & & & & iPP \\
\hline VAL & 37.34 & 386 & iP & 19 & 41.78 & 0.7 & 1 & & & & is \\
\hline & & & is & 25 & 30.00 & & $1 \mathrm{CO} 2$ & & 50.05 & 80 & $i P c$ \\
\hline DMN & 37.46 & 96 & iPc & 19 & 42.80 & 0.2 & 1 & & & & es \\
\hline$K K N$ & 37.50 & 96 & ¡Pc & 19 & 42.60 & -0.3 & I BTo & & 50.41 & 66 & $i P_{C}$ \\
\hline NR I & 37.71 & 25 & iPC & 19 & 43.80 & 0.0 & 1 & & & & PP \\
\hline & $2.7 \mathrm{~s}$ & 2048 & 3. $.80 \mathrm{~nm}$ & & & $5 \mathrm{mb}$ & 1 & & & & $s$ \\
\hline & $19 \mathrm{~s}$ & 113 & 3.00 um & & & & I TIK & & 51.29 & 24 & ePc \\
\hline & & & iPPP & 21 & 38.00 & & 1 & & $2.2 \mathrm{~s}$ & 1260 & $.00 \mathrm{~nm}$ \\
\hline & & & $\triangle P C P$ & 22 & 82.00 & & 1 & & & & ePP \\
\hline & & & is & 25 & 32.00 & & 1 & & & & es \\
\hline & & & eSS & 28 & 10.00 & & I & & & & eSPP \\
\hline PKI & 37.71 & 96 & $i P c$ & 19 & 44.48 & -0.4 & 1 & & & & ess \\
\hline & $1.4 \mathrm{~s}$ & 1530 & $9.00 \mathrm{~nm}$ & & & $6 \mathrm{mb}$ & $\mathrm{HHC}$ & & 51.38 & 65 & iPc \\
\hline SFS & 37.73 & 280 & $i P c$ & 19 & 46.00 & 1.5 & | & & & & $\mathrm{s}$ \\
\hline & & & $i$ & 19 & 53.00 & & I TUP & & 51.82 & 47 & ePc \\
\hline & & & iPP & 20 & 22.00 & & 1 & & $2.4 \mathrm{~s}$ & 820 & $.00 \mathrm{~nm}$ \\
\hline & & & i & 21 & 20.00 & & I ALE & & 52.13 & 351 & eP \\
\hline & & & $\mathrm{i}$ & 22 & 24.00 & & I & & $0.7 \mathrm{~s}$ & 53 & $.80 \mathrm{~nm}$ \\
\hline & & & is & 25 & 22.00 & & KMI & & 52.22 & 87 & iPct \\
\hline & & & isS & 28 & 31.00 & & 1 & & $9.0 \mathrm{~s}$ & 11 & $.68 \mathrm{~nm}$ \\
\hline IFR & 38.05 & 275 & $\mathrm{iPc}$ & 19 & 47.50 & 0. 1 & 1 & $E$ & $17 \mathrm{~s}$ & & $.20 u m$ \\
\hline HYB & 38.80 & 115 & iPc & 19 & 53.00 & -0.7 & | & & & & $\mathrm{pP}$ \\
\hline & $1.0 \mathrm{~s}$ & 470 & $.00 \mathrm{~nm}$ & & -4 & $2 \mathrm{mb}$ & I & & & & PCP \\
\hline & & & e & 20 & 14.50 & & I & & & & PP \\
\hline
\end{tabular}




\begin{tabular}{|c|c|c|c|c|c|c|c|c|c|c|c|}
\hline & & & & $s P$ & 23 & 12.50 & & 1 & $\mathbf{N}$ & $18 \mathrm{~s}$ & 15 \\
\hline & & & & is & 31 & 16.00 & & $i$ & $E$ & $18 \mathrm{~s}$ & 178 \\
\hline & & & & PS & 31 & 41.50 & & 1 & & & \\
\hline & & & & ScS & 32 & 44.00 & & i & & & \\
\hline MBC & & 63.10 & 355 & eP & 22 & 55.50 & -0.7 & i & & & \\
\hline & & $1.1 \mathrm{~s}$ & 81 & $1.00 \mathrm{~nm}$ & & & $.8 \mathrm{mb}$ & IMIY & & 71.99 & 53 \\
\hline SEY & & 63.12 & 29 & iPc & 22 & 56.60 & 0.1 & I PET & & 72.07 & 35 \\
\hline & & $2.5 \mathrm{~s}$ & 4910 & $0.00 \mathrm{~nm}$ & & & $.2 \mathrm{mb} x$ & 1 & & $5.0 \mathrm{~s}$ & 415 \\
\hline & & & & ePP & 25 & 13.50 & & i & & & \\
\hline & & & & is & 31 & 27.60 & & i & & & \\
\hline & & & & ePPS & 31 & 54.60 & & 1 & & & \\
\hline IPM & & 63.57 & 108 & ePd & 22 & 57.20 & $-2.9 x$ & 1 & & & \\
\hline & & $1.6 \mathrm{~s}$ & 175 & $5.00 \mathrm{~nm}$ & & 6 & $.2 \mathrm{mb}$ & I HVD & & 72.26 & 195 \\
\hline & & & & e & 25 & 05.30 & & i & & $1.5 \mathrm{~s}$ & 19 \\
\hline VLA & & 63.78 & 55 & iPd & 22 & 58.00 & $-3.2 x$ & I OCP & & 72.29 & 86 \\
\hline & & & & IPP & 25 & 23.00 & & I SKR & & 72.54 & 38 \\
\hline & & & & IPPP & 26 & 48.00 & & 1 & $z$ & $18 \mathrm{~s}$ & 72 \\
\hline & & & & is & 31 & 34.00 & & i & $N$ & $18 \mathrm{~s}$ & 65 \\
\hline & & & & IPPS & 32 & 04.00 & & i & $E$ & $18 \mathrm{~s}$ & 92 \\
\hline$M G D$ & & 64.18 & 32 & ¿Pc & 23 & 11.60 & $8.1 x$ & 1 & & & \\
\hline & & $1.3 \mathrm{~s}$ & 340 & $0.00 \mathrm{~nm}$ & & & $.3 \mathrm{mb}$ & 1 & & & \\
\hline & $z$ & $19 \mathrm{~s}$ & 314 & $4.00 \mathrm{um}$ & & & $.5 M s Z$ & 1 & & & \\
\hline & $N$ & $19 \mathrm{~s}$ & 32 & $2.00 \mathrm{um}$ & & & & 1 & & & \\
\hline & $E$ & $19 \mathrm{~s}$ & 299 & $9.00 \mathrm{um}$ & & & & I PGP & & 72.89 & 87 \\
\hline & & & & ePP & 25 & 23.00 & & I IMA & & 73.22 & 7 \\
\hline & & & & is & 31 & 34.00 & & I PPR & & 73.51 & 91 \\
\hline & & & & iscs & 32 & 58.00 & & I KKM & & 73.96 & 96 \\
\hline$Q Z H$ & & 64.33 & 78 & iPc & 23 & 04.00 & -0.9 & I & & $1.2 \mathrm{~s}$ & 88 \\
\hline & & & & $\mathbf{s P}$ & 23 & 28.00 & & I GRM & & 74.69 & 193 \\
\hline STJ & & 64.52 & 312 & eP & 23 & 68.20 & 2.3 & I & & $1.4 \mathrm{~s}$ & 256 \\
\hline ANP & & 66.37 & 76 & iPt & 23 & 20.00 & 1.8 & 1 & $z$ & $22 \mathrm{~s}$ & 24 \\
\hline & & & & is & 32 & 12.00 & & I $\mathrm{COL}$ & & 74.81 & 4 \\
\hline PI & & 66.59 & 112 & ePd & 23 & 19.00 & -0.6 & i & $z$ & $19 \mathrm{~s}$ & 37 \\
\hline & & $1.5 \mathrm{~s}$ & 300 & $0.00 \mathrm{~nm}$ & & 6 & $.2 \mathrm{mb}$ & 1 & & & \\
\hline$\langle G M$ & & 66.98 & 108 & ePd & 23 & 21.70 & -0.4 & FBA & & 74.81 & 4 \\
\hline$S L R$ & & 66.99 & 194 & iPc- & 23 & 21.60 & -0.4 & I SUR & & 75.00 & 199 \\
\hline & & $1.0 \mathrm{~s}$ & 100 & $0.00 \mathrm{~nm}$ & & 5 & $.9 \mathrm{mb}$ & 1 & & $0.7 \mathrm{~s}$ & 100 \\
\hline & $z$ & $17 \mathrm{~s}$ & 104 & $4.00 \mathrm{um}$ & & 7 & $.1 M s z X$ & I LGP & & 75.23 & 85 \\
\hline PRE & & 67.02 & 194 & e (P) & 23 & 22.00 & -0.3 & 1 & & $1.0 \mathrm{~s}$ & 166 \\
\hline & & $0.8 \mathrm{~s}$ & 430 & $0.00 \mathrm{~nm}$ & & 6 & $.6 m b$ & I YKC & & 75.82 & 349 \\
\hline KSR & & 67.38 & 195 & iPc & 23 & 23.50 & -1.0 & I & & $1.1 \mathrm{~s}$ & 45 \\
\hline BP I & & 67.46 & 194 & iPc & 23 & 23.70 & -1.4 & I YKA & & 75.82 & 349 \\
\hline $\mathrm{SCH}$ & & 67.54 & 324 & ePc & 23 & 24.90 & -0.2 & I RSNT & & 75.84 & 349 \\
\hline & & $1.2 \mathrm{~s}$ & 222 & $2.00 \mathrm{~nm}$ & & 6 & $.2 \mathrm{mb}$ & I & & $2.0 \mathrm{~s}$ & 1016 \\
\hline EVA & & 67.60 & 193 & $e^{P}$ & 23 & 25.70 & -0.3 & I TTA & & 76.05 & 8 \\
\hline & & $1.5 \mathrm{~s}$ & 380 & $0.06 \mathrm{~nm}$ & & 6 & $.3 \mathrm{mb}$ & I TUH & & 76.29 & 200 \\
\hline$I L T$ & & 67.79 & 16 & iPd & 23 & 26.00 & -0.4 & I CER & & 76.32 & 200 \\
\hline & & $2.6 \mathrm{~s}$ & 1900 & $0.00 \mathrm{~nm}$ & & & $.8 \mathrm{mb}$ & i & & $1.3 \mathrm{~s}$ & 80 \\
\hline & $z$ & $20 \mathrm{~s}$ & 145 & $5.00 \mathrm{um}$ & & 7 & $.2 M s Z$ & I BNH & & 76.47 & 318 \\
\hline & $N$ & $20 \mathrm{~s}$ & 84 & $4.00 \mathrm{um}$ & & & & I MNT & & 77.03 & 319 \\
\hline & $E$ & $20 \mathrm{~s}$ & 51 & $1.00 \mathrm{um}$ & & & & I TOA & & 77.69 & 4 \\
\hline & & & & ePcP & 23 & 50.00 & & I svw & & 77.87 & 9 \\
\hline & & & & ePP & 25 & 56.00 & & I PMR & & 78.01 & 5 \\
\hline & & & & ePPP & 27 & 30.00 & & 1 & $z$ & $20 \mathrm{~s}$ & $4 \ell$ \\
\hline YSS & & 68.01 & 47 & $i P_{c}$ & 23 & 29.00 & 0.8 & I OT T & & 78.13 & 320 \\
\hline & & 6.05 & $* * * *$ & $* * * * n m$ & & & . $2 \mathrm{mb} \times$ & I & & $1.2 \mathrm{~s}$ & 94 \\
\hline & & & & ePCP & 23 & 56.40 & & I RSNY & & 78.16 & 319 \\
\hline & & & & ePPP & 27 & 42.00 & & i & & 2.05 & 472 \\
\hline & & & & is & 32 & 32.00 & & I SMY & & 78.40 & 28 \\
\hline & & & & ePPS & 33 & 05.00 & & 1 & 2 & $19 \mathrm{~s}$ & 112 \\
\hline & & & & escs & 33 & 26.00 & & I CGP & & 78.90 & 88 \\
\hline & & & & eSS & 36 & 58.00 & & 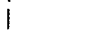 & & $0 \mathrm{~s}$ & 95 \\
\hline 102 & & 68.08 & 190 & iPc & 23 & 25.60 & $-3.1 x$ & I PCA & & 79.90 & 1 \\
\hline NGS & & 68.11 & 65 & eP & 23 & 29.00 & 0.0 & I DAV & & 80.44 & 89 \\
\hline & & & & es & 32 & 34.00 & & FFC & & 80.46 & 340 \\
\hline WZ & & 68.97 & 196 & $i P d$ & 23 & 33.50 & -0.9 & 1 & & $1.0 \mathrm{~s}$ & 45 \\
\hline & & $1.6 \mathrm{~s}$ & 130 & $0.00 \mathrm{~nm}$ & & 6 & $.6 \mathrm{mb}$ & I RSON & & 81.32 & 333 \\
\hline HK & & 69.61 & 62 & ePc & 23 & 33.20 & -1.4 & I & & $2.0 \mathrm{~s}$ & 377 \\
\hline SAP & & 69.55 & 51 & eP & 23 & 40.00 & 2.3 & I KDC & & 81.51 & 8 \\
\hline & & & & es & 32 & 52.00 & & I LHC & & 1.73 & 330 \\
\hline EK & & 3.63 & 94 & $i P_{C}$ & 23 & 38.50 & 0.1 & 1 & & $0.9 \mathrm{~s}$ & 103 \\
\hline $\mathrm{K} I \mathrm{M}$ & & 70.61 & 196 & iPc & 23 & 43.00 & -1.4 & I ELF & & 82.45 & 322 \\
\hline & & $0.6 \mathrm{~s}$ & 207 & $7.00 \mathrm{~nm}$ & & 6 & $.4 \mathrm{mb}$ & LDN & & 51 & 322 \\
\hline BLF & & 70.67 & 195 & iPc & 24 & 02.50 & $17.8 x$ & I DLA & & 82.83 & 322 \\
\hline$O B I$ & & 70.75 & 50 & eP & 23 & 48.00 & $3.0 x$ & I SIT & & 82.95 & 359 \\
\hline & & & & e & 56 & 08.00 & & MN I & & 83.48 & 94 \\
\hline BAG & & 70.83 & 85 & ePt & 23 & 44.00 & -2.1 & EDM & & 84.44 & 345 \\
\hline & & $1.8 \mathrm{~s}$ & 636 & $3.00 \mathrm{~nm}$ & & 6 & $.4 \mathrm{mb}$ & MRG & & 4.50 & 319 \\
\hline CVP & & 70.99 & 83 & ePc & 23 & 46.00 & -0.8 & SES & & 86.64 & 343 \\
\hline MAT & & 71.49 & 58 & iPc & 23 & 48.00 & -1.7 & I & & $1.4 \mathrm{~s}$ & 545 \\
\hline & & $1.2 \mathrm{~s}$ & 156 & $5.00 \mathrm{~nm}$ & & 6 & $.6 \mathrm{mb}$ & ITR & & 88.51 & 257 \\
\hline & & & & es & 33 & 10.00 & & i & & & \\
\hline INK & & 71.65 & 358 & eP & 23 & 49.50 & -0.5 & 1 & & & \\
\hline & & $1.5 \mathrm{~s}$ & 176 & $5.00 \mathrm{~nm}$ & & 5 & $.9 \mathrm{mt}$ & NEW & & 89.89 & 346 \\
\hline UUR & & 71.94 & 46 & iPd & 23 & 55.50 & $3.4 x$ & | & $z$ & $20 s$ & 68 \\
\hline & & 6.05 & $* * *$ & $* * * n \mathrm{~nm}$ & & 7 & $.0 \mathrm{mb} x$ & FDF & & 90.42 & 290 \\
\hline & & $15 \mathrm{~s}$ & 46 & $5.70 \mathrm{um}$ & & & $.9 M s z X$ & & & & \\
\hline
\end{tabular}


FCH $\quad 126.66 \quad 255$ iPKPd 3134.20

PEL 126.86255 IPKPC 3134.40

ROCH $127.02 \quad 255$ IPKPd 3134.50

CHCH 127.21254 IPKP 3136.00

KOU 127.30 B6 IPKPC 3134.40

LNV $127.78 \quad 255$ IPKP 3134.10

DRV $\quad 129.27 \quad 149$ ePKP $31 \quad 37.00$

NOU $\quad 129.95 \quad 86$ IPKPC 3142.90

$\begin{array}{llll}Z & 20 \mathrm{~s} & 39.70 \mathrm{um} & \end{array}$

\begin{tabular}{lcccccc|c} 
& & $e$ & 44 & 53.80 & & TP \\
SBA & 136.22 & 165 & ePKP & 31 & 45.50 & $-3.3 x$ &
\end{tabular}

AFI $140.34 \quad 58$ e(PKP) $32 \quad 04.00 \quad 5.8 \times \quad$ WDC

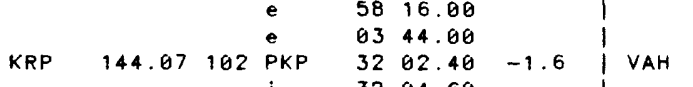

$\begin{array}{lllll} & & \mathrm{i} & 32 & 04.60 \\ \text { TCW } \quad 144.09 & 107 & \text { PKP } & 32 & 27.50 \\ \text { WEL } & 144.46 & 107 & 01.80\end{array}$

\begin{tabular}{l|l}
1.9 & \\
2.0 & ARN
\end{tabular}

1.6 SLD

$3.0 \times$ RMT

1.0 PR

0.2

1.2 SCCM

MO

WEL $\quad 144.46107$ PKP 3158.06

$\begin{array}{lll}Z & 22 \mathrm{~s} & 35.60 \mathrm{um} \\ \mathrm{N} & 22 \mathrm{~s} & 13.30 \mathrm{um} \\ \mathrm{E} & 21 \mathrm{~s} & 21.50 \mathrm{um}\end{array}$

e $\quad 32 \quad 25.00$

GNZ $\quad 146.15 \quad 102$ ePKP $32 \quad 08.70$

PMO $153.28 \quad 22$ ePKP $32 \quad 25.00$ $1.4 \mathrm{~s} \quad 105.00 \mathrm{~nm}$

TVO $155.45 \quad 27$ ePKP $32 \quad 28.00$

$1.4 \mathrm{~s} \quad 195.00 \mathrm{~nm}$

S.D. $=1.2$ on 406 of 463 obs

\& Nov 16, $198316 \mathrm{~h} 13 \mathrm{~m} 00.07 \mathrm{~s}$ $19.430 \mathrm{~N} \quad 155.454 \mathrm{~W}$

DEPTH $=12.0 \mathrm{~km}$ (geophysicist) HAWAII

6.7Msz ( 41 obs.)

(613) SBB

<HVO-P>. Six people injured and I COR consideroble domoge (VIII) in I RVR ports of Kopopolo, Howoii

Volconoes Notionol Pork,

Volcono, Kou and Puno districts

ond Hilo. Londslides ond

rockfolls occurred, telephone

and electrical service distupted

ond woter moins broken in

severol oreos of the island.

Totol domoge estimoted ot 6 to

6.5 million dollors. Also felt

on Moui, Dohu ond Kouoi.

\begin{tabular}{l}
$A$ \\
$M L$ \\
$M L X$ \\
$D$ \\
$C P K$ \\
$M$ \\
$N$ \\
$O$ \\
$O$ \\
$R$ \\
$K$ \\
$A$ \\
$H$ \\
$H$ \\
$E$ \\
$P$ \\
$K$ \\
\hline \\
$P$ \\
$P$ \\
$M$ \\
$K$ \\
$K A$ \\
$H$ \\
$H$ \\
$M$ \\
$M$ \\
$W H$ \\
$K K$ \\
$H O$ \\
$O$
\end{tabular}

$0.06 \quad 186 \mathrm{iPc}$

$13 \quad 02.56-0.2$

$0.09 \quad 43 \mathrm{iPc}$

$0.11 \quad 74 \mathrm{iPd}$

0.11146 iPc

$0.12 \quad 106 \mathrm{iPd}$

$0.15293 \mathrm{iPd}$

$0.16 \quad 95 \mathrm{iPd}$

$0.17104 \mathrm{iPd}$

$0.1710 ; \mathrm{iPd}$

$0.18 \quad 121 \mathrm{iPd}$

$0.19108 \mathrm{iPd}$

$0.19134 \mathrm{iPc}$

$0.20 \quad 95 \mathrm{iPd}$

$0.23103 \mathrm{iPd}$

$0.24221 \mathrm{iPc}$

$0.26 \quad 124 \quad \mathrm{iPd}$

$0.27182 \mathrm{iPc}$

$0.28103 \mathrm{iPd}$

$0.33115 \mathrm{iPd}$

$0.35359 \mathrm{iPc}$

$0.35196 \mathrm{P}$

$0.38 \quad 79 \mathrm{iPd}$

$0.39104 \mathrm{iPd}$

$0.57294 \mathrm{P}$

$3.05309 \mathrm{eP}$

$3.29314 \mathrm{eP}$

$34.2150 \mathrm{eP}$

$1.2 \mathrm{~s} \quad 290.00 \mathrm{~nm}$

$\begin{array}{lll}\text { EPCP } & 22 & 30.20 \\ \text { ES } & 25 & 19.00\end{array}$

$\begin{array}{llllll} & & & \mathrm{e} & 27 & 00.00 \\ \mathrm{FHC} & 34.28 & 45 \mathrm{eP} & 1952.80 \\ \mathrm{SAO} & 34.38 & 53 \mathrm{eP} & 19 & 49.40\end{array}$

$\begin{array}{lllll}\text { SAO } & 34.38 & 53 \mathrm{eP} & 19 & 49.40 \\ & & \mathrm{PCP} & 22 & 30.00 \\ & & & \end{array}$

MHC $\begin{array}{lll}3 & 03.38 & 0.1\end{array}$

\begin{tabular}{lll|l}
13 & 03.03 & -0.2 & I PAE
\end{tabular}

$03.40 \quad 0.0$

\begin{tabular}{llr|r}
13 & 03.90 & 0.0 & I TVO
\end{tabular}

\begin{tabular}{lll|l}
13 & 03.92 & -0.2 & GSC
\end{tabular}

$304.00-0.2$ I BFW

$1304.46-0.3$

$\begin{array}{lll}13 & 04.73 & -0.5\end{array}$

$\begin{array}{lll}13 & 04.83 & -0.6\end{array}$

$\begin{array}{lll}13 & 05.67 & -0.5\end{array}$

$1306.68-0.4$

$\begin{array}{lll}13 & 04.60 & -2.8\end{array}$

$\begin{array}{lll}13 & 07.63 & -0.3\end{array}$

$\begin{array}{llll}13 & 07.47 & -0.8\end{array}$

$10.70-0.8$

$\begin{array}{lll}3 & 44.00 & -4.9\end{array}$

\begin{tabular}{lll|l}
3 & 44.30 & -8.1 & MSU
\end{tabular}

\begin{tabular}{lll|l}
13 & 49.80 & & GCA \\
19 & 49.20 & 1.5 & PCA
\end{tabular}

$1 \mathrm{mb}$

I PMR

RMU

4.6 RMU

$0.2: \begin{aligned} & \mathrm{HPI} \\ & \mathrm{TB}\end{aligned}$ $\begin{array}{lll}3 & 02.90 & -0.3\end{array}$

$\begin{array}{lll}13 & 05.52 & -0.2\end{array}$
ePcP 2231.00

$34.53 \quad 52 \mathrm{eP} \quad 1951.40$

$34.66 \quad 52 \mathrm{eP} \quad 1953.50$

$34.76 \quad 47 \mathrm{eP} \quad 1954.00$

$34.76 \quad 54 \mathrm{ePd} \quad 1953.80$

$34.77 \quad 56 \mathrm{ePCP} \quad 2231.00$

$34.8755 \mathrm{eP} \quad 1955.10$

$35.02 \quad 167$ iP 1956.00

$1.6 \mathrm{~s} 1900.00 \mathrm{~nm}$

$35.03346 \mathrm{eP}$

$35.06 \quad 167$ iP

$1.6 \mathrm{~s} 1900.00 \mathrm{~nm}$

$1954.90^{6.7 \mathrm{mb}}$

$35.15 \quad 46 \mathrm{eP}^{\mathrm{P}}$

e $\quad 2025.00$

$\begin{array}{lllll}35.30 & 167 \mathrm{IPP} & 22 & 32.00 \\ 19 & 58.20\end{array}$

$1.6 \mathrm{~s} \quad 1330.00 \mathrm{~nm}$

35.31166 iP

$1.6 \mathrm{~s} \quad 1330.00 \mathrm{~nm}$

1958.30

$6.6 \mathrm{mb}$

$35.56 \quad 51$ iPd 2000.50

2028.40

ePCP $22 \quad 33.20$

$35.68 \quad 47 \mathrm{eP} \quad 2001.50$

$35.73 \quad 45 \mathrm{eP} \quad 20 \quad 04.00$

$35.77 \quad 53 \mathrm{ePd} \quad 20 \quad 01.80$

$36.05355 \mathrm{eP} \quad 2004.40$

$36.15 \quad 58$ iPd 2006.00

$1.2 \mathrm{~s} \quad 300.00 \mathrm{~nm}$

IPP 2130.00

IPCP 2237.00

$36.26 \quad 58 \mathrm{eP} \quad 2006.00$

$36.31 \quad 56 \mathrm{eP} \quad 20 \quad 06.50$

$36.37 \quad 56 \mathrm{eP} \quad 20 \quad 06.50$

$36.41338 \mathrm{e}(P) \quad 2005.30$

$\begin{array}{lllll}36.56 & 58 \mathrm{iPd} & 20 & 08.80\end{array}$

$36.65 \quad 40$ iPd 2014.90

$36.75 \quad 59 \mathrm{eP} \quad 20 \quad 09.00$

$36.82 \quad 207$ eP $20 \quad 12.00$ e(PPP) 2136.00 e(S) 2540.00

$\begin{array}{llll}36.84 & 55 \mathrm{eP}^{\mathrm{P}} \quad 20 \quad 10.00\end{array}$

2012.00
$e \quad 2017.00$

$\begin{array}{llll}36.90 \quad 56 & e & 29 & 17.00\end{array}$

$\begin{array}{llll}37.03 & 60 \mathrm{eP} & 20 & 12.00\end{array}$

e $\quad 2014.00$

$37.95 \quad 61 \mathrm{eP} \quad 2012.00$

$\begin{array}{lll}20 & 12.70 & 0.2\end{array}$

$1.6 \mathrm{~s} \quad 950.00 \mathrm{~nm}$

37.20170 iP $20 \quad 15.00$

1.9

$\begin{array}{ll}1.6 \mathrm{~s} & 950.00 \mathrm{~nm} \quad 6.3 \mathrm{mb}\end{array}$

37.31171 iP $2015.90^{6.3} 1.9$

$\begin{array}{lll}1.6 \mathrm{~s} & 930.00 \mathrm{~nm} & 6.3 \mathrm{mb}\end{array}$

$\begin{array}{lllll}37.48 & 170 \text { iP } \quad 20 & 17.40 & 1.9\end{array}$

$1.6 \mathrm{~s} 2080.00 \mathrm{~nm}$

$37.5257 \mathrm{eP}^{2} \quad 2016.00^{6.7 \mathrm{mb}}$

$\begin{array}{lllll}37.76 & 37 \mathrm{eP} & 20 & 20.80 & 3.1\end{array}$

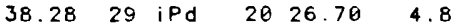

$1.7 \mathrm{~s} 2090.00 \mathrm{~nm}$

$38.6136 \mathrm{eP}$

$38.64 \quad 61 \mathrm{eP}$

$38.79 \quad 49 \mathrm{ePd}$

$38.90 \quad 34 \mathrm{eP}$

$40.44 \quad 17$ eP

$1.2 \mathrm{~s} \quad 439.39 \mathrm{~nm}$

40.61186 iP

$40.77 \quad 332 \mathrm{eP} \quad 2044.30 \quad 1.8$

$z 20 \mathrm{~s} \quad 95.00 \mathrm{um}$

$2026.70^{6.6 m b} 2.0$

$20 \quad 26.70 \quad 1.4$

$\begin{array}{lll}20 & 27.70 & 1.2\end{array}$

$41.2153 \mathrm{eP}$

$41.35 \quad 35 \mathrm{eP}$

$1.1 \mathrm{~s} \quad 686.00 \mathrm{~nm}$

$41.64360 \mathrm{iPc}$

$41.90 \quad 51 \mathrm{ePd}$

$41.92 \quad 54 \quad \mathrm{iPC}$

$42.0156 \mathrm{eP}$

$42.0511 \mathrm{eP}$

$42.21 \quad 38 \mathrm{eP}$

42.36

$1.5 \mathrm{~s}$

4 eP

$20 \mathrm{~s} 120.00 \mathrm{um}$

42.5256 eP

$42.83 \quad 46$ iP

$42.92 \quad 172$ iP

$1.6 \mathrm{~s} \quad 1710.00 \mathrm{~nm}$

$2047.50^{6.6 M 52}$

$\begin{array}{lll}20 & 49.00 & 1.7\end{array}$

$2050.90^{6.3 \mathrm{mb}}$

$2053.80 \quad 1.7$

\begin{tabular}{lll|l}
2054.00 & 1.5 & ATO
\end{tabular}

$2055.10 \quad 2.0$ | $11 C$

$2054.80 \quad 1.7 \quad 111$

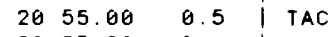

$\begin{array}{lll}20 & 55.80 & 0.4\end{array}$

$\lim _{\text {I ITX }}$

6.8Msz ATX

$2058.50 \quad 1.2$

$\begin{array}{lll}21 & 01.90 & 2.0 \\ 21 & 04.80 & 4.4\end{array}$

KUR

202

$\begin{array}{llllll}43.36 & 39 & \mathrm{IPC} & 21 & 04.80 & 0.8\end{array}$

$\begin{array}{llllll}43.36 & 39 \text { IPC } & 21 & 04.70 & 0.8\end{array}$

43.47360 iPc $2104.60 \quad 0.1$

$\begin{array}{lllll}43.50 & 47 \mathrm{eP} & 21 & 07.40 & 2.1\end{array}$

$\begin{array}{lllll}44.03 & 43 \mathrm{eP} & 21 & 11.40 & 1.9 \\ 44.03 & 43 \mathrm{eP} & 21 & 10.20 & 0.6\end{array}$

$\begin{array}{lllll}44.36 & 46 \mathrm{eP} & 21 & 13.20 & 0.9\end{array}$

$\begin{array}{llllll}44.97 & 48 & \mathrm{eP} & 21 & 17.00 & -0.2\end{array}$

$1.3 \mathrm{~s} \quad 392.00 \mathrm{~nm} \quad 6.2 \mathrm{mb}$

$45.26216 \mathrm{ePC} \quad 2122.50 \quad 3.1$

$\begin{array}{llll}45.54 & \text { ePP } 21 \quad 29.00 & 22 \mathrm{kmx}\end{array}$

$\begin{array}{rrrrr}45.54 & 217 \mathrm{eP} & 21 & 27.00 & 5.4 \\ 45.60 & 354 \mathrm{eP} & 21 & 22.70 & 1.2\end{array}$

$\begin{array}{lllll}45.73 & 5 \text { eP } \quad 21 & 22.00 & -0.6\end{array}$

$18 \mathrm{~s} \quad 95.50 \mathrm{um} 28 \quad 6.8 \mathrm{Msz}$

$\begin{array}{lllll} & \mathrm{eS} & 28 & 10.00 & \\ 45.73 & 5 \mathrm{eP} & 21 & 23.10 & 0.5\end{array}$

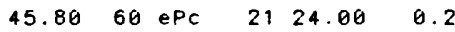

$\begin{array}{llll}218 \mathrm{~s} & 61.90 \mathrm{um} & 6.6 \mathrm{Msz}\end{array}$

$\begin{array}{lllll}46.63 \quad 1 \mathrm{PC} & 21 & 29.30 & -0.5\end{array}$

$\begin{array}{llllll}46.72 & 38 \mathrm{eP} & 21 & 31.00 & 0.3\end{array}$

$\begin{array}{lll}1.6 \mathrm{~s} & 179.00 \mathrm{~nm} & 5.9 \mathrm{mb}\end{array}$

$\begin{array}{llllll}46.75 & 34 & \text { iPd } & 21 & 30.50 & -0.3\end{array}$

$1.5 \mathrm{~s} \quad 310.00 \mathrm{~nm} \quad 6.1 \mathrm{mb}$

$\begin{array}{llllll}46.78 & 154 \text { iP } \quad 2133.40 \quad 2.1\end{array}$

$1.6 \mathrm{~s} \quad 1710.00 \mathrm{~nm} \quad 6.9 \mathrm{mb}$

$47.34 \quad 54 \mathrm{e}^{\mathrm{P}} \quad 2136.00 \quad-0.1$

$1.2 \mathrm{~s} 2830.00 \mathrm{~nm} \quad 7.2 \mathrm{mb}$

$z 20 \mathrm{~s} 62.50 \mathrm{um} \quad 6.6 \mathrm{Msz}$

$\begin{array}{lllll}47.47 & 54 \text { eP } & 21 & 39.10 & 2.2\end{array}$

$\begin{array}{rrr}1.5 \mathrm{~s} & 1970.00 \mathrm{~nm} & 7.0 \mathrm{mb} \\ 20 \mathrm{~s} & 95.00 \mathrm{um} & 6.8 \mathrm{Msz}\end{array}$

$\begin{array}{llllll}48.68 & 325 & \text { iPc } & 21 & 47.50 & 1.7\end{array}$

$2.0 \mathrm{~s} \quad 1600.00 \mathrm{~nm} \quad 6.7 \mathrm{mb}$

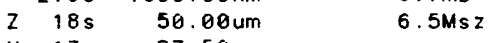

N $17 \mathrm{~s} \quad 27.50 \mathrm{um}$

ESP $28 \quad 53.00$

eSCS 3138.00

$\begin{array}{llllll}\text { SKR } & 49.20321 \mathrm{iPC} & 21 & 50.80 & 0.9\end{array}$

$0.8 \mathrm{~s} \quad 480.00 \mathrm{~nm} \quad 6.6 \mathrm{mb}$

$z 17 \mathrm{~s} \quad 40.50 \mathrm{um} \quad 6.5 \mathrm{MszX}$

$\begin{array}{lll}\mathrm{N} & 17 \mathrm{~s} & 21.30 \mathrm{um} \\ \mathrm{E} & 16 \mathrm{~s} & 41.80 \mathrm{um}\end{array}$

EPP 2344.40

EPPP $24 \quad 31.00$

$\begin{array}{lll}\text { is } & 24 & 31.00 \\ \text { is } & 29 & 00.00\end{array}$

RSSD $\quad 49.21 \quad 48$ eP $\quad 21 \quad 49.30 \quad-1.1$

$\begin{array}{lll}1.5 \mathrm{~s} & 735.00 \mathrm{~nm} & 6.5 \mathrm{mb}\end{array}$

50.64349 iPC $22 \quad 00.50 \quad-0.2$

$\begin{array}{lll}1.6 \mathrm{~s} & 1100.00 \mathrm{~nm} & 22 \\ 7.6 \mathrm{mb}\end{array}$

N $18 \mathrm{~s} 63.00 \mathrm{um}$

IPP 2356.00

ePPP $24 \quad 48.00$

is $29 \quad 12.00$

eses 3148.00

$\begin{array}{lllll}50.76 & 10 \mathrm{eP} & 22 & 01.00 & -0.6\end{array}$

$0.6 \mathrm{~s} \quad 203.00 \mathrm{~nm} \quad 6.2 \mathrm{mb}$

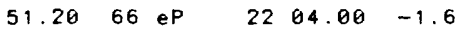

$1.0 \mathrm{~s} \quad 180.00 \mathrm{~nm} \quad 6.0 \mathrm{mb}$

$220 \mathrm{~s} 92.20 \mathrm{um} \quad 6.8 \mathrm{Msz}$

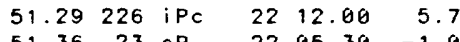

$\begin{array}{lllll}51.36 & 23 \mathrm{eP} & 22 & 05.30 & -1.0\end{array}$

\begin{tabular}{llll}
$1.5 \mathrm{~s}$ & $1510.00 \mathrm{~nm}$ & \multicolumn{6}{c}{$6.7 \mathrm{mb}$} \\
51.37 & $23 \mathrm{eP} \quad 2206.70^{0.4}$
\end{tabular}

$\begin{array}{llllll}51.41 & 23 & \text { ePd } \quad 22 & 06.20 & -0.4\end{array}$

$0.3 \mathrm{~s} \quad 116.00 \mathrm{~nm} \quad 6.3 \mathrm{mb}$

$\begin{array}{lllll}51.87 & 58 \mathrm{eP} & 22 & 10.80 & 0.2\end{array}$

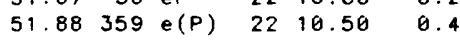

$\begin{array}{lllll}52.42 & 241 \mathrm{eP} & 22 & 18.00 & 3.0\end{array}$

$52.45 \quad 60$ eP $\quad 22 \quad 18.40 \quad 3.4$

$1.8 \mathrm{~s} \quad 1390.00 \mathrm{~nm} \quad 6.6 \mathrm{mb}$

$\begin{array}{lllll}52.47 & 241 \mathrm{ePC} & 22 & 18.00 & 2.7\end{array}$ 


\begin{tabular}{|c|c|c|c|c|c|c|c|c|c|c|c|}
\hline & $N$ & $16 s$ & & 4. 50 um & & & & I ELF & 65.53 & 51 & $\mathrm{P}$ \\
\hline & $E$ & $16 \mathrm{~s}$ & & $3.50 \mathrm{um}$ & & & & i LON & 65.64 & 51 & $\mathbf{P}$ \\
\hline & & & & ePcP & 23 & 30.00 & & | PRM & 65.65 & 61 & eP \\
\hline & & & & ePP & 24 & 24.00 & & I GFM & 65.85 & 58 & eP \\
\hline & & & & is & 29 & 54.00 & & I YAK & 65.92 & 330 & $\mathrm{i} P \mathrm{c}$ \\
\hline & & & & eSS & 33 & 24.00 & & 1 & $1.5 \mathrm{~s}$ & 740 & $0.00 \mathrm{~nm}$ \\
\hline$\| P$ & & 53.11 & 80 & iP & 22 & 21.70 & 1.2 & i & & & iPP \\
\hline Oco & & 53.17 & 60 & eP & 22 & 20.80 & 0.5 & I & & & iPPP \\
\hline FFC & & 53.47 & 35 & eP & 22 & 20.00 & -2.2 & 1 & & & is \\
\hline & & $1.1 \mathrm{~s}$ & & $2.00 \mathrm{~nm}$ & & & $.6 \mathrm{mb}$ & 1 & & & iPS \\
\hline $\mathrm{PCO}$ & & 53.60 & 58 & eP & 22 & 22.70 & -0.8 & 1 & & & iss \\
\hline $11 \mathrm{~T}$ & & 53.74 & 80 & ip & 22 & 26.20 & 1.2 & $1 \mathrm{MOJ}$ & 66.02 & 311 & $P C$ \\
\hline 510 & & $54 \cdot 12$ & 60 & eP & 22 & 26.90 & -0.4 & 1 & & & $S$ \\
\hline TUL & & 54.54 & 59 & iPd- & 22 & 30.70 & 0.3 & | WEL & 66.42 & 204 & $\mathbf{P}$ \\
\hline & & $1.7 \mathrm{~s}$ & 1600 & $0.00 \mathrm{~nm}$ & & & $.8 \mathrm{mb}$ & 1 & & & $\mathrm{~s}$ \\
\hline & $z$ & 215 & 49 & 7. 50 um & & & $.6 \mathrm{Msz}$ & 1 & & & SKS \\
\hline HKT & & 54,66 & 67 & iP & 22 & 31.50 & 0.3 & 1 & & & e \\
\hline GBO & & 55.04 & 59 & eP & 22 & 32.90 & -1.2 & I NAV & 66.51 & 57 & eP \\
\hline$R L O$ & & 55.17 & 59 & eP & 22 & 34.10 & -0.9 & I JSC & 66.53 & 60 & eP \\
\hline $\mathrm{BHO}$ & & 55.31 & 61 & IPd & 22 & 35.00 & -1.0 & I BLA & 66.81 & 57 & eP- \\
\hline MGD & & 55.54 & 331 & iPc & 22 & 37.40 & 0.1 & 1 & $1.7 \mathrm{~s}$ & 2540 & $0.00 \mathrm{~nm}$ \\
\hline & & $1.9 \mathrm{~s}$ & 1470 & $0.00 \mathrm{~nm}$ & & & $.7 \mathrm{mb}$ & 1 & $18 s$ & 46 & $6.90 \mathrm{um}$ \\
\hline & & & & iPPP & 25 & 54.00 & & 1 & & & is \\
\hline & & & & is & 30 & 27.40 & & I TIK & 67.19 & 341 & $i P c$ \\
\hline & & & & iSS & 34 & 03.00 & & 1 & $1.9 \mathrm{~s}$ & 1250 & $0.00 \mathrm{~nm}$ \\
\hline VHO & & 55.55 & 82 & iP & 22 & 40.00 & 1.8 & 1 & $18 \mathrm{~s}$ & 34 & $4.00 \mathrm{um}$ \\
\hline NOU & & 55.74 & 224 & iPc & 22 & 39.00 & -0.1 & 1 & $19 \mathrm{~s}$ & 10 & $0.00 \mathrm{um}$ \\
\hline KOU & & 55.99 & 227 & iPc & 22 & 42.10 & 1.2 & 1 & $19 \mathrm{~s}$ & 35 & $5.00 \mathrm{um}$ \\
\hline SEY & & 56.00 & 334 & iPc & 22 & 40.10 & -0.4 & 1 & & & ePcP \\
\hline & & $1.7 \mathrm{~s}$ & 1000 & $0.00 \mathrm{~nm}$ & & 6 & $.6 \mathrm{mb}$ & 1 & & & ePP \\
\hline & & & & ePP & 24 & 44.60 & & i & & & es \\
\hline & & & & is & 30 & 33.60 & & 1 & & & EPPS \\
\hline RAB & & 56.59 & 251 & iPct & 22 & 44.60 & -0.9 & 1 & & & escs \\
\hline YSS & & 56.91 & 314 & $\mathrm{IPC}$ & 22 & 48.00 & 0.7 & I MVI & 67.20 & 291 & eP \\
\hline & & $1.5 \mathrm{~s}$ & 500 & $0.00 \mathrm{~nm}$ & & & $.3 \mathrm{mb}$ & 1 & & & eS \\
\hline & 2 & $17 \mathrm{~s}$ & 60 & 0.00 um & & & $.8 \mathrm{Ms} z \mathrm{X}$ & I DHN & 67.85 & 51 & eP \\
\hline & $\mathbf{N}$ & $17 \mathrm{~s}$ & 17 & $.50 \mathrm{um}$ & & & & I CVL & 68.25 & 56 & eP \\
\hline & $E$ & $17 \mathrm{~s}$ & 29 & $7.60 \mathrm{um}$ & & & & $1 \mathrm{CN} 2$ & 69.06 & 310 & iPc \\
\hline & & & & is & 30 & 48.00 & & 1 & & & es \\
\hline & & & & eSSS & 36 & 40.80 & & INY & 9.15 & 51 & $P$ \\
\hline MIY & & 56.93 & 305 & eP & 22 & 47.00 & -0.5 & I CTA & 69.31 & 239 & i Pc \\
\hline & & & & $S$ & 30 & 46.00 & & 1 & $1.8 \mathrm{~s}$ & 445 & $5.00 \mathrm{~nm}$ \\
\hline GUA & & 57.25 & 274 & ePt & 22 & 50.00 & -0.2 & $!$ & & & is \\
\hline & & $0.8 \mathrm{~s}$ & 263 & $3.00 \mathrm{~nm}$ & & & $.3 m b$ & 1 & & & $i(S S)$ \\
\hline & & & & PP & 22 & 56.80 & $22 \mathrm{kmX}$ & I CTAO & 69.31 & 239 & eP \\
\hline & & & & es & 30 & 51.50 & & 1 & $1.4 \mathrm{~s}$ & 134 & $4.00 \mathrm{~nm}$ \\
\hline GUMO & & 57.28 & 274 & ePt & 22 & 51.00 & 0.6 & I OTT & 69.32 & 48 & eP \\
\hline & & $1.5 \mathrm{~s}$ & 1590 & $0.00 \mathrm{~nm}$ & & & $.8 \mathrm{mb}$ & 1 & $2.0 \mathrm{~s}$ & 1200 & $0.00 \mathrm{~nm}$ \\
\hline & & & & PP & 22 & 57.20 & $20 \mathrm{kmX}$ & I PTN & 69.94 & 49 & eP \\
\hline CB I & & 57.31 & 290 & $\mathbf{P}$ & 22 & 51.50 & 1.0 & I RSNY & 70.27 & 49 & eP \\
\hline RSON & & 57.39 & 42 & eP & 22 & 48.30 & -2.4 & 1 & $1.5 \mathrm{~s}$ & 869 & $9.00 \mathrm{~nm}$ \\
\hline & & $1.5 \mathrm{~s}$ & 913 & $3.80 \mathrm{~nm}$ & & & $.6 \mathrm{mb}$ & I PRIN & 70.77 & 53 & eP \\
\hline OLY & & 58.07 & 60 & eP & 22 & 54.20 & -1.4 & I MNT & 70.77 & 48 & eP \\
\hline POW & & 58.28 & 59 & eP & 22 & 54.50 & -2.6 & 1 & $1.5 \mathrm{~s}$ & 780 & $0.00 \mathrm{~nm}$ \\
\hline TSK & & 58.41 & 301 & eP & 22 & 57.90 & -0.1 & I ALE & 71.27 & 8 & eP \\
\hline KYS & & 58.42 & 300 & eP & 23 & 03.90 & 5.8 & 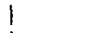 & $0.7 \mathrm{~s}$ & 205 & $5.00 \mathrm{~nm}$ \\
\hline$F \vee M$ & & 58.84 & 57 & iP & 23 & 00.20 & -0.8 & I TUP & 71.35 & 322 & ePc \\
\hline & & $2.0 \mathrm{~s}$ & 2230 & $.00 \mathrm{~nm}$ & & & $.9 \mathrm{mb}$ & i & $2.0 \mathrm{~s}$ & 810 & $0.00 \mathrm{~nm}$ \\
\hline SRY & & 59.11 & 300 & eP & 23 & 02.80 & -0.2 & | FRB & 71.41 & 28 & eP \\
\hline OYM & & 59.15 & 300 & eP & 23 & 04.60 & 1.3 & $1 \mathrm{MSZ}$ & 72.02 & 207 & eP \\
\hline DDR & & 59.16 & 301 & eP & 23 & 03.90 & 0.5 & 1 & & & $(p P)$ \\
\hline$N \mid$ I & & 59.21 & 303 & eP & 23 & 06.00 & 2.5 & BNH & 72.57 & 48 & eP \\
\hline LST & & 59.44 & 59 & eP & 23 & 05.00 & -0.2 & I DL2 & 72.69 & 306 & iPc \\
\hline MBC & & 59.76 & 9 & ePc & 23 & 05.40 & -1.4 & I & & & PP \\
\hline & & $1.3 \mathrm{~s}$ & 1300 & $.00 \mathrm{~nm}$ & & & $.9 \mathrm{mb}$ & 1 & & & $\mathrm{~s}$ \\
\hline ELC & & 59.81 & 58 & eP & 23 & 07.20 & -0.5 & 1 & & & sS \\
\hline MAT & & 59.93 & 302 & iPct & 23 & 08.90 & 0.3 & RIV & 73.42 & 224 & eP \\
\hline & & $0.3 \mathrm{~s}$ & 403 & $3.00 \mathrm{~nm}$ & & & $.0 \mathrm{mb}$ & $\mathrm{SCH}$ & 73.60 & 37 & eP \\
\hline & $z$ & $20 \mathrm{~s}$ & 19 & $9.00 \mathrm{um}$ & & & $2 M s z$ & 1 & $1.8 \mathrm{~s}$ & 834 & $4.00 \mathrm{~nm}$ \\
\hline & & & & es & 31 & 22.00 & & $1 B O D$ & 73.96 & 326 & iPc \\
\hline LHC & & 59.97 & 45 & eP & 23 & 06.00 & -2.6 & 1 & $1.4 \mathrm{~s}$ & 1000 & $0.00 \mathrm{~nm}$ \\
\hline & & $1.8 \mathrm{~s}$ & 2500 & $.00 \mathrm{~nm}$ & & & $\theta m b$ & $M I M$ & 73.99 & 47 & eP \\
\hline PWLA & & 60.88 & 60 & eP & 23 & 14.00 & -1.1 & CBM & 74.28 & 45 & eP \\
\hline ALOT & & 60.91 & 246 & eP & 23 & 19.00 & 3.5 & I SSE & 74.72 & 298 & i P+ \\
\hline CRZ & & 61.55 & 210 & $\mathrm{P}$ & 23 & 21.80 & 2.4 & d & $6.0 \mathrm{~s}$ & & $3.90 \mathrm{~nm}$ \\
\hline OSK & & 62.14 & 300 & P+ & 23 & 25.00 & 1.3 & 1 & $24 \mathrm{~s}$ & 29 & $9.60 \mathrm{um}$ \\
\hline & & & & $\mathrm{s}$ & 31 & 55.00 & & 1 & $18 \mathrm{~s}$ & & $6.86 \mathrm{um}$ \\
\hline LMG & & 62.27 & 48 & eP & 23 & 7.00 & 4.1 & 1 & $22 \mathrm{~s}$ & 22 & $2.00 \mathrm{um}$ \\
\hline LAT & & 62.29 & 251 & eP & 23 & 8.00 & 3.2 & 1 & & & i \\
\hline KRP & & 63.24 & 205 & $P$ & 23 & 29.00 & -1.6 & 1 & & & $i$ \\
\hline & & & & $(P P)$ & 25 & 37.00 & & 1 & & & $p P$ \\
\hline PMG & & 63.38 & 248 & ePt & 23 & 31.00 & -0.9 & i & & & SP \\
\hline & $z$ & $19 \mathrm{~s}$ & & $.00 \mathrm{um}$ & & & $.6 \mathrm{Msz}$ & 1 & & & PP \\
\hline SSS & & 63.44 & 84 & eP & 23 & 32.80 & 0.3 & 1 & & & $s$ \\
\hline$T K L$ & & 64.31 & 59 & $e(P)$ & 23 & 36.30 & -1.6 & 1150 & 75.10 & 241 & eP \\
\hline SHK & & 64.59 & 300 & eP & 23 & 41.50 & 1.7 & I YOU & 75.54 & 225 & eP \\
\hline OLA & & 65.37 & 51 & $\mathbf{P}$ & 23 & 45.30 & 0.7 & I CMS & 75.71 & 229 & eP \\
\hline
\end{tabular}


$16 d \quad 16 h$

\begin{tabular}{|c|c|c|c|c|c|c|c|c|c|c|c|}
\hline & & & & EPPP & 30 & 16.00 & & 1 & & & \\
\hline & & & & is & 35 & 24.00 & & $i$ & & & \\
\hline & & & & iSKS & 35 & 28.00 & & i & & & \\
\hline & & & & ePS & 36 & 10.00 & & i & & & \\
\hline & & & & ess & 40 & 44.00 & & 12080 & & 92.86 & 106 \\
\hline В 10 & & 80.93 & 310 & $\mathrm{iPc}$ & 25 & 17.00 & 0.9 & 1 & & $2.2 \mathrm{~s}$ & 436 \\
\hline & & & & PP & 28 & 24.50 & & I KIR & & 92.93 & 2 \\
\hline & & & & es & 35 & 19.50 & & I LPB & & 92.97 & 106 \\
\hline BFD & & 81.69 & 226 & eP & 25 & 18.00 & 1.3 & 1 & & $2.0 \mathrm{~s}$ & 706 \\
\hline ASPA & & 81.09 & 241 & eP & 25 & 17.00 & -0.1 & I & $z$ & $18 \mathrm{~s}$ & \\
\hline LGN & & 81.26 & 82 & $i(P)$ & 25 & 19.00 & 1.0 & 1 & & & \\
\hline UAV & & 81.85 & 84 & eP & 25 & 24.00 & 2.6 & 1 & & & \\
\hline ZAK & & 82.18 & 321 & eP & 25 & 13.40 & -8.8 & 1 & & & \\
\hline & & & 770 & $3.00 \mathrm{~nm}$ & & 6 & $.5 \mathrm{mb}$ & I APA & & 93.03 & 357 \\
\hline & & & & ePP & 28 & 27.08 & & I & & $1.5 \mathrm{~s}$ & 190 \\
\hline & & & & is & 35 & 40.00 & & 1 & $z$ & $21 \mathrm{~s}$ & 23 \\
\hline & & & & ess & 41 & 02.00 & & 1 & & & \\
\hline SDV & & 82.25 & 83 & iPc & 25 & 25.10 & 1.6 & 1 & & & \\
\hline & & $0.7 \mathrm{~s}$ & & 7. $10 \mathrm{~nm}$ & & & $8 \mathrm{mb}$ & 1 & & & \\
\hline ADE & & 82.61 & 229 & $i P c$ & 25 & 30.50 & 5.8 & 1 & & & \\
\hline & & $1.0 \mathrm{~s}$ & 124 & $1.00 \mathrm{~nm}$ & & & $.8 \mathrm{mb}$ & I ANT & & 93.29 & 114 \\
\hline TOV & & 82.75 & 82 & $e P$ & 25 & 24.00 & -1.9 & i & & & \\
\hline HKC & & 83.16 & 291 & iP & 25 & 31.50 & 3.7 & 1 & & & \\
\hline & & & & is & 36 & 16.00 & & 1500 & & 93.44 & 359 \\
\hline LM2 & & 83.29 & 105 & iP & 25 & 36.50 & 2.0 & 1 & & & \\
\hline NNA & & 83.45 & 105 & iP & 25 & 29.70 & e. 3 & 1 & & & \\
\hline & & $2.0 \mathrm{~s}$ & 1488 & $0.00 \mathrm{~nm}$ & & & $.8 \mathrm{mb}$ & I TRT & & 94.31 & 263 \\
\hline SJG & & 83.51 & 73 & ip & 25 & 28.70 & -1.0 & I WMO & & 94.61 & 320 \\
\hline & & $1.0 s$ & 178 & $3.00 \mathrm{~nm}$ & & 6 & $.2 \mathrm{mb}$ & 1 & & & \\
\hline & $z$ & $20 \mathrm{~s}$ & 18 & $3.40 \mathrm{um}$ & & 6 & $.5 \mathrm{Msz}$ & I SEM & & 94.94 & 328 \\
\hline GZH & & 83.64 & 292 & $\mathrm{PC}$ & 25 & 31.50 & 1.2 & 1 & & $5.0 \mathrm{~s}$ & 1710 \\
\hline & & & & $P P$ & 28 & 42.50 & & i & & & \\
\hline & & & & es & 35 & 46.50 & & i & & & \\
\hline XAN & & 83.83 & 304 & $P C$ & 25 & 31.00 & -0.2 & I TLL & & 95.11 & 120 \\
\hline STJ & & 84.11 & 42 & eP & 25 & 32.50 & 0.3 & I ROCH & & 95.87 & 123 \\
\hline CAR & & 85.19 & 81 & eP & 25 & 39.46 & 1.1 & I TACH & & 96.15 & 123 \\
\hline & & $0.6 \mathrm{~s}$ & 37 & $7.39 \mathrm{~nm}$ & & & $.8 \mathrm{mb}$ & I PEL & & 96.19 & 123 \\
\hline & $z$ & $20 \mathrm{~s}$ & 21 & $1.10 \mathrm{um}$ & & & $.5 \mathrm{Msz}$ & I NAU & & 96.40 & 248 \\
\hline & $\mathbf{N}$ & $20 \mathrm{~s}$ & 16 & $5.00 \mathrm{um}$ & & & & $\mathrm{I} \mathrm{PCH}$ & & 96.48 & 123 \\
\hline & $\mathbf{E}$ & $20 \mathrm{~s}$ & 25 & $5.00 \mathrm{um}$ & & & & I $\mathrm{FCH}$ & & 96.56 & 123 \\
\hline KKM & & 86.45 & 275 & ePd & 25 & 48.00 & 3.4 & I KJF & & 96.61 & 359 \\
\hline UER & & 87.00 & 324 & iPC & 25 & 47.50 & 1.1 & 1 & & $1.1 \mathrm{~s}$ & 91 \\
\hline & & $1.9 \mathrm{~s}$ & 800 & $9.00 \mathrm{~nm}$ & & 6 & $.6 \mathrm{mb}$ & 1 & $z$ & $20 \mathrm{~s}$ & 20 \\
\hline & & & & is & 36 & 16.60 & & i & & & \\
\hline L.ZH & & 87.02 & 307 & iPct & 25 & 48.00 & 0.9 & 1 & & & \\
\hline & & $5.0 \mathrm{~s}$ & 5870 & $9.80 \mathrm{~nm}$ & & & $.1 \mathrm{mb} X$ & i & & & \\
\hline & $N$ & $13 \mathrm{~s}$ & 19 & 7. 50 um & & & & i & & & \\
\hline & & & & $\mathrm{pP}$ & 25 & 56.00 & $25 \mathrm{kmX}$ & I & & & \\
\hline & & & & ePP & 29 & 03.00 & & I & & & \\
\hline & & & & S & 36 & 16.00 & & I YJA & & 97.08 & 111 \\
\hline & & & & sS & 36 & 36.00 & & I PCT & & 97.14 & 288 \\
\hline & & & & SS & 41 & 53.00 & & | & & & \\
\hline uKS & & 87.09 & 263 & $\mathrm{iPc}$ & 25 & 49.50 & 2.0 & $M D Z$ & & 97.56 & 122 \\
\hline WBN & & 88.17 & 242 & eP & 25 & 56.00 & 3.5 & I $\mathrm{CHG}$ & & 97.73 & 293 \\
\hline PAG & & 88.22 & 74 & eP & 25 & 54.00 & 1.8 & 1 & & $1.0 \mathrm{~s}$ & 16 \\
\hline GYA & & 88.25 & 297 & $\mathrm{PC}$ & 25 & 54.00 & 0.9 & 1 & & & \\
\hline & & & & sP & 26 & 20.00 & & I SLA & & 97.87 & 113 \\
\hline & & & & $s$ & 36 & 24.00 & & $\mid$ NST & & 97.90 & 290 \\
\hline & & & & PS & 37 & 08.00 & & SUF & & 98.12 & 359 \\
\hline SEG & & 88.26 & 73 & eP & 25 & 51.00 & -2.1 & 1 & & $0.9 \mathrm{~s}$ & 10 \\
\hline MGG & & 88.59 & 74 & eP & 25 & 55.00 & 0.3 & I DRV & & 98.14 & 201 \\
\hline GTA & & 88.67 & 311 & $i P_{c}$ & 25 & 55.70 & 0.8 & I SVE & & 98.24 & 341 \\
\hline & & & & PP & 29 & 26.40 & & 1 & & $1.9 \mathrm{~s}$ & 180 \\
\hline & & & & is & 36 & 28.00 & & I & & & \\
\hline & & & & PS & 37 & 47.00 & & 1 & & & \\
\hline $\mathrm{CD} 2$ & & 89.02 & 302 & iPC & 25 & 57.00 & 0.4 & BDT & & 98.29 & 292 \\
\hline & & & & $S$ & 36 & 28.50 & & $i$ & & $1.8 \mathrm{~s}$ & 201 \\
\hline AKU & & 89.22 & 16 & iP & 26 & 03.50 & 6.7 & LEM & & 98.75 & 266 \\
\hline & & $1.4 \mathrm{~s}$ & 270 & $.08 \mathrm{~nm}$ & & & $.3 m b$ & 1 & & & \\
\hline REY & & 89.31 & 19 & eP & 26 & 03.10 & 5.8 & I BER & & 98.95 & 10 \\
\hline ARE & & 89.88 & 107 & eP & 26 & 03.00 & 1.9 & I NB2 & & 99.10 & 7 \\
\hline ELT & & 90.25 & 328 & IPC & 26 & 02.80 & 1.0 & 1 & & $1.0 \mathrm{~s}$ & 12 \\
\hline & & $2.0 \mathrm{~s}$ & 830 & $.00 \mathrm{~nm}$ & & & $6 \mathrm{mb}$ & ARU & & 99.20 & 342 \\
\hline & & & & iSKS & 36 & 34.00 & & 1 & & $1.8 \mathrm{~s}$ & 340 \\
\hline TRN & & 90.28 & 79 & eP & 26 & 02.00 & -0.6 & 1 & $z$ & $21 \mathrm{~s}$ & 29 \\
\hline NVS & & 90.74 & 330 & iPc & 26 & 05.50 & 1.5 & 1 & N & $23 \mathrm{~s}$ & 17 \\
\hline & & $1.6 \mathrm{~s}$ & 690 & $.00 \mathrm{~nm}$ & & & $.7 \mathrm{mb}$ & 1 & E & $17 \mathrm{~s}$ & 14 \\
\hline KEV & & 91.04 & 359 & eP & 26 & 06.00 & 0.8 & I & & & \\
\hline & & $1.3 \mathrm{~s}$ & 188 & $3.00 \mathrm{~nm}$ & & 6 & $.3 m b$ & 1 & & & \\
\hline & & & & $\mathrm{i}$ & 26 & 10.00 & & 1 & & & \\
\hline & & & & ePP & 29 & 44.00 & & i & & & \\
\hline & & & & es & 36 & 40.00 & & I LSA & & 99.38 & 306 \\
\hline & & & & ess & 43 & 88.00 & & I NNT & & 99.44 & 287 \\
\hline TRO & & 91.09 & 2 & eP & 26 & 86.00 & 0.6 & I HFS & & 00.23 & 5 \\
\hline KMI & & 92.03 & 297 & $\mathrm{PCt}$ & 26 & 12.00 & 1.1 & 1 & & $1.7 \mathrm{~s}$ & 144 \\
\hline & E & $16 \mathrm{~s}$ & & $.86 u m$ & 26 & 7000 & $07 \mathrm{~km} \times$ & $!$ & $z$ & $18 \mathrm{~s}$ & 21 \\
\hline & & & & $\mathrm{pP}$ & 26 & 38.00 & $97 \mathrm{kmX}$ & I KON & & 00.26 & 8 \\
\hline
\end{tabular}


$16 \mathrm{~d} \quad 16 \mathrm{~h}$

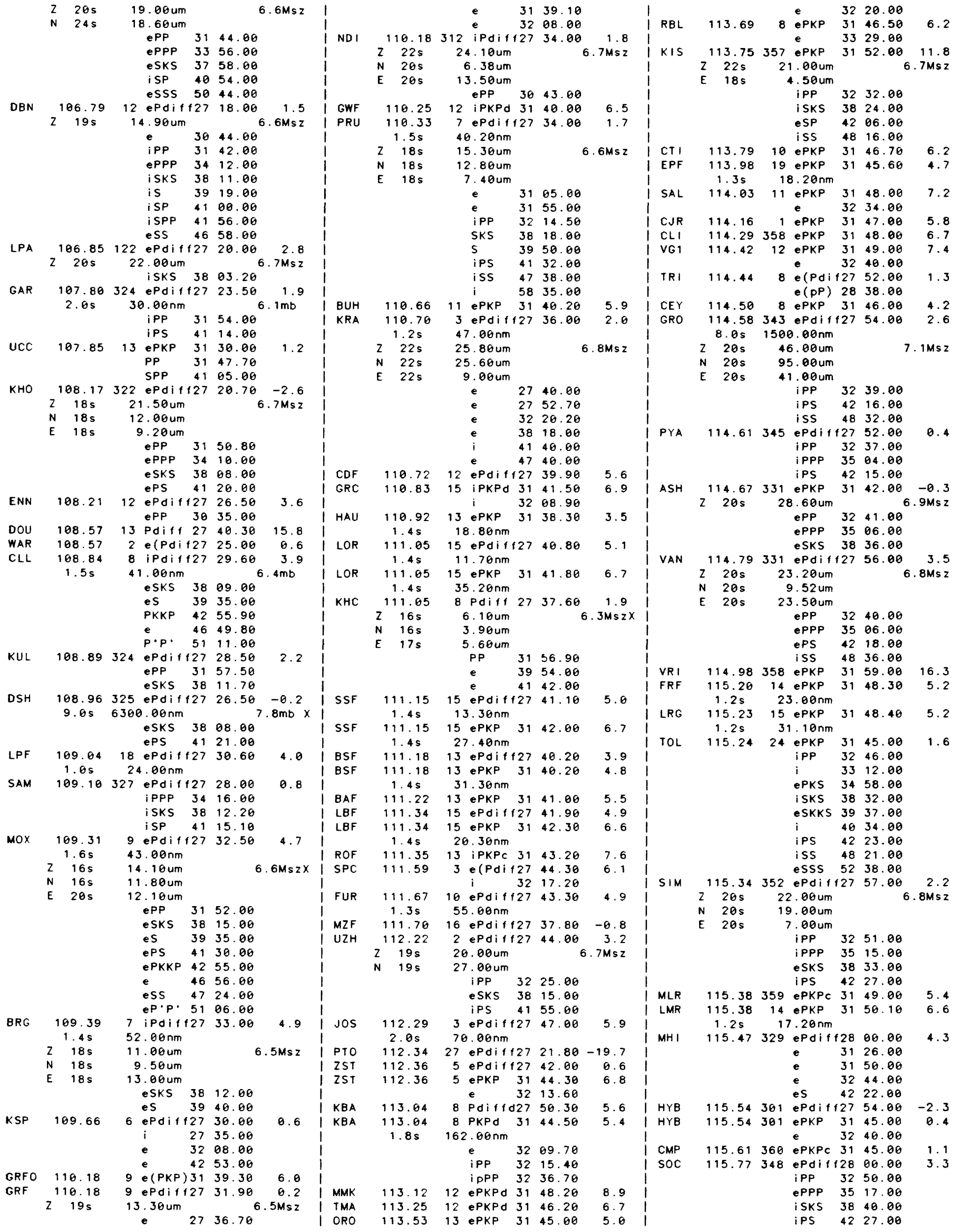




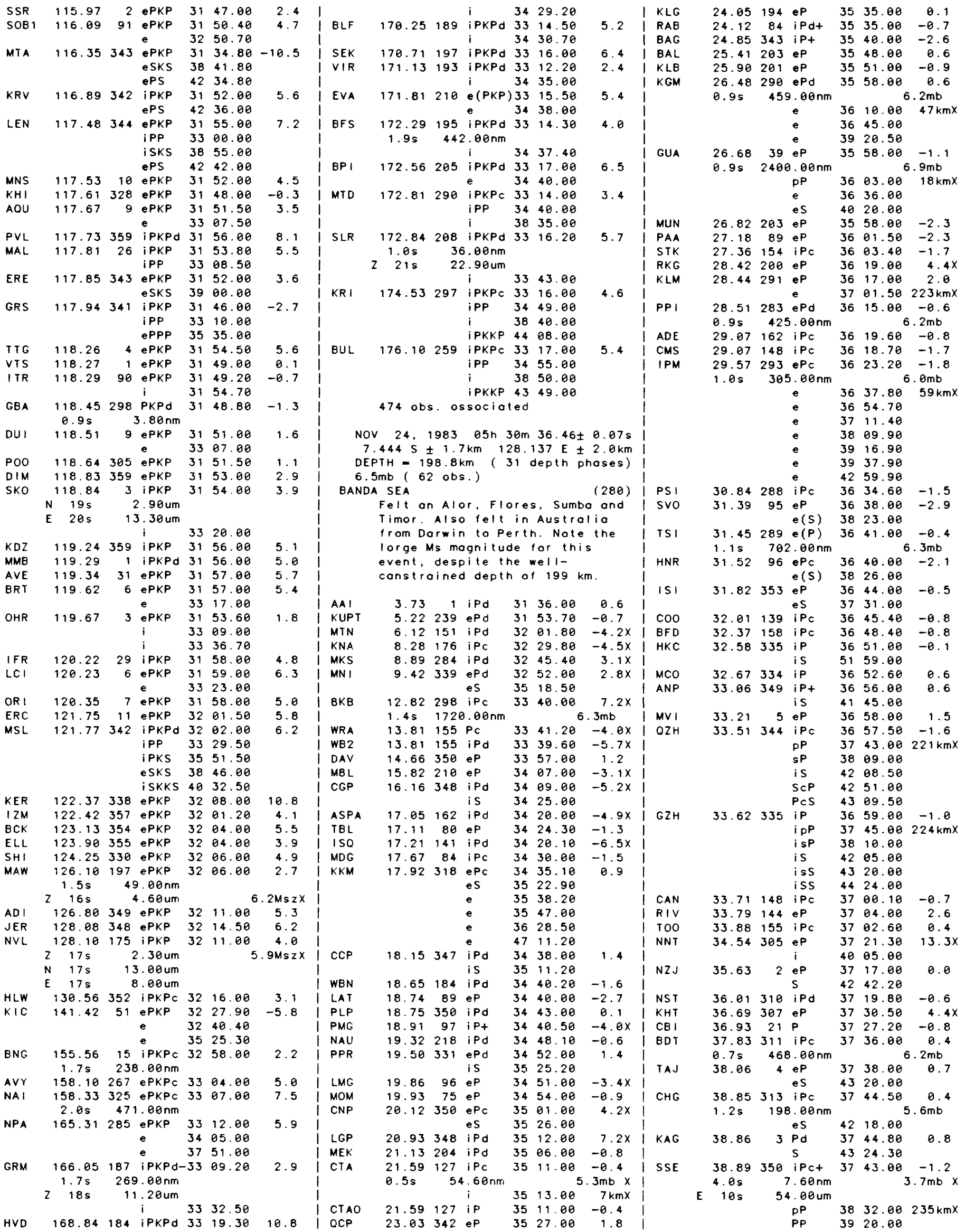




\begin{tabular}{|c|c|c|c|c|c|c|c|c|c|c|c|}
\hline & & & PCP & 39 & 40.00 & & I FUK & 43.93 & 9 & eP & 38 \\
\hline & & & $i$ & 40 & 21.00 & & I DDR & 44.44 & 13 & eP & 38 \\
\hline & & & SCP & 43 & 13.00 & & I СHO & 44.57 & 15 & eP & 38 \\
\hline & & & is & 43 & 32.00 & & $i$ & & & es & 44 \\
\hline & & & SS & 46 & 25.00 & & I TIA & 44.64 & 347 & $\mathrm{Pc}$ & 38 \\
\hline & & & $\mathbf{i}$ & 47 & 58.00 & & $i$ & & & PP & 40 \\
\hline MYZ & 39.26 & 4 & eP & 37 & 47.00 & -0.3 & 1 & & & $\mathrm{~S}$ & 44 \\
\hline & & & es & 43 & 21.00 & & I $\mathrm{CD} 2$ & 44.72 & 330 & IPC & 38 \\
\hline GYA & 39.62 & 329 & $\mathrm{Pc}$ & 37 & 50.00 & -0.5 & i & & & PP & 39 \\
\hline & & & s & 43 & 42.00 & & 1 & & & is & 44 \\
\hline NOU & 39.72 & 116 & iPc & 37 & 50.50 & -0.7 & I MAT & 44.76 & 12 & eP & 38 \\
\hline & & & is & 43 & 46.50 & & i & & & es & 44 \\
\hline FKJ & 39.92 & 1 & Po & 37 & 54.80 & 2.1 & I TSK & 44.86 & 14 & eP & 38 \\
\hline & & & es & 43 & 43.00 & & I XAN & 45.10 & 337 & iPc & 38 \\
\hline NOB & 39.95 & 5 & eP & 37 & 52.00 & -0.8 & 1 & & & SP & 39 \\
\hline & & & S & 43 & 47.00 & & 1 & & & $P P$ & 40 \\
\hline NGS & 39.99 & 2 & $P C$ & 37 & 53.20 & 0.0 & 1 & & & $\mathrm{~s}$ & 45 \\
\hline & & & $S$ & 43 & 48.40 & & I MIT & 45.10 & 14 & eP & 38 \\
\hline WHN & 40.02 & 341 & iPc & 37 & 53.00 & -0.5 & i & & & es & 44 \\
\hline & & & pP & 38 & 42.00 & $234 \mathrm{kmX}$ & I WAJ & 45.35 & 10 & eP & 38 \\
\hline & & & SP & 39 & 05.00 & & 1 & & & $\mathrm{~s}$ & 45 \\
\hline & & & PP & 39 & 35.00 & & I AIK & 46.20 & 11 & eP & 38 \\
\hline & & & s & 43 & 49.50 & & I FKS & 46.42 & 14 & eP & 38 \\
\hline & & & SCS & 47 & 32.00 & & 1 & & & es & 45 \\
\hline KUM & 40.10 & 3 & eP & 37 & 56.00 & 1.9 & $10 L 2$ & 46.51 & 353 & $P$ & 38 \\
\hline & & & es & 43 & 49.00 & & 1 & & & $\mathrm{pP}$ & 39 \\
\hline ASZ & 40.21 & 6 & eP & 37 & 54.00 & -1.0 & 1 & & & sP & 39 \\
\hline & & & e & 43 & 54.30 & & 1 & & & PP & 40 \\
\hline NJ2 & 40.26 & 348 & PC & 37 & 55.20 & -0.3 & 1 & & & S & 45 \\
\hline & & & PP & 38 & 43.00 & $227 \mathrm{kmx}$ & 1 & & & sS & 46 \\
\hline & & & $S$ & 43 & 51.00 & & I YAM & 46.86 & 13 & eP & 38 \\
\hline PVC & 40.44 & 109 & iPc & 37 & 56.20 & -0.9 & I SEN & 47.00 & 14 & eP & 38 \\
\hline$O 1 T$ & 40.59 & 4 & ePd & 38 & 00.00 & 1.9 & 1 & & & $S$ & 45 \\
\hline & & & es & 44 & 00.00 & & I ISN & 47.25 & 14 & eP & 38 \\
\hline KMI & 40.74 & 323 & iPct & 38 & 01.00 & 1.2 & 1 & & & es & 45 \\
\hline & $1.0 \mathrm{~s}$ & 48 & $.10 \mathrm{~nm}$ & & & $5.0 \mathrm{mb} \times$ & I TIY & 47.26 & 343 & $P C$ & 38 \\
\hline & $14 \mathrm{~s}$ & 108 & 3.00 um & & & & I & & & $p P$ & 39 \\
\hline & & & PP & 38 & 32.00 & $139 \mathrm{kmx}$ & 1 & & & PP & 40 \\
\hline & & & SP & 38 & 44.00 & & I & & & $s$ & 45 \\
\hline & & & PP & 39 & 37.00 & & I OFU & 47.96 & 14 & eP & 38 \\
\hline & & & $\mathrm{s}$ & 44 & 01.00 & & 1 & & & S & 45 \\
\hline & & & sS & 44 & 20.00 & & $|A K|$ & 48.21 & 12 & eP & 39 \\
\hline FKK & 40.85 & 3 & eP & 38 & 01.00 & 0.7 & 1 & & & es & 45 \\
\hline & & & es & 43 & 57.00 & & $|\mathrm{BJ}|$ & 48.52 & 348 & $P C$ & 39 \\
\hline MRT & 40.87 & 8 & eP & 38 & 01.00 & 0.5 & I & $2.0 \mathrm{~s}$ & 1850 & $0.00 \mathrm{~nm}$ & \\
\hline & & & es & 44 & 01.00 & & 1 & $19 s$ & 55 & 5.50 um & \\
\hline KOC & 41.09 & 7 & eP & 38 & 01.00 & -1.2 & I & & & PP & 39 \\
\hline & & & e & 38 & 50.00 & $233 \mathrm{kmx}$ & I & & & SP & 39 \\
\hline SHN & 41.26 & 4 & ePd & 38 & 03.00 & -0.6 & 1 & & & $P P$ & 40 \\
\hline & & & es & 44 & 05.00 & & 1 & & & PPP & 41 \\
\hline SHJ & 41.31 & 10 & $e^{P}$ & 38 & 04.00 & 0.0 & I & & & es & 45 \\
\hline & & & e & 38 & 47.00 & $291 \mathrm{~km}$ & 1 & & & sS & 46 \\
\hline 120 & 41.43 & 1 & eP & 38 & 03.00 & -2.0 & I & & & Ses & 48 \\
\hline & & & es & 44 & 11.00 & & I MIY & 48.57 & 14 & eP & 39 \\
\hline TKS & 41.73 & 8 & eP & 38 & 07.00 & -0.5 & 1 & & & $\mathrm{~S}$ & 45 \\
\hline & & & e & 44 & 14.00 & & I LZH & 48.95 & 334 & iPct & 39 \\
\hline HIR & 41.78 & 5 & eP & 38 & 07.00 & -0.8 & I & $14 \mathrm{~s}$ & 33 & $3.00 \mathrm{um}$ & \\
\hline & & & es & 44 & 14.00 & & 1 & & & $i p P$ & 39 \\
\hline TKM & 41.91 & 7 & eP & 38 & 08.00 & -0.9 & i & & & PP & 40 \\
\hline & & & es & 44 & 15.00 & & 1 & & & PPP & 42 \\
\hline SHK & 41.97 & 6 & ePc & 38 & 08.00 & -1.4 & 1 & & & PcS & 44 \\
\hline & & & es & 44 & 15.00 & & I & & & is & 45 \\
\hline OKA & 42.25 & 7 & ep & 38 & 12.00 & 0.3 & I CRZ & 48.95 & 130 & eP & 39 \\
\hline & & & e & 44 & 19.00 & & I NDF & 49.07 & 107 & ePc & 39 \\
\hline HMD & 42.27 & 5 & eP & 38 & 07.00 & $-4.8 x$ & I HAC & 49.30 & 13 & eP & 39 \\
\hline & & & e & 38 & 59.00 & $248 \mathrm{kmx}$ & i & & & es & 46 \\
\hline OSK & 42.43 & 9 & eP & 38 & 14.00 & 0.8 & VUN & 50.06 & 107 & $i P d$ & 39 \\
\hline & & & es & 44 & 16.00 & & I SVA & 50.06 & 107 & ePc & 39 \\
\hline OSA & 42.47 & 9 & $e^{P}$ & 38 & 15.00 & 1.5 & I HAK & 50.36 & 12 & eP & 39 \\
\hline$H I M$ & 42.51 & 8 & eP & 38 & 14.00 & 0.3 & 1 & & & e & 46 \\
\hline & & & es & 44 & 24.00 & & I HHC & 50.42 & 344 & IPC & 39 \\
\hline KYO & 42.83 & 9 & eP & 38 & 17.00 & 0.6 & 1 & & & PP & $4 i$ \\
\hline & & & es & 44 & 28.00 & & 1 & & & $\mathrm{~s}$ & 46 \\
\hline MTS & 42.92 & 6 & $P$ & 38 & 19.80 & 2.7 & I VLA & 50.44 & 4 & iPd & 39 \\
\hline TOT & 43.10 & 7 & eP & 38 & 19.00 & 0.4 & 1870 & 50.64 & 342 & $\mathrm{iPc}$ & 39 \\
\hline & & & e & 44 & 32.00 & & 1 & & & $p P$ & 40 \\
\hline $\mathrm{HIK}$ & 43.17 & 10 & eP & 38 & 20.00 & 0.9 & 1 & & & SP & 40 \\
\hline & & & es & 44 & 33.00 & & 1 & & & $s$ & 46 \\
\hline NAG & 43.19 & 11 & eP & 38 & 22.00 & 2.7 & I CN2 & 51.06 & 357 & iPc & 39 \\
\hline TYK & 43.21 & 8 & eP & 38 & 20.00 & 0.6 & 1 & & & $i p P$ & 40 \\
\hline TAT & 43.63 & 14 & eP & 38 & 36.00 & $13.2 x$ & 1 & & & $\mathbf{S P}$ & 40 \\
\hline 110 & 43.69 & 11 & eP & 38 & 26.00 & 2.6 & | & & & $\mathrm{s}$ & 46 \\
\hline & & & e & 38 & 41.00 & $58 \mathrm{kmx}$ & I SUT & 51.20 & 11 & ep & 39 \\
\hline SAI & 43.69 & 6 & eP & 38 & 23.00 & -0.3 & I & & & $s$ & 46 \\
\hline & & & s & 44 & 40.40 & & I LSA & 51.26 & 318 & iPe & 39 \\
\hline KYS & 43.90 & 14 & eP & 38 & 22.90 & -2.1 & 1 & & & $p P$ & 40 \\
\hline OYM & 43.90 & 13 & eP & 38 & 23.80 & -1.3 & 1 & & & $S$ & 46 \\
\hline
\end{tabular}


$24 d \quad 05 h$

\begin{tabular}{|c|c|c|c|c|c|c|c|c|}
\hline & $\mathrm{N}$ & $21 \mathrm{~s}$ & & $4.20 u m$ & & & & 1 \\
\hline & E & $21 \mathrm{~s}$ & & $4.00 \mathrm{um}$ & & & & 1 \\
\hline & & & & ePcP & 41 & 30.00 & & I NVS \\
\hline & & & & es & 49 & 30.00 & & $i$ \\
\hline & & & & eSP & 49 & 50.00 & & i \\
\hline & & & & escs & 50 & 30.00 & & SEY \\
\hline & & & & ess & 53 & 46.00 & & i \\
\hline KSH & & 67.07 & 318 & iPc & 41 & 11.00 & 0.4 & 1 \\
\hline & & & & $P P$ & 42 & 00.00 & $209 \mathrm{~km} x$ & 1 \\
\hline PRZ & & 67.20 & 322 & iPd & 41 & 11.80 & 0.4 & I \\
\hline & & $1.8 \mathrm{~s}$ & 3800 & $0.00 \mathrm{~nm}$ & & & $6.8 \mathrm{mb}$ & \\
\hline & & & & es & 49 & 54.00 & & SBA \\
\hline & & & & eScS & 50 & 52.00 & & ! \\
\hline NRN & & 68.11 & 320 & iPc & 41 & 16.00 & -1.3 & SAM \\
\hline TLG & & 68.26 & 322 & $\mathrm{iPc}$ & 41 & 18.20 & 0.4 & 1 \\
\hline & & $1.7 \mathrm{~s}$ & 1070 & $0.00 \mathrm{~nm}$ & & & $5.3 \mathrm{mb}$ & i \\
\hline & & & & ipP & 42 & 08.00 & $212 \mathrm{kmx}$ & I MAW \\
\hline & & & & ePP & 43 & 54.00 & & I ADK \\
\hline & & & & is & 50 & 07.00 & & I KHI \\
\hline AAA & & $\begin{array}{l}68.53 \\
8.0 \mathrm{~s}\end{array}$ & $\begin{array}{r}322 \\
47\end{array}$ & $\begin{array}{l}\text { iPc } \\
.00 \mathrm{~nm}\end{array}$ & 41 & $18.80_{4}$ & $\begin{array}{l}-0.7 \\
4 m b x\end{array}$ & MHI \\
\hline KHO & & 69.13 & 315 & $i P_{c}$ & 41 & 22.00 & -1.4 & HON \\
\hline & & $6.0 \mathrm{~s}$ & $* * * *$ & $* * * n \mathrm{~nm}$ & & & $.2 \mathrm{mb} \times$ & I KIP \\
\hline & $z$ & $18 \mathrm{~s}$ & 75 & $5.00 \mathrm{um}$ & & & $.0 \mathrm{Msz}$ & I AVY \\
\hline & N & $18 \mathrm{~s}$ & & $3.40 \mathrm{um}$ & & & & I TIK \\
\hline & $E$ & $18 \mathrm{~s}$ & & $5.60 u m$ & & & & 1 \\
\hline & & & & ePP & 43 & 53.00 & & i \\
\hline & & & & es & 50 & 14.60 & & i \\
\hline YAK & & 69.26 & 1 & $i P c$ & 41 & 22.30 & -1.1 & i \\
\hline & & $0.8 \mathrm{~s}$ & 3790 & $0.00 \mathrm{~nm}$ & & & $.2 \mathrm{mb}$ & ASH \\
\hline & & & & ipP & 42 & 08.20 & $193 \mathrm{~km}$ & \\
\hline & & & & ePP & 44 & 05.20 & & 1 \\
\hline & & & & ePPP & 45 & 43.20 & & 1 \\
\hline & & & & is & 50 & 17.20 & & 1 \\
\hline & & & & eSP & 50 & 31.00 & & i \\
\hline & & & & ises & 51 & 05.60 & & I VAN \\
\hline & & & & ess & 54 & 43.20 & & 1 \\
\hline$M G D$ & & 69.69 & 12 & $i P c$ & 41 & 25.20 & -0.9 & 1 \\
\hline & & $0.8 \mathrm{~s}$ & 1560 & $.00 \mathrm{~nm}$ & & 6 & $5.8 \mathrm{mb}$ & | TBI \\
\hline & & & & iPCP & 41 & 45.00 & & I \\
\hline & & & & $i p P$ & 42 & 10.00 & $188 \mathrm{kmX}$ & 1 \\
\hline & & & & isp & 42 & 32.00 & & I NKI \\
\hline & & & & IPP & 44 & 08.00 & & I AFR \\
\hline & & & & is & 50 & 20.20 & & $i$ \\
\hline FRU & & 69.72 & 321 & iPc & 41 & 26.80 & 0.8 & I PAE \\
\hline & & $2.4 \mathrm{~s}$ & 6900 & $.00 \mathrm{~nm}$ & & & $.0 \mathrm{mb}$ & i \\
\hline & $z$ & $25 \mathrm{~s}$ & 64 & $4.50 \mathrm{um}$ & & & 5. $8 \mathrm{MszX}$ & I PPT \\
\hline & $\mathrm{N}$ & $24 \mathrm{~s}$ & 76 & $5.78 u m$ & & & & i \\
\hline & E & $24 \mathrm{~s}$ & & $1.70 \mathrm{um}$ & & & & I PPN \\
\hline & & & & ePCP & 41 & 52.30 & & i \\
\hline & & & & epP & 42 & 13.00 & $195 \mathrm{~km}$ & I TVO \\
\hline & & & & ePPP & 45 & 48.00 & & i \\
\hline & & & & es & 50 & 23.00 & & I KAT \\
\hline & & & & escs & 51 & 12.00 & & 1 \\
\hline & & & & ess & 54 & 48.00 & & 1 \\
\hline ELT & & 70.07 & 335 & iPc & 41 & 27.20 & -1.3 & i \\
\hline & & $1.0 \mathrm{~s}$ & 2910 & $.00 \mathrm{~nm}$ & & & $.0 \mathrm{mb}$ & 1 \\
\hline & & & & is & 50 & 23.00 & & 1 \\
\hline ANR & & 70.12 & 318 & iPc & 41 & 28.40 & -0.8 & SHI \\
\hline & & $1.6 \mathrm{~s}$ & 7100 & $.00 \mathrm{~nm}$ & & 7 & $.1 \mathrm{mb}$ & NRI \\
\hline & & & & is & 50 & 28.20 & & 1 \\
\hline KUL & & 70.58 & 314 & iPc & 41 & 30.10 & -1.9 & 1 \\
\hline & & $7.8 \mathrm{~s}$ & 8000 & $.00 \mathrm{~nm}$ & & & $.5 \mathrm{mb} \times$ & \\
\hline & & & & ePP & 44 & 04.30 & & 1 \\
\hline & & & & IPS & 51 & 23.80 & & i \\
\hline GAR & & 70.68 & 316 & $i P_{c}$ & 41 & 31.50 & -1.2 & I PMO \\
\hline & & $1.2 \mathrm{~s}$ & & $.00 \mathrm{~nm}$ & & & $3.3 \mathrm{mb} \times$ & 1 \\
\hline & & & & iPcP & 41 & 45.10 & & $i$ \\
\hline & & & & iPPP & 45 & 52.30 & & I VAH \\
\hline & & & & is & 50 & 32.30 & & i \\
\hline & & & & iscs & 51 & 18.30 & & i \\
\hline & & & & iss & 55 & 10.00 & & I TPT \\
\hline RAR & & 70.68 & 110 & $\mathbf{P}$ & 41 & 32.00 & -0.9 & i \\
\hline & & & & $s$ & 50 & 36.00 & & i \\
\hline SEM & & 70.94 & 330 & $i P c$ & 41 & 33.40 & -0.4 & I SPA \\
\hline & & $7.8 \mathrm{~s}$ & $* * * * *$ & $* * * n m$ & & & $1 \mathrm{mb} \times$ & $i$ \\
\hline & & & & ipP & 42 & 21.60 & $203 \mathrm{~km}$ & i \\
\hline & & & & is & 50 & 35.00 & & I RUV \\
\hline SMY & & 71.52 & 27 & eP & 41 & 38.60 & 1.4 & 1 \\
\hline DSH & & 71.56 & 315 & i Pc & 41 & 37.00 & -0.9 & i \\
\hline & $z$ & $21 \mathrm{~s}$ & 114 & $.00 \mathrm{um}$ & & & $.1 \mathrm{Msz}$ & TEH \\
\hline TAS & & 72.43 & 317 & $\mathrm{iPc}$ & 41 & 42.30 & -0.6 & I ILT \\
\hline & & $1.4 \mathrm{~s}$ & $* * * *$ & $* * * n m$ & & & $.4 \mathrm{mb}$ & 1 \\
\hline & $z$ & $18 \mathrm{~s}$ & & $.00 u m$ & & & $.8 M s z$ & i \\
\hline & & & & ePP & 44 & 28.30 & & | \\
\hline & & & & es & 50 & 57.00 & & SVE \\
\hline
\end{tabular}

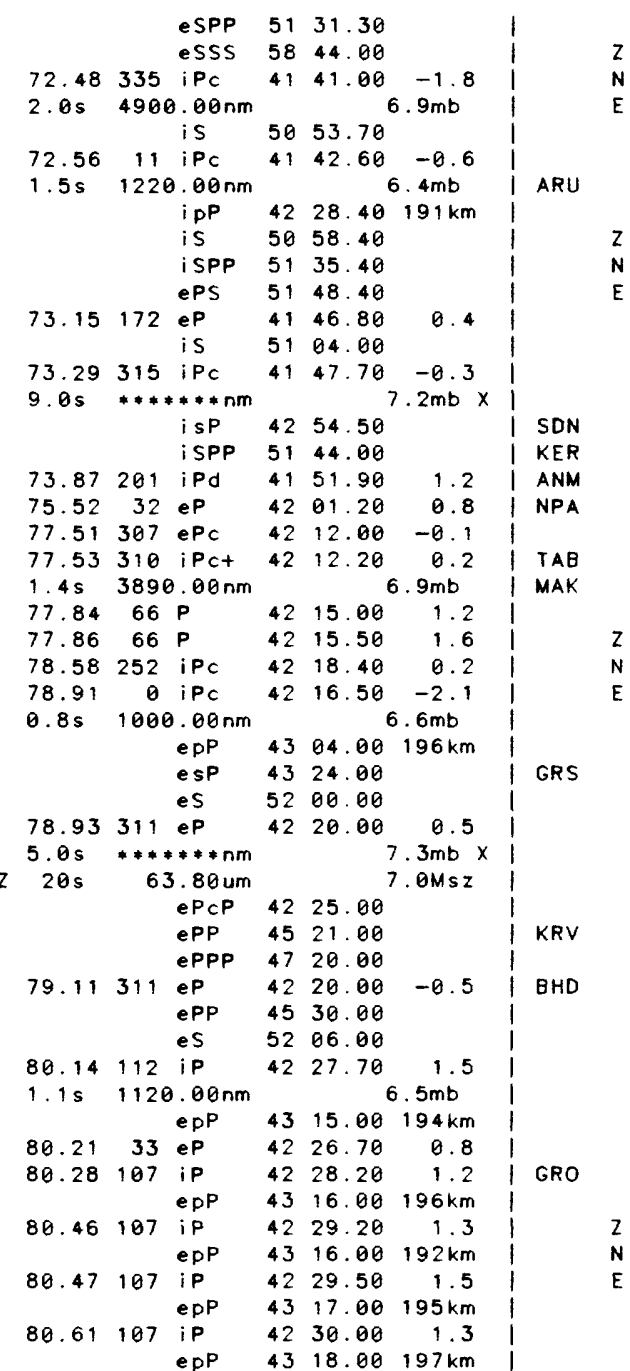

$80.76107 \mathrm{epP}$

$1.2 \mathrm{~s} \quad 785.00 \mathrm{~nm}$

$80.86312 \quad \mathrm{PC} \quad 42 \quad 31.50 \quad 1.8$ TTA

$8.0 \mathrm{~s} * * * * * * * \mathrm{~nm} \quad 7.3 \mathrm{mb} \times$ I MSL

epP $4322.50210 \mathrm{kmx}$

ePP $45 \quad 30.00$

eS 5226.50

ESPP 5327.00

$\begin{array}{lllll}81.30301 & P & 42 & 31.00 & -1.4\end{array}$

$\begin{array}{ccccc}81.47347 \mathrm{iPc} & 4231.20 \quad-1.0 \\ 0.9 \mathrm{~s} & 580.00 \mathrm{~nm} & 6.3 \mathrm{mb}\end{array}$

$\begin{array}{ccc}0.9 \mathrm{~s} & 580.00 \mathrm{~nm} & 6.3 \mathrm{mb} \\ & \mathrm{epP} & 43 \quad 14.00^{6} 173 \mathrm{kmx}\end{array}$

eS 5229.00

ESPP 5333.00

eSS 5743.00

\begin{tabular}{cc|c|c}
$1.2 \mathrm{~s}$ & $1570.00 \mathrm{~nm}$ & $6.6 \mathrm{mb}$ & BKR
\end{tabular}

$82.54104 \mathrm{epP} \quad 4328.00200 \mathrm{~km}$

$\begin{array}{lllll}82.54 & 104 & \text { iP } & 42 & 40.00 \quad 1.2 \\ 1.2 \mathrm{~s} & 685.00 \mathrm{~nm} & 6.3 \mathrm{mb}\end{array}$

epP $4329.00201 \mathrm{~km}$

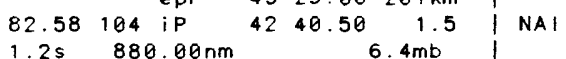

$\begin{array}{ccc}1.2 \mathrm{~s} \quad 880.00 \mathrm{~nm} & 6.4 \mathrm{mb} \\ & \mathrm{epP} & 4330.00203 \mathrm{~km}\end{array}$

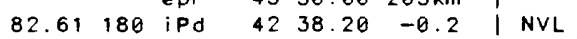

$1.0 \mathrm{~s} \quad 70.00 \mathrm{~nm} \quad 5.3 \mathrm{mb} \mathrm{x}$

$z 18 \mathrm{~s} 50.90 \mathrm{um}$

82.78104 iP $4241.20 .9 \mathrm{M}^{62}$

$1.2 \mathrm{~s} \quad 490.00 \mathrm{~nm} \quad 6.1 \mathrm{mb}$ \begin{tabular}{llll|l} 
ePP $43 \quad 31.00204 \mathrm{~km}$ & PYA
\end{tabular}

$\begin{array}{lllll}83.68 & 307 \mathrm{eP} & 42 & 48.00 & 3.6 \mathrm{X}\end{array}$

$\begin{array}{llllll}83.92 & 18 & \mathrm{iPc} & 42 & 44.00 & -0.8\end{array}$

$1.2 \mathrm{~s} \quad 1500.00 \mathrm{~nm}$

ipP $43 \quad 34.00204 \mathrm{~km}$

iPPP 4759.00

$84.19329 \mathrm{iPc} \quad 42 \quad 46.00 \quad-0.3$

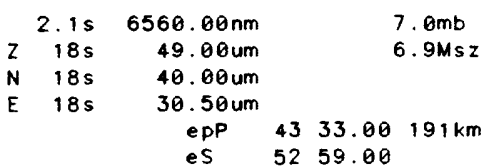

$\begin{array}{llllll}85.08 & 328 \mathrm{iPc} & 42 & 50.00 & -0.7\end{array}$

$\begin{array}{rrr}8.0 \mathrm{~s} & * * * * * * \mathrm{~nm} & 7.0 \mathrm{mb} X \\ 22 \mathrm{~s} & 44.00 \mathrm{um} & 6.8 \mathrm{Msz}\end{array}$

N $18 \mathrm{~s} \quad 17.00 \mathrm{um}$

E $18 \mathrm{~s} \quad 32.00 \mathrm{um}$

EPP $43 \quad 38.50 \quad 197 \mathrm{~km}$

ESP 4358.00

$\begin{array}{lll}\text { ePP } & 46 \quad 14.00\end{array}$

ePPP $48 \quad 02.00$

$\begin{array}{lllll}85.68 & 33 \mathrm{eP} & 42 & 53.10 & -0.6\end{array}$

86.81305 eP $\quad 4253.00 \quad-6.9 x$

$\begin{array}{llllll}86.86 & 23 & \text { eP } & 42 & 58.90 & -0.4\end{array}$

$87.02 \quad 255$ iPd $4303.00 \quad 1.9$

$88.14309 \mathrm{iPt} \quad 4307.00 \quad 0.8$

$88.28314 \mathrm{iPc} \quad 4307.50 \quad 1.0$

$6.0 \mathrm{~s} \quad * * * * * * \mathrm{~nm} \quad 7.3 \mathrm{mb} x$

$Z 17 \mathrm{~s} \quad 43.00 \mathrm{um} \quad 6.9 \mathrm{MszX}$

N $21 \mathrm{~s} \quad 30.00 \mathrm{um}$

$15 \mathrm{~s} \quad 34.00 \mathrm{um}$

iPP $46 \quad 35.00$

is $\quad 5339.00$

$\begin{array}{lllllll}88.40 & 310 \mathrm{iPC} & 43 & 07.20 & -0.3\end{array}$

5. Os *******nm $7.0 \mathrm{mb} x$ ipP $4359.00210 \mathrm{kmX}$ iPP $46 \quad 44.00$

eSKS $53 \quad 19.00$

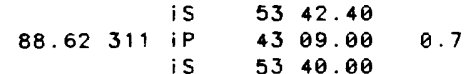

$88.85304 \mathrm{ePd} \quad 43 \quad 14.00 \quad 4.6 \mathrm{X}$

ePP $46 \quad 49.00$

e $\quad 4926.50$

is 5345.50

ePS $54 \quad 48.00$

i $\quad 5505.00$

i $\quad 5615.00$

$\begin{array}{llllll}89.60 & 314 & \mathrm{iPc} & 43 & 12.00 & -0.7\end{array}$

$1.5 \mathrm{~s} 2300.00 \mathrm{~nm} \quad 6.9 \mathrm{mb}$

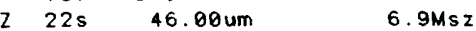

N $23 \mathrm{~s} \quad 52.00 \mathrm{um}$

E $16 \mathrm{~s} \quad 6.50 \mathrm{um}$

ipP $4402.00 \quad 202 \mathrm{~km}$

is 5342.00

iSP 5450.00

$\begin{array}{llllll}89.88 & 28 \text { eP } & 43 & 14.00 & 0.3\end{array}$

$\begin{array}{llllll}89.91310 \text { iPC } \quad 4313.50 & -0.9\end{array}$

$\begin{array}{lllll}90.20 \quad 26 \mathrm{eP} & 43 & 14.70 & -0.5\end{array}$

90.35306 ePd $43 \quad 16.00 \quad-0.4$

i $\quad 4320.00 \quad 12 \mathrm{kmX}$

i 4409.00

$\begin{array}{llll}\text { iPPP } \quad 46 \quad 16.00 & 00\end{array}$

is $\quad 5005.50$

iSS 5326.50

iSSS 5456.50

LEN $\quad 90.50 \quad 311$ iPC $43 \quad 19.00 \quad 1.8$

$2.0 \mathrm{~s} \quad 900.00 \mathrm{~nm}$

IPP 4703.00

iPPP 4900.00

90.53279 iP $43 \quad 19.50 \quad 1.5$

$\begin{array}{lllll}90.61 & 32 \mathrm{eP} & 43 & 17.20 & 0.2\end{array}$

$90.90312 \mathrm{iPc} \quad 4320.006 .6 \mathrm{mb}$

\begin{tabular}{ccc}
$1.6 \mathrm{~s} \quad 1100.00 \mathrm{~nm}$ & \multicolumn{1}{c}{$\begin{array}{c}6.6 \mathrm{mb} \\
\mathrm{ipP}\end{array}$} \\
& $44 \quad 10.00 \quad 202 \mathrm{~km}$
\end{tabular}

iPP $46 \quad 54.00$

is 5352.00

$91.16 \quad 269$ iPc $43 \quad 23.00 \quad 2.1$

$1.0 \mathrm{~s} \quad 30.00 \mathrm{~nm} \quad 5.3 \mathrm{mb} \times$

91.39197 is $\quad 4321.00 \quad 0.5$

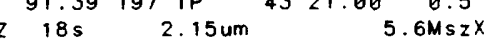

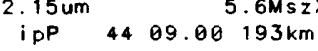

isP 4430.00

iSKS $53 \quad 42.00$

91.59314 iPC $43 \quad 21.00 \quad-0.9$

$2.0 \mathrm{~s} \quad 1650.00 \mathrm{~nm}$

IDP $4412.00206 \mathrm{~km}$

eSSS 0356.00

$\begin{array}{llllll}91.99 & 24 \mathrm{eP} & 43 & 22.40 & -1.1\end{array}$

$93.04 \quad 28$ eP $4326.90 \quad-1.2$

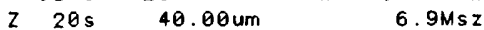


$24 d \quad 05 h$

\begin{tabular}{|c|c|c|c|c|c|c|c|c|}
\hline RKT & & 93.40 & 114 & iP & 43 & 33.60 & $3.0 x$ & APA \\
\hline $\mathrm{OC}$ & & $\begin{array}{l}1.05 \\
93.93\end{array}$ & 313 & $\begin{array}{l}0.00 \mathrm{~nm} \\
\mathrm{eP}\end{array}$ & & 30.50 & $\begin{array}{l}6.3 m b \\
-2.2\end{array}$ & $A P$ \\
\hline & & $3.0 \mathrm{~s}$ & 1500 & $0.00 \mathrm{~nm}$ & & & $6.6 \mathrm{mb}$ & 1 \\
\hline & $z$ & $24 \mathrm{~s}$ & & $5.00 \mathrm{um}$ & & & 7. $0 \mathrm{Msz} \mathrm{X}$ & \\
\hline & & & & ipP & 44 & 20.00 & $199 \mathrm{~km}$ & I HLW \\
\hline & & & & iSKS & 53 & 40.00 & & \\
\hline & & & & is & 54 & 18.00 & & SIT \\
\hline & & & & eSP & 55 & 44.00 & & INK \\
\hline & & & & iSPP & 56 & 20 & & \\
\hline MTD & & 94.10 & 253 & eP & 43 & 33 & -1.1 & \\
\hline & & & & ePP & 48 & 90 & & PUL \\
\hline & & & & iPKKP & 00 & 00 & & \\
\hline$\angle H E$ & & 94.10 & 351 & iPc & 43 & 34.00 & 1. 2 & 1 \\
\hline & & $5.0 \mathrm{~s}$ & 6000 & $0.00 \mathrm{~nm}$ & & & 7. $0 \mathrm{mb} \times$ & 1 \\
\hline & $z$ & $22 \mathrm{~s}$ & 125 & 5. 00 um & & & $7.3 M S z$ & 1 \\
\hline & & $22 \mathrm{~s}$ & & $0.00 \mathrm{um}$ & & & & 1 \\
\hline & c & $22 \mathrm{~s}$ & & $6.00 \mathrm{um}$ & & & & \\
\hline & & & & $i p P$ & 44 & 21.00 & $188 \mathrm{kmX}$ & 1 \\
\hline & & & & iPP & 47 & 23.00 & & SUR \\
\hline & & & & iSKS & 53 & 54.00 & & | \\
\hline & & & & is & 54 & 27.00 & & \\
\hline $\mathrm{COL}$ & & 94.17 & 25 & eP & 43 & 31.00 & -2.3 & I GPA \\
\hline & & $1.3 \mathrm{~s}$ & 591 & $1.00 \mathrm{~nm}$ & & & $6.6 \mathrm{mb}$ & I KEV \\
\hline & $z$ & $23 \mathrm{~s}$ & & 5.00 um & & & 7. $1 M \leqslant z X$ & 1 \\
\hline & & & & es & 54 & 17.00 & & 1 \\
\hline BA & & 94.17 & 25 & eP & 43 & $\theta$ & -2.2 & 1 \\
\hline$O A$ & & 50 & 28 & $e(P)$ & 43 & 0 & -0.4 & 1 \\
\hline EV & & 94.75 & 243 & iPd & 43 & & 1.4 & 1 \\
\hline SLR & & 95.60 & 243 & iPd & 43 & 20 & 0.3 & 1 \\
\hline & & $0.9 \mathrm{~s}$ & 53 & $3.80 \mathrm{~nm}$ & & & $5.8 \mathrm{mb}$ & ALT \\
\hline & $z$ & $22 \mathrm{~s}$ & 44 & $4.40 \mathrm{um}$ & & & $6.9 \mathrm{MSZ}$ & I ELL \\
\hline BPI & & 95.73 & 243 & iPd & 43 & 42.30 & 0.7 & I KJF \\
\hline & & & & $i$ & 43 & 55.00 & $42 \mathrm{kmx}$ & 1 \\
\hline NN & & 95.79 & 314 & iPC & 43 & 39.00 & -2.1 & I \\
\hline & & $9.0 \mathrm{~s}$ & 8000 & $0.00 \mathrm{~nm}$ & & & $7.0 \mathrm{mb} \times$ & \\
\hline & N & $25 s$ & 114 & $4.00 u m$ & & & & I \\
\hline & $E$ & $22 \mathrm{~s}$ & & 5.00 um & & & & \\
\hline R! & & & & ePPS & 57 & 01.00 & & HRT \\
\hline & & 95.95 & 252 & eP & 43 & 00 & 0.4 & 1500 \\
\hline & & & & iPP & 48 & & & 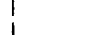 \\
\hline GI & & 96.21 & 302 & eP & 43 & 0 & 2.6 & 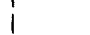 \\
\hline UL & & 96.32 & 249 & iPc & 43 & 44.00 & -0.3 & I ISK \\
\hline & & & & es & 54 & 0 & & KIS \\
\hline & & & & ePKKP & 00 & 8 & & 1 \\
\hline JER & & 96.36 & 301 & eP & 43 & 0 & 2.4 & 1 \\
\hline dos & & 96.38 & 325 & eP & 43 & 43.00 & -0.5 & 1 \\
\hline & & $3.0 \mathrm{~s}$ & 7000 & $0.00 \mathrm{~nm}$ & & & $7.5 \mathrm{mb}$ & 1 \\
\hline & $z$ & $17 \mathrm{~s}$ & & $2.80 u m$ & & & $7.1 M s z X$ & I DST \\
\hline & $\mathbf{N}$ & $20 \mathrm{~s}$ & & 1.00 um & & & & I SUF \\
\hline & $E$ & $18 s$ & & $.20 u m$ & & & & KBS \\
\hline & & & & epP & 44 & 31.00 & $193 \mathrm{~km}$ & I CFR \\
\hline & & & & IPP & 47 & .00 & & PSN \\
\hline & & & & iPPP & 49 & 44 & & I YER \\
\hline & & & & eSKS & 54 & 04.00 & & $T L B$ \\
\hline & & & & is & 54 & 45 & & $M B C$ \\
\hline & & & & iSP & 56 & 0 & & I \\
\hline & & & & isS & 01 & 00 & & PPE \\
\hline FS & & 96.68 & 242 & IPd & 43 & 43.70 & -2.1 & $\mathrm{CLI}$ \\
\hline & & $0.8 \mathrm{~s}$ & 403 & $3.00 \mathrm{~nm}$ & & & $6.8 \mathrm{mb}$ & MFT \\
\hline LF & & 96.74 & 240 & iPd & 43 & 47.50 & 1.4 & I NUR \\
\hline & & & & i & 44 & 48.00 & $248 \mathrm{kmX}$ & 1 \\
\hline MI & & 96.74 & 300 & eP & 43 & 48.00 & 2.2 & I \\
\hline SR & & 96.79 & 243 & ep & 43 & 44.90 & -1.5 & $112 M$ \\
\hline BN & & 96.93 & 325 & iPc & 43 & 44.90 & -1.1 & I VR I \\
\hline & & $1.2 \mathrm{~s}$ & 720 & $.00 \mathrm{~nm}$ & & & $6.9 \mathrm{mb}$ & I ISR \\
\hline & & $28 s$ & 105 & 5.00 um & & & 7. $2 M s z X$ & I JMB \\
\hline & & $28 s$ & & $3.00 u m$ & & & & 1 \\
\hline & 2 & $28 \mathrm{~s}$ & 101 & .00 um & & & & TRO \\
\hline & & & & ipP & 44 & 36.00 & $206 \mathrm{~km}$ & $E Z N$ \\
\hline & & & & iPP & 47 & 50 & & MLR \\
\hline & & & & iSKS & 54 & 08.00 & & BUC 1 \\
\hline & & & & IPS & 56 & 32.00 & & $D I M$ \\
\hline & & & & IPPS & 57 & 00 & & KDZ \\
\hline & & & & is & 01 & 00 & & PVL \\
\hline & & & & isSS & 05 & & & LVV \\
\hline VD & & .00 & 238 & iPd & 43 & & 2.3 & 1 \\
\hline$A$ & & 20 & 30 & eP & 43 & 00 & -0.3 & 1 \\
\hline$W Z$ & & 97.90 & 241 & ep & 43 & 00 & -0.3 & 1 \\
\hline IM & & 98.05 & 314 & $e P c$ & 43 & 49.00 & -2.3 & 1 \\
\hline & 2 & $28 s$ & 87 & $.00 u$ & & & 7. $1 \mathrm{MszX}$ & 1 \\
\hline & & $28 s$ & 132 & 2.000 & & & & 1 \\
\hline & E & $28 s$ & 130 & .00 um & & & & \\
\hline & & & & ipp & 44 & 39.00 & $202 \mathrm{~km}$ & I \\
\hline & & & & IPP & 47 & 59.00 & & \\
\hline & & & & ef & 50 & 0 & & \\
\hline
\end{tabular}

iSKS $54 \quad 13.00$ \begin{tabular}{lll|l}
$1.2 \mathrm{~s}$ & $260.00 \mathrm{~nm}$ & $6.5 \mathrm{mb}$ & CJR
\end{tabular} \begin{tabular}{cll|l} 
iSKS 5416.80 & MMB \\
iS & 5459.00 & ATH
\end{tabular} $\begin{array}{lllll}99.54 & 299 \text { iPc } & 4404.00 & 5.5 \times\end{array}$ \begin{tabular}{lllll|l}
$23 \mathrm{~s}$ & $4.00 u m$ & $5.9 M s z X$ & \\
99.76 & $33^{\mathrm{eP}}$ & 43 & $59.90^{5}$ & 1.1 & VTS
\end{tabular} \begin{tabular}{lllll|l}
99.97 & $22 \mathrm{ePC}$ & 43 & 57.80 & -1.8 & WAR
\end{tabular} $1.3 \mathrm{~s} \quad 223.00 \mathrm{~nm}$ PP $4449.00207 \mathrm{~km}$ VAY 100.33329 iPdiff44 $05.00 \quad 3.7 \times$ UPP $6.0 \mathrm{~s} 3300.00 \mathrm{~nm} \quad 7.0 \mathrm{mb} \times$ \begin{tabular}{llll|l}
$Z$ & $22 \mathrm{~s}$ & $51.00 \mathrm{um}$ & $7.0 \mathrm{MSZ}$ & $\mathrm{SSR}$ \\
$\mathrm{N}$ & $22 \mathrm{~s}$ & $54.00 \mathrm{um}$ & & $\mathrm{JOS}$
\end{tabular} E $22 \mathrm{~s} \quad 54.00 \mathrm{um}$ E $24 \mathrm{~s} \quad 54.00 \mathrm{um}$ iPP $\quad 48 \quad 18.00$ iSKS $55 \quad 16.00$ es 5524.00

100.44235 iPdiff 4407.00 $1.1 \mathrm{~s} \quad 304.00 \mathrm{~nm}$ $221 \mathrm{~s} \quad 95.304 \mathrm{~m}$ $100.73 \quad 310$ ePdiff44 $04.80 \quad 1.1$ 100.79340 ePdiff44 $03.00 \quad-0.2$ $1.0 \mathrm{~s} \quad 102.00 \mathrm{~nm}$ $\begin{array}{lll}i & 44 & 14.00 \\ \text { ePP } & 47 & 40.00 \\ \text { eSKS } & 54 & 24.00 \\ \text { eS } & 55 & 20.00 \\ \text { iPKKP } & 00 & 27.50\end{array}$ 100.87308 ePdiff $44 \quad 10.00$ 100.99306 iPdiff4406.00 101.20334 iPdiff44 03.00

$1.0 \mathrm{~s} \quad 264.00 \mathrm{~nm}$ i $\quad 4415.20$ iPP $\quad 48 \quad 26.00$ ESKS 5436.00 iPKKP 0016.00 101.22310 ePdiff44 09.00 101.26337 iPdif $144 \quad 05.60$ iSKP $47 \quad 24.40$ EPKKP $00 \quad 14.00$ 101.69310 ePdit $\$ 4406.30$ 101.72316 iPdiff44 10.00 $5.0 \mathrm{~s} \quad 1400.00 \mathrm{~nm}$

(O)

JOS

SPC

$4.2 x$
$6.7 \mathrm{mb}$
$7.3 \mathrm{Msz}$
1.1
-0.2
$6.3 \mathrm{mb}$

KRA

KRA

$$
\text { KRA } 2
$$
$6.8 \mathrm{mb} \times \mid \mathrm{HFS}$ \begin{tabular}{lllll|l}
98.67 & 337 iPc & 43 & 59.30 & $5.6 x$ & $\mathrm{COZ}$
\end{tabular} IPP $48 \quad 10.90$

$\begin{array}{cccc}0.6 \mathrm{~s} & \text { B. } 00 \mathrm{~nm} & 5.9 \mathrm{mb}\end{array}$

105.35316 ePdiff $4428.00 \quad 4.0 X$ 105.68311 ePdiff44 $28.00 \quad 2.4$ 105.90307 iPdiff44 $24.00-2.6 x$ iPP $\quad 48 \quad 56.00$ iSKS 5500.00

105.99312 ePdiff44 $30.00 \quad 3.1 x$ 106.32322 ePdiff44 $35.00606 x$ es 4905.00 106.57311 iPdiff44 $32.00 \quad 2.5 x$ 106.65330 iPdiff44 $33.10 \quad 3.8 x$ $106.83 \quad 315$ ePdiff44 $32.00 \quad 1.4$ 107.19318 ePdiff44 $33.00 \quad 0.9$ $2.0 \mathrm{~s} \quad 130.00 \mathrm{~nm} \quad 6.7 \mathrm{mb}$ 107.28319 elPdif44 $34.60 \quad 1.9$ Z $20 \mathrm{~s} \quad 64.50 \mathrm{um} \quad 7.2 \mathrm{Msz}$ $07.34 \quad 320$ i $\quad 4907.30$ $\begin{array}{llll}36 \mathrm{~s} & 254.00 \mathrm{um} & 33.00 \quad 0.3 \\ \mathrm{Z} & 7.5 \mathrm{MszX}\end{array}$ $N$ 46s $373.00 u m$ $725 \quad 552.00 u m$

$\begin{array}{lrrr}e & 44 & 35.00 & \\ \mathrm{i} & 44 & 39.60 & \\ \mathrm{e} & 44 & 45.00 & \\ \mathrm{e} & 44 & 47.50 & \\ \mathrm{i} & 49 & 12.00 & \\ \text { is } & 55 & 11.00 & \\ \mathrm{i} & 57 & 43.00 & \\ \text { iPdiff } 44 & 32.00 & -1.0 \\ \mathrm{i} & 48 & 41.90 & \\ \mathrm{i} & 49 & 07.00 & \end{array}$

$\begin{array}{rrrr}107.35312 & \text { iPdiff44 } & 32.00 \\ & i & 48 & 41.90\end{array}$ 4907.00

$107.70 \quad 41$ ePdiff $44 \quad 41.20 \quad 6.9 x$ $107.70 \quad 41$ ePKP $48 \quad 49.00 \quad 7.7 x$ 107.70318 e(Pdif44 $39.00 \quad 4.5 x$ 107.92311 ePdiff4439.09

4757.10 5502.70 108.35318 e(Pdif 4439.00 108.3550 ePdiff 4440.50 108.3550 ePKP 4851.00 108.46352 iPdiff44 $35.20 \quad-1.9$ $0.7 \mathrm{~s} \quad 8.22 \mathrm{~nm} \quad 6.1 \mathrm{mb}$

108.47331 i 4914.00 diff44 $36.28-1.3$ $0.6 \mathrm{~s} 11.00 \mathrm{~nm} \quad 6.3 \mathrm{mb}$ 108.77318 epdiff 4444.00 $\begin{array}{ccc}\text { iSKS } 5432.00 \\ \text { iS } & 5530.00\end{array}$ 102.02309 epdif $\$ 4408.00$ 102.07333 iPdiff4407.70 102.18350 iPdiff 4409.90 102.27315 ePdif $\{44 \quad 02.00$ 102.29313 iPdif $f 44 \quad 15.00$ 102.30306 ePdiff44 12.60 $102.38 \quad 314$ ePdiff44 16.00 $102.46 \quad 13$ ePdiff $44 \quad 09.50$ $0.7 \mathrm{~s} \quad 64.00 \mathrm{~nm}$ $8 \mathrm{Msz} X$ SRO 102.61316 ePdiff $4405.00^{6.4 m b}-6.8 \times \mid Y K C$ $102.83 \quad 316$ ePdiff44 $29.00 \quad 16.2 X$ 103.04310 ePdiff $44 \quad 16.00$ 103.08330 iPdiff44 15.10 $1.0 \mathrm{~s} \quad 94.00 \mathrm{~nm}$ 4423.90

103.10308 ePdif $\{44 \quad 17.30$ 103.25315 ePdiff $44 \quad 17.00$ $103.41315 \mathrm{ePdi} f 4420.00$ 103.51312 iPdiff44 15.00 iPP $48 \quad 40.00$ $103.58 \quad 340$ ePdiff $44 \quad 15.50$ 103.79309 ePdiff $44 \quad 18.00$ 103.82315 ePdiff $44 \quad 16.50$ 103.82314 ePdiff $44 \quad 18.00$ 104.27311 ePdiff 4430.00 104.46311 iPdiff44 19.00 104.51313 iPdiff44 23.00 104.75319 iPdiff44 27.50 $9.2 \mathrm{~s} \quad 4200.00 \mathrm{~nm}$

$220 \mathrm{~s} \quad 36.30 \mathrm{um}$

$N 20 \mathrm{~s} \quad 46.20 \mathrm{um}$ E $195 \quad 28.00 u m$ $\begin{array}{lll}\text { EPP } \quad 48 \quad 52.00 & 0\end{array}$ ESKS 5528.00 is $\quad 5557.58$ iSP $57 \quad 29.00$ iPS 5753.00 $104.83 \quad 1$ ePdiff44 21.00 $-1.4$ $-1.3$ \begin{tabular}{l|l}
0.7 & TTG
\end{tabular} $-8.3 x$ 4. $6 x$ 1.9 YKA 5. $2 X \mid Y K A$ $\begin{array}{lll}\text { e } & 4530.00 \\ \text { e } & 4754.00 \\ \text { e } & 4924.00\end{array}$ e $\quad 4801.00$ e(S) 5447.00 108.9626 ePdiff $44 \quad 43.40$ $108.96 \quad 26$ ePKP $48 \quad 44.80$ $109.02 \quad 26$ ePdiff $44 \quad 45.00$ $1.1 \mathrm{~s} \quad 66.00 \mathrm{~nm}$

$109.02 \quad 26$ ePKP $48 \quad 43.00 \quad-0.4$ $0.8 \mathrm{~s} \quad 115.00 \mathrm{~nm}$

$109.33 \quad 333$ Pdiff $44 \quad 41.70$ $0.8 \mathrm{~s} \quad 22.90 \mathrm{~nm}$

$109.47 \quad 50$ ePdiff $44 \quad 45.30 \quad 2.9 x$

109.4750 ePKP $48 \quad 52.00 \quad 7.0 X$ ESP 5856.50 $4.5 \times \mid 0.8 \mathrm{~s} \quad 72.00 \mathrm{~nm}$

2.3 KSP

$-0.9$

0.0

\begin{tabular}{l|l}
0.8 & ZST
\end{tabular}

$-0.91$

\begin{tabular}{c|c}
0.8 & \\
$10.7 \times$ & BNG
\end{tabular} $-1.2$

2. $7 x \mid$ VKA

$6.3 \times$ 6. $9 \mathrm{Msz}$ i VKA 109.50319 ePdiff $44 \quad 46.90$ e $\quad 4820.20$ 4858.20 109.96272 ifdiff44 $48.30 \quad 3.1 X$ $0.5 \mathrm{~s} \quad 142.00 \mathrm{~nm}$

110.00319 ePdiff44 49.00 $4.5 \mathrm{~s} \quad 739.00 \mathrm{~nm}$ pP $\quad 4538.00$ SP $\quad 4559.00$

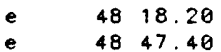
$\begin{array}{llllll}\text { VKA } \quad 110.00 & 319 \text { iPKPd } 48 & 57.90 & 12.1 \mathrm{X}\end{array}$ $z 20 \mathrm{~s} \quad 12.40 \mathrm{um}$ 6. $5 \mathrm{Msz}$
108.91312 elPdif 4441.00

$\begin{array}{lll}\text { iPP } & 49 & 26.80\end{array}$

is $56 \quad 46.00$ is 5807.00 


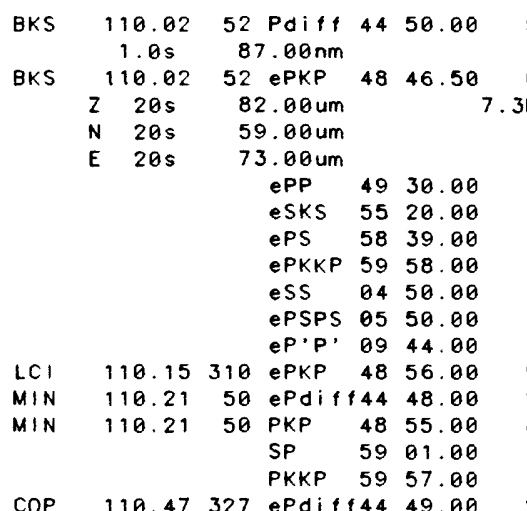

$\begin{array}{llll}\text { COP } \quad 110.47 \quad 327 \text { ePdiff44 } 49.00 & 0\end{array}$ $z 20 \mathrm{~s} 56.78 \mathrm{um} 5651.00^{7}$

MHC $\quad 110.54 \quad 53$ ePdiff44 48.50

MHC 110.5453 ePKP 4854.20

PRU 110.76321 ePdiff44 52.50

$\begin{array}{cccc}Z & 19 \mathrm{~s} & 50.30 \mathrm{um} & 4.6 \mathrm{X} \\ \mathrm{N} & 20 \mathrm{~s} & 57.48 \mathrm{um} & 7.1 \mathrm{Msz}\end{array}$

$\begin{array}{lll}\mathrm{N} & 20 \mathrm{~s} & 57.40 \mathrm{um} \\ \mathrm{E} & 20 \mathrm{~s} & 31.50 \mathrm{um}\end{array}$

$\begin{array}{lll}e & 45 & 43.00 \\ e & 48 & 32.00 \\ i & 48 & 59.50 \\ \text { PP } & 49 & 36.00 \\ \text { e } & 5622.00\end{array}$

PKKP $58 \quad 41.00$

BRG $110.92 \quad 322$ ePdiff44 53.10

$$
1.6 \mathrm{~s} \quad 70.00 \mathrm{~nm}
$$

EPP $\quad 45 \quad 44.40$

BRN 110.96324 ePdiff44 50.00 $\begin{array}{lll}\text { EPP } & 45 & 43.00\end{array}$ SKS $55 \quad 37.00$

CLL 111.38322 Pdiff 4450.00

PP 4540.00 $\begin{array}{lll}\text { iPKP } & 48 & 59.70 \\ \text { iPP } & 49 & 40.00\end{array}$ PKKP 0004.80

JAS $111.42 \quad 52$ ePdi $\$ 4450.30$

JAS 111.4252 ePKP $48 \quad 50.90$ $\begin{array}{ll}1.1 \mathrm{~s} & 5.50 \mathrm{~nm}\end{array}$

$111.46 \quad 54$ ePdiff44 54.00

PRI $\quad 111.46 \quad 54$ ePKP $48 \quad 56.50$

KMR 111.48319 ePdiff44 58.00 PP $45 \quad 46.00$

KMR 111.48319 (PKP) $48 \quad 36.00$

KHC 111.58320 Pdiff 4456.50 $\begin{array}{lll}N & 27 \mathrm{~s} & 49.00 \mathrm{um}\end{array}$

E $24 \mathrm{~s} \quad 37.60 u m$

e $\quad 4845.50$

e $\quad 48 \quad 56.50$

PP $\quad 4942.60$

e $\quad 5032.00$

SKS $55 \quad 30.00$

$\begin{array}{lll}\text { e } & 56 & 32.00 \\ \text { PKKP } & 59 & 04.00\end{array}$

LJU $\quad 111.65317$ ePdiff44 55.00

CEY 111.79316 e(Pdif44 58.00

CEY 111.79316 e(Pdif 4458.00

CEY 111.79316 iPKP 4858.50

NEW $1111.91 \quad 41$ ePdiff44 53.00

NEW 111.9141 ePKP 4851.00

MUD 111.94328 iPdiff44 57.70

$0.8 \mathrm{~s} \quad 23.00 \mathrm{~nm}$

SGO $112.12 \quad 311$ ePKP $48 \quad 30.00$

FRI $\quad 112.12 \quad 53$ ePdift44 57.00

FRI $\quad 112.12 \quad 53$ ePKP $48 \quad 57.00$

RBL $\quad 112.22 \quad 317$ ePdif $\$ 45 \quad 00.50$

KBA $\quad 112.22 \quad 318$ ePdiff44 55.00

$\begin{array}{lll}i p P & 45 & 45.00\end{array}$

KBA $\quad 112.22 \quad 318$ iPKPd $48 \quad 53.40$

$$
1.3 \mathrm{~s} \quad 278.00 \mathrm{~nm}
$$

$\begin{array}{lll}\text { iPP } & 49 & 35.20\end{array}$

ipPKP 4941.80

112.25316 iPdiI $50 \quad 06.40$

iPdiff44 54.00

TRI $112.25 \quad 316$ IPKP $48 \quad 48.80$ $\begin{array}{lll}\text { iPP } & 49 & 01.60\end{array}$ eSKS $5523.4 \theta$

$6.9 \mathrm{x}$

$-12.6 x$

3. $2 x$

\section{$5.1 \times$ \\ 0.4

(

BER

MOX

Mox

i

2. 1
$8 \times$

$2.6 \times 1$ HAM

IMSz I DUI

\begin{tabular}{l|l}
1.1 & EDM \\
$6 D M$
\end{tabular}

$6.9 \times$

4.6X GRF

$\begin{array}{lcccc}\text { GRF } & 112.92 & 321 \text { ePdiff } 44 & 58.00 \\ & z & 21 \mathrm{~s} & 49.80 \mathrm{um} & \end{array}$

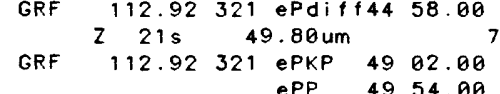

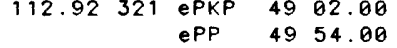
$\begin{array}{lll}\text { ePP } & 49 & 54.00 \\ \text { eS } & 57 & 15.00\end{array}$ e $\quad 58 \quad 32.00$

LDM $\quad 113.02 \quad 40$ ePKP $48 \quad 52.00$

I MNA $113.13 \quad 51$ ePdifi45 03.00

MNA

I CLX

$4.5 \times \mid \begin{aligned} & \text { ISA } \\ & \text { ISA }\end{aligned}$

ISA

1.3 FUR

$-0.6 \mid \mathrm{POI}$

CWC

$11.5 \times$ CWC

GIB

-0.9 CT

2.1 I MNS

$2.5 \times$

$7.4 \times$ I PAS

4. $9 \times$

i

MWC

MWC

OGA

ROP

\begin{tabular}{l|l}
$3.0 \times$ & RDP \\
$5.4 \times$ & RMP
\end{tabular}

$\begin{array}{l:l}5.4 \times & \text { RMP } \\ 1.3 & \end{array}$

\begin{tabular}{l|l|l}
0.1 & CLC \\
-0.1 & CLC
\end{tabular}

$1.6 \quad$

4. $8 \times$ RVR

I RVR

\begin{tabular}{r|l}
$20.0 x$ & \\
$2.8 X$ & FIR
\end{tabular}

$6.9 \times$

$5.9 \times$

\begin{tabular}{l|l}
0.3 & INS
\end{tabular}

$113.13 \quad 51$ ePKP $49 \quad 00.30$

$113.21 \quad 41$ ePKP $48 \quad 51.40$

$113.28 \quad 54$ ePdift45 06.00

$113.28 \quad 54$ ePKP $48 \quad 53.00$

$\begin{array}{llll} & \text { e } & 49 & 52.00\end{array}$

$0.95 \quad 779$ ePKP

$Z 20 \mathrm{~s} \quad 37.20 \mathrm{um}$

113.49313 ePKP $48 \quad 50.00$

$113.49 \quad 54$ ePdiff45 15.00

113.4954 ePKP 4858.00

$\begin{array}{clll} & \text { e } & 49 & 51.00 \\ 113.52308 \text { ePKP } & 48 & 56.00\end{array}$

113.60317 ePdit 44506.00 e $\quad 4852.00$

$113.72 \quad 313$ ePdit145 06.00

e 4852.00

113.7356 ePdiff45 06.00 $1.0 \mathrm{~s} \quad 100.00 \mathrm{~nm}$

$$
\begin{array}{lll}
\text { e } & 4554.00 \\
\mathrm{e} & 4854.00 \\
\mathrm{e} & 5227.00 \\
\text { is } & 5529.00 \\
\text { eS } & 5951.00 \\
\text { eS } & 00 & 50.00
\end{array}
$$

113.8156 ePditt45 06.00

$\begin{array}{lllll}113.81 & 56 \text { ePKP } & 46 & 16.00 \\ 1134.00\end{array}$

$\begin{array}{rl}e & 4900.00\end{array}$ e $\quad 4950.00$

113.81318 iPKPd $48 \quad 54.40$ $1.0 \mathrm{~s} \quad 500.00 \mathrm{~nm}$

113.82312 ePKP $48 \quad 40.50$ $113.82 \quad 312$ ePdiff45 02.00

$$
\text { e } 4950.00
$$$$
\text { e } \quad 5540.00
$$

$113.97 \quad 54$ ePdiff45 16.00

$113.97 \quad 54$ ePKP $48 \quad 55.00$

$\begin{array}{llr}114.40 & 56 \text { ePdifi45 } & 10.00\end{array}$

$114.40 \quad 56$ ePKP 4856.00

e $\quad 4950.00$

114.43315 ePdit $\$ 4502.00$

iPP $\quad 49 \quad 06.00$

iSKS 5531.00

is 5650.00

114.46322 ePdit 14511.80

e 4854.30

SAL

WIT

e 4845.00

114.62325 ePditt45 19.00

$48 \quad 57.00$

$114.62 \quad 325$ IPKPd $49 \quad 07.90$ ePP $\quad 50 \quad 03.00$ $\begin{array}{lll}\text { EPPP } 52 \quad 27.50 & 030\end{array}$

EPKKP 5942.50

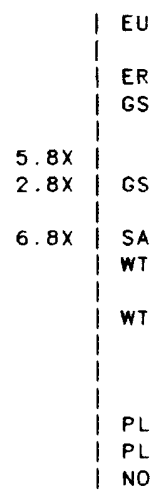

114.6250 iPdift45 09.00 $5.77 \mathrm{~nm}$

$114.63 \quad 308$ ePKP $48 \quad 55.50$ 114.6855 ePditf 4509.00 4520.00 $\begin{array}{ccccc} & \text { e } & 46 & 23.00 \\ 114.68 & 55 \text { ePKP } & 48 & 56.00\end{array}$ $\begin{array}{cccc}114.6855 & \text { ePKP } & 48 & 56.00 \\ \text { e } & 50 & 03.00\end{array}$

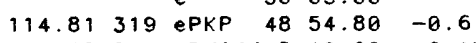
114.83324 ePdiff45 12.00 e 4856.50

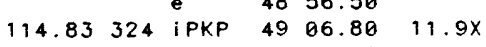
\begin{tabular}{llll} 
EPP & 50 & 04.00 \\
\hline
\end{tabular} $\begin{array}{lll}\text { ePPP } & 52 & 18.00\end{array}$ ePKKP $59 \quad 24.50$

$114.91 \quad 57$ ePdiff45 15.00 $\begin{array}{lllll}114.91 & 57 & \text { ePKP } \quad 48 & 56.00\end{array}$ $115.08 \quad 54$ ePdiff45 11.50 $115.08 \quad 54$ ePKP $48 \quad 56.60$ $\begin{array}{llll}115.09 & 37 \text { ePdif } f 45 & 06.00 & -1.2\end{array}$ $\begin{array}{llll}115.09 & 321 \text { ePdiff45 } 10.80 \quad 3.5 x\end{array}$ $\begin{array}{llllll}115.09 & 321 & \text { ePKP } \quad 48 & 54.50 & -1.1\end{array}$ $\begin{array}{lllll}115.16 & 57 & \text { ePdif }\{45 & 25.00 & 17.1 \times\end{array}$ $115.16 \quad 57$ ePKP $48 \quad 58.00$ e $\quad 4903.00$

$\begin{array}{llllll}115.21 & 320 \text { ePKP } & 58 & 10.00 & 54.50 & -1.3\end{array}$ 115.36319 ePKP $48 \quad 55.00 \quad-1.1$ $\begin{array}{lllll}115.38 \quad 321 & \text { iPdiff45 } & 12.40 & 3.9 x\end{array}$ $\begin{array}{lllll}115.63 & 316 & \text { elPdit45 } & 05.00 & -4.6 x\end{array}$ $\begin{array}{lllll}115.63 & 316 \text { ePKP } \quad 4858.50 & 1.9\end{array}$ $\begin{array}{lllll}115.63 & 43 & \text { ePdiff } 45 & 11.60 & 1.6\end{array}$ $\begin{array}{lllll}115.73 & 52 & \text { ePdiff } & 10.00 & -0.5\end{array}$ $\begin{array}{llllll}115.73 & 52 & \text { ePKP } & 48 & 56.20 & -1.1\end{array}$ $\begin{array}{lll}115.73 & 325 \text { elPdif45 } 10.00 & 0.1\end{array}$ $Z 22 \mathrm{~s} 22.20 \mathrm{um}$

ePP $\quad 4951.00$ e $\quad 5026.00$ IPPP $52 \quad 18.00$ ISKKS 5651.00 ePS $59 \quad 32.00$

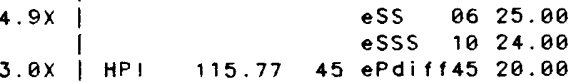

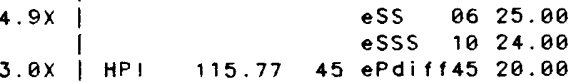

\begin{tabular}{l|llll}
$5.2 X$ & HPI 115.77 & 45 ePKP 49 & 00.20
\end{tabular}

I CDF $115.78 \quad 321$ iPKPC 4857.10

4.8X ENN 115.79323 ePdiff45 16.50

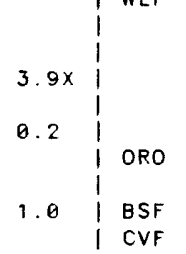

$-12.9 \times$

0.3 HAU

$13.4 \times \quad$ UCC

1.1 UCC

$5.5 \times$

1.3

$-2.3$

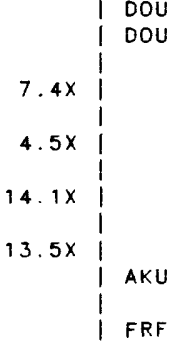
e 4856.50

115.79323 ePKP $49 \quad 09.00$ $\begin{array}{lll}\text { EPP } & 50 & 08.08\end{array}$ $\begin{array}{lll}\text { EPPP } & 52 & 34.00\end{array}$ ePKKP 5930.00

I

ECH
WLF

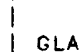

$\begin{array}{llll}115.92 & 320 & \text { ePdiff } & 18.40 \\ 116.05 & 322 & \text { Pdifft45 } & 21.00\end{array}$

4958.00

S $\quad 5759.00$

PKKP $59 \quad 36.00$

SS $06 \quad 24.00$ SSP 0641.00

$\begin{array}{llll}116.19 & 317 \text { e(Pdif } 45 \quad 11.50\end{array}$

e 4856.50

116.28320 iPKPC 4857.10

116.42314 iPKPC $48 \quad 58.40$

$0.8 \mathrm{~s} \quad 106.00 \mathrm{~nm}$

116.50320 iPKPC $48 \quad 57.70$

$1.0 \mathrm{~s} 108.00 \mathrm{~nm}$

$116.64 \quad 57$ ePditt45 21.70

$116.70 \quad 324$ Pdift+45 21.00

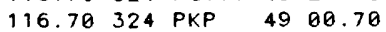

e $\quad 50 \quad 01.00$

PP $\quad 50 \quad 11.60$

PPP 5252.00

e $\quad 5350.00$

PKKP $59 \quad 37.00$

116.83323 Pdiff+45 21.00

116.83323 PKP 4858.70

e $\quad 4906.10$

\begin{tabular}{l}
4910.70 \\
\hline
\end{tabular}$\quad 5009.00$

PPP $52 \quad 55.00$

e 5354.00

PPS 5750.00

\begin{tabular}{lll}
\hline & 59 & 02.00
\end{tabular}

$\begin{array}{cc}117.29 & 345 \text { iPKPC } \\ 1.0 \mathrm{~s} & 208.00 \mathrm{~nm}\end{array}$

$\begin{array}{llllll}117.71 \quad 316 & \text { iPKPC } 49 \quad 00.20 & -0.4\end{array}$

9. $3 x$

$8 x$

0.1
$6.3 x$

$12.3 x$

$7.5 x$

9. $6 x$

$-0.8$

$-0.8$

$-0.5$

7. $2 x$

$6.8 x$

2. 2

6. $2 \mathrm{x}$

0.0

1.1

I FRF 
LMR

$\begin{array}{llllll} & 117.94 & 316 & \text { iPKPC } & 49 & 00.80\end{array}$

i 5025.00

FFC 118.2830 ePKP 4901.00 $0.8 \mathrm{~s} \quad 51.00 \mathrm{~nm}$

LOR $\quad 118.33 \quad 320$ iPKPC $49 \quad 01.30$

LBF $\quad 118.36320$ iPKPC $49 \quad 01.20$

BDW $118.50 \quad 45$ ePdif 4533.30

$1.0 \mathrm{~s} \quad 4.60 \mathrm{~nm}$

BDW $\quad 118.50 \quad 45$ ePKP $49 \quad 02.10$ $0.8 \mathrm{~s} \quad 155.00 \mathrm{~nm}$

$\begin{array}{llllll}\text { GCA } & 118.52 & 52 & \text { ePKP } & 49 & 03.40\end{array}$

SMF $\quad 118.57 \quad 320$ IPKPC $49 \quad 01.70$

SSB $\quad 118.59 \quad 318$ ePKP $49 \quad 01.90$

SSF $\quad 118.63 \quad 320$ IPKPC $49 \quad 02.00$

EKA 118.66331 PKP 4903.00

$0.7 \mathrm{~s} \quad 22.30 \mathrm{~nm}$

GRC 118.82321 IPKPC 4903.10

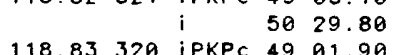

AVF $\quad 118.83 \quad 320$ IPKPC 4901.90

RMU $118.98 \quad 52$ ePKP $49 \quad 04.30$

REY $\quad 119.50345$ ePKP 4904.80

MZF $\quad 119.54 \quad 320$ IPKPC 4903.70

$0.85 \quad 54.80 \mathrm{~nm}$

TCF $\quad 119.75 \quad 320$ IPKPC $49 \quad 04.20$

PV10 120.1450 ePKP $49 \quad 66.10$

LSF $\quad 120.20 \quad 320 \quad$ iPKPC $49 \quad 04.70$

LDF $\quad 120.26 \quad 323$ iPKPC $49 \quad 06.60$

CAF $\quad 120.36 \quad 318$ iPKPC $49 \quad 05.70$

FLN 120.40323 IPKPC 4906.60

$1.2 \mathrm{~s} \quad 156.00 \mathrm{~nm}$

$\begin{array}{llllll}\text { PVO7 } & 120.42 & 50 & \text { ePKP } & 49 & 06.70\end{array}$

RJF 120.57319 IPKPC $49 \quad 06.00$

$\begin{array}{llllll}\text { PVO6 } & 120.59 & 50 & \text { ePKP } 49 & 07.00\end{array}$

$\begin{array}{lllllll}\text { GRR } & 120.79 & 323 & \text { IPKPC } & 49 & 07.60\end{array}$

LPO $\quad 121.03 \quad 318$ IPKPC 4907.10

LPF $\quad 121.06 \quad 323$ iPKPC 4906.70

MFF $121.12 \quad 321$ iPKPC $49 \quad 06.80$

LFF $\quad 121.23 \quad 319$ IPKPC $49 \quad 07.40$

$1.2 \mathrm{~s} \quad 144.00 \mathrm{~nm}$

$\begin{array}{llllll}\text { DMU } & 121.27 & 331 \text { iPKPC } & 49 & 06.70 & -0.4\end{array}$

$1.0 \mathrm{~s} \quad 1120.00 \mathrm{~nm}$

DLE 121.44330 iPKPC 4906.20

$1.0 \mathrm{~s} \quad 450.00 \mathrm{~nm}$

$121.77 \quad 330$ iPKPc $49 \quad 09.20$

$0.9 \mathrm{~s} \quad 290.00 \mathrm{~nm}$

RSSD $121.82 \quad 42$ ePKP $49 \quad 08.50$

ECP $\quad 121.97 \quad 329$ IPKPC $49 \quad 09.60$

$2.0 \mathrm{~s} 1140.00 \mathrm{~nm}$

ECB $\quad 122.08 \quad 329$ iPKPC $49 \quad 10.40$

$1.7 \mathrm{~s} \quad 585.00 \mathrm{~nm}$

EPF $\quad 122.22 \quad 317$ iPKPC $49 \quad 88.90$

ABA 122.31309 iPKP $49 \quad 10.00$

$\begin{array}{llllll}\text { GOL } \quad 122.47 & 47 & \text { ePKP } & 49 & 10.20\end{array}$

$z 21 \mathrm{~s} 30.00 \mathrm{um} \quad 6.9 \mathrm{Ms}$

GLD $122.57 \quad 47$ ePKP $49 \quad 10.80 \quad 0.5$

$1.3 \mathrm{~s} \quad 460.00 \mathrm{~nm}$

$221 \mathrm{~s} 31.10 \mathrm{um} \quad 6.9 \mathrm{Msz}$

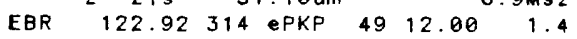

$\begin{array}{llllll} & & & \text { EPP } & 50 & 52.00 \\ 4 & 123.05 & 53 & \text { ePKP } & 49 & 12.00\end{array}$

$Z 20 \mathrm{~s} \quad 53.20 \mathrm{um}$

$7.2 \mathrm{Msz}$

$\begin{array}{llllllll}\text { OFD } & 123.63 & 309 & \text { IPKP } & 49 & 10.00 & -2.2\end{array}$

VAL 124.06330 PKP 4918.60

LGR $\quad 124.37 \quad 317$ ePKP $49 \quad 15.00$

i $\quad 4927.50$

$\begin{array}{llllll} & & \text { IPP } & 51 & 06.00 \\ \text { ALM } & 126.48 & 311 & \text { iPKPC } & 49 & 16.90\end{array}$ $1.8 \mathrm{~s} \quad 22.20 \mathrm{~nm}$

iPP $\quad 4920.00$ is 5123.30

$\begin{array}{lllll} & & \text { iS } & 51 & 23.30 \\ \text { rOL } \quad 126.49315 & \text { ePKP } & 49 & 16.00\end{array}$

CRT $\quad 127.20 \quad 312$ IPKP $49 \quad 19.70$

$\begin{array}{llllll} & & & \text { i } & 49 & 23.30 \\ \text { ALR } \quad 127.23 & 310 & \text { IPKP } & 49 & 31.00\end{array}$

STS 128.14320 EPKP 4921.00

$\begin{array}{lll}128.38 \quad \text { eSn } & 5226.00\end{array}$ $\begin{array}{lllll}28.38 & 31 & \text { ePKP } & 49 & 11.00\end{array}$ $0.9 \mathrm{~s} \quad 453.00 \mathrm{~nm}$

129.04318 PKP 4922.50

RRO

4928.80

4933.80

5238.40

SFS

129.41312 IPKPC 4930.00

0.4

$-0.6$
$-0.5 \quad$

$6.3 \times$

0.8

$\begin{array}{lll}\text { iPP } & 51 & 57.00 \\ \text { iPKS } & 53 & 04.00 \\ \text { ePPP } & 55 & 28.00 \\ i & 94 & 14.00 \\ i S S & 09 & 00.00 \\ i & 16 & 05.00\end{array}$

129.6856 ePKP

$.00^{7}$

0

$\begin{array}{rrr}129.68 & 308 & \text { iPKP } \\ 129.82 & 49 \text { ePKP }\end{array}$

4927.00
5232.40

OCO

$\begin{array}{rr}e & 5232.40 \\ i & 5237.60\end{array}$

LIS 130.56316 ePKP 4926.00

$130.92 \quad 48$ iPKP+ $49 \quad 27.48$

$0.8 \mathrm{~s} \quad 337.00 \mathrm{~nm}$

$Z 21 \mathrm{~s} \quad 27.80 \mathrm{um}$

SC

\begin{tabular}{lll|l}
131.27 & e $5234.90^{6.9 M s z}$ & FDF \\
GUV
\end{tabular}

$\begin{array}{lllllll}0.72 & 11 \text { ePKP } 49 & 17.00 & -9.3 x & \text { ATB }\end{array}$

RLO

GBO

PP $52 \quad 34.00$

$\begin{array}{llll}131.37 & 48 & \text { e(PKP) } 49 \quad 27.60\end{array}$

$131.39 \quad 48$ e(PKP) $49 \quad 28.90$

$132.26 \quad 74$ ePKP $49 \quad 21.20$

$132.48 \quad 72$ ePKP 4921.40

132.4970 ePKP

132.6071 IPKP

4928.20

$\begin{array}{llllll}132.87 & 71 & \text { ePKP } & 49 & 22.80 & -8.0 x\end{array}$

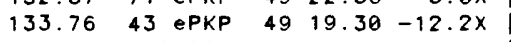

$1.0 \mathrm{~s} \quad 490.00 \mathrm{~nm}$

$Z 19 \mathrm{~s} \quad 5.30 \mathrm{um}$

$5.30 \mathrm{um}$
ePKP

$\begin{array}{rr}6.3 M s z \\ 49 & 19.0 \theta^{-12.6 x}\end{array}$

IPKP

5251.00

$135.20 \quad 73$ iPKP

$4925.00-10.1 x$

TACH $\quad 135.30 \quad 157$ IPKP $4923.70-10.9 x$

BACH $135.74 \quad 157$ ePKP $4926.00 \quad-9.5 x$

-0.1 FCH

$\begin{array}{llllll}135.83 & 158 & \text { iPKPC } 49 & 28.00 & -8.1 \times\end{array}$

$\begin{array}{llllll}135.85 & 157 \text { iPKPc } 49 & 23.90 & -11.8 \times\end{array}$

$\begin{array}{lllllll}\text { OTT } & 136.92 & 25 & \text { ePKP } 49 & 25.50 & -11.7 X\end{array}$

$1.0 \mathrm{~s} \quad 312.00 \mathrm{~nm}$

$0.8 \mathrm{~s} \quad 597.00 \mathrm{~nm}$

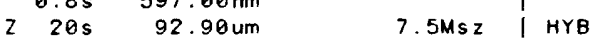

MNT $137.67 \quad 23$ ePKP $49 \quad 29.00 \quad-9.7 x$

$1.6 \mathrm{~s} \quad 420.00 \mathrm{~nm}$

\begin{tabular}{|lcccc|c} 
& $1.6 \mathrm{~s}$ & $420.00 \mathrm{~nm}$ & & POO \\
CEN 137.97 & $158 \mathrm{ePKP}$ & 49 & 27.00 & $-12.8 \times$ & AVY
\end{tabular}

$\begin{array}{llllllll}\text { RSNY } 138.10 & 24 & \text { ePKP } & 49 & 29.30 & -10.2 \times & \text { ISI }\end{array}$

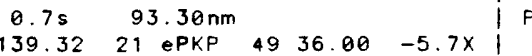

\begin{tabular}{lllll|l}
140.01 & 1 ePKP 49 & 35.00 & $-7.8 \times$ & PPI
\end{tabular}

$0.9 \mathrm{~s} \quad 287.00 \mathrm{~nm}$

140.43306 iPKP 4945.20

iPP

iSKS $56 \quad 19.50$

JSC $\quad 141.96 \quad 41$ ePKP 4941.00

$142.78 \quad 76$ ePKP

142.8177 ePKP

$\begin{array}{ll}49 & 41.00 \\ 49 & 44.00\end{array}$

$\begin{array}{ll}49 & 44.00 \\ 49 & 51.40\end{array}$

145.84151 iPKP 4955.50

146.3960 iPKP+ 4955.00

$\begin{array}{lllll}147.68 & 156 & \text { ePKPC } & 49 & 41.00 \\ 148.89 & 83 & \text { ePKP } & 50 & 00.90\end{array}$

$148.09 \quad 83$ ePKP $50 \quad 00.90$

148.31128 ePKP 5000.00

148.54128 iPKP 4958.50

$0.4 \mathrm{~s} \quad 21.20 \mathrm{~nm}$

$\begin{array}{lll}\text { e } & 50 & 02.50 \\ \text { es } & 5206.00\end{array}$

$\begin{array}{ccccc}149.11 & 194 \text { eS } & 52 & 06.00 \\ \text { ePKP } & 50 & 00.90\end{array}$

5004.00
$1 \quad 5006.30$

LPB $151.27 \quad 146$ PKPC+ $50 \quad 04.20$

$2 \begin{array}{ccc}19 \mathrm{~s} & 146 \mathrm{PKPC+} \\ 2 & 6.88 \mathrm{um}\end{array}$

$\begin{array}{llll}50 & 18.38\end{array}$

$\begin{array}{llllllll} & & & \text { SS } & 06 & 13.00 & & \text { NST } \\ \text { ZOBO } & 151.47 & 146 & \text { iPKP } & 50 & 04.10 & 0.8 & \text { BDT }\end{array}$

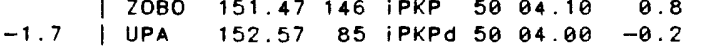

$$
\begin{array}{r|rr}
0.5 & 1.0 \mathrm{~s} & 60.00 \mathrm{~nm} \\
20 \mathrm{~s} & 4.61 \mathrm{um}
\end{array}
$$

$11.9 \times \quad i^{2} 20 \mathrm{~s} \quad 4.61 \mathrm{um} \quad 5211.80$

0.3

$-9.9 \times$ I PSO

$0.1 \quad$ BAO

$153.87 \quad 103$ ePKP $50 \quad 07.50$

156.75189 PKP $50 \quad 10.00$
157.75

$\begin{array}{llllll} & 157.75 & 96 & \text { iPKP } & 50 & 12.00\end{array}$

$\begin{array}{llllll}\text { BOCO } & 157.76 & 96 & \text { iPKP } & 50 & 12.00 \\ \text { BMG } & 158.95 & 90 & \text { ePKP } & 50 & 25.00\end{array}$

I TR

$\begin{array}{lrl}158.95 & 90 \text { ePKP } \\ 159.06 & 220 \text { ePKP }\end{array}$

$\begin{array}{ll}50 & 25.00 \\ 50 & 12.80\end{array}$

160.17214 ePKP 5014.50
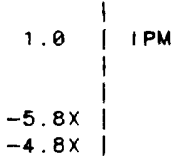

$2.6 \times$

1.5

0.

1.2 KLM

$3.2 \times$

2.2 NPA

0.2

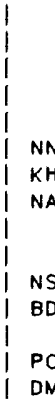

PCT

DMN

KKN

0.91

0.0 A 0.4

0.3 MTD

0.2 KR I
$12.4 \times$ I SHI

$\begin{array}{lllll}160.64 & 81 & \text { ePKP } 50 & 24.30 & 10.1 X\end{array}$

$0.4 \mathrm{~s} \quad 281.00 \mathrm{~nm}$

$\begin{array}{lllll}160.87 & 85 & \text { ePKP } 50 & 24.20 & 9.5 \mathrm{x}\end{array}$

$\begin{array}{llllll}161.36 & 84 & \text { ePKP } & 50 & 15.80 & 0.6\end{array}$

$\begin{array}{lllll}162.12 & 81 & \text { iPKPd } 50 & 16.58 & 0.7\end{array}$

$1.0 \mathrm{~s} \quad 97.50 \mathrm{~nm}$

$\begin{array}{lllll}162.51 & 51 & \text { IPKPC } 50 & 15.60 & -0.4\end{array}$

$0.9 \mathrm{~s} \quad 303.00 \mathrm{~nm}$

$219 \mathrm{~s} 34.70 \mathrm{um}$

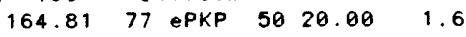

$\begin{array}{llllll}166.35 & 45 & \text { ePKP } & 50 & 18.00 & -1.5\end{array}$

$\begin{array}{llllll}167.03 & 46 & \text { ePKP } & 50 & 19.50 & -0.4\end{array}$

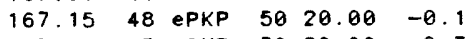

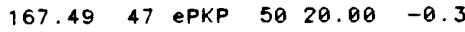

$\begin{array}{lllll}167.81 & 49 & \text { ePKP } 50 & 20.00 & -0.5\end{array}$

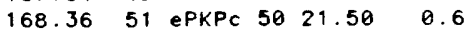

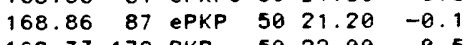

$\begin{array}{lllll}169.33 & 178 \text { PKP } 50 \quad 22.00 & 0.5\end{array}$

$\begin{array}{lllll}170.86 & 71 \text { IPKPC } 50 \quad 22.50 & 0.6\end{array}$

$1.2 \mathrm{~s} \quad 237.00 \mathrm{~nm}$

S.D. $=1.2$ on 414 of 588 obs.

Nov $30,198317 \mathrm{~h} 46 \mathrm{~m} 00.76 \pm 0.08 \mathrm{~s}$ $6.821 \mathrm{~S} \pm 2.0 \mathrm{~km} \quad 72.107 \mathrm{E} \pm 1.6 \mathrm{~km}$ DEPTH $=10.0 \mathrm{~km}$ (geophysicist)

$6.7 \mathrm{mb}$ ( 91 obs.) $7.5 \mathrm{Msz}$ ( 22 obs.)

CHAGOS ARCHIPELAGO REGION

(426)

Some domage (VI) to buildings ond piers on Diego Gorcio. Five

lagoon ond significont wave

domoge near the southeostern tip of the island. Forty $\mathrm{cm}$. Isunami ot Victorio, Seychelles. Lorge zone of discolored seo water

observed 35 to 40 nautical miles northwest of Diego Garcio.

$\begin{array}{llllll}17.76 & 18 & \mathrm{IPC} & 50 & 12.00 & 1.8\end{array}$ $20.97 \quad 15$ Pd $5047.10 \quad 0.5$ $1.0 \mathrm{~s} \quad 252.00 \mathrm{~nm} \quad 5.5 \mathrm{mb} \times$

$24.91 \quad 15$ i Pd $51 \quad 25.50 \quad 0.8$

$\begin{array}{llllll} & \text { eS } & 56 & 89.50 \\ 25.25 & 4 & \text { iPd } & 51 & 29.7 \theta & 1.0\end{array}$

26.59241 iPc $5142.80 \quad 1.4$

$28.3370 \mathrm{ePd} 5201.00 \quad 4.0 \mathrm{X}$

$\begin{array}{ccccc}28.39 & 71 \mathrm{ePc} & 51 & 58.50 & 0.9 \\ 0.8 \mathrm{~s} & 72.50 \mathrm{~nm} & 5.5 \mathrm{mb} \times\end{array}$

$28.9279 \mathrm{ePC} \quad 5203.50^{1.2}$

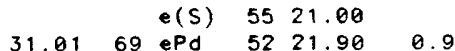

$\begin{array}{ccccc}31.01 & 69 \mathrm{ePd} & 52 & 21.90 & 0.9 \\ 0.7 \mathrm{~s} & 69.40 \mathrm{~nm} & & 5.7 \mathrm{mb}\end{array}$

e 5226.10

5240.00

$53 \quad 11.10$

$53 \quad 39.90$

5435.00

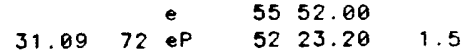

$32.37 \quad 75 \mathrm{eP}$

5233.50

$54 \quad 46.10$

5242.00

5400.00

5501.00

5533.00

$\begin{array}{rrrrr} & \text { eS } & 59 & 15.00 & \\ & \text { e } & 84 & 06.08 & \\ 33.55 & 55 \text { eP } & 53 & 08.00 & 16.8 \mathrm{x}\end{array}$

$\begin{array}{lllll}33.92 & 51 \mathrm{eP} & 52 & 53.50 & 7.1 \mathrm{x}\end{array}$

$\begin{array}{lllll}35.63 & 277 \text { iPd } & 53 & 03.00 & 1.7 \\ 1.0 \mathrm{~s} & 120.00 \mathrm{~nm} & & 5.7 \mathrm{mb}\end{array}$

5304.00

$\begin{array}{lllll}35.64 & 51 \mathrm{ePn} & 53 & 01.00 & -0.1\end{array}$

$\begin{array}{lllll}35.75 & 48 \mathrm{eP} & 53 & 82.00 & 0.0\end{array}$

$1.5 \mathrm{~s} \quad 2170.00 \mathrm{~nm}$

$36.07 \quad 54 \mathrm{eP}$

$36.46 \quad 20 \quad \mathrm{PPO}$

$36.5320 \quad \mathrm{PPd}$

$36.6920 \mathrm{iPd}$

$36.73 \quad 46$ iPd

$1.1 \mathrm{~s} \quad 127.00 \mathrm{~nm}$

es

$5304.80^{6.8 \mathrm{mb}}$

$\begin{array}{lll}53 & 09.30 & -1.1\end{array}$

$\begin{array}{lll}53 & 09.50 & 0.6\end{array}$

$\begin{array}{lll}53 & 10.90 & 0.8\end{array}$ 


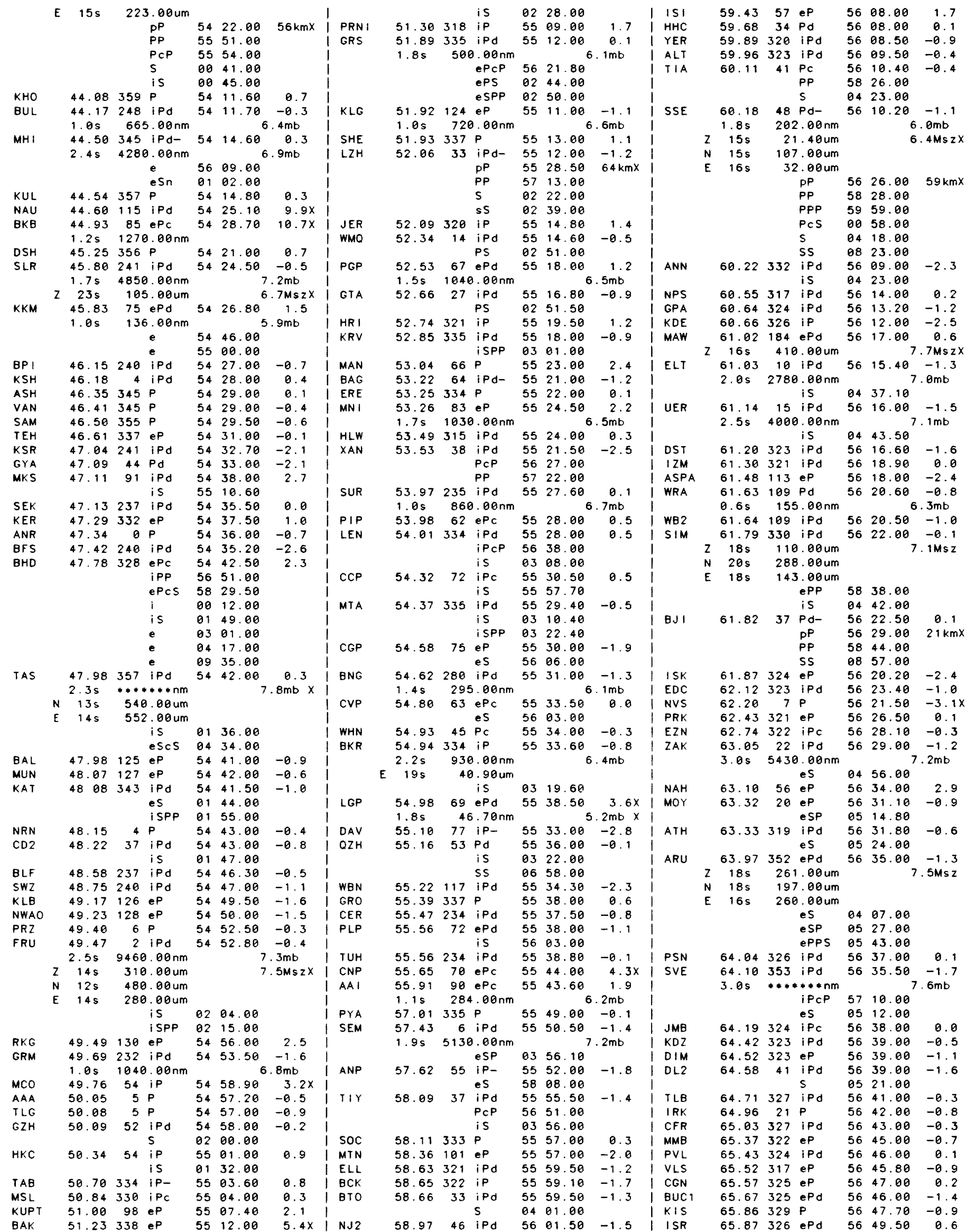




$\begin{array}{lrrl}\text { KZN } & 65.88 & 320 & \text { iPd } \\ \text { MVI } & 65.91 & 58 & \mathrm{eP} \\ \text { PPE } & 65.98 & 328 & \mathrm{ePC} \\ \text { VRI } & 66.24 & 327 & \mathrm{iPC} \\ \text { VTS } & 66.27 & 323 \mathrm{iPd} \\ \text { CLI } & 66.38 & 328 \mathrm{iPC} \\ \text { MLR } & 66.43 & 326 \mathrm{iPc} \\ \text { ISO } & 66.54 & 110 \mathrm{eP} \\ \text { IAS } & 66.65 & 329 \mathrm{eP} \\ \text { FKJ } & 66.68 & 50 \mathrm{eP} \\ & & & \mathrm{eS} \\ \text { CMP } & 66.79 & 326 & \mathrm{iPd}\end{array}$

CMP $\quad 66.79326$ iPd

$\begin{array}{lll}\text { OHR } & 66.87 & 325 \mathrm{eP} \\ \text { OHR } & 66.93 & 320 \mathrm{iP}\end{array}$

$66.97 \quad$ iPS

SKO

$1.5 \mathrm{~s} \quad 400.00 \mathrm{~nm}$

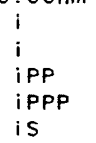

$\mathrm{COZ}$

NGS

SEO

$67.48 \quad 51 \quad \mathrm{eP}$

67.48 eP

iPd-

$1.6 \mathrm{~s} \quad 3360.00 \mathrm{~nm}$

$Z 17 \mathrm{~s} \quad 476.00 \mathrm{um}$

N $17 \mathrm{~s} \quad 246.00 \mathrm{um}$

E $17 \mathrm{~s} \quad 178.00 \mathrm{um}$

TA es

120

$67.5653 \mathrm{eP}$

$67.67 \quad 49 \mathrm{eP}$ es

KUM

MYZ

68.36325 eS

LCI $68.38318 \mathrm{iPC}$

SSR

68.46339 iPd

$Z$ 20s $302.00 \mathrm{um}$

E $18 \mathrm{~s} 260.00 \mathrm{um}$

$\begin{array}{lll}\text { iPP } & 59 & 38.00 \\ \text { is } & 96 & 04.00\end{array}$

isS $\quad 10604.00$

68.4690 iPd 5708.0

CJR $68.46326 \mathrm{eP}$

MOS $68.55340 \mathrm{P}$

TTG

$P C P$
$P P P$

ES

SHN

68.8050 ePd 5708.00

MS

STK

BR

OR

$\mathrm{CN} 2$

CE I

AS 2

GI

LVV

LVV $\quad 70.13329 \mathrm{P}$

SGO

UZH

5659.00

5702.00

5937.00

0544.00

0617.00

5656.00

665.00

5701.90

0556.80

5705.00

5705.00

0609.00

5708.00

$\begin{array}{ll}06 & 12.00 \\ 57 & 06.00\end{array}$

0612.00

5705.00

$\begin{array}{ll}57 & 04.50 \\ 10 & 30.50\end{array}$

5705.00

5703.60

5708.00

$\begin{array}{ll}57 & 04.30 \\ 25 & 22.38\end{array}$

5704.08

5725.00

0121.50

0611.50

$68.99315 \mathrm{eP}$

$69.02 \quad 121 \mathrm{eP}$

$69.03 \quad 51 \mathrm{eP}$

5717.00

5707.00

5703.08

0605.00

5710.00

69.14318 iPd

ipP 5731.50

0008.50

5709.40

5949.00

5714.00

5714.00

$06 \quad 29.00$

5715.00

5716.00

0632.00

$57 \quad 15.00$

$\begin{array}{ll}57 & 16.00 \\ 59 & 59.50\end{array}$

70.31327 IPd

5716.00

$$
67.00321 \mathrm{iPd}
$$

68.44324 iP

$1.2 \mathrm{~s} \quad 2210.00 \mathrm{~nm}$

$N$ 20s $210.00 \mathrm{um}$

\begin{tabular}{lll|l}
56 & 48.50 & -0.5 & BFD
\end{tabular}

$\begin{array}{lll}56 & 55.00 & 5.6 \times\end{array}$

\begin{tabular}{lll|l}
56 & 49.00 & -0.5 & I ERC
\end{tabular}

\begin{tabular}{lll|l}
56 & 51.00 & -0.1 & MTS
\end{tabular}

\begin{tabular}{rrr|l}
56 & 50.00 & -1.4 & \\
56 & 52.00 & 0.0 & Jos
\end{tabular}

\begin{tabular}{lll|}
56 & 53.00 & 0.5
\end{tabular}

$5650.00-3.6 \times$ DU

$\begin{array}{lll}56 & 54.00 & 0.3\end{array}$

$5701.00 \quad 6.9 \times$ | OKA

557.00

$\begin{array}{lll}50.00 & -4.7 \times \text { SPC }\end{array}$

$\begin{array}{lll}55.00 & -0.2\end{array}$

$\begin{array}{ll}56 & 53.70 \\ 06 & 14.20\end{array}$

$56 \quad 54.50$

$\begin{array}{lll}56 & 54.00 & -2.1\end{array}$

0635.00

$Z 17 \mathrm{~s} \quad 210.00 \mathrm{um}$

IPCP $57 \quad 31.00$

iPP 5954.00

is 0626.00

$\begin{array}{cc}71.74 & 327 \mathrm{eP} \\ 18 \mathrm{~s} & 351.00 \mathrm{um}\end{array}$
$.4 \mathrm{mb}$

HIM

I SRO

WKY

$A O U$

KRA

$\begin{array}{rrr}1.8 \mathrm{~s} & 1630.00 \mathrm{~nm} \\ \mathrm{z} & 18 \mathrm{~s} & 160.00 \mathrm{um}\end{array}$

$70.33127 \mathrm{eP}$

$70.35 \quad 50$ eP

$71.01314 \mathrm{eP}$

71.19327 iPd 0646.00

$1.4 \mathrm{~s} \quad 370.00 \mathrm{~nm}$

$\begin{array}{lllll}71.33 & 318 \mathrm{eP} & 57 & 23.50 & 0.7\end{array}$

$71.35 \quad 50$ ePP $\theta 016.00$

$\begin{array}{cl}5 & 5726.00 \\ e & 0728.00\end{array}$

5724.40

iPP
e

0021.70

$72.0150 \mathrm{eP}$

$72.05 \quad 325 \mathrm{eP}$

$72.14 \quad 51 \mathrm{eP}$

2531.00

$57 \quad 29.00$

0651.00

5725.50

5725.00

0705.00

72.35318 iPd 5729.00

$72.40328 \mathrm{ePd}$

5727.60

$18 \mathrm{~s} \quad 160.00 \mathrm{um}$

5730.10

5734.10

5742.30

5746.30

5959.00

$3 \mathrm{mb}$

$.8 M \operatorname{six}$

0213.00

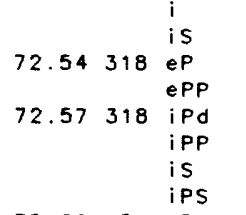

0650.00

5729.00

5729.50

$00 \quad 01.90$

0654.00

0732.00

$72.60 \quad 121 \mathrm{eP}$

$72.62 \quad 39 \mathrm{Pd}$

$72.66 \quad 51 \mathrm{eP}$

5729.00

5730.00

5733.00

$06 \quad 55.10$

72.71127 iPd-

5730.20

72.76318 iPd 5731.50

72.83109 iPd

$1.0 \mathrm{~s} \quad 123.00 \mathrm{~nm}$

5735.00

5739.50

5857.00

5929.00

0121.00

9207.00

5731.00

$72.83 \quad 109$ iP

$72.84318 \quad \mathrm{iPd}$

5732.00

$72.86 \quad 50$ eP

$\begin{array}{ll}57 & 32.00\end{array}$

1. Bs $440.00 \mathrm{~nm}$

$72.90 \quad 27$ iPd $5730.10 \quad-1$

$2.0 \mathrm{~s} 1010.00 \mathrm{~nm}$

$72.94325 \mathrm{eP}$

$5731.80-0.4$

$73.11330 \mathrm{eP}-$

0023.60

5732.00

5745.00

e

0115.00

73.29322 iPd

5734.10

$-5.9 \times \mid$ CEY

- 6 HIK

0.9

$73.3550 \mathrm{eP}$

5735.00

73.37322 iPd 5734.80

$0.6 \mathrm{~s} \quad 3.90 \mathrm{~nm}$

5737.50

EPP $\quad 0019.00$

ES $\quad 0655.00$

$73.42 \quad 325$ ePd 5734.00

5738.80

ipP $\quad 57 \quad 43.50$

0.3

$-0.3$

TR I

$73.68 \quad 322$

$\begin{array}{ll}57 & 49.00 \\ 57 & 35.60\end{array}$

$00 \quad 26.90$

EPPP $02 \quad 07.00$

ES 0656.00

ESP $07 \quad 33.00$

ESS 1200.00

$.5 \mathrm{MszX}$

eSSS

$73.7950 \mathrm{eP}$

1542.00

I GIF

$74.07 \quad 197$ iP

$\begin{array}{ll}57 & 39.00 \\ 57 & 40.00\end{array}$

\begin{tabular}{c|c}
-1.9 & \\
0.5 & RBL \\
0.6 & \\
$6.0 x$ & PUL \\
& \\
-1.4 & \\
$3 \mathrm{mb}$ & \\
0.7 &
\end{tabular}

$\begin{aligned} 74.15322 & \text { eP } \\ & \text { ePP }\end{aligned}$

5756.00

$5739.50 \quad 0.1$

$\begin{array}{lllll}1.85 & 3500 & & \end{array}$

$7 \quad 23 \mathrm{~s} 3500.00 \mathrm{~nm}$

$\mathrm{N} 23 \mathrm{~s} \quad 230.00 \mathrm{um}$

E $23 s \quad 160.00$ um

iPCP 5754.00

EPP $\quad 0029.00$

iPPP $02 \quad 17.00$

is 0710.00

$-0.9 \quad$

PMG

$\begin{array}{lll}74.22 & 98 \text { iPd }\end{array}$

$\begin{array}{ll}07 & 10.00 \\ 57 & 39.20\end{array}$

$74.28 \quad 51 \mathrm{eP} \quad 5742.00$

e 0724.00

$74.39-319$ eP

5740.50

$5741.00-0.3$

iPP $\quad 0040.00$

is 0708.00

$\begin{array}{lllllll}\text { KBA } & 74.58 & 323 \text { iPd } 5741.20 & -0.8\end{array}$

$2.7 \mathrm{~s} 6230.00 \mathrm{~nm}$

$57 \quad 45.60$

$\begin{array}{lll}i & 5749.50\end{array}$

$74.59324 \mathrm{iP}-\quad 5741.30-0.5$

$74.6450 \mathrm{eP} \quad 5749.00$

0726.50

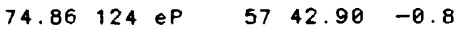

$75.0174 \mathrm{eP}-\quad 5743.00-1.8$

$1.5 \mathrm{~s} \quad 1410.00 \mathrm{~nm}$

$75.0474 \mathrm{eP}-5743.00 \quad-2.0$

$0.9 \mathrm{~s} \quad 840.00 \mathrm{~nm}$

$Z 18 \mathrm{~s} \quad 84.50 \mathrm{um}$ 


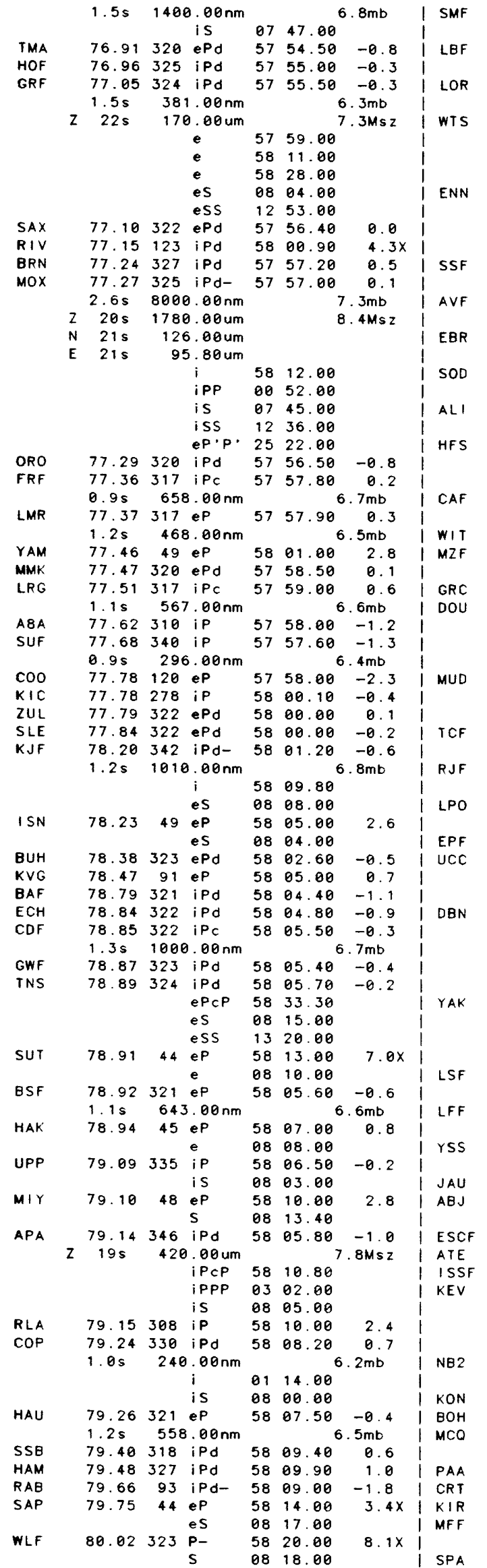

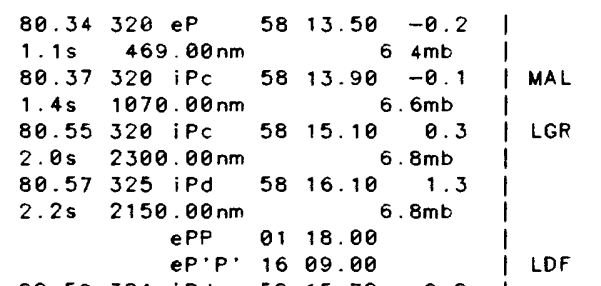

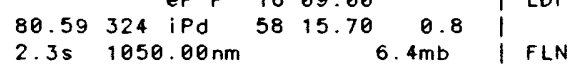

\begin{tabular}{cc|c|c}
$i$ & 58 & 20.10 & \\
ePP & 01 & 23.50 & TOL
\end{tabular}

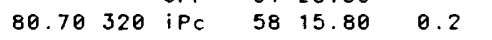

$\begin{array}{lll}0.8 \mathrm{~s} & 735.00 \mathrm{~nm} & 6.7 \mathrm{mb} \\ 80.70 \quad 320 \mathrm{eP} & 58 & 15.50^{-0.1}\end{array}$

$1.5 \mathrm{~s} \quad 752.00 \mathrm{~nm} \quad 6.5 \mathrm{mb}$

80.74313 eP $\quad 58 \quad 15.00 \quad-0.9$

80.75344 ip $\quad 08 \quad 39.00$

i $\quad 5824.80$
80.76311 ipt 5816.50

$\begin{array}{ccccc}80.76311 \mathrm{iPt} & 58 & 16.50 & 0.4 \\ & \mathrm{eS} & 09 & 04.50 & \\ 80.90334 \mathrm{eP} & 58 & 16.20 & -0.2\end{array}$

$0.8 \mathrm{~s} \quad 136.00 \mathrm{~nm} \quad 6.0 \mathrm{mb}$

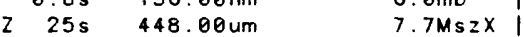

$80.92317 \mathrm{eP} 5817.901 .0$ | LPF

\begin{tabular}{cc|c|c}
$1.4 \mathrm{~s}$ & $735.00 \mathrm{~nm}$ & $6.5 \mathrm{mb}$ & \\
$80.98 \quad 326 \mathrm{ePd}$ & 58 & $19.00^{2.1}$ & SBA
\end{tabular}

\begin{tabular}{lllll|l}
80.98 & 326 ePd & 58 & 19.00 & 2.1 & SBA \\
81.01 & $319 \mathrm{eP}$ & 58 & 17.70 & 0.4 & TRO
\end{tabular}

$1.3 \mathrm{~s} 607.00 \mathrm{~nm} \quad 6.5 \mathrm{mb}$ SFS

$\begin{array}{llllll}81.06 & 320 \mathrm{iPc} & 58 & 18.40 & 0.9\end{array}$

$\begin{array}{lllll}81.12323 \mathrm{P} & 58 & 18.10 & 0.4\end{array}$

$0.8 \mathrm{~s} \quad 234.00 \mathrm{~nm}$

$\begin{array}{ccc}e & 0136.00 \\ S & 0830.00\end{array}$

$\begin{array}{llllll}81.22 & 330 & \text { iPd } & 58 & 19.90 & 1.8\end{array}$

$0.9 \mathrm{~s} \quad 160.00 \mathrm{~nm}$

$\begin{array}{llllll}81.28 & 319 & 01 & 27.00 & \\ 1.75 & 58 & 19.50 & 0.8\end{array}$

\begin{tabular}{cc|c}
$1.7 \mathrm{~s} 650.00 \mathrm{~nm}$ & $6.4 \mathrm{mb}$ & KUR
\end{tabular}

$81.41318 \mathrm{iPc} 5820.50^{6.4 \mathrm{mb}} 1.1$

$1.4 \mathrm{~s} 1470.00 \mathrm{~nm} \quad 6.8 \mathrm{mb}$ T TIK

$\begin{array}{lllllll}81.44 & 317 \quad \mathrm{PPC} & 58 & 20.70 & 1.2 & \text { SVO }\end{array}$

$0.8 \mathrm{~s} 610.00 \mathrm{~nm} \quad 6.7 \mathrm{mb}$ | HNR

$81.51315 \mathrm{eP} \quad 5820.80^{0.7 \mathrm{mb}} 0.8$

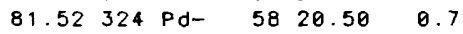

$\begin{array}{lll}e & 5823.70\end{array}$

$\begin{array}{lllll}81.54325 \mathrm{iP}- & 0828.00 & 20.00 & 0.2\end{array}$

$20 \mathrm{~s} 90.00 \mathrm{um} \quad$ 7.1Msz

\begin{tabular}{c|c|c} 
is & 0834.00 & PTO \\
iSCS 0846.00 &
\end{tabular}

eSS $13 \quad 39.00$
.524

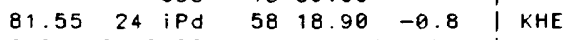

$2.0 \mathrm{~s} 2190.00 \mathrm{~nm}$

$$
\begin{array}{lll}
\text { is } & 08 & 28.70^{6} \\
\text { iSSS } & 17 & 15.90
\end{array}
$$

81.71319 iPC $58 \quad 21.70 \quad 0.8$

$0.8 \mathrm{~s} 485.00 \mathrm{~nm} \quad 6.6 \mathrm{mb}$ DLE

$\begin{array}{llllll}81.82 & 317 \text { iPC } 58 \quad 22.70 & 1.2 & \text { DMU }\end{array}$

$0.9 \mathrm{~s} \quad 771.00 \mathrm{~nm} \quad 6.8 \mathrm{mb}$

\begin{tabular}{llllll|l}
81.98 & 41 & iPd 58 & 22.00 & -0.2 & MSZ
\end{tabular}

$1.4 \mathrm{~s} \quad 950.00 \mathrm{~nm} \quad 6.7 \mathrm{mb}$

$82.01315 \mathrm{eP} \quad 5824.70 \quad 1.9$

$82.09 \quad 44 \mathrm{eP}^{\mathrm{P}} \quad 5823.00 \quad 0.1 \quad$ KOU

\begin{tabular}{rrrrr|r|r}
82.17 & $315 \mathrm{eS}$ & 08 & 43.00 & & MGD \\
\hline & 58 & 25.10 & 1.7 & VAL
\end{tabular}

$\begin{array}{lllll}82.17 & 315 \mathrm{eP} & 58 & 25.10 & 1.7 \\ 82.26 & 315 \mathrm{eP} & 58 & 25.70 & 1.8\end{array}$

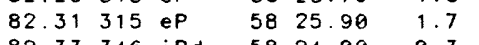

$82.33 \quad 346$ iPd- $5824.00 \quad 0.3$

$1.1 \mathrm{~s} 540.00 \mathrm{~nm}$

$\begin{array}{ccc}i & 5833.20\end{array}$

$82.41335 \mathrm{eS} \quad 0840.00$

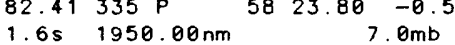

82.46333 iPd $5824.40^{7.0 m b}-0.1$ NOU

$82.48315 \mathrm{eP} \quad 5827.40 \quad 2.3$ | SEY

$\begin{array}{llllll}82.67 & 144 \mathrm{eP} & 58 & 30.00 & 4.3 \mathrm{X}\end{array}$

$1.3 \mathrm{~s} \quad 1.20 \mathrm{~nm}$

$82.73 \quad 95 \mathrm{eP}^{2} \quad 5825.00^{3.9 \mathrm{mb} X}$

$82.83 \quad 309$ iPd $5828.70 \quad 1.7$ TEN

$\begin{array}{lllll}82.85 & 343 \text { iP } & 58 & 26.60 & 0.1\end{array}$

$\begin{array}{lllll}82.92 & 319 \mathrm{iPC} & 58 & 2790 & 0.7\end{array}$

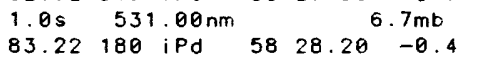

$1.0 \mathrm{~s} \quad 508.00 \mathrm{~mm}$

$6.7 \mathrm{mb}$

$22 \mathrm{~s} 61.60 \mathrm{um} \quad 6.9 \mathrm{Msz}$

83.35308 iPd 58

$\begin{array}{llllll}83.36 & 314 & \mathrm{iPd} \quad 58 & 31.40 & 1.8\end{array}$

$2.2 \mathrm{~s} \quad 5.90 \mathrm{~nm}$

5846.00

iPP 0146.00

$\begin{array}{lll}\text { iPP } & 01 & 46.00 \\ \text { is } & 08 & 52.00\end{array}$

$83.51320 \mathrm{eP} \quad 58 \quad 30.70 \quad 0.5$

$1.5 \mathrm{~s} \quad 1220.00 \mathrm{~nm} \quad 6.9 \mathrm{mb}$

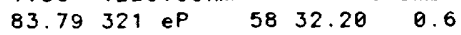

$1.5 \mathrm{~s} 1080.00 \mathrm{~nm} \quad 6.8 \mathrm{mb}$

83.83311 iP- $5833.50 \quad 1.5$ 5843.00 5850.00

IPP 0204.00

$\begin{array}{lll}\text { IPPP } 0402.00 & 02.00\end{array}$

is $\quad 0858.00$

i $\quad 0919.00$

iPS 0951.00

isS 1409.00

$\begin{array}{lll}\text { iSSS } & 18 & 13.00 \\ \text { i } & 24 & 58.00\end{array}$

$\begin{array}{lllll}83.92 & 320 \text { eP } \quad 58 \quad 32.60 & 0.4\end{array}$

$1.5 \mathrm{~s} \quad 1480.00 \mathrm{~nm} \quad 7.0 \mathrm{mb}$

$83.93 \quad 320$ iPc $58 \quad 32.80 \quad 0.5$

$1.2 \mathrm{~s} \quad 1350.00 \mathrm{~nm} \quad 7.0 \mathrm{mb}$

$84.36 \quad 168 \mathrm{eP} \quad 58 \quad 35.00 \quad 1.0$

$\begin{array}{llllll}84.38 & 344 & \text { iP } & 58 & 34.00 & -0.2\end{array}$

84.70308 iPd $5834.00-2.4$

i $\quad 5846.00$

i 5900.00

IPP $02 \quad 04.00$

IPPP $05 \quad 01.00$

is 0910.00

iSS $15 \quad 10.00$

iSSS 1900.00

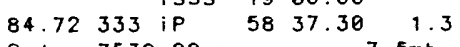

$2.4 \mathrm{~s} \quad 7530.00 \mathrm{~nm} \quad 7.5 \mathrm{mb}$

$84.89 \quad 43 \mathrm{iPd} \quad 58 \quad 36.00 \quad-1.1$

86.52 is 0908.00

$\begin{array}{llllll}86.52 & 15 P & 58 & 44.00 & -0.8\end{array}$

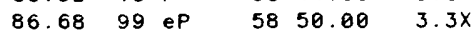

$86.7899 \mathrm{ePc}-58 \quad 46.00-1.2$

$\begin{array}{llll}8.3 \mathrm{~s} & 538.00 \mathrm{~nm} & 6.6 \mathrm{mb}\end{array}$

$\begin{array}{lll}\text { PP } & 01 & 10.00\end{array}$

PPP $03 \quad 12.00$

PS 0520.00

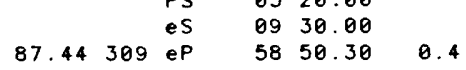

$87.49312 \mathrm{iPd} \quad 5851.50 \quad 1.4$

is 0750.00

iPS $08 \quad 40.00$

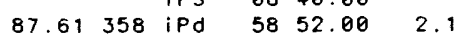

$1.7 \mathrm{~s} \quad 3070.00 \mathrm{~nm} \quad 7.3 \mathrm{mb}$

$\begin{array}{lll}\text { EPP } & 02 & 15.00\end{array}$

iPPP 0412.00

$\begin{array}{llllll}88.68 & 324 \mathrm{eP} & 58 & 53.00 & -2.5\end{array}$

$\begin{array}{llllll}88.68 & 324 & \text { eP } & 58 & 53.00 & -2.5 \\ 89.03 & 584.20 & -3.0 x\end{array}$

$2.1 \mathrm{~s} 5600.00 \mathrm{~nm} \quad 7.5 \mathrm{mb}$

$89.37135 \mathrm{eP} \quad 5859.00 \quad 0.0$

(PP) $59 \quad 10.90 \quad 38 \mathrm{kmX}$

$\begin{array}{lll}(P P) & 59 & 10.90 \\ i & 59 & 17.90\end{array}$

$\begin{array}{llllll}89.66 & 111 & \mathrm{iPC} & 59 & 03.20 & 2.4\end{array}$

$\begin{array}{llllll}90.26 & 29 P & 59 & 00.00 & -2.8\end{array}$

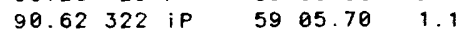

$\begin{array}{rrrrr} & \text { is } & 09 & 40.00 & \\ 91.43 \quad 39 \text { eP } & 59 & 08.40 & 0.1\end{array}$

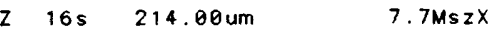

N $18 \mathrm{~s} \quad 54.00 \mathrm{um}$

E $18 \mathrm{~s} \quad 75.60 \mathrm{um}$

iPPP $04 \quad 42.20$

is $10 \quad 12.00$

iPPS 1153.00

$\begin{array}{llllll}91.44 & 113 & \text { IPPS } & 11 & 53.00 & \\ 9 & 1.69 & 09.00 & 0.0\end{array}$

$\begin{array}{lrllll}91.62 & 27 & \mathrm{iPd} & 59 & 08.80 & -0.2\end{array}$

$2.1 \mathrm{~s} \quad 1320.00 \mathrm{~nm} \quad 6.9 \mathrm{mb}$

$\begin{array}{ccccc} & \text { eSKS } & 09 & 44.80 & \\ & \text { ePS } & 11 & 23.00 & \\ 91.76298 & \text { eP } & 59 & 12.00 & 1.6 \\ & \text { ePP } & 03 & 04.00 & \end{array}$

S $\quad 1028.00$

$\begin{array}{lllll}93.32 & 37 \mathrm{iPd} \quad 59 & 15.00 & -2.0\end{array}$

$2.0 \mathrm{~s} \quad 830.00 \mathrm{~nm} \quad 6.8 \mathrm{mb}$ 


\begin{tabular}{|c|c|c|c|c|c|c|c|c|c|c|c|c|}
\hline & & & ePP & 03 & 06.00 & & | OT & 132.53 & 329 & ePKP & 05 & 19.00 \\
\hline & & & eSKS & 09 & 50.00 & & I EDM & 133.52 & 5 & ePKP & 05 & 09.50 \\
\hline & & & es & 10 & 20.00 & & SEG & 133.67 & 286 & ePKP & 05 & 25.00 \\
\hline & & & ePS & 11 & 43.00 & & MON & 133.68 & 285 & ePKP & 05 & 30.50 \\
\hline & & & ess & 16 & 36.00 & & PAG & 133.88 & 286 & ePKP & 05 & 25.00 \\
\hline vC & 93.82 & 108 & $\mathrm{IPC}$ & 59 & 16.60 & $-3.4 x$ & RN & 133.92 & 278 & ePKP & 5 & 22.40 \\
\hline $\mathrm{CW}$ & 94.62 & 132 & $\mathbf{P}$ & 59 & 22.20 & -1.1 & & $1.0 \mathrm{~s}$ & 100 & $0.00 \mathrm{~nm}$ & & \\
\hline$R Z$ & 94.83 & 125 & eP & 59 & 28.00 & $3.6 x$ & PA & 133.93 & 287 & ePKP & 05 & 23.50 \\
\hline VEL & 94.96 & 132 & $\mathbf{P}$ & 59 & 24.00 & -0.9 & PB & 134.30 & 239 & PKP & 05 & 04.00 \\
\hline & $z \quad 18 s$ & 137 & $7.00 \mathrm{um}$ & & & $.5 \mathrm{Msz}$ & & & & $i$ & 85 & 26.00 \\
\hline & $N \quad 18 \mathrm{~s}$ & 82 & $2.50 \mathrm{um}$ & & & & i & & & $\mathrm{pP}$ & 07 & 49.00 \\
\hline & & & $5.28 \mathrm{um}$ & & & & 2080 & 134.45 & 239 & ePKP & 05 & 14.00 \\
\hline & & & PP & 83 & 20.00 & & & $1.0 \mathrm{~s}$ & 155 & $5.00 \mathrm{~nm}$ & & \\
\hline & & & PPP & 05 & 30.00 & & & $z 20 \mathrm{~s}$ & & $3.50 \mathrm{um}$ & & \\
\hline & & & SKS & 89 & 56.00 & & I RSON & 134.54 & 347 & ePKP & 85 & 19.30 \\
\hline & & & e & 10 & 48.00 & & I PMO & 134.81 & 119 & iPKP & 85 & 25.20 \\
\hline VG & 95.64 & 132 & $\mathbf{P}$ & 59 & 27.80 & -0.3 & i & $1.2 \mathrm{~s}$ & 95 & $5.00 \mathrm{~nm}$ & & \\
\hline KU & 96.25 & 335 & iP & 59 & 35.70 & $5.5 x$ & VAH & 134.93 & 119 & IPKP & 05 & 23.30 \\
\hline & $2.1 \mathrm{~s}$ & 907 & $.00 \mathrm{~nm}$ & & & $.9 \mathrm{mb}$ & & $1.2 \mathrm{~s}$ & & $0.00 \mathrm{~nm}$ & & \\
\hline$A G$ & 96.79 & 347 & $\mathrm{iPd}$ & 59 & 32.50 & 0.0 & I TPT & 135.05 & 119 & iPKP & 85 & 23.90 \\
\hline & $0.8 \mathrm{~s}$ & & $.40 \mathrm{~nm}$ & & & $.7 \mathrm{mb}$ & & $1.2 \mathrm{~s}$ & 80 & $0.00 \mathrm{~nm}$ & & \\
\hline & & & $\mathrm{i}$ & 03 & 36.00 & & RUV & 135.17 & 119 & IPKP & 05 & 23.40 \\
\hline GNZ & 98.04 & 130 & $\mathbf{P}$ & 59 & 40.10 & 1.2 & i & $1.2 \mathrm{~s}$ & 110 & $0.00 \mathrm{~nm}$ & & \\
\hline & & & $e(S)$ & 83 & 55.00 & & GUV & 135.57 & 274 & ePKP & 05 & 24.20 \\
\hline $1 L T$ & 103.46 & 22 & iPdiff & 100 & 03.00 & 0.5 & I $P G C$ & 136.28 & 15 & ePKP & 05 & 24.50 \\
\hline & $1.6 \mathrm{~s}$ & 100 & $0.00 \mathrm{~nm}$ & & 6 & $.3 \mathrm{mb}$ & SES & 136.49 & 3 & ePKP & 05 & 15.50 \\
\hline & & & iPP & 84 & 22.00 & & I PNT & 136.51 & 11 & ePKP & 05 & 13.00 \\
\hline & & & iSKS & 10 & 44.80 & & & $2.0 \mathrm{~s}$ & 276 & $5.00 \mathrm{~nm}$ & & \\
\hline & & & es & 11 & 40.00 & & SJG & 137.83 & 289 & ePKP & 05 & 19.00 \\
\hline & & & IPS & 13 & 28.00 & & & $z 20 s$ & 110 & $0.00 u m$ & & 7 \\
\hline I TR & 109.05 & 258 & ePdiff & 100 & 41.10 & $12.4 x$ & I NEW & 137.96 & 9 & ePKP & 05 & 20.00 \\
\hline RDJ & 110.25 & 243 & iPdiff & 100 & 36.80 & $3.0 x$ & $i$ & & & e & 08 & 21.00 \\
\hline$M B C$ & 110.33 & 3 & ePdift & 100 & 36.00 & $3.0 x$ & LDM & 04 & 7 & ePKP & 05 & 19.70 \\
\hline & $0.8 \mathrm{~s}$ & & $1.00 \mathrm{~nm}$ & & & & UTO & 3 & 332 & ePKPc & 05 & 23.50 \\
\hline IMA & 112.97 & 19 & ePditf & 100 & 52.00 & $6.9 x$ & I CAR & 139.36 & 278 & iPKPc & 05 & 31.80 \\
\hline AFI & 113.35 & 108 & ePKP & 04 & 56.00 & $14.4 x$ & I & $s$ & 88 & $8.00 \mathrm{~nm}$ & & \\
\hline & & & e & 85 & 40.00 & & OR & 05 & 17 & ePKP & 05 & $\theta 0$ \\
\hline & & & e & 15 & 12.00 & & RKT & 17 & 139 & iPKP & 05 & 36.40 \\
\hline & & & e & 21 & 36.00 & & & $1.2 \mathrm{~s}$ & 105 & $5.00 \mathrm{~nm}$ & & \\
\hline & & & e & 33 & 00.00 & & I BLA & 140.75 & 324 & ePKP- & 05 & 22.80 \\
\hline VAO & 113.50 & 241 & $e(P d i f$ & 81 & 04.00 & $15.6 x$ & & & & e & 05 & 29.80 \\
\hline & & & e & 01 & 11.10 & & & & & e & 85 & 46.80 \\
\hline INK & 116.27 & 10 & ePKP & 84 & 39.00 & $-6.6 x$ & & & & eP & 08 & 27.60 \\
\hline & $1.5 \mathrm{~s}$ & 373 & $.00 \mathrm{~nm}$ & & & & RM & 140.95 & 5 & ePKP & 05 & 25.70 \\
\hline AO & 116.63 & 249 & $e(P K P)$ & 04 & 10 & 1.0 & SSD & 142.69 & 355 & ePKP & 05 & 31.30 \\
\hline LPA & 117.25 & 225 & ePKP+ & 84 & 56.00 & $7.5 x$ & SDV & 143.04 & 276 & ePKP & 05 & 34.80 \\
\hline & $z 205$ & 163 & $.00 u m$ & & & $.6 \mathrm{Msz}$ & FHC & 143.24 & 21 & ePKP & 05 & 32.80 \\
\hline PMR & 117.43 & 21 & PKP & 04 & 47.60 & -0.4 & i JsC & 143.24 & 322 & iPKP & 05 & 34.80 \\
\hline & $z 20 \mathrm{~s}$ & 250 & $.00 \mathrm{um}$ & & & $.8 \mathrm{Msz}$ & AV & 143.55 & 275 & ePKP & 05 & 37.00 \\
\hline $\mathrm{SCH}$ & 121.95 & 333 & ePKPC & 04 & 56.50 & -0.4 & N & 143.64 & 278 & ePKP & 05 & 41.70 \\
\hline & $1.0 \mathrm{~s}$ & 122 & $.00 \mathrm{~nm}$ & & & & DC & 143.92 & 19 & ePKP & 05 & 34.60 \\
\hline & & & PP & 05 & 32.50 & & BDW & 144.16 & 2 & ePKP & 05 & 36.20 \\
\hline$T B$ & 3 & 262 & PKP & 05 & 00.00 & -1.1 & $M I N$ & 144.43 & 18 & ePKP & 05 & 30 \\
\hline CA & 7 & 225 & $e(P K P)$ & 85 & 03.00 & 1.6 & FVM & 16 & 335 & iPKP & 5 & \\
\hline YKA & 4.22 & 4 & ePKP & 85 & 03.40 & 2.4 & MG & 145.44 & 273 & ePKP & 05 & $\partial \theta$ \\
\hline RSNT & .24 & 4 & ePKP & 04 & 59.50 & -1.5 & $B O C O$ & 146.25 & 268 & ePKP & 05 & 43.00 \\
\hline YKC & 124.24 & 4 & ePKPd & 05 & 00.50 & -0.5 & & $1.5 \mathrm{~s}$ & 1310 & $.00 \mathrm{~nm}$ & & \\
\hline & $1 \mathrm{~s}$ & 169 & $.00 \mathrm{~nm}$ & & & & $B O G$ & 146.27 & 268 & IPKP & 5 & \\
\hline FA & 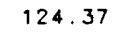 & 9 & ePKPC & 05 & & & & 5 & 21 & iP & 05 & \\
\hline DN & $\theta$ & 4 & $K P$ & 05 & .50 & & EUR & 146.68 & 11 & $i($ PKP) & 85 & 5 \\
\hline$z$ & 2 & 1 & $i(P K P)$ & 85 & .90 & & JAS & 146.99 & 18 & ePKP & 05 & \\
\hline $\mathrm{CH}$ & 45 & 9 & ¡PKPC & 05 & 09.50 & 2 & GLD & 147.13 & 356 & ePKP & 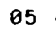 & 44.50 \\
\hline$A C$ & 6 & 219 & ePKP & 5 & .00 & $x$ & i & $z \quad 18 \mathrm{~s}$ & 79 & $9.10 \mathrm{um}$ & & \\
\hline EN & 8 & 222 & ePKP & 5 & .00 & 2 . & $-1 C$ & 147.14 & 20 & ePKP & 05 & 45.50 \\
\hline y) & & 8 & iPKPd & $\theta$ & 59 & & $\mathrm{DL}$ & 147.19 & 356 & ePKP & 05 & 44.50 \\
\hline EL & 82 & 219 & IPKPC & 5 & .00 & -2.1 & & $20 \mathrm{~s}$ & 65 & $5.00 \mathrm{um}$ & & \\
\hline $\mathrm{ACH}$ & & 220 & ePKP & 5 & $5 a$ & -5.1 & & 147.19 & 15 & ePKP & 05 & \\
\hline Cc & & 1 & e & 05 & .0 & -0 & & .53 & 279 & & 05 & \\
\hline LL & & 2 & ef & 05 & 1 & -1 & & .73 & 21 & & 05 & \\
\hline JA & & 234 & ePr & 85 & 8 & -9 & $|F R|$ & 148.09 & 18 & & 05 & \\
\hline TBI & & 128 & ePKP & 05 & 11 & & & 148.58 & 340 & & 05 & \\
\hline & & 455 & $.00 \mathrm{~nm}$ & & & & & 148.56 & 20 & & 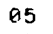 & \\
\hline H & & 326 & ip & 05 & & & & & a & & 05 & \\
\hline UT & & & & & & & & & & & -05 & 10 \\
\hline$=c$ & 8 & 355 & ePKP & 85 & 16.00 & -0.1 & & $1.3 \mathrm{~s}$ & 6060 & & & \\
\hline & & & & & & & & 140 & 132 & & & \\
\hline$R$ & 13 & 121 & ePKP & 85 & 22.00 & $.7 x$ & I PSO & 149.01 & 261 & iP & 05 & \\
\hline & & 160 & & & & & & & 341 & & 05 & \\
\hline$E$ & 132.18 & 121 & ePKP & 05 & 22.00 & $x$ & RM & & 5 & & 85 & \\
\hline & & & $.00 \mathrm{~nm}$ & & & & $\sigma$ & & 6 & iP & 05 & \\
\hline IT & 132.23 & 121 & ePKP & 85 & 22.00 & $4 x$ & CLC & 149.80 & 16 & ePKP & 05 & 53 \\
\hline & & & & & & & ort & & & 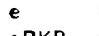 & 05 & \\
\hline & 132.37 & 1216 & ePKP & 85 & 21.00 & $3.1 x$ & OC & & 343 & & 05 & \\
\hline & & & & & & & & & 5 & & 05 & \\
\hline & $\mathrm{s}$ & $\begin{array}{c}121 \\
18 \theta\end{array}$ & $.00 \mathrm{~nm}$ & & & & & 91.16 & 18 & & $\begin{array}{l}05 \\
05\end{array}$ & $\begin{array}{l}5 \\
5\end{array}$ \\
\hline $\mathrm{N}$ & 132.49 & 328 & i PKP & 05 & 18.80 & .4 & & & & . & 05 & 58.00 \\
\hline
\end{tabular}




\begin{tabular}{|c|c|c|c|c|c|c|c|c|c|c|c|}
\hline LPO & 35.06 & 18 & eP & 18 & 23.70 & -0.4 & I WLF & & 41.05 & 19 & $\mathrm{P}+$ \\
\hline & $1.2 \mathrm{~s}$ & 963 & $3.00 \mathrm{~nm}$ & & & $.5 \mathrm{mb}$ & 1 & & & & PP \\
\hline LFF & 35.16 & 18 & eP & 18 & 24.80 & -0.1 & $i$ & & & & $S$ \\
\hline CAF & 35.56 & 19 & $\mathrm{eP}$ & 18 & 27.80 & -0.5 & I GAP & & 41.08 & 25 & eP \\
\hline & $1.1 \mathrm{~s}$ & 696 & $6.00 \mathrm{~nm}$ & & 6 & $4 m b$ & 1 & & $0.8 \mathrm{~s}$ & 476 & $6.00 \mathrm{~nm}$ \\
\hline RJF & 35.72 & 18 & eP & 18 & 29.10 & -0.6 & | CEY & & 41.30 & 30 & ePc \\
\hline & $1.2 \mathrm{~s}$ & 347 & $7.00 \mathrm{~nm}$ & & 6 & $1 \mathrm{mb}$ & I ATB & & 41.30 & 251 & iP \\
\hline GIB & 35.81 & 39 & $\mathrm{eP}$ & 18 & 31.00 & 0.4 & I RBL & & 41.40 & 28 & IPd \\
\hline & & & ePP & 19 & 10.50 & & I UCC & & 41.50 & 17 & Pd- \\
\hline LMR & 35.84 & 25 & $i P c$ & 18 & 30.70 & 0.0 & i & & $1.2 \mathrm{~s}$ & 880 & $0.00 \mathrm{~nm}$ \\
\hline & $1.2 \mathrm{~s}$ & 900 & $0.00 \mathrm{~nm}$ & & & $.5 \mathrm{mb}$ & 1 & & & & PP \\
\hline LRG & 35.88 & 25 & $\mathrm{iPc}$ & 18 & 31.10 & 0.1 & I & & & & $s$ \\
\hline & $1.2 \mathrm{~s}$ & 810 & $0.00 \mathrm{~nm}$ & & & $.5 \mathrm{mb}$ & I LJU & & 41.57 & 30 & iPc \\
\hline FRF & 36.08 & 25 & iPc & 18 & 32.60 & -0.2 & I & & & & iPP \\
\hline & $1.2 \mathrm{~s}$ & 594 & $4.00 \mathrm{~nm}$ & & & $.3 \mathrm{mb}$ & 1 & & & & es \\
\hline MFF & 36.43 & 16 & eP & 18 & 35.50 & -0.2 & I DLE & & 41.70 & 6 & $i P d$ \\
\hline & 1.15 & 548 & $8.00 \mathrm{~nm}$ & & 6 & $.3 \mathrm{mb}$ & 1 & & $0.9 \mathrm{~s}$ & $152 \theta$ & $0.00 \mathrm{~nm}$ \\
\hline LSF & 36.58 & 18 & $\mathrm{eP}$ & 18 & 36.60 & -0.3 & I FUR & & 41.71 & 25 & $i P c$ \\
\hline & $1.0 \mathrm{~s}$ & 438 & $8.00 \mathrm{~nm}$ & & 6 & $2 \mathrm{mb}$ & 1 & & $1.4 \mathrm{~s}$ & 145 & $5.00 \mathrm{~nm}$ \\
\hline TCF & 36.82 & 18 & eP & 18 & 38.90 & -0.1 & 1 & $z$ & $19 \mathrm{~s}$ & 46 & $6.60 \mathrm{um}$ \\
\hline MSI & 36.90 & 40 & eP & 18 & 42.50 & $2.9 x$ & 1 & & & & es \\
\hline LPF & 37.54 & 14 & eP & 18 & 45.00 & 0.0 & ITG & & 41.71 & 37 & $e(P)$ \\
\hline & 1.25 & 437 & $7.00 \mathrm{~nm}$ & & & $1 \mathrm{mb}$ & 1 & & & & e \\
\hline RDP & 37.59 & 33 & eP & 18 & 46.50 & 1.0 & 1 & & & & e \\
\hline RMP & 37.62 & 33 & iPd & 18 & 48.00 & 2.3 & I KBA & & 41.77 & 28 & iPd \\
\hline & & & iPP & 20 & 12.00 & & 1 & & $2.0 \mathrm{~s}$ & 1340 & $0.00 \mathrm{~nm}$ \\
\hline & & & is & 24 & 36.00 & & 1 & & & & $\mathrm{i}$ \\
\hline$A \vee F$ & 37.63 & 19 & eP & 18 & 46.10 & 0.4 & 1 & & & & $\mathrm{i}$ \\
\hline & $1.1 \mathrm{~s}$ & 454 & $4.00 \mathrm{~nm}$ & & & $2 \mathrm{mb}$ & 1 & & & & ipPP \\
\hline SMF & 37.66 & 20 & eP & 18 & 46.00 & 0.0 & 1 & & & & $\mathrm{i}$ \\
\hline & $1.1 \mathrm{~s}$ & 537 & $7.00 \mathrm{~nm}$ & & & $2 \mathrm{mb}$ & 1 & & & & $\mathrm{i}$ \\
\hline SSF & 37.92 & 19 & $i P c$ & 18 & 48.60 & 0.5 & I OHR & & 1.88 & 40 & iP \\
\hline GRR & 37.92 & 14 & iPc & 18 & 48.30 & 0.2 & I ENN & & 41.94 & 18 & iPd \\
\hline & 0.75 & 343 & $3.00 \mathrm{~nm}$ & & & $2 \mathrm{mb}$ & I & & $1.2 \mathrm{~s}$ & 589 & $9.00 \mathrm{~nm}$ \\
\hline LBF & 38.00 & 20 & iPc & 18 & 48.90 & 0.0 & 1 & & & & $\mathrm{i}$ \\
\hline & $1.0 \mathrm{~s}$ & 422 & $2.00 \mathrm{~nm}$ & & & $2 m b$ & 1 & & & & ePP \\
\hline MNS & 38.02 & 32 & $e^{P}$ & 18 & 49.00 & 0.0 & $1 \mathrm{KZN}$ & & 42.05 & 41 & eP \\
\hline LOR & 38.21 & 19 & iPc & 18 & 50.80 & 0.2 & I ATH & & 42.19 & 45 & iPd \\
\hline & $0.7 \mathrm{~s}$ & 315 & $5.00 \mathrm{~nm}$ & & & $.2 \mathrm{mb}$ & 1 & & & & ePP \\
\hline S6O & 38.23 & 36 & eP & 18 & 51.50 & 0.7 & 1 & & & & is \\
\hline VGI & 38.23 & 26 & eP & 18 & 52.80 & 2.1 & I DMU & & 42.26 & 6 & iPd \\
\hline EMS & 38.27 & 23 & ePd & 18 & 51.60 & 0.3 & 1 & & $1.1 \mathrm{~s}$ & 2280 & $0.00 \mathrm{~nm}$ \\
\hline POI & 38.29 & 32 & $i P c$ & 18 & 51.50 & 0.3 & 1 NPS & & 42.39 & 50 & eP \\
\hline LDF & 38.29 & 14 & iPc & 18 & 51.30 & 0.1 & 1 LII & & 42.41 & 42 & eP \\
\hline & $1.1 \mathrm{~s}$ & 847 & $7.00 \mathrm{~nm}$ & & & $4 \mathrm{mb}$ & I SKO & & 42.76 & 39 & $\mathrm{iPc}$ \\
\hline CSI & 38.31 & 38 & eP & 18 & 51.50 & -0.1 & 1 & & $1.3 \mathrm{~s}$ & 850 & $0.00 \mathrm{~nm}$ \\
\hline ORO & 38.32 & 25 & $e^{P}$ & 18 & 51.00 & -0.7 & I & & & & $\mathrm{i}$ \\
\hline$F \mid R$ & 38.32 & 29 & eP & 18 & 53.50 & 2.0 & 1 & & & & iPP \\
\hline & & & is & 24 & 50.00 & & 1 & & & & is \\
\hline$F \perp N$ & 38.36 & 14 & eP & 18 & 51.50 & -0.3 & 1 & & & & isS \\
\hline & $1.2 \mathrm{~s}$ & 630 & $0.00 \mathrm{~nm}$ & & 6 & $2 m b$ & I GRG & & 42.81 & 41 & ePc \\
\hline AOU & 38.37 & 33 & $e P$ & 18 & 52.50 & 0.4 & I KMR & & 42.85 & 27 & iP- \\
\hline$D 1 x$ & 38.46 & 24 & ePd & 18 & 53.60 & 0.5 & 1 & & & & i \\
\hline DUI & 38.47 & 34 & $\mathrm{i} P \mathrm{C}$ & 18 & 56.50 & $3.7 x$ & 1 & & & & e \\
\hline MMK & 38.66 & 24 & ePd & 18 & 55.10 & 0.4 & I DBN & & 42.89 & 17 & iPd \\
\hline ALP & 38.76 & 32 & $e(P)$ & 18 & 54.50 & -0.7 & 1 & $z$ & 225 & & $8.00 \mathrm{um}$ \\
\hline TMA & 39.07 & 25 & ePd & 18 & 57.10 & -1.0 & 1 & & & & ePP \\
\hline SAL & 39.38 & 27 & eP & 19 & 00.00 & -0.4 & 1 & & & & is \\
\hline BRT & 39.54 & 38 & $i P c$ & 19 & 02.50 & 0.7 & 1 & & & & ess \\
\hline ROF & 39.61 & 22 & $i P_{c}$ & 19 & 02.80 & 0.5 & I GRF & & 42.90 & 24 & iPc \\
\hline VDL & 39.63 & 25 & ePd & 19 & 01.80 & -0.9 & i & & $1.4 \mathrm{~s}$ & 866 & $6.00 \mathrm{~nm}$ \\
\hline BBS & 39.65 & 22 & $\mathrm{P}$ & 19 & 02.40 & -0.3 & 1 & 2 & $19 \mathrm{~s}$ & & $1.00 \mathrm{um}$ \\
\hline BSF & 39.70 & 21 & $i P c$ & 19 & 02.90 & -0.3 & 1 & & & & ePP \\
\hline LLS & 39.74 & 24 & ePd & 19 & 03.00 & -0.6 & I & & & & es \\
\hline BAF & 39.77 & 22 & $\mathbf{P}$ & 19 & 02.80 & -1.0 & I THE & & 43.00 & 42 & eP \\
\hline ZUL & 40.00 & 23 & ePd & 19 & 05.30 & -0.3 & I VAY & & 43.11 & 41 & eP \\
\hline VAL & 40.06 & 3 & iP & 19 & 06.90 & 1.0 & I PAIG & & 43.11 & 43 & ePc \\
\hline & & & is & 25 & 12.00 & & I WET & & 43.14 & 25 & iPc \\
\hline oss & 0.07 & 25 & ePd & 19 & 05.50 & -0.8 & 1 & & & & es \\
\hline VLS & 40.15 & 43 & eP & 19 & 06.50 & -0.4 & I KNT & & 43.23 & 41 & ePd \\
\hline $\mathrm{ECH}$ & 40.16 & 22 & $\mathbf{P}$ & 19 & 06.40 & -0.4 & WTS & & 43.28 & 18 & ePd \\
\hline FEL & 0.18 & 23 & $\mathbf{P}$ & 19 & 06.40 & -0.8 & 1 & & $1.4 \mathrm{~s}$ & 1350 & $0.00 \mathrm{~nm}$ \\
\hline SAX & 0. 19 & 24 & ePd & 19 & 06.50 & -0.9 & 1 & & & & $\mathrm{i}$ \\
\hline CTI & 40.22 & 27 & eP & 19 & 07.00 & -0.5 & 1 & & & & iPP \\
\hline SLE & 40.28 & 23 & ePd & 19 & 07.50 & -0.3 & I KHC & & 43.42 & 26 & $i P d$ \\
\hline CDF & 40.36 & 21 & $\mathbf{P}$ & 19 & 08.40 & -0.2 & 1 & & $1.0 \mathrm{~s}$ & 232 & $2.00 \mathrm{~nm}$ \\
\hline OGA & 40.58 & 26 & iPc & 19 & 09.80 & -0.8 & 1 & & $10 \mathrm{~s}$ & & $6.60 u m$ \\
\hline$B U H$ & 40.93 & 22 & ePd & 19 & 13.20 & 0.0 & I & $\mathrm{E}$ & $14 \mathrm{~s}$ & & 9.90 um \\
\hline DOU & 40.94 & 18 & $\mathrm{Pd}$ & 19 & 13.10 & -0.1 & 1 & & & & PP \\
\hline & $1.4 \mathrm{~s}$ & 3910 & $0.00 \mathrm{~nm}$ & & & $9 \mathrm{mb}$ & 1 & & & & $\$$ \\
\hline & & & PP & 20 & 52.30 & & 1 & & & & e \\
\hline & & & $\mathrm{S}$ & 25 & 05.00 & & I OUR & & 43.52 & 43 & eP \\
\hline TRI & 40.95 & 29 & iPd & 19 & 12.80 & -0.5 & I SRS & & 43.66 & 41 & $\mathrm{ePc}$ \\
\hline & & & iPP & 20 & 46.00 & & 1 MOX & & 43.81 & 23 & iPd- \\
\hline & & & is & 25 & 28.00 & & 1 & & $2.2 \mathrm{~s}$ & 1400 & $0.00 \mathrm{~nm}$ \\
\hline & & & iss & 28 & 26.00 & & I & $z$ & $14 \mathrm{~s}$ & 29 & $9.00 \mathrm{um}$ \\
\hline$G W F$ & 40.96 & 21 & $\mathbf{P}$ & 19 & 13.20 & -0.2 & | & $\mathbf{N}$ & $18 \mathrm{~s}$ & 32 & $2.00 \mathrm{um}$ \\
\hline
\end{tabular}


$220 \quad 04 \mathrm{~h}$

\begin{tabular}{|c|c|c|c|c|c|c|c|c|c|c|c|c|}
\hline KRA & & $\begin{array}{l}46.96 \\
1.35\end{array}$ & $\begin{array}{l}29 \\
590\end{array}$ & $\begin{array}{c}\text { iPd } \\
0.00 \mathrm{~nm}\end{array}$ & 20 & $\begin{array}{r}01.30_{6} \\
\end{array}$ & $\begin{array}{l}-0.5 \\
.5 m b\end{array}$ & 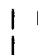 & NB2 & & $\begin{array}{l}52.33 \\
0.9 \mathrm{~s}\end{array}$ & $\begin{array}{l}15 \\
350\end{array}$ \\
\hline & $\mathbf{N}$ & $20 s$ & & 1.60 um & & & & $i$ & REY & & 52.53 & 355 \\
\hline & $\mathbf{E}$ & $20 \mathrm{~s}$ & & $1.00 \mathrm{um}$ & & & & i & & & & \\
\hline & & & & e & 20 & 12.90 & & $i$ & MTD & & 52.91 & 122 \\
\hline & & & & e & 20 & 15.60 & & $i$ & & & & \\
\hline & & & & $i$ & 20 & 38.10 & & $i$ & UPP & & 53.20 & 19 \\
\hline & & & & $\mathbf{i}$ & 21 & 55.00 & & 1 & & & $1.1 \mathrm{~s}$ & 700 \\
\hline & & & & es & 26 & 45.00 & & 1 & & & & \\
\hline & & & & $i$ & 30 & 21.00 & & $i$ & $A K U$ & & 53.85 & 358 \\
\hline UZH & & 47.21 & 32 & iPd & 20 & 04.50 & 0.8 & i & & & $1.5 \mathrm{~s}$ & 778 \\
\hline & & $1.3 \mathrm{~s}$ & 1300 & $0.00 \mathrm{~nm}$ & & & $5.9 \mathrm{mb}$ & $i$ & SWZ & & 54.11 & 136 \\
\hline & $z$ & $14 \mathrm{~s}$ & & $9.00 \mathrm{um}$ & & & 5. $4 M s=X$ & $i$ & ANN & & 54.33 & 43 \\
\hline & $N$ & $14 \mathrm{~s}$ & & 3.00um & & & & $i$ & & & $2.0 \mathrm{~s}$ & 4000 \\
\hline & $E$ & $14 \mathrm{~s}$ & & $2.00 \mathrm{um}$ & & & & i & & $z$ & $18 \mathrm{~s}$ & 15 \\
\hline & & & & iPP & 21 & 50.00 & & $i$ & & $\mathrm{~N}$ & $19 \mathrm{~s}$ & 17 \\
\hline & & & & es & 26 & 56.00 & & $i$ & & $E$ & $19 \mathrm{~s}$ & 18 \\
\hline IST & & 47.22 & 44 & IPd & 20 & 00.00 & $-3.9 x$ & i & & & & \\
\hline & & & & $i$ & 29 & 54.00 & & 1 & & & & \\
\hline ISK & & 47.27 & 44 & ePn & 20 & 03.80 & -0.5 & $i$ & & & & \\
\hline$M L R$ & & 47.42 & 37 & iPc & 20 & 05.00 & -0.6 & i & & & & \\
\hline HRT & & 47.58 & 45 & $i P_{n}$ & 20 & 06.30 & -0.6 & $i$ & TUH & & 54.66 & 146 \\
\hline ISR & & 47.59 & 38 & eP & 20 & 06.50 & -0.4 & $i$ & CER & & 54.79 & 146 \\
\hline VAO & & 47.67 & 223 & iPd & 20 & 07.80 & 0.0 & i & BFS & & 54.93 & 135 \\
\hline & & & & i & 20 & 09.30 & & i & & & $1.6 \mathrm{~s}$ & 2270 \\
\hline & & & & e & 20 & 23.90 & & $i$ & ARO & & 55.13 & 84 \\
\hline GPA & & 47.81 & 46 & iP & 20 & 08.30 & -0.4 & i & TOV & & 55.22 & 274 \\
\hline MUD & & 47.84 & 17 & iPd & 20 & 08.20 & -0.4 & i & SLR & & 55.26 & 133 \\
\hline & & $0.7 \mathrm{~s}$ & 360 & $0.00 \mathrm{~nm}$ & & & $.6 \mathrm{mb}$ & i & & & $0.9 \mathrm{~s}$ & 765 \\
\hline VRI & & 48.08 & 37 & iP & 20 & 11.00 & 0.3 & I & & $z$ & $20 s$ & 25 \\
\hline COP & & 48.18 & 19 & iPd- & 20 & 11.90 & 0.6 & $i$ & $B P 1$ & & 55.35 & 134 \\
\hline & & $0.7 \mathrm{~s}$ & 356 & $6.00 \mathrm{~nm}$ & & & $.6 \mathrm{mb}$ & $i$ & PRY & & 55.42 & 135 \\
\hline & & & & is & 27 & 10.00 & & 1 & SOC & & 55.54 & 45 \\
\hline$T L B$ & & 48.23 & 39 & IPo & 20 & 12.00 & 0.2 & $i$ & & & $1.4 \mathrm{~s}$ & 700 \\
\hline BSO & & 48.23 & 59 & eP & 20 & 12.00 & 0.0 & $i$ & & & & \\
\hline STJ & & 48.39 & 325 & eP & 20 & 12.00 & -1.0 & 1 & & & & \\
\hline$B A C$ & & 48.56 & 37 & eP & 20 & 12.00 & -2.3 & $i$ & & & & \\
\hline CFR & & 48.60 & 39 & ePd & 20 & 13.00 & -1.6 & $\mathrm{i}$ & & & & \\
\hline AS I & & 48.75 & 59 & eP & 20 & 16.00 & -0.2 & i & & & & \\
\hline CLI & & 48.77 & 37 & iPd & 20 & 15.50 & -0.5 & $i$ & VIR & & 55.73 & 136 \\
\hline PPE & & 48.79 & 37 & ePc & 20 & 16.00 & -0.1 & $i$ & BLF & & 55.94 & 138 \\
\hline$B \backslash R$ & & 48.83 & 37 & eP & 20 & 18.00 & 1.6 & 1 & NUR & & 56.07 & 22 \\
\hline LVV & & 48.84 & 32 & iPd & 20 & 16.30 & -0.1 & 1 & & & $0.7 \mathrm{~s}$ & 88 \\
\hline & & $5.0 \mathrm{~s}$ & 8800 & $0.00 \mathrm{~nm}$ & & & $.1 \mathrm{mb} \times$ & $i$ & & & & \\
\hline & & & & iPP & 22 & 07.00 & & i & & & & \\
\hline & & & & iPPP & 22 & 54.00 & & i & & & & \\
\hline & & & & is & 27 & 14.00 & & 1 & & & & \\
\hline & & & & iss & 30 & 43.00 & & 1 & SDV & & 56.16 & 273 \\
\hline JER & & 48.93 & 58 & ePd & 20 & 17.50 & 0.0 & $i$ & EVA & & 56.30 & 133 \\
\hline & & & & es & 27 & 24.00 & & $i$ & SEK & & 56.46 & 136 \\
\hline GUV & & 48.95 & 270 & ep & 20 & 17.60 & -0.2 & $i$ & HVD & & 56.48 & 139 \\
\hline$K D E$ & & 48.99 & 45 & eP & 20 & 16.10 & -1.6 & $i$ & $L G N$ & & 56.61 & 274 \\
\hline 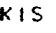 & & 49.94 & 37 & iPd & 20 & 24.00 & -0.9 & $i$ & UAV & & 56.70 & 273 \\
\hline & & $6.0 \mathrm{~s}$ & 3600 & $0.00 \mathrm{~nm}$ & & & $.5 \mathrm{mb} \times$ & $i$ & BHD & & 56.84 & 58 \\
\hline & $z$ & $18 \mathrm{~s}$ & 24 & $4.00 \mathrm{um}$ & & & $2 \mathrm{Msz}$ & i & & & & \\
\hline & $N$ & $19 \mathrm{~s}$ & & $3.50 \mathrm{um}$ & & & & i & & & & \\
\hline & $E$ & $19 \mathrm{~s}$ & & $5.00 \mathrm{um}$ & & & & i & & & & \\
\hline & & & & iPP & 22 & 19.00 & & 1 & & & & \\
\hline & & & & is & 27 & 25.00 & & 1 & & & & \\
\hline BER & & 50.41 & 12 & ip & 20 & 28.00 & -0.3 & 1 & & & & \\
\hline & & $1.0 \mathrm{~s}$ & & $0.50 \mathrm{~nm}$ & & 3 & $.4 \mathrm{mb} \times$ & i & & & & \\
\hline KON & & 50.72 & 15 & eP & 20 & 30.30 & -0.4 & i & CBM & & 57.49 & 319 \\
\hline SJG & & 51.07 & 284 & iPd & 20 & 33.00 & -1.0 & i & BKR & & 57.73 & 48 \\
\hline & & $0.7 \mathrm{~s}$ & 151 & $1.00 \mathrm{~nm}$ & & & $.0 \mathrm{mb}$ & i & & & $1.4 \mathrm{~s}$ & 1900 \\
\hline & $z$ & $20 s$ & & $9.22 \mathrm{um}$ & & 5 & $8 M S Z$ & i & & & & \\
\hline KR I & & 51.30 & 123 & ipd & 20 & 35.00 & -0.9 & i & & & & \\
\hline & & & & iPP & 22 & 36.00 & & i & & & & \\
\hline AAE & & 51.46 & 88 & eP & 20 & 38.50 & 1.1 & i & LEN & & 57.75 & 49 \\
\hline NA I & & 51.68 & 101 & eP & 20 & 42.00 & $3.1 x$ & i & & & $2.0 \mathrm{~s}$ & 1900 \\
\hline & & $2.0 s$ & 1290 & $0.00 \mathrm{~nm}$ & & & $.5 \mathrm{mb}$ & 1 & & $\mathbf{N}$ & $16 \mathrm{~s}$ & \\
\hline SIM & & 52.26 & 42 & eP & 20 & 41.00 & -1.6 & i & & & & \\
\hline & & $6.0 \mathrm{~s}$ & 6000 & $0.00 \mathrm{~nm}$ & & & $.7 \mathrm{mb} \times$ & i & & & & \\
\hline & $Z$ & $26 s$ & & $7.50 u m$ & & & $.0 M s z x$ & 1 & & & & \\
\hline & $N$ & $20 s$ & & $1.00 \mathrm{um}$ & & & & 1 & PUL & & 57.77 & 24 \\
\hline & $E$ & $20 s$ & & 3.00um & & & & i & & & $3.0 s$ & 2600 \\
\hline & & & & ePCP & 21 & 49.00 & & i & & $z$ & $14 \mathrm{~s}$ & \\
\hline & & & & ePP & 22 & 41.00 & & 1 & & $\mathrm{~N}$ & $14 \mathrm{~s}$ & \\
\hline & & & & EPPP & 23 & 47.00 & & 1 & & $E$ & $14 \mathrm{~s}$ & \\
\hline & & & & es & 27 & 57.00 & & $i$ & & & & \\
\hline & & & & ess & 31 & 47.00 & & i & & & & \\
\hline BUL & & 52.27 & 127 & iPd & 20 & 42.00 & $-1 \cdot 1$ & i & & & & \\
\hline & & & & ipp & 21 & 00.00 & $71 \mathrm{kmX}$ & i & & & & \\
\hline & & & & IPP & 22 & 49.00 & & i & & & & \\
\hline & & & & is & 28 & 07.00 & & 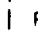 & & & 57.98 & 45 \\
\hline AR & & 52.33 & 274 & $e^{P}$ & 20 & 40.00 & $-3.7 x$ & 1 & & & $2.0 s$ & 2640 \\
\hline & & $0.7 \mathrm{~s}$ & & $7.40 \mathrm{~nm}$ & & & $3 \mathrm{mb} \times$ & 1 & & $z$ & $27 \mathrm{~s}$ & 18 \\
\hline & & & & IPP & 22 & 00.00 & & 1 & & $\mathrm{~N}$ & $27 s$ & .0 \\
\hline
\end{tabular}




\begin{tabular}{|c|c|c|c|c|c|c|c|c|c|c|c|c|}
\hline RSNY & & 61.17 & 314 & iP & 21 & 46.40 & 0.3 & I RFA & & 69.69 & 226 & ePc \\
\hline & & $1.5 \mathrm{~s}$ & 200 & $0.00 \mathrm{~nm}$ & & & $\theta m b$ & I FCH & & 70.27 & 228 & iPC \\
\hline TRO & & 61.28 & 12 & iP & 21 & 45.70 & -0.8 & I BACH & & 70.42 & 228 & eP \\
\hline SOD & & 61.46 & 17 & ¡P & 21 & 46.20 & -1.5 & I PEL & & 70.46 & 228 & iPc \\
\hline I NY & & 62.17 & 312 & iP & 21 & 52.00 & -0.9 & I SAN & & 70.60 & 228 & iP \\
\hline & & & & is & 30 & 20.00 & & I PWLA & & 70.67 & 303 & eP \\
\hline OTT & & 62.17 & 315 & ePd & 21 & 52.50 & -0.3 & $\mathrm{I} \mathrm{CHCH}$ & & 70.83 & 228 & IPO \\
\hline & & $1.2 \mathrm{~s}$ & 107 & $7.00 \mathrm{~nm}$ & & & $.9 \mathrm{mb}$ & I TACH & & 70.90 & 228 & eP \\
\hline BAK & & 62.21 & 51 & iPc & 21 & 56.00 & $2.8 x$ & I LNV & & 71.39 & 228 & iPd \\
\hline & & $0.4 \mathrm{~s}$ & 7500 & $0.00 \mathrm{~nm}$ & & & $2 \mathrm{mb} X$ & I SVE & & 71.49 & 34 & ePd \\
\hline & & & & is & 30 & 22.00 & & I & & $3.0 \mathrm{~s}$ & 2170 & $0.00 \mathrm{~nm}$ \\
\hline$S L A$ & & 62.38 & 234 & ePd & 21 & 53.00 & -1.7 & I & $z$ & $16 \mathrm{~s}$ & 22 & $2.00 \mathrm{um}$ \\
\hline IR 4 & & 62.42 & 56 & eP & 21 & 53.70 & -1.2 & 1 & $N$ & $16 \mathrm{~s}$ & 16 & $5.00 \mathrm{um}$ \\
\hline NA 12 & & 62.61 & 307 & eP & 21 & 55.40 & -0.5 & 1 & E & $16 \mathrm{~s}$ & 12 & 2.60 um \\
\hline LPA & & 62.71 & 220 & eP- & 21 & 58.00 & 1.5 & 1 & & & & ePcP \\
\hline & 2 & $20 s$ & 22 & $2.76 \mathrm{um}$ & & & $3 M S z$ & 1 & & & & ePP \\
\hline$C V L$ & & 63.06 & 307 & eP & 21 & 57.30 & -1.6 & I & & & & ePPP \\
\hline KEV & & 63.23 & 15 & iPd & 21 & 58.00 & -1.5 & 1 & & & & es \\
\hline & & $0.8 \mathrm{~s}$ & 469 & $9.00 \mathrm{~nm}$ & & & $.7 \mathrm{mb}$ & 1 & & & & ess \\
\hline & & & & $i$ & 22 & 03.00 & & I ELC & & 71.57 & 305 & iPd \\
\hline & & & & ePP & 24 & 08.00 & & I LHC & & 71.76 & 317 & iPd \\
\hline & & & & EPPP & 26 & 00.00 & & I & & $2.0 \mathrm{~s}$ & 907 & $.00 \mathrm{~nm}$ \\
\hline & & & & eS & 36 & 20.00 & & I & & & & PP \\
\hline SHI & & 63.50 & 63 & eP & 21 & 59.80 & $-3 \cdot 1 x$ & I FVM & & 72.50 & 306 & eP \\
\hline APA & & 63.54 & 18 & IPC & 22 & 01.20 & -0.3 & 1 & & $1.8 \mathrm{~s}$ & 458 & $3.00 \mathrm{~nm}$ \\
\hline & & $2.0 \mathrm{~s}$ & 990 & $0.00 \mathrm{~nm}$ & & & $.7 \mathrm{mb}$ & I POW & & 73.18 & 304 & eP \\
\hline & 2 & $18 s$ & 45 & $5.00 \mathrm{um}$ & & & $.7 \mathrm{Msz}$ & I RSON & & 74.70 & 320 & iP \\
\hline & $N$ & $17 \mathrm{~s}$ & 12 & 2.00 um & & & & I & & $1.5 \mathrm{~s}$ & 423 & $3.00 \mathrm{~nm}$ \\
\hline & $\mathbf{E}$ & $17 \mathrm{~s}$ & & 1.00 um & & & & I FCC & & 75.04 & 328 & eP \\
\hline & & & & iPCP & 22 & 42.70 & & I SAM & & 75.27 & 52 & ip \\
\hline & & & & iPPP & 25 & 49.00 & & 1 & & $2.2 \mathrm{~s}$ & 1400 & $0.00 \mathrm{~nm}$ \\
\hline & & & & is & 30 & 34.06 & & 1 & $N$ & $16 s$ & 23 & $3.10 \mathrm{um}$ \\
\hline ARE & & 63.84 & 245 & $\mathbf{P}$ & 22 & 02.00 & -2.7 & 1 & $E$ & $16 s$ & 14 & $.60 \mathrm{um}$ \\
\hline PSO & & 64.12 & 266 & iP & 22 & 06.00 & -0.7 & 1 & & & & ePP \\
\hline CAC & & 64.19 & 238 & $e(P)$ & 22 & 07.00 & 0.2 & 1 & & & & ePPP \\
\hline FRB & & 64.37 & 336 & eP & 22 & 06.00 & -1.0 & 1 & & & & is \\
\hline BLA & & 64.54 & 306 & eP & 22 & 08.90 & 0.2 & 1 & & & & iSP \\
\hline UPA & & 64.84 & 274 & iPc & 22 & 11.00 & 0.1 & I KHE & & 75.39 & 9 & iPd \\
\hline & & $1.0 \mathrm{~s}$ & 104 & $1.00 \mathrm{~nm}$ & & & $0 \mathrm{mb}$ & 1 & & $1.5 \mathrm{~s}$ & 750 & $.00 \mathrm{~nm}$ \\
\hline & $z$ & $20 s$ & & $5.67 \mathrm{um}$ & & & $8 \mathrm{Msz}$ & 1 & 2 & $18 s$ & 22 & $2.00 \mathrm{um}$ \\
\hline & $N$ & $20 \mathrm{~s}$ & & $3.87 \mathrm{um}$ & & & & I & $N$ & $19 \mathrm{~s}$ & & $9.10 \mathrm{um}$ \\
\hline NAV & & 64.85 & 306 & eP & 22 & 10.30 & -0.4 & | & $E$ & $19 \mathrm{~s}$ & & $9.10 \mathrm{um}$ \\
\hline DAG & & 64.96 & 359 & iPd & 22 & 09.80 & -0.8 & I & & & & ePcP \\
\hline & & $0.9 \mathrm{~s}$ & 185 & $5.00 \mathrm{~nm}$ & & & $3 \mathrm{mb}$ & 1 & & & & iPP \\
\hline & & & & $i$ & 30 & 47.00 & & 1 & & & & ePPP \\
\hline & & & & $i$ & 35 & 07.00 & & 1 & & & & es \\
\hline JSC & & 65.05 & 302 & eP & 22 & 11.90 & -0.1 & 1 & & & & eScS \\
\hline FM & & 65.59 & 304 & eP & 22 & 15.60 & 0.0 & 1 & & & & eSs \\
\hline LDN & & 65.68 & 312 & $P$ & 22 & 14.40 & -1.4 & I COM & & 75.95 & 284 & ip \\
\hline ELF & & 65.79 & 312 & $\mathbf{P}$ & 22 & 15.20 & -1.4 & I RLO & & 76.28 & 305 & iPd \\
\hline ANT & & 65.88 & 237 & eP- & 22 & 17.00 & -0.4 & I BHO & & 76.30 & 303 & iPd \\
\hline & & & & eS & 31 & 04.00 & & I NSLM & & 76.60 & 300 & ¡P \\
\hline & & & & e & 42 & 52.00 & & I DSH & & 76.74 & 53 & iPd \\
\hline PRM & & 65.96 & 302 & iPd & 22 & 17.50 & -0.3 & 1 & $z$ & $12 \mathrm{~s}$ & & $5.00 \mathrm{um}$ \\
\hline KAT & & 66.97 & 52 & i Pd & 22 & 25.00 & 0.8 & 1 & $\mathbf{N}$ & $12 \mathrm{~s}$ & & 5.00 um \\
\hline & & $6.0 \mathrm{~s}$ & 6700 & $0.00 \mathrm{~nm}$ & & & $\theta \mathrm{mb} x$ & I & $E$ & $12 \mathrm{~s}$ & & $7.00 \mathrm{um}$ \\
\hline & & & & ePcP & 22 & 55.00 & & 1 & & & & is \\
\hline & & & & ePP & 24 & 54.00 & & I TUL & & 76.92 & 304 & iPd- \\
\hline & & & & is & 31 & 19.00 & & 1 & & 1.65 & 924 & $4.00 \mathrm{~nm}$ \\
\hline & & & & ePPS & 31 & 50.00 & & 1 & $z$ & $19 \mathrm{~s}$ & & $9.20 \mathrm{um}$ \\
\hline & & & & ess & 35 & 40.00 & & 1 & & & & e \\
\hline TKL & & 67.16 & 304 & eP & 22 & 25.50 & 0.0 & I & & & & e(s) \\
\hline VBA & & 67.21 & 220 & iPd & 22 & 7.00 & 1.3 & 1 TAS & & 76.92 & 50 & eP \\
\hline$V Y$ & & 67.69 & 116 & ePc & 22 & 9.00 & 0.8 & 1 & & $2.0 \mathrm{~s}$ & 900 & $9.00 \mathrm{~nm}$ \\
\hline CEN & & 68.27 & 229 & eP & 22 & 3.00 & 0.5 & I & E & $14 \mathrm{~s}$ & & $9.00 \mathrm{um}$ \\
\hline VAN & & 68.35 & 54 & iPd & 22 & 31.00 & -1.9 & 1 & & & & ePP \\
\hline & & $8.0 \mathrm{~s}$ & 7070 & $.00 \mathrm{~nm}$ & & & $9 m b x$ & 1 & & & & ePPP \\
\hline & $z$ & $16 \mathrm{~s}$ & & $.30 \mathrm{um}$ & & & $2 M s z X$ & I & & & & es \\
\hline & $E$ & $16 \mathrm{~s}$ & & . $16 \mathrm{um}$ & & & & i & & & & eScS \\
\hline & & & & ePP & 25 & 04.00 & & 1 & & & & eSs \\
\hline & & & & es & 31 & 34.00 & & I KUL & & 77.56 & 54 & eP \\
\hline & & & & ess & 35 & 56.00 & & i & & $4.2 \mathrm{~s}$ & 2300 & $3.00 \mathrm{~nm}$ \\
\hline ASH & & 68.55 & 54 & eP & 22 & 34.00 & -0.1 & 1 & $N$ & $13 \mathrm{~s}$ & 26 & $5.00 \mathrm{um}$ \\
\hline & & $5.0 \mathrm{~s}$ & 5660 & $.00 \mathrm{~nm}$ & & & $0 \mathrm{mb} x$ & 1 & $E$ & $12 \mathrm{~s}$ & 11 & $.30 u m$ \\
\hline & & & & es & 31 & 37.00 & & I & & & & is \\
\hline & & & & ePS & 32 & 02.00 & & I HKT & & 77.56 & 298 & ip \\
\hline & & & & ePPS & 32 & 14.00 & & I GAR & & 77.90 & 52 & eP \\
\hline & & & & eScS & 32 & 33.00 & & 1 & & $2.0 \mathrm{~s}$ & 900 & $3.06 \mathrm{~nm}$ \\
\hline SCP & & 68.64 & 304 & iP & 22 & 34.50 & -0.3 & 1 & $\mathrm{~N}$ & $18 \mathrm{~s}$ & &.$B B u m$ \\
\hline & & $1.5 \mathrm{~s}$ & 744 & $.00 \mathrm{~nm}$ & & & $.7 \mathrm{mb}$ & I & & & & es \\
\hline & $z$ & $22 \mathrm{~s}$ & 20 & $.60 u m$ & & & $3 M s z$ & 1 & & & & eSKS \\
\hline $\mathrm{KHI}$ & & 68.74 & 58 & ePc & 22 & 34.80 & -0.8 & 1 & & & & eSS \\
\hline MDZ & & 69.00 & 228 & $i(P)$ & 22 & 38.60 & 1.6 & 1000 & & 78.32 & 304 & eP \\
\hline$T L L$ & & 69.13 & 231 & eP & 22 & 37.00 & -1.1 & I KHO & & 79.01 & 54 & eP \\
\hline MHI & & 69.44 & 56 & iPd & 22 & 38.80 & -1.0 & 1 & & $2.0 \mathrm{~s}$ & 4800 & $3.00 \mathrm{~nm}$ \\
\hline & & $1.0 \mathrm{~s}$ & 320 & $\begin{array}{l}.00 \mathrm{~nm} \\
\mathrm{eS}\end{array}$ & 31 & & $4 \mathrm{mb}$ & 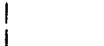 & & & & $\begin{array}{l}\text { ePP } \\
\text { ePPP }\end{array}$ \\
\hline
\end{tabular}




\begin{tabular}{|c|c|c|c|c|c|c|c|c|c|c|c|}
\hline & & & & es & 34 & 39.00 & & 1 & $20 s$ & & 1.80 um \\
\hline EDM & & 86.40 & 324 & ePd & 24 & 13.20 & 0.4 & 1 & & & $7.80 \mathrm{um}$ \\
\hline BDW & & 86.42 & 313 & ePd & 24 & 2.50 & -0.9 & f & & & ePP \\
\hline LRM & & 87.71 & 316 & ePd & 24 & 20.50 & 0.9 & 1 & & & eSKS \\
\hline BUT & & 87.74 & 316 & eP & 24 & 22.10 & 2.4 & 1 & & & es \\
\hline TMI & & 87.97 & 314 & eP & 24 & 20.70 & -0.2 & & & & eSP \\
\hline$M Z X$ & & 88.02 & 293 & eP & 24 & 22.80 & 1.7 & & & & eSS \\
\hline GBA & & 88.19 & 77 & $\mathrm{Pc}$ & 24 & 21.30 & -0.8 & & & & EPSPS \\
\hline & & $0.9 \mathrm{~s}$ & 117 & $7.00 \mathrm{~nm}$ & & & $.2 \mathrm{mb}$ & I ZAK & 97.23 & 35 & iPd \\
\hline HYB & & 88.47 & 73 & ePd & 24 & 23.50 & 0.0 & 1 & $2.2 \mathrm{~s}$ & 270 & $0.00 \mathrm{~nm}$ \\
\hline & & 1. & 350 & $9.00 \mathrm{~nm}$ & & & $.6 \mathrm{mb}$ & 1 & & & eSKS \\
\hline HPI & & 88.70 & 314 & eP & 24 & 25.00 & 0.5 & I FHC & 97.28 & 314 & eP \\
\hline RMU & & 8 & 308 & ip & 24 & 26.20 & 1.4 & 1800 & 98.49 & 25 & $e^{P}$ \\
\hline KOD & & .90 & 80 & eP & 24 & 27.20 & 1.3 & I & $1.2 \mathrm{~s}$ & & $0.00 \mathrm{~nm}$ \\
\hline MSU & & 89.35 & 389 & eP & 24 & 28.30 & 0.7 & $1 / L T$ & 99.73 & 354 & eP \\
\hline DUG & & 89.40 & 311 & eP & 24 & 27.50 & -0.2 & 1 & $20 \mathrm{~s}$ & & $3.00 \mathrm{um}$ \\
\hline I NK & & 89.56 & 341 & iPd & 24 & 27.90 & 0.2 & 1 & $20 \mathrm{~s}$ & & $9.80 \mathrm{um}$ \\
\hline & & $1.8 \mathrm{~s}$ & 554 & $4.00 \mathrm{~nm}$ & & $\epsilon$ & $.5 m b$ & 1 & $20 \mathrm{~s}$ & & $7.00 \mathrm{um}$ \\
\hline WMO & & 89.83 & 45 & Pd & 24 & 30.50 & 0.9 & 1 & & & ePP \\
\hline & & & & ePP & 27 & 52.00 & & 1 & & & eSKS \\
\hline & & & & $s$ & 35 & 25.00 & & 1 & & & eSP \\
\hline NEW & & 90.10 & 319 & eP & 24 & 29.00 & -1.6 & 1 & & & eSS \\
\hline & $z$ & $18 \mathrm{~s}$ & & 5.80 um & & & $1 \mathrm{Msz}$ & I GTA & 99.87 & 46 & eP \\
\hline PNT & & 91.32 & 321 & eP & 24 & 37.00 & 0.8 & I YAK & 100.95 & 17 & ePdiff \\
\hline & & $2.0 \mathrm{~s}$ & 526 & $0.00 \mathrm{~nm}$ & & & $.5 \mathrm{mb}$ & 1 & $20 \mathrm{~s}$ & & $1.00 \mathrm{um}$ \\
\hline UER & & 91.46 & 37 & iPd & 24 & 36.00 & -0.7 & 1 & $20 \mathrm{~s}$ & & 9.50 um \\
\hline & & & & es & 35 & 35.50 & & 1 & & & iPP \\
\hline & & & & ESPP & 37 & 16.00 & & 1 & & & ePPP \\
\hline EUR & & 91.94 & 311 & iP & 24 & 40.00 & 0.5 & 1 & & & iSKS \\
\hline & & $0.8 \mathrm{~s}$ & 33 & $3.60 \mathrm{~nm}$ & & & $.8 \mathrm{mb}$ & 1 & & & es \\
\hline KKN & & 92.19 & 61 & IPd & 24 & 40.90 & 0.0 & I SPA & 101.78 & 180 & ePdiff \\
\hline PKI & & 92.36 & 61 & iP & 24 & 41.60 & -0.2 & 1 & $2.0 \mathrm{~s}$ & & $2.90 \mathrm{~nm}$ \\
\hline & & $0.7 \mathrm{~s}$ & 110 & $3.00 \mathrm{~nm}$ & & & $.4 \mathrm{mb}$ & I TUP & 103.15 & 26 & ePdiff \\
\hline BMN & & 92.57 & 312 & ePd & 24 & 42.30 & 0.0 & I LZH & 104.18 & 48 & ePdiff \\
\hline & & 2.05 & 629 & $9.46 \mathrm{~nm}$ & & & $.7 \mathrm{mb}$ & 1 & & & $P P$ \\
\hline GLA & & 92.87 & 304 & eP & 24 & 44.00 & 0.3 & I SEY & 104.68 & 7 & ePdiff \\
\hline$T I K$ & & 92.99 & 11 & eP & 24 & 43.50 & 0.1 & 1 & $21 s$ & & 9.80 um \\
\hline & & $1.2 \mathrm{~s}$ & 80 & $3.00 \mathrm{~nm}$ & & &.$\theta \mathrm{mb}$ & 1 & $20 \mathrm{~s}$ & & $1.48 \mathrm{um}$ \\
\hline & & & & ePP & 28 & 22.00 & & 1 & & & iPP \\
\hline & & & & ePPP & 30 & 30.00 & & 1 & & & ePS \\
\hline & & & & eSKS & 35 & 14.00 & & D2 & 186.35 & 53 & Pdiff \\
\hline & & & & es & 35 & 51.00 & & I $\mathrm{CHG}$ & 106.81 & 66 & ePdiff \\
\hline & & & & ePS & 37 & 08.00 & & I BDT & 187.36 & 68 & e (PKP) \\
\hline & & & & eSPP & 37 & 34.00 & & 1 & 1.15 & & $9.30 \mathrm{~nm}$ \\
\hline BRW & & 93.41 & 349 & eP & 24 & 47.20 & 1.8 & I TIY & 109.37 & 43 & ePKP \\
\hline LON & & 93.60 & 319 & eP & 24 & 46.80 & 0.0 & | BJI & 110.45 & 39 & ePdiff \\
\hline GSC & & 93.80 & 307 & eP & 24 & 48.00 & 0.0 & 1 & $19 \mathrm{~s}$ & & $7.00 \mathrm{um}$ \\
\hline & & & & e & 28 & 33.00 & & I & $20 \mathrm{~s}$ & & $5.00 \mathrm{um}$ \\
\hline ANA & & 93.88 & 310 & eP & 24 & 48.60 & 0.2 & I & & & e \\
\hline CLC & & 94.26 & 308 & ep & 24 & 53.00 & $2.9 x$ & 1 & & & $P P$ \\
\hline VPEM & & 94.39 & 308 & eP & 24 & 52.20 & 1.4 & 1 & & & PPP \\
\hline$P L M$ & & 94.42 & 305 & ep & 24 & 52.00 & 1.0 & 1 & & & PS \\
\hline & & & & e & 28 & 48.00 & & GYA & 110.55 & 56 & PKF \\
\hline CWC & & 94.44 & 388 & eP & 24 & 52.00 & 1.0 & 251 & 111.35 & 82 & EPKP \\
\hline & & & & e & 28 & 35.00 & & I IPM & 12.92 & 80 & ePKPd \\
\hline GAR & & 94.47 & 305 & eP & 24 & 51.00 & 0.0 & I CN2 & 113.20 & 31 & Pdiff \\
\hline RVR & & 94.64 & 306 & ep & 24 & 52.00 & 0.2 & I CN2 & 113.20 & 31 & PKP \\
\hline SBB & & 94.78 & 307 & eP & 24 & 52.00 & -0.5 & i & & & $P P$ \\
\hline & & & & e & 28 & 38.00 & & 1 & & & SKS \\
\hline I SA & & 94.99 & 308 & eP & 24 & 54.00 & 0.6 & | TIA & 113.31 & 42 & ePKP \\
\hline & & & & e & 28 & 43.00 & & | PPI & 113.58 & 85 & ePKP \\
\hline MWC & & 95.11 & 386 & ef & 24 & 55.00 & 0.8 & I NJ2 & 116.91 & 45 & PKPd \\
\hline & & & & e & 28 & 03.00 & & i & & & IPP \\
\hline PAS & & 95.22 & 386 & eP & 24 & 55.00 & 0.6 & HK & 123.81 & 34 & ePKP \\
\hline & & $1.6 \mathrm{~s}$ & 100 & $.08 \mathrm{~nm}$ & & & $.0 \mathrm{mb}$ & MAT & 124.99 & 28 & IPKPC \\
\hline & & & & e & 28 & 44.00 & & 1 & $20 s$ & & 5.85 um \\
\hline$=R I$ & & 95.51 & 389 & eP & 24 & 55.10 & -0.6 & $D R$ & 125.88 & 27 & ePKP \\
\hline JAS & & 95.74 & 311 & ePd & 24 & 57.00 & 0.2 & I TSK & 126.11 & 26 & ePKP \\
\hline & & & & ePP & 28 & 45.80 & & I $B A G$ & 126.63 & 59 & ePKP \\
\hline IIN & & 95.76 & 313 & eP & 24 & 57.20 & 0.2 & | TRT & 126.86 & 91 & IPKPC \\
\hline $\mathrm{COL}$ & & 96.12 & 342 & eP & 24 & 58.00 & 0.0 & I & $0.8 \mathrm{~s}$ & 63 & $3.60 \mathrm{~nm}$ \\
\hline & & $0.9 \mathrm{~s}$ & 10 & 1. $10 \mathrm{~nm}$ & & & $.3 \mathrm{mb} \times$ & I CVP & 127.03 & 57 & ePKPC \\
\hline & $z$ & $19 \mathrm{~s}$ & & 1. $60 \mathrm{um}$ & & & $.5 M s z$ & $1 O C P$ & 127.90 & 61 & ePKP \\
\hline & & & & es & 37 & 36.00 & & PPR & 127.92 & 68 & ePKP \\
\hline F8A & & 96.12 & 342 & $e(P)$ & 24 & 58.30 & 0.3 & I MUN & 129.76 & 122 & ePKP \\
\hline WDC & & 96.34 & 314 & ePd & 24 & 58.00 & -1.4 & I NWAO & 130.40 & 123 & ePKP \\
\hline PRI & & 96.51 & 309 & iPd & 25 & 00.90 & 0.4 & BAL & 130.45 & 120 & ePKP \\
\hline LSA & & 96.62 & 58 & $\mathrm{P}$ & 25 & 02.00 & 0.5 & MEK & 132.71 & 115 & ePKP \\
\hline MHC & & 96.84 & 310 & eP & 25 & 82.40 & 0.5 & 1 & $0.8 \mathrm{~s}$ & 61 & $1.00 \mathrm{~nm}$ \\
\hline IRK & & 96.89 & 33 & eP & 25 & 01.20 & -0.5 & RUV & 135.07 & 260 & iPKP \\
\hline & & $2.4 \mathrm{~s}$ & 200 & $.00 \mathrm{~nm}$ & & & $3 \mathrm{mb}$ & 1 & $1.2 \mathrm{~s}$ & 86 & $0.00 \mathrm{~nm}$ \\
\hline & & & & eSKS & 35 & 40.00 & & TPT & 135.29 & 261 & IPKP \\
\hline & & & & es & 36 & 20.60 & & 1 & $1.2 \mathrm{~s}$ & 190 & $0.00 \mathrm{~nm}$ \\
\hline & & & & eSP & 37 & 44.60 & & VAH & 135.31 & 260 & iPKP \\
\hline & & & & EPPS & 38 & 28.00 & & 1 & $1.2 \mathrm{~s}$ & 158 & $0.00 \mathrm{~nm}$ \\
\hline BKS & & 97.09 & 311 & ePd & 25 & 03.60 & 0.7 & PMO & 135.56 & 261 & IPKP \\
\hline & Z & $20 s$ & & $.00 \mathrm{um}$ & & & . $1 \mathrm{Msz}$ & & $1.2 \mathrm{~s}$ & 135 & $5.00 \mathrm{~nm}$ \\
\hline
\end{tabular}




\begin{tabular}{|c|c|c|c|c|c|c|c|c|c|c|c|}
\hline & & & & $i s n$ & 54 & 31.50 & & 1 & & & iPP \\
\hline SAM & & 4.42 & 319 & iPnd & 53 & 46.10 & -0.9 & I MSL & 22.20 & 278 & ePd \\
\hline & & & & isn & 54 & 39.40 & & $i$ & & & is \\
\hline ANR & & 4.53 & 16 & $P$ & 53 & 47.50 & -0.9 & I ABS & 22.27 & 292 & $i P c$ \\
\hline NAM & & 4.64 & 9 & iPnd & 53 & 49.80 & 0.2 & i & & & EPCP \\
\hline & & & & isn & 54 & 44.40 & & I PYA & 22.41 & 298 & $\mathrm{iPc}$ \\
\hline TAS & & 5.05 & 347 & iPnd & 53 & 54.50 & -0.5 & 1 & $1.0 \mathrm{~s}$ & 3500 & $0.00 \mathrm{~nm}$ \\
\hline & & & & isn & 54 & 49.00 & & 1 & & & iPP \\
\hline KSH & & 5.14 & 52 & ipd & 53 & 58.00 & 1.8 & 1206 & 23.05 & 294 & ip \\
\hline NRN & & 6.48 & 38 & iPnd & 54 & 12.00 & -1.8 & I GTA & 23.09 & 74 & iPc \\
\hline & & & & isn & 55 & 23.80 & & 1 & & & $\mathrm{~s}$ \\
\hline QUE & & 6.96 & 208 & ipd- & 54 & 20.00 & 0.2 & 1 & & & $\mathrm{PP}$ \\
\hline FRU & & 7.09 & 24 & iPnd & 54 & 21.00 & -0.3 & I SOC & 24.74 & 296 & eP \\
\hline & & & & i Sn & 55 & 41.00 & & I MOY & 26.32 & 45 & iPc \\
\hline PRZ & & 8. 48 & 42 & iPnc & 54 & 39.00 & -0.7 & I ANN & 26.61 & 299 & $i P c$ \\
\hline & $\mathbf{N}$ & $10 \mathrm{~s}$ & 396 & $5.00 \mathrm{um}$ & & & & 1 & $1.0 \mathrm{~s}$ & 2700 & $0.00 \mathrm{~nm}$ \\
\hline I LG & & 8.55 & 35 & iPnd & 54 & 38.60 & -1.8 & I & & & es \\
\hline & $z$ & $14 \mathrm{~s}$ & 520 & $.00 \mathrm{um}$ & & & & 1 & & & iPP \\
\hline & & & & $i S_{n}$ & 56 & 18.00 & & I LZH & 26.62 & 81 & iPct \\
\hline MHI & & 9.08 & 273 & iPnd & 54 & 45.20 & -2.2 & 1 & & & is \\
\hline & & $0.4 \mathrm{~s}$ & 2370 & $.00 \mathrm{~nm}$ & & & $.8 \mathrm{mb}$ & 1 & & & iss \\
\hline & & & & esn & 56 & 04.00 & & | & & & iss \\
\hline NDI & & 9.43 & 143 & iPd & 54 & 51.00 & -0.8 & 1 & & & isP \\
\hline & & $0.5 \mathrm{~s}$ & 585 & $5.00 \mathrm{~nm}$ & & 6 & $.1 \mathrm{mb}$ & I ZAK & 27.21 & 49 & i Pc \\
\hline & & & & is & 56 & 25.00 & & I & $2.0 \mathrm{~s}$ & 4340 & $0.00 \mathrm{~nm}$ \\
\hline ASH & & 10.02 & 283 & $i P c$ & 54 & 57.50 & -1.9 & I & & & es \\
\hline & & & & is & 56 & 49.00 & & I & & & ePP \\
\hline KHI & & 10.15 & 261 & iPd & 55 & 01.00 & -0.4 & I KVT & 7.36 & 291 & ip \\
\hline VAN & & 10.22 & 283 & eP & 54 & 59.00 & $-3.0 x$ & $\mathrm{CD} 2$ & 27.93 & 92 & ip \\
\hline & & $3.0 \mathrm{~s}$ & $* * * *$ & $* * * n m$ & & 7 & $5 \mathrm{mb}$ & I & & & $\mathrm{S}$ \\
\hline KAT & & 11.80 & 288 & iPd & 55 & 19.00 & $-3.2 x$ & 1 & & & $\mathbf{s P}$ \\
\hline WMO & & 14.92 & 55 & iPd & 56 & 00.60 & -0.7 & I IRK & 28.45 & 45 & $\mathrm{P}$ \\
\hline & & & & s & 58 & 45.00 & & | HR I & 28.81 & 274 & iP \\
\hline DMN & & 14.99 & 122 & $e^{P}$ & 56 & 01.30 & -1.1 & I SIM & 28.87 & 299 & $P$ \\
\hline KKN & & 14.99 & 121 & eP & 56 & 01.10 & -1.3 & I MGI & 29.30 & 273 & ip \\
\hline PKI & & 15.22 & 121 & $e^{P}$ & 56 & 04.10 & $-1 \cdot 2$ & I KMI & 29.57 & 103 & iP \\
\hline SEM & & 15.59 & 23 & iPd & 56 & 07.40 & -2.0 & I & & & is \\
\hline & & $2.3 s$ & 8540 & $.00 \mathrm{~nm}$ & & 6 & . Bmb & I & & & PP \\
\hline TEH & & 15.68 & 273 & ep & 56 & 13.00 & 2.3 & 1 & & & SP \\
\hline IR 4 & & 16.15 & 272 & iPd & 56 & 17.10 & 0.7 & 1 MOS & 29.61 & 321 & iPc \\
\hline $\mathrm{SHI}$ & & 16.69 & 251 & iPd & 56 & 22.50 & -0.5 & 1 & & & es \\
\hline LNK & & 17.53 & 284 & eP & 56 & 29.00 & -2.9 & I & & & iss \\
\hline POO & & 18.00 & 170 & iPd & 56 & 38.60 & 1.5 & I JER & 9.70 & 272 & ePc \\
\hline LSA & & 18.34 & 105 & $i P c$ & 56 & 43.50 & 2.4 & OBN & 9.89 & 320 & $P$ \\
\hline & & & & $S$ & 00 & 00.50 & & I BSO & 30.44 & 271 & ip \\
\hline MAK & & 19.09 & 297 & $i P c$ & 56 & 48.70 & 0.6 & I KDE & 30.83 & 291 & ip \\
\hline & & $6.0 \mathrm{~s}$ & 8200 & $0.00 \mathrm{~nm}$ & & & $.4 \mathrm{mb} \times$ & В ВТО & 30.84 & 70 & iPc \\
\hline & $z$ & $13 \mathrm{~s}$ & 352 & .00 um & & & & I & & & $\mathrm{S}$ \\
\hline & $N$ & $14 \mathrm{~s}$ & 456 & .00 um & & & & I XAN & 31.14 & 83 & ¿Pc \\
\hline & $E$ & $14 \mathrm{~s}$ & 713 & 5.00 um & & & & 1 & & & $S$ \\
\hline & & & & is & 00 & 10.80 & & BDT & 31.43 & 120 & iPc \\
\hline KER & & 19.38 & 271 & ip & 56 & 52.50 & 1.1 & I GPA & 1.76 & 290 & $i P c$ \\
\hline GRS & & 19.49 & 287 & $P$ & 56 & 53.60 & 1.1 & I BCK & 1.96 & 284 & iP \\
\hline TAB & & 19.51 & 282 & ePc & 56 & 54.00 & 1.3 & I HHC & 31.99 & 69 & $i P c$ \\
\hline & & & & $i$ & 56 & 56.00 & & ! & & & $\mathrm{S}$ \\
\hline HYB & & 20.13 & 158 & iPd & 57 & 00.00 & 1.1 & 1 & & & $p P$ \\
\hline & & & & is & $\theta 0$ & 28.00 & & I GYA & 32.05 & 98 & $\mathrm{PC}$ \\
\hline NAK & & 20.21 & 286 & iPc & 57 & 00.50 & 1.0 & I & & & $\mathrm{s}$ \\
\hline GRO & & 20.41 & 298 & iPc & 57 & 02.50 & 1.1 & I ALT & 32.07 & 287 & ip \\
\hline & & $1.0 \mathrm{~s}$ & 5700 & $.00 \mathrm{~nm}$ & & & $.1 \mathrm{mb}$ & I HRT & 32.19 & 291 & iP \\
\hline & & & & is & $\theta 0$ & 39.00 & & I ELL & 32.63 & 283 & iPc \\
\hline NVS & & 26.42 & 21 & eP & 57 & 00.00 & -1.4 & I ISK & 2.63 & 291 & $i P c$ \\
\hline MTA & & 20.80 & 293 & iPc & 57 & 06.80 & 1.5 & I KIS & 32.67 & 302 & iPc \\
\hline & & & & es & 00 & 43.00 & & 1 & $19 \mathrm{~s}$ & 168 & 3.00 um \\
\hline ERE & & 20.92 & 288 & $i P c$ & 57 & 09.00 & 2.3 & ! & & & is \\
\hline STE & & 21.05 & 291 & iPc & 57 & 09.20 & 1.2 & 1 & & & $i p P$ \\
\hline LEN & & 21.44 & 290 & iPc & 57 & 16.00 & $4.2 x$ & KHT & 32.87 & 124 & $\mathrm{iPc}$ \\
\hline & & $3.0 \mathrm{~s}$ & $* * * *$ & $* * * n m$ & & & $.7 \mathrm{mb} \quad x$ & TIY & 33.10 & 75 & $\mathrm{iPc}$ \\
\hline SVE & & 21.52 & 345 & iPc & 57 & 13.00 & 0.7 & ! & & & SP \\
\hline & 2 & $15 \mathrm{~s}$ & 815 & .00 um & & & $2 M s=X$ & 1 & & & $\mathrm{PcP}$ \\
\hline & $\mathbf{N}$ & $15 \mathrm{~s}$ & 638 & .00 um & & & & 1 & & & $\mathrm{~s}$ \\
\hline & $E$ & $15 s$ & 235 & .00 um & & & & i & & & $S C P$ \\
\hline & & & & is & $\theta 0$ & 53.00 & & 1 & & & sS \\
\hline & & & & iPP & 57 & 53.00 & & 1 & & & PP \\
\hline ARU & & 21.63 & 341 & iPc & 57 & 14.50 & 1.2 & I PSN & 33.10 & 296 & $\mathrm{iPC}$ \\
\hline & $z$ & $18 \mathrm{~s}$ & 458 & $.00 \mathrm{um}$ & & & $9 M s z$ & I DST & 3.13 & 289 & $\mathrm{iPc}$ \\
\hline & $\mathrm{N}$ & $20 \mathrm{~s}$ & 299 & .00 um & & & & I HLW & 3.47 & 270 & iPc \\
\hline & $E$ & $16 s$ & 443 & .00 um & & & & I PPE & 33.49 & 301 & iPd \\
\hline & & & & is & 01 & 00.50 & & I DMK & 33.53 & 293 & iPd \\
\hline & & & & ess & 01 & 52.00 & & I EDC & 33.61 & 290 & iPd \\
\hline & & & & ePP & 57 & 52.50 & & I CLI & 33.73 & 301 & iPc \\
\hline & & & & esp & 58 & 20.50 & & I YER & 33.82 & 284 & $i P c$ \\
\hline AKH & & 21.76 & 292 & iPd & 57 & 19.60 & $4.7 x$ & BAC & 34.00 & 301 & eP \\
\hline BKR & & 21.76 & 293 & iPC & 57 & 16.80 & 1.9 & | VRI & 34.11 & 300 & $i P c$ \\
\hline & & & & is & 01 & 03.70 & & I ISR & 34.24 & 299 & $i P c$ \\
\hline BHD & & 21.84 & 270 & iPc & 57 & 16.50 & 0.9 & JMB & 34.35 & 294 & iPd \\
\hline & & & & is & 01 & 03.00 & & I I ZM & 34.37 & 287 & $i P c$ \\
\hline
\end{tabular}


$30 d \quad 23 h$

\begin{tabular}{|c|c|c|c|c|c|c|c|c|c|c|c|}
\hline & $Z$ & $18 \mathrm{~s}$ & 145 & 5.00 um & & & $6.8 \mathrm{Msz}$ & 1 & & & \\
\hline & & & & $\mathrm{i}$ & 04 & .44 .00 & & 1 & & & \\
\hline & & & & is & 05 & 24.00 & & I PRU & & 42.20 & 307 \\
\hline & & & & $\mathrm{i}$ & 59 & 45.30 & & i & & $2.0 \mathrm{~s}$ & 5820 \\
\hline PSZ & & 38.76 & 364 & iPc & 59 & 46.60 & 1.6 & 1 & & & \\
\hline OHR & & 38.81 & 293 & $I P C$ & 59 & 44.10 & -1.3 & 1 & & & \\
\hline & & & & $i$ & 01 & 30.50 & & I ORI & & 42.27 & 292 \\
\hline & & & & is & 05 & 24.10 & & I OZH & & 42.32 & 92 \\
\hline GZH & & 38.98 & 98 & IPc & 59 & 48.00 & 1.1 & 1 & & & \\
\hline & & & & $p P$ & 00 & 35.50 & $226 \mathrm{kmx}$ & 1 & & & \\
\hline & & & & SP & 01 & 00.00 & & 1 & & & \\
\hline & & & & PP & 01 & 27.00 & & I BRG & & 42.53 & 308 \\
\hline & & & & is & 05 & 30.00 & & 1 & & & \\
\hline & & & & sS & 06 & 51.00 & & 1 & & & \\
\hline BUD & & 39.37 & 303 & iPc & 59 & 50.00 & 0.2 & 1 & & & \\
\hline & & $2.0 s$ & 5250 & $0.00 \mathrm{~nm}$ & & & $6.8 \mathrm{mb}$ & 1 & & & \\
\hline VLS & & 39.56 & 288 & ep & 59 & 50.50 & -1.0 & I LJU & & 42.58 & 301 \\
\hline $\mathrm{MCO}$ & & 39.64 & 99 & iP & 59 & 54.90 & 2.6 & 1 & & & \\
\hline NJ2 & & 39.69 & 82 & $\mathrm{IPC}$ & 59 & 53.50 & 0.9 & 1 & & & \\
\hline & & & & i & 00 & 34.00 & & 1 & & & \\
\hline & & & & SCP & 05 & 23.80 & & 1 & & & \\
\hline & & & & is & 05 & 43.00 & & I KMR & & 42.62 & 304 \\
\hline & & & & ScS & 09 & 36.20 & & I CEY & & 2.69 & 301 \\
\hline TTG & & 39.73 & 295 & iPc & 59 & 52.50 & -0.3 & I KHC & & 42.88 & 306 \\
\hline & & & & $\epsilon$ & 00 & 00.21 & & 1 & & $1.0 \mathrm{~s}$ & 378 \\
\hline & & & & EPPP & 01 & 33.00 & & 1 & $z$ & $12 \mathrm{~s}$ & 81 \\
\hline & & & & is & 05 & 40.00 & & 1 & $\mathrm{~N}$ & $12 \mathrm{~s}$ & 169 \\
\hline SOD & & 39.75 & 335 & iP & 59 & 52.70 & 0.1 & 1 & $E$ & $14 \mathrm{~s}$ & 113 \\
\hline ULC & & 39.81 & 294 & $i P c$ & 59 & 52.40 & -1.2 & 1 & & & \\
\hline & & & & es & 05 & 39.80 & & 1 & & & \\
\hline AAE & & 39.82 & 235 & eP & 59 & 56.50 & 2.3 & 1 & & & \\
\hline SRO & & 39.83 & 303 & iPc & 59 & 53.70 & 0.1 & 1 & & & \\
\hline & & & & es & 05 & 36.10 & & I BRN & & 42.93 & 311 \\
\hline DL2 & & 39.94 & 71 & $\mathrm{iPc}$ & 59 & 55.50 & 0.9 & $\$ 60$ & & 43.00 & 293 \\
\hline & & & & $i$ & 00 & 37.00 & & I HFS & & 43.02 & 322 \\
\hline & & & & S & 05 & 49.00 & & I & & $0.7 \mathrm{~s}$ & 7060 \\
\hline HKC & & 40.04 & 98 & ip & 59 & 57.50 & 2.0 & $C L L$ & & 43.10 & 309 \\
\hline & & & & is & 06 & 20.00 & & $!$ & & $2.5 \mathrm{~s}$ & 7800 \\
\hline BRY & & 40.18 & 296 & iPC & 59 & 56.40 & -0.3 & I & & & \\
\hline & & & & es & 05 & 48.30 & & 1 & & & \\
\hline $\mathrm{HCY}$ & & 40.29 & 295 & IPC & 59 & 56.60 & -0.8 & I & & & \\
\hline & & & & es & 05 & 47.70 & & | TRI & & 43.16 & 301 \\
\hline ZST & & 40.62 & 304 & eP & 00 & 00.00 & -0.1 & 1 & & & \\
\hline & & & & e & 09 & 07.50 & & 1 & & & \\
\hline SNY & & 40.81 & 66 & iPc & 00 & 01.70 & 0.0 & 1 & & & \\
\hline & & & & SCP & 05 & 30.00 & & 1 & & & \\
\hline & & & & is & 85 & 50.00 & & 1 & & & \\
\hline KEV & & 40.82 & 338 & iPct & 00 & 02.00 & 0.6 & I & & & \\
\hline BLY & & 40.89 & 299 & eP & 00 & 04.00 & 1.6 & 1 & & & \\
\hline UPP & & 41.03 & 322 & iPc & 00 & 02.90 & -0.3 & I RBL & & 43.19 & 302 \\
\hline & & & & is & 05 & 55.00 & & I WET & & 43.34 & 306 \\
\hline KSP & & 41.04 & 308 & iPc & 00 & 03.80 & 0.3 & 1 & & & \\
\hline & & $0.9 \mathrm{~s}$ & 1020 & $0.00 \mathrm{~nm}$ & & & $.3 \mathrm{mb}$ & $\mid \mathrm{RCI}$ & & 43.35 & 290 \\
\hline & & & & iPP & 01 & 46.00 & & COP & & 43.37 & 315 \\
\hline VIE & & 41.11 & 304 & iPt & 00 & 05.20 & 1.1 & I & & & \\
\hline & & & & isP & 01 & 19.00 & & I DUI & & 43.39 & 295 \\
\hline & & & & iPP & 01 & 48.00 & & 1 & & $1.6 \mathrm{~s}$ & 4100 \\
\hline & & & & (PPP) & 02 & 26.00 & & | MSI & & 43.39 & 290 \\
\hline & & & & is & 06 & 04.80 & & BHG & & 43.49 & 304 \\
\hline VKA & & 41.14 & 304 & iPc & 00 & 05.10 & 0.7 & $K L M$ & & 43.69 & 132 \\
\hline & & $1.0 \mathrm{~s}$ & 1720 & $0.00 \mathrm{~nm}$ & & & $.5 \mathrm{mb}$ & 1 & & $0.7 \mathrm{~s}$ & 117 \\
\hline & & & & $i$ & 00 & 06.00 & & ! & & & \\
\hline & & & & $i(s P)$ & 01 & 22.00 & & ALP & & 43.80 & 297 \\
\hline & & & & IPP & 01 & 48.00 & & HOF & & 43.88 & 308 \\
\hline & & & & i & 05 & 34.00 & & AQU & & 44.01 & 296 \\
\hline & & & & is & 06 & 01.50 & & I MOX & & 44.02 & 308 \\
\hline & & & & iss & 09 & 05.00 & & I & & $1.0 \mathrm{~s}$ & 2030 \\
\hline BRT & & 41.53 & 293 & iPc & 00 & 07.08 & -0.6 & 1 & & $15 \mathrm{~s}$ & 163 \\
\hline $\mathrm{CN} 2$ & & 41.83 & 62 & iPc & 00 & 09.80 & -0.3 & I & N & $13 \mathrm{~s}$ & 175 \\
\hline SSE & & 41.89 & 82 & iPct & 00 & 11.50 & 0.9 & 1 & E & $13 \mathrm{~s}$ & 143 \\
\hline & & $5.0 \mathrm{~s}$ & 49 & $9.30 \mathrm{~nm}$ & & & $3 \mathrm{mb} \times$ & 1 & & & \\
\hline & & $13 \mathrm{~s}$ & 101 & 1.00 um & & & & 1 & & & \\
\hline & & & & $(p P)$ & 00 & 59.00 & $224 \mathrm{kmx}$ & 1 & & & \\
\hline & & & & SP & 01 & 12.00 & & 1 & & & \\
\hline & & & & PP & 01 & 49.00 & & ! & & & \\
\hline & & & & i & 03 & 02.00 & & I & & & \\
\hline & & & & PCS & 05 & 57.00 & & 1 & & & \\
\hline & & & & is & 86 & 13.00 & & I YAK & & 44.18 & 35 \\
\hline & & & & sS & 07 & 21.00 & & 1 & & $4.0 \mathrm{~s}$ & $* * * *$ \\
\hline & & & & SS & 09 & 20.00 & & 1 & $N$ & $13 \mathrm{~s}$ & 132 \\
\hline & & & & $(S c S)$ & 09 & 36.00 & & 1 & & & \\
\hline IPM & & 42.14 & 132 & ePc & 00 & 14.20 & 1.3 & 1 & & & \\
\hline & & & & e & 00 & 38.10 & & I & & & \\
\hline & & & & $e(S)$ & 00 & 56.60 & & I POI & & 44.30 & 296 \\
\hline & & & & e & 01 & 12.90 & & NB2 & & 44.34 & 323 \\
\hline & & & & e & 01 & 26.20 & & $!$ & & $1.1 \mathrm{~s}$ & 3740 \\
\hline & & & & e & 01 & 58.90 & & GRF & & 44.36 & 367 \\
\hline
\end{tabular}




\begin{tabular}{|c|c|c|c|c|c|c|c|c|c|c|c|}
\hline & & & e & 09 & 04.00 & & 1 & & & $\mathrm{pP}$ & 02 \\
\hline$D$ IX & 47.46 & 302 & ePt & 00 & 54.20 & -0.8 & WAJ & 51.90 & 68 & ePc & 01 \\
\hline BSF & 47.53 & 305 & iPc & 00 & 54.70 & -0.7 & 1 & & & e & 10 \\
\hline ENN & 47.60 & 309 & iPc & 00 & 55.30 & -0.4 & I OSK & 51.96 & 72 & $\mathrm{Pc}$ & 01 \\
\hline & $0.8 \mathrm{~s}$ & 422 & $2.00 \mathrm{~nm}$ & & & $.9 \mathrm{mb}$ & 1 & & & es & 08 \\
\hline & & & $i$ & 01 & 01.00 & & I LPO & 52.05 & 302 & iPc & 01 \\
\hline & & & epP & 01 & 42.50 & $217 \mathrm{kmX}$ & 1 & $0.6 \mathrm{~s}$ & 656 & $3.00 \mathrm{~nm}$ & \\
\hline HAU & 47.79 & 305 & iPc & 00 & 56.80 & -0.5 & I FLN & 52.07 & 307 & iPc & 01 \\
\hline & $1.0 \mathrm{~s}$ & 1500 & $.00 \mathrm{~nm}$ & & & $.4 \mathrm{mb}$ & 1 & $1.2 \mathrm{~s}$ & 2890 & $3.00 \mathrm{~nm}$ & \\
\hline & & & PP & 01 & 43.10 & $212 \mathrm{kmX}$ & I MVI & 52.10 & 84 & eP & 01 \\
\hline MYK & 47.79 & 88 & eP & 00 & 58.00 & 0.5 & I HIK & 52.16 & 71 & eP & 01 \\
\hline$D B N$ & 47.87 & 311 & iPc & 00 & 58.00 & 0.2 & I EKA & 52.22 & 316 & Pd & 01 \\
\hline & & & isP & 02 & 06.00 & & 1 & $1.0 \mathrm{~s}$ & 1110 & $0.00 \mathrm{~nm}$ & \\
\hline & & & iPP & 02 & 53.00 & & LFF & 52.29 & 302 & iPc & 01 \\
\hline & & & ePPP & 03 & 54.00 & & 1 & & & $p P$ & 02 \\
\hline & & & is & 07 & 37.00 & & GRR & 52.41 & 307 & $i P c$ & 01 \\
\hline & & & e & 08 & 37.00 & & MFF & 52.41 & 305 & $i P c$ & 01 \\
\hline & & & i & 08 & 49.00 & & SHJ & 52.48 & 73 & iPc & 01 \\
\hline & & & $\mathbf{i}$ & 09 & 02.00 & & ! & & & e & 08 \\
\hline & & & iscs & 10 & 15.00 & & I SUT & 52.56 & 60 & $P c$ & 01 \\
\hline & & & ess & 11 & 06.00 & & 1 & & & e & 10 \\
\hline & & & i & 11 & 32.00 & & LPF & 52.62 & 307 & iPc & 01 \\
\hline NGS & 48.17 & 76 & Pc & 01 & 01.00 & 0.7 & I WAK & 52.76 & 57 & eP & 01 \\
\hline & & & es & 09 & 10.00 & & 1 & & & e & 10 \\
\hline$B A G$ & 48.29 & 101 & iPct & 01 & 02.00 & 0.4 & I YSS & 53.00 & 55 & iPc & 01 \\
\hline & & & is & 07 & 27.00 & & 1 & $1.2 \mathrm{~s}$ & 2430 & $0.00 \mathrm{~nm}$ & \\
\hline DOU & 48.55 & 308 & Pc & 01 & 03.20 & 0.2 & ! & $16 s$ & 74 & $+.10 u m$ & \\
\hline & & & $\mathrm{pP}$ & 01 & 48.60 & $207 \mathrm{kmX}$ & 1 & $16 \mathrm{~s}$ & 60 & $.30 \mathrm{um}$ & \\
\hline & & & $\mathrm{S}$ & 07 & 50.50 & & 1 & $16 \mathrm{~s}$ & 82 & ?.10um & \\
\hline & & & $\operatorname{ses}$ & 10 & 31.10 & & ! & & & ePPP & 04 \\
\hline CVP & 48.56 & 98 & ePd & 01 & 04.00 & 0.5 & 1 & & & is & 08 \\
\hline & & & es & 01 & 17.00 & & 1 & & & eSP & 09 \\
\hline FRF & 48.56 & 299 & iPc & 01 & 02.60 & -0.6 & EPF & 53.13 & 300 & iPc & 01 \\
\hline UCC & 48.58 & 309 & iPc & 01 & 03.00 & -0.2 & 1 & $0.6 \mathrm{~s}$ & 12 & $.50 \mathrm{~nm}$ & \\
\hline & $4.0 \mathrm{~s}$ & 2740 & $.00 \mathrm{~nm}$ & & & $.0 \mathrm{mb} \times$ & I RMJ & 53.17 & 58 & $\mathrm{Pc}$ & 01 \\
\hline KBS & 48.67 & 347 & iPt & 01 & 04.60 & 1.0 & I MAT & 53.18 & 68 & iPct & 01 \\
\hline LMR & 48.71 & 299 & iPc & 01 & 03.60 & -0.8 & 1 & & & es & 08 \\
\hline & $1.0 \mathrm{~s}$ & 656 & $.00 \mathrm{~nm}$ & & & $.0 \mathrm{mb}$ & I HAK & 53.23 & 61 & $\mathrm{Pc}$ & 01 \\
\hline LRG & 48.79 & 299 & iPc & 01 & 04.30 & -0.6 & 1 & & & e & 08 \\
\hline NAI & 49.00 & 228 & iPd & 01 & 09.00 & 1.9 & I SAP & 53.24 & 60 & Pc & 01 \\
\hline & $1.8 \mathrm{~s}$ & 1770 & $.00 \mathrm{~nm}$ & & & $.2 \mathrm{mb}$ & 1 & & & S & 08 \\
\hline HMD & 49.10 & 73 & eP & 01 & 07.00 & -0.4 & 1110 & 53.28 & 70 & $P c$ & 01 \\
\hline & & & e & 09 & 23.00 & & ! & & & e & 02 \\
\hline$A G$ & 49.14 & 77 & eP & 01 & 08.00 & 0.2 & ABA & 53.28 & 292 & iP & 01 \\
\hline 017 & 49.35 & 75 & $\mathrm{PC}$ & 01 & 10.50 & 1.2 & 1 & & & is & 09 \\
\hline & & & es & 08 & 15.00 & & I NII & 53.31 & 66 & ePd & 01 \\
\hline$H I R$ & 49.57 & 73 & eP & 01 & 09.00 & -2.0 & I ASA & 53.73 & 59 & Pc & 01 \\
\hline LBF & 49.58 & 304 & iPc & 01 & 09.80 & -1.2 & | EBR & 53.73 & 298 & eP & 01 \\
\hline LOR & 49.59 & 305 & $\mathrm{iPc}$ & 01 & 10.00 & -1.1 & I & & & e & 04 \\
\hline & $0.9 \mathrm{~s}$ & 563 & $.00 \mathrm{~nm}$ & & & $.0 \mathrm{mb}$ & 1 & & & e & 88 \\
\hline & & & $p P$ & 01 & 56.80 & $213 \mathrm{kmx}$ & I & & & (S) & 08 \\
\hline $5 \mathrm{Al}$ & 49.61 & 70 & Pc & 01 & 11.00 & -0.3 & I CNP & 53.75 & 102 & ePc & 01 \\
\hline NOB & 49.64 & 76 & $\mathrm{Pc}$ & 01 & 11.20 & -0.4 & I & & & es & 02 \\
\hline & & & es & 07 & 46.00 & & 1 KOF & 53.77 & 69 & eP & 01 \\
\hline DP & 49.69 & 102 & eP & 01 & $21.0 B$ & $8.9 x$ & DDR & 54.12 & 69 & eP & 01 \\
\hline MYZ & 49.69 & 76 & eP & 01 & 12.00 & 0.0 & YAM & 54.15 & 66 & $\mathrm{PC}$ & 01 \\
\hline SHK & 49.70 & 73 & iPc & 01 & 11.80 & -0.2 & HAC & 54.20 & 63 & iPc & 01 \\
\hline SMF & 49.75 & 304 & iPc & 01 & 11.60 & -0.6 & MRK & 54.23 & 64 & iPc & 01 \\
\hline TAJ & 49.82 & 78 & eP & 01 & 12.00 & -1.0 & 1 & & & es & 09 \\
\hline SSF & 49.87 & 304 & iPc & 01 & 12.30 & -0.9 & I OYM & 54.37 & 69 & eP & 01 \\
\hline$A \vee F$ & 50.04 & 304 & iPc & 01 & 13.60 & -0.8 & AJI & 54.41 & 70 & ePd & 01 \\
\hline OKA & 50.59 & 72 & eP & 01 & 19.00 & 0.2 & I FKS & 54.42 & 66 & $\mathrm{Pc}$ & 01 \\
\hline & & & e & 08 & 16.00 & & 1 & & & es & 09 \\
\hline MZF & 50.69 & 304 & iPc & 01 & 19.10 & -0.3 & I UTS & 54.42 & 68 & $\mathrm{PC}$ & 01 \\
\hline KOC & 50.72 & 74 & eP & 01 & 20.00 & 0.3 & 1 & & & e & 09 \\
\hline TKM & 50.84 & 73 & eP & 01 & 20.00 & -0.6 & I URA & 54.54 & 60 & eP & 01 \\
\hline & & & es & 09 & 39.00 & & I SEN & 54.55 & 65 & Pc & 01 \\
\hline$T C F$ & 50.93 & 304 & iPc & 01 & 20.70 & -0.5 & 1 & & & es & 09 \\
\hline & & & $\mathrm{pP}$ & 02 & 07.30 & $210 \mathrm{kmx}$ & DLE & 54.62 & 314 & iPc & 01 \\
\hline $\mathrm{HIM}$ & 51.14 & 72 & Pc & 01 & 23.00 & 0.1 & ! & $1.2 \mathrm{~s}$ & 2400 & $.00 \mathrm{~nm}$ & \\
\hline & & & es & 08 & 40.00 & & I SEY & 4.63 & 34 & iPc & 01 \\
\hline MRT & 51.33 & 74 & $P$ & 01 & 25.00 & 0.6 & $!$ & $1.4 \mathrm{~s}$ & 1720 & $.00 \mathrm{~nm}$ & \\
\hline & & & e & 08 & 32.00 & & I & & & iPP & 03 \\
\hline TKS & 51.33 & 73 & iPc & 01 & 25.10 & 0.8 & 1 & & & is & 09 \\
\hline CAF & 51.38 & 302 & iPc & 01 & 24.10 & -0.6 & I & & & iss & 10 \\
\hline LSF & 51.39 & 304 & iPc & 01 & 23.80 & -0.9 & I & & & iss & 12 \\
\hline KOB & 51.56 & 72 & $P c$ & 01 & 26.50 & 0.4 & I TOK & 54.66 & 69 & $\mathbf{P}$ & 01 \\
\hline & & & e & 09 & 54.00 & & I DMU & 54.67 & 315 & $i P_{C}$ & 01 \\
\hline RJF & 51.65 & 303 & iPc & 01 & 26.20 & -0.5 & I & $1.2 \mathrm{~s}$ & 2900 & $.00 \mathrm{~nm}$ & \\
\hline SET & 51.72 & 291 & iP & 01 & 27.00 & -0.4 & I YOK & 54.68 & 69 & ePc & 01 \\
\hline WKY & 51.72 & 72 & iPc & 01 & 28.00 & 0.7 & I TSK & 54.72 & 68 & eP & 01 \\
\hline & & & es & 08 & 33.10 & & I OSH & 54.73 & 70 & $\mathrm{P}$ & 01 \\
\hline SA & 51.83 & 72 & $P c$ & 01 & 28.50 & 0.5 & I ISN & 54.78 & 65 & $\mathrm{Pc}$ & 01 \\
\hline & & & es & 08 & 24.00 & & ! & & & es & 09 \\
\hline KYO & 1.87 & 71 & Pc & 01 & 28.50 & 0.2 & I DAG & 54.80 & 344 & iPc & 01 \\
\hline & & & es & 08 & 33.00 & & 1 & $1.4 \mathrm{~s}$ & 2560 & $.00 \mathrm{~nm}$ & \\
\hline DF & 38 & 97 & iPc & 01 & 27.00 & $-1 \cdot 3$ & 1 & & & is & 09 \\
\hline
\end{tabular}




\begin{tabular}{|c|c|c|c|c|c|c|c|c|c|c|c|}
\hline & & & eSS & 14 & 36.00 & & LAT & B2.96 & 104 & $i P c$ & 04 \\
\hline UN! & 60.80 & 112 & $e^{P}$ & $\theta 2$ & 31.00 & -0.7 & KVG & 83.51 & 98 & iPc & 04 \\
\hline & $1.2 \mathrm{~s}$ & 2710 & $0.00 \mathrm{~nm}$ & & & $.8 \mathrm{mb}$ & I SIT & 84.33 & 14 & ePc & 04 \\
\hline-15 & 61.40 & 299 & $i P c$ & 02 & 35.30 & -0.1 & I FCC & 84.36 & 352 & $i P C$ & 84 \\
\hline AVE & 62.52 & 292 & iPc & 02 & 41.60 & -1.4 & ASPA & 84.36 & 125 & $i P c$ & 84 \\
\hline$I L T$ & 63.92 & 23 & $P$ & 02 & 51.50 & -0.1 & PMG & 4.77 & 106 & $e P c$ & 04 \\
\hline ITD & 64.55 & 222 & iPd & 02 & 56.00 & -0.3 & LMG & 5.24 & 105 & $e^{P}$ & 84 \\
\hline & & & is & 11 & 12.00 & & 1150 & 86.25 & 119 & iPc & 04 \\
\hline RI & 65.67 & 224 & iPd & 03 & 03.00 & -0.6 & I FFC & 89.03 & 356 & $i P c$ & 05 \\
\hline & & & is & 11 & 29.00 & & I CTA & 90.68 & 114 & iPct & 05 \\
\hline A! & 66.70 & 114 & iPc & 03 & 08.70 & -1.3 & i & & & $i$ & 06 \\
\hline GOH & 67.04 & 341 & $i P c$ & 03 & 11.10 & -0.4 & 1 & & & $i$ & 08 \\
\hline & $2.0 \mathrm{~s}$ & 1480 & $0.00 \mathrm{~nm}$ & & & $.4 \mathrm{mb}$ & i & & & is & 15 \\
\hline & & & is & 11 & 48.00 & & I EDM & 90.68 & 2 & $e P c$ & 05 \\
\hline $\mathrm{BCC}$ & 67.45 & 3 & IPC & 03 & 14.30 & 0.4 & i & & & $P P$ & 86 \\
\hline UL & 68.91 & 223 & iPd- & 03 & 23.50 & -0.2 & PHC & 91.80 & 11 & ePc & 85 \\
\hline & $0.7 \mathrm{~s}$ & 959 & $9.00 \mathrm{~nm}$ & & 6 & $.6 \mathrm{mb}$ & & $1.1 \mathrm{~s}$ & 1570 & $0.00 \mathrm{~nm}$ & \\
\hline & & & is & 12 & 04.90 & & I RSON & 92.05 & 350 & iPc & 85 \\
\hline MY & 69.17 & 39 & ePd & 03 & 23.80 & -0.9 & i & $1.8 \mathrm{~s}$ & 1740 & $0.00 \mathrm{~nm}$ & \\
\hline UMO & 69.35 & 88 & $e^{P}$ & 03 & 24.00 & -2.4 & i & & & PP & 06 \\
\hline & $1.5 \mathrm{~s}$ & 4778 & $0.00 \mathrm{~nm}$ & & 7 & $.8 m b$ & I BNH & 92.35 & 334 & ip & 05 \\
\hline SUA & 69.41 & 88 & eP & $\theta 3$ & 24.58 & -2.4 & I MNT & 92.36 & 336 & eP & 05 \\
\hline & $1.5 \mathrm{~s}$ & 3670 & $0.00 \mathrm{~nm}$ & & & $.9 \mathrm{mb}$ & i & $1.6 \mathrm{~s}$ & 540 & $0.00 \mathrm{~nm}$ & \\
\hline 2 & $20 \mathrm{~s}$ & & $1.80 u m$ & & & $.8 \mathrm{MsZ}$ & I OTT & 93.14 & 337 & ePc & 05 \\
\hline TEN & 71.50 & 292 & iPc & 03 & 37.50 & -1.7 & i & $1.0 \mathrm{~s}$ & 303 & $3.00 \mathrm{~nm}$ & \\
\hline & & & iPP & 06 & 15 & & I RSNY & 93.51 & 336 & $e^{P}$ & 05 \\
\hline & & & is & 12 & 41.40 & & & $1.8 \mathrm{~s}$ & 1080 & $0.00 \mathrm{~nm}$ & \\
\hline AU & 72.27 & 137 & $i P c$ & 03 & 43.80 & 0.2 & & & & $P P$ & 06 \\
\hline I MA & 72.28 & 17 & $i P c$ & 03 & 43.00 & -0.4 & ES & 3.57 & 1 & iPc & 85 \\
\hline SLR & 73.64 & 219 & $i P c$ & 03 & 10 & 0.3 & LHC & 70 & 347 & iPc & 85 \\
\hline & $1.8 \mathrm{~s}$ & 5730 & $0.00 \mathrm{~nm}$ & & & $.8 m b$ & i & $1.1 \mathrm{~s}$ & 738 & $8.00 \mathrm{~nm}$ & \\
\hline IBL & 73.66 & 133 & IPd & 03 & 51.80 & 0.0 & I PNT & 94.17 & 7 & $i P c$ & 05 \\
\hline EVA & 73.87 & 218 & iPc & 03 & 53.70 & 0. & I PGC & 94.39 & 9 & ePc & 05 \\
\hline INK & 74.06 & 9 & iPc & 03 & 53.60 & 0.2 & -1 & $1.5 \mathrm{~s}$ & 207 & $7.00 \mathrm{~nm}$ & \\
\hline & $0.9 \mathrm{~s}$ & 756 & $0.00 \mathrm{~nm}$ & & & $.4 \mathrm{mb}$ & I VSG & 94.58 & 98 & $P$ & 05 \\
\hline PP 1 & 74.13 & 219 & iPc & 03 & 54.50 & -0.1 & svo & 94.61 & 98 & $\mathrm{P}$ & 05 \\
\hline TTA & 74.19 & 20 & iPc & $\theta 3$ & 54.70 & 0. & HNR & 94.88 & 98 & ePt & 85 \\
\hline ADK & 74.23 & 36 & ePd & 03 & 51.90 & -2.8 & 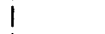 & & & $e(\mathrm{~S})$ & 16 \\
\hline KSR & 74.46 & 220 & $i P c$ & 03 & $\theta$ & $-\theta$. & TK & 4.89 & 126 & $i P_{C}$ & 85 \\
\hline 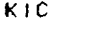 & 74.58 & 267 & iPC & 03 & 56.00 & -1.3 & DE & 5.07 & 130 & $\mathrm{iPc}$ & 05 \\
\hline & & & e & 13 & 06.30 & & 1 & $0.9 \mathrm{~s}$ & 269 & $9.00 \mathrm{~nm}$ & \\
\hline $\mathrm{COL}$ & 74.62 & 16 & $i P_{C}$ & 03 & 56.70 & -0.1 & I NEW & 95.42 & 5 & $i P_{C}$ & 05 \\
\hline & & & PP & 06 & 57.00 & & i & $20 \mathrm{~s}$ & 68 & $0.00 \mathrm{um}$ & \\
\hline BA & 74.62 & 16 & iPc & 03 & 56.90 & 0.1 & & & & e & 06 \\
\hline ITN & 75.02 & 119 & $i P c$ & 03 & 0 & -1 & LF & 6.80 & 340 & $P$ & 05 \\
\hline PRY & 75.83 & 219 & $e^{P}$ & 03 & 5 & -0 & DN & 91 & 340 & $\mathrm{P}$ & 5 \\
\hline FRB & 75.10 & 343 & $i P c$ & 03 & 59.00 & $-\theta$. & uS & 44 & 124 & iPc & 5 \\
\hline FS & 75.36 & 220 & iPc & 04 & 8 & -1 & & & 2 & IPC & 5 \\
\hline NA & 75.39 & 122 & iPd & 04 & 01.80 & 0.0 & RM & 98.11 & 2 & iPc & 05 \\
\hline & $0.5 \mathrm{~s}$ & 710 & $0.00 \mathrm{~nm}$ & & & $.7 \mathrm{mb}$ & I บто & 98.84 & 341 & iPc & 05 \\
\hline IAY & 75.49 & 104 & iPc & 04 & 02.00 & -0.5 & $B F D$ & 98.86 & 130 & $e^{P}$ & 05 \\
\hline vw & 75.75 & 21 & ePC & 04 & & 0. & RSSD & 99.72 & 356 & $e^{P}$ & 85 \\
\hline SEK & 76.09 & 218 & $i P c$ & 04 & s & 0 & PI & 100.19 & 3 & ePdiff & 106 \\
\hline$V I R$ & 7 & 219 & $e^{P}$ & 04 & e & 0 & & & 125 & ePdiff & 106 \\
\hline$w z$ & 7 & 221 & iPc & 04 & & o. & i & 95 & 129 & EPdiff & 106 \\
\hline$k 1$ & 7 & 32 & epd & 04 & e & -2 & $B D$ & 20 & 0 & iPdiff & 106 \\
\hline MR & & 18 & iPc & 04 & & -0 . & i & $1.8 \mathrm{~s}$ & 480 & $0.00 \mathrm{~nm}$ & \\
\hline EK & 77.17 & 137 & iPC & 84 & 10 & -0.4 & CAN & 101.87 & 125 & ePdiff & $f 06$ \\
\hline OA & 41 & 17 & ePc & 04 & & 0. & & & & $i$ & 10 \\
\hline BLF & 7 & 219 & iPc & 04 & . & -0 & $\mathrm{HC}$ & 99 & 11 & ePdift & 106 \\
\hline NW & 7 & 103 & ePc & 04 & 2 & 1. & LA & 04 & 337 & iPdiff & 106 \\
\hline DN & 78.68 & 27 & $\mathrm{P}$ & 04 & . & -1 & & $1.0 \mathrm{~s}$ & 80 & $0.00 \mathrm{~nm}$ & \\
\hline$A L$ & .38 & 141 & iP & & & -1 & $r$ & $\theta$ & 126 & ePdifi & \\
\hline KDC & 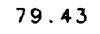 & 22 & if & + & & 0 & WOC & 102.43 & 10 & ePdi & 106 \\
\hline NUN & 3 & 142 & if & & & $-\theta$ & & & & ePP & 10 \\
\hline 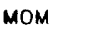 & & 100 & iPc & & & & & & & EPKKP & 22 \\
\hline$C A$ & .52 & 15 & ePc & 84 & & e & & & & e & 23 \\
\hline RM & & 216 & $i P c$ & 04 & & & & & & $e P$ & 106 \\
\hline 2 & Os & 25 & $5.50 \mathrm{um}$ & & 6 & $.6 \mathrm{Ms}$ & 1. & .79 & 10 & $e P d$ & \\
\hline LB & 80.70 & 141 & iPc & 04 & 9.50 & -6 & MAW & 103 & 183 & ePdiff & 106 \\
\hline & $0.8 \mathrm{~s}$ & 606 & $6.00 n$ & & 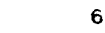 & $.4 \mathrm{~m}$ & & 1.1 & 136 & $6.00 \mathrm{~nm}$ & \\
\hline$G$ & 81.15 & 103 & ePc & & & & & & & es & 10 \\
\hline YKA & & 3 & $e^{P}$ & & & 1 & FVM & 10 & 345 & ePdiff & 106 \\
\hline RSNT & 8 & 3 & $e^{P}$ & 84 & & $\theta$ & & & 131 & $1.00 \mathrm{~nm}$ & \\
\hline$B N$ & & 131 & iP & & & & & 1 & 44 & 4.4 & \\
\hline WAO & 1 & 142 & i & 04 & & $-\theta$ & LD & 4 & 7 & $e^{P}$ & 106 \\
\hline LG & & 138 & IPC & 04 & & -0 & & I. & 010 & 0.0 & \\
\hline$R A$ & 11 & 122 & $P c$ & 04 & & 0.2 & 1 & 215 & 104 & 4.0 & \\
\hline & & 285 & 5.08 & & & $.0 \mathrm{mb}$ & $\mathrm{OL}$ & 34.19 & 357 & eP & 106 \\
\hline $\mathrm{CH}$ & 11 & 337 & ePc & 84 & .50 & 0.0 & 1 & 2 & 446 & $6.00 \mathrm{~nm}$ & \\
\hline & & 1930 & 0.00 & & & $.7 \mathrm{mb}$ & EUR & 104 & 5 & & 60 \\
\hline 86 & 82.12 & 122 & $i P C$ & 04 & $\theta$ & 0.4 & & $\theta$. & 92 & 2.7 & \\
\hline & & & $i$ & 14 & & & $S C P$ & & 340 & & 106 \\
\hline & & & eSKKS & 34 & 11.20 & & & $0.6 \mathrm{~s}$ & 27 & 7. $10 \mathrm{~nm}$ & \\
\hline T J & 4 & 325 & $e^{P}$ & 04 & & - & & & 7 & ef & 6 \\
\hline & & 999 & $9.00 \mathrm{~nm}$ & & & $.2 \mathrm{~m}$ & BKS & 13 & 11 & ePdiff & $f 06$ \\
\hline & 85 & 2 & iPc & 04 & 0 & 1.5 & & 30 & 20 & $.00 \mathrm{~nm}$ & \\
\hline
\end{tabular}




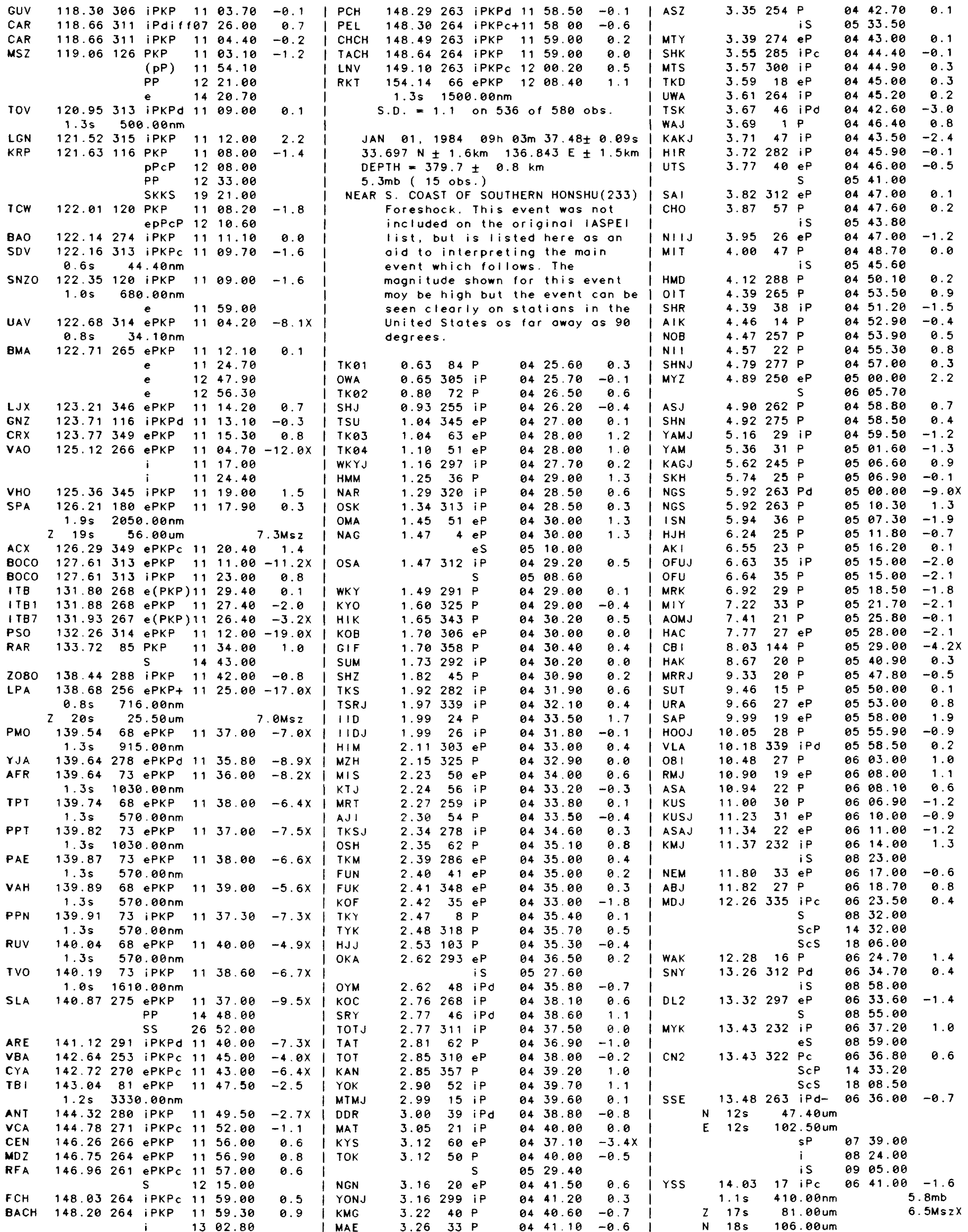


01d 69h

\begin{tabular}{|c|c|c|c|c|c|c|c|c|c|c|c|c|}
\hline & $E$ & $17 \mathrm{~s}$ & & $9.00 \mathrm{um}$ & & & & I ZAK & 29.66 & 314 & $\mathrm{eP}$ & 09 \\
\hline & & & & is & 69 & 07.00 & & 1 & $1.8 \mathrm{~s}$ & 2710 & $0.00 \mathrm{~nm}$ & \\
\hline KUR & & 14.31 & 33 & eP & 06 & 46.10 & 0.6 & 1 & $14 \mathrm{~s}$ & 49 & $9.10 \mathrm{um}$ & \\
\hline & & $0.8 \mathrm{~s}$ & $* * * *$ & $* * * * \mathrm{~nm}$ & & & $7.3 m b \times$ & 1 & $14 \mathrm{~s}$ & 24 & 4. 70 um & \\
\hline & & & & is & 09 & 25.00 & & 1 & $14 \mathrm{~s}$ & & $3.70 \mathrm{um}$ & \\
\hline$|S|$ & & 14.48 & 233 & iP & 06 & 47.30 & -0.2 & 1 & & & ePP & 10 \\
\hline & & & & is & 09 & 21.50 & & 1 & & & is & 13 \\
\hline NJ2 & & 15.21 & 269 & $\mathrm{PC}$ & 06 & 53.70 & -1.5 & 1 & & & ess & 15 \\
\hline & & & & $S$ & 69 & 34.00 & & I IRK & 29.82 & 318 & $e P c$ & 09 \\
\hline & & & & SCP & 14 & 36.70 & & 1 & $1.9 \mathrm{~s}$ & 1540 & $0.00 \mathrm{~nm}$ & \\
\hline & & & & ScS & 18 & 17.50 & & 1 & $16 s$ & 62 & $2.80 \mathrm{um}$ & \\
\hline ANP & & 15.80 & 242 & iP- & 07 & 03.00 & 1.4 & 1 & $16 \mathrm{~s}$ & & $.90 u m$ & \\
\hline & & & & es & 69 & 48.00 & & 1 & $16 \mathrm{~s}$ & & 5. 50 um & \\
\hline UGL & & 15.86 & 13 & iPC & 07 & 03.10 & 1.1 & 1 & & & epP & 10 \\
\hline$T \mid A$ & & 16.36 & 284 & $\mathrm{Pd}$ & 07 & 05.70 & -1.6 & 1 & & & ePcP & 12 \\
\hline & & & & $\mathbf{S P}$ & 08 & 35.00 & & 1 & & & es & 13 \\
\hline & & & & $s$ & 09 & 57.00 & & 1 & & & ess & 15 \\
\hline & & & & $\mathrm{SCP}$ & 14 & 38.80 & & I GTA & 30.14 & 292 & $P$ & 09 \\
\hline & & & & ScS & 18 & 19.30 & & 1 & & & PP & 10 \\
\hline BJI & & 17.69 & 297 & $\mathrm{PC}$ & 07 & 19.50 & -1.2 & 1 & & & $S$ & 13 \\
\hline & & & & $\mathrm{i}$ & 08 & 31.00 & & 1 & & & SCP & 15 \\
\hline & & & & $\mathbf{S P}$ & 08 & 59.00 & & I & & & SCS & 19 \\
\hline & & & & es & 10 & 26.00 & & I KMI & 30.80 & 263 & $\mathrm{Pd}$ & 09 \\
\hline & & & & ScS & 18 & 22.50 & & I SEY & 30.83 & 14 & eP & 09 \\
\hline $\mathrm{OZH}$ & & 18.13 & 246 & PPd & 07 & 25.60 & 0.5 & 1 & $1.1 \mathrm{~s}$ & 1150 & $0.00 \mathrm{~nm}$ & \\
\hline & & & & is & 10 & 20.00 & & 1 & $16 \mathrm{~s}$ & 87 & 7.00 um & \\
\hline WHN & & 19.30 & 267 & $\mathrm{Pd}$ & 07 & 37.00 & 0.3 & 1 & $16 \mathrm{~s}$ & & 1.00 um & \\
\hline$T I Y$ & & 20.21 & 288 & $P$ & 07 & 43.80 & -1.7 & 1 & & & I pP & 10 \\
\hline CVP & & 20.86 & 224 & ePc & 07 & 52.00 & 0.2 & 1 & & & IPCP & 12 \\
\hline & & & & es & 68 & 59.00 & & 1 & & & is & 14 \\
\hline $\mathrm{HHC}$ & & 21.30 & 297 & $e^{P}$ & 87 & 55.80 & -0.3 & 1 & & & is S & 16 \\
\hline GUMO & & 21.31 & 158 & eP & 07 & 58.00 & 1.8 & I MOY & 31.48 & 316 & eP & 09 \\
\hline GUA & & 21.37 & 158 & eP & 07 & 58.00 & 1.2 & 1 & $1.5 \mathrm{~s}$ & 1420 & $0.00 \mathrm{~nm}$ & \\
\hline & & $1.0 \mathrm{~s}$ & 320 & $0.00 \mathrm{~nm}$ & & & $.7 \mathrm{mb}$ & I KKM & 33.52 & 219 & ePd & 09 \\
\hline & & & & es & 11 & 26.00 & & I & $1.0 \mathrm{~s}$ & 268 & $8.70 \mathrm{~nm}$ & \\
\hline SZP & & 21.79 & 226 & iPc & 07 & 59.00 & -1.7 & 1 & & & e & 09 \\
\hline В TO & & 22.41 & 296 & $\mathbf{P}$ & 08 & 07.00 & 0.4 & 1 & & & e & 10 \\
\hline & & & & $\$$ & 11 & 46.00 & & I MNI & 34.00 & 202 & ePd & 09 \\
\hline$B A G$ & & 22.60 & 224 & iPd- & 08 & 06.00 & -2.6 & 1 & & & es & 10 \\
\hline & & & & is & 11 & 36.00 & & I UER & 35.56 & 313 & ePd & 10 \\
\hline XAN & & 23.16 & 279 & $P$ & 08 & 12.00 & -1.5 & 1 & $1.5 \mathrm{~s}$ & 1070 & $0.00 \mathrm{~nm}$ & \\
\hline & & & & $\mathrm{S}$ & 11 & 51.00 & & 1 & & & is & 15 \\
\hline GZH & & 23.17 & 249 & $\mathrm{Pc}$ & 08 & 12.50 & -1.0 & I CHG & 36.85 & 256 & iPd & 10 \\
\hline & & & & is & 11 & 56.00 & & 1 & $1.1 \mathrm{~s}$ & & $1.65 \mathrm{~nm}$ & \\
\hline MAN & & 23.73 & 221 & eP & 08 & 20.00 & 1.3 & I & & & es & 15 \\
\hline & & & & es & 12 & 11.00 & & I PCT & 37.20 & 248 & eP & 10 \\
\hline TUP & & 23.92 & 335 & iPd & 08 & 19.00 & -1.1 & 1 & & & e & 15 \\
\hline & & $0.8 \mathrm{~s}$ & 1080 & $0.00 \mathrm{~nm}$ & & 6 & $5.3 m b x$ & I BDT & 37.64 & 254 & eP & 10 \\
\hline & & & & es & 12 & 04.00 & & I ADK & 37.89 & 47 & eP & 10 \\
\hline PGP & & 24.76 & 219 & ePd & 88 & 08.50 & $-19.5 x$ & 1 & & & $i$ & 10 \\
\hline & & $1.0 \mathrm{~s}$ & 1408 & $3.00 \mathrm{~nm}$ & & & & $|A A|$ & 38.07 & 194 & ePd & 10 \\
\hline PET & & 24.81 & 32 & eP & 08 & 28.00 & -0.2 & 1 & & & is & 11 \\
\hline & & $1.0 \mathrm{~s}$ & 1200 & $0.00 \mathrm{~nm}$ & & & $0.2 m b x$ & I TIK & 38.24 & 356 & eP & 10 \\
\hline & Z & $16 \mathrm{~s}$ & & $5.00 \mathrm{um}$ & & & 5. $1 \mathrm{MszX}$ & 1 & $0.9 \mathrm{~s}$ & 1350 & $0.00 \mathrm{~nm}$ & \\
\hline & $N$ & $13 \mathrm{~s}$ & & $1.00 \mathrm{um}$ & & & & 1 & $19 \mathrm{~s}$ & & 4.00 um & \\
\hline & $E$ & $13 \mathrm{~s}$ & & $3.00 \mathrm{um}$ & & & & 1 & $18 \mathrm{~s}$ & & $0.00 \mathrm{um}$ & \\
\hline & & & & es & 12 & 28.00 & & 1 & $18 \mathrm{~s}$ & & 3.00um & \\
\hline $\mathrm{CCP}$ & & 26.11 & 210 & iPd & 08 & 41.00 & 0.7 & 1 & & & epP & 11 \\
\hline & & & & es & 09 & 55.50 & & 1 & & & ePP & 12 \\
\hline GYA & & 27.03 & 263 & $P$ & 08 & 47.00 & -1.6 & 1 & & & ePcP & 12 \\
\hline & & & & $S$ & 12 & 56.00 & & 1 & & & es & 15 \\
\hline LZH & & $27 \cdot 10$ & 284 & IPd & 08 & 44.00 & $-5.2 x$ & 1 & & & ess & 17 \\
\hline & & $7.0 \mathrm{~s}$ & $* * * *$ & $* * * * n m$ & & & $5.7 \mathrm{mb} \times$ & I KVG & 38.40 & 157 & eP & 10 \\
\hline & $N$ & $12 \mathrm{~s}$ & 59 & 3. 30 um & & & & I WMO & 39.13 & 300 & iPd & 10 \\
\hline & $E$ & 125 & & $3.60 u m$ & & & & BKB & 39.56 & 212 & ePc & 10 \\
\hline & & & & $P P$ & 08 & 59.00 & $62 \mathrm{kmx}$ & 1 & $1.1 \mathrm{~s}$ & 6888 & $8.10 \mathrm{~nm}$ & \\
\hline & & & & $\mathrm{IPCP}$ & 12 & 25.00 & & I NNT & 39.76 & 247 & eP & 10 \\
\hline & & & & is & 12 & 54.00 & & | ELT & 40.58 & 314 & iPC & 10 \\
\hline & & & & iss & 13 & 19.00 & & 1 & & & is & 16 \\
\hline CGP & & 27.51 & 207 & iPc & 08 & 52.50 & -0.2 & I NVS & 42.53 & 317 & iPc & 10 \\
\hline MGD & & 27.96 & 15 & iPc & 08 & 56.00 & -0.3 & 1 & $1.2 \mathrm{~s}$ & 2760 & $0.00 \mathrm{~nm}$ & \\
\hline & 2 & $15 \mathrm{~s}$ & & 2.00 um & & & $.4 M s z X$ & I & & & is & 16 \\
\hline & $\mathrm{N}$ & $15 \mathrm{~s}$ & 100 & 0.00 um & & & & I ILT & 42.58 & 23 & iPc & 10 \\
\hline & E & $15 s$ & & $3.00 \mathrm{um}$ & & & & 1 & $2.0 \mathrm{~s}$ & $* * * *$ & $* * * * \mathrm{~nm}$ & \\
\hline & & & & esp & 10 & 50.00 & & 1 & & & ipP & 12 \\
\hline & & & & is & 13 & 16.00 & & 1 & & & IPCP & 12 \\
\hline & & & & escs & 18 & 53.00 & & 1 & & & ePP & 12 \\
\hline $\mathrm{CD} 2$ & & 28.04 & 273 & $\mathbf{P}$ & 08 & 56.60 & -0.7 & 1 & & & is & 16 \\
\hline & & & & $P \subset P$ & 11 & 58.50 & & I & & & escs & 20 \\
\hline & & & & is & 13 & 13.00 & & I SNG & 42.62 & 240 & iPc & 11 \\
\hline DAV & & 28.46 & 204 & iPd- & 09 & 01.00 & -0.1 & 1 & $0.9 \mathrm{~s}$ & 521 & $1.01 \mathrm{~nm}$ & \\
\hline & & & & is & 13 & 21.00 & & 1 & & & is & 16 \\
\hline YAK & & 28.70 & 353 & iPd & 09 & 02.00 & -0.8 & | NKI & 42.77 & 46 & eP & 10 \\
\hline & & $0.8 \mathrm{~s}$ & 2430 & $0.00 \mathrm{~nm}$ & & & $6 \mathrm{mb} \times$ & I PMG & 43.97 & 165 & eP & 11 \\
\hline & & & & is & 13 & 26.00 & & SEM & 44.10 & 310 & eP & 11 \\
\hline PR & & 29.05 & 219 & $\begin{array}{l}\text { ePc } \\
\text { is }\end{array}$ & $\begin{array}{l}08 \\
09\end{array}$ & $\begin{array}{l}59.90 \\
40.70\end{array}$ & $-6.3 x$ & 1 & 2.15 & 5050 & $\begin{array}{l}0.00 \mathrm{~nm} \\
\mathrm{ePCP}\end{array}$ & 12 \\
\hline
\end{tabular}




\begin{tabular}{|c|c|c|c|c|c|c|c|c|c|c|}
\hline & & & & iPP & 14 & 28.50 & & 1 & & \\
\hline & & & & is & 19 & 28.90 & & 1 & & \\
\hline & & & & & 21 & 35.50 & & 1 & & \\
\hline $\mathrm{COL}$ & & 53.89 & 31 & eP & 12 & 25.00 & 0.3 & I KEV & & 65.22 \\
\hline & & & & is & 19 & 39.00 & & $\mathrm{i}$ & $z$ & $18 s$ \\
\hline FBA & & 53.89 & 31 & eP & 12 & 24.38 & -0.4 & i & & \\
\hline & & & & $\mathrm{i}$ & 12 & 25.30 & & $i$ & & \\
\hline DSH & & 54.06 & 296 & ¡Pd & 12 & 26.00 & -0.4 & 1 & & \\
\hline & & $5.0 \mathrm{~s}$ & $* * * *$ & $* * * * n m$ & & & $6.8 \mathrm{mb} \times$ & $i$ & & \\
\hline & $z$ & $20 s$ & & $3.00 \mathrm{um}$ & & & 5. $4 \mathrm{MsZ}$ & 1 & & \\
\hline & & & & is & 19 & 36.00 & & I STK & & 65.38 \\
\hline ISO & & 54.17 & 177 & eP & 12 & 25.00 & -2.1 & I CMS & & 65.39 \\
\hline CTA & & 54.23 & 169 & iP & 12 & 27.30 & -0.3 & I KLG & & 65.76 \\
\hline & & $1.0 \mathrm{~s}$ & 565 & $5.00 \mathrm{~nm}$ & & & $5.9 \mathrm{mb} \times$ & I SOD & & 66.56 \\
\hline & & & & $\mathrm{i}$ & 13 & 44.00 & & I BAL & & 66.73 \\
\hline & & & & is & 19 & 35.00 & & I KLB & & 67.39 \\
\hline HYB & & 54.36 & 268 & ePd & 12 & 27.00 & -1.8 & I KJF & & 67.85 \\
\hline & & $1.0 \mathrm{~s}$ & 970 & $3.00 \mathrm{~nm}$ & & & $6.1 \mathrm{mb} x$ & i & & \\
\hline & & & & e & 12 & 30.80 & & I KJF & & 67.85 \\
\hline & & & & es & 19 & 36.00 & & i & $z$ & $16 \mathrm{~s}$ \\
\hline & & & & eP'P. & 42 & 20.00 & & i & & \\
\hline TOA & & 54.86 & 34 & $\mathbf{P}$ & 12 & 31.80 & 0.0 & I & & \\
\hline SAM & & 55.06 & 298 & ePc & 12 & 32.00 & -1.5 & i & & \\
\hline & & $2.0 \mathrm{~s}$ & 2800 & $3.00 \mathrm{~nm}$ & & & $5.3 \mathrm{mb} \times$ & 1 & & \\
\hline & & & & iPP & 14 & 46.60 & & 1 & & \\
\hline & & & & is & 19 & 48.00 & & 1 & & \\
\hline & & & & isss & 26 & 82.20 & & I YOU & & 68.48 \\
\hline $\mathrm{KHE}$ & & 55.16 & 349 & iPc & 12 & 33.00 & -0.6 & i & & \\
\hline & & $1.3 \mathrm{~s}$ & 2000 & $3.00 \mathrm{~nm}$ & & & $5.3 \mathrm{mb} \times$ & I YKA & & 68.54 \\
\hline & & & & ¿PcP & 13 & 38.00 & & I IRT & & 68.57 \\
\hline & & & & iPP & 14 & 40.00 & & 1 & & \\
\hline & & & & IPPP & 16 & 02.00 & & I YKC & & 68.60 \\
\hline & & & & is & 19 & 50.00 & & I & & $1.0 \mathrm{~s}$ \\
\hline & & & & iscs & 21 & 45.00 & & I DAG & & 68.75 \\
\hline SVE & & 55.21 & 319 & ePc & 12 & 34.50 & 0.2 & I & & $0.9 \mathrm{~s}$ \\
\hline & & 2.05 & 3060 & $3.00 \mathrm{~nm}$ & & & $5.3 \mathrm{mb} \times$ & 1 & & \\
\hline & $z$ & $16 s$ & 59 & $7.50 \mathrm{um}$ & & & 5. $8 \mathrm{Ms} z \mathrm{X}$ & I NWAO & & 68.79 \\
\hline & $N$ & $16 \mathrm{~s}$ & & $.50 \mathrm{um}$ & & & & I SUF & & 69.25 \\
\hline & $E$ & $16 s$ & & 3.50 um & & & & I KRV & & 69.35 \\
\hline & & & & $i p P$ & 13 & 55.08 & $386 \mathrm{kmx}$ & i & & \\
\hline & & & & ePP & 14 & 40.00 & & I CAN & & 69.59 \\
\hline & & & & is & 19 & 46.00 & & 1 & & \\
\hline & & & & ises & 21 & 42.80 & & $i$ & & \\
\hline & & & & ess & 23 & 42.80 & & i & & \\
\hline ARU & & 56.40 & 319 & eP & 12 & 41.08 & -1.5 & 1 & & \\
\hline & & 7.05 & 7000 & $3.08 \mathrm{~nm}$ & & & $5.2 \mathrm{mb} \times$ & GRS & & 69.87 \\
\hline & $z$ & $16 s$ & 42 & $.50 \mathrm{um}$ & & & $.6 \mathrm{Ms} z \mathrm{X}$ & 1 & & $2.0 s$ \\
\hline & $N$ & $20 \mathrm{~s}$ & & $3.00 \mathrm{um}$ & & & & 1 & & \\
\hline & $E$ & $16 s$ & & $1.00 \mathrm{um}$ & & & & 1 & & \\
\hline & & & & epp & 14 & 04.00 & $398 \mathrm{kmx}$ & $i$ & & \\
\hline & & & & is & 20 & 06.00 & & I MTA & & 69.90 \\
\hline & & & & iscs & 21 & 50.00 & & 1 & $N$ & $20 \mathrm{~s}$ \\
\hline & & & & ess & 24 & 00.00 & & 1 & $E$ & $20 \mathrm{~s}$ \\
\hline MBL & & 56.91 & 199 & eP & 12 & 48.00 & 1.6 & 1 & & \\
\hline ASPA & & 57.11 & 183 & eP & 12 & 45.00 & -2.8 & 1 & & \\
\hline GBA & & 57.21 & 265 & $P d$ & 12 & 46.90 & -1.8 & I SHI & & 69.90 \\
\hline & & $0.9 \mathrm{~s}$ & 101 & $.50 \mathrm{~nm}$ & & & $5.3 \mathrm{mb}$ & I PYA & & 69.95 \\
\hline POO & & 57.75 & 272 & iPd & 12 & 51.80 & -0.7 & $i$ & & \\
\hline PCA & & 58.03 & 36 & $\mathbf{P}$ & 12 & 53.40 & -0.5 & $i$ & & \\
\hline OUE & & 58.37 & 287 & iP- & 12 & 56.00 & -0.7 & 1 & & \\
\hline & & & & es & 20 & 32.50 & & i & & \\
\hline$K O D$ & & 58.97 & 261 & iPd & 13 & 01.80 & 0. 7 & 1 & & \\
\hline I NK & & 59.04 & 26 & eP & 13 & 01.00 & 0.5 & I WAM & & 70.43 \\
\hline & & $0.7 \mathrm{~s}$ & 525 & $5.00 \mathrm{~nm}$ & & & 5. $1 \mathrm{mb} x$ & 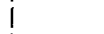 & & \\
\hline $\mathrm{NAU}$ & & 59.51 & 203 & IPd & 13 & 02.40 & -1.8 & 1 & & \\
\hline WBN & & 60.30 & 191 & eP & 13 & 08.00 & -1.5 & I TAB & & 70.54 \\
\hline $\mathrm{MBC}$ & & 68.75 & 15 & $e P$ & 13 & 10.50 & -1.4 & 1 & & \\
\hline SIT & & 61.33 & 38 & $\mathbf{P}$ & 13 & 17.10 & 1.2 & I NUR & & 71.09 \\
\hline MHI & & 61.77 & 297 & iPd & 13 & 19.80 & 0.5 & 1 & & $0.4 \mathrm{~s}$ \\
\hline & $z$ & 215 & & $.54 \mathrm{um}$ & & & $.4 \mathrm{MsZ}$ & 1 & $z$ & $19 \mathrm{~s}$ \\
\hline & & & & es & 21 & 16.00 & & 1 & & \\
\hline ASH & & 61.99 & 299 & $e^{P}$ & 13 & 22.00 & 1.5 & 1 & & \\
\hline & & $2.5 \mathrm{~s}$ & 5500 & $.00 \mathrm{~nm}$ & & & $.6 \mathrm{mb} \times$ & 1 & & \\
\hline & & & & esp & 15 & 23.00 & & 1 & & \\
\hline & & & & ePP & 15 & 44.00 & & 1 & & \\
\hline & & & & iPPP & 17 & 14.00 & & 1 & & \\
\hline VAN & & 62.17 & 299 & eP & 13 & 22.00 & 0.3 & 1 & & \\
\hline & $z$ & $18 \mathrm{~s}$ & 25 & $.40 u m$ & & & $.4 \mathrm{MsZ}$ & $i$ & & \\
\hline & $N$ & $18 \mathrm{~s}$ & 16 & $.60 \mathrm{um}$ & & & & PGC & & 71.33 \\
\hline & $\mathbf{E}$ & $18 \mathrm{~s}$ & 28 & $3.80 \mathrm{um}$ & & & & KER & & 71.74 \\
\hline & & & & ipp & 14 & 40.00 & $357 \mathrm{kmX}$ & I GMW & & 72.27 \\
\hline & & & & es & 21 & 20.00 & & 1 & & \\
\hline MEK & & 62.44 & 198 & iPc & 13 & 21.10 & -2.5 & isoc & & 72.29 \\
\hline APA & & 64.21 & 335 & ePc & 13 & 34.50 & 0.0 & 1 & & $6.0 \mathrm{~s}$ \\
\hline & & $1.0 \mathrm{~s}$ & 2130 & $.80 \mathrm{~nm}$ & & & $5.7 \mathrm{mb} \times$ & 1 & & \\
\hline & $z$ & $20 \mathrm{~s}$ & 14 & $.06 \mathrm{um}$ & & & . $1 M S Z$ & 1 & & \\
\hline & & & & iPCP & 14 & 09.00 & & 1 & & \\
\hline & & & & & & & & & & \\
\hline
\end{tabular}




\begin{tabular}{|c|c|c|c|c|c|c|c|c|c|c|c|}
\hline & & & $\mathbf{i}$ & 15 & 21.10 & & 1 & & & i & 17 \\
\hline BSO & 82.39 & 302 & eP & 15 & 21.00 & 1.8 & 1 & & & i & 19 \\
\hline AS & 82.54 & 54 & iPd & 15 & 22.00 & 2.0 & I ALO & 89.59 & 47 & eP & 15 \\
\hline & & & epp & 16 & 49.00 & $379 \mathrm{kmX}$ & i & $1.0 \mathrm{~s}$ & & $5.00 \mathrm{~nm}$ & \\
\hline & & & esP & 17 & 38.00 & & I MSI & 90.06 & 318 & eP & 15 \\
\hline & & & ePP & 18 & 38.00 & & I ACO & 92.65 & 42 & eP & 16 \\
\hline & & & epPP & 20 & 10.00 & & 1 & $1.8 \mathrm{~s}$ & 4090 & $9.80 \mathrm{~nm}$ & \\
\hline & & & ePPP & 20 & 38.00 & & I ISSF & 94.38 & 330 & eP & 16 \\
\hline & & & is & 25 & 08.00 & & I RLO & 95.29 & 40 & ePc & 16 \\
\hline & & & EPS & 26 & 24.00 & & I WLO & 95.56 & 43 & ePc & 16 \\
\hline & & & epscs & 26 & 59.00 & & 1 & $1.2 \mathrm{~s}$ & 1511 & $1.50 \mathrm{~nm}$ & \\
\hline & & & esscs & 27 & 44.00 & & I EBR & 95.64 & 328 & eP & 16 \\
\hline & & & esss & 33 & 00.00 & & 1 & & & e & 20 \\
\hline & & & eSSS & 34 & 25.00 & & 1 & & & e & 22 \\
\hline & & & e & 41 & 39.00 & & 1 & & & e & 26 \\
\hline & & & e & 43 & 30.00 & & 1 & & & e & 28 \\
\hline & & & e & 44 & 32.00 & & I MNT & 96.48 & 21 & iP & 16 \\
\hline MWC & 82.58 & 54 & eP & 15 & 21.00 & 0.6 & 1 & $1.6 \mathrm{~s}$ & 580 & $0.00 \mathrm{~nm}$ & \\
\hline BDW & 82.65 & 43 & eP & 15 & 20.50 & -0.2 & I ВНО & 96.71 & 41 & eP & 16 \\
\hline & $0.8 \mathrm{~s}$ & & $7.01 \mathrm{~nm}$ & & 4 & $.5 \mathrm{mb}$ & i & & & i & 16 \\
\hline$E Z N$ & 82.69 & 313 & eP & 15 & 19.80 & -0.8 & I $A B A$ & 97.69 & 324 & eP & 16 \\
\hline GSC & 82.78 & 52 & eP & 15 & 21.00 & -0.3 & I AVE & 105.37 & 330 & iPKP & 21 \\
\hline$v$ & 82. & 317 & eP & 15 & 23.00 & 2.0 & I PRY & 119.71 & 256 & ePdiff & 18 \\
\hline $1 Z M$ & 82.99 & 312 & iPc & 15 & 23.10 & 0.9 & 1 & & & $\mathrm{i}$ & 22 \\
\hline MM8 & 3 & 316 & iPc & 15 & 24.00 & 1.2 & I BFS & 120.25 & 256 & IPKPC & 21 \\
\hline RVR & 7 & 54 & eP & 15 & 24.00 & 0.9 & 1 & $0.5 \mathrm{~s}$ & 302 & $2.82 \mathrm{~nm}$ & \\
\hline BK & 9 & 48 & eP & 15 & 23.50 & o. 1 & I SWZ & 121.55 & 257 & $e(P K P)$ & 21 \\
\hline HOF & 83.25 & 328 & iPc & 15 & 25.10 & 1.8 & I SPA & 123.52 & 180 & IPKPd & 21 \\
\hline & & & i & 15 & 26.90 & & 1 & $1.0 \mathrm{~s}$ & 399 & $9.50 \mathrm{~nm}$ & \\
\hline & & & es & 25 & 14.00 & & I & $18 \mathrm{~s}$ & 12 & $2.37 \mathrm{um}$ & \\
\hline KHC & 83.34 & 326 & $\mathbf{P}$ & 15 & 23.50 & -0.2 & I UPA & 125.27 & 46 & ePKPc & 21 \\
\hline & 1.05 & 642 & $2.00 \mathrm{~nm}$ & & & $.3 \mathrm{mb} \times$ & 1 & $0.8 \mathrm{~s}$ & 179 & $9.10 \mathrm{~nm}$ & \\
\hline & $12 \mathrm{~s}$ & & 9.80 um & & & $4 M s z X$ & 1 & $20 \mathrm{~s}$ & & $7.45 \mathrm{um}$ & \\
\hline & $12 \mathrm{~s}$ & & $6.60 \mathrm{um}$ & & & & 1 & & & $\mathrm{i}$ & 22 \\
\hline & $14 \mathrm{~s}$ & & 9.20 um & & & & 1 & & & $\mathbf{i}$ & 23 \\
\hline & & & i & 15 & 27.50 & & 1 & & & $\mathrm{i}$ & 24 \\
\hline & & & $i p P$ & 16 & 57.50 & $413 \mathrm{kmX}$ & I SOV & 130.25 & 37 & ePKP & 21 \\
\hline & & & e & 18 & 50.50 & & I CAR & 130.57 & 31 & ePKP & 21 \\
\hline & & & $\mathrm{S}$ & 25 & 15.00 & & I TRN & 132.61 & 25 & ePKP & 22 \\
\hline & & & sS & 27 & 49.00 & & I PSO & 132.71 & 50 & ePKP & 21 \\
\hline & & & e & 30 & 57.00 & & I NVL & 132.81 & 202 & ePKP & 21 \\
\hline$P L M$ & 83.89 & 54 & eP & 15 & 26.00 & -1.0 & 1 & & & iPP & 24 \\
\hline GRF & 84.00 & 328 & iPc & 15 & 29.00 & 2.0 & I & & & eSP & 34 \\
\hline & & & ic & 15 & 31.00 & & I GUV & 134.59 & 28 & ePKP & 22 \\
\hline & & & e & 15 & 33.40 & & I PCH & 157.25 & 97 & ePKPc & 22 \\
\hline & & & e & 15 & 40.00 & & 1 & & & $i$ & 23 \\
\hline & & & epp & 17 & 01.60 & $405 \mathrm{kmX}$ & $\mathrm{FCH}$ & 157.39 & 97 & iPKPC & 22 \\
\hline & & & eSKS & 25 & 24.00 & & 1 & & & i & 23 \\
\hline BAR & 84.42 & 54 & eP & 15 & 31.00 & 1.6 & $1 \mathrm{BAO}$ & 161.49 & 15 & $e(P K P)$ & 22 \\
\hline MMU & 84.52 & 47 & ip & 15 & 12.10 & $-18.1 x$ & i & $5.0 .=1$ & 1.2 & on 346 & 5 of \\
\hline RSON & 84.76 & 29 & eP & 15 & 29.40 & -1.3 & I & & & & \\
\hline & $0.6 \mathrm{~s}$ & & $3.35 \mathrm{~nm}$ & & & $.7 \mathrm{mb}$ & JAN & 01 , & 1984 & $09 \mathrm{~h} 0$ & $3 m$ \\
\hline & & & e & 15 & 31.46 & & 33. & $681 \mathrm{~N} \pm$ & \pm 1.3 & $3 \mathrm{~km} \quad 13$ & 36.79 \\
\hline OHR & 85.12 & 317 & ip & 15 & 31.00 & -1.8 & DEP & $2 T H=38$ & 83.3 & \pm 0.6 & $\mathrm{~km}$ \\
\hline & & & $i$ & 15 & 36.10 & & 6.6 & $5 \mathrm{mb}(77$ & 7 obs & s.) & \\
\hline & & & es & 25 & 18.60 & & NEAR & S. COAS & ST OF & F SOUTHE & HERN \\
\hline RMU & 85.43 & 48 & eP & 15 & 38.10 & $3.6 x$ & 1 & FeIt (I & IV JN & $M A$ ) of & Tate \\
\hline ATH & 85.44 & 313 & $\mathrm{iPc}$ & 15 & 36.00 & 1.7 & 1 & Yokohom & mo, $T$ & Tokyo, L & Utsu \\
\hline & & & is & 25 & 24.00 & & | & Onohomo & $0:(1$ & $1 \| 1 \mathrm{JMA}$ & A) on \\
\hline GLA & 85.44 & 53 & eP & 15 & 35.00 & 0.5 & 1 & ot Chic & chibu & $\mathrm{u}, \mathrm{Kumo}$ & gayo \\
\hline TR I & 85.69 & 324 & $i P_{c}$ & 15 & 37.30 & 1.9 & 1 & Mito, S & Shiro & okowo, & Chit \\
\hline & & & $i p p$ & 17 & 04.00 & $375 \mathrm{kmX}$ & 1 & Nikko, & Fuku & ushimo & ond \\
\hline & & & iPP & 18 & 59.40 & & 1 & Felt fr & $\mathrm{rom} H$ & Hokkoido & do to \\
\hline & & & i pPP & 20 & 20.00 & & 1 & nor theo & oster & rn Kyush & hu 0 \\
\hline & & & is & 25 & 24.00 & & I & Bonin 1 & Is 100 & nos. & \\
\hline & & & iSP & 26 & 20.00 & & I & & & & \\
\hline & & & iss & 28 & 02.00 & & I OWA & 0.63 & 308 & ip & 04 \\
\hline & & & iss & 31 & 08.00 & & 1 & & & s & 05 \\
\hline & & & isss & 34 & 58.00 & & I TKOI & 0.67 & 83 & eP & 04 \\
\hline CTI & 86.47 & 325 & iPc & 15 & 41.20 & 1.9 & I TKOZ & 0.84 & 71 & $P$ & 04 \\
\hline & & & $i p p$ & 17 & 10.00 & $384 \mathrm{kmX}$ & I SHJ & 0.89 & 255 & $P$ & 04 \\
\hline GOL & 87.05 & 43 & iP & 15 & 44.50 & 2.1 & 1 & & & s & 05 \\
\hline & $0.8 \mathrm{~s}$ & & $4.46 \mathrm{~nm}$ & & 4 & $.4 \mathrm{mb}$ & I TSU & 1.04 & 347 & ip & 04 \\
\hline GLD & 87.10 & 43 & iP & 15 & 45.00 & 2.5 & I & & & es & 05 \\
\hline & $1.2 \mathrm{~s}$ & 84 & $4.85 \mathrm{~nm}$ & & & $.5 \mathrm{mb}$ & I TKO3 & 1.09 & 63 & $P$ & 04 \\
\hline POI & 88.35 & 322 & iPc & 15 & 50.00 & 1.9 & I WKYJ & 1.13 & 299 & $P$ & 04 \\
\hline & & & e & 17 & 21.00 & & I TKO4 & 1.14 & 52 & $\mathbf{P}$ & 04 \\
\hline & & & e & 19 & 26.00 & & I NAR & 1.28 & 322 & eP & 04 \\
\hline LHC & 88.47 & 29 & eP & 15 & 48.50 & -0.1 & HMM & 1.28 & 37 & eP & 04 \\
\hline & $1.2 \mathrm{~s}$ & 3531 & $1.00 \mathrm{~nm}$ & & & $.1 \mathrm{mb} \times$ & 1 & & & es & 05 \\
\hline ORO & 88.49 & 327 & eP & 15 & 50.50 & 1.6 & I OSK & 1.32 & 315 & iP & 04 \\
\hline & & & $i$ & 17 & 20.80 & & OSA & 1.45 & 314 & iP & 04 \\
\hline RMP & 89.03 & 322 & iPc & 15 & 52.00 & 0.7 & 1 & & & $s$ & 05 \\
\hline & & & iPP & 19 & 01.00 & & WKY & 1.46 & 292 & ¡P & 04 \\
\hline & & & is & 25 & 52.00 & & 1 & & & is & 05 \\
\hline & & & iPS & 26 & 40.00 & & NAG & 1.49 & 6 & iP & 04 \\
\hline$P F$ & 89.06 & 322 & iPc & 15 & 53.50 & 1.9 & & & & es & 05 \\
\hline
\end{tabular}




\begin{tabular}{|c|c|c|c|c|c|c|c|c|c|c|c|}
\hline TKD & 3.62 & 19 & $\mathrm{P}$ & 04 & 49.50 & 0.4 & I HOOJ & 10.08 & 29 & $\mathbf{P}$ & 06 \\
\hline & & & $\mathrm{s}$ & 05 & 43.00 & & I NGO & 10.40 & 230 & iP & 06 \\
\hline$H I R$ & 3.68 & 282 & ¡P & 04 & 49.80 & 0.1 & 1 & & & is & 08 \\
\hline & & & $S$ & 85 & 44.80 & & I OВ । & 10.51 & 27 & $P$ & 06 \\
\hline WAJ & 3.70 & 1 & iP & 04 & 50.70 & 0.8 & 1 & & & $\mathrm{~S}$ & 68 \\
\hline & & & es & 05 & 45.00 & & I NAH & 10.85 & 229 & iP & 86 \\
\hline KAKJ & 3.75 & 47 & P & 04 & 49.00 & -1.4 & ! & & & is & 08 \\
\hline SAI & 3.80 & 313 & iP & 04 & 51.40 & 0.6 & I RMJ & 10.92 & 19 & $\mathbf{P}$ & 06 \\
\hline & & & $\mathrm{s}$ & 05 & 47.20 & & I & & & $\mathrm{s}$ & 08 \\
\hline UTS & 3.81 & 41 & iP & 04 & 49.50 & -1.5 & I ASA & 10.97 & 22 & P & 86 \\
\hline $\mathrm{CHO}$ & 3.91 & 57 & eP & 04 & 51.00 & -0.9 & I & & & $\mathrm{s}$ & 08 \\
\hline$N \backslash 1 \mathrm{~J}$ & 3.98 & 26 & eP & 04 & 52.00 & -0.6 & I KUS & 11.03 & 30 & iP & 06 \\
\hline$M \perp T$ & 4.04 & 47 & $e^{P}$ & 04 & 52.00 & -1.2 & 1 & & & $S$ & 08 \\
\hline HMD & 4.09 & 289 & iP & 04 & 54.00 & 0.3 & I KUSJ & 11.26 & 31 & $P$ & 06 \\
\hline & & & is & 05 & 51.20 & & ASAJ & 11.37 & 22 & iP & 86 \\
\hline 017 & 4.34 & 266 & $\mathrm{P}$ & 04 & 57.30 & 1.1 & NEM & 11.83 & 33 & iP & 06 \\
\hline NOB & 4.42 & 257 & P & 04 & 57.20 & 0.2 & 1 & & & is & 08 \\
\hline & & & is & 05 & 56.00 & & $A B J$ & 11.85 & 27 & iP & 06 \\
\hline SHR & 4.43 & 38 & eP & 04 & 56.00 & -1.1 & $!$ & & & es & 68 \\
\hline & & & es & 05 & 53.00 & & I WAK & 12.31 & 16 & $\mathbf{P}$ & 06 \\
\hline A IK & 4.49 & 15 & $\mathrm{P}$ & 04 & 56.90 & -0.8 & I OKH & 20.34 & 11 & IPd & 07 \\
\hline & & & is & 05 & 55.80 & & I & $3.0 \mathrm{~s}$ & $* * * *$ & $* * * n \mathrm{~nm}$ & \\
\hline Nil & 4.60 & 23 & $e^{P}$ & 04 & 59.00 & 0.2 & 1 & $11 \mathrm{~s}$ & 44 & 4. $10 \mathrm{um}$ & \\
\hline & & & $s$ & 06 & 00.40 & & I & $11 \mathrm{~s}$ & 139 & $9.00 \mathrm{um}$ & \\
\hline ONA & 4.68 & 45 & P & 04 & 58.00 & -1.7 & 1 & & & is & 11 \\
\hline & & & is & 05 & 56.70 & & I SKR & 22.09 & 34 & eP & 08 \\
\hline SHNJ & 4.75 & 277 & $\mathrm{P}$ & 05 & 00.90 & 0.5 & $!$ & $1.3 \mathrm{~s}$ & $* * * *$ & $\cdots * n \mathrm{~nm}$ & \\
\hline ASJ & 4.86 & 262 & $\mathrm{P}$ & 05 & 02.80 & 1.1 & $?$ & $14 \mathrm{~s}$ & 120 & $0.00 \mathrm{um}$ & \\
\hline & & & $\mathrm{s}$ & 06 & 09.00 & & ? & $16 \mathrm{~s}$ & & $1.80 u m$ & \\
\hline SHN & 4.88 & 275 & P & 05 & 02.40 & 0.7 & 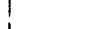 & $14 \mathrm{~s}$ & 129 & $9.00 \mathrm{um}$ & \\
\hline FKS & 5.05 & 35 & iP & 05 & 02.50 & -1.0 & $!$ & & & is & 11 \\
\hline & & & $\mathrm{s}$ & 06 & 05.00 & & I $\mathrm{HKC}$ & 22.91 & 246 & ip & 08 \\
\hline KUM & 5.17 & 262 & eP & 05 & 05.00 & 0.2 & 1 & & & is & 11 \\
\hline & & & $S$ & 06 & 13.80 & & $1 \mathrm{MCO}$ & 23.49 & 247 & ip & 08 \\
\hline YAMJ & 5.20 & 29 & eP & 05 & 05.00 & -0.1 & I OCP & 23.71 & 221 & eP & 08 \\
\hline$F K K$ & 5.35 & 271 & $\mathbf{P}$ & 05 & 06.80 & 0.0 & $18 O D$ & 28.59 & 335 & iPc & 09 \\
\hline & & & $\mathrm{s}$ & 06 & 14.10 & & 1 & $0.9 \mathrm{~s}$ & 1060 & $0.00 \mathrm{~nm}$ & \\
\hline YAM & 5.40 & 31 & eP & 05 & 07.00 & -0.3 & $K M I$ & 30.76 & 263 & iP- & 89 \\
\hline & & & es & 06 & 12.00 & & 1 & $14 \mathrm{~s}$ & 76 & 5. Boum & \\
\hline$S A G$ & 5.44 & 267 & eP & 05 & 08.00 & 0.3 & 1 & & & DP & 10 \\
\hline & & & $\mathrm{s}$ & 06 & 21.00 & & 1 & & & SP & 10 \\
\hline KAGJ & 5.58 & 245 & $P$ & 05 & 08.80 & -0.5 & 1 & & & $\mathrm{PCP}$ & 13 \\
\hline SEN & 5.65 & 35 & ¡P & 05 & 08.90 & -1.2 & I & & & 5 & 13 \\
\hline & & & is & 06 & 16.50 & & 1 & & & sS & 15 \\
\hline KAG & 5.67 & 250 & P & 05 & 10.10 & -0.1 & 1 & & & $S C P$ & 15 \\
\hline & & & is & 06 & 23.40 & & I & & & PCS & 16 \\
\hline TAJ & 5.72 & 241 & iP & 05 & 10.90 & 0.1 & $!$ & & & ScS & 19 \\
\hline & & & is & 06 & 24.70 & & I SMY & 32.70 & 43 & ePd & 09 \\
\hline SKH & 5.77 & 26 & $P$ & 05 & 11.40 & 0.1 & I JAY & 36.19 & 173 & ePc & 10 \\
\hline NGS & 5.88 & 263 & $\mathbf{P}$ & 5 & 13.10 & 0.5 & 1 & $1.3 \mathrm{~s}$ & 1332 & $2.80 \mathrm{~nm}$ & \\
\hline ISN & 5.98 & 36 & eP & 85 & 12.00 & -1.6 & MOM & 36.91 & 162 & ePd & 10 \\
\hline & & & is & 86 & 20.90 & & NST & 37.58 & 251 & iPd & 10 \\
\hline 120 & 6.25 & 277 & iP & 05 & 18.00 & 1.3 & ADK & 37.93 & 47 & $i P c$ & 10 \\
\hline & & & es & 06 & 34.00 & & I LSA & 38.80 & 277 & iPd & 10 \\
\hline HJH & 6.27 & 25 & eP & 05 & 16.00 & -0.9 & I & & & PP & 11 \\
\hline$A K I$ & 6.58 & 23 & iP & 05 & 20.30 & -0.1 & КHT & 39.30 & 251 & eP & 10 \\
\hline & & & S & 06 & 38.10 & & B BKB & 39.53 & 212 & ePd & 10 \\
\hline OFUJ & 6.67 & 35 & eP & 05 & 20.00 & -1.4 & 1 & $1.1 \mathrm{~s}$ & 5550 & $060 \mathrm{~nm}$ & \\
\hline & & & (S) & 06 & 37.70 & & I MDG & 3964 & 166 & ePc & 10 \\
\hline OFU & 6.68 & 35 & iP & 85 & 20.00 & -1.5 & I RAB & 40.38 & 156 & iPCt & 10 \\
\hline & & & is & 86 & 34.70 & & I LAT & 41.26 & 165 & ePd & 10 \\
\hline $\mathrm{FKJ}$ & 6.74 & 264 & $P$ & 05 & 22.70 & 0.4 & I MKS & 42.02 & 206 & iPd & 10 \\
\hline & & & $\mathrm{s}$ & 06 & 44.00 & & NKI & 42.82 & 46 & IPd & 11 \\
\hline MRK & 6.96 & 29 & $\mathbf{P}$ & 05 & 23.50 & -1.2 & LMG & 43.71 & 164 & eP & 11 \\
\hline & & & S & 06 & 42,30 & & $K L M$ & 44.72 & 235 & eP & 11 \\
\hline$M I Y$ & 7.26 & 33 & P & 05 & 26.20 & -1.9 & 1 & $0.8 \mathrm{~s}$ & 175 & $5.20 \mathrm{~nm}$ & \\
\hline & & & is & 06 & 46.80 & & I KUPN & 44.84 & 164 & eP & 11 \\
\hline AOMJ & 7.44 & 22 & $\mathbf{P}$ & 05 & 30.00 & -0.2 & I PRZ & 46.00 & 299 & $i P C$ & 11 \\
\hline AOM & 7.80 & 23 & iP & 05 & 34.20 & -0.2 & 1 & $5.0 \mathrm{~s}$ & $* * *$ & $* * * n m$ & \\
\hline & & & $\mathrm{S}$ & 07 & 01.80 & & 1 & $16 \mathrm{~s}$ & 47 & $7.00 \mathrm{um}$ & \\
\hline HAC & 7.81 & 28 & iP & 05 & 33.00 & -1.4 & 1 & $16 s$ & 33 & $3.00 \mathrm{um}$ & \\
\hline & & & is & 86 & 58.00 & & 1 & $16 s$ & 37 & 7.00 um & \\
\hline CBI & 8.05 & 143 & $\mathrm{P}$ & 05 & 33.00 & $-4.3 x$ & 1 & & & epP & 12 \\
\hline NZJ & 8.19 & 232 & iP & 05 & 37.90 & -1.0 & 1 & & & is & 17 \\
\hline & & & is & 07 & 13.60 & & 1 & & & escs & 20 \\
\hline HAK & 8.70 & 20 & $\mathrm{P}$ & 05 & 44.60 & -0.3 & $!$ & & & ess & 21 \\
\hline & & & $\mathrm{S}$ & 07 & 18.50 & & TSI & 46.58 & 238 & ePd & 11 \\
\hline MVI & 9.19 & 213 & $P$ & 65 & 49.50 & -1.2 & 1 & $0.8 \mathrm{~s}$ & 1001 & 1. $.50 \mathrm{~nm}$ & \\
\hline MRRJ & 9.36 & 20 & $P$ & 65 & 51.90 & -0.7 & $T L G$ & 46.61 & 300 & ip & 11 \\
\hline SUT & 9.49 & 16 & P & 05 & 53.80 & -0.3 & $!$ & & & ipp & 12 \\
\hline & & & is & 07 & 40.00 & & 1 & & & is & 17 \\
\hline URA & 9.69 & 27 & eP & 05 & 57.00 & 0.5 & AAA & 46.93 & 300 & ip & 11 \\
\hline & & & $\mathrm{S}$ & 07 & 40.40 & & $!$ & & & iPCP & 12 \\
\hline SAP & 10.02 & 19 & eP & 06 & 00.00 & -0.3 & I & & & is & 17 \\
\hline & & & e & 06 & 21.80 & & 851 & 47.59 & 243 & ePd & 11 \\
\hline & & & es & 07 & 49.00 & & $!$ & $1.0 \mathrm{~s}$ & 1053 & $3.70 \mathrm{~nm}$ & \\
\hline & & & escs & 18 & 03.00 & & NRN & 47.94 & 298 & iPd & 11 \\
\hline
\end{tabular}




\begin{tabular}{|c|c|c|c|c|c|c|c|c|c|c|c|}
\hline & & & & is & 23 & 04.00 & & 1 & $17 \mathrm{~s}$ & & $4.80 u m$ \\
\hline ERE & & 70.78 & 306 & $i P_{C}$ & 14 & 20.40 & 1.4 & $i$ & 175 & & 4.60um \\
\hline & & & & ipP & 15 & 46.00 & $385 \mathrm{kmX}$ & $i$ & & & i \\
\hline & & & & is & 23 & 05.00 & & 1 & & & $\mathrm{i}$ \\
\hline LEN & & 70.94 & 307 & $i P c$ & 14 & 20.00 & -0.1 & $i$ & & & $i$ \\
\hline & & $1.6 \mathrm{~s}$ & 1500 & $0.00 \mathrm{~nm}$ & & & $6.4 \mathrm{mb}$ & $i$ & & & $\mathrm{i}$ \\
\hline & $N$ & $18 \mathrm{~s}$ & 32 & 2. $20 \mathrm{um}$ & & & & 1 & & & is \\
\hline & $E$ & $18 \mathrm{~s}$ & 22 & 2. $40 \mathrm{um}$ & & & & I MUD & 79.83 & 333 & iPd \\
\hline & & & & is & 23 & 05.00 & & i & $1.0 \mathrm{~s}$ & 192 & $2.00 \mathrm{~nm}$ \\
\hline TOO & & 71.35 & 173 & $i P c$ & 14 & 21.50 & -0.6 & I $M D B$ & 79.88 & 319 & iPd \\
\hline GMW & & 72.31 & 44 & eP & 14 & 28.60 & 0.9 & I CEI & 79.94 & 321 & eP \\
\hline BFW & & 72.60 & 45 & iPd & 14 & 30.30 & 0.8 & I GPA & 79.95 & 312 & iPc \\
\hline JMI & & 72.82 & 349 & eP & 14 & 30.40 & 0.2 & HRT & 80.03 & 313 & ip \\
\hline NUE & & 73.01 & 128 & $P$ & 14 & 33.20 & 1.2 & I CMP & 80.06 & 318 & ePc \\
\hline & & & & $S$ & 16 & 12.00 & & I BuC1 & 80.07 & 317 & $I P C$ \\
\hline ANN & & 73.12 & 313 & $i P c$ & 14 & 31.50 & -0.9 & | HP | & 80.08 & 44 & ip \\
\hline & & $1.8 \mathrm{~s}$ & 4600 & $0.00 \mathrm{~nm}$ & & & $6.8 \mathrm{mb}$ & 1 & & & $e$ \\
\hline & $\mathrm{N}$ & $21 \mathrm{~s}$ & 43 & 3. 40 um & & & & I SPC & 80.09 & 323 & eP \\
\hline & $E$ & $17 \mathrm{~s}$ & 21 & 1.00 um & & & & i & & & $i(P P)$ \\
\hline & & & & ipP & 16 & 00.00 & $397 \mathrm{kmX}$ & i & & & $i(P P)$ \\
\hline & & & & ePP & 17 & 23.00 & & i & & & $e(s)$ \\
\hline & & & & ePPP & 19 & 13.00 & & I PHAM & 80.12 & 54 & iP \\
\hline & & & & is & 23 & 25.50 & & CGN & 80.20 & 317 & $i P_{c}$ \\
\hline LON & & 73.29 & 45 & iPd & 14 & 33.80 & 0.3 & I ISK & 80.26 & 313 & eP \\
\hline SHW & & 73.33 & 45 & ip & 14 & 35.80 & 2.0 & $1 \mathrm{COZ}$ & 80.42 & 319 & $i P c$ \\
\hline BHD & & 74.21 & 300 & ePd & 14 & 38.50 & -0.3 & I DMK & 80.60 & 314 & iPd \\
\hline & & & & e & 16 & 06.00 & & I ALT & 80.79 & 311 & ip \\
\hline & & & & $\mathrm{i}$ & 23 & 39.00 & & I DEV & 80.85 & 320 & iPd \\
\hline & & & & e & 25 & 17.50 & & I JMB & 80.90 & 315 & iPd \\
\hline UPP & & 74.25 & 333 & $i P_{C}$ & 14 & 37.50 & -1.0 & | TMI & 81.01 & 44 & iP \\
\hline & & $1.6 \mathrm{~s}$ & 7900 & $0.00 \mathrm{~nm}$ & & & $7.1 \mathrm{mb}$ & I MGI & 11.03 & 303 & eP \\
\hline & & & & i & 16 & 02.40 & & i PSZ & 1.10 & 323 & eP \\
\hline & & & & is & 23 & 37.00 & & SYP & 81.15 & 54 & $e P$ \\
\hline & & & & $\mathrm{i}$ & 26 & 05.00 & & I BRN & 81.15 & 329 & ePc \\
\hline$S I M$ & & 75.01 & 314 & $i P c$ & 14 & 43.00 & -0.1 & $i$ & & & epP \\
\hline & $z$ & $20 \mathrm{~s}$ & 14 & 4.00 um & & & $6.3 M s z$ & i & & & es \\
\hline & $\mathrm{N}$ & $20 s$ & 22 & $2.00 \mathrm{um}$ & & & & PVL & 81.30 & 317 & $\mathrm{iPC}$ \\
\hline & $E$ & $20 \mathrm{~s}$ & 19 & 9.00 um & & & & DST & 81.39 & 312 & $i P c$ \\
\hline NEW & & 75.15 & 42 & iPc & 14 & 44.10 & 0.2 & RAR & 81.41 & 122 & $P$ \\
\hline & & & & e & 16 & 12.00 & & 1 & & & $s$ \\
\hline CRZ & & 75.66 & 150 & $P$ & 14 & 47.30 & 0.6 & EDC & 81.42 & 313 & iP \\
\hline & & & & e & 16 & 21.00 & & I GNZ & 81.50 & 149 & $\mathrm{Pc}$ \\
\hline & & & & $P P$ & 17 & 33.00 & & $i$ & & & $(p P)$ \\
\hline & & & & $S$ & 23 & 58.00 & & i & & & PP \\
\hline NO & & 75.69 & 45 & iP & 14 & 48.00 & 1.1 & i & & & $S$ \\
\hline$\times 1 S$ & & 76.88 & 318 & eP & 14 & 53.00 & -0.4 & I JER & 81.58 & 302 & ePd \\
\hline & & $2.0 \mathrm{~s}$ & 1800 & $0.00 \mathrm{~nm}$ & & & $6.5 \mathrm{mb}$ & DIM & 81.74 & 315 & $e P$ \\
\hline & $z$ & $16 \mathrm{~s}$ & 15 & $5.00 u m$ & & & $6.4 M s 2 X$ & I HAM & 81.76 & 331 & iPd \\
\hline & $\mathrm{N}$ & $16 \mathrm{~s}$ & 10 & 3.80 um & & & & BRG & 81.92 & 327 & iPct \\
\hline & $\mathrm{E}$ & $16 \mathrm{~s}$ & 34 & $4.70 u m$ & & & & $i$ & $1.8 \mathrm{~s}$ & 2300 & $0.00 \mathrm{~nm}$ \\
\hline & & & & ipP & 16 & 18.00 & $375 \mathrm{kmX}$ & i & & & $i p P$ \\
\hline & & & & IPPP & 19 & 45.00 & & 1 & & & is \\
\hline & & & & is & 24 & 04.00 & & i & & & ess \\
\hline ONO & & 77.32 & 336 & iP & 14 & 55.30 & -0.3 & i & & & ePKKP \\
\hline IAS & & 77.50 & 319 & $e P$ & 14 & 57.00 & 0.2 & 1 & & & $e^{\prime} P^{\prime}$ \\
\hline LVV & & 77.79 & 322 & $i P c$ & 14 & 58.50 & 0.2 & I SRO & 81.97 & 323 & $\mathrm{iPc}$ \\
\hline & $z$ & $17 \mathrm{~s}$ & 56 & 6.70 um & & & 7. OMszX & i & & & e \\
\hline & $\mathrm{N}$ & $18 \mathrm{~s}$ & 74 & 4. 50 um & & & & I & & & is \\
\hline & $E$ & $18 \mathrm{~s}$ & 43 & 3. $20 u m$ & & & & i & & & e \\
\hline & & & & ipP & 16 & 25.00 & $382 \mathrm{kmx}$ & i & & & e \\
\hline & & & & is & 24 & 19.00 & & $\mathrm{CLL}$ & 82.03 & 328 & $\mathrm{iPc}$ \\
\hline & & & & ess & 29 & 25.00 & & $i$ & $1.7 \mathrm{~s}$ & 2100 & $0.00 \mathrm{~nm}$ \\
\hline LI & & 78.04 & 318 & iPc & 15 & 01.00 & 1.2 & $i$ & & & $i p P$ \\
\hline PPE & & 78.05 & 318 & iPd & 15 & 02.00 & 2.2 & $i$ & & & is \\
\hline BER & & 78.18 & 338 & iP+ & 15 & 04.00 & $3.8 x$ & ( & & & PKKP \\
\hline$C F R$ & & 78.36 & 317 & $i P c$ & 15 & 00.00 & -1.4 & i & & & $P \cdot P$. \\
\hline$A R N$ & & 78.52 & 53 & iP & 15 & 03.60 & 1.0 & I TCW & 82.13 & 152 & $P$ \\
\hline KDE & & 78.68 & 312 & eP & 5 & 02.00 & -1.3 & i & & & (s \\
\hline VRI & & 78.76 & 318 & iPd & 5 & 03.00 & -0.6 & & & & $\mathrm{~s}$ \\
\hline$T L B$ & & 78.78 & 316 & iPc & 15 & 04.00 & 0.3 & $\angle D$ & 2.22 & 16 & $\mathrm{iPC}$ \\
\hline$A K U$ & & 79.06 & 350 & IPC & 15 & 06.10 & 1.3 & I PRU & 82.28 & 327 & $\mathrm{iPc}$ \\
\hline & & $1.6 \mathrm{~s}$ & 3400 & $.00 \mathrm{~nm}$ & & & 6. $9 \mathrm{mb}$ & 1 & $1.5 \mathrm{~s}$ & 2165 & $5.20 \mathrm{~nm}$ \\
\hline & & & & $i$ & 16 & 38.40 & & 1 & $16 \mathrm{~s}$ & 21 & 1. $50 \mathrm{um}$ \\
\hline JECI & & 79.06 & 44 & iPC & 15 & 06.50 & 0.9 & i & $15 \mathrm{~s}$ & & g. 50 um \\
\hline cro & & 79.09 & 318 & $i P c$ & 15 & 05.00 & -0.4 & i & $17 \mathrm{~s}$ & & 9.50 um \\
\hline COP & & 79.14 & 332 & iPd & 15 & 06.10 & 0.7 & i & & & ipP \\
\hline & & & & $i$ & 16 & 32.00 & & 1 & & & e \\
\hline & & & & is & 24 & 32.00 & & $i$ & & & is \\
\hline SN & & 3 & 316 & iPc & 5 & & 0.9 & & & & sS \\
\hline ISR & & 9 & 317 & iPc & 15 & 20 & 0.4 & 1 & & & $i$ \\
\hline $\mathrm{UZH}$ & & 79.41 & 322 & $i P c$ & 15 & 06.00 & -1.0 & ELL & 2.28 & 09 & iPc \\
\hline & $z$ & $16 \mathrm{~s}$ & 53 & $3.00 u m$ & & & $7 . \theta M s z X$ & IST & 82.31 & 324 & $i P_{c}$ \\
\hline & & & & is & 24 & 33.00 & & i & & & e \\
\hline ALR & & 79.42 & 318 & $i P C$ & 15 & 07.00 & -0.3 & $i$ & & & e \\
\hline KRA & & 79.68 & 324 & iPc & 15 & 08.30 & 0.0 & MOUT & 82.35 & 45 & iP \\
\hline & & $0.8 \mathrm{~s}$ & 969 & $.00 \mathrm{~nm}$ & & & $6.6 \mathrm{mb}$ & WEL & 82.38 & 152 & iP \\
\hline & $z$ & $18 \mathrm{~s}$ & 38 & 3.40 um & & & $5.8 M s z$ & 1 & & & SP \\
\hline
\end{tabular}




\begin{tabular}{|c|c|c|c|c|c|c|c|}
\hline & $19 \mathrm{~s}$ & & $6.40 \mathrm{um}$ & & & & BSF \\
\hline & & & $\mathrm{i}$ & 17 & 10.30 & & i \\
\hline & & & $\mathrm{i}$ & 18 & 59.20 & & I HAU \\
\hline & & & is & 25 & 24.80 & & \\
\hline LJU & 85.06 & 324 & $\mathrm{iPc}$ & 15 & 35.20 & -0.6 & I PRO \\
\hline & & & e & 17 & 06.20 & & ROF \\
\hline & & & e & 19 & 03.00 & & I DLE \\
\hline & & & es & 25 & 20.00 & & \\
\hline FUR & 85.08 & 327 & $\mathrm{iPc}$ & 15 & 36.00 & 0.1 & I FOG \\
\hline & $1.4 \mathrm{~s}$ & 1895 & $5.00 \mathrm{~nm}$ & & & $6.7 \mathrm{mb}$ & I ALP \\
\hline & & & $\mathbf{i}$ & 17 & 06.00 & & I MMK \\
\hline & & & es & 25 & 34.00 & & I DUI \\
\hline TTG & 85.09 & 319 & eP & 15 & 35.50 & -0.5 & i \\
\hline & & & ePcP & 15 & 40.50 & & | OR I \\
\hline & & & $\mathrm{i}$ & 17 & 17.20 & & 1 \\
\hline & & & ePP & 18 & 34.00 & & $1 \mathrm{AOU}$ \\
\hline & & & es & 25 & 21.00 & & ! \\
\hline $\begin{array}{l}\text { RBL } \\
\text { CEY }\end{array}$ & 85.25 & 325 & IPC & 15 & 35.50 & -1.3 & 1 \\
\hline CEY & 85.33 & 324 & - IPC & 15 & 36.30 & -0.9 & I FIR \\
\hline HLW & 85.39 & 303 & IPC & 15 & 38.00 & 0.3 & \\
\hline & & & e & 17 & 00.00 & & $101 x$ \\
\hline & & & e & 18 & 05.00 & & 1560 \\
\hline & & & es & 25 & 25.00 & & i \\
\hline ENN & 85.42 & 331 & iPc & 15 & 37.00 & -0.4 & i \\
\hline & $1.5 \mathrm{~s}$ & 2107 & $7.00 \mathrm{~nm}$ & & & $5.8 \mathrm{mb}$ & MNS \\
\hline & & & epP & 17 & 14.50 & $428 \mathrm{kmX}$ & 1 \\
\hline RMU & 85.47 & 48 & ip & 15 & 41.10 & 2.9 & EMS \\
\hline GLA & 85.48 & 53 & eP & 15 & 37.00 & -1.2 & LOR \\
\hline GAP & 85.68 & 326 & eP & 15 & 38.60 & -0.3 & i \\
\hline SCE & 85.71 & 326 & IPC & 15 & 38.00 & -1.2 & I LBF \\
\hline ucc & 86.01 & 332 & Pct & 15 & 40.40 & 0.1 & \\
\hline & $1.8 \mathrm{~s}$ & 811 & $1.00 \mathrm{~nm}$ & & & 5. $3 \mathrm{mb}$ & GRC \\
\hline BUH & 86.06 & 329 & iPc & 15 & 39.90 & -0.8 & \\
\hline GWF & 86.09 & 329 & iPc & 15 & 40.20 & -0.6 & SSF \\
\hline OGA & 86.15 & 326 & IPC & 15 & 40.80 & -0.6 & \\
\hline WLF & 86.18 & 330 & P+ & 15 & 44.00 & 2.9 & I SMF \\
\hline & & & pP & 17 & 11.00 & $376 \mathrm{kmX}$ & \\
\hline & & & e & 18 & 04.00 & & I FLN \\
\hline & & & s & 25 & 32.00 & & \\
\hline & & & $\mathrm{i}$ & 25 & 44.00 & & I LDF \\
\hline & & & e & 28 & 20.00 & & \\
\hline PMO & 86.43 & 111 & iP & 15 & 44.30 & 1.6 & $\mathrm{SCH}$ \\
\hline AFR & 86.47 & 114 & iP & 15 & 44.90 & 2.0 & i \\
\hline & $13 \mathrm{~s}$ & 3910 & $0.00 \mathrm{~nm}$ & & & $.1 \mathrm{mb}$ & AVF \\
\hline DOU & 86.47 & 331 & $P d$ & 15 & 42.70 & 0.2 & i \\
\hline & & & $P P$ & 17 & 12.00 & $386 \mathrm{kmx}$ & I ALO \\
\hline & & & e & 18 & 09.00 & & i \\
\hline & & & $s$ & 25 & 32.00 & & 1 \\
\hline & & & e & 25 & 46.00 & & i \\
\hline & & & e & 28 & 25.00 & & $\mathrm{MCO}$ \\
\hline SLE & 86.63 & 328 & ePt & 15 & 42.50 & -0.9 & \\
\hline TPT & 86.63 & 110 & ip & 15 & 45.30 & 1.6 & GRR \\
\hline & $1.3 \mathrm{~s}$ & 4020 & $0.00 \mathrm{~nm}$ & & & $.1 \mathrm{mb}$ & \\
\hline SAX & 86.64 & 327 & $e P+$ & 15 & 43.20 & -0.6 & LPF \\
\hline PPT & 86.64 & 114 & $i p$ & 15 & 45.90 & 2.1 & \\
\hline & $1.3 \mathrm{~s}$ & 3565 & $5.00 \mathrm{~nm}$ & & & $7.1 \mathrm{mb}$ & $M Z F$ \\
\hline CDF & 86.67 & 329 & iPc & 15 & 43.00 & -0.7 & \\
\hline & $1.6 \mathrm{~s}$ & 960 & $0.10 \mathrm{~nm}$ & & & $.4 \mathrm{mb}$ & CVF \\
\hline oss & 86.69 & 326 & $\mathrm{ePt}^{-}$ & 15 & 43.50 & -0.4 & \\
\hline PAE & 86.69 & 114 & iP & 15 & 46.00 & 2.0 & TCF \\
\hline & $1.3 \mathrm{~s}$ & 2750 & $0.00 \mathrm{~nm}$ & & & $.0 \mathrm{mb}$ & \\
\hline PPN & 86.73 & 113 & iP & 15 & 46.00 & 1.8 & FRF \\
\hline & $1.3 \mathrm{~s}$ & 3095 & $5.00 \mathrm{~nm}$ & & & $.0 \mathrm{mb}$ & \\
\hline VAH & 86.77 & 111 & iP & 15 & 45.80 & 1.4 & LSF \\
\hline & $1.3 \mathrm{~s}$ & 2065 & $5.00 \mathrm{~nm}$ & & & $.8 \mathrm{mb}$ & \\
\hline $\mathrm{ECH}$ & 86.87 & 329 & iPC & 15 & 43.60 & -0.9 & LRG \\
\hline $2 U \mathrm{~L}$ & 86.89 & 328 & ePt & 15 & 43.80 & -0.9 & \\
\hline RUV & 86.94 & 110 & iP & 15 & 46.70 & 1.5 & LMR \\
\hline & $1.3 \mathrm{~s}$ & 3215 & $5.00 \mathrm{~nm}$ & & & $.0 \mathrm{mb}$ & \\
\hline IVo & 87.02 & 114 & iP & 15 & 47.80 & 2.2 & CDR \\
\hline & $1.3 \mathrm{~s}$ & 3910 & $0.00 \mathrm{~nm}$ & & & $.1 \mathrm{mb}$ & \\
\hline LLS & 87.08 & 327 & ePt & 15 & 45.10 & -0.7 & \\
\hline $\mathrm{GOL}$ & 87.09 & 43 & ip & 15 & 47.00 & 0.9 & \\
\hline & $1.5 \mathrm{~s}$ & 1022 & $2.01 \mathrm{~nm}$ & & & $.5 \mathrm{mb}$ & \\
\hline & & & e & 17 & 16.00 & & GIB \\
\hline GLD & 87.14 & 43 & ip & 15 & 47.10 & 0.9 & \\
\hline & $1.5 \mathrm{~s}$ & 1906 & $6.25 \mathrm{~nm}$ & & & $.7 \mathrm{mb}$ & MFF \\
\hline & & & e & 17 & 21.00 & & \\
\hline VDL & 87.15 & 327 & ePt & 15 & 45.50 & -0.7 & RJF \\
\hline BAF & 87.23 & 329 & $i P_{C}$ & 15 & 45.40 & -1.0 & \\
\hline BRT & 87.27 & 319 & eP & 15 & 47.50 & 1.0 & CAF \\
\hline & & & e & 17 & 18.00 & & \\
\hline & & & e & 19 & 16.00 & & AAE \\
\hline DMU & 87.29 & 339 & $\mathrm{iPC}$ & 15 & 46.40 & $\begin{array}{ll}-\theta & 1\end{array}$ & ERC \\
\hline & $1.7 \mathrm{~s}$ & 3120 & $0.00 \mathrm{~nm}$ & & & $.9 \mathrm{mb}$ & LFF \\
\hline SAL & 87.31 & 325 & eP & 15 & 45.50 & -1.2 & \\
\hline
\end{tabular}

\begin{tabular}{|c|c|c|c|c|c|c|}
\hline 7.32 & 329 & iPc & 15 & 5.80 & .0 & LPO \\
\hline $.4 \mathrm{~S}$ & 55 & 1. $30 \mathrm{~nm}$ & & 6 & $.2 \mathrm{mb}$ & \\
\hline 7.38 & 329 & $i P_{c}$ & 15 & 46.20 & -0.8 & ACO \\
\hline $1.4 \mathrm{~s}$ & 498 & $0.00 \mathrm{~nm}$ & & 6 & $.2 \mathrm{mb}$ & \\
\hline 87.39 & 323 & eP & 15 & 46.50 & -0.5 & MLS \\
\hline 87.39 & 329 & iPc & 15 & 46.00 & -1.1 & EPF \\
\hline 2 & 339 & i Pc & 1 & 48.10 & -0.4 & \\
\hline $1.8 \mathrm{~s}$ & 3200 & $0.00 \mathrm{~nm}$ & & & $.9 \mathrm{mb}$ & RRO \\
\hline 9.77 & 320 & eP & 15 & 9.80 & 0.9 & \\
\hline 87.87 & 322 & iPc & 15 & 49.40 & 0.1 & OCO \\
\hline 88.17 & 327 & ePt & 15 & 0.30 & -0.8 & PCR \\
\hline 88.20 & 321 & iPc & 15 & 50.00 & -0.9 & $\mathrm{ACM}$ \\
\hline & & e & 17 & 24.00 & & S10 \\
\hline 88.26 & 318 & iPc & 15 & 51.00 & -0.2 & TUL \\
\hline & & & & $\begin{array}{l}24.06 \\
51.08\end{array}$ & -0.3 & \\
\hline
\end{tabular}

$88.31324 \mathrm{eP}$

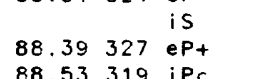

88.53319 iPc $1551.60-0.6$ I LDN

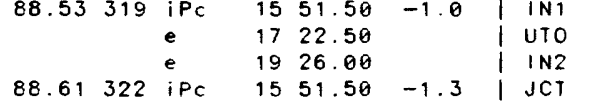

\begin{tabular}{cccc|c|c} 
& iPc & 15 & 51.50 & -1.3 & JCT \\
$e$ & 1722.00 & & \\
$e$ & 1929.00 & & \\
$88.62328 \mathrm{ePt}$ & 15 & 52.40 & -0.7 & BNH
\end{tabular}

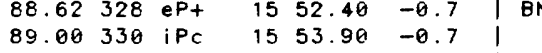

$1.4 \mathrm{~s} 551.30 \mathrm{~nm} \quad 6.2 \mathrm{mb}$ |ALI 89.18330 iPC

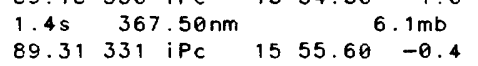

is $26 \quad 16.40$

$89.32 \quad 330$ iPc $15 \quad 55.30 \quad-0.7$

$1.4 \mathrm{~s} \quad 612.50 \mathrm{~nm}$

89.51330 iPc $1556.10^{6.3 m b}-0.9$

$1.6 \mathrm{~s} \quad 880.10 \mathrm{~nm} \quad 6.4 \mathrm{mb}$

89.51333 iPC $1556.20-0.7$

$1.6 \mathrm{~s} 800.10 \mathrm{~nm}$

$89.52333 \quad \mathrm{iPC} \quad 15 \quad 56.20 \quad-0.7$

$1.6 \mathrm{~s} 1120.10 \mathrm{~nm} \quad 6.5 \mathrm{mb}$ PTO

$\begin{array}{lllll}89.55 & 13 \mathrm{ePC} & 15 & 57.00 & -0.1\end{array}$

$1.2 \mathrm{~s} \quad 657.00 \mathrm{~nm}$

89.59330 iPc $15 \quad 56.70-0.6$

$1.6 \mathrm{~s} 1120.10 \mathrm{~nm} \quad 6.5 \mathrm{mb}$

89.6347 iPC+ $1559.00 \quad 1.0$ MTE

\begin{tabular}{cc|c|c}
$1.0 \mathrm{~s}$ & $40000 \mathrm{~nm}$ & $6.2 \mathrm{mb}$ & CRT
\end{tabular}

$218 \mathrm{~s} 13.23 \mathrm{um} \quad 6.4 \mathrm{Msz}$

\begin{tabular}{ccccc|}
89.88 & $167 \mathrm{ePP}$ & 17 & 28.00 & $382 \mathrm{kmX}$ \\
$8 \mathrm{P}$ & 15 & 59.00 & 0.9
\end{tabular}

\begin{tabular}{lll|l}
$1.6 \mathrm{~s}$ & $1.10 \mathrm{~nm}$ & $3.5 \mathrm{mb} X$ & RKT
\end{tabular}

$89.96 \quad 333 \quad \mathrm{iPc} \quad 15 \quad 58.60 \quad-0.4$

$\begin{array}{lll}1.6 \mathrm{~s} & 1680.20 \mathrm{~nm} & 6.7 \mathrm{mb} \\ 90.33 & 333 \mathrm{iPc} & 1600.60^{-0.1}\end{array}$

$1.6 \mathrm{~s} 2080.30 \mathrm{~nm} \quad 6.8 \mathrm{mb}$

90.37330 iPc $16 \quad 01.10 \quad 0.1$

\begin{tabular}{llll}
$1.6 \mathrm{~s}$ & $1440.20 \mathrm{~nm}$ & \multicolumn{1}{c}{$6.6 \mathrm{mb}$} \\
90.38 & $324 \mathrm{iPc}$ & 15 & $59.90^{-1.2}$
\end{tabular}

$\begin{array}{lll}1.6 \mathrm{~s} & 368.60 \mathrm{~nm} & 6.0 \mathrm{mb}\end{array}$

$\begin{array}{llllll}90.47 & 330 & \text { iPc } & 16 & 01.00 & -0.4\end{array}$

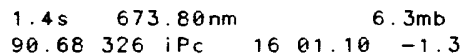

$\begin{array}{ll}1.6 \mathrm{~s} & 480.00 \mathrm{~nm} \quad 6.2 \mathrm{mb}\end{array}$

$90.78 \quad 331$ iPc $16 \quad 02.30 \quad-0.5$

$1.4 \mathrm{~s} \quad 1225.10 \mathrm{~nm} \quad 6.6 \mathrm{mb}$

$\begin{array}{llllllll}90.89 & 326 & \text { iPC } & 16 & 02.60 & -0.7\end{array}$

$1.6 \mathrm{~s} \quad 832.10 \mathrm{~nm} \quad 6.4 \mathrm{mb}$

$\begin{array}{llllll}90.91326 & \text { iPC } & 16 & 02.60 & -0.9\end{array}$

$1.6 \mathrm{~s} 720.10 \mathrm{~nm} \quad 62.3 \mathrm{mb}$

90.99327 ePC $\quad 1602.70$

ipP $1733.90392 \mathrm{kmx}$

iPP 2050.00

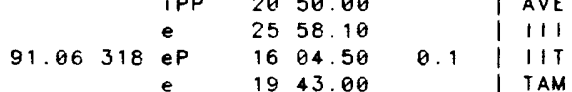

\begin{tabular}{lllll|l}
$91.16 \quad 332 \mathrm{iPC}$ & 19 & 43.00 & & & I TAM \\
91.60 & 0.0 & VHO
\end{tabular}

$1.6 \mathrm{~s} 3040.40 \mathrm{~nm} \quad 7.0 \mathrm{mb}$ BNG

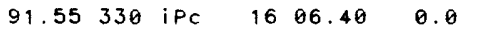

$1.6 \mathrm{~s} 1680.20 \mathrm{~nm} \quad 6.7 \mathrm{mb}$ | MTD

91.63330 iPc $16 \quad 07.00 \quad 0.2$ I MTD

\begin{tabular}{lll}
$1.6 \mathrm{~s}$ & $1120.10 \mathrm{~nm}$ & \multicolumn{6}{c}{$6.6 \mathrm{mb}$} \\
91.66 & $282 \mathrm{eP}^{\mathrm{P}} \quad 1608.00^{0.3}$
\end{tabular}

91.80319 eP $\quad 1606.50-1.1 \quad$ KRI

$92.17330 \mathrm{iPc} \quad 16 \quad 99.50 \quad 0.3$ $\begin{array}{llllll}92.19 & 330 \mathrm{iPc} & 16 & 09.30 & 0.0\end{array}$

\begin{tabular}{lllll}
$1.6 \mathrm{~s}$ & $1200.10 \mathrm{~nm}$ & \multicolumn{3}{c}{$6.6 \mathrm{mb}$} \\
92.69 & $42 \mathrm{eP}$ & 16 & $12.30^{0.5}$
\end{tabular}

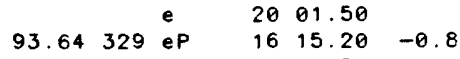

93.89330 iPC $16 \quad 16.30-1.0$

$1.6 \mathrm{~s} \quad 280.00 \mathrm{~nm} \quad 6.1 \mathrm{mb}$

$\begin{array}{lllll}94.03 \quad 42 \mathrm{eP} & 16 & 19.30 & 1.3\end{array}$

$\begin{array}{llllll}94.47 & 42 \mathrm{e} & 16 & 21.00 & \\ 94.56 & 16 & 20.60 & 0.6\end{array}$

$94.56248 \mathrm{eP}-1621.90 \quad 1.1$

$\begin{array}{lllll}94.59 & 30 \mathrm{ePc} & 16 & 20.70 & 0.3\end{array}$

$\begin{array}{lllll}94.95 & 41 \mathrm{eP} & 16 & 22.60 & 0.4\end{array}$

$95.10 \quad 40 \mathrm{iPct} 16 \quad 22.50 \quad-0.4$

$2.9 \mathrm{~s} \quad 4138.80 \mathrm{~nm} \quad 7.1 \mathrm{mb}$

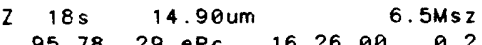

$\begin{array}{lllll}95.78 & 29 \mathrm{ePc} & 16 & 26.00 & 0.2 \\ 95.93 & 27 \mathrm{P} & 16 & 27.00 & 0.5\end{array}$

$\begin{array}{llllll}95.96 & 22 & \mathrm{eP} & 16 & 26.00 & -0.5\end{array}$

$1.0 \mathrm{~s} \quad 219.00 \mathrm{~nm} \quad 6.3 \mathrm{mb}$

$\begin{array}{lllll}96.12 & 27 \mathrm{P} & 16 & 28.00 & 0.7\end{array}$

$96.30 \quad 31 \mathrm{ePc} \quad 16 \quad 28.00 \quad-0.1$

$\begin{array}{lllll}96.34 & 29 \mathrm{ePc} & 16 & 30.50 & 2.2\end{array}$

$\begin{array}{lllll}96.40 & 32 \mathrm{ePc} & 16 & 28.80 & 0.2\end{array}$

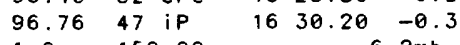

$\begin{array}{lll}1.0 \mathrm{~s} & 150.00 \mathrm{~nm} & 6.2 \mathrm{mb}\end{array}$

$\begin{array}{llllll}98.03 & 20 & \text { iP } & 1821.00 & 1640.00 & 4.0 x\end{array}$

$\begin{array}{ccccc}98.08327 \mathrm{iPt} & 18 & 09.00 & \\ 96.50 & 4.3 \mathrm{x}\end{array}$

$\begin{array}{lll}98.08 & 327 \text { IPP } 2043.50\end{array}$

$\begin{array}{lllll}98.34 & 331 \mathrm{ePC} & 16 & 37.00 & -0.5\end{array}$

$1.5 \mathrm{~s} \quad 7.00 \mathrm{~nm} \quad 4.8 \mathrm{mb} \times$

i $\quad 18 \quad 12.00$

IPPP $22 \quad 16.00$

ISKS $26 \quad 36.00$

is $27 \quad 28.00$

iPS 2850.00

iSS $34 \quad 10.00$

ISS $34 \quad 3755.00$

$\begin{array}{lllll}98.67 & 7 \mathrm{eP} & 16 & 40.50 & 1.7\end{array}$

$\begin{array}{lllll}99.01334 \mathrm{P} & 1640.20 & -0.3\end{array}$ $\begin{array}{lll}\text { is } & 26 & 40.00 \\ \text { iPS } & 27 & 30.00\end{array}$

$99.02 \quad 274 \mathrm{eP} \quad 16 \quad 42.00 \quad 0.9$

$2.0 \mathrm{~s} \quad 352.94 \mathrm{~nm} \quad 6.3 \mathrm{mb}$

$99.26 \quad 333 \mathrm{ePd} \quad 16 \quad 41.50 \quad-0.2$

$100.42 \quad 329$ ePditfic $45.50-1.2$

$100.48 \quad 23$ ePdiffl6 $47.80 \quad 1.0$

$\begin{array}{rrrrr} & \text { PP } & 18 & 20.00 \\ 101.06 \quad 110 & \text { ePdiff } 16 & 53.00 & 3.2 x\end{array}$

$\begin{array}{lll}1.3 \mathrm{~s} & 120.00 \mathrm{~nm} & 6.3 \mathrm{mb}\end{array}$

101.16329 iPdiffl6 $50.20 \quad 0.3$ IPP 2056.00
iS is 2741.00 iPS $29 \quad 36.00$

BLA 101.4130 ePdift16 $54.10 \quad 3.0 x$ $2.0 \mathrm{~s} \quad 876.47 \mathrm{~nm} \quad 54.107 .0 \mathrm{mb}$ 101.59218 iPdiff17 $00.00 \quad 8.5 \times$ 102.14330 ePdiffl6 $54.00 \quad-0.2$ e $\quad 1820.00$ IPP 2106.00 ePPP $22 \quad 30.00$ ES $\quad 26 \quad 33.00$ IPS $28 \quad 05.00$ iSP 3010.00 iSS 3500.00 iSSS 3900.00

104.6254 Pdiff 1709.68 $104.66 \quad 54$ ePdiffi7 10.50 105.0154 ePdiff17 16.00 $105.18 \quad 54$ ePdiff $17 \quad 14.00$ 105.36329 iPdiff 1709.00 $105.48 \quad 55$ ePdiffi7 13.00 $105.81 \quad 54$ ePdiff17 17.00 $107.16 \quad 313$ ePdiff $17 \quad 16.50$ $108.13 \quad 54$ iPdiff 1733.00 110.55290 iPdiffl7 32.00

$0.9 \mathrm{~s} \quad 10.00 \mathrm{~nm}$

111.59264 ePdiff17 40.00 111.59264 iPKP 2134.00 IPP $22 \quad 21.00$ iPKKP $32 \quad 28.00$

113.25265 iPKP 2136.00 IPP $22 \quad 33.00$ iPKKP $32 \quad 22.00$
$3.5 x$ 4. $3 x$

$5.4 x$ 0.3 3. $3 x$ $5.7 x$ $11.5 x$ $-0.2$

$3.2 x$ 1.2 
$01 d \quad 09 \mathrm{~h}$

KRI $\quad 113.25265$ ePdiff17 $43.00 \quad-1.2$

MAW 114.92204 ePdiff18 $01.00 \quad 10.8 \mathrm{x}$ $1.0 \mathrm{~s} \quad 103.00 \mathrm{~nm}$

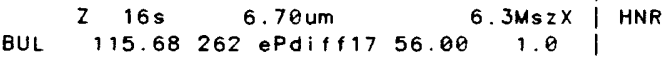

\begin{tabular}{cc|c|c|}
$1.0 \mathrm{~s} 15.00 \mathrm{~nm}$ & & \\
BUL 1.0 & VSG
\end{tabular}

$\begin{array}{lllll}\text { BUL } 115.68 & 262 & \text { IPKP } & 21 & 40.00 \\ & & \text { iPP } & 22 & 52.00\end{array}$ \begin{tabular}{lll} 
iPKK & 22 & 52.00 \\
\hline & 32 & 15.00
\end{tabular}

EVA 118.19256 e(Pdif18 16.00

BLF $\quad 121.60254$ i $21 \quad 45.00$

GRM $\quad 122.89249$ iPKPd $21 \quad 53.50$ $1.2 \mathrm{~s} \quad 171.88 \mathrm{~nm}$

$z 20 \mathrm{~s} \quad 12.06 \mathrm{um}$

JG $\quad 124.04 \quad 27 \quad 23 \quad 30.50$

KIC $\quad 124.04 \quad 27$ ePdiff18 34.00

KIC 3136.00

BPA $126.51 \quad 22$ iPKPd 2200.40

SUR 127.10253 iPKPd 2202.80 $1.3 \mathrm{~s} \quad 230.77 \mathrm{~nm}$

$z \quad 18 \mathrm{~s} \quad 30.41 \mathrm{um}$

$\begin{array}{llllll}127.23 & 22 & \text { IPKPd } 22 & 01.90\end{array}$

$\begin{array}{llllll}\text { PAG } & 127.52 & 23 & \text { ePKP } & 22 & 02.60 \\ & 127.5 \% & 22 & 02.90\end{array}$

MGG $\quad 127.74 \quad 22$ ePKP 2203.00

MON $\quad 128.28 \quad 23$ ePKPd 2204.30

CER $\quad 128.63252$ ePKP $22 \quad 05.50$ $1.0 \mathrm{~s} \quad 160.00 \mathrm{~nm}$

$\begin{array}{llll}128.89 & 37 & \text { ePKP } 22 & 12.40\end{array}$

$\begin{array}{llllll}\text { FDF } & 128.91 & 23 & \text { iPKPC } & 22 & 05.55 \\ \text { BIM } & 129.13 & 23 & \text { EPKP } & 22 & 06.05\end{array}$

MVM $129.16 \quad 22$ EPKP 2206.15

TOV $129.92 \quad 35$ iPKPd 2208.50

$0.9 \mathrm{~s} \quad 168.50 \mathrm{~nm}$

CAR $130.60 \quad 31$ ePdiff19 02.00

$\begin{array}{lllll} & & \text { EPP } 21 & 16.00 \\ \text { BOG } \quad 132.02 \quad 43 \text { ePdiffig } & 09.00\end{array}$ $\begin{array}{lll}\text { EPP } 22 & 12.00\end{array}$

ATB $148.61 \quad 17$ iPKP 2240.50

ARE $\quad 149.32 \quad 63$ IPKPC $22 \quad 42.50$

CAI $152.40 \quad 347$ ePKPC $22 \quad 47.10$

$1.3 \mathrm{~s} \quad 829.81 \mathrm{~nm}$

$\begin{array}{llllll}\text { ANT } & 154.21 & 74 & \text { iPKP } & 22 & 49.50\end{array}$

$\begin{array}{llllll}\text { CON } \quad 155.48 & 106 & \text { iPKP } 2250.00\end{array}$

$\begin{array}{llllll}\text { SOB } 1 & 155.55 & 354 & \text { ePKP } & 23 & 51.60\end{array}$

LNV $\quad 156.59 \quad 99$ ePKP 2252.00

$\begin{array}{llllll} & & & \mathrm{i} & 23 & 24.40 \\ \mathrm{TACH} & 156.94 & 98 & \text { iPKPC } & 22 & 53.50\end{array}$

$\begin{array}{lllll}\text { TACH } 156.94 & 98 & \text { iPKPC } & 22 & 53.50 \\ & & i & 23 & 27.50\end{array}$

PEL $\quad 157.08 \quad 96$ IPKP 2254.00

$\begin{array}{llllll} & & & & 23 & 28.60 \\ \text { YJA } \quad 157.26 & 65 & \text { EPKPC } & 22 & 53.80\end{array}$

BACH $157.27 \quad 97$ ePKP $22 \quad 54.50$

HJA $\quad 157.91 \quad 68$ ePKPC 2255.00

CEN $\quad 158.40 \quad 91$ (PKP) 2259.00

MOZ $158.56 \quad 95$ EPKP 2253.90

SLA $158.63 \quad 71$ iPKPC 2254.50

CFA $158.83 \quad 91$ ePKPd 2255.60

$\begin{array}{lllllll}\text { CYA } & 159.97 & 81 & \text { iPKPC } & 22 & 55.50\end{array}$

BAO $161.52 \quad 15$ IPKP 2258.80

$\begin{array}{llllll}T C A & 161.81 & 88 & \text { ePKPC } 22 & 57.00\end{array}$

VBA 164.17111 iPKPA 2300.00

$$
\text { e } \quad 2357.50
$$

2745.00

LPA $\quad 167.76 \quad 100 \quad$ iPKPd+23 03.20

$1.2 \mathrm{~s} 2000.00 \mathrm{~nm}$

VAO $\quad 168.86 \quad 18$ ePKPC $23 \quad 04.90$ $\begin{array}{lll}24 & 19 & 20\end{array}$

BMA $\quad 169.02 \quad 5$ ePKPC 2305.20 S.D. $=0.9$ on 432 of 456 obs

\begin{tabular}{l|l}
-0.7 & VSG \\
SVO \\
PAA \\
PA. & LOSU \\
-0.2 & RAB \\
PVC
\end{tabular}

Islands, ( $V \mid$ ) at Honiaro and

(111) ot Rabaul. New Britain.$$
\begin{array}{lllll|l}
0.77317 \text { iPC } & 33 & 34.00 & -1.2 & \text { MUN } \\
\text { RKG } \\
\text { KKM }
\end{array}
$$

1.06314 iP $\quad 3347.00$

1.07322 iPd

6.15306 ePc

$9.40278 \mathrm{eP}$

$10.06304 \mathrm{eP}-$

$10.82 \quad 136$ iPd

$11.12161 \mathrm{iPC}$

$12.11269 \mathrm{eP}$

$12.12307 \mathrm{eP}$

$12.21274 \mathrm{P}$

$13.15271 \mathrm{P}$

$13.52 \quad 156 \mathrm{iPC}$

$13.74283 \mathrm{P}$

16.99232 iPd-

$17.88290 \mathrm{eP}$

18.18 $117 \mathrm{iPc}$

18.76202 iP

$19.17117 \mathrm{ePc}$
$2.4 \mathrm{~s} \quad 3921.00 \mathrm{~nm}$

$19.21117 \mathrm{eP}$

$21.00289 \mathrm{ePC}$

$1.0 \mathrm{~s} \quad 221.60 \mathrm{~nm}$

$\begin{array}{ll}39.00 & -1.2\end{array}$

\begin{tabular}{rrr|}
33 & 39.00 & -1.3 \\
34 & 54.50 & 1.1
\end{tabular}

\begin{tabular}{lll|l}
3607.00 & IRT
\end{tabular}

$\begin{array}{lll}35 & 38.00 & -0.7\end{array}$

\begin{tabular}{lll|l}
35 & 48.00 & 0.1 & $B A G$
\end{tabular}

$3557.00-1.3$ | NAH

\begin{tabular}{rrr|r}
36 & 02.70 & 0.3 & NGO
\end{tabular}

$\begin{array}{lll}36 & 15.00 & -0.8\end{array}$

$3615.00-1.0$ | NZJ

$\begin{array}{lll}36 & 17.00 & -0.3\end{array}$

$\begin{array}{lll}36 & 29.00 & -0.7\end{array}$

\begin{tabular}{lll|l}
36 & 35.50 & 0.9 & I KMJ
\end{tabular}

$\begin{array}{lll}36 & 37.00 & -0.6\end{array}$

\begin{tabular}{lll|l}
37 & 18.60 & -1.1 & I AFR
\end{tabular}

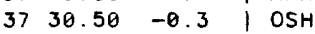

$\begin{array}{lll}37 & 35.20 & 0.7\end{array}$

$3741.90 \quad 0.2$ KYS

$3746.00-0.7$

$6.2 \mathrm{mb}$ | PPT

-0.5 SVA

-0.4 I JAY

\begin{tabular}{l|l}
-0.6 & \\
-0.4 & COO
\end{tabular}

$0.5 \mid$\begin{tabular}{l|l}
150 \\
RIV
\end{tabular}

$6.5 \times$

-0.4 CMS

-0.3 | YOU

-0.3 | WB2

0.5 WRA

0.7 CAN

\begin{tabular}{l|l}
1.0 & AFI \\
-0.8 & STK
\end{tabular}

\begin{tabular}{l|l}
-0.8 & STK \\
-0.2 & GUA
\end{tabular}

o. 2

0.4

-0.4 GUMO

\begin{tabular}{l|l}
0.4 & \\
& PJG \\
I WAM
\end{tabular}

0.0 WAM

ASPA
MTN

\begin{tabular}{l|l}
1.3 & NUE \\
TOO
\end{tabular}

\begin{tabular}{l|l}
0.0 & KNA \\
1.5 & BFD
\end{tabular}

\begin{tabular}{l|l}
1.5 & BFD \\
1.2 & ADE
\end{tabular}

4. $7 \times$ |AAI

$-0.5$

-0.4 T TCW

0.9 | WEL

\begin{tabular}{r|l}
-0.6 & \\
0.8 & SNZO
\end{tabular}

-0.9 WBN

0.1 KUPT

MN I

0.5

0.5 I DAV

\begin{tabular}{l|l}
1.0 & RAR \\
1.2 & MKS
\end{tabular}

s. $\mathrm{CB}$ I

FEB $07,198421 \mathrm{~h} 33 \mathrm{~m} 20.34 \pm 0.08 \mathrm{~s} / \mathrm{KLG}$ $9.998 \mathrm{~S} \pm 2.1 \mathrm{~km} 160.477 \mathrm{E} \pm 2.0 \mathrm{~km}$ । MEK

DEPTH $=10.9 \mathrm{~km}$ (15 depth phases) | PAP

$6.7 \mathrm{mb}$ ( 60 obs.) $7.5 \mathrm{Msz}$ ( 31 obs.) ।

SOLOMON ISLANDS , (193) / MCO

Damoge in southern Guadalcanal KLB

and some landslides reported.

Felt throughout the Solomon $\begin{array}{lllllll}98 & 200 & \text { iPd } & 38 & 16.70 & 0.4 & \text { AJl }\end{array}$

22.79240 iPd $3824.40 \quad 0.1$

$25.22 \quad 198 \mathrm{eP} \quad 3853.00 \quad 5.3 \times$ | 151

$0.8 \mathrm{~s} * * * * * * * \mathrm{~nm} \quad 8.1 \mathrm{mb} X$

$25.34210 \mathrm{eP} \quad 3849.00 \quad 0.2 \quad$ YOK

$\begin{array}{lllllll}26.59 & 203 \text { iPc } & 39 & 01.40 & 0.9 & \text { IVO }\end{array}$

27.07246 iPd $3903.20-1.8 \quad$ SHZ

$27.08246 \mathrm{Pd} 3903.30-1.8$ TAJ

$0.9 \mathrm{~s} \quad 199.50 \mathrm{~nm}$

$27.29201 \mathrm{iPC} \quad 3908.10 \quad 1.1 \quad$ OYM

$\begin{array}{lllll}i & 3913.00 & 17 \mathrm{~km} / \text { TOK }\end{array}$

$27.41101 \mathrm{iPc}$

$28.02326 \mathrm{eP}$

\begin{tabular}{ll|l}
12.70 & -1.0 & I SRY
\end{tabular}

e

$3920.80^{6.9 \mathrm{mb}} 28 \mathrm{kmx}$

\begin{tabular}{lllll|l} 
& $e(S)$ & 44 & 04.50 & & TSK \\
28.09326 eP & 39 & 13.60 & -0.7 & MIT
\end{tabular}

$1.7 \mathrm{~s} \quad 7487.81 \mathrm{~nm}$

$28.09326 \mathrm{eP}$

$28.12200 \mathrm{iPC}$

$3920.20^{7.21}$

\begin{tabular}{l|l}
$2 \mathrm{mb}$ & \\
$23 \mathrm{~km} X$ & $A S Z$
\end{tabular}

\begin{tabular}{rrr|l}
39 & 13.60 & -0.7 & \\
39 & 15.70 & 1.3 & DDR
\end{tabular}

\begin{tabular}{rrr|r}
39 & 15.70 & 1.3 & DDR \\
39 & 20.60 & $17 \mathrm{~km}$ & 110
\end{tabular}

$28.77238 \mathrm{eP}$

28.90262 eP

$29.99111 \mathrm{eP}$

$30.60204 \mathrm{eP}$

$31.41256 \mathrm{eP}$

$31.53208 \mathrm{eP}$

$31.82215 \mathrm{iPd}$

$32.57154 \mathrm{P}$

$\begin{array}{ll}32.64 & 279 \mathrm{eP} \\ 0.85 & 127.30 \mathrm{~nm}\end{array}$

$\begin{array}{lll}0.85 & 127.30 \mathrm{P} \\ 33.39 & 161 \mathrm{P}\end{array}$

$33.62 \quad 160 \quad \mathrm{iPt}$

$\begin{array}{lll}39 & 18.00 & -2.4\end{array}$

\begin{tabular}{lll|l}
39 & 19.00 & -2.6 & MYZ
\end{tabular}

\begin{tabular}{lll|l}
39 & 28.90 & -2.5 & I UTS
\end{tabular}

$\begin{array}{lll}39 & 37.00 & 0.4\end{array}$

\begin{tabular}{lll|l}
39 & 42.00 & -1.9 & OSK
\end{tabular}

\begin{tabular}{lll|l}
39 & 44.00 & -0.8 & KAG
\end{tabular}

\begin{tabular}{lll|l}
39 & 46.80 & -0.6 & $K O C$
\end{tabular}

$\begin{array}{lll}39 & 52.50 & -1.3\end{array}$

$3951.40-3.3 \times \quad$ OSA

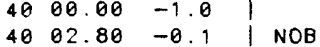

45.27 .00

SCS 5029.00

33.62160 e(P)

$\begin{array}{ll}50 & 29.00 \\ 40 & 03.00\end{array}$

35.86239 iPd

$36.29266 \mathrm{eP}$

$40 \quad 20.90$

4030.00

$37.25286 \mathrm{eP}$

$1.05 \quad 249.60 \mathrm{~nm}$

4645.00

$38.68295 \mathrm{eP}$

$\begin{array}{lll}40 & 32.00 & -2.2\end{array}$

$39.77111 \mathrm{P}$

40.90274 ePd

$40.92335 \mathrm{P}$

41.72235 iPC

42.87242 iPd

43.82299 ePd

$1.5 \mathrm{~s} \quad 12.80 \mathrm{~nm}$

$44.40 \quad 181 \mathrm{eP}$

$44.97235 \mathrm{eP}$

$45.52 \quad 237 \mathrm{eP}$

$45.58 \quad 322$
$4633.90^{5.9 \mathrm{mb}}$ PMO

4700.00

4105.50

4103.30

4251.70

\begin{tabular}{r|r}
0.9 & \\
-1.2 & $\mathrm{VAH}$
\end{tabular}

\begin{tabular}{lll|l}
410.00 & -1.1 & TPT
\end{tabular}

132.80

\begin{tabular}{l|l}
-0.9 & \\
$4.5 \times$ & $01 T$
\end{tabular}

$5 \mathrm{mb} \times$

$4138.00 \quad 5.5 \times$ | RUV

\begin{tabular}{ccc|c}
41 & 40.00 & 2.5 & \\
41 & 36.00 & $-5.9 x$ & OKA
\end{tabular}

4136.00
4147.00

$4.6 \times$
$1.1 \mathrm{~s} 2592.41 \mathrm{~nm}$

$5.9 \mathrm{mb}$ | GIF

\begin{tabular}{lll|l|l}
40 & 45.00 & -1.2 & \\
40 & 56.00 & 0.9 & MAT
\end{tabular}

$45.83233 \mathrm{eP}$

46.34235 eP

$46.40 \quad 232$ eP

$46.88288 \mathrm{eP}$

$1.2 \mathrm{~s} \quad 412.20 \mathrm{~nm}$

$\begin{array}{lll}41 & 44.50 & 1.4\end{array}$

$4142.00-2.3$

$4150.00 \quad 1.6$

$4154.00 \quad 5.2 x$

$151.20-1.8$

$4202.90^{0} 42 \mathrm{kmX}$

$\begin{array}{lllll}47.30 & 269 \text { ePd } & 42 & 14.40 & \\ 41 & 55.40 & -0.8\end{array}$

$1.0 \mathrm{~s} \quad 62.30 \mathrm{~nm}$

5. $7 \mathrm{mb} x$

$47.40303 \mathrm{eP}-$

$4154.00-3.1 x$

$48.08319 \mathrm{P}$

48. $16320 \mathrm{P}$

$48.50 \quad 323 \mathrm{eP}$

$4204.30 \quad 2.2$

$48.50323 \mathrm{eP}$

$49 \quad 15.00$

4209.00

4916.00

$48.72316 \mathrm{eP}$

4211.00

4210.00

4921.80

$48.78 \quad 105$ iP

4209.80

$48.80337 \mathrm{Pt}$

4209.40

$\begin{array}{ll}49 & 19.90\end{array}$

48.89338 eP

4210.30

48.97105 iP

$\begin{array}{ll}42 & 10.30 \\ 42 & 11.20\end{array}$

$48.98 \quad 105$ iP

$1.0 \mathrm{~s} \quad 1705.00 \mathrm{~nm}$

$49.11339 \mathrm{eP}$

$\begin{array}{lll}42 & 11.30 \quad 2.1\end{array}$

$4210.00 \quad 0.1$

$49.15337 \mathrm{eP}$

$\begin{array}{lll}42 & 12.50 & 2.3\end{array}$

$\begin{array}{lll}42 & 11.00 & 0.8\end{array}$ 


\begin{tabular}{|c|c|c|c|c|c|c|c|c|c|c|c|}
\hline KUM & 51.17 & 328 & $e P$ & 42 & 25.00 & -0.6 & 1 & & & & \\
\hline HON & 51.29 & 52 & $P$ & 42 & 28.00 & 1.2 & 1 & & & & \\
\hline$K I P$ & 51.34 & 52 & $P$ & 42 & 28.00 & 0.8 & i & & & & \\
\hline SEN & 51.37 & 340 & $e P$ & 42 & 37.00 & $9.9 x$ & & & & & \\
\hline & & & $S$ & 49 & 55.50 & & 1 & VLA & & 58.94 & 336 \\
\hline ISN & 51.39 & 341 & eP & 42 & 28.00 & 0.7 & 1 & & & & \\
\hline & & & $\epsilon$ & 49 & 41.00 & & 1 & WHN & & 59.88 & 314 \\
\hline$O P A$ & 51.49 & 52 & $P$ & 42 & 27.00 & -1.3 & 1 & $K L M$ & & 60.01 & 279 \\
\hline$H I R$ & 51.52 & 330 & eP & 42 & 30.00 & 1.7 & 1 & & & & \\
\hline & & & $\epsilon$ & 49 & 55.00 & & 1 & PPI & & 60.48 & 275 \\
\hline SHK & 51.53 & 331 & $e^{P}$ & 42 & 20.00 & $-8.5 x$ & 1 & & & & \\
\hline YAM & 51.55 & 340 & eP & 42 & 9.00 & 0.5 & 1 & SKR & & 60.53 & 357 \\
\hline & & & $\in S$ & 49 & 59.00 & & 1 & & & $6.0 \mathrm{~s}$ & $* * * *$ \\
\hline NGS & 51.56 & 327 & eP & 42 & 0.00 & 1.4 & 1 & & & & \\
\hline & & & $\mathrm{s}$ & 49 & 56.20 & & 1 & & & & \\
\hline MOK & 51.58 & 52 & $P$ & 42 & 30.00 & 1.0 & 1 & & & & \\
\hline ANP & 51.61 & 313 & eP- & 42 & 2.00 & 2.7 & 1 & DL2 & & 60.62 & 326 \\
\hline & & & es & 49 & 56.00 & & 1 & IPM & & 60.96 & 281 \\
\hline TOT & 51.63 & 333 & $e P$ & 42 & 32.00 & $2.9 x$ & 1 & & & $1.4 \mathrm{~s}$ & 160 \\
\hline & & & es & 49 & 55.00 & & 1 & & & & \\
\hline FKK & 51.94 & 328 & Pd & 42 & 2.50 & 1.0 & 1 & & & & \\
\hline & & & S & 49 & 53.50 & & 1 & MDJ & & 61.12 & 335 \\
\hline SHN & 51.94 & 329 & ePt & 42 & 33.00 & 1.5 & ! & & & & \\
\hline & & & es & 50 & 01.00 & & 1 & $T \mid A$ & & 61.46 & 321 \\
\hline A I K & 52.10 & 338 & $e^{P}$ & 42 & 33.00 & 0.4 & 1 & SNY & & 61.70 & 329 \\
\hline FKJ & 52.10 & 326 & $\mathrm{P}+$ & 42 & 34.50 & 1.8 & 1 & & & & \\
\hline & & & S & 50 & 01.50 & & 1 & CN2 & & 62.28 & 332 \\
\hline MTS & 52.10 & 332 & iPt & 42 & 35.20 & 2.5 & 1 & & & & \\
\hline & & & S & 50 & 03.00 & & 1 & PSI & & 62.57 & 278 \\
\hline HMD & 52.12 & 330 & eP & 42 & 34.00 & 1.2 & 1 & & & $1.6 \mathrm{~s}$ & 337 \\
\hline & & & es & 50 & 04.00 & & 1 & PET & & 62.78 & 359 \\
\hline$M I Y$ & 52.29 & 342 & eP & 42 & 6.00 & 1.9 & 1 & & & $2.5 \mathrm{~s}$ & 2570 \\
\hline & & & es & 50 & 06.00 & & 1 & & & & \\
\hline MKH & 52.53 & 56 & P & 42 & 36.00 & -0.2 & 1 & RKT & & 62.80 & 111 \\
\hline MRK & 52.59 & 341 & eP & 42 & 35.00 & -1.3 & 1 & & & $1.6 \mathrm{~s}$ & 2585 \\
\hline & & & es & 50 & 13.00 & & I & TSI & & 63.10 & 279 \\
\hline AKI & 52.96 & 340 & eP & 42 & 40.00 & 1.0 & 1 & PCT & & 63.47 & 292 \\
\hline & & & es & 50 & 23.00 & & 1 & & & $1.5 \mathrm{~s}$ & 710 \\
\hline 120 & 52.99 & 327 & eP & 42 & 40.00 & 0.6 & 1 & & & & \\
\hline & & & e & 50 & 13. & & 1 & SMY & & 63.56 & 9 \\
\hline HAC & 53.24 & 342 & eP & 42 & 40.00 & -1.1 & 1 & & 2 & $20 s$ & 300 \\
\hline & & & es & 50 & 20.00 & & 1 & & & & \\
\hline $\mathrm{OZH}$ & 53.60 & 311 & iPc & 42 & 45.00 & 1.0 & 1 & & & & \\
\hline URA & 54.38 & 344 & eP & 42 & 50.00 & 0.6 & 1 & GYA & & 63.58 & 306 \\
\hline & & & es & 50 & 35.00 & & 1 & NNT & & 64.37 & 289 \\
\hline HAK & 54.65 & 342 & eP & 42 & 51.00 & -0.4 & 1 & BJI & & 64.49 & 324 \\
\hline & & & e & 50 & 40.00 & & 1 & & $z$ & $20 \mathrm{~s}$ & 199 \\
\hline KUS & 54.73 & 346 & eP & 42 & 52.00 & 0.0 & 1 & & $E$ & $19 \mathrm{~s}$ & 148 \\
\hline & & & es & 50 & 41.00 & & 1 & & & & \\
\hline NEM & 54.78 & 347 & eP & 42 & 50.00 & -2.4 & I & & & & \\
\hline & & & es & 50 & 43.00 & & 1 & & & & \\
\hline OB। & 54.97 & 345 & eP & 42 & 54.00 & 0.2 & 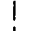 & & & & \\
\hline & & & $\epsilon$ & 50 & 43.00 & & 1 & & & & \\
\hline SSE & 55.57 & 319 & $P+$ & 42 & 56.60 & -1.7 & 1 & & & & \\
\hline & 6.05 & 29 & $.20 \mathrm{~nm}$ & & & $.5 \mathrm{mb} \times$ & 1 & & & & \\
\hline & $22 \mathrm{~s}$ & 316 & $.90 \mathrm{um}$ & & & $.4 \mathrm{Msz}$ & 1 & ADK & & 64.70 & 15 \\
\hline E & $20 \mathrm{~s}$ & 188 & $3.70 \mathrm{um}$ & & & & 1 & & & & \\
\hline & & & sP & 43 & 11.00 & $86 \mathrm{kmX}$ & 1 & & & & \\
\hline & & & $\mathrm{i}$ & 43 & 18.00 & & 1 & NST & & 64.96 & 292 \\
\hline & & & $\mathrm{PCP}$ & 44 & 06.00 & & 1 & TIY & & 65.34 & 320 \\
\hline & & & S & 50 & 37.50 & & 1 & XAN & & 65.64 & 314 \\
\hline & & & PS & 50 & 48.50 & & 1 & KHT & & 66.09 & 291 \\
\hline & & & sS & 50 & 53.00 & & 1 & KMI & & 66.18 & 303 \\
\hline & & & i & 57 & 09.50 & & 1 & & 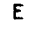 & $17 \mathrm{~s}$ & 127 \\
\hline $\mathrm{HKC}$ & 55.59 & 306 & $\in P$ & 43 & 01.00 & 2.4 & 1 & & & & \\
\hline SAP & 55.62 & 343 & ef & 42 & 55.00 & $-3.5 x$ & 1 & & & & \\
\hline & & & $\in S$ & 50 & 47.00 & & 1 & & & & \\
\hline SUT & 55.69 & 342 & eP & 43 & 02.00 & $3.0 x$ & 1 & & & & \\
\hline & & & es & 50 & 55.00 & & 1 & & & & \\
\hline $\mathrm{ABJ}$ & 55.73 & 346 & e & 43 & 00.00 & 0.7 & 1 & BSI & & 66.70 & 280 \\
\hline & & & es & 50 & 47.00 & & 1 & $\mathrm{CHG}$ & & 67.08 & 295 \\
\hline ASA & 55.99 & 344 & $e^{P}$ & 43 & 02.00 & 0.9 & I & & & $0.6 \mathrm{~s}$ & 10 \\
\hline & & & $\in S$ & 50 & 50.00 & & 1 & СHTO & & 67.08 & 295 \\
\hline $\mathrm{MCO}$ & 15.99 & 305 & eP & 43 & 09.00 & $7.5 x$ & 1 & $\mathrm{HHC}$ & & 67.75 & 322 \\
\hline KUR & 56.14 & 349 & iPd & 43 & 06.00 & $3.9 x$ & 1 & $\mathrm{CD} 2$ & & 67.89 & 309 \\
\hline & $1.0 \mathrm{~s}$ & 770 & $.00 \mathrm{~nm}$ & & & $.7 \mathrm{mb}$ & 1 & & & & \\
\hline & & & isP & 43 & 28.50 & & 1 & NK I & & 67.95 & 19 \\
\hline & & & ePPP & 46 & 26 & & 1 & & & & \\
\hline & & & is & 50 & 40.00 & & 1 & & & & \\
\hline RMJ & 56.36 & 344 & eP & 43 & 07.00 & $3.3 x$ & 1 & BTO & & 68.55 & 321 \\
\hline & & & $\in S$ & 51 & 06.00 & & 1 & & & & \\
\hline GZH & 56.64 & 306 & eP & 43 & 05.50 & -0.7 & 1 & $\mathrm{LZH}$ & & 70.26 & 314 \\
\hline NJ2 & 57.71 & 318 & $\mathrm{PC}$ & 43 & 14.00 & 0.4 & 1 & & & $2.5 \mathrm{~s}$ & 1461 \\
\hline & & & $\mathrm{s}$ & 51 & 10.00 & & 1 & & $\mathrm{~N}$ & $11 \mathrm{~s}$ & 14 \\
\hline GM & 8.14 & 279 & ePc & 43 & 17.20 & 0.3 & 1 & & $E$ & $17 \mathrm{~s}$ & 85 \\
\hline YSS & 58.93 & 346 & $I P c$ & 43 & 21.60 & -0.2 & 1 & & & & \\
\hline & $1.0 \mathrm{~s}$ & 240 & 1.0 & & & $.3 \mathrm{mb}$ & 1 & & & & \\
\hline
\end{tabular}


$07 d \quad 21 \mathrm{~h}$

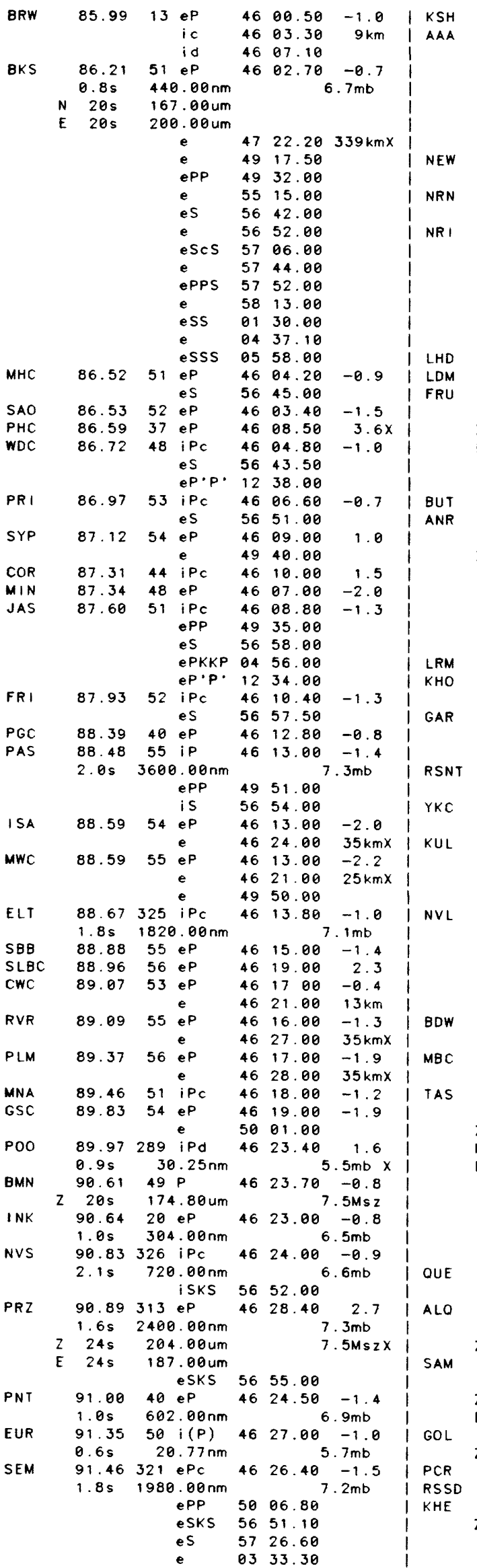

$92.06310 \mathrm{Pc} \quad 4633.00 \quad 1.9 \quad$ $92.10313 \quad \mathrm{iPc}$ $5.4 \mathrm{~s} \quad 6.48 \mathrm{~nm}$ $Z 21 \mathrm{~s} 108.00 \mathrm{um}$ N $21 \mathrm{~s} \quad 95.00 \mathrm{um}$ $21 \mathrm{~s} \quad 12.80 \mathrm{um}$ iSKS $\begin{array}{lllll} & i & 0341.20 \\ 92.37 & 42 \mathrm{eP} & 4631.00\end{array}$ $1.2 \mathrm{~s} \quad 645.00 \mathrm{~nm}$ $\begin{array}{llllll}92.43 & 311 & \text { eP } & 46 & 32.80 & -0.2\end{array}$ $93.20 \quad 340$ eSKS 5704.00

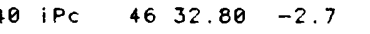

$1.8 \mathrm{~s} \quad 400.00 \mathrm{~nm} \quad 6.5 \mathrm{mb}$ $\begin{array}{lll}\text { iPP } & 50 & 19.00 \\ \text { iSKS } & 57 & 08.00\end{array}$ is 5741.00 ePS 5859.00 \begin{tabular}{llllll|l}
93.39 & $42 \mathrm{IPC}$ & 46 & 37.70 & 0.7 & IIT \\
ACO
\end{tabular} $\begin{array}{llllll}93.58 & 42 \mathrm{IPC} & 46 & 38.70 & 0.9\end{array}$ 93.70313 eP $46 \quad 38.00 \quad-0.5$ $2.0 \mathrm{~s} \quad 1260.00 \mathrm{~nm}$ $219 \mathrm{~s} 120.00 \mathrm{um}$ E $19 \mathrm{~s} \quad 131.00 \mathrm{um}$

$$
\begin{gathered}
19 s \quad 131.00 \mathrm{um} \\
\text { is } 5747.00
\end{gathered}
$$

$95.01 \quad 44$ ePc $\quad 46 \quad 45.90$

$95.02310 \mathrm{eP}$

$2.5 \mathrm{~s} \quad 930.00 \mathrm{~nm}$

$\begin{array}{lll}46 & 46.80 & 1.3\end{array}$

E $20 \mathrm{~s} \quad 130.00 \mathrm{um}$

ePP $50 \quad 38.00$

ESKS $57 \quad 17.00$

eS 5751.00

eSP 5911.00

\begin{tabular}{lllll|l}
95.05 & $45 \mathrm{ePC}$ & 46 & 43.70 & -1.2 & ALE
\end{tabular} $95.17307 \mathrm{iPC} \quad 46 \quad 45.40 \quad-0.2 \quad$ $6.6 \mathrm{~s} \quad 3300.00 \mathrm{~nm}$ $96.33308 \mathrm{eP}$

$2.4 \mathrm{~s} \quad 700.00 \mathrm{~nm}$ $\begin{array}{llll} & \mathrm{eS} & 57 & 52.50 \\ 96.45 & 28 \mathrm{P} & 46 & 48.60\end{array}$ $1.1 \mathrm{~s} \quad 378.00 \mathrm{~nm} \quad 48.60-1.9$ $96.50 \quad 28 \mathrm{eP} \quad 46 \quad 48.00 \quad-2.8$ $\begin{array}{lll}0.9 \mathrm{~s} & 372.00 \mathrm{~nm} & 6.9 \mathrm{mb} \\ 96.63 & 307 \mathrm{eP} & 4651.10^{-0.9}\end{array}$ $3.1 \mathrm{~s} 3700.00 \mathrm{~nm} \quad 7.4 \mathrm{mb} \times$ IPP $50 \quad 52.10$ ISKS 5724.30 $96.66190 \mathrm{eP} \quad 4653.00 \quad 1.4 \quad F C C$

$1.8 \mathrm{~s} \quad 320.00 \mathrm{~nm}$ eSKS eS 5810.00 ESP 5936.00 ESPP 0025.00

$\begin{array}{llllll}96.67 & 48 P & 46 & 50.00 & -2.3\end{array}$

1. $1 \mathrm{~s} 261.00 \mathrm{~nm}$

$97.31 \quad 14 \mathrm{eP}$

$0.8 \mathrm{~s} \quad 119.00 \mathrm{~nm}$

$97.40311 \mathrm{eP} \quad 46 \quad 56.20 \quad 0.9$

$2.0 \mathrm{~s} 1000.00 \mathrm{~nm} \quad 7.1 \mathrm{mb}$

$Z 22 \mathrm{~s} \quad 160.00 \mathrm{um}$

N $18 \mathrm{~s} \quad 57.00 \mathrm{um}$

E $21 \mathrm{~s} 162.00 \mathrm{um}$ ePP $50 \quad 55.20$ EPPP 5255.20 ESKS $57 \quad 24.20$ ES 5806.20 ESPP 0012.20

97.98299 IPC 4700.00 eS 5747.00

$98.1456 \mathrm{iPct} 4658.00$

$1.0 \mathrm{~s} \quad 151.25 \mathrm{~mm}$

$222 \mathrm{~s} 240.74 \mathrm{um}$

$98.97309 \mathrm{eP}$

$5.5 \mathrm{~s} 2400.00 \mathrm{~nm}$

$\begin{array}{rrr}24 \mathrm{~s} & 210.00 \mathrm{um} & 7.6 \mathrm{Msz} X\end{array}$

$4703.00 \quad 0.5$

$\begin{array}{llllll}99.48 & 51 & \text { e(P) } & 47 & 03.00 & -2.2\end{array}$ $Z 20 \mathrm{~s} 300.00 \mathrm{um} \quad 7.8 \mathrm{Msz}$

100.04246 ePdif $f 47 \quad 12.00 \quad 3.8 \times \quad$ KEV

$101.82 \quad 351$ ePdiff47 $16.40 \quad 1.9$

$z \quad 19 \mathrm{~s} 130.00 \mathrm{um}$

7. $5 \mathrm{MsZ}$

iSKS 5753.00

es $58 \quad 48.00$
DAG
KEV
KEV

0557.00

$102.45 \quad 36$ ePdiff47 19.00 102.5573 Pdiff 4729.00 102.9072 Pdiff 4730.00 $102.93 \quad 72$ Pdiff $4730.60 \quad 9.5 x$ $103.18 \quad 72 \mathrm{Pditf} 47 \quad 36.00 \quad 13.8 \mathrm{X}$ $103.33 \quad 61$ ePdiff47 $24.00 \quad 1.6$ $1.0 \mathrm{~s} \quad 55.00 \mathrm{~nm} \quad 6.3 \mathrm{mb}$ $220 \mathrm{~s} 113.48 \mathrm{um} \quad 7.4 \mathrm{MsZ}$ 103.69326 ePdiff47 $21.00 \quad-2.2$ $5.0 \mathrm{~s} 2300.00 \mathrm{~nm} \quad 7.2 \mathrm{mb} \times$ $\begin{array}{lrr}Z & 20 \mathrm{~s} & 230.00 \mathrm{um} \\ \mathrm{N} & 20 \mathrm{~s} & 36.00 \mathrm{um}\end{array}$ E $20 \mathrm{~s} \quad 183.00 \mathrm{um}$ PPP 5346.00 eS 5900.00 ePS 0046.00 e $\quad 0622.00$

$\begin{array}{lllll}103.72 & 73 \text { Pdiff } 4735.00 & 10.4 x\end{array}$ $104.20 \quad 55$ ePdiff47 $29.60 \quad 3.6 x$ $0.8 \mathrm{~s} \quad 37.50 \mathrm{~nm} \quad 6.3 \mathrm{mb}$

$104.67 \quad 305$ ePdiff47 $30.00 \quad 1.7$ $\begin{array}{lll}\text { e } & 50 & 48.00 \\ \text { es } & 01 & 19.00\end{array}$

$\begin{array}{llllll}\text { ARU } & 104.84 & 326 & \text { ePdiff47 } & 29.00 & 0.7\end{array}$

$2.8 \mathrm{~s} \quad 400.00 \mathrm{~nm} \quad 6.8 \mathrm{mb}$

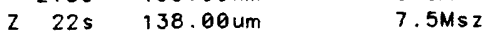

$N 20 \mathrm{~s} \quad 58.00 \mathrm{um}$

E $22 \mathrm{~s} \quad 112.00 \mathrm{um}$

APP 5151.50

eSKS 5808.00 eS 5904.00 ePS 0106.00

$\begin{array}{llllll}\text { ATX } & 104.99 & 61 & \text { Pdiff } 47 & 30.00 & 0.3\end{array}$ PP 5200.00

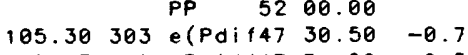
$\begin{array}{lllll}105.43 \quad 5 \mathrm{ePdiff} 47 \quad 31.00 & 0.5\end{array}$ $1.5 \mathrm{~s} \quad 159.00 \mathrm{~nm} \quad 6.8 \mathrm{mb}$ $\begin{array}{llll}105.62 & 307 \text { ePdiff47 } 34.00 \quad 1.7\end{array}$ ePP 5203.00 EPPP $54 \quad 15.50$ ESPP 0154.00

VAN 105.81307 ePdiff47 $34.60 \quad 1.4$ $\begin{array}{lll}22 \mathrm{~s} & 130.00 \mathrm{um} & 7.4 \mathrm{Msz}\end{array}$ ePP 5154.00 EPPP 5408.00 eSKS 5814.00 eS 5916.00 ePS 0108.00 $106.49 \quad 32$ ePdiff47 $36.00 \quad 0.4$ 106.8956 ePdiff $47 \quad 45.70 \quad 7.7 x$ $1.0 \mathrm{~s} \quad 31.50 \mathrm{~nm} \quad 6.3 \mathrm{mb}$ $Z 21 \mathrm{~s} 260.40 \mathrm{um} \quad 7.8 \mathrm{MsZ}$ $\begin{array}{llllll}\text { KAT } & 107.24 \quad 308 \text { ePdiff } 47 \quad 43.00 \quad 3.5 x\end{array}$ $6.0 \mathrm{~s} 2000.00 \mathrm{~nm} \quad 7.3 \mathrm{mb} \mathrm{x}$ $Z 17 \mathrm{~s} \quad 39.00 \mathrm{um}$ N $17 \mathrm{~s} \quad 44.00 \mathrm{um}$ E 175 68.00um $\begin{array}{lll}\text { ePP } \quad 52 & 15.00\end{array}$ iSKS 5821.00

$\begin{array}{lllll}\text { BHO } 107.64 \quad 57 \text { ePdiff47 } & 52.50 \quad 11.1 \times\end{array}$

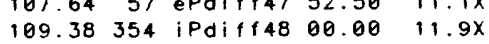
$\begin{array}{llll}110.84 & 42 \text { ePdiff } 48 \quad 05.00 & 9.7 X\end{array}$ $\begin{array}{lllll}110.84 & 42 & \text { ePKP } 52 & 10.00 & 14.8 X\end{array}$ $2.2 \mathrm{~s} \quad 853.00 \mathrm{~nm}$

$112.00 \quad 79$ ePdiff48 $04.00 \quad 2.7 x$ 112.07309 e 5246.50 $0015.1 x$ $\begin{array}{lllll}112.81 & 341 \text { ePdif } f 48 \quad 09.20 \quad 5.6 X\end{array}$ $\begin{array}{lllll}112.81341 \text { ePKPC } 5201.80 \quad 3.5 \mathrm{X} & 010\end{array}$ $Z 21 \mathrm{~s} \quad 179.00 \mathrm{um}$

N $21 \mathrm{~s} \quad 39.00 \mathrm{um}$ E $22 \mathrm{~s} \quad 114.00 \mathrm{um}$ IPP $52 \quad 44.00$ ESKS $58 \quad 38.00$ IPS $02 \quad 17.00$ EPPS 0327.00 i $\quad 0828.00$

$\begin{array}{llll}113.25 & 360 \text { ipdiff48 } & 16.00 & 10.7 x\end{array}$ 5235.00 is 0029.00

$113.43 \quad 344$ ePdift $48 \quad 15.00 \quad 8.8 x$ 113.43344 iPKP 5203.10 EPP $52 \quad 44.00$ epPPP $55 \quad 44.00$ EPS 0216.00 IPKKP 0251.00 


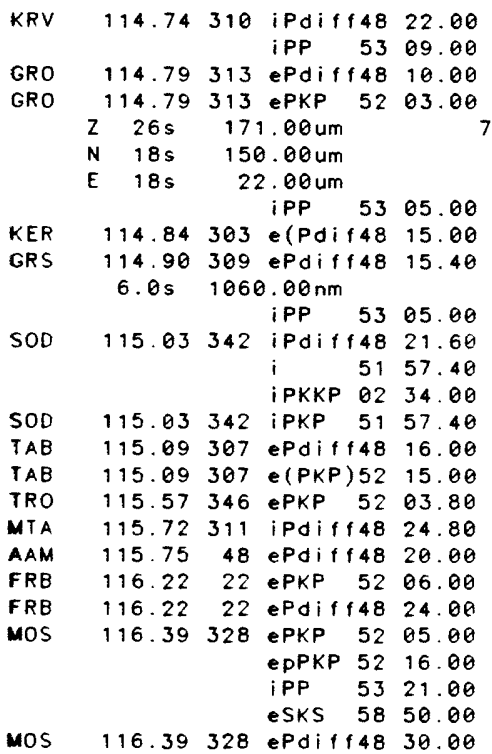
$2.0 \mathrm{~s} \quad 200.00 \mathrm{~nm}$

PYA $116.53 \quad 314$ iPdiff48 31.30 $1.3 \mathrm{~s} 260.00 \mathrm{~nm}$ EPP $53 \quad 20.00$

LEN $\quad 116.58 \quad 311$ ePKP $52 \quad 06.00$ LEN 116.58311 ePdiff48 25.00 4 os $2700.00 \mathrm{~nm}$

KJF $\quad 116.60339$ iPdiff48 28.00 $1.0 \mathrm{~s} \quad 30.00 \mathrm{~nm}$

$\begin{array}{lll}i & 48 & 32.00 \\ i & 52 & 06.10 \\ \text { EPP } & 53 & 20.00 \\ \text { ESPP } & 53 & 56.00 \\ \text { EPPPP } & 56 & 20.00 \\ \text { ESKS } & 58 & 52.00 \\ \text { ES } & 01 & 12.00 \\ \text { EPKKP } & 02 & 34.00 \\ \text { EPS } & 02 & 52.00\end{array}$

BKR $\quad 116.66 \quad 312$ ePdiff 482626.00 Z $24 \mathrm{~s} \quad 134.00 \mathrm{um}$

PP $53 \quad 10.00$

GDH $\quad 116.88 \quad 13$ ePdiff $48 \quad 26.00$

OBN $\quad 117.15 \quad 328$ ePdiff $48 \quad 24.00$ $\begin{array}{rl}1.7 \mathrm{~s} & 130.00 \mathrm{~nm} \\ 220 \mathrm{~s} & 147.00 \mathrm{um}\end{array}$

$\begin{array}{lll}2 & 20 \mathrm{~s} & 147.00 \mathrm{um} \\ \mathrm{N} & 20 \mathrm{~s} & 105.00 \mathrm{um}\end{array}$

E $20 \mathrm{~s} \quad 126.00 \mathrm{um}$

APPP $55 \quad 48.00$

iSKS 5900.00 ESS $09 \quad 16.00$

BHD $117.20 \quad 302$ ePKPd $52 \quad 88.00$ ePP $53 \quad 23.08$ \begin{tabular}{l}
$e \quad 5458.00$ \\
$e \quad 5556.00$ \\
\hline
\end{tabular} eS $58 \quad 35.00$ e $\quad 00 \quad 59.00$

$\begin{array}{llllll}\text { ELF } & 117.27 & 46 & \text { PKP } & 52 & 08.35\end{array}$

LDN $117.39 \quad 47$ PKP 5208.35

MSL $\quad 117.83 \quad 306$ IPKP $52 \quad 20.50$ 5327.00

MSL $117.83 \quad 306$ ePdiff48 31.00

SUF 118.05338 ePKP 5206.00

SUF 118.05338 ePdiff48 35.00

PUL 118.05334 ePKP 5211.00

$Z 20 \mathrm{~s} 120.00 \mathrm{um}$

N $20 \mathrm{~s} \quad 80.00 \mathrm{um}$

$\begin{array}{lll}\text { PPP } & 53 \quad 27.00\end{array}$ ESKS 5909.00 e $\quad 0940.00$

SOC $118.98 \quad 314$ ePdiff $48 \quad 32.00$

BLA $119.10 \quad 53$ ePKP 5220.80 $\begin{array}{lll}\text { PP } 53 & 35.00\end{array}$

VCA $\quad 119.25131$ e(PKP) $52 \quad 15.00$ NUR 119.96337 IPKP 5214.00 $\begin{array}{ll}119.96 & 337 \text { iPKP } \\ 1.0 \mathrm{~s} \quad 68.00 \mathrm{~nm} & \end{array}$

$120.04 \quad 125$ ePdiff $48 \quad 41.00$

TCA

$120.40 \quad 135$ e(PKP) $52 \quad 09.00$ e $\quad 0005.00$

$9.2 \times \quad$ OTT $-2.9 x$ 0.1 SEK 7. $5 \operatorname{Msz} 2$

UPA
UPA

1.4
1.6

$8.2 \times$ $8.2 x$

\section{$-5.3 x$}

$1.4 \mid \mathrm{BPI}$ 11. 1X I PSO \begin{tabular}{l|l}
0.2 & MNT
\end{tabular} $7.7 \times$ $2.7 \times$ BFS

$1.8 \times$ 5.2X I ARE \begin{tabular}{l|l}
-0.6 & $K V T$ \\
& FSA
\end{tabular} I MNK $10.3 \times$ $10.6 \times$

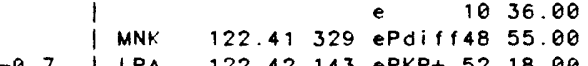
-0.7 LPA 122.42143 ePKP+ 5218.00

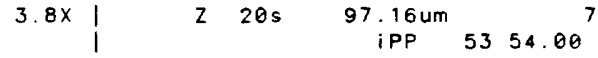
$7.5 \times$ SIM 122.42317 ePdiff49 84.00 $Z 26 \mathrm{~s} \quad 166.00 \mathrm{um}$ $\begin{array}{lrr}N & 28 \mathrm{~s} & 89.00 \mathrm{um} \\ \mathrm{E} & 28 \mathrm{~s} & 269.00 \mathrm{um}\end{array}$ EPP 5352.00 ESKS $59 \quad 16.00$ ESPP 0508.00 e 1044.00

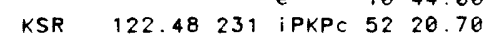
$0.9 \mathrm{~s} \quad 16.15 \mathrm{~nm}$

MTD 122.89243 IPKPd $52 \quad 21.00$ IPPKP $52 \quad 33.00$ $4.5 x$ $.5 \mathrm{MszX}$ PKKP O2 25.00

\section{$4.4 \times 1$
0.9}

SUR $7.6 \mathrm{Msz}$ UPP $1.2 \mathrm{~s} \quad 140.63 \mathrm{~nm}$ $218 \mathrm{~s} \quad 88.83 \mathrm{um}$ 123.03339 iPKP $52 \quad 17.8$ $i \quad 5403.00$ $05 \quad 13.70$

123.15 47 Pdiff 4856.00

$123.42 \quad 128$ e(PKP) $52 \quad 22.00$ 123.96238 iPKPd $52 \quad 23.00$ $Z 21 \mathrm{~s} 296.06 \mathrm{um}$

$\begin{array}{lll}\mathrm{N} & 21 \mathrm{~s} & 146.95 \mathrm{um} \\ \mathrm{E} & 21 \mathrm{~s} & 222.22 \mathrm{um}\end{array}$

i PPKP $52 \quad 36.00$ $\begin{array}{lll}\text { iPP } 54 & 16.00\end{array}$ IPKKP O2 17.00

\begin{tabular}{l:|l} 
& \\
& \\
& \\
0.4 & JER \\
$9.4 \times$ & LPB \\
$2.9 \times$ & LPB \\
1.8 & KDE \\
& BOG \\
$4.3 \times$ & \\
$-5.1 \times$ & IAS
\end{tabular}

124.24342 PKP 5220.60 $1.6 \mathrm{~s} \quad 506.67 \mathrm{~nm}$ $\begin{array}{llll}125.19 & 118 & \text { Pdifft+49 } & 06.00 \\ 125.52 & 314 & \text { ePKP } 52 & 30.00\end{array}$
124.18341 (PKP) $52 \quad 20.40$ $0.7 \mathrm{~s} \quad 39.00 \mathrm{~nm}$ $0.9 \mathrm{~s} \quad 79.10 \mathrm{~nm}$

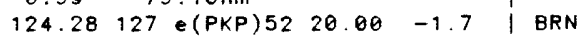
124.38359 iPKP $52 \quad 22.20$

124.74126 e(PKP) $52 \quad 25.00$

$124.77 \quad 303$ ePKP $52 \quad 36.00$

$124.98 \quad 321$ ePKP $52 \quad 21.00$ $1.8 \mathrm{~s} \quad 200.00 \mathrm{~nm}$

$226 \mathrm{~s} \quad 115.00 \mathrm{um}$

E $26 \mathrm{~s} 98.00 \mathrm{um}$

i PPKP $52 \quad 33.00$

$\begin{array}{lll}\text { IPP } & 54 & 10.00\end{array}$

ISKS 5934.00

125.09302 ePKP $52 \quad 35.50$ $\begin{array}{cccc}125.19 & 118 \text { ePKP } & 52 & 25.00 \\ \text { SKS } & 59 & 32.00\end{array}$ $\begin{array}{lrr}5 \% & 32.00 \\ \text { SKS } & 50.00\end{array}$

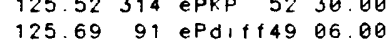
ePP $\quad 52 \quad 25.50$ $125.70 \quad 322$ ePKP $52 \quad 25.00$

\begin{tabular}{r|l}
1.6 & KONO \\
& REY \\
1.8 & PPE \\
CFR \\
BER \\
0.7 & LVV \\
$6.8 M S Z$ & \\
&
\end{tabular}

$125.84 \quad 342$ EPKP $52 \quad 25.40$ $125.92 \quad 1$ IPKP 5224.70 126.11321 ePKP $52 \quad 27.00$ 126.23320 ePKPC $52 \quad 27.00$ 126.41345 EPKP 5228.00 126.43326 iPKP $52 \quad 25.20$ $\begin{array}{lll}\text { ePP } & 54 & 16.00\end{array}$ eSKS 5922.00 EPS 0421.00

TLB $\quad 126.56319$ EPKPC $52 \quad 28.00$ $\begin{array}{llllll}\text { TLB } & 126.56 & 319 & \text { EPKPC } & 52 & 28.00 \\ \text { ODB } & 126.66 & 321 \text { EPKP } & 52 & 44.00\end{array}$ $\begin{array}{lllll}126.66 & 321 & \text { EPKP } & 52 & 44.00 \\ 126.66 & 313 & \text { ePKP } & 52 & 28.10\end{array}$ 126.82321 ePKP $52 \quad 28.00$ 127.19321 ePKPC $52 \quad 29.00$ 127.27320 EPKPC $52 \quad 30.00$

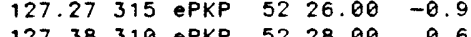
$\begin{array}{lllll}127.48 & 321 \text { ePKP } 52 \quad 28.00 & 0.6\end{array}$ $\begin{array}{llll}127.88 & 316 \text { ePKP } 5227.60 & -0.5\end{array}$

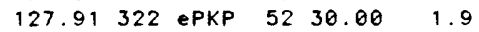
$\begin{array}{lllll}127.95 & 319 \text { ePKP } 52 \quad 35.00 \quad 6.9 x\end{array}$ $\begin{array}{llll}127.99 & 338 \text { ePKP- } 52 \quad 35.00 \quad 7.2 X\end{array}$

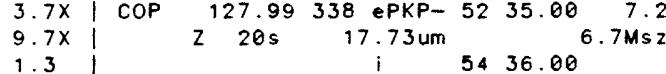
$1.3 \quad$

\begin{tabular}{l|lllllll}
$3.0 X$ & UZH & 128.03 & 326 & IPKP & 52 & 29.40 & 1.3
\end{tabular} $2.1 \mathrm{~s} \quad 1450.00 \mathrm{~nm}$

$z 20 \mathrm{~s} 120.00 \mathrm{um}$ I PPKP 5240.00 iPP $54 \quad 27.50$ ISKS 5930.00

$8.5 X) K C T$ 0.3 DST \begin{tabular}{l|l}
$.5 \mathrm{Msz}$ & $\mathrm{ELL}$ \\
& $\mathrm{CMP}$
\end{tabular} 17.1X CJR 7.6MszX | EDC

I KRA

\subsection{KRA} MUD $10.2 \times \quad \mathrm{HLW}$ \begin{tabular}{l|l}
$5 M s z$ & $S P C$ \\
-0.3 &
\end{tabular} DRA 5. $8 \times$ JOS 1.8 | UAV 1.6 Y YER

\begin{tabular}{l|l} 
IIMS & IZM \\
SOV \\
SOV
\end{tabular}
SDV EZN I PSZ

0.0 KSP
0.0 \begin{tabular}{l|l}
1.6 & TIM \\
& BUD \\
1.8 & TOV
\end{tabular} \begin{tabular}{l|l}
$13.6 \times$ & HAM \\
-1.3 & SRO
\end{tabular} -1.3
$4 \mathrm{Msz} X$

\begin{tabular}{c|c} 
& BRG \\
$12.4 \times$ & \\
0.9 & \\
& \\
$5.5 x$ & $\mathrm{CLL}$ \\
$6.4 \mathrm{X}$ & $\mathrm{CLL}$ \\
$3.3 \mathrm{X}$ & $\mathrm{ZST}$ \\
&
\end{tabular}
BRG 128.07314 IPKP $52 \quad 29.20$ 128.11313 iPKPC $52 \quad 29.10$ 128.12309 iPKPC 5239.50 128.15321 EPKPC $52 \quad 33.00$ 128.31323 ePKP $52 \quad 31.80$ 128.38314 IPKP $52 \quad 29.80$ 128.47328 ePKP $52 \quad 27.70$ $1.8 \mathrm{~s} \quad 227.00 \mathrm{~nm}$

$$
\begin{aligned}
& 218 \mathrm{~s} \quad 98.50 \mathrm{um} \\
& \text { - } \quad 5231.40
\end{aligned}
$$$$
\text { e } 5231.40
$$$$
\text { e } \quad 5248.40
$$$$
\text { e } \quad 5435.00
$$
s2

$\begin{array}{lllll}128.47 & 328 \text { ePdiff } 49 & 24.40 & 10.8 \times\end{array}$

128.54340 iPKP $52 \quad 36.20 \quad 7.4 X$ $1.0 \mathrm{~s} \quad 72.00 \mathrm{~nm}$

128.72300 ePKP 5230.00 ES 5442.00 128.82327 ePKP 5230.40 128.92321 ePKP $52 \quad 33.00 \quad 3.0 x$

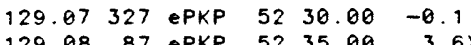
$\begin{array}{lllll}129.23 & 310 \text { IPKP } 52 & 32.20 & 1.3\end{array}$ 129.54312 ePKP $52 \quad 32.00 \quad 0.6$

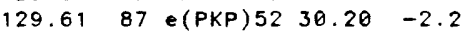
$\begin{array}{lllll}129.61 & 87 & \text { iPKPC } 52 & 34.30 & 1.9\end{array}$ $0.7 \mathrm{~s} \quad 57.10 \mathrm{~nm}$

$129.67 \quad 314$ ePKPd $52 \quad 31.30 \quad-0.2$ $\begin{array}{lllll}129.75 & 326 & \text { e(PKP) } 52 \quad 32.00 & 0.4\end{array}$

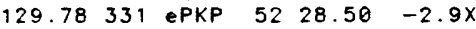
$1.8 \mathrm{~s} \quad 544.00 \mathrm{~nm}$

$\begin{array}{lll}i & 5233.00 \\ i & 5443.00\end{array}$

$\begin{array}{llll}130.08 \quad 334 \text { EPKP } 52 \quad 32.00 & 0\end{array}$ $130.22 \quad 323$ iPKP $52 \quad 32.4 \theta$ 130.49326 ePKPd $52 \quad 34.00$

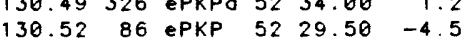

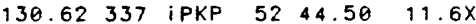
$\begin{array}{llll}130.67 & 327 & \text { ePKP } 52 \quad 37.20\end{array}$ $\begin{array}{cccc}30.67327 & \text { ePKP } & 52 & 37.20 \\ e & 53 & 14.00 \\ e & 54 & 46.00\end{array}$

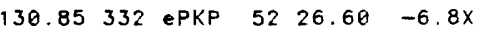
$Z 20 \mathrm{~s} \quad 115.00 \mathrm{um}$ i $\quad 5235.30$ 5244.60 - $\quad 5301.00$ iSKP $55 \quad 58.50$ iPKS $56 \quad 10.00$

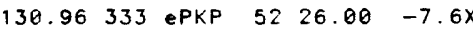
130.96333 iPKP $52 \quad 35.30$ ESKP 5558.00 $\begin{array}{cccccc}1.3 & 131.08328 \text { ePKP } & 5233.00 \\ & & i & 52 & 37.00\end{array}$ 


$$
\begin{array}{lll}
\text { EPP } & 54 & 44.00 \\
\text { e } & 01 & 09.50 \\
\text { ES } & 16 & 00.00
\end{array}
$$

$\begin{array}{llllll}1 T B 7 & 131.43 & 137 & \text { e(PKP) } 52 \quad 40.70\end{array}$

VKA $131.44 \quad 329$ ePKP $52 \quad 35.80$ $\begin{array}{lll}i & 52 & 52.00 \\ i & 53 & 42.20\end{array}$ e $\quad 5404.60$ IPP 5500.30 $i \quad 5538.80$

$\begin{array}{lllll} & & i & 56 & 02.40 \\ \text { VAY } \quad 131.61318 \text { ePKP } & 52 & 34.30\end{array}$

SKO 132.04319 IPKP $52 \quad 37.00$ $1.8 \mathrm{~s} 1200.00 \mathrm{~nm}$

5246.40 $56 \quad 04.00$

MOX $\quad 132.05 \quad 334$ ePKP $\quad 5233.00$ $2.0 \mathrm{~s} \quad 529.00 \mathrm{~nm}$

$\begin{array}{lll}i & 52 & 38.00 \\ i & 52 & 47.50 \\ i & 53 & 10.00 \\ i P P & 54 & 58.00 \\ i P K S & 56 & 10.00 \\ e & 57 & 00.00 \\ \text { eSS } & 12 & 50.00\end{array}$

HOF $\quad 132.17 \quad 333$ iPKPC $52 \quad 38.60$

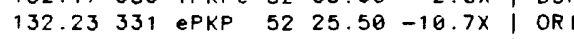
$1.5 \mathrm{~s} \quad 437.00 \mathrm{~nm}$

$N \quad 25 \mathrm{~s} \quad 59.60 \mathrm{um}$

E $24 \mathrm{~s} \quad 53.50 \mathrm{um}$

e 5238.00

e $\quad 5247.00$

ATH $132.28 \quad 313$ ePKP $52 \quad 40.00$

WIT 132.34339 e(PKP) $52 \quad 39.50$ e $52 \quad 49.00$

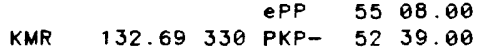

$\begin{array}{llllll}\text { OHR } & 132.88 & 318 & \text { IPKP } 52 & 35.50\end{array}$

$\begin{array}{llllll} & & & i & 56 & 05.00 \\ \text { GRF } \quad 132.92 & 333 & \text { ePKP } & 52 & 38.00\end{array}$

WTS 132.95338 ePKP 5236.50 e 5240.00 e $\quad 5250.00$ $\begin{array}{lll}\text { e } & 54 & 43.50 \\ \text { ePP } & 55 & 05.00\end{array}$

ZAG $\quad 133.16 \quad 326$ ePKP $52 \quad 36.00$ EPKS 5600.00

TTG $\quad 133.19321$ ePKP $52 \quad 39.00$

STJ $133.23 \quad 31$ ePKP $52 \quad 47.50$

CAR $\quad 133.38 \quad 85$ ePdiff49 40.00

DBN $\quad 133.45339$ e(PKP) $52 \quad 32.00$ $24 \mathrm{~s} 77.00 \mathrm{um}$ $\begin{array}{lll}\text { e } & 53 & 20.00 \\ \text { ePKS } 56 & 00.00\end{array}$

BNS $\quad 133.64 \quad 337$ IPKPC $52 \quad 43.70$ $1.7 \mathrm{~s} \quad 400.00 \mathrm{~nm}$

TNS 133.71335 ePKP $52 \quad 41.00$

$\begin{array}{llllll} & & & \text { ePP } & 55 & 16.00 \\ \text { LJU } & 133.80 & 327 & \text { ePKP } & 52 & 37.00\end{array}$ $i \quad 5251.10$ $\begin{array}{lll}\text { e } & 53 & 10.10 \\ \text { es } & 56 & 12.20\end{array}$

FUR $\quad 133.99 \quad 332$ iPKPC $52 \quad 42.10$ $223 \mathrm{~s} 227.00 \mathrm{um}$

RBL 134.05328 EPKP $52 \quad 44.50$

CEY $\quad 134.06 \quad 327$ ePKP $52 \quad 39.30$ i 5251.90

$\begin{array}{lllll} & & \text { eS } & 56 & 13.20 \\ \text { SJG } & 134.16 & 75 \text { ePKP } & 52 & 33.00\end{array}$

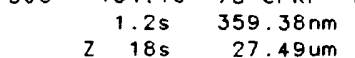

ENN $134.27 \quad 338$ ePKP $52 \quad 40.50$

e $\quad 5242.50$

e $\quad 5251.00$

134.43527 ePP $55 \quad 10.00$
ipPP $52 \quad 41.00$

TRI $\quad 134.43 \quad 327$ IPKP $52 \quad 41.90$ $i \quad 5429.00$ i(PKS) $56 \quad 17.90$ $i(P K S) 57 \quad 13.10$
$i P^{1} \quad 57 \quad 34.50$ UCC $\quad 134.82339$ PKP $\quad 5234.56$

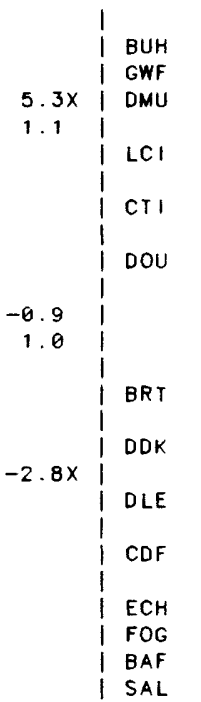

HAU

DUI

SGO

$3.4 \times 1$

$3.3 x$ | $5 G G$

I POI

2.0 FIR

-2.2 MNS

0.6 $7 \mathrm{MSZ}$ ORO -0.9 VAL I RMP

$-2.0$

\begin{tabular}{c|c}
2.0 & VAO \\
0.9 & $\vdots$
\end{tabular}

9. $5 x$ LOR

$3.4 X \mid$ LBF

$-6.3 x$

$3 M s=X$

FLN
SSF

$5.0 \times$ LDF

2.0 BPA

I SMF

-2.2 AVF

I GRR

MEG

$2.5 \times \mid M G G$

. $8 M 5 Z X \mid L P F$

4. $7 X$ | CVF

$\begin{array}{lll}-0.5 & \text { MZF } \\ \text { TCF }\end{array}$

I BMA

$-7.7 \times$

\begin{tabular}{l|l}
$.0 M S Z$ & $F R F$
\end{tabular}

\section{ISF \\ LRG \\ LMR}

1.5 CDR

MFF

I RJF

$-6.5 \times \quad \begin{aligned} & \text { CAF } \\ & \text { LFF } \\ & \text { LPO }\end{aligned}$ e 5510.50 134.99334 ePKPO $52 \quad 40.40$ $135.06 \quad 349$ ePKP $52 \quad 34.80$ $1.2 \mathrm{~s} \quad 280.00 \mathrm{~nm}$ 135.13319 ePKP 5251.00 ePP $55 \quad 24.50$ $135.30 \quad 329$ ePKP $52 \quad 48.00$ EPP 5524.00 135.31338 PKP 5237.40 $\begin{array}{lll}\text { e } & 5246.40 \\ \text { e } & 5513.50\end{array}$ $\begin{array}{lll}e & 56 & 26.00 \\ e & 0624.00\end{array}$ $135.32 \quad 320$ еPKP $52 \quad 48.00$ PPP 5526.00 135.45349 ePKP $52 \quad 39.06$ $1.0 \mathrm{~s} \quad 145.00 \mathrm{~nm}$

$135.58 \quad 349$ ePKP $52 \quad 39.00$ $1.2 \mathrm{~s} \quad 280.00 \mathrm{~nm}$ 135.59335 ePKP $52 \quad 35.90$ $1.4 \mathrm{~s} \quad 156.80 \mathrm{~nm}$ $\begin{array}{lllll}135 & 80 & 334 & \text { EPKP } 52 \quad 43.00\end{array}$ 136.03322 IPKPd 5252.00 $136.16 \quad 334$ ePKP $52 \quad 43.00$ 136.17329 ePKP 136.25334 ePKP 5237.50 $136.26 \quad 319$ ePKP $52 \quad 46.00$ $\begin{array}{rlrr} & \text { iPP } & 5535.50\end{array}$ 5237.80 136.57322 ePKP $52 \quad 51.50$

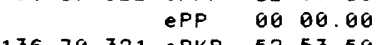
$136.70 \quad 321$ ePKP 5253.50 IPP 5533.00 136.78322 ePKP 5244.50 $136.90 \quad 325$ e 5534.00 $137.05 \quad 325$ ePKP 5245.00 $137.17 \quad 324$ ePKP $52 \quad 43.50$ $137.36 \quad 330$ ePKP $52 \quad 47.50$ iPP 5532.50 137.39331 ePKP $52 \quad 40.00$ 137.50352 PKP 5253.00 $137.52 \quad 324$ ePKP

137.54324 EPKP EPP

$137.60 \quad 141$ ePKP 5542.00 $\begin{array}{lll}55 & 46.50 \\ P & 52 & 47.40\end{array}$ $\begin{array}{lll}\text { e } & 525510.70\end{array}$ 137.90336 еPKP 5241.00 138.09336 ePKP 5241.30 138.19 337 iPKPC $52 \quad 46.60$ $\begin{array}{lll}i & 5248.70 \\ i & 5626.40\end{array}$ 138.20341 ePKP 5242.70 138.21336 еРKP 5241.70 $138.23 \quad 341$ ePKP 5242.90 $1.2 \mathrm{~s} \quad 101.10 \mathrm{~nm}$ 138.3276 еPKP $52 \quad 42.00$ $\begin{array}{llll}138.42 & 336 & \text { ePKP } 52 \quad 42.20\end{array}$ 138.49336 ePKP $52 \quad 42.00$ 138.64341 ePKP $52 \quad 44.00$ $138.69 \quad 77$ ePKP $52 \quad 49.00$ 138.8578 ePKP $52 \quad 49.00$ 138.9077 EPKP $52 \quad 49.50$ $139.02 \quad 341$ ePKP $52 \quad 44.80$ 139.15328 ePKP 139.27336 еPKP $139.36 \quad 337$ ePKP 139.53144 ePKP

$52 \quad 43.20$ 5243.10 5243.80 $52 \quad 49.40$ $\begin{array}{cll}e & 5301.20 \\ e & 5344.40\end{array}$ $139.56 \quad 330$ ePKP 5244.00 $1.3 \mathrm{~s} \quad 187.70 \mathrm{~nm}$ $139.65 \quad 337$ ePKP $52 \quad 44.00$ $139.78 \quad 331$ ePKP $52 \quad 44.90$ $139.80 \quad 330$ еPKP $52 \quad 44.60$ 139.87319 ePKP $53 \quad 03.00$ $139.90 \quad 331$ ePKPC 5251.10 139.96339 ePKP $52 \quad 45.10$ 140.44337 ePKP $52 \quad 45.30$ $1.5 \mathrm{~s} \quad 274.70 \mathrm{~nm}$

140.54336 ePKP $52 \quad 45.10$

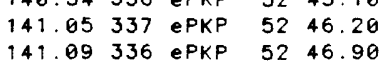

141.09336 ePKP
235

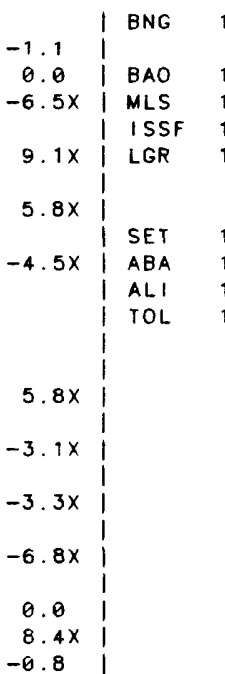

141.85264 ePKPC $52 \quad 48.00 \quad-7.1 \times$ $1.0 \mathrm{~s} \quad 17.50 \mathrm{~nm}$

$\begin{array}{llllll}142.01 & 132 \text { iPKPC } 52 \quad 48.00 & -7.4 x\end{array}$ $142.56 \quad 335$ ePKP $52 \quad 53.90 \quad-1.6$ 143.26337 ePKP $5255.10 \quad-1.7$ $\begin{array}{llllll}144.39 & 338 & \text { IPKP } 5300.00 & 1.4\end{array}$

IPKKP 5310.00
IPP 56 $\begin{array}{lll}\text { IPP } & 56 \quad 27.50\end{array}$

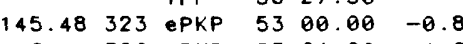
146.41326 ePKP $5301.00 \quad-1.2$ 147.00332 IPKP- $5301.00 \quad-2.0$ 147.21338 iPKP+ $53 \quad 05.00 \quad 1.6$ iPKKP $53 \quad 17.50$ $\begin{array}{lll}53 & 45.00\end{array}$ 5535.00 5626.00 i PP $\quad 56 \quad 41.00$ i $\quad 5715.00$ iSKS 0046.00 0208.06 ESKKS 0319.00 IPS $07 \quad 12.00$ i $08 \quad 37.00$ iSS $\quad 15 \quad 37.00$ iSSS 2106.00

$6.3 \times$ PTO $-6.5 X \mid$ MTE 2.0 COI \begin{tabular}{l|l|l}
$-6.1 \times$ & PRL \\
\hline
\end{tabular} $\begin{array}{ccc}149.13 & 333 \text { iPKPO } 5310.60 \\ 1.8 \mathrm{~s} & 11.20 \mathrm{~nm} & \end{array}$ $1.8 \mathrm{~s} \quad 11.20 \mathrm{~nm}$

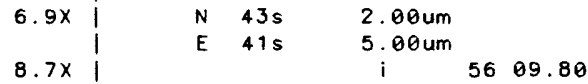
$\begin{array}{llll}8.7 \times & i & 0319.20\end{array}$ \begin{tabular}{l|lllllll}
-0.6 & CRT & 149.34 & 335 & IPKP & 53 & 10.60 & $3.7 \times$ \\
& | MTH & 149.77 & 344 & ePKPC & 53 & 15.00 & $7.6 \times$
\end{tabular} \begin{tabular}{l|lrlllr}
-0.2 & LIS & 149.93 & 344 & iPKPd 53 & 12.30 & $4.7 X$ \\
$-6.4 X$ & MAL & 150.08 & 335 & iPKP+ 53 & 06.00 & -1.9
\end{tabular}

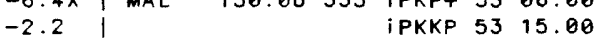
$\begin{array}{lll}\text { IPP } & 57 \quad 24.00\end{array}$ IPS $07 \quad 02.00$ $-6.2 \times$ ALR $7.0 \times 1$ $0.6 \quad$ ISFS

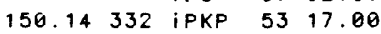
5324.50 $53 \quad 35.00$

$151.02 \quad 337$ IPKPC $53 \quad 12.00$ 5340.00 5358.00 $0.2 i \begin{array}{lll}i & 53 & 58.00 \\ & i & 5510.00 \\ & \text { iPP } & 5624.00\end{array}$ ESKS 0010.00 EPS 0705.00

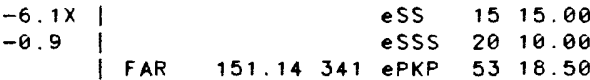

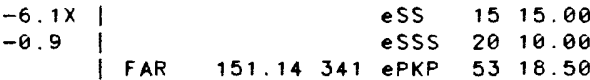
$\begin{array}{lllllll}\text { IFAR } & 151.14 & 341 & \text { ePKP } & 53 & 18.50 & 9.0 \times\end{array}$ $-4.7 \times$

$-5.9 x$ | TAM $-4.6 x$ I ITR $-6.6 \times \mid$ FUL $-5.8 x \mid$ TEN $-6.1 \times$
$-4.3 \times$ \begin{tabular}{l|llllll}
-0.2 & MBO 174.96330 & IPKPC $53 \quad 34.10$ & 2.2
\end{tabular} $-0.5 \mid$ S.D. $=1.4$ on 376 of 548 abs. $-0.1 \quad$

$-4.1 \times$ MAR $05.198403 \mathrm{~h} 33 \mathrm{~m} 50.85 \pm 0.05 \mathrm{~s}$ $-6.2 \mathrm{X} \quad 8.148 \mathrm{~N} \pm 1.0 \mathrm{~km} \quad 123.775 \mathrm{E} \pm 1.2 \mathrm{~km}$ $-6.4 \times$ DEPTH $=646.1 \pm 0.6 \mathrm{~km}$ $-5.9 \times$ I $6.6 \mathrm{mb}$ ( 99 abs.)

-1.3 MINDANAO, PHILIPPINE ISLANDS

Felt (I I RF) an Mindonaa, Leyte ond Ponay.

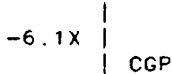

$-6.2 \times \mid$ DAV $-5.5 x \mid$ PLP $-5.9 \times \mid P D P$ $12.2 \times$ 0.4 | PPR $-5.6 \times \mid$ $-6.3 x$ PGP MN I \begin{tabular}{l|l}
$-6.8 x$ & \\
$-6.5 x$ & OCP
\end{tabular} $-5.9 \times$ I MAN

$\begin{array}{rrlrrr}0.96 & 71 & \text { iPc } & 35 & 07.50 & -0.4 \\ 2.07 & 120 & \text { iPd } & 35 & 11.50 & 0.4 \\ 3.22 & 22 & \text { iPd } & 35 & 13.00 & -2.3 \\ 3.91 & 335 & \text { iPc } & 35 & 23.20 & 3.8 \times \\ & & \text { iS } & 35 & 46.50 & \\ 5.24 & 288 & \text { iPd } & 35 & 31.90 & 3.2 \times \\ & & \text { iS } & 36 & 29.60 & \\ 6.00 & 333 & \text { iPc } & 35 & 35.00 & 0.3 \\ 6.74 & 171 & \text { iPC } & 35 & 41.30 & 0.4 \\ & & \text { ES } & 36 & 45.00 & \\ 6.97 & 338 & \text { iP } & 35 & 25.00 & -17.9 x \\ 6.99 & 338 & \text { iPd } & 35 & 44.00 & 0.9\end{array}$

$\begin{array}{llllll}0.96 & 71 \text { iPC } & 35 & 07.50 & -0.4\end{array}$

$\begin{array}{lll}35 & 11.50 & 0.4\end{array}$ $8 x$ $2 x$ 


\begin{tabular}{|c|c|c|c|c|c|c|c|c|c|c|c|c|}
\hline KKM & 7.79 & 255 & iPd & 35 & 50.30 & 0.1 & I NNT & 24.05 & 283 & i Pc & 38 & 21.80 \\
\hline$B A G$ & 8.79 & 339 & iPct & 36 & 00.00 & 0.6 & $K A G$ & 24.15 & 14 & iPd & 38 & 21.30 \\
\hline$S Z P$ & 9.89 & 341 & i $\mathrm{Pc}$ & 36 & 09.00 & -0.5 & i & & & $e$ & 48 & 09.00 \\
\hline $8 K B$ & 11.65 & 217 & iPd & 36 & 28.60 & 2.4 & $|\mathrm{KS}|$ & 24.17 & 242 & iPd & 38 & 22.00 \\
\hline & $1.0 \mathrm{~s}$ & 9814 & $4.70 \mathrm{~nm}$ & & & $8 m b$ & 1 & & & is & 42 & 01.50 \\
\hline AA I & 12.56 & 159 & $i \mathrm{Pc}$ & 36 & 38.20 & $3.3 x$ & I NJ2 & 24.22 & 350 & $\mathrm{Pc}$ & 38 & 23.00 \\
\hline MKS & 13.95 & 198 & iPc & 36 & 49.80 & 1.5 & 1 & & & sP & 41 & 01.50 \\
\hline & $1.0 \mathrm{~s}$ & $* * *$ & $* * * n m$ & & & $7 \mathrm{mb} \times$ & 1 & & & SCP & 44 & 21.30 \\
\hline TWG & 14.82 & 350 & $\mathbf{i} \mathbf{P}_{\mathrm{c}}$ & 36 & 55.90 & -0.6 & 1 & & & $\mathrm{~s}$ & 45 & 01.00 \\
\hline & & & es & 37 & 09.00 & & I KNA & 24.25 & 168 & iPd & 38 & 22.00 \\
\hline TWF 1 & 15.30 & 351 & iPc & 37 & 00.10 & -1.0 & I NST & 24.29 & 290 & eP & 38 & 25.00 \\
\hline$T W K$ & 15.36 & 349 & iPc & 37 & 02.40 & 0.7 & I GYA & 24.41 & 320 & Pc & 38 & 24.00 \\
\hline$|s|$ & 16.18 & 1 & iPd & 37 & 10.10 & 1.5 & 1 & & & sP & 41 & 05.00 \\
\hline & & & is & 39 & 48.20 & & 1 & & & S & 42 & 03.00 \\
\hline TLE & 16.36 & 147 & $i P_{C}$ & 37 & 87.10 & $-4.8 x$ & I TBL & 24.43 & 119 & iPd & 38 & 25.00 \\
\hline MrK & 16.61 & 5 & eP & 37 & 15.00 & 1.7 & I MYZ & 24.70 & 16 & eP & 38 & 26.00 \\
\hline & & & es & 39 & 52.00 & & I & & & es & 42 & 07.00 \\
\hline $\mathrm{HKC}$ & 16.84 & 328 & iP & 37 & 16.30 & 0.8 & I FKJ & 24.88 & 10 & $P d$ & 38 & 28.10 \\
\hline & & & is & 39 & 36.00 & & I & & & s & 42 & 08.90 \\
\hline ANP & 17.08 & 353 & iP- & 37 & 16.00 & -1.8 & I NGS & 25.11 & 12 & $P$ & 38 & 30.80 \\
\hline & & & is & 39 & 36.00 & & 1 & & & $\mathrm{~s}$ & 42 & 13.20 \\
\hline 112 & 17.32 & 310 & iPc & 37 & 20.80 & 0.8 & | PSI & 25.32 & 259 & ePc & 38 & 32.70 \\
\hline $\mathrm{OZH}$ & 17.42 & 344 & iPc & 37 & 21.50 & 0.6 & I KUM & 25.37 & 14 & $P d$ & 38 & 31.60 \\
\hline & & & $\mathbf{S P}$ & 39 & 25.00 & & 1 & & & es & 42 & 07.00 \\
\hline :ZH & 17.91 & 327 & iPc & 37 & 26.30 & 0.9 & I KHT & 25.53 & 287 & iPc & 38 & 35.00 \\
\hline VS I & 18.06 & 191 & ePc & 37 & 25.00 & -1.9 & I MOM & 25.64 & 112 & i Pc & 38 & 35.50 \\
\hline & & & es & 38 & 20.00 & & I MDG & 25.67 & 121 & ePd & 38 & 3.80 \\
\hline UPT & 18.18 & 181 & iPc & 37 & 27.90 & 0.0 & $|C B|$ & 25.67 & 41 & i P & 38 & 33.20 \\
\hline KUG & 18.19 & 181 & $i \mathrm{Pc}$ & 37 & 27.90 & -0.1 & I & & & 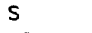 & 41 & 24.40 \\
\hline & & & e & 40 & 26.70 & & I SAG & 25.70 & 13 & eP & 38 & 35.00 \\
\hline & & & e & 44 & 04.20 & & I BDT & 25.77 & 293 & iPc & 38 & 35.50 \\
\hline & & & e & 47 & 41.20 & & I & $0.8 \mathrm{~s}$ & 122 & $2.00 \mathrm{~nm}$ & & \\
\hline MJ & 18.32 & 9 & iPd & 37 & 30.30 & 1.2 & $A S Z$ & 25.91 & 18 & $P d$ & 38 & 37.20 \\
\hline & & & $\mathrm{s}$ & 40 & 23.20 & & 1 & & & is & 42 & 24.60 \\
\hline NAH & 18.35 & 11 & iPd & 37 & 30.60 & 1.2 & 1011 & 26.00 & 15 & ePd & 38 & 37.00 \\
\hline & & & $\mathrm{S}$ & 40 & 14.60 & & 1 & & & es & 42 & 03.00 \\
\hline NGO & 18.78 & 12 & iPd & 37 & 34.90 & 1.6 & I FKK & 26.03 & 13 & $\mathbf{P d}$ & 38 & 37.50 \\
\hline & & & S & 40 & 22.20 & & 1 & & & es & 42 & 07.00 \\
\hline MVI & 18.96 & 21 & iPd & 37 & 35.20 & 0.2 & I KMI & 26.22 & 313 & iPct & 38 & 41.00 \\
\hline & & & S & 40 & 32.50 & & 1 & $12 \mathrm{~s}$ & & $9.70 u m$ & & \\
\hline TRT & 19.27 & 215 & iPc & 37 & 38.70 & 0.8 & 1 & & & is & 41 & 35.00 \\
\hline SJI & 19.80 & & is & 38 & 18.00 & & I CHG & 26.33 & 296 & iPct & 38 & 41.80 \\
\hline VZJ & 20.83 & 217 & ePd & 37 & 42.90 & 0.1 & 1 & $0.6 \mathrm{~s}$ & 150 & $.00 \mathrm{~nm}$ & & \\
\hline & & 14 & s & 37 & 52.50 & 0.5 & 1 & & & es & 42 & 46.00 \\
\hline$G M$ & 21.26 & 254 & iPc & 37 & 56.70 & 0.8 & $\begin{array}{l}\text { CHTO } \\
1 Z U\end{array}$ & 26.33 & 296 & iPc & 38 & 41.80 \\
\hline & $0.7 \mathrm{~s}$ & 123 & $3.40 \mathrm{~nm}$ & & & $.5 \mathrm{mb} \times$ & 1 & 26.42 & 10 & $\begin{array}{l}\text { P } \\
\text { e }\end{array}$ & $\begin{array}{l}38 \\
42\end{array}$ & $\begin{array}{l}42.00 \\
32.00\end{array}$ \\
\hline & & & $i$ & 38 & 43.00 & & I SHN & 26.52 & 13 & $\mathrm{Pd}$ & 38 & 41.50 \\
\hline & & & $\mathrm{i}$ & 44 & 09.00 & & 1 & & & es & 42 & 30.00 \\
\hline UMO & 21.40 & 74 & iPd & 37 & 56.60 & -0.6 & I MRT & 26.77 & 20 & $P d$ & 38 & 44.40 \\
\hline JG & 21.40 & 74 & iPd & 37 & 56.50 & -0.7 & i & & & e & 42 & 37.00 \\
\hline & & & i & 58 & 13.00 & & I KOC & 26.84 & 18 & ¡Pd & 38 & 45.60 \\
\hline SUA & 21.43 & 74 & ePd & 37 & 56.60 & -0.9 & 1 & & & $\mathbf{i}$ & 42 & 37.30 \\
\hline & $0.9 \mathrm{~s}$ & 2789 & $.92 \mathrm{~nm}$ & & 6 & $.7 \mathrm{mb}$ & | MTY & 26.88 & 17 & eP & 38 & 45.00 \\
\hline & & & i & 40 & 32.00 & & I HIR & 27.28 & 16 & $\mathbf{P}$ & 38 & 48.50 \\
\hline$z z$ & 21.92 & 127 & iPc & 38 & 02.50 & 0.5 & 1 & & & $\mathrm{~s}$ & 42 & 41.00 \\
\hline MTN & 22.10 & 161 & iPc & 38 & 02.00 & -1.5 & I LAT & 27.43 & 122 & iPc & 38 & 50.50 \\
\hline TNG & 22.23 & 231 & iPc & 38 & 03.30 & -1.4 & I SHK & 27.51 & 16 & iPd & 38 & 50.10 \\
\hline$K L M$ & 22.58 & 258 & ¡P & 38 & 09.00 & 1.2 & 1 & & & es & 42 & 46.60 \\
\hline & $0.6 \mathrm{~s}$ & 212 & $.40 \mathrm{~nm}$ & & 5 & $9 \mathrm{mb}$ & I SHJ & 27.51 & 22 & $P d$ & 38 & 50.80 \\
\hline & & & is & 41 & 04.40 & & I & & & $\mathrm{s}$ & 42 & 47.20 \\
\hline $\mathrm{CT}$ & 22.85 & 289 & iPc & 38 & 11.80 & 1.6 & I TKS & 27.65 & 20 & $P d$ & 38 & 52.30 \\
\hline & & & e & 41 & 34.10 & & 1 & & & es & 42 & 52.00 \\
\hline & & & e & 44 & 14.00 & & 1 TKM & 27.71 & 19 & eP & 38 & 52.00 \\
\hline & & & e & 59 & 49.00 & & 1 & & & es & 42 & 49.00 \\
\hline KLI & 22.87 & 236 & ePc & 38 & 09.80 & -0.6 & I WKY & 27.99 & 20 & iPd & 38 & 55.20 \\
\hline & & & es & 41 & 28.80 & & 1 & & & $\mathrm{~s}$ & 42 & 58.00 \\
\hline IPM & 22.88 & 262 & ePc & 38 & 10.80 & 0.3 & I OKA & 28.00 & 18 & iPd & 38 & 55.00 \\
\hline & & & $\mathrm{i}$ & 38 & 31.80 & & 1 & & & es & 42 & 55.00 \\
\hline & & & e & 39 & 10.40 & & I HIM & 28.40 & 19 & $P d$ & 38 & 58.40 \\
\hline & & & $\mathrm{i}$ & 39 & 39.80 & & 1 & & & es & 43 & 01.00 \\
\hline & & & e & 40 & 23.80 & & I KOB & 28.42 & 20 & eP & 38 & 59.00 \\
\hline & & & is & 41 & 35.30 & & 1 & & & e & 42 & 04.00 \\
\hline & & & $\mathrm{i}$ & 44 & 12.30 & & I MTS & 28.48 & 16 & iPd & 38 & 59.20 \\
\hline$S E$ & 22.96 & 354 & iPd- & 38 & 11.50 & 0.5 & i & & & s & 43 & 06.20 \\
\hline & $9.0 \mathrm{~s}$ & 17 & $.00 \mathrm{~nm}$ & & & $6 \mathrm{mb} \times$ & I OSK & 28.52 & 21 & $P$ & 38 & 59.50 \\
\hline & $11 \mathrm{~s}$ & & $.00 \mathrm{um}$ & & & & 1 & & & es & 43 & 01.00 \\
\hline & & & $\mathbf{s P}$ & 40 & 49.00 & & I OSA & 28.53 & 21 & $P$ & 38 & 59.90 \\
\hline & & & is & 41 & 27.00 & & 1 & & & $\mathrm{~s}$ & 43 & 02.90 \\
\hline & & & ScS & 48 & 26.00 & & I TIA & 28.59 & 349 & Pd & 39 & 00.20 \\
\hline SNG & 22.97 & 269 & i P & 38 & 12.00 & 0.7 & 1 & & & $P \subset P$ & 41 & $5: .10$ \\
\hline & $1.4 \mathrm{~s}$ & 5116 & $.28 \mathrm{~nm}$ & & & $9 \mathrm{mb}$ & 1 & & & PcS & 45 & 33.20 \\
\hline & & & is & 41 & 38.00 & & 1 & & & S & 46 & 03.00 \\
\hline IAJ & 23.46 & 16 & iPd & 38 & 15.90 & 0.5 & 1 & & & ScS & 48 & 25.80 \\
\hline & & & s & 41 & 48.00 & & I WITU & 28.65 & 116 & ePc & 39 & 01.00 \\
\hline $\mathrm{HN}$ & 23.96 & 340 & $\mathrm{iPc}$ & 38 & 21.50 & 1.7 & I TOT & 28.85 & 18 & eP & 39 & 02.00 \\
\hline & & & $\mathbf{s P}$ & 40 & 59.00 & & 1 & & & es & 43 & 03.00 \\
\hline
\end{tabular}




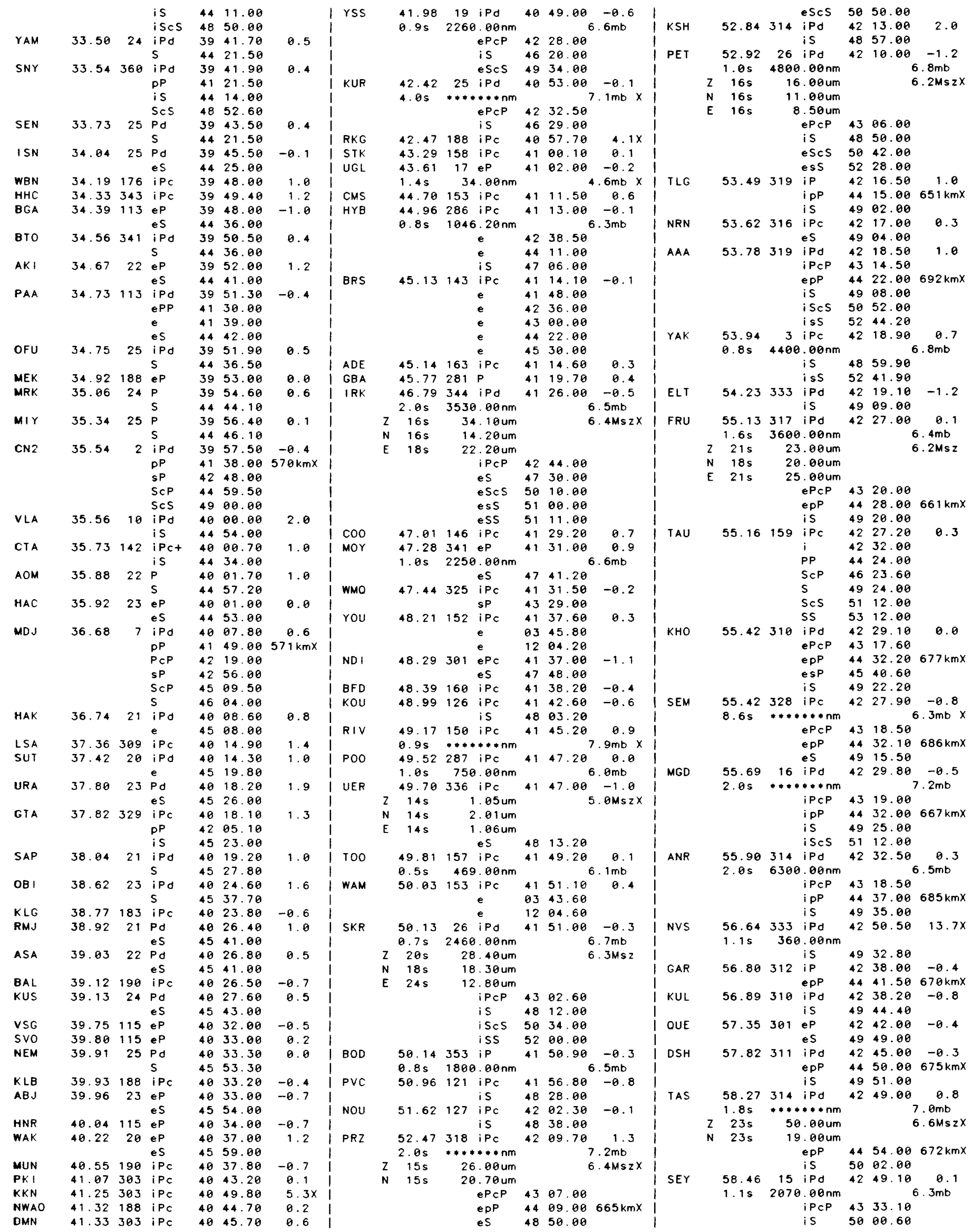




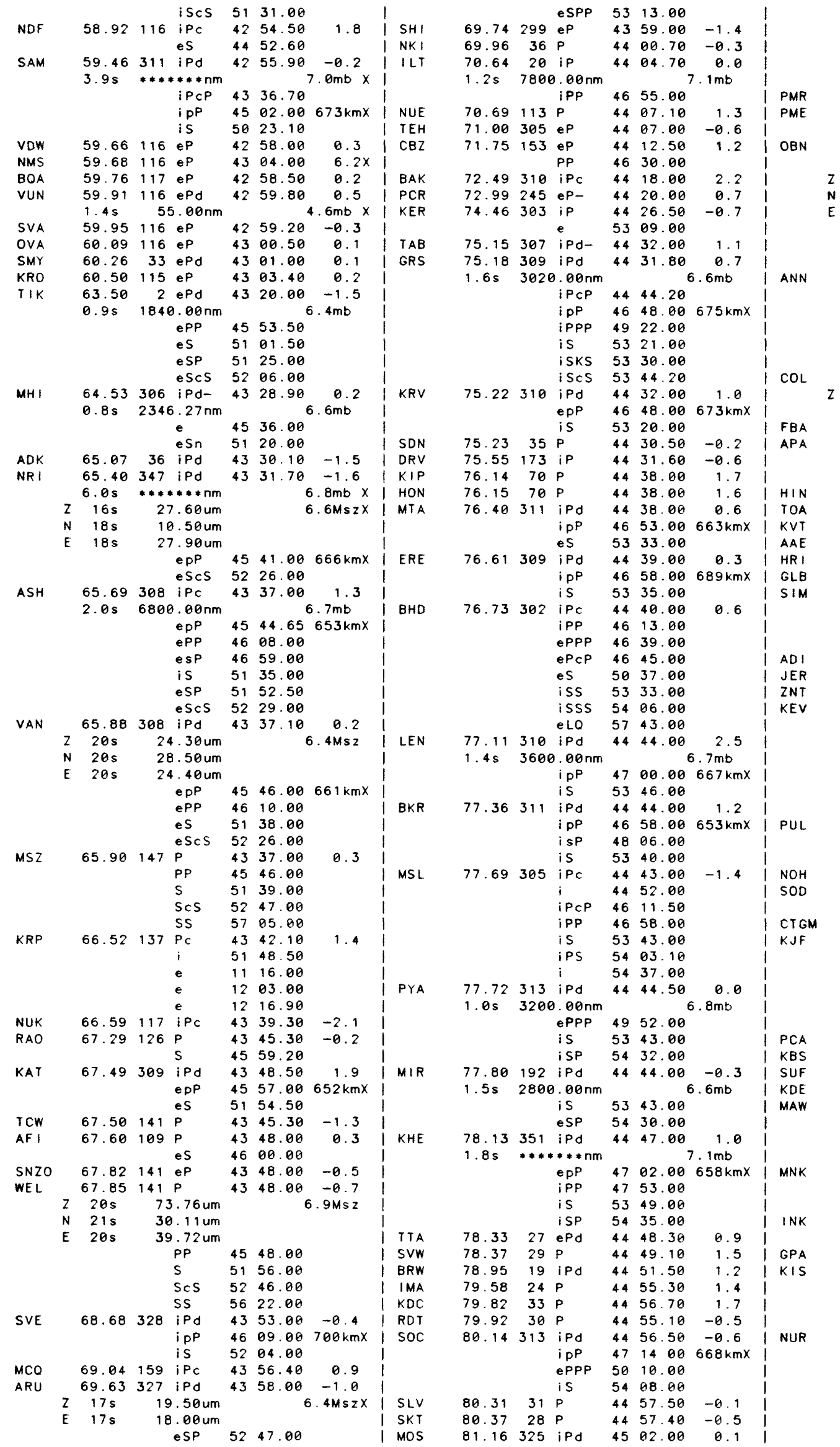

iscs 5131.00

\begin{tabular}{|c|c|c|c|c|c|c|c|c|c|c|c|c|}
\hline & & & & iscs & 51 & 31.00 & & $!$ & & & eSPP & 53 \\
\hline NDF & & 58.92 & 116 & iPC & 42 & 54.50 & 1.8 & | SHI & 69.74 & 299 & eP & 43 \\
\hline & & & & es & 44 & 52.60 & & | NK I & 69.96 & 36 & $P$ & 44 \\
\hline SAM & & 59.46 & 311 & iPd & 42 & 55.90 & -0.2 & I ILT & 70.64 & 20 & $i p$ & 44 \\
\hline & & $3.9 \mathrm{~s}$ & $* * *$ & $* * * * n m$ & & & $.0 \mathrm{mb} \quad \mathrm{X}$ & 1 & $1.2 \mathrm{~s}$ & 7800 & $.00 \mathrm{~nm}$ & \\
\hline & & & & $i P C P$ & 43 & 36.70 & & 1 & & & iPP & 46 \\
\hline & & & & ipP & 45 & 02.00 & $673 \mathrm{kmx}$ & I NUE & 70.69 & 113 & $P$ & 44 \\
\hline & & & & is & 50 & 23.10 & & I TEH & 71.00 & 305 & eP & 44 \\
\hline VDW & & 59.66 & 116 & eP & 42 & 58.00 & 0.3 & I CBZ & 71.75 & 153 & eP & 44 \\
\hline NMS & & 59.68 & 116 & eP & 43 & 04.00 & $6.2 x$ & 1 & & & $P P$ & 46 \\
\hline$B O A$ & & 59.76 & 117 & eP & 42 & 58.50 & 0.2 & BAK & 72.49 & 310 & iPc & 44 \\
\hline VUN & & 59.91 & 116 & ePd & 42 & 59.80 & 0.5 & I PCR & 72.99 & 245 & eP- & 44 \\
\hline & & $1.4 \mathrm{~s}$ & 55 & $5.00 \mathrm{~nm}$ & & & $.6 \mathrm{mb} \times$ & I KER & 74.46 & 303 & $i p$ & 44 \\
\hline SVA & & 59.95 & 116 & eP & 42 & 59.20 & -0.3 & 1 & & & e & 53 \\
\hline OVA & & 60.09 & 116 & eP & 43 & 00.50 & 0.1 & TAB & 75.15 & 307 & iPd- & 44 \\
\hline SMY & & 60.26 & 33 & ePd & 43 & 01.00 & 0.1 & I GRS & 75.18 & 309 & iPd & 44 \\
\hline KRO & & 60.50 & 115 & eP & 43 & 03.40 & 0.2 & I & $1.6 \mathrm{~s}$ & 3020 & $.00 \mathrm{~nm}$ & \\
\hline TIK & & 63.50 & 2 & ePd & 43 & 20.00 & -1.5 & I & & & $i P \subset P$ & 44 \\
\hline & & $0.9 \mathrm{~s}$ & $184 e$ & $0.00 \mathrm{~nm}$ & & & $.4 \mathrm{mb}$ & ? & & & ipP & 46 \\
\hline & & & & ePP & 45 & 53.50 & & 1 & & & iPPP & 49 \\
\hline & & & & es & 51 & 01.50 & & 1 & & & is & 53 \\
\hline & & & & eSP & 51 & 25.00 & & I & & & iSKS & 53 \\
\hline & & & & escs & 52 & 06.00 & & ! & & & ises & 53 \\
\hline MHI & & 64.53 & 306 & iPd- & 43 & 28.90 & 0.2 & KRV & 75.22 & 310 & iPd & 44 \\
\hline & & $0.8 \mathrm{~s}$ & 2346 & $6.27 \mathrm{~nm}$ & & & $.6 \mathrm{mb}$ & ! & & & epp & 46 \\
\hline & & & & e & 45 & 36.00 & & 1 & & & is & 53 \\
\hline & & & & eSn & 51 & 20.00 & & SDN & 75.23 & 35 & $\mathrm{P}$ & 44 \\
\hline ADK & & 65.07 & 36 & IPd & 43 & 30.10 & -1.5 & I DRV & 75.55 & 173 & ip & 44 \\
\hline NRI & & 65.40 & 347 & iPd & 43 & 31.70 & -1.6 & KIP & 76.14 & 70 & $\mathbf{P}$ & 44 \\
\hline & & $6.0 \mathrm{~s}$ & $* * * *$ & $* * * * n \mathrm{~nm}$ & & & $.8 \mathrm{mb} \quad X$ & HON & 76.15 & 70 & $\mathbf{P}$ & 44 \\
\hline & $z$ & $16 \mathrm{~s}$ & 27 & $7.68 \mathrm{um}$ & & & $6 M s=X$ & I MTA & 76.40 & 311 & iPd & 44 \\
\hline & $N$ & $18 \mathrm{~s}$ & & $0.58 \mathrm{um}$ & & & & ! & & & $i p p$ & 46 \\
\hline & $E$ & $18 s$ & 27 & $7.90 \mathrm{um}$ & & & & 1 & & & es & 53 \\
\hline & & & & epp & 45 & 41.00 & $666 \mathrm{kmx}$ & I ERE & 76.61 & 309 & iPd & 44 \\
\hline & & & & eScs & 52 & 26.00 & & 1 & & & ipp & 46 \\
\hline ASH & & 65.69 & 308 & IPC & 43 & 37.00 & 1.3 & I & & & is & 53 \\
\hline & & & 6800 & $0.60 \mathrm{~nm}$ & & & $.7 \mathrm{mb}$ & I BHD & 76.73 & 302 & iPc & 44 \\
\hline & & & & epP & 45 & 44.65 & $653 \mathrm{kmX}$ & 1 & & & iPP & 46 \\
\hline & & & & $\begin{array}{l}\text { ePP } \\
\text { esp }\end{array}$ & 46 & 08.00 & & ! & & & ePPP & 46 \\
\hline & & & & $\begin{array}{l}\text { esp } \\
\text { is }\end{array}$ & $\begin{array}{l}46 \\
51\end{array}$ & $\begin{array}{l}59.00 \\
35.00\end{array}$ & & 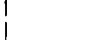 & & & $\begin{array}{l}\text { ePCP } \\
\text { es }\end{array}$ & $\begin{array}{l}46 \\
50\end{array}$ \\
\hline & & & & eSP & 51 & 52.50 & & i & & & iss & 53 \\
\hline & & & & escs & 52 & 29.00 & & I & & & isss & 54 \\
\hline VAN & & 65.88 & 308 & iPd & 43 & 37.10 & 0.2 & I & & & eLO & 57 \\
\hline & $z$ & $20 \mathrm{~s}$ & 24 & $4.38 \mathrm{um}$ & & & $.4 \mathrm{Msz}$ & I LEN & 77.11 & 310 & iPd & 44 \\
\hline & $N$ & $28 s$ & & $8.50 u m$ & & & & I & $1.4 \mathrm{~s}$ & 3600 & $3.00 \mathrm{~nm}$ & \\
\hline & $\mathbf{E}$ & $20 \mathrm{~s}$ & 24 & $4.40 u m$ & & & & ! & & & ipP & 47 \\
\hline & & & & epP & 45 & 46.00 & $661 \mathrm{kmx}$ & 1 & & & is & 53 \\
\hline & & & & ePP & 46 & 10.00 & & I BKR & 77.36 & 311 & iPd & 44 \\
\hline & & & & es & 51 & 38.00 & & 1 & & & $i p P$ & 46 \\
\hline & & & & escs & 52 & 26.00 & & 1 & & & $i s p$ & 48 \\
\hline MSZ & & 65.90 & 147 & $\mathrm{P}$ & 43 & 37.00 & 0.3 & 1 & & & is & 53 \\
\hline & & & & $P P$ & 45 & 46.00 & & I MSL & 77.69 & 305 & iPc & 44 \\
\hline & & & & $\mathrm{s}$ & 51 & 39.00 & & 1 & & & $i$ & 44 \\
\hline & & & & $\operatorname{ses}$ & 52 & 47.00 & & 1 & & & IPCP & 46 \\
\hline & & & & SS & 57 & 85.08 & & 1 & & & iPP & 46 \\
\hline KRP & & 66.52 & 137 & $P_{c}$ & 43 & 42.10 & 1.4 & 1 & & & is & 53 \\
\hline & & & & $i$ & 51 & 48.50 & & 1 & & & IPS & 54 \\
\hline & & & & e & 11 & 16.00 & & I & & & $i$ & 54 \\
\hline & & & & e & 12 & 03.00 & & I PYA & 77.72 & 313 & iPd & 44 \\
\hline & & & & e & 12 & 16.90 & & I & $1.0 \mathrm{~s}$ & 3200 & $0.00 \mathrm{~nm}$ & \\
\hline NUK & & 66.59 & 117 & $i P c$ & 43 & 39.30 & -2.1 & I & & & ePPP & 49 \\
\hline RAO & & 67.29 & 126 & $P$ & 43 & 45.30 & -0.2 & I & & & is & 53 \\
\hline & & & & $\$$ & 45 & 59.20 & & I & & & iSP & 54 \\
\hline KAT & & 67.49 & 309 & $i P d$ & 43 & 48.50 & 1.9 & $M I R$ & 77.80 & 192 & iPd & 44 \\
\hline & & & & epP & 45 & 57.00 & $652 \mathrm{kmX}$ & 1 & $1.5 \mathrm{~s}$ & 2800 & $0.00 \mathrm{~nm}$ & \\
\hline & & & & es & 51 & 54.50 & & 1 & & & is & 53 \\
\hline TCW & & 67.50 & 141 & $P$ & 43 & 45.30 & -1.3 & 1 & & & eSP & 54 \\
\hline AF I & & 67.60 & 109 & $P$ & 43 & 48.00 & 0.3 & I KHE & 78.13 & 351 & iPd & 44 \\
\hline & & & & es & 46 & 00.00 & & 1 & $1.8 \mathrm{~s}$ & $* * * *$ & $* * * * \mathrm{~nm}$ & \\
\hline SNZO & & 67.82 & 141 & eP & 43 & 48.00 & -0.5 & 1 & & & epp & 47 \\
\hline WEL & & 67.85 & 141 & $P$ & 43 & 48.00 & -0.7 & 1 & & & iPP & 47 \\
\hline & $z$ & $20 \mathrm{~s}$ & 73 & $3.76 \mathrm{um}$ & & & $.9 M s z$ & ! & & & is & 53 \\
\hline & $N$ & $21 \mathrm{~s}$ & 30 & $0.11 \mathrm{um}$ & & & & I & & & iSP & 54 \\
\hline & $E$ & $20 \mathrm{~s}$ & & $9.72 \mathrm{um}$ & & & & I TTA & 78.33 & 27 & ePd & 44 \\
\hline & & & & $P P$ & 45 & 48.00 & & I sVw & 78.37 & 29 & $\mathrm{P}$ & 44 \\
\hline & & & & $s$ & 51 & 56.00 & & I BRW & 78.95 & 19 & iPd & 44 \\
\hline & & & & ScS & 52 & 46.00 & & 1 IMA & 79.58 & 24 & $\mathrm{P}$ & 44 \\
\hline & & & & SS & 56 & 22.00 & & I KDC & 79.82 & 33 & $P$ & 44 \\
\hline SVE & & 68.68 & 328 & iPd & 43 & 53.00 & -0.4 & I RDT & 79.92 & 30 & $\mathrm{P}$ & 44 \\
\hline & & & & $i p p$ & 46 & 09.00 & $700 \mathrm{kmX}$ & SOC & 80.14 & 313 & iPd & 44 \\
\hline & & & & is & 52 & 04.00 & & I & & & $i p p$ & 47 \\
\hline MCQ & & 69.04 & 159 & iPc & 43 & 56.40 & 0.9 & 1 & & & ePPP & 50 \\
\hline ARU & & 69.63 & 327 & iPd & 43 & 58.00 & -1.0 & 1 & & & is & 54 \\
\hline & $z$ & $17 \mathrm{~s}$ & 19 & $9.50 \mathrm{um}$ & & 6 & $4 M s z X$ & SLV & 80.31 & 31 & $\mathrm{P}$ & 44 \\
\hline & $E$ & $17 \mathrm{~s}$ & 18 & $8.00 \mathrm{um}$ & & & & SKT & 80.37 & 28 & $P$ & 44 \\
\hline & & & & eSP & 52 & 47.00 & & MOS & 81.16 & 325 & iPd & 45 \\
\hline
\end{tabular}

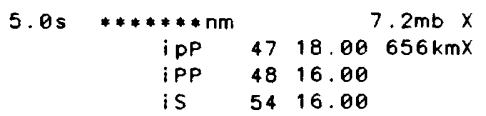

iSP 5508.00

$\begin{array}{lllll}B 1.5129 P & 4503.50 & -0.1\end{array}$

$\begin{array}{llllll}81.56 & 29 & \text { iPd } 45 & 03.60 & -0.2\end{array}$

$1.0 \mathrm{~s} \quad 3250.00 \mathrm{~nm} \quad 6.8 \mathrm{mb}$

81.76325 iPd $45 \quad 04.50 \quad-0.5$

$2.0 \mathrm{~s} \quad * * * * * * \mathrm{~nm} \quad 7.7 \mathrm{mb} \mathrm{x}$

$18 \mathrm{~s} \quad 14.50 \mathrm{um}$

$23 \mathrm{~s} \quad 7.40 \mathrm{um}$

$23 \mathrm{~s} \quad 15.00 \mathrm{um}$

esP $48 \quad 36.00$

is 5425.00

eSS 0008.00

$81.82314 \mathrm{iPd} \quad 4504.50 \quad-1.0$

$0.8 \mathrm{~s} \quad 4500.00 \mathrm{~nm} \quad 4721.50660 \mathrm{~km}$

iPP 4830.00

is $\quad 5421.00$

iSKS 5424.00

$82.02 \quad 26$ iPd $4505.90 \quad-0.2$

$18 \mathrm{~s} 23.71 \mathrm{um}$

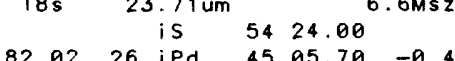

$82.70 \quad 337$ iPd $4508.70-0.8$

$1.2 \mathrm{~s} \quad 3400.00 \mathrm{~nm} \quad 6.8 \mathrm{mb}$

$82.84 \quad$ is 5428.00

$\begin{array}{lllll}30 \mathrm{P} & 45 & 11.70 & 1.3\end{array}$

$\begin{array}{lllll}89 & 28 P & 45 & 11.70 & 1.1\end{array}$

83.00310 iPd $4512.30 \quad 0.7$

83.86278 eP $\quad 4518.00 \quad 1.4$

83.95303 iP $\quad 4517.50 \quad 1.0$

$\begin{array}{lllll}84.06 & 29 P & 45 & 16.80 & 0.4\end{array}$

84.07314 iPd $4516.00 \quad-0.7$

IPPP 5044.00

iSKS 5436.00

iSP 5548.00

$\begin{array}{lllll}84.40303 \text { iP } & 4519.50 & 0.9\end{array}$

84.57301 iPd $4521.50 \quad 2.0$

84.66302 iP $\quad 4521.00 \quad 1.2$

84.76340 iPd- $4518.80 \quad-0.7$

$0.5 \mathrm{~s} \quad 926.30 \mathrm{~nm} \quad 6.7 \mathrm{mb}$

$\begin{array}{lll}4522.40 & \\ \text { epP } & 4732.00629 \mathrm{kmx}\end{array}$

$\begin{array}{lll}\text { EPP } & 47 & 32.00 \\ \text { esP } & 48 & 36.00\end{array}$

esPP 5152.00

eS 5440.00

84.77330 iPd $45 \quad 20.00 \quad 0.3$

$2.0 \mathrm{~s} * * * * * * * \mathrm{~nm} \quad 25 \mathrm{mb}$

ipP $47 \quad 40.00 \quad 672 \mathrm{kmx}$

84.88300 iP $\quad 4523.00 \quad 2.0$

$\begin{array}{lllll}85.29 & 337 \text { iPd } 4521.30 & -0.8\end{array}$

IPKKP 0321.20

$\begin{array}{llllll}85.31 & 29 P & 45 & 23.60 & 1.0\end{array}$

85.36334 iPd- $4521.90 \quad-0.6$

ePP $47 \quad 38.00 \quad 646 \mathrm{kmx}$

esP $48 \quad 46.00$

e SPP 5152.00

iSKS $54 \quad 43.50$

eS 5500.00

$\begin{array}{lllll}85.97 \quad 30 \mathrm{iPd} & 45 & 26.20 & 0.6\end{array}$

86.17350 iPd $4525.50 \quad-0.7$

$\begin{array}{llllll}86.31333 \text { iP } & 4526.30 & -0.8\end{array}$

86.45311 ip $\quad 4526.10 \quad-2.1$

$\begin{array}{lllll}86.88 & 200 \mathrm{eP} & 4529.00 & -0.6\end{array}$

$1.1 \mathrm{~s} \quad 225.00 \mathrm{~nm} \quad 5.8 \mathrm{mb}$

epP $45 \quad 35.00 \quad 19 \mathrm{kmx}$

$\begin{array}{ccc}\text { eS } & 4745.00\end{array}$

87.13324 iPd $4530.00 \quad-1.1$

is 5506.00

iSP 5616.00

$87.16 \quad 21$ iPd $45 \quad 30.80 \quad-0.2$

$\begin{array}{llll}\mathrm{PP} & 4628.00235 \mathrm{~km} x\end{array}$

$4531.50-1.4$

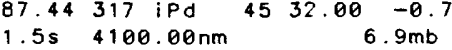

is 5516.00

iSP $56 \quad 26.00$

87.44331 iPd- $4532.00 \quad-0.4$

$1.1 \mathrm{~s} \quad 1175.60 \mathrm{~nm} \quad 6.5 \mathrm{mb}$

epP $4744.00616 \mathrm{kmX}$

e $\mathrm{PP} \quad 48 \quad 48.00$

ESPP $52 \quad 12.08$

iSKS 5456.50

eS $55 \quad 16.00$ 
05d 03h

BCK
HLW
CFR
ISK
ELL
MBC
BIR
TLB
PPE
PSN
CTT
DST
AFR

\begin{tabular}{|c|c|c|}
\hline 87.62 & 307 & iP \\
\hline 88.10 & 300 & IPd \\
\hline 88.23 & 315 & $i P c$ \\
\hline 88.25 & 311 & $i P c$ \\
\hline 88.27 & 307 & IPd \\
\hline 88.30 & 12 & iPc \\
\hline 88.40 & 316 & eP \\
\hline 88.41 & 315 & iPd \\
\hline 88.42 & 316 & IPC \\
\hline 88.46 & 314 & IPd \\
\hline 88.70 & 311 & eP \\
\hline
\end{tabular}

\begin{tabular}{lll:l:}
45 & 31.10 & -2.8 & \\
45 & 35.00 & -1.1 & \\
45 & 36.00 & -0.4 & \\
45 & 35.80 & -0.8 & \\
45 & 36.00 & -0.9 & \\
46 & 36.50 & $60.3 \times$ & \\
45 & 38.00 & 0.8 & \\
45 & 37.00 & -0.2 & \\
45 & 37.00 & -0.3 & \\
45 & 38.00 & 0.6 & \\
45 & 37.50 & -1.2 & JOS \\
45 & 38.10 & -1.2 & \\
45 & 43.90 & $3.2 \times$ & SPC \\
& \multicolumn{1}{c}{$6.1 \mathrm{mb}$} & \\
45 & 41.70 & 2.0 & \\
55 & 09.20 & & \\
45 & 41.00 & 0.9 & \\
45 & 44.70 & $3.1 \times$ & \\
45 & 40.30 & -1.0 & HFS
\end{tabular}

$88.81310 \mathrm{iPd}$

$\begin{array}{ll}89.06 & 108 \text { iP } \\ 1.05 & 335\end{array}$

89.09172 P

SBA

SIT

PPT

EDC
PAE

ISR

PPN

ALE

cro

TVO

$1.0 \mathrm{~s} 290.00 \mathrm{~nm}$

MLR

JMB $\quad 89.70316$ iPd

$\begin{array}{llll}89.83 & 313 \text { IPC } \\ \text { BUC1 } & 89.87315 & \text { iPd }\end{array}$

CGN $89.92315 \mathrm{ePd}$

LVV

$90.07320 \mathrm{iPd}$

iSP

T日

MSR

CMP

EZN

PRK

PVL
TPT

VAH

KDZ

BMP

UPP

UPP

DRA

CJR

WAR

$90.24 \quad$ is 0204.70

$0.8 \mathrm{~s} \quad 60.00 \mathrm{~nm}$

$90.36 \quad 317$ ePd

$90.37316 \mathrm{ePd}$

$\begin{array}{ll}90.48 & 105 \text { iP } \\ 1.0 \mathrm{~s} & 150.00 \mathrm{~nm}\end{array}$

$90.52310 \mathrm{iPC}$

90.64312 iP

90.67310 iPd

90.71314 iPd

$90.74 \quad 105$ iP

$1.0 \mathrm{~s} \quad 95.00 \mathrm{~nm}$

90.76105 iP

$1.0 \mathrm{~s} \quad 85.00 \mathrm{~nm}$

90.89312 iPd

90.89318 ePc

90.99105 iP

$1.0 \mathrm{~s} \quad 80.00 \mathrm{~nm}$

$1.3 \mathrm{~s} 4200.00 \mathrm{~nm}$

$$
\text { is }
$$

$91.04315 \mathrm{eP}$

91.04317 iP

91.27313 IPO

$91.34323 \mathrm{eP}-$

e

UZH

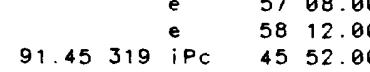

$\begin{array}{lll}Z & 17 \mathrm{~s} & 18.00 \mathrm{um} \\ \mathrm{N} & 17 \mathrm{~s} & 8.00 \mathrm{um}\end{array}$

$\begin{array}{rrr}\mathrm{N} & 17 \mathrm{~s} & 8.00 \mathrm{um} \\ \mathrm{E} & 17 \mathrm{~s} & 15.00 \mathrm{um}\end{array}$

$4812.00658 \mathrm{kmX}$

is 5554.00

iSP 5714.00

\section{NPS}

VTS

DAG

91.95306 iPd

92.09312 IPd

92.25313 iPd

eS

\begin{tabular}{lll|l}
45 & 53.20 & -0.6 & BRN \\
45 & 53.09 & -1.3 & BLY
\end{tabular}

$\begin{array}{ll}48 & 22.00 \\ 49 & 42.09\end{array}$

$92.52352 \mathrm{iPd}$

$0.6 \mathrm{~s} 20.00 \mathrm{~nm}$

\begin{tabular}{l|l}
-1.3 & BLY \\
-0.9 & PRU
\end{tabular}

i

$5602.00^{5.4 \mathrm{mb} \times 1}$

KRA

$\begin{array}{rr}1.5 \mathrm{~s} & 2683.00 \mathrm{~nm} \\ 24 \mathrm{~s} & 39.20 \mathrm{um} \\ 24 \mathrm{~s} & 25.40 \mathrm{um} \\ 24 \mathrm{~s} & 40.00 \mathrm{um}\end{array}$

$$
24 \mathrm{~s} \quad 4.00 \mathrm{um}
$$

4558.40

4559.40

i $\quad 4602.40$

4603.90

i $\quad 4813.00$

$\begin{array}{lllll}92.62320 \text { if } & 5601.00 & \\ 1.95 & 45 & 57.20 & 0.7\end{array}$

$1.2 \mathrm{~s} \quad 280.00 \mathrm{~nm}$

$92.62320 \mathrm{eP}$
$1.6 \mathrm{~s} \quad 1477.00 \mathrm{~nm}$

$6 \mathrm{mb}$
0.8

4822.60

$\begin{array}{cr}i & 4822.60 \\ i & 4958.90 \\ e & 5518.00 \\ i & 5642.00\end{array}$

92.74316 iP $\quad 4557.00$

$\begin{array}{ll}92.76 & 332 \mathrm{eP} \\ 1.3 \mathrm{~s} & 4722.00 \mathrm{~nm}\end{array}$

92.88317 iPd

92.89309 IPd

93.00312 iPd

93.19319 iPd

$93.55333 \mathrm{P}$

57.00
5
55.80

$\begin{array}{ll}93.66 & 313 \text { iPd } \\ 1.5 \mathrm{~s} & 1900.00 \mathrm{~nm}\end{array}$

$\begin{array}{lll}i & 00 \mathrm{~nm} & \\ \text { i } & 46 & 03.00 \\ \text { ePP } & 48 & 05.00 \\ \text { iPPP } & 48 & 56.00 \\ \text { iS } & 53 & 00.20 \\ \text { iSS } & 56 & 09.00 \\ \text { iSSS } & 57 & 38.50\end{array}$

93.83311 IPd $46 \quad 00.00 \quad-2.4$ | HAM

93.87319 iPd $4601.70-0.6 \quad$ HOF

$1.4 \mathrm{~s} \quad 360.00 \mathrm{~nm}$

6. $3 \mathrm{mb}$

94.24319 ePd $46 \quad 83.4 \theta \quad-0.5$

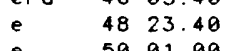

e $\quad 5001.00$

e $55 \quad 03.4 \theta$

e(S) $56 \quad 19.4 \theta$

$\begin{array}{lllll}94.34312 \text { ip } & 4600.20\end{array}$

$\begin{array}{ll}46 & 00.20 \\ 48 & 55.10\end{array}$

94.40254 iPd

4603.00

4829.00

$\begin{array}{ll}i & 5001.00 \\ i & 5047.00 \\ i & 0303.00\end{array}$

$\begin{array}{ll}i & 5001.00 \\ i & 5047.00 \\ i & 0303.00\end{array}$

94.57323 iPd

$1.3 \mathrm{~s} 2463.00 \mathrm{~nm}$

$\begin{array}{ccc}i & 4837.00 \\ i & 50 & 10.50\end{array}$

4605.50

4606.50

94.90320 ePd

$\begin{array}{rrr}e & 4640.00 \\ e & 5010.00\end{array}$

$1.3 \mathrm{~s} \quad 1615.39 \mathrm{~nm}$

Z $20 \mathrm{~s} \quad 17.62 \mathrm{um}$

$i \quad 4825.00$

95.11314 is $\quad 5740.00$

e(S) 5534.00

95.24310 IPd

95.35320 iP-

i

$46 \quad 07.00$

$46 \quad 09.70$

4611.80

4930.20

ipd

$95.38320 \mathrm{IPd}$

4609.10

$46 \quad 11.90$

$50 \quad 16.10$

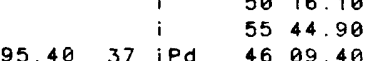

$0.8 \mathrm{~s} \quad 334.00 \mathrm{~nm}$

95.49201 iP

95.77325 iPd

95.84325 iPd

95.89317 iP

95.91322 iPd

$1.5 \mathrm{~s} \quad 1529.00 \mathrm{~nm}$

$z \quad 18 s$

N $18 \mathrm{~s}$

15. $40 \mathrm{um}$

11. 90 um

$\begin{array}{lll}i & 46 & 23.40 \\ \text { ePP } & 50 & 16.00\end{array}$

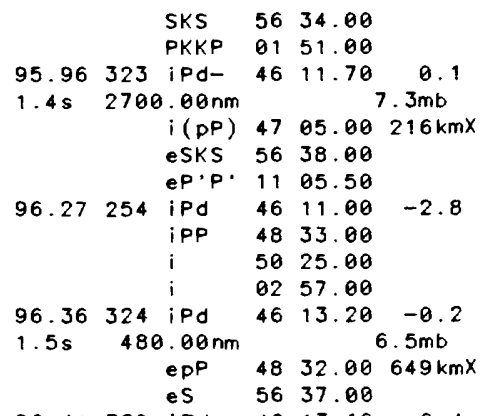

$\begin{array}{llllll}96.41 & \text { ES } & 56 & 37.00 & \\ \text { iPd } & 46 & 13.10 & -0.4\end{array}$

$1.1 \mathrm{~s} 1100.00 \mathrm{~nm}$

$96.43334 \mathrm{eP}$

$96.64312 \mathrm{iPd}$

$96.68 \quad 23 \mathrm{eP}$

$96.74 \quad 23$ ePd

$1.6 \mathrm{~s} \quad 1132.00 \mathrm{~nm}$

96.83320 ip-

$4613.00^{7.0 m b}-0.5$

$\begin{array}{lll}46 & 13.50 & -1.4\end{array}$

$\begin{array}{lll}46 & 15.20 \quad 0.5\end{array}$

$46 \quad 15.00 \quad 0.1$

$4616.30^{6.9 m b} 0.7$

4848.00

$50 \quad 26.80$

$58 \quad 07.60$

BRT

WET

$\begin{array}{lll}46 & 16.00 & -0.6\end{array}$

$\begin{array}{rll}97.03313 \mathrm{eP} & 4616.00 \\ & \mathrm{e} & 5028.00\end{array}$

97.22322 IPd

$46 \quad 17.50 \quad 0.0$

97.25318 iPd

ePP

$46 \quad 17.10$

4844.80

4915.00

e

5552.09

$97.27 \quad 327$ iPd

$97.39323 \mathrm{iPd}$

$46 \quad 18.00$

97.42324 iPd- 5646.00

$1.652315 .80 \mathrm{~nm}$

0.2

$z \quad 18 \mathrm{~s} \quad 15.40 \mathrm{um}$

$\mathrm{N} 16 \mathrm{~s} \quad 14.40 \mathrm{um}$

E $16 \mathrm{~s} \quad 8.20 \mathrm{um}$

$\begin{array}{llll}\text { EPP } & 48 \quad 38.00 & 653 \mathrm{kmX}\end{array}$

IPP $\quad 5028.00$

iPPP $52 \quad 36.00$

iSPP $53 \quad 36.00$

iSKS 5648.00

isP $58 \quad 12.00$

isSP 0212.00

isS 0345.00

eSSS 0720.00

e

1105.00

$97.42 \quad 318$ iPd $46 \quad 18.10 \quad-0.4$

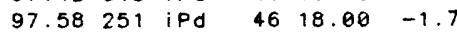

$0.9 \mathrm{~s} \quad 315.13 \mathrm{~nm}$

IPP $48 \quad 40.00$

5030.00

5053.00

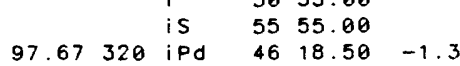

$1.2 \mathrm{~s} \quad 468.80 \mathrm{~nm}$

4620.70

4720.00

4851.20

4950.90

5008.30

$50 \quad 20.20$

$\begin{array}{lll}50 & 42.80\end{array}$

5156.70

$55 \quad 57.30$

0252.00

BHG
EVA

$97.73 \quad 320$ IPd

$\begin{array}{lll}46 & 19.40 & -0.4\end{array}$

97.77244 iPd

$1.0 \mathrm{~s} \quad 388.00 \mathrm{~nm}$

$97.82 \quad 312 \mathrm{eP}$

4845.50

$6.7 \mathrm{mb}$

97.87318 iPd

4620.00

5033.00

$\begin{array}{lll}46 & 19.50 & -0.9\end{array}$

4838.00

4940.00

5031.10

5338.00

5650.00

$58 \quad 04.90$

0218.00

$\begin{array}{rrlll}98.02312 & \text { iPc } & 46 & 20.80 & -0.5\end{array}$

4621.40 


\begin{tabular}{|c|c|c|c|c|c|c|c|c|c|c|c|}
\hline & $z$ & $21 s$ & & 5.56 um & & & $6.5 \mathrm{Msz}$ & 1 & & & 49 \\
\hline & & & & ePP & 50 & 34.00 & & i & & & iPP \\
\hline & & & & eSKS & 56 & 53.00 & & i & & & 52 \\
\hline & & & & $e(S)$ & 58 & 19.40 & & 1 & & & 53 \\
\hline SPA & & 98.09 & 180 & eP & 46 & 21.70 & 0.6 & 1 & & & 57 \\
\hline & & $1.0 \mathrm{~s}$ & & $0.50 \mathrm{~nm}$ & & & $5.4 \mathrm{mb} \times$ & i & & & 58 \\
\hline & $z$ & $20 s$ & & 8. $29 \mathrm{um}$ & & & $6.6 \mathrm{Msz}$ & 1 & & & 59 \\
\hline SLR & & 98.41 & 245 & iPd & 46 & 22.20 & -1.2 & I & & & 80 \\
\hline & & $1.6 \mathrm{~s}$ & 766 & $6.67 \mathrm{~nm}$ & & & $6.8 \mathrm{mb}$ & 1 & & & 02 \\
\hline & $z$ & $20 s$ & 15 & $5.96 \mathrm{um}$ & & & $6.5 \mathrm{Msz}$ & I GWF & 100.46 & 323 & iPdif $f 46$ \\
\hline & & & & $\mathrm{i}$ & 48 & 44.60 & & I BLF & 100.52 & 242 & iPdiff 46 \\
\hline PGC & & 98.56 & 38 & eP & 46 & 23.80 & 0.4 & i & $0.6 \mathrm{~s}$ & & 2. $14 \mathrm{~nm}$ \\
\hline FUR & & 98.57 & 321 & iPd & 46 & 23.46 & -6.2 & 1 & & & 48 \\
\hline & & & & $i$ & 46 & 25.96 & & I PNT & 100.59 & 37 & ePdif $\$ 46$ \\
\hline & & & & e & 56 & 39.06 & & 1 & $1.0 \mathrm{~s}$ & 178 & $8.00 \mathrm{~nm}$ \\
\hline & & & & e & 56 & 00.56 & & I ENN & 100.66 & 325 & iPdiff 46 \\
\hline & & & & e & 02 & 23.00 & & 1 & $1.3 \mathrm{~s}$ & 1295 & $5.00 \mathrm{~nm}$ \\
\hline BPI & & 98.67 & 245 & ep & 46 & 23.00 & -1.6 & 1 & & & 50 \\
\hline & & $1.6 s$ & 150 & $0.00 \mathrm{~nm}$ & & & $6.3 \mathrm{mb}$ & I & & & 58 \\
\hline & & & & $\mathrm{i}$ & 48 & 47.50 & & 1 & & & 59 \\
\hline DUI & & 98.75 & 314 & eP & 46 & 26.00 & 1.6 & CDF & 100.91 & 323 & ePdiff 46 \\
\hline$M C W$ & & 98.92 & 38 & $P$ & 46 & 24.70 & -0.4 & ILF & 101.03 & 324 & Pdiff-46 \\
\hline MSI & & 99.04 & 311 & eP & 46 & 26.50 & 0.7 & 1 & & & SKS \\
\hline & & & & e & 50 & 45.00 & & 1 & & & 57 \\
\hline OVO & & 99.07 & 314 & eP & 46 & 25.50 & -6.4 & I & & & 58 \\
\hline CTI & & 99.13 & 319 & iPd & 46 & 25.00 & -1.2 & I ECH & 101.07 & 322 & iPdiff 46 \\
\hline & & & & e & 48 & 54.00 & & I FHC & 101.12 & 46 & ePdiff 46 \\
\hline & & & & e & 50 & 44.00 & & 1 & & & ePP \\
\hline & & & & $e(S)$ & 56 & 02.50 & & I AKU & 101.21 & 345 & iPdif 46 \\
\hline NPL & & 99.16 & 314 & ep & 46 & 25.70 & -6.5 & 1 & $1.3 \mathrm{~s}$ & 792 & $2.31 \mathrm{~nm}$ \\
\hline SEK & & 99.20 & 243 & IPd & 46 & 25.80 & -1.1 & 1 & & & 49 \\
\hline & & $0.9 \mathrm{~s}$ & 184 & $4.87 \mathrm{~nm}$ & & & $6.4 \mathrm{mb}$ & 1 & & & 50 \\
\hline & & & & i & 48 & 51.00 & & 1 & & & 82 \\
\hline OGA & & 99.22 & 320 & iPd & 46 & 26.10 & -0.6 & I VG1 & 101.22 & 319 & ePdiff 46 \\
\hline$A Q U$ & & 99.26 & 315 & iPd & 46 & 26.50 & -0.3 & 1 & & & $e(S)$ \\
\hline & & & & e & 49 & 03.00 & & I BAF & 101.33 & 322 & iPdiff 46 \\
\hline & & & & e & 50 & 45.50 & & I BSF & 101.46 & 322 & ePdiff 46 \\
\hline & & & & $e(S)$ & 56 & 10.00 & & I ROF & 101.46 & 322 & iPdiff 46 \\
\hline WiT & & 99.32 & 327 & iPd & 46 & 27.80 & 1.1 & I UCC & 101.52 & 326 & PdiffC46 \\
\hline & & & & e & 49 & 03.00 & & 1 & $1.4 \mathrm{~s}$ & 4327 & $7.00 \mathrm{~nm}$ \\
\hline & & & & e & 58 & 35.00 & & 1 & & & 48 \\
\hline GMW & & 99.39 & 39 & $\mathrm{P}$ & 46 & 28.06 & 0.8 & 1 & & & 51 \\
\hline TNS & & 99.45 & 324 & iPd & 46 & 22.40 & $-5.1 x$ & 1 & & & PPP \\
\hline & & & & e & 49 & 00.00 & & 1 & & & 54 \\
\hline & & & & e & 50 & 41.00 & & 1 & & & SP \\
\hline & & & & e & 56 & 06.50 & & HAU & 101.65 & 323 & ePdif $f 46$ \\
\hline & & & & $e$ & 57 & 09.30 & & 1 & $1.2 \mathrm{~s}$ & 139 & $9.40 \mathrm{~nm}$ \\
\hline & & & & e & 58 & 33.30 & & I ORO & 101.66 & 320 & e(Pdif 46 \\
\hline & & & & e & 59 & 31.00 & & 1 & & & $e(s)$ \\
\hline BFW & & 99.53 & 40 & $\mathrm{P}$ & 46 & 28.40 & 0.5 & I DOU & 101.72 & 325 & Pdiff 46 \\
\hline WTS & & 99.61 & 326 & iPd & 46 & 28.40 & 0.4 & 1 & $1.2 \mathrm{~s}$ & 6366 & $6.00 \mathrm{~nm}$ \\
\hline & & $1.4 \mathrm{~s}$ & 2231 & $1.00 \mathrm{~nm}$ & & & $7.4 \mathrm{mb}$ & 1 & & & 46 \\
\hline & & & & $\mathbf{i}$ & 50 & 46.20 & & 1 & & & sP \\
\hline & & & & e & 58 & 21.56 & & 1 & & & PP \\
\hline MNS & & 99.75 & 315 & $i p d$ & 46 & 27.50 & -1.3 & 1 & & & PPP \\
\hline & & & & e & 49 & 04.00 & & 1 & & & SKS \\
\hline & & & & e & 50 & 4150 & & 1 & & & 57 \\
\hline & & & & e (S) & 56 & 64.50 & & 1 & & & 58 \\
\hline BFS & & 99.84 & 244 & IPd & 46 & 29.00 & -6.7 & 1 & & & $P \cdot P \cdot$ \\
\hline & & $0.9 \mathrm{~s}$ & 126 & $6.05 \mathrm{~nm}$ & & 6 & 5. $3 \mathrm{mb}$ & I EDM & 102.16 & 31 & iPdiff 46 \\
\hline & & & & I & 48 & 54.36 & & I WDC & 102.22 & 46 & iPdiff 46 \\
\hline BNS & & 99.86 & 325 & iPKPd & 46 & 29.20 & 0.0 & 1 & & & ePP \\
\hline & & & & iPP & 48 & 46.06 & & 1 & & & eSKS \\
\hline & & & & $\mathrm{i}$ & 49 & 55.06 & & 1 & & & eSP \\
\hline RMP & & 99.94 & 315 & iPd & 46 & 29.06 & -0.7 & ! & & & $e P^{\prime} P^{\prime} 11$ \\
\hline & & & & iPP & 50 & 48.06 & & SVF & 02.36 & 317 & iPdiff 46 \\
\hline & & & & iPS & 59 & 44.60 & & I RMT & 62.38 & 46 & Pdiff 46 \\
\hline RDP & & 99.95 & 315 & eP & 46 & 29.06 & -0.8 & NEW & 102.54 & 37 & ePdiff 46 \\
\hline & & & & e & 50 & 44.50 & & $\mathrm{GDH}$ & 102.76 & 359 & ePdiff 46 \\
\hline SAL & & 100.02 & 319 & iPdiff & $f 46$ & 30.00 & 0.1 & $!$ & $1.4 \mathrm{~s}$ & 237 & $.21 \mathrm{~nm}$ \\
\hline & & & & e & 49 & 05.50 & & 1 & & & 49 \\
\hline & & & & e & 50 & 50.06 & & I EKA & 102.97 & 332 & Pdiffc46 \\
\hline & & & & $e(S)$ & 56 & 88.00 & & 1 & $1.4 \mathrm{~s}$ & 511 & $1.70 \mathrm{~nm}$ \\
\hline COR & & 90.20 & 42 & iPdiff & $f 46$ & 16.00 & $-14.8 x$ & I MIN & 102.97 & 45 & ePdif $f 46$ \\
\hline BUH & & 100.23 & 323 & ePdiff & $\{46$ & 30.60 & -6.3 & I ORV & 03.35 & 46 & Pdiff 46 \\
\hline$F \mid R$ & & 100.23 & 317 & iPdiff & $\{46$ & 30.50 & -0.4 & I FRF & 103.39 & 318 & iPdiff 46 \\
\hline & & & & $\mathrm{S}$ & 56 & 06.00 & & I REY & 163.42 & 345 & ePdiff 46 \\
\hline FIR & & 100.23 & 317 & iPdift & $f 46$ & 40.56 & $9.6 x$ & 1 & & & 51 \\
\hline & & & & ePP & 49 & 28.06 & & BKS & 103.44 & 48 & ePdi +46 \\
\hline & & & & is & 56 & 86.00 & & I & $1.1 \mathrm{~s}$ & 230 & $9.00 \mathrm{~nm}$ \\
\hline GIB & & 100.26 & 311 & elPdif & $f 46$ & 30.00 & -1.4 & 1 & & & epP \\
\hline & & & & e & 50 & 12.00 & & I LHD & 103.46 & 36 & ePdif $f 46$ \\
\hline GRM & & 100.42 & 238 & iPdiff & $f 46$ & 31.00 & -1.1 & I LOR & 103.48 & 323 & ePdiff 46 \\
\hline & & $0.9 \mathrm{~s}$ & 151 & $1.26 \mathrm{~nm}$ & & & $.4 \mathrm{mb}$ & 1 & $1.2 \mathrm{~s}$ & 278 & $3.96 \mathrm{~nm}$ \\
\hline DEN & & 100.46 & 327 & iPdiff & $f 46$ & 33.00 & 1.3 & I LDM & 103.50 & 36 & iPdiff 46 \\
\hline & $z$ & $18 \mathrm{~s}$ & 16 & $5.20 \mathrm{um}$ & & & $6 M s z$ & 1 LBF & 103.55 & 322 & ePdiff46 \\
\hline & & & & e & 48 & .00 & & VDM & 103.56 & 325 & ePdiff46 \\
\hline
\end{tabular}


$05 d 03 h$

PAS 107.9150 ePKP $5020.00-48.6 \times$ $\begin{array}{llll}\text { EPPP } 5343.00 & \\ \text { SSP } & \text { I RRO }\end{array}$

PAS 107.9150 iPKP $5040.00-28.6 \times$

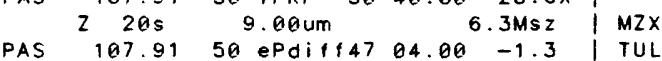

$1.4 \mathrm{~s} \quad 100.00 \mathrm{~nm} \quad 6.5 \mathrm{mb}$

SBE 107.9650 PKP $5036.00-32.8 \times$ TUL EPPP $53 \quad 43.00$ ESP $\quad 003.00$

SBE $\quad 107.96 \quad 50$ ePdiff 4705.00

MWC 107.9650 ePdif 4706.00

MWC 107.9650 PKP 5109.00 EPPP $53 \quad 45.00$ ESP 0003.00

ATE 108.35320 ePKP 5112.10

VAL $108.38 \quad 332$ ePdiff47 06.00 is 5711.09

MADF $108.39 \quad 321$ ePKP $51 \quad 12.10$

GSC $108.47 \quad 49$ ePdif 4708.00

GSC $108.47 \quad 49$ PKP $51 \quad 13.00$ EPPP $53 \quad 41.00$ ESP 5950.00

RVR $\quad 108.58 \quad 50$ ePdif 4711.00

RVR 108.5850 PKP 5104.00 EPPP $53 \quad 46.00$ eSP 0009.00

EBR $108.70318 \mathrm{ePdiff4709.00}$ e 5113.00 e $\quad 5153.00$
e $\quad 5456.00$

ABA $108.80 \quad 313$ ePKP 5109.00

SLEC 109.05 51 ePdif $\{47 \quad 12.60$

SLBC $109.05 \quad 51$ ePKP $51 \quad 11.50$ es 5156.00

PLM $109.23 \quad 51$ ePdiff47 12.00

PLM $109.23 \quad 51$ PKP $51 \quad 12.00$ $\begin{array}{lll}\text { ePPP } & 53 & 59.00 \\ \text { ESP } & 00 & 07.00\end{array}$

SNA $109.28 \quad 197$ ePdiff 4728.00

$13.33 \mathrm{~nm}$

TPC 109.5350 PKP $5022.00-49.8 \times \quad$ IIP EPPP $53 \quad 47.00$ ESP $A Q 19.00$

TPC $\quad 109.5350$ ePdiff47 12.00

$\begin{array}{llll}\text { GAR } & 109.64 & 51 \mathrm{ePdiff47} & 14.00\end{array}$ 109.6451 PKP $5059.00-13.0 \times$ VHO ePPP $53 \quad 47.00$ ESP $Q 0808.00$

LGR 109.81321 ePdiff47 19.00

BDW $109.87 \quad 39$ Poiff 4716.00 1 . As 45 . an

ALI 110.56316 iPdiff47 16.00

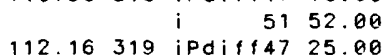
112.22299 iPdiff47 25.00

$\begin{array}{llllll}\text { TAM } & 112.22 & 299 & \text { iPdiff } 47 & 20.50 \\ \text { ALM } & 112.66 & 315 & \text { iPKPC } 51 & 19.50\end{array}$ $1.3 \mathrm{~s} 10.00 \mathrm{~nm}$

5209.00 5224.10 5857.60 CRT $\quad 113.28316$ iPKPd 5101.80

MAL $114.07316 \mathrm{Pdiff} 4733.00$ 5120.00 5229.00 0100.00

SFS $\quad 115.44 \quad 317$ ePdiff47 40.00 5124.00 5210.00 5538.00 5818.00 0110.00 0524.00

LIS $\quad 116.09320$ ePKP 5124.00

ALO $\quad 116.10 \quad 45$ ePdiff $47 \quad 43.00$ $220 \mathrm{~s} \quad 14.54 \mathrm{um}$

$\begin{array}{lllllll}\text { IFR } & 116.23 & 314 & \text { iPKPC } 51 & 25.50\end{array}$

LHC $\quad 116.61 \quad 24$ ePdif $47 \quad 44.00$

LHC $\quad 116.6124$ iPKPd 5124.40 $1.2 \mathrm{~s} 1172.00 \mathrm{~nm}$

$\mathrm{SCH} \quad 116.65 \quad 7 \mathrm{ePdi} f 4752.00$

ACO $\quad 119.93 \quad 39$ elPdif48 03.80 0.7s $7.80 \mathrm{~nm}$

ACO $\quad 119.93 \quad 39$ ePKP 5128.50 5131.40

1.9 | CR6

1.4 RFA \begin{tabular}{l|l}
-0.6 & $R L O$
\end{tabular}

\begin{tabular}{l|l}
0.2 & $R L O$ \\
0.0 & GBO
\end{tabular}

JCT

$2.9 \times, \mathrm{JCT}$

\begin{tabular}{c|c}
-0.9 & OT \\
$2.8 \times$ &
\end{tabular}

0.1

$3.2 \times \mid M N T$

STJ

$2.8 \times$

$\begin{array}{lll}-5.9 \times & A T X \\ \text { HKT } & \text { ATI }\end{array}$

$\left\{\begin{array}{l}\text { KIC } \\ \text { I }\end{array}\right.$

a. 4

I TEN

GMTN

\begin{tabular}{c|}
-1.2 \\
$2.2 x$
\end{tabular}

0.7 OXM

I 110

0.6 BLA

0.6

II

5.5X OZC

I CR5

\begin{tabular}{l|l}
-0.9 & $\mathrm{CZC}$ \\
& $\mathrm{COM}$
\end{tabular}

a. 9 TMU

$-4.4 \times$ SRA

1.9 SJS

LCR

I VBA

UPA

$-17.9 \times$

0.4

$\sum_{R F A}$

SAN

I PEL

$1 \mathrm{FCH}$

I SJG 152.1321 IPKPd 5229.00

$1.4 \mathrm{~s} \quad 976.74 \mathrm{~nm}$

$-0.1 / Z$ 20s $10.64 \mathrm{um} \quad 6.6 \mathrm{MsZ}$

JACH $152.19 \quad 153$ iPKPC $52 \quad 29.60$

I MDZ 152.75156 IPKP $52 \quad 31.00$

LPA 153.32177 iPKPd-52 32.00 $1.0 \mathrm{~s} \quad 1152.09 \mathrm{~nm}$

$$
2 \quad 22 \mathrm{~s} \quad 22.96 \mathrm{um}
$$

5. $2 X$ ZON

RTLL

$\begin{array}{lllll}99 & 155 & \text { ePKP } 52 \quad 32.00\end{array}$

$154.11 \quad 156$ ePKPd $52 \quad 29.40$

$\begin{array}{llll}154.26 & 155 \text { ePKPC } 52 \quad 29.60\end{array}$

$\begin{array}{llll}154.36 & 13 & \text { ePKPd } 52 & 32.30\end{array}$

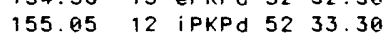
$6 \mathrm{Msz}$
5. $4 \times \mid S F G$ -1.0 | PAG PAG MDN 1 LGN VCA CRM BIM \begin{tabular}{lll|l}
$1.0 \mathrm{~s}$ & $1288.20 \mathrm{~nm}$ & $\mathrm{MVM}$ \\
$19 \mathrm{~s}$ & $10.60 \mathrm{um}$ & $6.5 \mathrm{Ms} 2$ & $\mathrm{PSO}$
\end{tabular}

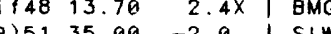
\begin{tabular}{c|c}
-2.0 & SLW \\
$4.3 \times$ & TOV
\end{tabular} -2.3 U UAV $2.5 \times$ \begin{tabular}{c|c}
$2.5 X$ & \\
-0.1 & SDV
\end{tabular}

CYA FUQ a.1 5. $3 \times$ I CAR $-4.8 \times$ AN ANT 1.3 RDJ $18.0 \times$ SLA $2.4 \times$ 1 ITR HJA VAO YJA LPB BAO

155.26 11 ePKP $52 \quad 34.00$ $\begin{array}{llll}155.38 & 13 & \text { ePKP } 52 \quad 34.50\end{array}$ $\begin{array}{llll}155.56 & 12 & \text { ePKPd } 52 \quad 34.30\end{array}$ $156.13 \quad 12$ ePKP $52 \quad 35.00$ $156.45 \quad 40$ ePKP $52 \quad 41.4 \theta$ $156.61 \quad 153$ iPKPd 5236.40 $\begin{array}{llll}156.75 & 12 & \text { ePKP } 52 \quad 36.20\end{array}$ $156.78 \quad 12$ ePKP $52 \quad 35.90$ $156.98 \quad 12$ ePKP $52 \quad 36.40$ $156.97 \quad 12$ ePKP 5236.10 $157.01 \quad 67$ ePKP $52 \quad 39.00$ $157.40 \quad 48$ ePKP $52 \quad 37.00$ $157.49 \quad 12$ ePKP 5237.00 $\begin{array}{llll}157.64 & 37 & \text { iPKPd } 52 \quad 37.00\end{array}$

$157.68 \quad 42$ iPKPd $52 \quad 37.70$ $0.6 \mathrm{~s} \quad 171.40 \mathrm{~nm}$

$157.81 \quad 41$ iPKPd $52 \quad 37.70$ $0.7 \mathrm{~s} \quad 300.00 \mathrm{~nm}$

$157.88 \quad 157$ ePKPd $52 \quad 36.80$ $\begin{array}{llllll}157.91 & 53 & \text { IPKP } & 52 & 37.00 & -0.6\end{array}$ 158.1555 ePKP $5238.00 \quad 0.1$ $\begin{array}{lllll}158.62 \quad 30 & \text { iPKPd } 52 \quad 38.00 & -0.1\end{array}$ $1.0 \mathrm{~s} \quad 168.00 \mathrm{~nm}$

IPP 5318.00

159.40140 IPKPd-52 $40.00 \quad 1.5$

160.74219 is 0244.50 SS 0303.00

162.38267 iPKP $52 \quad 42.10$ $\begin{array}{lllll}162.76 & 210 \text { ePKP } 52 & 42.90 & 0.8\end{array}$

$1.4 \mathrm{~s} \quad 300.60 \mathrm{~nm}$

$\begin{array}{lll}\text { e } & 52 \quad 45.90\end{array}$ 165.72127 iPKPd-52 47.00 $1.0 \mathrm{~s} 1470.00 \mathrm{~nm}$ ISKS 0324.00 169.04227 iPKPd $5246.80 \quad-0.4$ S.D. $=1.0$ on 600 of 683 obs.

MAR 19, 1984 20h $28 \mathrm{~m} 38.24 \pm 0.05 \mathrm{~s}$ $40.341 \mathrm{~N} \pm 1.5 \mathrm{~km} \quad 63.359 \mathrm{E} \pm 0.8 \mathrm{~km}$ DEPTH $=14.5 \mathrm{~km}$ ( 26 depth phoses)

$6.5 \mathrm{mb}$ (119 obs.) 7.0Msz ( 28 obs.) UZBEK SSR (339) At leost 100 people injured and extensive domoge $(\mid x)$ in the Gazli orea. Domoge ( ||$|-v| l l)$ ot Dzhongeldy ond ( $V \mid l)$ in the Bukharo orea. Felt (VII) ot Chordzhau: (VI) at Navoi ond Mory; (V-VI) at Samorkond: (V) ot Dzhizak and Ashkhabod; (IV) ot Tashkent ond Dushanbe: (111IV) ot Andizhan; (111) at Leninobad, Uro-Tyube, Chimkent and Dzhambul; $(11-111)$ at Kulyob, Khorog and Frunze. Felt olso at Mashhod, Iran.

\begin{tabular}{|c|c|c|c|c|c|}
\hline \multirow[t]{3}{*}{2.87} & 102 & iPnd & 29 & 24.90 & 0. 6 \\
\hline & & is* & 30 & 03.70 & \\
\hline & & isg & 30 & 13.30 & \\
\hline \multirow[t]{3}{*}{4.56} & 111 & iPnc & 29 & 49.00 & 0.7 \\
\hline & & ePg & 30 & 08.00 & \\
\hline & & $\mathrm{eSg}$ & 31 & 14.00 & \\
\hline \multirow[t]{2}{*}{4.57} & 240 & iPnd & 29 & 49.00 & 0.6 \\
\hline & & esn & 30 & 42.00 & \\
\hline \multirow[t]{3}{*}{4.61} & 76 & iPnc & 29 & 49.00 & -0.1 \\
\hline & & eSn & 30 & 39.20 & \\
\hline & & $i \mathrm{Sg}$ & 31 & 14.00 & \\
\hline \multirow[t]{2}{*}{4.73} & 241 & ePnc & 29 & 49.70 & -1.1 \\
\hline & & eSn & 30 & 44.30 & \\
\hline 5.85 & 218 & iPCt & 29 & 55.50 & 0.2 \\
\hline \multirow[t]{2}{*}{5.53} & 102 & ePn & 30 & 02.20 & 0.0 \\
\hline & & es* & 31 & 22.50 & \\
\hline \multirow[t]{3}{*}{5.54} & 114 & iPn & 30 & 03.00 & 0.8 \\
\hline & & $\mathrm{iPg}$ & 30 & 28.40 & \\
\hline & & isn & 31 & 11.40 & \\
\hline 5.58 & 260 & iPnc & 30 & 03.50 & 0.7 \\
\hline \multirow[t]{3}{*}{6.87} & 84 & iPnc & 30 & 19.80 & -1.1 \\
\hline & & esn & 31 & 42.00 & \\
\hline & & $\mathrm{eSg}$ & 32 & 25.00 & \\
\hline 98 & 112 & iPne & 30 & 22.10 & -0.6 \\
\hline
\end{tabular}




\begin{tabular}{|c|c|c|c|c|c|c|c|c|c|}
\hline $\mathrm{KHI}$ & & 7.24 & 213 & iPt & 30 & 25.30 & -0.9 & 1 & MOS \\
\hline \multirow[t]{2}{*}{ FRU } & & 8.80 & 70 & $i P c$ & 30 & 45.40 & -2.5 & i & \\
\hline & & & & is & 32 & 25.00 & & 1 & \\
\hline NRN & & 9.64 & 79 & iPc & 30 & 55.80 & $-3.9 x$ & 1 & \\
\hline & & & & es & 32 & 46.00 & & 1 & \\
\hline KSH & & 9.74 & 91 & iPC & 30 & 59.00 & -2.0 & 1 & $\mathrm{OBN}$ \\
\hline BAK & & 10.27 & 275 & $i P d$ & 31 & 06.00 & -2.1 & 1 & \\
\hline & $N$ & $10 \mathrm{~s}$ & 105 & $5.00 \mathrm{um}$ & & & & 1 & \\
\hline & $E$ & $10 \mathrm{~s}$ & & $\theta .20 \mathrm{um}$ & & & & 1 & \\
\hline & & & & es & 33 & 07.00 & & 1 & \\
\hline TEH & & 10.50 & 248 & ePc & 31 & 09.50 & -1.9 & 1 & \\
\hline QUE & & 10.55 & 163 & iPd- & 31 & 11.50 & -0.6 & 1 & \\
\hline & & & & es & 33 & 08.00 & & 1 & HR I \\
\hline$A A A$ & & 10.56 & 69 & iPd & 31 & 10.60 & -1.5 & 1 & POO \\
\hline & & & & is & 33 & 10.40 & & 1 & KDE \\
\hline$A A A$ & & 10.56 & 69 & $P$ & 31 & 14.00 & 1.9 & 1 & JER \\
\hline TLG & & 10.87 & 70 & $i P c$ & 31 & 12.10 & $-4 \cdot 3 x$ & 1 & LSA \\
\hline & & & & is & 33 & 22.00 & & 1 & PRN I \\
\hline SHE & & 11.22 & 276 & $P$ & 31 & 27.00 & $5.9 x$ & 1 & GPA \\
\hline PRZ & & 11.50 & 74 & eP & 31 & 22.40 & -2.6 & 1 & HRT \\
\hline & & $1.0 \mathrm{~s}$ & 1500 & $9.00 \mathrm{~nm}$ & & 7 & $.2 \mathrm{mb}$ & 1 & $B C K$ \\
\hline & & & & es & 33 & 30.00 & & 1 & KIS \\
\hline MAK & & 12.21 & 288 & $I P C$ & 31 & 29.20 & $-5.2 x$ & 1 & \\
\hline & & $1.0 \mathrm{~s}$ & 9500 & $\partial .00 \mathrm{~nm}$ & & & . Omb $x$ & 1 & \\
\hline & & & & is & 33 & 46.80 & & 1 & \\
\hline KRV & & 12.97 & 277 & ip & 31 & 39.00 & $-5.6 x$ & 1 & \\
\hline & & & & is & 34 & 04.00 & & 1 & \\
\hline GRS & & 13.10 & 272 & $i P c$ & 31 & 41.00 & $-5.5 x$ & 1 & \\
\hline & & $1.8 \mathrm{~s}$ & 970 & $9.00 \mathrm{~nm}$ & & 6 & $.6 \mathrm{mb}$ & 1 & \\
\hline & & & & es & 34 & 04.00 & & 1 & \\
\hline$T A B$ & & 13.41 & 266 & iPCt & 31 & 47.70 & -2.9 & I & ISK \\
\hline GRO & & 13.52 & 289 & $i P c$ & 31 & 47.00 & $-4.8 x$ & 1 & CFR \\
\hline & & & & is & 34 & 21.00 & & 1 & PSN \\
\hline$T I F$ & & 14.08 & 282 & $P$ & 31 & 55.20 & $-4 \cdot 1 x$ & 1 & $T L B$ \\
\hline MTA & & 14.08 & 282 & $i P c$ & 31 & 55.20 & $-4.0 x$ & 1 & HYB \\
\hline KER & & 14.24 & 250 & eP & 32 & 01.00 & -0.5 & 1 & \\
\hline ERE & & 14.40 & 275 & $i P c$ & 32 & 00.00 & $-3.6 x$ & 1 & \\
\hline & & & & is & 34 & 45.60 & & i & $B\} R$ \\
\hline LEN & & 14.84 & 278 & eP & 32 & 07.00 & -2.4 & 1 & DST \\
\hline & & $2.0 \mathrm{~s}$ & 1800 & $0.00 \mathrm{~nm}$ & & 6 & $.2 \mathrm{mb}$ & 1 & IAS \\
\hline BKR & & 15.04 & 282 & iPd & 32 & 09.00 & $-3.0 x$ & 1 & KCT \\
\hline & Z & $18 \mathrm{~s}$ & 4494 & $4.00 u m$ & & & & 1 & FOC \\
\hline & & & & is & 34 & 54.80 & & 1 & BNT \\
\hline PYA & & 15.49 & 290 & $i P C$ & 32 & 12.50 & $-5.2 x$ & 1 & $\mathrm{ODB}$ \\
\hline & & $1.0 \mathrm{~s}$ & 1450 & $9.00 \mathrm{~nm}$ & & 6 & $.2 \mathrm{mb}$ & 1 & EDC \\
\hline & $z$ & $13 \mathrm{~s}$ & 35 & $5.00 u m$ & & & $.7 M \leq 2 X$ & 1 & $B A C$ \\
\hline & $\mathrm{N}$ & $13 \mathrm{~s}$ & 27 & $7.00 \mathrm{um}$ & & & & 1 & VRI \\
\hline & $E$ & $13 \mathrm{~s}$ & 14 & $4.00 \mathrm{um}$ & & & & 1 & I SR \\
\hline & & & & es & 35 & 06.00 & & I & KGT \\
\hline SEM & & 15.53 & 44 & ePc & 32 & 13.20 & $-4.9 x$ & 1 & cro \\
\hline & & & & es & 35 & 03.00 & & 1 & $J M B$ \\
\hline NDI & & 16.28 & 131 & iPd & 32 & 23.80 & $-4.0 x$ & 1 & MNK \\
\hline & & $1.0 \mathrm{~s}$ & 800 & $3.00 \mathrm{~nm}$ & & 5 & $.8 \mathrm{mb}$ & 1 & \\
\hline & & & & is & 35 & 16.00 & & 1 & \\
\hline ARU & & 16.37 & 351 & ePc & 32 & 24.00 & $-4.9 x$ & I & \\
\hline & & & & es & 35 & 22.00 & & 1 & \\
\hline SVE & & 16.58 & 355 & iPc & 32 & 27.00 & $-4.5 x$ & 1 & MLR \\
\hline & & & & is & 35 & 31.00 & & 1 & BUC 1 \\
\hline BHD & & 16.74 & 251 & IPd & 32 & 33.00 & -0.6 & 1 & CGN \\
\hline & & & & $i$ & 34 & 42.00 & & 1 & $12 M$ \\
\hline & & & & is & 35 & 37.00 & & 1 & GTA \\
\hline & & & & $i P C P$ & 37 & 27.00 & & 1 & SHL \\
\hline & & & & i LO & 37 & 49.00 & & 1 & \\
\hline & & & & $\mathrm{i}$ & 38 & 36.00 & & 1 & HLW \\
\hline & & & & iss & 44 & 34.00 & & 1 & MOY \\
\hline SOC & & 17.86 & 288 & $I P C$ & 32 & 46.00 & -1.7 & 1 & \\
\hline & & $2.0 \mathrm{~s}$ & 2900 & $0.00 \mathrm{~nm}$ & & 6 & $.1 \mathrm{mb}$ & 1 & $E Z N$ \\
\hline & & & & es & 36 & 08.00 & & I & PUL \\
\hline WMO & & 18.38 & 71 & IPC & 32 & 52.00 & -2.2 & 1 & \\
\hline ANN & & 19.67 & 292 & $i P c$ & 33 & 07.00 & -2.6 & 1 & \\
\hline & & $1.5 \mathrm{~s}$ & $*$ & $* * * * n \mathrm{~m}$ & & 7 &.$\theta \mathrm{mb}$ & 1 & \\
\hline & & & & is & 36 & 49.00 & & 1 & \\
\hline NVS & & 19.72 & 36 & ePc & 33 & 06.50 & $-3.4 x$ & 1 & \\
\hline & & 1. & 2800 & $3.00 \mathrm{~nm}$ & & & $.5 \mathrm{mb}$ & 1 & \\
\hline & & & & is & 36 & 40.50 & & 1 & LRR \\
\hline ELT & & 20.20 & 43 & $i P_{C}$ & 33 & 12.80 & -2.2 & 1 & $D i M$ \\
\hline & & & & is & 36 & 55.00 & & 1 & CMP \\
\hline$K V T$ & & 20.69 & 281 & iPc & 33 & 19.70 & -0.6 & 1 & PVL \\
\hline $\operatorname{sim}$ & & 21.94 & 292 & iPc & 33 & 31.00 & -1.8 & | & $K D Z$ \\
\hline & Z & $20 \mathrm{~s}$ & 408 & $3.00 \mathrm{um}$ & & 6 & $.8 M s z$ & 1 & DRA \\
\hline & $\mathrm{N}$ & $20 \mathrm{~s}$ & 680 & $0.00 \mathrm{um}$ & & & & i & $P L D$ \\
\hline & $E$ & $20 \mathrm{~s}$ & 384 & $4.00 \mathrm{um}$ & & & & 1 & LVV \\
\hline & & & & iPP & 33 & 54.00 & & 1 & \\
\hline & & & & is & 37 & 30.00 & & 1 & \\
\hline UN & & 21.99 & 119 & $i P C$ & 33 & 33.60 & -0.1 & I & CJR \\
\hline $\mathrm{N}$ & & 21.99 & 118 & iPc & 33 & 33.20 & -0.5 & 1 & BMR \\
\hline$<1$ & & 22.2 & 118 & $i P C$ & 33 & 36.00 & -0.1 & & \\
\hline
\end{tabular}

$2.0 \mathrm{~s} 6200.00 \mathrm{~nm}$

EPP $34 \quad 12.00$

$\begin{array}{lll}\text { iPCP } & 37 & 34.00 \\ \text { iS } & 37 & 47.00\end{array}$

\begin{tabular}{llll|l}
$23.12319 \mathrm{iPC}$ & 3344.00 & -0.3 & SRS
\end{tabular}

$1.7 \mathrm{~s} 1700.00 \mathrm{~nm} \quad 6.3 \mathrm{mb}$.

$Z 18 \mathrm{~s} \quad 1008.00 \mathrm{~mm}$

$\mathrm{N} 18 \mathrm{~s} \quad 1113.00 \mathrm{um}$

E $18 \mathrm{~s} 987.00 \mathrm{um}$

ePP 3412.00

23.15261 is $\quad 3756.00$

2.0 I PAIG

$\begin{array}{llllll}154 & \mathrm{IPC} & 33 & 50.70 & 1.9 & \mathrm{SOH}\end{array}$

24.12283 iP $3353.70 \quad-0.5$ | IRK

24.24258 iPc

$25.02106 \mathrm{Pc}$

$25.10255 \mathrm{eP}$

25. 12281 iP

25.51282 iP

25.59274 iP

$25.67297 \mathrm{iPd}$

$Z 21 \mathrm{~s} 363.00 \mathrm{um}$

$\begin{array}{lll}\mathrm{N} & 19 \mathrm{~s} & 305.00 \mathrm{um} \\ \mathrm{E} & 22 \mathrm{~s} & 482.00 \mathrm{um}\end{array}$

$22 \mathrm{~s} \quad 482.00 \mathrm{um}$

$$
\begin{array}{lll}
\text { iPP } & 34 & 53.00 \\
\text { iPPP } & 34 & 58.00 \\
\text { iS } & 38 & 39.00 \\
\text { iSS } & 38 & 45.00 \\
\text { iSSS } & 48 & 00.00
\end{array}
$$

$25.92 \quad 283 \quad \mathrm{PPd} \quad 34 \quad 11.80$

26.16292 iPC $34 \quad 13.50$

$26.23289 \mathrm{iPC} \quad 34 \quad 15.00$

$26.27291 \mathrm{IPC} \quad 34 \quad 14.70$

$26.36 \quad 146 \quad \mathrm{PC} \quad 34 \quad 16.00$

$1.0 \mathrm{~s} 2280.00 \mathrm{~nm}$

26.51295 is

$26.53280 \mathrm{iPC}$

$26.57297 \mathrm{eP}$

$26.60281 \mathrm{iP}$

$26.83294 \quad \mathrm{iP}$

$26.90282 \mathrm{iPC}$

$26.92294 \mathrm{eP}$

$26.95282 \quad \mathrm{PPd}$

$27.01295 \mathrm{eP}$

$27.14294 \mathrm{eP}$

$27.30292 \mathrm{iPc}$

27.34282 iP

$27.53294 \mathrm{iPc}$

27.53287 iPd

27.54312 iPC

IPC 3426.00

$\begin{array}{llll}\text { EPP } & 35 & 12.00\end{array}$

ePPP $35 \quad 32.00$

EPCP $37 \quad 48.00$ eS

$27.71293 \mathrm{iPd}$

3903.00

3428.00

$27.72 \quad 291 \mathrm{iPc}$

$27.76290 \mathrm{ePc}$

$27.86278 \quad \mathrm{iPc}$

$27.87 \quad 80 \quad \mathrm{iPc}$

$27.96 \quad 113$ iP

$28.07 \quad 258$ is

$28.18 \quad 54 \quad \mathrm{iPC}$

$1.4 \mathrm{~s} \quad 310.00 \mathrm{~nm}$

$\begin{array}{llllll}28.21 & 281 & \mathrm{PPd} & 34 & 32.00 & -0.3\end{array}$

$\begin{array}{llllll}34.00 & 324 & \text { iPc } & 34 & 33.00 & 0.0\end{array}$

$1.3 \mathrm{~s} 5800.00 \mathrm{~nm}$

3429.00

3428.50

3429.00

3430.40

3429.80

3908.00

430.00

-0.5
$-3.4 x$

2.0
2.2

$-0.5$

$-1.4$

\begin{tabular}{|c|c|c|c|c|c|c|}
\hline & & esp & 34 & 40.00 & & NRI \\
\hline & & is & 39 & 20.00 & & \\
\hline 28.32 & 293 & $P$ & 34 & 33.00 & -0.3 & \\
\hline 28.33 & 286 & iP & 34 & 34.00 & 0.7 & \\
\hline 28.36 & 293 & ePc & 34 & 34.00 & 0.4 & \\
\hline 28.47 & 288 & $i P_{c}$ & 34 & 35.00 & 0.4 & \\
\hline 28.57 & 285 & $i P c$ & 34 & 36.00 & 0.5 & OHR \\
\hline 28.95 & 292 & eP & 34 & 44.00 & 5. $1 x$ & \\
\hline 28.97 & 286 & EP & 34 & 39.00 & 0.0 & BUD \\
\hline 29.04 & 302 & iPc & 34 & 39.90 & 0.2 & KOD \\
\hline & & IPPP & 35 & 50.00 & & \\
\hline & & is & 39 & 23.60 & & SRO \\
\hline 29.29 & 296 & ep & 34 & 42.50 & a. 5 & \\
\hline 29.33 & 298 & ePc & 34 & 43.00 & 0.7 & \\
\hline
\end{tabular}

$-1.0$

$Z \quad 18 \mathrm{~s} \quad 480.00 \mathrm{um}$

N $18 \mathrm{~s} \quad 200.00 \mathrm{um}$

E $18 \mathrm{~s} \quad 420.00 \mathrm{um}$
$1.7 \mathrm{~s} \quad 363.00 \mathrm{~nm} \quad 5.9 \mathrm{mb}$ $29.47 \quad 57 \quad$ iPC $\quad 34 \quad 44.60 \quad 1.1$ $1.7 \mathrm{~s} \quad 2120.00 \mathrm{~nm} \quad 6.7 \mathrm{mb}$ $29.77 \quad 286$ iPC $34 \quad 46.00 \quad-0.4$ 29.97285 ePn $34 \quad 48.00 \quad-0.1$ $29.98 \quad 288$ iPC $3449.00 \quad 0.9$ 30.04298 eP $3452.00 \quad 3.4 X$ 30.14300 IPC $34 \quad 49.00 \quad-0.5$ $Z 19 \mathrm{~s} 168.00 \mathrm{um} \quad 6.7 \mathrm{MsZ}$ EPP $35 \quad 45.50$ IPPP $35 \quad 55.00$ $\begin{array}{lllll} & \text { is } & 39 & 50.00 & \\ 30.17282 & \text { iPnc } & 34 & 49.20 & -0.6\end{array}$ 30.21284 iPnc $3450.00 \quad-0.3$ $\begin{array}{llllll}30.30 & 53 & \text { iPC } & 34 & 50.00 & -0.9\end{array}$ $2.0 \mathrm{~s} \quad 970.00 \mathrm{~nm} \quad 6.3 \mathrm{mb}$ $z 10 \mathrm{~s} 605.00 \mathrm{um} \quad 7.5 \mathrm{MszX}$ $N$ 10s $185.00 \mathrm{um}$

E $10 \mathrm{~s} 585.00 \mathrm{um}$ $\begin{array}{lll}\text { ePP } 35 \quad 42.00 & 0\end{array}$ EPCP 3758.00 es 3950.00 eSSS 4147.00

THE $\quad 30.54284$ IPnC $3452.00 \quad-1.1$ ATH $\quad 30.66279$ IPC $3454.2 \theta \quad 0.0$ $\begin{array}{lllllll} & & & \text { IS } & 40 & 00.00 & \\ \text { VAY } & 30.68 & 285 & \text { IPC } & 34 & 54.20 & -0.1\end{array}$ TIM $30.99294 \mathrm{iPC} \quad 3457.20 \quad 0.2$ WAR $31.14307 \mathrm{ePt} 3457.00-1.2$

$3536.00189 \mathrm{kmX}$ 4000.00 40
4028.00
4100.00 e 4100.00

31.19323 iPct $34 \quad 58.10 \quad-0.5$ $0.8 \mathrm{~s} \quad 953.50 \mathrm{~nm}$ $3501.2011 \mathrm{~km}$ eS $40 \quad 04.00$ $\begin{array}{llllll}31.31 & 300 & \text { iPc } & 34 & 59.80 & 0.0\end{array}$

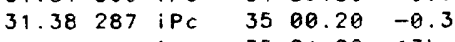
i $\quad 3504.00 \quad 13 \mathrm{~km}$ iPP $36 \quad 06.00$

is $\quad 40 \quad 05.50$

$\begin{array}{lllll}31.48 & 328 \text { iPC } & 35 & 00.80 & -0.4\end{array}$ $0.6 \mathrm{~s} 290.18 \mathrm{~nm} \quad 6.4 \mathrm{mb}$ $\begin{array}{lllll}31.49 & 301 \text { eP } & 35 & 02.20 & 0.5\end{array}$ $1.4 \mathrm{~s} 5274.00 \mathrm{~nm} \quad 7.2 \mathrm{mb}$ 31.61331 iPCt $3501.40-0.9$ $0.6 \mathrm{~s} \quad 824.00 \mathrm{~nm} \quad 6.8 \mathrm{mb}$ i $\quad 3506.00 \quad 16 \mathrm{~km}$ ePPP $37 \quad 00.00$ es 4000.00

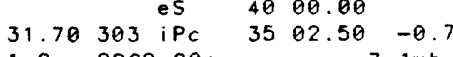
$1.0 \mathrm{~s} 2862.00 \mathrm{~nm} \quad 7.1 \mathrm{mb}$ i $\quad 3507.00 \quad 16 \mathrm{~km}$ is 4069.00

$\begin{array}{llllllll}0.2 & P S Z & 31.76 & 298 \mathrm{ePC} & 35 & 04.00 & 0.2\end{array}$ $\begin{array}{llllll}1.2 & \text { APA } & 31.84339 \mathrm{iPC} & 3503.70 & -0.6\end{array}$ \begin{tabular}{r|ccc}
0.4 & $0.7 \mathrm{~s}$ & $860.00 \mathrm{~nm}$ & $6.8 \mathrm{mb}$
\end{tabular} $Z 11 \mathrm{~s} 670.00 \mathrm{um}$ $\begin{array}{lll}\text { IPP } & 36 & 05.00 \\ \text { IPCP } & 37 & 52.00\end{array}$ $\begin{array}{lllll} & \text { is } & 40 & 13.00 & \\ 31.90 & 84 & \text { iPCt } & 3506.00 & 0.7\end{array}$

$7.0 \mathrm{~s} \quad 8979.00 \mathrm{~nm}$ N $11 \mathrm{~s} \quad 20.90 \mathrm{um}$ E $13 \mathrm{~s} 407.00 \mathrm{um}$ PP $\quad 35 \quad 16.00 \quad 36 \mathrm{kmx}$ PPP $36 \quad 25.00$ PcP 3759.00 S $\quad 40 \quad 09.00$ $55 \quad 4027.00$ $\begin{array}{ccccc}31.92 & 16 \text { iPc } & 35 & 05.00 & 0.0 \\ 1.3 \mathrm{~s} & 1000.00 \mathrm{~nm} & 6.6 \mathrm{mb}\end{array}$ $\mathrm{N} 14 \mathrm{~s} \quad 135.00 \mathrm{um}$

E $13 \mathrm{~s} 168.00 \mathrm{um}$ ePPP $36 \quad 20.00$ $\begin{array}{llllll} & \text { is } & 40 & 14.00 & \\ 32.03286 & \text { if } & 35 & 05.30 & -1.0\end{array}$ i $\quad 3509.70 \quad 15 \mathrm{~km}$ $\begin{array}{llllll}32.37 & 298 \quad \mathrm{IPC} & 35 & 09.10 & 0.0\end{array}$ $\begin{array}{llllll}32.49 & 153 \mathrm{iPC} & 35 & 11.60 & 0.8\end{array}$ $1.0 \mathrm{~s} \quad 806.00 \mathrm{~nm} \quad 6.6 \mathrm{mb}$

$\begin{array}{llllll}32.83 & 298 & \mathrm{IPC} & 35 & 13.20 & 0.2\end{array}$

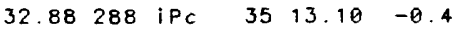




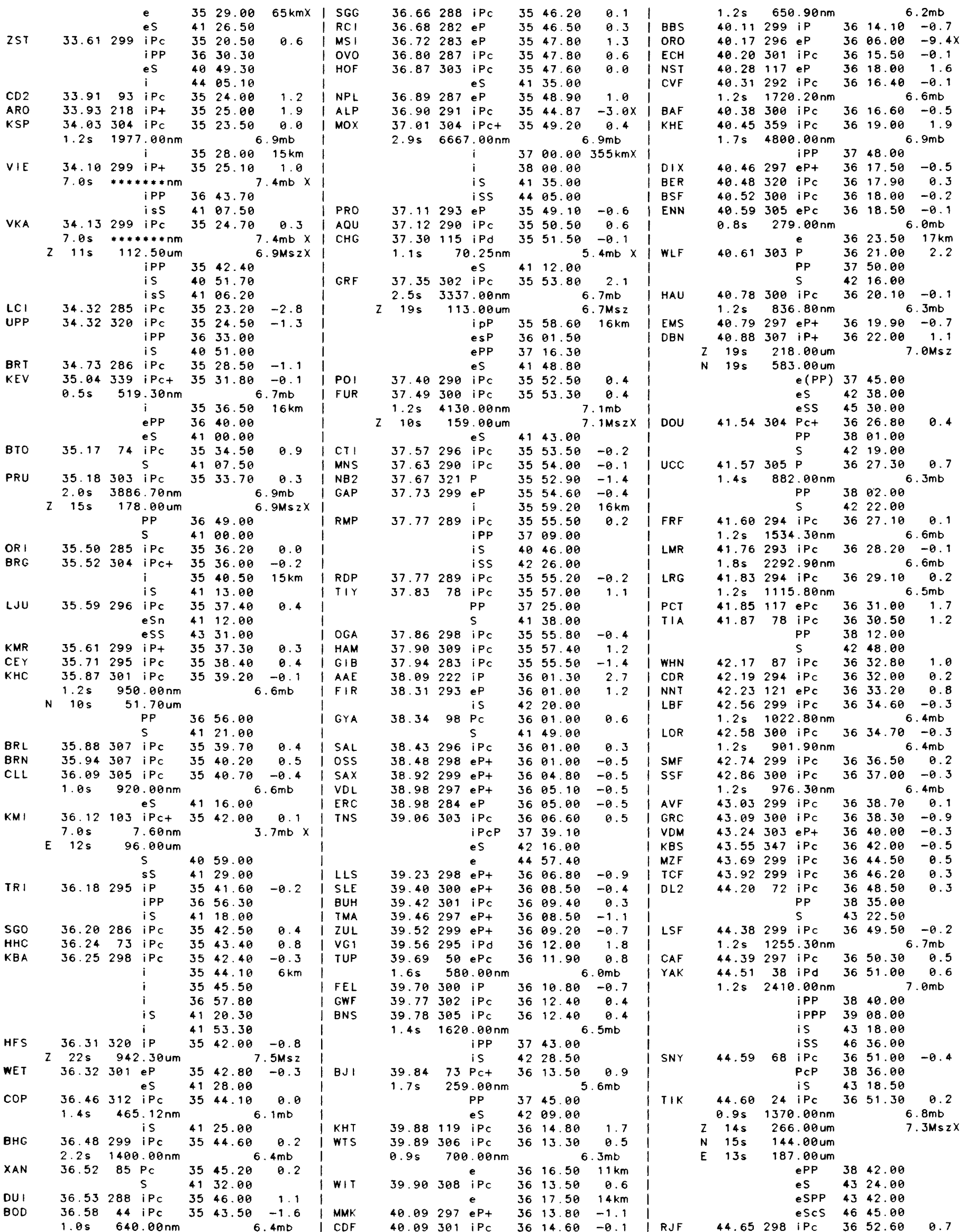




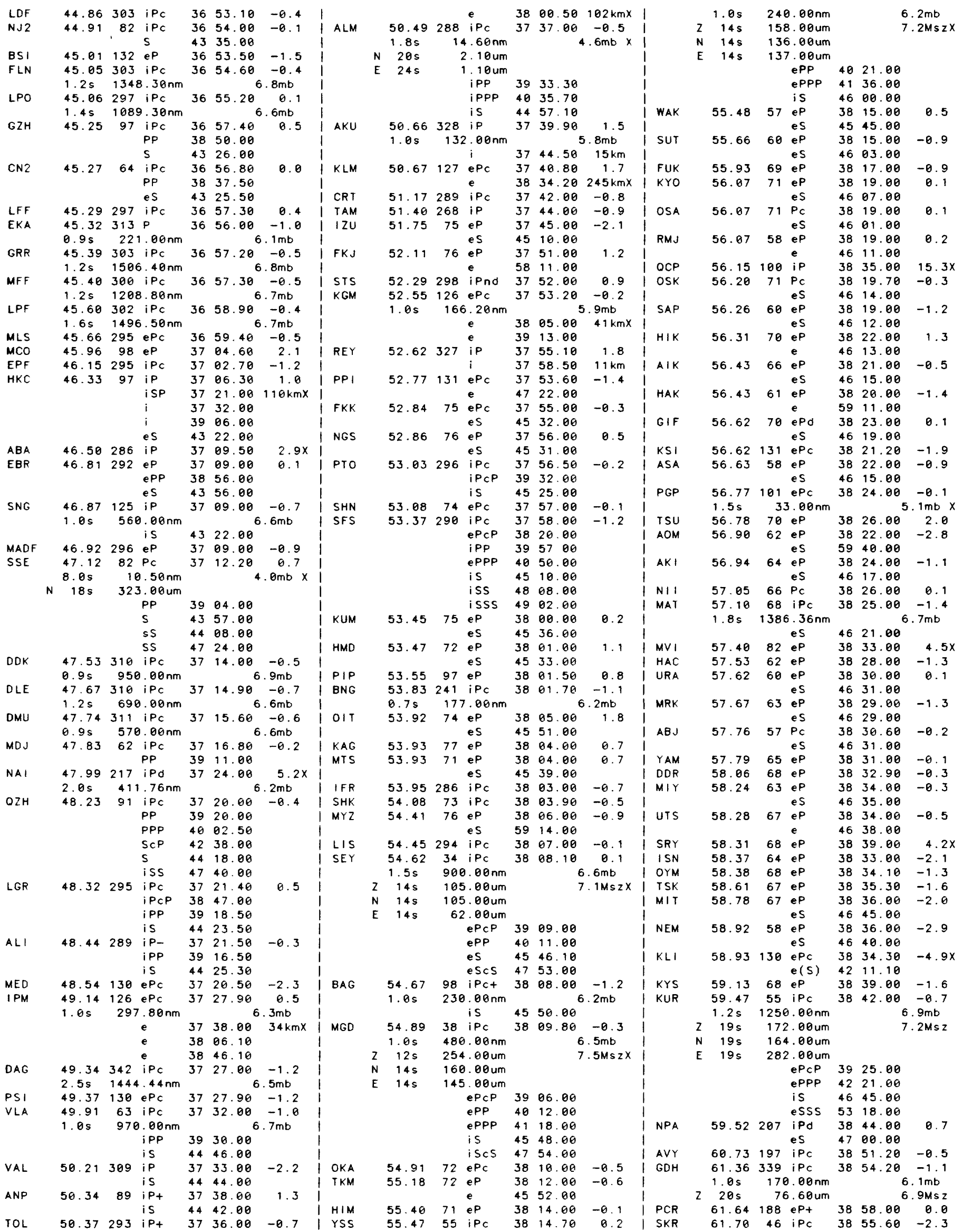


$19 d \quad 20 h$

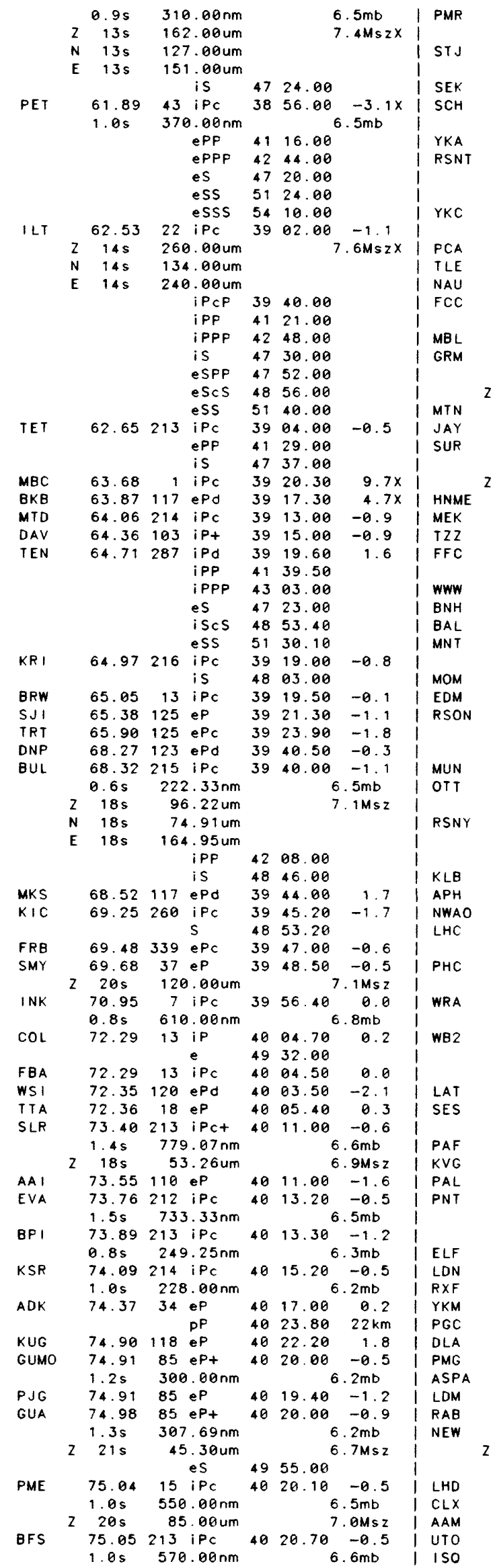

\begin{tabular}{lccccc|l}
75.05 & $15 \mathrm{iP}$ & $4 \theta$ & $2 \theta .80$ & 0.2 & BUT \\
& $\mathrm{pP}$ & $4 \theta$ & 24.80 & $13 \mathrm{~km}$ & LRM \\
75.71 & $321 \mathrm{eP}$ & $4 \theta$ & 24.00 & -0.6 & ALOA \\
$0.9 \mathrm{~s}$ & $477.00 \mathrm{~nm}$ & & $6.6 \mathrm{mb}$ & RSSD \\
75.96 & $212 \mathrm{iPC}$ & 40 & 25.00 & -1.3 &
\end{tabular}

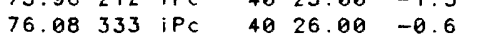

$0.8 \mathrm{~s} 859.00 \mathrm{~nm} \quad 6.9 \mathrm{mb} / \mathrm{COR}$

$77.50359 \mathrm{eP} \quad 4034.70 \quad 0.4 \quad$ BLA

$\begin{array}{cccc}77.51 & 359 \mathrm{iPc} & 40 & 34.00 \quad-0.3 \\ 1.3 \mathrm{~s} & 1475.81 \mathrm{~nm} & 6.9 \mathrm{mb}\end{array}$

\begin{tabular}{llllll}
77.51 & $359 \mathrm{PP}$ & 40 & 39.60 & $18 \mathrm{~km}$ \\
\hline $\mathrm{PC}$ & 40 & $34.4 \theta$ & 0.0
\end{tabular}

$0.5 \mathrm{~s} \quad 27500 \mathrm{~nm}$

$78.05 \quad 12 \mathrm{eP} \quad 40 \quad 38.80^{6.6 \mathrm{mb}} 1.3$

$78.18 \quad 108$ ePd $40 \quad 42.00 \quad 3.2 \times$

79.17132 iPc $40 \quad 44.90 \quad 0.9$ ।

$79.49348 \mathrm{iPC} \quad 40 \quad 45.30 \quad 0 . \theta$

$0.8 \mathrm{~s} \quad 571.00 \mathrm{~nm} \quad 6.6 \mathrm{mb}$

80.64128 iPd $4051.90 \quad 0.0 \quad \mathrm{FVM}$

$\begin{array}{lllll}80.79 & 211 \mathrm{iPc} & 40 & 52.50 & 0.0 \\ 0.6 \mathrm{~s} & 286.67 \mathrm{~nm} & 6.5 \mathrm{mb}\end{array}$

$218 \mathrm{~s} \quad 18.04 \mathrm{um} \quad 6.5 \mathrm{Msz}$

$81.98114 \mathrm{eP} \quad 4058.00-1.0 \quad$ RSCP

$82.00100 \mathrm{ePC} \quad 4104.00 \quad 4.7 \times$ । WDC

$\begin{array}{lllll}82.37 & 215 \mathrm{IFC} \quad 4101.90 & 0.9\end{array}$

$\begin{array}{lll}1.1 \mathrm{~s} & 405.06 \mathrm{~nm} & 6.4 \mathrm{mb}\end{array}$

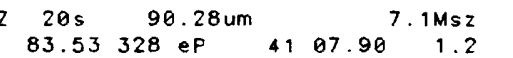

$84.07132 \mathrm{eP} \quad 4109.50-0.1$

84.19102 OP $4111.30 \quad 0.7$

84.48352 ePc $41 \quad 11.60 \quad 0.3 \quad$ GOL

$0.9 \mathrm{~s} \quad 214.00 \mathrm{~nm}$

84.9199 eP $\quad 4115.30^{6.4 m b}$

86.13329 iP $4121.00 \quad 1.2$ DUG

$86.17136 \mathrm{eP} \quad 4119.40-0.6$ | HNR

$\begin{array}{lllll}86.25 & 331 \text { iPC } 4121.00 & 0.7\end{array}$

$1.0 \mathrm{~s} \quad 310.00 \mathrm{~nm}$

$6.5 \mathrm{mb}$

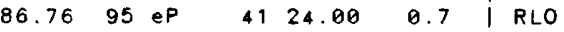

$86.77358 \mathrm{eP} \quad 4123.50 \quad 0.7$ । MNA

86.98346 ifC $4123.40-0.5$ G8O

$0.8 \mathrm{~s} 103.52 \mathrm{~nm} \quad 6.1 \mathrm{mb} / \mathrm{ACO}$

$\begin{array}{cccccc}86.99 & \text { PP } & 41 & 27.50 & 13 \mathrm{~km} \\ 8 P & 41 & 23.00 & -1.0\end{array}$

$87.10332 \mathrm{ePC} \quad 4125.100 .6$

D. $9 \mathrm{~s} 319.00 \mathrm{~nm}$

87.40331 eP $4126.80 \quad 0.8 \quad$ BKS

$0.9 \mathrm{~s} \quad 350.42 \mathrm{~nm}$

$87.50136 \mathrm{eP}$

PP $4130.90^{6.6 \mathrm{mb}}$

88.00331 eP

$88.27137 \mathrm{eP}$

$\begin{array}{lll}4125.60 & -0.8\end{array}$

$\begin{array}{llll}1129.00 & -1.1\end{array}$

$\begin{array}{llll}41 & 30.30 & -0.2\end{array}$

$88.847 \mathrm{eP}^{2} \quad 4133.50^{6.4 \mathrm{mb}} 0.8$

$1.0 \mathrm{~s} \quad 340.00 \mathrm{~nm} \quad 6.6 \mathrm{mb}$

89.10 $117 \mathrm{Pd} 4138.30$ 4.0X ADE

$1.1 \mathrm{~s} \quad 217.60 \mathrm{~nm}$

89.11117 IFC $4133.90-0.5 \quad$ RMU

\begin{tabular}{ccc|c} 
ES & 4354.78 & -0.5 & RMU \\
iPCP & 45 & 19.80 & BHO
\end{tabular}

\begin{tabular}{llll|l}
$89.4399 \mathrm{eP}$ & 45 & 19.80 & & \\
81.00 & $-5.0 \times$ & CLC
\end{tabular}

$\begin{array}{lllllll}89.43 & 99 & \mathrm{eP} & 41 & 31.00 & -5.0 \times & \mathrm{CLC} \\ 89.51356 & \mathrm{iPC} & 41 & 36.10 & 0.1 & \text { ISA }\end{array}$

$0.7 \mathrm{~s} 674.00 \mathrm{~nm} / \mathrm{mb}$ ALO

$89.51176 \mathrm{eP} \quad 4140.00 \quad 4.5 \mathrm{X}$

\begin{tabular}{lllll|l}
89.69 & $94 \mathrm{eP}$ & 41 & 37.50 & 0.2 & GSC
\end{tabular}

$90.20329 \mathrm{eP} \quad 4140.00 \quad 0.7$ I FDF

\begin{tabular}{lllll|l}
90.69 & 2 eP & 4142.00 & 0.6 & SJG
\end{tabular}

$1.0 \mathrm{~s} \quad 408.00 \mathrm{~nm}$

$90.94335 \stackrel{P P}{P}$

$91.03335 \mathrm{P}$

$91.17359 \mathrm{iPC}$

91.18359 iP

$91.195 \mathrm{eP}$

$91.32335 \mathrm{P}$

$91.33101 \mathrm{ePt}$

$91.37 \quad 120 \mathrm{iPC}$

91.58359 iPC

$91.7894 \mathrm{eP}$

$91.78 \quad 0 \mathrm{IPC}$

$22 \mathrm{~s} \quad 50.00 \mathrm{um}$

$91.78359 \quad \mathrm{iP}$

$91.82359 \mathrm{iPC}$

$92.48336 \mathrm{ePc}$

93.04336 iPd

$93.21114 \mathrm{eP}^{P}$
$4205.0084 \mathrm{kmX}$ SBB \begin{tabular}{llc|l|l|}
41 & 43.00 & 0.3 & SYP
\end{tabular} $4123.00-20.1 \times$ | MWC $4144.00 \quad 0.2$ | PAS $4144.40 \quad 0.5 \quad$ PAS $\begin{array}{lll}41 & 44.50 & 0.8\end{array}$ \begin{tabular}{lll|l}
4143.00 & -1.4 & PLM
\end{tabular} $4144.00 \quad-0.8$ | BRS $\begin{array}{lll}4145.00 & 0.2\end{array}$ \begin{tabular}{lll|l}
41 & 46.10 & 0.5 & SOB
\end{tabular} \begin{tabular}{lll|l}
4146.00 & -0.9 & I GLA
\end{tabular}

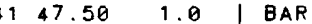
$4151.00^{6.9 M s z} 11 \mathrm{~km}$ | MAW $4146.80 \quad 0.2 \quad$ I CAN \begin{tabular}{lll|l}
4147.80 & 0.9 & SYO
\end{tabular} $4149.90 \quad 0.1$ I CAR \begin{tabular}{lll|l}
41 & 47.50 & $-4.9 x$ & TAU \\
41 & 53.00 & -0.3 &
\end{tabular} $\begin{array}{lllll}93.95 \quad 357 \mathrm{eP} & 4158.40 & 1.6\end{array}$ 94.14357 IPC $\quad 4158.50 \quad 0.8$
$94.32 \quad 109$ $\begin{array}{lllll}94.32 & 100 \text { eP } \quad 4159.00 & 0.5\end{array}$ $\begin{array}{llllll}95.16 & 351 \mathrm{iPc} & 42 & 03.20 & 0.8 \\ 1.3 \mathrm{~s} & 556.45 \mathrm{~nm} & & 6.8 \mathrm{mb}\end{array}$ PP $4207.30 \quad 13 \mathrm{~km}$ $\begin{array}{llllll}95.24 & 5 \mathrm{PP} & \mathrm{iPd} & 42 & 05.00 & 2.5\end{array}$ $95.97332 \mathrm{ePt} 4207.50 \quad 1.5$ $Z 22 \mathrm{~s} 101.48 \mathrm{um} \quad 7.3 \mathrm{Msz}$ $\begin{array}{llllll}97.02 & 355 & \mathrm{PPC} & 42 & 11.30 & 0.4\end{array}$ $1.1 \mathrm{~s} \quad 116.94 \mathrm{~nm} \quad 6.4 \mathrm{mb}$ $\begin{array}{llll}\text { PP } & 42 & 16.70 & 17 \mathrm{~km} \\ \mathrm{iPd} & 42 & 14.90 & 1.8\end{array}$ $\begin{array}{llll}97.55 & 110 \mathrm{iPd} & 42 & 14.90 \quad 1.8\end{array}$ $1.25 \quad 50.78 \mathrm{~nm} 45 \quad 07.00$ i (PP) $46 \quad 10.00$ is 5250.00

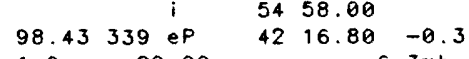

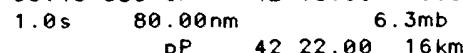

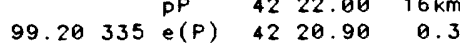
$0.7 \mathrm{~s} \quad 27.14 \mathrm{~nm} \quad 6.0 \mathrm{mb}$ $99.28 \quad 5 \quad \mathrm{eP}^{P} \quad 4221.00 \quad 0.1$ $\begin{array}{lllll}99.61 & 0 \mathrm{ePP} & 47 & 24.00 & \\ \mathrm{iP} & 42 & 24.10 & 1.5\end{array}$

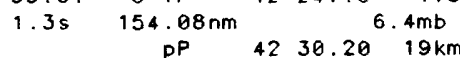
$\begin{array}{llllll}99.61 & 351 \mathrm{PP} & 42 & 30.20 & 19 \mathrm{~km} \\ 1.5 & 42 & 23.20 & 0.5\end{array}$ $1.5 \mathrm{~s} \quad 428.13 \mathrm{~nm} \quad 6.8 \mathrm{mb}$ $\begin{array}{lllll}99.68 & 351 & \text { e } \quad 42 \quad 23.00 & -0.1\end{array}$ $\begin{array}{lll}1.2 \mathrm{~s} & 117.21 \mathrm{~nm} \quad 6.3 \mathrm{mb}\end{array}$

$Z 19 \mathrm{~s} \quad 41.98 \mathrm{um} \quad 7.0 \mathrm{Msz}$ $99.77 \quad 357$ eP $\quad 42 \quad 24.50 \quad 1.2$ 101.0493 ePdiff $42 \quad 28.00-1.1$ $\begin{array}{lll}\text { EPP } & 46 & 40.00\end{array}$

101.31342 ePdiff $42 \quad 29.60 \quad-0.3$ $101.59 \quad 1$ ePditt42 $32.40 \quad 1.1$ 101.65342 elPdit42 $28.50 \quad-2.9 x$ 101.67346 elPdif42 $31.70 \quad 0.2$ $101.73 \quad 343$ ePdiff42 $32.30 \quad 0.6$

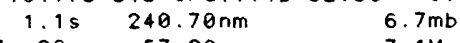
$220 \mathrm{~s} 57.20 \mathrm{um} \quad 7.1 \mathrm{Msz}$

$101.91 \quad 122$ ePdiff42 $34.00 \quad 1.4$ $101.99 \quad 5$ iPdiff42 $41.50 \quad 8.7 x$ $\begin{array}{ccc}1.0 \mathrm{~s} & 109.00 \mathrm{~nm} & 6.4 \mathrm{mb} \\ 20 \mathrm{~s} & 15.00 \mathrm{~mm} & 6.5 \mathrm{Ms}\end{array}$

$\begin{array}{lll}Z & 20 \mathrm{~s} & 15.00 \mathrm{um} \\ \mathrm{N} & 20 \mathrm{~s} & 37.00 \mathrm{um}\end{array}$

E $20 \mathrm{~s} \quad 48.00 \mathrm{um}$ PPPS $56 \quad 00.00$ ESS 0120.00

$\begin{array}{lllll}102.01 & 3 \text { ePdiff } 42 & 33.80 & 0.8\end{array}$ ePKKP $59 \quad 05.00$ EP.P. 0700.00

$102.07 \quad 126$ ePdiff42 $35.00 \quad 1.7$

$1.0 \mathrm{~s} \quad 40.00 \mathrm{~nm} \quad 6.0 \mathrm{mb}$ $102.78 \quad 355$ ePdiff $42 \quad 37.90 \quad 1.3$ 102.98342 el Pdit42 $36.00 \quad-1.3$ $1.6 \mathrm{~s} \quad 243.60 \mathrm{~nm} \quad 6.7 \mathrm{mb}$ $104.21 \quad 1$ ePdiff42 $49.00 \quad 6.1 \mathrm{x}$ 104.352 ePdift $4250.00 \quad 6.5 \mathrm{X}$ 104.50351 ePdiff42 $45.00 \quad 0.6$ $719 \mathrm{~s} \quad 53.82 \mathrm{um} \quad 7.1 \mathrm{Msz}$ 104.73 DePdiff42 $46.00 \quad 0.8$ 104.83304 ePdiff $42 \quad 47.50 \quad 1.5$ 105.26310 elPdit42 $48.00 \quad 0.1$

$z 20 \mathrm{~s} \quad 37.59 \mathrm{um} \quad 6.9 \mathrm{Msz}$ $105.33 \quad 1$ ePdiff42 $51.00 \quad 3.1 x$ 105.443 ePdiff43 $02.00 \quad 13.5 x$ $105.79 \quad 1$ epdiff42 $54.00 \quad 3.9 x$ $\begin{array}{lll}105.87 \quad 1 \text { ePdiff43 } 06.00 \quad 15.8 x & 000\end{array}$ $\begin{array}{llllll}105.87 & 1 \text { ePKP } 47 \quad 16.00 & 13.2 X\end{array}$ 106.67 epdiff4 $58.00 \quad 4.0 x$ 106.75112 iPdiff42 $58.50 \quad 4.2 x$ eS $53 \quad 34.00$ $106.76 \quad 272$ ePdiff43 $11.70 \quad 17.1 \mathrm{x}$ $\begin{array}{lllll}106.95 & 358 \text { ePdiff43 } 03.00 \quad 7.9 x\end{array}$ $107.34 \quad 0$ epdiff43 $05.00 \quad 8.2 x$ 107.58343 (Pdiff $4300.00 \quad 2.1 x$ $107.62 \quad 180$ ePKP $47 \quad 10.00 \quad 5.0 x$ $\begin{array}{lllll}108.88 & 120 \text { ePKP } & 47 & 11.00 & 2.5\end{array}$ $\begin{array}{llllll}110.46 \quad 189 & \text { ePKP } 47 \quad 10.40 & 0.0\end{array}$

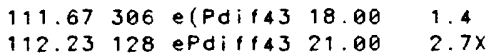




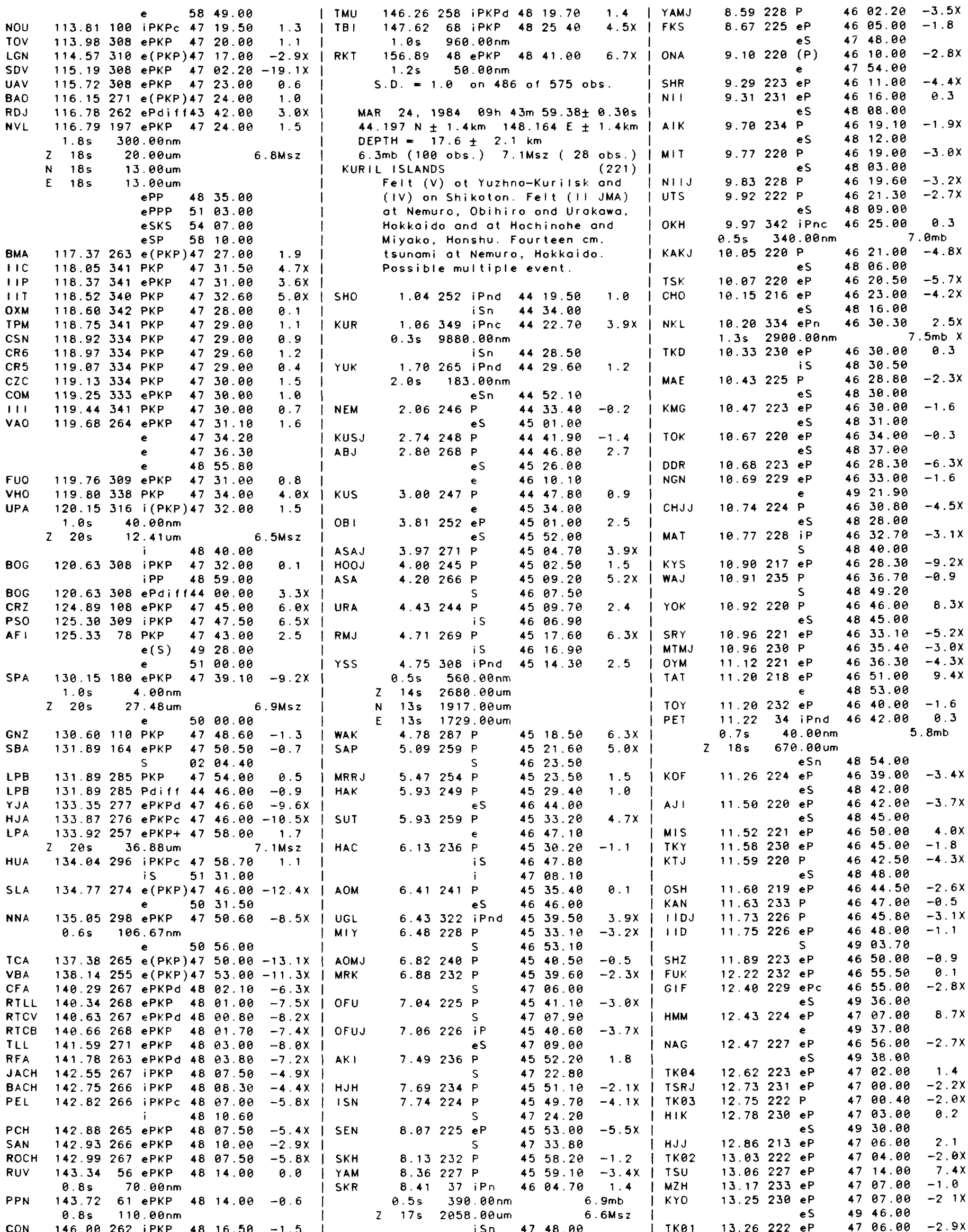




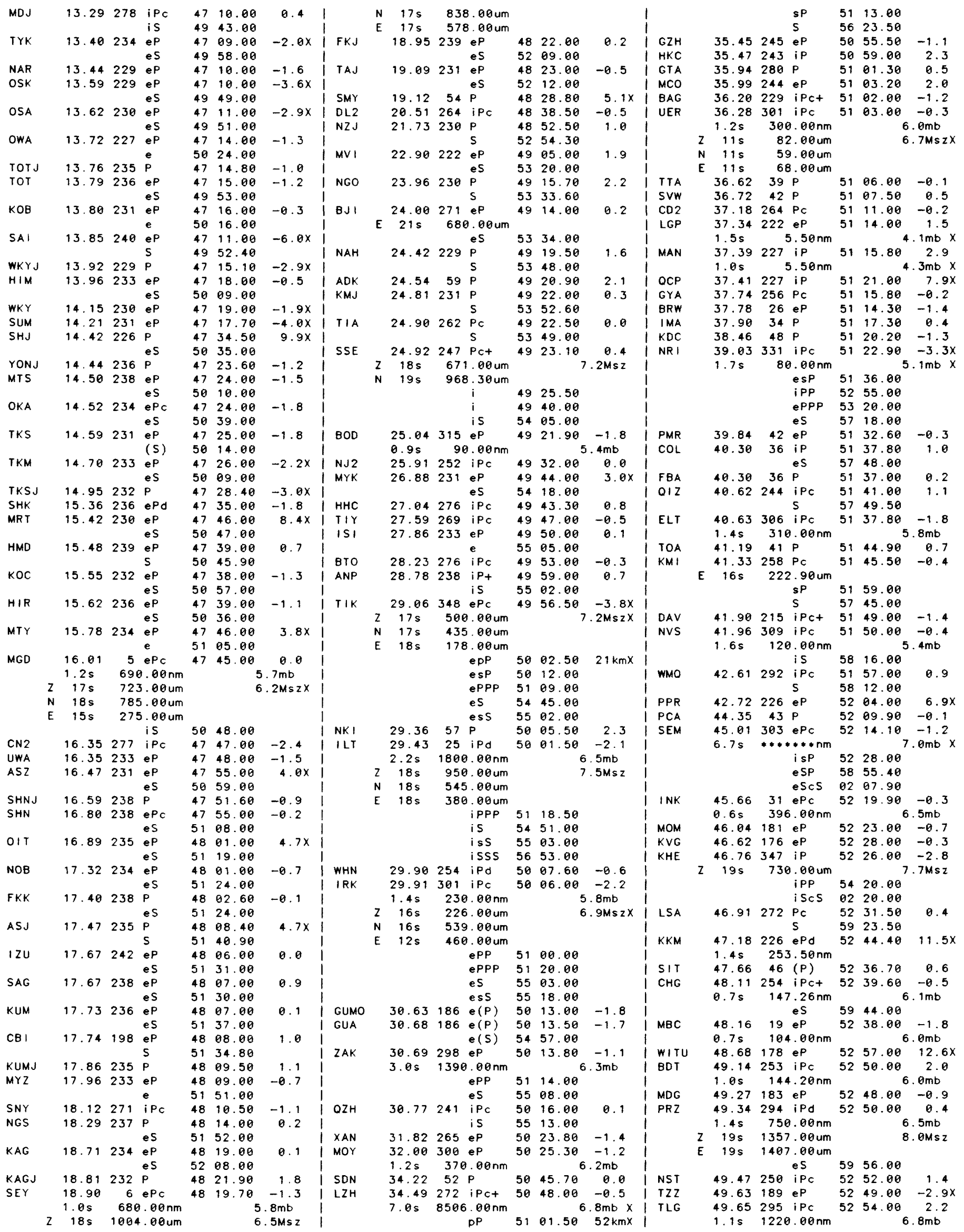




\begin{tabular}{|c|c|c|c|c|c|c|c|c|c|c|c|}
\hline & Z & $16 \mathrm{~s}$ & 439 & $9.00 \mathrm{um}$ & & & $7.6 \mathrm{MszX}$ & 1 & $19 \mathrm{~s}$ & 358 & $0.00 \mathrm{um}$ \\
\hline & & & & is & 80 & 00.00 & & i & & & IPCP \\
\hline HON & & 49.69 & 99 & $P$ & 53 & 02.60 & $9.8 x$ & 1 & & & IPP \\
\hline AAA & & 49.94 & 295 & iPc & 52 & 55.40 & 1.4 & 1 & & & iPPP \\
\hline & $z$ & $15 s$ & 486 & $6.00 \mathrm{um}$ & & & $7.6 \mathrm{Ms} 2 \mathrm{X}$ & I NOI & 57.45 & 280 & iPc \\
\hline & $\mathrm{N}$ & $15 \mathrm{~s}$ & 720 & $0.00 \mathrm{um}$ & & & & I & $0.6 \mathrm{~s}$ & 386 & $6.67 \mathrm{~nm}$ \\
\hline & $E$ & $15 s$ & 532 & $2.00 \mathrm{um}$ & & & & 1 & $18 s$ & & $0.21 \mathrm{um}$ \\
\hline & & & & is & 00 & 04.00 & & 1 & $18 \mathrm{~s}$ & & B. $49 u m$ \\
\hline$B G A$ & & 50.51 & 171 & eP & 52 & 57.50 & -1.1 & 1 & $18 \mathrm{~s}$ & & 7.49 um \\
\hline LAT & & 50.62 & 181 & eP & 52 & 58.00 & -1.3 & 1 & & & is \\
\hline PAA & & 56.70 & 171 & $e(P)$ & 53 & 01.00 & 1.0 & I DSH & 57.65 & 294 & iPc \\
\hline NRN & & 51.42 & 294 & $e^{P}$ & 53 & 05.10 & -0.5 & 1 & $6.5 \mathrm{~s}$ & $* * * *$ & $* * * * \mathrm{~nm}$ \\
\hline & & & & es & 00 & 24.00 & & 1 & $16 \mathrm{~s}$ & 825 & $5.00 \mathrm{um}$ \\
\hline FRU & & 51.65 & 296 & iP & 53 & 07.80 & 0.8 & I & & & es \\
\hline & & $8.0 \mathrm{~s}$ & $* * * *$ & $* * * * \mathrm{~nm}$ & & & $7.3 \mathrm{mb} \times$ & I KGM & 57.73 & 236 & ePc \\
\hline & $z$ & $15 \mathrm{~s}$ & 650 & 0.00 um & & & 7. $8 \mathrm{MszX}$ & I PGC & 57.76 & 52 & $e^{P}$ \\
\hline & $N$ & $15 s$ & 750 & $0.00 \mathrm{um}$ & & & & I KLM & 57.89 & 239 & eP \\
\hline & $E$ & $16 \mathrm{~s}$ & 658 & B. 00 um & & & & I SAM & 58.20 & 296 & IPC \\
\hline & & & & IPCP & 54 & 16.00 & & i & $1.0 \mathrm{~s}$ & 3400 & $0.00 \mathrm{~nm}$ \\
\hline & & & & is & 00 & 28.00 & & 1 & $16 s$ & 560 & $0.00 \mathrm{um}$ \\
\hline & & & & iss & 03 & 59.00 & & 1 & & & IPP \\
\hline KKN & & 52.22 & 274 & $e^{P}$ & 53 & 10.90 & -0.8 & 1 & & & IPPP \\
\hline PKI & & 52.25 & 274 & $e P$ & 53 & 11.20 & -0.9 & 1 & & & is \\
\hline & & $0.9 \mathrm{~s}$ & 710 & $0.00 \mathrm{~nm}$ & & & $6.6 \mathrm{mb}$ & I APA & 58.30 & 336 & iPc \\
\hline KSH & & 52.40 & 292 & $i \mathrm{Pc}$ & 53 & 13.00 & 0.1 & 1 & $1.2 \mathrm{~s}$ & 160 & $0.00 \mathrm{~nm}$ \\
\hline DMN & & 52.45 & 274 & eP & 53 & 12.20 & -1.3 & 1 & $19 s$ & 360 & $0.00 \mathrm{um}$ \\
\hline LMG & & 52.85 & 180 & eP & 53 & 11.00 & $-5.3 x$ & 1 & $19 \mathrm{~s}$ & 180 & $0.00 \mathrm{um}$ \\
\hline SVE & & 53.33 & 317 & iPc & 53 & 18.00 & -1.3 & 1 & $19 \mathrm{~s}$ & 279 & $9.00 \mathrm{um}$ \\
\hline & & $2.8 \mathrm{~s}$ & 900 & $0.00 \mathrm{~nm}$ & & & $6.2 \mathrm{mb}$ & 1 & & & iPCP \\
\hline & $z$ & $16 \mathrm{~s}$ & 1017 & $7.00 \mathrm{um}$ & & & 8. $0 M s z X$ & 1 & & & is \\
\hline & $\mathrm{N}$ & $16 \mathrm{~s}$ & 304 & $4.00 \mathrm{um}$ & & & & I KUPT & 58.50 & 209 & $e(P)$ \\
\hline & $E$ & $16 s$ & 464 & $4.00 \mathrm{um}$ & & & & I KUG & 58.52 & 209 & eP \\
\hline & & & & ePcP & 54 & 28.00 & & I KEV & 58.55 & 340 & ipt \\
\hline & & & & ePP & 55 & 15.00 & & 1 & $0.8 \mathrm{~s}$ & & $6.00 \mathrm{~nm}$ \\
\hline & & & & es & 00 & 44.00 & & 1 & & & $\mathrm{i}$ \\
\hline PMG & & 53.36 & 181 & eP & 53 & 20.00 & 0.2 & 1 & & & isp \\
\hline & & & & es & 00 & 50.00 & & 1 & & & ePPP \\
\hline ANR & & 54.14 & 295 & iPc & 53 & 25.46 & -0.1 & I & & & es \\
\hline & & $1.2 \mathrm{~s}$ & 1200 & $0.00 \mathrm{~nm}$ & & & $6.8 \mathrm{mb}$ & I GMW & 58.73 & 52 & eP \\
\hline & $z$ & $15 \mathrm{~s}$ & 520 & $0.00 \mathrm{um}$ & & & $7.7 M \leq z X$ & 1 & & & e \\
\hline & $N$ & $15 \mathrm{~s}$ & 850 & $0.00 \mathrm{um}$ & & & & I MTN & 58.85 & 200 & eP \\
\hline & $E$ & $15 \mathrm{~s}$ & 500 & $0.00 \mathrm{um}$ & & & & I DAG & 59.03 & 357 & iPc \\
\hline & & & & isp & 53 & 41.50 & & 1 & $0.8 \mathrm{~s}$ & & $4.63 \mathrm{~nm}$ \\
\hline & & & & is & 01 & 01.30 & & 1 & & & $i$ \\
\hline KBS & & 54.47 & 351 & eP & 53 & 29.90 & 2.4 & 1 & & & $i$ \\
\hline $\mathrm{PHC}$ & & 54.49 & 51 & eP & 53 & 27.56 & -0.4 & I MED & 59.39 & 242 & $e(P)$ \\
\hline & & $1.0 \mathrm{~s}$ & 221 & $1.00 \mathrm{~nm}$ & & & 6. $1 \mathrm{mb}$ & I PNT & 59.53 & 49 & eP \\
\hline ARU & & 54.52 & 317 & iPc & 53 & 26.70 & -1.3 & I SHW & 59.79 & 53 & $e P$ \\
\hline & & $2.8 \mathrm{~s}$ & 1800 & $0.00 \mathrm{~nm}$ & & & $6.6 \mathrm{mb}$ & I COR & 60.06 & 56 & IPd \\
\hline & 2 & $16 \mathrm{~s}$ & 822 & $2.00 \mathrm{um}$ & & & $7.9 M s z X$ & 1500 & 60.33 & 338 & iP \\
\hline & $N$ & $16 \mathrm{~s}$ & 370 & $0.08 \mathrm{um}$ & & & & I & & & $i$ \\
\hline & $E$ & $16 \mathrm{~s}$ & 653 & $3.00 \mathrm{um}$ & & & & 1 & & & $\mathrm{i}$ \\
\hline & & & & ePP & 55 & 26.00 & & I & & & e \\
\hline & & & & eSP & 01 & 11.00 & & I DNP & 60.49 & 218 & eP \\
\hline YKA & & 55.02 & 34 & $e^{P}$ & 53 & 31.00 & -0.7 & I TRO & 60.56 & 342 & eP \\
\hline RSNT & & 55.04 & 34 & eP & 53 & 30.30 & -1.4 & EDM & 60.58 & 43 & ePC \\
\hline & & $1.2 \mathrm{~s}$ & 275 & $5.86 \mathrm{~nm}$ & & & $6.2 \mathrm{mb}$ & | TRT & 60.81 & 221 & iPd \\
\hline & & & & e & 55 & 42.00 & & I NEW & 61.49 & 49 & P \\
\hline$Y K C$ & & 55.08 & 34 & eP & 53 & 31.00 & -1.1 & I NEW & 61.49 & 49 & eP \\
\hline & & $0.6 \mathrm{~s}$ & 137 & $7.00 \mathrm{~nm}$ & & & $6.2 \mathrm{mb}$ & I & $20 s$ & & $0.00 \mathrm{um}$ \\
\hline SNG & & 55.39 & 243 & iPct & 53 & 35.60 & 0.1 & 1 & & & e \\
\hline & & $1.0 \mathrm{~s}$ & 420 & $0.00 \mathrm{~nm}$ & & & $6.4 \mathrm{mb}$ & 1 & & & e \\
\hline & $z$ & $22 \mathrm{~s}$ & 33 & $3.70 \mathrm{um}$ & & & $6.4 \mathrm{Msz}$ & I FHC & 61.79 & 59 & $e(P)$ \\
\hline & $N$ & $22 \mathrm{~s}$ & 48 & $3.89 \mathrm{um}$ & & & & I YKM & 61.90 & 48 & $i P c$ \\
\hline & $E$ & $20 \mathrm{~s}$ & & $1.56 \mathrm{um}$ & & & & I PNO & 62.11 & 52 & eP \\
\hline & & & & es & 01 & 17.00 & & I RXF & 62.21 & 48 & iP \\
\hline MKS & & 55.55 & 215 & iPc & 53 & 36.00 & 0.0 & I KNA & 62.23 & 201 & eP \\
\hline TAS & & 55.83 & 297 & iPc & 53 & 37.00 & -0.9 & $\mathrm{KJF}$ & 62.28 & 335 & iP \\
\hline & & $1.5 \mathrm{~s}$ & 2040 & $0.08 \mathrm{~nm}$ & & & $6.9 \mathrm{mb}$ & I & $0.9 \mathrm{~s}$ & 152 & 2. $10 \mathrm{~nm}$ \\
\hline & $E$ & $15 \mathrm{~s}$ & 702 & $2.00 \mathrm{um}$ & & & & 1 & & & $i$ \\
\hline & & & & ePP & 55 & 50.00 & & 1 & & & ePPP \\
\hline & & & & es & 01 & 28.00 & & 1 & & & es \\
\hline GAR & & 56.40 & 294 & iP & 53 & 41.00 & -1.1 & 1 & & & e \\
\hline & & $2.5 \mathrm{~s}$ & 1020 & $0.00 \mathrm{~nm}$ & & & $6.4 \mathrm{mb}$ & I LHD & 62.34 & 49 & $\mathrm{iPc}$ \\
\hline & & & & is & 01 & 30.50 & & I LOM & 62.35 & 48 & iPc \\
\hline $\mathrm{KHO}$ & & 56.41 & 292 & $\mathrm{iPC}$ & 53 & 41.40 & -0.9 & $1 C L X$ & 62.60 & 48 & iPc \\
\hline & $z$ & $30 \mathrm{~s}$ & 370 & $0.00 \mathrm{um}$ & & & 7. $3 M s z X$ & I KSI & 62.71 & 233 & $e^{P}$ \\
\hline & $N$ & $30 \mathrm{~s}$ & 299 & $9.00 \mathrm{um}$ & & & & 1 & & & e \\
\hline & $E$ & $30 \mathrm{~s}$ & 153 & $3.00 \mathrm{um}$ & & & & 1 WDC & 62.81 & 59 & ePc \\
\hline & & & & es & 01 & 31.00 & & 1 & & & $\mathrm{i}$ \\
\hline & & & & escs & 03 & 27.00 & & 1 & & & $i$ \\
\hline IPM & & 57.12 & 240 & ePd & 53 & 48.00 & 0.7 & I & & & $\mathrm{i}$ \\
\hline & & $1.9 \mathrm{~s}$ & 231 & $1.40 \mathrm{~nm}$ & & & $5.9 \mathrm{mb}$ & 1 & & & eP'P' \\
\hline & & & & e & 53 & 58.30 & & RMT & 63.12 & 60 & eP \\
\hline KUL & & 57.37 & 293 & ePc & 53 & 48.50 & -0.4 & 1 & & & $i$ \\
\hline & & $8.0 \mathrm{~s}$ & $* * * *$ & $* * * * \mathrm{~nm}$ & & & $7.2 \mathrm{mb} \times$ & I HYB & 63.52 & 269 & ePc \\
\hline & $N$ & $17 \mathrm{~s}$ & 175 & 5.00 um & & & & I & $0.8 \mathrm{~s}$ & 500 & $0.00 \mathrm{~nm}$ \\
\hline
\end{tabular}


$24 d 09 h$

KOU

BM

POO
PRI
FRI
MNA
GBA
KHI
NDF
EUR
VUN
SVA
MAK

$\begin{array}{llllll}\text { POO } & 66.17 & 273 & \mathrm{iPc} & 54 & 47.30 \\ \text { PRI } & 66.67 & 62 & \mathrm{e}(\mathrm{P}) & 54 & 50.00\end{array}$

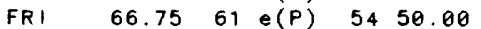

MNA $\quad 66.8159 \mathrm{eP} \quad 5451.00 \quad-1.2$

$66.852961 .00 \mathrm{~nm}$

$5452.3 \theta^{6.2 m b}$

$67.48 \quad 56$ ip

$0.2 \mathrm{~s} \quad 25.68 \mathrm{~nm}$

$5508.2013 .6 \times 1$ BKR

$67.82149 .68 \mathrm{~nm} \quad 6.0 \mathrm{mb}$

$7.0 \mathrm{~s} * * * * * * \mathrm{~nm} \quad 5501.10 \quad 1.5$

$Z 21 \mathrm{~s} \quad 245.00 \mathrm{um}$

N $21 \mathrm{~s} 402.00 \mathrm{um}$

E $18 \mathrm{~s} \quad 253.00 \mathrm{um}$

ipP $\quad 55 \quad 10.30 \quad 30 \mathrm{kmx}$

iPCP 5525.80

is 0357.10

CWC 68.1160 iPd 5502.80

$\begin{array}{lll} & e & 5513.00 \\ & e & 5604.00\end{array}$

SYP $\quad 68.12 \quad 63 \mathrm{eP} \quad 5503.00$

NOU $68.27 \quad 162$ iPd 5503.00

is 0404.00

$\begin{array}{lllll}\text { WKTM } & 68.29 & 61 \mathrm{eP} & 5501.30\end{array}$

BAK 68.32306 iPd 5506.00

$6.05 * * * * * n \mathrm{~nm}$

EP 5518.00

ISA $\quad 68.35 \quad 61 \mathrm{eP} \quad 5500.00$

AF

$68.37 \quad 138 \mathrm{eP}$

ess

0356.00

$\begin{array}{ll}55 & 05.00 \\ 0420.00\end{array}$

UPP

$68.55 \quad 17 \mathrm{ePC}$

08.67336 iP

5501.00

$\begin{array}{llll}55 & 01.80 & -1.4\end{array}$

$5504.60^{6.1}$

5513.40

0401.10

$1 \mathrm{mb}$

is

$68.78 \quad 194 \mathrm{iPC}$

$0.8 \mathrm{~s} \quad 43.00 \mathrm{~nm}$

5502.58

$-1$

GRO

$2.0 \mathrm{~s} \quad 1700.00 \mathrm{~nm}$

$68.8160 \mathrm{eP}$

$68.89 \quad 54 \mathrm{eP}$

$68.90306 \mathrm{P}$

$69.05 \quad 50 \mathrm{eP}$

$69.38 \quad 61 \mathrm{eP}$

$69.45339 \mathrm{P}$

$69.5162 \mathrm{eP}$

$1.5 \mathrm{~s} 600.00 \mathrm{~nm}$

5758.00

ePPP $\quad 00 \quad 02.00$

eS 0400.00

ESS $\theta 916.00$

MWC

$.5362 \mathrm{eP}$

69.56338 ip

$0.5 \mathrm{~s} \quad 88.00 \mathrm{~nm}$

5510.00

0414.00

$55 \quad 07.20$

-0.3
$0.9 \mathrm{mb}$

$6.9 \mathrm{mb}$

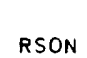

BRS

2.3

BAR

1.9 LEN

-0.2
$4.6 x$

$3 \mathrm{mb} \times 1$

$-1.8$

3.0

ERE GCA

REY

SOC

TAB

RMU

ANN

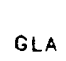

$5505.00 \quad 0.0$ |WBN

$5451.60-14.6 \times$ । NAU

\begin{tabular}{lll|l}
507.00 & -1.2 & $\mathrm{GOL}$
\end{tabular}

$-1.3$

$.5 \mathrm{mb}$

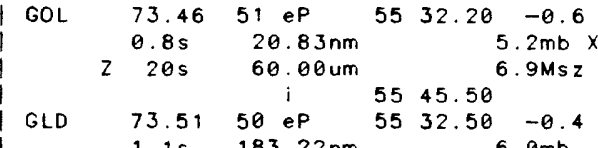

$\left\{\begin{array}{ccccc} & 0.8 \mathrm{~s} & 20.83 \mathrm{~nm} & 5.2 \mathrm{mb} x \\ 20 \mathrm{~s} & 60.00 \mathrm{um} & 6.9 \mathrm{Msz} \\ & & \mathrm{i} & 5545.50 & \\ \text { GLD } & 73.51 & 50 \mathrm{eP} & 5532.50 & -0.4\end{array}\right.$

$1.1 \mathrm{~s} \quad 183.22 \mathrm{~nm}$

$220 \mathrm{~s} 68.00 \mathrm{um}$

$73.68317 \mathrm{eP}$

$6.0 \mathrm{~s} \quad * * * * * * * \mathrm{~nm}$

$Z \quad 17 \mathrm{~s} \quad 450.00 \mathrm{um}$

$\begin{array}{lll}\mathrm{N} & 17 \mathrm{~s} & 414.00 \mathrm{um} \\ \mathrm{E} & 17 \mathrm{~s} & 215.00 \mathrm{um}\end{array}$

$$
\text { IPCP }
$$

e $\quad 5520.00$

PYA

69.76312

$3.0 \mathrm{~s} 2500.00 \mathrm{~nm}$

isP $55 \quad 23.00$

iSP 0435.00

MNK $\quad 69.92327 \mathrm{IPC} \quad 5508.00$ ePP 5740.00

AKU $\quad 69.94 \quad 354$ iPC 5511.90

$1.0 \mathrm{~s} \quad 448.00 \mathrm{~nm}$

$218 \mathrm{~s} \quad 118.21 \mathrm{um}$

$511.90 \quad 1.0$

$70.1262 \mathrm{eP}$

KRV

70.21308 eP

es

$5511.00^{7.2 \mathrm{Msz}}$

$\begin{array}{lll}55 & 12.00 & -1.0\end{array}$

0417.00

MTA

$\begin{array}{rrrr}70.37 & 309 & & \\ 2 & 14.00 & 0.0\end{array}$

N $20 \mathrm{~s} \quad 136.00 \mathrm{um}$

E $20 \mathrm{~s} \quad 136.00 \mathrm{um}$

is 0426.00
7369336 iPC
0.55

$0.5 \mathrm{~s} \quad 154.93 \mathrm{~nm}$

$73.69329 \mathrm{ePt}^{\mathrm{i}}$
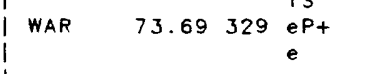

MUD
KER
LVV
E $20 \mathrm{~s} \quad 720.00 \mathrm{um}$

iPP $\quad 5828.00$

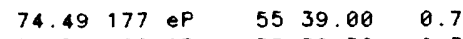
$\begin{array}{lllll}74.59 & 322 \text { iPc } 5538.50 & -0.3\end{array}$ $\begin{array}{rrl}2.5 \mathrm{~s} & 2300.00 \mathrm{~nm} & 6.8 \mathrm{mb} \\ 17 \mathrm{~s} & 776.00 \mathrm{um} & 8.1 \mathrm{Msz}\end{array}$

$\begin{array}{lll}Z & 17 \mathrm{~s} & 776.00 \mathrm{um} \\ \mathrm{N} & 17 \mathrm{~s} & 475.00 \mathrm{um}\end{array}$

E $17 \mathrm{~s} \quad 309.00 \mathrm{um}$

$$
\begin{array}{ccccc} 
& \text { iPP } & 58 & 24.00 \\
\text { is } & 05 & 10.00 & \\
74.92 & 36 \mathrm{eP} & 55 & 39.50 & -1.2 \\
0.9 \mathrm{~s} & 264.00 \mathrm{~nm} & & 6.3 \mathrm{mb}
\end{array}
$$

$75.03323 \mathrm{eP} \quad 5543.00 \quad 1.7$

$\begin{array}{lllll}75.35 & 182 \mathrm{eP} & 5543.00 & -0.2\end{array}$

$\begin{array}{lllll}75.52 & 314 & \text { iP } & 5545.00 & 0.7\end{array}$

$\begin{array}{lllll}75.86 & 329 \text { iPC } \quad 55 \quad 45.40 & -0.6\end{array}$

$0.9 \mathrm{~s} \quad 1420.00 \mathrm{~nm} \quad 7.0 \mathrm{mb}$

$z \quad 16 \mathrm{~s} \quad 181.50 \mathrm{um}$

5547.70

7. $5 \mathrm{Ms} 2 \mathrm{X}$

$55 \quad 57.50$

$\begin{array}{ll}56 & 17.70\end{array}$

is 0534.00

$\begin{array}{lllll}75.95 & \mathrm{e} & 11 & 04.00 & \\ \mathrm{eP} & 55 & 46.00 & -0.6\end{array}$

$\begin{array}{rrrrr}76.10 & 326 \text { iPd } & 5547.50 & 0.1 \\ 17 \mathrm{~s} & 468.00 \mathrm{um} & & 7,9 \mathrm{Msz}\end{array}$

EPP $58 \quad 33.00$

is $\quad 0530.00$

$76.1555 \mathrm{iPC+} 5547.00 \quad-1.2$

$\begin{array}{lll}1.0 \mathrm{~s} & 85.00 \mathrm{~nm} & 5.8 \mathrm{mb} \\ 20 \mathrm{~s} & 74.47 \mathrm{um} & 7.0 \mathrm{Ms}\end{array}$

5600.00

$76.24334 \mathrm{eP} \quad 5549.00$

76.31321 ePd 5549.00

76.35336 iPC 5550.40

76.36303 iPd 5550.50

EPP 5852.00

EPPP 0040.00

ES $\quad 0532.50$

ESS 1055.00

$76.38325 \mathrm{ePd} \quad 5552.00 \quad 3.0 \mathrm{x}$

$\begin{array}{lllll}76.42 & 322 \mathrm{eP} & 55 & 50.00 & 0.7\end{array}$

$76.53 \quad 331$ iPC $5549.50 \quad-0.3$

$1.0 \mathrm{~s} \quad 1110.00 \mathrm{~nm}$

id 5550.20

id $\quad 5601.60$

is 0533.20

76.70322 iPd $5552.00 \quad 1.1$

$76.79326 \mathrm{eP} \quad 5555.00 \quad 3.7 \mathrm{X}$

76.82321 iPC $5552.00 \quad 0.5$

$76.90327 \mathrm{ePc} \quad 55 \quad 52.00 \quad 0.1$

$1.2 \mathrm{~s} \quad 10.00 \mathrm{~nm} \quad 4.8 \mathrm{mb} \times$

$76.98 \quad 20$ ePd $55 \quad 50.80 \quad-1.5$

$0.6 \mathrm{~s} \quad 54.00 \mathrm{~nm} \quad 5.8 \mathrm{mb}$

77.05322 iPd $55 \quad 53.00 \quad 0.0$

77.06322 iPd $5554.00 \quad 1.1$

$\begin{array}{ll}77.25 & 333 \mathrm{iPc} \quad 55 \quad 52.90 \quad-0.9\end{array}$

$1.7 \mathrm{~s} 3000.00 \mathrm{~nm}$

$\begin{array}{lll}i & 56 & 19.00 \\ \text { eS } & 05 & 40.00\end{array}$

$\begin{array}{lll}\text { eS } & 0540.00 \\ i & 0608.00\end{array}$

$77.31332 \mathrm{iPC}+5553.30 \quad-0.8$

$2.6 \mathrm{~s} \quad 1250.00 \mathrm{~nm} \quad 6.5 \mathrm{mb}$ i 5606.60 P.P. 2322.00

$77.45320 \mathrm{iPC} \quad 5557.00 \quad 2.1$

$77.63 \quad 323$ iPC $56 \quad 00.00 \quad 4.0 x$

$77.70177 \mathrm{eP} \quad 5556.00 \quad-0.2$

77.71316 eP $\quad 5556.00 \quad-0.5$

$\begin{array}{lllll}77.85 & 337 \mathrm{eP} & 5558.50 & 1.5\end{array}$

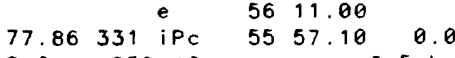

$2.0 \mathrm{~s} \quad 859.40 \mathrm{~nm} \quad 6.5 \mathrm{mb}$

$721 \mathrm{~s} \quad 523.80 \mathrm{um} \quad 7.8 \mathrm{Msz}$

$\begin{array}{llllll}55 & 56.00 & 1.5 & \mathrm{i} & 5610.00\end{array}$

\begin{tabular}{l|lll}
55 & 56.00 & $\mathrm{i}$ & 5610.00 \\
5828.00 & $\mathrm{~S}$ & 0548.00
\end{tabular}

$\begin{array}{llllll}\text { EKA } & 77.90 & 344 \mathrm{PC} & 55 & 58.00 & 0.7\end{array}$

0028.00

0119.00

\begin{tabular}{l|l|l}
0332.00 & BUC1 \\
0504.00 & BUC1
\end{tabular}

$0.6 \mathrm{~s} \quad 70.90 \mathrm{~nm} \quad 5.9 \mathrm{mb}$

77.91322 IPC $5540.00-17.5 x$

$\begin{array}{lllll}77.91 & 322 & \text { IPC } & 5557.00 & -0.5\end{array}$

$\begin{array}{lllll}78.05 & 324 & \text { ePd } 56 \quad 02.00 & 3.7 x\end{array}$

78.10180 eP $\quad 5558.60 \quad 0.1$

$\begin{array}{llllll} & \text { epP } & 56 & 12.20 & 47 \mathrm{kmx} \\ 78.27 & 333 \mathrm{iP} & 55 & 59.00 & -0.4\end{array}$

$1.6 \mathrm{~s} \quad 1574.00 \mathrm{~nm} \quad 6.8 \mathrm{mb}$

$218 \mathrm{~s} 264.00 \mathrm{um}$

N $18 \mathrm{~s} 201.00 \mathrm{um}$

E $18 \mathrm{~s} \quad 142.00 \mathrm{um}$ 


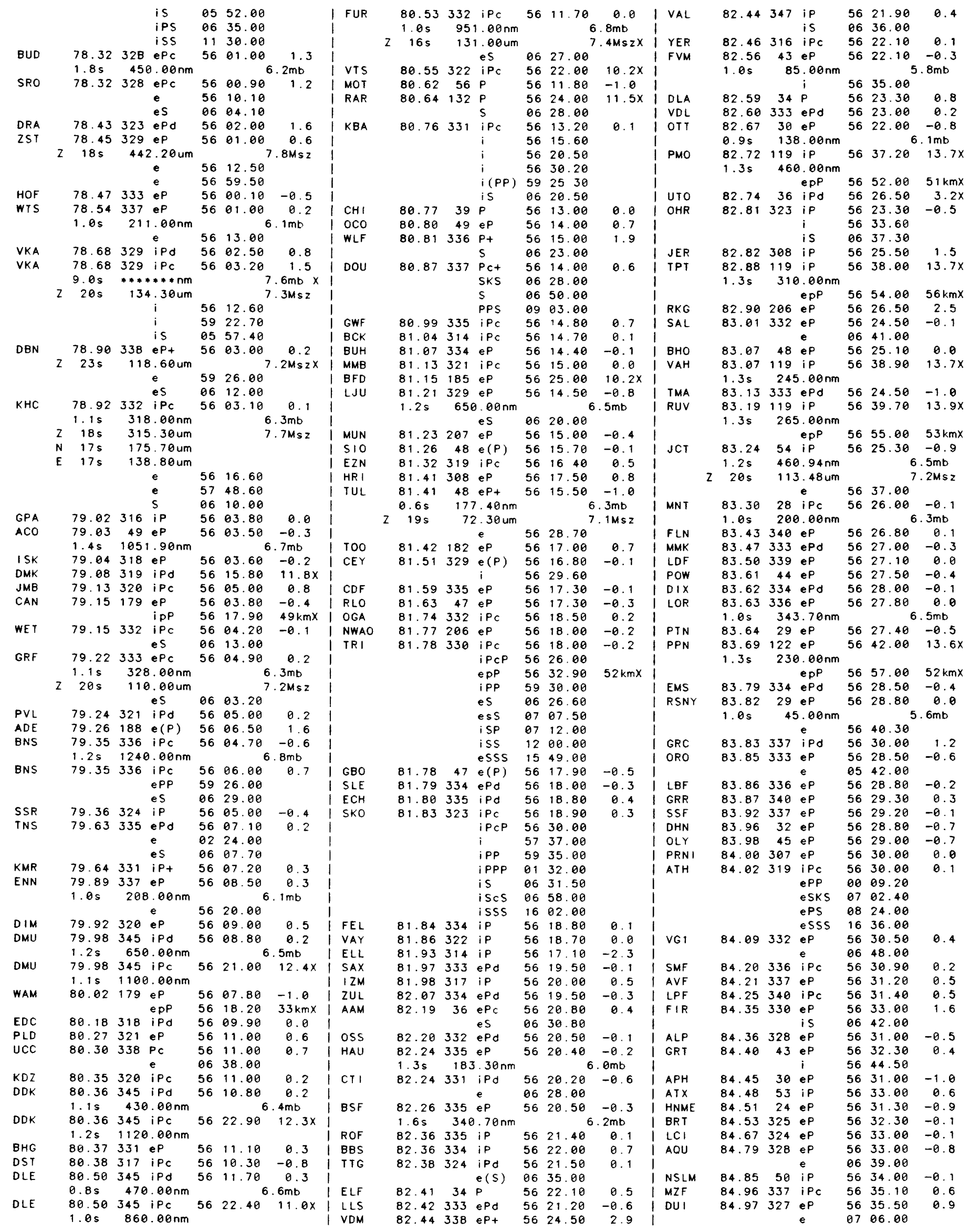


$24 d \quad 09 h$

\begin{tabular}{|c|c|c|c|c|c|c|c|c|c|c|c|}
\hline TCF & 85.00 & 337 & iPc & 56 & 35.10 & 0.4 & 1 & & & es & 08 \\
\hline & $1.6 \mathrm{~s}$ & 777 & 7. $46 \mathrm{~nm}$ & & & $.7 \mathrm{mb}$ & I CRT & 95.04 & 338 & eP & 57 \\
\hline$M \mid M$ & 85.01 & 25 & eP & 56 & 34.30 & -0.4 & I SFS & 96.42 & 339 & $\mathrm{ePc}$ & 57 \\
\hline & & & e & 56 & 47.80 & & 1 & & & & 57 \\
\hline MNS & 85.03 & 329 & eP & 56 & 34.00 & -0.8 & 1 & & & iPP & 9 \\
\hline & & & e & 07 & 01.00 & & 1 & & & iSKS & 08 \\
\hline LSF & 85.22 & 337 & eP & 56 & 36.20 & 0.4 & 1 & & & is & 10 \\
\hline SGG & 5.24 & 327 & EP & 56 & 36.49 & 0.3 & 1 & & & PS & 12 \\
\hline$M F F$ & 5.34 & 339 & eP & 56 & 36.80 & 0.4 & 1 & & & SP & 15 \\
\hline KRP & 85.41 & 159 & eP & 56 & 40.00 & $3.4 x$ & I & & & SS & 19 \\
\hline & & & (PC & 56 & 56.00 & & 1 & & & SSS & 26 \\
\hline & & & $(p P)$ & 57 & 03.00 & $85 \mathrm{kmx}$ & I AAE & 97.33 & 290 & eP & 57 \\
\hline RMP & 85.52 & 328 & iPc & 56 & 38.00 & 0.6 & I TA & 105.07 & 325 & iPdiff & $f 58$ \\
\hline & & & IPP & 00 & 32.00 & & AVY & 110.32 & 263 & ePKP & 02 \\
\hline & & & i SKS & 07 & 20.00 & & I SJG & 110 & 35 & e(Pdif & $f 58$ \\
\hline DRI & 85.54 & 325 & eP & 56 & 38.50 & 1.1 & 1 & $20 s$ & 42 & $2.55 \mathrm{um}$ & \\
\hline RDP & 85.56 & 328 & eP & 56 & 37.00 & -0.6 & I BNG & 113.82 & 303 & ePdiff & $f 59$ \\
\hline & & & e & $\theta 6$ & 36 & & LGN & 8 & 4 & eP & 02 \\
\hline SGO & 5.57 & 326 & $e(P)$ & 56 & 10 & -0. & 1 & 30 & 225 & if & f59 \\
\hline$R$ & 5.09 & 337 & e & 56 & 0 & $\theta$ & 1 & 5 & 32 & ef & f59 \\
\hline FRF & .12 & 333 & 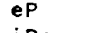 & 56 & 40.20 & -0 & 30 & 57 & 44 & eP & 02 \\
\hline CVF & .25 & 331 & iPc & 56 & 40.30 & -0.7 & I & 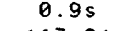 & 83 & $3.30 \mathrm{~nm}$ & \\
\hline & $1.3 \mathrm{~s}$ & 140 & $0.00 \mathrm{~nm}$ & & &.$\theta m b$ & $\mathrm{CH}$ & 117.24 & 51 & ePKP & 02 \\
\hline CAF & 86.28 & 337 & eP & 56 & 42.20 & 1.0 & BOG & 38 & 50 & ePKP & 03 \\
\hline CDR & 86.30 & 334 & ePc & 56 & 41.30 & 0.1 & PSO & 11 & 55 & KP & 02 \\
\hline & & & e & 56 & 42.00 & & MTD & 120.51 & 276 & $K P$ & $\theta 2$ \\
\hline & & & $i$ & 6 & 43. & & 1 & & & ipPKP & 03 \\
\hline & & & $\mathrm{i}$ & 6 & 52. & & 1 & & & iPP & 4 \\
\hline & & & $\mathrm{i}$ & 7 & 10 & & GUV & 3 & 57 & KP & 02 \\
\hline & & & e & 07 & $\theta$ & & KR I & 95 & 278 & 2 & 2 \\
\hline RG & 6.30 & 333 & $\mathrm{i}$ & 56 & $\theta$ & $\theta$ & | & & & $K P$ & 3 \\
\hline LMR & .36 & 333 & iP & 56 & $\theta$ & $\theta$ & 1 & & & $P$ & 4 \\
\hline$H L W$ & 6.44 & 309 & iPc & 5 & 4.00 & 1.9 & SBA & 122.31 & 175 & ePKP & 2 \\
\hline & & & e & 07 & 10 & & & & & $S$ & 4 \\
\hline $2+1$ & .65 & 337 & e & 56 & 80 & $\theta$. & $\mathrm{K} \backslash \mathrm{C}$ & 124.06 & 327 & PKP & $\theta 2$ \\
\hline & 86.72 & 181 & eP & 6 & $\theta$ & 3.1 & | & & & e & 03 \\
\hline & & & e & 57 & 10.00 & & 1 & & & e & 04 \\
\hline & & & e & 07 & 06.00 & & & & & e & 12 \\
\hline & & & e & 07 & 20.00 & & BUL & 124.83 & 275 & ePKP & 02 \\
\hline $\begin{array}{l}\text { LPO } \\
\text { STJ }\end{array}$ & $\begin{array}{l}6.75 \\
6.78\end{array}$ & 337 & eP & 6 & 4.20 & 0.8 & & & & ipPKP & 03 \\
\hline STJ & $\begin{array}{l}86.78 \\
1.0 s\end{array}$ & 14 & eP & 6 & 43.50 & 0.0 & 1 & & & iPP & 05 \\
\hline & $1.0 \mathrm{~s}$ & 344 & $4.00 \mathrm{~nm}$ & & & $.5 \mathrm{mb}$ & I MAW & 128.25 & 209 & ePKP & 03 \\
\hline SCP & 86.79 & 41 & eP & 56 & 43.20 & -0.6 & I EVA & 128.44 & 269 & ePdiff & 100 \\
\hline & $1.0 \mathrm{~s}$ & 180 & 0.00 & & 6 & $.2 \mathrm{mb}$ & SL & 128.55 & 270 & e(Pdi) & 100 \\
\hline & & & i & 56 & 57.00 & & NNA & 129.86 & 64 & ePKP & 03 \\
\hline in & 9 & 31 & ep & 56 & 70 & $\theta$ & I & $0.5 \mathrm{~s}$ & & $2.25 \mathrm{~nm}$ & \\
\hline$A V$ & .55 & 37 & eP & 56 & 7.50 & $\theta$ & 1 & & & e & 05 \\
\hline LA & 87.80 & 37 & $P$ & 56 & 49.50 & $\theta$. & BFS & 130.32 & 270 & ePKP & 93 \\
\hline MNG & 87.94 & 160 & $P$ & 56 & 49.70 & 0.8 & 1 & $1.0 \mathrm{~s}$ & & $3.00 \mathrm{~nm}$ & \\
\hline & & & $(p P)$ & 57 & 53.80 & $262 \mathrm{kmx}$ & SEK & 130.55 & 268 & IPKPd & 83 \\
\hline & & & $i$ & 58 & 13.90 & & I & $0.8 \mathrm{~s}$ & 33 & $3.58 \mathrm{~nm}$ & \\
\hline 18 & 88.29 & 325 & $e(P)$ & 57 & 03.50 & $12.4 x$ & I HUA & 130.88 & 63 & IPKPd & 03 \\
\hline LS & 88.37 & 336 & eP & 56 & .70 & 0.4 & I & & & is & 06 \\
\hline NEL & 88.39 & 160 & $P$ & 7 & 00.80 & $9.8 x$ & SPA & 134.00 & 180 & ePKP & 03 \\
\hline & $22 \mathrm{~s}$ & 171 & $1.85 \mathrm{c}$ & & & & 1 & $1.0 \mathrm{~s}$ & & 5. & \\
\hline & $22 \mathrm{~s}$ & & .78 um & & & & 1 & $20 \mathrm{~s}$ & & .21 um & \\
\hline & & & $S$ & 07 & 26.00 & & GRM & 134.05 & 263 & e (PKP) & 03 \\
\hline EPF & 51 & 337 & $i P c$ & 56 & 52.20 & 0.2 & 1 & $0.7 \mathrm{~s}$ & 34 & $7.25 \mathrm{~nm}$ & \\
\hline & $1.3 s$ & 86 & $5.60 \mathrm{~nm}$ & & & $.9 \mathrm{mb}$ & 1 & $22 \mathrm{~s}$ & & $3.07 \mathrm{um}$ & \\
\hline PRM & 89.54 & 40 & eP & 56 & 56.90 & -0.1 & I LPB & 138.83 & 60 & PKP & 03 \\
\hline & & & e & 57 & 10.30 & & 1 & $23 \mathrm{~s}$ & & 5.23 um & \\
\hline LGR & 89.82 & 339 & eP & 56 & 55.00 & $-3.2 x$ & 1 & & & i & 03 \\
\hline & & & $\mathrm{i}$ & 57 & 11.50 & & ITR & 144.24 & 11 & ePKP & 03 \\
\hline & & & iPP & 00 & 000 & & & & & e & 03 \\
\hline & & & $i$ & 67 & .00 & & & & & e & 83 \\
\hline$z$ & .21 & 66 & eP & 7 & 70 & 3. $2 x$ & 1 & & & e & 93 \\
\hline$B R$ & .51 & 336 & eP & & 00 & -0.3 & SOB1 & 144.27 & 15 & ePKP & 03 \\
\hline IC & .52 & 60 & $P$ & & & 1. & & & & e & 13 \\
\hline$x_{N}$ & .60 & 61 & $P$ & & & s & & & & e & 3 \\
\hline$A C$ & 8 & 61 & iP & & & .9 & & & & e & 03 \\
\hline TPA & .23 & 61 & ip & & & .2 & JA & .64 & 63 & & 03 \\
\hline 111 & 2 & 62 & ef & & .00 & 1.3 & HJA & .45 & 64 & $\mathrm{Pc}$ & 03 \\
\hline PTO & 2.52 & 343 & ePKF & 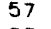 & 09.50 & -1.1 & NVL & 145.88 & 204 & EPKP & 03 \\
\hline TOL & 92.64 & 339 & iPt & 57 & 11.00 & -0.2 & 1 & $21 \mathrm{~s}$ & & $.00 \mathrm{um}$ & \\
\hline & & & $i$ & 57 & 26.00 & & & $21 \mathrm{~s}$ & & um & \\
\hline & & & $\mathrm{i}$ & 58 & 50.00 & & I & $21 s$ & & 3.00 um & \\
\hline & & & iPP & 01 & 00.00 & & TLL & 146.27 & 79 & IPKP & 03 \\
\hline & & & iSKS & 08 & 92.00 & & SLA & 146.45 & 66 & IPKPd & 03 \\
\hline & & & is & 08 & 16.00 & & VCA & 147.24 & 74 & EPKPC & 03 \\
\hline & & & i( & 09 & 50.00 & & $\mathrm{ROCH}$ & 147.67 & 83 & iPKP & 03 \\
\hline & & & is & 14 & 44.00 & & $J A C H$ & 147.82 & 82 & iPKP & 03 \\
\hline & & & is & 18 & 35.00 & & LNV & 147.89 & 85 & EPKP & 03 \\
\hline २० & 7 & 289 & iPt & 57 & 14.30 & 2.2 & PEL & 148.00 & 83 & ¡PKPC & 03 \\
\hline L 1 & 8 & 36 & iPt & 57 & 15.00 & 1.8 & SAN & 148.17 & 84 & iPKP & 03 \\
\hline & & & iPP & 01 & & & $\mathrm{BACH}$ & 148.24 & 83 & ePKP & 03 \\
\hline 70 & 3 & $6 \theta$ & iP & 57 & .00 & 3. $4 x$ & BA & 148.40 & 31 & IPKPC & 03 \\
\hline LM & 95.04 & 337 & eP & 57 & 30 & $5.6 x$ & RTCB & 44 & 79 & ePKPC & 03 \\
\hline
\end{tabular}


Table 4-215

\section{Explanation of Entries in This Table}

Hypocentral coordinates are determined by a modified Geiger's method and may be constrained by reported first arriving P-waves, Pdiff, and the DF branch of PKP. Data are corrected for station elevation and for the ellipticity of the Earth. Outliers may be truncated (ie., removed from the calculation) either automatically or manually. The solution is allowed to converge between rounds of automatic truncation to insure a unique result. Convergence is aided by step length damping.

The formal standard errors of the computed hypocentral coordinates (Flinn, 1965) are based on the assumption of independent normally distributed random errors in the raw data, and tend to be underestimates. Care should be exercised in interpreting these numbers in terms of absolute location accuracy because of unmodeled biases. Analysis of events with independently known coordinates indicates that most PDE determinations are accurate to a few tenths of a degree in epicentral position and $25 \mathrm{~km}$ in depth. For special studies, we urge that inquiry be made to this office for possible recomputation of hypocenters of interest, using more complete instrumental data.

Restricted focal depths occur in four instances. If at any point in the computation the depth becomes negative, the solution is automatically restricted at $33 \mathrm{~km}$ and indicated by "NORMAL DEPTH". If the unrestricted depth computation is unsatisfactory, and in the judgment of the reviewing geophysicist the earthquake probably has a shallow focus, a solution may be held at $33 \mathrm{~km}$. These are also indicated by "NORMAL DEPTH". The geophysicist may restrain the depth at any value indicated by evidence from available seismograms. These are indicated by, for example, "DEPTH $=100 \mathrm{KM}$ (GEOPHYSICIST)". If two or more $\mathrm{pP}$ phases are identified, and in general, yield depths within $10 \mathrm{~km}$ of the mean, then the depth is automatically restricted to this value and denoted by, for example, "DEPTH $=51 \mathrm{KM}$ (5 DEPTH PHASES)" . pP phases may also appear as unidentified second arrivals with associated travel-time residuals. Hypocentral coordinates derived from other sources, such as the California Institute of Technology, the University of California at Berkeley, and ERDA are noted on the EDR.

Two types of magnitude are computed: body-wave magnitude $\left(m_{\mathrm{b}}\right)$ and surface-wave magnitude $\left(\mathrm{M}_{\mathrm{SZ}}\right)$. Each is a $25 \%$ trimmed mean of individual station values. Station magnitudes not used in the trimmed mean are marked with an $\mathrm{X}$. This includes station magnitudes of either type which deviate significantly from the mean and surface-wave magnitudes determined from horizontal amplitudes. Body-wave magnitudes are computed according to the formula $\log (A / T)+Q$, derived by Gutenberg and Richter (1956), where $A$ is the Pwave amplitude in micrometers, $T$ is the period in seconds, and $Q$ is the depth-distance factor. Surface-wave magnitudes are computed from the formula $\log (A / T)+1.66 \log (\Delta)+3.3$, where $A$ is the maximum vertical surface-wave amplitude in micrometers, $T$ is the period in seconds, and $\Delta$ is the epicentral distance in degrees. Surface-wave magnitudes are determined only for earthquakes whose focal depths (taking into account the computed standard deviations) are potentially less than $50 \mathrm{~km}$, for stations having $20^{\circ} \leq \Delta \leq 160^{\circ}$, and for reported periods of $18 \leq T \leq 22 \mathrm{~s}$. No correction for focal depth is used in the $\mathrm{M}_{S}$ calculation. Body-wave magnitudes are not determined from PKP arrivals or for stations having $\Delta \leq 5^{\circ}$. Amplitude values stated in this report are in nanometers $(\mathrm{nm})$ for body-waves and micrometers $(\mu \mathrm{m})$ for surface-waves.

The travel-time residual (observed - computed) is based on the 1940 Jeffreys-Bullen P and 1968 Bolt PKP travel-time tables. Phases not used in the computation are marked by an X. The azimuth from the epicenter to the station is measured clockwise from north. The epicentral distance is the central angle in degrees.

\section{Hypocenter Symbols}

\& Indicates that parameters of the hypocenter were supplied or determined by a computational procedure not normally used by the National Earthquake Information Service (NEIS). The source or nature of the determination is indicated by a 2 to 5 letter code enclosed by angle brackets and appearing in the first line of comments. A "-P" appended to the code indicates that the computation is preliminary. These codes are included with the list of abbreviations in the PDE Monthly Listing.

Note: On printers available to the NEIS for this publication, the symbol for degrees $\left({ }^{\circ}\right)$ appears as "" . 
Table 4-216

\section{References}

Bolt, Bruce A. (1968), Estimation of PKP Travel Times, Bull. Seis. Soc. Am., 58, pp. 1305-1324.

Flinn, E. A. (1965), Confidence Regions and Error Determination for Seismic Event Locations, Rev. Geophys., 3, no. 1, pp. 157-185.

Gutenberg, B. and C. F. Richter (1956), Magnitude and Energy of Earthquakes, Ann. di Geofisica, 9, no. 1, pp. 1-15.

Jeffreys, Harold and K. E. Bullen (1940), Seismological Tables, British Assoc. for the Advancement of Science, Gray Milne Trust. 